\title{
Electric Sales and Revenue
}

\section{3}

\section{January 1995}

\section{ENEROY INFORMATION ADMINISTRATION}

Office of Coal, Nuclear, Electric and Alternate Fuels

U.S. Department of Energy

Washington, DC $205 \mathrm{B5}$ 


\section{Contacts}

Questions regarding the contents of this document may be directed to:

Survey Managentent Division

Energy Information Administration, EI-52

U.S. Department of Energy

1000 Independence Avenue, \$.W.

Washington, DC 20585

Fax phone number (202) 254-5765

Questions of a gencral nature may be directed to:

Howard Walton (202/254-5500),

e-Mail HWALTONOLIA.DOE.GOV

Director of the Survey Management Division;
Neal Moerschel $(202 \sqrt{254-5640) \text {, }}$

e-Nai NMOLIRSCI@ELDOE.GOV

Chief of the Etectric Data Systems Branch;

Specific information on sales, revenue, and average revenue may be directed to:

Linda M. Bromley (202/254-5653),

e-Mail: LBROMLEY@LLDOE.GOV

or

Channele M. Donalit (202/254-8571), e-Mail: CDONALD@EIA.DOI.GOV 


\section{DISCLAIMER}

This report was prepared as an account of work sponsored by an agency of the United States Government. Neither the United States Government nor any agency thereof, nor any of their employees, make any warranty, express or Implled, or assurnes any legal liability or responsibility for the accuracy, completeness, or usefulness of any information, apparatus, product, or process disclosed, or represents that its use would not infringe privately owned rights. Reference herein to any specific commercial product, process, or service by trade narne, trademark, manufacturer, or otherwise does not necessarily constitute or imply its endorsement, recommendation, or favoring by the United States Government or any agency thereof. The views and opinions of authors expressed herein do not necessarily state or reflect those of the United States Government or any agency thereof. 


\section{DISCLAIMER}

Portions of this document may be illegible in electronic image products. Images are produced from the best available original document. 


\section{Preface}

The Eleciric Sales and Reveme is prepared by the Survey Mfanagement Division, Office of Coal, Nuclear, Elextric and Alternate Fucls; Encrgy Information Administration (EIA); U.S. Department of Energy. This publication provides infomation about sales of electricity, its associated reventte, and the average revenue per kilowatthour sold to residential, commercial, industrial, and other consumers throughout the United States.

The sales, revenue, and average revenue per kilowatthour data provided in the Electirc Sales and Reventoe are based on annual data reported by electric utilities for the cakendar year encling December 31, 1993. Operating revenue includes energy charges, demand charges, consumer service charges, enviromental surcharges, tuei adjustments, and other misecilantsous charges. The revenue does not include taxes, such as sales and excise taxes, that ars assessed on the consumer and collected through the utility. Average revente per kilowatthour is dicfined as the cost per unit of electricity sold and is calculated by dividing retail sales into the associated clectric nevenuc. Because cicctriv rates vary bisxd on enctuy usage, average nevenuw per kilowatthour are affected by changes in the volume of sales. The sales of electricity, associated revenue, and average revenue per kilowatthour data provided in this report are presented at the national, Census division, State, and electric utility levels.

The electric power industry is experiencing the emer. acnos of a more competitive, market-driven approach that will likely result in considerable change in the industry over the next several years. The industry change will be reflected in the sales, reventhe, and avctage reverus per kilowatthour data presented in this publication. The Energy Policy Act of 1992 (El'ACT) removed some constraints on ownership of eleetric generntion facilities and encouraged incrensed competition in the wholesale clectric power business. An incrcased interest in peffomance-based ratemaking and retail wheeling, as well as cuts in common dividends and employec layofis, all point to an increasingly more competitive electric power industry. Presently, electric rates are based on embedded historical costs. Future electric ratcs are likely to be more dynamic and reflec- tive of the current cost of providing service. Unbundling of electric power services and prices that reflect time-of-day and seasonal variations will becone more common with the increased interaction of the consumer with the utility.

\section{Target Audience}

In the private sector, users of the Electric Sales and Revemie include researchors, analysts, and ultimately the policy- and decision-making members of electric entility companies. Other users include financial and investment institutions, economic development organizations, special interest groups, lobbyists, electric power associations, and the news media.

In the public setor, the usets include analysts, researchers, statisticians, and other professionals ctroaged in regulatory, policy, and program areas for Federal, State, and local governments. The Congress, other leg. islative bodies, public service commissions, and other special government groups are also interssted in general trerds related to cloctricity at Strate and national levels Data int this report can be ussed in analytic studies to evaluate now legisiation and regulatory atternatives, and to forecas future demand for electric powor.

\section{Coverage of Sources}

Data published in the Electric Sales and Reventse are compiled from the Form EIA-\$61, "Annual lelectric Uitijty Report." The Form EIA-861 is a census of electric vitilities in the United States, its territories, and Puerto Rico. The form collects infomation on the production and sale of electricity $5 \mathrm{rom}$ approximately 3,200 elcatric utilities (Figure I).The form is described in more detial in Appendix B, "Tecknical Notes. "Cop. ies of the form and its instructions may be obtained fron the National Energy Information Center (NE1C).

Data for the four power authorities of American Samoa, Guan, Puerto Rico, and the Virgin islands ate included in Table 14 through Table 17, but not in the national, Census division, and State level statistics. 



\section{Contents}

Proftle $\ldots \ldots \ldots \ldots \ldots \ldots \ldots \ldots \ldots+\ldots \ldots \ldots+\ldots \ldots \ldots \ldots \ldots \ldots \ldots \ldots \ldots \ldots \ldots \ldots \ldots \ldots+\ldots \ldots \ldots, \quad 1$

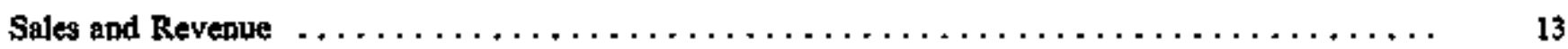

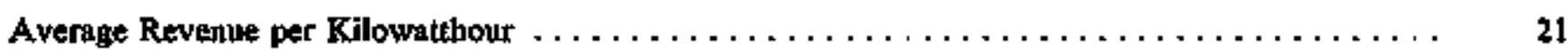

Appendices

A. Electric Utilities Serving Ultimate Consumers in More Than One State $\ldots \ldots \ldots \ldots \ldots .213$

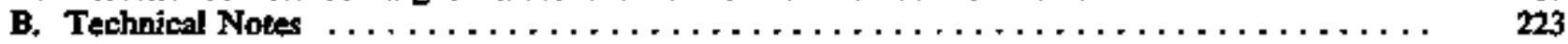

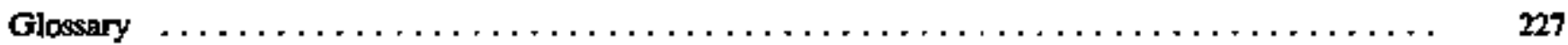


1. U.S. Sales to Ultimate Consumers and Assochated Revenue by Sector, $1993 \ldots \ldots \ldots \ldots$.

2 U.S. Average Revenue per Kilowatehour by Class of Ownership and by Sector, 1993 . . . .

U.S. Sales to Ultimate Consumers and Assoctated Revenue by Sector, 1989 Through 1993 . .

U.S. Sales to Ultimate Consumers and Associated Revenue by Class of Ownership, 1989

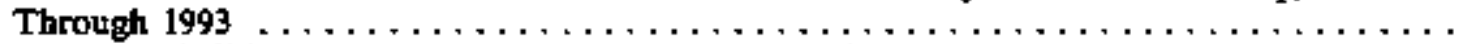

5. Number of Ultimate Consumers by Sector, Census Division, and State, $1993 \ldots \ldots \ldots \ldots$.

6. Sales to Ultimate Consumers by Sector, Census Division, and State, $1993 \ldots \ldots \ldots \ldots \ldots$

7. Rovente from Sales to Ultimate Consumers by Sector, Censes Division, and State, 1993 .. .

8. Number of Ultimate Consumers by Class of Ownership, Census Division, and State, 1993 ..

9. Sales to Ultimate Consumers by Class of Ownership, Census Division, and State, 1993 ....

10. Revenue from Sales to Ultimate Consumers by Class of Ownership, Census Division, and

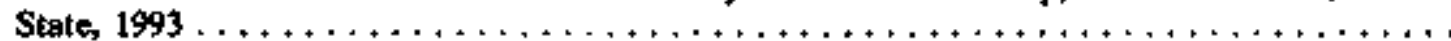

11. U.S. Average Revenue per Kilowatthour by Sector and Class of Owhership, 1989 Through

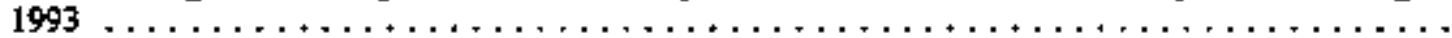

12. Average Revenue per Kilowatthour by Sector, Census Division, and State, $1993 \ldots \ldots \ldots$.

13. Average Revenue per Kilowatthour by Class of Ownership, Census Division, and State,

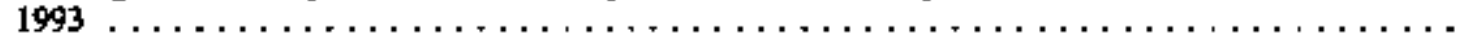

14. Class of Ownership, Number of Ultimate Consumers, Revente, Sales, and Average Revenue per Kilowatthour for the Residential Sector by State and Utitity, $1993 \ldots \ldots \ldots \ldots \ldots \ldots$

15. Class of Ownership, Number of Ultimate Consumers, Revenue, Sales, and Average Revenue per Kilowatthour for the Commercial Sector by State and Utility, $1993 \ldots \ldots \ldots \ldots \ldots$.

16. Class of Ownership, Number of Ultimate Consumers, Revenue, Sales, and Average Revenue per Kilowatthour for the Industrial Sector by State and Utility, $1993 \ldots \ldots \ldots \ldots \ldots$.

17. Class of Ownership, Number of Ultimate Consumers, Revenue, Sales, and Average Revenue per Kilowatthour for All Sectors by \$tate and Utility, $1993 \ldots \ldots \ldots \ldots \ldots \ldots$

A1. Electric Utilities Serving Ultimate Consumers in More Than One State, $1993 \ldots \ldots \ldots \ldots$. B1. Unit-of-Measure Equivalents for Electricity $\ldots \ldots \ldots \ldots \ldots \ldots \ldots \ldots \ldots \ldots$

\section{Illustrations}

1. Number of Electric Utilities by Class of Ownership, $1993 \ldots \ldots \ldots \ldots \ldots \ldots \ldots \ldots$

Number of Ultimate Consumers Served by Sector, $1993 \ldots \ldots \ldots \ldots \ldots \ldots \ldots$ Number of Ultimate Consumers Served by Class of Ownership, $1993 \ldots \ldots \ldots \ldots \ldots$. Share of Ultimate Consumers Served by Sector and Class of Ownership, $1993 \ldots \ldots \ldots \ldots$ Sales to Ultimate Consumers by Sector, $1993 \ldots \ldots \ldots \ldots \ldots \ldots \ldots \ldots \ldots \ldots$ Revenue from Sales to Ultimate Consumers by Sector, $1993 \ldots \ldots \ldots \ldots \ldots \ldots \ldots$ Share of Sales to Ultimate Consumers and Associated Revenue by Sector, 1993 . . . . . . . Average Revenue per Kilowatthour by Sector, $1993 \ldots \ldots \ldots \ldots \ldots \ldots \ldots$ Sales to Ultimate Consumers by Class of Owreerstup, $1993 \ldots \ldots \ldots \ldots \ldots \ldots \ldots$

11. Share of Sales to Ultimate Consumers and Associated Revenue by Class of Ownership 1993 Average Revenue per Kilowhtthour by Class of Ownership, $1993 \ldots \ldots \ldots \ldots \ldots \ldots \ldots \ldots, 11$

13. Average Revenue per Kilowatthour for the Residential Sector by State, 1993 . . . . . . . .

14. Avernge Revenue per Kilowatthour for the Commercial Sector by State, $1993 \ldots \ldots \ldots \ldots$

15. Average Revenue per Kilowatthour for the Industrial Sector by \$tate, $1993 \ldots \ldots \ldots \ldots$ 


\section{Profile}

This chapter contains two sections. The "Background" section provides a discussion on (1) sales of electricity, its assoclated revenue, and the average revenue per kilowatthour sold to ultimate consumers, (2) the classes of electric utility ownership, and (3) the classes of service or seetors. The "Year at a Glance" section provides a summary of pertinent statistice related to sales of eleotricity, associated revenne, and average revenue per kilowatthour.

\section{Background}

Sales, Retenue, and Average Rerenue per Kilowatthow. Electric power is an integral part of our way of life. It is indispensabje to the factory, the commercial establishment, the home, and even most recreational facilities. The unavailability of electric power causes inconvenience to customers as well as economic loss due to reduced industrial production. Electric utilities are responsible for ensuring an adequate and reliable source of electricity to all consumes in their service territories at a reasonable cost.

Electric utilities are regulated by local, State, and Federal authorities. Generally, interstate activities (those that cross State lines) are snbject to Federal regulation, while intrastate activities are subject to State regulation. Wholesalo rates (sales and purchases among telectric utilities), licensing of hydroelectric facilitiés, questions of nuclear safety and high-level nuclear waste disposal, and environmental regulation are federally mandated. Approval for most plant and transmission line construction and retail rate levels are State regulatory iunctions.

The primary electritity jurisdiction of the State utility commissions tocludes the almost $\mathbf{3 0 0}$ generally lacge, vertically integrated, investor-owned utilities who own over 75 percent of the nation's generating and transmission capactity and serve over 75 percent of the load. There are over 2,000 publicly owned utilities serving almost 15 percent of the koad and approximately 950 consumer-owned rural cooperatives. Approximately 20 States regulate cooperatives and 7 States regulate municipal utilities. Many State legistatures leave it to the local municipal officials or cooperative members to provide control.

The service territory of an electric atility is geographically distinct and is usually composed of many differ. ent combinations of consumers. Electric utilities classify their consumers by different factors, shch as level of electrical demand, rate schedule, Standard Industrial Classification (SIC) code, distribution voltage, accounting methods, end-use applications, and other social and economic characteristics. Electric ntilities use consumer classifications for planning (logd growth, peak demands), and for determining their sales and revenue requirements (cost of service) in order to derive their rates.

Average revenue per kilowatthour is defined as the cost per unit of electricity sold and is calculated by dividing retail electric revenue by the corresponding sales of ejectricity. The average revenue per kilowatthour is calculated for all consumers and for each sector (residential, commercial, industrial, and other sales).

Utilities typically employ a number of rate schedules within a single sector. These alternative rate schedules reflect the varying consumption levels and patterns of these consumers and their associated impact on the utility's costs of providing electrical service. The average revenue per kilowatthour reported in this publication by sector represents a weighted average of elis. tomer revenue and sales within that sector and across sectors for all consumers.

The electric revenue used to derive the average revenue per kilowatthour is the operating revenue roported by the utility. Operating revenue includes energy charges, demand charges, consumer service charges, environmental surcharges, fuel adjustments, and other miscellaneous charges.

Utility operating revenoes include, amoons ofher costs of service, State and Federal income taxes and caxes other than income taxes paid by the utility. The Federal component of these traxes are, for the most part, "payroll" taxes. State and lockal authortites thx the value of plant (property taxes), the amount of revenues (pross receipts taxes), purchases of materigls and services (sales and use taxes), and a potentially long list of other tanes that vary extensively by taxing anthority. Taxes deducted from empolyees' pay, such as Federail income taxes and employees' share of social security taxes are bot a part of the utility's "thx costs," but are paid to the taxting anthorities in the name of the employees. These taxes are included in the utility's cost of gervice (j.e., revenue exquirements) and are included in the 
amounts recovered from the customer in rates and reported in operating revenues.

Electric utilities, like other business enterprises, are required by various taxing authorities to collect and remit taxes assessed on its customers. In this regard, the utility serves as an agent for the taxing authority. Taxes assosied on the consumer, such as a gross receipts tax or sales tax, are referred to as "pass through" taxes. These taxes do pot represent a cost to the utilty and are not recorded in the operating revenues of the utility. However, taxing authorities differ in whether a specific tax is assessed to the utility or to the consumer, which in turn determines whether of not the tax is included in the electric utility's operating revenues.

Average revenue per kilowathour is affected by changes in the rate scheduies used by the electicic utilities and by changes in the volume of electrictty sales. Because fixed charges remain constant regardless of the volume of sales, average revenue per kilowatthonr will decreage as the volume of sales increases. A change in average reventue per kilowatthour may occur whert the volume of electricity saies changes (because of an increase/decrease in the use of electricity by the individual consumer or an increase/decrease in the number of ultimate consumers) across all stetors or within a specific sector. Gencrally, the rate schedules used by electric utilities are designed so that revenue increases more slowly as the volume of sales increases, lowering the average revenue per kilowatthour. The net effect of these factors on the average revenue per kilowatthour presented in this pubtication has not been determined. Average revenue per kilowatthour is in part impacted by class of utility ownership and by class of service (sector). Consequently, data are pre. sented in this publication by class of ownership and sector.

Qlasses of Omership. The electric power industry in the United States is a combination of approximately 3,200 investor-owned, publicly owned, Federal, and cooperative electric utilities. Historically, investorowned electric utilities have been most successful in serving large, consolidated markets where economiesof-scale afforded the lowest prices. However, publicly owned, Federal, and cooperative electric utilities all have a role in producing, transmitting, and distributing electricity.

Like all private businesses, the fundamental objective of an investor-owned utility is to produce a return for its investors. The investor-owned electric utility either distributes its profits to stockholders as dividends or reirvests these profits. It is granted a service monopoly in certain geographic areas and is obliged to serve all consumers. As franchised monopolies, these utilities are regulated and required to charge reasonable prices, to charge comparable prices to similar classifications of consumers, and to give consumers access to services under similat conditions. Most investor-owned electric utilities are operating companies that provide besic ser- vices for the generation, transmission, or distribution of electricity. The majority of investor-owned utilities perform all three functions. There are approximately 270 investor-owned electric utilities operating in all States except Nebraska. The electric utilities in Nebraska consist primarily of muaicipal systems and public power districts. Investor-owned electric utilities, while representing only 8 percent of the total number of electric utilities, account for more than 75 percent of all electric utility generating capability, generation, sales, and revenue in the United States.

The approximately 2000 publicly owned electric utiljibs represent 62 percent of the number of electric utilities, supply approximately 10 percent of generation and generating capability, and represent approximately 13 percent of the sales and revenuc. Publicly owned electric utifities are gonprofit local government agencies established to provide service to their comnuntties and nearby consumers at cost, returning excess funds to the consumer in the form of community contributions, more economic and efficient facilities, and lower rates. Publicly owned electric utilities mclude municipals, public power districts. State authorities, irrigation districts, and other State organizations. Most municipal ueilities simply distribute power, although some larger ones produce and transmit electricity as well. They obtain their financing from municipal treasuries and from revenue bonds secured by proceeds from the sale of electricity. Public power districts and projects are concentrated in Nebraska (which has no investorowted electric utilities), Washington, Oregon, Arizona, and California. Voters in a public utility district (PџD) elect commissioners or directors to govern the district independent of any municipel government. State authorities, like the Power Authority of the State of New York or the South Carolina Public Service Authority, are agencies of their respective State governments. Irrigation districts may have still other forms of organization. In the Salt River Project, votes for the board of directors are apportioned according to the size of landtioldings.

Cooperative electric utilities comprise approximately 30 percent of the number of electric utilities and represent 8 percent of sates and reveuue and about 4 per. cent of generation and generating capability. Cooperative electric utilities are owned by their members and are established to provide electricity to those members. These electric utilitics operate in rural areas with a lower concentration of consumers because these areas are historically viewed as uneconomical operations for investor-owned electric utitities. They are inoorporated under State law and are usually directed by an elected board of directors, which in turn selects a manager. The Rural Electrification Administration, the National Rural Utilities Cooperative Finance Corporation, the Federal Financing Bank, and the Bank for Cooperatives are the most important sources of debt financing for electric cooperatives. There are approximately 950 electric ooperatives currently doing business in 47 States. Cooperative electric utilities do not 
operate in Conpecticut, Hawaii, and Rhode Island, or in the District of Colnmbia.

The 10 Federal electric utilities in the United States while less than 1 percent of the number of electric utilities, provide almost 10 percent of generating capability and generation, but less than 2 percent of sales to ultimate consumers and the associsted revenue. The Federal electric utilities are the Department of Defense-U.S. Army Corps of Engincess; the Department of Energy: Ataska, Bonneville, Sontheastern, Southwestern, and Western Area Power Administrations; the Department of Interior: U.S. Bureau of Inditan Affairs and the U.S. Burean of Reclamation; the Department of State-International Boundary and Water Commission; and the Tennessee Valley Authority. The Federal electric utilities are primarily generators and wholesalers of electricity rather than distributors to ultimate consumers. Federal electric utilities operate approximately 180 power plants. Most of the power plants are Federal hydroelectric projects initially de. signted for flood control and irrigation purposes. Federal power is not sold for profit, but to recover the cost of operations. Most Federal generation is sold for resale to municipals, cooperative electric utilities, and other nonprofit preference customers, as requised by law. Three Federal agencies operate Federal generating facilities: the Tennessee Valley Authority (TVA), the largest Federal producer; the U.S. Army Corps of Engineers (USCE); and the U.S. Bureau of Reclama. tion (USBR). The TVA markets its own power while the generation by the USCE, except for the North Central Division (St. Mary's Falls at Sanlt St. Marie, Michigan), and the USBR is marketed by four of the Federal power marketing administrations (PMA): Borneville, Southeastern, Southwestern, and Western Area. The four PMA's also purchase energy for resale from other electrito utilities in the United States and Cantada. Alaska, the fffth PMA, operates its own power plants and distributes power to ultitnate consumers. The majority of electricity generated by Federal electric utilities is sold for resale, not to ultimate consumers.

Clusses of Service (Sectors). Electricity consumers are divided into classes of service or sectors (residential, commercial, industrial, and other) based on the type of service they receive. Sectorial classifification of consurmers is determined by each electric utility and is based on different criteria such as demand level, rates, Standard Industrital Classification (SIC) code, accountisi methods, end-use activity and other social and economic characteristics, Reclassification of consumers, usually between the commercial and industrial sectors, may opcur from year-to-year dne to changes in demand level, econonic factors, or other factors. For this reason, the commereial antd inderstrial sectors are discussed collectively as the "nonresidential" sector in some dis- cussions in this publication. The residential sector re. mains relatively stable frord year to year.

The residential sector inclutes electricity supplied to private household stablishments and apartment buildings where energy is consusped primarily for space heating, water heating, air conditioning, lighting, re. frigeration, cooking, and clothes drying.

The commercial sector includes electricity supplied to nonmanufacturing business establishments and sometimes small marufacturing facilities, including hotels, motels, restausants, wholesale businesses, and retall stores and health, social, and educational institutions. An electric utility may classify commercial consumers as those consumers whose demand and/or usage exceds specified limits. The limits are set by the electric utility based on different rate schedules.

The industrial sector includes electricity supplied to manufacturing, construction, mining, agriculture, gishing, and forestry establishruents - Standard Industrtal Classification (SIC) codes $t$ through 39. An electric utility may classify industrial consumers using these SIC codes, or it may classify industrial consumers based on demand and/or nsage exceeding speciłted limits. As in the commercial sector, the limits are set by the electric utility based on different rate schedules.

Sales to other consumers include public street and highway lighting, railroads and railways, municipalities, divisions or agencies of State and Federal Oovernments under special contracts or agreements, and other utility departments, as defined by the pertinent regulatory agency and/or electric utility. Sales to these cousumers, as well as the residential, commercial, and industrial consumers may be classinied differently by each electsic utility because of different rates, demand levels, and the economite activities of the consumers.

\section{The Year at a Glance}

In 1993, total sales of electricity to nltimate consumers were 2,861 billion kilowatthours and totai revenue was $\$ 198$ billion (Table 1). Sales to the residential sector in 1993 were 995 billion kilowathours, representing 35 percept of total sales to ultimate consumers, and revenue was $\$ 83$ billion, representing 42 percent of the total assoctated revente. The nonresidential sector ${ }^{2}$ (i.e., the commercial and industrial sectors) had sales of 1,772 billion kilowatthours, representing 62 percent of sales to ultimate consumers, and $\$ 109$ billion in revenue, representing 55 percent. The average revenue per kilowatthour of electricity sold to ultimate consumers in the United States in 1993 was 6.93 cents (Table 2). Average revenue per kilowatthour in 1993 for the res-

\footnotetext{
Changes for the nnpresidentill sector (nonresidentivl tefers to the combercial and industrial sectors only) are discussed as cantion must be used in unalyzing year to year shanges in the commercial and industrial sectors because electrife ptilities may have tecilassified their consumers between the commercial and indostrial cector.
} 
idential and nonresidential sectors was 8.32 and 6.15 cents, respectively.

Averape Revenue by Sector and Ommership. Because Federal electric utilities have access to relatively lowcost financing and mastly utilize less expetasive bydroelectric facilities, they have the lowest average revenue per kilowathour, at 2.77 cents, of all the ownership classes for all sectors. The average revernue per kilowatthour of publicly owned electric utilities in the residential sector, at 6.63 cents, was 14 percent below that for cooperative electric utilities at 7.73 cents and 24 percent below that of investor-awned electric utilities at 8.77 cents (Table 2). The average revenue per kilowatthour of publicly owned electric ubilities in the nonresidential sectors, fit 5.67 cents, was 2 percent be. low that for cooperative electric utilities at 5.83 cents and 10 percent below that for javestor-owned electric utilities at 6.34 cests.

Sales by Sector. Sales of electricity rose approximately 4 percent in 1993 to 2,861 billion kilowatthours. Sales of electricity to the residential sector increased by 6 percent (59 billion kjlowatthours) in 1993. Sales of electricity to the nonresidential sector increased 2 percent (38 bitlion kilowatthonrs) fn 1993.

Retenue by Sector. The total revenue from sales of electricity increased 5 percent in 1993 to $\$ 198$ billion (Table 3). Revenue increased by 8 percent for the residential sector in 1993 . The average revenue per kilowatchoor for the residential sector increased by 1 percent fiom 8.21 cents per kjowatthour, in 1992, to 8.32 cents per kilowatthour in 1993. Revenue for the noruesidential sector increased by 3 percent in 1993 . The average revenue per kilowatthon for the nonresidential sector increased by I percent from 6.07 cents per kilowatthour, in 1992, to 6.15 cents per kilowatthour in 1993 .

Sales by Ownership. Among the ownership classes, investor-owned electric utilities accounted for 76 percent (2,187 billion kilowatthonrs) of total U.S. sales of electricity in 1993 (Table 4). Publicly owned electric utilities accounted for 14 percent ( 497 billion kilowatthours); electric cooperative utilities accounted for 8 percent (22) billion kjowatthours); and Federal electric utilities accounted for 2 percent (46 billion kilowatthours) of total U.S. sales in 1993. Investor. owned electric utilities reported a 4-percent increase (75 billion kilowatthours) in sales to ułtimate consumers and publicly owned utilities, a 3-peccent increase (12 billion kilowatthours) in 1993, compared with 1992. Sales by cooperative electric utilities tocreased by 7 percent ( 14 billion kilowatthours). Federal electric utiities reported a 5-percent decrease in sales ( 2 billion kilowatthours).

Rerenne by Owmerahip. All ownership classes reported increases in reverue from sales of electricity in 1993, compared with 1992. Publicly owned and cooporatively owned electric utilities each reported increases of 4 percent and 7 percent, respectively. Investorowned electric utilities reported a 5-percent increase in 1993 revenue, white Federal electric utilities reported a less than I-percent torease (Table 4).

Average Revende by Omwership. In 1993, Federal electric utilities continned to have the lowest average revenue per kilowathour at 2.77 cents, an 6-percent increase from 1992 (Table 11). Federal electric utitities generally have lower average revenue per kilowatthour because they are exenpt from income tax, have access to lower interest loans, and generate less expensive hydroclectric power. In addition, many Federal bydroelectric projects are multi-purpose projects, and only a portion of the costs are allocated to electricity.

Inverstor-owned utilities had an average revenue per kllowatthour of 7.16 cents in 1993, a 2-percent increage from 1992 (Table 11). Average revente per kilowatthour for publicly owned electric utilities was 6.09 cents, and for cooperative electric utlities, it was 7.00 cents, both lower than the average revenue per kilowatthour of investor-owned utilities. Publicly owned utilities bave access to low-cost capital and are not allowed to charge a return for profit. Although cooperative electric utilities have access to low-cost loans through the Rural Electrification Admintstration. the bigh average revenue per kilowatthour by this ownership group is at least partly attributable to the ligh cost of operating in remote rural aress having a Jow concentration of consumers and to providing service to a higher percentage of residential customers than other classes of utilities. The lower load factor of residential customers, compared with other sectors (i.e., commercial and industriaf), has an associated higher allocation of demand cost due to a lower utilization of generating equipment. In addition, many cooperatives do not generate electricity, but as full requirement customers of electric utilities with generating facilities, must pay requirement demand charges. 
Tabłe 1. U.S. Sales to Utimate Consumers and Assoclated Revenue by Sector, 1993

\begin{tabular}{|c|c|c|c|c|c|}
\hline Item & Ractionida & Commaralat & Indulutrili & other' & Aft sectos: \\
\hline Seres (m|lon kliomitthouris) - & 694,781 & 794,573 & 87.164 & 04,044 & $2,881,462$ \\
\hline 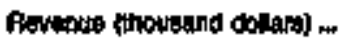 & $02,918,824$ & $61,521,269$ & $47,357,145$ & $6,527,851$ & $198,220,189$ \\
\hline
\end{tabular}

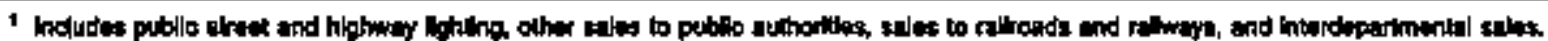

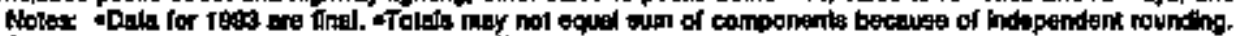

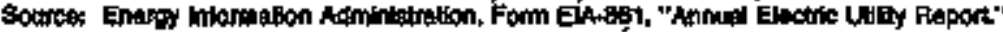

Table 2. U.S. Average Revenese per Kilowatthour by Class of Ownership and by sector, 1993 (Cents)

\begin{tabular}{|c|c|c|c|c|c|}
\hline Iber & Roosidentitial & Commareled & Indurititad & Other' & All Seckore \\
\hline 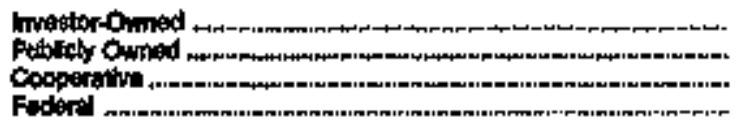 & $\begin{array}{l}8.77 \\
6.63 \\
7.73 \\
6.77\end{array}$ & $\begin{array}{l}7.91 \\
6.77 \\
7.41 \\
6.17\end{array}$ & $\begin{array}{l}4.86 \\
4.85 \\
4.84 \\
2.88\end{array}$ & $\begin{array}{l}7.33 \\
7.10 \\
7.02 \\
1.62\end{array}$ & $\begin{array}{l}7.18 \\
6.00 \\
7.00 \\
2.77\end{array}$ \\
\hline 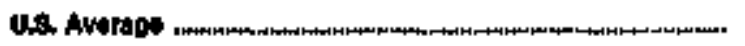 & 8.32 & 7.74 & 485 & fus & 893 \\
\hline
\end{tabular}

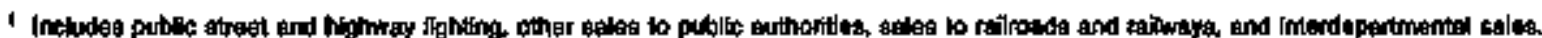

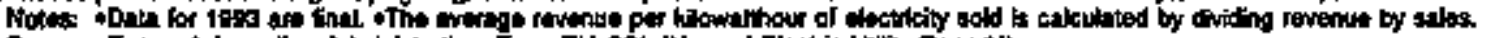

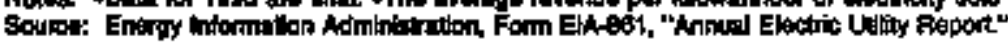


Figure 1. Number of Electrtc Utilities by Class of Ownershlp, 1993

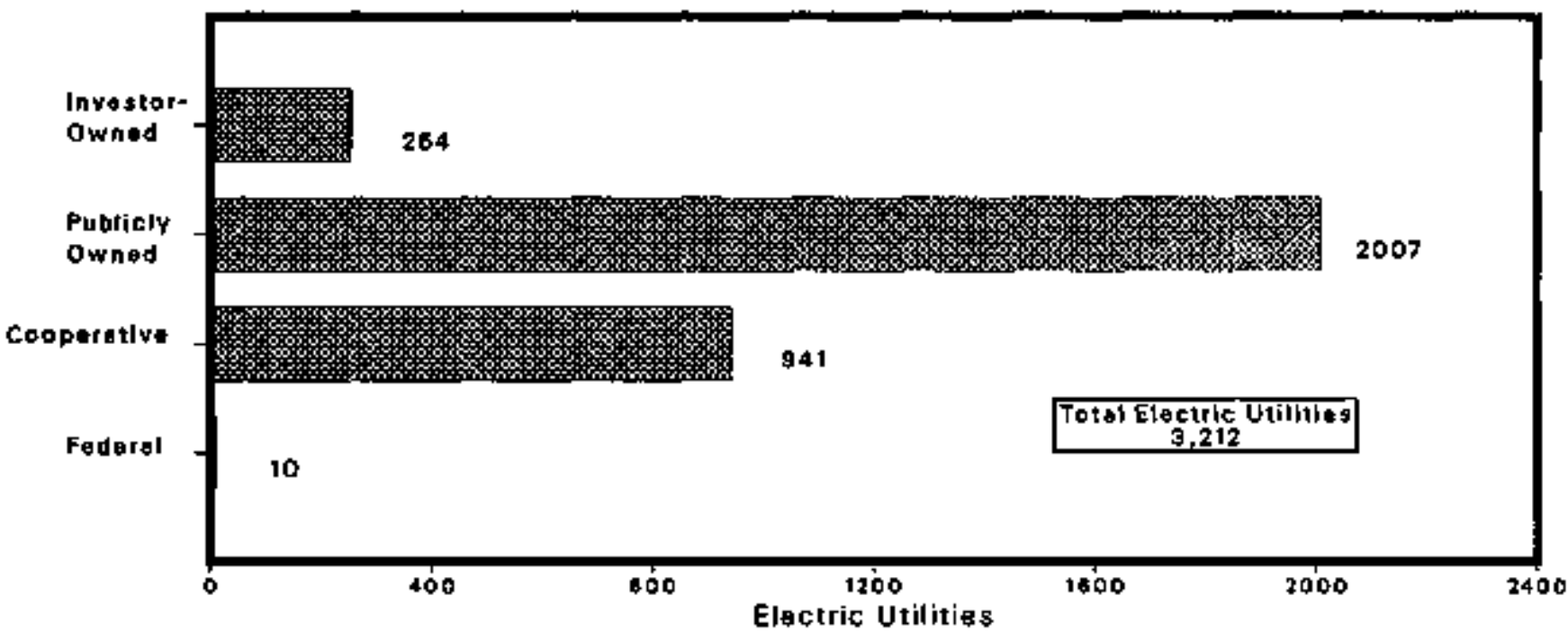

Note: Data for 1993 are final.
Source: Energy Information Administration, form ElA-861, 'Andual Electric Utility Regort.'

Figure 2. Number of Utimate Consumers Served by Sector, 1993

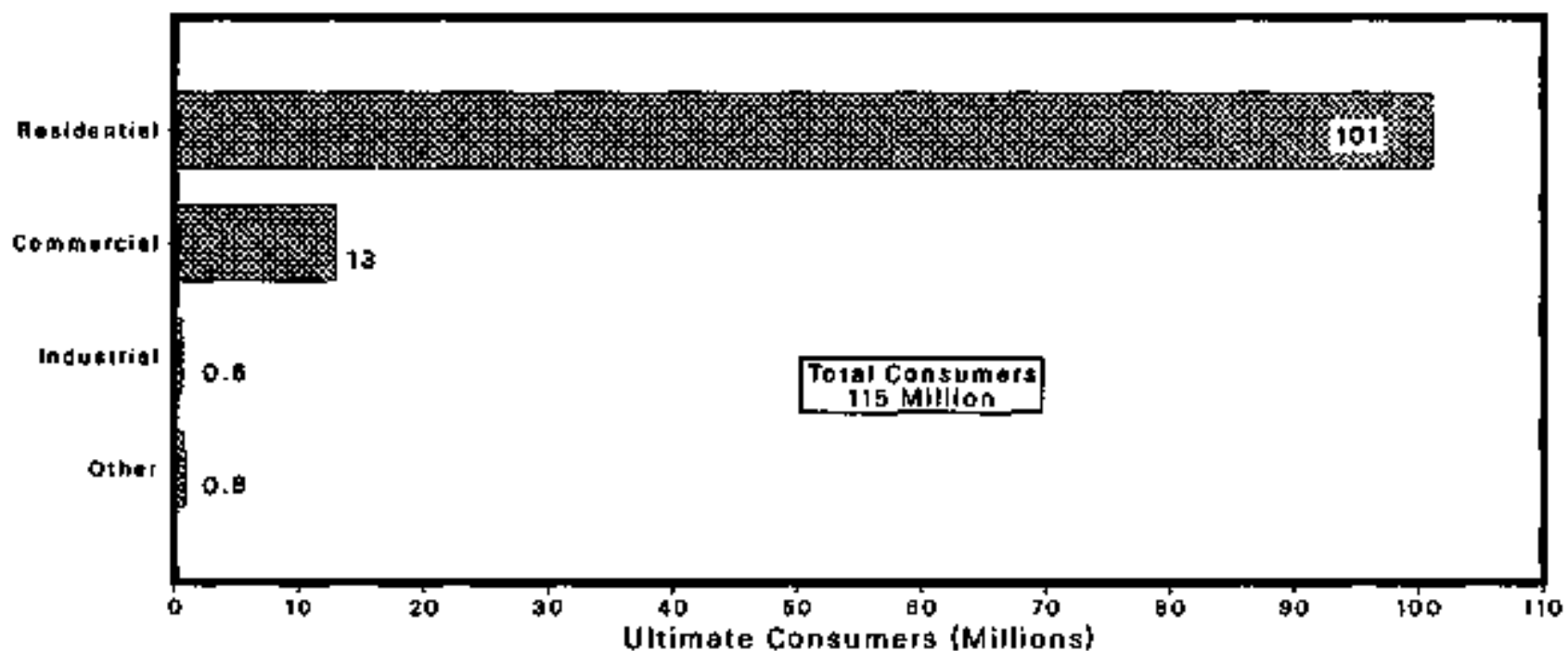

Notes: Data for 1993 are final. Other includes public stret and highway lighting. other sales to public authorities. sales to railrosds and ratiways, end interdepartmenisl sales.

- The number of ultimate consumers is an average of the number of consumers at the close of

each month. Totals may not equal sum of components because of independent rounding.

Source: Energy Information Administration. Form ElA-B61. Annual Electric Utility Report. 
Figute 3. Number of Uitmate Consumers Served by Class of Ownershp, 1993

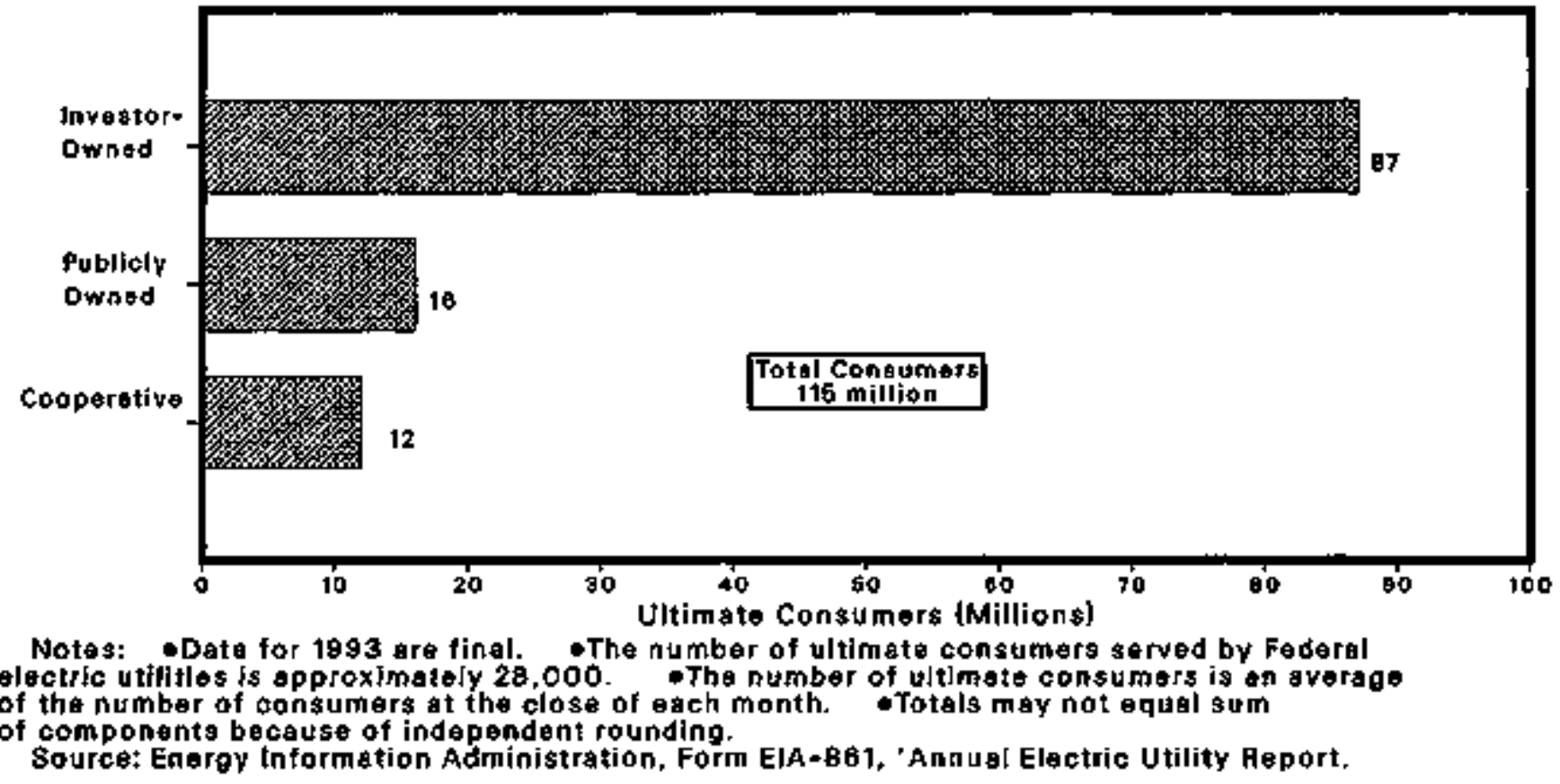

Fipure 4. Share of Ultmate Consumers Served by Sector and Class of Ownership, 1993

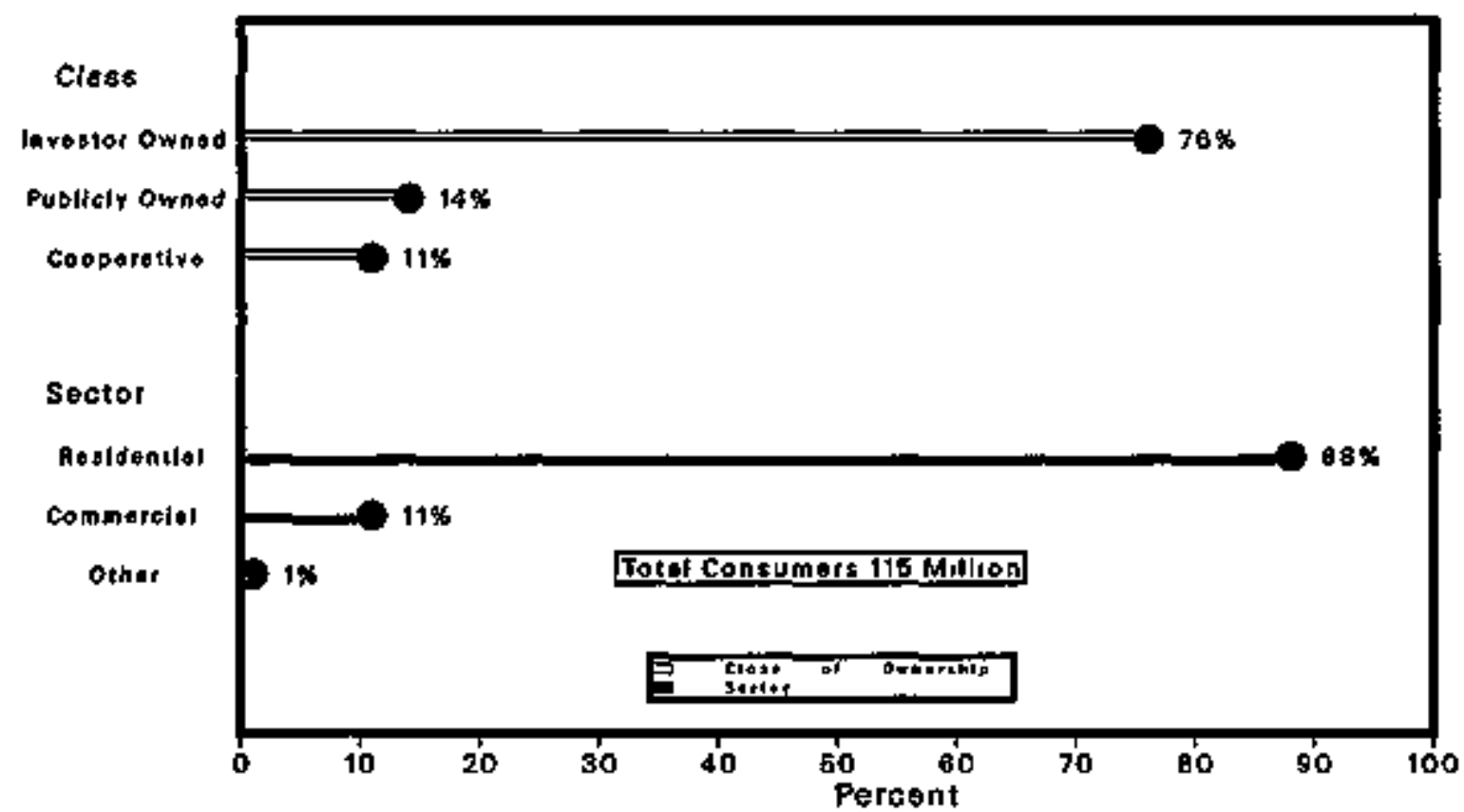

Nates: -Date for 1993 are final. Othar includas oublic strat and highwsy liphting. other actes to public authorities, sales to reilroads and railways, end interdepertmental osles.

- The share of tadustrial consumers is 0.4B percent. the share of uttimete consumars served by Foderal electic uttlities is 0.025 percent. The number of ultimete consumers is an average of the rumber of consumers at the close of tach month. Hotals may not aqual sum components because of independent rounding.

Source: Enerav Information Administration. Form ElA-B61. 'Annual Elactric Utility Renort." 
Figure 6. Sales to Utimate Consumers by Sector, 1993

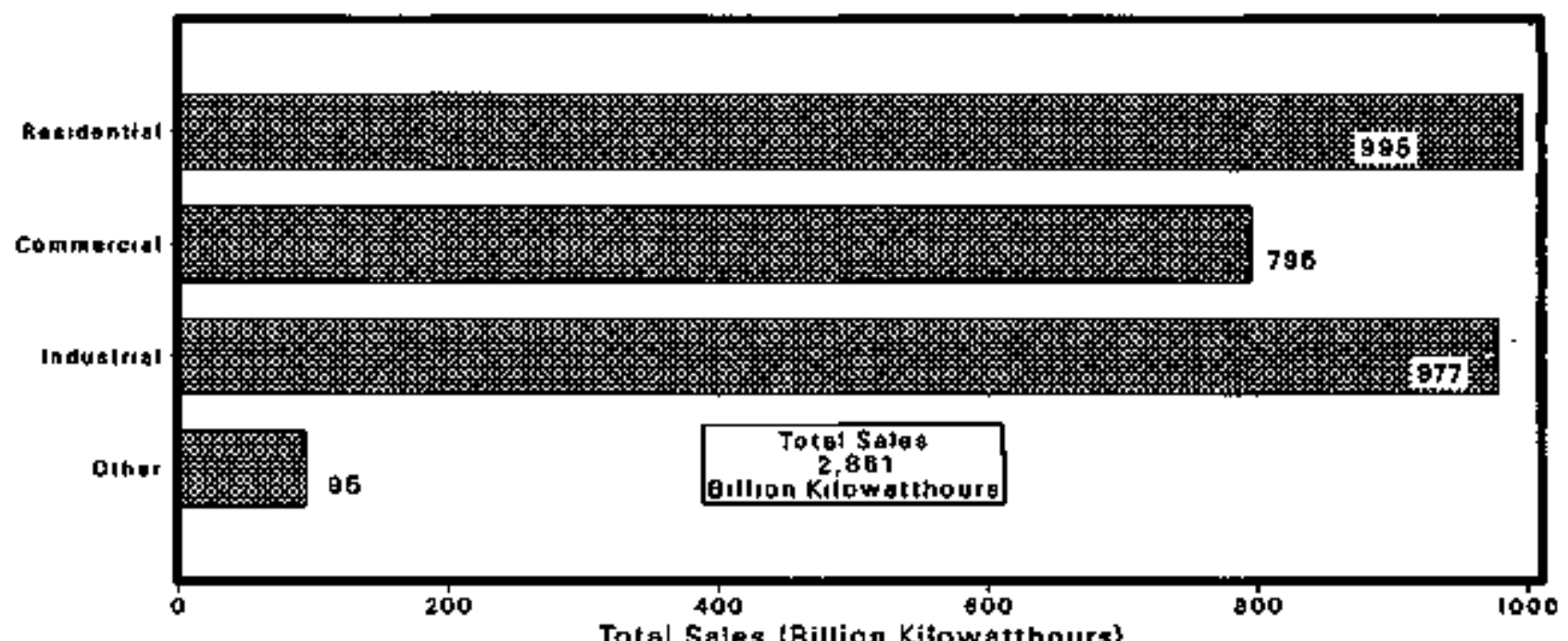

No1es. - Data for 1903 are final. Othar includes public straet and highwey lighting, other salea to publc authorities, sales to ratiroeds and railways, and interdepartmental sales. $\bullet$ Todals may not equaf sum of components because of independent rounding.

Source: Energy Information Adminiotration, Form Ela-B61. 'Annuel Electric Utility Report.

Figure 6. Feverue from Sales to Ultimate Consumers by Sector, 1993

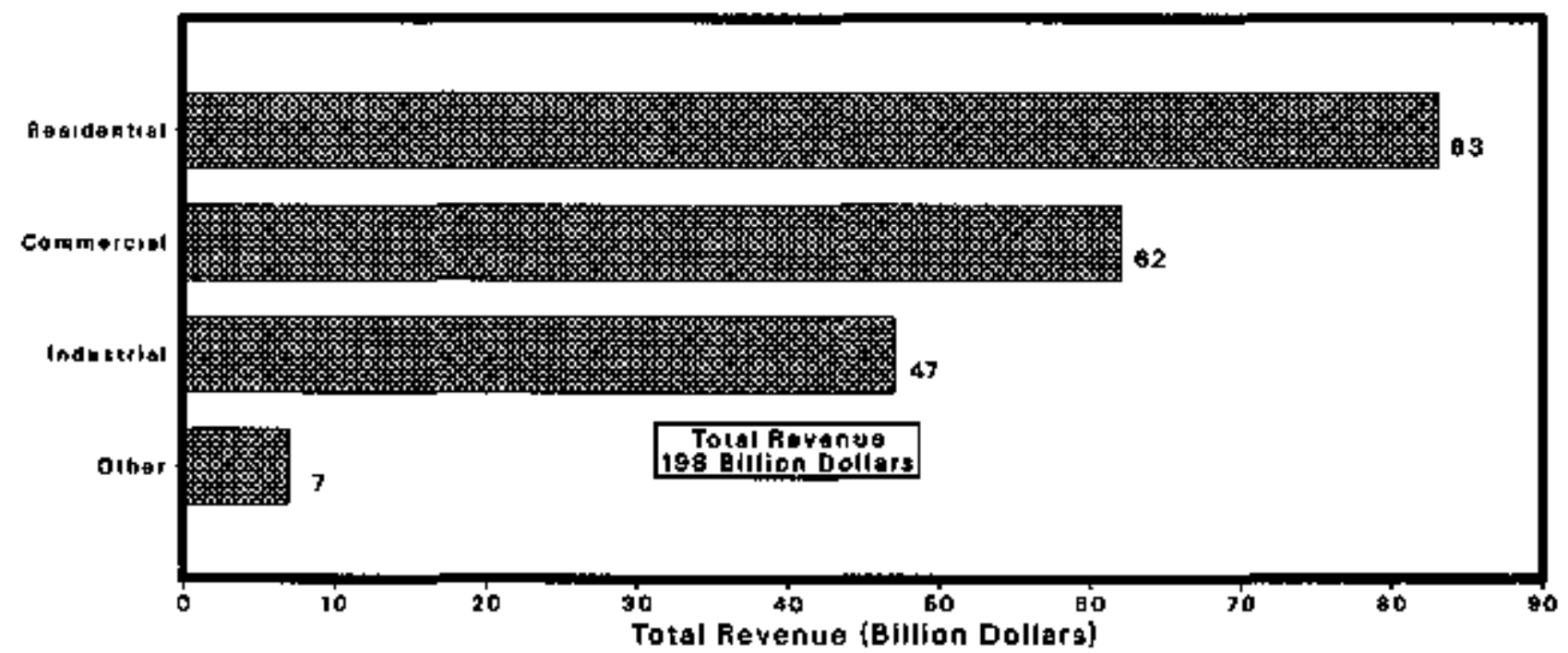

Notes: Data for 1993 are final. Other includes public streat and highway jighting, other sales to public authoritias, sales to fafirosds and reilways, and interdepartmantel salas.

- Totals may not equal sum of components besause of indepeadent rounding.

Source: Energy Information Administration, Form ElA-8b1, "Annual Elactric Utility Report. 
Figure 7. Share of Sales to Utimate Consumers and Associated Revenus by Sector, 1993

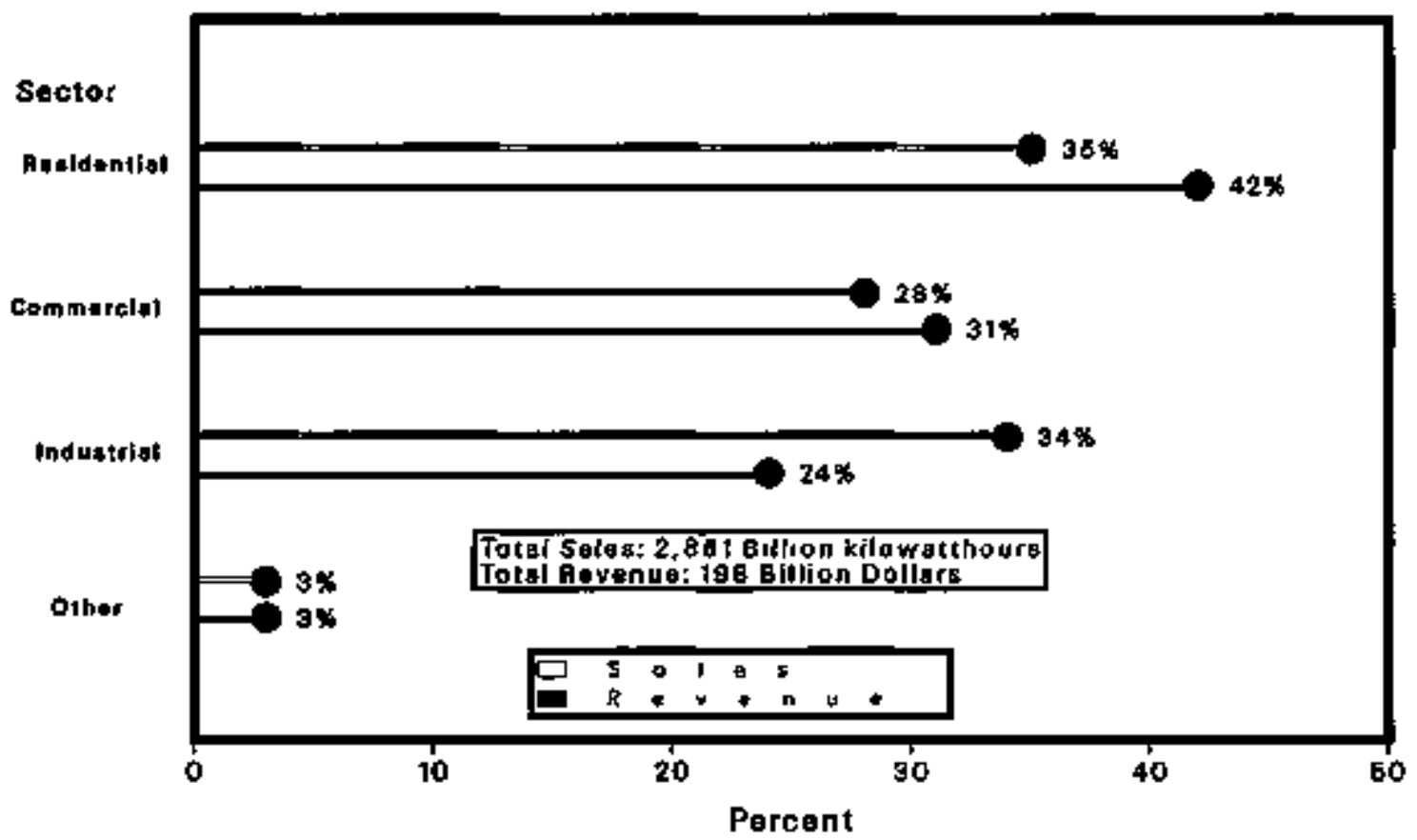

Notes: Dota for 1993 are tinal. OOther inciudes public streat and highway lighting, other sales to public authorities, sales to railroads and railways, and interdapartmental salas.

-Totsla may not equal sum of components because of independent rounding.

Source: Energy Informetion Adminjstration, Form ElA-B61. Annual Eleckrie Utility Ropork."

Figure 8. Average Revenue per Kllowathour by Sector, 1993

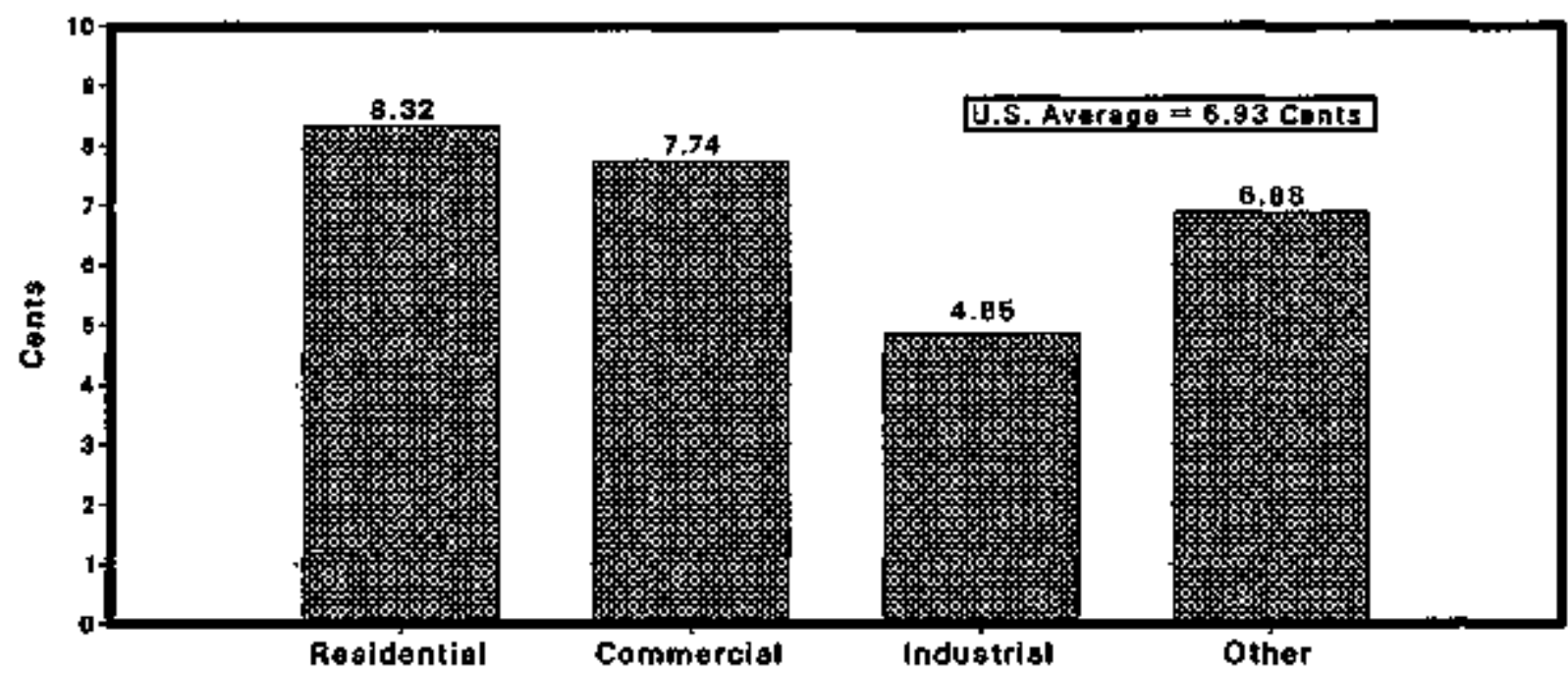

Notes: -Data for 1983 are final. OOther includes public strest and highway lighting, other sales to publit authorities. siles to cailroads and reilweys, and taterdepartmental salos.

-The sverage revenue per kilowatthour of electrictty sold is calculated by dividing ravenue by sales.

Source: Energy Information Administration, Form ElA-861. 'Annusi Electric Utility Report.' 
Figure 9 . Sales to Utimate Consumers by Class of Ownership, 1993

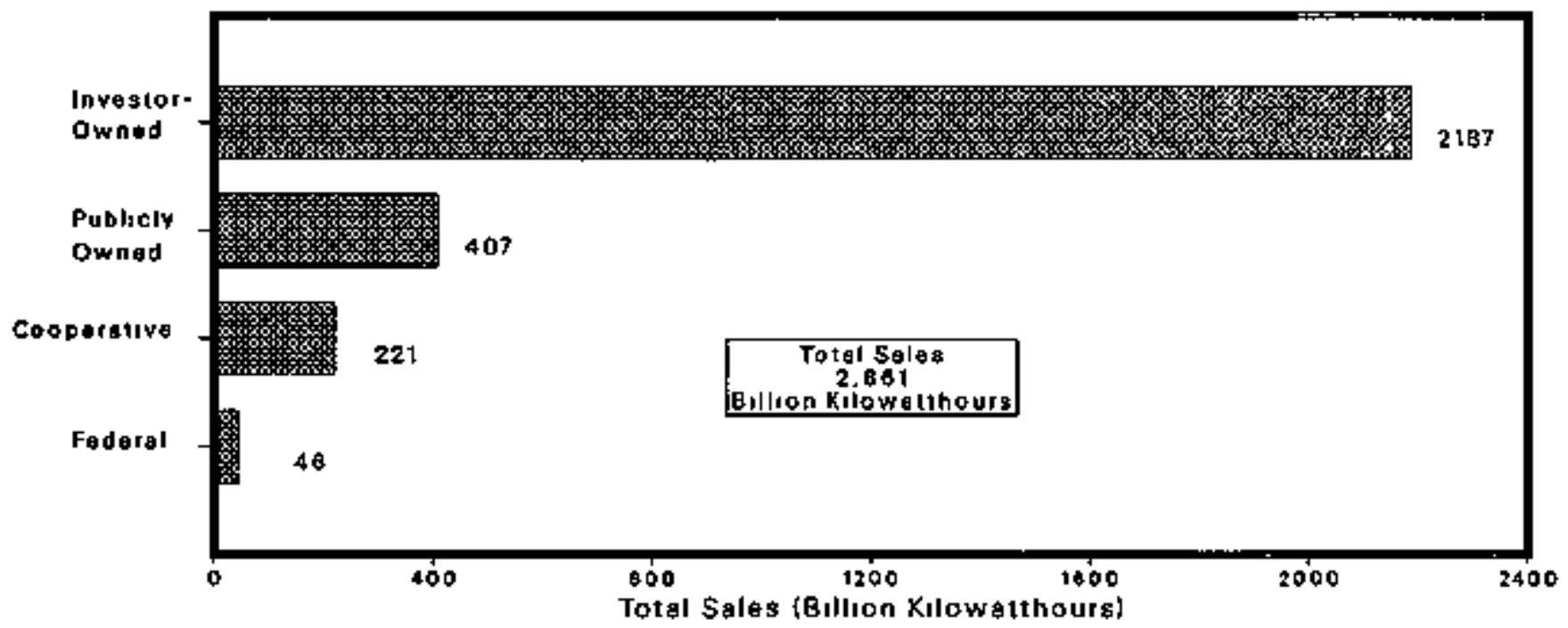

Notes - Date for 1993 are final toials mar not equal sum of components because of Independent rounding

Sourge Energy Information Administration, Form ElA-861. Annual Electric Utility Raport

Figure 10. Revenue from Sales to Uitimate Consumers by Class of Ownership, 1993

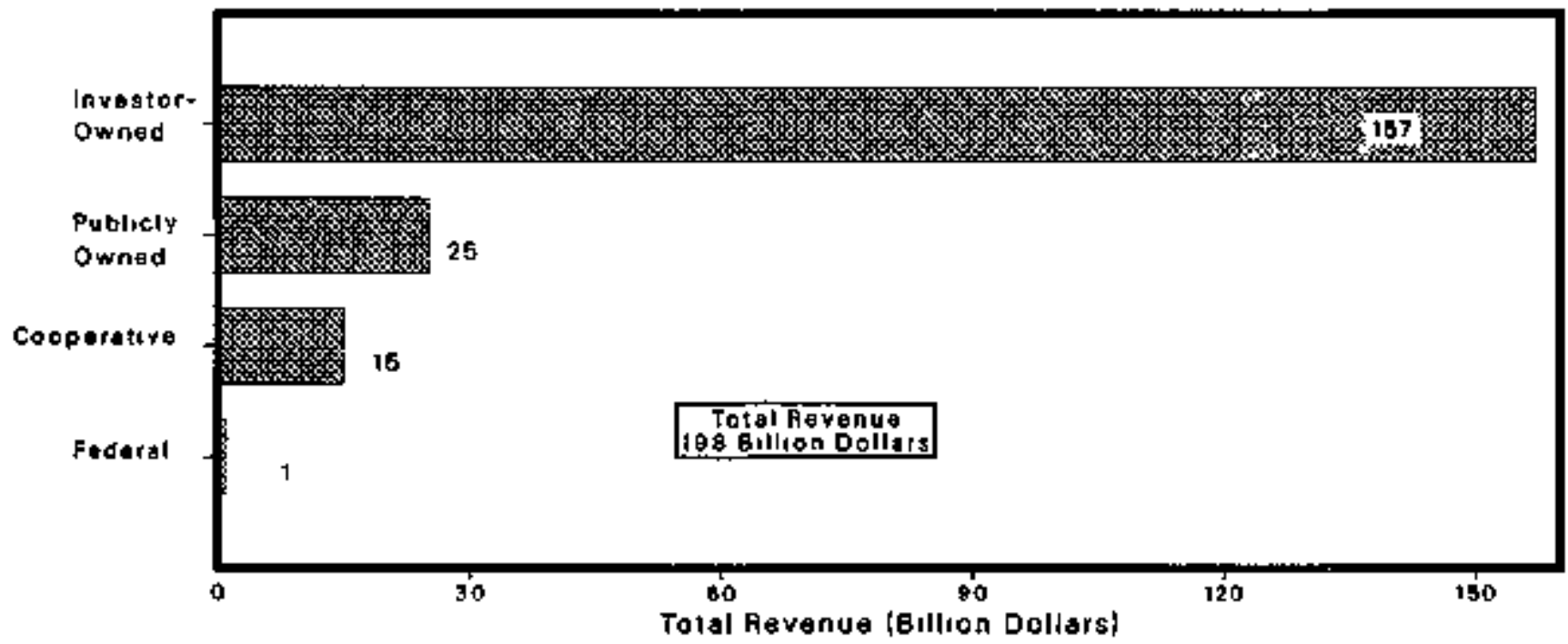

Notes Date for 1993 are fingl Totals may not equal sum of components because of indopendent rounding

Source Energy Information Administrotton. Form ElA-861, Annual Electro Utility Repor1 
Figure 11. Share of Sales to Ultimate Consumers and Aswociated Revenue by Class of Ownershlp, 1993

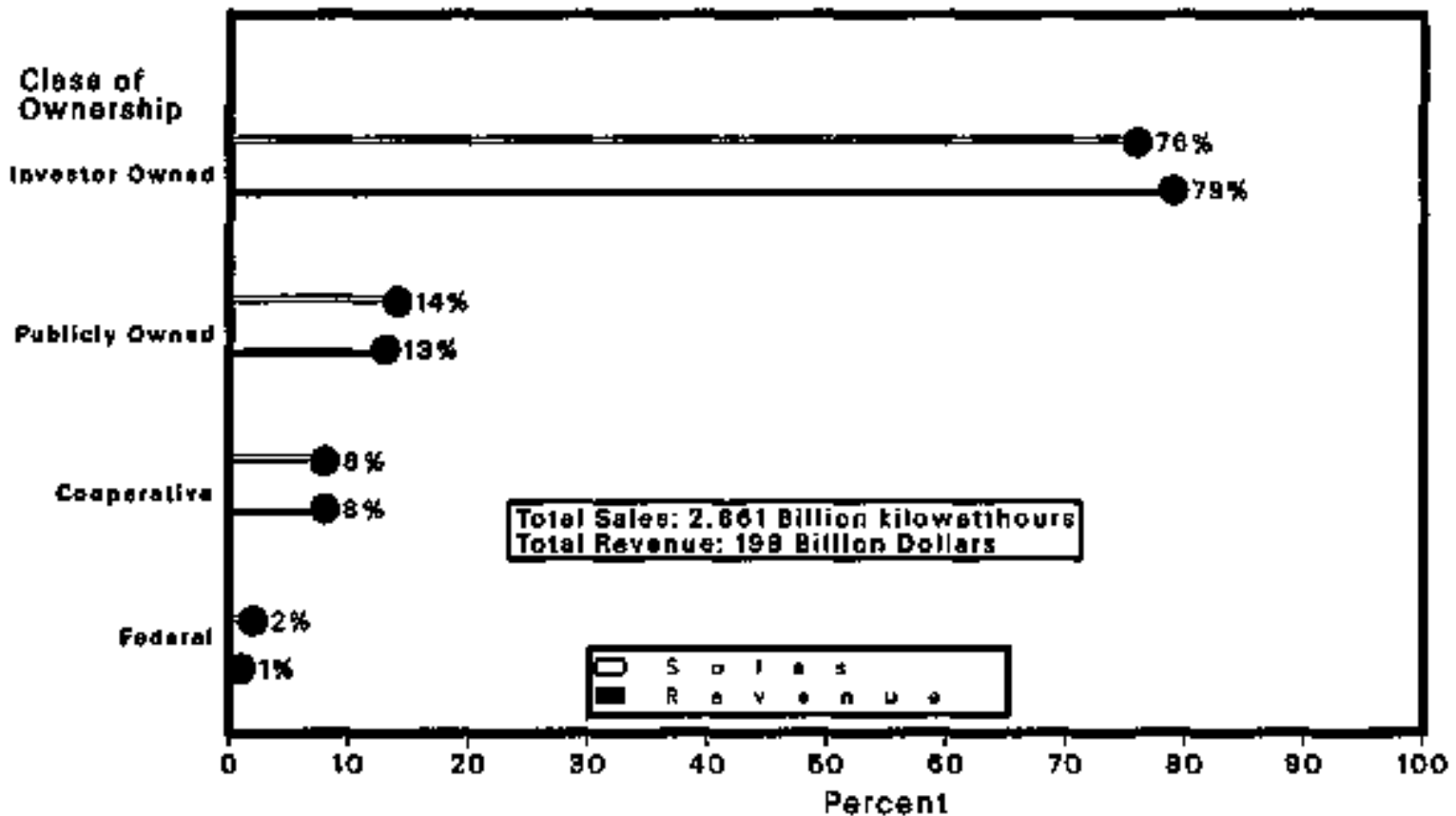

Notea: -Data for 1993 are final. - Tolels may not equal sum of components because of independent rounding.

Source: Energy Information Ađ̆ministration, Form ElA-B61, 'Annuel Elactstc Utillty Report.'

Figure 12 Average Revenue per Klowatthour by Class of Ownership, 1998

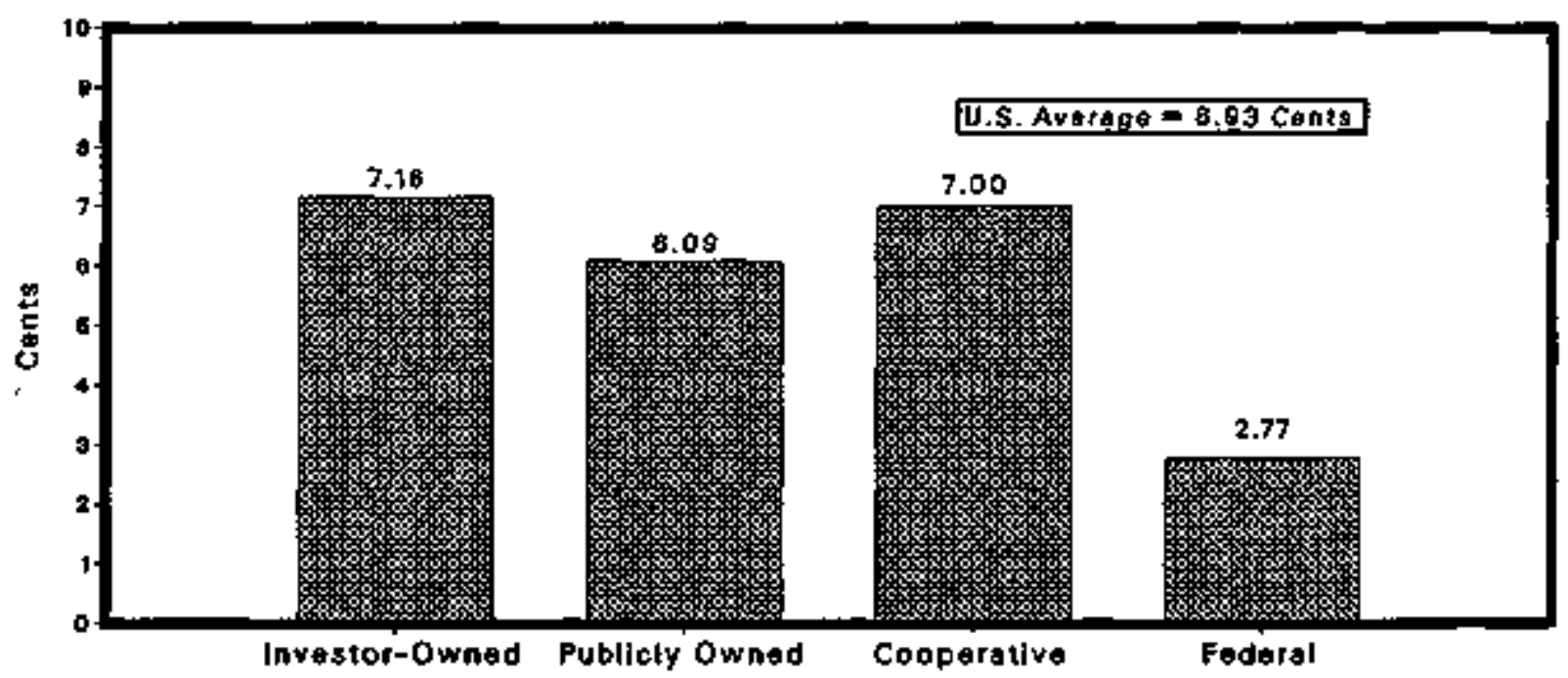

Notes: Dota for 1993 are finel. -The svarege revenute per kilowatthour of electricity sold is caloulated by dividing revenue by sales.

Source: Enaroy tnformation Administration, Form EIA-B61, 'Annual Elactric Utility Report.' 



\section{Sales and Revenue}

This chapter provides a discussion on revenue requite ments, the allocation of revente across sectors, and rate schedules. Also provided are summary statistics on the sales of electricity to ultimate consumers and the associated revenue. This information is provided at the national, Census division, and State level.

\section{Cost of Electricity}

The process of determining the cost of electricity generally follows three stages: (1) revenue requirements are determined, (2) the revenue requirement is allo. cated to different classes of service (sectors), and (3) rate schedules for each sector are established.

Repemue Requirements. Revenue requirements are set to reimburse the electric utility for providing electric service. The revenue requirements reflect the anticipated cost of providing services for some period of time in the future, usually a year. Revenue reçuirements include investment depreciation, expenses for the production, transmission, and distribution systems, as well as sales costs, consumer costs, Federal and other taxes, and administration and genteral expenses. The revenue requirements also include an allowance for the profit of the electric utility, if applicable.

Allocation of Revepue. The cost of service to partictlar sectors and the mix of consumers served in the sectors provide guideltines for determining how much of the revepue must be collected from each sector. For example, the cost of service to the residential sector is usually higher than the cost to the commercial or industrial sectors because of the large number of consumers served, amount of distribution lines needed to provide the service, and the low consumption levels. The economic activity or type of business of the ulti. mate consumer is not necessarily the messs by which electric utitities classify their consumers to determine revenue requirements and set. rates. Electric utilities, in many instances, deternine their revente reculirements and set their rates based on the level of demand and/or comsumption of electricity.

The residential sector represents 88 percent of the total number of consumers but only accounts for 35 percent of the total sales of electricity and 42 percent of the revenue from those sales. The industrial sector consists of less than 1 percent of the total number of consumers but accounts for 34 percent of the sales and 24 percent of the revenue. The conmercial sector consists of 11 percent of the consumers and accounts for 28 percent of total sales and 31 percent of the revenue. The remaining share of sales is accounted for by the "other" sector, whick includes public street and highway lighting railroads and railways, muntcipalities, djvistons or agencies of State and Federal Governments under spectal contracts or agreements, and other utility departments, as defined by the pertinent regulatory agency and/or electrte utility. Investor-owned electric utilities serve approximately three-fourths of the total number of ultimate consumers. Publicly owned electric utilities and electric cooperatives serve nearly all the remaining ultimate consumers, because federally owned electric utilities have very few retail customers and sell almost exclusively to other electric utilities.

Rate Scheidules. In general, the rate schedules for electric vtilities are designed to reimburse utilities for operation, generation, maintenance, rental fees for equipment, and demand and service charges. Operation, generation, maintenance, and rental fees for equipment are usually lixed charges that are assessed montbly ar annually. Demand charges are often fixxed, but they can vary with the peak deraand of the cuscomer. Energy charges vary with the amount of electric energy delivered to consumers. A more detailed discusston of these charges is contained in the chapter, "Average Revemue per Kilowatthour."

The rate schedules developed by electric utilities are unique to each utility. The rates reflect any number of consideralions that are important to the economic welfare of the electric utility and may reflect other interests as well, such as residential rates that provide economical service to low-income families. Generally, commercial or industrial billings are determined more by the levels of demand and consumption of electricity than by the type of econonic activity in which the consumer is engaged.

For instance, while commercial and industrial consurrers are billed accorting to a particular rate schedule that frequently reflects the type of business indicated by that schedule, there are exceptions. A light mamsfacturing establishment with a manufacturing process that consumes little energy could be billed at a commercial rate because its consumption is not high enough to fall into the industrial schedule. Conversely, a very large commercial building might have sufficiently large consumption to be billed at the industrial rate. 
Sectoral clessification of consurners is based on different criterja such as demand level, rates, Standard Industrial Classification (S1C) code, accountug methods, end-use actuvty, and other social and economic characteristics Reclassification of consumers, usualty between the commerctal and udustnal sectors, may occur from year-to-year due to changes in demand level or economic or other factors

The anthonty for the approval of rate schedules is based on the class of utdity ownershop Investor-owned electrtc utulutes are regulated by State publtc service commissions and the Federal Energy Regulatory Commission Publicly owned and cooperatuve electric utlIttes, in most States, are regulated by their consumer- owners through locally elected or appointed officials A more detaled discussion on vility classes of ownership ts contarned in the "Background" section of the Profile chapter in thes publication

Date Sources. Tables 3 through 10 contatn data on the retall sales of electncity, its associated revenwe, and the average number of constrmers at the national, Census and state levels by sector and class of utility ownershop Statistics contanned in Tables 3 through 10 are basted on data collected on the Form EIA-861, "Annual Electne Utility Report " Data for the four power authonties of American Samo, Guam, Puerto Rico, and the Vurgin Islands are not neluded to the summary statustics

Table 3. U.S. Sales to Ulitmate Consumers and Associated Revenue by Sector, t989 Through 1993

\begin{tabular}{|c|c|c|c|c|c|}
\hline Itant & 1790 & 1002 & 1901 & 1990 & 1906 \\
\hline 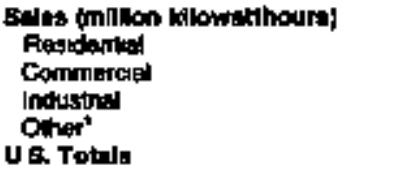 & $\begin{array}{r}984781 \\
764.573 \\
977.154 \\
94,944 \\
2,261,468\end{array}$ & $\begin{array}{r}906,999 \\
761,271 \\
972,714 \\
90,442 \\
2,763,945\end{array}$ & $\begin{array}{r}966,417 \\
765,664 \\
946580 \\
94360 \\
2,762,003\end{array}$ & $\begin{array}{r}924,019 \\
751,027 \\
945522 \\
91,969 \\
2,712,585\end{array}$ & $\begin{array}{r}906,646 \\
725,031 \\
095,850 \\
89,766 \\
2,046,000\end{array}$ \\
\hline 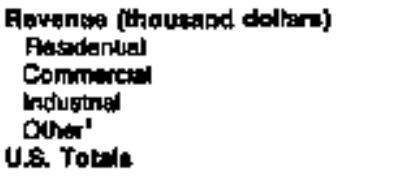 & $\begin{array}{r}82,813,624 \\
61,521259 \\
47,367,144 \\
6,527,651 \\
196,520,189\end{array}$ & $\begin{array}{r}7 B, 846,956 \\
58,942,581 \\
48,983,176 \\
6,296,819 \\
196,480,312\end{array}$ & $\begin{array}{r}76,027,977 \\
57,655,250 \\
46,737,197 \\
6,158,269 \\
188,358,700\end{array}$ & $\begin{array}{r}72,370408 \\
55,116,678 \\
44,856,817 \\
5,680,681 \\
174,242,794\end{array}$ & $\begin{array}{r}09,240,395 \\
5,2,227,813 \\
40,718,690 \\
5,609,485 \\
1 \pi, 798,598\end{array}$ \\
\hline
\end{tabular}

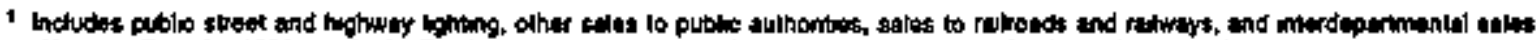

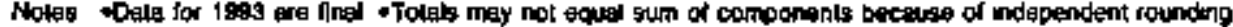

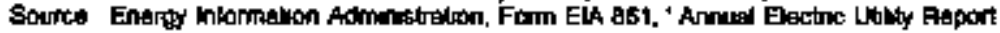

Table 4. U.S. Sales to Ultimate Consumers and Assoclated Revenue by Class of Ownership, 1989 Through 1998

\begin{tabular}{|c|c|c|c|c|c|}
\hline Itam & 1000 & ter & 1491 & 1900 & 1904 \\
\hline 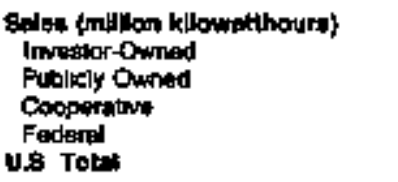 & $\begin{array}{r}2186,899 \\
406,996 \\
221,206 \\
46,370 \\
2,861,462\end{array}$ & $\begin{array}{r}2,112,271 \\
395,998 \\
208,999 \\
48,766 \\
2,763,365\end{array}$ & $\begin{array}{r}2,110,5028 \\
399,448 \\
205,093 \\
52,944 \\
2,762,008\end{array}$ & $\begin{array}{r}2,071,0969 \\
385,859 \\
200,048 \\
54,867 \\
2,712,545\end{array}$ & $\begin{array}{r}2,031,700 \\
372,233 \\
190,201 \\
5,676 \\
2,446,809\end{array}$ \\
\hline 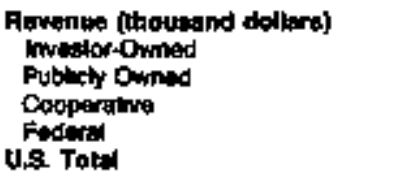 & $\begin{array}{r}165,660,700 \\
24,761,626 \\
15,491,712 \\
1,262,854 \\
t=8,270,180\end{array}$ & $\begin{array}{r}149,016,317 \\
23,726,382 \\
14,461,065 \\
1,275,819 \\
181,400,312\end{array}$ & $\begin{array}{r}147,592,609 \\
29,149,881 \\
14,147,164 \\
1,470,079 \\
184,358,703\end{array}$ & $\begin{array}{r}140,159,375 \\
22,703,165 \\
13,699,961 \\
1,67 a, 251 \\
170,242,700\end{array}$ & $\begin{array}{r}134,169,257 \\
21,849,504 \\
13,040,659 \\
1,0 \% 7,598 \\
170,793,508\end{array}$ \\
\hline
\end{tabular}

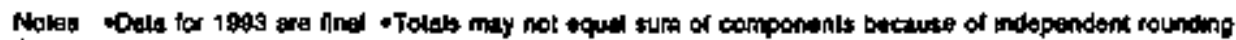

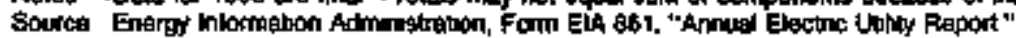


Table 5. Number of Utimate Consumers by Sector, Census Divkslon, and State, 1993

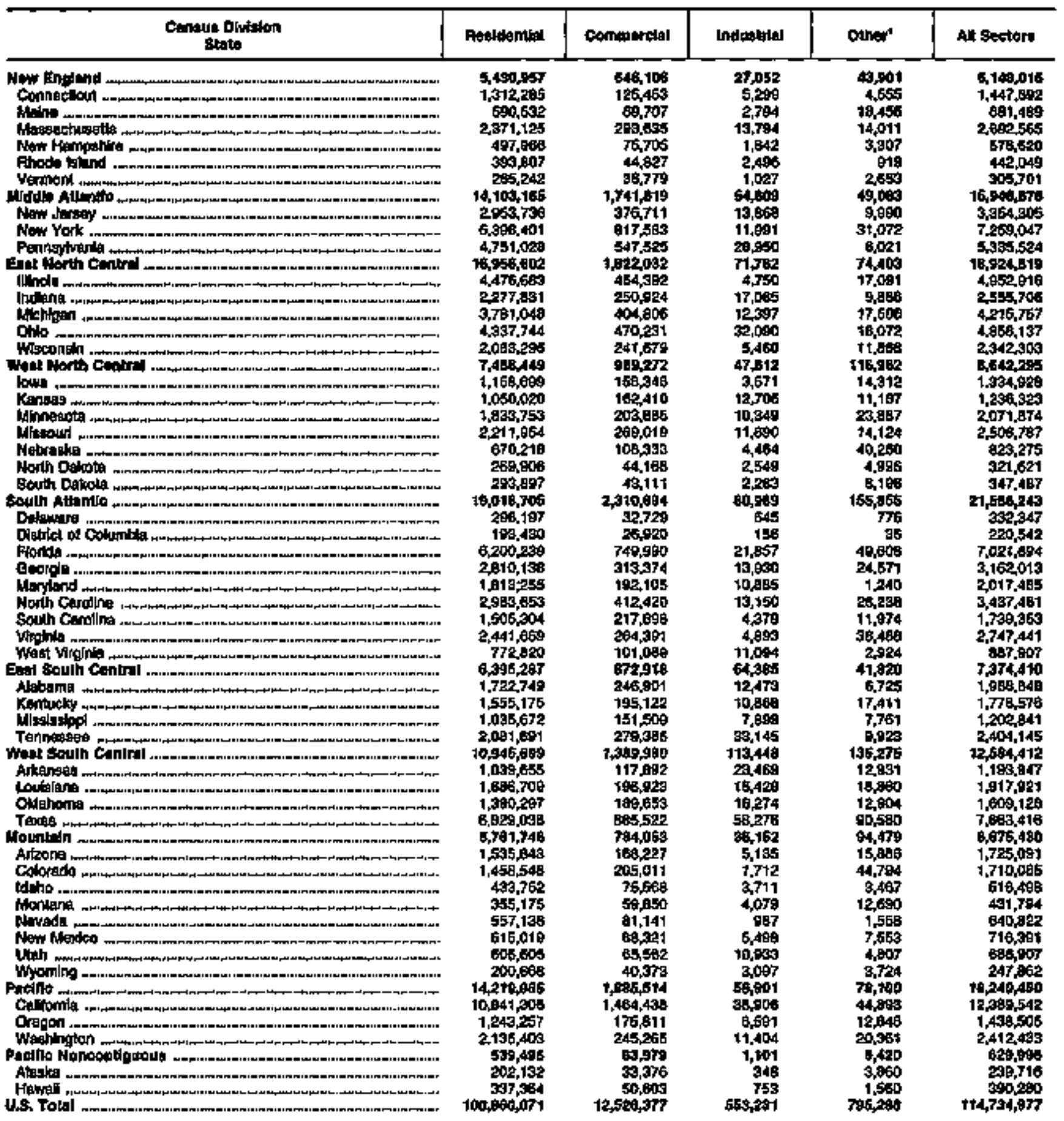

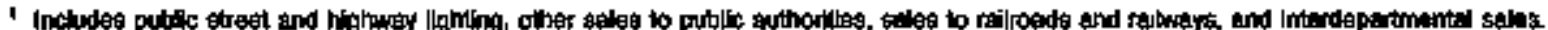

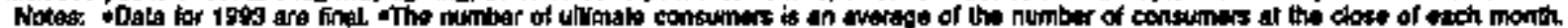

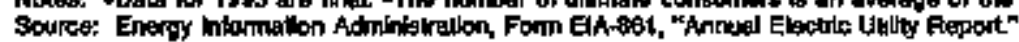


Table 6. Sales to Ultimate Consumers by Sector, Census Division, and State, 1993 (Million Kolowatthours)

\begin{tabular}{|c|c|c|c|c|c|}
\hline $\begin{array}{c}\text { Consul Chition } \\
\text { State }\end{array}$ & Fadidenthal & Gonmurek & Intuabrial & Other' & All 8oslor: \\
\hline 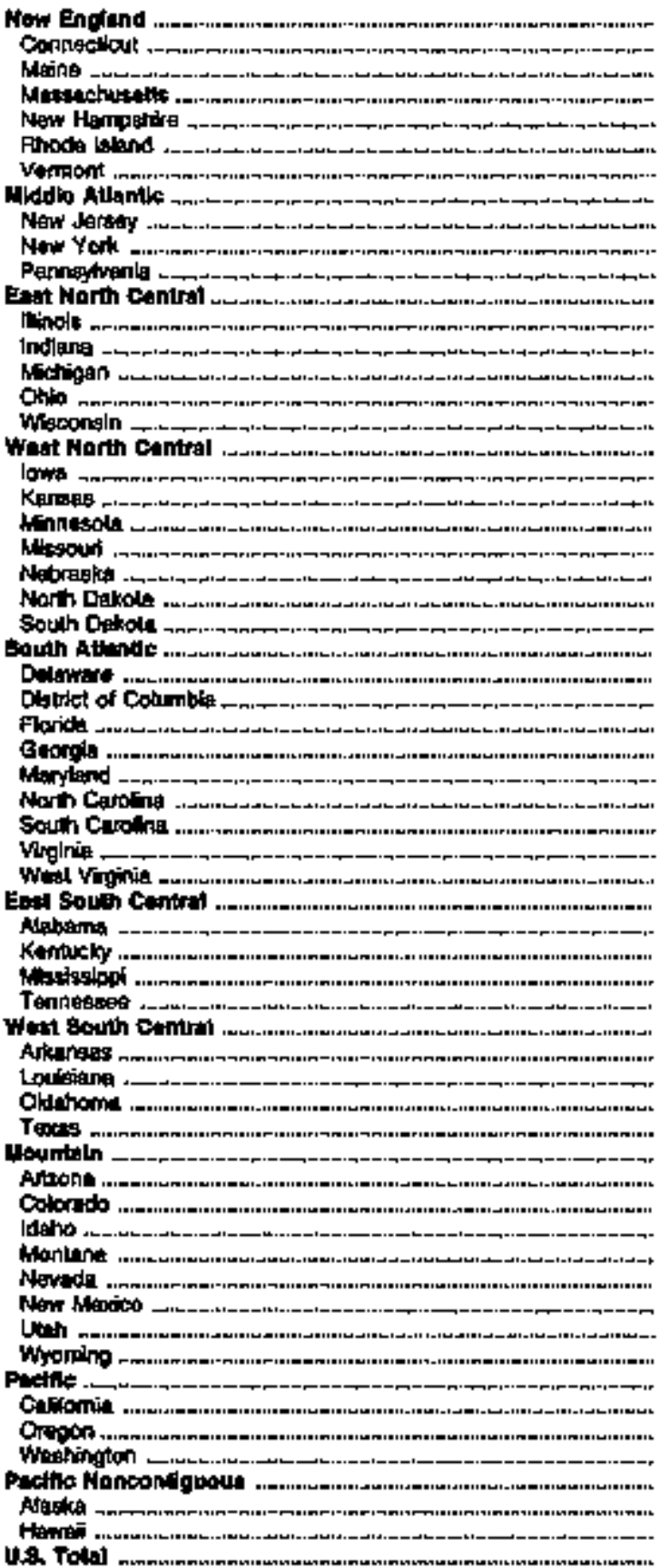 & 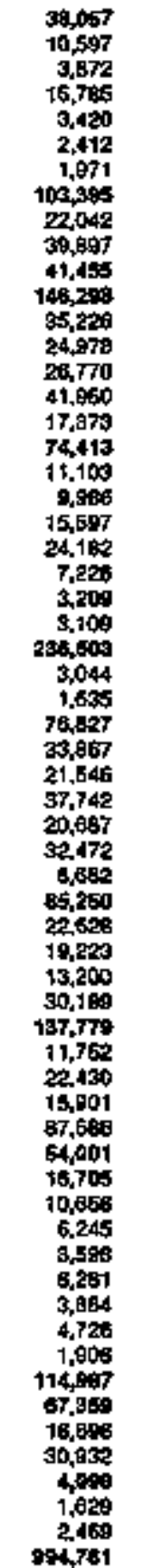 & 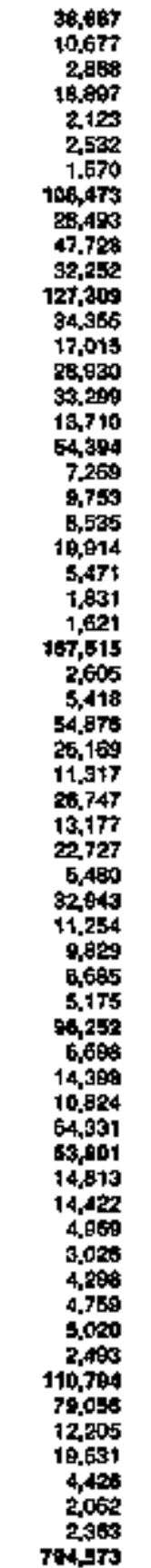 & 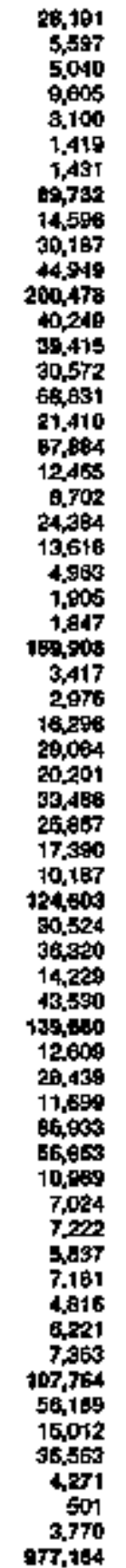 & 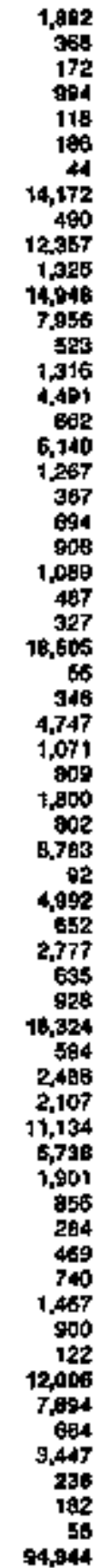 & 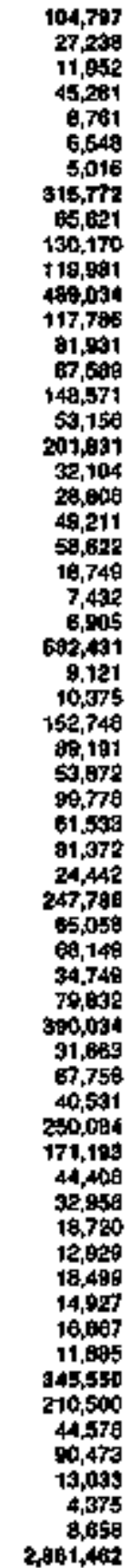 \\
\hline
\end{tabular}

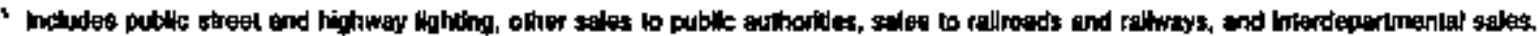

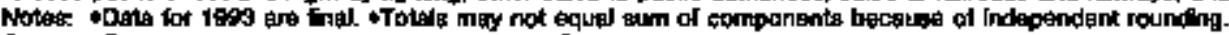

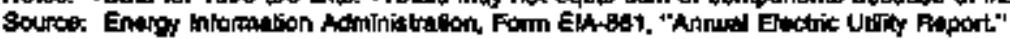


Table 7. Revenue from Sales to Ultimate Consumers by Sector, Census Division, and State, 1993 (Thousand Dollars)

\begin{tabular}{|c|c|c|c|c|c|}
\hline $\begin{array}{c}\text { Gontous Dhialos } \\
\text { Stels }\end{array}$ & Protdentilal & Corimeretel & Induatrial & Other' & An trecters \\
\hline 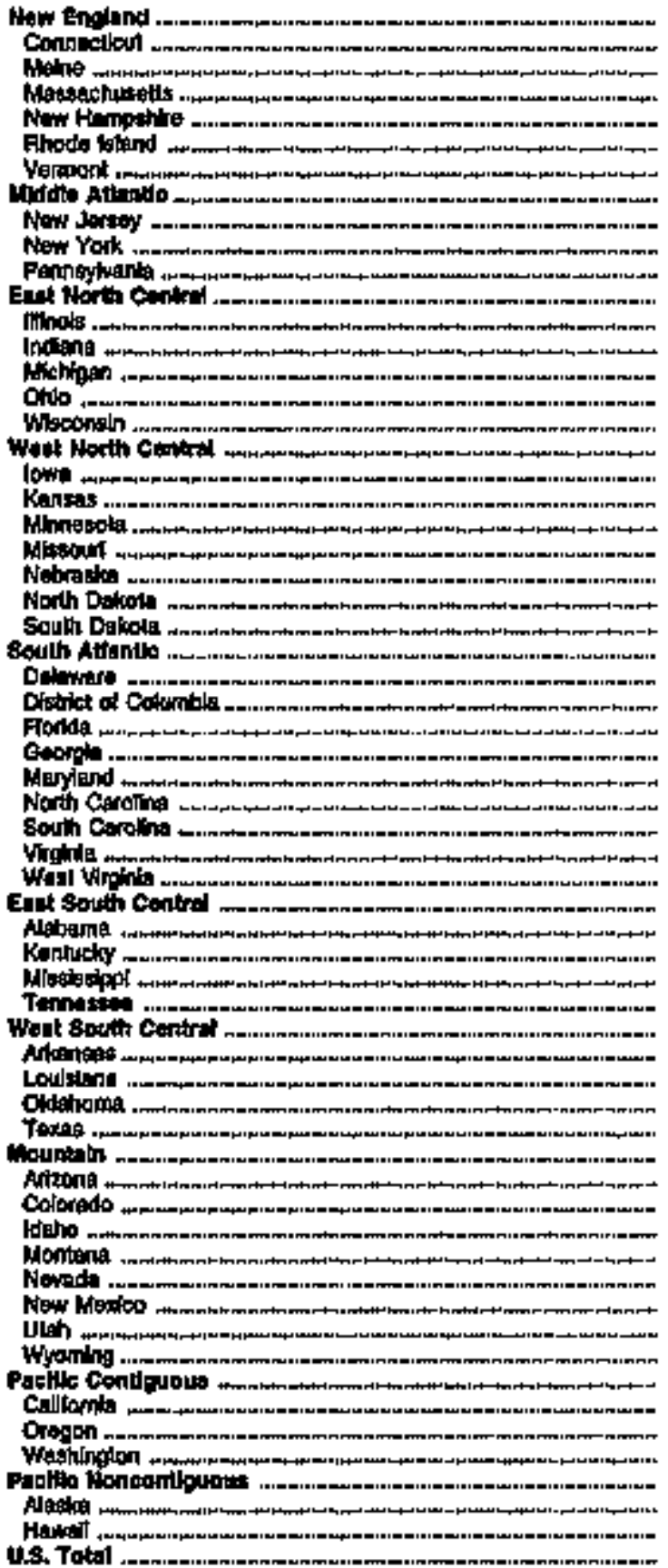 & 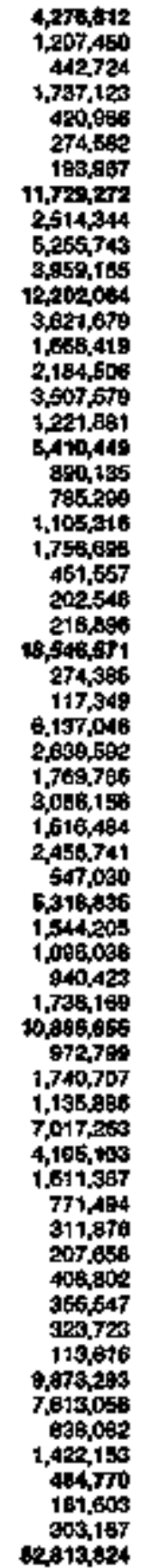 & 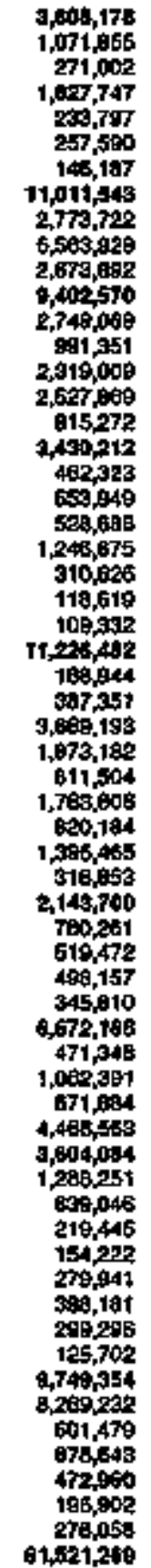 & 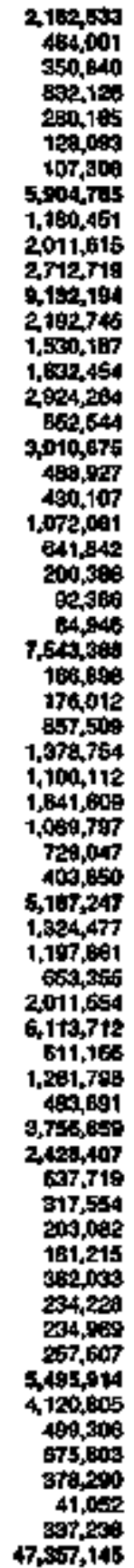 & 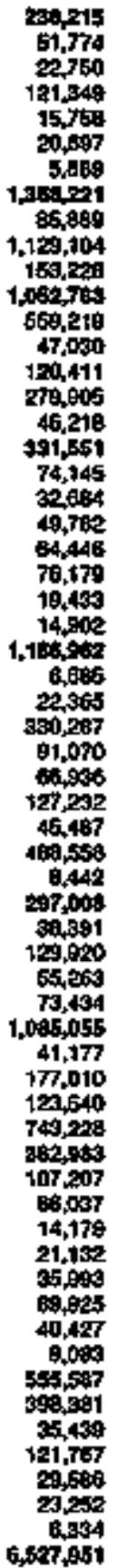 & 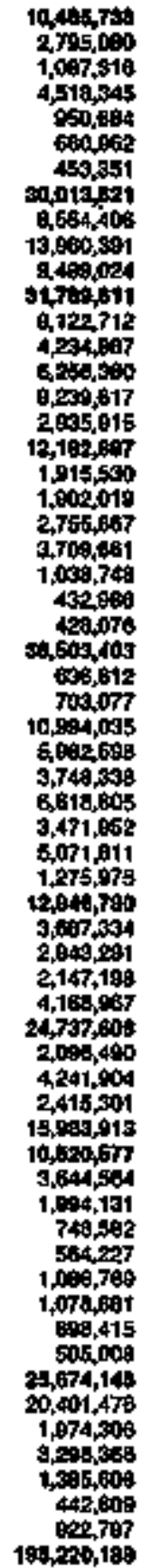 \\
\hline
\end{tabular}

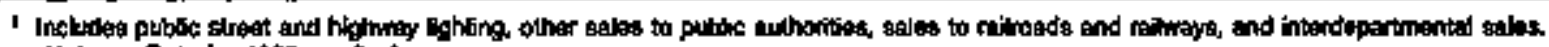

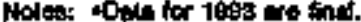

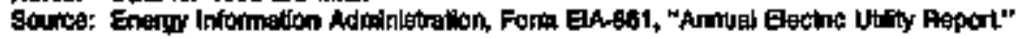


Table 8. Number of Ultimate Cansumers by Class of Owmership, Census Division, and State, 1993

\begin{tabular}{|c|c|c|c|c|c|}
\hline $\begin{array}{c}\text { Ceapus Dhiplon } \\
\text { strote }\end{array}$ & Inweatar-Ownes & Putuchy Owned & Cooperathe & Fipdartid & AH CH:and \\
\hline 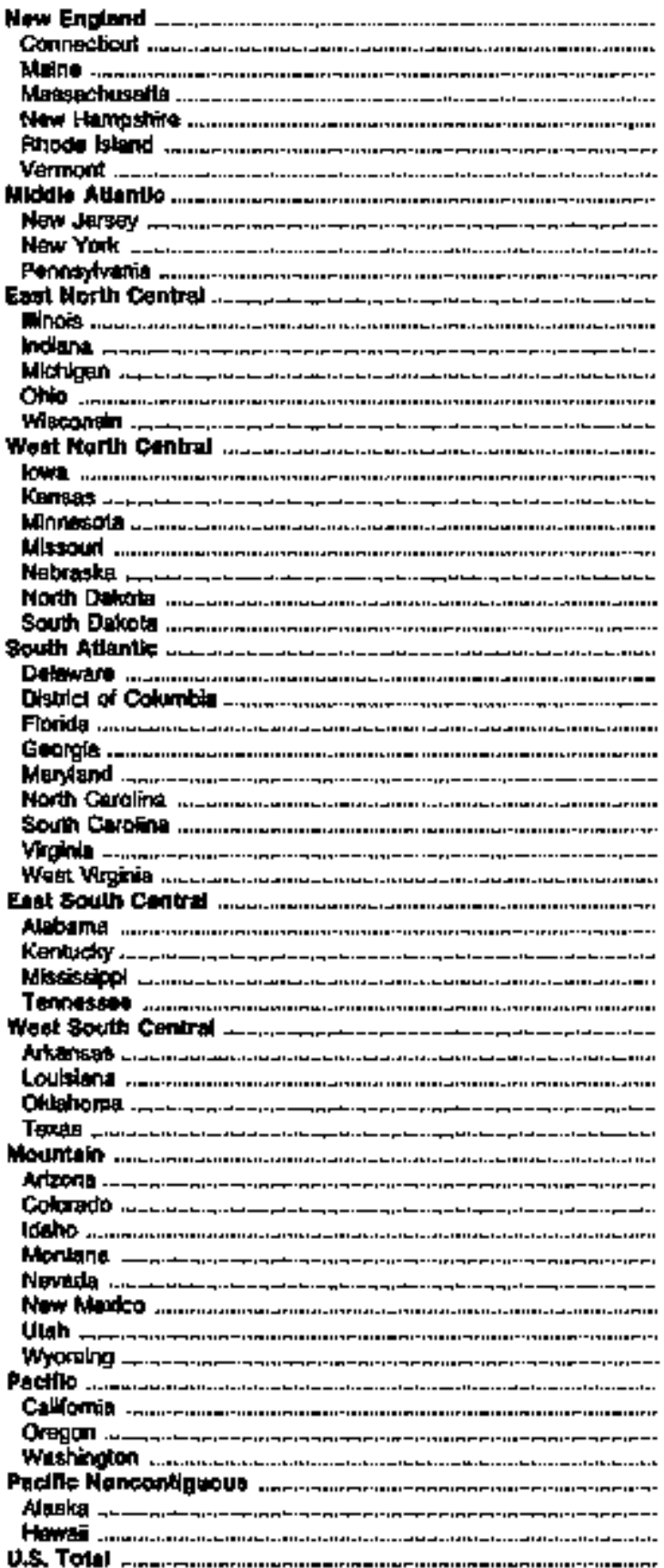 & 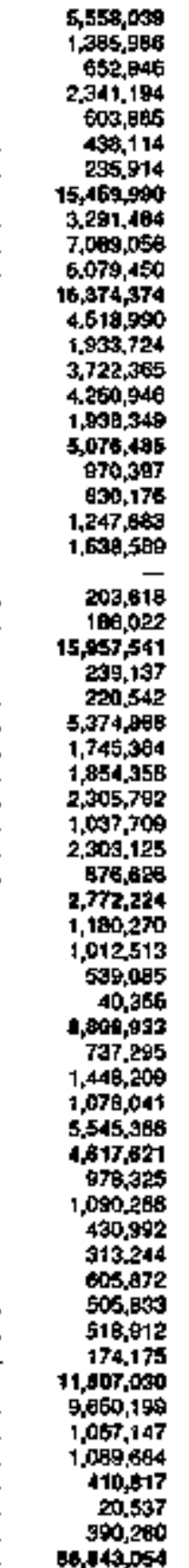 & 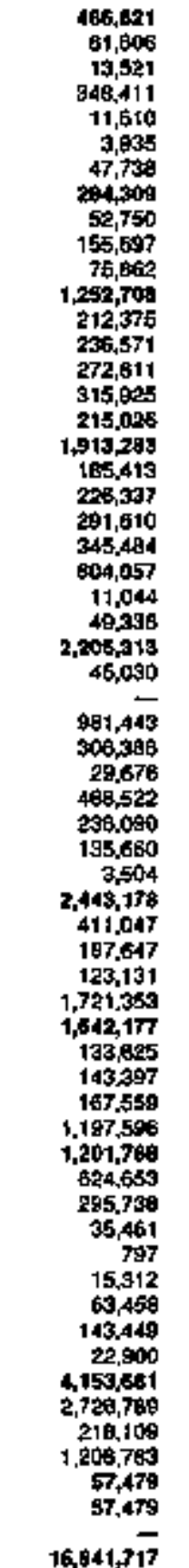 & 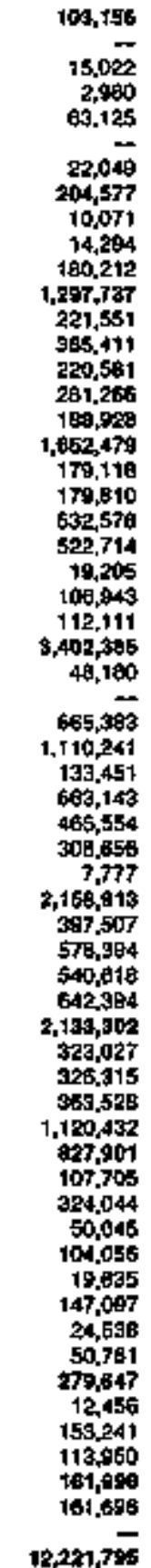 & 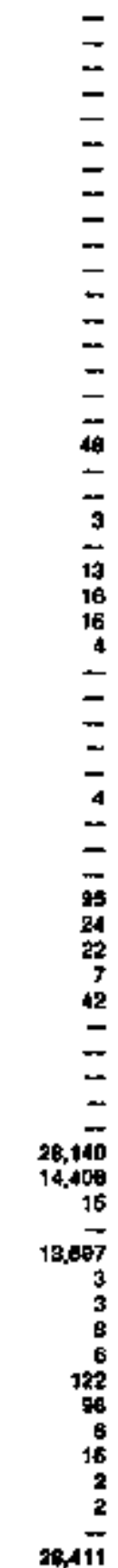 & 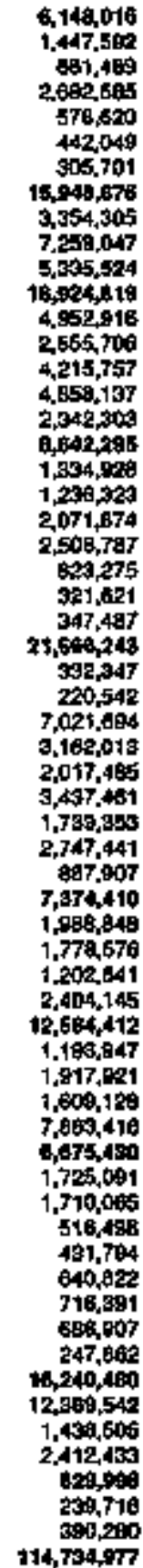 \\
\hline
\end{tabular}

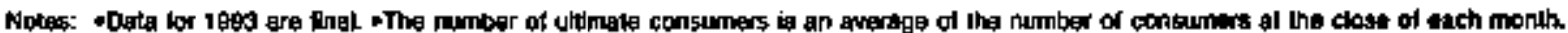

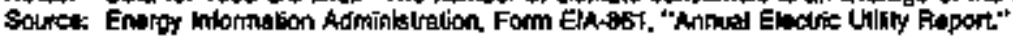


Table 9 . Sales to Uitimate Consumers by Cass of Ownerahip, Census Division, and Btate, 1993 (Million K'lowatthours)

\begin{tabular}{|c|c|c|c|c|c|}
\hline $\begin{array}{l}\text { cennua Toilolon } \\
\text { sth }\end{array}$ & Invetitor-Ominted & Popilfty Ominded & Cospitrtitted & Fodtural & A: Ctrises \\
\hline 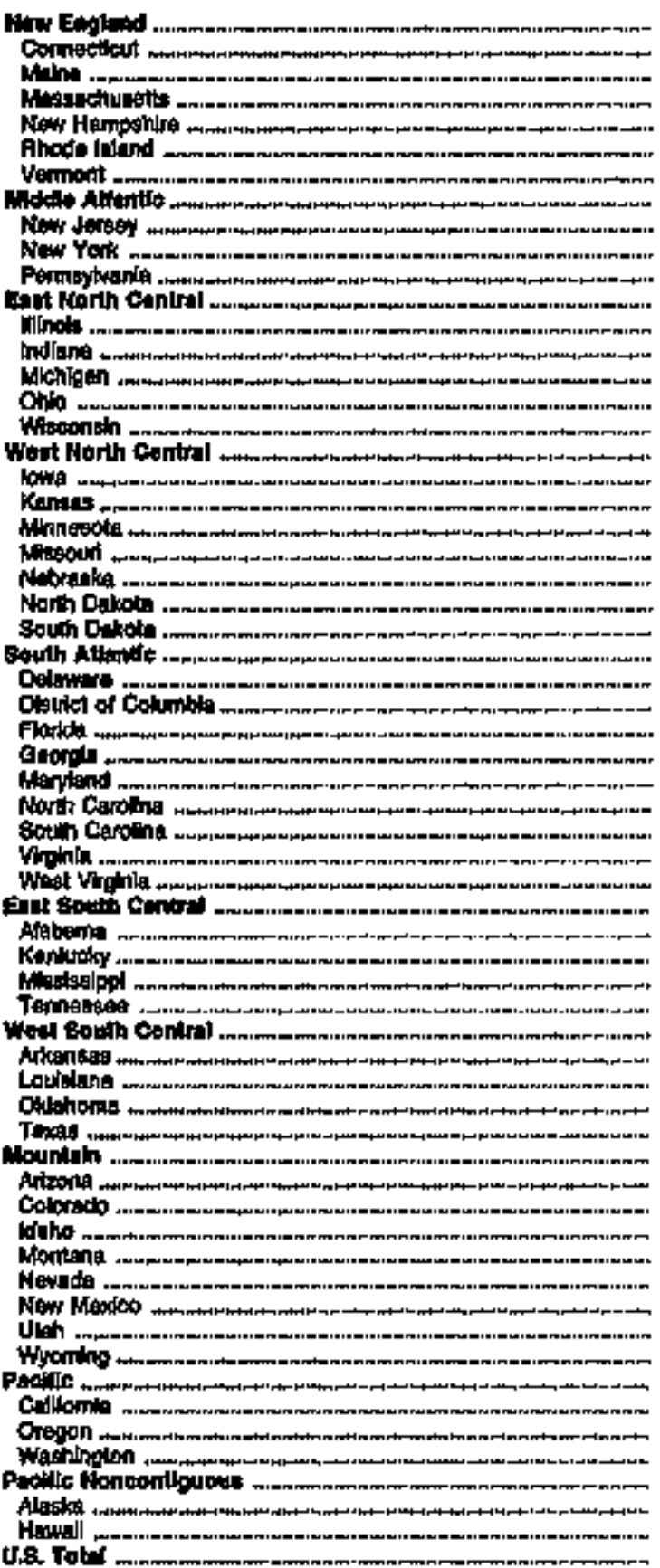 & 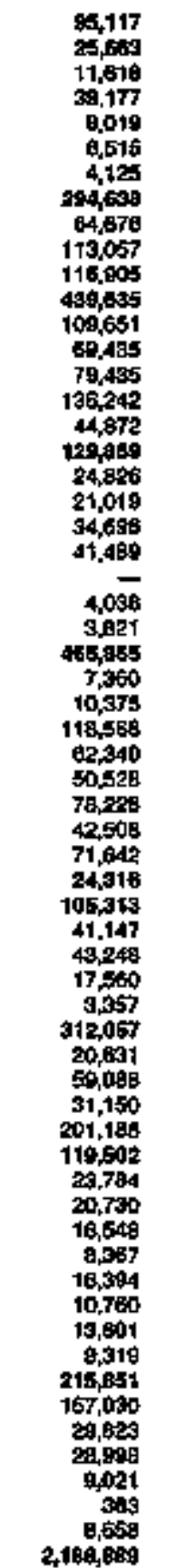 & 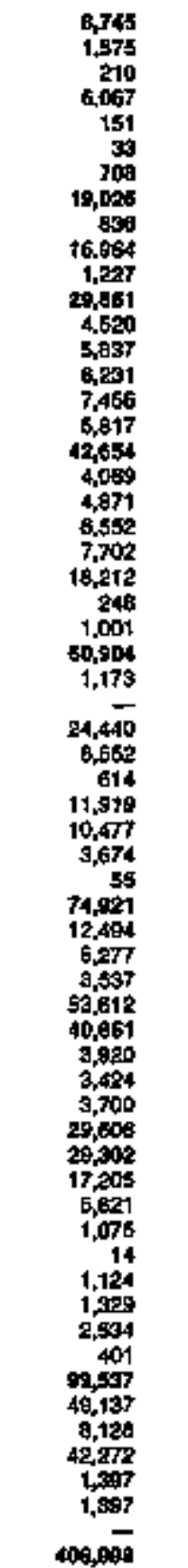 & 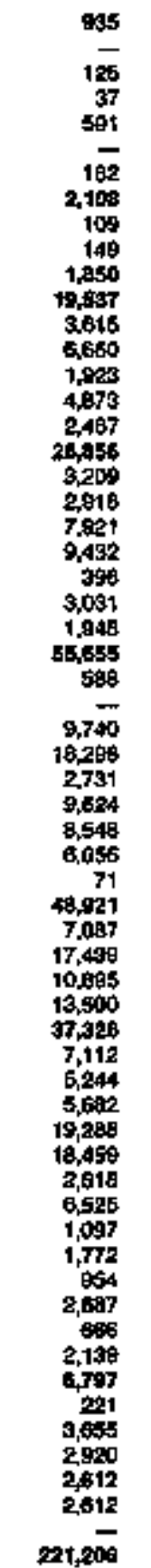 & 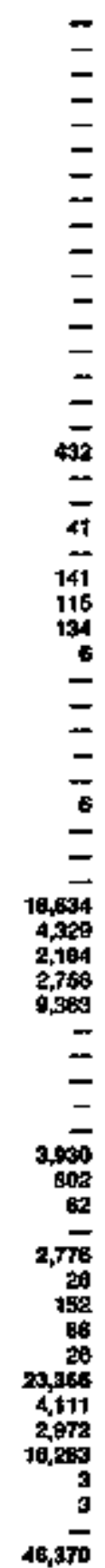 & 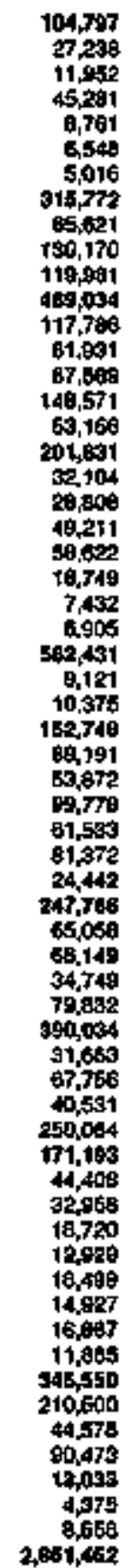 \\
\hline
\end{tabular}

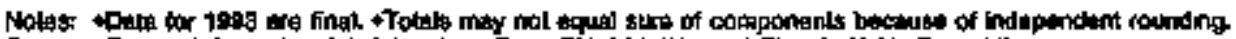

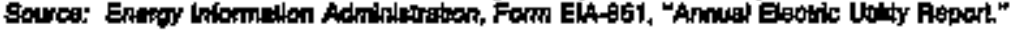


Table 10. Revenue from Sales to Ulimate Consumers by Class of Dwnership, Census Diviston, and State, 1993

(Thousand Dollars)

\begin{tabular}{|c|c|c|c|c|c|}
\hline 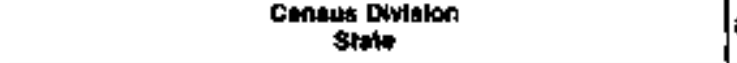 & Intratar-Orinded & Pubdlaly Omed & Cooptratsy & Fidfertid & An Curoster \\
\hline 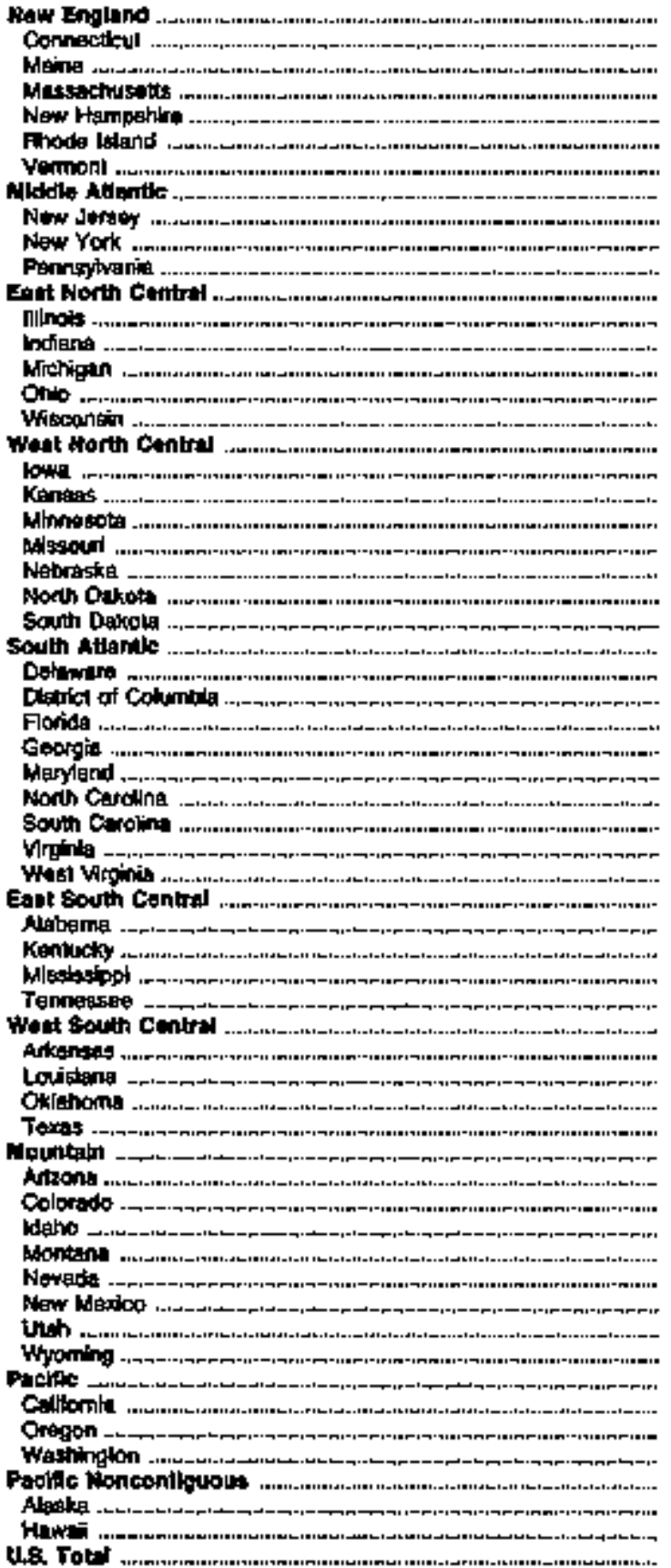 & 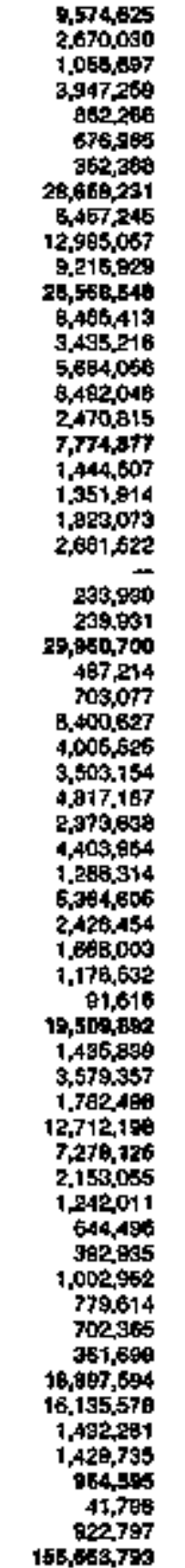 & 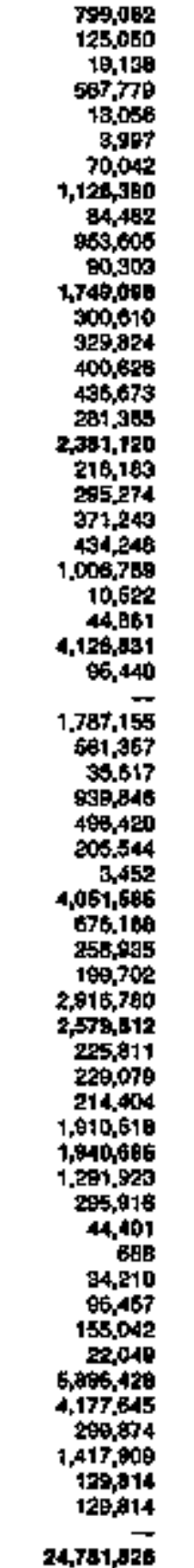 & 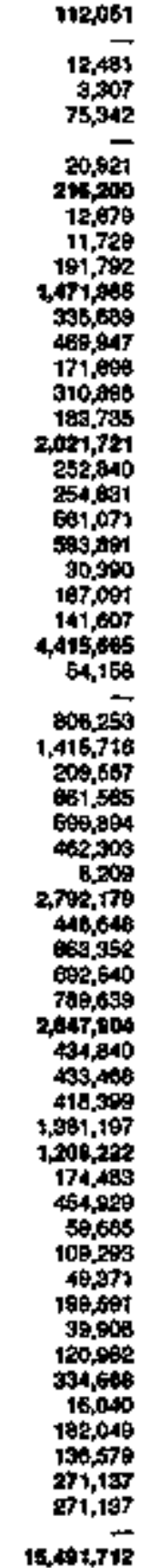 & 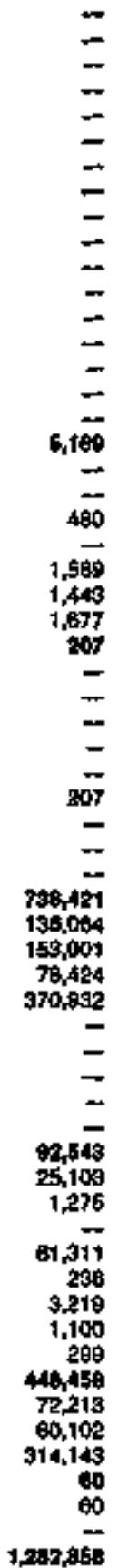 & 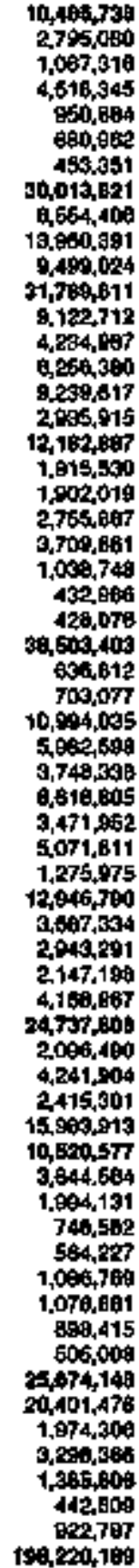 \\
\hline
\end{tabular}

Notec Dala for 1893 ane Anal

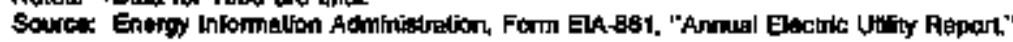




\section{Average Revenue per Kllowatthour}

This chapter provides summary statistics on the average revenue per killowatthour at the national, Census division, State, and electric utility levels.

\section{Average Revenue per Kllowathour at the Nattonal, Regional, and State Level}

The average revenue per kilowatthour by sector and State is calculated by dividing total annual revenue by total annual retail sales for each sector and State. The resulting statistic is the average revenue per kilowathour sold by consumer sector. Many rate schedules have a minimum service charge and most have a declinisig rate per unit of consumption as consumption increases, Rate schedules for the commercial end tndastrtal sectors ajmost always have a demand charge in addition to an energy charge. These charges lower the average revenue per kilowathour as the consumption increases.

The average revenue for the residential sector is generally higher than for any ather sector, due in part to the relatively small consumption level per consumer and the relatively lower load factor. The load factor is average lood expressed as the percentage of the peak load. Generally, a consumer whose average load is low relative to its maximum demand is more costly to serve than a consumer whose load factor is higher. Residential consumers typicalty have lower load factors than industrial consumers.

Average revenue for the residential and commercial sectors increased fairly consistently from 1989 to 1993 , but the average revenue for the industrial sector re. mained relatively flat. The residential sector's average reverue climbed 0.67 cents per kilowatthour, from 7.65 cents in 1989 to 8.32 cents in 1993; and the commercial. sector climbed 0.54 cents per klowatthour, from 7.20 cents in 1989 to 7.74 cents in 1993. The other sector showed an increase in average revenue of 0.63 cents per klowatthour, from 6.25 is 1989 to 6.88 cents in 1993. The industttal sector increased by 0.13 cents to 4.85 cents in 1993, from 4.72 eents in 1989. The relatively smal] change in average revenue for the itdustrial sector indicates that some State utility commissions, during 1989 to 1993, probably approved lower rates for industrial consulmers to better reflect the cost of services (Table 11).
The average revenue by ownership class also exhibits some interesting trends. Both investor-owned and publicly owned utlities bhow upward trends in average revenue, while the cooperatives remain relatively lat. In 1993, the investor-owned utility average revenue surpassed the cooperative's average revenue for the second year in the last jive-year period. The average revenue for Federal utilities continnes to decline falling from 3.64 cents in 1989 to 2.77 cents in 1993.

Utility Demand-Side Maragement (DSM) programs designed to reduce system peak demands and energy requirements have become importsint components of utility load and resource plans. DSM programs are designed to modify consumption patterns and increase the efficient use of electricity. As a result, these programs may play a cole in changing the consumers' average consumption and average revenue per kilowatthour. The recently enacted Energy Policy Act of 1992 contains a section that promotes conservation and energy efficiency by encouraging utilities to $e x$ pand DSM programs. It requires utilities and public ut lity cornmissions to consider requiring two new Fed. eral standards that encourage DSM: (1) integrated resource planning, which includes consideration of demand-side options with supply-side options, and (2) cost recovery for energy efficiency prograns that allows their profitability to be at least as attractive strpply-side option profitability.

Public concerns and stricter Federal and State envirofunental standards have forced electric utilities to reduce the release of air pollutants from power plents. The Clean Air Act Amendments of 1990 will result in the installation of additional pollution control and/or monitoring equipunent and potential shifting to relatively higher cost, lower salfur fuels that will eventually affect utility revenue requirements and may increse electricity rates.

Federal electric ntilities generally have the lowest average revenue per kilowatthour for the ownership classes because they have access to rebatively low-cost financing and generally utilize less expensive hydro. electric facilities. Because publicly owned electric utilities also have acoss to relatively low-cost jnancing and are nonprofit entities, they have lower average revenue per kilowatthour than investor-owned electrite utilities. Although cooperative electric utilities have econonic advantages similar to those of the publicly owned electric utilities, they generally serve sparsely 
Table 11. U.5. Average Revenue per Kilowatthour by Sector and Class of Ownership, 1989 Through 1993

(Cents)

\begin{tabular}{|c|c|c|c|c|c|}
\hline Item & 1993 & 1902 & 1991 & 1950 & 1996 \\
\hline 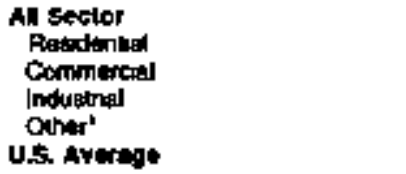 & $\begin{array}{l}830 \\
774 \\
485 \\
689 \\
6.85\end{array}$ & $\begin{array}{l}121 \\
786 \\
483 \\
674 \\
6.62\end{array}$ & $\begin{array}{l}604 \\
759 \\
480 \\
5 \$ 1 \\
6.75\end{array}$ & $\begin{array}{l}768 \\
734 \\
474 \\
640 \\
6.57\end{array}$ & $\begin{array}{l}705 \\
720 \\
472 \\
624 \\
6.46\end{array}$ \\
\hline 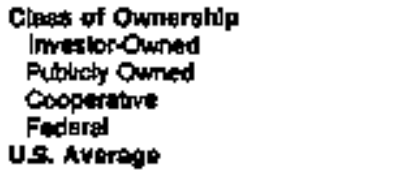 & $\begin{array}{l}716 \\
609 \\
700 \\
277 \\
600\end{array}$ & $\begin{array}{l}705 \\
600 \\
699 \\
280 \\
6.12\end{array}$ & $\begin{array}{l}698 \\
689 \\
680 \\
279 \\
675\end{array}$ & $\begin{array}{l}677 \\
698 \\
662 \\
307 \\
657\end{array}$ & $\begin{array}{l}660 \\
602 \\
680 \\
364 \\
6.45\end{array}$ \\
\hline
\end{tabular}

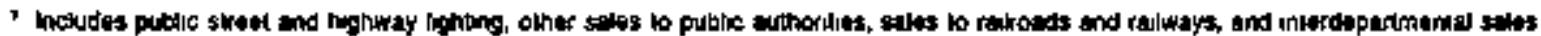

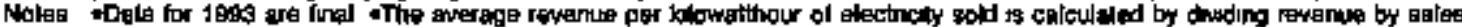

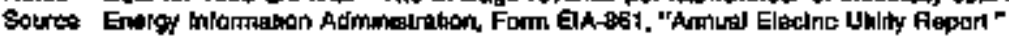

populated areas and provide service to a higher percentage of rura] residential customers than other classes of utilities. As a consequence, cooperative electric utilities generally have a higher average revenue per kilowatthour than do publicly owned utiluties.

Because of the type and avalability of capacity and the cost of fuel, the average revenue per kilowatthour differs across U. S. Census divisons. Petroleura is generaily a more expensive energy source than coal and natural gas. Therefore, the New England and Middle Atlantic Census Divisions tend to have an average rev. enue per kulowatthour that is bugher than the national average because of their reljance on petroleurn, whereas the East and West South Central Divisions rely on gas-fired generation and the East North Central and South Atlantic Census Divisions rely on coal-fired generation. Because the Mountain Census Division relies on less expensive hydroelectric generation, the average revenue per kilowathour in this region is usually below the national average for all classes of consumers. The Census divisıons where Federal hydroelectric facilities provide significant amounts of electricity, such as the East South Central Census Division, also have lower than average revenue per kilowatthour. ${ }^{2}$

Tables II through 13 contain summary statistics on the average revenue per kilowatthour of electricity sold at the national, Census division, and State level by sector and class of utility ownership. Data for the four power authorities of American Samoa, Guam, Puerto Rico, and the Virgin Islands are not included in the sommary statistics. 
Fgure 13. Average Revenue per Kilowatthour for the Residential Sector by State, 1993

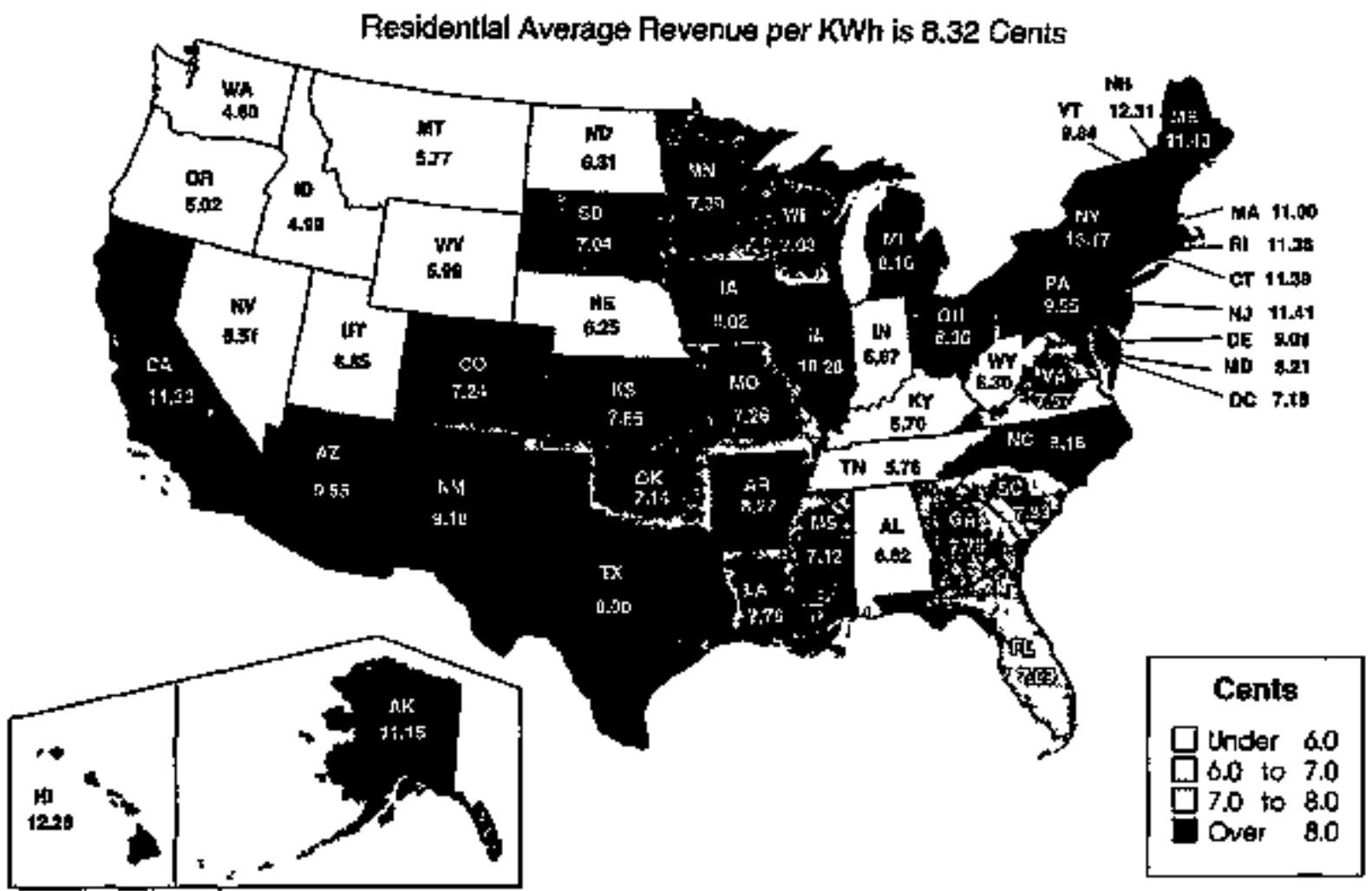

KWh = KNlowallhour.

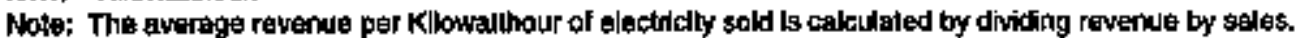

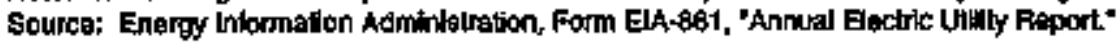

Figure 14. Average Revenue per Kllowatthour for the Commercied Sector by State, 1993

Commercial Average Reverue per KWh is 7.74 Cents

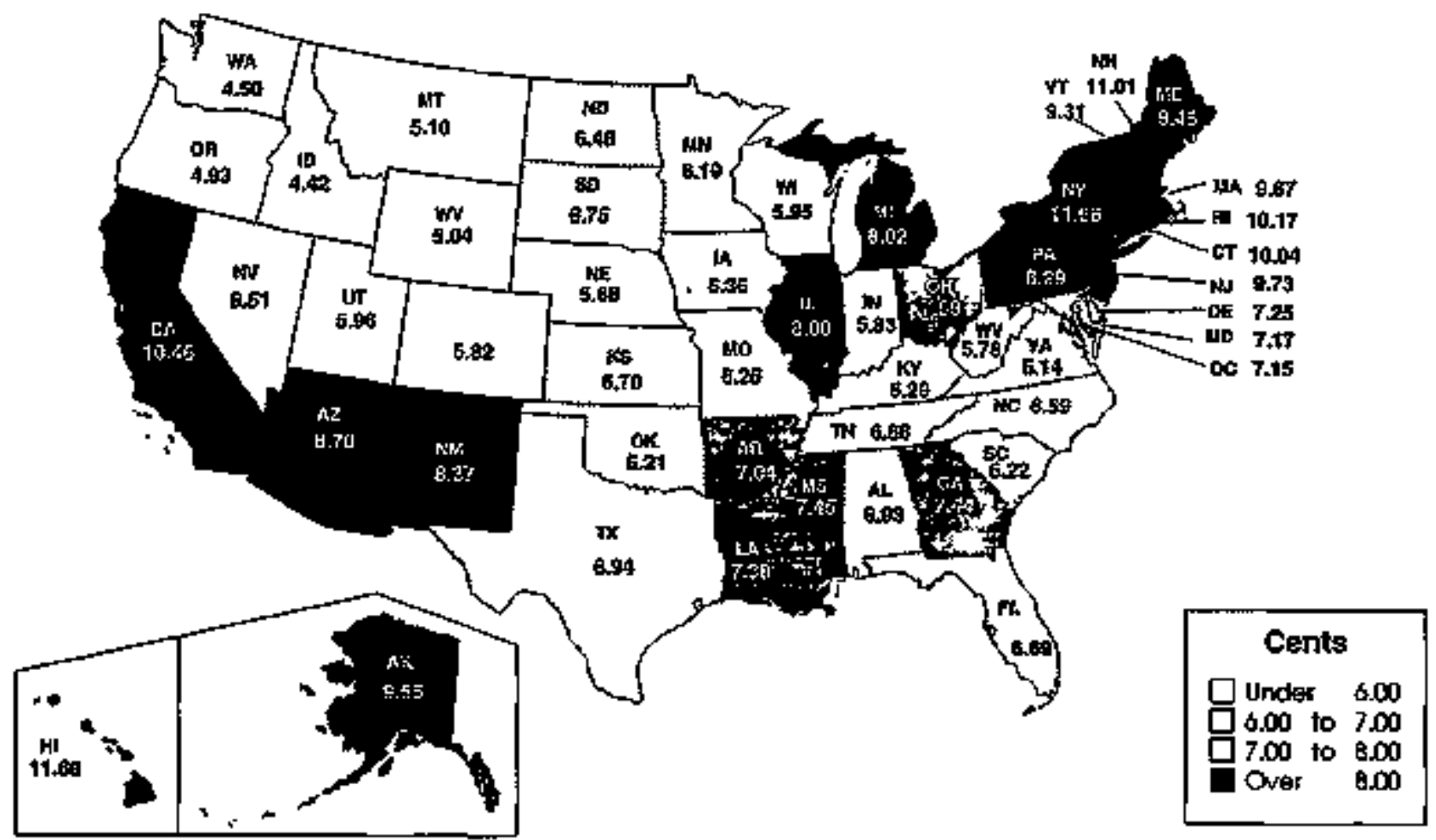

KWh = Klowalthour.

Note: The average reverule per Kilowaltiour of electricily sold is calculabed by diniding rewathe by sales.

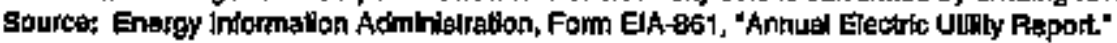


Flgure 16. Average Revenue per Kilowatthour for the Industrial Sector by State, 1993

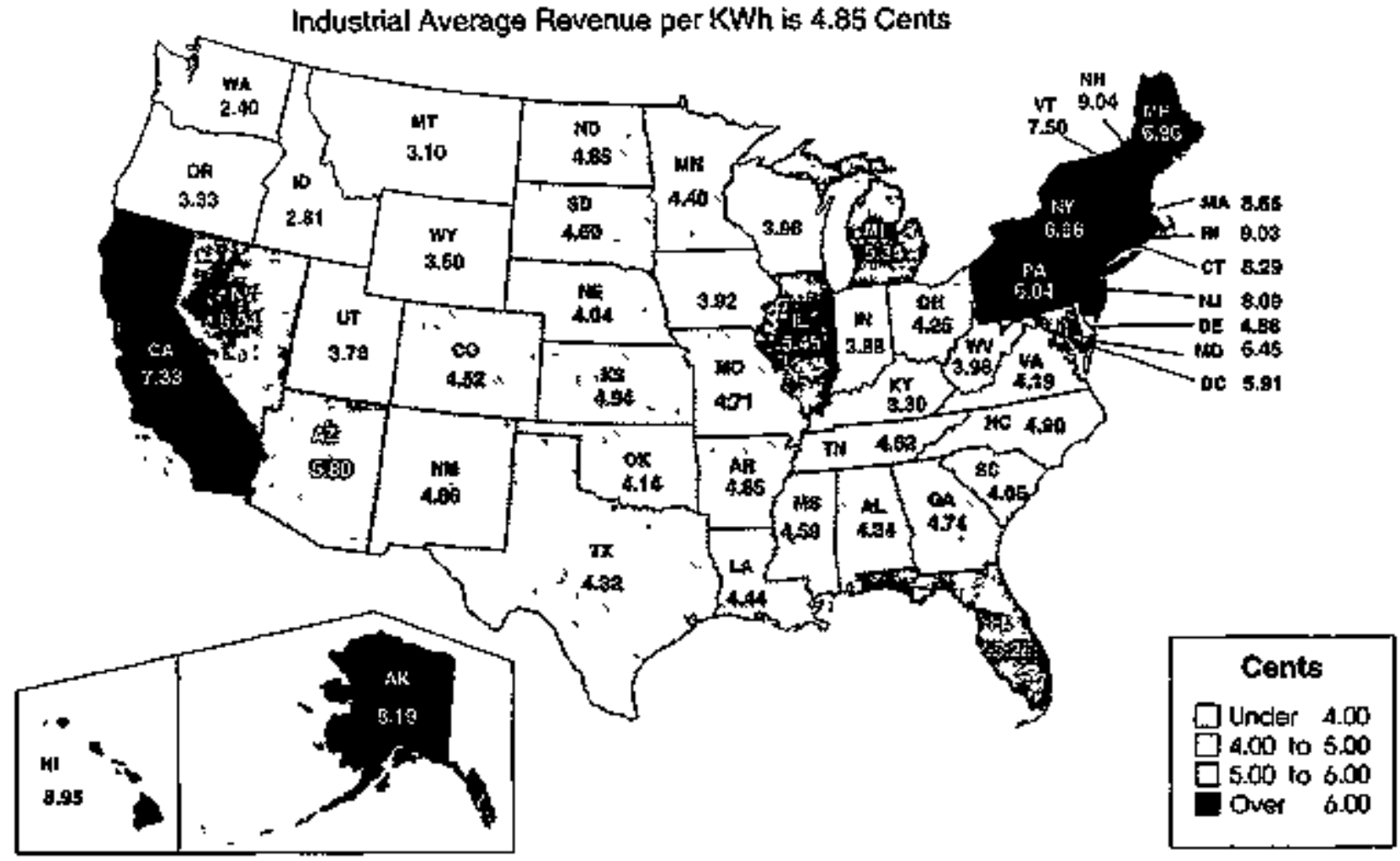

KWh = Kilowralthour.

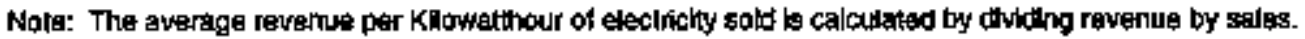

Source: Energy Inlormalion Admirustration, Ford EAA-861, "Annual Electric Uality Report."

Figure 16. Average Revenue per Kilowatthour for All Sectors by State, 1998

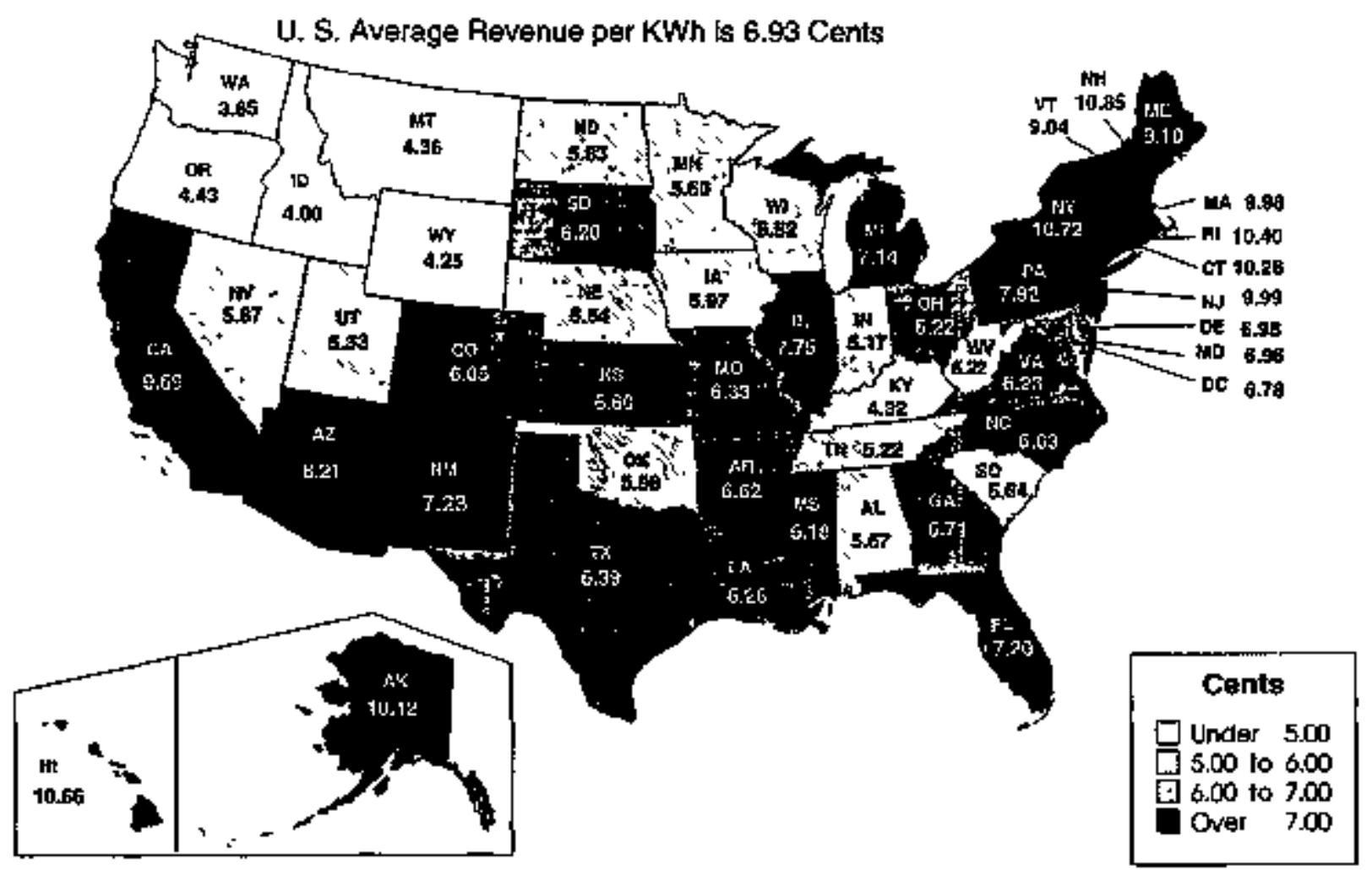

KWh $=$ Kliowatthour,

Note: The average revenue per Kilowathour of electrically sold is calculated by diving reverue by gales.

Source: Energy Information Adrinisiration, Fom ElA-361, "Annual Electric ulitity Report: 
Table 12. Average Revenue per Kllowatthour by Sector, Census Division, and State, 1993 (Cents)

\begin{tabular}{|c|c|c|c|c|c|}
\hline $\begin{array}{c}\text { Crinut Dirialon } \\
\text { stats }\end{array}$ & Poatidental & Commereted & Indunitrte & Qundr' & Al suctort \\
\hline 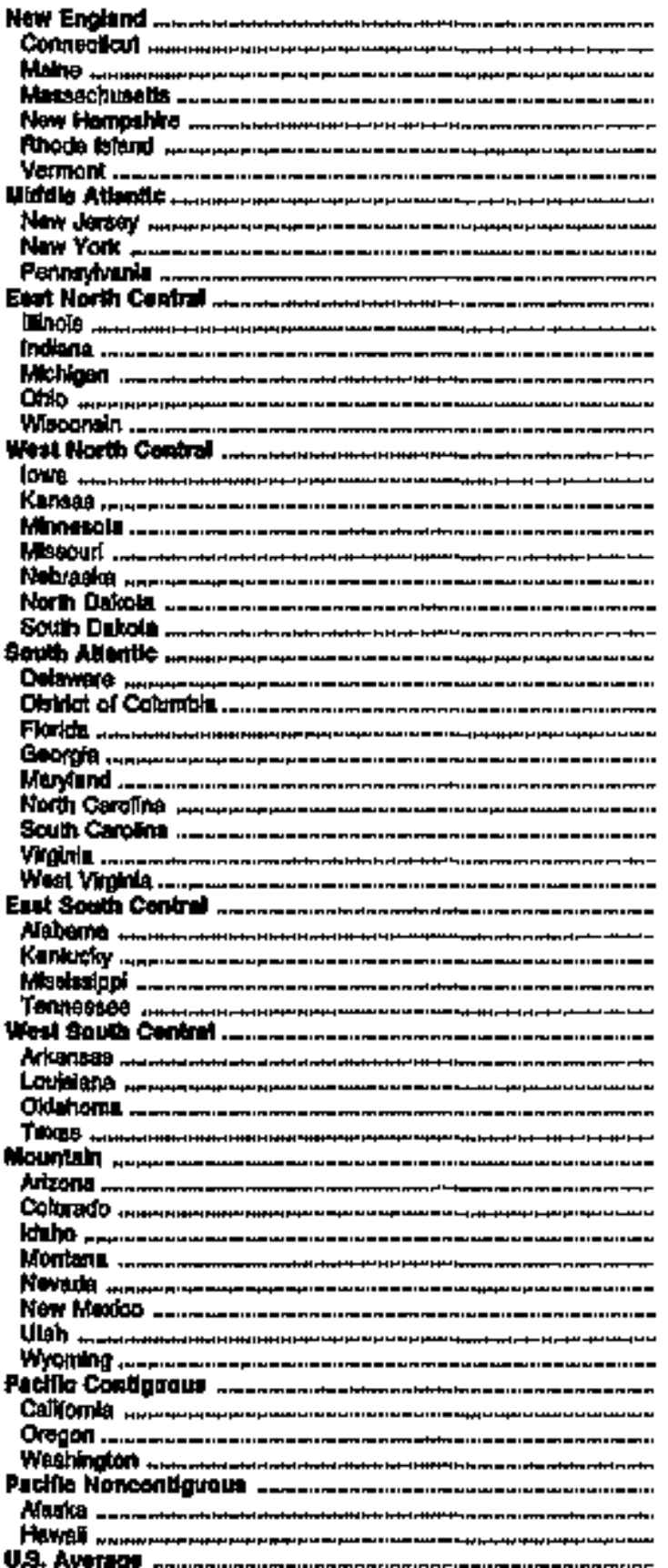 & 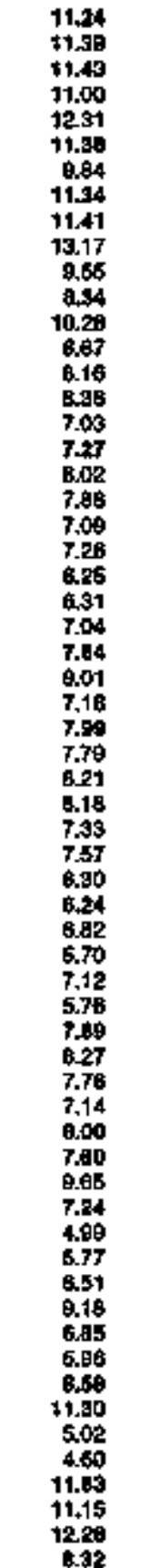 & 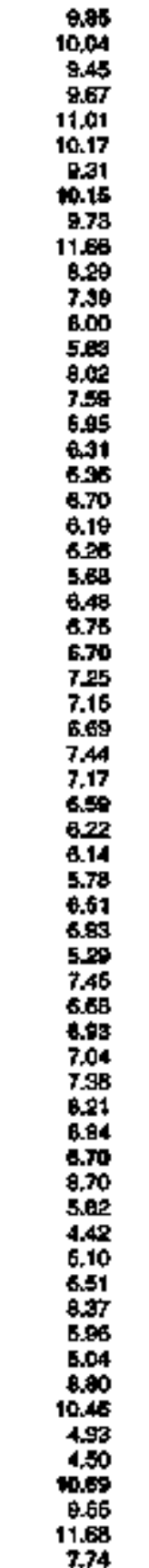 & 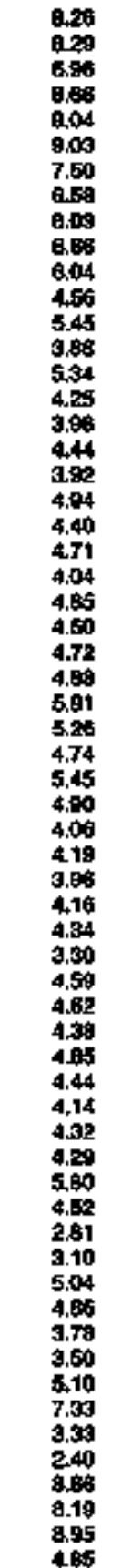 & 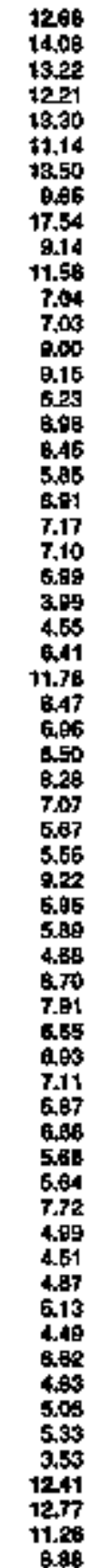 & 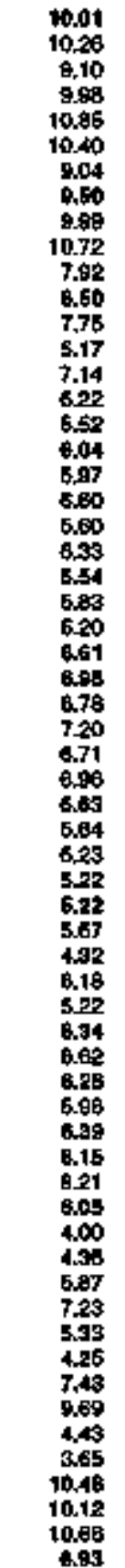 \\
\hline
\end{tabular}

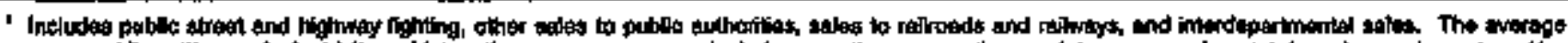

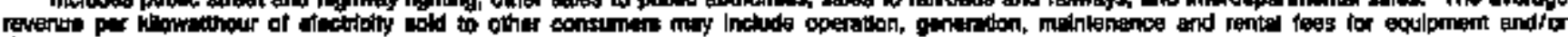

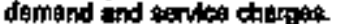

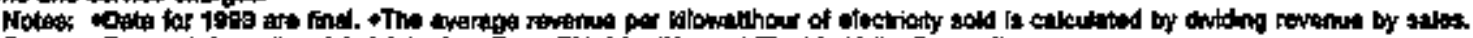

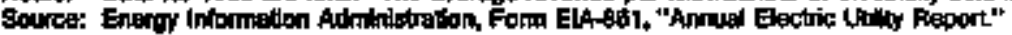


Table 13. Average Revenute per Kilowatthour by Class of Ownership, Census Divlsion, and State, 1993

(Cents)

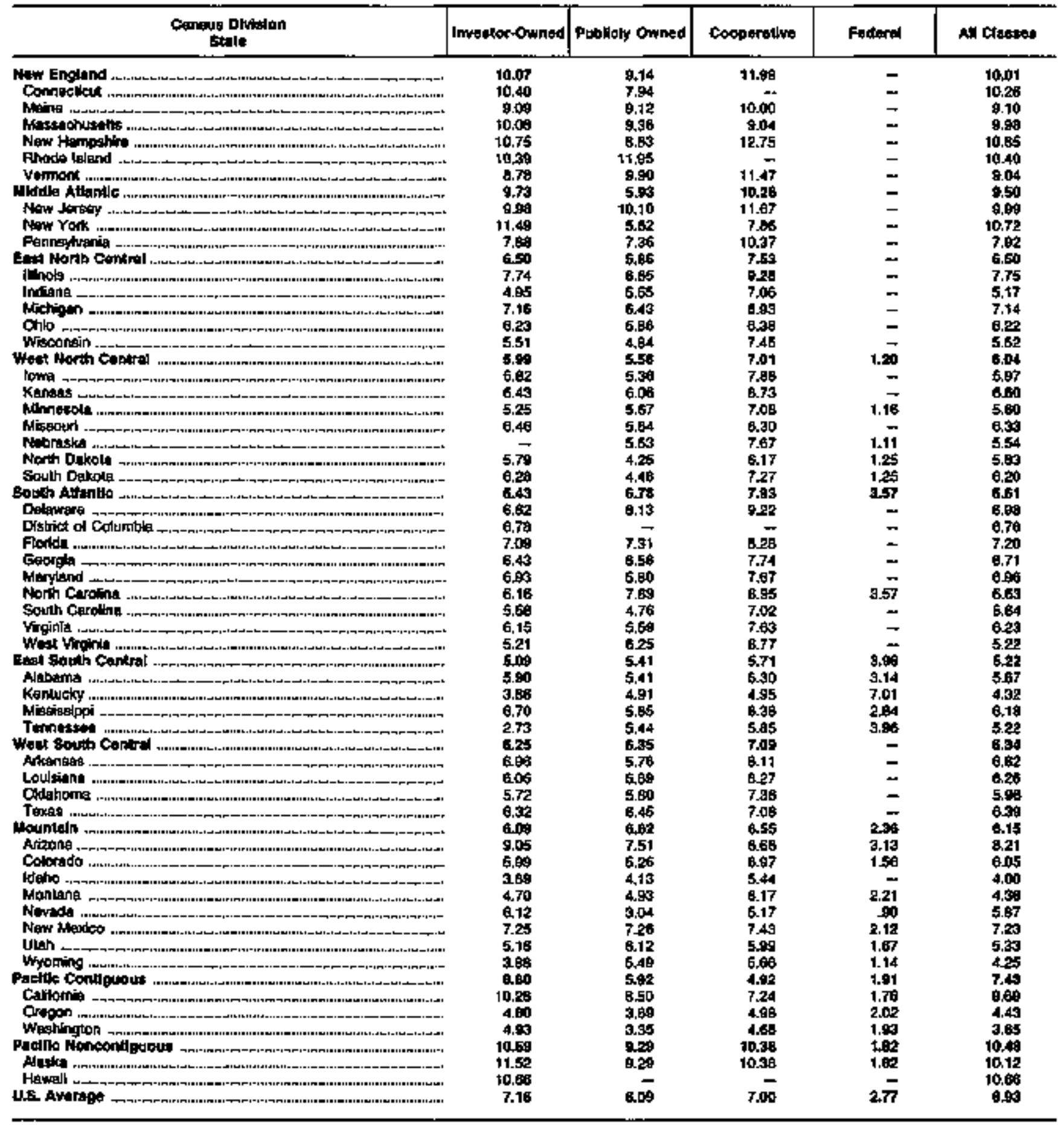

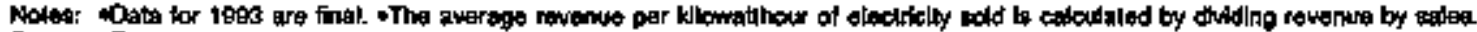

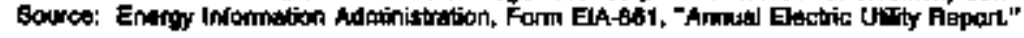




\section{Average Revente per Kllowatthour at the Electric Uthty Level}

The average revenue per kilowathour of electricity sold for each utility is calculated in this report by dividing the total annual revenue of the electric utillty by its total annual sales of electricity across all sectors. Because the average revenue per kilowatthour varies between sectors, the difference in average revenue per kilowatthour between utilities may represent a difference to their proportion of sales in the different sectors. Accordingly, the average revenue for a given utility does not reflect the differences that may exist in its revenue structure across sectors. The average revenue per kilowat thour at the utility level is usually between 4 and 12 cents per kilowatthour, but the average may be larger or smatler for a particular sector, ownership type, or State.

Cooperative electric utilities are owned by their members and are established to provide electricity to those Inembers. These electric utilities operate in rural areas with a lower concentration of consumers because these areas were historically viewed as uneconotnical operations for investor-owned electric utílities. Cooperative electric utilitites generally bave broader service areas with smaller consumer bases than other ownership types and, as a consequence, they tend to have higher average revenue per kilowatthour. Cooperative electric utilities that serve consumess in multiple States tend to have even broader service areas and higher average reventse per kilowatthour. Average cents per kilowatthour are not provided in Tables 14 through 17 for those coaperative electric utilities or other electric utilities with revenue below $\$ 100,000$ or sales beJow 1,000 megawatthours.

For example, the Columbus Electric Cooperative, Incorporated, in New Mexico serves consumers in New Mexico as well as in Arizona. The residential average revenue per kilowatthour in Table 14 is 13.1 cents in New Mexico and 12.7 cents in Arizora. The commercial average revenue per kilowatthour in Table 15 is 11,6 cents for New Mexico. The commercial ayerage revenue per kílowatthour is not provided for Arizona because the electric utility reported either less than $\$ 100,000$ of revenue or 1 million kilowatthours of sales in this State.

Of the 83 reporting electric utilities in Alaska, 81 utilities have average revenue per kilowatthour exceeding the U.S. average. This occurs in part due to the Jack of economites of scale in Alaska and because of the high costs associated with genefation and distribution. The electric utilities that bave higher costs charge higher rates for electricity to recover these costs from their consumers. The Power Cost Equalization (PCE) subsidy by the State of Alaska was initially destigned to compensate consumers served by electric utilitites located in rural areas where transmission access is litm- ited and electric power generation is with expensive diesel gas turbhes. The Alaska Energy Authority (AEA) administers the subsidy with funds from a State statute since Alaska has no State jucorne tar. Electric utilities qualitying for the PCE program are required to notify consumers of the cost of electric service and the cost minus the State equalization. Consumers receiving the subsidy do not actually pay the high rates. The subsidy is allocated to thectric utilities based on consumer sector and demand for electricity within each sector. In Tables 14 through 17, the PCE subsidy bas not bees subtracted from the total revenue of those electric uttlities receiving cormpensation for high enexgy costs.

Electric utilities operating on islands with high seasonal populations also tend to have much higher average revenue per kilowatthour. Higher demand charges are needed to recover the fixed equipment and operating costs associated with generating electricity at infrequent times. These electric utilities are few and are primarily located on the East Coast and in the Great Lakes area.

Data on the number of consumers, revenue, sales, and average revenue per kilowatthour for individual electric utilities are contained in Tables 14 through 17. The average revenue per kilowatthour presented in Tables 14 through 17 are based on an average for each of the end-use sectors. When an electric utility provided service to ultimate consumers in more than one State, that utility is listed under each State it serviced. The electric utlities in Table 14 through 17 that provided service to altimate consumers in more than one State and their percent of sales in each State are shown to Appendix A. Tables 14 through 16 contain the respective consumers, sales, revenue, and average revenue per kilowatthour by each sector for alt electric utilities. Table 17 contains the respective sales, revenue, and average revenue for the stun of al] sectors. This inclades the residential, conmercial, and industrial sectors, as well as public street and highway lighting, interdepartmental sales, sales to railroads and ratilways, and sales to other public anthorities.

Electric revenue and sales data are reported by electric utilities in thousand dollars and thousand kilowathours. Average revenue per kilowatthour for electric utilities with either less than $\$ 100,000$ of revenue or less than 1 million kilowatthours of sales is not provided becalise the significance of these data is not sufficient to make the ratio meaningful. See Appendix $B$ for further information.

Data Sources. Statistics contained in Tables 11 through 17 are based on data collected on the Form EIA-861. "Annual Electric Utility Report." Data for the four power authoritic of Amertican Samos, Guan, Puerto Rico, and the Virgin Islands are inciuded only in $\mathrm{Ta}$. bles 14 through 17 , but not in the summary statistics. 
Tahle 14. Class of Ownershlp, Number of Ultimate Consumers, Revenue, Sales, and Average Revenue per Kllowatthour for the Residential Sector by State and Utility, 1993

\begin{tabular}{|c|c|c|c|c|c|}
\hline Elate Uality & $\begin{array}{c}\text { Cla:es } \\
\text { of } \\
\text { Cwinurahlp }\end{array}$ & $\begin{array}{c}\text { Number } \\
\text { of } \\
\text { Contumbert }\end{array}$ & $\begin{array}{c}\text { Rovthus } \\
\text { (thouland doilass) }\end{array}$ & $\begin{array}{c}\text { setes } \\
\text { thoupgand } \\
\text { ulowatthourt) }\end{array}$ & $\begin{array}{c}\text { Averago Aewenus } \\
\text { per klowatthour" } \\
\text { (comtol }\end{array}$ \\
\hline 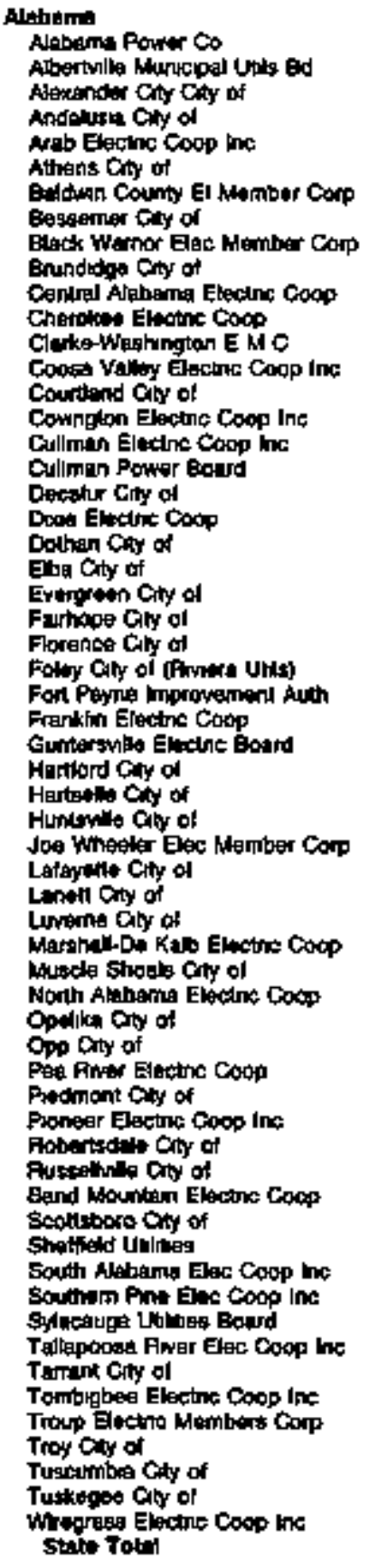 & 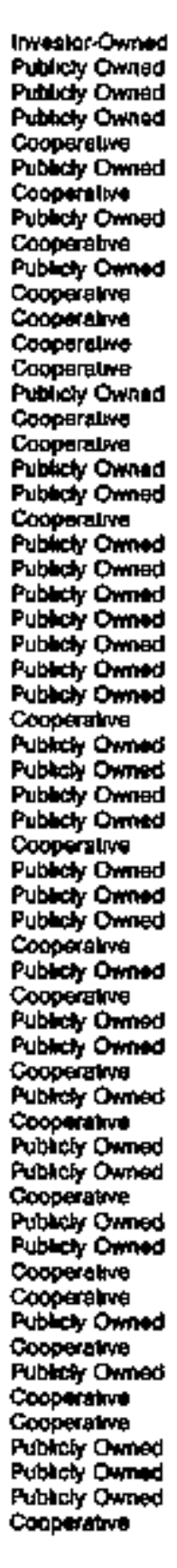 & 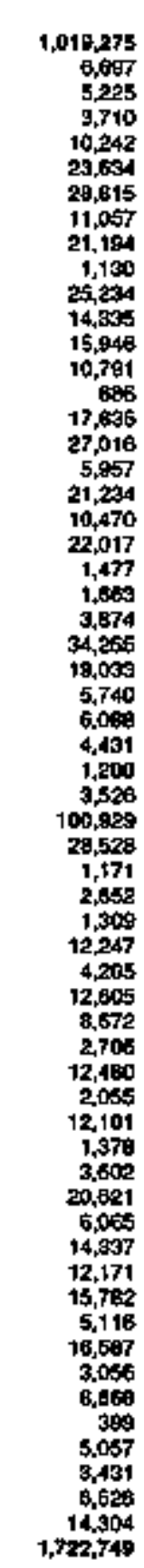 & 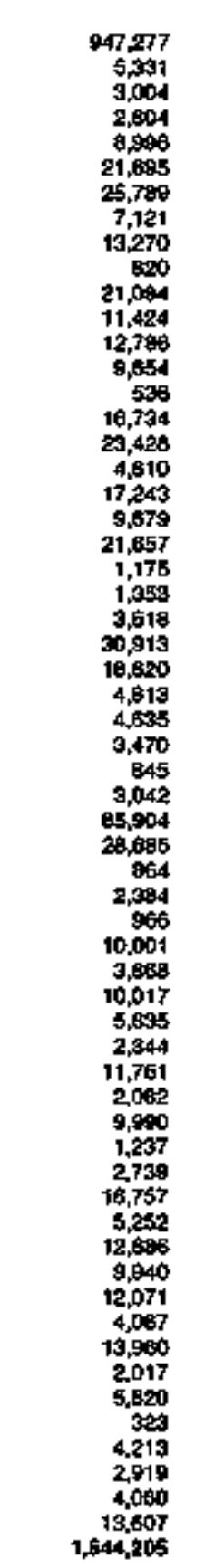 & 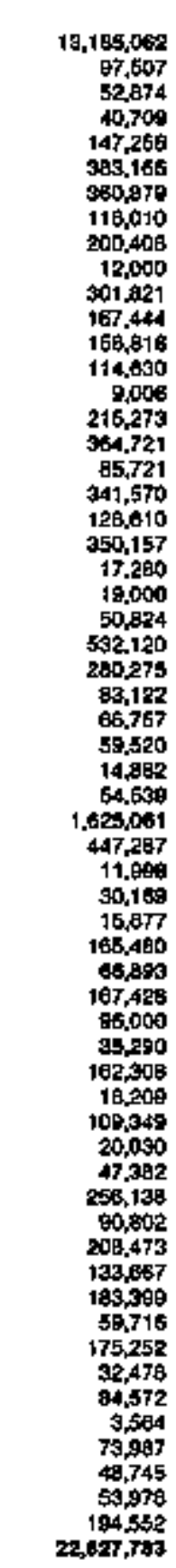 & 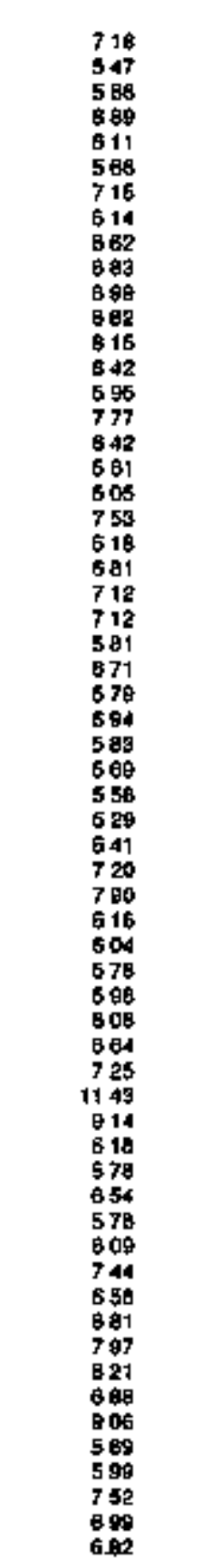 \\
\hline 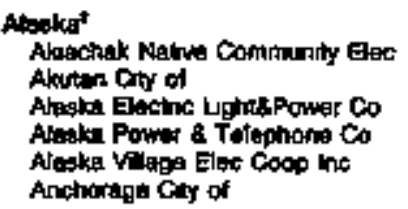 & 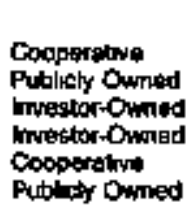 & $\begin{array}{r}138 \\
30 \\
11,052 \\
1,721 \\
4,408 \\
23,080\end{array}$ & $\begin{array}{r}198 \\
67 \\
10,977 \\
1.713 \\
7,376 \\
14,052\end{array}$ & $\begin{array}{r}368 \\
160 \\
127,329 \\
9,156 \\
17,445 \\
145,354\end{array}$ & $\begin{array}{l}17 \\
17 \\
862 \\
1671 \\
428 \\
967\end{array}$ \\
\hline
\end{tabular}

Sen foctrolas ax end ol lable 
Table 14. Class of Ownership, Number of Ultimate Consumers, Revenue, Stles, and Average Fevenue per Klowathour for the Residentlal Sector by State and Utility, 1993 (Continued)

\begin{tabular}{|c|c|c|c|c|c|}
\hline $\begin{array}{c}\text { stato } \\
\text { elaterto Utinty }\end{array}$ & $\begin{array}{c}\text { chito } \\
\text { of } \\
\text { omingrath }\end{array}$ & $\begin{array}{c}\text { Numer } \\
\text { of } \\
\text { Contamara }\end{array}$ & Roweras & 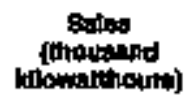 & 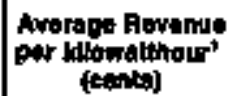 \\
\hline
\end{tabular}

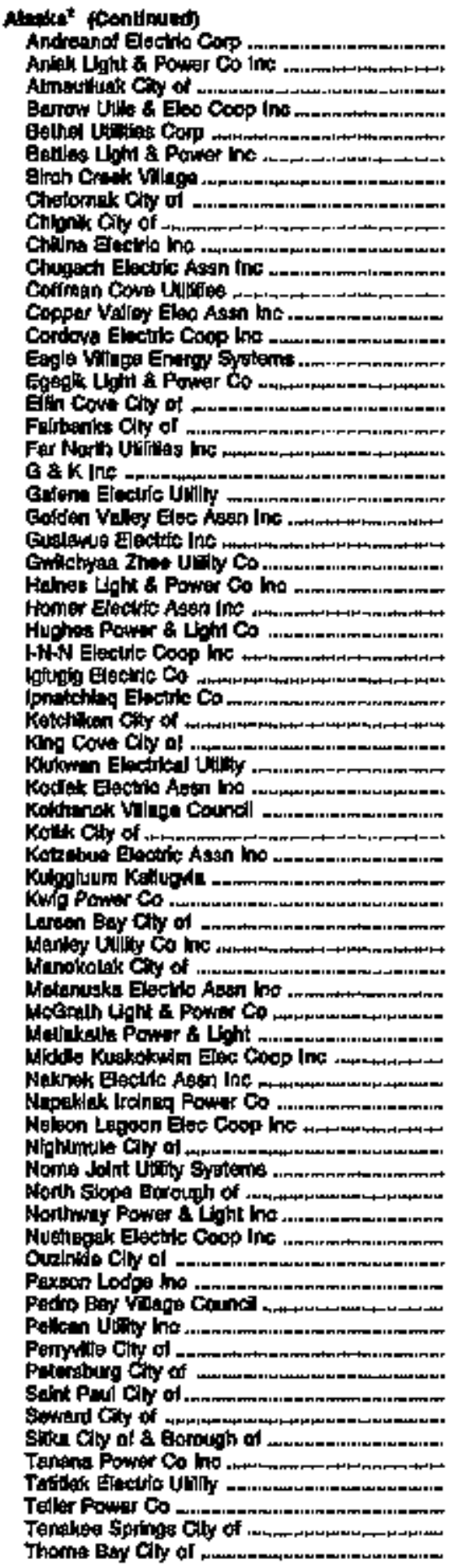

Itrestorominted Inestor-Owned Putichy Ouned Cocosidstive

investur-Owned

Irvestorsowisted

Putricty onmod

Publity Orrad

Puthicty Owmed

Invastorowited

Cooperalvo

Publicty Oumed

Cospontivo

Cooporame

Publich Ownod

Irivestertowned

Pubilcty ormind

Publicty Oumad

Imvecturomitom

Imwastor-Owne:

Publchr Ownod

Coopenthe

investar-0umed

investor-Oined

inwestor-Owned

Couparatine

invellor.0ined

Cooperatue

Pulbisty Owned

Pubichy ommo

Fublich Owmed

Pableky Owine

Publich Ownod

Copoporaliva

futilety Owined

Fublicty Owned

Coopurative

irvestor-Onned

Acibict owned

Puttiely Owned

mresslor-Omined

Dublesty Owned

Copperatue

tmvistor+Ome

Publecty Owned

Cooperathe

copoting

trweskor-Onned

Cooperathes

Pulbily Owned

Fublut Dwned

Publisty owised

investor-Omnod

Cooperatwa

Publesty Owned

Cocporative

Publietry Owned

invalor Onmod

Publicht Dwmed

Pistich Owned

Publiat Owned

Fublith Ownod

Pulglect Owned

Invétion-Oment

invetor-Oinnod

timestor-Omed

Fublity Oumbid

Publes Owned

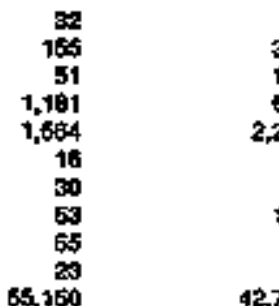

65,250

2351

933

$\mathbf{5 0}$

24

4,473

48

202

23,849

200

239

$18,+69$

28

176

$\mathbf{5 , 7 0 0}$

5,700

36

4.080

37

114

172

139

6

52

27,764

160

ses

136

543

36

32

1,379

sas

96
856

71

4

193

34

1.181

138
1,410

2.951

451

36

84

194
2,2

350

615

32

12

61

788

80
2789

1,282

41
121

$\begin{array}{r}121 \\ 22 \\ \hline\end{array}$

2,558

35

152

241

21,095
279

294

701

15.464

12

20

69

4,501

70

4,224

57

143

1,577

129

28

65

150
3.456

,

275

645
197

79

156

100

55

2,422

901

1,031

78

3

106

81

1,035

370

$1,5=6$

3,025

258

B1

140
$5 \mathrm{~B}$

241
85

243

7,270

0,170

67

20
914

$2 T s$

66

453,487

359

14,689

5.616

20

89

$20,9 \%$

7

419

309,166

602

40 in

130,143

89

34

170
55,006

t.107

400

$27, a 52$

102

300

6,155

514
517

190

177

997

253,375

acs

B.950

336
3678

208

145

6.098

9,741

387
6.157

24

68

$15 \%$

10322

1,160

14,139

38,498

657

168

25

175

92

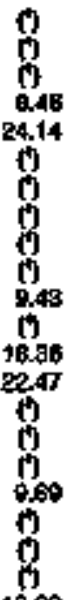

i)

17.12

11.97

$\phi$

0

635

(1)

15

20.75

(1)

10

(1)

10.82

1.5

ti)

21.7)

8

175

24.16

19

H

8

10.03

11.01

780

4

8

Soe foohrotes of end of table. 
Table 14. Class of Ownership, Number of Ultimate Consumers, Revenue, Sales, and Average Revenus per Kilowatthour for the Residential Sector by State and Utilty, 1993 (Continued)

\begin{tabular}{|c|c|c|c|c|c|}
\hline Etente & $\begin{array}{l}\text { Claps } \\
\text { of } \\
\text { ownerahip }\end{array}$ & $\begin{array}{l}\text { Namber } \\
\text { of } \\
\text { Consuners }\end{array}$ & $\begin{array}{c}\text { Rewerpus } \\
\text { [thoutsand doltats] }\end{array}$ & 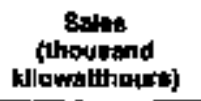 & $\begin{array}{l}\text { Aworape Rovemue } \\
\text { per kllowathour } \\
\text { (onnk) }\end{array}$ \\
\hline 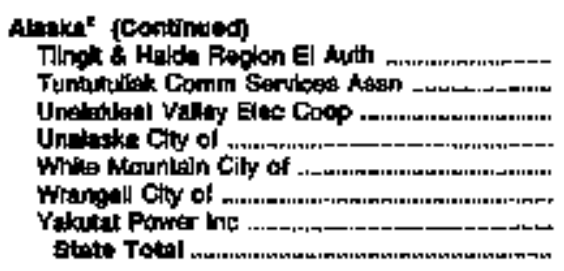 & 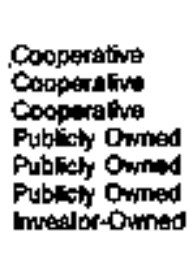 & $\begin{array}{r}1,025 \\
70 \\
246 \\
412 \\
84 \\
840 \\
939 \\
242,132\end{array}$ & $\begin{array}{r}1,721 \\
132 \\
964 \\
828 \\
96 \\
679 \\
377 \\
191,608\end{array}$ & $\begin{array}{r}5,695 \\
266 \\
1,468 \\
3,651 \\
257 \\
6,633 \\
1,654 \\
1,629,036\end{array}$ & $\begin{array}{c}30.27 \\
11 \\
20.00 \\
10.20 \\
10.24 \\
22.79 \\
11.16\end{array}$ \\
\hline 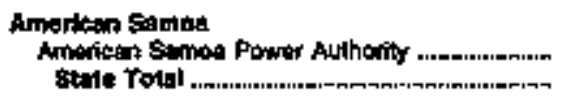 & Publisty Onned & $\begin{array}{l}7,2900 \\
7,260\end{array}$ & $\begin{array}{l}4.465 \\
4,485\end{array}$ & $\begin{array}{l}27.904 \\
27.904\end{array}$ & $\begin{array}{l}18.00 \\
16.00\end{array}$ \\
\hline 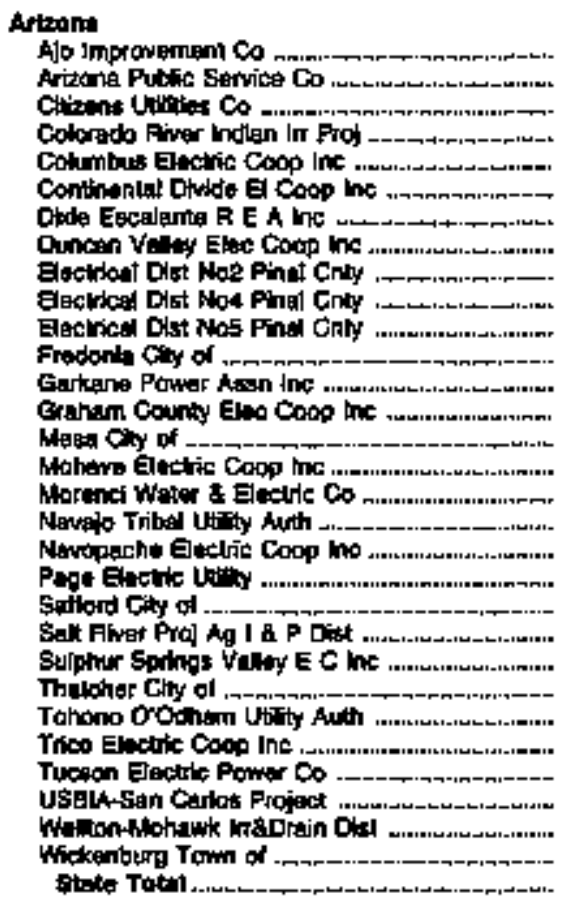 & 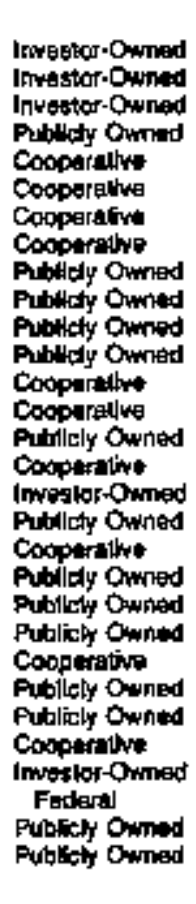 & 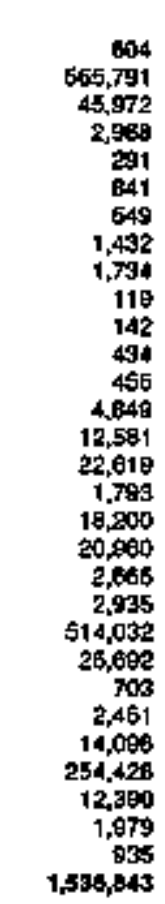 & 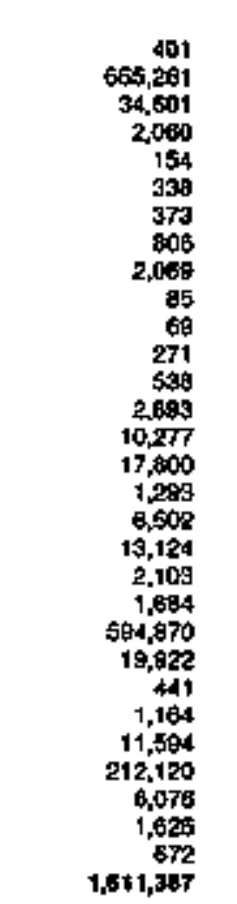 & 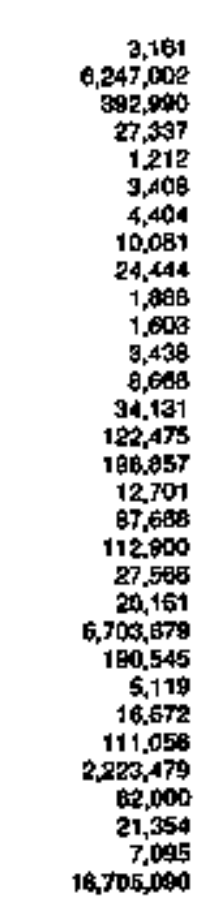 & 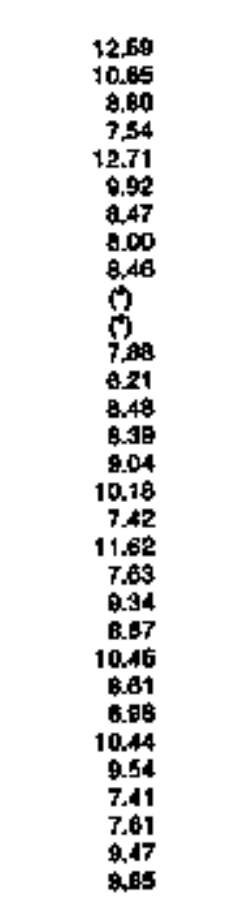 \\
\hline 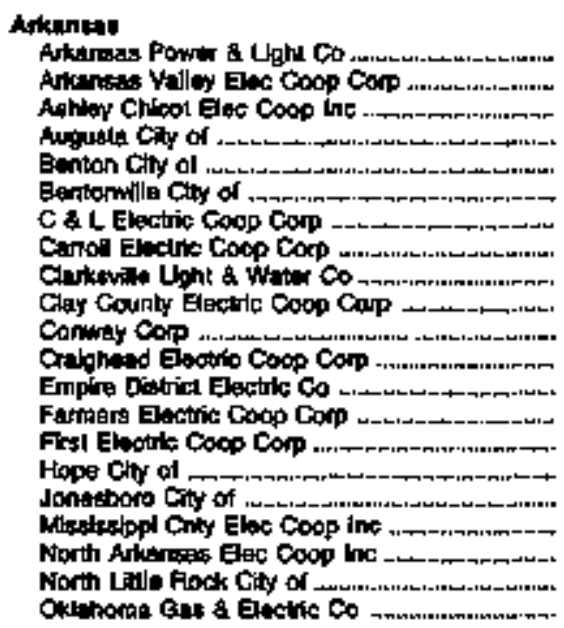 & 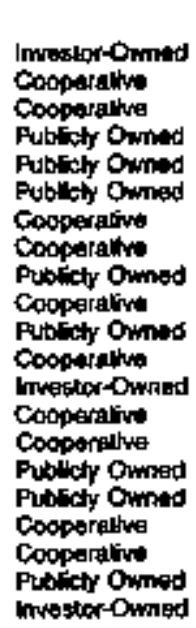 & $\begin{array}{r}505,291 \\
32,038 \\
3,335 \\
1,000 \\
7,542 \\
4,637 \\
16,927 \\
39,409 \\
2,669 \\
6,097 \\
12,142 \\
17,679 \\
2,586 \\
4,033 \\
50,698 \\
5,618 \\
20,113 \\
3,094 \\
22,023 \\
31,236 \\
16,159\end{array}$ & 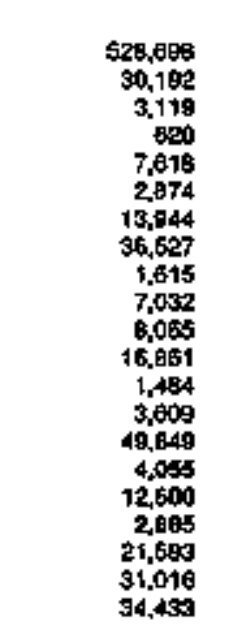 & 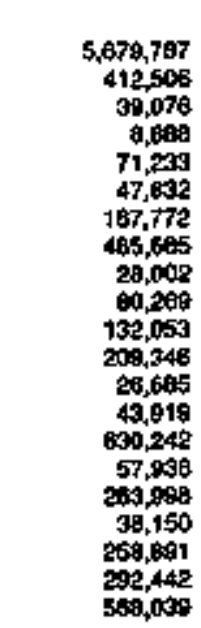 & $\begin{array}{r}8.31 \\
7.32 \\
3.90 \\
6.90 \\
10.69 \\
6.01 \\
6.31 \\
7.52 \\
5.77 \\
8.76 \\
6.11 \\
8.06 \\
5.56 \\
6.22 \\
7.91 \\
7.00 \\
4.77 \\
7.50 \\
8.34 \\
10.61 \\
6.06\end{array}$ \\
\hline
\end{tabular}

Serd foptuoles al and of labla. 
Table 14. Class of Ownershlp, Mumber of Ultimate Consumers, Revenue, Saltes, and Average Revenue per Kllowatthour for the Residential Sector by State and Utility, 1993 (Continued)

\begin{tabular}{|c|c|c|c|c|c|}
\hline 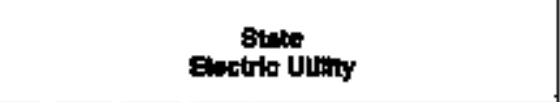 & $\begin{array}{c}\text { Clistip } \\
\text { of } \\
\text { Crinerathip }\end{array}$ & $\begin{array}{c}\text { Bhind } \\
\text { of } \\
\text { contumert }\end{array}$ & Rrewenus & $\begin{array}{c}\text { solvt } \\
\text { phoutpand } \\
\text { thowrathoure) }\end{array}$ & 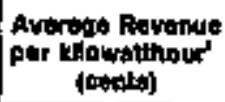 \\
\hline 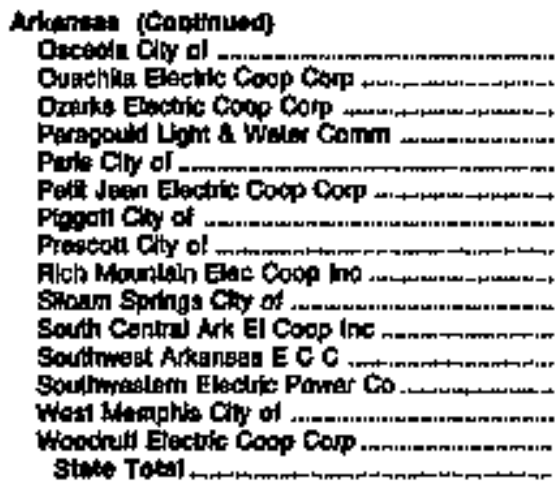 & 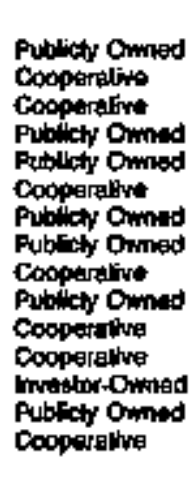 & $\begin{array}{r}3,177 \\
7,695 \\
20,647 \\
0,741 \\
1,500 \\
13,148 \\
1,655 \\
1,437 \\
5,891 \\
3,736 \\
7,090 \\
19,405 \\
74,589 \\
0,068 \\
12,704 \\
1,030,656\end{array}$ & 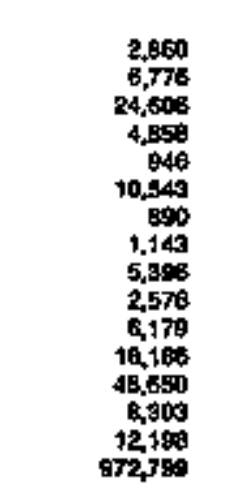 & $\begin{array}{r}49,625 \\
90,009 \\
342,2280 \\
125,165 \\
19,000 \\
129,325 \\
19,289 \\
11,501 \\
63,795 \\
39,478 \\
77,544 \\
232,023 \\
762,050 \\
130,650 \\
137,307 \\
11,764,761\end{array}$ & $\begin{array}{l}5.70 \\
8.46 \\
7.19 \\
3.89 \\
5.28 \\
8.15 \\
4.61 \\
9.92 \\
8.40 \\
8.59 \\
7.97 \\
7.04 \\
6.39 \\
8.38 \\
8.89 \\
9.27\end{array}$ \\
\hline 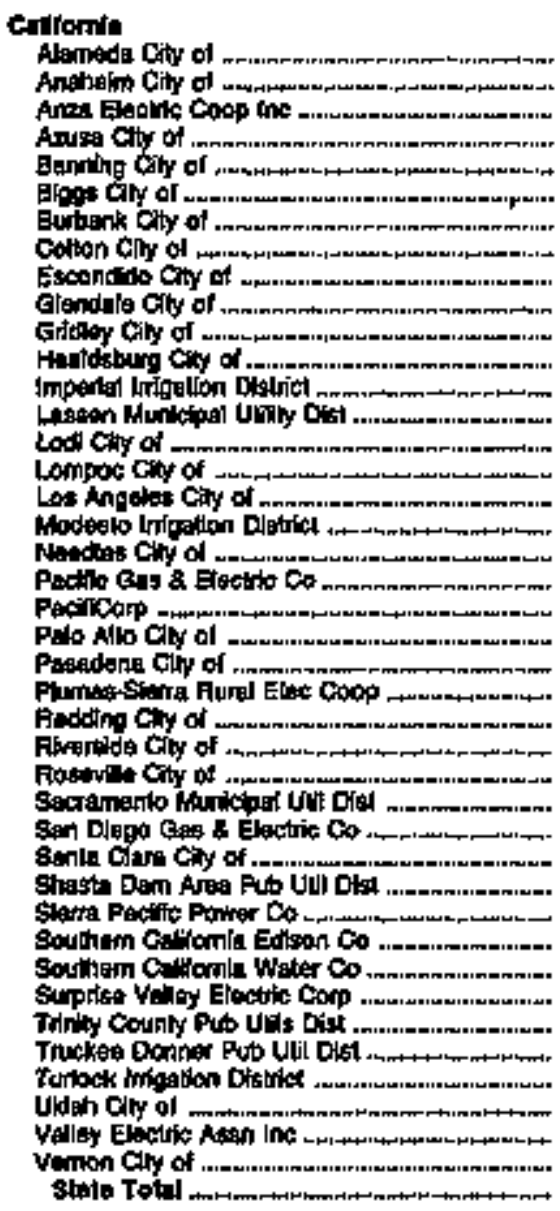 & 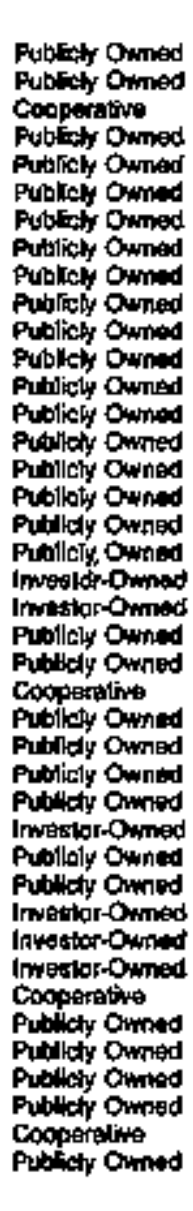 & 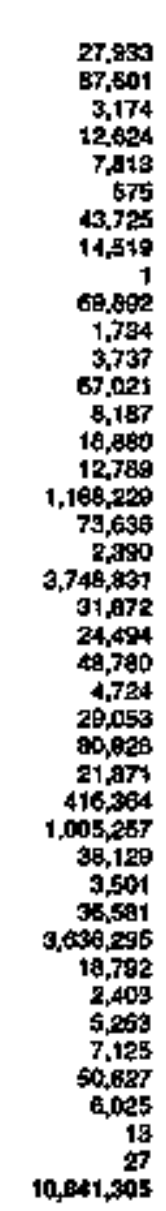 & 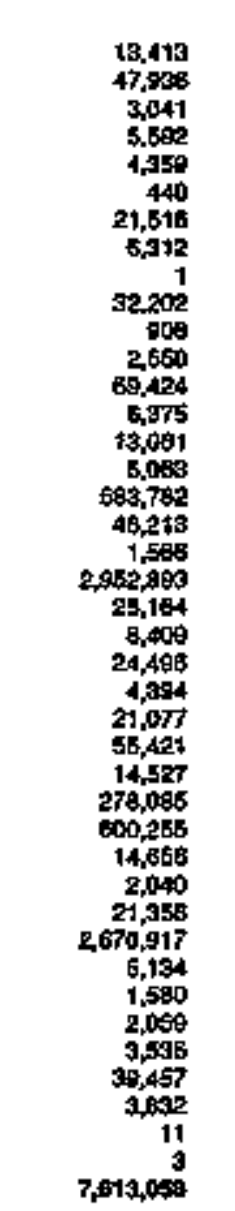 & 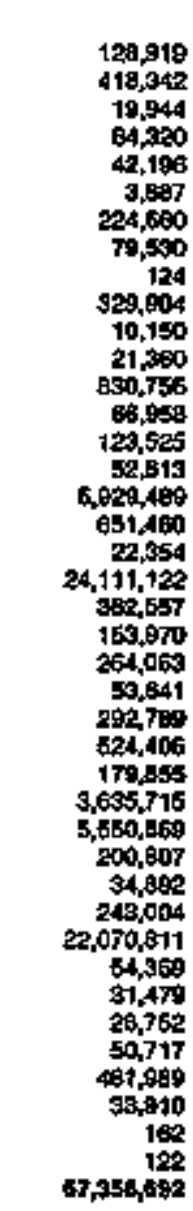 & 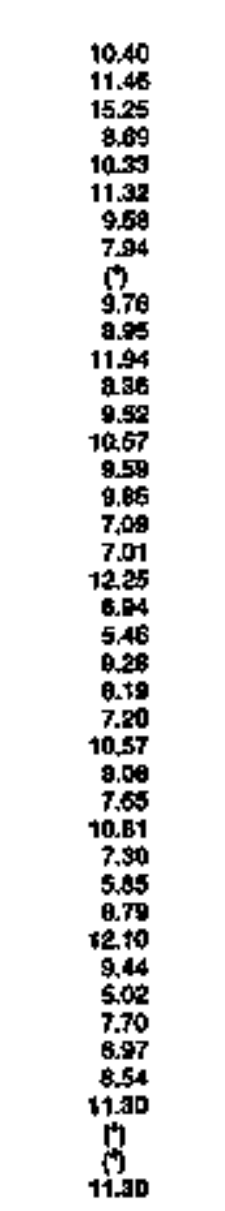 \\
\hline 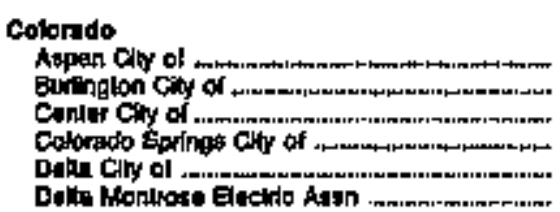 & 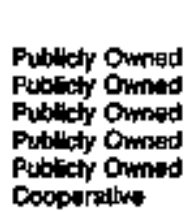 & $\begin{array}{r}1,470 \\
1,280 \\
776 \\
131,475 \\
1,849 \\
18,592\end{array}$ & $\begin{array}{r}1,189 \\
723 \\
312 \\
55,102 \\
14,201\end{array}$ & $\begin{array}{r}16,160 \\
8,243 \\
5,170 \\
951,650 \\
11,000 \\
160,258\end{array}$ & $\begin{array}{l}6.87 \\
8.77 \\
5.09 \\
5.79 \\
8.06 \\
\text { B.88 }\end{array}$ \\
\hline
\end{tabular}

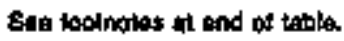


Table 14. Class of Ownership, Number of Uttimate Consumers, Revenue, Sales, and Average Revenue por Klowatthour for the Residential Sector by State and Utility, 1993 (Continuted)

\begin{tabular}{|c|c|c|c|c|c|}
\hline $\begin{array}{c}\text { Slate } \\
\text { Electric Iil|hy }\end{array}$ & $\begin{array}{l}\text { Colpest } \\
\text { of } \\
\text { Omeoratip }\end{array}$ & $\begin{array}{c}\text { Curmber } \\
\text { of } \\
\text { Cangenarg }\end{array}$ & $\begin{array}{c}\text { Rortulus } \\
\text { (thousand doltars) }\end{array}$ & 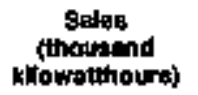 & $\begin{array}{c}\text { Avention Fownut } \\
\text { pw kllowalthour' } \\
\text { (Genta) }\end{array}$ \\
\hline 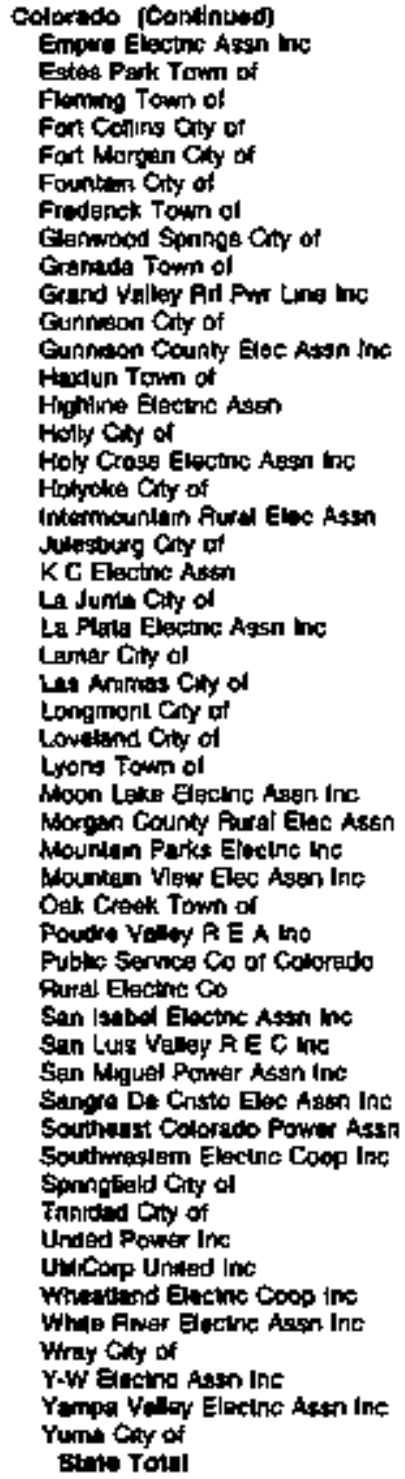 & 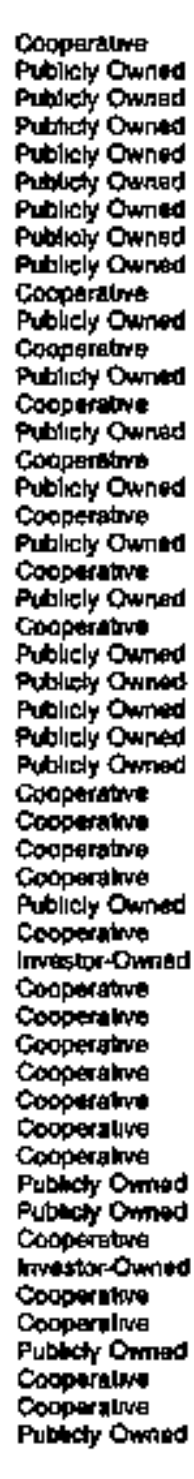 & 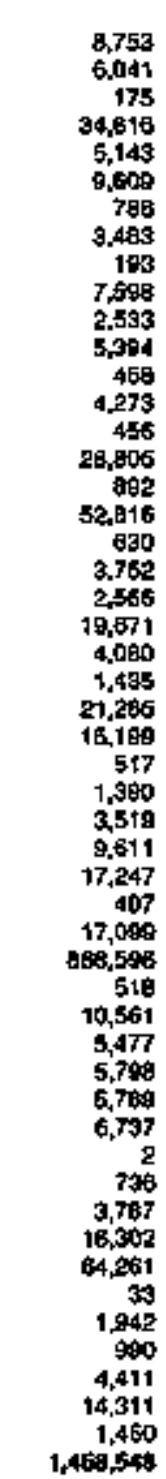 & 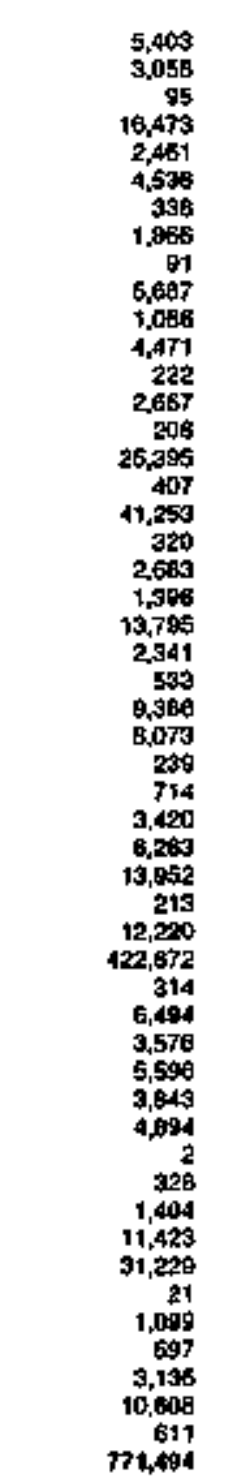 & 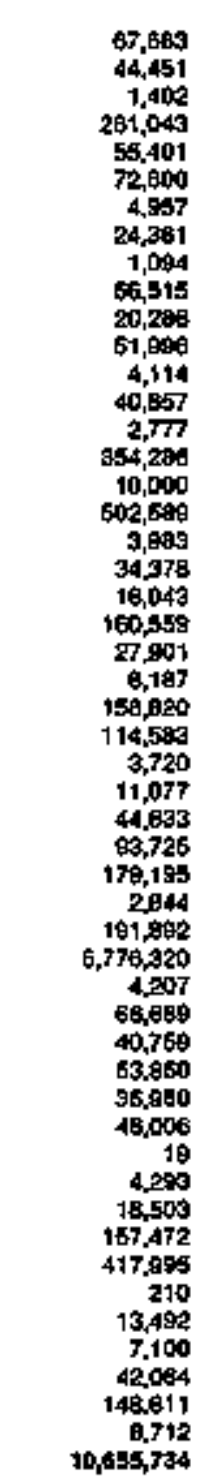 & 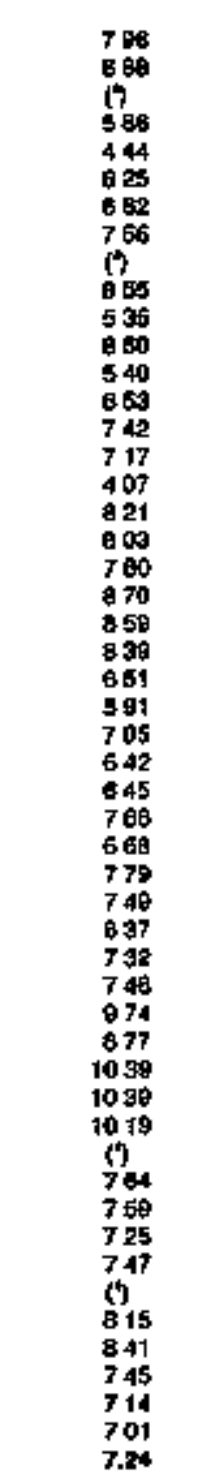 \\
\hline 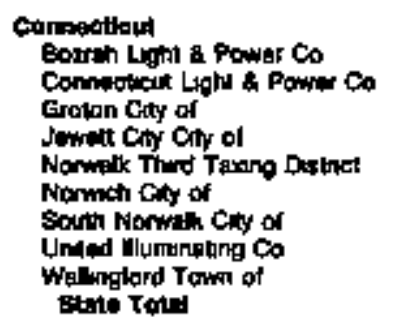 & 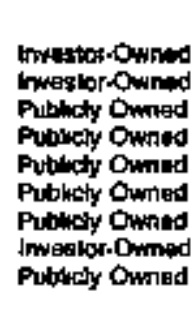 & $\begin{array}{r}1,966 \\
289,345 \\
9,817 \\
1,674 \\
2,950 \\
15,684 \\
4,574 \\
273,752 \\
18,724 \\
1,312,285\end{array}$ & $\begin{array}{r}1,720 \\
928,441 \\
9,151 \\
1,180 \\
2,460 \\
10,420 \\
2,722 \\
298,185 \\
19,171 \\
1,207,460\end{array}$ & $\begin{array}{r}17,048 \\
8,986,454 \\
106,379 \\
10,915 \\
25,167 \\
102,006 \\
32,065 \\
1,844,041 \\
10,768 \\
10,8 \%, 044\end{array}$ & $\begin{array}{r}1009 \\
1119 \\
868 \\
1081 \\
977 \\
1022 \\
928 \\
1298 \\
609 \\
11.39\end{array}$ \\
\hline
\end{tabular}

Sea foptnotiles at end ol labke 
Table 14. Class of Ownership, Number of Uttmate Consumers, Revenue, Sales, and Average Revente per Kilowatthour for the Residential Sector by State and Uitity, 1993 (Continued)

\begin{tabular}{|c|c|c|c|c|c|}
\hline $\begin{array}{c}\text { Stais } \\
\text { Enotyle Uthty }\end{array}$ & 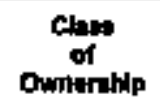 & $\begin{array}{c}\text { Number } \\
\text { of } \\
\text { contumers }\end{array}$ & $\begin{array}{c}\text { Favtinte } \\
\text { (thoutend toliturt) }\end{array}$ & 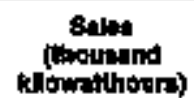 & $\begin{array}{l}\text { Averege Awwenus } \\
\text { par klowithour' } \\
\text { (owitg) }\end{array}$ \\
\hline
\end{tabular}

Delawere

Clavlox) Tom of

Dalaware Endirdo Coop the

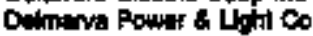

Lewor C C of of

Anddedsinm Toun of

Nillord cly of .

Newiak City of

suaford Ciy of

Sming Town of

sict

Oniriot of Cotunida

Potomtag Electrix Power Co strte tot.

Fardda

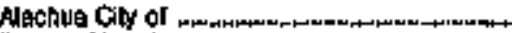

Barlow Cily of

clountaly cly of

Bubhingll Coty of

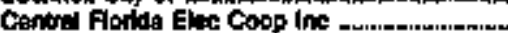

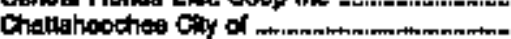

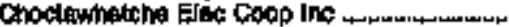

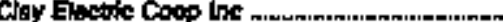

Clewiston Chy of

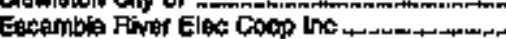

Fodid Kays El Coop Asth ho ....

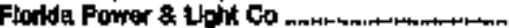

Flatida Patio Cop.

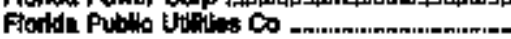

Fort Mlaseda City of

Fort Fierce yolian Auth

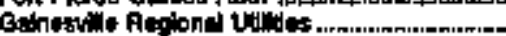

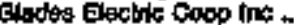

Grean Cove Bping city or

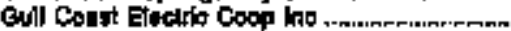

Cut Fower Co.

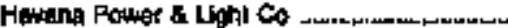

Hompilated Ciny of

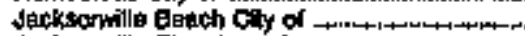

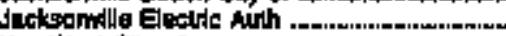

Ker Whest city of

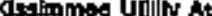

Lice Worti Chy of

Intheland Cily of

Los Count begtio Coop ino

Legbuta Ciny of t-

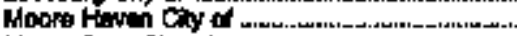

toun Dora City of .

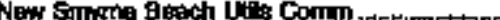

Nowbery Coy of -

Deala Chy of

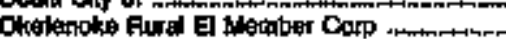

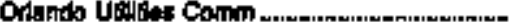

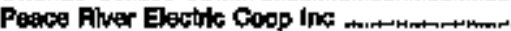

Ountoy chy of

St Cloud Chy of ...-.....--...-n..........--...

Sterke Ciny of

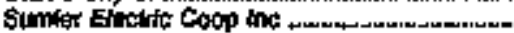

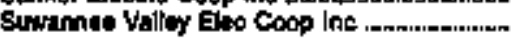

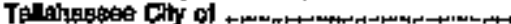

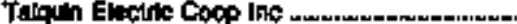

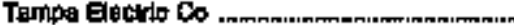

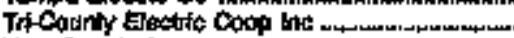

Vero Buich Cily of
Puthicy Ompad

Cocperative

Inwoptor-Owned

Pubifty Owitad

Puticty Omped

Pubsicy Ounted

Publtoty Ouned

Putict Omied

Punikly Chinded

Putilly Oined

Putecty Oniled

Intentior-Ounted

Publaty Omas

Putrich Cinnod

Pubecty Ounsd

Publictr Otrned

Cooperative

Publich Oimed

Conperalive

Coopenathe

Publacy Cimed

Copperalive

Cooperthe

nwertarownod

Inveglom-Gined

intoticor-guned

Pulnedy Ormed

Publicty Cimad

Publest Gimed

Croperartwe

Pubichy Onnes

Coopuralue

invegtarowned

Pubicty Comed

Putrect Omined

Pubilcty Onmerd

Pubncty Owned

Pubiledy Cinned

Pubrely Omind

Pubsey Owined

Pulticty Omied

Coopatitiva

Pubicky Omind

Pubrasy Omited

Publich Ommed

Publedy Orined

Publicy Omited

Pubich Gimod

Cosperellue

Pubicty Cimed

Couperallye

Pulpicly Owred

Fubricty Cined

Puhrey Ormad

cocperativa

Cogperilut

Putify Owned

Cooperaluse

lawetorowned

cosperativa

Puluery Oined

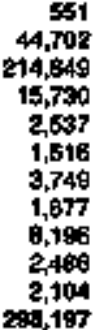

459
46,720
165,094
13,720
1,726
1,472
0,766
1,402
5,764
2317
1,906
27,996

4 ats

409519

$2,168,194$

146,847

22,006

14,727

5.710

14,757

70,450

24,031

18,647

$3,043,025$

183480

108,430

117,349

$117,3 \$ 9$

$1,634,0007$

$1,604,07$

7.19

1, 727

1,075

20,773

1,139

21,8300

9.167

3,215

8,409

23,450

2,974,6ee

$1.078,658$

19,470

2,315

19,738

69,306

9,490

2069

12,010

271,596

1,022

0.764

21,313

270,B18

20,400

30,200

द. 219

79,498

113,384

14,741

71

3003

17,213

715

32,067

6,250

704,978

16,478

3,658

13,430

1,071

60,945

14,260

6,17

35,871

420,051

11, 342

21,864

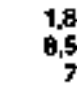

1,805
0,500

720

17684

455

20.688

107, B.45

3,27

6,775

108

2.850410

1,048,602

17,504

2,284

17.549

$49,0 \%=$

$9,3+2$

2,042

11,178

244.85?

940

1056

20,699

209,379

19062

31,213

17.914

78.446

101011

12,102

891

3,765

15,394

62

31.40?

10.572

00350

16966

3,0a2

14.467

1,610

$87.9+1$

16,290

70,115

39355

464,006

10.421

2015
21,161

211,132

6,580

196,000

12,243

272,179

$1,265,674$

43, 207?

80,524

200000

$30,350,002$

$19,972,584$

206,120

23 하의

205,658

630,540

85,060

24.408

135,390

3,7 12,961

10.011

70 s.8

267,055

$3,850,560$

219,035

303,692

779.810

$1,011,768$

$1,277,788$

150,258

7,3997

39, 180

174,570

8,625

\$69,346

120,681

$1.256,278$

to: 127

40335

160,004

19,604

748,714 
Table 14. Cbss of Ownership, Number of Ultimate Consamers, Revenue, Sales, and Average Revenue per KIlowathour for the Residential sector by State and UtIllty, 1998 (Continued)

\begin{tabular}{|c|c|c|c|c|c|}
\hline $\begin{array}{c}\text { statt } \\
\text { Jeolirio Uninty }\end{array}$ & $\begin{array}{c}\text { Casas } \\
\text { of } \\
\text { ommersho }\end{array}$ & $\begin{array}{c}\text { Nomber } \\
\text { of } \\
\text { Conmunats }\end{array}$ & 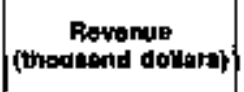 & $\begin{array}{l}\text { solet } \\
\text { (thotwand } \\
\text { Idlowetihoura) }\end{array}$ & 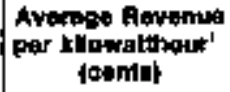 \\
\hline
\end{tabular}

Forida (Comlnued)

weveriula ony of

Wesl Flonds $1 \mathrm{C}$ Cowp Mosn Ine

Wilkblon ciny of

Whalacoocheie Pher Elect Coop 8take Total

\section{Geongla}

Anwith Gity of

Adet Ciny of

Nhany Waler Gá 8 Ught Comer

Altartahe Eectito Marbibe Corp

Amcolok Elogine Hember Corp

Bexroporalle City ar

Elaken City of

Blue Fudos Mountan E M C

Gnnson Town of

Elrond City or

Caro Oty of

Calluam Giny of

Carilla city of

Canoocher Electunc Mlamber Corp

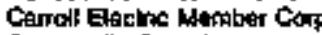

Corterswile Chy of

Centrat Gepraga El Member Corp

Chattenooga Cty of

Crickemang City of

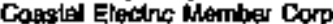

Cobb Eloutre Momborsho corp

Collens Park City of

Colquit Elocinc litembert Corp

Caramerce Oty of

Coninaton Chy of

Cowelo.Fapette El Member Comp

Crap Counly Pourt Comm

Dolion bay at

Doends City of

Domita Chy of

Eesi Pounl City of

Ehervon City of

Eltult Gaty of

Exceisor Elecinc Uember corp

Fenthurn Coty of

Fiboorald wit Lol \& Gond Comm

Flini Elecing Maenbersine Carp

Forsyth City of

Fort Vallary UVlity Comm

Eoorro Powir Co

Crady County Elec Mlember Corp

Eranivine Chy ol

Graystane Power Corro

enfin tity on

Hebersham Eactro Hembor Corp Hemptor Oty of

Hert Elochtic Member Cotp

Henwood Elocine Hamber Corp

Hogansuly Caty of

Inwh Eactine Membersho Corp

Jackson City of

Jackson Electnc Mlember Corp

Jaterson Elactinc hember Corp

Lin Fayelte Ciny of

the Erange Gty of

Lamar Elecinc Merabotsho Carp

Laweoncenvile Galy of

thlite Comulges El Member Carp

Ninstiald Town of

Mangtin Crity of

hadda Georgis El Meraber Gorp
Publety Owmed

Cooperalure

Publety Owmad

Cocperalrye

Publch Omingd

Publucty Drwed

Publucty Oimiod

cooparalme

Congerilare

Publichy Oinnod

Pubicly Qwised

Cocpertave

Publicly Ountod

Pubickly Owraed

Puticty Ownea

Publicly Owwits

Puldichy Owner

Cocpentivit

Cooperatowa

Publichy Owned

Cocoperations

Publely Dured

Pubuchy Owned

Cooperature

Cooperatrie

Publichy Owmed

Cooperativa

Publety Owned

Publich Owined

cooperalus

publety Owined

Pubtar Dmed

Publaty Omed

Publety Owmed

Publaty Omined

Publeck Dwned

Publuctr Ormod

Copperaluse

Publaty Ointed

Publuch Ormod

Copoeralive

Publichy Owned

Publuctr Omined

Inweglor-Owmed

Cooparaline

Publucty Oimod

Copperabere

Publich Owned

Cooperrawe

Publichy Owned

Cooperalve

Cooperatire

Pubdicky Owatad

Cooparrine

Publicly Owned

Comperatrot

Cooperative

Publely Owned

Publidy Onerted

Cooperato

Publicly Owmed

Copoptratrot

Publaty Owrued

Publitly Owined

Gopaptiaturt

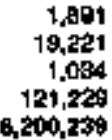

1.798

17.610

最5

171,660

B, 137,040

2,017

1,661

31,800

13, 189

22, 152

2,454

2,045

$13,5 \times 9$

불

$1, \operatorname{sich}$

3,624

2.835

$100 \%$

13.395

20,400

4,125

24,000

0,062

735

8,058

108.771

S.441

36,856

2170

8.488

36,225

B. 335

9,135

उot

3,930

15,650

3,544

675

13,400

$1, \mathrm{Bg}$

4,077

44,002

2,000

4,060

$1,431,642$

12,692

450

51,364

11 , 1

10,212

1,124

21,943

280

1,325

0,469

1.184

94,516

24,976

3.167

10,044

11,401

6,585

7,960

$2 \times 2$

32,576

\$, 989

\subsection{5}

1,632

25,491

19,578

21,120

1,637

1,865

8,055

殗

1,105

2,952

1,648

1,551

15.019

27.105

3,382

24,578

6.2. 40

501

B. 905

1)4,164

6,961

36,307

1.943

6.704

40.396

6. 842

5,040

274

3,849

9,136

2.410

590

13,279

1.260

4,794

40,493

1.100

2,509

$1,291,025$

13,092

280

54,429

0.913

16.165

600

19.721

159

0.6\%7

1,005

60,40

27,158

2620

7,000

11,354

5419

7,447

4,392

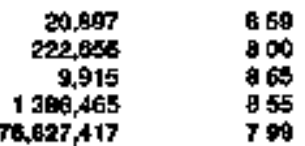

47, 068

21,061

371,416

154,352

200,772

17.149

27,400

110,304

1.796

18, dㄹㄹ

46,030

24.143

19. 的向

167.259

307.97

40,040

262079

147,606

0,747

9.9.308

$1,290,313$

94,370

468,463

19,975 
Table 14. Class of Ownerahip, Number of Uitimate Consumers, Revenub, Sales, and Average Revenue per K'lowatthour for the Residentla' Sector by State and Utility, 1993 (Contimued)

\begin{tabular}{|c|c|c|c|c|c|}
\hline 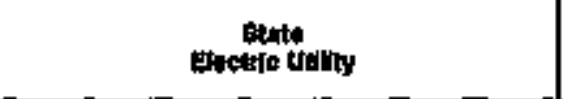 & 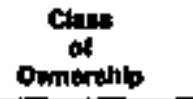 & 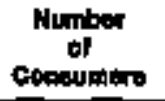 & [thousund toilary) & 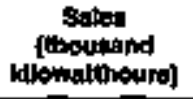 & 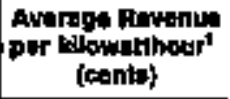 \\
\hline 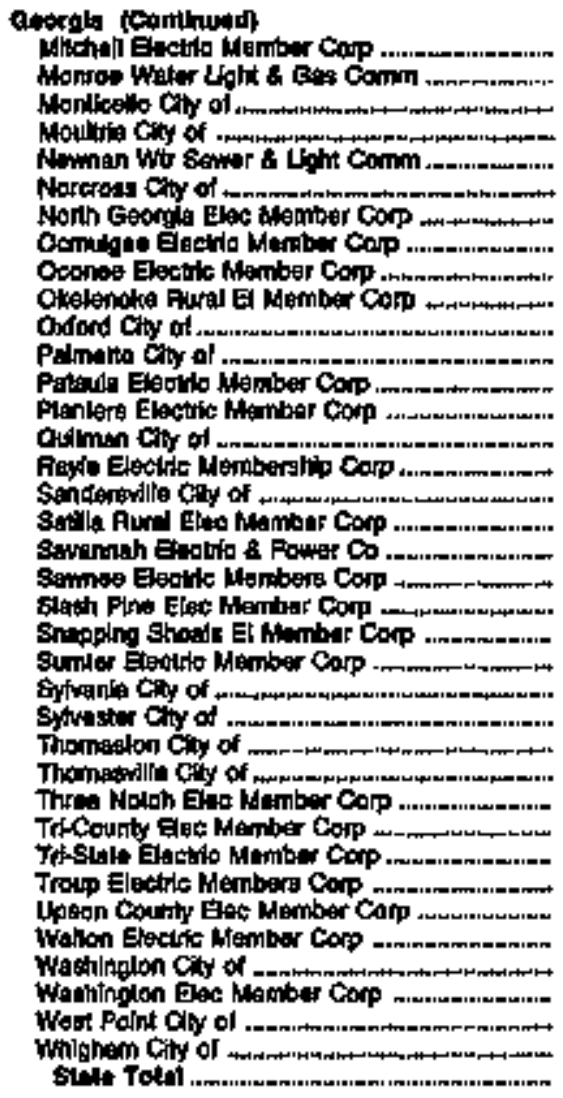 & 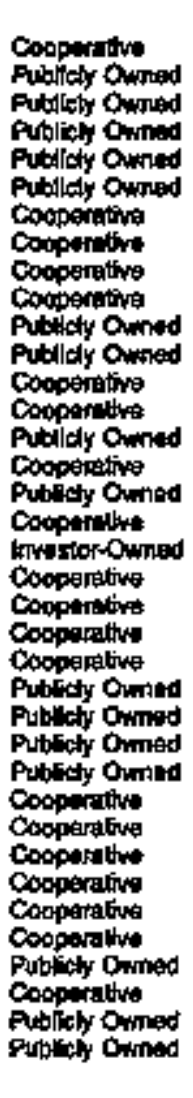 & 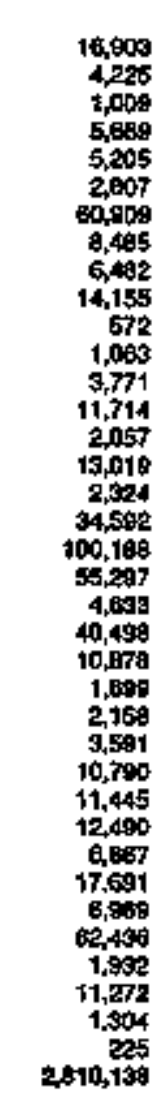 & 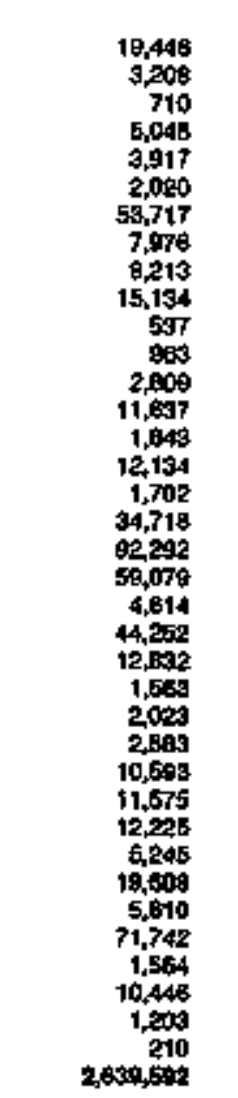 & 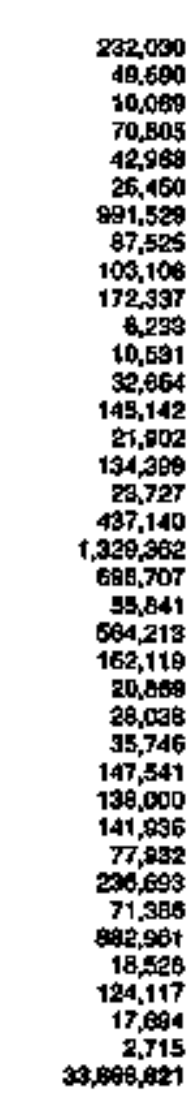 & 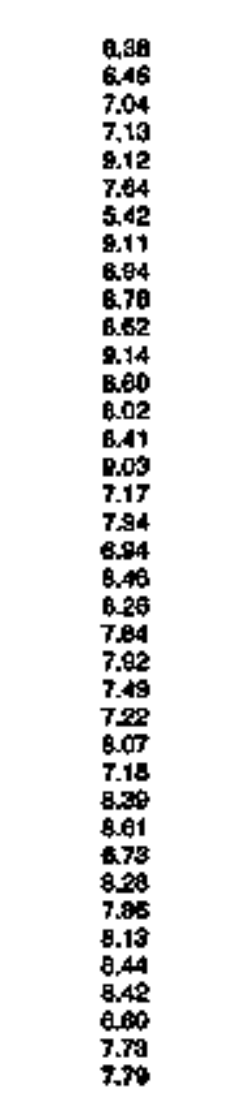 \\
\hline 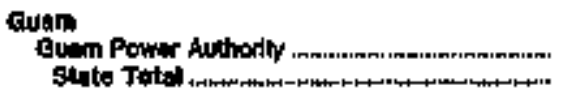 & Pubien Ownod & $\begin{array}{l}31,750 \\
31,750\end{array}$ & $\begin{array}{l}39,559 \\
30,559\end{array}$ & $\begin{array}{l}420,714 \\
420,714\end{array}$ & $\begin{array}{l}0.40 \\
0.40\end{array}$ \\
\hline 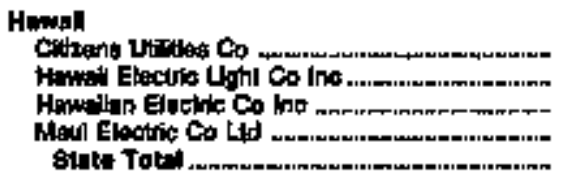 & 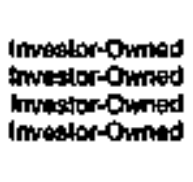 & $\begin{array}{r}10,707 \\
46,713 \\
277,916 \\
43,958 \\
3 \$ 7,384\end{array}$ & $\begin{array}{r}10,505 \\
47,070 \\
186,952 \\
98,632 \\
3 \times 3,187\end{array}$ & $\begin{array}{r}128,205 \\
310,649 \\
1.725,608 \\
304,141 \\
2,459,579\end{array}$ & $\begin{array}{l}15.20 \\
16.16 \\
11.41 \\
13.03 \\
12.00\end{array}$ \\
\hline 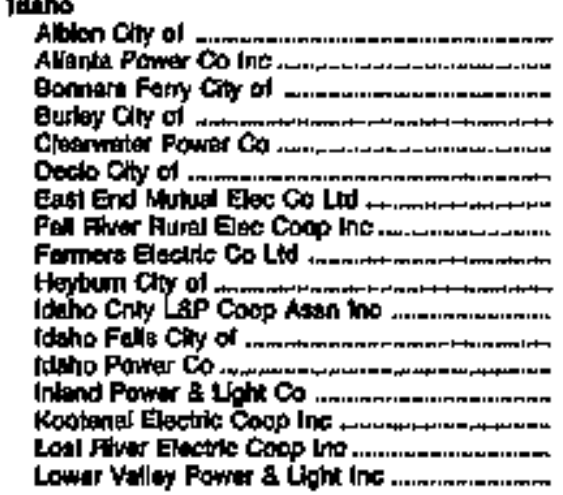 & 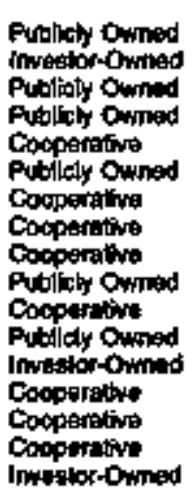 & $\begin{array}{r}167 \\
12 \\
1,589 \\
3,344 \\
6,597 \\
86 \\
380 \\
5,172 \\
04 \\
970 \\
2,177 \\
18,213 \\
240,278 \\
775 \\
10,550 \\
1,428 \\
908\end{array}$ & $\begin{array}{r}77 \\
29 \\
998 \\
1,892 \\
6,270 \\
56 \\
552 \\
4,588 \\
67 \\
569 \\
2,020 \\
12,104 \\
173,124 \\
687 \\
8,0,07 \\
1,056 \\
489\end{array}$ & $\begin{array}{r}1,610 \\
74 \\
24,480 \\
44,514 \\
01,519 \\
2,200 \\
12,947 \\
69,200 \\
2,093 \\
17,416 \\
28,450 \\
29,914 \\
3,58,898 \\
17,318 \\
145,387 \\
23,587 \\
1,527\end{array}$ & $\begin{array}{l}7 \\
6 \\
4.01 \\
4.47 \\
6.85 \\
69 \\
4.50 \\
7.44 \\
69 \\
3.24 \\
7.12 \\
4.13 \\
4.80 \\
6.07 \\
6.02 \\
4.51 \\
5.78\end{array}$ \\
\hline
\end{tabular}

Foe tootnokes al end wil table. 
Table 14. Class of Ownership, Number of Ultimate Consumers, Revenue, Sales, and Average Revenue per Kilowatthour for the Residential Sector by State and Utility, 1993 (Continued)

\begin{tabular}{|c|c|c|c|c|c|}
\hline $\begin{array}{c}\text { Silote } \\
\text { Jhotrito Itnthy }\end{array}$ & clate & $\begin{array}{c}\text { Number } \\
\text { of } \\
\text { Corteumert }\end{array}$ & $\begin{array}{c}\text { hovintus } \\
\text { (thousand dolkare) }\end{array}$ & 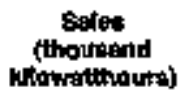 & 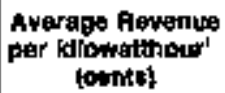 \\
\hline
\end{tabular}

Ioliti (Gominumed)

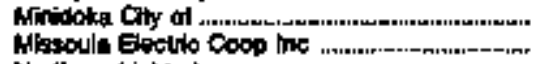

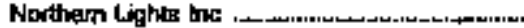

Pacinicon

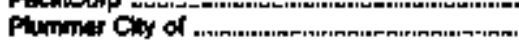

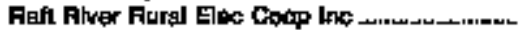

Fupar cony of

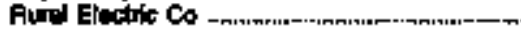

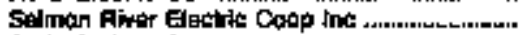

Sodv SpAnos Chy 어 -...................................

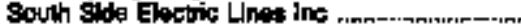

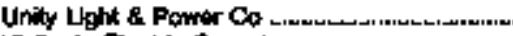

Vingle Eloctio coop ino

Weshingtan Waler Pown to

Weidef Gify of

State Tote ..............................................

Wholit

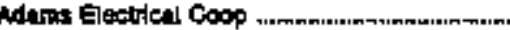

Albeny City of nn---r

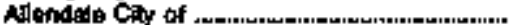

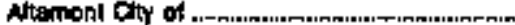

Balavia City of

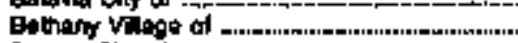

Ereoes City ol .

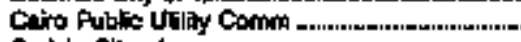

Cable City ol

Coniml City at

Catsy Ciny of . .

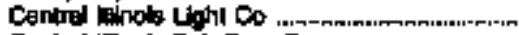

Centuel runos Pit Serv Co

Chethem Villages of .........-n.................-..............

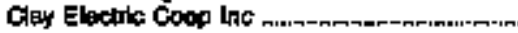

Cinlon Cown's Elac Codp he

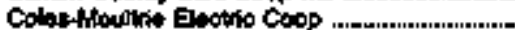

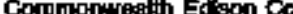

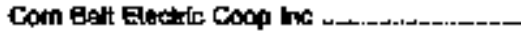

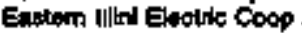

Edpar Elaghis Coon Aagn

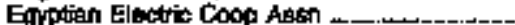

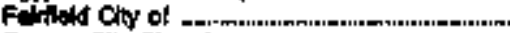

Femmar City clty of .

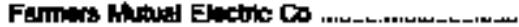

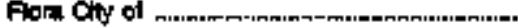

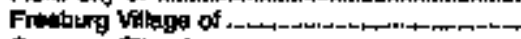

Ganseded ciny of

Cescova Ciny of 7 -

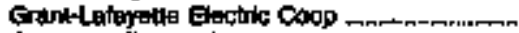

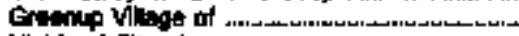

Highiland City of

ITnols Fowt Co

Itinols Rurd Eladilo Co ..............................

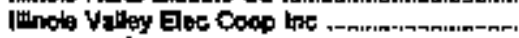

Intertilalt Ptrmer Co.

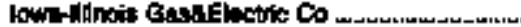

Jo-dearmith Elactrit CaOp Inx

Lind Vilhe of

I J N EProtic Coso Ino

Mlabcouteh City of ..., num

MeDonough Power Coop ..............................

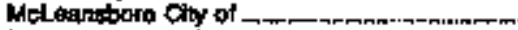

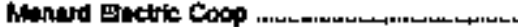

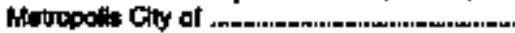

Monhos Gaunly Glex Coop bye

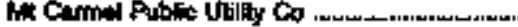

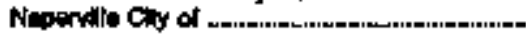

Publichy Owned

Cooperativo

Copporallive

Inweslor-Omned

Putilyty Owated

Cosperalfye

Publiely Owned

Cooparative

Cosparalion

Publicly Owned

Coogentive

Cosparative

Coopereltwe

Inweglor owntad

Pubricly Onised

Cooperalive

Putulely Owmed

Putich Owand

Pubridy Ominad

Putulchy Owmed

Putioly Owrat

Pubricty Oined

Puhlchy Owned

Putrichy Owated

Pubtict Onwed

Publely Owmad

Putrichy Owated

Inwolor owned

Itwegtor-0mined

Putrichy Ownded

Cooperalive

Coppotitirs

Cocporative

Inwosior owned

Coppercelóne

Cooporabe

Cooperalle

Cooperiline

Putlicly Owrind

Pubtoly Owaed

Conperabive

Publicly Ownded

Pubilely Owned

Puticty Ownad

Publlaly Owined

Cooperithe

Puticty Owand

Potlely Oumind

Imeslor-Ommed

Cocperatite

Cooperalue

Inwealor-Ommed

Inweslor-Owned

Cooporatire

Pubilkly Owned

Corpenatime

Publoty Owned

Pubtlely Owmed

Cosperative

Pubbich Owined

Copperativa

Publicly Owited

Cooperative

Invisolit-Cmmed

Pustrely Owmed

49
25
9,659
46,113
613
1,058
2,373
2,046
1,657
1,292
653
1,515
14
67,153
1,950
433,762

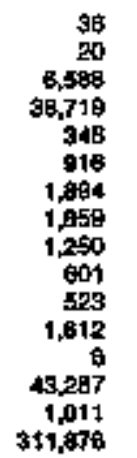

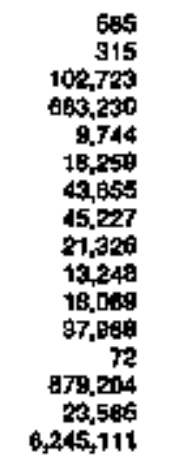

1

\section{7\$8}

352

$1,0 \Leftrightarrow 0$

7.189

608

1,476

1,245

1,738

1,416

3.104

1,568

$18 \mathrm{~g}, \mathrm{A2} 9$

271205

2,435

2,626

4,358

$7,1 \mathrm{AB}$

2,998,500

13.395

11,973

4,662

10.900

2,742

968

1,116

2,550

1,210

2,726

5,460

75

4,167

498,391

0,517
5,666

5,665

72,900

4,682

4,6802
574

7.450

$1, \mathrm{AOS}$

2,225

4.513

2,609

7,807

g,212

4,608

4,584

33,385

9,003

158

150

gas

4,348

$40^{20}$

1,252

713
1,125

1.123
1.107

1.761

g84

119,700

245.510

1,724

3,192

7.187

B.sog

2,341,165

17.169

$17.65 \%$

B.072

11.045

1,659

50内

1.517

1,527

1,018

$1.4 A B$

2610

14
422

3,453

408116

10,562

10,567
7.191

1,074

47,962

4,490

335

8.572

1,046

1,226

5,007

050

e,tes

1.850

6.969

3 .

23.483 
Table 14. Chass of Ownerahip, Numbet of Ulitmate Consumers, Rovenue, Sales, and Average Revenue per Kilowathour for the Resildentlat Sector by State and Utility, ig9s (Continued)

\begin{tabular}{|c|c|c|c|c|c|}
\hline $\begin{array}{c}\text { stale } \\
\text { Elocint ditlty }\end{array}$ & clates & 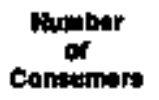 & Alhourend doflem & $\begin{array}{c}\text { 8ales } \\
\text { (thoutions }\end{array}$ & 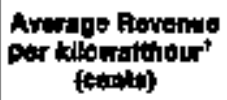 \\
\hline 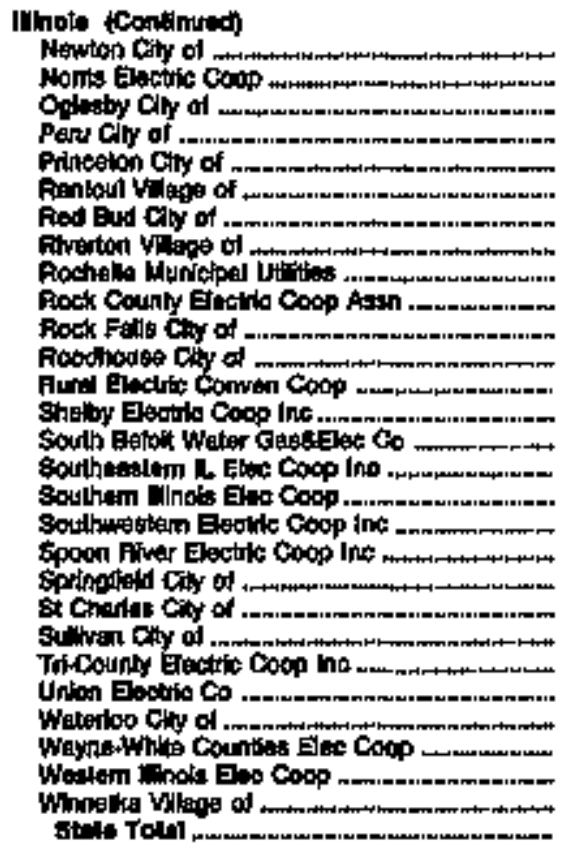 & 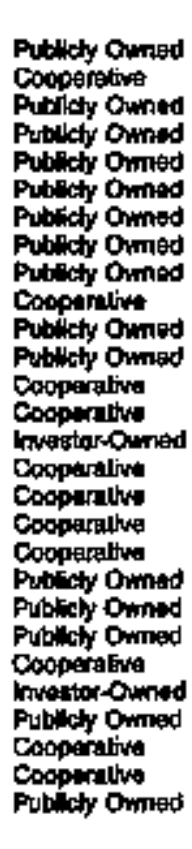 & 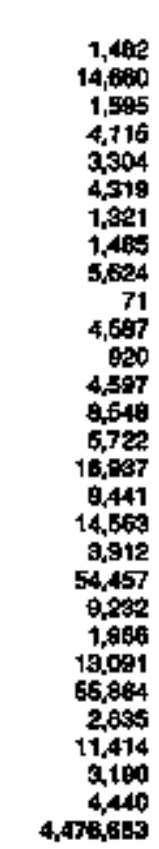 & 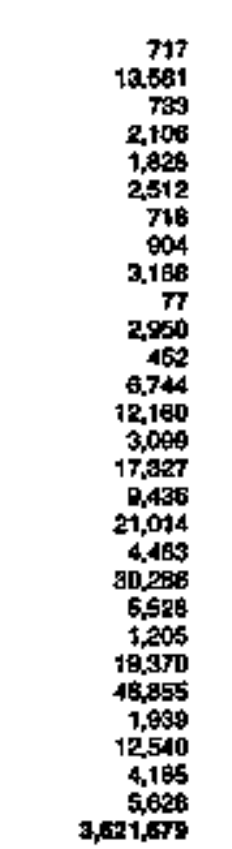 & 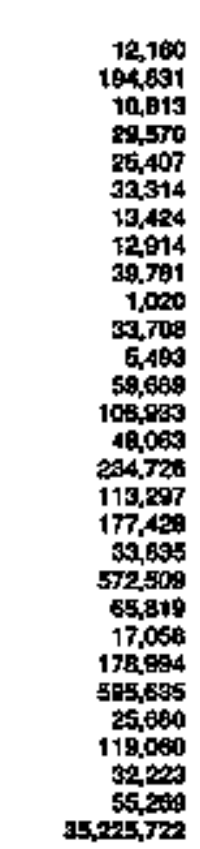 & 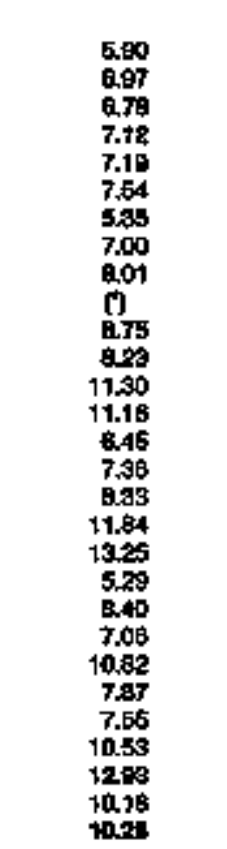 \\
\hline 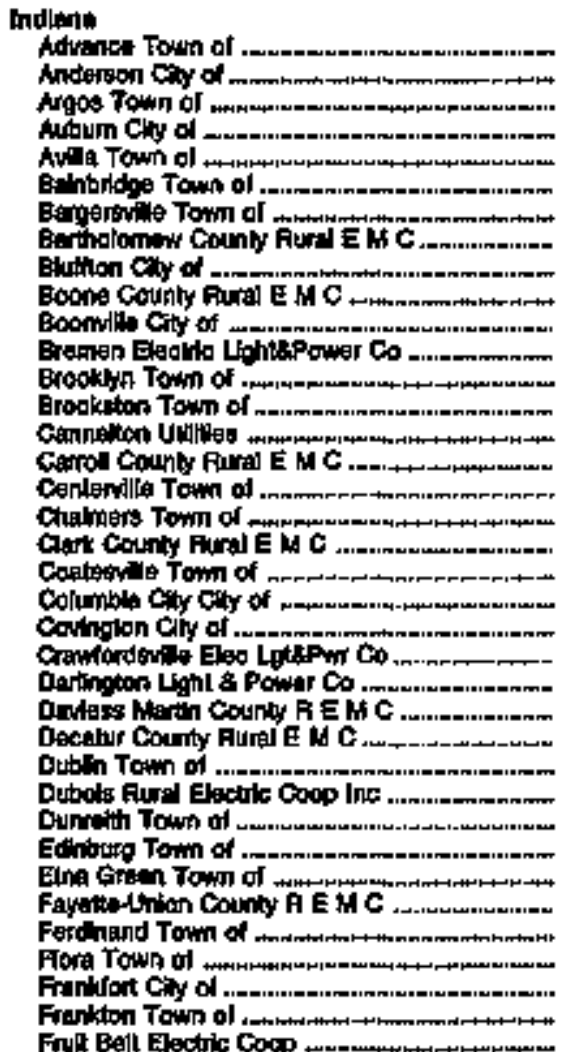 & 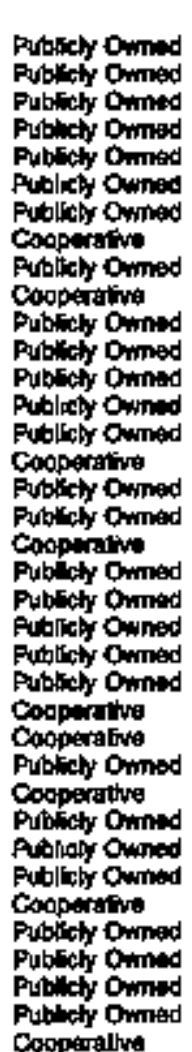 & 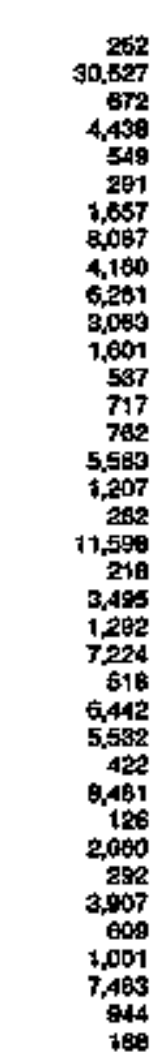 & 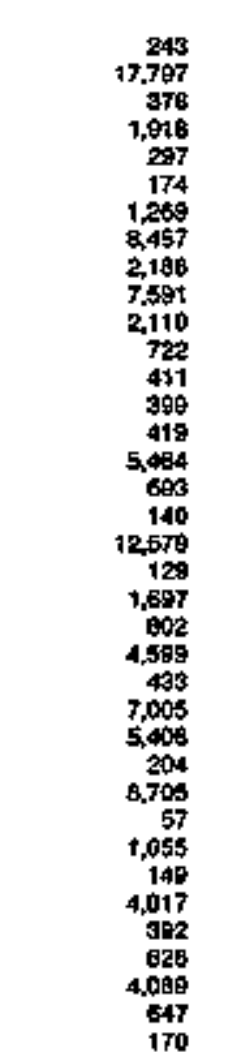 & 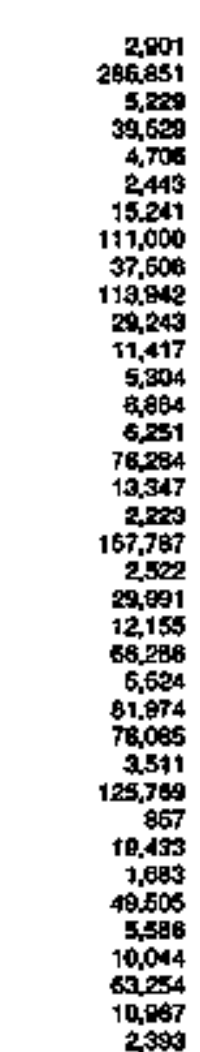 & 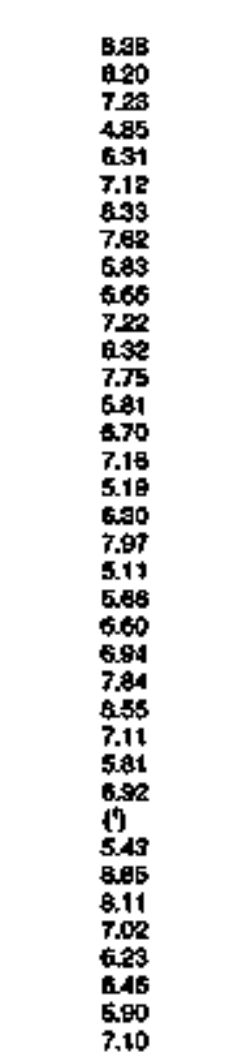 \\
\hline
\end{tabular}

Sie foohotes at and of table. 
Table 14. Class of Ownership, Number of Ulitimate Consumers, Revenue, Salos, and Average Revenue per Kilowatthour for the Residential Sector by State and Utillty, 1993 (Continued)

\begin{tabular}{|c|c|c|c|c|c|}
\hline Slate & 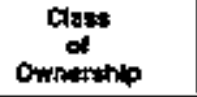 & $\begin{array}{l}\text { Number } \\
\text { od } \\
\text { Contsmert }\end{array}$ & 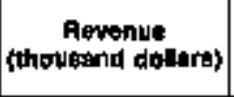 & $\begin{array}{l}\text { salw: } \\
\text { (thouband } \\
\text { idlowglthoura\} }\end{array}$ & 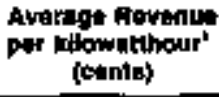 \\
\hline 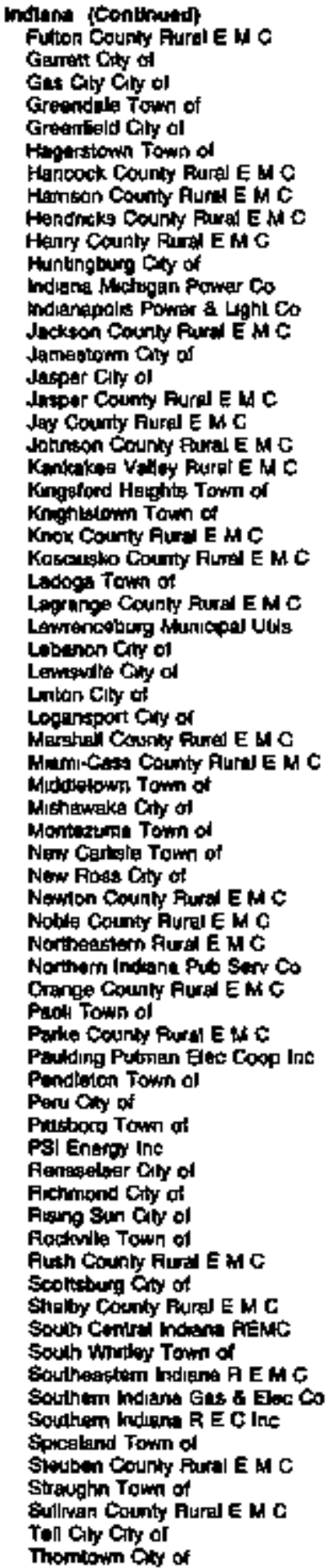 & 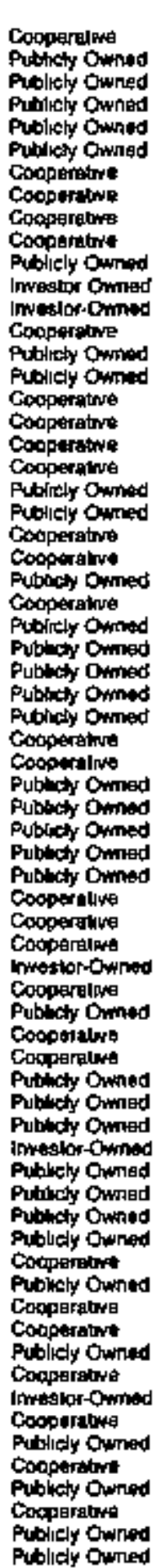 & 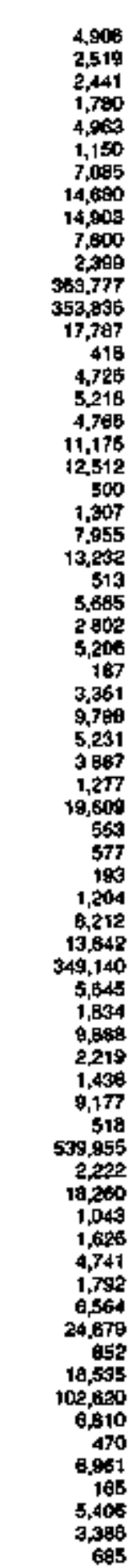 & 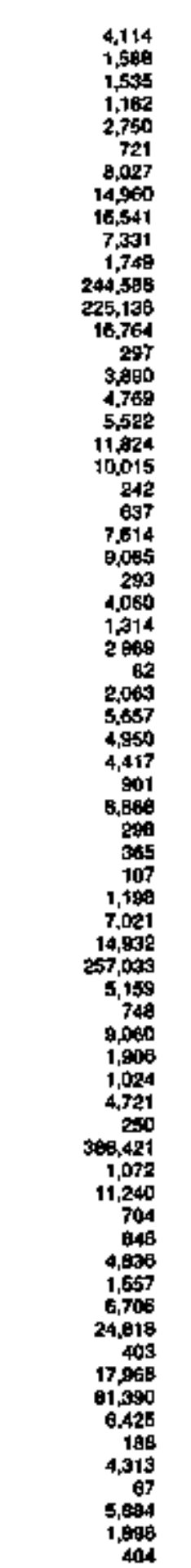 & 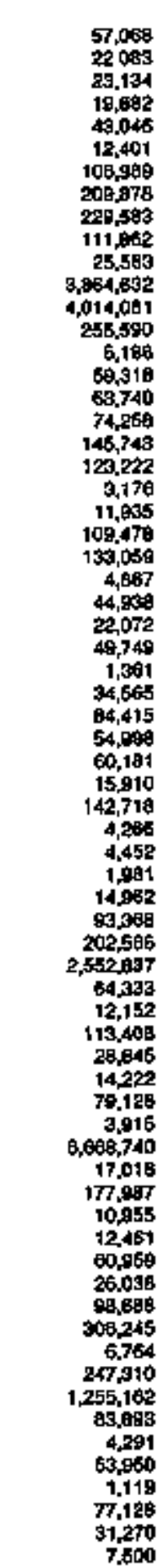 & 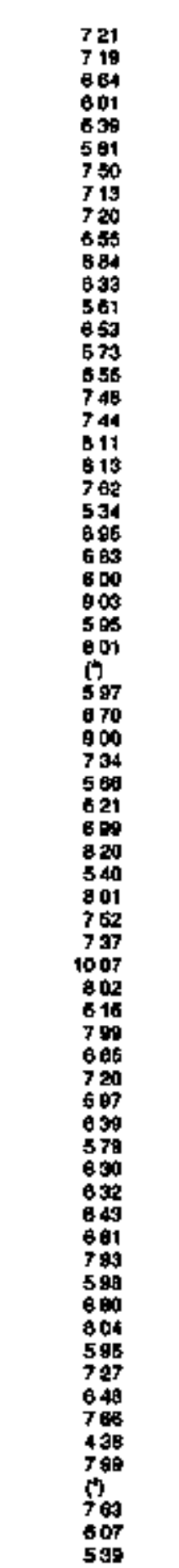 \\
\hline
\end{tabular}

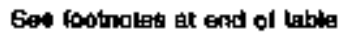


Teble 14. Class of Ownerahlp, Mumber of Ultimate Consumers, Revenue, Sales, and Average Revenue per Kllowatthour for the Residential Sector by State and Utillty, 1993 (Continued)

\begin{tabular}{|c|c|c|c|c|c|}
\hline 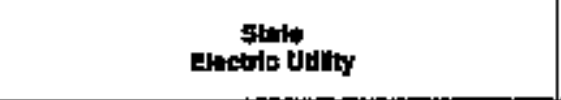 & $\begin{array}{c}\text { Clan } \\
\text { of } \\
\text { Ownarahlp }\end{array}$ & $\begin{array}{l}\text { Punber } \\
\text { of } \\
\text { contumitum }\end{array}$ & 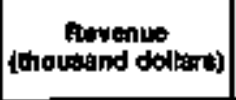 & 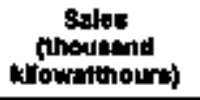 & 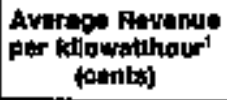 \\
\hline 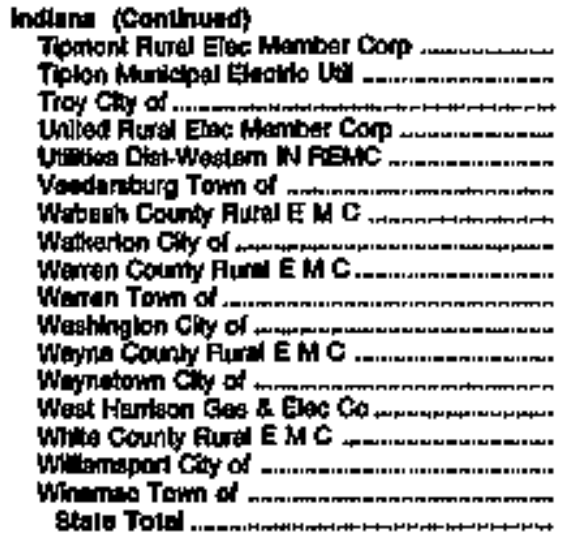 & 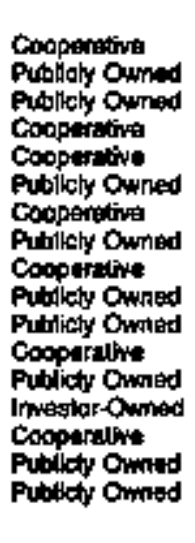 & 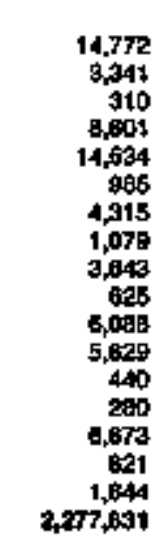 & 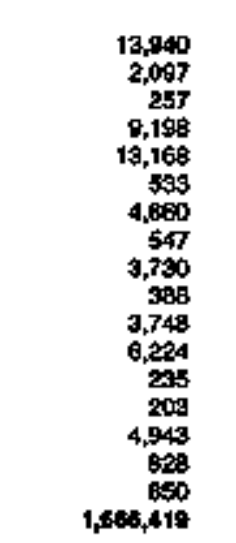 & 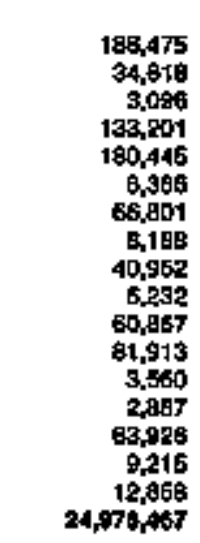 & $\begin{array}{l}7.46 \\
8.08 \\
6.30 \\
6.81 \\
7.90 \\
6.36 \\
7.26 \\
6.68 \\
9.11 \\
6.23 \\
6.16 \\
7.80 \\
6.60 \\
7.09 \\
7.79 \\
6.99 \\
6.81 \\
0.67\end{array}$ \\
\hline 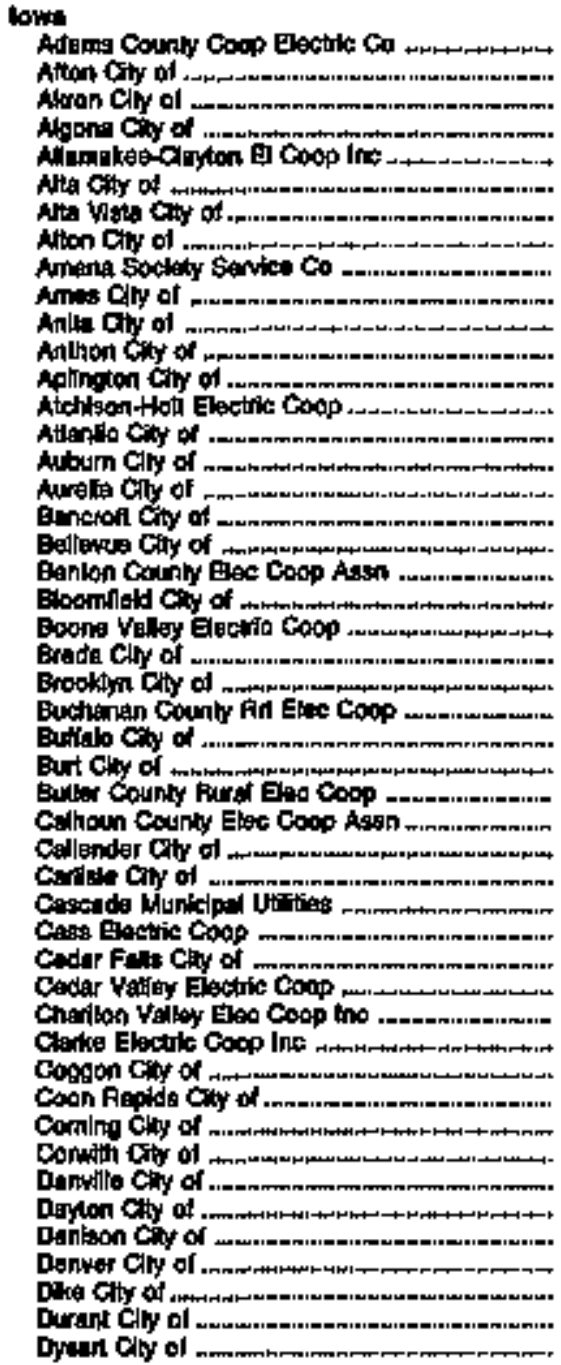 & 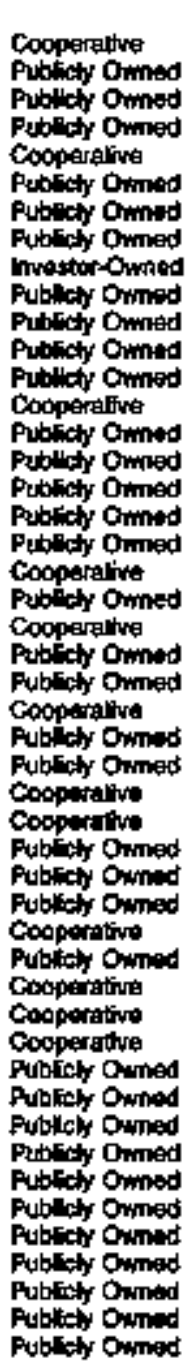 & 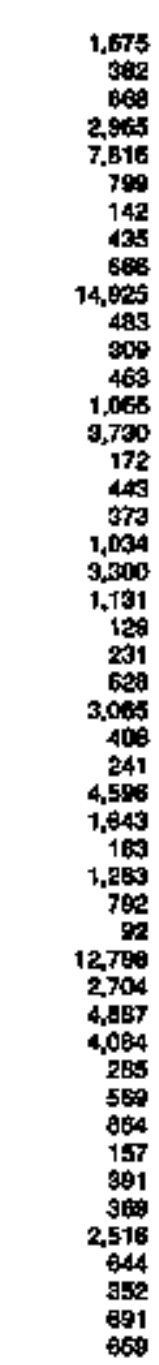 & 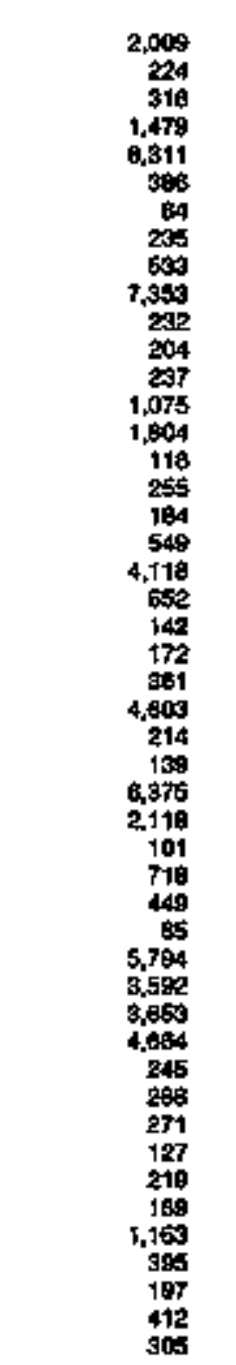 & 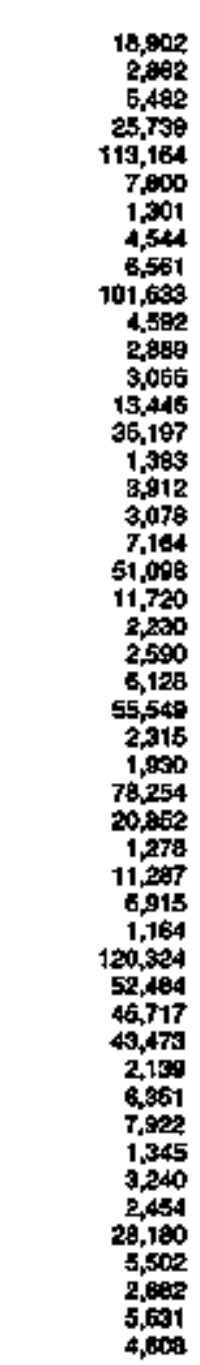 & 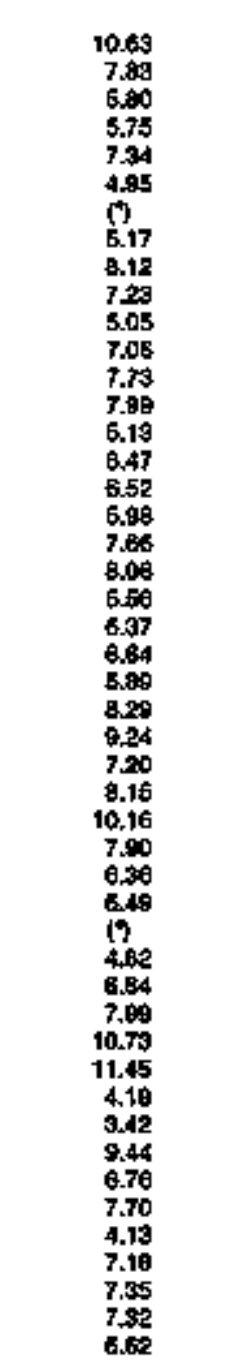 \\
\hline
\end{tabular}

Sta looluotos at end of tante. 
Table 14. Class of Ownership, Number of Ultimate Consumers, Revenue, Sales, and Average Revenue per Kllowatthour for the Residential Sector by State and Utility, 1993 (Continued)

\begin{tabular}{|c|c|c|c|c|c|}
\hline 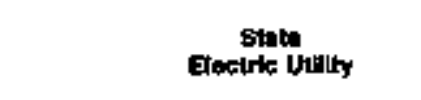 & 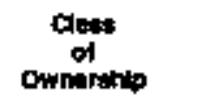 & $\begin{array}{l}\text { number } \\
\text { of } \\
\text { Conaumers }\end{array}$ & $\begin{array}{c}\text { Rewembit } \\
\text { (tholpard dollers) }\end{array}$ & 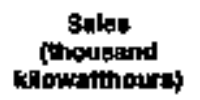 & 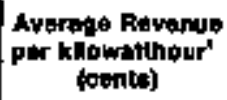 \\
\hline 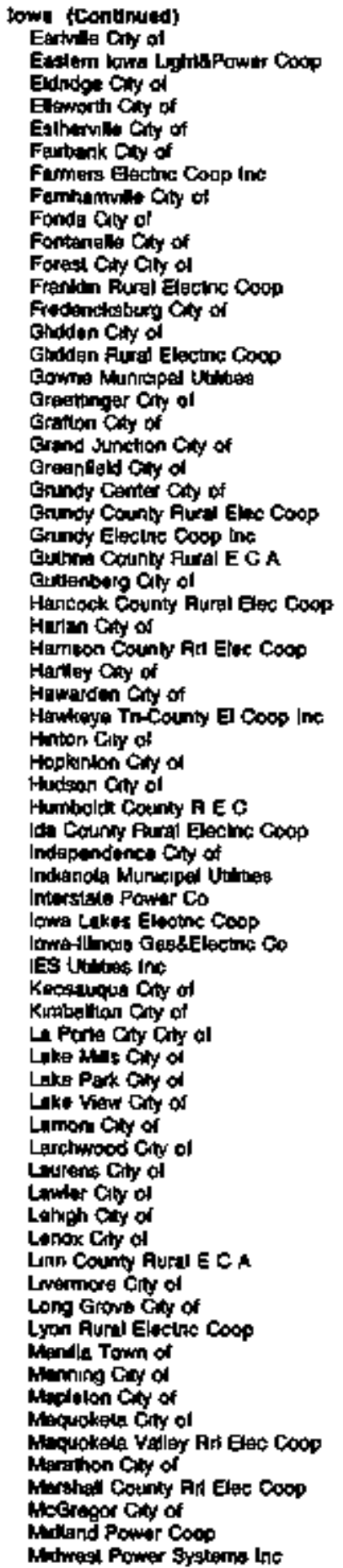 & 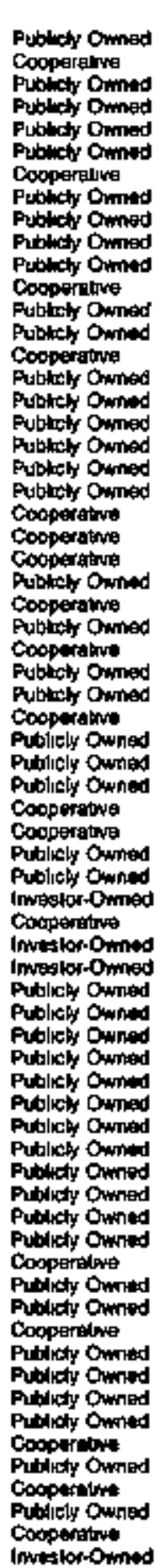 & 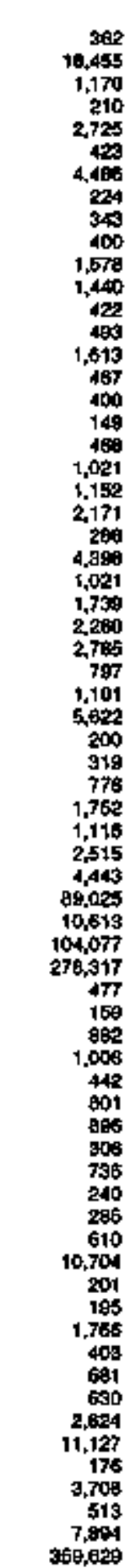 & 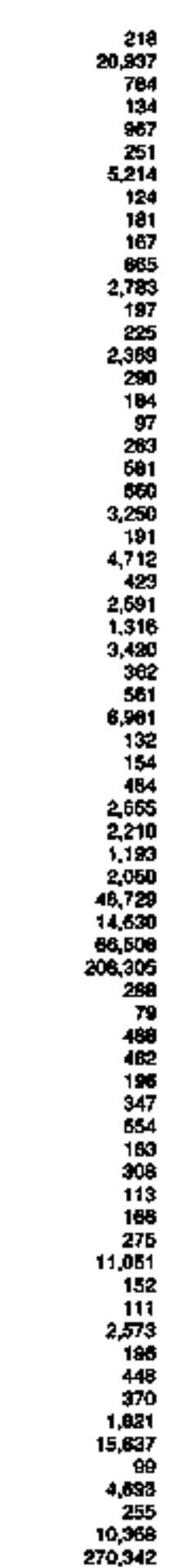 & 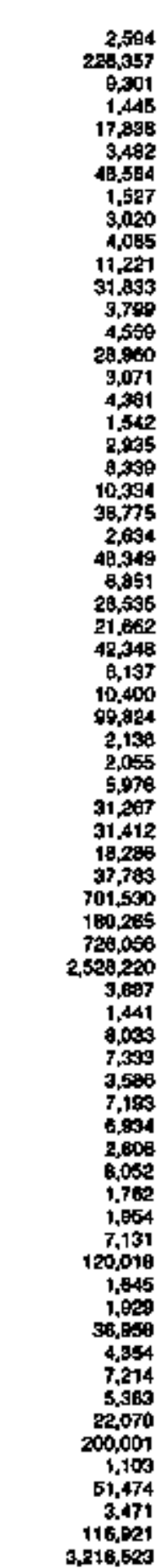 & 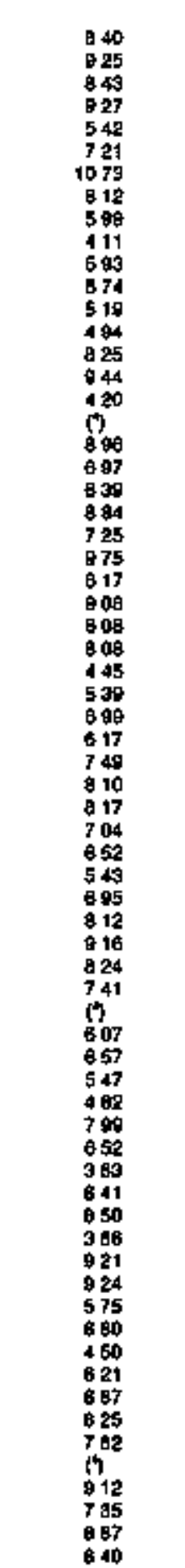 \\
\hline
\end{tabular}

Sop tootnotes at end of table 
Table 14. Class of Ownership, Number of Ultimate Consumers, Revonue, Sales, and Average Revenue per Kllowatthour for the Residential Sector by State and Utility, 1993 (Continued)

\begin{tabular}{|c|c|c|c|c|c|}
\hline Sinco & $\begin{array}{c}\text { Ctitiog } \\
\text { od } \\
\text { Owhority }\end{array}$ & 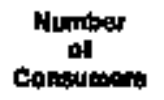 & Povonus & 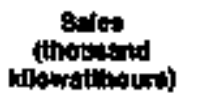 & 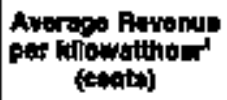 \\
\hline 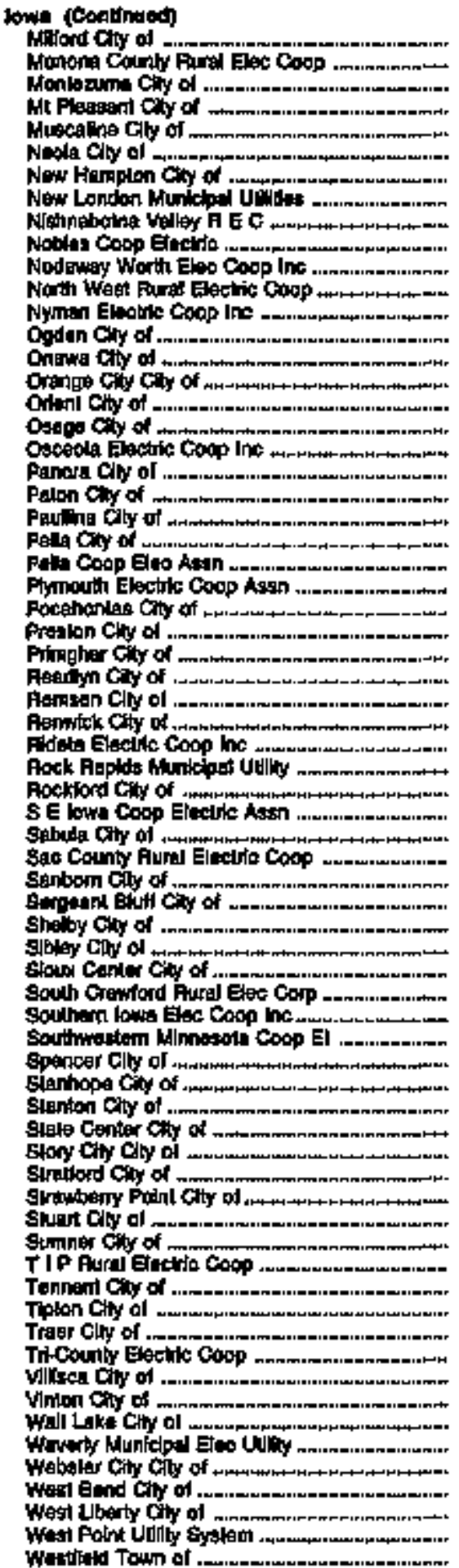 & 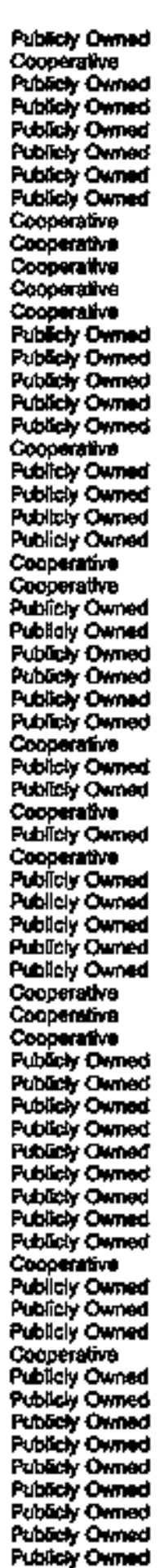 & 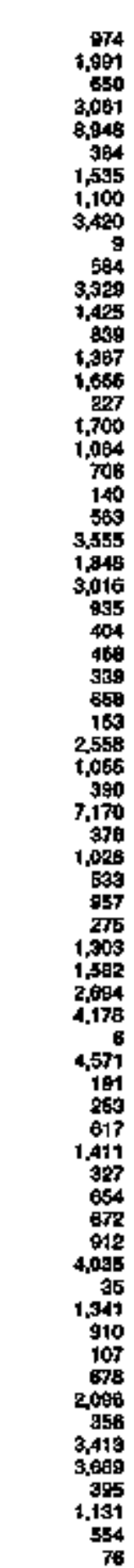 & 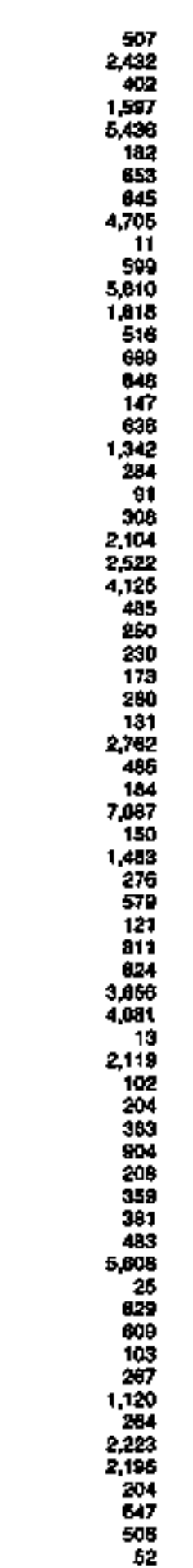 & 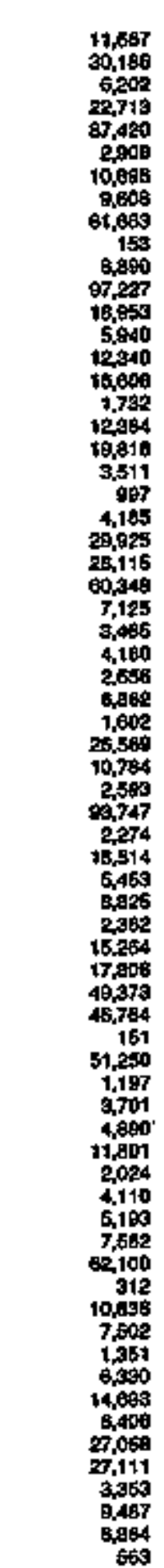 & 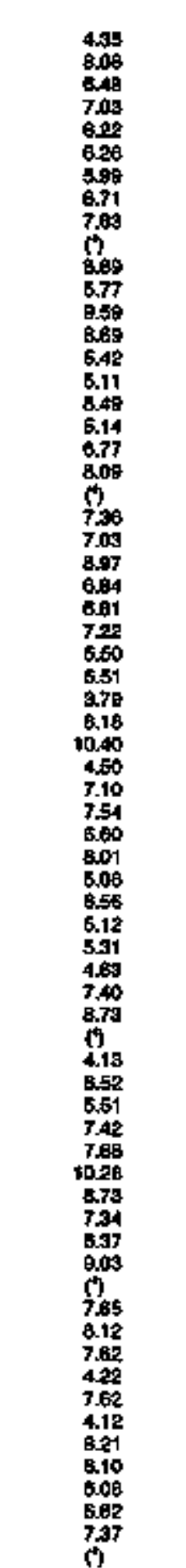 \\
\hline
\end{tabular}

Som foctholes et end of lable. 
Table 14. Class of Ownership, Number of Uitumate Consumers, Revenue, Sales, and Average Revenue per Kilowatthour for the Residential Sector by State and Utitity, 1993 (Continued)

\begin{tabular}{|c|c|c|c|c|c|}
\hline Sithe & $\begin{array}{c}\text { Close } \\
\text { of } \\
\text { Orimerthlp }\end{array}$ & $\begin{array}{c}\text { Number } \\
\text { ol } \\
\text { Conesmars }\end{array}$ & $\begin{array}{c}\text { Fewemt } \\
\text { \{thouseand doulares }\end{array}$ & 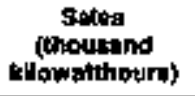 & 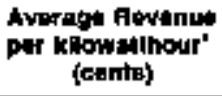 \\
\hline 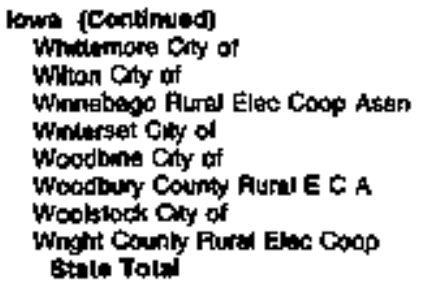 & 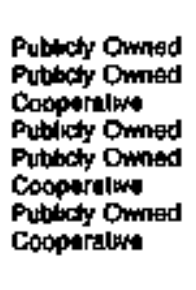 & $\begin{array}{r}251 \\
1,099 \\
1,059 \\
1,767 \\
816 \\
2,801 \\
104 \\
1,800 \\
1,1590909\end{array}$ & $\begin{array}{r}147 \\
716 \\
2,579 \\
664 \\
906 \\
3,731 \\
67 \\
2,747 \\
604,136\end{array}$ & $\begin{array}{r}2169 \\
9,382 \\
35,116 \\
12,591 \\
7,205 \\
42,365 \\
958 \\
30,0893 \\
11,103,104\end{array}$ & $\begin{array}{l}878 \\
765 \\
734 \\
800 \\
426 \\
881 \\
17 \\
619 \\
8.02\end{array}$ \\
\hline 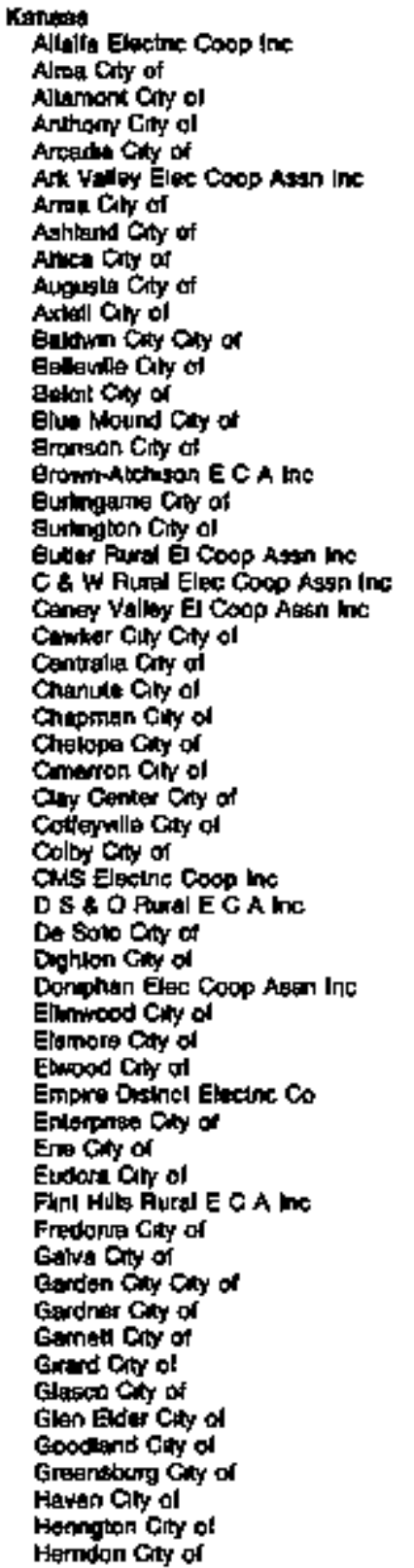 & 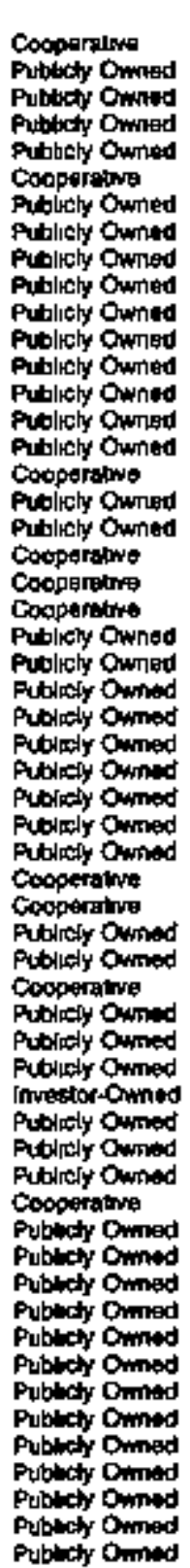 & 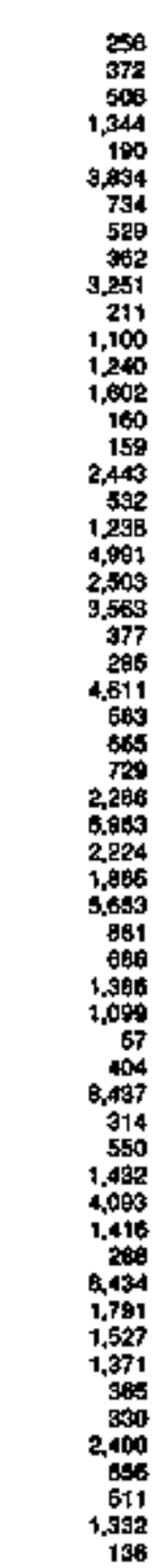 & 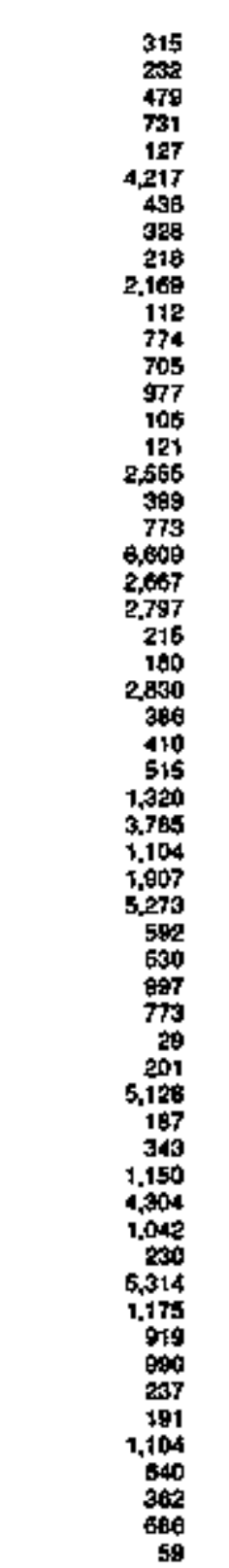 & 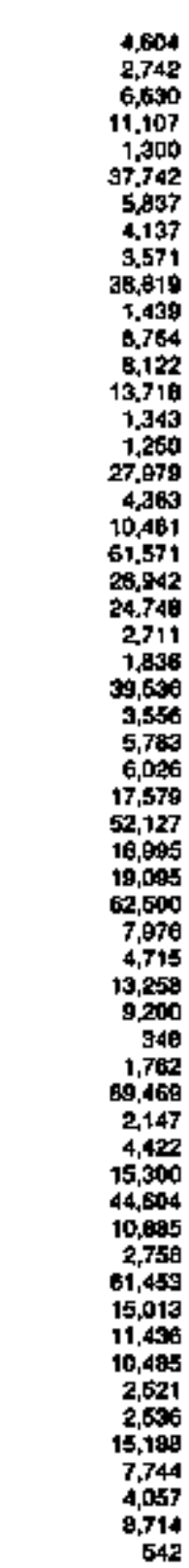 & 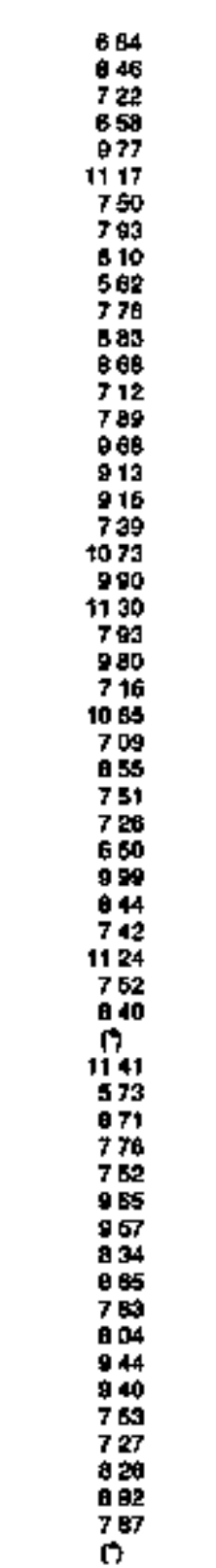 \\
\hline
\end{tabular}

Set loolnotes al and of table 
Table 14. Class of Ownership, Number of Uttmate Consumers, Revenue, Sales, and Average Revenue per Kilowatthour for the Residential Seclor by State and Utility, 1993 (Continued)

\begin{tabular}{|c|c|c|c|c|c|}
\hline 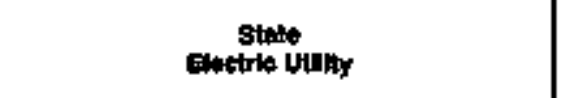 & $\begin{array}{c}\text { ctasts } \\
\text { od }\end{array}$ & $\begin{array}{c}\text { Humber } \\
\text { at } \\
\text { Conaumars }\end{array}$ & Rovenuld & 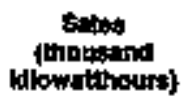 & 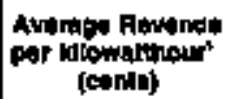 \\
\hline 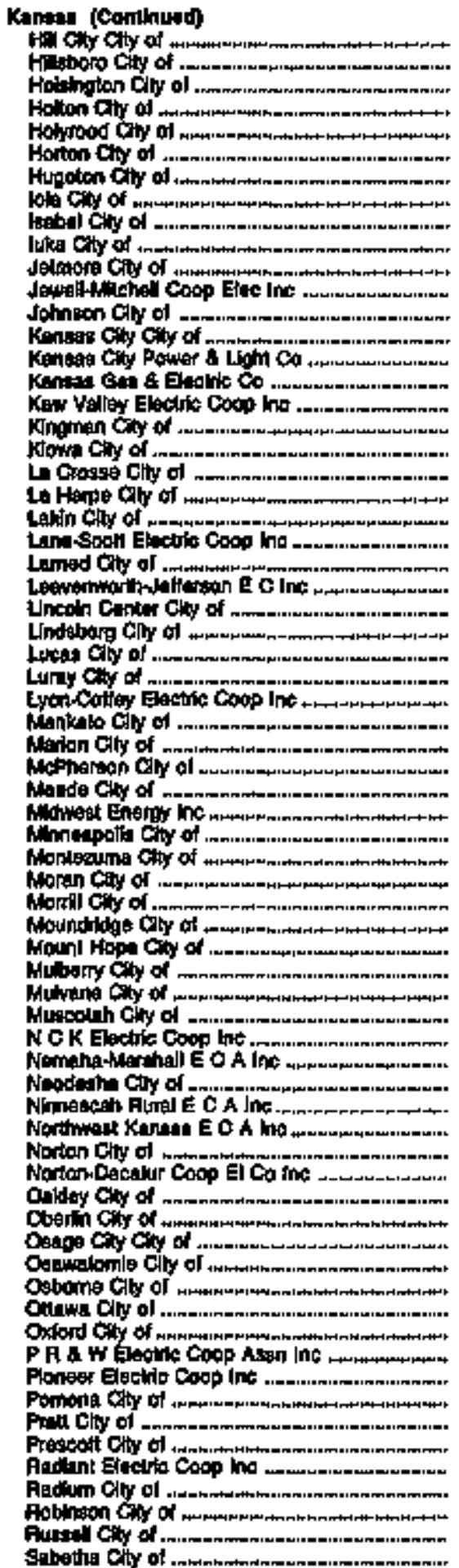 & 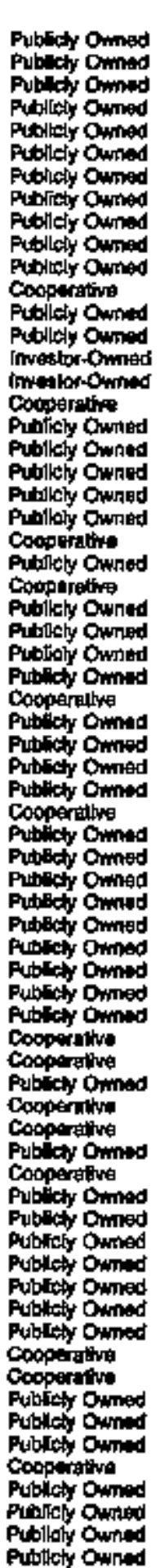 & 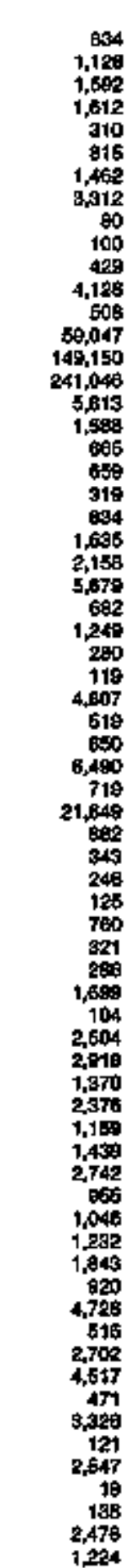 & 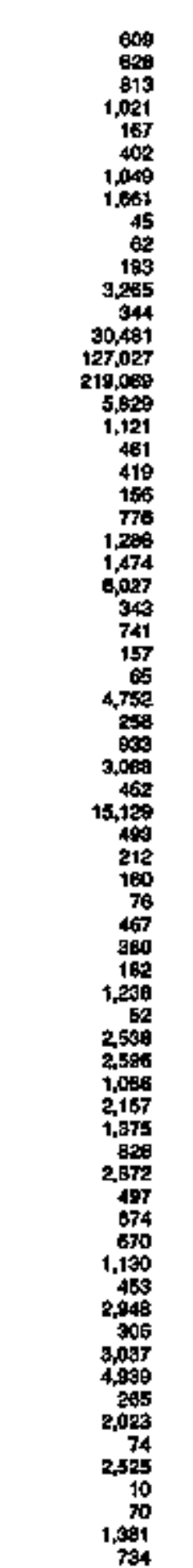 & 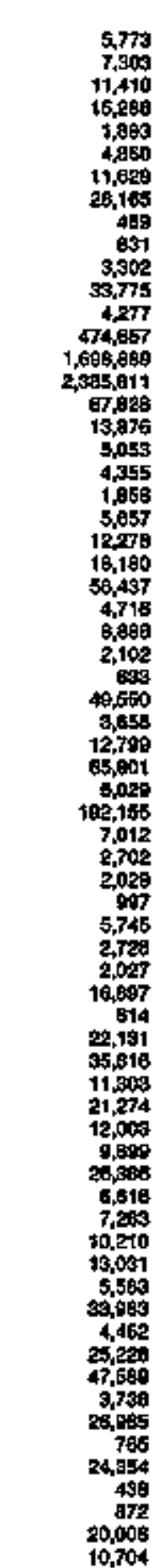 & 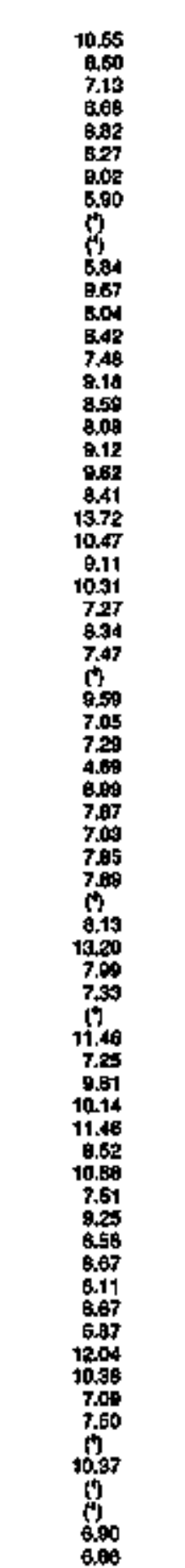 \\
\hline
\end{tabular}

Sas foolnotes at end of table. 
Table 14. Class of Ownerstip, Number of Ultimate Consumers, Revenue, Sales, and Average Revonue por Kllowatthour for the Resldential Sector by State and Utility, 1993 (Continuad)

\begin{tabular}{|c|c|c|c|c|c|}
\hline $\begin{array}{c}\text { bials } \\
\text { Elestrls Utilty }\end{array}$ & $\begin{array}{c}\text { ctoba } \\
\text { of } \\
\text { Onweraplp }\end{array}$ & $\begin{array}{l}\text { Number } \\
\text { of } \\
\text { centumernt }\end{array}$ & (thouband dollere) & 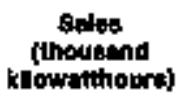 & 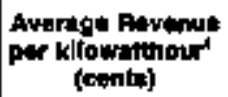 \\
\hline 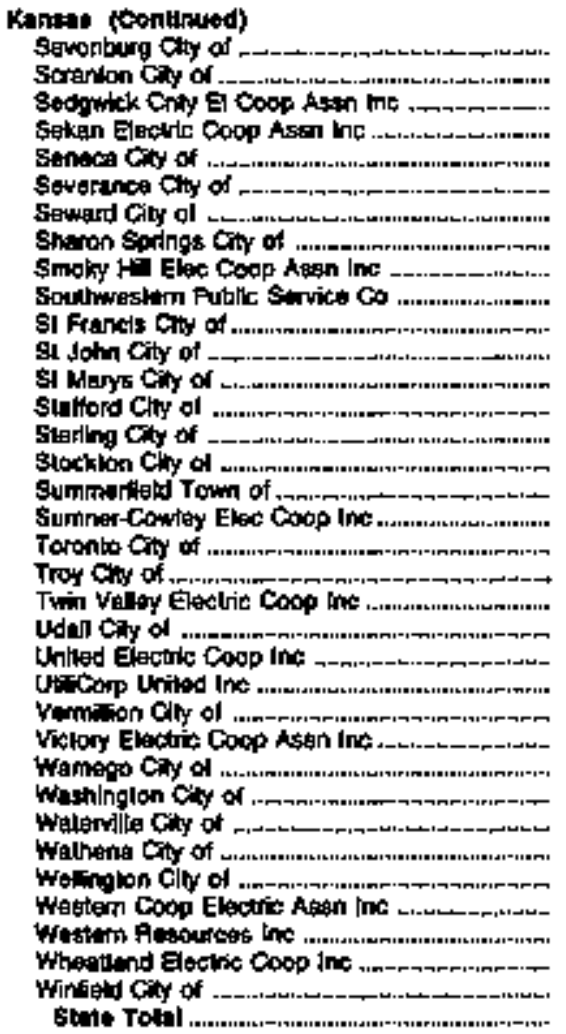 & 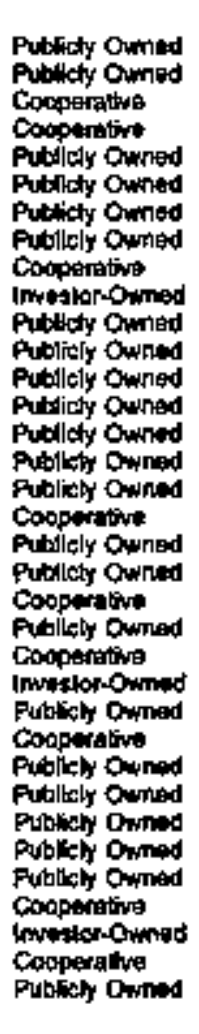 & 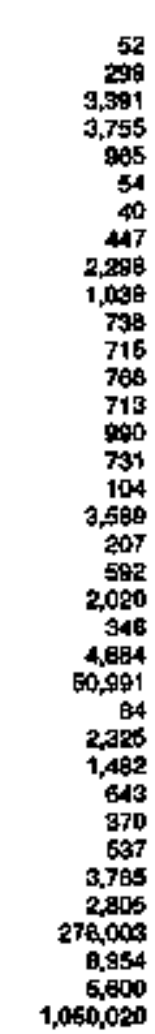 & 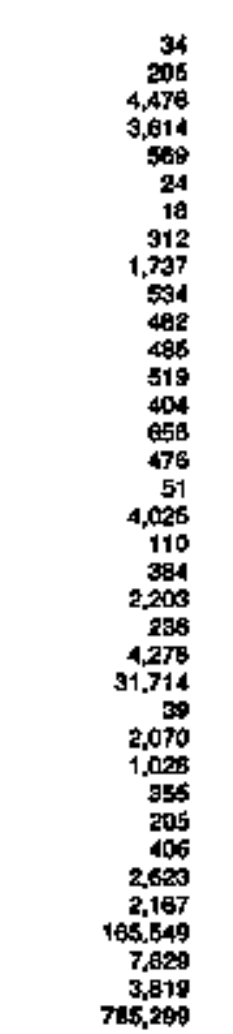 & 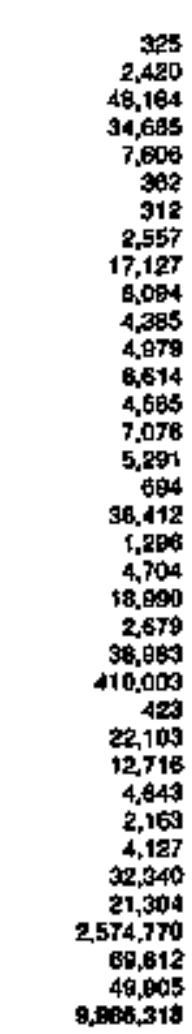 & 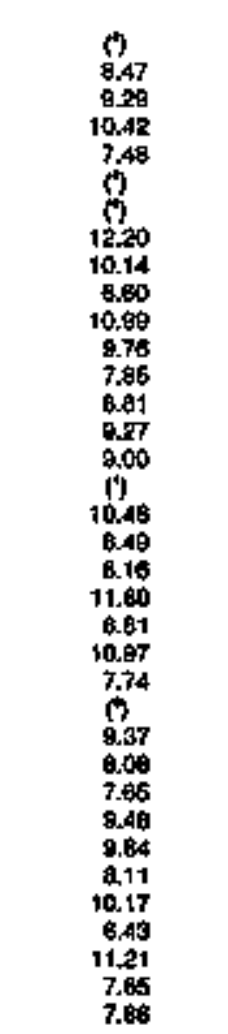 \\
\hline 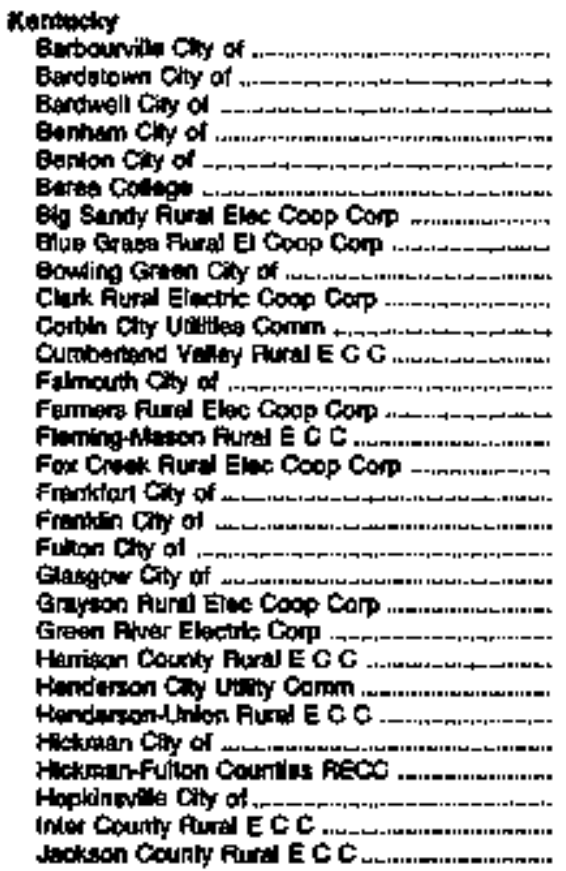 & 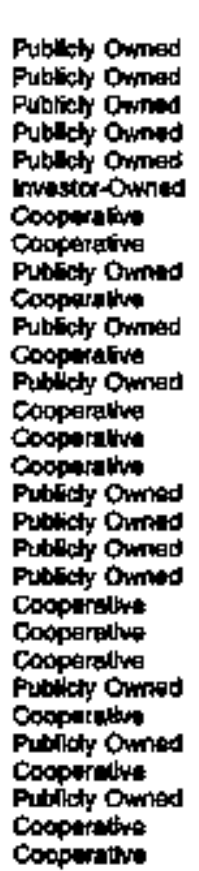 & 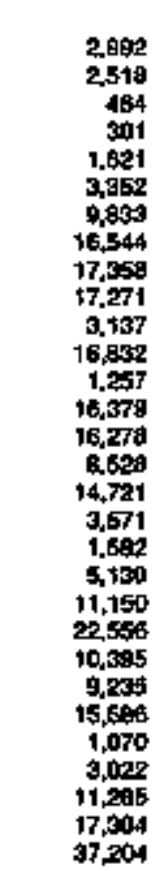 & 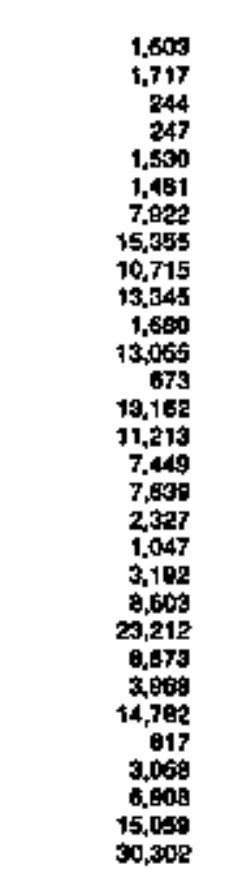 & 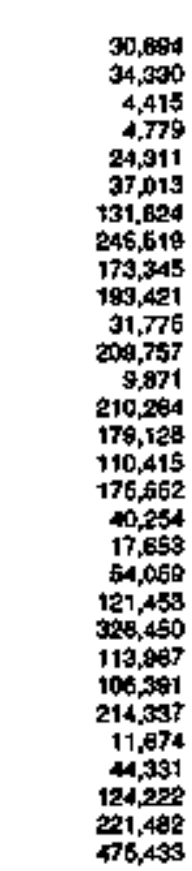 & 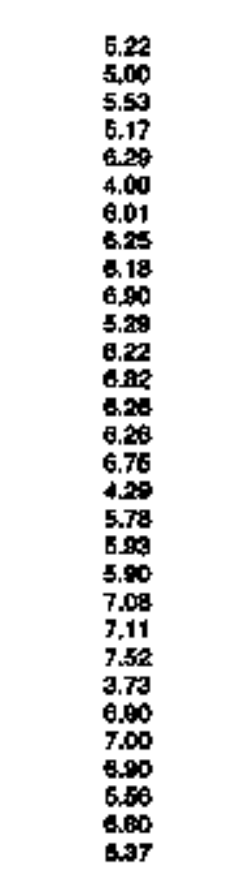 \\
\hline
\end{tabular}

Seg foutroitan at end of tabla. 
Table 14. Class of Ownership, Number of Lttmate Consumers, Revenue, Sales, and Average Revenue per Kllowatthour for the Residentlal Sector by stete and Utility, 1893 (Continued)

\begin{tabular}{|c|c|c|c|c|c|}
\hline $\begin{array}{c}\text { steto } \\
\text { Eecide villty }\end{array}$ & $\begin{array}{c}\text { of } \\
\text { Owinestiplp }\end{array}$ & $\begin{array}{c}\text { Humbor } \\
\text { of }\end{array}$ & $\begin{array}{c}\text { Fovente } \\
\text { (thousend dolbra) }\end{array}$ & $\begin{array}{c}\text { 8ales } \\
\text { (tholritind } \\
\text { ldlawatipound }\end{array}$ & 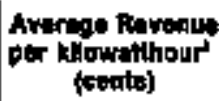 \\
\hline 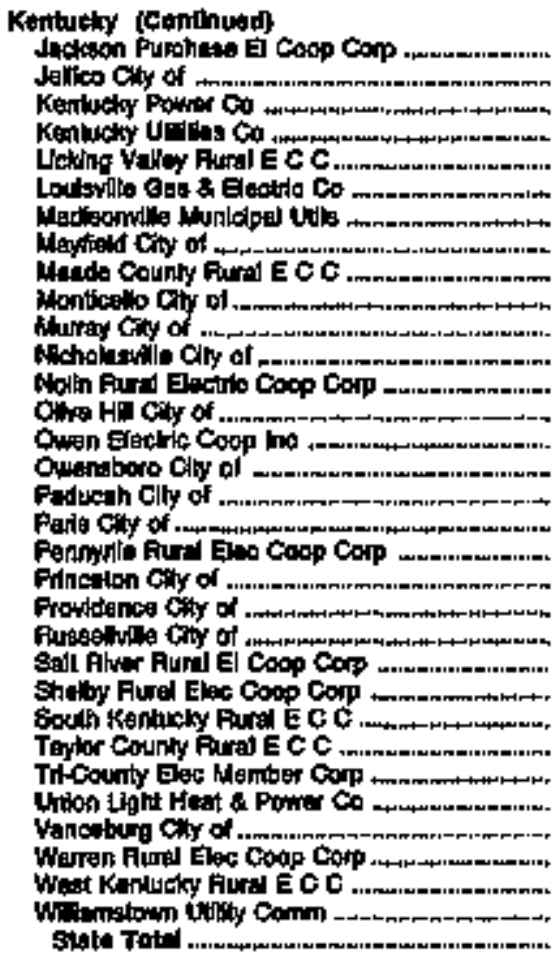 & 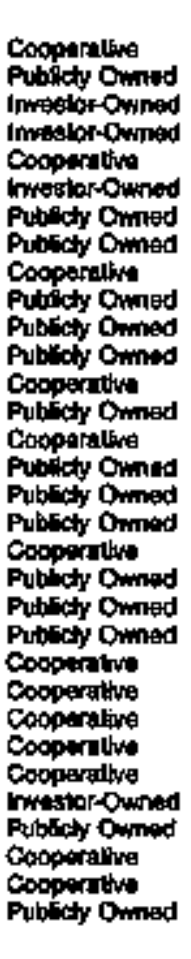 & 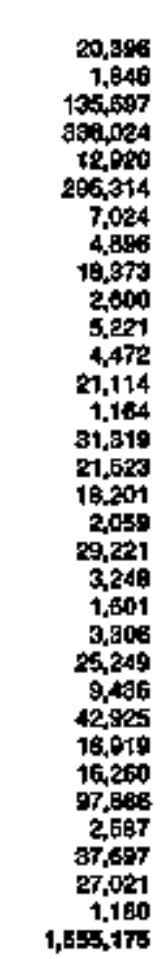 & 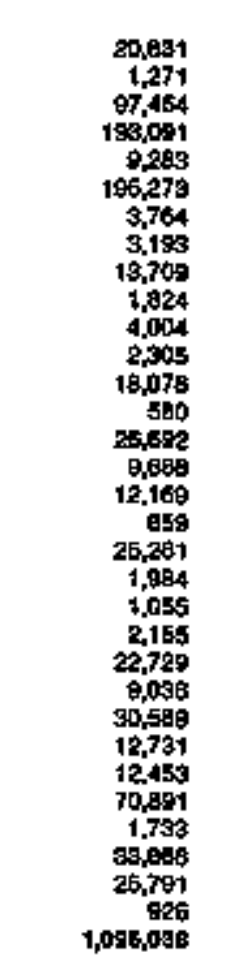 & 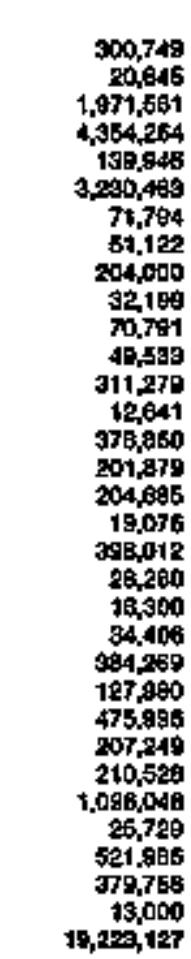 & 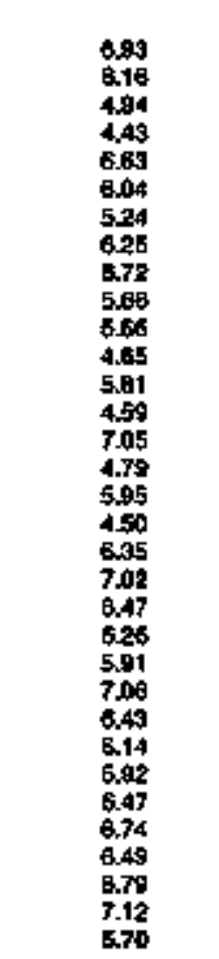 \\
\hline 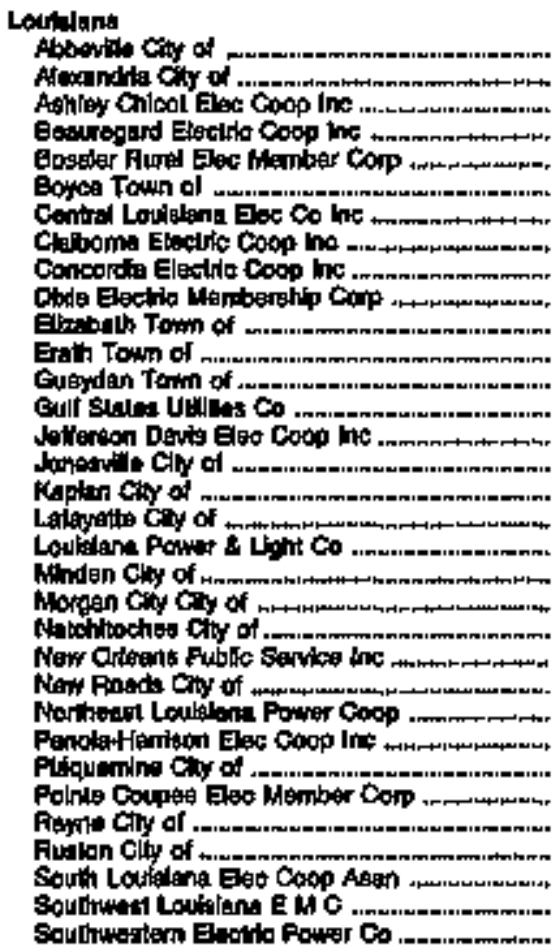 & 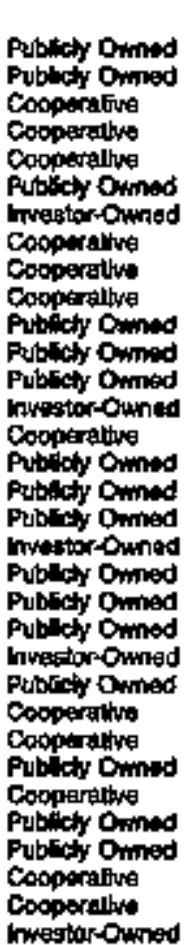 & 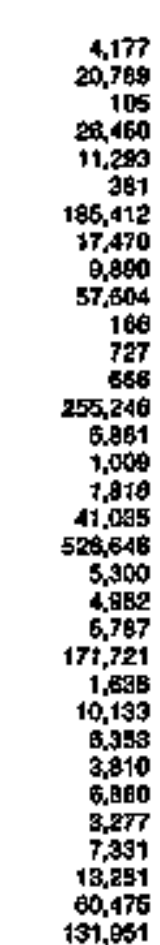 & 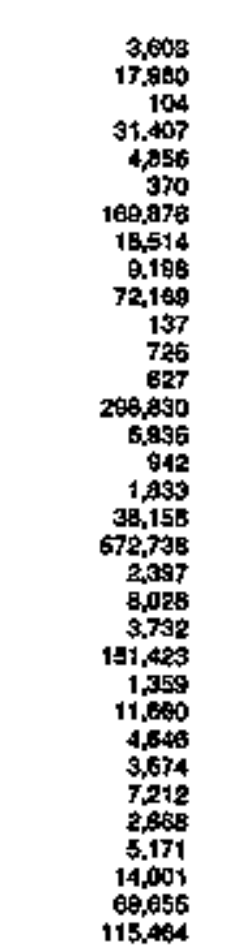 & 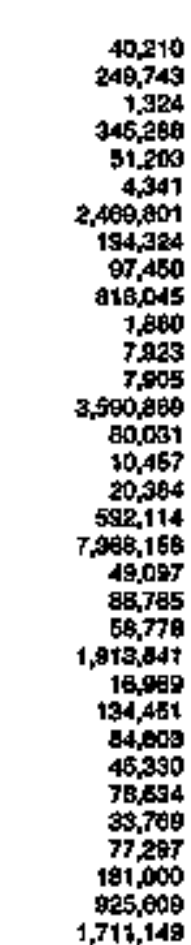 & 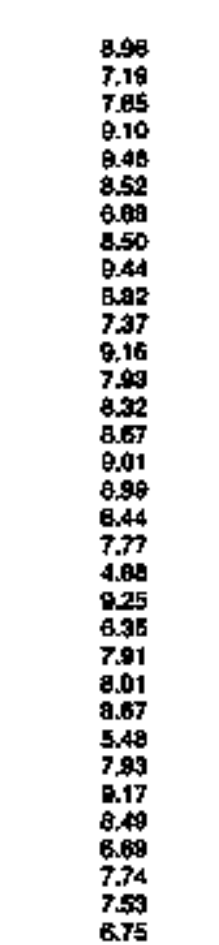 \\
\hline
\end{tabular}

See toothotea al end of tabla. 
Table 14. Class of Ownership, Number of Utimate Consumers, Revenue, Sales, and Average Revenue per Kilowatthour for the Residential Sector by State and Utility, 1993 (Continuted)

\begin{tabular}{|c|c|c|c|c|c|}
\hline $\begin{array}{c}\text { Stat: } \\
\text { Eracter| Udilty }\end{array}$ & 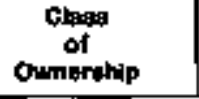 & $\begin{array}{c}\text { Number } \\
\text { of } \\
\text { Congumara }\end{array}$ & $\begin{array}{c}\text { Revanus } \\
\text { (thousend dolurt) }\end{array}$ & 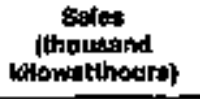 & 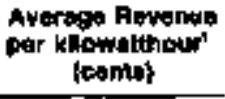 \\
\hline 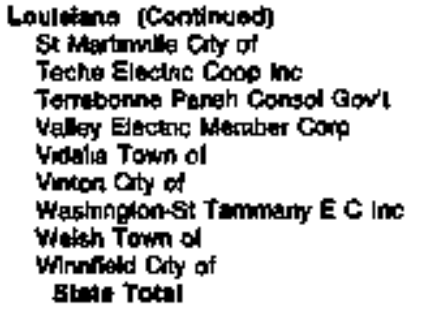 & 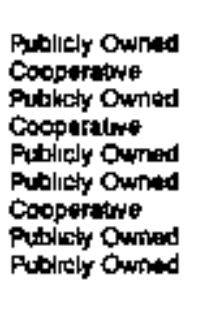 & $\begin{array}{r}2,422 \\
7,964 \\
7,501 \\
31,347 \\
2,161 \\
1,301 \\
28,8006 \\
2,002 \\
2,348 \\
1,698,709\end{array}$ & $\begin{array}{r}2,189 \\
6,827 \\
6,401 \\
31,179 \\
1,854 \\
1,482 \\
37,300 \\
1,079 \\
2,127 \\
1,740,707\end{array}$ & $\begin{array}{r}27,9695 \\
104,883 \\
106,961 \\
928,906 \\
25,780 \\
14,396 \\
110,280 \\
19,778 \\
32,428 \\
22,490,248\end{array}$ & $\begin{array}{r}780 \\
942 \\
608 \\
945 \\
720 \\
1030 \\
902 \\
546 \\
650 \\
7.76\end{array}$ \\
\hline 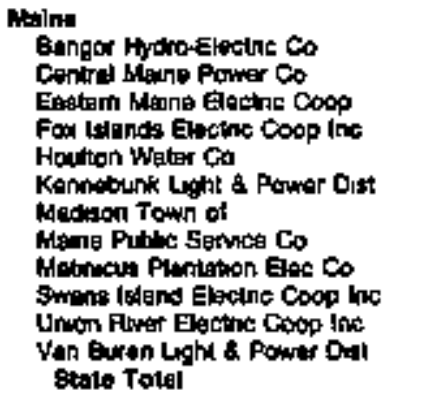 & 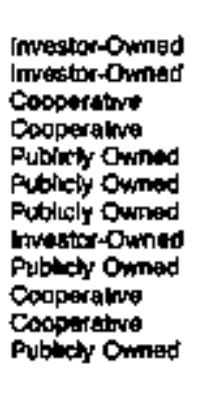 & $\begin{array}{r}68,347 \\
451,497 \\
9,846 \\
1,355 \\
3,844 \\
3,953 \\
2,021 \\
98,220 \\
100 \\
489 \\
1,690 \\
1,231 \\
590,152\end{array}$ & $\begin{array}{r}64,123 \\
349,189 \\
5,639 \\
953 \\
2,422 \\
3,210 \\
1,448 \\
10.670 \\
48 \\
396 \\
949 \\
736 \\
442,724\end{array}$ & $\begin{array}{r}516,342 \\
3,031,981 \\
58,134 \\
4,896 \\
26,139 \\
93,909 \\
14,718 \\
17,738 \\
183 \\
1,730 \\
6,251 \\
7,889 \\
3,972,290\end{array}$ & $\begin{array}{r}1242 \\
1132 \\
1061 \\
2064 \\
069 \\
949 \\
612 \\
1113 \\
19 \\
1642 \\
1518 \\
600 \\
11.43\end{array}$ \\
\hline 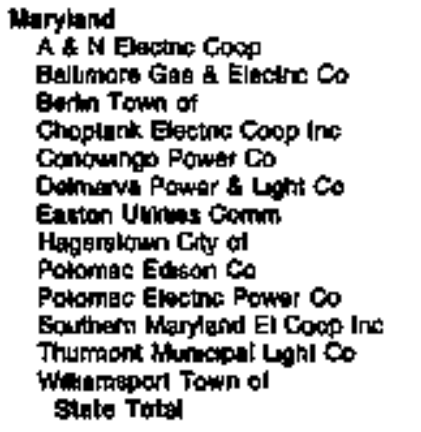 & 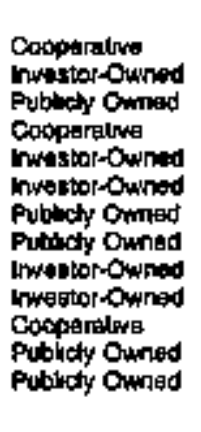 & $\begin{array}{r}3526 \\
6 \% 2,074 \\
1,431 \\
32,121 \\
32,219 \\
107,810 \\
5,920 \\
15,502 \\
169,297 \\
399,652 \\
96,405 \\
1,770 \\
739 \\
1,813,250\end{array}$ & $\begin{array}{r}251 \\
031,649 \\
1,196 \\
36,600 \\
41,601 \\
100,646 \\
4,562 \\
6,789 \\
161,729 \\
960,747 \\
102,310 \\
1,278 \\
499 \\
1,700,788\end{array}$ & $\begin{array}{r}2,630 \\
10,814,235 \\
14,343 \\
393,490 \\
399,640 \\
1,160,230 \\
69,469 \\
113,010 \\
2,291,092 \\
5,105,180 \\
1,320,290 \\
25,300 \\
9,119 \\
21,649,122\end{array}$ & $\begin{array}{r}085 \\
078 \\
702 \\
979 \\
1054 \\
049 \\
667 \\
604 \\
682 \\
761 \\
771 \\
505 \\
537 \\
6.21\end{array}$ \\
\hline 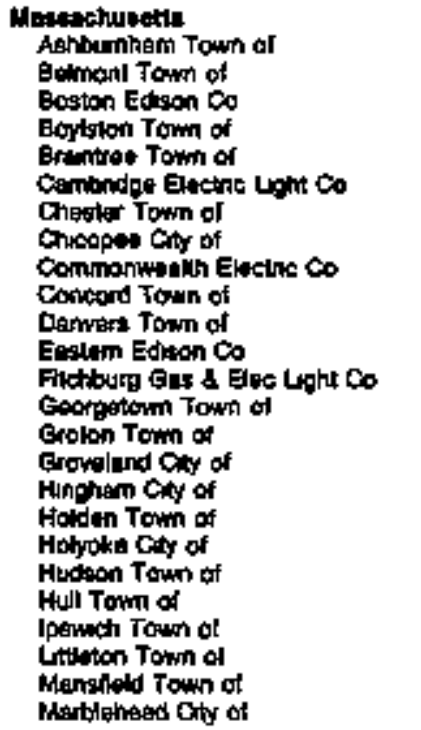 & 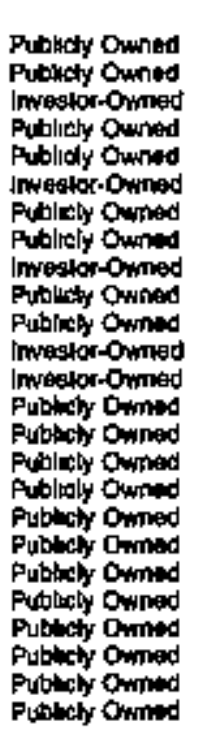 & 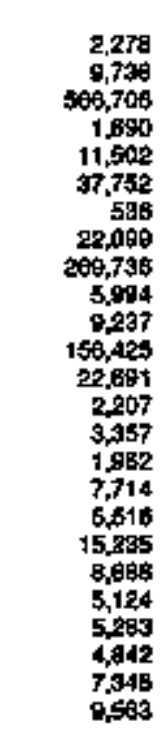 & 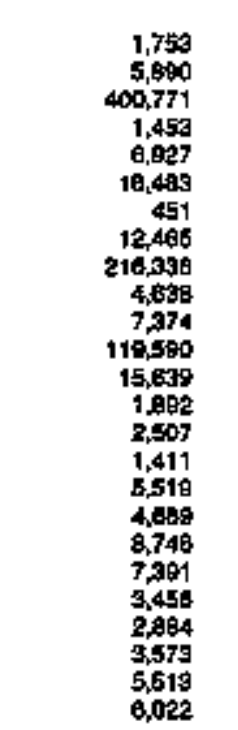 & 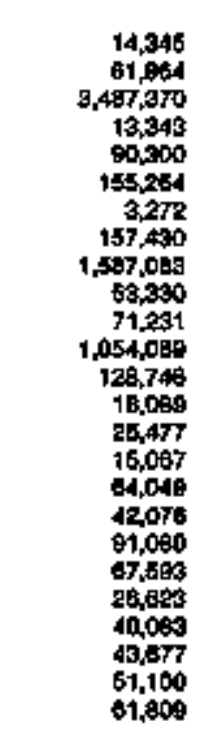 & $\begin{array}{r}1222 \\
952 \\
1149 \\
1099 \\
767 \\
1100 \\
1378 \\
790 \\
1369 \\
670 \\
1095 \\
1135 \\
1215 \\
1048 \\
964 \\
936 \\
862 \\
1114 \\
960 \\
1099 \\
1298 \\
720 \\
818 \\
1070 \\
674\end{array}$ \\
\hline
\end{tabular}

Sow lootnotes al and of teblo 
Table 14. Clase of Ownershlp, Number of Ultimate Consumers, Rovenue, Salea, and Average Revenue per KClowatthour for the Residentlal Sector by State and Ut'lity, 1993 (Continued)

\begin{tabular}{|c|c|c|c|c|c|}
\hline $\begin{array}{l}\text { Stite } \\
\text { Enotific villity }\end{array}$ & $\begin{array}{c}\text { ciat: } \\
\text { of } \\
\text { Ormeithip }\end{array}$ & $\begin{array}{c}\text { of } \\
\text { ofmber } \\
\text { Cormunar: }\end{array}$ & Fiortenve & $\begin{array}{c}\text { selto } \\
\text { (whodend } \\
\text { ktowathourt) }\end{array}$ & 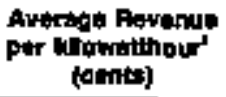 \\
\hline 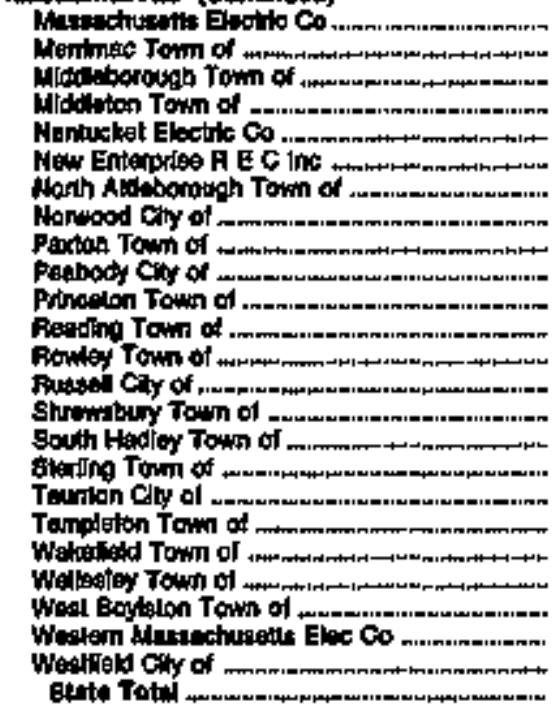 & 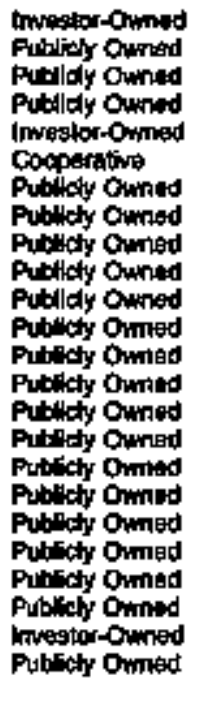 & 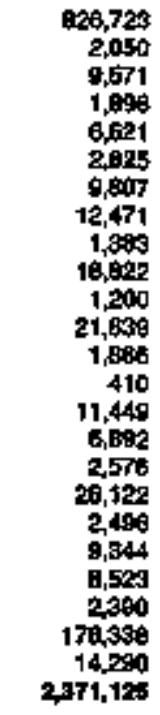 & 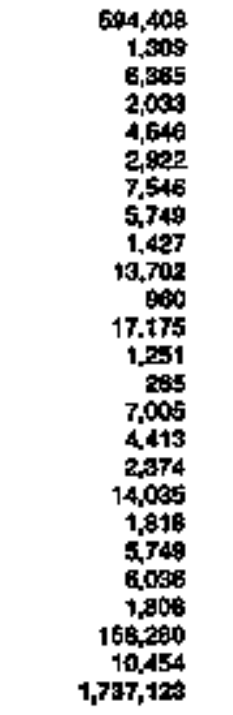 & 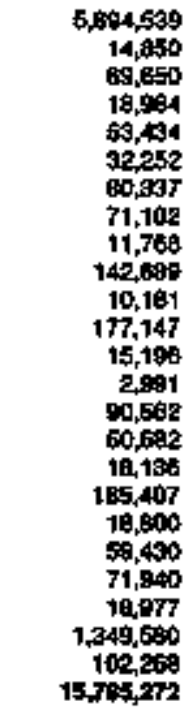 & $\begin{array}{r}10.44 \\
8.91 \\
9.14 \\
10.72 \\
8.69 \\
9.06 \\
8.39 \\
6.09 \\
12.13 \\
9.60 \\
9.49 \\
9.70 \\
9.20 \\
9.53 \\
7.74 \\
9.71 \\
13.09 \\
7.57 \\
9.67 \\
9.67 \\
6.89 \\
9.59 \\
11.73 \\
10.20 \\
11.00\end{array}$ \\
\hline 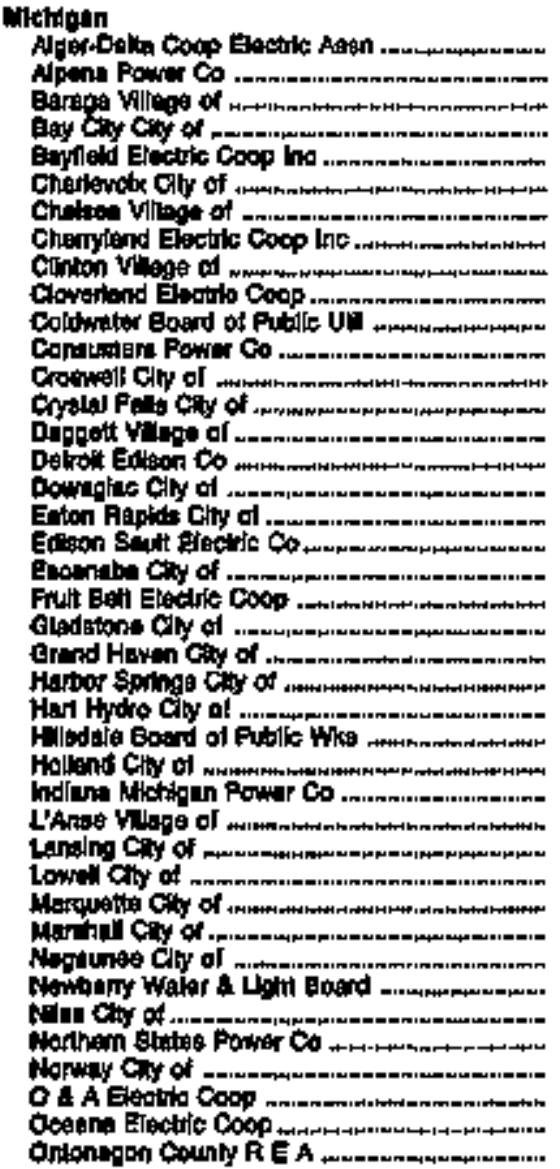 & 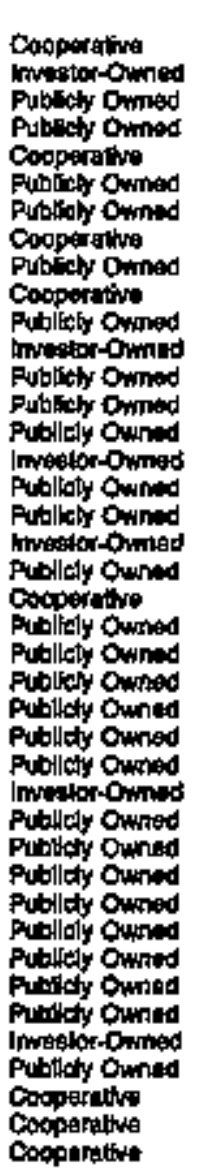 & 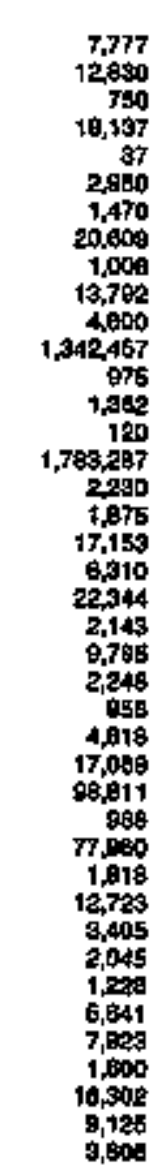 & 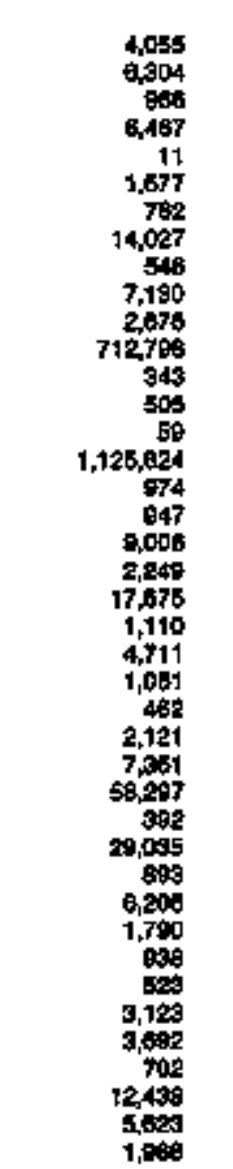 & 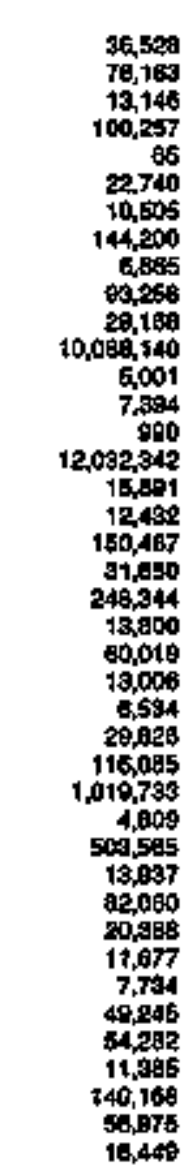 & 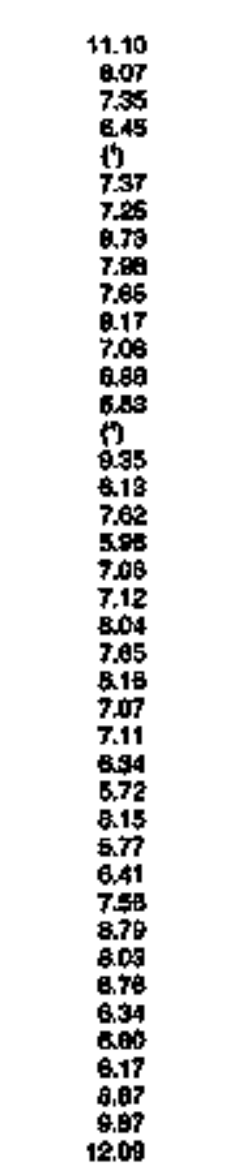 \\
\hline
\end{tabular}

ses forkngles at and of table. 
Table 14. Class of Owmorship, Number of Ultimate Consumers, Aevenue, Seles, and Average Revenue per Kilowatthour for the Residentlal Soclor by State and Utiltit, 1993 (Contlnued)

\begin{tabular}{|c|c|c|c|c|c|}
\hline Stale & 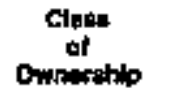 & $\begin{array}{l}\text { Notmber } \\
\text { of } \\
\text { Contumas: }\end{array}$ & $\begin{array}{c}\text { Revitinue } \\
\text { (Thodiend dollara) }\end{array}$ & $\begin{array}{c}\text { sutis: } \\
\text { (thouspand } \\
\text { hlowathoura] }\end{array}$ & 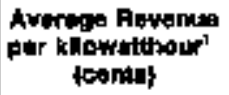 \\
\hline 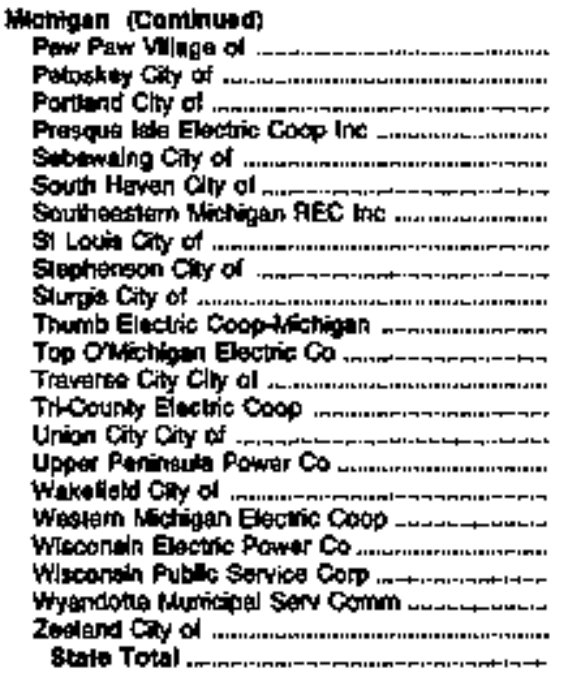 & 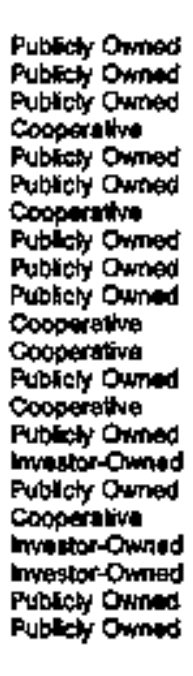 & 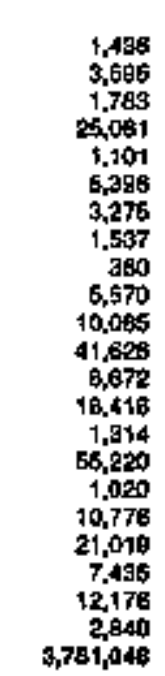 & $\begin{array}{r}541 \\
1,355 \\
1,005 \\
12,047 \\
515 \\
2,948 \\
2,378 \\
737 \\
168 \\
2,965 \\
3,929 \\
25,469 \\
2,521 \\
14,388 \\
687 \\
22,033 \\
408 \\
5,010 \\
11,503 \\
3,591 \\
3,108 \\
1,145 \\
2,184,006\end{array}$ & 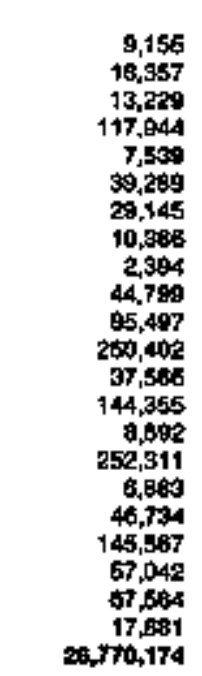 & $\begin{array}{r}5.91 \\
7.38 \\
7.80 \\
10.88 \\
6.85 \\
7.25 \\
6.18 \\
7.10 \\
6.47 \\
6.82 \\
6.72 \\
9.76 \\
6.71 \\
9.06 \\
7.90 \\
9.05 \\
6.90 \\
10.72 \\
7.83 \\
6.19 \\
9.04 \\
6.40 \\
8.16\end{array}$ \\
\hline 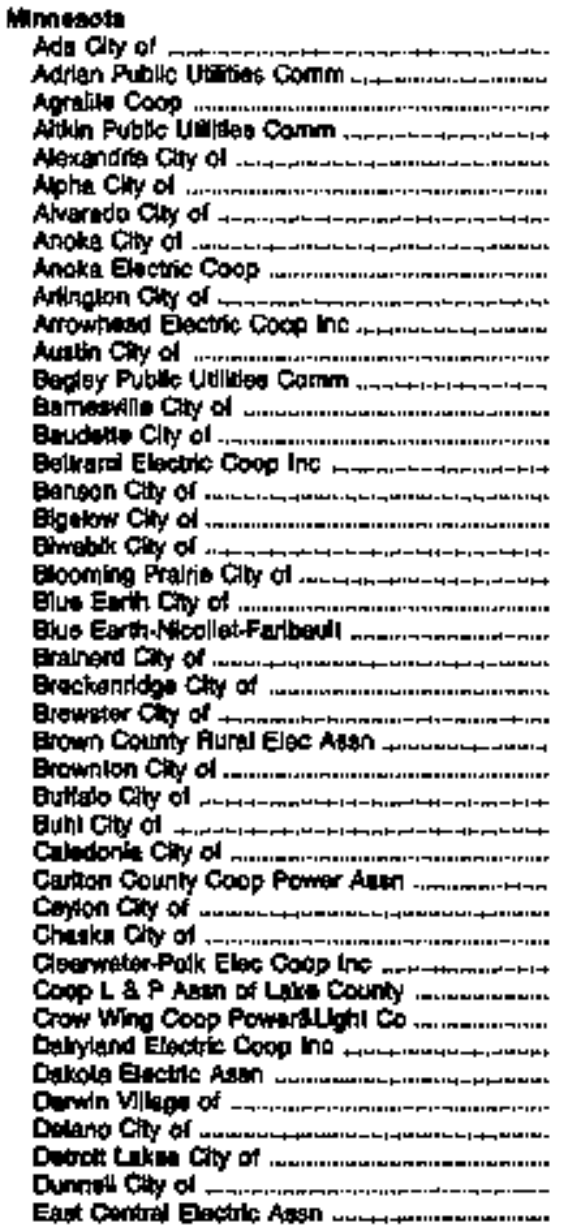 & 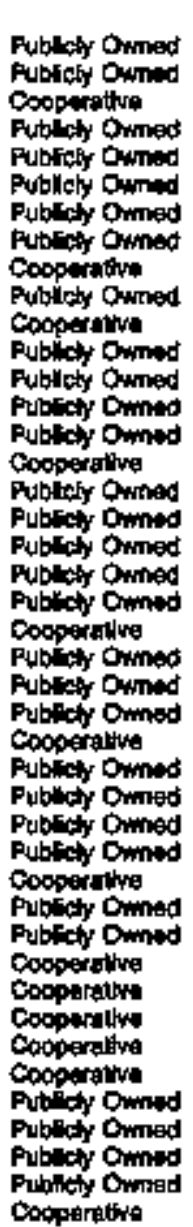 & 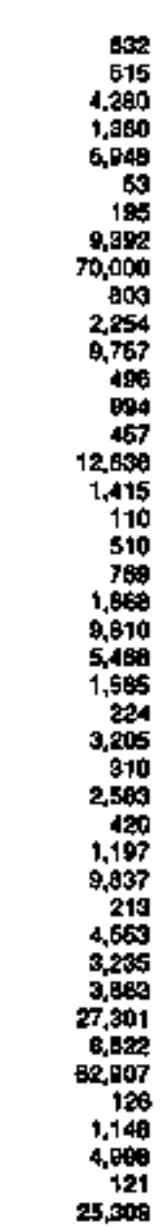 & 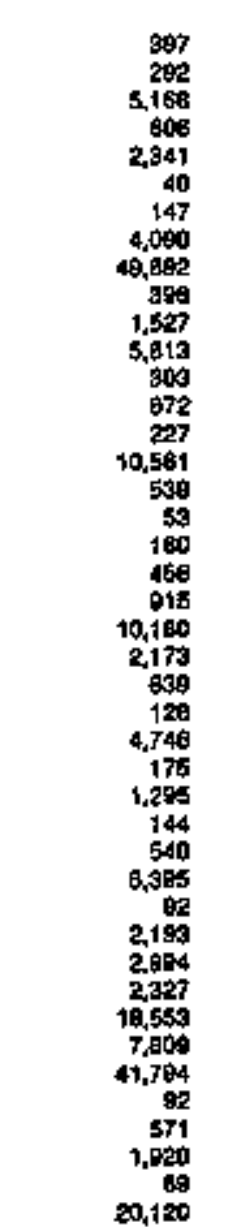 & 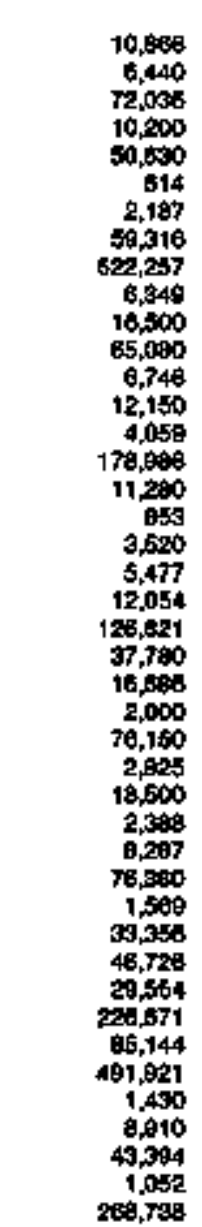 & 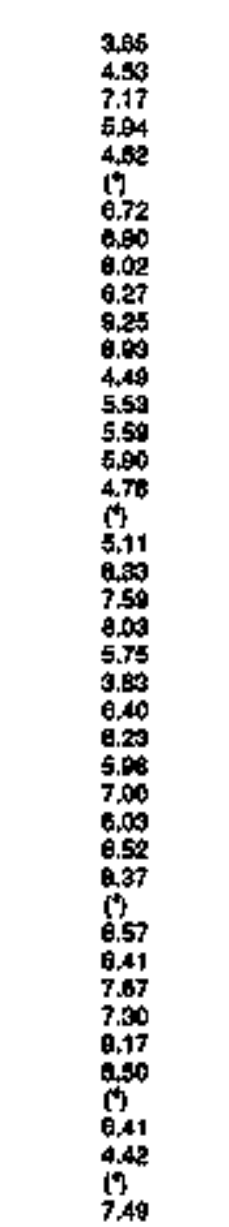 \\
\hline
\end{tabular}

See 1 botnoten al and of table. 
Table 14. Class of Owmership, Number of Litimate Consumers, Revenue, sales, and Average Ravenuto per Kllowatthour for the Rogidentlal Sinctor by State and Utily, 1998 (Continued)

\begin{tabular}{|c|c|c|c|c|c|}
\hline $\begin{array}{c}\text { Stat } \\
\text { enctio ubily }\end{array}$ & 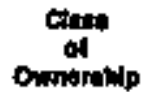 & $\begin{array}{l}\text { Mumbar } \\
\text { of } \\
\text { Consumare }\end{array}$ & Fovithe & 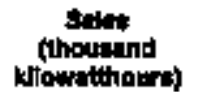 & 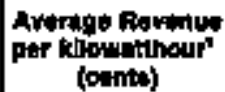 \\
\hline
\end{tabular}

Uhnarota (Conthnod)

Eepl Grend Forth Caty of .

Erron tory of

Ebow lake Clly of

Ey Ciny of

Fintoric col of

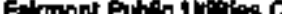

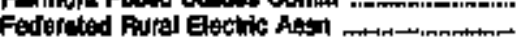

Foption Con of

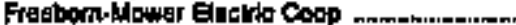

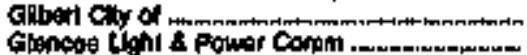

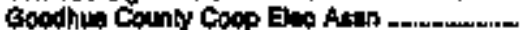

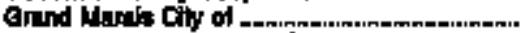

Griond Raplds Publlo Itti Coinm

Gromite Fath chy of .

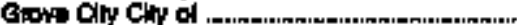

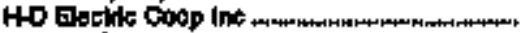

Haloted Ciny ot .

Harmeny Cary of

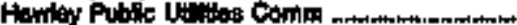

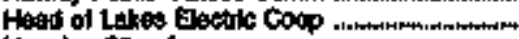

Henning coly of

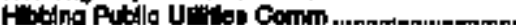

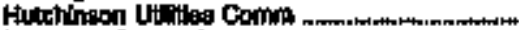

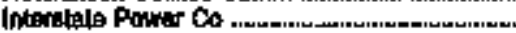

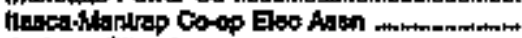

Jactison City of

Jentwilla Cy of

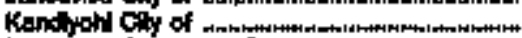

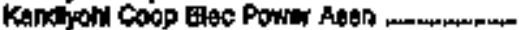

Kawoll Cly al

Katsion bify of .

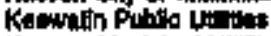

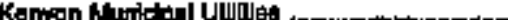

Lake city Chy of

Inse Cryatil Ciny of

Lake Patk City of

Lake Realon Coop El Eo Aatn .

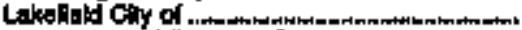

Lanesbato Pubile Uimily Comm

La guver Chy of .

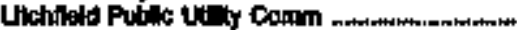

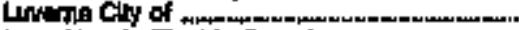

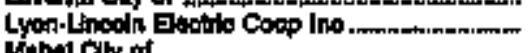

Mabal Caly of

Madatle Ciby ol .

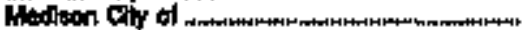

Hershell clly of

Mol and Coap Power Anm .

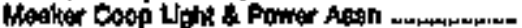

Nalroes Publo Uth

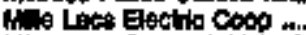

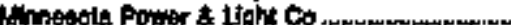

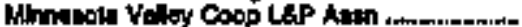

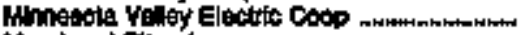

Hoophas Cily of

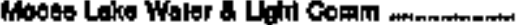

Mora tity of

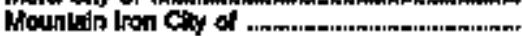

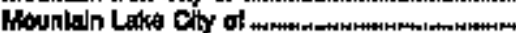

Nethrinets Cly of

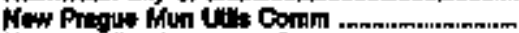

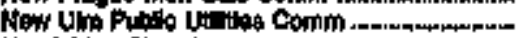

Newroldun City of

Neatevitio ciny or

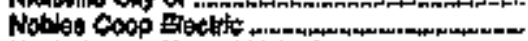

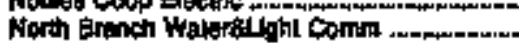

Publicty Omed

Pibilcty Oinned

Publledy Oined

Putulaty Ompred

Puticts Ommed

Publichy Onmed

Pubilcty Oimad

Cooperafive

Puticty Ouned

Cooperntws

Pubichy Onmerd

Pabicty Ommod

Cooperativa

Putuch Ownot

Publicty Ommed

Pubicty onined

Fublest Ownos

Cooperafive

Puticty Omed

Publicty owned

Fusilut Dwmad

Cooparative

Publety Owned

Puthaty Ownod

Fublist Owmad

imestor Oned

Cooperatve

Puituctr Ombad

Putaliok owned

Publikw Dwmed

Cooperalve

Fublety Ownes

Publect owned

Pulbety owned

Publich Owned

Pastest Oimad

Pubich Ownod

Fublich owmed

Cosporatu

Fublinty Drmad

Publety Ommad

Pustioly Owned

Fulbilety Ownod

Pulblety Owned

Cooperatwe

Futblist Owmed

Publesty Owined

Putblity Dwmad

Pulbectr Ownot

Coponithe

Copperative

Pribilety Owned

cooperstive

muasturowned

Cooperatur

Cooperative

Pustrely Ownod

Publetr Owmod

Fublist Ownad

Publicty Owned

Publech Dwned

Publefy Owned

Publicty owned

Publaty ownod

Pusilety Ownos

Puldets Onmed

Cooperaltre

Pulbety Owned
9.506

107

3,976

1,900

576

5,042

4,514

5,439

876

a.070

3,722

784
4,498

1,300

205

12
283

405

700

376

B. 240

4,714

30,449

7,312

1,697

ert

200

t,420

580

700

2,056

880
283

10.54

768

146

2430

1,984

3,600

1555

$\mathbf{g} 8$

846
5,016

5 s19

6.3185

$7,0 \mathrm{ga}$

10,804

1 \%

6,005

16,215

11,258

645

1,249

eas

806

1989

5,400

180

55
4,618

4,618
948
1,791

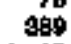

2,19?

ex5

276

2.816

5,115

$\$ 72$

6914

322

919

5,178

209

2,391

148

25

204

486

437

187

3000

1,612

18,4eg

4,306

537

405

120
-47

200

75

321

350

1,167

428

185

11,148

294

186

798

1,040

sy

4,045

128

40

377
2,010

e.014

a,04

58

5,674

4254

0.540

16,094

4,620

29

59

358

41

188

7t?

2,605

89

-447
479

42,100

$1,18 \%$

4,40

31.256

13,709

6,455

35,717

97,412

11,032

02,778

4089

15,610

64,685

5,020

37,506

10,7:5

2,246

256

3,000

8,340

4,351

3,51B

18,144

37 SAl5

228,792

es, 065

o.sor

B.,155.

3,015

apoas

2,208 
Table 14. Class of Ownership, Number of Uitimate Consumers, Revenue, Sales, and Average Revenue per Kilowatthour for the Residential Sector by State and Utikty, 1993 (Continued)

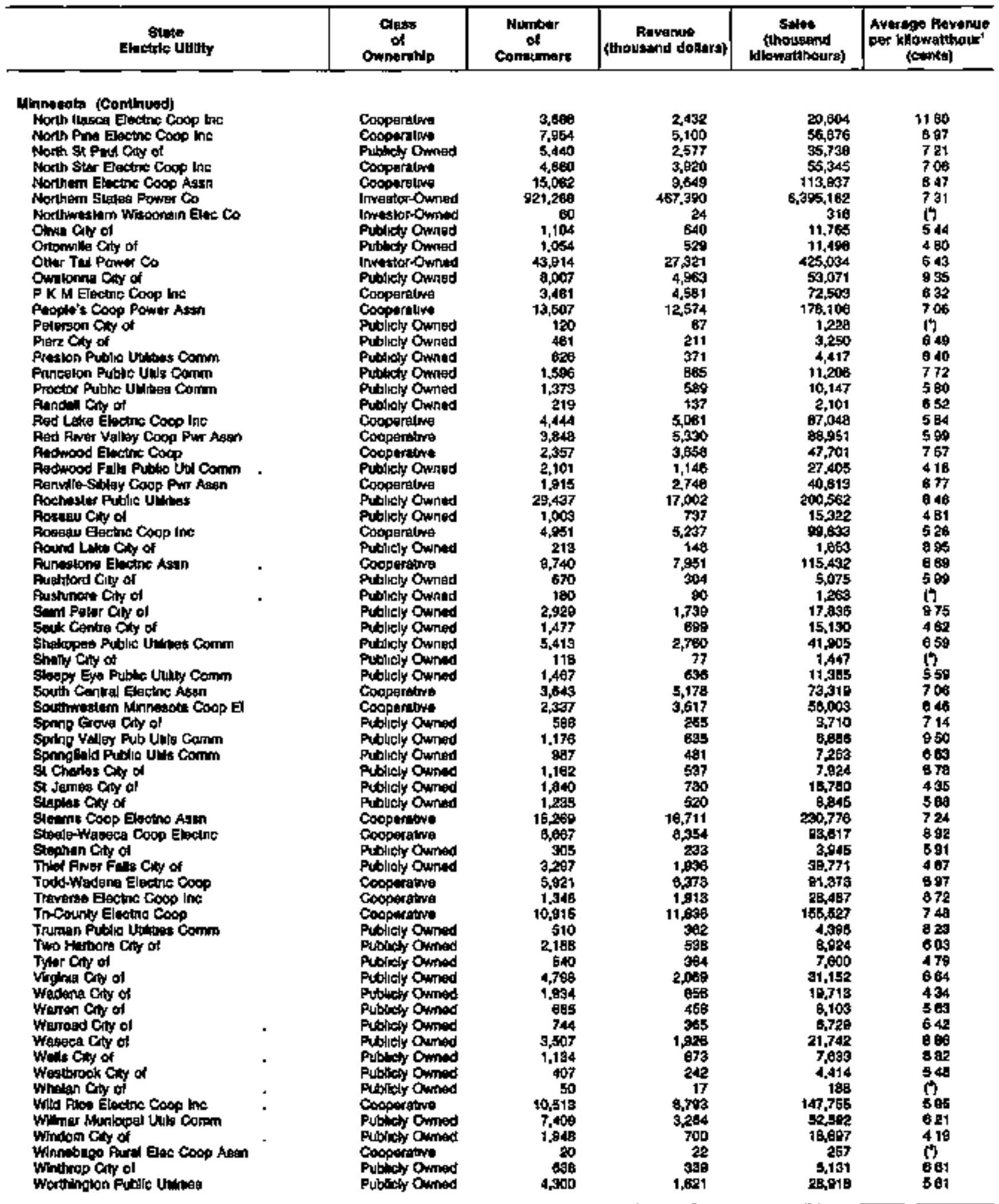

Sac lootnoks al and of tebio 
Table 14. Class of Ownerahip, Number of Uitimate Consumers, Revenus, Sales, and Average Reverue per Kllowatthour for the Residentlal Sector by State and Utilly, 1998 (Continued)

\begin{tabular}{|c|c|c|c|c|c|}
\hline 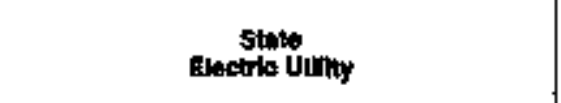 & 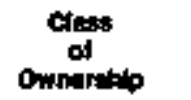 & $\begin{array}{l}\text { Number } \\
\text { of } \\
\text { Con:unions }\end{array}$ & $\begin{array}{c}\text { Ruvinut } \\
\text { (thousand dotara) }\end{array}$ & $\begin{array}{l}\text { Suled } \\
\text { (thoustind } \\
\text { idloinatibours) }\end{array}$ & 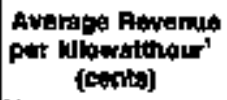 \\
\hline 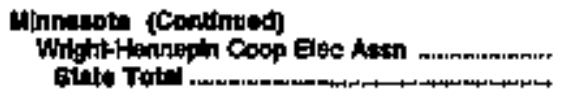 & Cooponitive & $\begin{array}{r}25,142 \\
1,838,750\end{array}$ & $\begin{array}{r}20,609 \\
1,105,348\end{array}$ & $\begin{array}{r}274,679 \\
15,504,685\end{array}$ & $\begin{array}{l}7.55 \\
7.09\end{array}$ \\
\hline 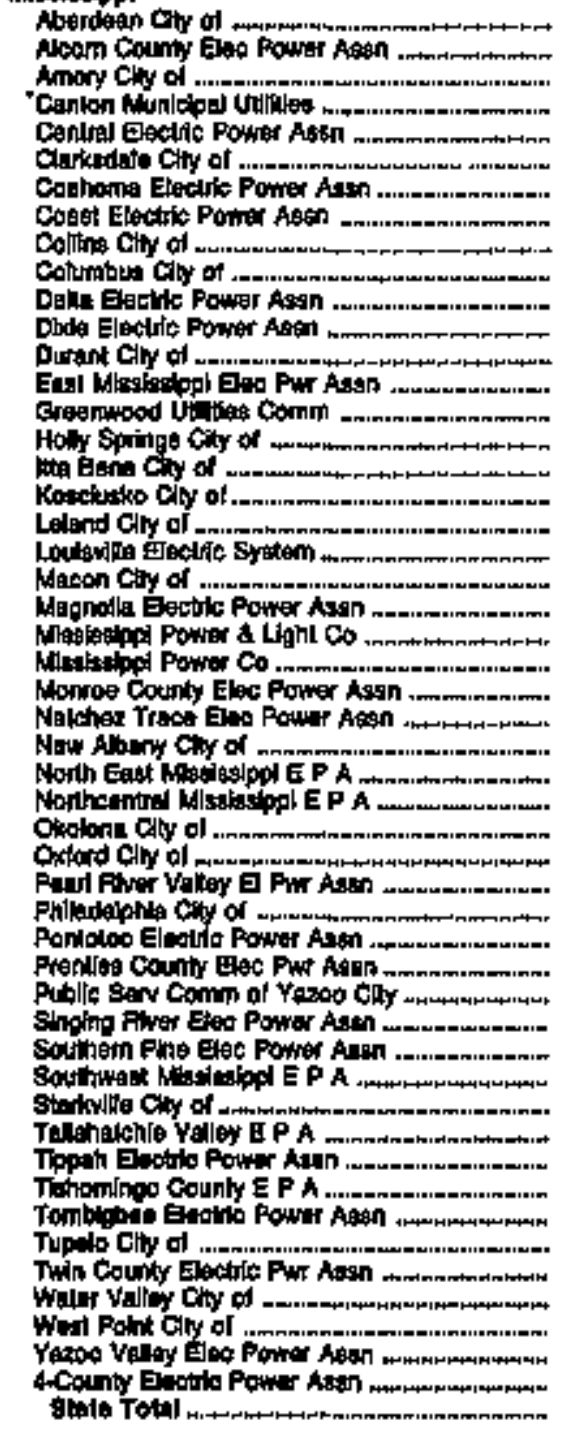 & 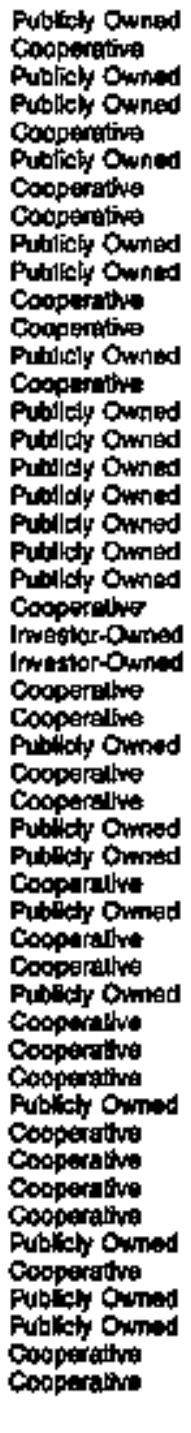 & 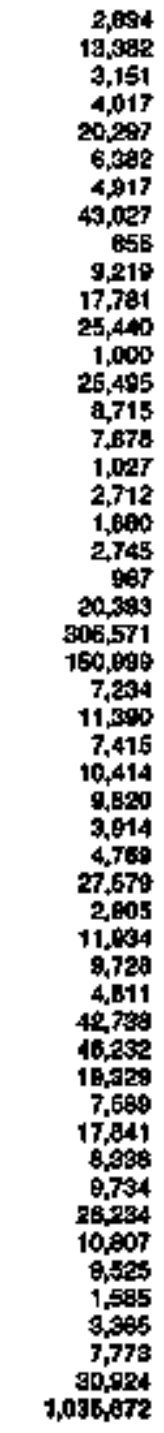 & 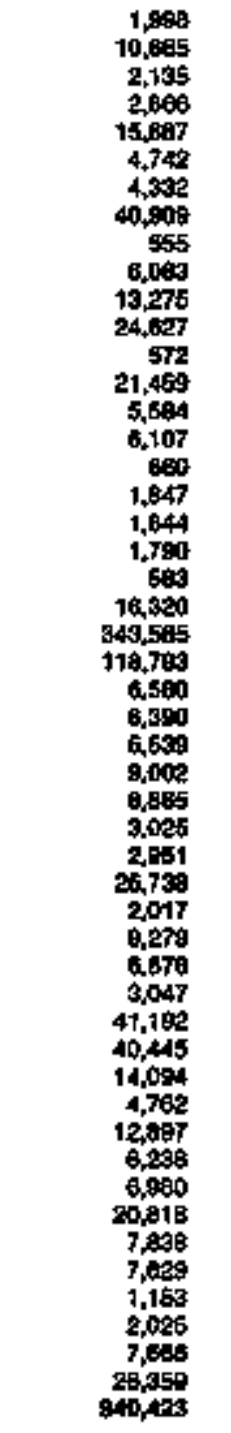 & 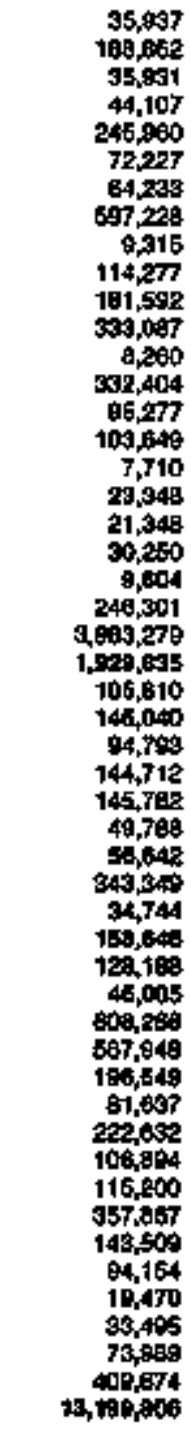 & 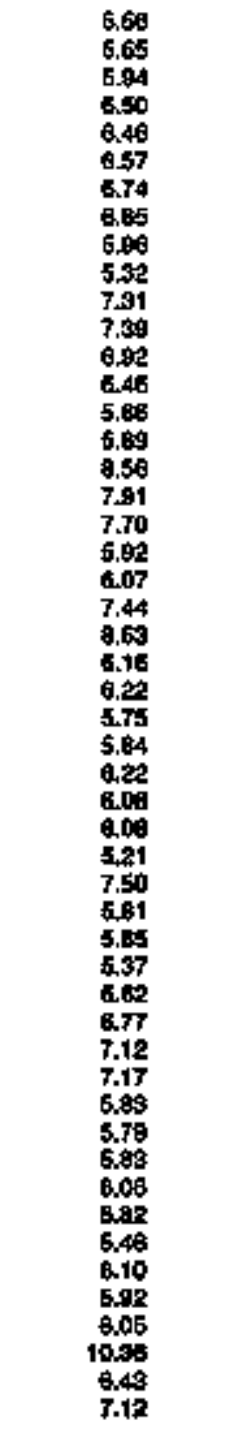 \\
\hline 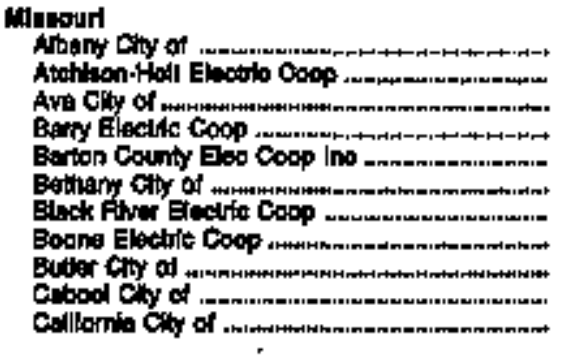 & 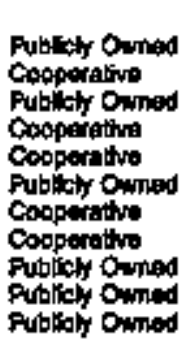 & $\begin{array}{r}870 \\
2,315 \\
1,144 \\
6,590 \\
4,072 \\
1,495 \\
17,708 \\
20,141 \\
1,900 \\
1,776\end{array}$ & $\begin{array}{r}441 \\
2,114 \\
773 \\
5,103 \\
4,198 \\
747 \\
14,679 \\
19,108 \\
1,158 \\
1,017 \\
1,017\end{array}$ & $\begin{array}{r}7,055 \\
27,395 \\
9,074 \\
77,042 \\
54,493 \\
11,243 \\
291,455 \\
270,050 \\
13,000 \\
7,055 \\
18,200\end{array}$ & $\begin{array}{l}5.04 \\
7.72 \\
7.75 \\
6.54 \\
7.69 \\
6.64 \\
6.08 \\
7.08 \\
7.81 \\
7.38 \\
7,97\end{array}$ \\
\hline
\end{tabular}

Sal loolnotes al and of table. 
Tabje 14. Class of Ownerahip, Number of Uitimate Consumers, Revenue, Sales, and Average Revenue per Kllowatthour for the Residential Seclor by State and Utility, 1993 (Continued)

\begin{tabular}{|c|c|c|c|c|c|}
\hline $\begin{array}{c}\text { sinte } \\
\text { Elattels Utylty }\end{array}$ & $\begin{array}{l}\text { clate } \\
\text { of } \\
\text { onmersilp }\end{array}$ & $\begin{array}{c}\text { Numbar } \\
\text { ad } \\
\text { Contopinary }\end{array}$ & 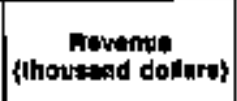 & 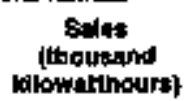 & 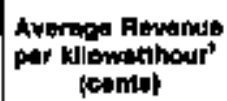 \\
\hline
\end{tabular}

Missour rominued

Cellawray Electric Codop .....--_........................

Canduron City of .

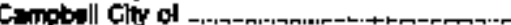

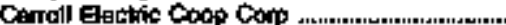

Cemallon Board of Publl Wha

Conthang Cily all

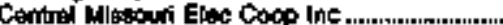

Contracis City of

Chilicothe City of .

Qtirens Eloctric corp

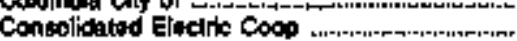

Grane City of

Citantorid Erectitic cose me

Cubs Cily of

Cunve River Elathic Goog lac

Cotho Emate coop mo wus

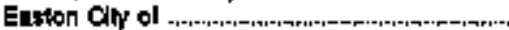

El Corado springa City of

Emplis Oiturict Gincirto Co

Evmes' Eloctric Coop be

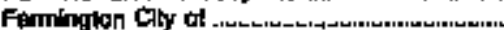

Frejeits cisy of

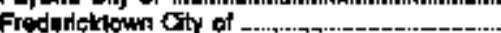

Fultan City of

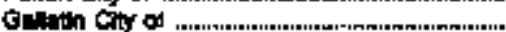

Galk City of

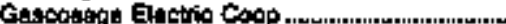

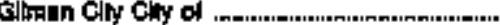

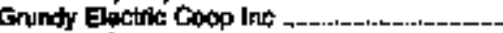

hennibel cay of ..

Hatsomfle City of

Herrogrn Coty of

thoolntrila City of

Hoution Civ of

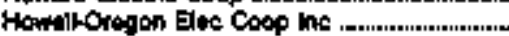

Huninewell chy of

Matepindence City of

Interounty Eloolrio Cosp hat .......................

Jackion ctiby of

Kenoles City of

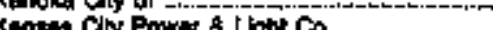

Kennoll chy of

Ninuwood Cory of

is Piata cory of

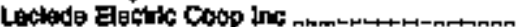

Lemar Cay of w.

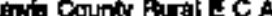

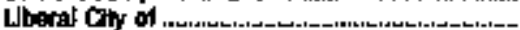

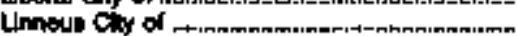

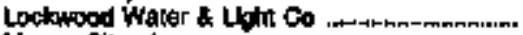

Mnoon City ol ..................................................

Mecon Elestrie Coop

Matom City of

Mrniliold ciny of

Markatl coy of

Mundili chy of

vinan ciny of ...

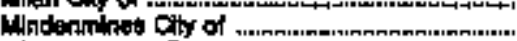

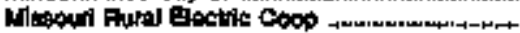

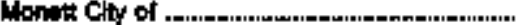

Monroe Chy City of

Mosit Verion Gity of
Coopuralive

Publity Oumed

Putilaty Dmind

Coopentulve

Pulpildy Omined

Pubicty Omed

Coopuraltiv

Pulplidy Ouned

Pubresy Cimad

Coogurathin

Coxparative

Publoly Orwaed

Cogpariolus

Publaty Omiad

Cooperatus

Fublich Oumad

Cooperallive

Cooporative

Pultich Owind

Putcidy Omind

Inwerorowner

Coopheraltwe

Puscicly Oimad

Publicy Ormes

Publety Omined

Pubsely Owined

Pubucty Onved

Puticty Owited

Cocpention

Publck Omed

Cooperallive

Pubsicty Omed

Publich Owand

Puticly Owated

Publity Owined

Putecty Owrod

Cospertative

Coopentive

Putuly Owied

Publety Omrag

Cooptintion

Publely Owand

Publely Cwrated

Iwrenlor-Owned

Puthly Owand

Pubilety Owined

Pubroty Owned

Cocperative

Publicty Owined

Publloky Owined

Cacpriatipo

Publely Owstad

Publloly Ownod

Putulyty Owrod

Pubicky Owned

Cooperatir:

Publety Owned

Publety Owned

Publichy Owned

Publey Owoted

Publlaly Ownet

Pubiely Oworod

Publthy Ownad

Publldy Owned

cangerathe

Putildy Owined

Publloty Ownod

Publlaty Owined
B.64?

2,063

549

B,872

1.925

5.884

7,360

1526

3.785

18,376

24,891
6,047

576

13, 的5

1.104

31.007

21,37

1,889

1, 70

9.089

3,885

1,04 ?

1,041

4,048

100

8,816

20

$6,5 \times 5$

7,480

3,120

1229

1,044

1,000

2,379

18,797

120

44,991

29,790

4,027

748

218,642

4,760

$0,3 \geq 0$

85

21,651

1.028

9,721

$\$, 200$

305
212

836

2.271

7,344

2,126

580

1,184

4,701
213

1,000

604

144

3,062

3,002

1,071

$1,5 \mathrm{gs}$
7,032

1240

877

4,072

868

253

7,015

1045

2,473
14,061

36

17,477

6,540

300

11,222

B17

39.176

19,774

77

50, 341

823

2,026

740

1,123

2,6re

เ10

119
4,739

200

4,114

3,760

1,035

946

1,2012

1,067

2,216

12,02

50

21,806

3,272

560

180 .

1,873

6,260

424

19,175

787

4,414

189

1 fit

300

1745

6.642

1,02

$5 \pi 7$

1,011

$2,8 \times 4$

197

$1,0 \mathrm{e}$

407
108

$\$, 274$

$1, \mathrm{Bac}^{\circ}$

767

105,866

17,2827

$8,0977.69$

69,659

16.530

42,460 \$,66

$100,071 \quad 7.01$

6.76

37,336 $\quad \mathbf{7 , 0 0}$

210.583

550.972

91,054

$4,1000 \quad 7,51$

155,052

$11,251 \quad 7.26$

$154,110 \quad 7,09$

$198580 \quad 794$

1,341

$12.001 \quad 7,10$

$1,0,07,007 \quad \$ .48$

4,000

$7,400 \quad 10.11$ 
Table 14. Class of Ownershlp, Number of Uttmate Coneumers, Revenue, Salos, and Average Revenue per Kllowatthour for the Residentlal Sector by State and Utifity, 1993 (Continued)

\begin{tabular}{|c|c|c|c|c|c|}
\hline State & $\begin{array}{c}\text { Clags } \\
\text { of } \\
\text { Onemofithp }\end{array}$ & $\begin{array}{c}\text { livabor } \\
\text { of } \\
\text { coneymara }\end{array}$ & 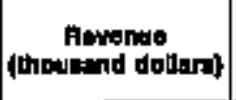 & 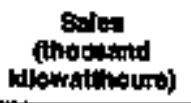 & 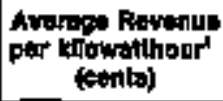 \\
\hline 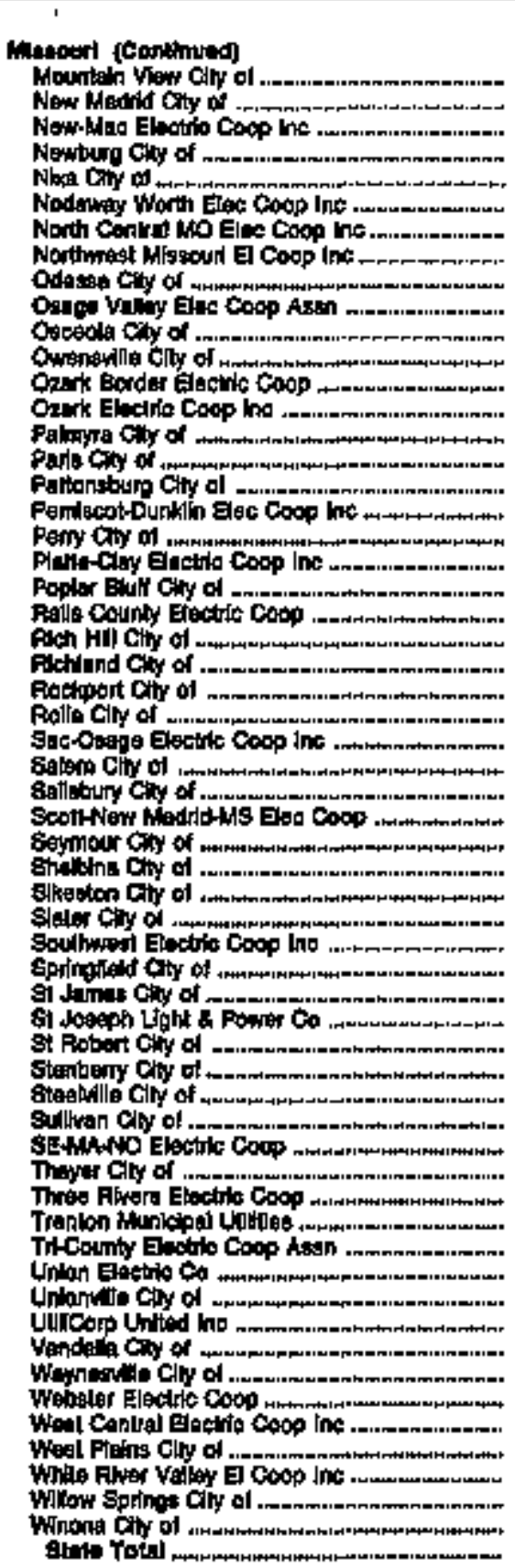 & 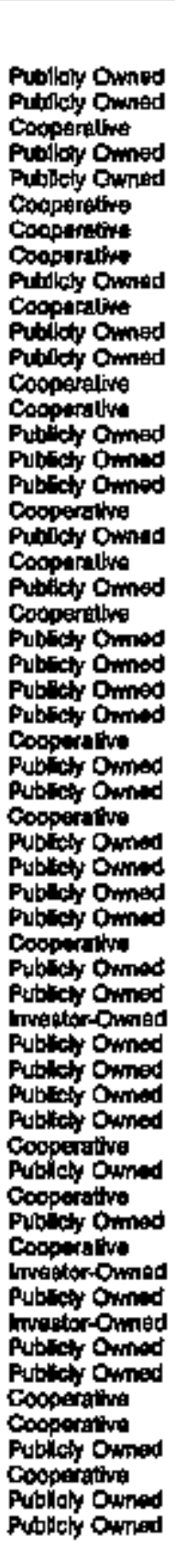 & 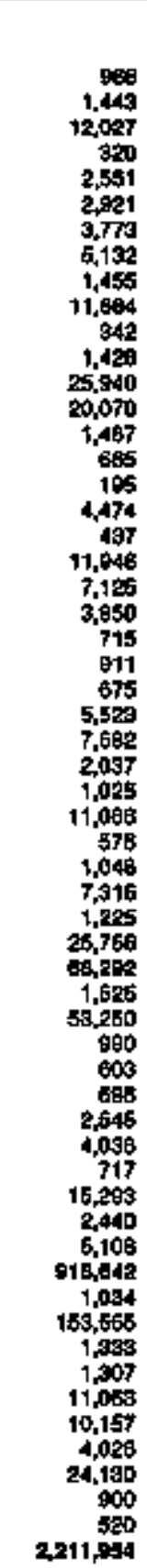 & 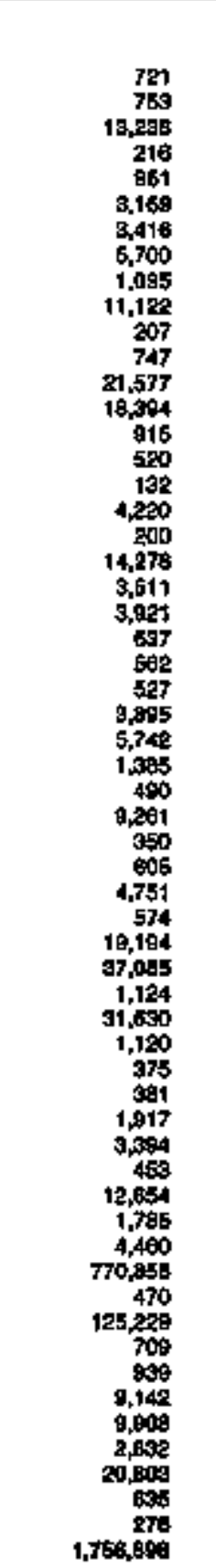 & 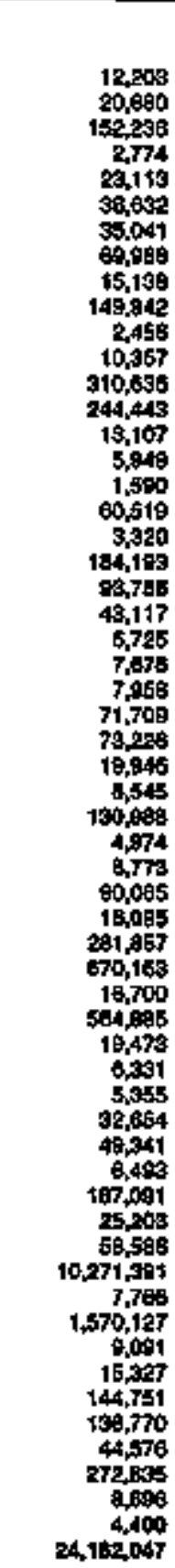 & 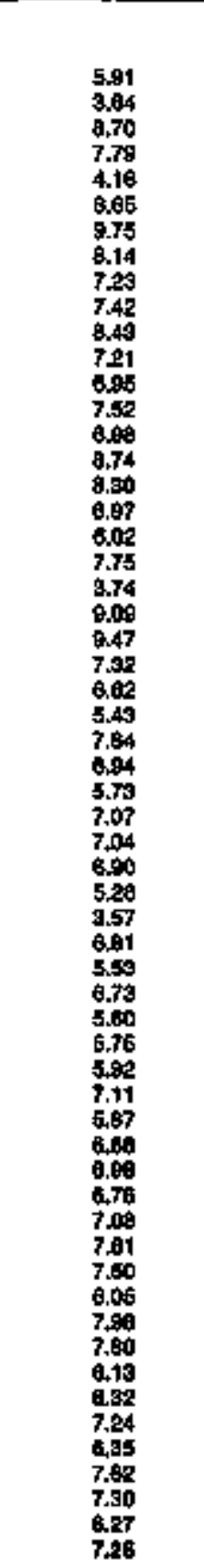 \\
\hline 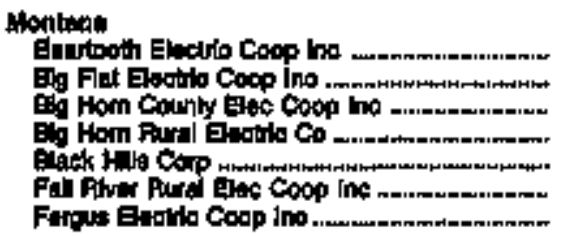 & 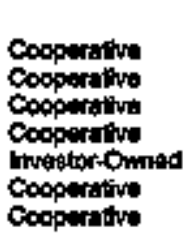 & $\begin{array}{r}2,300 \\
1,200 \\
2,540 \\
30 \\
15 \\
260 \\
4,500\end{array}$ & $\begin{array}{r}2,143 \\
890 \\
1,663 \\
24 \\
9 \\
744 \\
3,564\end{array}$ & $\begin{array}{r}33,603 \\
17,048 \\
28,640 \\
388 \\
110 \\
10,180 \\
54,089\end{array}$ & $\begin{array}{l}6.84 \\
5.86 \\
6.59 \\
17 \\
19 \\
7.31 \\
6.53\end{array}$ \\
\hline
\end{tabular}

\$te foolnotes an end of thille. 
Table 14. Class of Ownership, Number of Utimate Consumers, Revembe, Sales, and Average Revenute per Kllowatthour for the Residential Soctor by State and Utillty, 1993 (Continued)

\begin{tabular}{|c|c|c|c|c|c|}
\hline $\begin{array}{c}\text { getht } \\
\text { Aftoctide tally }\end{array}$ & $\begin{array}{c}\text { Clapt } \\
\text { oif } \\
\text { Oinnetentp }\end{array}$ & $\begin{array}{c}\text { Number } \\
\text { of } \\
\text { contumer: }\end{array}$ & $\begin{array}{c}\text { Fevende } \\
\text { (thotiand dollart) }\end{array}$ & 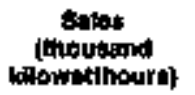 & $\begin{array}{l}\text { Average flowents } \\
\text { per lillowathour" } \\
\text { (comis) }\end{array}$ \\
\hline 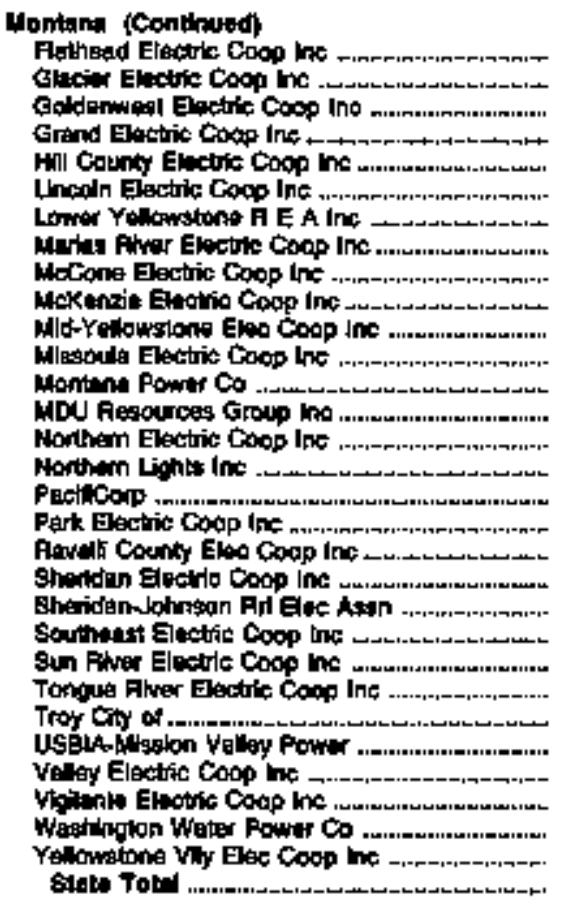 & 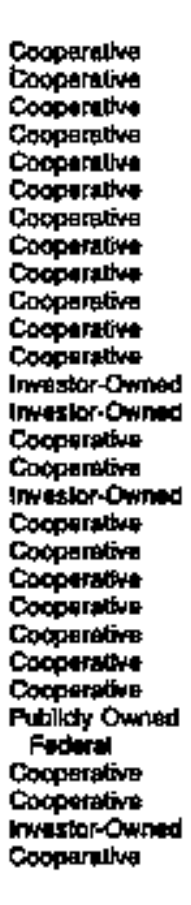 & 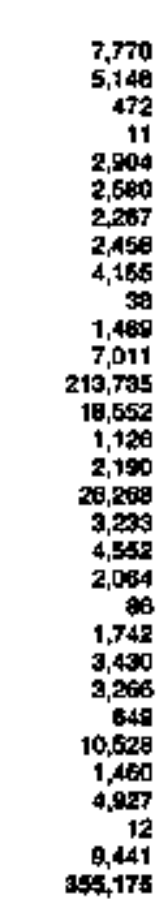 & 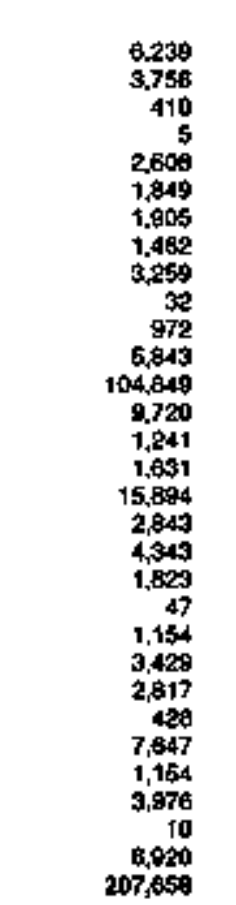 & 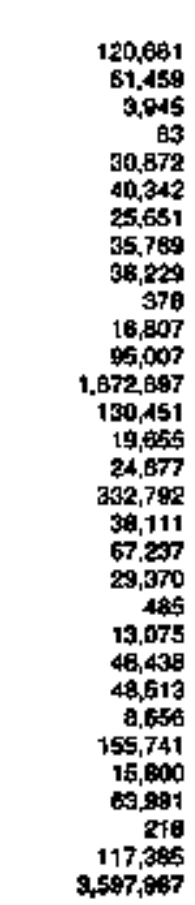 & $\begin{array}{l}5.17 \\
6.11 \\
10.39 \\
41 \\
8.45 \\
4.68 \\
7.48 \\
4.09 \\
0.00 \\
41 \\
5.78 \\
6.16 \\
5.50 \\
7.45 \\
6.31 \\
6.55 \\
4.78 \\
7.46 \\
6.45 \\
6.21 \\
16 \\
6.63 \\
7.38 \\
6.81 \\
4.94 \\
4.81 \\
7.30 \\
6.21 \\
19 \\
5.90 \\
5.77\end{array}$ \\
\hline 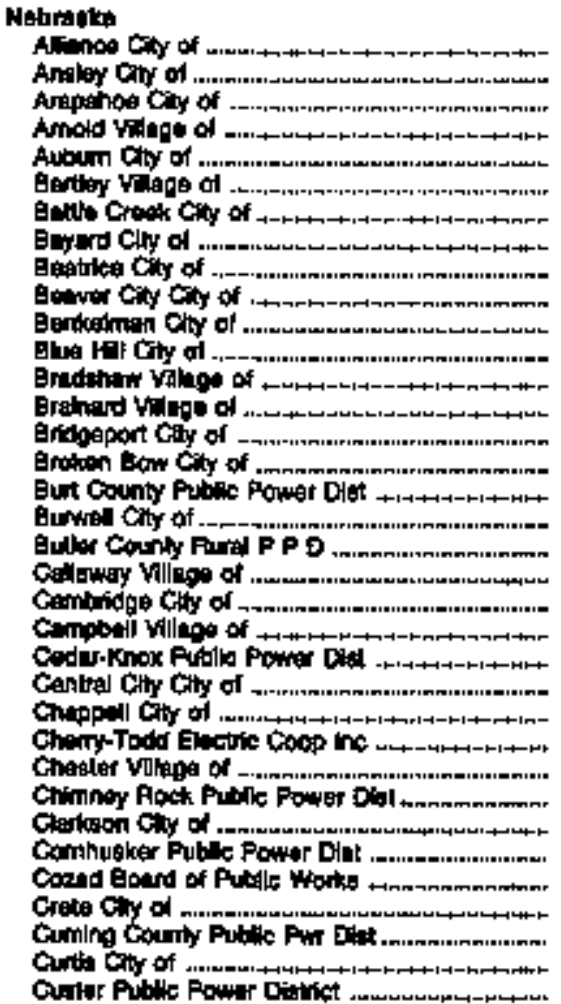 & 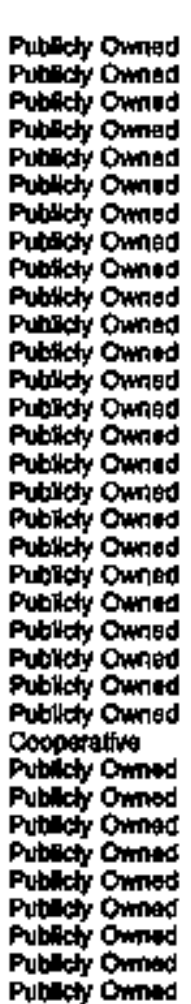 & 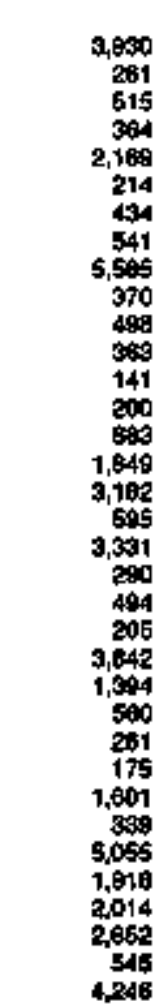 & 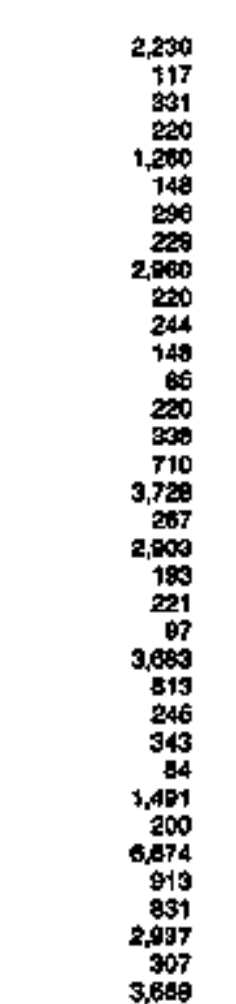 & 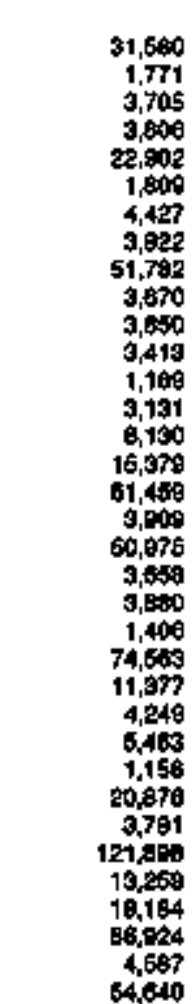 & 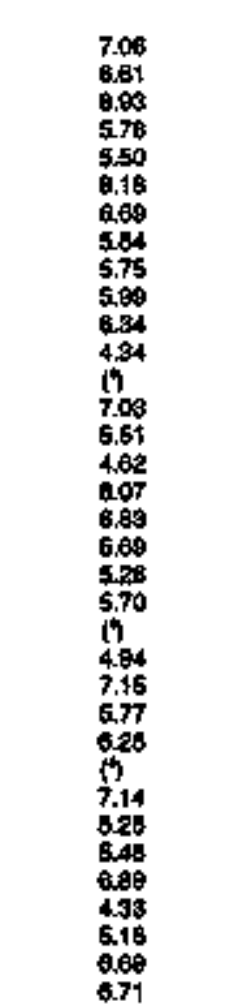 \\
\hline
\end{tabular}

Sac formales ax and ar tribla 
Tabje 14, Class of Ownership, Number of Uitimate Consumers, Revemus, Ssles, and Average Aevenute per Kllowatthour for the Fodidential Sector by State and Utility, 1993 (Continued)

\begin{tabular}{|c|c|c|c|c|c|}
\hline $\begin{array}{c}\text { Ebilo } \\
\text { suctiols llanty }\end{array}$ & $\begin{array}{c}\text { thes } \\
\text { of } \\
\text { ommarthing }\end{array}$ & $\begin{array}{c}\text { Nymber } \\
\text { of } \\
\text { Condumars }\end{array}$ & Rathosto & 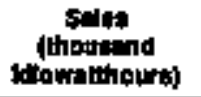 & 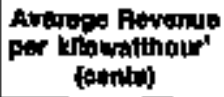 \\
\hline 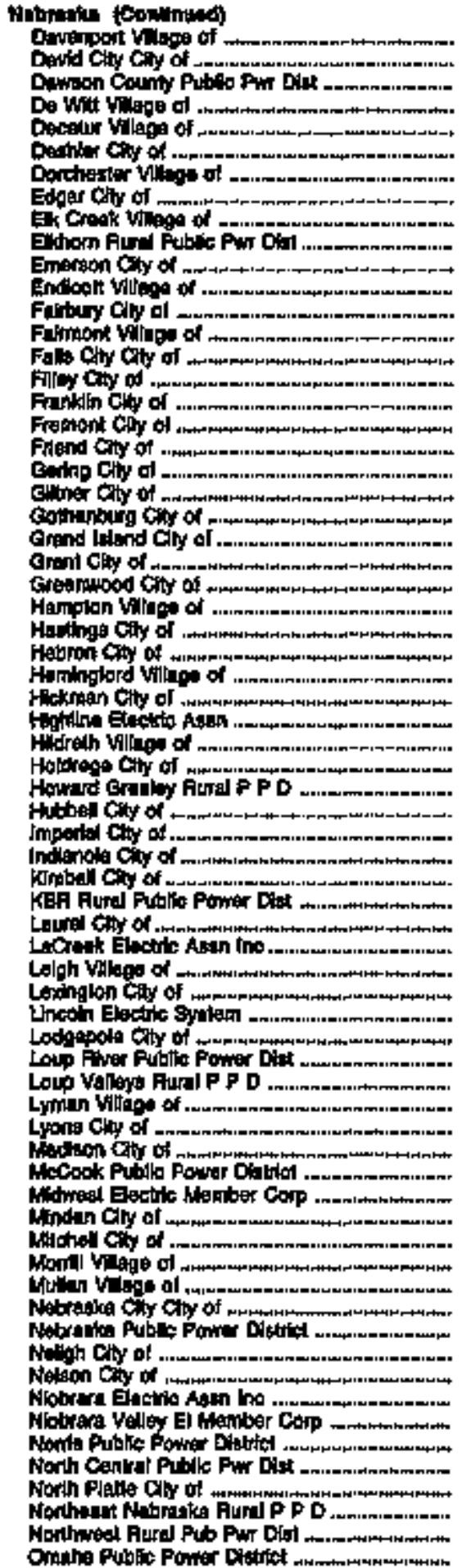 & 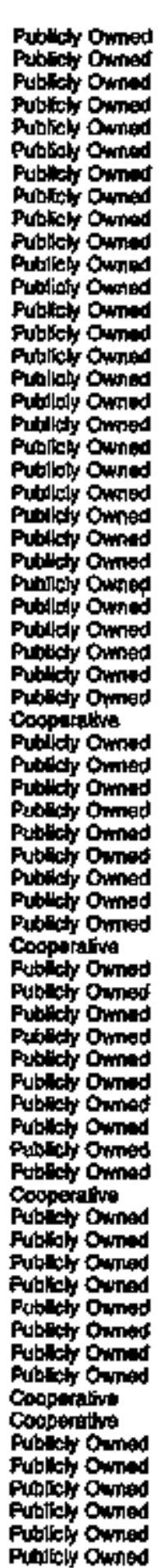 & 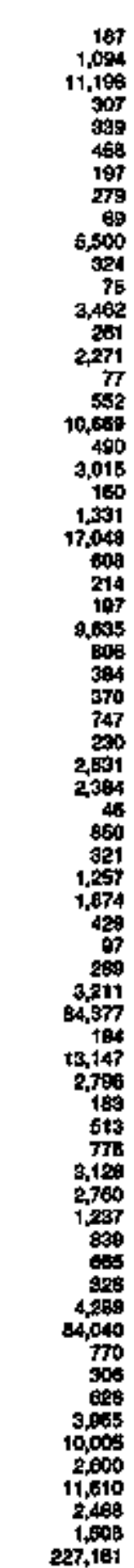 & 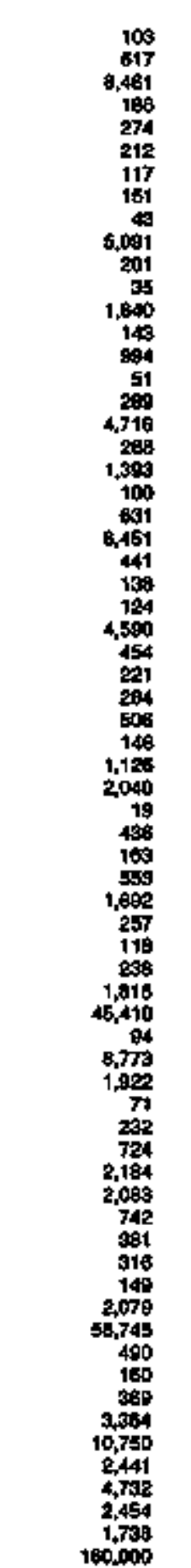 & 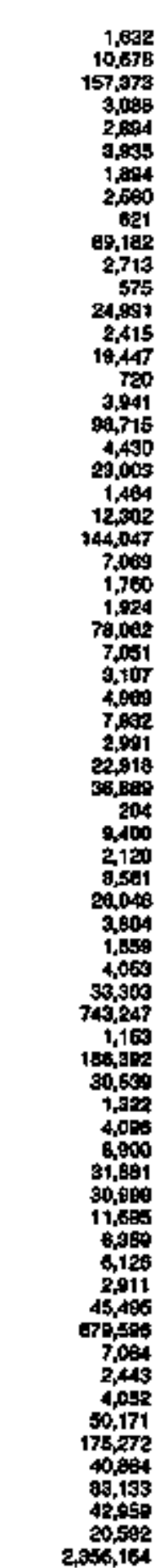 & 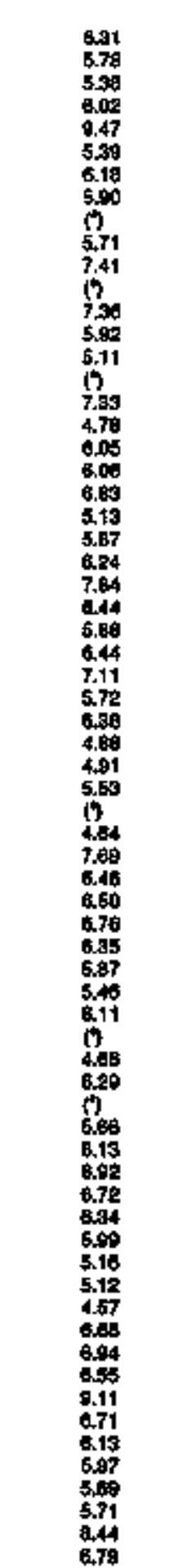 \\
\hline
\end{tabular}

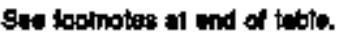


Table 14. Class of Ownership, Number of Uttimate Consumers, Revenue, Sales, and Average Rovenue per Kllowatthour for the Residential Sector by State and Utility, 1993 (Continued)

\begin{tabular}{|c|c|c|c|c|c|}
\hline $\begin{array}{c}\text { Stak } \\
\text { Elthtep Utity }\end{array}$ & $\begin{array}{c}\text { Clapos } \\
\text { of } \\
\text { Omprabs'o }\end{array}$ & $\begin{array}{c}\text { Rumber } \\
\text { ol } \\
\text { Concumast }\end{array}$ & Fewtenti & $\begin{array}{l}\text { 5thlas } \\
\text { (thousund } \\
\text { Idlowathours) }\end{array}$ & $\begin{array}{l}\text { Avanage Arvanum } \\
\text { per klowutthour' } \\
\text { (tent) }\end{array}$ \\
\hline 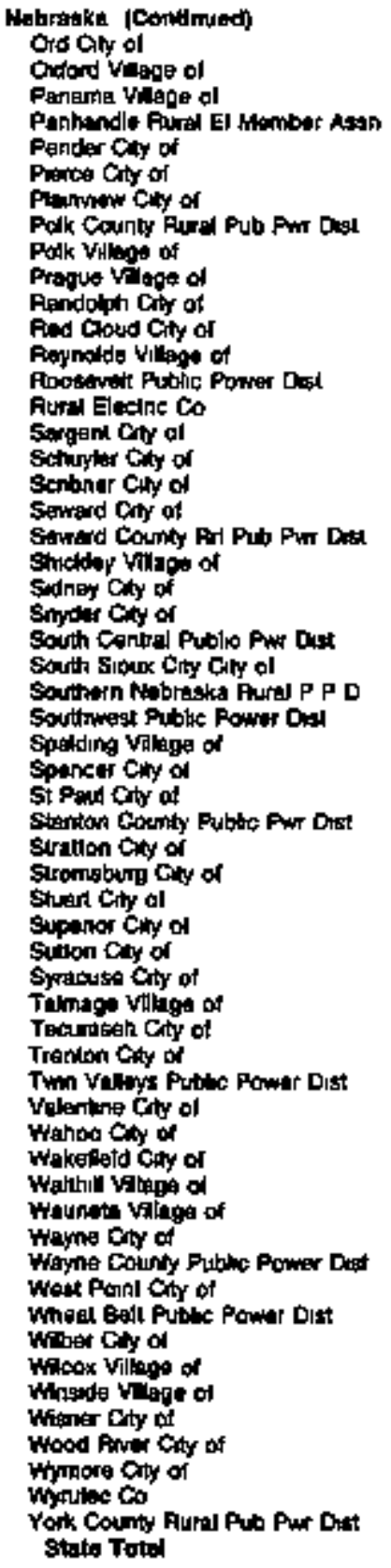 & 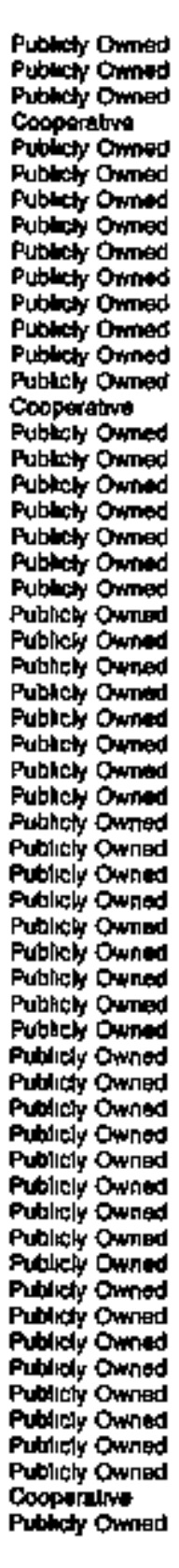 & 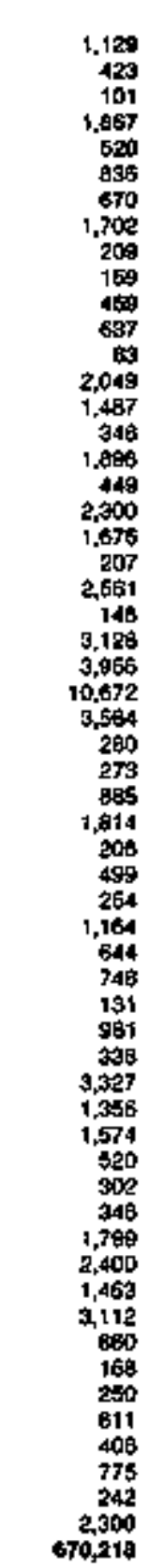 & 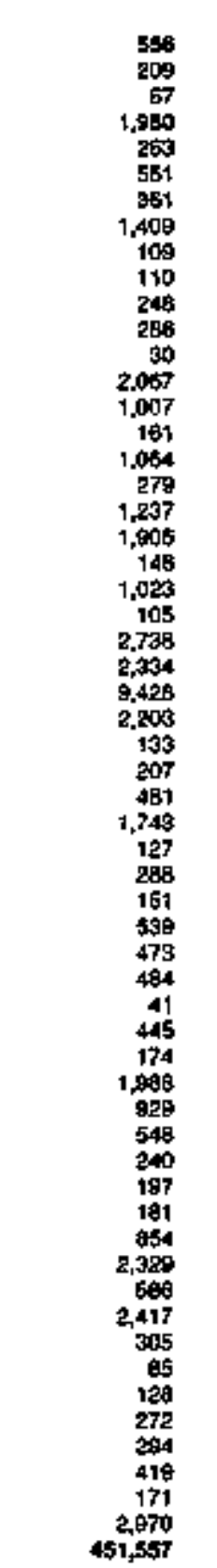 & 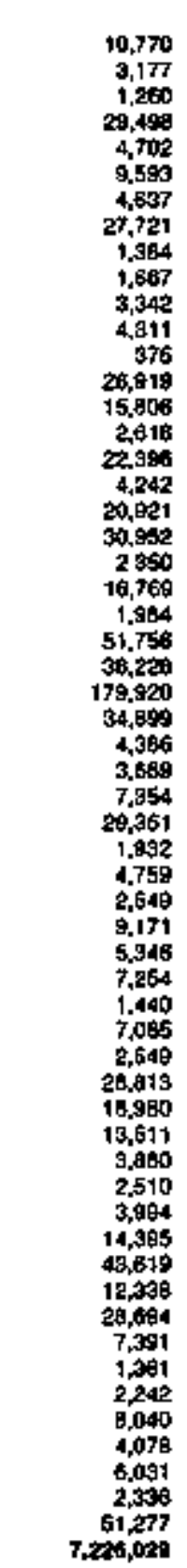 & 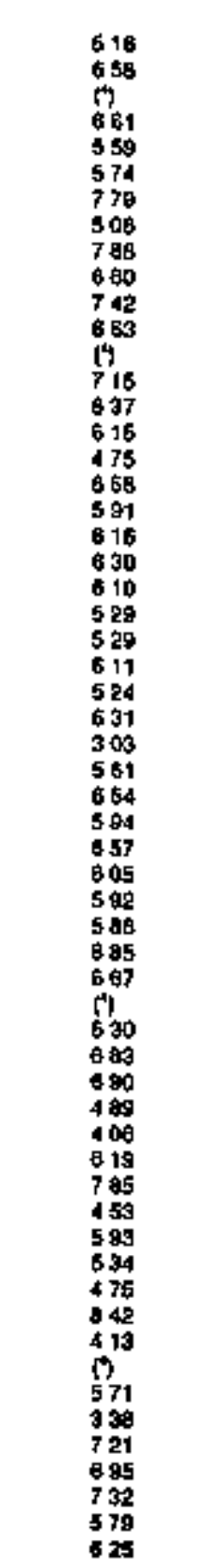 \\
\hline 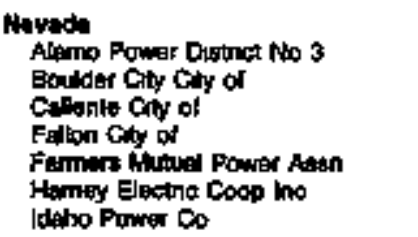 & 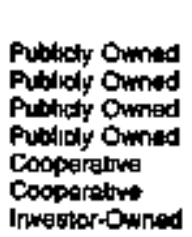 & $\begin{array}{r}458 \\
5,695 \\
439 \\
2,900 \\
47 \\
961 \\
994\end{array}$ & $\begin{array}{r}354 \\
3.377 \\
270 \\
1.481 \\
28 \\
605 \\
541\end{array}$ & $\begin{array}{r}6,129 \\
68,090 \\
6,475 \\
15,510 \\
805 \\
10,795 \\
13,739\end{array}$ & $\begin{array}{l}570 \\
302 \\
199 \\
965 \\
14 \\
469 \\
304\end{array}$ \\
\hline
\end{tabular}

8en tootroles at end of labla 
Table 14. Class of Ownorahlip, Number of Utimate Consumers, Rovenule, Sales, and Average Revente per Kllowathour for the Residentlat Sector by State and Utility, 1993 (Continued)

\begin{tabular}{|c|c|c|c|c|c|}
\hline state & 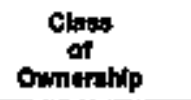 & $\begin{array}{l}\text { Sumber } \\
\text { of } \\
\text { connumera }\end{array}$ & 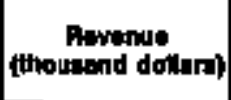 & $\begin{array}{l}\text { 8ofos } \\
\text { ithotitiond }\end{array}$ & 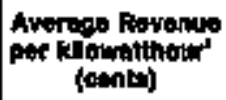 \\
\hline 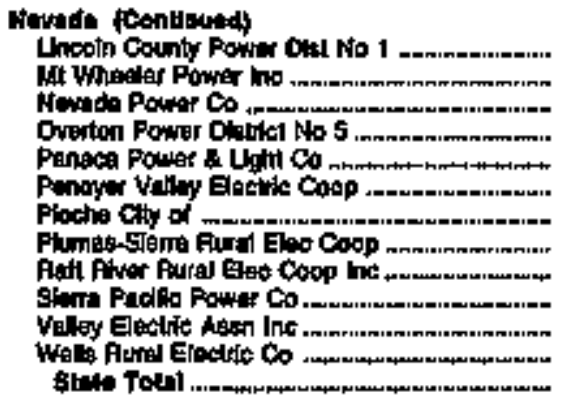 & 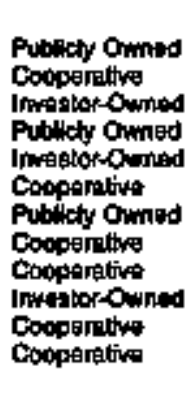 & $\begin{array}{r}89 \\
4,302 \\
344,797 \\
3,249 \\
291 \\
62 \\
296 \\
141 \\
\$ 50 \\
182,021 \\
6765 \\
3,310 \\
67,138\end{array}$ & 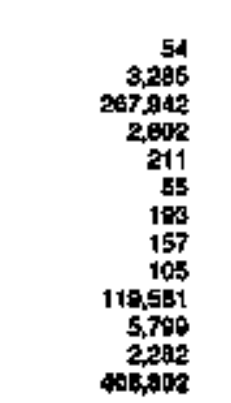 & $\begin{array}{r}091 \\
41,396 \\
4,51 t, 769 \\
\mathbf{5 2 , 7 1 0} \\
3,829 \\
481 \\
4,245 \\
2,054 \\
2,093 \\
1,404,792 \\
84,694 \\
33,174 \\
6,200,698\end{array}$ & 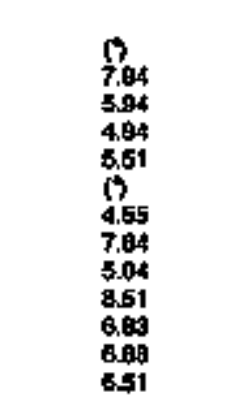 \\
\hline 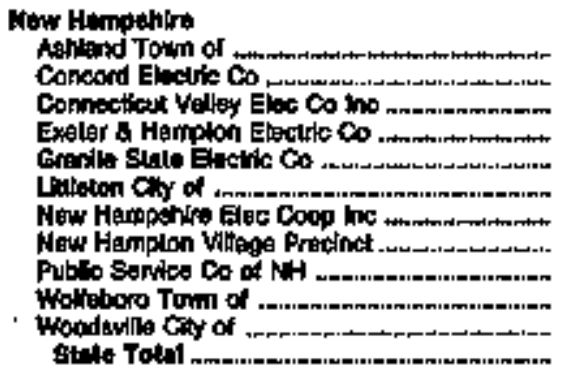 & 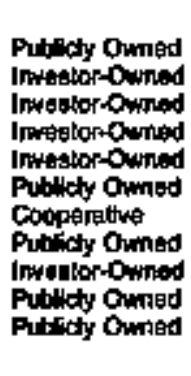 & $\begin{array}{r}864 \\
21,470 \\
0,899 \\
29,414 \\
29,640 \\
2,590 \\
55,579 \\
121 \\
343,490 \\
5,069 \\
510 \\
407,968\end{array}$ & $\begin{array}{r}526 \\
15,453 \\
6,748 \\
22,452 \\
22,948 \\
1,699 \\
49,146 \\
186 \\
290,097 \\
2,670 \\
380 \\
40,756\end{array}$ & 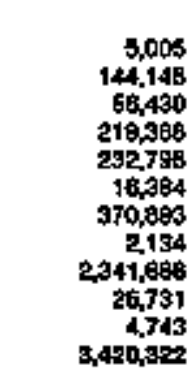 & $\begin{array}{r}10.51 \\
10.72 \\
11.08 \\
10.23 \\
9.61 \\
9.39 \\
13.25 \\
8.61 \\
12.77 \\
9.65 \\
8.25 \\
1231\end{array}$ \\
\hline 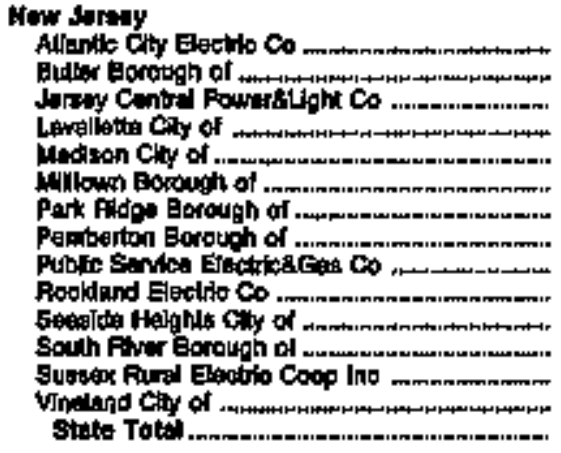 & 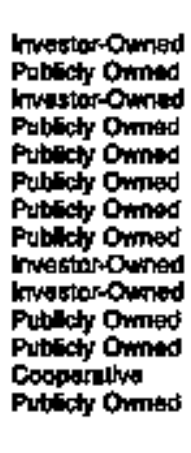 & $\begin{array}{r}05,107 \\
0,270 \\
806,436 \\
2,565 \\
6,480 \\
2,651 \\
3,041 \\
517 \\
1,690,350 \\
65,040 \\
1,594 \\
5,700 \\
10,032 \\
14,702 \\
2,968,736\end{array}$ & $\begin{array}{r}394,163 \\
7,651 \\
65,242 \\
7,790 \\
4,516 \\
2,404 \\
2,724 \\
414 \\
1.191,282 \\
67,068 \\
828 \\
2,608 \\
10,986 \\
13,090 \\
2,5+4,344\end{array}$ & 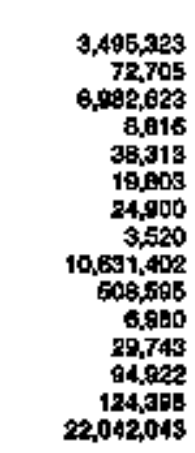 & $\begin{array}{r}11.29 \\
10.52 \\
11.69 \\
8.08 \\
11.79 \\
12.44 \\
8.99 \\
11.76 \\
11.11 \\
11.39 \\
11.79 \\
9.42 \\
11.57 \\
10.62 \\
11.41\end{array}$ \\
\hline 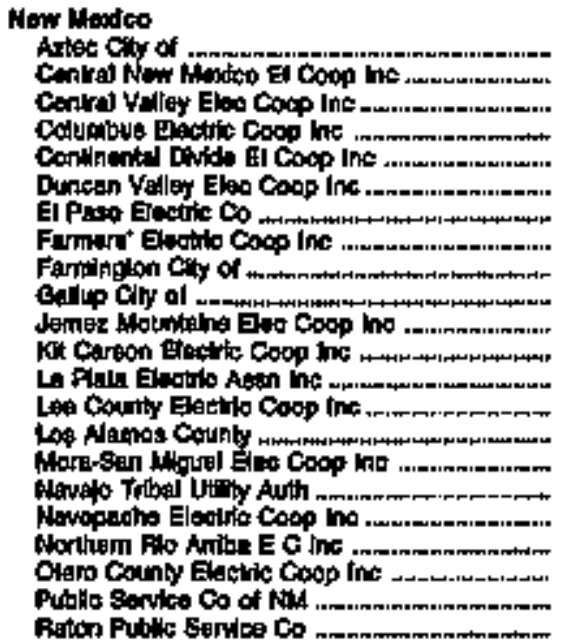 & 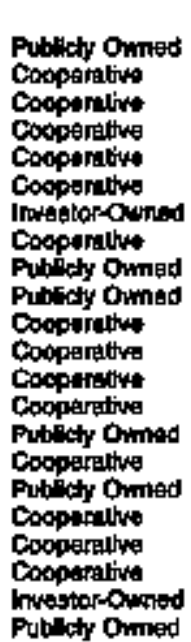 & 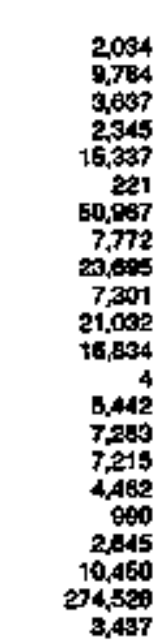 & 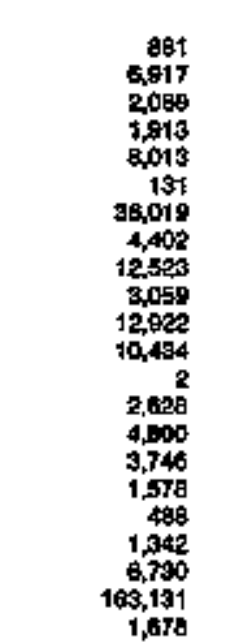 & 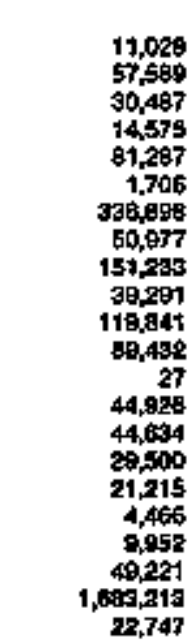 & $\begin{array}{r}7.99 \\
12.01 \\
6.79 \\
13.19 \\
9.96 \\
7.69 \\
10.69 \\
8.64 \\
8.29 \\
7.79 \\
10.78 \\
11.07 \\
19 \\
5.85 \\
10.75 \\
12.70 \\
7.44 \\
10.09 \\
13.49 \\
13.67 \\
9.89 \\
7.30\end{array}$ \\
\hline
\end{tabular}

Son foolnotes at and od labie. 
Table 14. Class of Ownership, Number of Ultimate Consumerts, Revenue, Sales, and Average Revenus per KHowatthour for the Residential Sector by state and Uttitity, 1993 (Continued)

\begin{tabular}{|c|c|c|c|c|c|}
\hline $\begin{array}{c}\text { Silote } \\
\text { Ekcirio Uanty }\end{array}$ & $\begin{array}{c}\text { Clane } \\
\text { of } \\
\text { Ownerath }\end{array}$ & $\begin{array}{l}\text { Number } \\
\text { of } \\
\text { Consinmerss }\end{array}$ & $\begin{array}{c}\text { Rervand } \\
\text { (thoutand dolkars) }\end{array}$ & 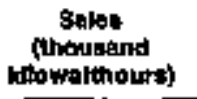 & $\begin{array}{c}\text { Average Aewenug } \\
\text { per klowitshour' } \\
\text { (osints) }\end{array}$ \\
\hline 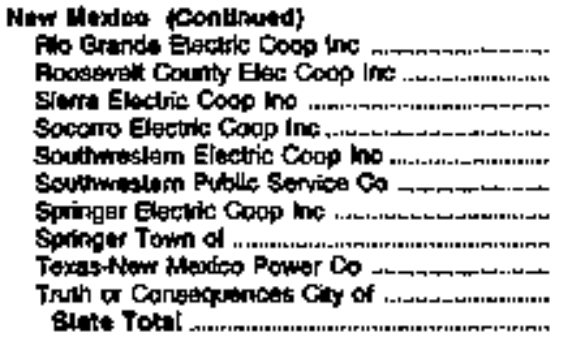 & 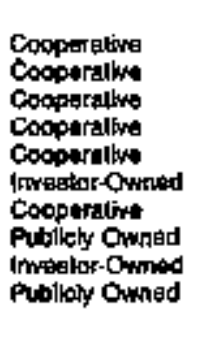 & $\begin{array}{r}205 \\
2,035 \\
2,505 \\
7,495 \\
1,065 \\
81,057 \\
2,040 \\
591 \\
35,505 \\
3,164 \\
615,019\end{array}$ & $\begin{array}{r}153 \\
1.717 \\
1,700 \\
4.254 \\
897 \\
40.9693 \\
1.281 \\
333 \\
18.604 \\
1.479 \\
356.647\end{array}$ & $\begin{array}{r}1.197 \\
25,496 \\
11.677 \\
11.023 \\
8,600 \\
693.623 \\
10,106 \\
2,573 \\
192,610 \\
15,272 \\
3,084,268\end{array}$ & 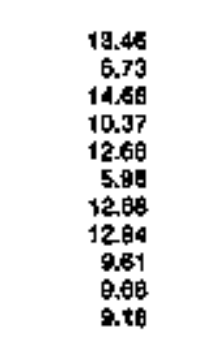 \\
\hline 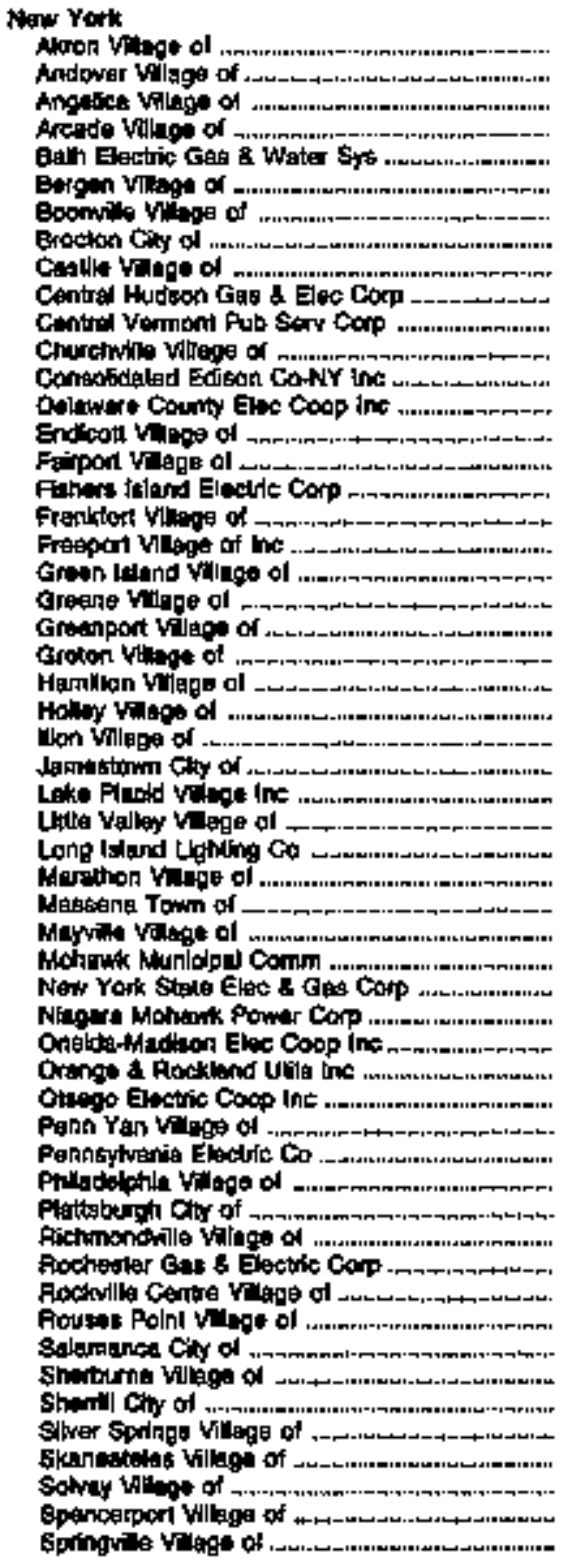 & 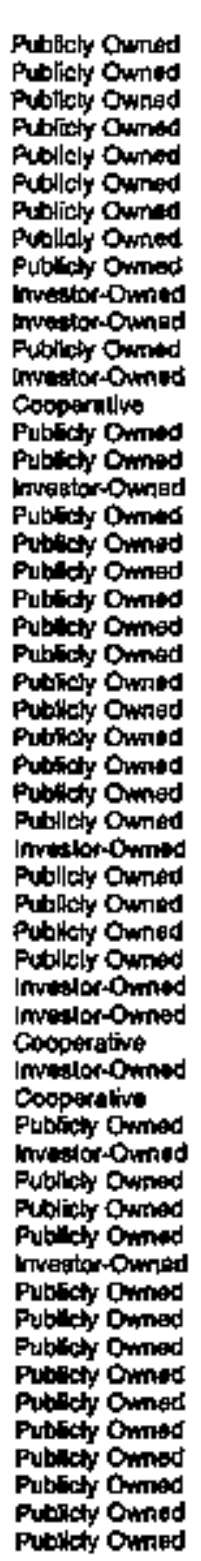 & 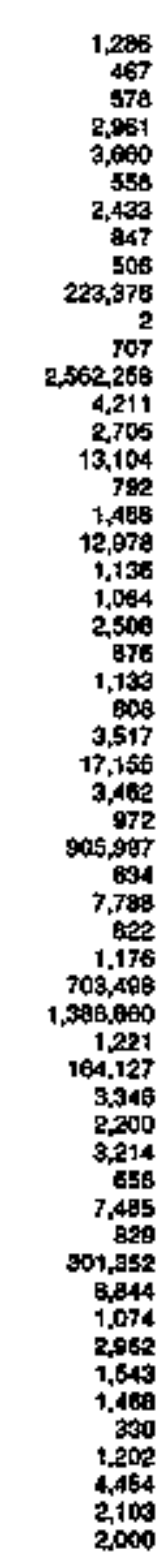 & 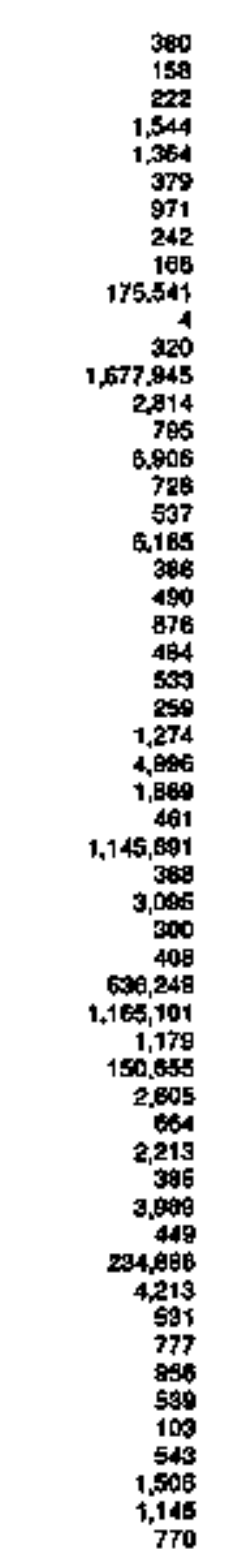 & 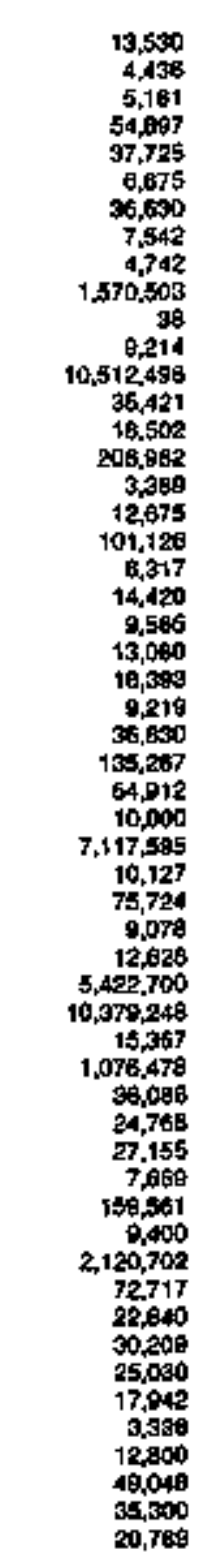 & 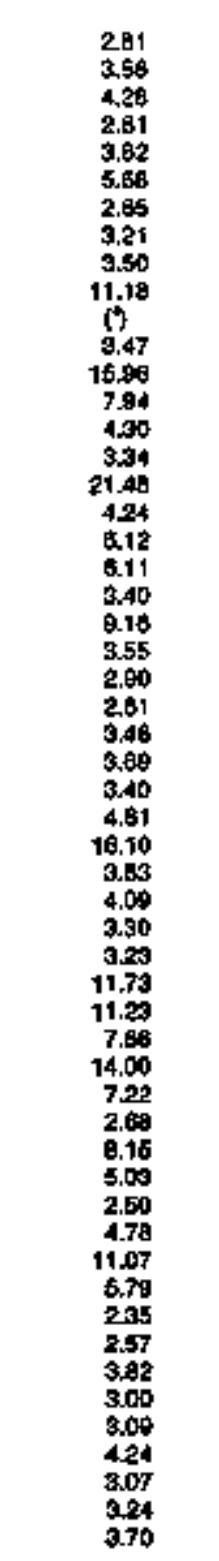 \\
\hline
\end{tabular}

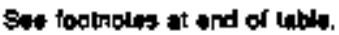


Teble 14. Class of Omnerghip, Number of Ultimate Consumers, Revenue, Sales, and Average Fevenute per Kilowathour for the Residentlal Sector by State and Utilly, 1993 (Continued)

\begin{tabular}{|c|c|c|c|c|c|}
\hline $\begin{array}{c}\text { 8iato } \\
\text { Elogthic Utulity }\end{array}$ & $\begin{array}{c}\text { Ctact } \\
\text { of } \\
\text { owroptaplo }\end{array}$ & $\begin{array}{c}\text { thrmber } \\
\text { of } \\
\text { Conaumins }\end{array}$ & Rowtonve & $\begin{array}{l}\text { sathe } \\
\text { (fhoucend } \\
\text { (tiorathoud) }\end{array}$ & 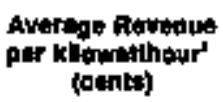 \\
\hline 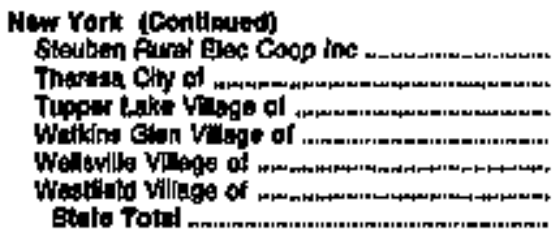 & 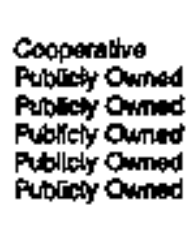 & $\begin{array}{r}4,895 \\
390 \\
2,710 \\
970 \\
8,241 \\
2,407 \\
6,35,401\end{array}$ & $\begin{array}{r}4,027 \\
200 \\
1,309 \\
349 \\
599 \\
638 \\
5245,743\end{array}$ & 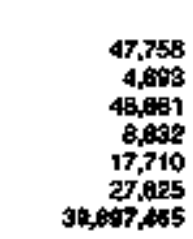 & $\begin{array}{r}9.49 \\
4.30 \\
2.99 \\
3.45 \\
3.30 \\
3.01 \\
+3.17\end{array}$ \\
\hline 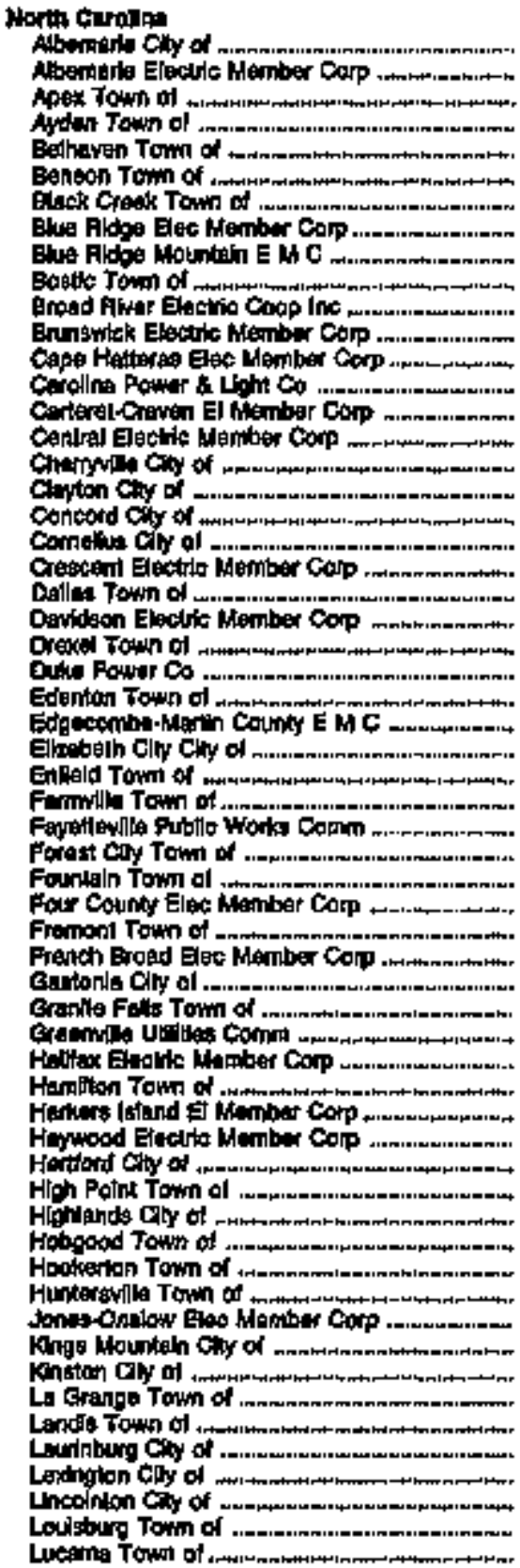 & 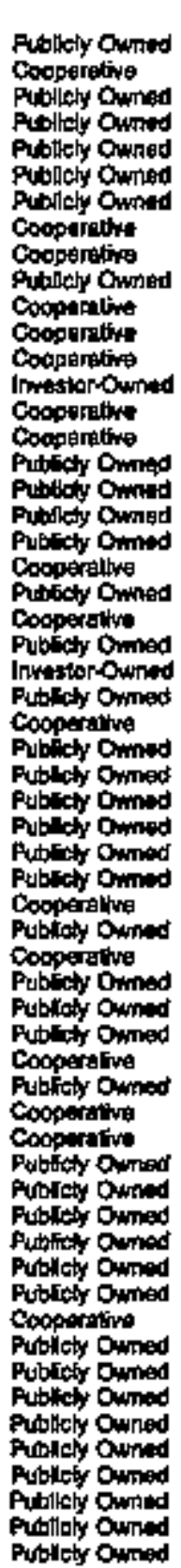 & 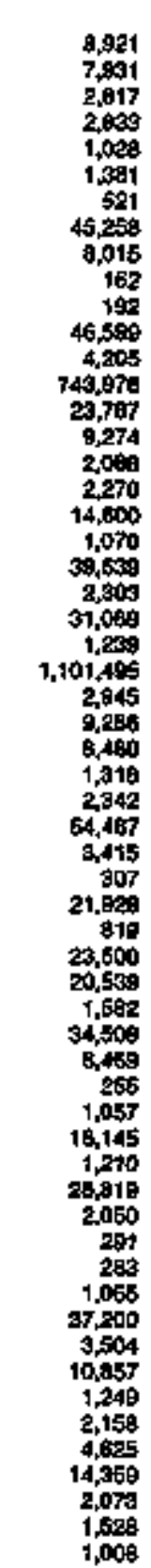 & 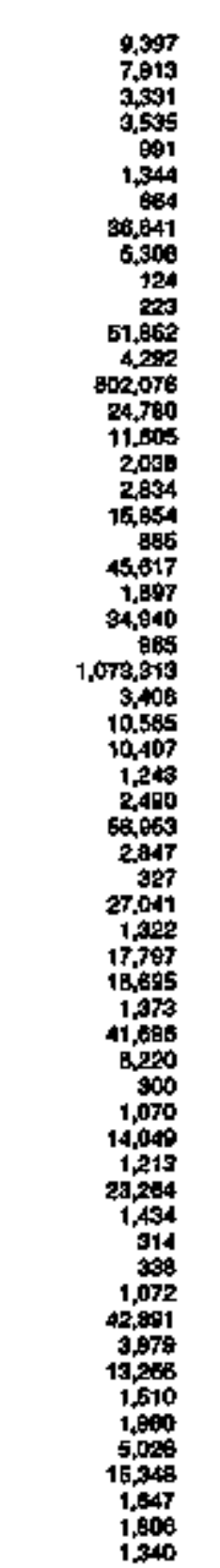 & 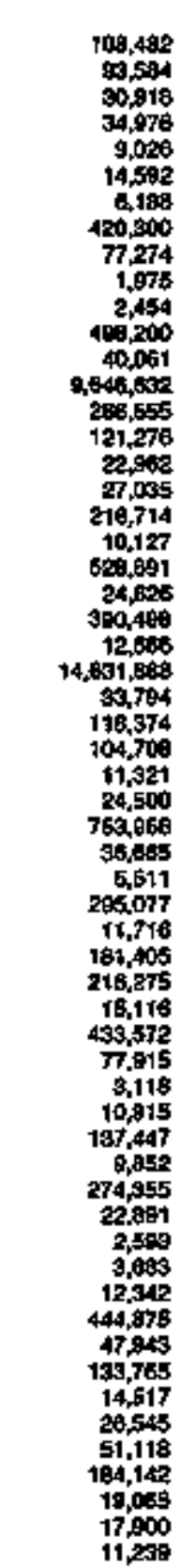 & 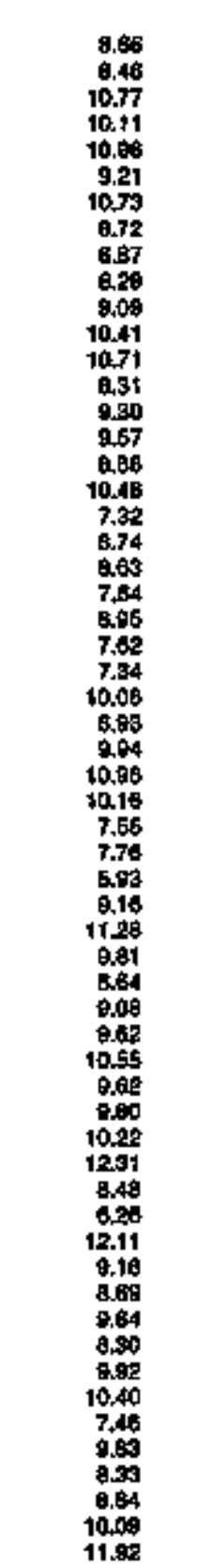 \\
\hline
\end{tabular}

See tootnotes al ond of table. 
Table 14. Class of Ownership, Number of Utimate Consumers, Revenue, Saton, and Average Revenue per KIlowatthour for the Residential Sector by State and Utility, 1993 (Continued)

\begin{tabular}{|c|c|c|c|c|c|}
\hline $\begin{array}{c}\text { ftril: } \\
\text { Eroctelo Uulity }\end{array}$ & $\begin{array}{c}\text { Clat: } \\
\text { od } \\
\text { Onintralp }\end{array}$ & $\begin{array}{c}\text { Number } \\
\text { of } \\
\text { Cipnotimero }\end{array}$ & Porthoin & 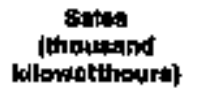 & 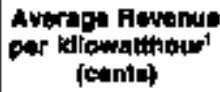 \\
\hline 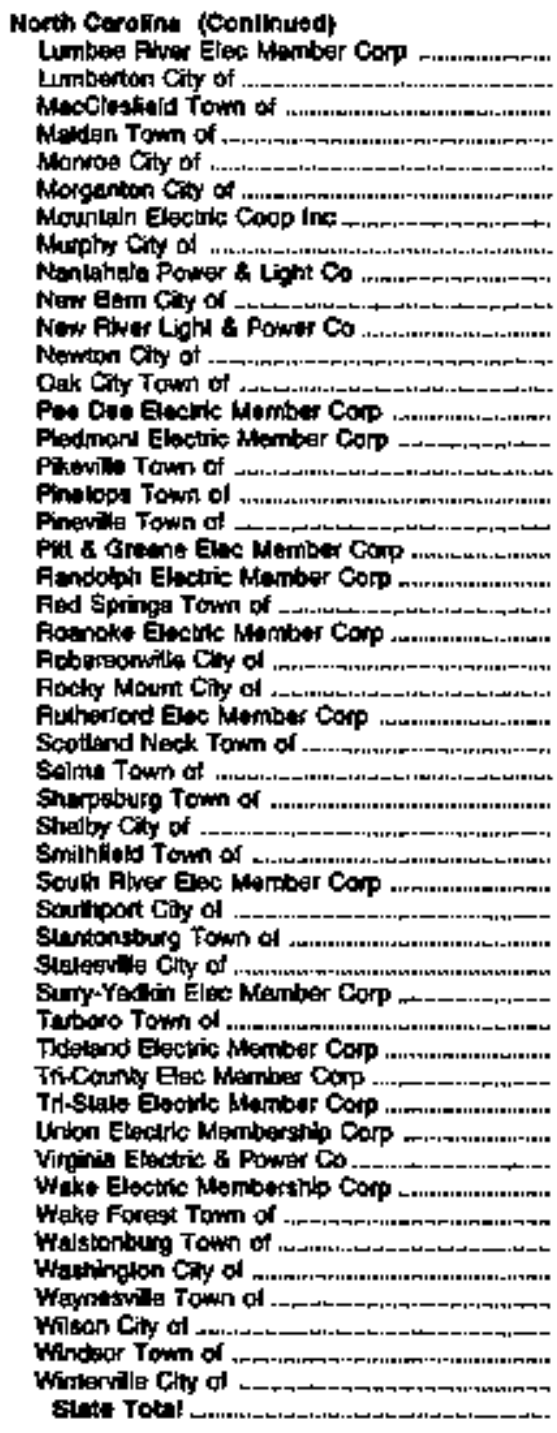 & 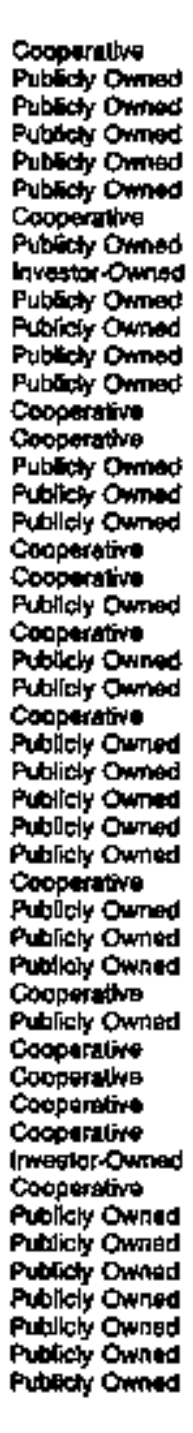 & 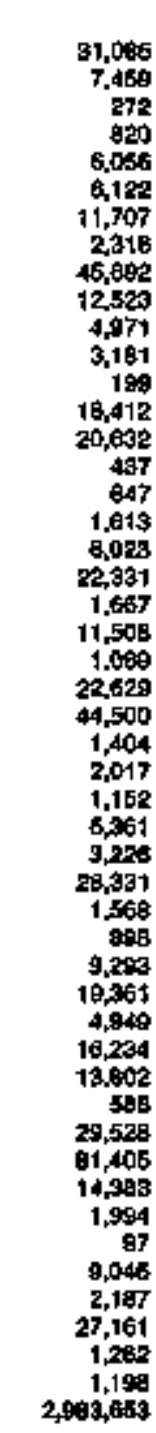 & 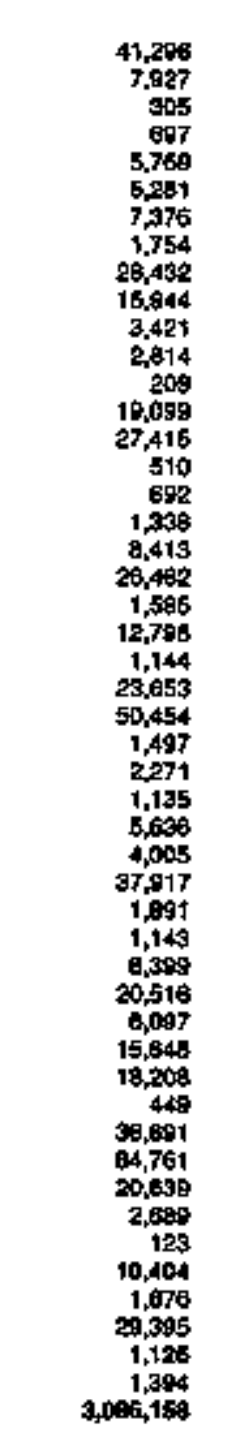 & 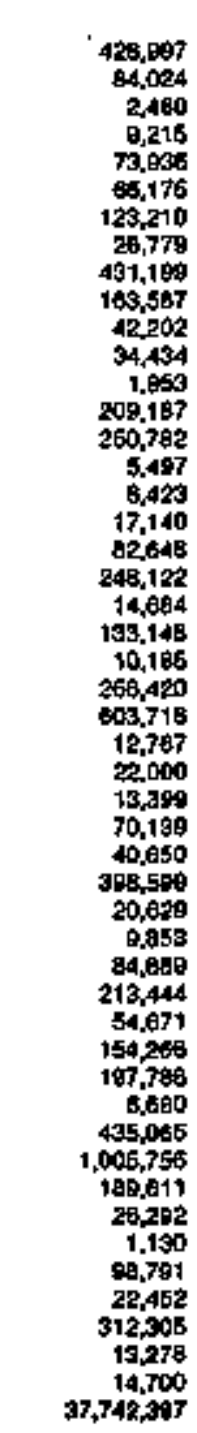 & 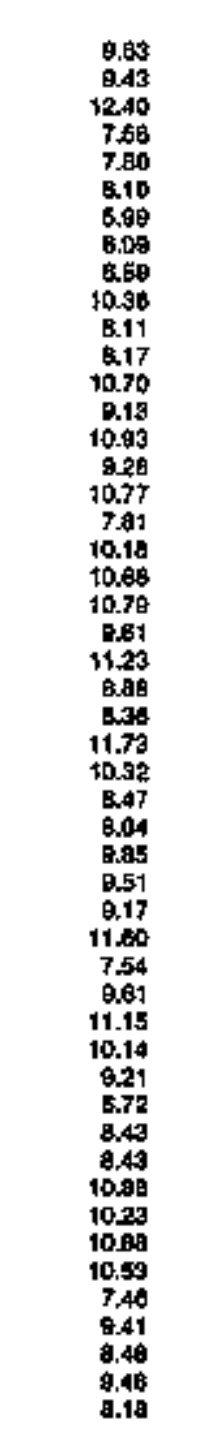 \\
\hline 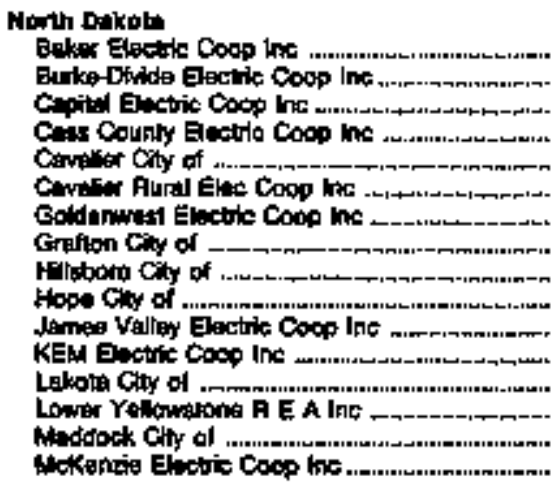 & 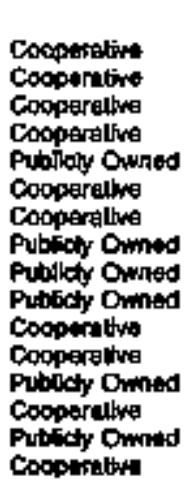 & 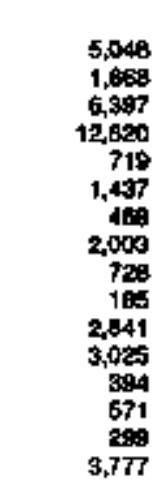 & $\begin{array}{r}6,239 \\
1,406 \\
4,860 \\
14,646 \\
380 \\
1,700 \\
404 \\
1,357 \\
497 \\
151 \\
3,706 \\
3,084 \\
246 \\
442 \\
360 \\
2,479\end{array}$ & 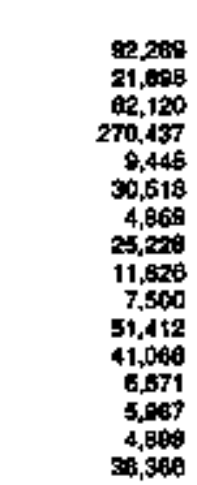 & $\begin{array}{l}6.76 \\
6.43 \\
7.82 \\
5.43 \\
4.02 \\
5.60 \\
6.30 \\
5.96 \\
4.27 \\
2.01 \\
7.21 \\
7.51 \\
3.89 \\
7.36 \\
7.19 \\
6.46\end{array}$ \\
\hline
\end{tabular}

Sow tootnoles at end of thible. 
Table 14. Class of Ownership, Number of Ultimate Consumert, Revenue, Bales, and Average Revenue per Kilowatthour for the Residential Sector by State and Vitilly, 1993 (Continued)

\begin{tabular}{|c|c|c|c|c|c|}
\hline 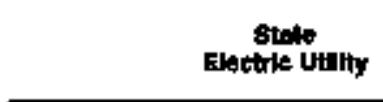 & $\begin{array}{c}\text { gians } \\
\text { of } \\
\text { onmenthlp }\end{array}$ & $\begin{array}{l}\text { Runber } \\
\text { of } \\
\text { tonumers }\end{array}$ & 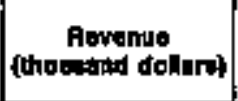 & 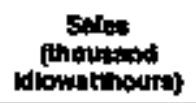 & 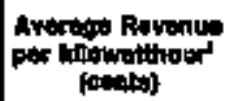 \\
\hline
\end{tabular}

Horth Datiols (Conkinued)

Henn Elitio Ctop In

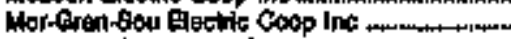

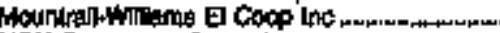

MoU Rasourcea Broup ino.

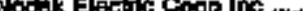

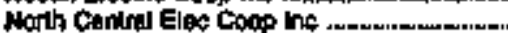

Nowinam siaks Fowar Do

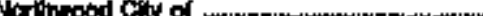

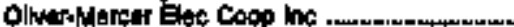

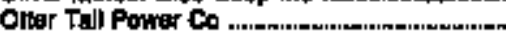

Pats Fiver etty of .

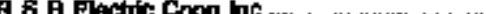

Stharon Clty of n....

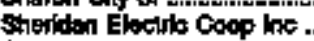

theyond Vellay Elex CoOp inc

Slape Elactrk Coop ho

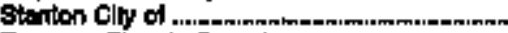

Trewarise Elecido Coop inc .

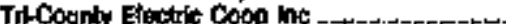

Veloy ciny cisy of

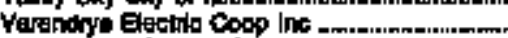

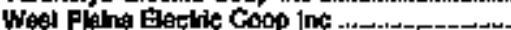

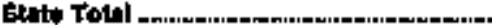

Qhila

Adam\$ furtal Eloctrio Cocp Ind ................

Ambenc Chy of

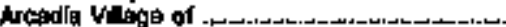

Arcanera cty of .

Begch City Clly of

Gathes Chy of

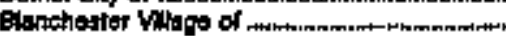

Biocindale Vilage id

Boring Cnim Con of

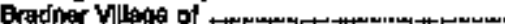

Erewale vition of

Bryin Clly of

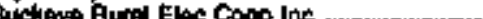

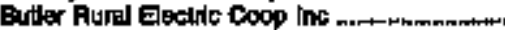

Cerey vinge of

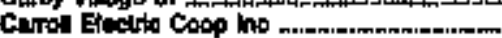

Cellna Chy of

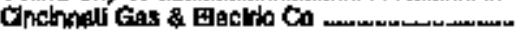

Clevolans Caty of

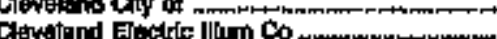

Chyda Chy of

Columblanta Vileoge of

Oolumbas Ciry of .

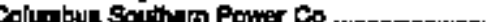

Culdtar Chy of

Cryahoge Falle Ciry of nm...m.

Quphel Ving of

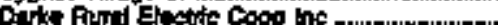

Deytor Power \& Uth Co

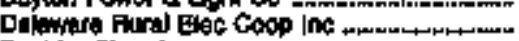

Deahler Chy of

Diver Cilly of

Eidorion vill

Eldorado vilage of

elmora vilade of

Fiontion Foner

Getion Cly of

Benos Vilang of

crocution with of of

Cloutite Villoge of .mm

Cration coly of

Geandich toing of
Cooperative

Casperaling

Cappativi:

imvistor-Owned

Coaperathe

Conpuative

irvestor-Gimad

Fublitly Ouried

Capartivt

Theitor-Onimed

Publing Ownod

Cacperatho

Putllely Ounnd

Cooporathe

Capperative

Cocpentatis:

Publldy Ouned

Goperative

Comperative

Publoty Ounied

Cocperatero

cocperation:

Cocpertivit

Publldy Ouned

Putiky Omiat

Publity Owined

Publithy Ouned

Publldy Ouned

Putilly Owred

Pubich Omind

Publuch Ounod

Publlaly Owriad

Publldy Owned

Puluchy Omied

Cocportive

Cooperative

Publlaty Ourtad

Coopapalive

Pubindy Ownad

invesloctowned

Publldy Ouned

Inwtiblor Owned

Putildy Owind

Publety Owast

Publlaty Ouned

infortor-Owned

Puticly Omiad

Publkchy Onined

Publthty Ounned

Cosperntive

Inwertorowned

Cocpparative

Publidy Ouned

Publldy Owned

Publdy Cimes:

Putnchy Dwined

Publlaty Ouned

Cocperratse

Cocpentive

Publly Owined

Publlety Ourned

Publldy Ouned

Publich Comed

Pulvicty Omed

Putitely Owined

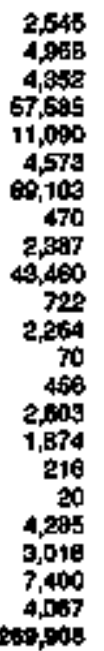

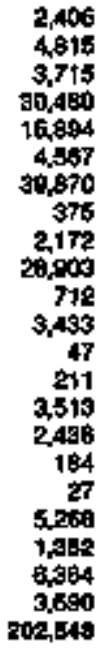

5,016

3.891

246

1, 817

1,200

1,761

258

9,279

478

Bag

4,379

15,168

7,654

1,528

$\mathrm{B}, 768$

5,004

52,598

62,608

as 111 1日

1,856

2,48

7,385

617,307

16,85

16,855
215

4,300

414,410

4,055

4.055
tes:
5.200

833

0

e, $78: 9$

5.007

5.809

645

$1,5 \%$

861
000

B44

\section{5,003}

1,065

139

899

427

567
1,509

129

4,460

200

534

2003

13,as4

9,961

713

6.857

3.263

492763

210,634

590.165

1,226

1.616

3.065

394,775

B.491

101

5,117

ง 73,760

4.325

305

297

386

as4

6,133

4,524

2,815
475

t.928

417

707
268

2906

60,178

60.304

4 sBge?

272446

71,644

EF5,144

7,763

37,094

$47 \mathrm{~B}=56$

11800

42337

1,200

g.650

she00

51.951

247

414

71,104

12060

87570

B7, 315

3,209,0s1

8. 7

628

697

6.94

$6 \mathrm{~s}$

9.37

581

4 .

5.73

6.0.

tios

8.11

(t)

897

7.44

b)

3.29
7.27

6.44

651

57.488

$34,36 \dot{0}$

2442

13,925

5,307

13, 108

20105

1,817 
Table 14. Class of Ownership, Number of Ultinate Consumers, Rovenue, Sales, and Average Revenue per Kilowatthour for the Residential Sector by State and Utility, 1993 (Continued)

\begin{tabular}{|c|c|c|c|c|c|}
\hline $\begin{array}{l}\text { state } \\
\text { Eleotrie Uilliky }\end{array}$ & $\begin{array}{c}\text { Clasa } \\
\text { of } \\
\text { ommitahip }\end{array}$ & $\begin{array}{l}\text { Number } \\
\text { al } \\
\text { conaumert }\end{array}$ & $\begin{array}{c}\text { Fowenue } \\
\text { (thousand dolfarn) }\end{array}$ & 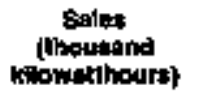 & 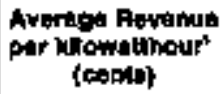 \\
\hline 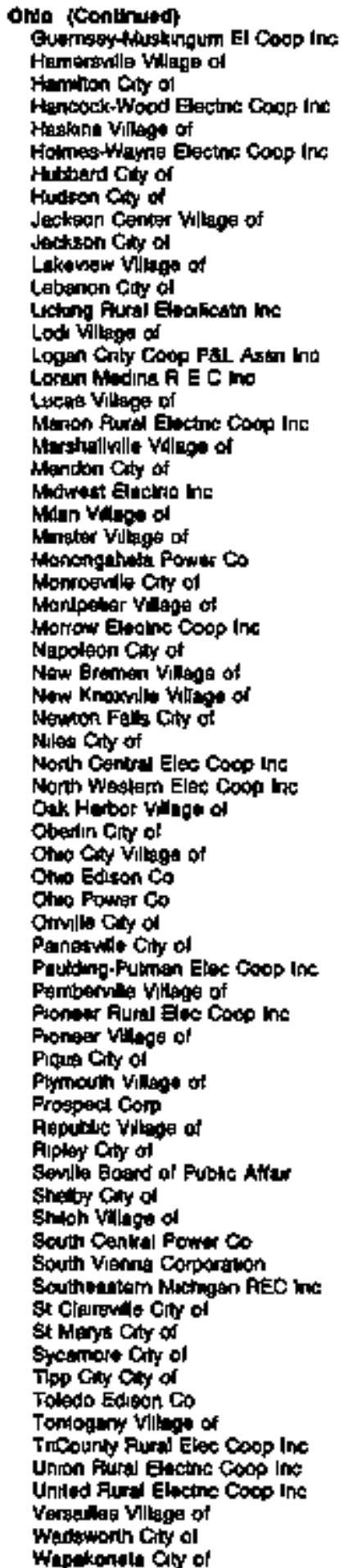 & 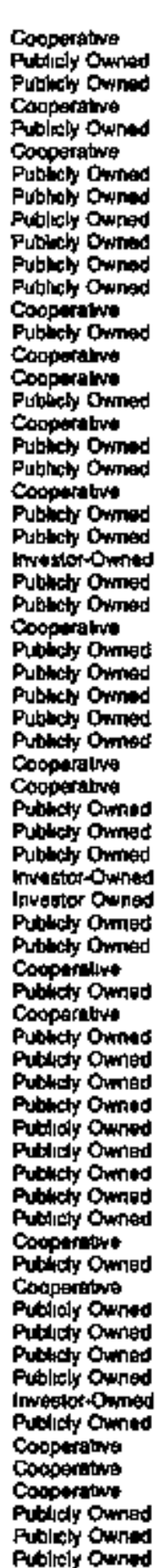 & 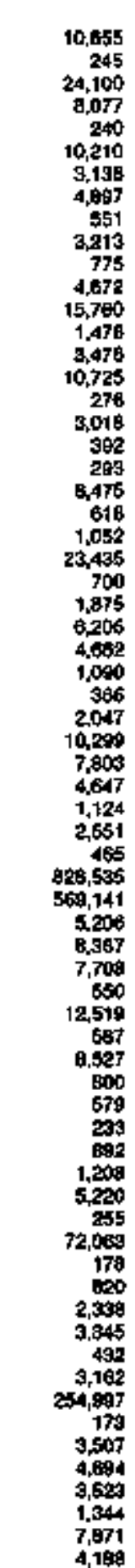 & 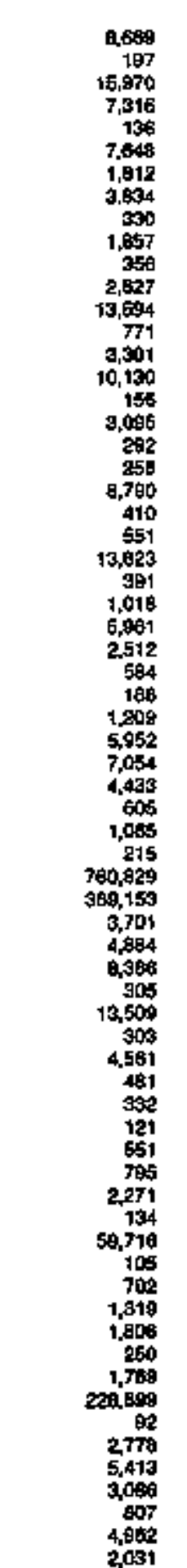 & 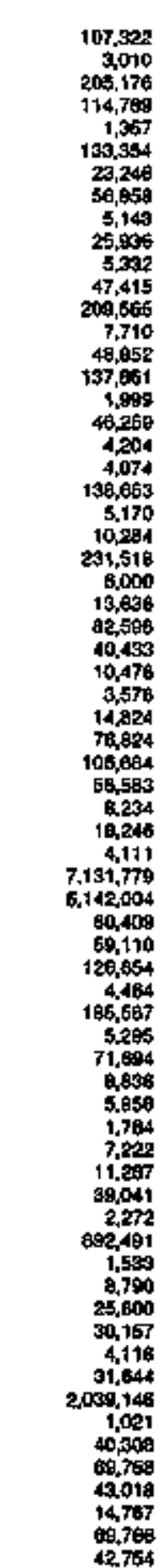 & 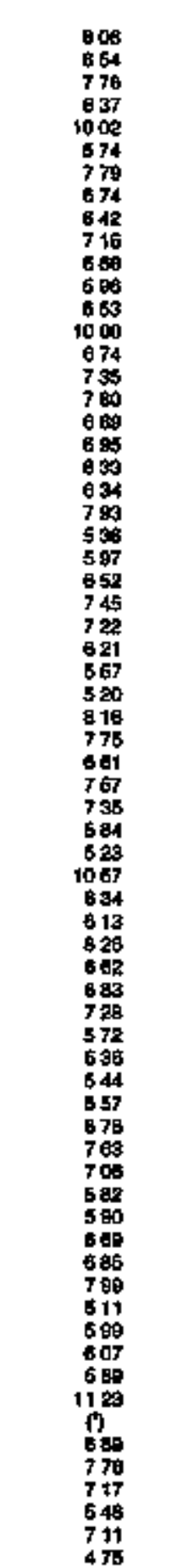 \\
\hline
\end{tabular}

Sod lootnoiltes al and af table 
Tabte 14. Class of Ownership, Number of Ultimate Consumers, Fevenue, Sales, and Average Revenue per Kllowatthour for the Residentlal Sector by State and Vtillty, t993 (Continued)

\begin{tabular}{|c|c|c|c|c|c|}
\hline 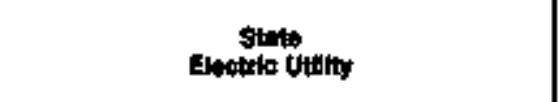 & ctises & $\begin{array}{c}\text { Munber } \\
\text { of } \\
\text { contulatiots }\end{array}$ & (thoubland dollert) & 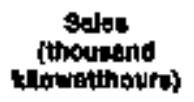 & $\begin{array}{c}\text { Average Revenue } \\
\text { per R.jowatilhour' } \\
\text { (Oente) }\end{array}$ \\
\hline 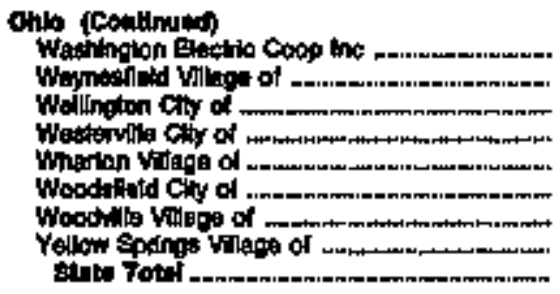 & 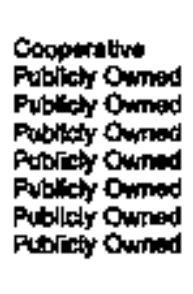 & $\begin{array}{r}7,740 \\
430 \\
1,798 \\
10,818 \\
180 \\
1,312 \\
607 \\
1,768 \\
4,307,744\end{array}$ & $\begin{array}{r}5,358 \\
338 \\
1,098 \\
4,024 \\
79 \\
694 \\
397 \\
651 \\
3,607,679\end{array}$ & $\begin{array}{r}65,816 \\
4,035 \\
13,144 \\
140,518 \\
1,202 \\
8,840 \\
5,924 \\
19,040 \\
41,950,455\end{array}$ & $\begin{array}{l}8.16 \\
6.99 \\
8.35 \\
5.84 \\
17 \\
6.72 \\
6.70 \\
4.89 \\
9.56\end{array}$ \\
\hline 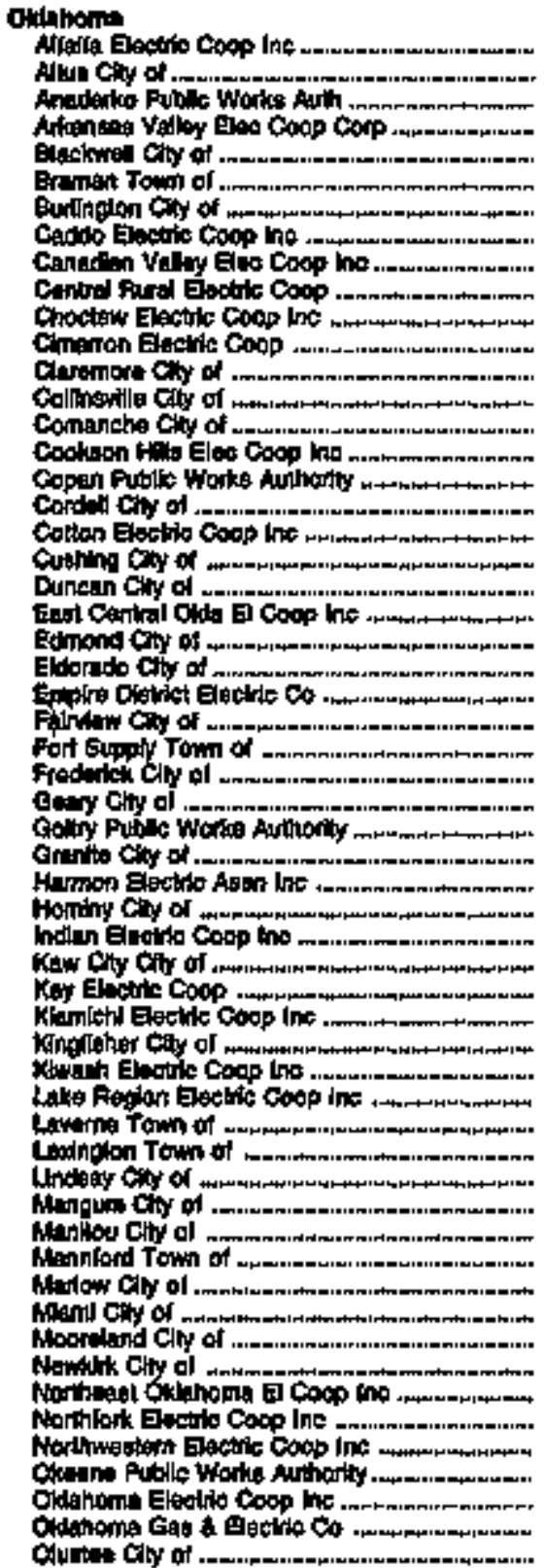 & 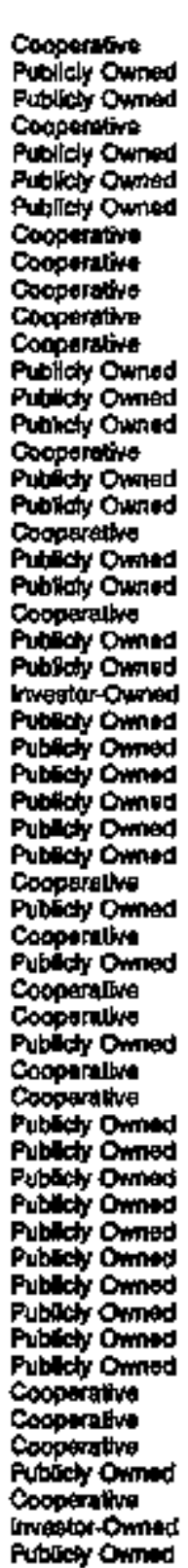 & 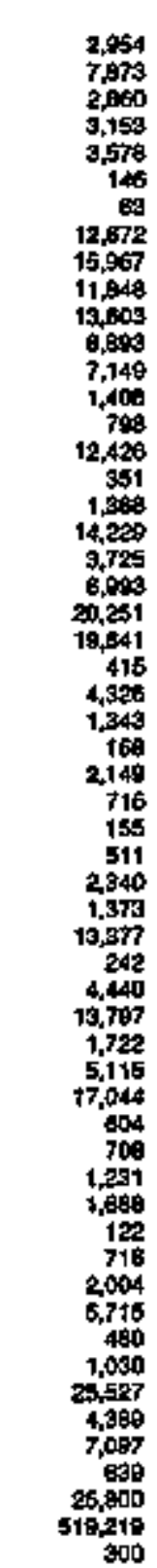 & 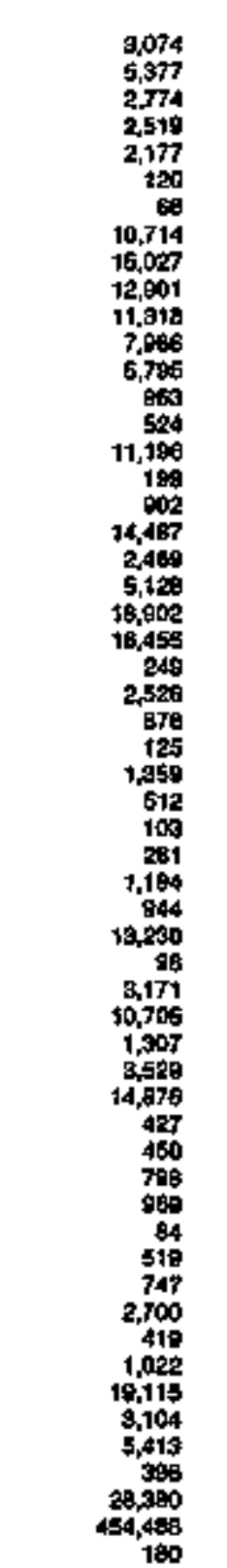 & 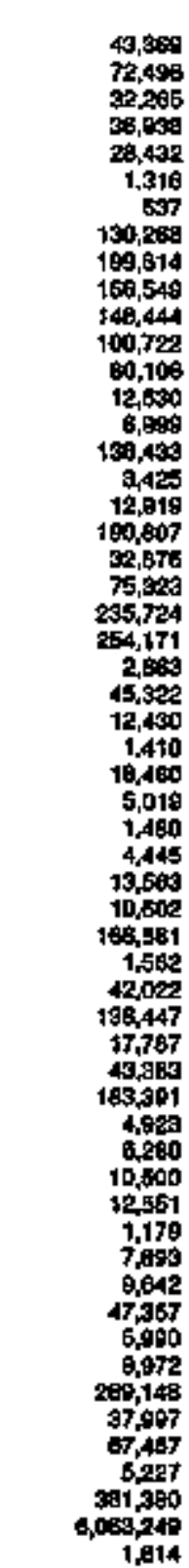 & 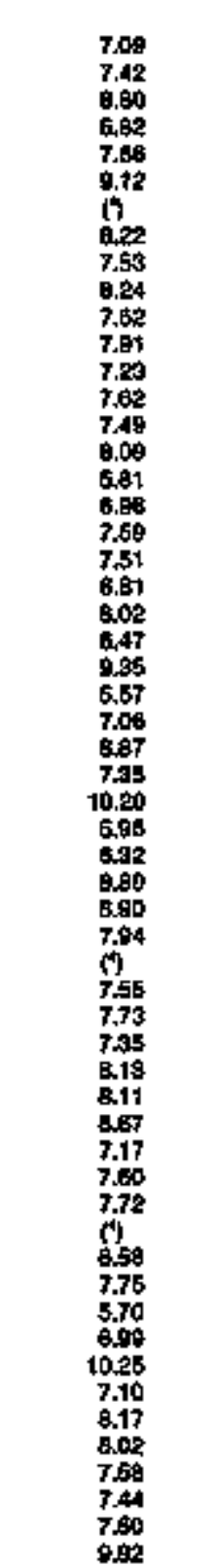 \\
\hline
\end{tabular}

Soa footnotes at and of tabla 
Table 14. Class of Ownershlp, Number of Uytunte Consumers, Revenue, Sales, and Average Revenue per Kllowatthour for the Rosidential Sector by State and Utillty, 1998 (Continued)

\begin{tabular}{|c|c|c|c|c|c|}
\hline 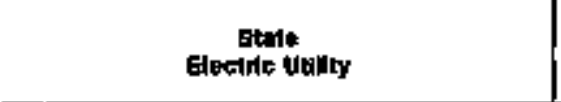 & 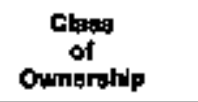 & $\begin{array}{l}\text { Number } \\
\text { of } \\
\text { Contoumara }\end{array}$ & 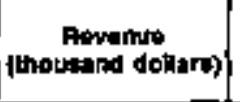 & 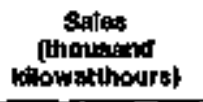 & $\begin{array}{l}\text { Avorege Perenut } \\
\text { per thowalthour } \\
\text { \{cento\} }\end{array}$ \\
\hline 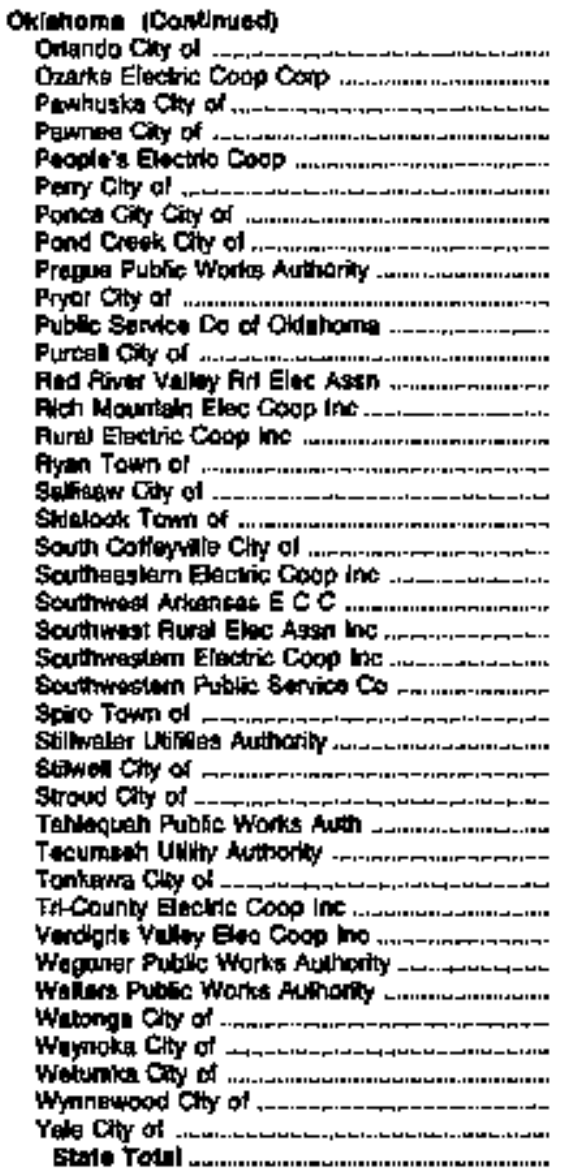 & 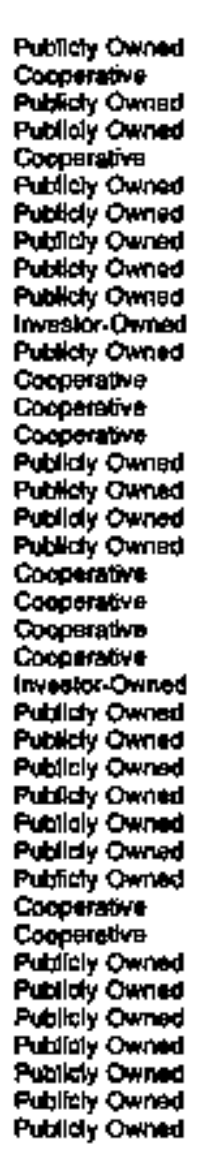 & 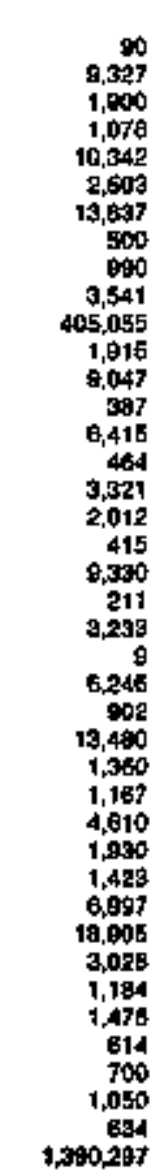 & 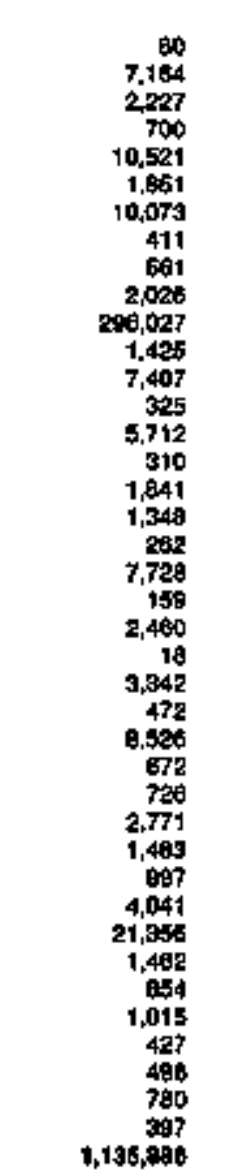 & 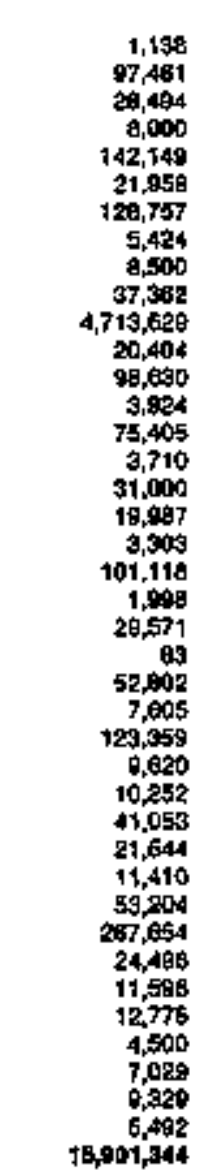 & 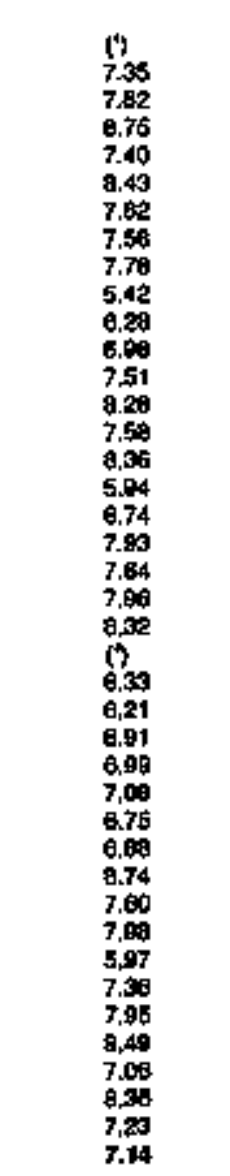 \\
\hline 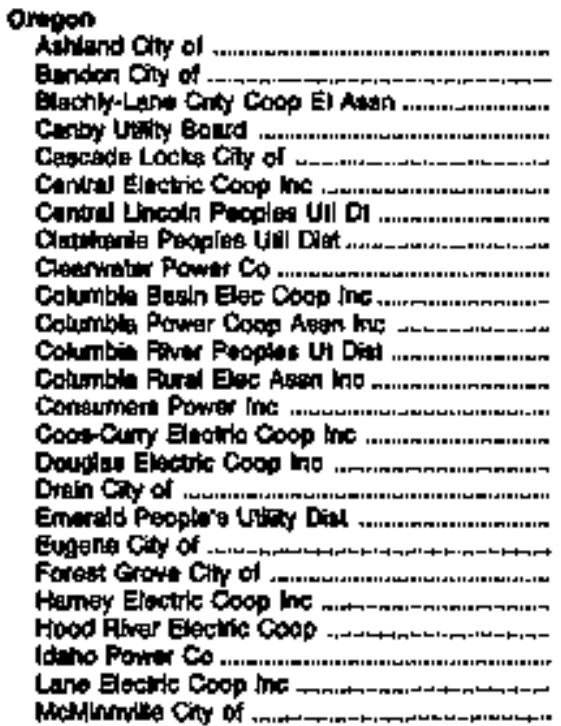 & 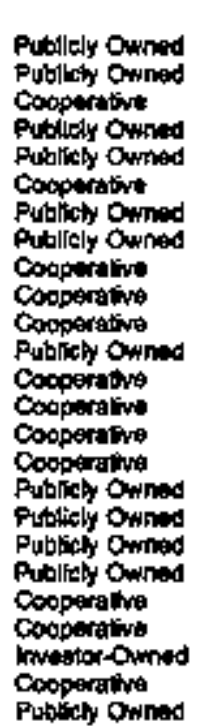 & $\begin{array}{r}7,210 \\
2,113 \\
2,778 \\
3,540 \\
448 \\
16,650 \\
26,760 \\
2,040 \\
128 \\
2,918 \\
1,147 \\
6,624 \\
56 \\
15,798 \\
12,463 \\
7,405 \\
498 \\
13,030 \\
42,927 \\
5,934 \\
650 \\
2,585 \\
11,903 \\
10,911 \\
8,630\end{array}$ & 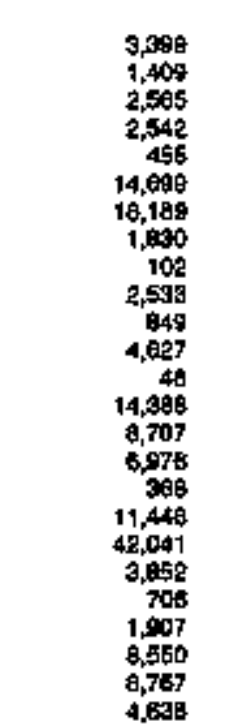 & 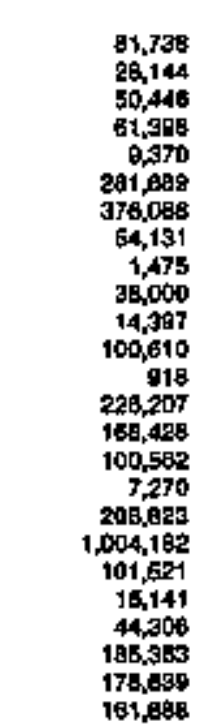 & 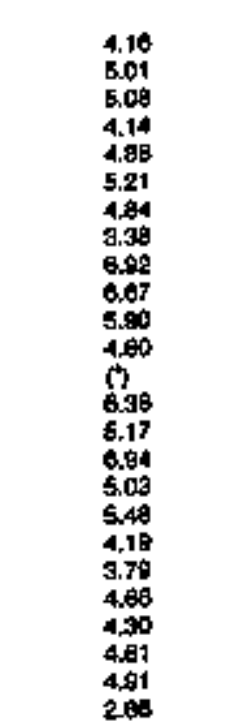 \\
\hline
\end{tabular}

Ses lootrigh al end of labke. 
Table 14. Class of Ownership, Number of Utimate Contumers, Revenue, Sales, and Average Reventue per Kllowatthour for the Residentlal Sector by State and Utillty, 1993 (Contlined)

\begin{tabular}{|c|c|c|c|c|c|}
\hline Statio & $\begin{array}{c}\text { cinot } \\
\text { of } \\
\text { Ommonhip }\end{array}$ & 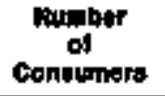 & 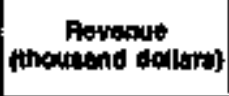 & 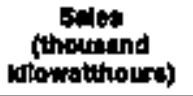 & 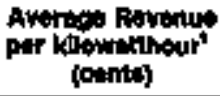 \\
\hline 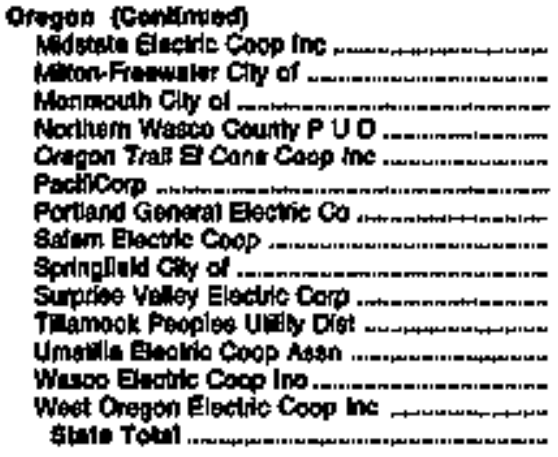 & 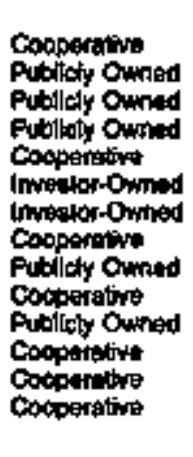 & 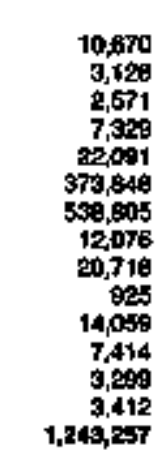 & 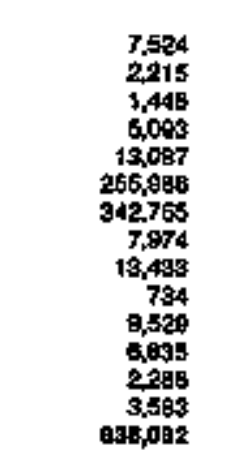 & 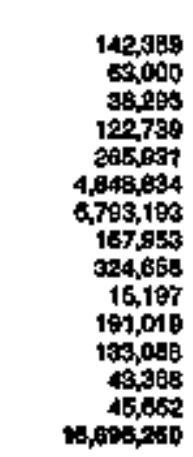 & $\begin{array}{l}5.28 \\
3.52 \\
3.77 \\
4.15 \\
4.92 \\
5.20 \\
5.05 \\
4.75 \\
4.14 \\
4.89 \\
4.99 \\
5.14 \\
5.97 \\
7.95 \\
5.92\end{array}$ \\
\hline 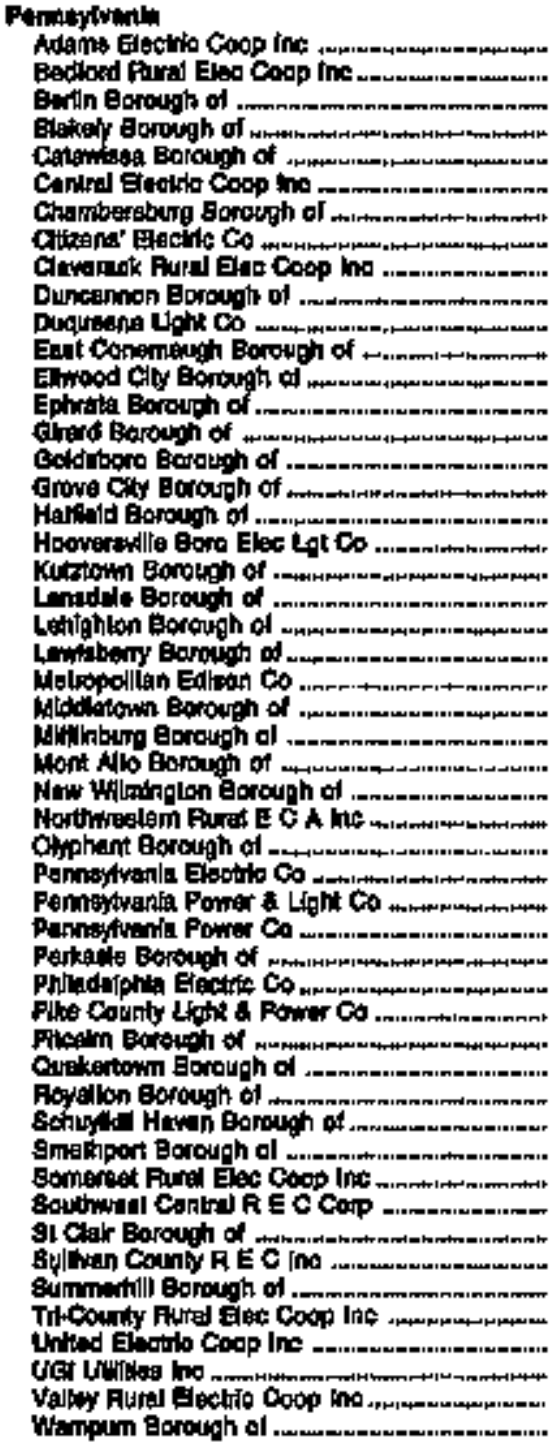 & 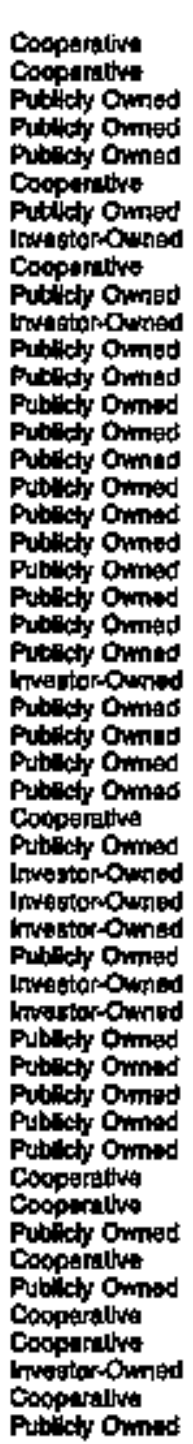 & 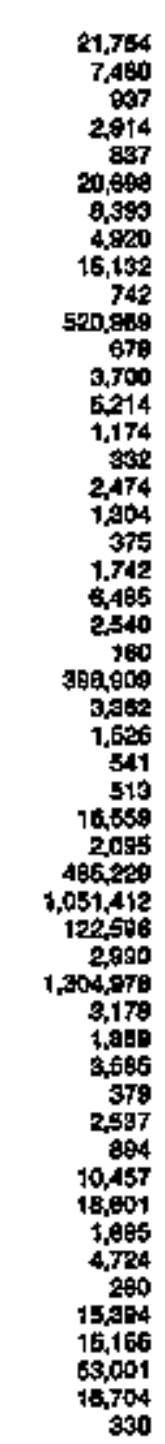 & 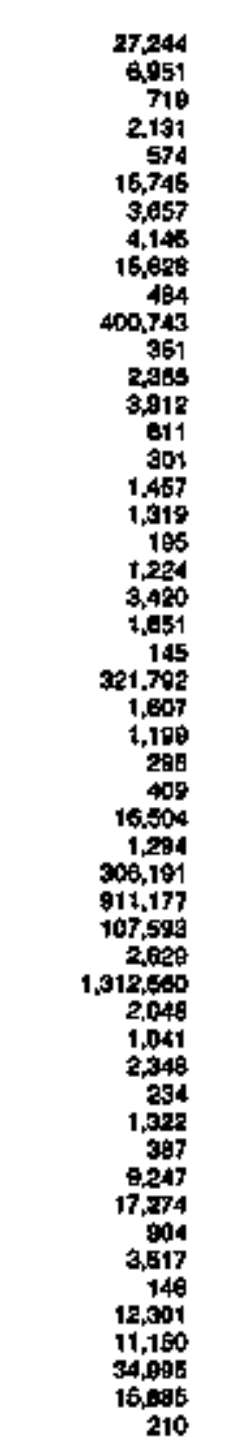 & 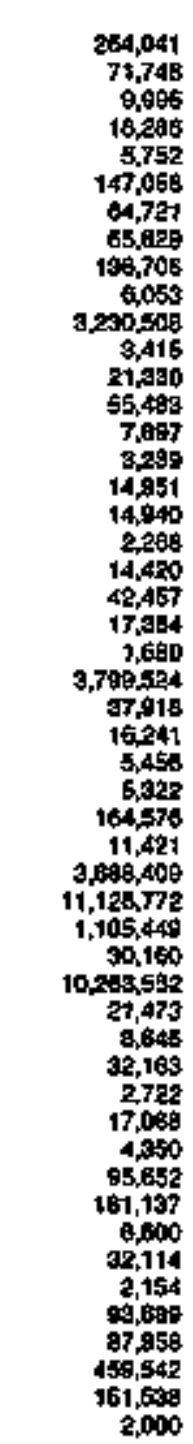 & 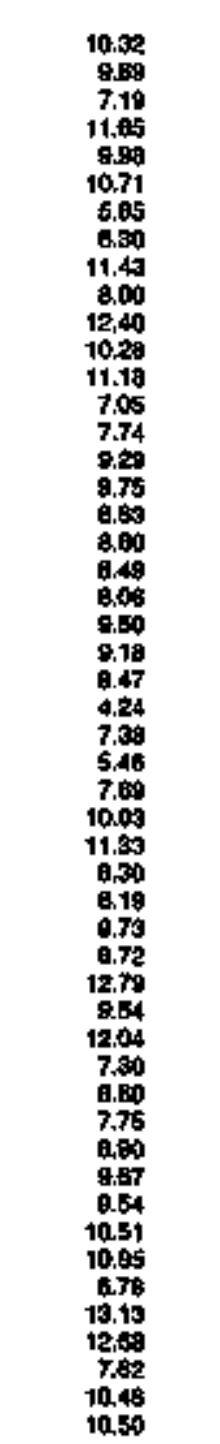 \\
\hline
\end{tabular}

Sas founolos at end of tabla 
Table 14. Class of Ownership, Number of Ultimate Consumers, Revenue, Sales, and Average Revenue per Kilowatthour for the Residentlal Sector by State and Utillty, 1993 (Contimued)

\begin{tabular}{|c|c|c|c|c|c|}
\hline Stat & $\begin{array}{l}\text { Clang } \\
\text { of } \\
\text { ouminghip }\end{array}$ & $\begin{array}{l}\text { Mumber } \\
\text { of } \\
\text { Conpunarn }\end{array}$ & $\begin{array}{c}\text { Rewenue } \\
\text { fthauctend dolltrits }\end{array}$ & $\begin{array}{l}\text { 5ettot } \\
\text { (thotiand } \\
\text { kelkwethoura) }\end{array}$ & 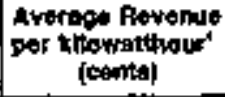 \\
\hline 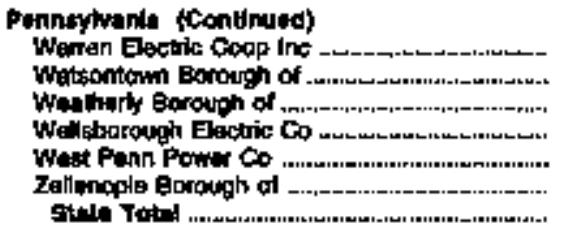 & 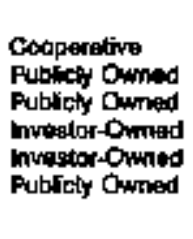 & $\begin{array}{r}7,701 \\
954 \\
1,110 \\
4,251 \\
506.566 \\
1,819 \\
4,756,028\end{array}$ & $\begin{array}{r}4,289 \\
609 \\
670 \\
2,069 \\
35,900 \\
1,168 \\
3,969,160\end{array}$ & $\begin{array}{r}3,302 \\
\$, 965 \\
10,018 \\
31,824 \\
\$, 479,746 \\
11,915 \\
41,466,4 \$ 6\end{array}$ & $\begin{array}{r}11.14 \\
10.21 \\
6.77 \\
6.55 \\
6.32 \\
9.65 \\
9.55\end{array}$ \\
\hline 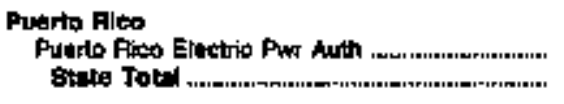 & Publichr Ormat & $\begin{array}{l}1,097,8 \mathrm{~dB} \\
2,0097, \mathrm{a2b}\end{array}$ & $\begin{array}{l}397,766 \\
309,755\end{array}$ & $\begin{array}{l}4,901,5,57 \\
4,900,507\end{array}$ & $\begin{array}{l}7.90 \\
7.90\end{array}$ \\
\hline 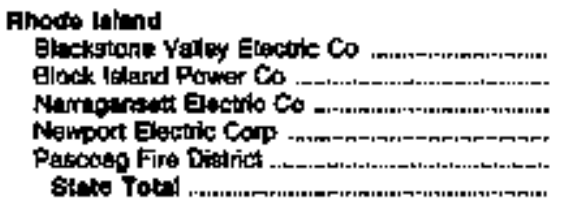 & 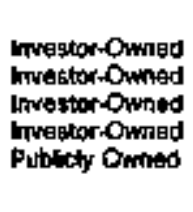 & $\begin{array}{r}74,053 \\
1,008 \\
286,923 \\
27,595 \\
3,587 \\
393,007\end{array}$ & $\begin{array}{r}45,462 \\
1344 \\
202,523 \\
23,570 \\
2,394 \\
274,542\end{array}$ & $\begin{array}{r}374,302 \\
2,487 \\
1,817,675 \\
198,428 \\
20,916 \\
2,4+1,008\end{array}$ & $\begin{array}{l}12.15 \\
25.69 \\
11.14 \\
12.00 \\
11.40 \\
11.90\end{array}$ \\
\hline 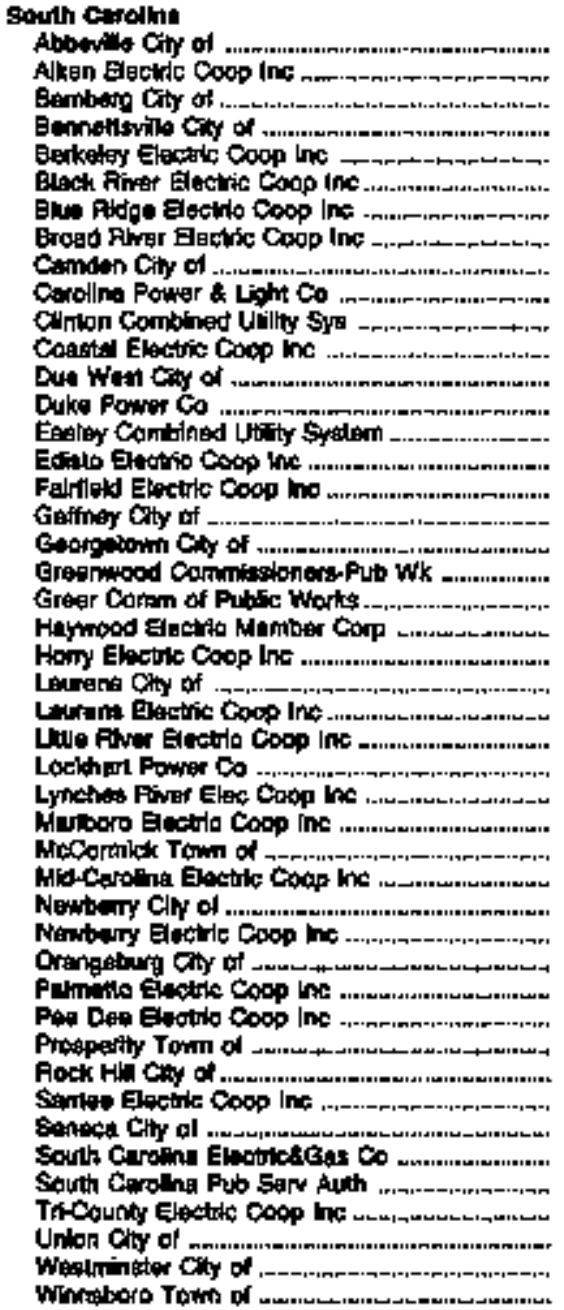 & 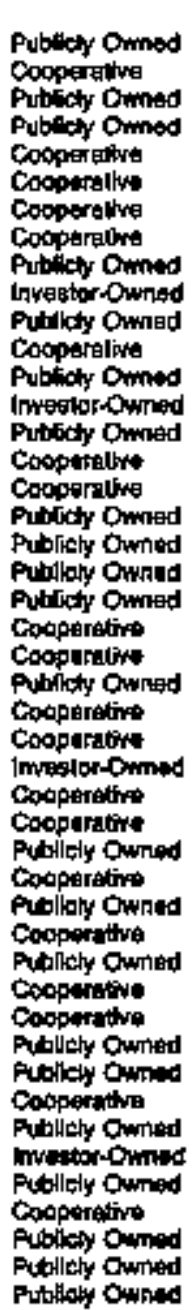 & 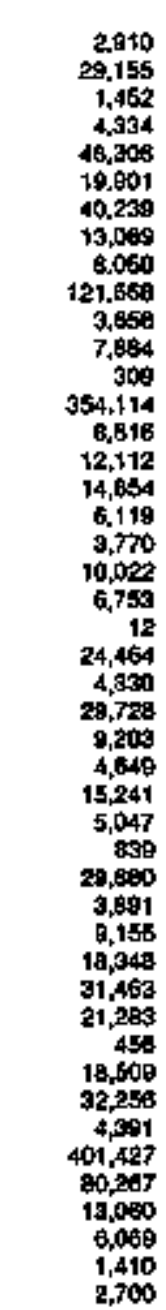 & 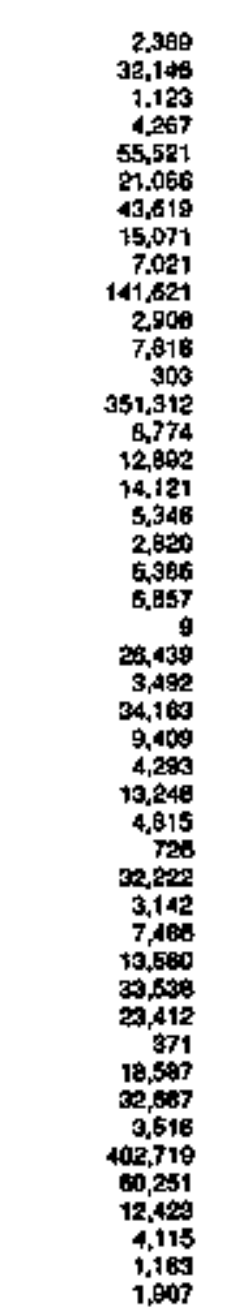 & 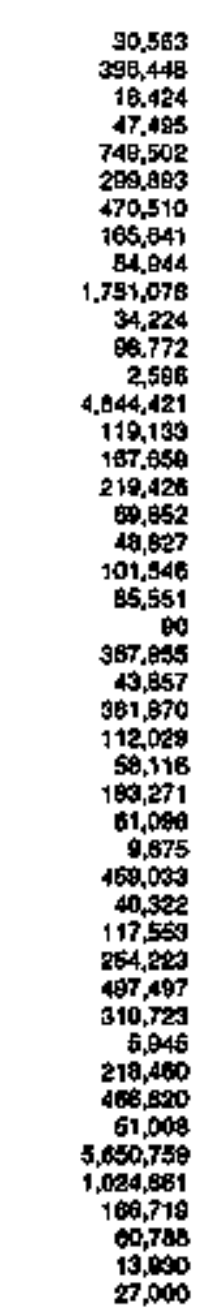 & 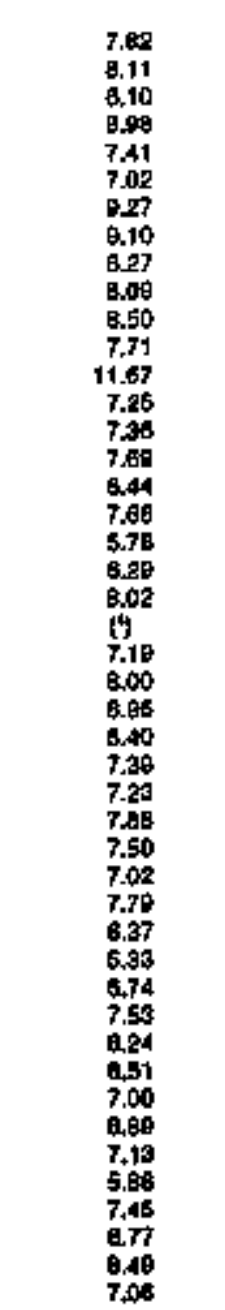 \\
\hline
\end{tabular}

Seg lootunoteg al end of table. 
Table 14. Class of Ownershjp, Number of Ulimate Consumers, Revenue, Sales, and Average Revenue per Kilowatthour for the Residential sector by State and Utility, 1993 (Continued)

\begin{tabular}{|c|c|c|c|c|c|}
\hline $\begin{array}{c}\text { Stake } \\
\text { Electipe Utoky }\end{array}$ & $\begin{array}{l}\text { Clans: } \\
\text { of } \\
\text { Pinntorithip }\end{array}$ & $\begin{array}{l}\text { Fumber } \\
\text { of } \\
\text { Oontinger }\end{array}$ & (thousand dobort) & 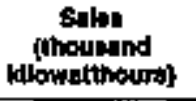 & 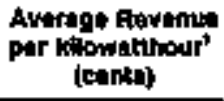 \\
\hline 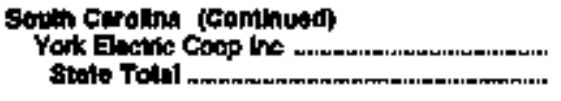 & Cooperatiste & $\begin{array}{r}19,919 \\
1,506,804\end{array}$ & $\begin{array}{r}25,692 \\
1,516, A 84\end{array}$ & $\begin{array}{r}297,162 \\
20,908,689\end{array}$ & $\begin{array}{l}9,51 \\
7,35\end{array}$ \\
\hline 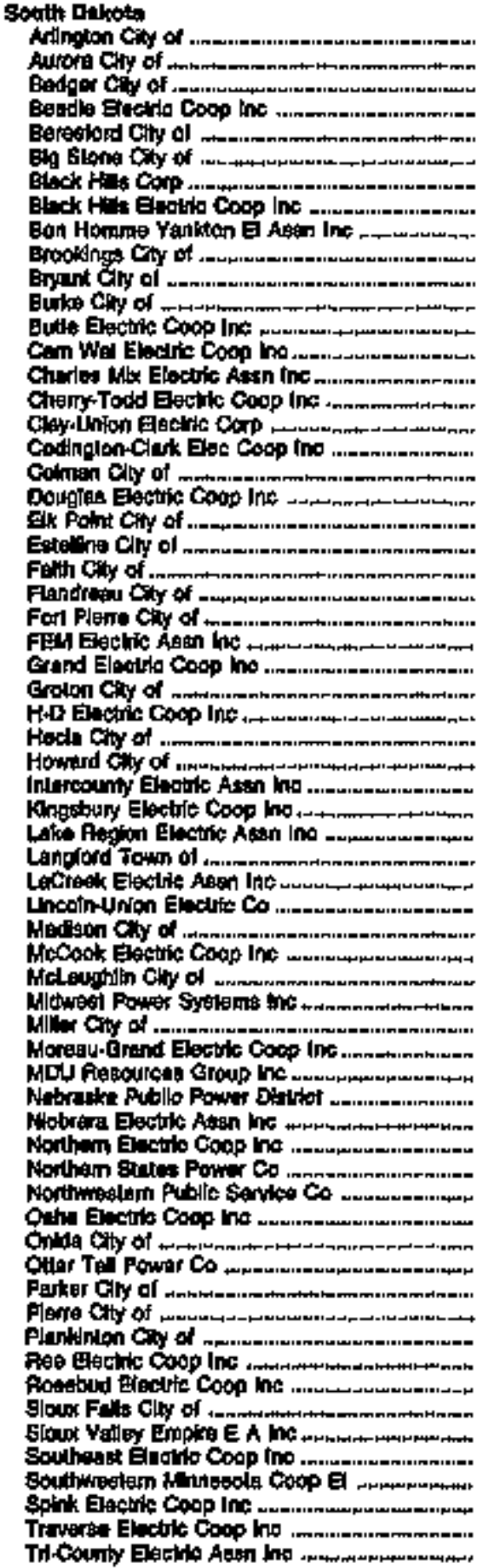 & 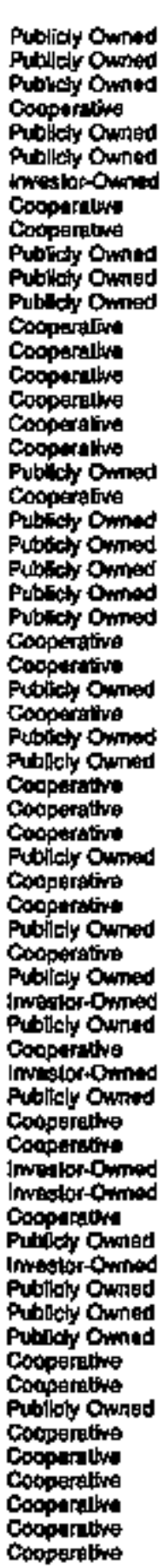 & 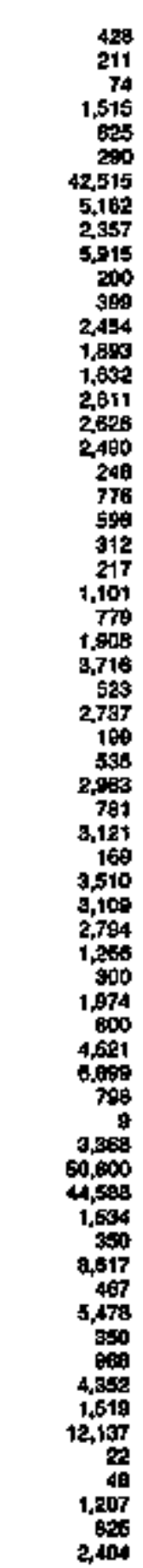 & 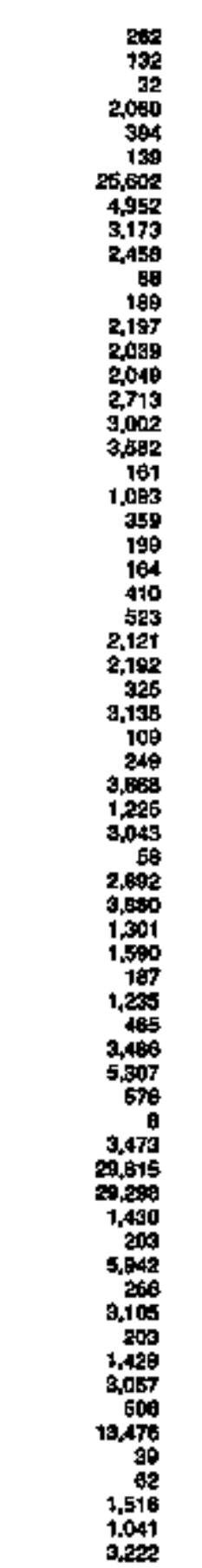 & 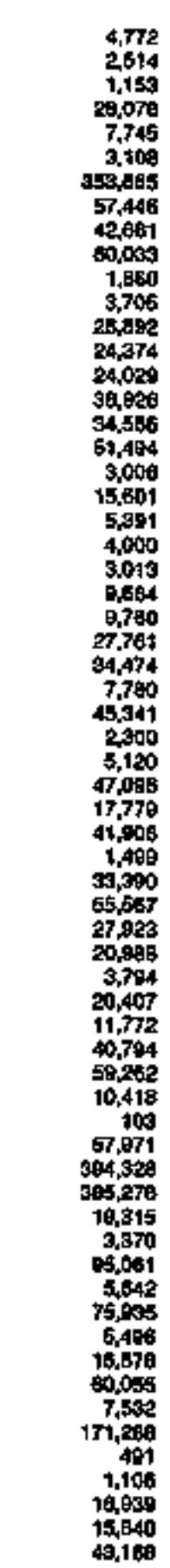 & 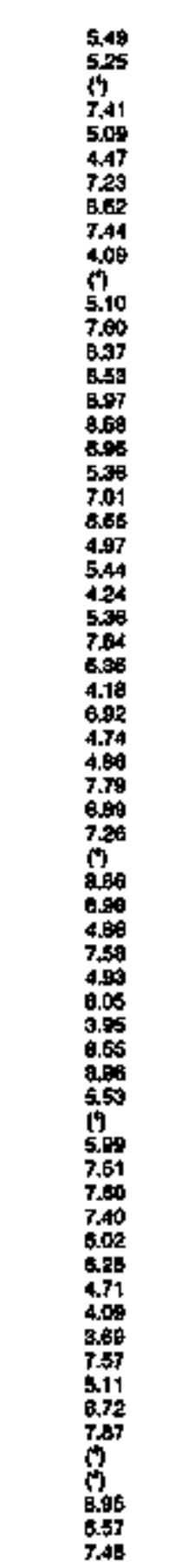 \\
\hline
\end{tabular}

Sea foothotes at and of lablo. 
Tahle 14. Class of Ownership, Number of Ultimate Consumers, Revenue, Sales, and Average Revenue por Kilowathour for the Residential Sector by State and Utility, 1993 (Continued)

\begin{tabular}{|c|c|c|c|c|c|c|}
\hline $\begin{array}{c}\text { Sinke } \\
\text { Fioclite Uillty }\end{array}$ & & $\begin{array}{c}\text { ciats } \\
\text { of } \\
\text { omiterthlp }\end{array}$ & $\begin{array}{l}\text { Humber } \\
\text { of } \\
\text { contumert }\end{array}$ & Ravenus & $\begin{array}{c}\text { salns } \\
\text { (thousend } \\
\text { Mllowathours) }\end{array}$ & 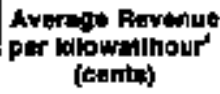 \\
\hline 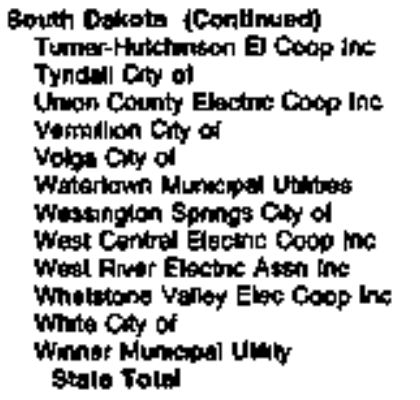 & - & 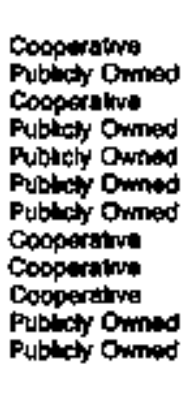 & 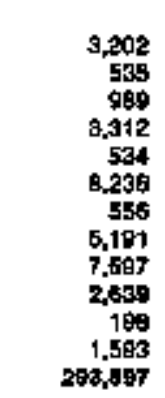 & 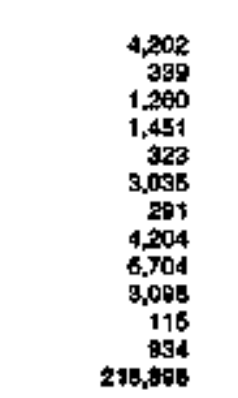 & $\begin{array}{r}64.451 \\
7,410 \\
15,846 \\
21,093 \\
5,956 \\
81.806 \\
8,238 \\
50,791 \\
78,259 \\
45,874 \\
1,673 \\
19,012 \\
3,109,114\end{array}$ & $\begin{array}{l}894 \\
1 \$ 7 \\
785 \\
660 \\
544 \\
372 \\
406 \\
620 \\
657 \\
676 \\
614 \\
101 \\
7.04\end{array}$ \\
\hline 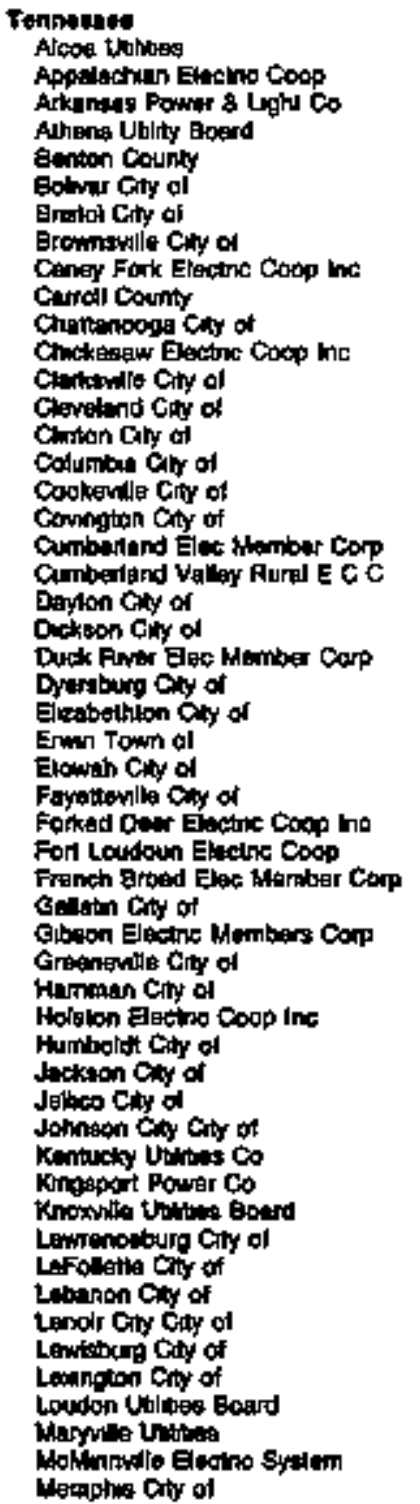 & $\begin{array}{l}\dot{.} \\
\dot{.} \\
\dot{*}\end{array}$ & 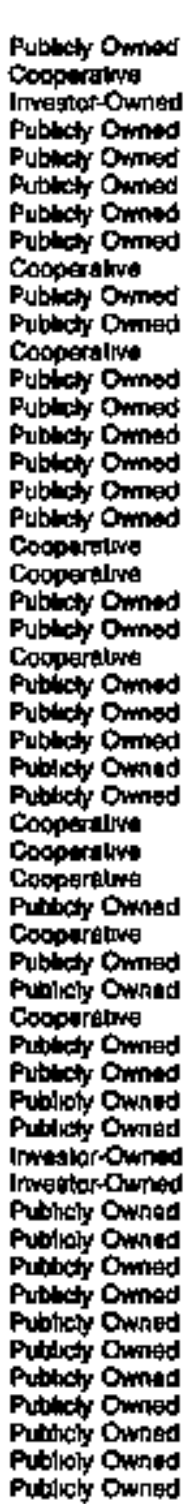 & 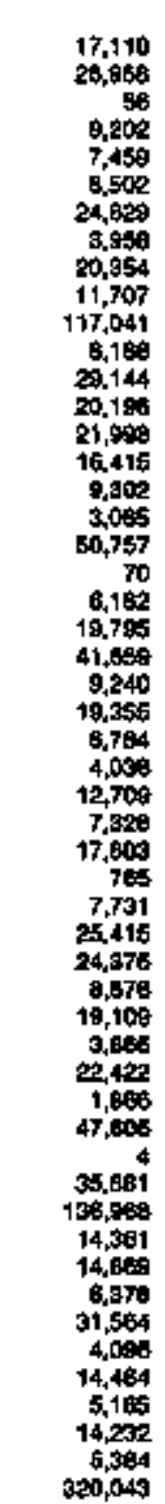 & 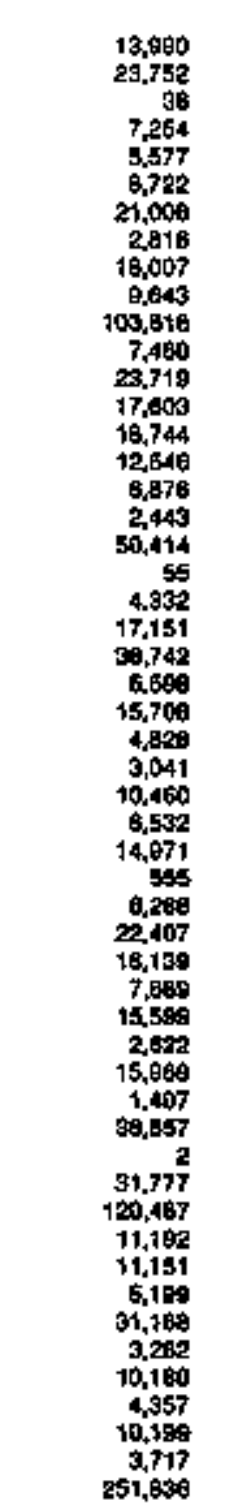 & 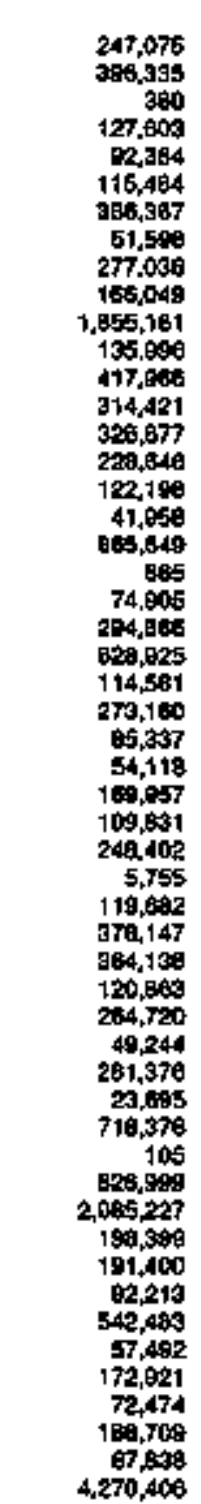 & 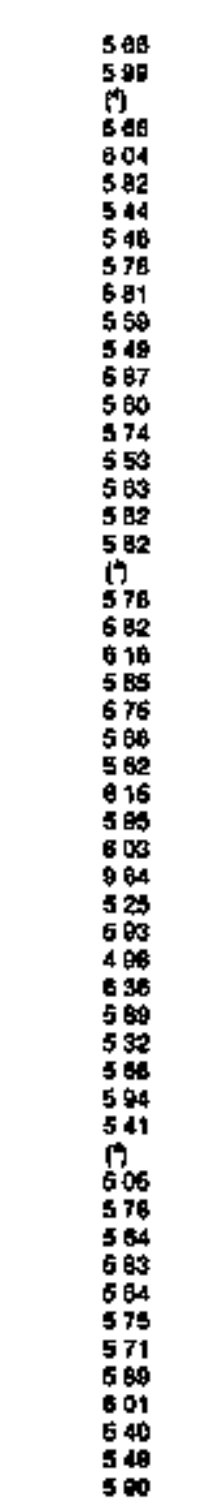 \\
\hline
\end{tabular}

8ut tooknotes at ind or tibit 
Table 14. Class of Ownershlp, Mumber of Ultimate Consumers, Revenue, Salos, and Average Revenue per Kllowatthour for the Residential sector by State and Utilty, 1998 (Continued)

\begin{tabular}{|c|c|c|c|c|c|}
\hline Etocidt thing & of & $\begin{array}{c}\text { Number } \\
\text { at } \\
\text { Con:umbtat }\end{array}$ & $\begin{array}{c}\text { Ravtulut } \\
\text { (thou:und dolkre) }\end{array}$ & 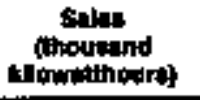 & 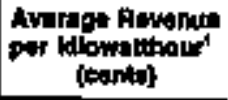 \\
\hline 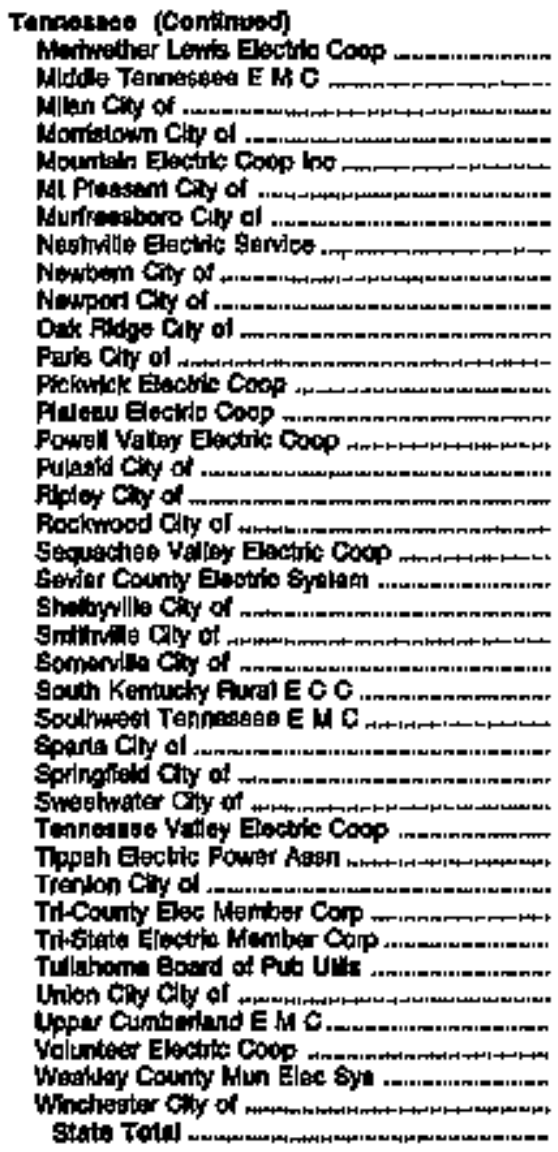 & 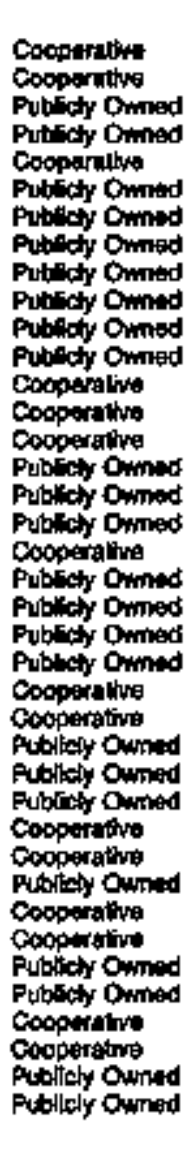 & 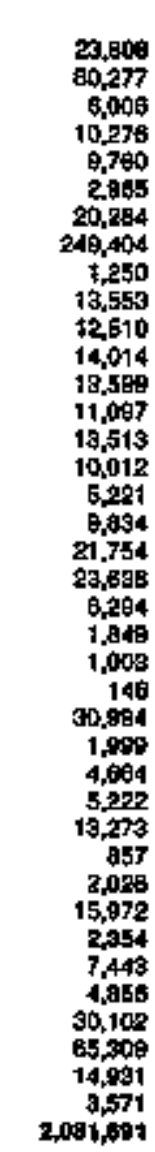 & 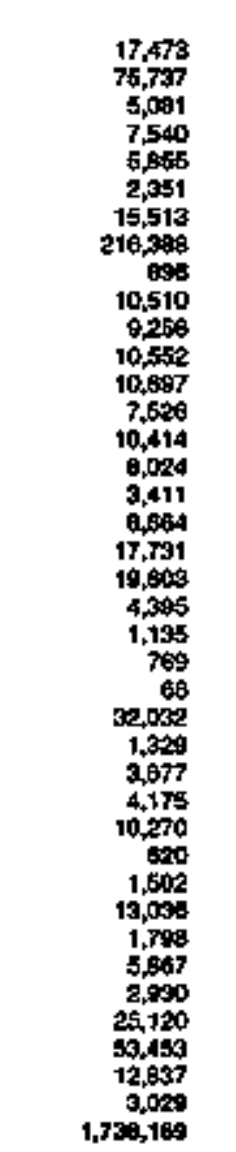 & 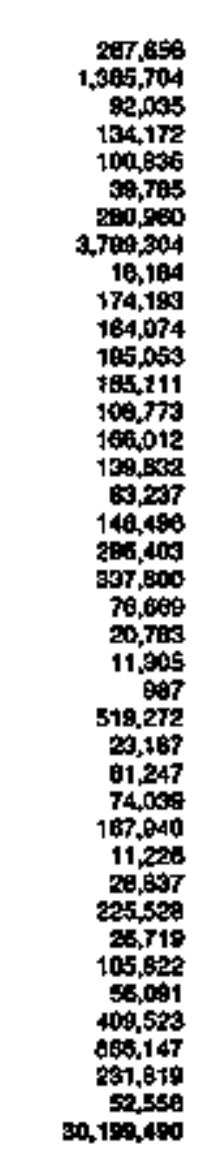 & 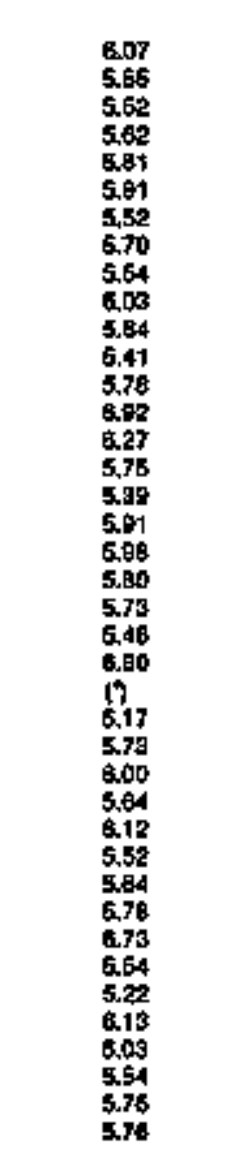 \\
\hline 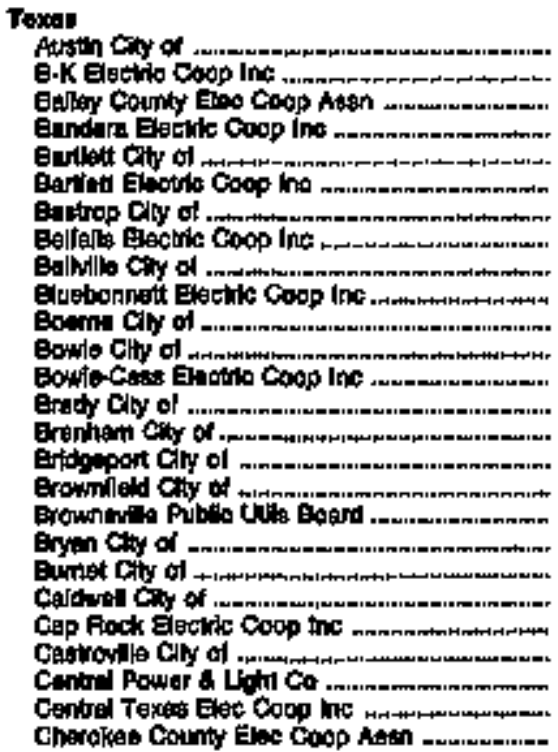 & 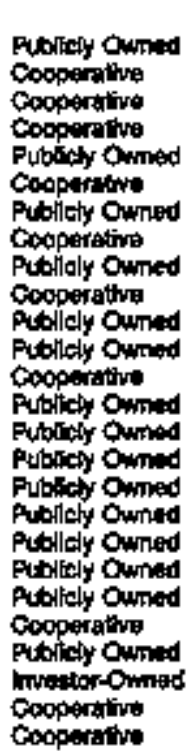 & 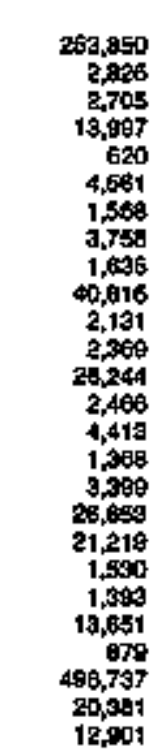 & 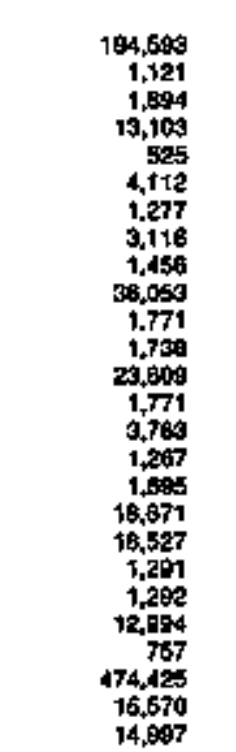 & 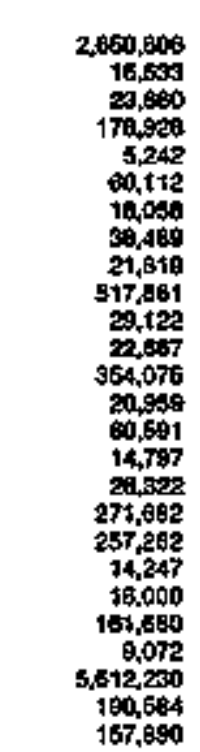 & 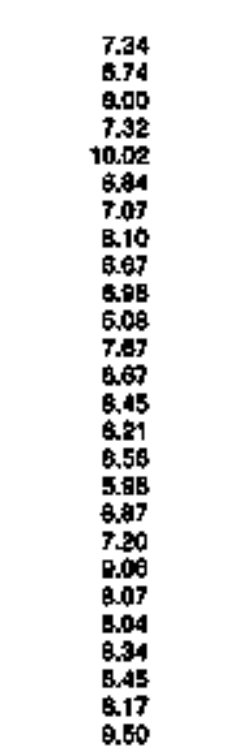 \\
\hline
\end{tabular}

8og footnokg at and of tabla. 
Table 14. Class of Omnership, Number of Ultimate Consumers, Revenue, Salea, and Average Revenue par Kilowatthour for the Residential Sector by State and Utillty, 1993 (Continued)

\begin{tabular}{|c|c|c|c|c|c|}
\hline $\begin{array}{c}\text { state } \\
\text { Elopte Utity }\end{array}$ & 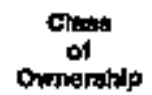 & $\begin{array}{c}\text { Number } \\
\text { af } \\
\text { consurithes }\end{array}$ & $\begin{array}{c}\text { Prewenut } \\
\text { (thournnd dollimes] }\end{array}$ & $\begin{array}{l}\text { Eakes } \\
\text { (Thouend } \\
\text { anowatthoures }\end{array}$ & 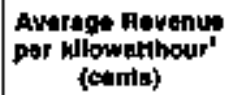 \\
\hline
\end{tabular}

\section{Toxus (Coninted)}

Colomin City of

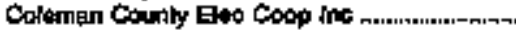

Colinge Station City of

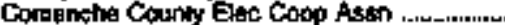

Conchid Velly Eled Coop mo

Cooke Counth Elec CoOp Agsi

Guero city of .

Oust Smith Electsto Coop inc -

Deeo Epor Teras Elex Coop lac

Dention City of

Oenton County Eles Coop Ine

Dewith Elactric Cocp lne

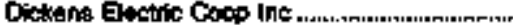

G Peso Electrte Co

Electra $C$ thy of

Eralh Count Elac Coop Asten

Fannin Couml Electric Goop

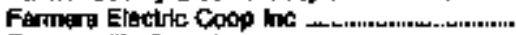

Farmartille $\mathrm{CH}$ of

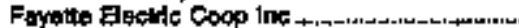

Falosip City of

Floresile Ciny of

Flondedes City al

Fort Betiones Enctic Coop the ......................

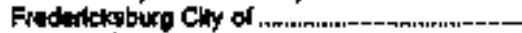

Gertand Cay of

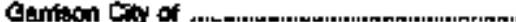

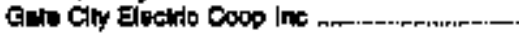

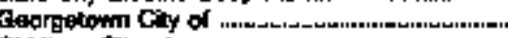

elidalinge $C$ aty of

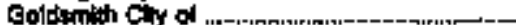

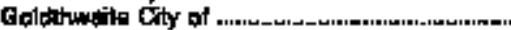

Gonzalea City of

Cranbury City of

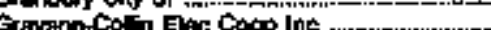

Bresiball Electure Coop ino

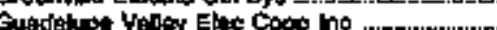

Gull suiges Utitiog Co

Helleitswifie Gity or

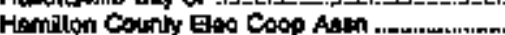

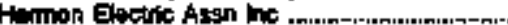

Hearne City of

Homphili Clity of ...

Pienpeatead Cay of

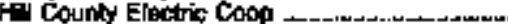

Honds Cry of ...

How

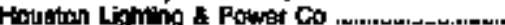

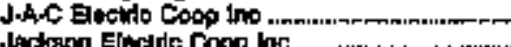

Jandegon Elactrie Coop bro

Jaspar City of

tator. Ntavton geo Coop ho

Johusson Courty Glec Coop Aver , .

Kemed Elevitit Cosp ine

Keutmen Courth Eloo Coop ho

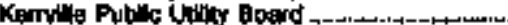

Kimbla Electte coop he

Kitywllo Llobe \& Power Co

Le Grenge Chy of

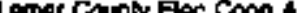

Lamb County Elotino Coop Inc -

Lampas:as City ol

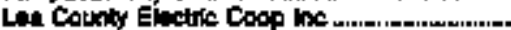

Leningtor Chy ol

Itinity City of

tot thous slocito $\cos$ ine

Unhoulon thy of
Pudilaty Oimed

Cooperaltwe

Putillely Owined

Cooperation

Cooperpilve

coopertily

Pubilly Owned

Cocoperativo

cooperathe

Puslety Owised

Cocpentive

Cooperative

Cooperativin

involor-Oined

Publicty Owned

Cooperature

Cooperative

cooperalme

Pubthy Owned

cooperalivi

Pubucty Ormed

Publuty Ombed

Publicty Oined

Cooperaltve

Puticly Omed

Publich Omed

Publucty Omned

Cooperalive

Putblicty Owned

Putulaty Onwed

Putricty Dimed

Publich Oined

Putllely Owined

Publichy Ownad

Cosperative

Cooperaline

Putlicty Owined

Cocperative

mpestor-Ommed

Pubsely Owned

Cooperateve

Cooperatha

Publest Owned

Publety Owned

Publlety Ownad

Cocontrative

Fublicly Owned

Cooperylute

invatoronened

Coopertitwe

Cocperallye

Pubticty Omed

Coopernitue

Cocoperature

Cooparalu

Cooperalue

Putbety Onmed

Coopurature

Putedlely Owried

Publoly Owined

Cooparative

Cooperalive

Publithy Owitud

Gooporathe

Puthly ty Owined

Putllaly Owned

Cocoerstus

Problesy Owined

\section{2,437}

5.164

18,469

10,497

6.203

6,205

2,691

4,530

29,376

25,099

29.753

6,677

2,599

191,010

1,041

14,778

6,386

22,202

1.137

B, 526

731

6.955

1,016

2405

3,703

56,860

435

754

?,001

1.700

115

02

2,369

1,433

13,418

2,810

9,879

27,7e5

261, 9055

1,276

10,440

220

1,256

467

1,575

10,484

2,200

14,138

$1,271,306$

2871

10 A

3,400

16.162

25,100

9.820

21,869

$\$ 3,67$

3,741

722

2260

7.669

2.834

2,978

1,002

404

2,701

3,361
1,546

2,771

14,185

7.104

4,436

5,900

2067

2. तre

24.441

21.703

36,739

4,659

1,065

112,165

120

13,791

a, 003

25, 7 er

903

512

8,508

495

1.과묭

2,129

67.805

415

699

6,456

1.281

113

1.891

1,75

15.480

1.772

1,274

26,813

280,800

752
6,856

62

1,539

400

1,122

10980

1,052

13,190

$1,579,176$

2,535

8,400

$\$, 091$

17,010

28,036

8,732

24,701

11,898

2,719

763

1,622

B,811

1,757

2,570

\$0.

3,260

2082

2,550

18.75:

33,302

202408

83.5त

53.044

104.700

31,869

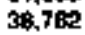

a96, 710

289.160

476,484

40,772

11,127

1,069,037

10,422

179,114 
Table 14. Clase of Ownerahy, Number of Uhimate Conaumers, Revenue, Salen, and Average Revenue per Kliowatthour for the Redidential Sector by State and Utility, 1993 (Continuod)

\begin{tabular}{|c|c|c|c|c|c|}
\hline 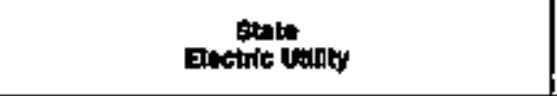 & $\begin{array}{c}\text { otad } \\
\text { of } \\
\text { omennithip }\end{array}$ & 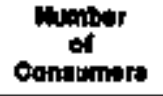 & 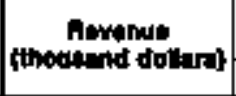 & 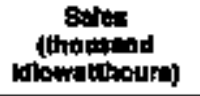 & 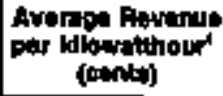 \\
\hline 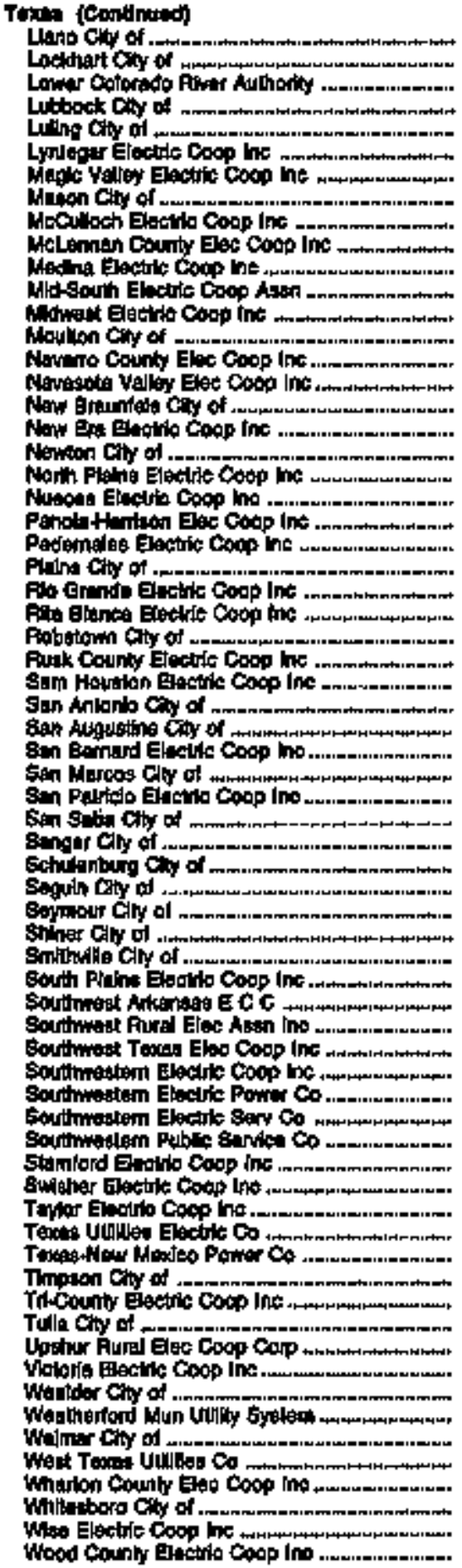 & 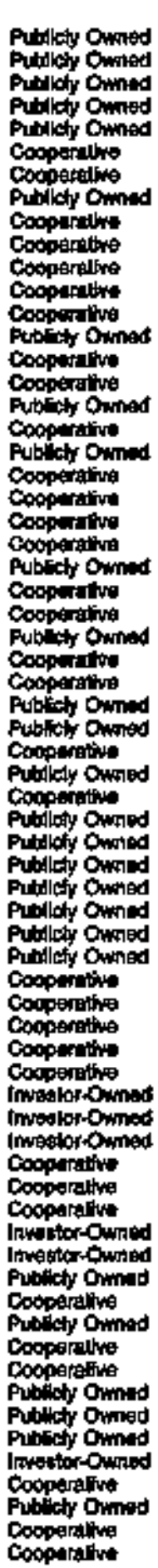 & 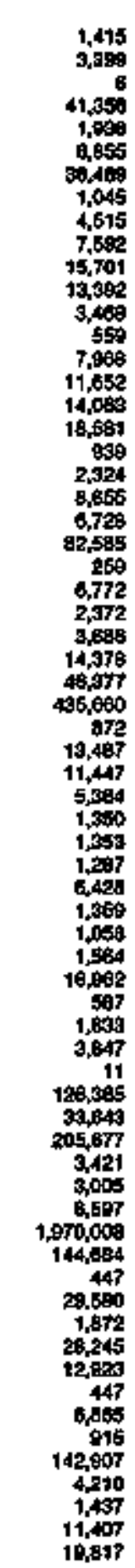 & 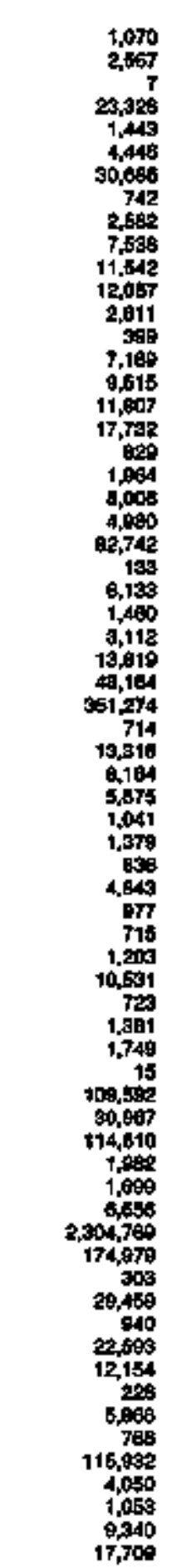 & 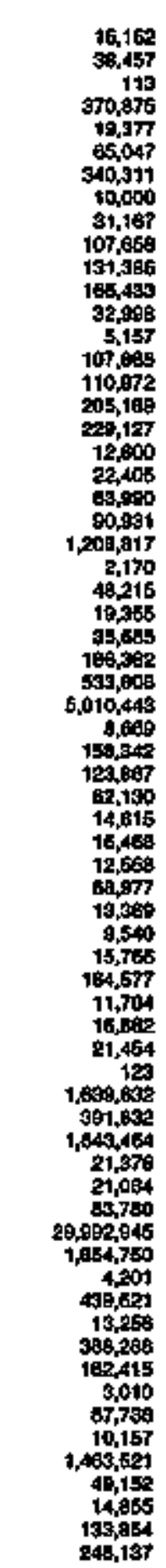 & 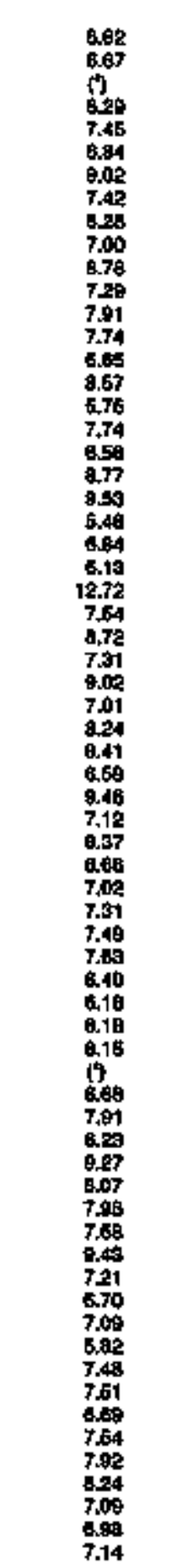 \\
\hline
\end{tabular}

Soe footnoles of end of tabla. 
Table 14. Class of Ownership, Number of Uttimate Consumers, Revenue, Sales, and Average Revenue per KHowatthour for the Residential Sector by State and Utility, 1999 (Continued)

\begin{tabular}{|c|c|c|c|c|c|}
\hline $\begin{array}{l}\text { Etate } \\
\text { Elociric tedulty }\end{array}$ & $\begin{array}{l}\text { Clags } \\
\text { of } \\
\text { Ownerantp }\end{array}$ & $\begin{array}{l}\text { Number } \\
\text { of } \\
\text { Conounert }\end{array}$ & $\begin{array}{c}\text { Aewenoe } \\
\text { (thotsentd dollaros) }\end{array}$ & 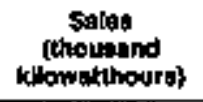 & $\begin{array}{l}\text { Avarage Revenua } \\
\text { pur latownathour' } \\
\text { (cants) }\end{array}$ \\
\hline 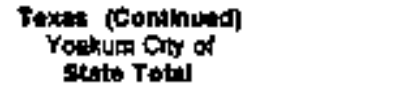 & Publucty Owned & $\begin{array}{r}2,510 \\
5,029,036\end{array}$ & $\begin{array}{r}1,968 \\
7,017,263\end{array}$ & $\begin{array}{r}24,497 \\
\text { का,698,723 }\end{array}$ & $\begin{array}{l}805 \\
\text { g.00 }\end{array}$ \\
\hline 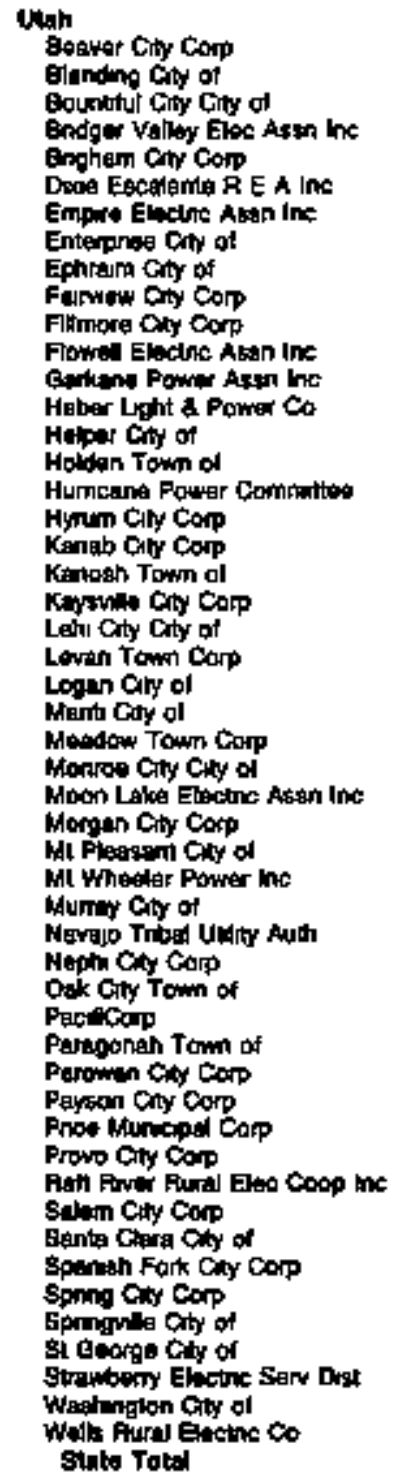 & 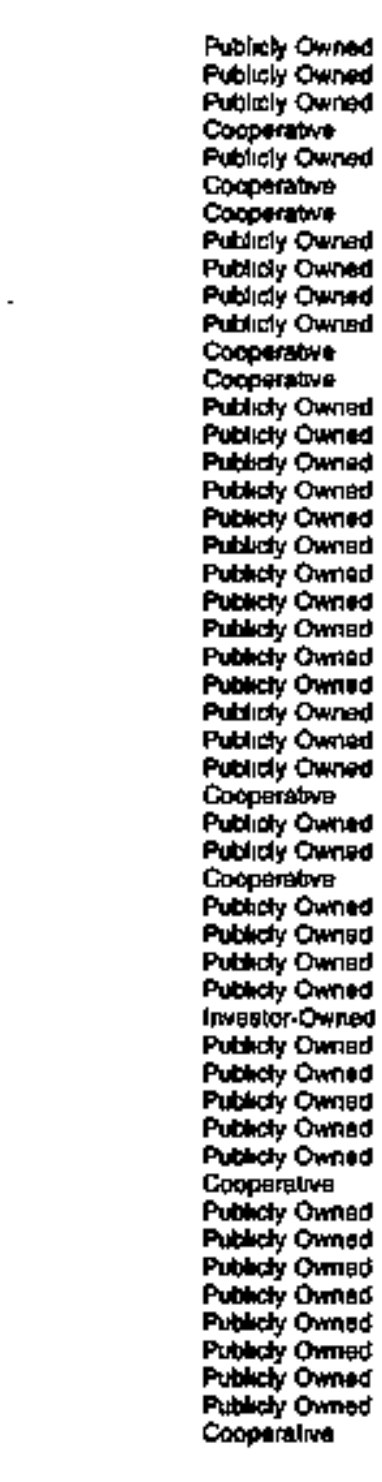 & 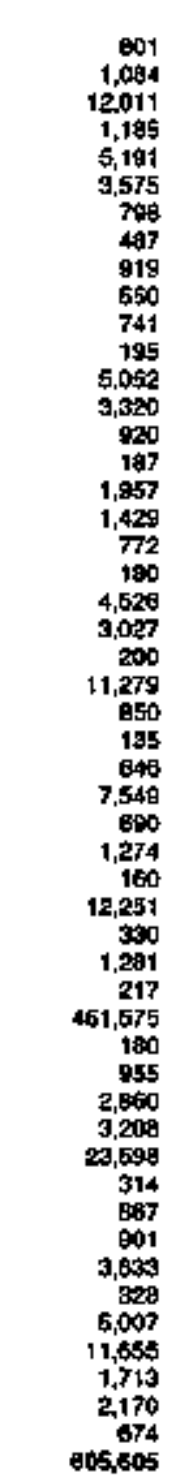 & 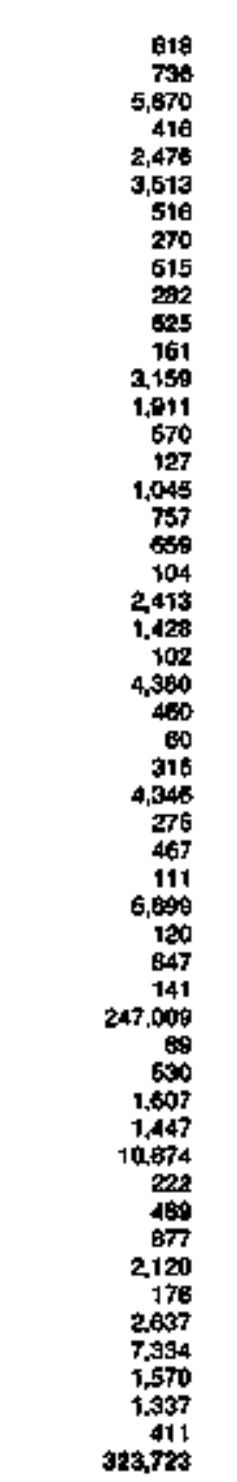 & 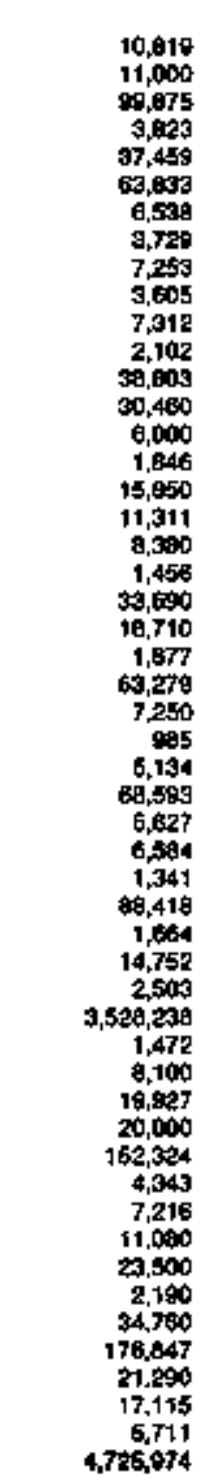 & 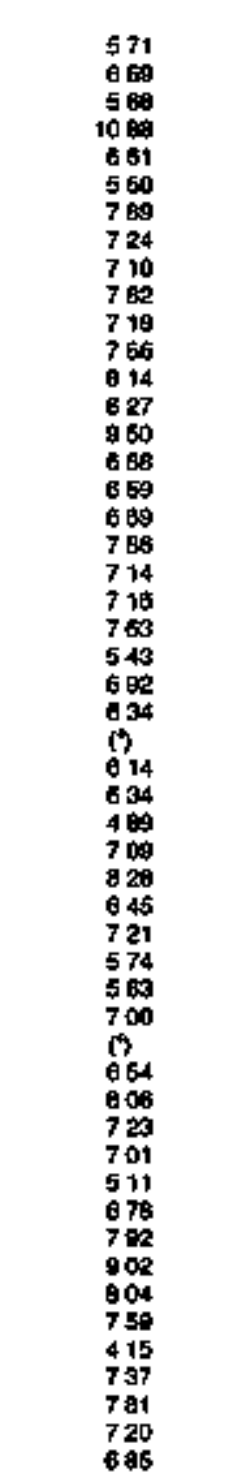 \\
\hline 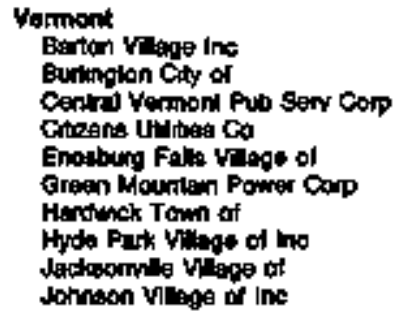 & 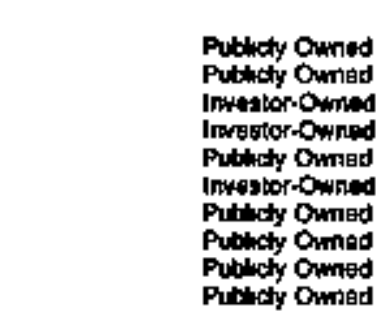 & $\begin{array}{r}1,609 \\
1+, 902 \\
1+1,637 \\
17,021 \\
1,213 \\
67,094 \\
3,362 \\
046 \\
546 \\
\$ 67\end{array}$ & $\begin{array}{r}778 \\
7.451 \\
00,789 \\
11,476 \\
1,004 \\
40,390 \\
2,244 \\
645 \\
345 \\
126\end{array}$ & $\begin{array}{r}10,610 \\
65,271 \\
876,920 \\
114,068 \\
11,539 \\
657,006 \\
21,660 \\
8,002 \\
3,006 \\
5,029\end{array}$ & $\begin{array}{r}711 \\
075 \\
1036 \\
986 \\
670 \\
087 \\
1027 \\
032 \\
1126 \\
722\end{array}$ \\
\hline
\end{tabular}

See footrotien of and of tablo 
Table 14. Class of Ownership, Number of Ultimate Conwumers, Revenue, Sales, and Average Revenute per Kllowatthour for the Rebidential Sector by State and Uallity, 1998 (Continued)

\begin{tabular}{|c|c|c|c|c|c|}
\hline 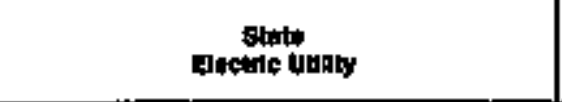 & CAnch & $\begin{array}{l}\text { Nuphor } \\
\text { of } \\
\text { Consumers }\end{array}$ & $\begin{array}{c}\text { ftowenus } \\
\text { (thoustand belitert) }\end{array}$ & 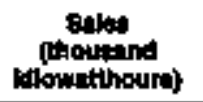 & 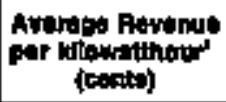 \\
\hline 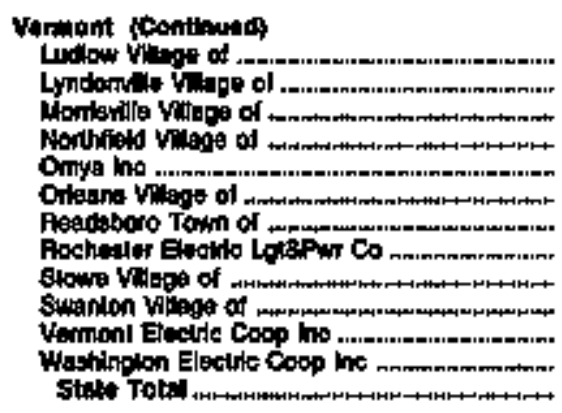 & 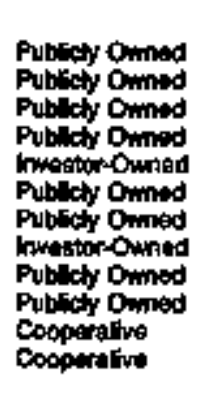 & $\begin{array}{r}2,783 \\
4,010 \\
2,690 \\
1,507 \\
004 \\
600 \\
267 \\
667 \\
2,467 \\
2,670 \\
13,204 \\
6,388 \\
206,242\end{array}$ & 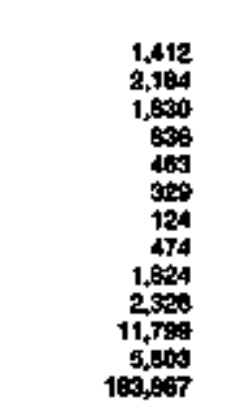 & 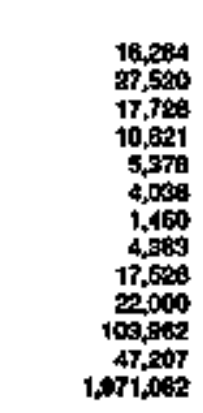 & $\begin{array}{r}8.67 \\
7.64 \\
10.38 \\
7.87 \\
0.81 \\
6.18 \\
6.49 \\
10.81 \\
10.41 \\
70.57 \\
11.35 \\
12.28 \\
0.94\end{array}$ \\
\hline 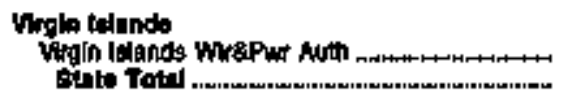 & Puthely Owned & $\begin{array}{l}39,710 \\
39,710\end{array}$ & $\begin{array}{l}25,434 \\
26,494\end{array}$ & $\frac{22 t, 449}{221,443}$ & $\begin{array}{l}11.94 \\
11.94\end{array}$ \\
\hline 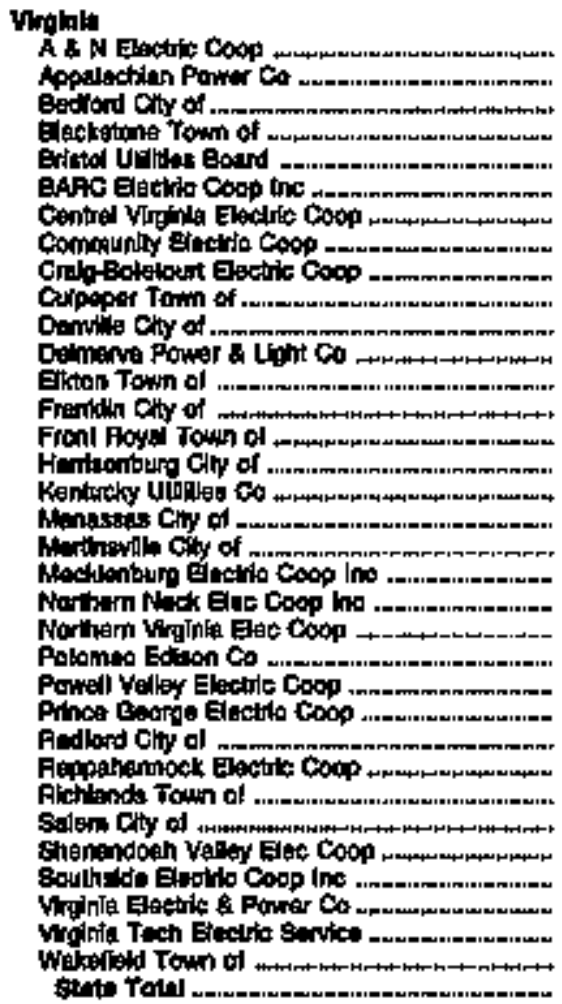 & 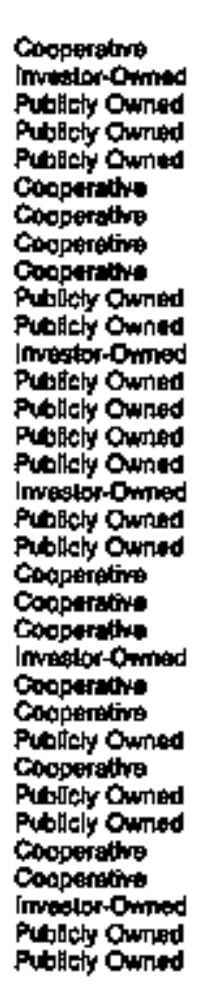 & 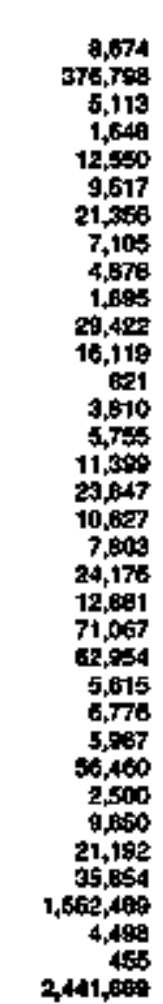 & 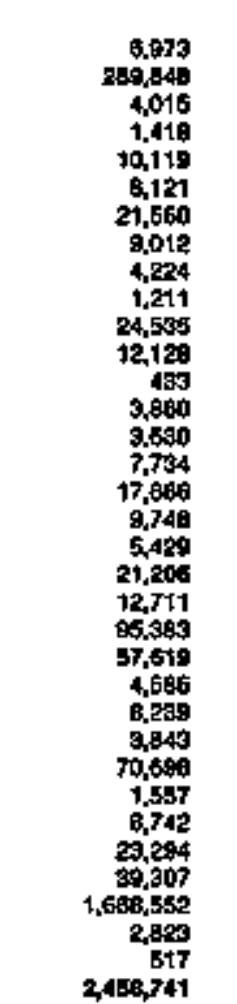 & 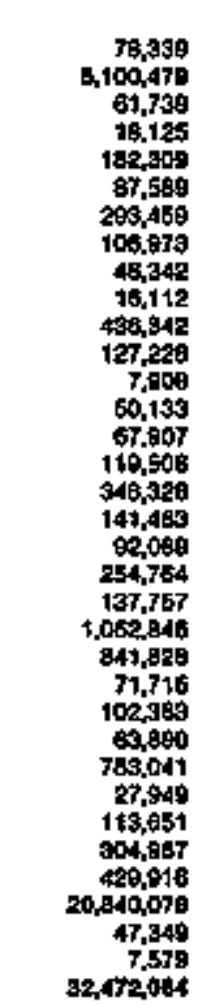 & 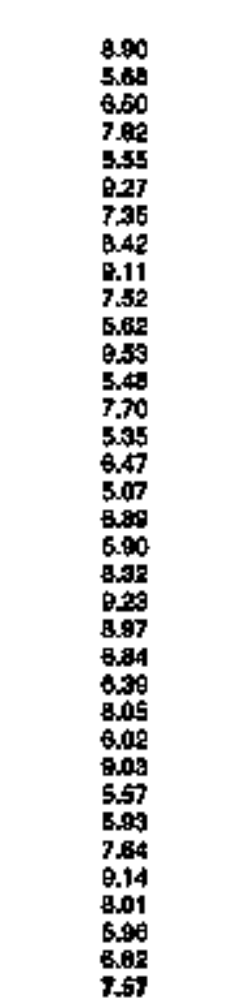 \\
\hline 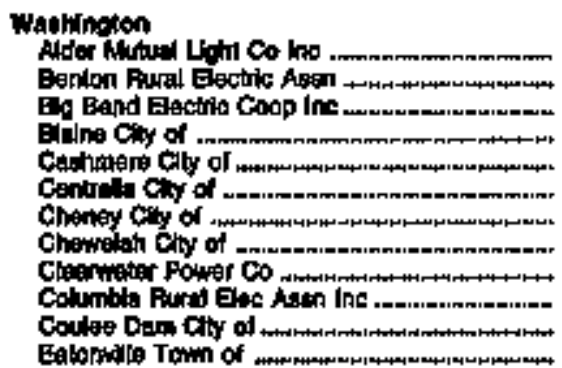 & 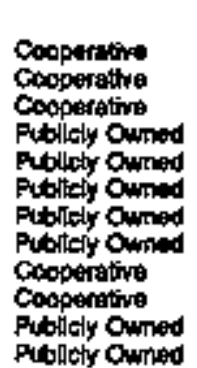 & $\begin{array}{r}176 \\
7,209 \\
3,928 \\
1,443 \\
934 \\
7,077 \\
2,779 \\
983 \\
5 \% 1 \\
1,777 \\
509 \\
607\end{array}$ & 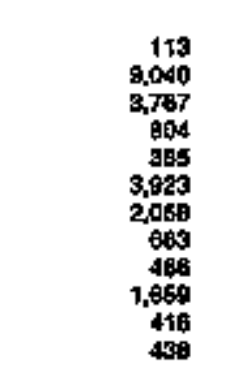 & $\begin{array}{r}2,409 \\
15,000 \\
84,087 \\
18,510 \\
20,009 \\
113,961 \\
43,410 \\
12,396 \\
8,769 \\
32,586 \\
11,985 \\
9,289\end{array}$ & $\begin{array}{l}4.09 \\
5.72 \\
4.43 \\
4.34 \\
1.92 \\
3.45 \\
4.76 \\
5.49 \\
8.98 \\
5.09 \\
3.05 \\
4.72\end{array}$ \\
\hline
\end{tabular}

Sos footnoleg at end of table 
Table 14. Class of Ownershlp, Number of Ultimate Consumers, Revenue, Salos, and Average Revenue per Kllowatthour for the Residential sector by State and UtIllty, 1993 (Continued)

\begin{tabular}{|c|c|c|c|c|c|}
\hline $\begin{array}{l}\text { Shple } \\
\text { Eecirle totury }\end{array}$ & $\begin{array}{c}\text { Clga: } \\
\text { of } \\
\text { Owneromp }\end{array}$ & $\begin{array}{c}\text { Number } \\
\text { of } \\
\text { Conoumars }\end{array}$ & $\begin{array}{c}\text { Revwenue } \\
\text { (Theubant dollira) }\end{array}$ & $\begin{array}{c}\text { 9alan } \\
\text { (thoustand } \\
\text { kdowathours) }\end{array}$ & $\begin{array}{l}\text { Average 月ovenua } \\
\text { por kilowallhour" } \\
\text { (cents) }\end{array}$ \\
\hline
\end{tabular}

Waghincton toontipued)

Eilansburs Ciny ol

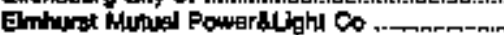

Fritutil Clty of

Inlend Powe s ingl Co.

Kontensal Electris Cout

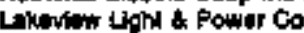

Linodin Elecink Coop Int

Milion chy of

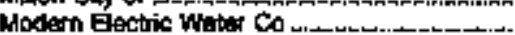

Neapalam Vallow Elog Coop tno .....................

Northam Unhts Inc.

Ohap Mutudid Lلdgh Co Inc

Okinogun Courty Elow Coop inc

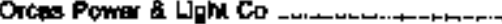

Pucticorp .

Perkland Ligh \& Water $\mathrm{C}$

Pecinsula Lijt co

Punt Sound pone

UD 1 of chion County

Pul No 1 or Cinlom Count

PUD No 1 의 Clati Counky

PUD No 1 of Cowlik Caumb

PUD No 1 of Dounda: Counbr

FuD No 1 of Ferry County

PUD rot 1 of Frantes Counly.

PUD No 1 od Kotaliag Gounty

PUD No t of Kacklal cotsnty

PUD No 1 of Letws Coesnby .

FUD to $t$ of Hason County

PUD Nis 1 of Ckinogen Coumb

PUD No $x$ of Pend Orime Cniny

FuD rlo 1 of \$kamania County

PUD kit 1 of Snohomish Colnty

PLO No i of Whridation Coun

PLO No 2 of Pretsc Counly.

PLO 3 of Maron Connty

Fichiland Gity of

Buston Town of

seaptita cly of

Stollacoln Town of .

Sumas tity of .

Tncomi Cly ol of

Tenner Electrlt Gaxp

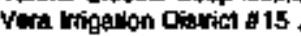

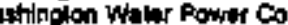

Tom of

Btate Total

Wọnt Vireinis

Apoalachian Powar co

Giack Oimond Pown Co ............................

Craig-Botalourt Electrite Coop

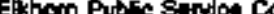

Harrogn Fared Elac Apin ling

KGmbel Litht \& Waler Co

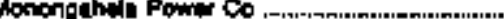

Mew Wertinowite Cly of

Finlpeis cisy of .

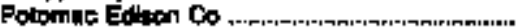

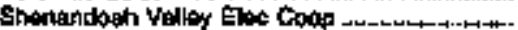

Undon Pown co

Uhited U, inl \& Fowar Co
Puhichy Owited

Cooperalue

Puldichy Owrepd

Cooparative

Cooperallwe

Cooperation

Cooparsilw

Publicty Omated

Pubichy Cimed

Coppartive

Cooperalive

Cooparalivo

Cooperaltre

Cooputally

Cooperellve

Invetions-Owhis

Cooperallu:

Copparpltre

Pubicky Omed

Invester prured

Pubficty Owand

Publlely Ownat

Publkly Owrad

Publlaly Owind

Putlaly Ownad

Putlicly Owied

Pubtioly Omined

Putilicty Ommed

Publicky Owinad

Puturich Omined

Pubsty Omided

Putscly Comiad

Pubsich Ormed

Putict, Omad

Putchly Owned

Putrictr Omed

Pubictr Omad

Pubraty Ommed

Pubticky Oimed

Putichy Omad

Pubicity Comed

Publicty Otmed

Publictr Omod

Pubrich Othed

Pubcely Omind

Pubficty Omed

Putricky Owised

Cooperalirs

Putilcty Ownod

Intestor-Owhed

Publieky Owned

Inwoglor-owned

Irwetilar-Owmed

Cocpentlve

Irvoglor-Owned

Irwegicar-Ownted

Cocperalve

Irwegtor-Ownod

Investor Owher

Pubtecy Oimed

Pulaticy Orined

Invertor Ouned

Cooperathe

Irresturolowind

Itwenter-Owarted
4.5600

10,2015

$20: 0$

21.40

37
6,415

t.679

320

2,261

6.523

1,084

2537

047

7.772

01.291

3.0日8

19.27s

7,968

706,123

29,250

26,978

21,119

107,700

3. 159

11.210

2,172

12084

3074

1,60

7.2600

19.51

$3, \mathrm{~B} 79$

14.069

5,821

3.80 :

189.979

1.560

25,004

12 d2a

21.8B7

14,106

$\$ 60$

00,030

2,440

349

121,979

230?

612

168,630

2,135,400

546.200

1.412

341

1,004

165
4,715

\$31

272,979

1.442

1,958

7038

$1.4+5$

1294

960
2.558

7,265

1.065

22,303

3,901

1.79

603

1,433

s.a21

090

11
2,251

70

7,203

68,315

1,976

18,970

5,990

511.980

25.185

12,450

17.229

78,504

18,650

4 너

1,038

11.099

22.712

1.784

5.651

13.886

2 的

7,277

3,040

2, 981

139.048

1.275

14,647

7,498

14,5e日

13,680

225

121,448

1,494

184

7.800

7.6B?

4,706

1 10, s52

$1,422,168$

200,571

764

196

牙要

124
3,113

250

171,318

694

775

65,012

1,501

632
674

55.877

190,078

34.647

392771

762

123,513

27,478

13,675

35,033

87.455

18,9:5

172

45.168

15,172

96.516

$1,499.701$

49,111

305,105

138, 138

$0,070,924$

619.8ds

617,528

344333

1,864,005

701.732

298,443 
Table 14. Clags of Ownership, Number of Ulitimate Consimers, Revenue, Sales, and Average Revenue per Kilowatthour for the Residential Sector by State and Utillty, 1998 (Continued)

\begin{tabular}{|c|c|c|c|c|c|}
\hline $\begin{array}{c}\text { stalo } \\
\text { Eloctidt Whaty }\end{array}$ & $\begin{array}{c}\text { CHats } \\
\text { of }\end{array}$ & $\begin{array}{c}\text { Number } \\
\text { of } \\
\text { Consumets }\end{array}$ & Rarrefore & 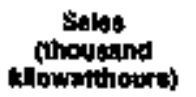 & 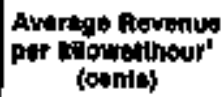 \\
\hline 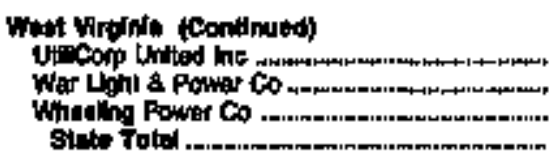 & 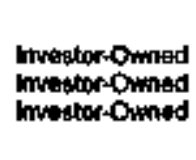 & $\begin{array}{r}21,502 \\
780 \\
38,052 \\
77,020\end{array}$ & $\begin{array}{r}15,940 \\
527 \\
24,329 \\
547,006\end{array}$ & $\begin{array}{r}199,612 \\
9,397 \\
360,098 \\
80.0937\end{array}$ & $\begin{array}{l}6.40 \\
5.61 \\
6.56 \\
6.50\end{array}$ \\
\hline 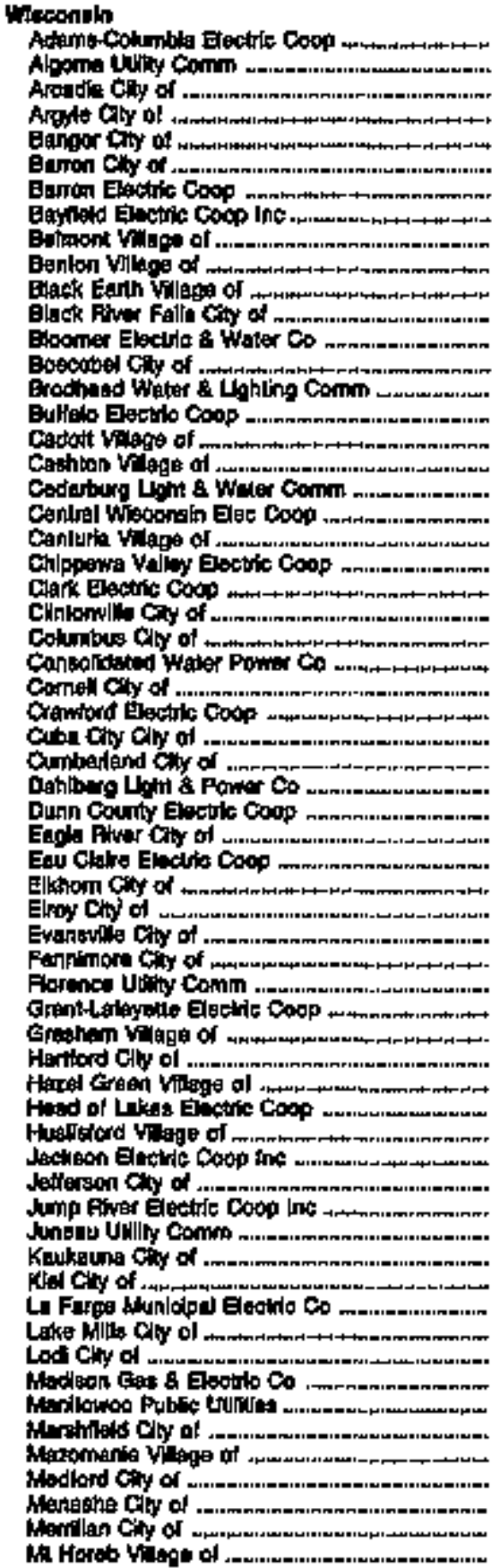 & 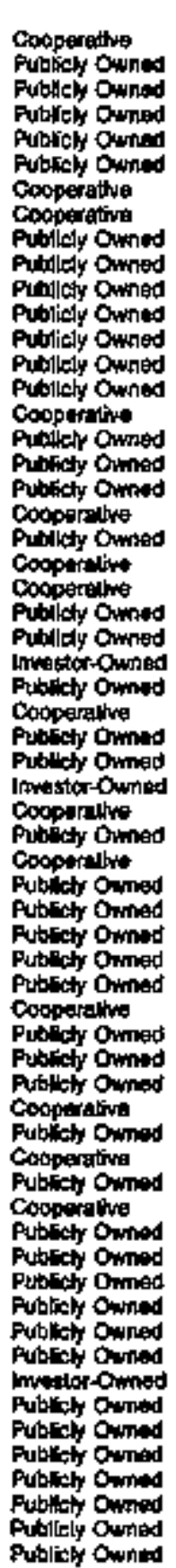 & 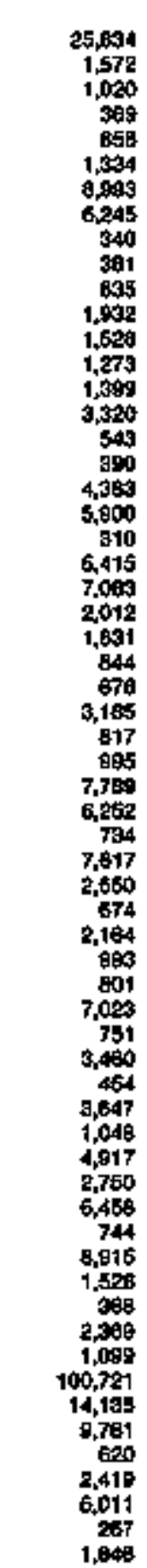 & 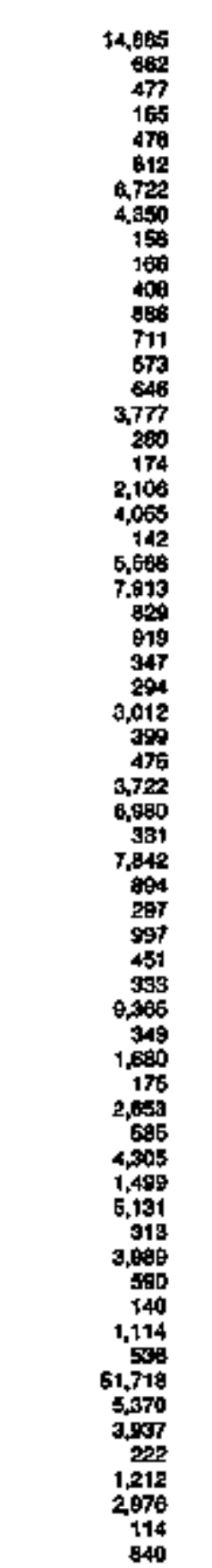 & 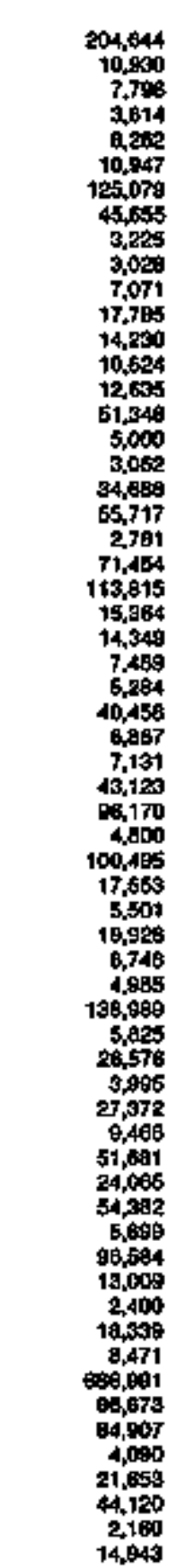 & 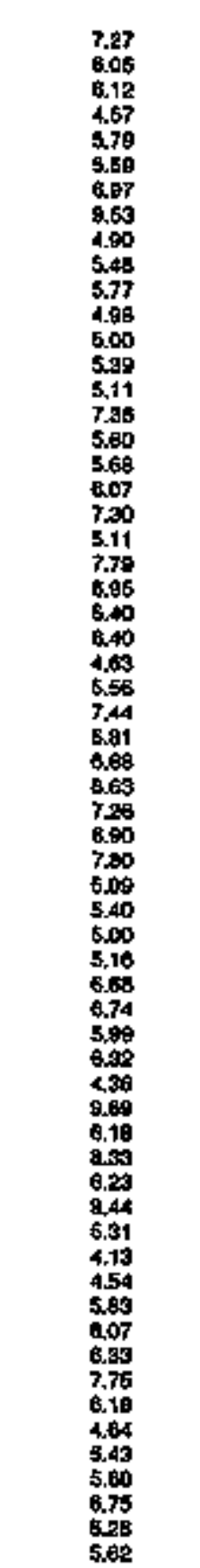 \\
\hline
\end{tabular}

Sea roornglas al and of tatto. 
Table 14. Class of Ownership, Number of Utimate Consumers, Revenus, Sales, and Average Revenue per Kllowathour for the Residentlal Sactor by State and Utllity, 1993 (Continued)

\begin{tabular}{|c|c|c|c|c|c|}
\hline $\begin{array}{c}\text { Stab } \\
\text { Elestrie toldty }\end{array}$ & $\begin{array}{c}\text { Clap: } \\
\text { of } \\
\text { Ownnerahtp }\end{array}$ & $\begin{array}{l}\text { Myspor } \\
\text { of } \\
\text { Conoumera }\end{array}$ & 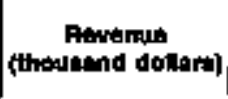 & 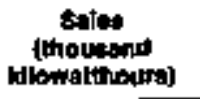 & 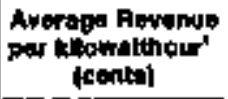 \\
\hline 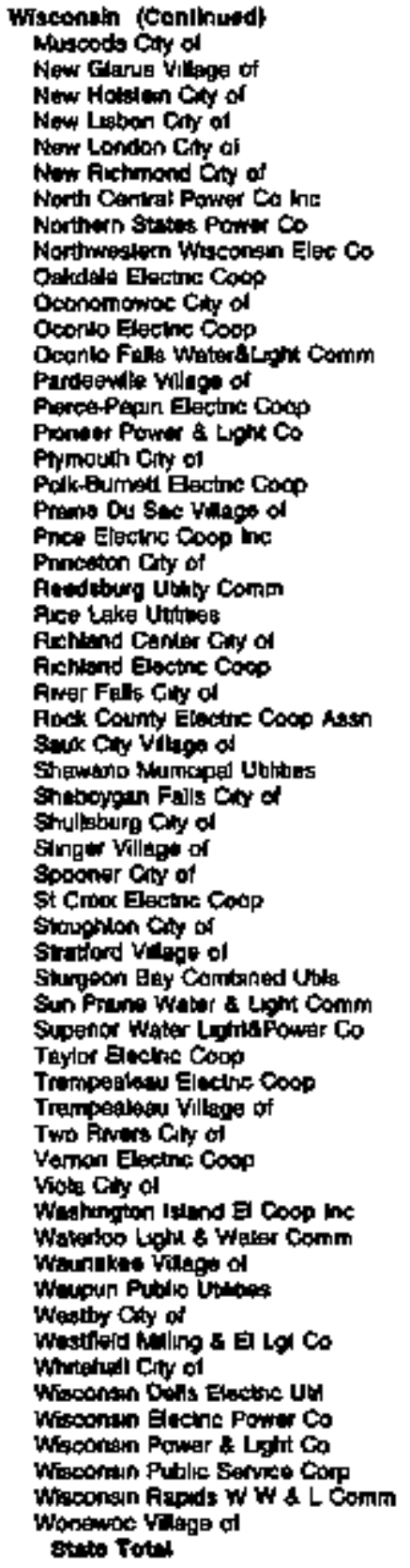 & 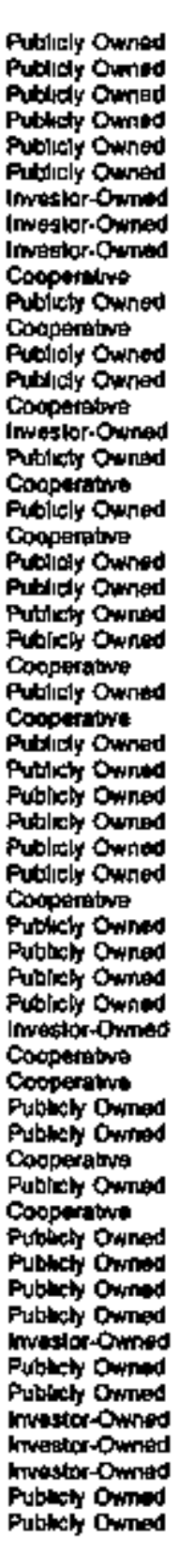 & 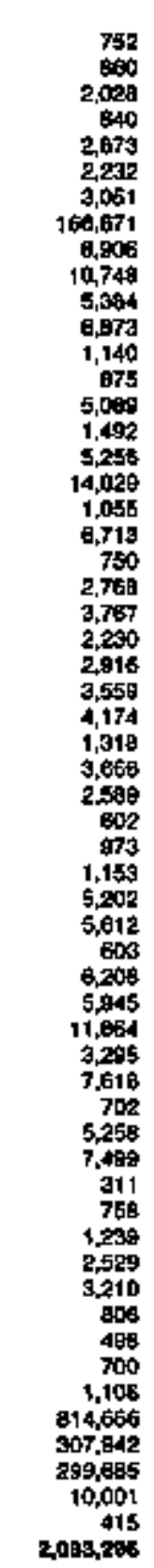 & 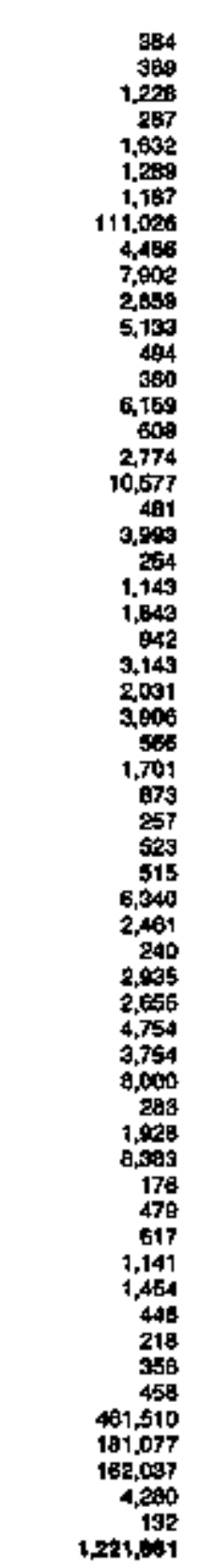 & 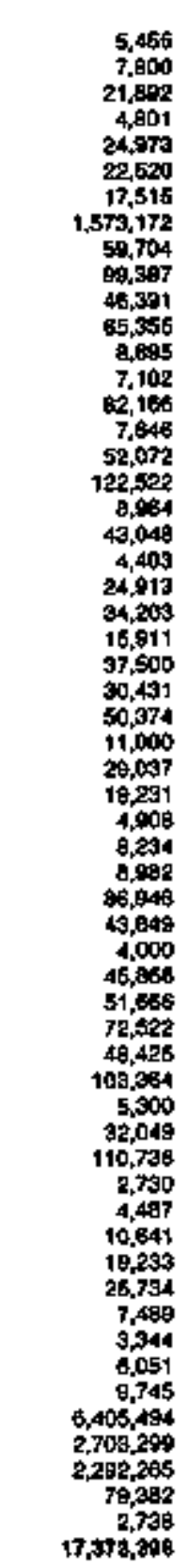 & $\begin{array}{l}704 \\
467 \\
565 \\
555 \\
654 \\
572 \\
678 \\
708 \\
748 \\
775 \\
616 \\
785 \\
555 \\
535 \\
750 \\
705 \\
533 \\
863 \\
537 \\
929 \\
577 \\
460 \\
539 \\
592 \\
839 \\
667 \\
775 \\
616 \\
566 \\
454 \\
524 \\
635 \\
573 \\
729 \\
561 \\
600 \\
640 \\
514 \\
696 \\
775 \\
774 \\
534 \\
608 \\
757 \\
645 \\
1069 \\
660 \\
593 \\
544 \\
599 \\
652 \\
569 \\
470 \\
720 \\
670 \\
707 \\
539 \\
402 \\
7.09\end{array}$ \\
\hline 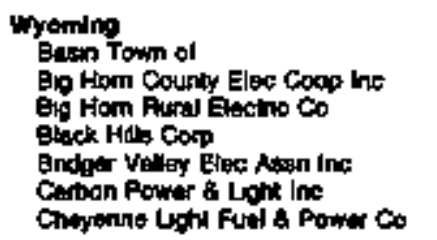 & 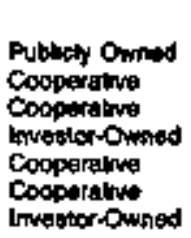 & $\begin{array}{r}481 \\
180 \\
1,810 \\
1,685 \\
2.740 \\
3.807 \\
27.797\end{array}$ & $\begin{array}{r}228 \\
146 \\
1,283 \\
1,166 \\
2,306 \\
2,702 \\
10,849\end{array}$ & $\begin{array}{r}3,201 \\
2,341 \\
18,412 \\
16,741 \\
26,805 \\
32,800 \\
1,00,200\end{array}$ & $\begin{array}{l}712 \\
632 \\
897 \\
090 \\
618 \\
627 \\
562\end{array}$ \\
\hline
\end{tabular}

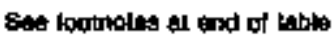


Table 14. Class of Ownershlp, Number of Uhtumate Consumers, Revenue, Sales, and Average Revenue per Kilowathour for the Residentlal Sector by State and Utility, 1993 (Continuted)

\begin{tabular}{|c|c|c|c|c|c|}
\hline Etest & $\begin{array}{c}\text { Clats } \\
\text { of } \\
\text { Ounturtalp }\end{array}$ & $\begin{array}{c}\text { Number } \\
\text { of } \\
\text { Ctontelatal }\end{array}$ & 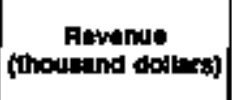 & 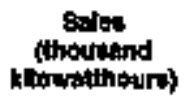 & 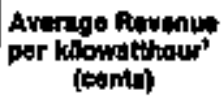 \\
\hline 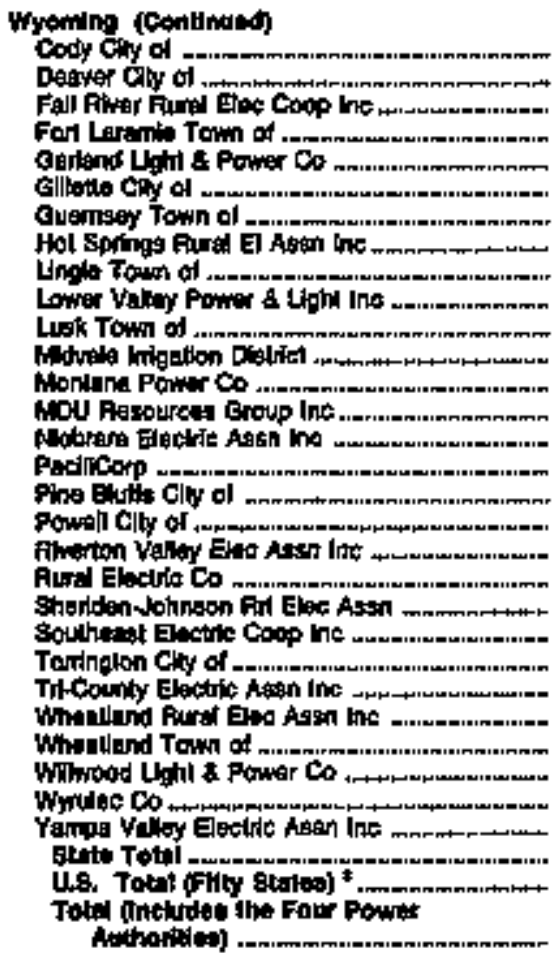 & 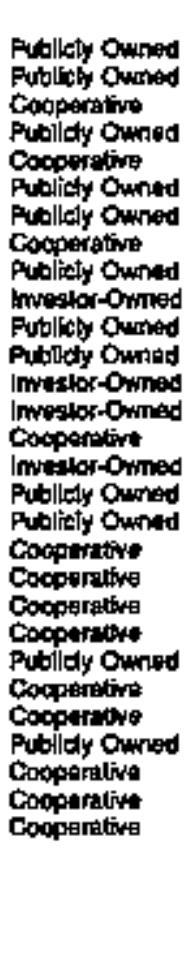 & 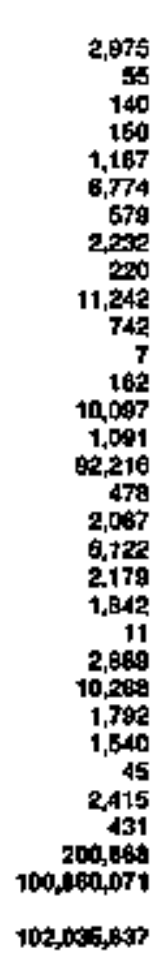 & 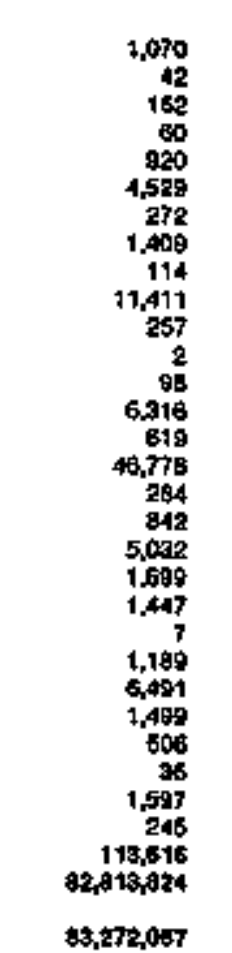 & 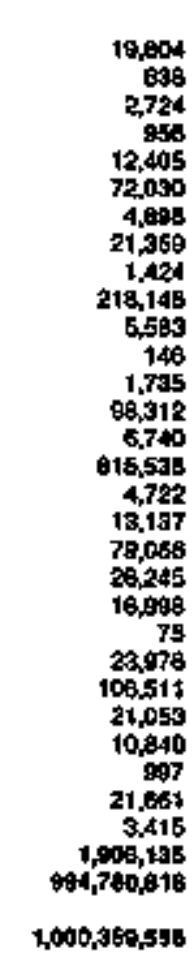 & 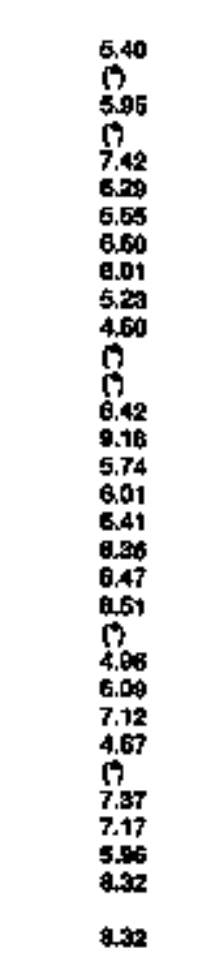 \\
\hline
\end{tabular}

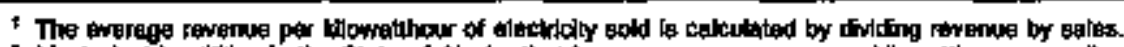

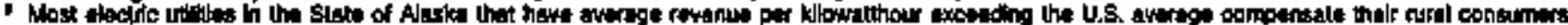

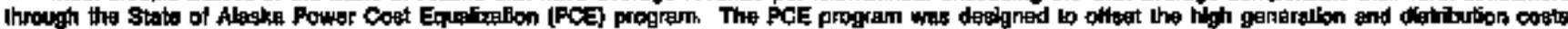

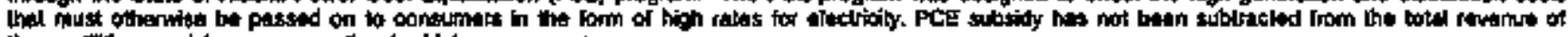

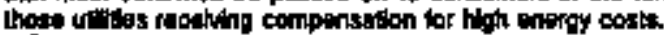

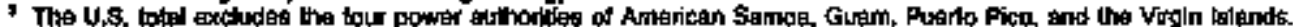

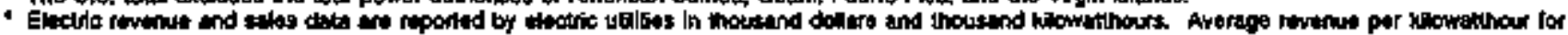

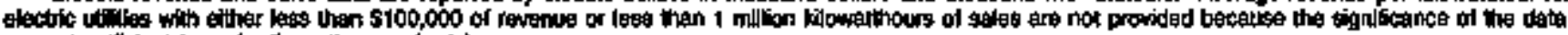

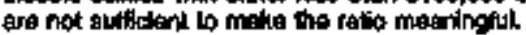

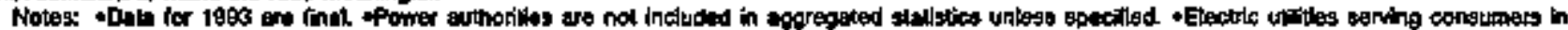
nore than one State gria llsted under each stata serwed.

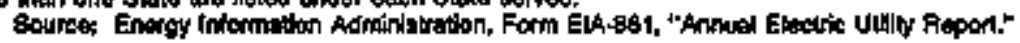


Table 16. Class of Ownership, Number of Ultimate Consumers, Rovenue, Sales, and Average Revenue per Kilowatthour for the Commerleal Sector by State and Utility, 1903

\begin{tabular}{|c|c|c|c|c|c|}
\hline $\begin{array}{c}\text { stals } \\
\text { clactrk Utity }\end{array}$ & $\begin{array}{c}\text { class } \\
\text { of } \\
\text { Dwmokthlp }\end{array}$ & $\begin{array}{c}\text { Mumber } \\
\text { of } \\
\text { Comsumers }\end{array}$ & [thousand dollert) & $\begin{array}{c}\text { Salat } \\
\text { (thouband } \\
\text { Idlonwtinours) }\end{array}$ & 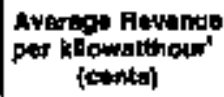 \\
\hline 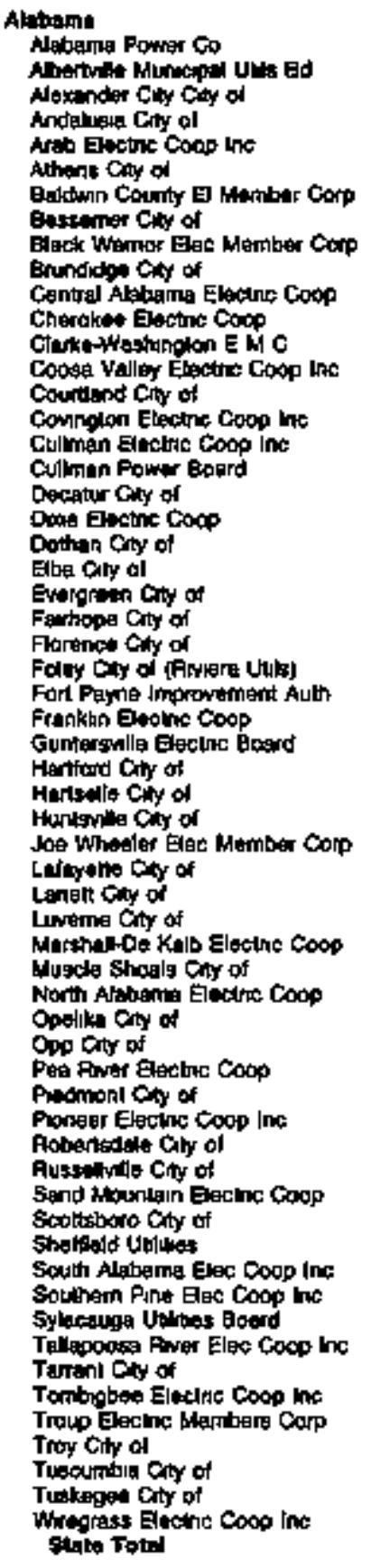 & 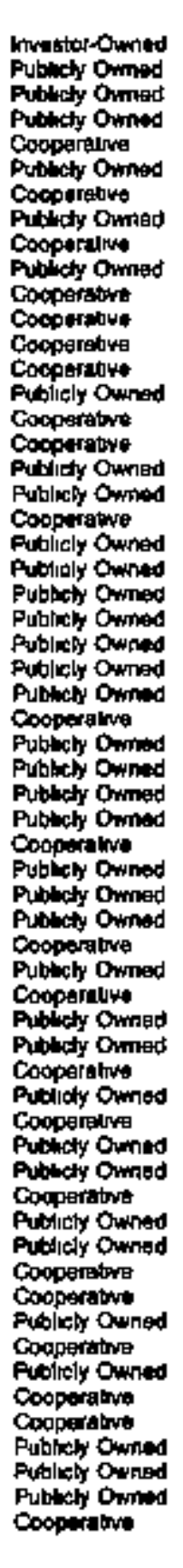 & 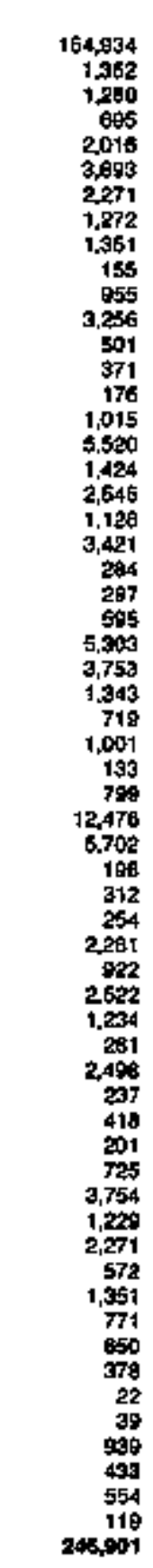 & 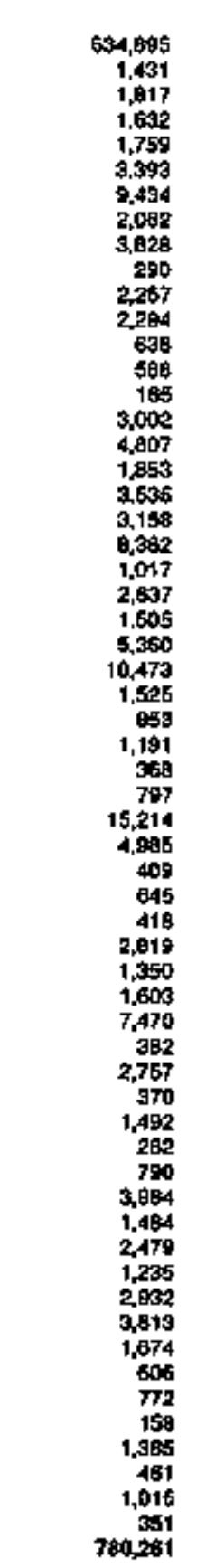 & 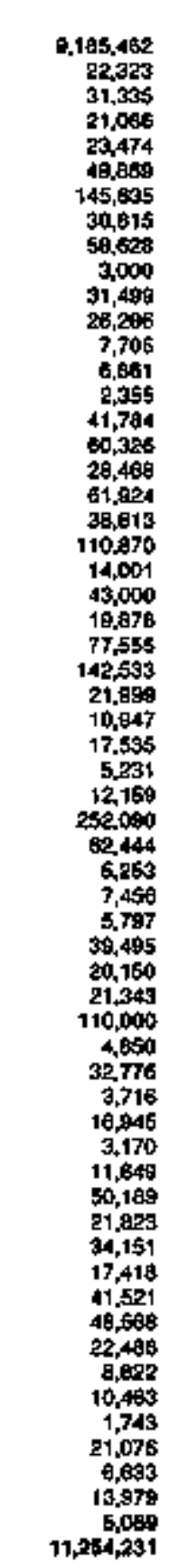 & 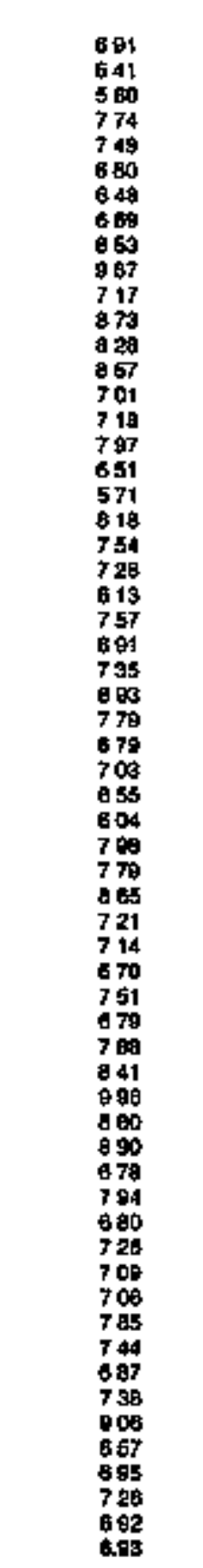 \\
\hline 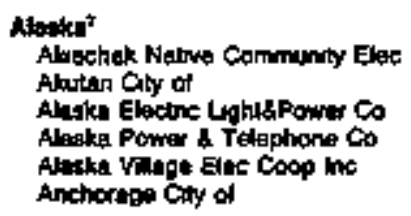 & 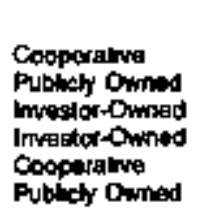 & $\begin{array}{r}29 \\
1 \\
1,198 \\
665 \\
395 \\
5,559\end{array}$ & $\begin{array}{r}118 \\
21 \\
2,078 \\
1,997 \\
2,173 \\
51,304\end{array}$ & $\begin{array}{r}232 \\
56 \\
98,083 \\
+1,915 \\
6,007 \\
648,679\end{array}$ & $\begin{array}{l}0 \\
6 \\
781 \\
1651 \\
3017 \\
702\end{array}$ \\
\hline
\end{tabular}

Beo foolnotes et end of bable 
Tabte 15. Class of Ownership, Number of Ultimate Congumers, Revenue, Sales, and Average Revenue per Kilowatthour for the Commerical Sector by State and Utillty, 1998 (Continued)

\begin{tabular}{|c|c|c|c|c|c|}
\hline 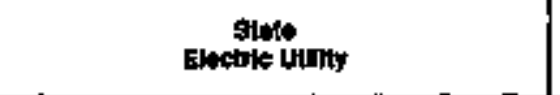 & $\begin{array}{c}\text { cinss } \\
\text { of } \\
\text { ommantiplo }\end{array}$ & $\begin{array}{c}\text { Number } \\
\text { of } \\
\text { Cogmingre }\end{array}$ & 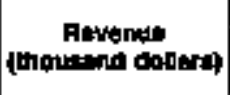 & $\begin{array}{c}\text { Bales } \\
\text { (thousand } \\
\text { kilomathoura) }\end{array}$ & 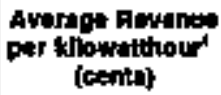 \\
\hline 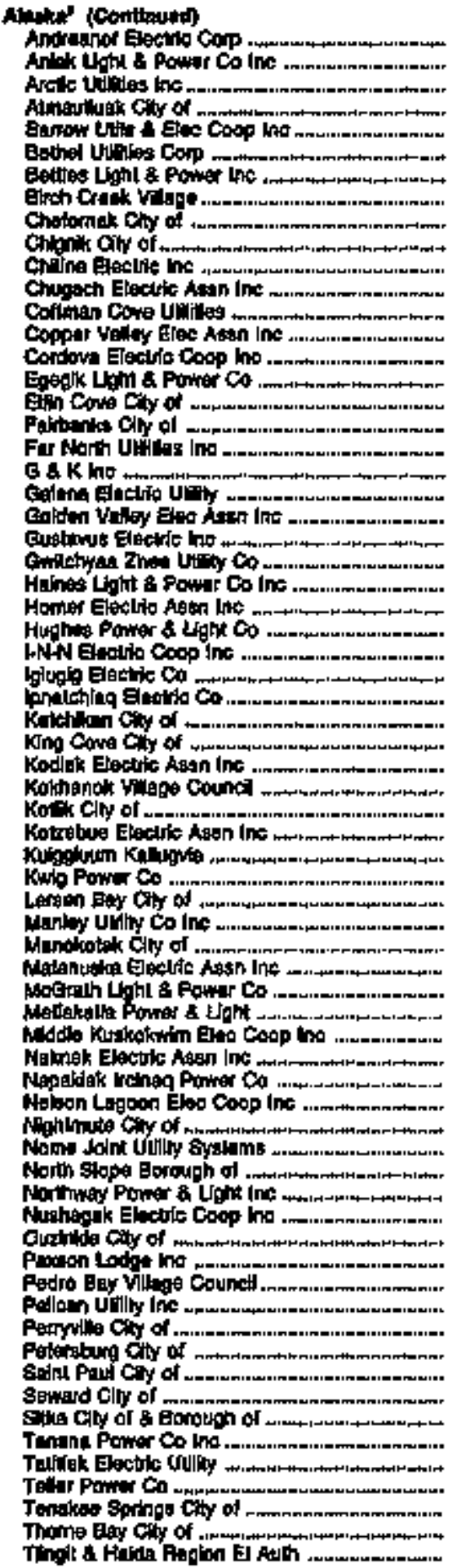 & 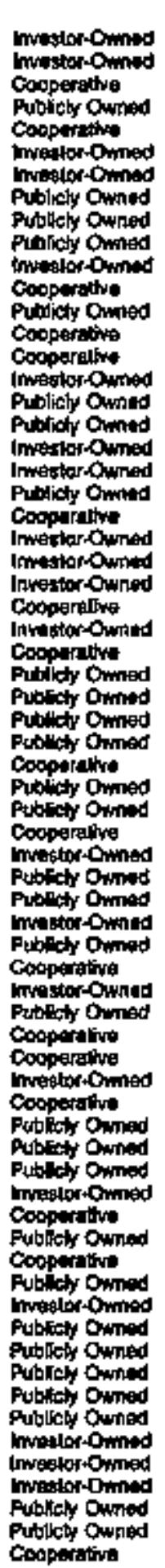 & 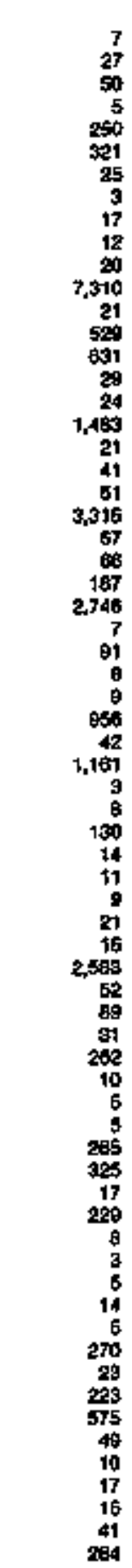 & 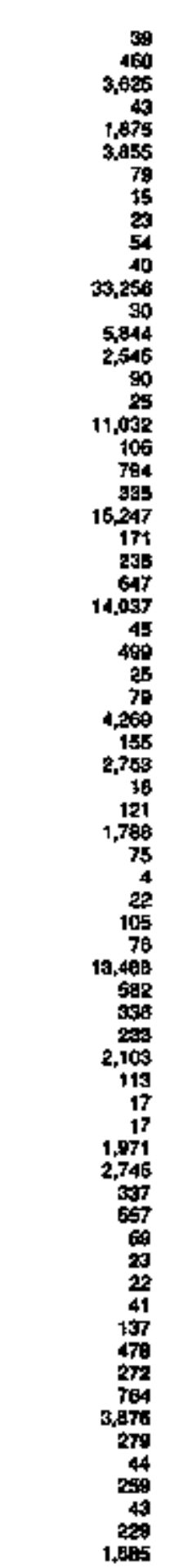 & 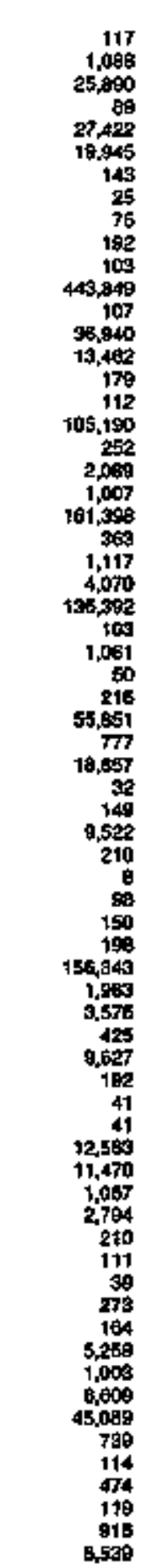 & 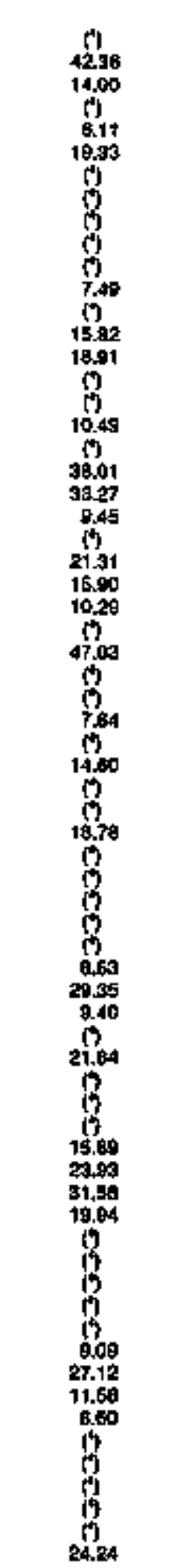 \\
\hline
\end{tabular}

see Iootnoleg at and of tabla. 
Table 15. Class of Ownership, Number of Uttimate Consumers, Revenue, Sales, and Average Revenue per Kilowatthour for the Commerical Sector by State and Utillily, 1993 (Continured)

\begin{tabular}{|c|c|c|c|c|c|}
\hline 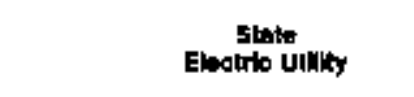 & $\begin{array}{l}\text { Gang } \\
\text { of } \\
\text { awrementp }\end{array}$ & $\begin{array}{l}\text { Number } \\
\text { of } \\
\text { Corpopinere }\end{array}$ & $\begin{array}{c}\text { Rewentwe } \\
\text { (thoustand dollerts) }\end{array}$ & $\begin{array}{l}\text { Ealow } \\
\text { (thouland } \\
\text { kllormathourty }\end{array}$ & $\begin{array}{l}\text { Averase Aominuma } \\
\text { per kllowathour' } \\
\text { (cents) }\end{array}$ \\
\hline 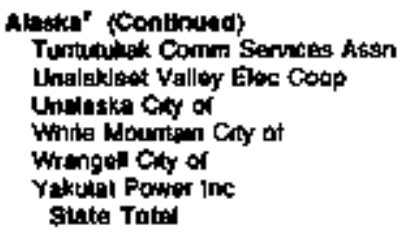 & 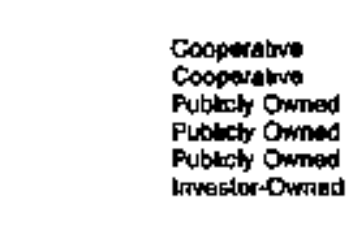 & $\begin{array}{r}5 \\
65 \\
87 \\
15 \\
377 \\
68 \\
33,376\end{array}$ & $\begin{array}{r}32 \\
498 \\
1,254 \\
99 \\
770 \\
607 \\
198,902\end{array}$ & $\begin{array}{r}69 \\
2,160 \\
6.909 \\
242 \\
8,109 \\
3,370 \\
2,062,399\end{array}$ & $\begin{array}{c}17 \\
2900 \\
19 \$ 7 \\
19 \\
950 \\
1979 \\
0.65\end{array}$ \\
\hline 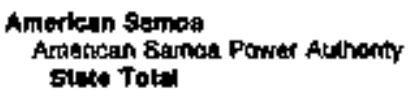 & Publiaty Dumid & $\begin{array}{l}695 \\
636\end{array}$ & $\begin{array}{l}1,798 \\
1,798\end{array}$ & $\begin{array}{l}9,769 \\
9,769\end{array}$ & $\begin{array}{l}18300 \\
10.30\end{array}$ \\
\hline 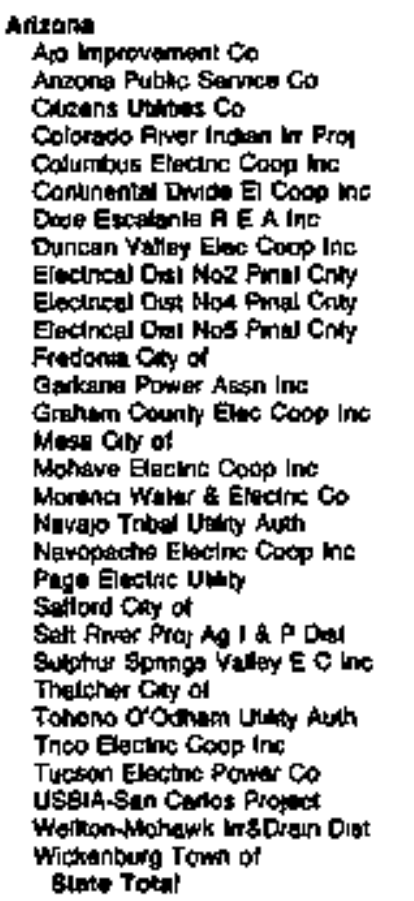 & 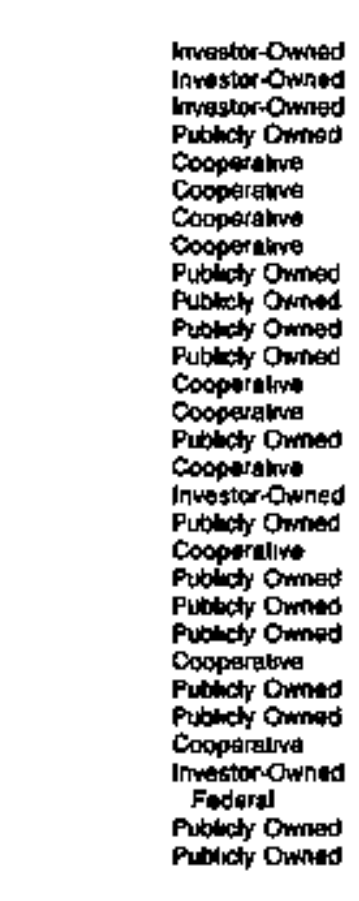 & $\begin{array}{r}114 \\
69,865 \\
6,300 \\
987 \\
33 \\
235 \\
81 \\
150 \\
277 \\
76 \\
75 \\
52 \\
140 \\
539 \\
2,051 \\
2,701 \\
446 \\
2,087 \\
2.281 \\
500 \\
510 \\
43,680 \\
5,501 \\
145 \\
371 \\
731 \\
26,839 \\
1,512 \\
538 \\
301 \\
158,227\end{array}$ & 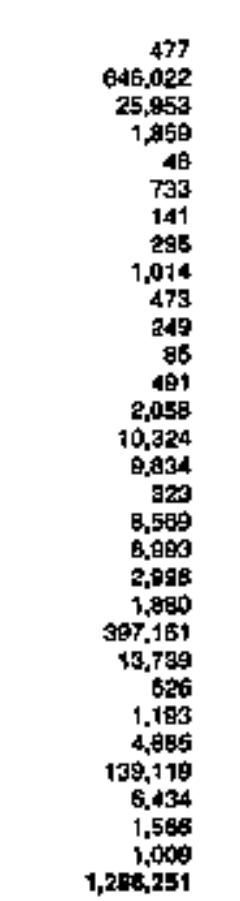 & 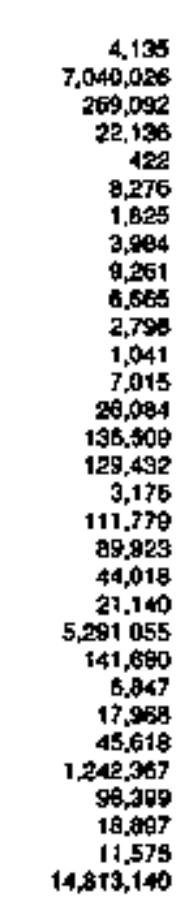 & $\begin{array}{r}1154 \\
918 \\
964 \\
840 \\
0 \\
986 \\
773 \\
740 \\
1095 \\
710 \\
891 \\
17 \\
700 \\
796 \\
758 \\
700 \\
1017 \\
767 \\
1000 \\
691 \\
399 \\
751 \\
870 \\
768 \\
664 \\
1027 \\
1120 \\
664 \\
820 \\
672 \\
1.70\end{array}$ \\
\hline 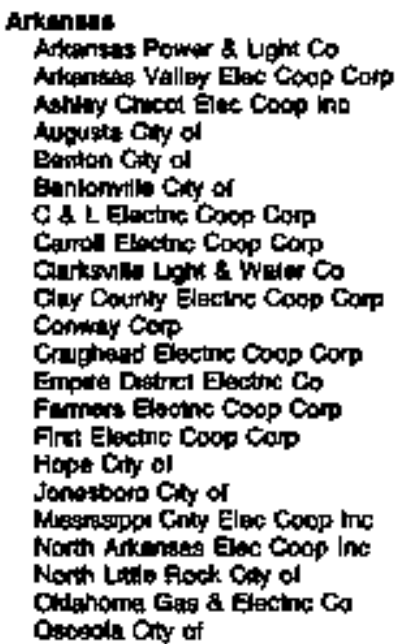 & 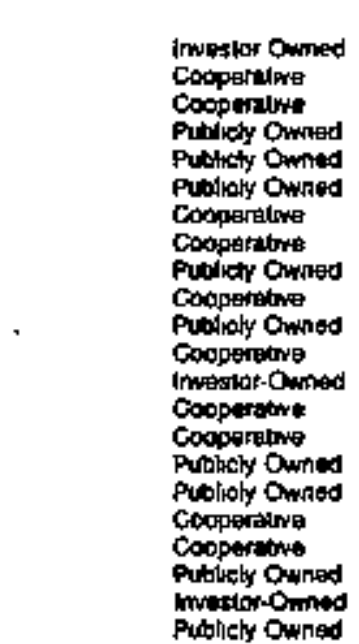 & 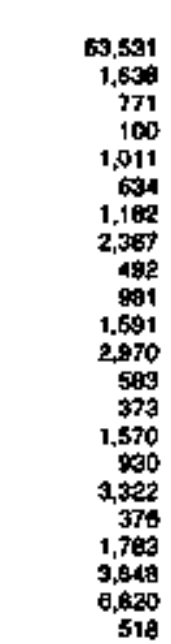 & 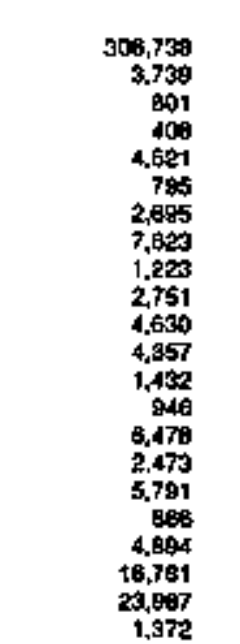 & 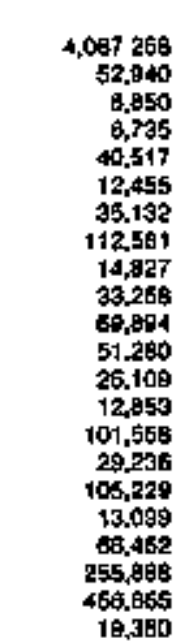 & $\begin{array}{r}754 \\
706 \\
906 \\
608 \\
1116 \\
638 \\
767 \\
877 \\
625 \\
627 \\
692 \\
850 \\
548 \\
736 \\
638 \\
848 \\
560 \\
581 \\
715 \\
686 \\
585 \\
708\end{array}$ \\
\hline
\end{tabular}

Soe loptnotes gil end of tibists 
Table 15. Clast of Ownership, Number of Ultimate Consumers, Revenus, Stolos, and Average Revenue per Kilowatthour for the Commerica' Sector by State and Utility, 1993 (Continued)

\begin{tabular}{|c|c|c|c|c|c|}
\hline 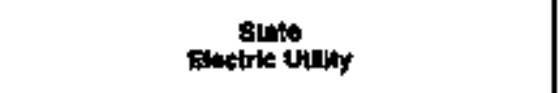 & $\begin{array}{c}\text { ciate } \\
\text { of } \\
\text { ommermip }\end{array}$ & $\begin{array}{l}\text { Nuribar } \\
\text { of } \\
\text { Gontumart }\end{array}$ & $\begin{array}{l}\text { Rovenus: } \\
\text { (thourmind dollars) }\end{array}$ & 8nles & 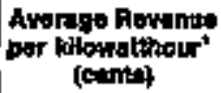 \\
\hline 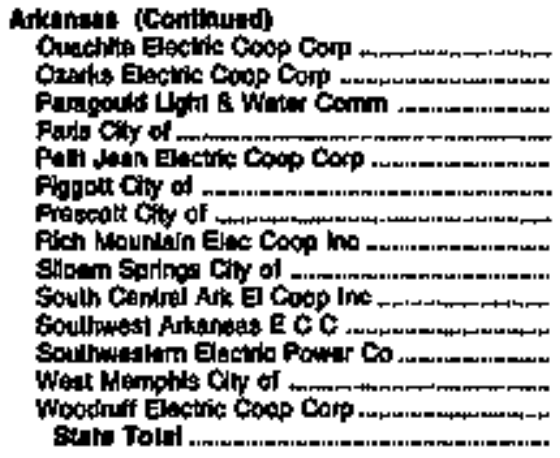 & 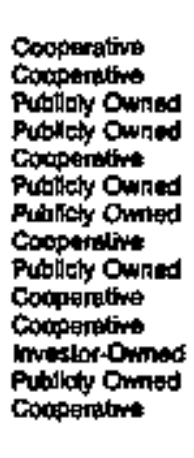 & 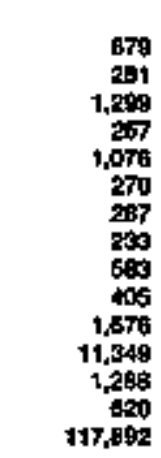 & $\begin{array}{r}4,247 \\
690 \\
1,954 \\
2,498 \\
139 \\
1,050 \\
695 \\
999 \\
791 \\
4,859 \\
34,742 \\
9,765 \\
2,997 \\
47,340\end{array}$ & 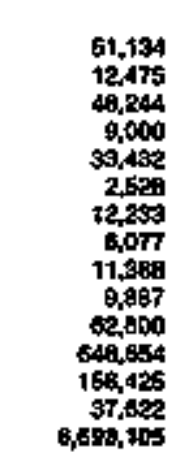 & $\begin{array}{l}9.31 \\
6.65 \\
4.05 \\
5.89 \\
7.44 \\
5.50 \\
6.68 \\
6.16 \\
6.25 \\
6.00 \\
7.60 \\
5.86 \\
6.28 \\
7.61 \\
7.04\end{array}$ \\
\hline 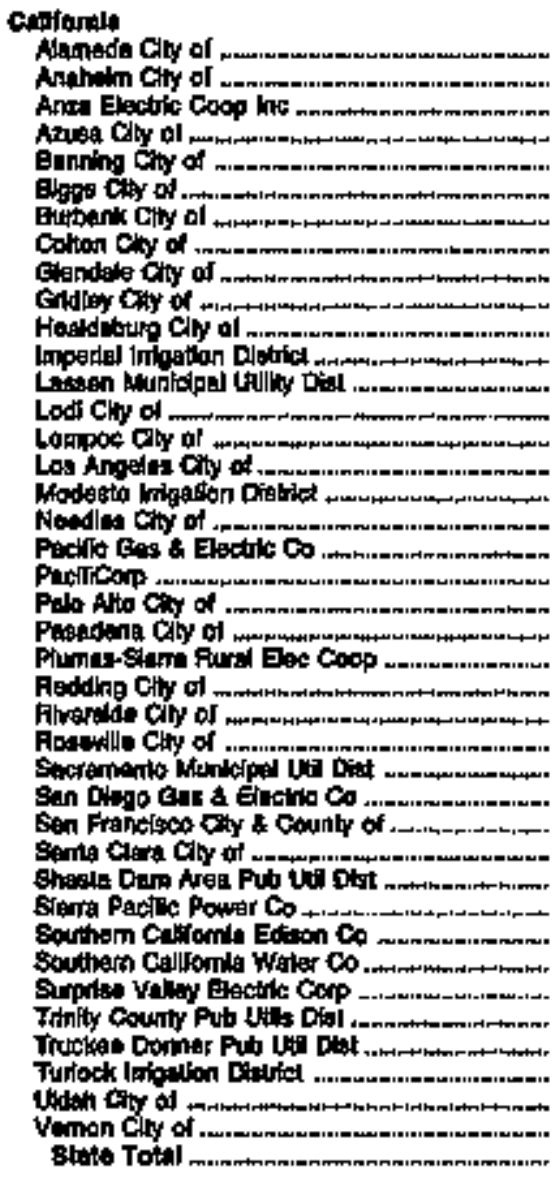 & 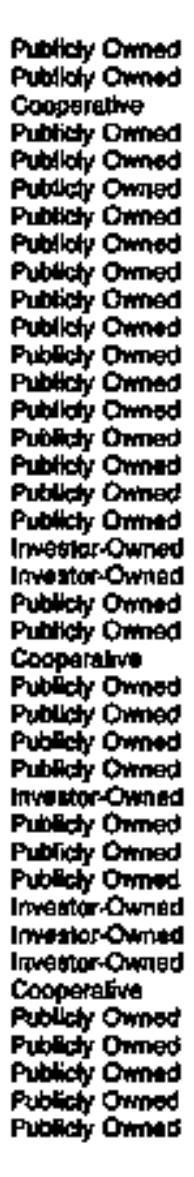 & 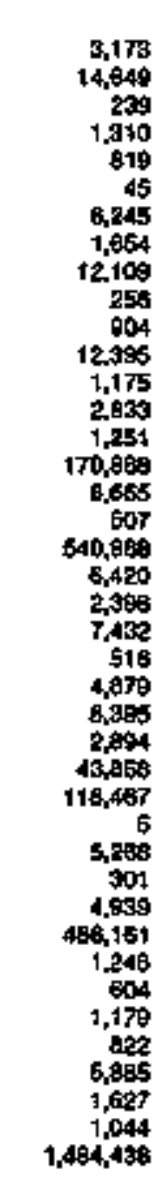 & 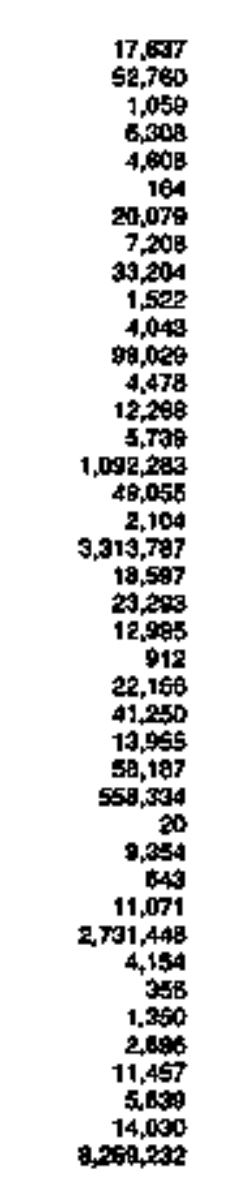 & 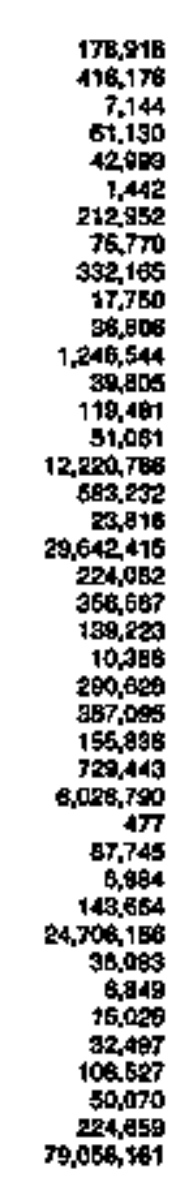 & 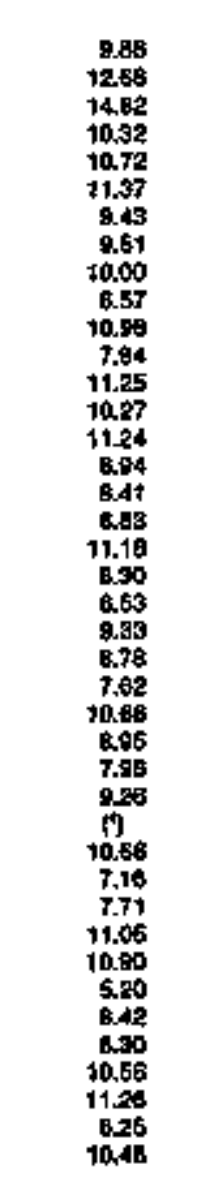 \\
\hline 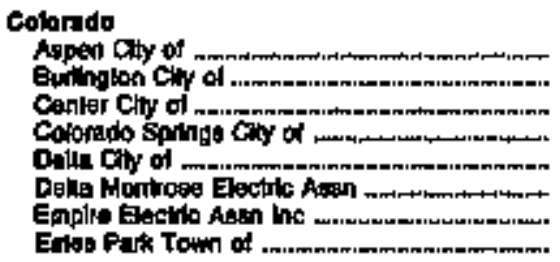 & 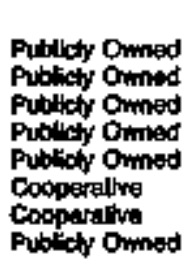 & $\begin{array}{r}834 \\
375 \\
130 \\
17,798 \\
\$ 11 \\
1,539 \\
1,712 \\
1.169\end{array}$ & $\begin{array}{r}2,728 \\
1,094 \\
109 \\
39+, 150 \\
192 \\
0,900 \\
2,629 \\
2,679\end{array}$ & $\begin{array}{r}38,158 \\
12,496 \\
3,040 \\
748,907 \\
5,700 \\
42,007 \\
31,156 \\
41,326\end{array}$ & $\begin{array}{l}7.18 \\
0.08 \\
6.22 \\
5.24 \\
8.11 \\
9.00 \\
6.11 \\
6.23\end{array}$ \\
\hline
\end{tabular}

Sac looknoles at and ol labine 
Tabte 15. Class of Ownership, Number of Ultimate Consumers, Revenue, Sales, and Average Reventue per Kllowatthour for the Commerical Sector by State and Utility, 1993 (Conthued)

\begin{tabular}{|c|c|c|c|c|c|}
\hline 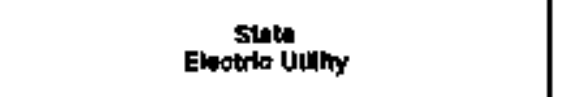 & $\begin{array}{c}\text { Clat: } \\
\text { of } \\
\text { owmershlp }\end{array}$ & $\begin{array}{c}\text { stumber } \\
\text { of } \\
\text { connumer }\end{array}$ & 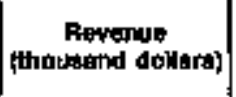 & $\begin{array}{l}\text { Sades } \\
\text { (1houband } \\
\text { ldionattiches) }\end{array}$ & $\begin{array}{l}\text { Awerage Rovenup } \\
\text { por bllowwetthour' } \\
\text { (cents) }\end{array}$ \\
\hline 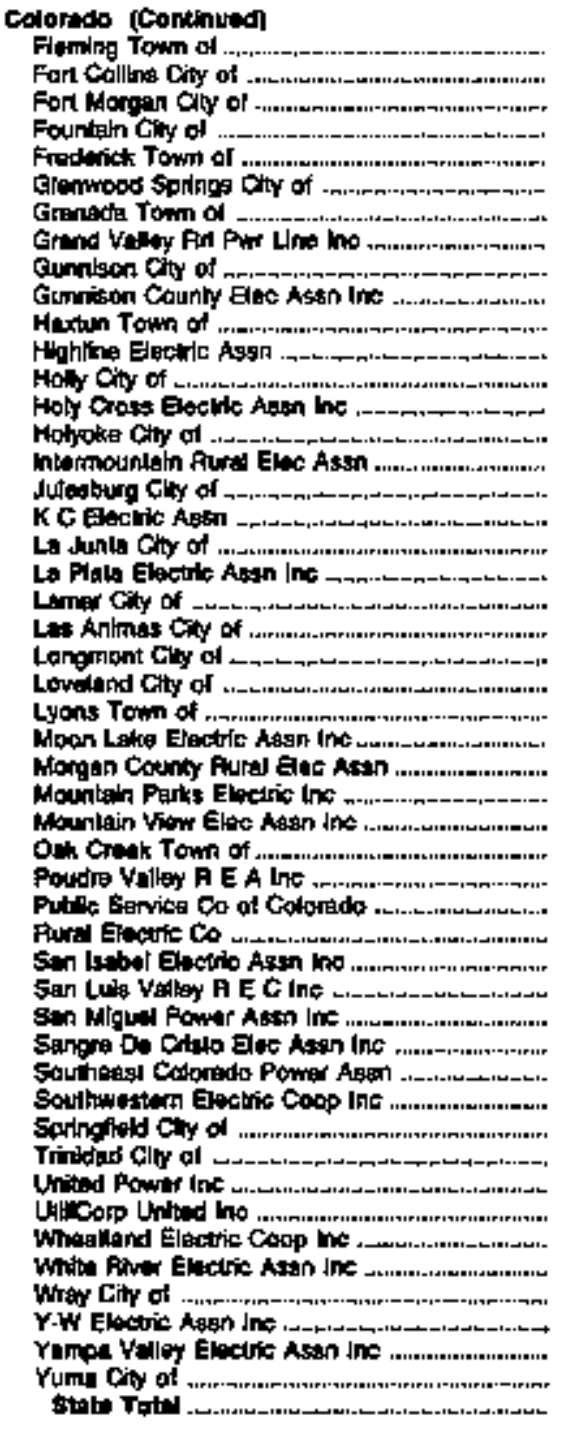 & 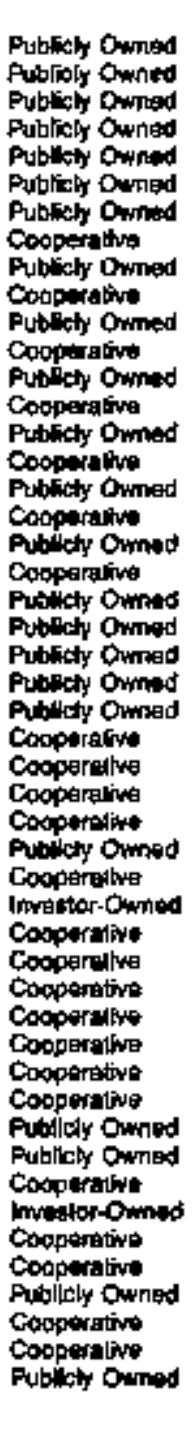 & 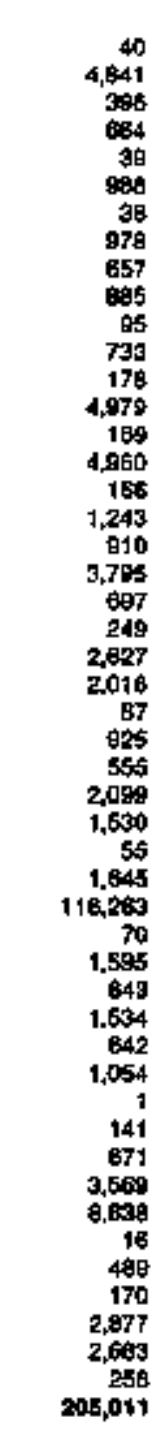 & 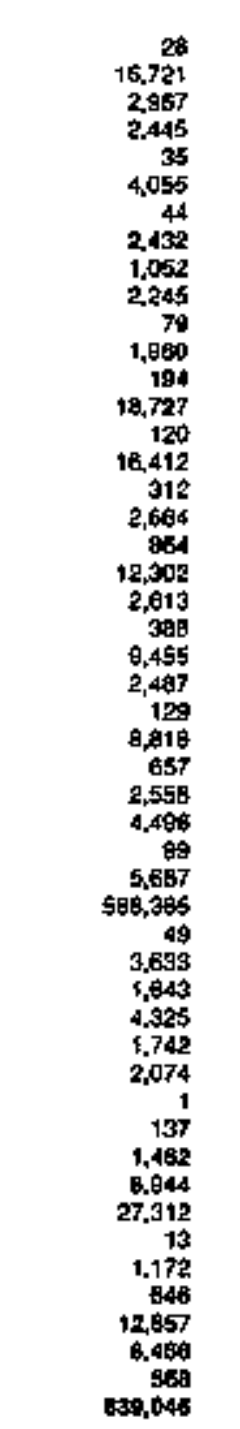 & 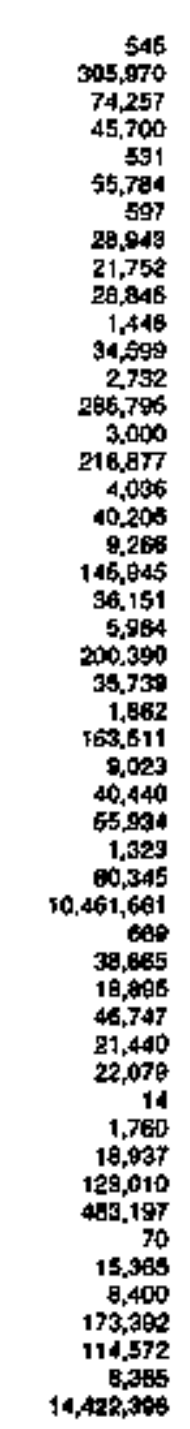 & 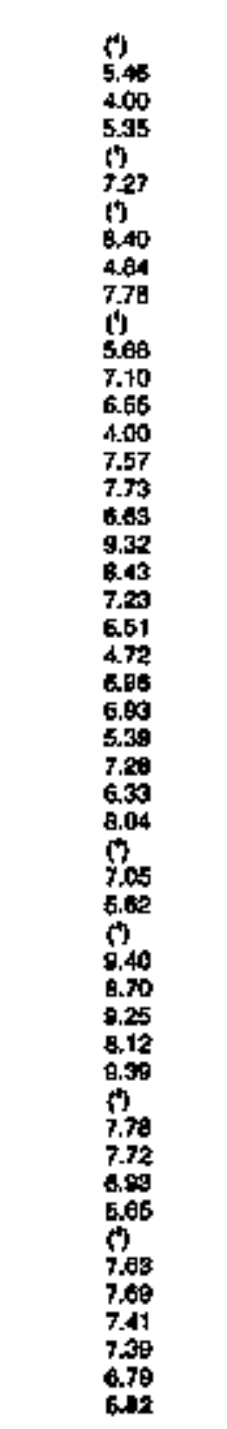 \\
\hline 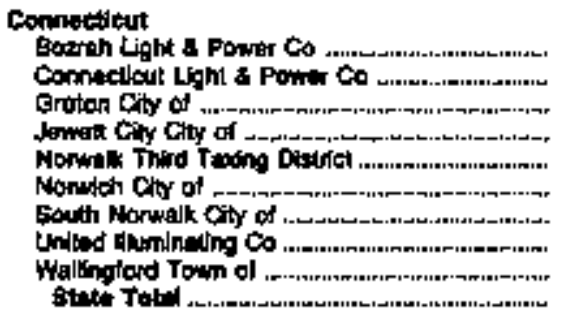 & 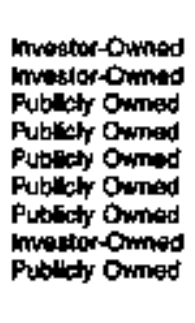 & $\begin{array}{r}198 \\
98,608 \\
1,408 \\
200 \\
490 \\
1,807 \\
1,009 \\
28,969 \\
2,790 \\
179,463\end{array}$ & 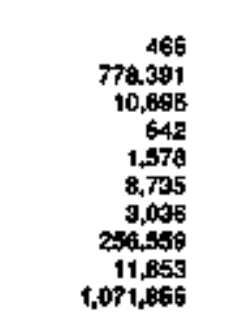 & $\begin{array}{r}4,114 \\
7,000,077 \\
193,785 \\
6,600 \\
14,028 \\
95,707 \\
24,761 \\
2,559,023 \\
140,048 \\
10,974,141\end{array}$ & $\begin{array}{r}11.90 \\
6.85 \\
7.69 \\
6.85 \\
10.84 \\
0.13 \\
12.26 \\
10.08 \\
0.46 \\
10.04\end{array}$ \\
\hline 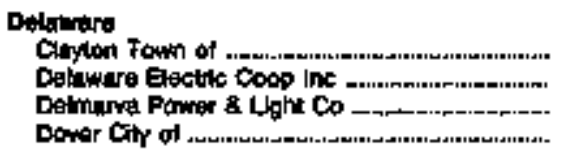 & 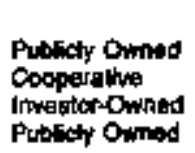 & $\begin{array}{r}80 \\
3,158 \\
23,888 \\
2,570\end{array}$ & $\begin{array}{r}180 \\
6.383 \\
155,983 \\
14,605\end{array}$ & $\begin{array}{r}1.600 \\
73,777 \\
2,242,473 \\
172,389\end{array}$ & $\begin{array}{l}8.60 \\
8.67 \\
6.69 \\
0.08\end{array}$ \\
\hline
\end{tabular}

Sog kolnotes at ind of labo. 
Table 15. Clasg of Ownership, Number of Ultimate Consumers, Revenue, Sallos, and Average Revenue per Kllowatthour for the Commerleal Sector by State and Utilty, 1993 (Continued)

\begin{tabular}{|c|c|c|c|c|c|}
\hline Etosts Uth & $\begin{array}{l}\text { ctint } \\
\text { of } \\
\text { Onwminhtp }\end{array}$ & $\begin{array}{l}\text { Numbtr } \\
\text { of } \\
\text { Conomemer: }\end{array}$ & 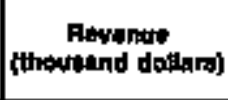 & 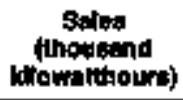 & 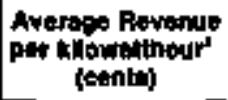 \\
\hline 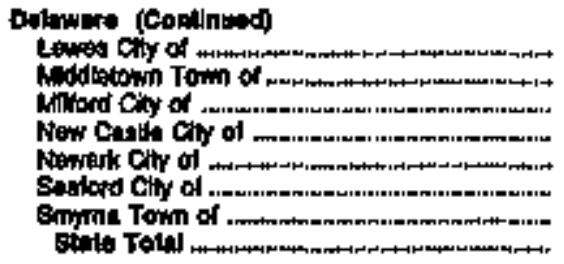 & 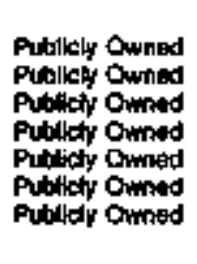 & $\begin{array}{r}330 \\
183 \\
618 \\
189 \\
1.036 \\
480 \\
264 \\
3,720\end{array}$ & 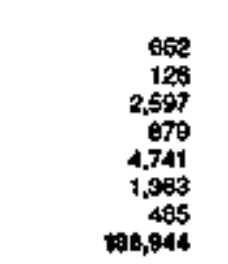 & $\begin{array}{r}7,378 \\
1,152 \\
27,153 \\
10,362 \\
51,420 \\
11,649 \\
4,843 \\
2,804,564\end{array}$ & $\begin{array}{r}8.97 \\
10.94 \\
6.56 \\
8.49 \\
9.28 \\
11.51 \\
10.01 \\
7.25\end{array}$ \\
\hline \multicolumn{6}{|l|}{ Divbrat of Columitla } \\
\hline 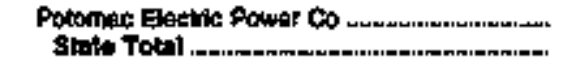 & Invattor-Ownted & $\begin{array}{l}26,920 \\
2+, 920\end{array}$ & $\begin{array}{l}307,351 \\
307,451\end{array}$ & $\begin{array}{l}5,4 \$ 7,566 \\
5,417,868\end{array}$ & $\begin{array}{l}7,16 \\
7.15\end{array}$ \\
\hline 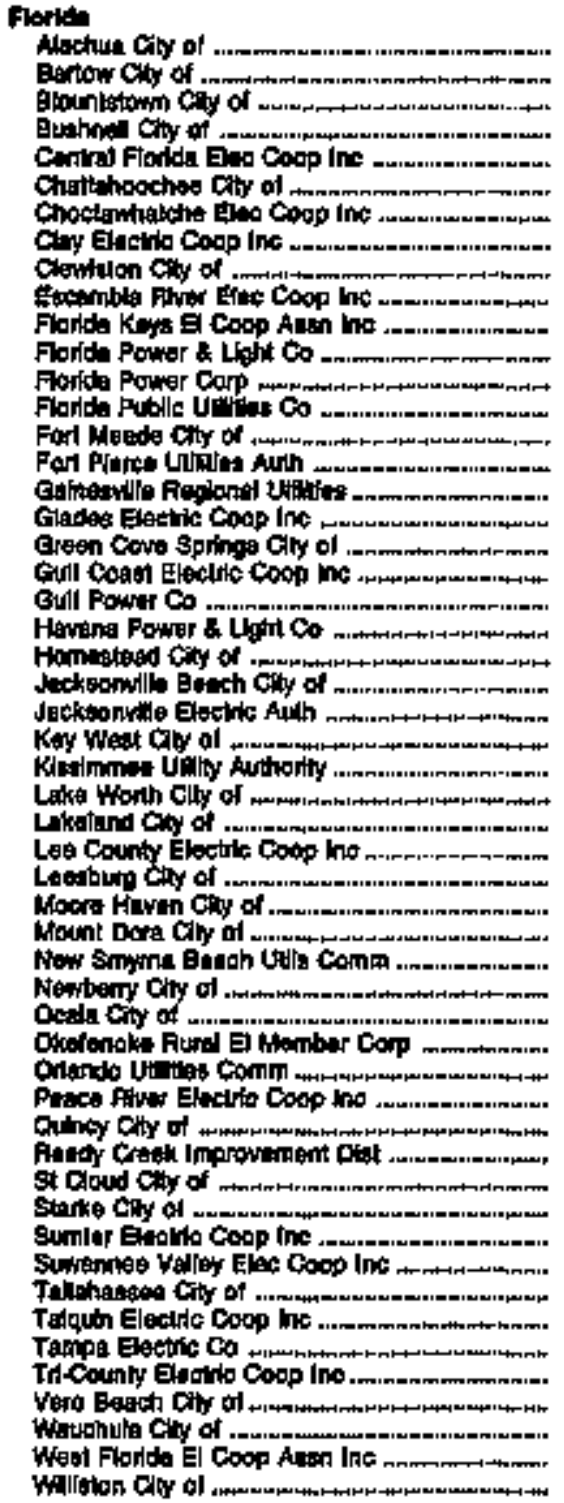 & 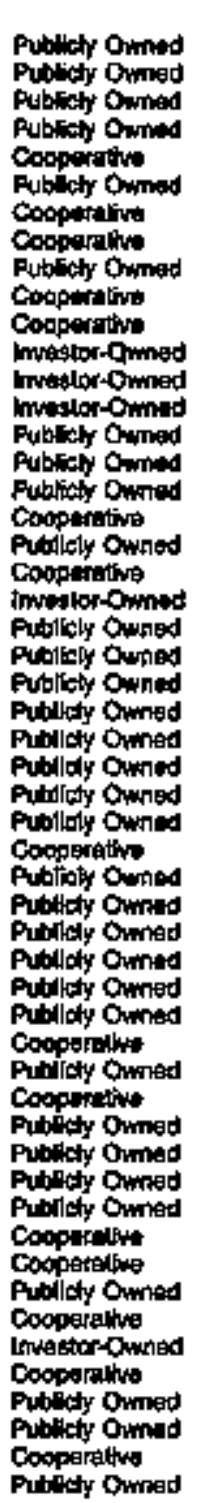 & 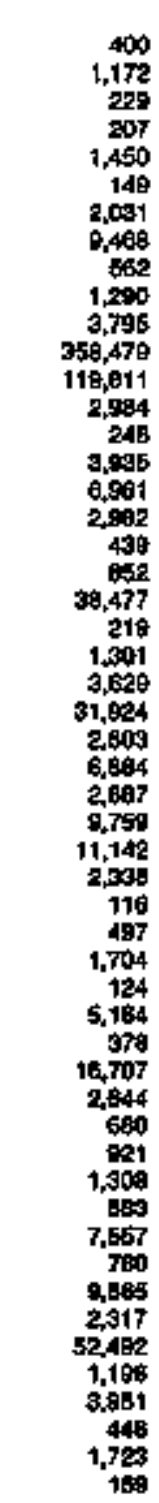 & 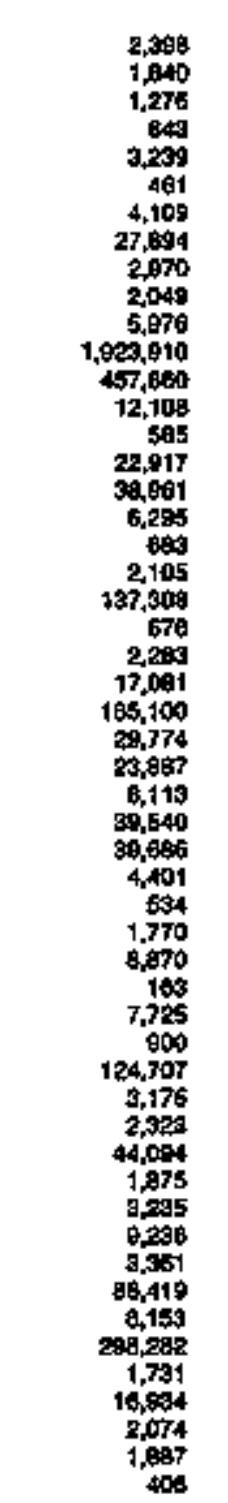 & 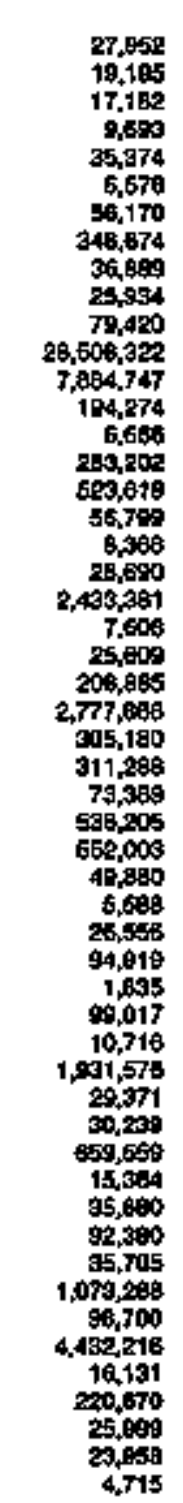 & 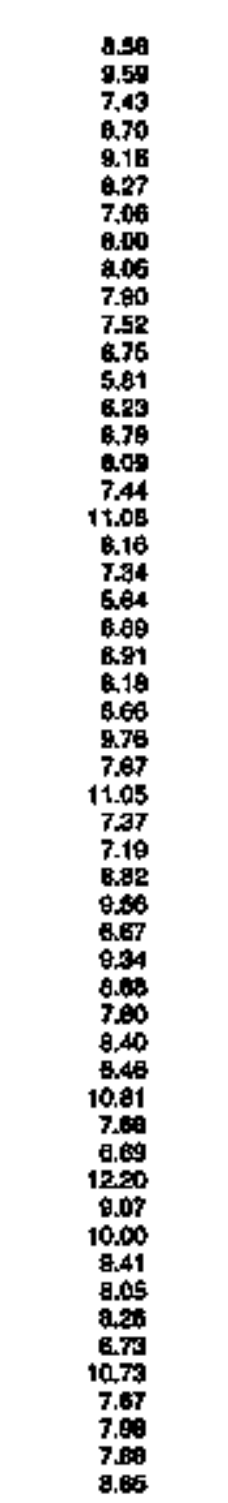 \\
\hline
\end{tabular}

Sog footnoles at and al labks. 
Teble 15. Class of Ownership, Number of Ultimate Congumers, Revenue, Sales, and Average Revenue per Kilowatthour for the Commerlcal Sector by State and Utulty, 1993 (Continuod)

\begin{tabular}{|c|c|c|c|c|c|}
\hline $\begin{array}{c}\text { Sigke } \\
\text { Electrle Utllay }\end{array}$ & clary & $\begin{array}{l}\text { Aumber } \\
\text { to } \\
\text { Comaventet }\end{array}$ & 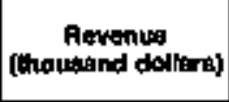 & 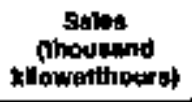 & 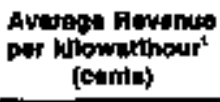 \\
\hline 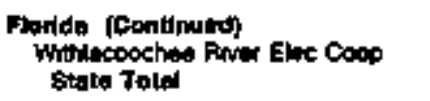 & Cooperativit & $\begin{array}{r}10,149 \\
745, \% 00\end{array}$ & $\begin{array}{r}35,029 \\
3,0189,190\end{array}$ & $\begin{array}{r}47,457 \\
64,975,460\end{array}$ & $\begin{array}{l}748 \\
680\end{array}$ \\
\hline 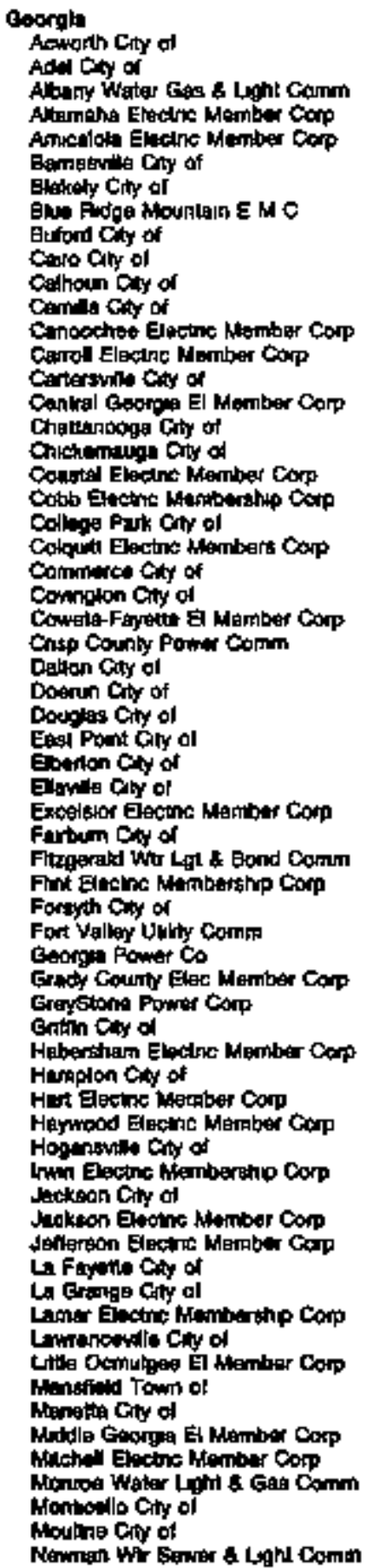 & 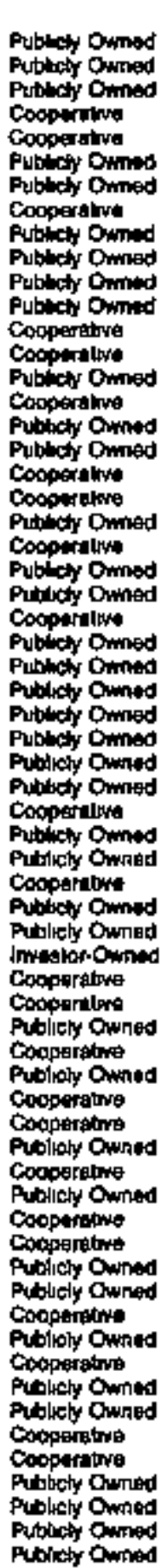 & 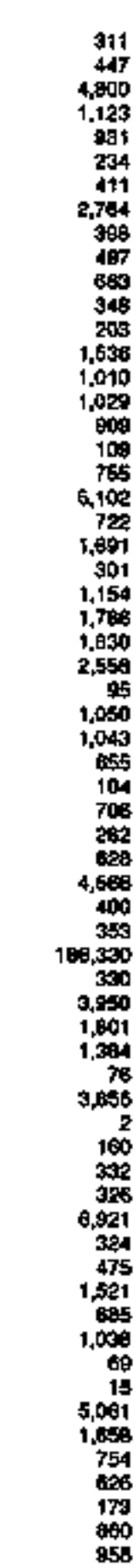 & 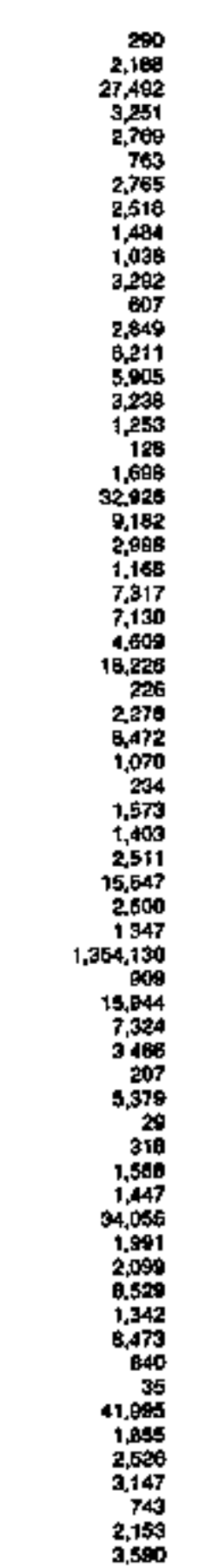 & 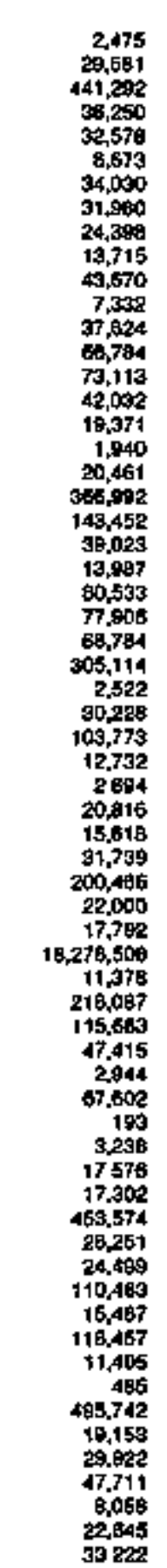 & 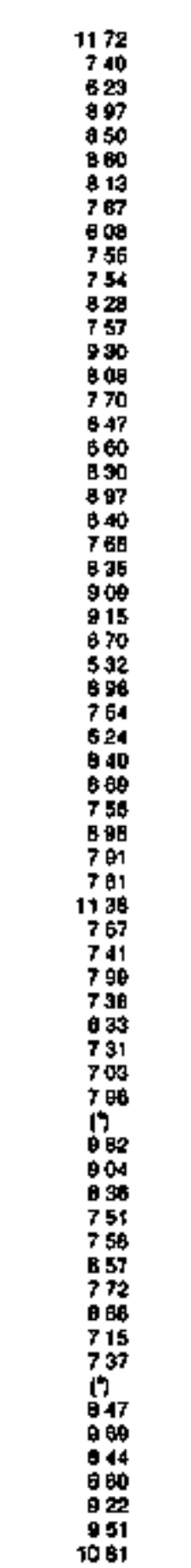 \\
\hline
\end{tabular}

890 footnoles al and of trble 
Table 15. Class of Ownerehip, Number of Uttimate Consumers, Revenue, Sales, and Average fevenue per Kllowatthour for the Commerloal Sector by State and Utilty, 1993 (Contimued)

\begin{tabular}{|c|c|c|c|c|c|}
\hline 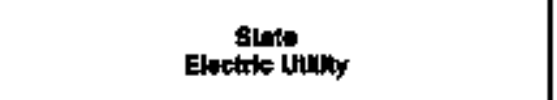 & $\begin{array}{l}\text { Cleser } \\
\text { oif } \\
\text { Oimerahip }\end{array}$ & $\begin{array}{l}\text { Chumber } \\
\text { od } \\
\text { Conationa }\end{array}$ & $\begin{array}{c}\text { Ruverit } \\
\text { (thousend dollare) }\end{array}$ & 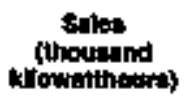 & $\begin{array}{l}\text { Averape ficwanue } \\
\text { par ldiowrithour' } \\
\text { (cemter) }\end{array}$ \\
\hline 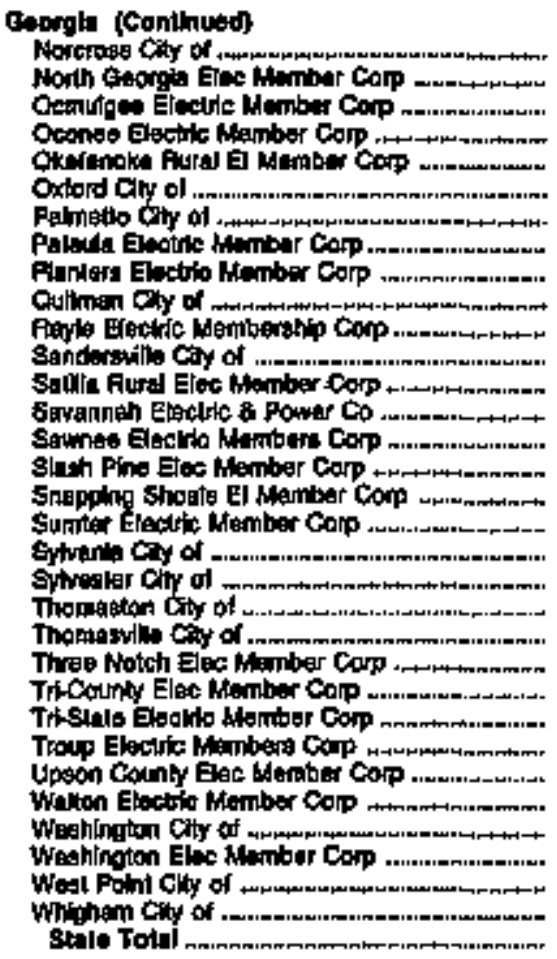 & 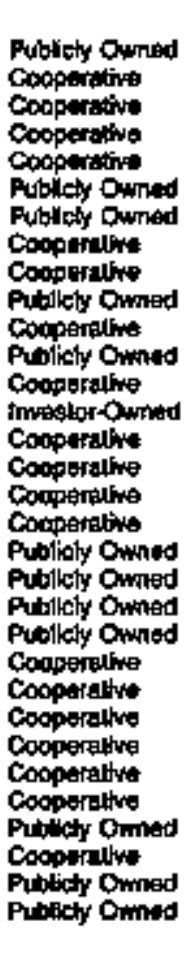 & 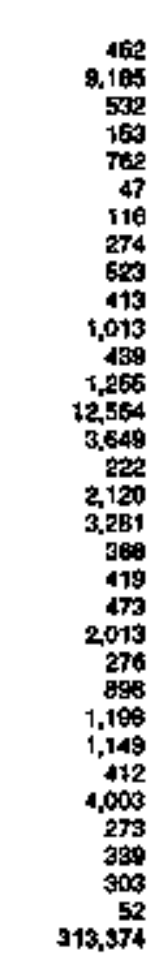 & 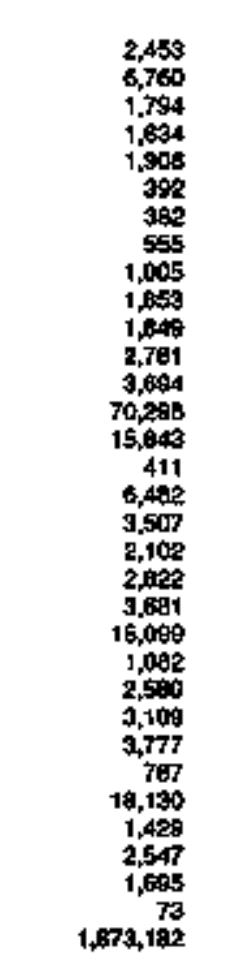 & 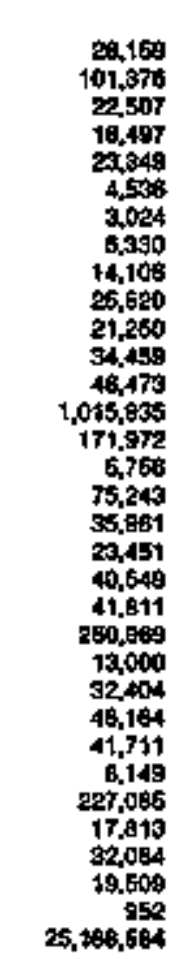 & 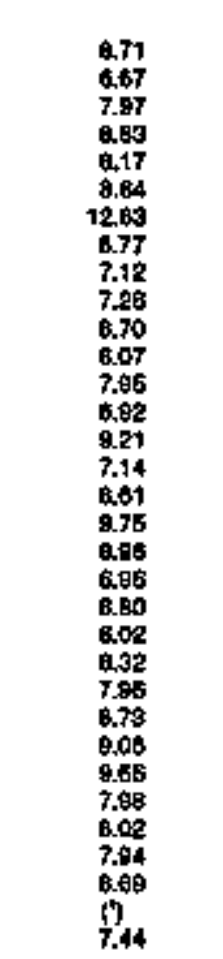 \\
\hline 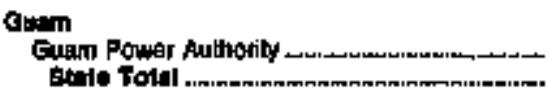 & Putialy Dimed & $\begin{array}{l}4,4 B 3 \\
4,496\end{array}$ & $\begin{array}{l}47,542 \\
47, \leqslant 82\end{array}$ & $\begin{array}{l}473,071 \\
473,071\end{array}$ & $\begin{array}{l}10.05 \\
10.06\end{array}$ \\
\hline 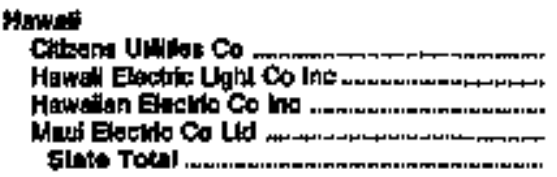 & 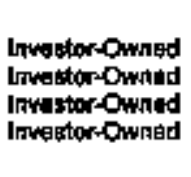 & $\begin{array}{r}3,447 \\
8,148 \\
30,073 \\
7,000 \\
90,005\end{array}$ & $\begin{array}{r}13,309 \\
43,317 \\
179,997 \\
39,436 \\
276,069\end{array}$ & $\begin{array}{r}78.618 \\
282,157 \\
1,701,172 \\
2,29+237 \\
2,365,224\end{array}$ & $\begin{array}{l}16.98 \\
18.82 \\
10.68 \\
13.84 \\
11.64\end{array}$ \\
\hline 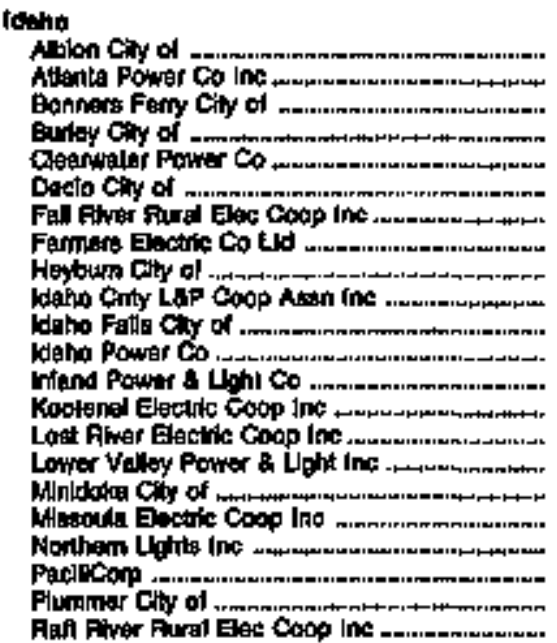 & 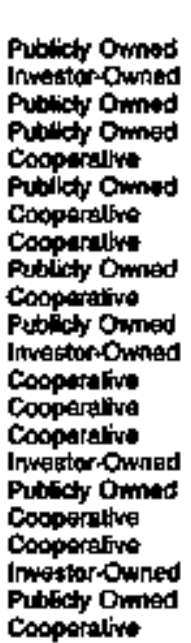 & $\begin{array}{r}7 \\
5 \\
483 \\
419 \\
500 \\
9 \\
1.048 \\
23 \\
67 \\
185 \\
2,557 \\
49,012 \\
17 \\
726 \\
152 \\
144 \\
5 \\
17 \\
528 \\
6,707 \\
89 \\
105\end{array}$ & $\begin{array}{r}16 \\
18 \\
616 \\
647 \\
595 \\
27 \\
1,460 \\
68 \\
234 \\
261 \\
10.619 \\
130.775 \\
79 \\
2,197 \\
147 \\
99 \\
9 \\
45 \\
1,613 \\
19.477 \\
267 \\
109\end{array}$ & 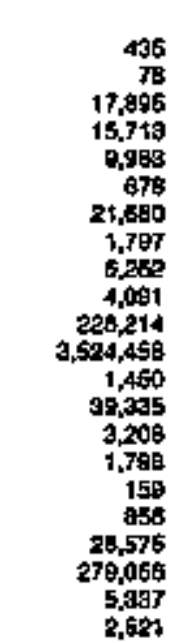 & 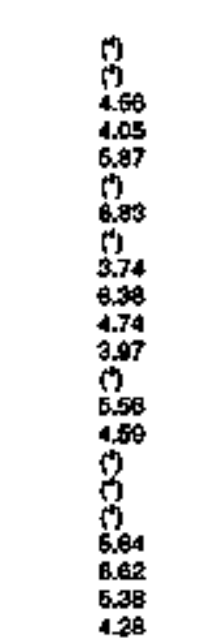 \\
\hline
\end{tabular}

See loolnotes al end of lable. 
Table 15. Class of Ownership, Number of Utimate Conoumars, Revenue, Sales, and Average Revenue per Kilowatthour for the Commerical Sector by State and Utillty, 1993 (Continued)

\begin{tabular}{|c|c|c|c|c|c|}
\hline 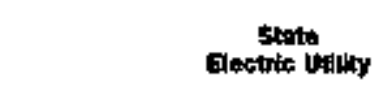 & $\begin{array}{c}\text { of } \\
\text { Ormoriplap }\end{array}$ & $\begin{array}{c}\text { Humbet } \\
\text { of } \\
\text { Contodiminer }\end{array}$ & (thousund dalkere) & $\begin{array}{l}\text { Sates } \\
\text { phoupand } \\
\text { kiliwathour }\end{array}$ & 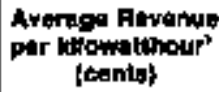 \\
\hline
\end{tabular}

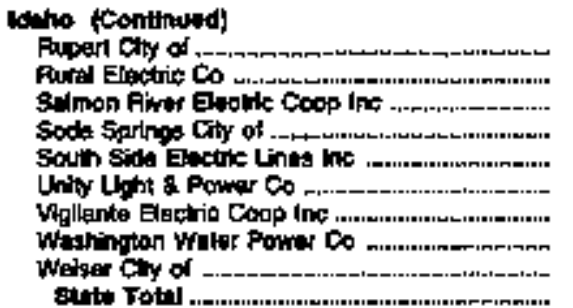

IUlabit.

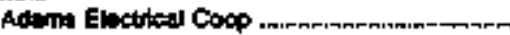

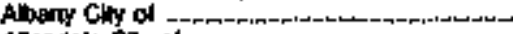

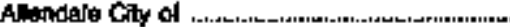

Allomont Cay of

Betpivi City of

Bethony vilan of

Bnotion cin of

Eushneil City of .

Gelo Pubic Utivy Cormm

Germ City of

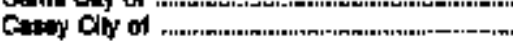

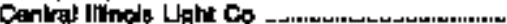

Canjal I

Ghatum vilugo ol .

(2)

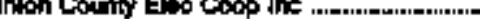

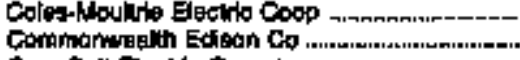

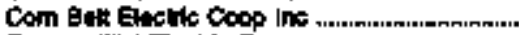

EAstem Minl Electri; Coco

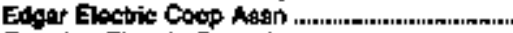

Explun Elverde Coop Assn

Fitifield City of

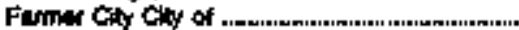

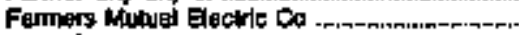

Fiora city of ..

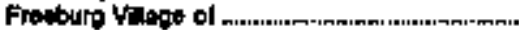

Gengseo Cly of ,

Ganevit City ol .

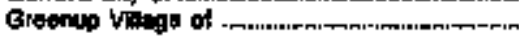

Histand City of

Illonols Rowet Co.

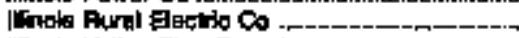

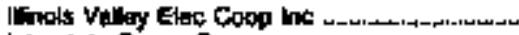

Iftersilelo Power Co ....................................

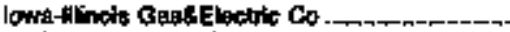

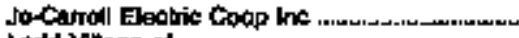

Ledd Vlang of

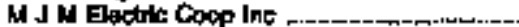

Merstall Ciny of .

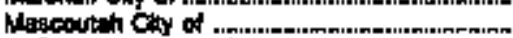

McDonsibh Pomat COOp

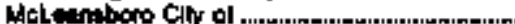

Nenand Elactric Coop

Metopenle Coty of

Mermen Count Enco Cop Ine

Wh Germet Pubte Utilty Co .

Napervila City of ....

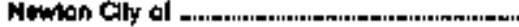

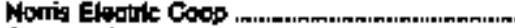

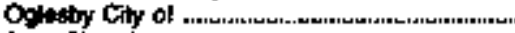

Peru Cary of

Pindention City of

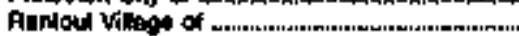

fos gud City of
Putaldy Owned

Coopentw:

Cooperative

Pubichy Omed

Coopenutwa

Cocpentivise

Cooperation

Imeglor-Oungad

Putlicty Oumed

Cooperative

Publisy Owied

Publloby Owned

Fulfichy Owned

Publeth Owmad

Publint Dwned

Publicty Owned

Publely Owned

Publucty Owned

Putecty Owned

Pubichy Owned

Pubicty Ommad

musetor Oumpos

Investor-Owhed

Pubitity Ommes

Cooperalve

Cooperaltwo

Cocperalive

Intrinor-Ounned

Cooperalwo

Cosperative

Coopuralive

Cooperaikve

Pubalcty Ouried

Publloty Oined

Cosperatbo

Puthlly Ouned

Pubulty Ourned

Publlcly Ownted

Fucollely Owine:

Pubildy Owaned

Publity Owroted

Imvislor-Owned

Copperativa

Copoptotive

Imestor-Omed

timester-Owned

Cocoptrative

Publusty Ownod

Cospeterative

Publey Omines

Fublect Owndod

Cooperative

Publicty Owned

cooperalive

Publestr Owined

Cooperalivit

investor-Ouned

Pudichy owmed

Publetr Ownod

Cooperalive

Putbichy Owned

Fublety Owned

Pubicty Oninto

Fublecty Ounes

Pusticty Ownet

339
410
398
270
177
213
5
10,797
290
74.968

1,513
976
776
583
694
570
3
35,620
933
210,445

29,660
22,420
14,1060
6,008
17,140
13,140
35
849,242
20,090
$4,060,602$

5.10
4.35
5.46
6.65
3.60
4.34
17
5.49
4.64
4.42

46

770

$\mathbf{5 3}$

579

5.65

213

784

539
1,515

703

1,927

649

$90,59 \%$

178,15d

411
877

2.006.

2,193

1. 102.662

5,35

2,303

975
2,159

2,159
1.615

255

297

692

358

$\$, 510$

3.131

974

269.379

1,559

1,012

4,032

$54,02 ?$

1,285

115

1,262

1,012

651

721

2,419

1.345

469

1,007

21,067

1,060

185

2048

766

B19

7,589

709

if 50

as, 605

2,500

11,61日

4,025

20,400

0.18

27.743

8,453

1.370.642

2.635,480

4,571

B.215

21.672

25,288

23,402689

57.262

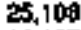

11.107

30,389

26,046

4,234

2,500

a. 140

4,575

22,417

49,732

4,980

$3,246,403$

13,450

12,439

B:, 163

463,204

19,367

1,374

12, 953

12,351

11.143

2,623

7.50

21,150

18.108

5,649

12,984

274,496

13,560

13,234

2,474

31,253

9,274

6,842

13,170

10.17

(1)

B.A7

6.84

8.52

6.75

7.02

7.43

7.66

6.57

604

1.69

10.88

2.63

6.10

8.36

8.35

7.10

8.45

0.02

10,31

8.50

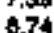

8.42

7.60

7.14

8.30

11.59

B.14

650

$72 x$

6.6A

Q.57

8.10

6.9.97

0.40

11.44

7.04

B. 7

77

7.08

6.84

B.16

7.48

B. 40

6.14

9.06

Q.17

Ser rooinotes and of labla. 
Tabse 15. Class of Ownership, Number of Uitimate Consumbre, Rovenub, Sales, and Average Revenue per Kllowatthour for the Commorical Sector by State and Utlity, 1993 (Continued)

\begin{tabular}{|c|c|c|c|c|c|}
\hline 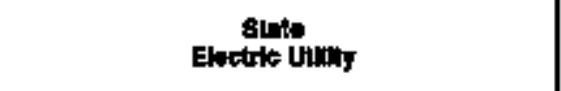 & Cing & $\begin{array}{c}\text { tumber } \\
\text { contumara }\end{array}$ & Athourend dolarel & 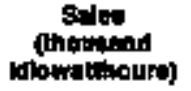 & 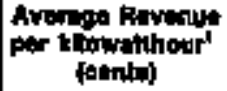 \\
\hline 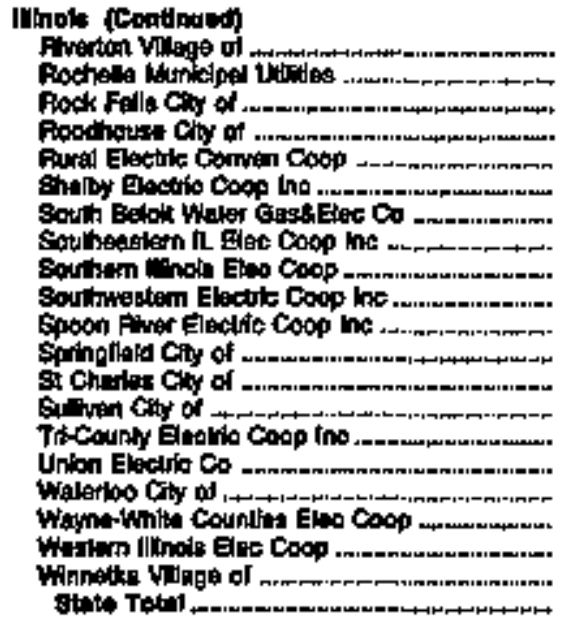 & 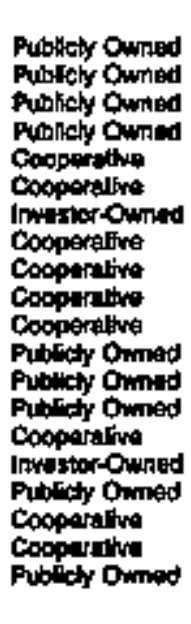 & 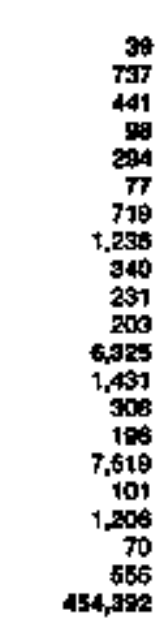 & 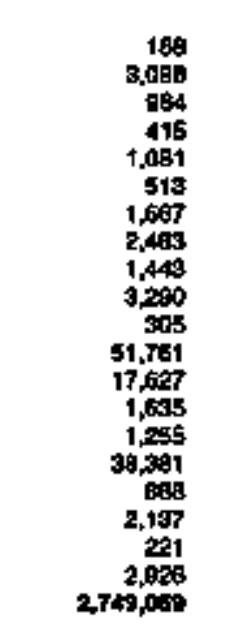 & 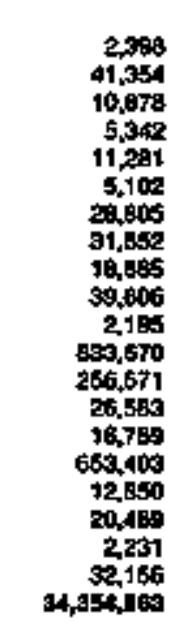 & $\begin{array}{r}7.89 \\
7.49 \\
8.06 \\
7.77 \\
7.58 \\
10.05 \\
5.79 \\
7.60 \\
7.84 \\
8.31 \\
13.90 \\
6.21 \\
6.67 \\
6.16 \\
7.49 \\
6.87 \\
9.91 \\
10.43 \\
9.91 \\
9.10 \\
0.00\end{array}$ \\
\hline 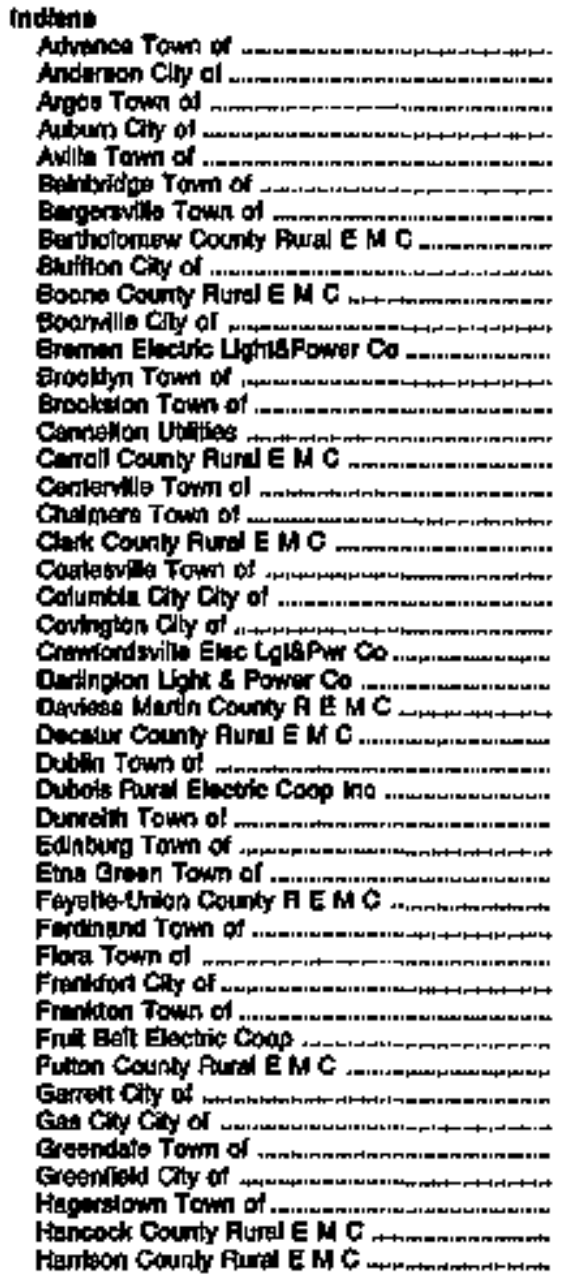 & 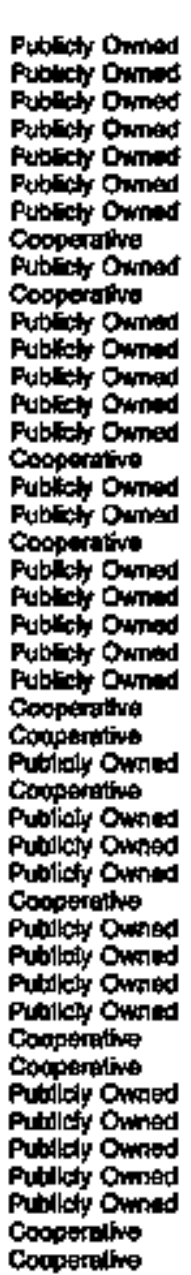 & 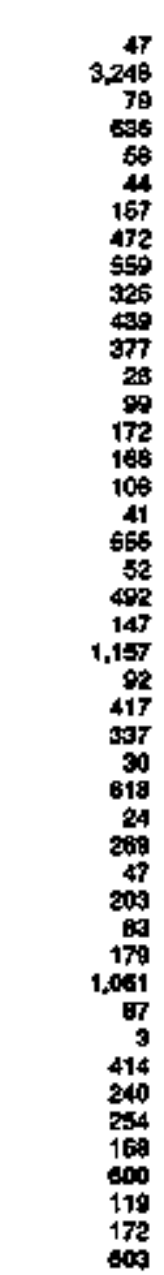 & 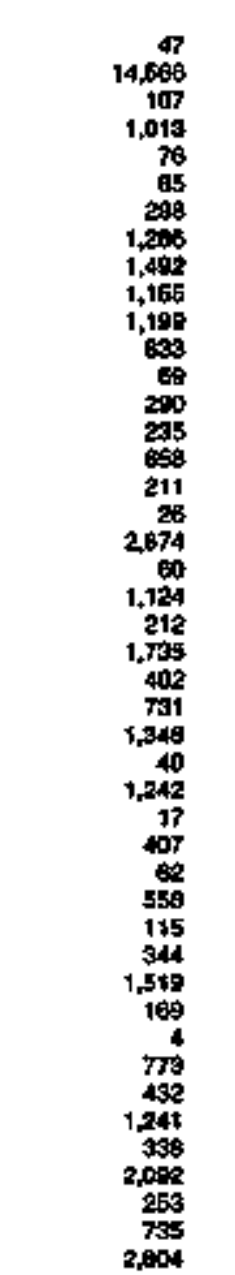 & 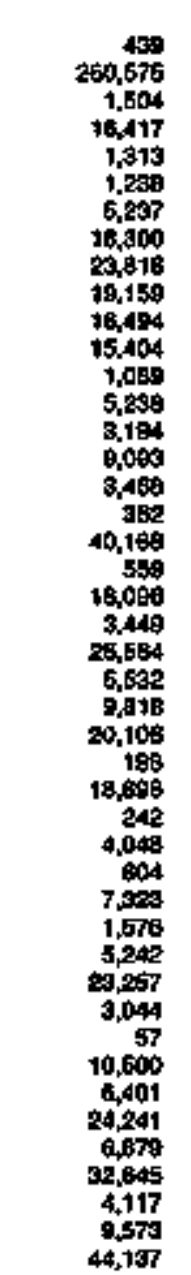 & 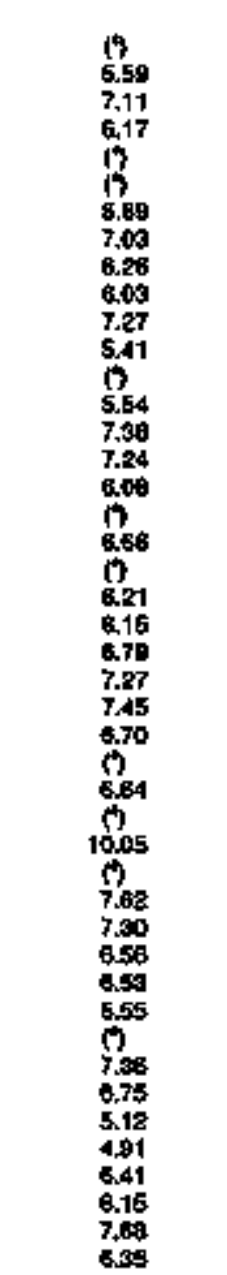 \\
\hline
\end{tabular}

Ser loolnotas al and of tabte. 
Table 15. Claste of Ownership, Number of Ultimate Consumers, Revenue, Sales, and Average Revenue per Kilowatthour for the Commerical sector by State and Utilty, 1993 (Continuod)

\begin{tabular}{|c|c|c|c|c|c|}
\hline 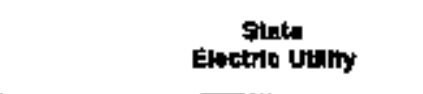 & $\begin{array}{c}\text { Gate } \\
\text { of } \\
\text { Onmortalp }\end{array}$ & $\begin{array}{c}\text { rumber } \\
\text { of } \\
\text { Conturatert }\end{array}$ & Firante & $\begin{array}{c}\text { Salos } \\
\text { (thoutiand } \\
\text { kllowathothrs) }\end{array}$ & $\begin{array}{c}\text { Avgrega Rovande } \\
\text { per klowathour } \\
\text { (coms) }\end{array}$ \\
\hline 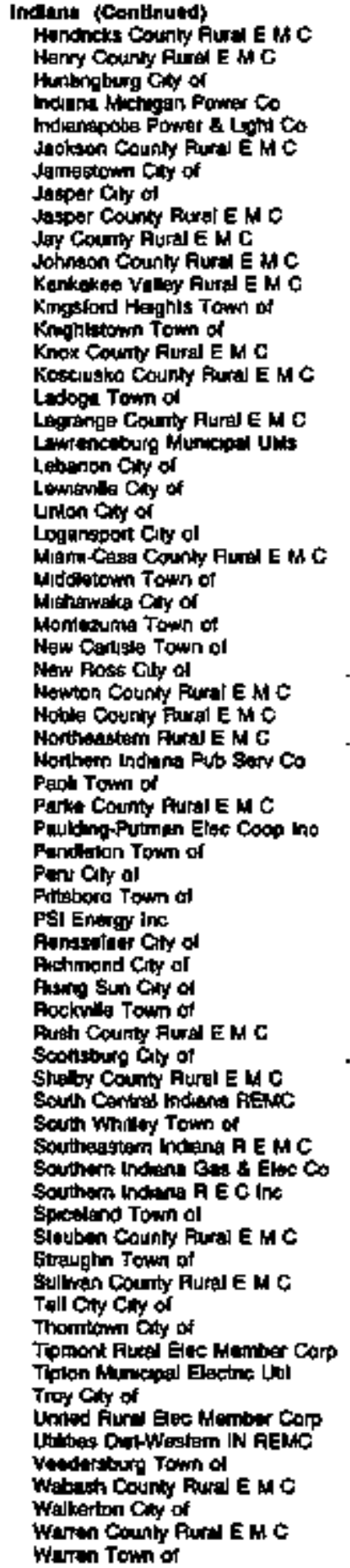 & 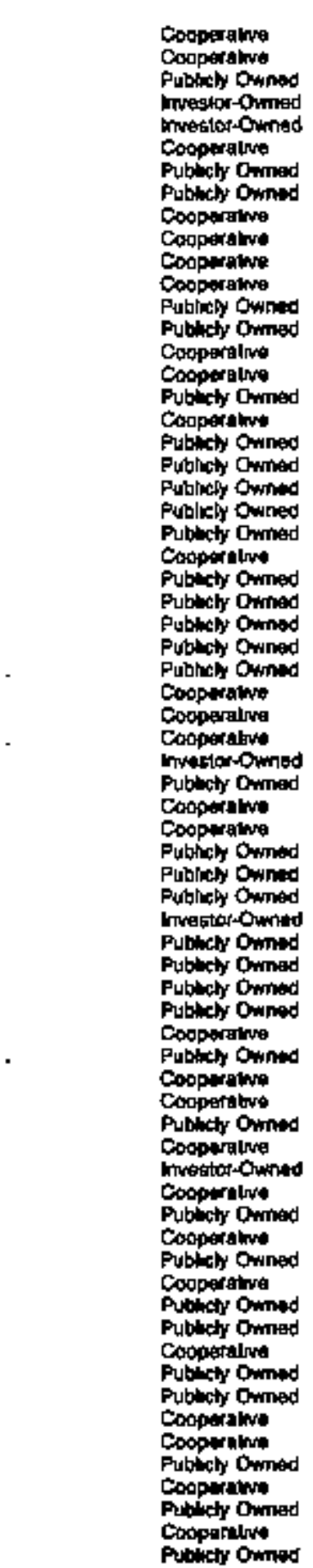 & 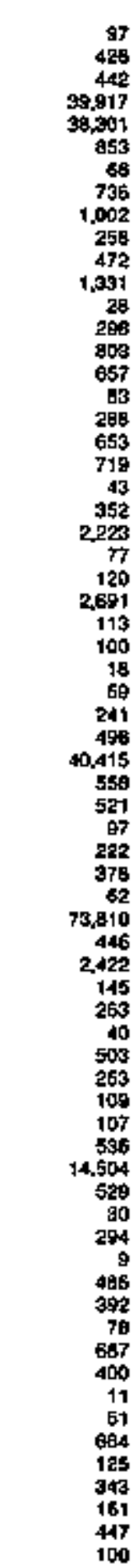 & 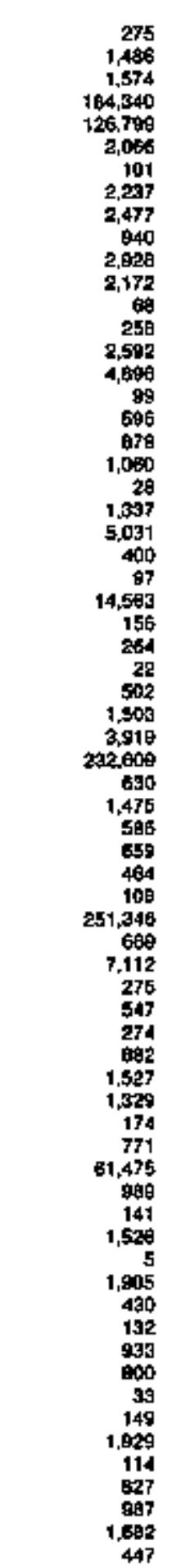 & 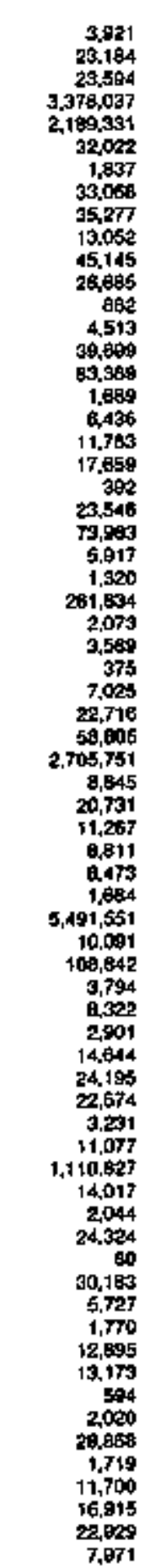 & 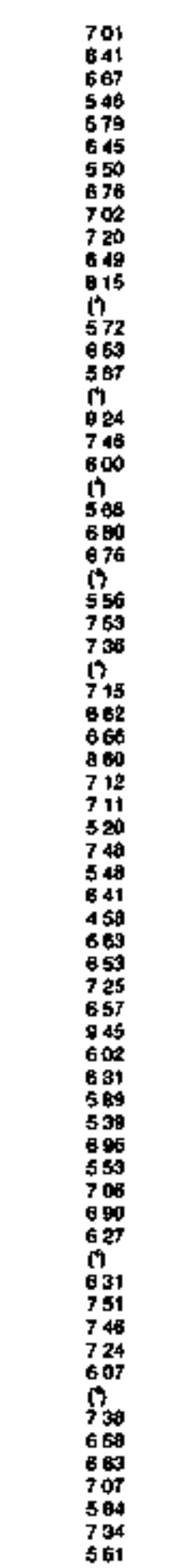 \\
\hline
\end{tabular}

See forttolet at end of lable 
Table 15. Class of Ownership, Number of Utimate Consuners, Fevenue, Sales, and Average Revenue per Kllowatthour for the Commerical Sector by State and Utîlty, 1993 (Continued)

\begin{tabular}{|c|c|c|c|c|c|}
\hline 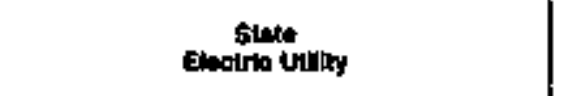 & orites & $\begin{array}{l}\text { Numbar } \\
\text { tof } \\
\text { Con:unet: }\end{array}$ & (browend doltary & stas & 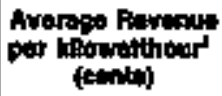 \\
\hline 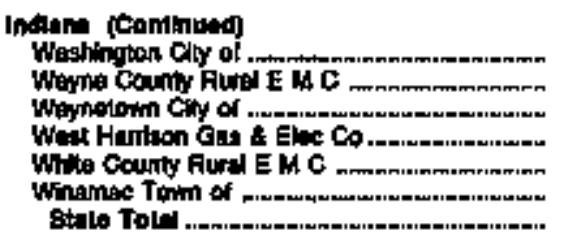 & 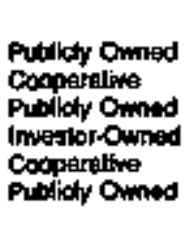 & $\begin{array}{r}80 \\
35 \\
60 \\
81 \\
27 \\
248 \\
260,424\end{array}$ & 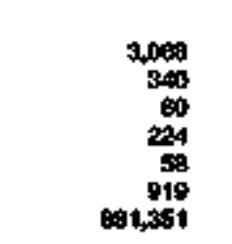 & $\begin{array}{r}55,707 \\
1,803 \\
1,160 \\
2,608 \\
758 \\
14,009 \\
17,016,036\end{array}$ & $\begin{array}{l}5.61 \\
7.08 \\
\$ \\
7.70 \\
40 \\
6.58 \\
6.05\end{array}$ \\
\hline 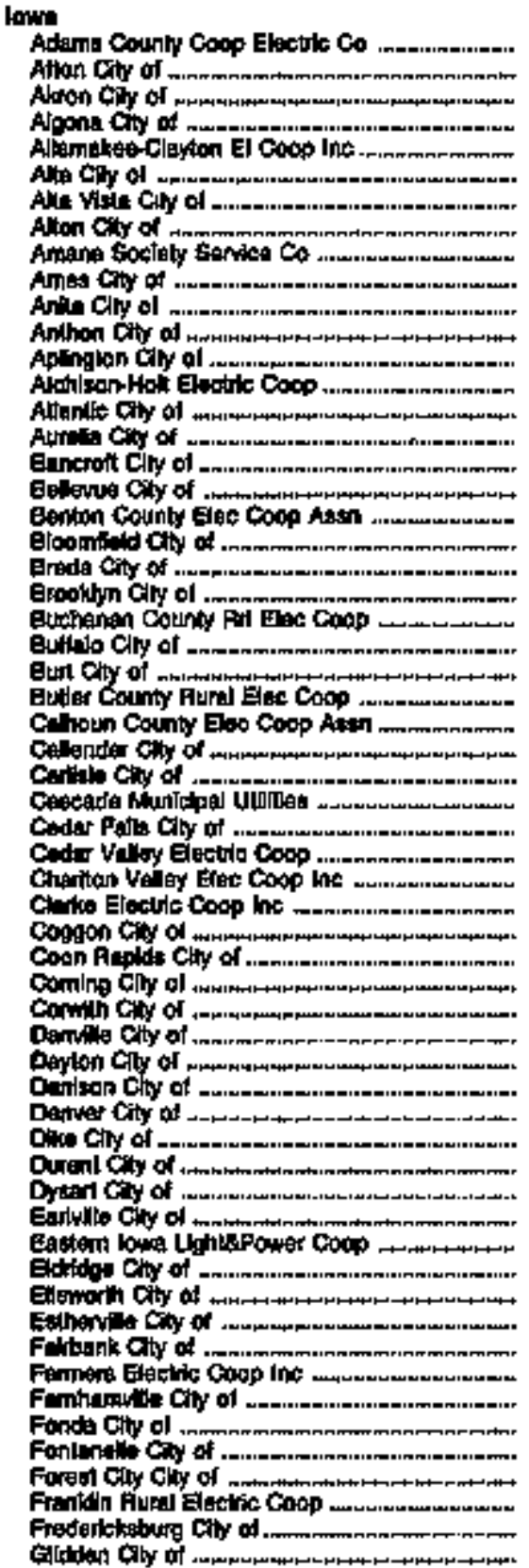 & 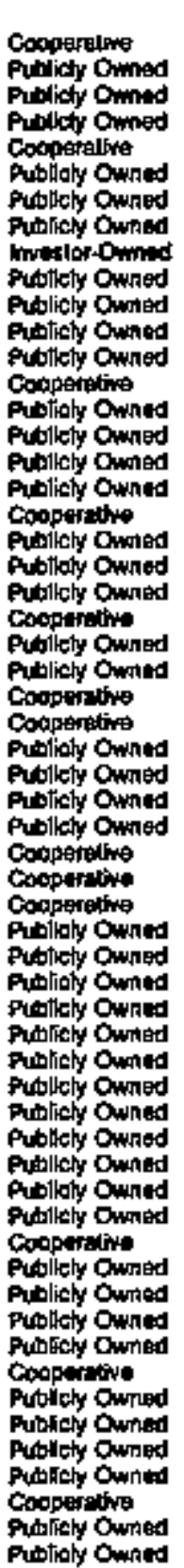 & 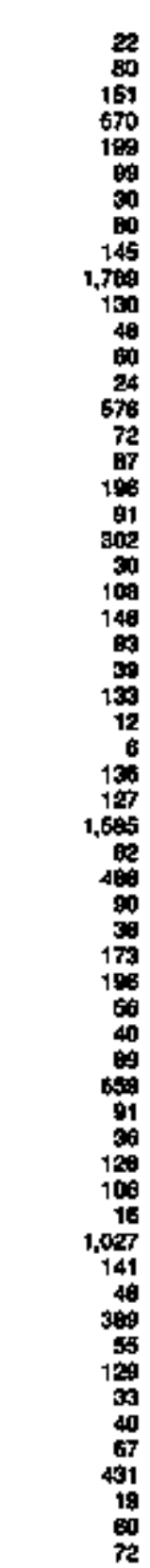 & 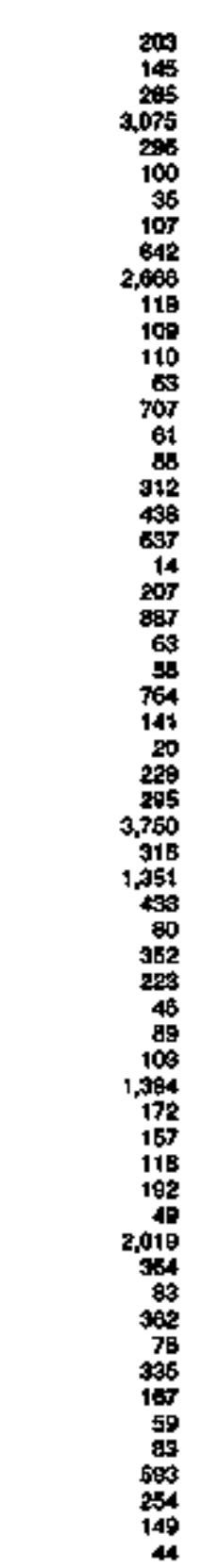 & 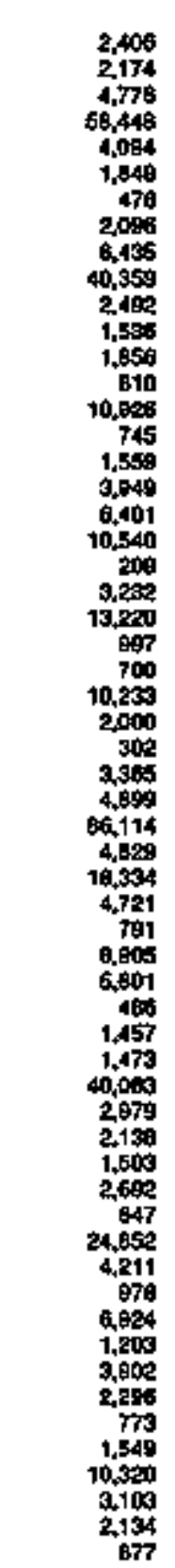 & 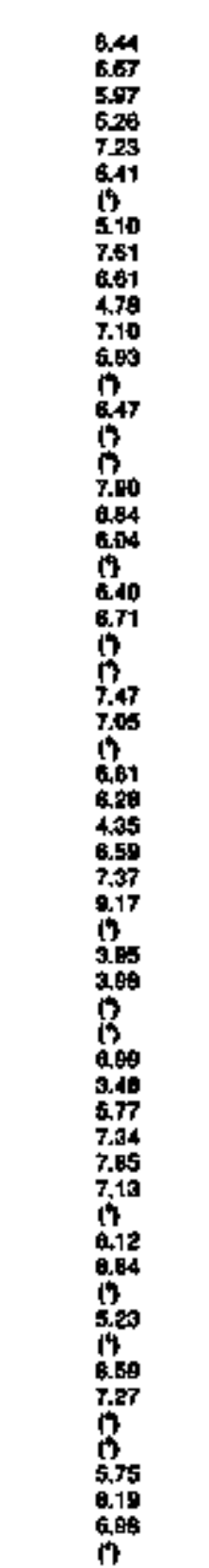 \\
\hline
\end{tabular}

Soe frotinotieg at end of tabla. 
Table 15. Class of Owner:thip, Number of Ultimate Consumert, Revenue, Sales, and Average Pevenue per Kllowatthour for the Commerlcal Sector by State and Utillty, 1993 (Continued)

\begin{tabular}{|c|c|c|c|c|c|}
\hline Electo & 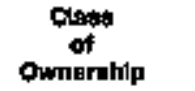 & $\begin{array}{c}\text { Numbet } \\
\text { ol } \\
\text { Conaumara }\end{array}$ & Ahourenve & 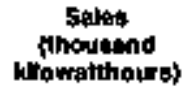 & $\begin{array}{l}\text { Avernge hovemst } \\
\text { per ldlownelhour' } \\
\text { [enmls) }\end{array}$ \\
\hline 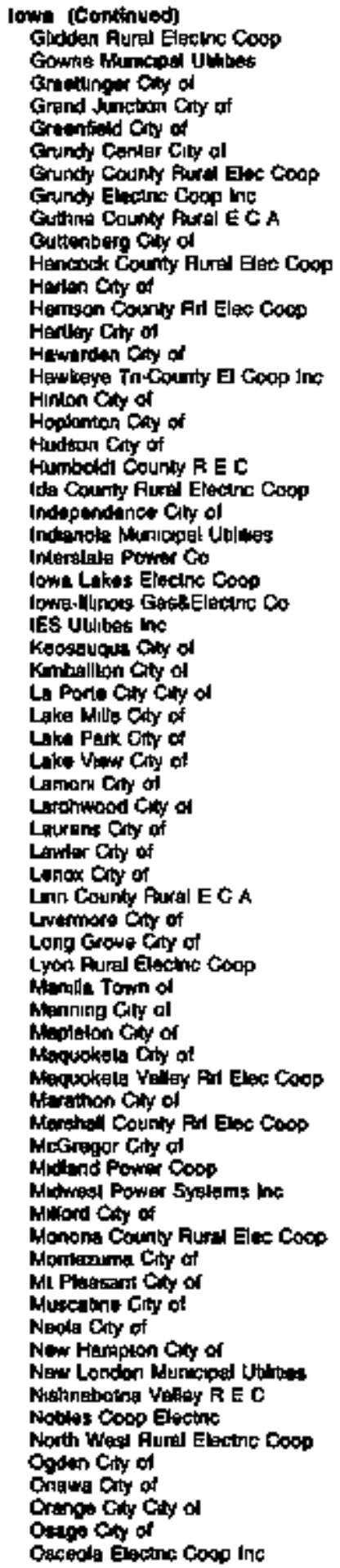 & 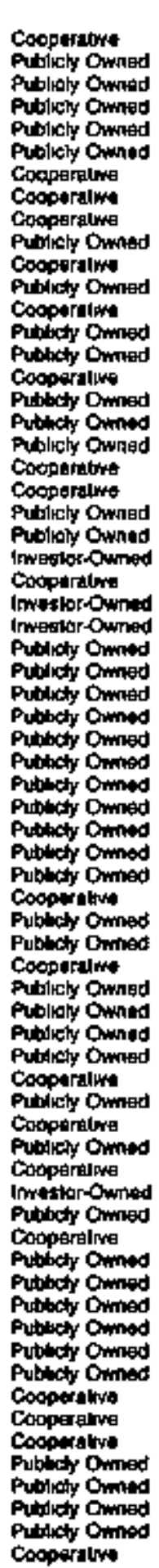 & 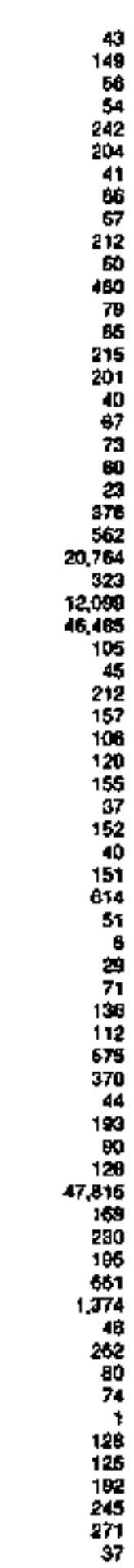 & 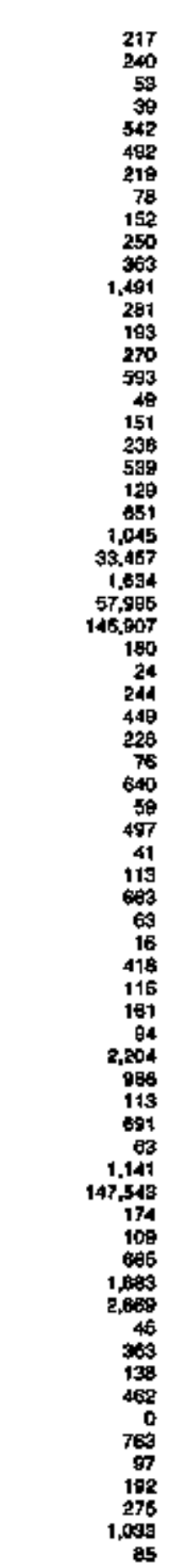 & 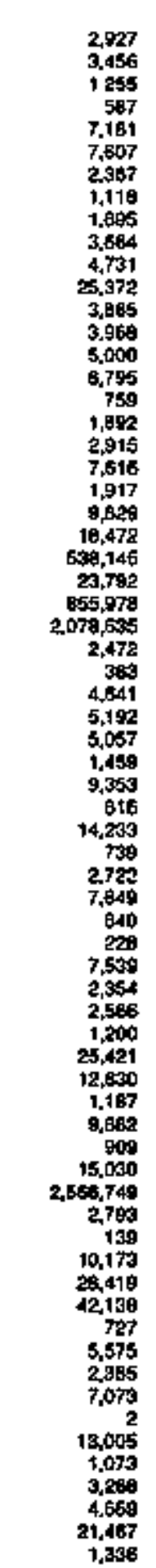 & 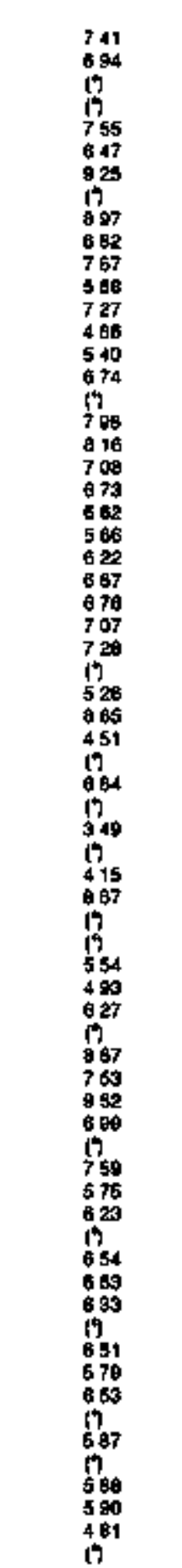 \\
\hline
\end{tabular}

Ses looknotes al and of tarie 
Table 16. Class of Ownership, Mumber of Litimbte Consumers, Revenue, Sales, and Average Revenue per KHowatthour for the Commerical Sector by State and tinity, 1993 (Continued)

\begin{tabular}{|c|c|c|c|c|c|}
\hline 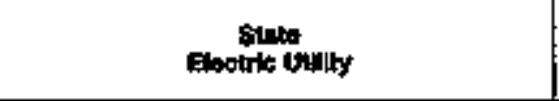 & of & 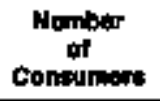 & Boventis & 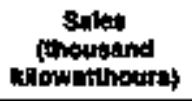 & 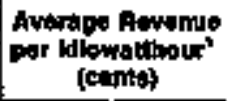 \\
\hline 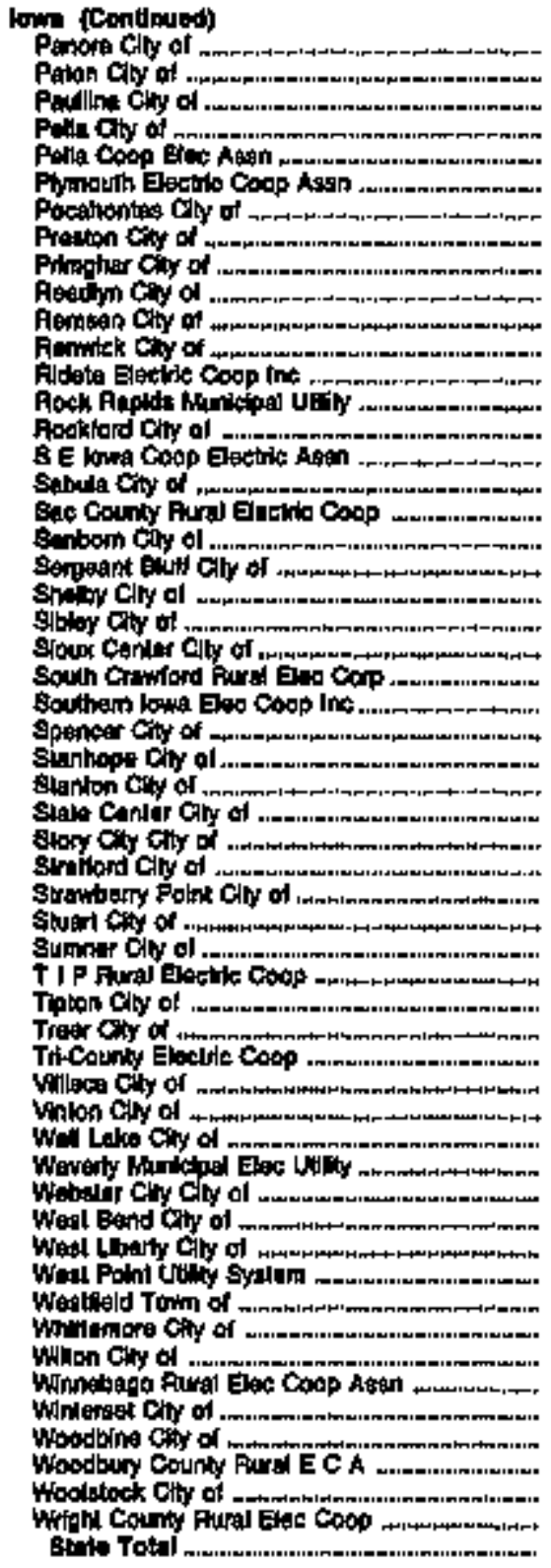 & 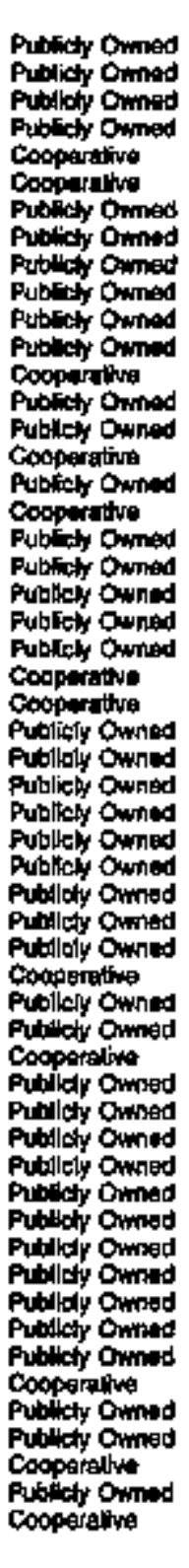 & 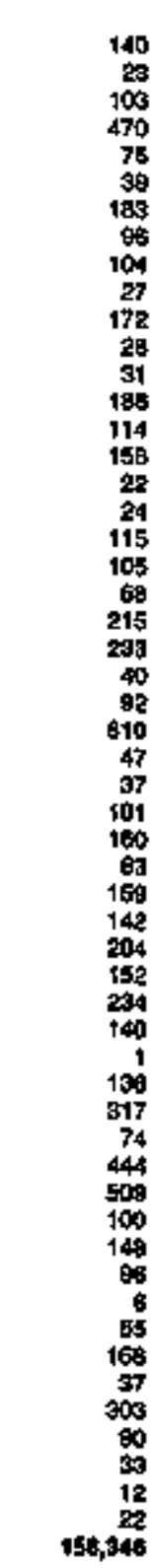 & 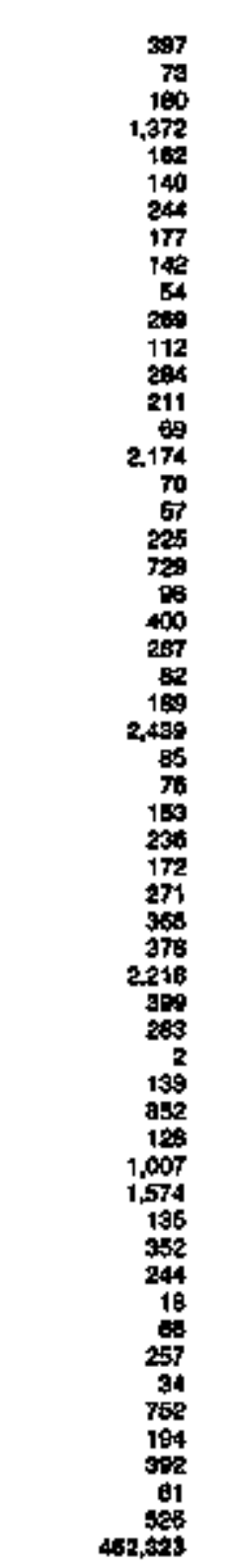 & 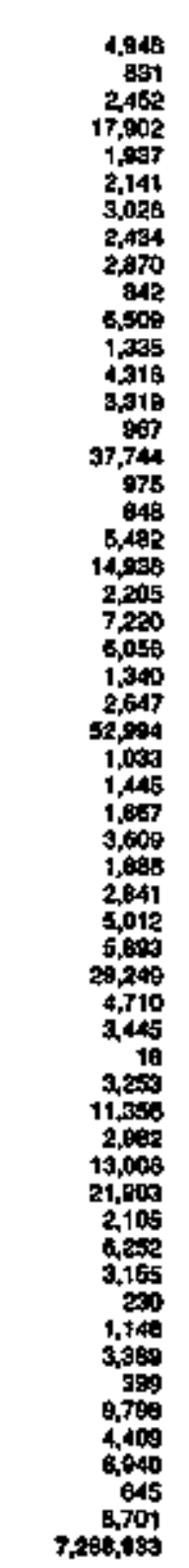 & 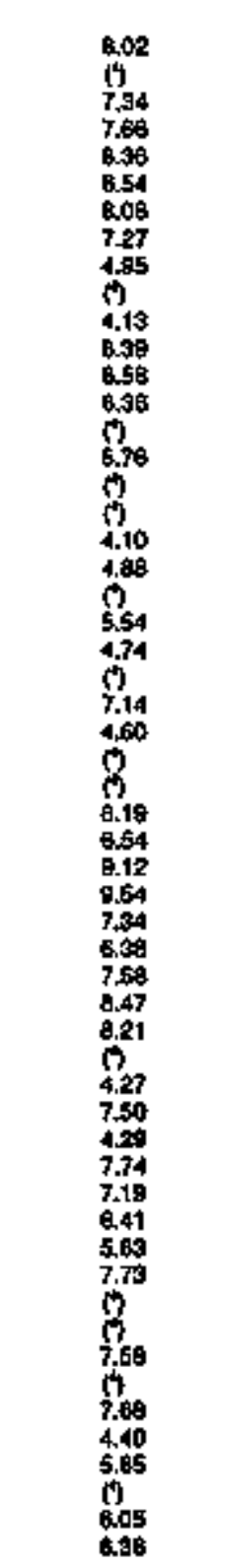 \\
\hline 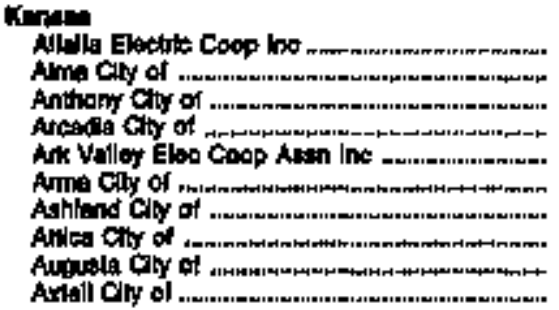 & 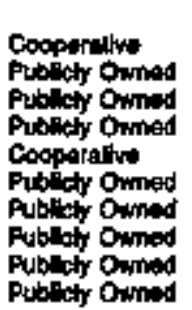 & $\begin{array}{r}554 \\
108 \\
263 \\
14 \\
647 \\
97 \\
157 \\
65 \\
35 \\
35\end{array}$ & $\begin{array}{r}472 \\
101 \\
607 \\
15 \\
1,301 \\
141 \\
275 \\
109 \\
1.189 \\
34\end{array}$ & 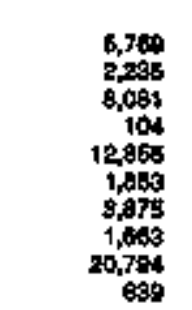 & 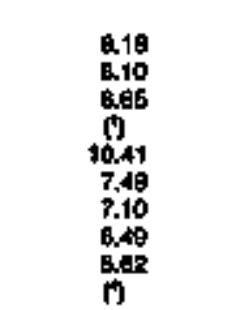 \\
\hline
\end{tabular}

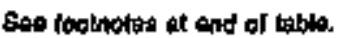


Table 15. Class of Ownership, Number of Ultimate Consumers, Revenue, Sales, and Average Revenue per Kllowatthour for the Commerlcal Sector by State and Utility, 1993 (Continued)

\begin{tabular}{|c|c|c|c|c|c|}
\hline $\begin{array}{c}\text { Stit: } \\
\text { Elogtok Uinhy }\end{array}$ & $\begin{array}{c}\text { Clape } \\
\text { of } \\
\text { twmerehlp }\end{array}$ & $\begin{array}{l}\text { tumber } \\
\text { of } \\
\text { condumarg }\end{array}$ & 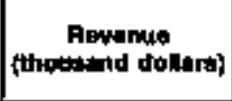 & 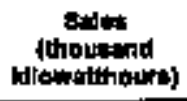 & 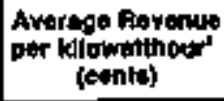 \\
\hline 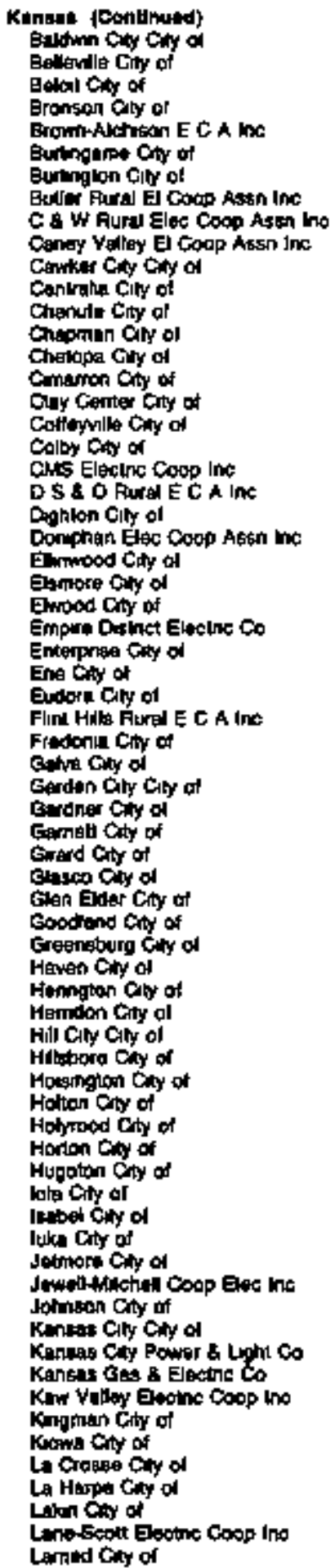 & 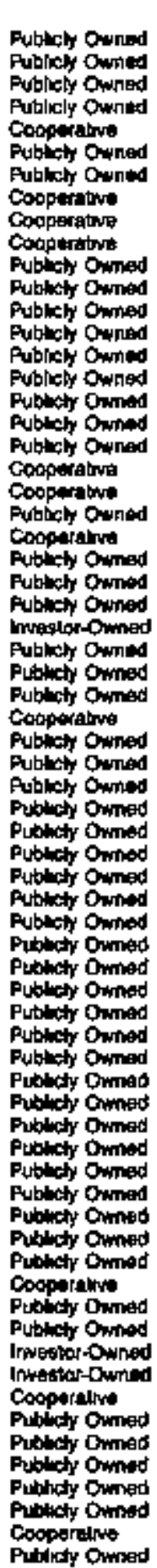 & 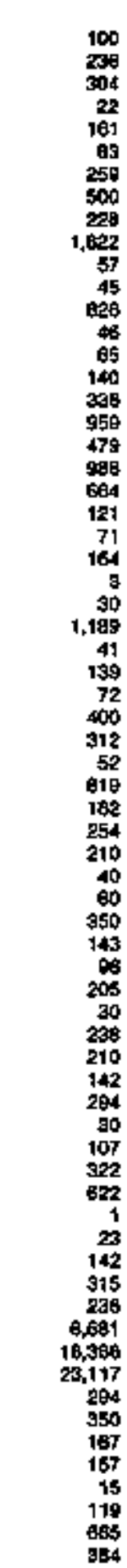 & 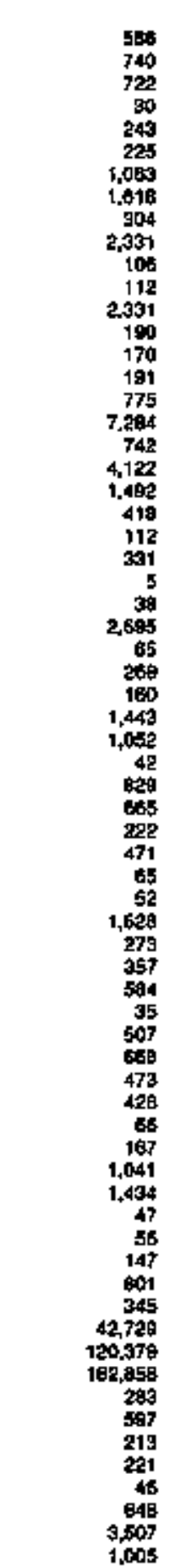 & 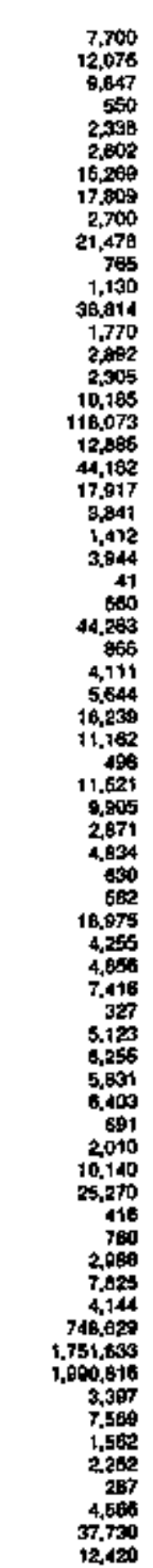 & 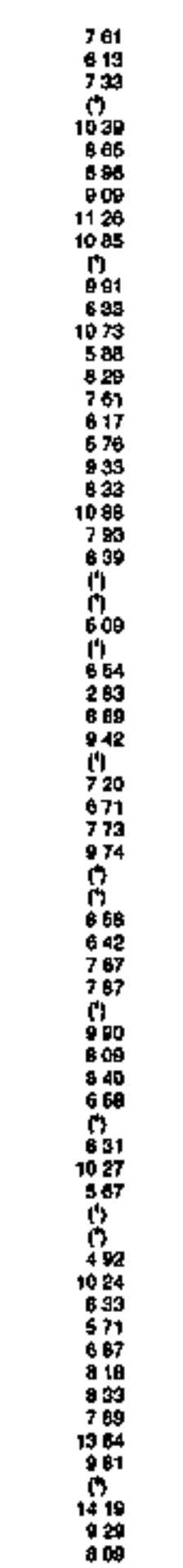 \\
\hline
\end{tabular}

Swe loolnotes at end 어 Inble 
Table 15. Class of Ownershlp, Number of Ultimate Consumers, Revente, Sales, and Average Revemie per Kilowatthour for the Commerloal Sector by State and Utolity, 1998 (Continued)

\begin{tabular}{|c|c|c|c|c|c|}
\hline Stalin & 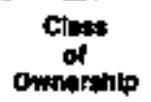 & $\begin{array}{c}\text { Ammber } \\
\text { of } \\
\text { contumert }\end{array}$ & 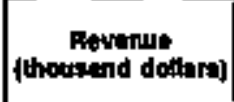 & 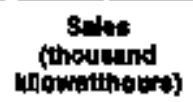 & 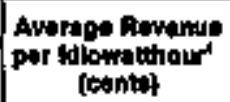 \\
\hline
\end{tabular}

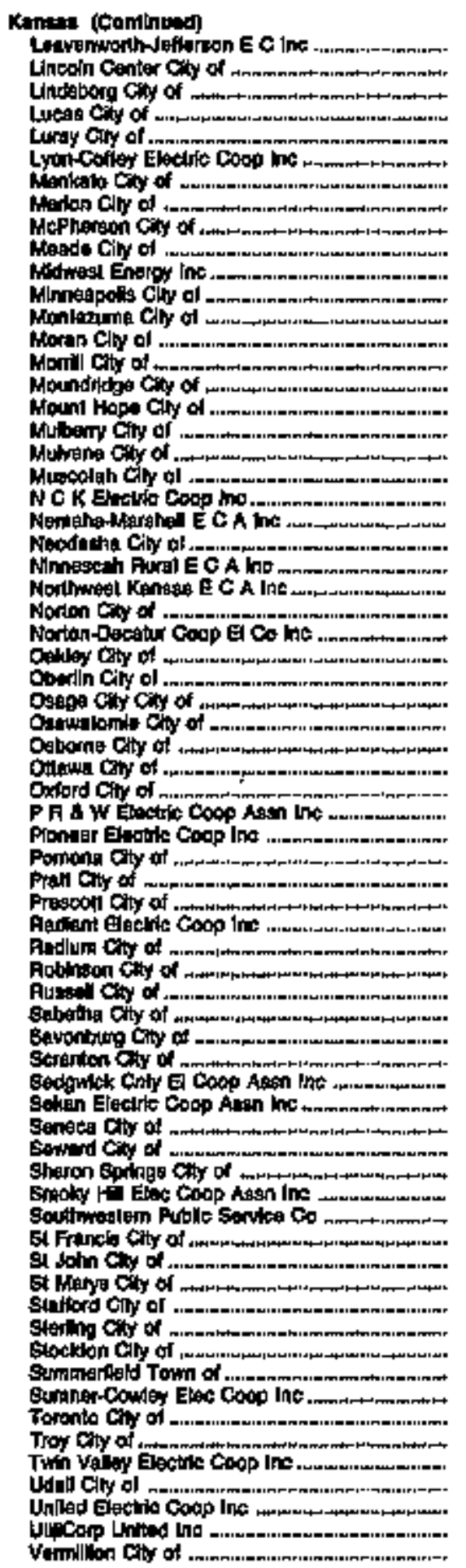

Cooparaling

Fulpiry Ouned

Putraty Owhed

Puticitr Ommed

Publtitr Omid

Cooperativin

Pubrition Oewnerd

Puthery Oumad

Pulacht Quned

Publith Ownd

Cooperathe

Fublict Owing

Pubicht Owhad

Pubrich Oimid

Fublet; Owingod

Publith Owhed

Publich owned

Fubiels ownex

Publictr Ownod

Publish Onmod

Cooperative

Copepertives

Publech owned

Cooperotintio

Conpertive

Publey Owned

Cooperaling

Aubten Owhed

Publely Owned

Publity Owhad

Fublicly ouned

Futbliebs Owised

fublesy Owned

Fublinty Owrod

Cooptrative

Cooperatha

Pularis O-ined

Publicly Ownted

Publicly Owred

Copperatín

Fublety owned

Publey Oringa

Pubick owned

Fublely Ownid

Publey Owned

Fublets Dewned

Copoperativi

Cooperative

Fubiely owned

Pubrest Owned

Publicly Owise

cooperalin

invertor-Dwmed

Fublich Ownax

Publist Owned

Fublety Ownad

Publopy Owned

Fublits owned

Publist Owned

Publits owned

Croperative

Publasy Owned

Fuling Orined

Cooperative

Fubileky owned

Cosperativis

imiaslor-Onmeo

Fubilety Gened

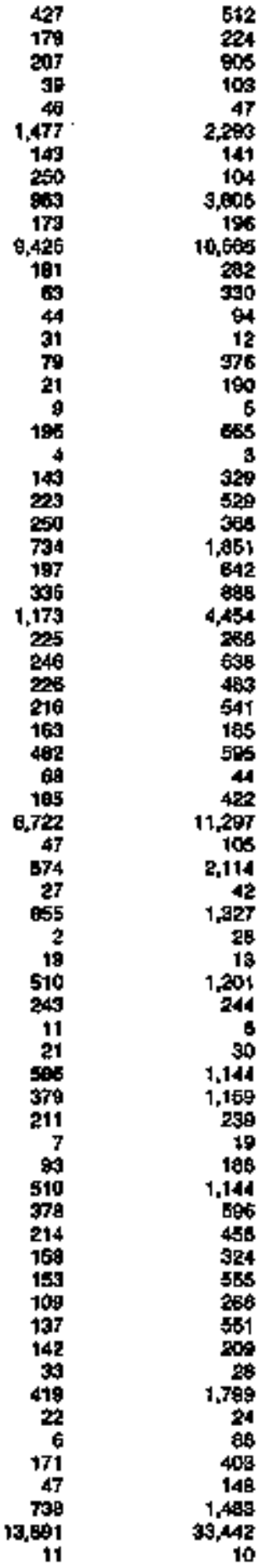

4,809
2,737

a.

218

7.36

(1)

2.62

$1,410 \quad 7.38$

05.940

2210 0.54

125,512

4.707

3.643

1,870

1.264

80

6.386

2,844

6.035

3.576

26,002

5,734

11,249

42659

3,702

6. 昗

7.288

7,350

1.649

8.870

629

4,093

112,160

1.737

30,013

18,669

165

16,727

3,630

50

12,530

13,854

3.089

601

1.647

12,381

9.574

4,141

3,460

6,657

2084

6.717

2.550

302
808

16.008

85

3,295

1.606

14807

477,659

107 
Table 15. Class of Owmership, Number of Ultimate Consumers, Revenue, Sales, and Average Revenue per Kilowatthour for the Commerical Sector by State and Utillty, 1993 (Continuted)

\begin{tabular}{|c|c|c|c|c|c|}
\hline $\begin{array}{c}\text { Btats } \\
\text { enectidc utilly }\end{array}$ & $\begin{array}{c}\text { Claps } \\
\text { of } \\
\text { Omperatip }\end{array}$ & $\begin{array}{l}\text { Munbar } \\
\text { of } \\
\text { conoumere }\end{array}$ & 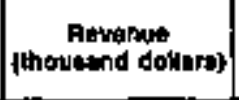 & $\begin{array}{l}\text { Sylwe } \\
\text { (1housend } \\
\text { dilowathours) }\end{array}$ & 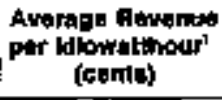 \\
\hline 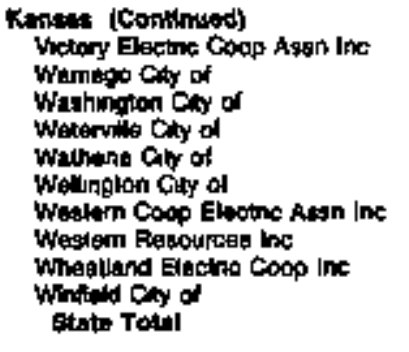 & 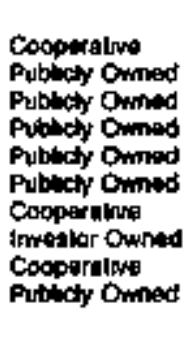 & $\begin{array}{r}371 \\
216 \\
138 \\
72 \\
97 \\
490 \\
1,299 \\
99,068 \\
5,020 \\
1.171 \\
182,410\end{array}$ & 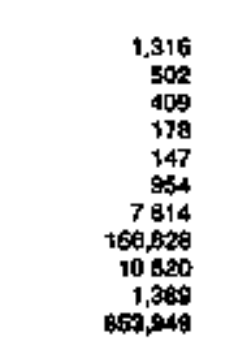 & $\begin{array}{r}14400 \\
6,513 \\
4,793 \\
2,297 \\
1,094 \\
11,996 \\
69,173 \\
3,109,539 \\
98,729 \\
18,744 \\
0,753,224\end{array}$ & $\begin{array}{l}914 \\
771 \\
893 \\
776 \\
602 \\
795 \\
964 \\
504 \\
1098 \\
741 \\
5.70\end{array}$ \\
\hline 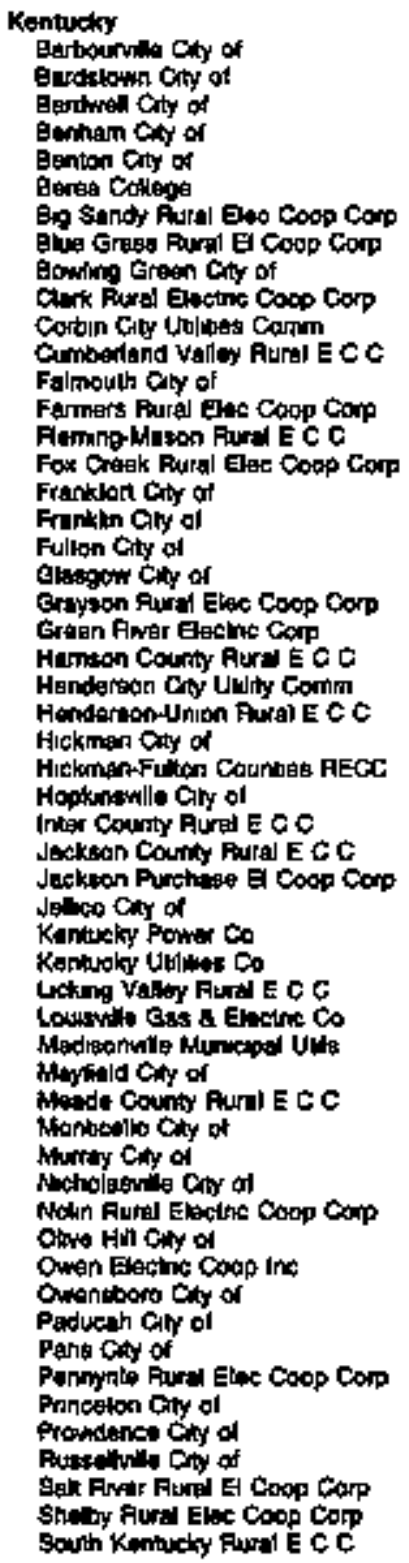 & 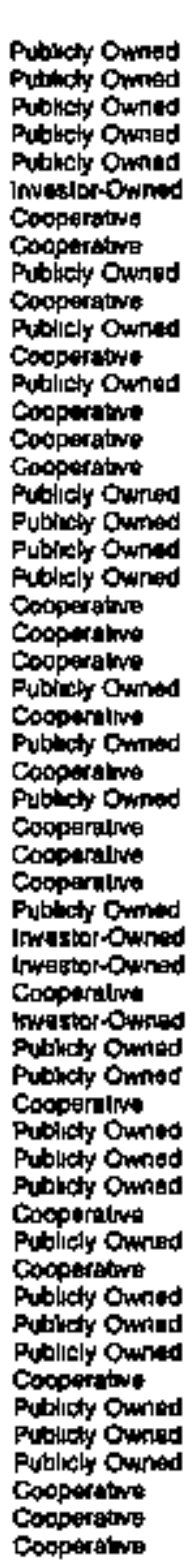 & 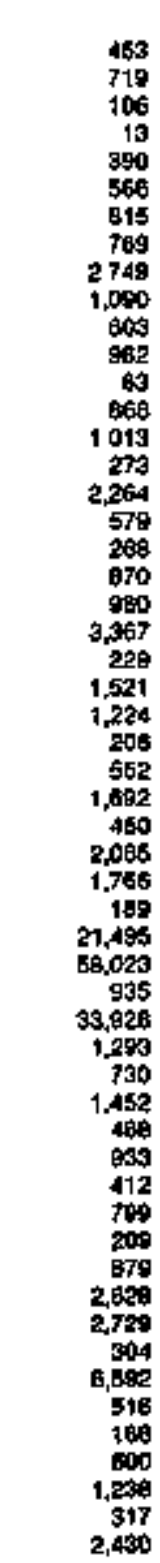 & 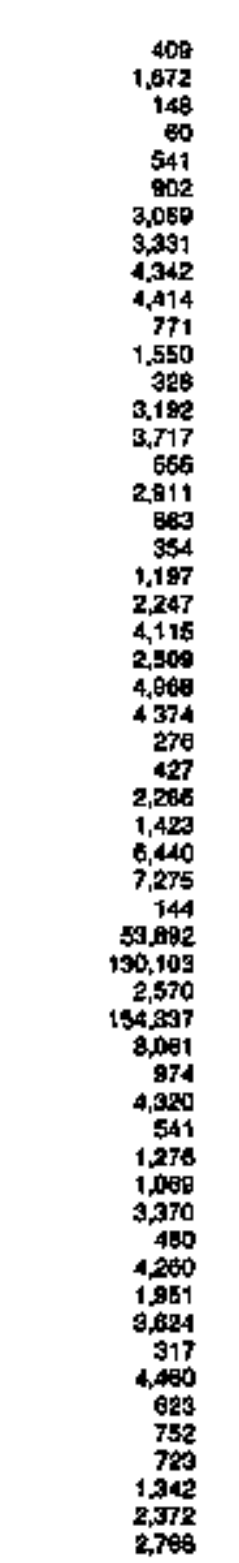 & 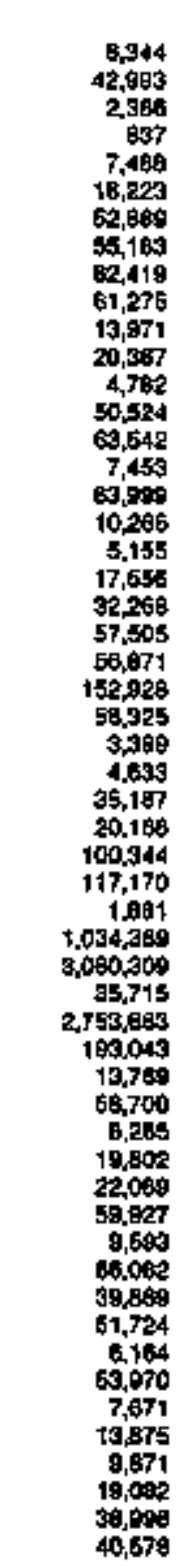 & 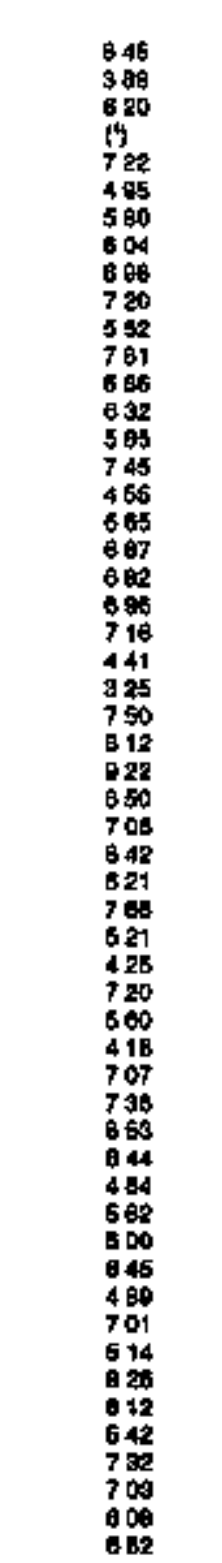 \\
\hline
\end{tabular}

sot foolnothe th and of lable 
Table 15. Claste of Ownershlp, Number of UItimate Consumers, Revenue, Sates, and Average Rovenue per Kllowatthour for the Commerical Sector by State and Uullity, 1993 (Continued)

\begin{tabular}{|c|c|c|c|c|c|}
\hline 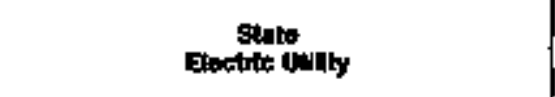 & ctasp & $\begin{array}{l}\text { Hunder } \\
\text { of } \\
\text { Congulaters }\end{array}$ & Rowenum & $\begin{array}{c}\text { Saloa } \\
\text { (thot:and } \\
\text { ldionintibours] }\end{array}$ & 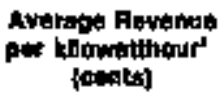 \\
\hline 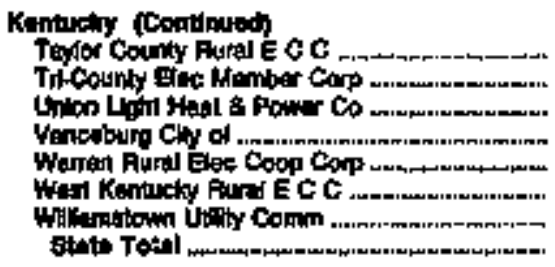 & 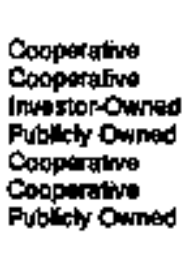 & $\begin{array}{r}1,790 \\
4,349 \\
10,701 \\
295 \\
5,192 \\
4,311 \\
188 \\
195,128\end{array}$ & $\begin{array}{r}4,023 \\
3,423 \\
47,510 \\
566 \\
4,400 \\
3,926 \\
679 \\
519,472\end{array}$ & $\begin{array}{r}65,416 \\
46,392 \\
772,691 \\
10,072 \\
54,132 \\
46,057 \\
9,0000 \\
9,028,996\end{array}$ & $\begin{array}{l}6.15 \\
7.30 \\
8.16 \\
5.92 \\
6.14 \\
8.62 \\
7.49 \\
5.98\end{array}$ \\
\hline 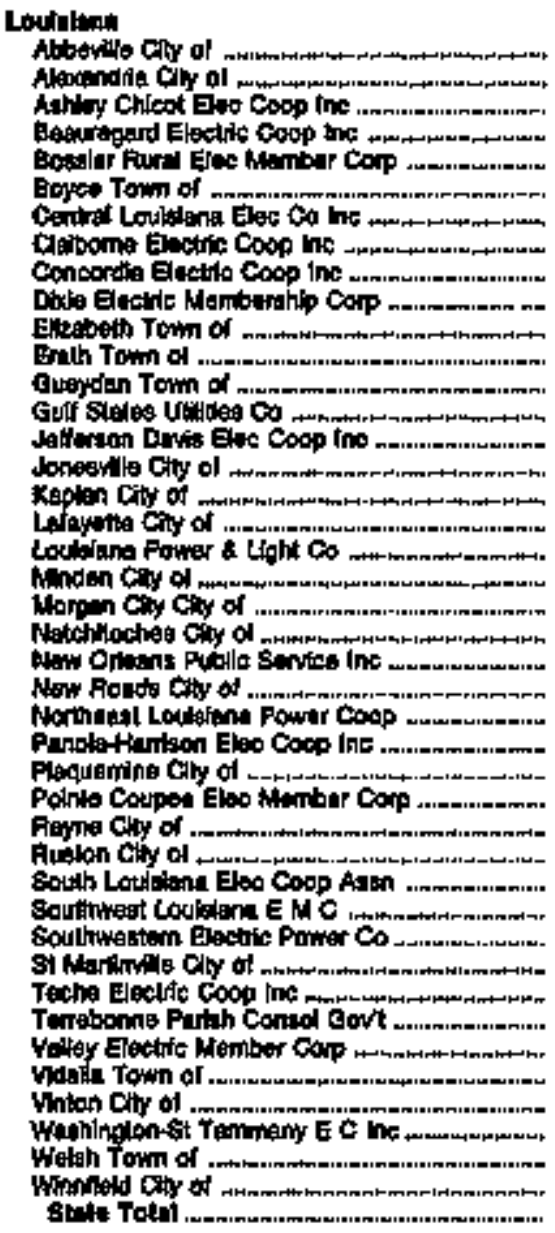 & 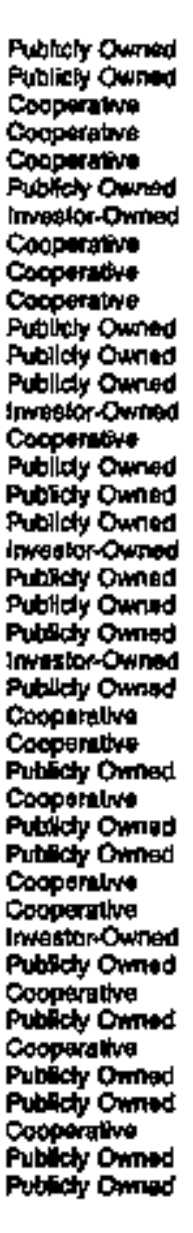 & 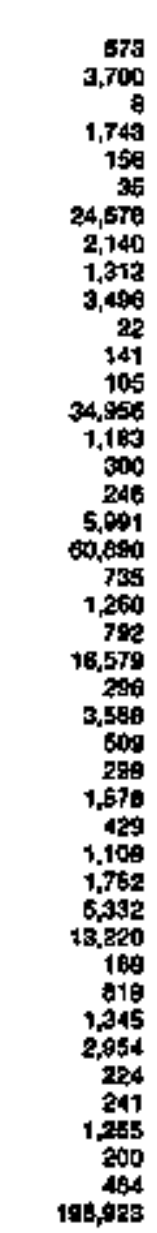 & 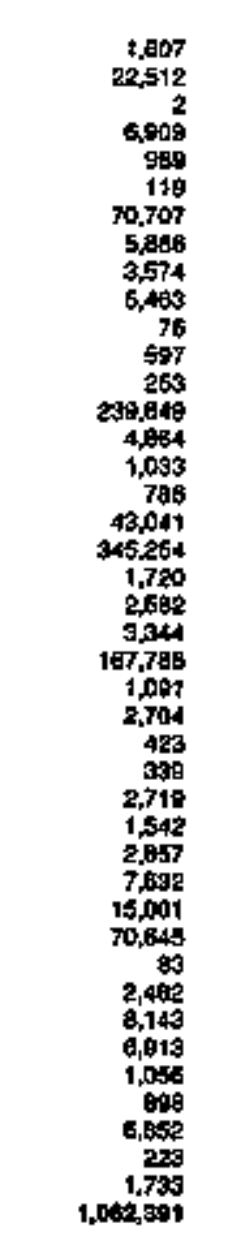 & 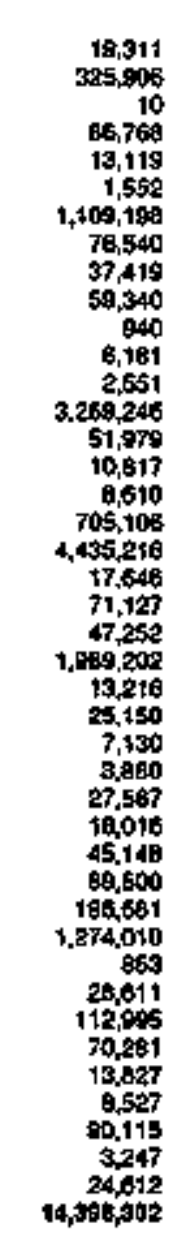 & 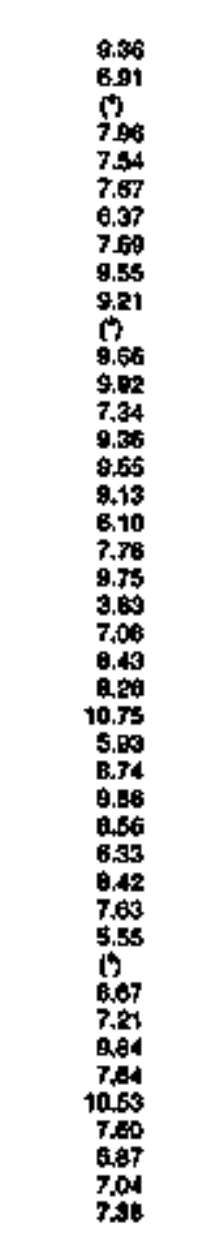 \\
\hline 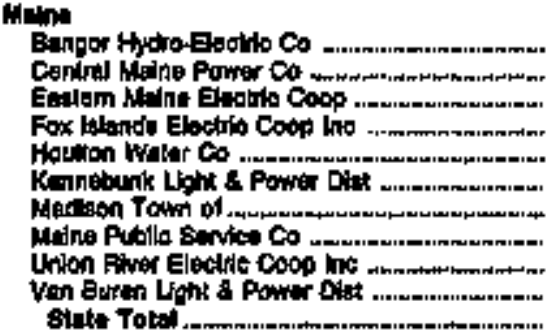 & 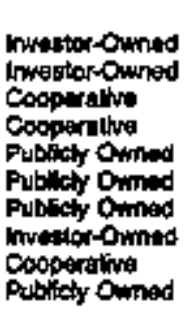 & $\begin{array}{r}12,575 \\
40,518 \\
1,294 \\
180 \\
725 \\
560 \\
205 \\
6,354 \\
60 \\
235 \\
65,707\end{array}$ & 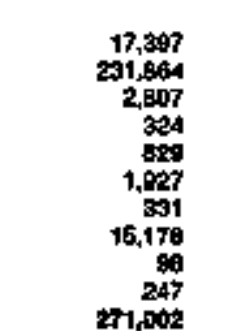 & $\begin{array}{r}149,672 \\
2,493,311 \\
28,095 \\
1,899 \\
8,004 \\
20,617 \\
3,202 \\
10,049 \\
671 \\
2,412\end{array}$ & $\begin{array}{r}11.83 \\
0.30 \\
10.00 \\
\$ 7.15 \\
0.64 \\
0.36 \\
\$ 0.34 \\
0.31 \\
10 \\
10.24 \\
0.45\end{array}$ \\
\hline
\end{tabular}

8mb rootiolos of end ol labia. 
Table 15. Class of Ownership, Number of Ultimate Consumers, Revenue, Seles, and Average Revenue per Kilowathour tor the Commerical Sector by State and Utibty, 1993 (Continued)

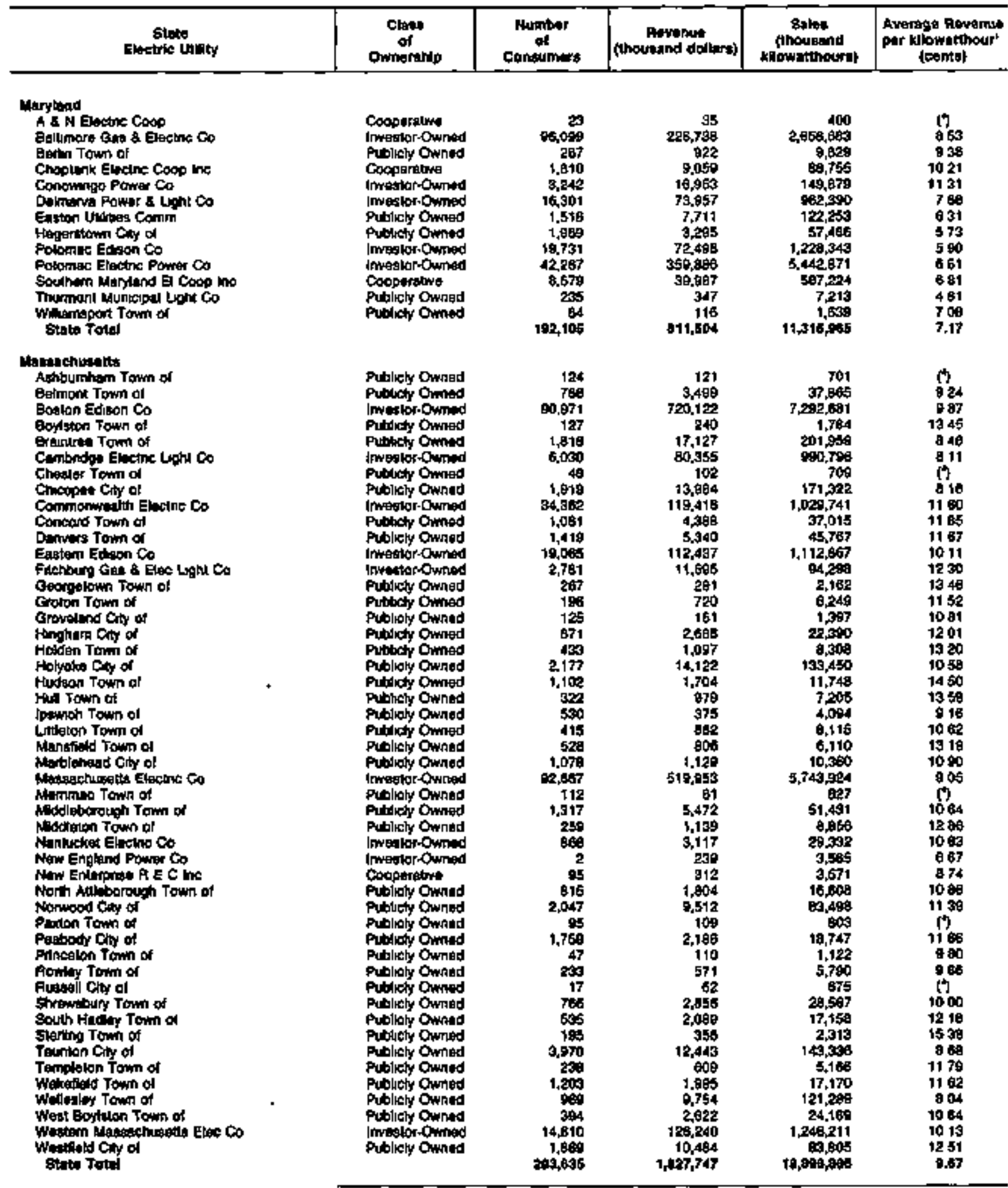

Ser foothotea al and of trbe 
Table 15. Class of Ownership, Number of Ultimate Consumers, Revenue, Sales, and Average Revenue per Kilowatthour for the Commerical Sector by State and Utility, 1993 (Continued)

\begin{tabular}{|c|c|c|c|c|c|}
\hline $\begin{array}{c}\text { Stata } \\
\text { enestito toidly }\end{array}$ & otias & $\begin{array}{c}\text { thimber } \\
\text { of } \\
\text { Cantumitim }\end{array}$ & 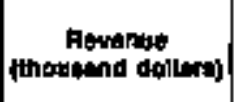 & $\begin{array}{l}\text { thlet } \\
\text { hlousind }\end{array}$ & 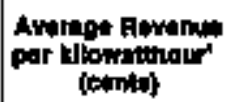 \\
\hline 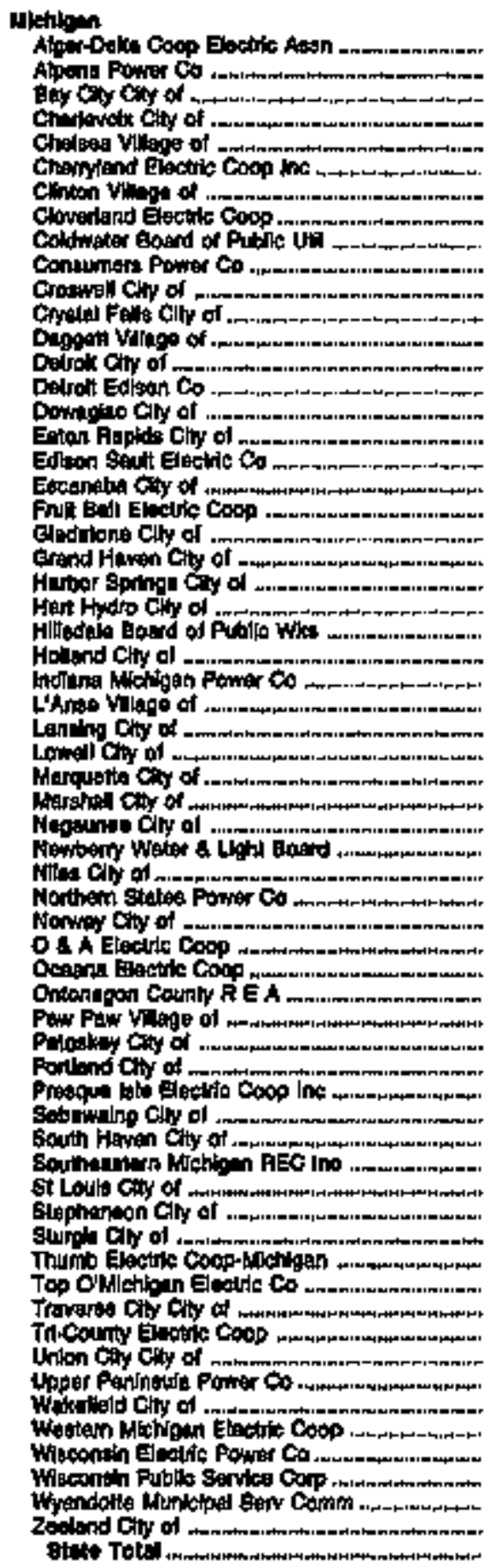 & 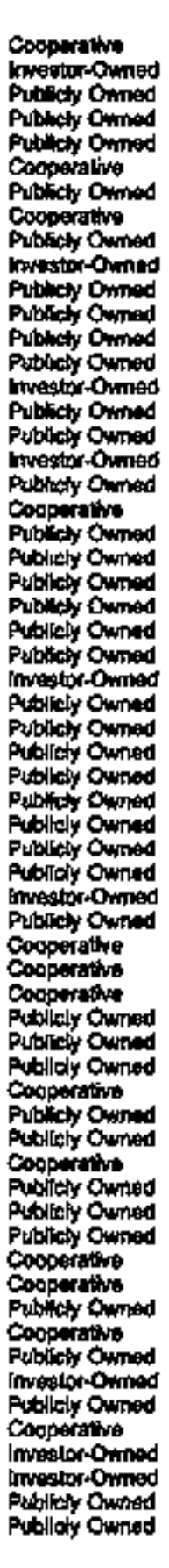 & 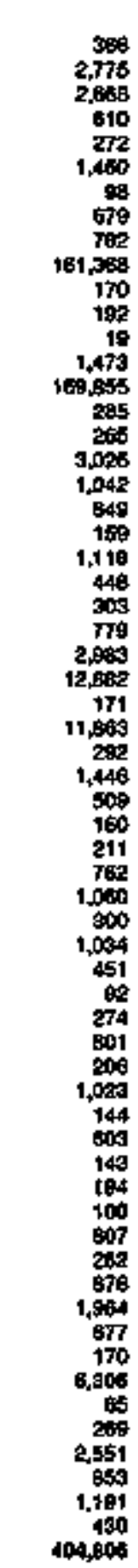 & 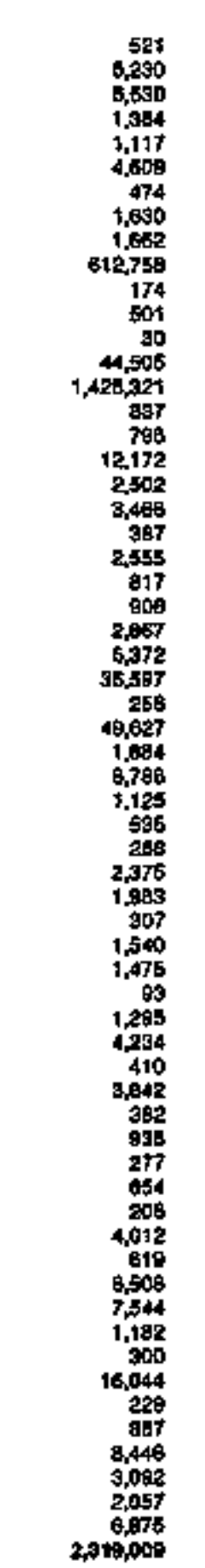 & 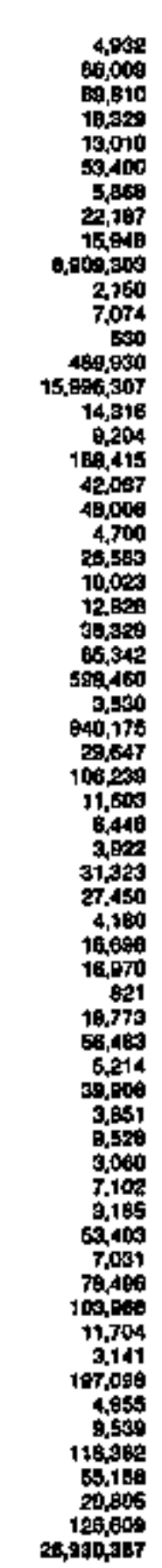 & 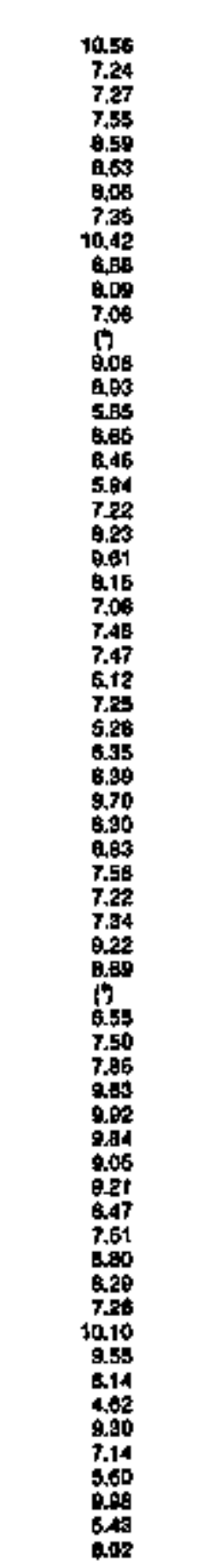 \\
\hline 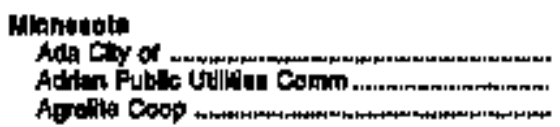 & 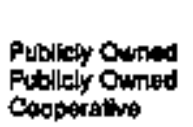 & $\frac{181}{70}$ & $\begin{array}{l}362 \\
115 \\
623\end{array}$ & $\begin{array}{l}7,007 \\
2,011 \\
0,142\end{array}$ & $\begin{array}{l}4.43 \\
5.68 \\
6.81\end{array}$ \\
\hline
\end{tabular}

sa looboles ex end ol luble. 
Table 15. Class of Ownership, Number of Ultimate Consumers, Revenue, Sales, and Average Revenue per Kllowatthour tor the Commerical Sector by State and Utility, 1993 (Continued)

\begin{tabular}{|c|c|c|c|c|c|}
\hline $\begin{array}{c}\text { State } \\
\text { Gaetiflc Uutity }\end{array}$ & $\begin{array}{c}\text { Clasa } \\
\text { of } \\
\text { Ommortohtp }\end{array}$ & $\begin{array}{l}\text { Number } \\
\text { of } \\
\text { conoumara }\end{array}$ & Ahousend dollars? & $\begin{array}{c}\text { Sales } \\
\text { (thoutind } \\
\text { Allowsthodros }\end{array}$ & 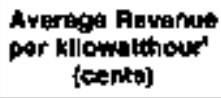 \\
\hline 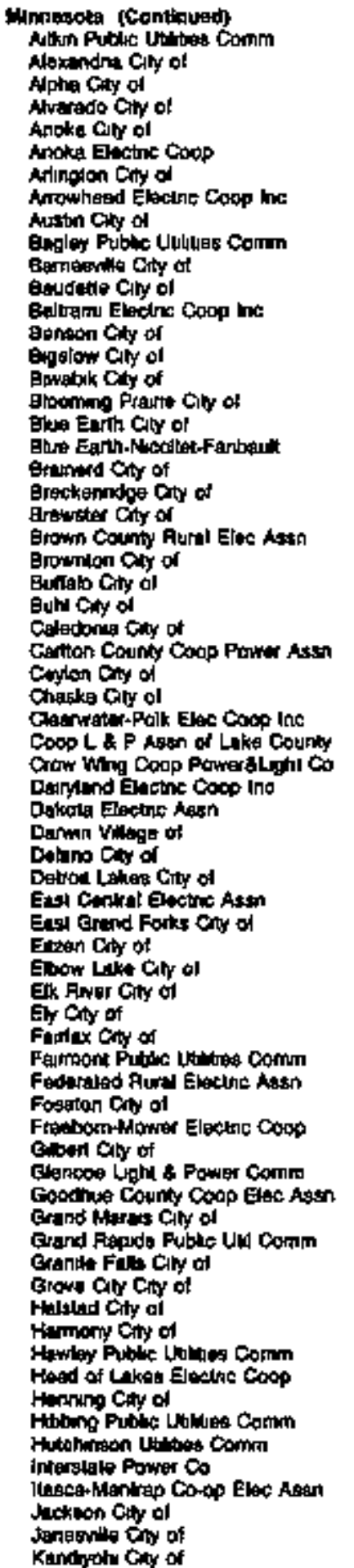 & 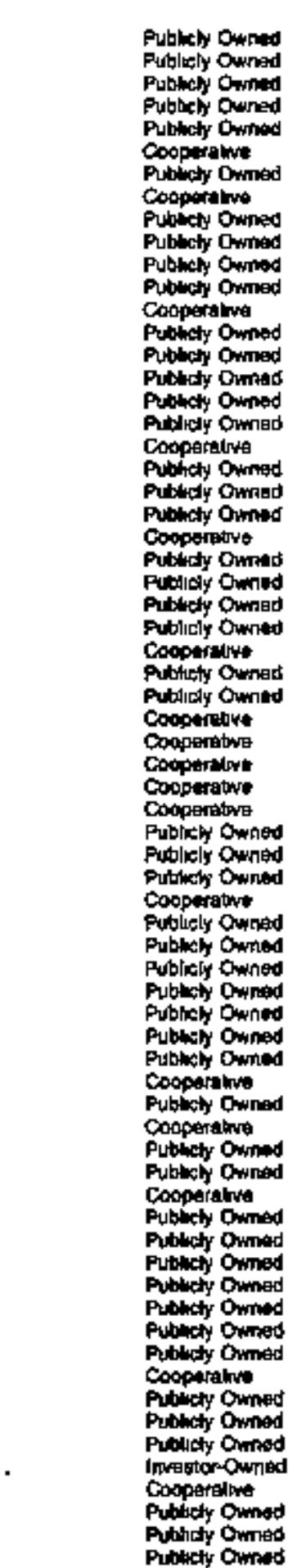 & 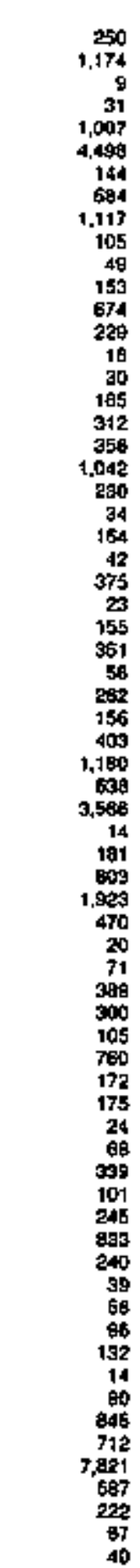 & 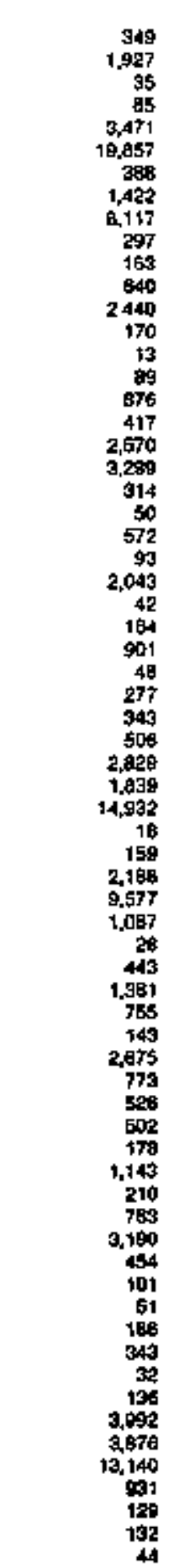 & 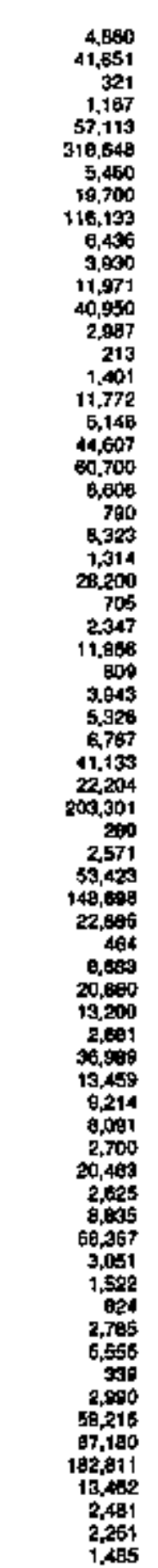 & 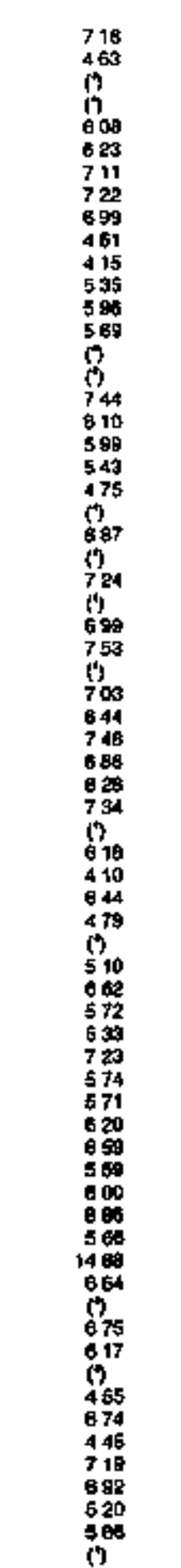 \\
\hline
\end{tabular}

Sog footnoles al and of rabte 
Teble 15. Class of Ownershlp, Number of Ultmate Constumers, Fevenue, Sales, and Averege Revenue per KIlowatthour for the Commerteal Sector by State and Utility, 1993 (Conthued)

\begin{tabular}{|c|c|c|c|c|c|}
\hline $\begin{array}{l}\text { Sinte } \\
\text { Eisctolo Uanity }\end{array}$ & $\begin{array}{c}\text { ctist } \\
\text { of } \\
\text { onmonilp }\end{array}$ & $\begin{array}{c}\text { A:mber } \\
\text { of } \\
\text { Copounents }\end{array}$ & Rrownes & 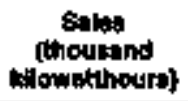 & 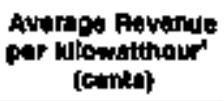 \\
\hline 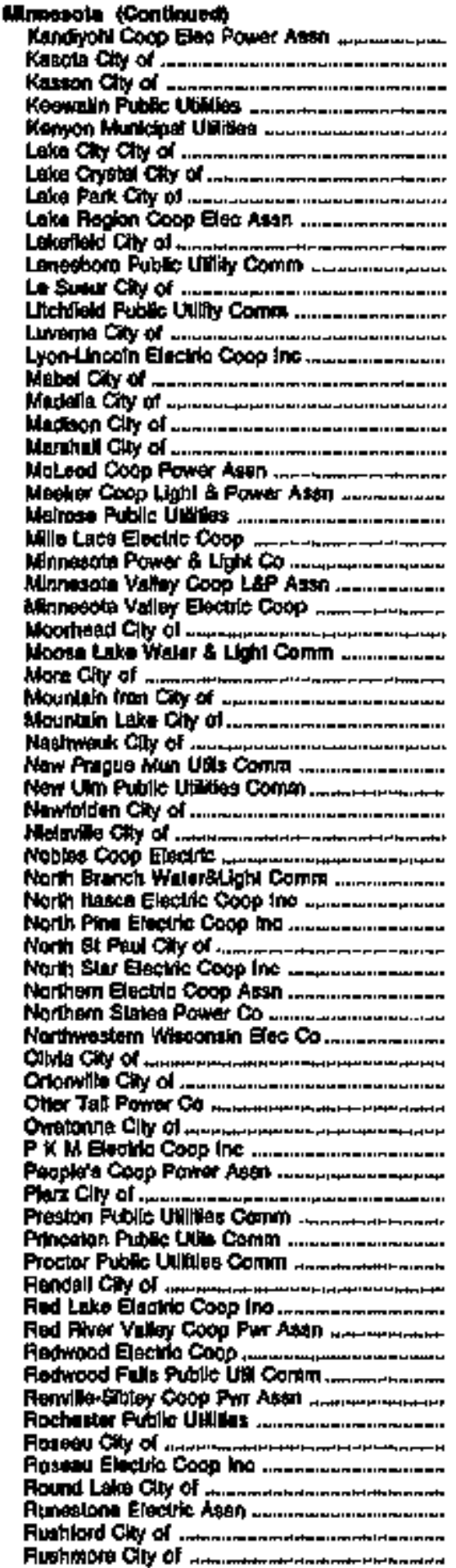 & 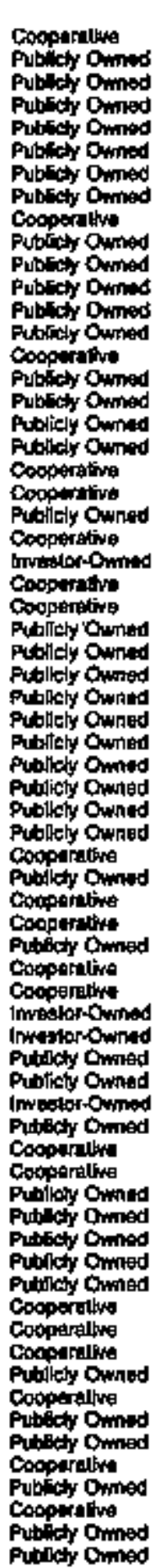 & 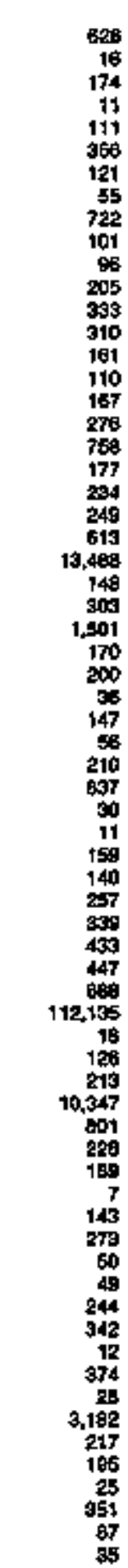 & 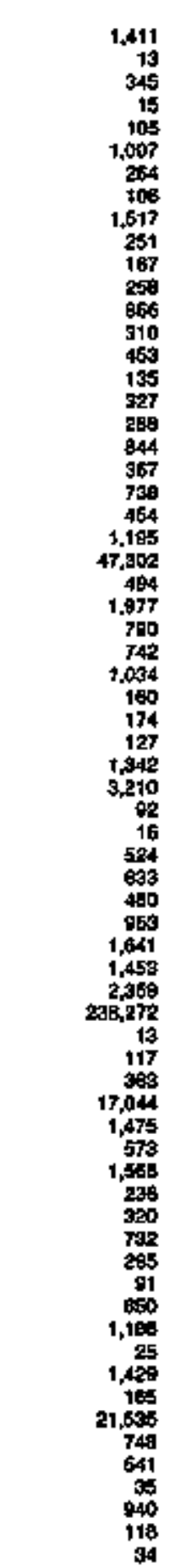 & 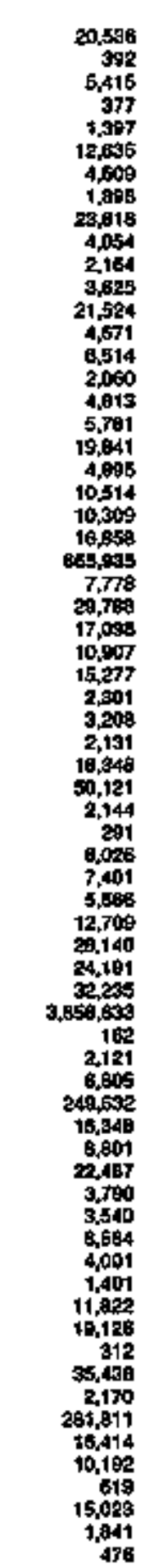 & 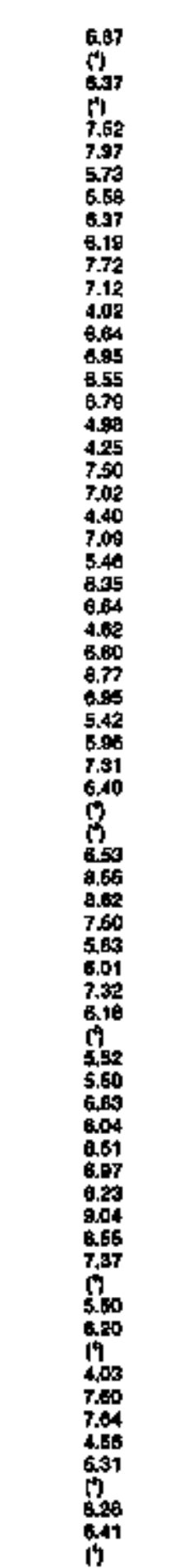 \\
\hline
\end{tabular}

Ser loolnotes al and of table. 
Table 15. Class of Ownership, Number of Ultimate Consumers, Revenue, Sales, and Average Revenue per KHowatthour for the Commerleal Sector by State and Utility, 1993 (Continued)

\begin{tabular}{|c|c|c|c|c|c|}
\hline $\begin{array}{c}\text { stint } \\
\text { Galectic Utifty }\end{array}$ & $\begin{array}{c}\text { Cilate } \\
\text { of } \\
\text { Onwainhlp }\end{array}$ & $\begin{array}{c}\text { Chinbur } \\
\text { of } \\
\text { Contumare }\end{array}$ & $\begin{array}{c}\text { Renend } \\
\text { (thousand dotera) }\end{array}$ & 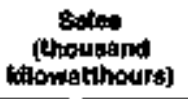 & $\begin{array}{c}\text { Average Revenus } \\
\text { par kllowalthaur } \\
\text { (cents) }\end{array}$ \\
\hline 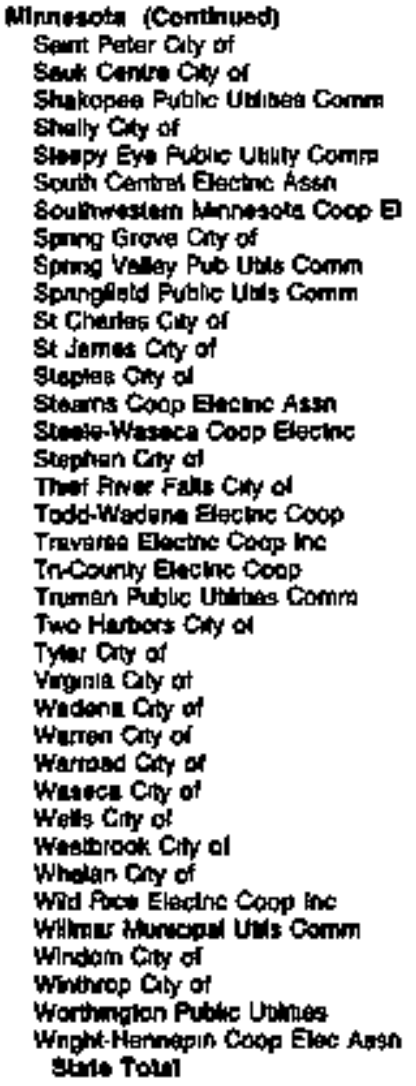 & 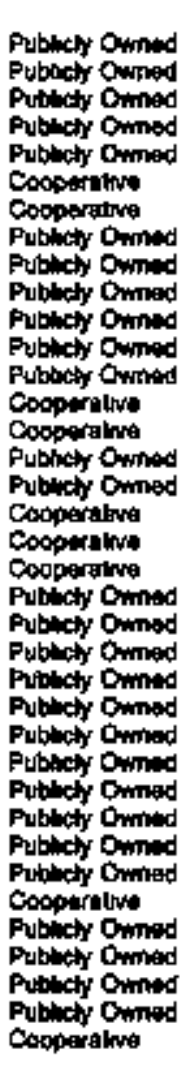 & 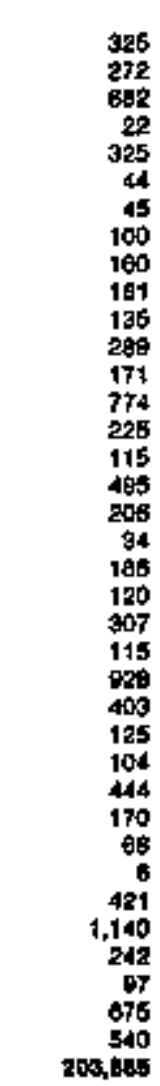 & 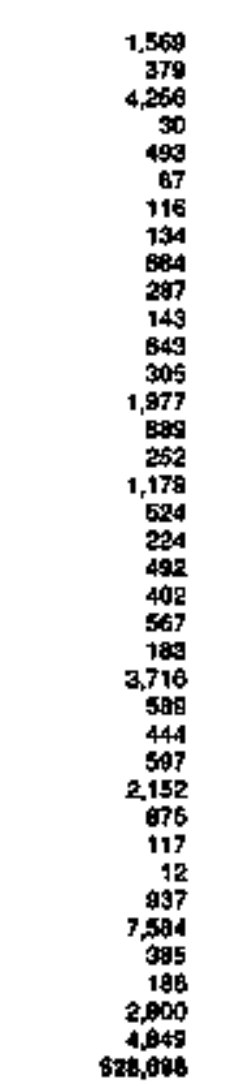 & 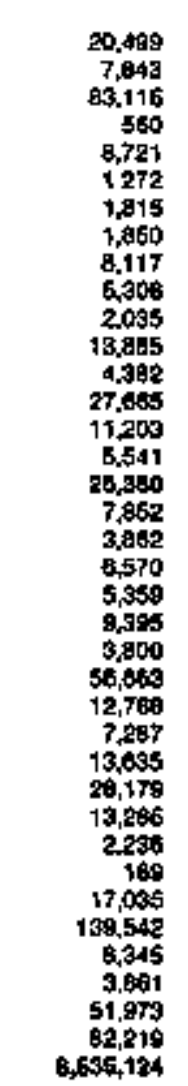 & 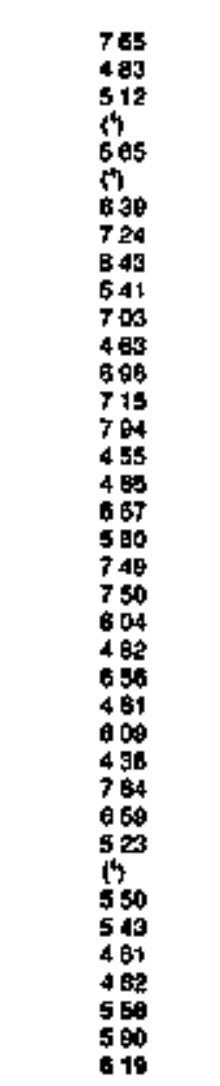 \\
\hline 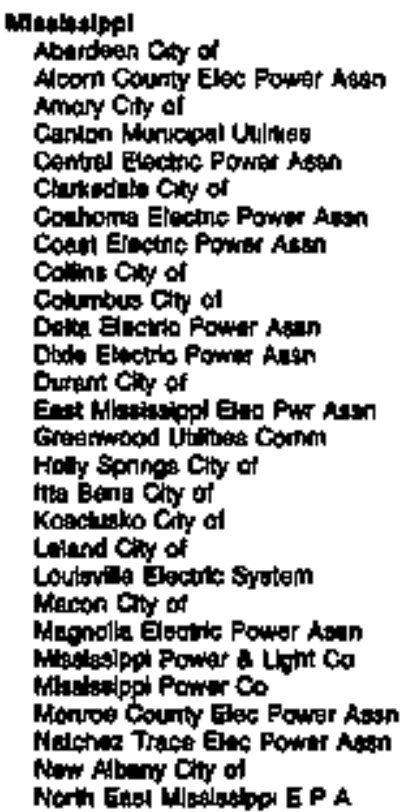 & 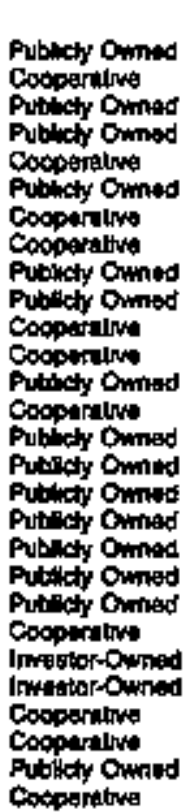 & 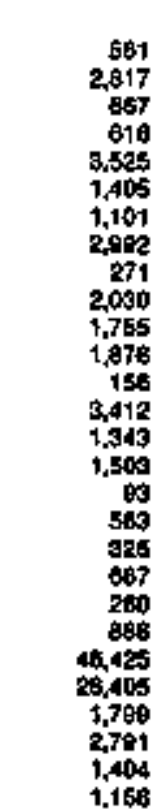 & 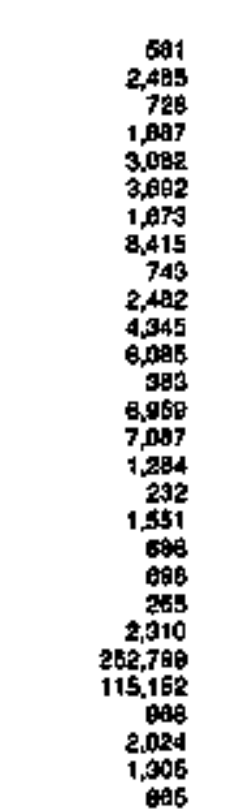 & 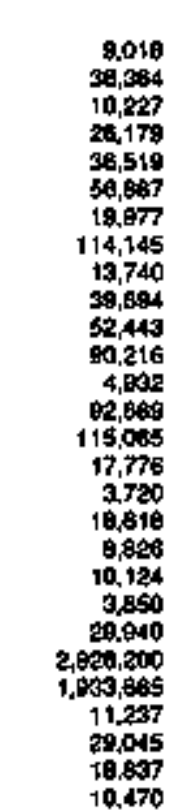 & 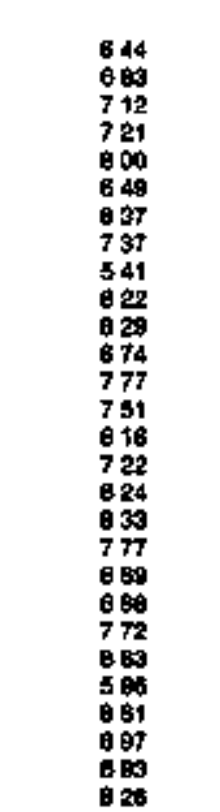 \\
\hline
\end{tabular}

Sos lootnote al end of table 
Table 15. Class of Ownershlp, Number of Uttimate Consumers, Revenue, Sales, and Average Revenue per Kllowatthour for the Commerical Sector by State and Uttlly, 1993 (Continued)

\begin{tabular}{|c|c|c|c|c|c|}
\hline 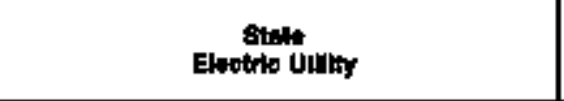 & $\begin{array}{c}\text { Clat: } \\
\text { of } \\
\text { Oininitap }\end{array}$ & $\begin{array}{c}\text { Rember } \\
\text { of } \\
\text { Conumber: }\end{array}$ & $\begin{array}{c}\text { Einwer } \\
\text { (thousand dolaral }\end{array}$ & 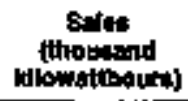 & 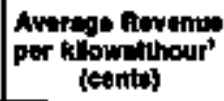 \\
\hline 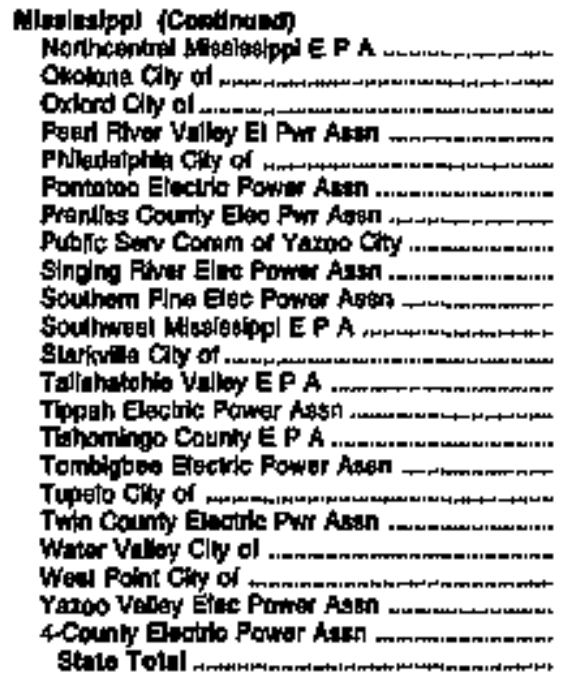 & 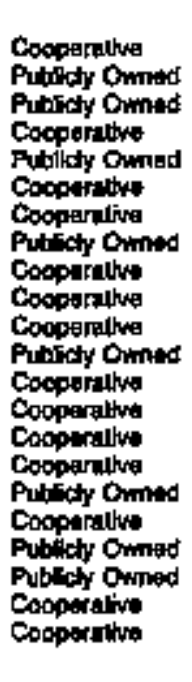 & 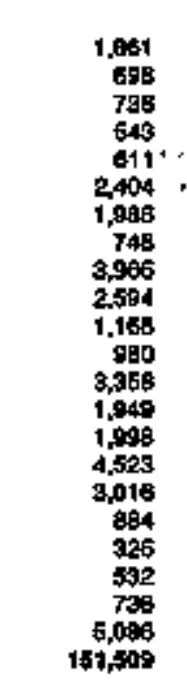 & 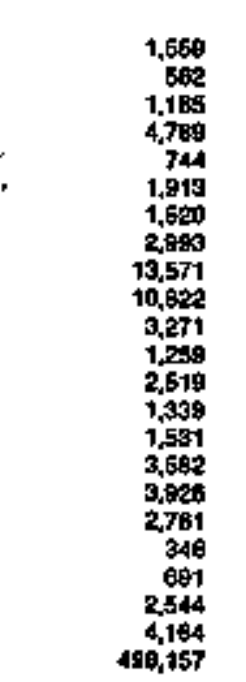 & 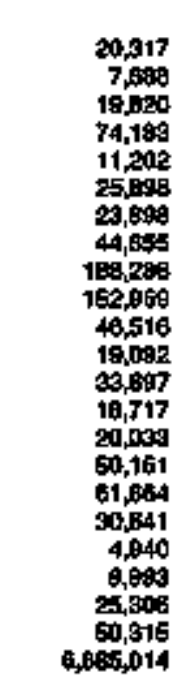 & 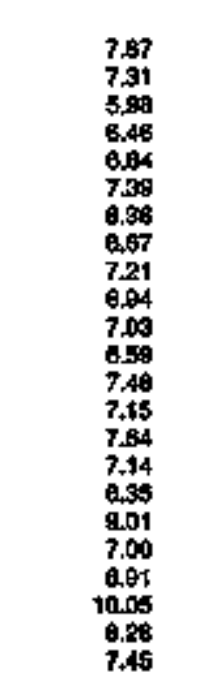 \\
\hline 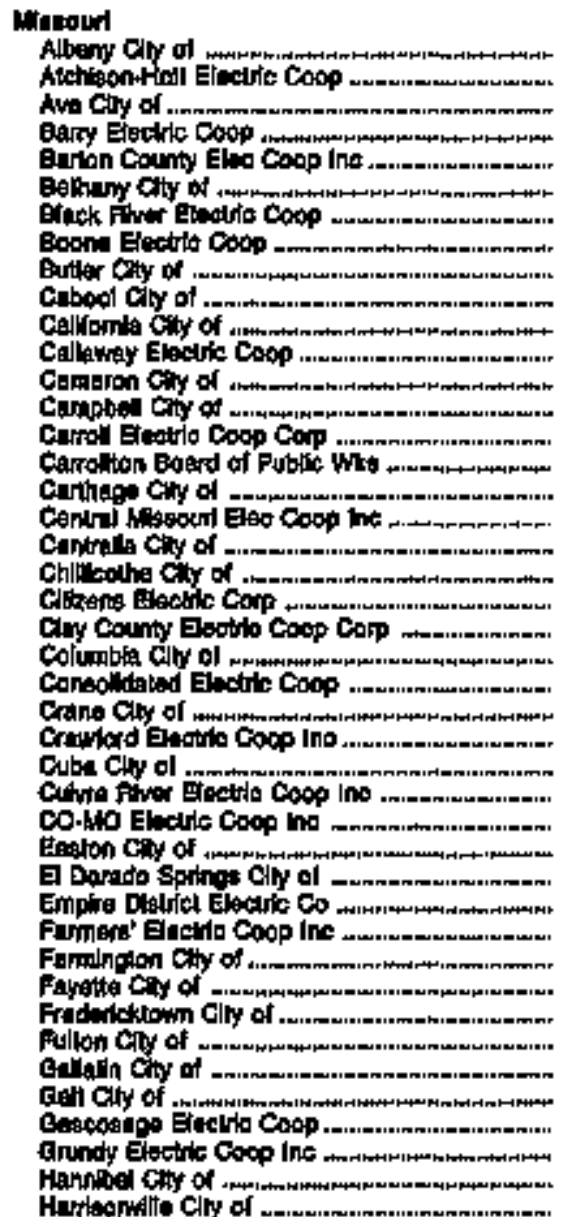 & 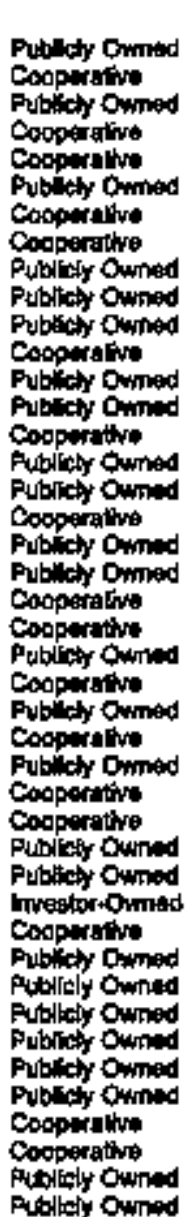 & 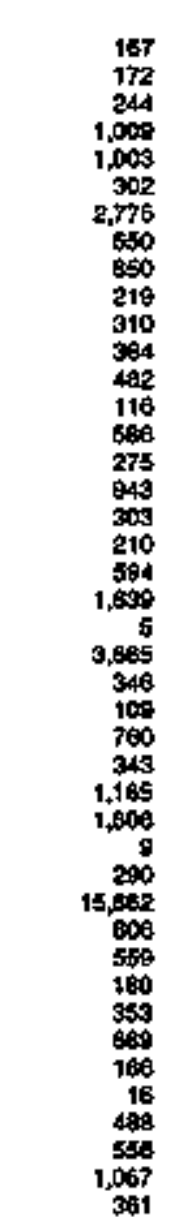 & 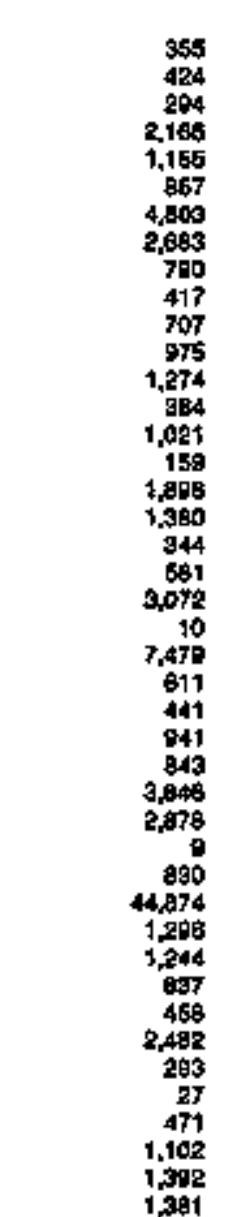 & 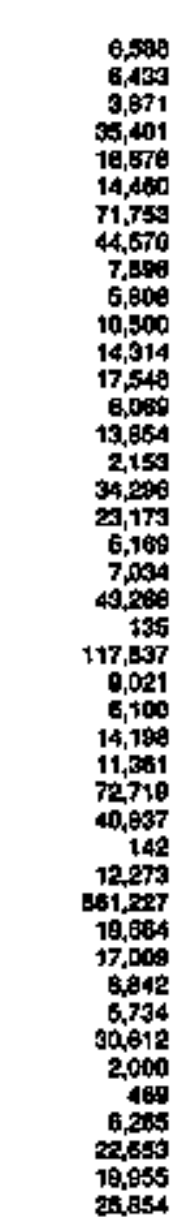 & 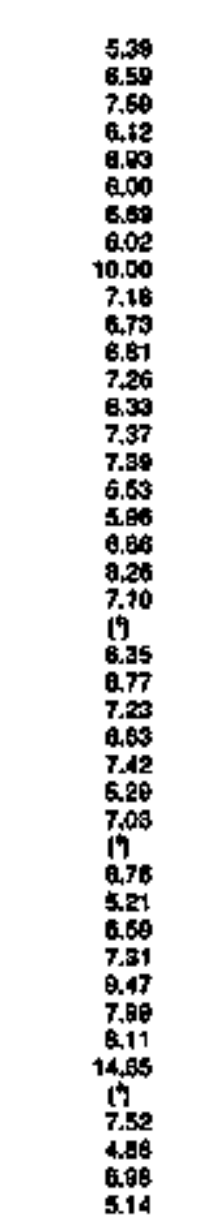 \\
\hline
\end{tabular}

5ea foolnotes at and of tobla 
Tobte 15. Class of Ownershlp, Number of Ultimate Consumers, Revenue, \$ales, and Average Revenute per Kilowatthour for the Commerlcal Sector by State and Utility, 1993 (Continued)

\begin{tabular}{|c|c|c|c|c|c|}
\hline $\begin{array}{c}\text { State } \\
\text { Elestric litity }\end{array}$ & 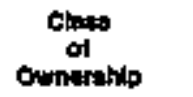 & $\begin{array}{c}\text { Numbar } \\
\text { af } \\
\text { Conumbes: }\end{array}$ & Gewnue & 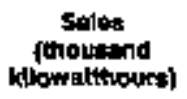 & $\begin{array}{l}\text { Awerage Ravione } \\
\text { per kliowatihour" } \\
\text { (ctinte) }\end{array}$ \\
\hline 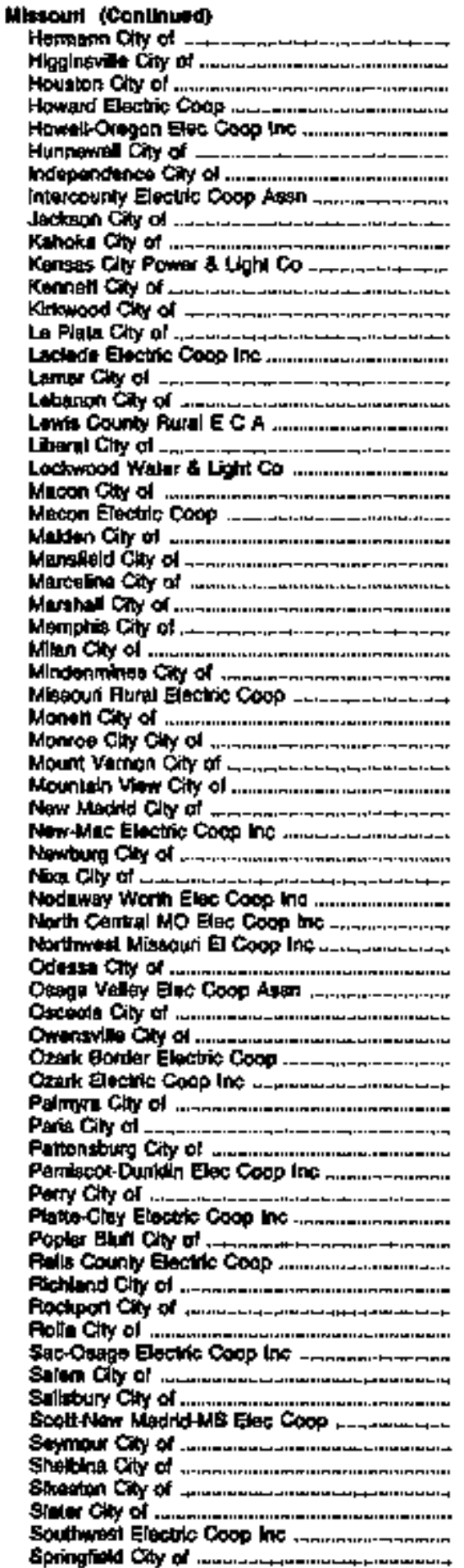 & 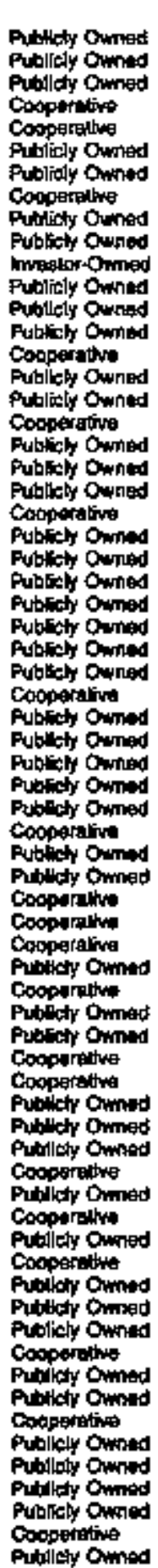 & 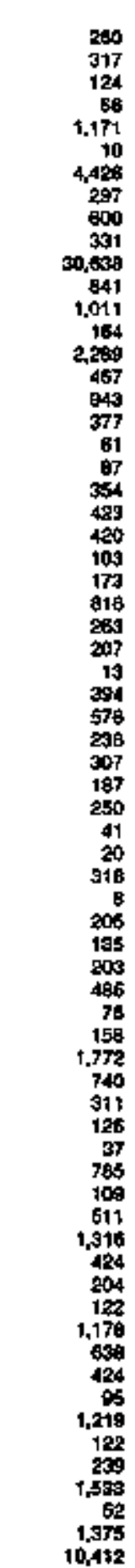 & 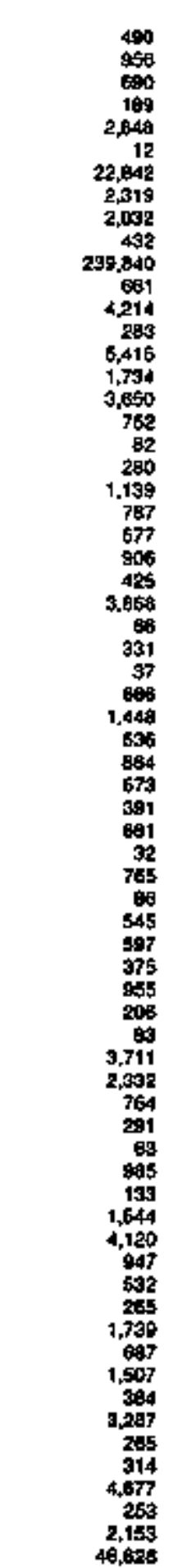 & 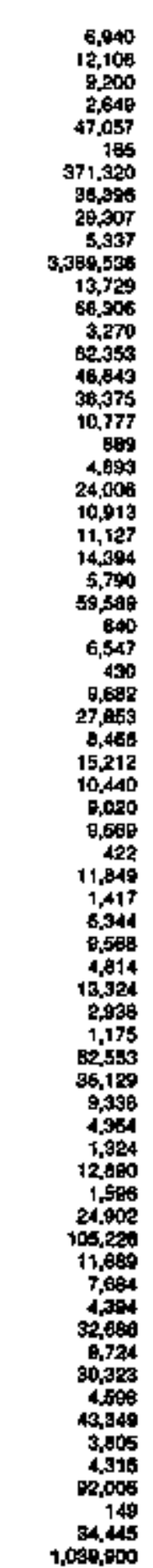 & 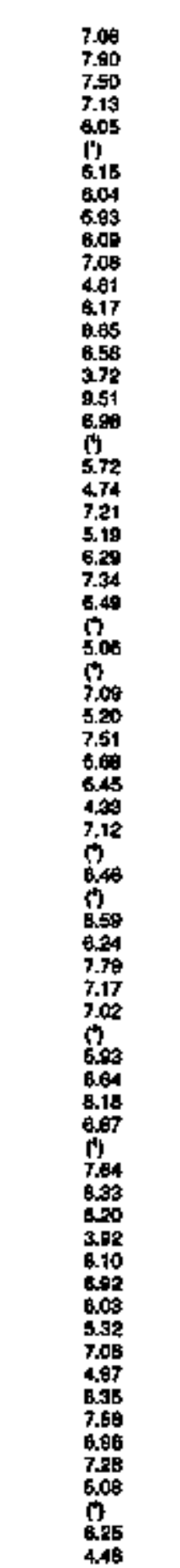 \\
\hline
\end{tabular}

See fooknolas at end ol lablo. 
Table 15. Class of Ownershlp, Number of Ulitmate Consumers, Revenue, 8sigs, and Average Revenue per Kllowatthour for the Cornmerical Sector by State and Utilty, 1993 (Continuted)

\begin{tabular}{|c|c|c|c|c|c|}
\hline 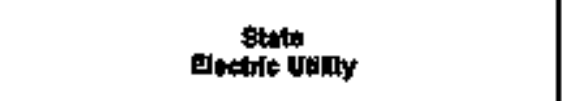 & 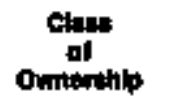 & $\begin{array}{c}\text { Rimbod } \\
\text { of } \\
\text { Comiumbrt }\end{array}$ & Rowitho & 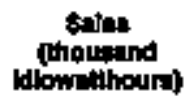 & 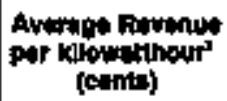 \\
\hline 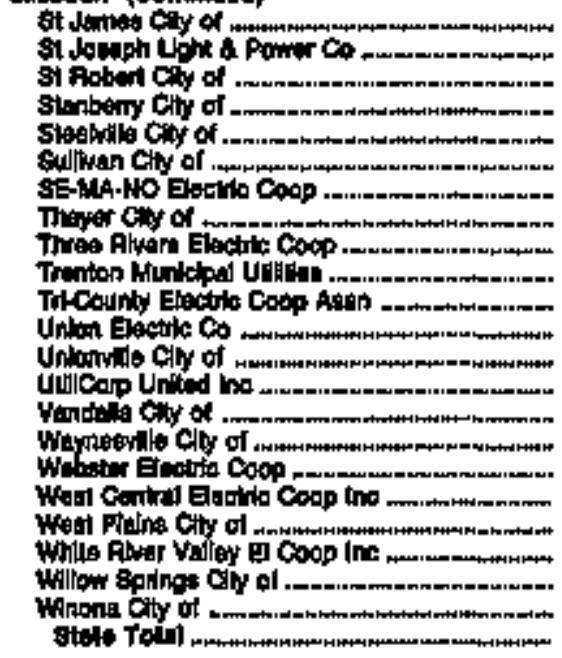 & 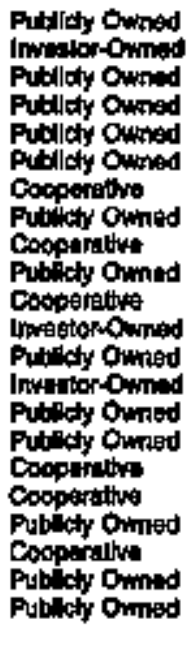 & 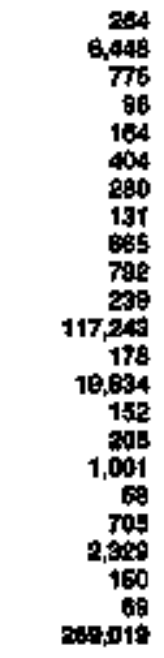 & 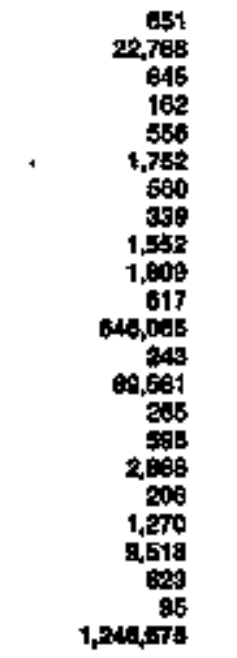 & 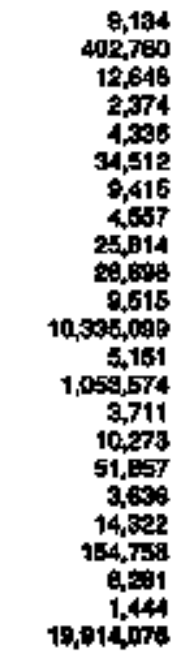 & 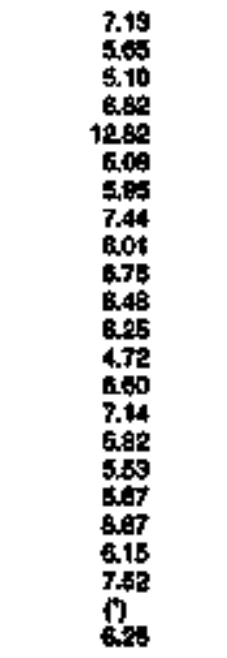 \\
\hline 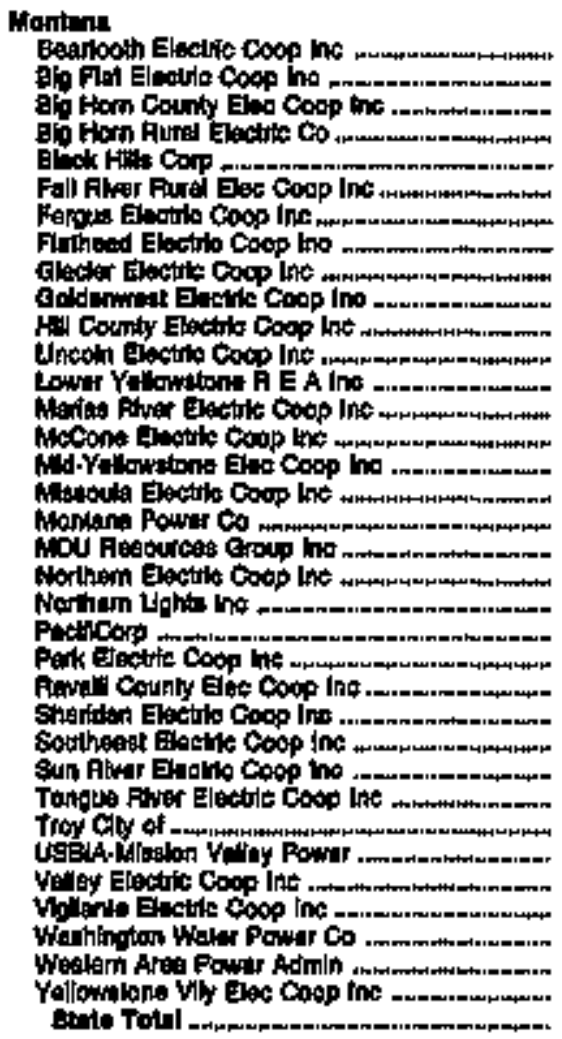 & 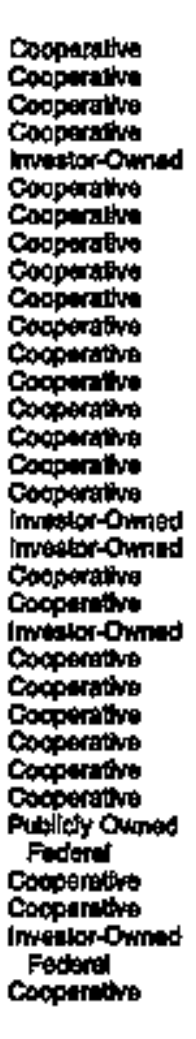 & 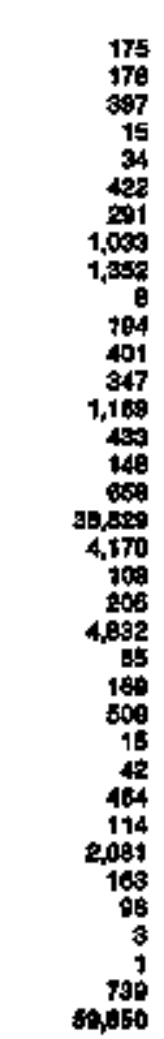 & 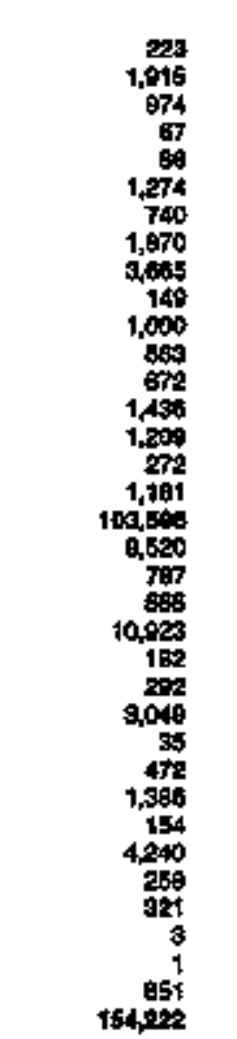 & 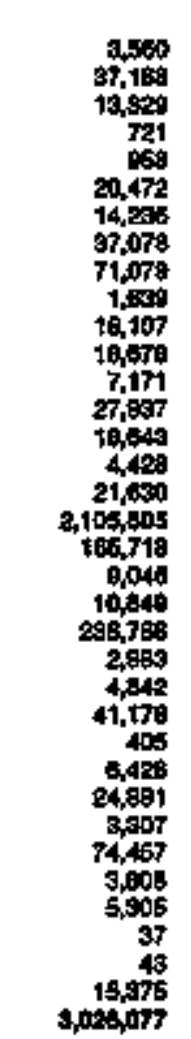 & 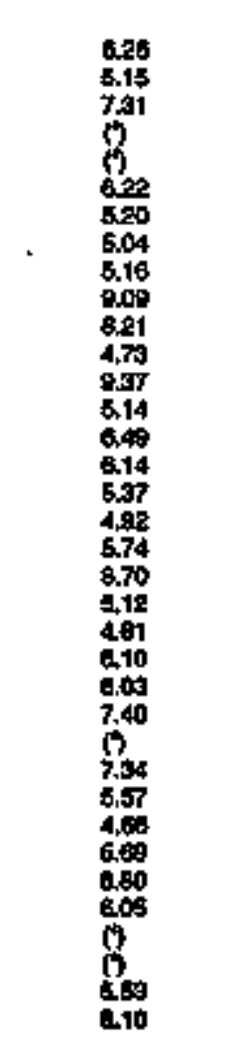 \\
\hline 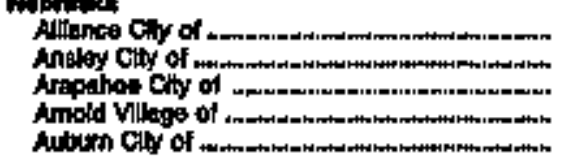 & 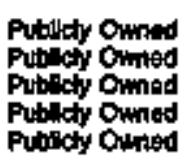 & $\begin{array}{r}685 \\
58 \\
120 \\
129 \\
488\end{array}$ & $\begin{array}{r}1,95 t \\
98 \\
280 \\
135 \\
678\end{array}$ & 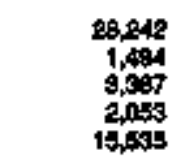 & 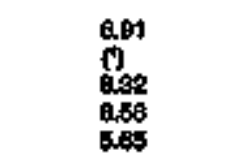 \\
\hline
\end{tabular}

soe toobotal al ond ox tablo. 
Table 15. Class of Ownership, Number of Uttimate Consumers, Revenue, Sales, and Average Revenue per Kilowatihour for the Commerleal Sector by State and Utllity, 1999 (Contionted)

\begin{tabular}{|c|c|c|c|c|c|}
\hline Sinba & 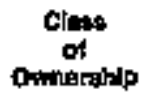 & $\begin{array}{c}\text { Number } \\
\text { od } \\
\text { Contimertim }\end{array}$ & $\begin{array}{c}\text { Bantrius } \\
\text { (thousand doliort) }\end{array}$ & $\begin{array}{l}\text { siles } \\
\text { (1haugend } \\
\text { flowathourta) }\end{array}$ & 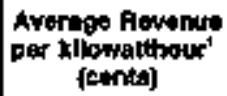 \\
\hline
\end{tabular}

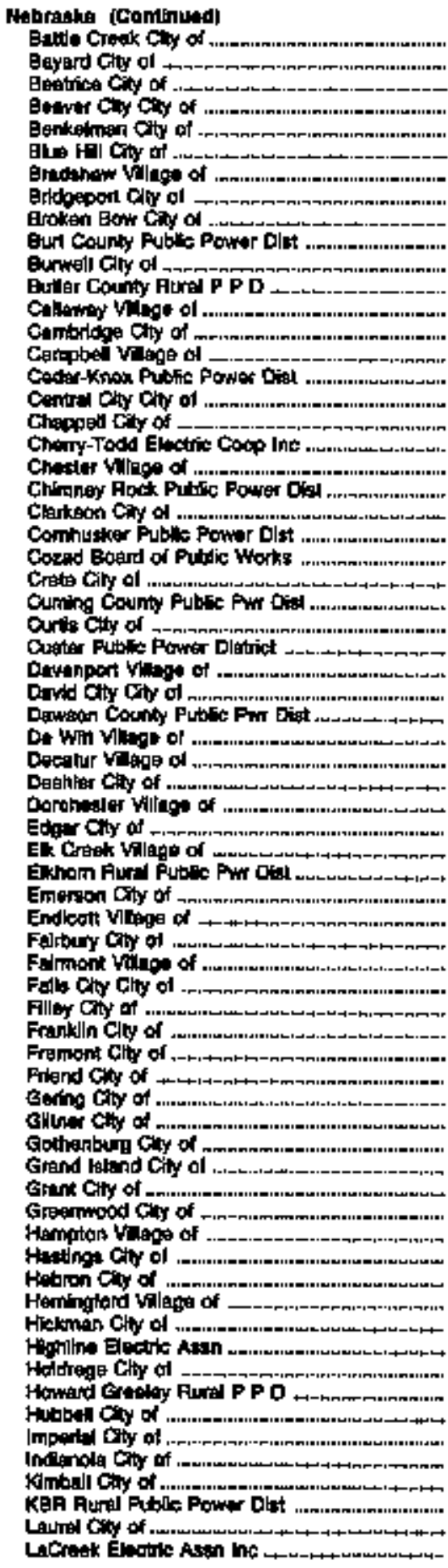

Publesty Onned

Publety Owned

Fublicts Owned

Fublery Owined

Publety Owned

Putblicty Owined

Publity Owned

Publtily Owned

Publecty owniad

Aublisty Owned

Puolloly Owied

Putulcly Owtied

Publlcly Owines

Publloly Owned

Pudlody Owred

Puthlisy Owined

Puticky Owned

Publety Owned

Gooperation

Publitly Owned

Publloly Owned

Pitrlely Owrted

Publlely Owned

Publlady Owited

Publdy Orwied

Publyty owned

Pubucty Ouned

Publucty Ouned

Publuty Ormed

Putsicty Oimed

Publecty Omed

Putbelty Omind

Puticty Ourad

Publedy Orwsed

Pulbichy Omad

Pubricty Omed

Publicty Owmed

Publucty Ormed

Pubsicty Orined

Pubichy Oined

Putuly Onned

Publicty Omed

Pubicty Ormad

Publuty Owrred

Fublicty Ommed

Pubcicty Ormed

Publecty Owned

Fubledr Ommed

Pulalkst Onmed

Plstesty Owned

Publetry owned

Fulpiesty Owmat

pubsely owned

Publely Owmod

Fublicity Owned

Publity Owned

Publest owned

Putlety owned

Cooperaliva

Pubfishy Owned

Publecty Owmed

Publisty Owned

Publest Owned

Pubtely Ownad

Fublicts owised

Putbey Owned

Publify Owned

Coopergtine

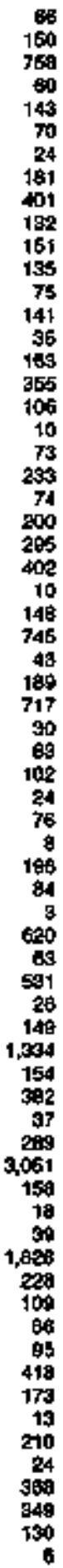

147

162
1.016

1.010
310

312

84

20

320

286

286
469

50

288

84

420

120
6

8
102
470

470

152
1.031

ext

925

945
194

665

65
202

1,903

1,931
76

$4 B$
131

131

110

अ5

159

ต15

127

579
17

253

1,961

240
617

111

402

6,749

207

132

3,494

493

139

210

1.104

1.104
258

15

739

107
469

409

124
3,230

25,640

17.403

1,035

4,740

1.635

6,668

13,484

4,570

4,200

s,0so

1,713

4,437

1,057

15,200

6,503

2.211

110
1,509

6,052

2776

20,000

11,077

16,ape

6.225

2,474

14,614

ass

3,228

1,192

300

2,486

1,792

1,625

476

5,947

2,513

25

2,255

11981

370

s,74:

38,054

4,218

8,002

1,574

9,818

119,857

4,201

1,835

1,120

06,549

9.130

1, 8

659

3.ses

29,408

3,065

160

14,134

1.619

7,760

7.436

3,264

817
4.54

6.97

B.AP

600

(1)

4.96

4.66

5.45

6.19

5,72

8.04

(b)

4.69

5.43

(t)

8.38

$5.4 a$

5.15

5.50

7.64

6.60

(1)

6.40

(4)

1).

510

6.77

it

500

6.33

1)

267

4.80

17

6.76

6.44

705

6.47

sea

6.24

7.10

5.

5.40

7.32

(1)

6.22

4.72

b. 35

5.53
6.01

acs

800

3,81

()

Sea lootnolus at and of tabia. 
Table 16. Class of Ownorship, Number of Uitimate Conoumers, Rovenute, Salos, and Average Revenue per Kilowatthour for the Comnerical Sector by State and Utility, 1993 (Contintued)

\begin{tabular}{|c|c|c|c|c|c|}
\hline Otab & ctitip & $\begin{array}{c}\text { Nomber } \\
\text { of } \\
\text { Conturatet }\end{array}$ & Reveras & $\begin{array}{l}\text { Sales } \\
\text { thortend }\end{array}$ & 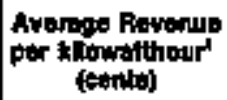 \\
\hline 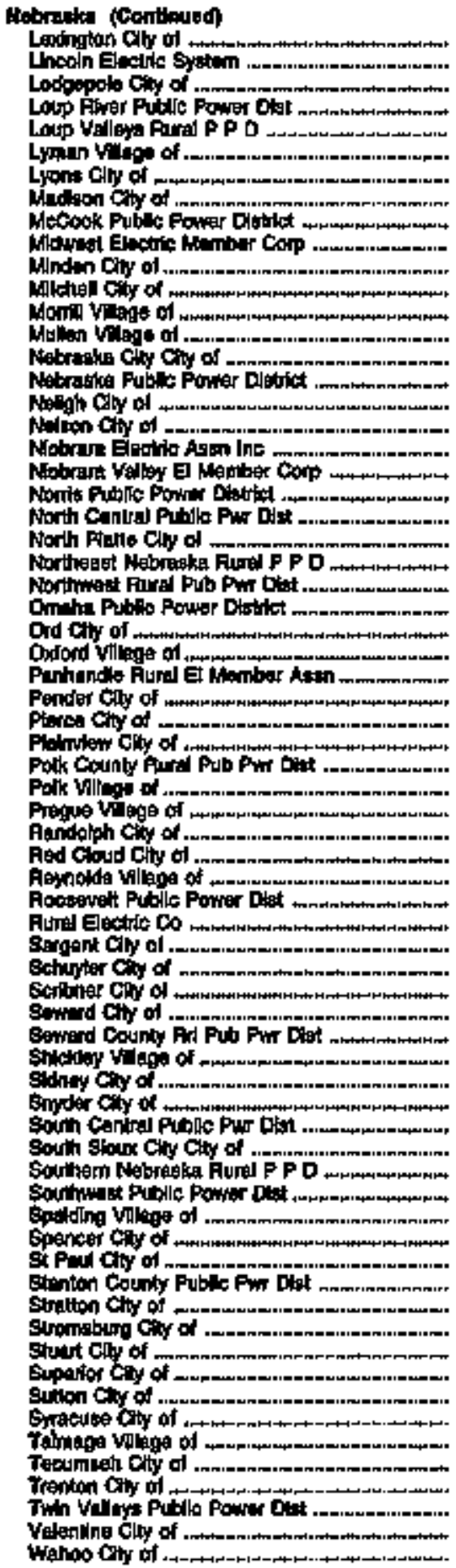 & 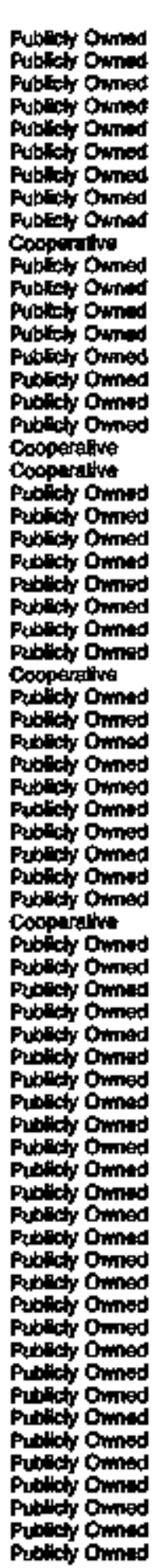 & 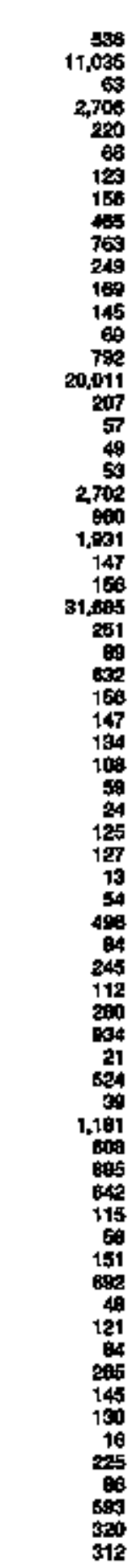 & 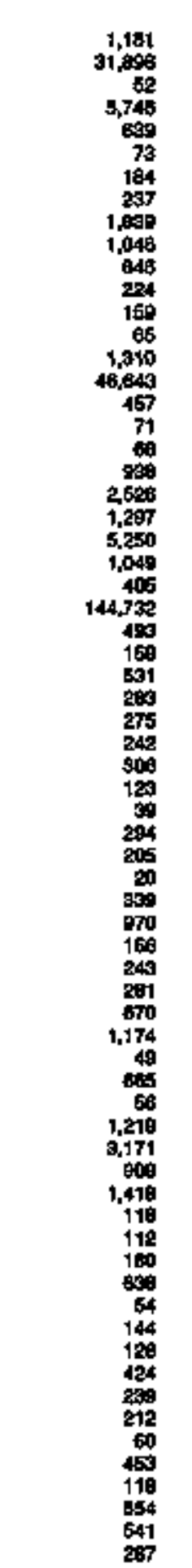 & 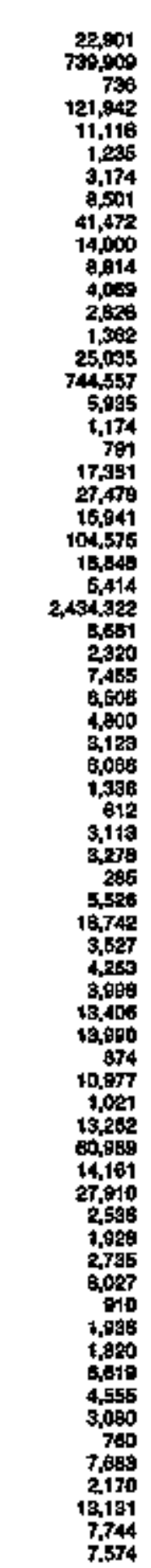 & 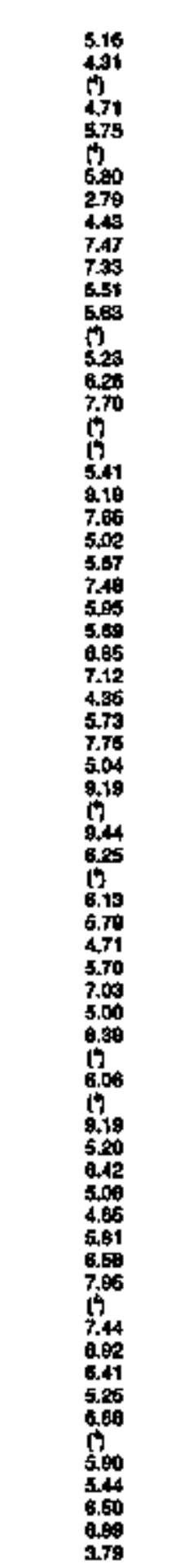 \\
\hline
\end{tabular}

Seo tooinotes al end of twbite. 
Table 15. Class of Ownership, Number of Ultimate Consumers, Revenue, Sales, and Average Revenue per Kllowatthour for the Commerical Sector by State and Utillty, 1993 (Continued)

\begin{tabular}{|c|c|c|c|c|c|}
\hline 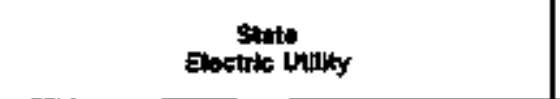 & $\begin{array}{c}\text { clatet } \\
\text { of } \\
\text { Dmonralp }\end{array}$ & $\begin{array}{c}\text { Namber } \\
\text { of } \\
\text { Contoumales }\end{array}$ & finolisand dollars' & 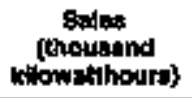 & 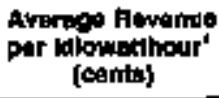 \\
\hline 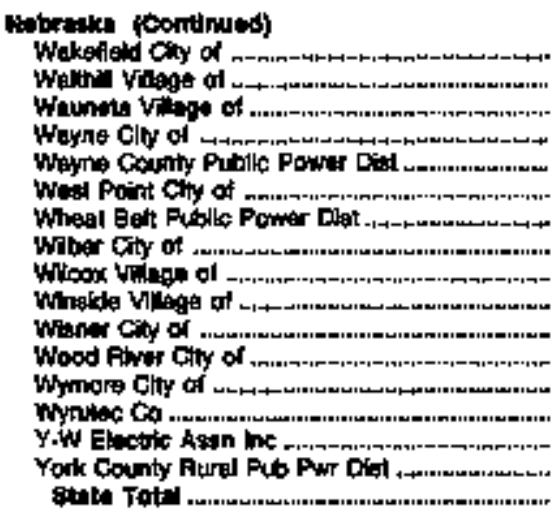 & 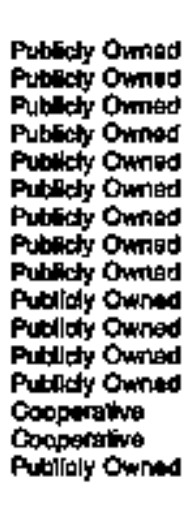 & $\begin{array}{r}62 \\
48 \\
66 \\
287 \\
210 \\
312 \\
420 \\
115 \\
52 \\
14 \\
139 \\
180 \\
148 \\
37 \\
68 \\
189 \\
108998\end{array}$ & 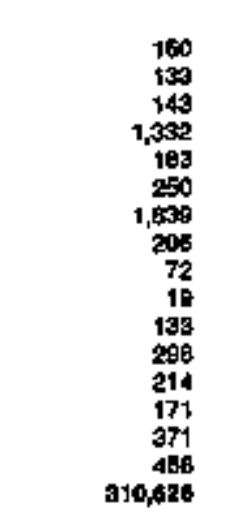 & 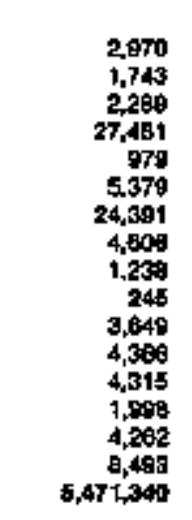 & $\begin{array}{l}5.39 \\
7.09 \\
6.25 \\
4.65 \\
69 \\
4.65 \\
6.72 \\
4.47 \\
69 \\
49 \\
3.64 \\
6.78 \\
4.98 \\
0.59 \\
0.70 \\
6.61 \\
6.96\end{array}$ \\
\hline 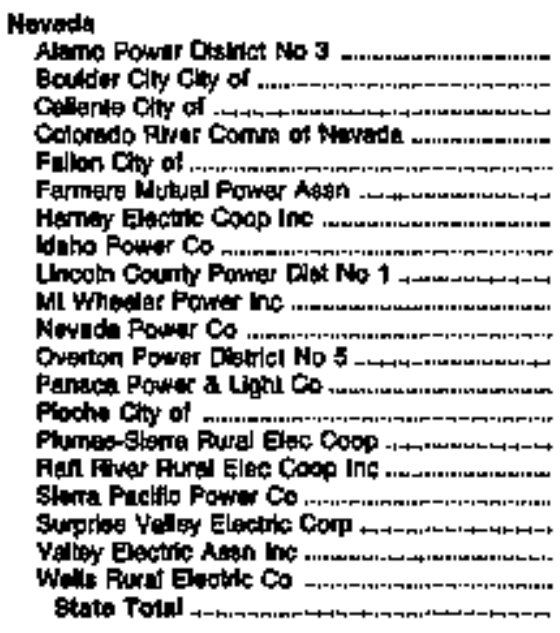 & 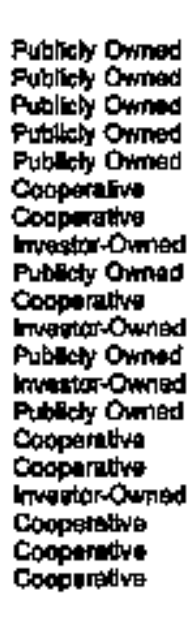 & 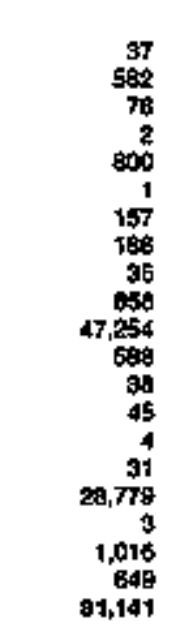 & 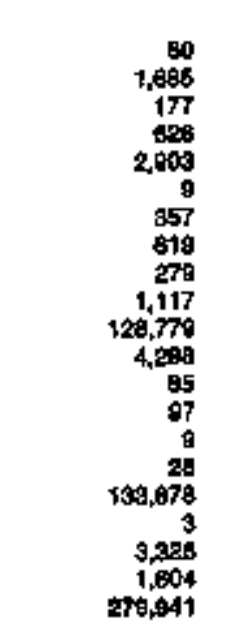 & 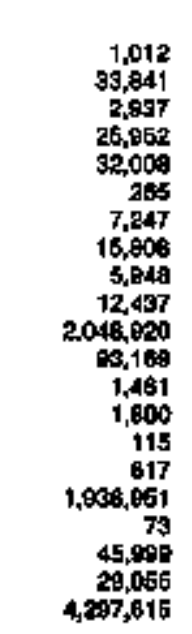 & 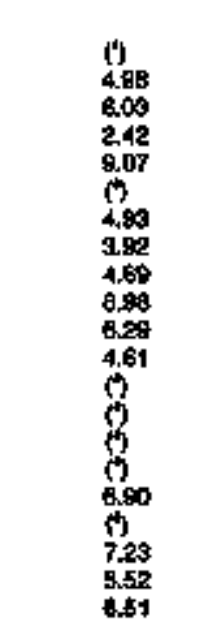 \\
\hline 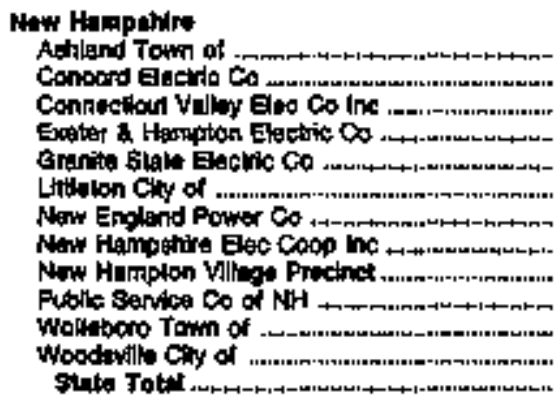 & 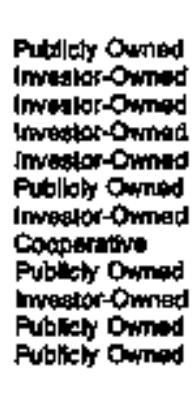 & 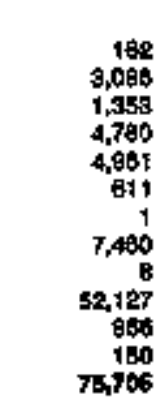 & $\begin{array}{r}804 \\
13,103 \\
6,520 \\
11,790 \\
20,874 \\
1,257 \\
106 \\
22,915 \\
147,19 \\
1,153 \\
834 \\
233,707\end{array}$ & 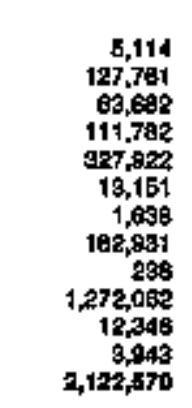 & $\begin{array}{r}0.88 \\
10.28 \\
10.25 \\
10.65 \\
6.84 \\
9.53 \\
6.41 \\
12.63 \\
17 \\
11.68 \\
9.54 \\
6.47 \\
11.01\end{array}$ \\
\hline 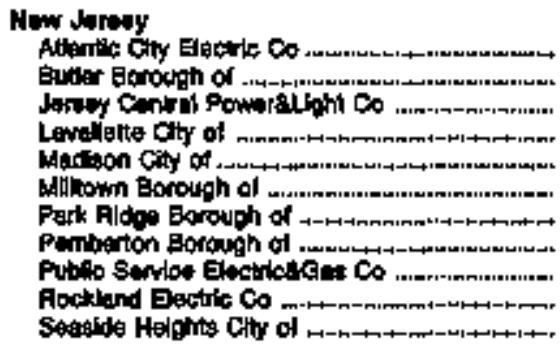 & 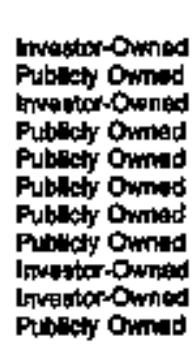 & 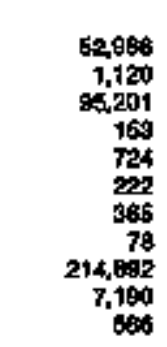 & $\begin{array}{r}314,867 \\
3,848 \\
908,641 \\
615 \\
9,206 \\
500 \\
2,804 \\
207 \\
1,687,213 \\
41,005 \\
1,073\end{array}$ & $\begin{array}{r}5,256,768 \\
38,978 \\
4,473,968 \\
7,298 \\
54,451 \\
6,480 \\
\$, 500 \\
2,309 \\
10,000,312 \\
769,227 \\
10,597\end{array}$ & $\begin{array}{r}9.87 \\
10.77 \\
10.79 \\
9.49 \\
10.89 \\
7.72 \\
8.00 \\
9.98 \\
8.32 \\
11.11 \\
10.64\end{array}$ \\
\hline
\end{tabular}

Seo rootneles al end of tabia. 
Table 15. Class of Ownership, Number of Uttmate Consumers, Revenue, Sales, and Average Revenue per Kllowathour for the Commerical Sector by State and Ltillty, 1993 (Continued)

\begin{tabular}{|c|c|c|c|c|c|}
\hline 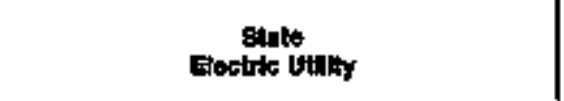 & $\begin{array}{c}\text { 하od } \\
\text { of }\end{array}$ & $\begin{array}{l}\text { tomber } \\
\text { con }\end{array}$ & (thousand dollores & 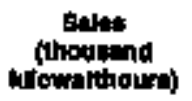 & 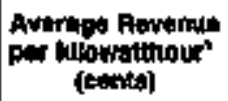 \\
\hline 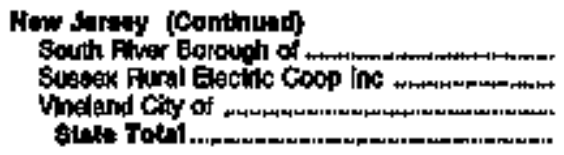 & 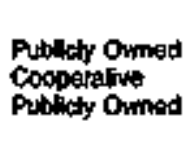 & $\begin{array}{r}640 \\
98 \\
2,318 \\
376,311\end{array}$ & $\begin{array}{r}1,294 \\
1,079 \\
10,118 \\
2,775,722\end{array}$ & $\begin{array}{r}9,069 \\
9,459 \\
90,379 \\
25,405,042\end{array}$ & $\begin{array}{r}14.16 \\
12.68 \\
11.80 \\
9.73\end{array}$ \\
\hline 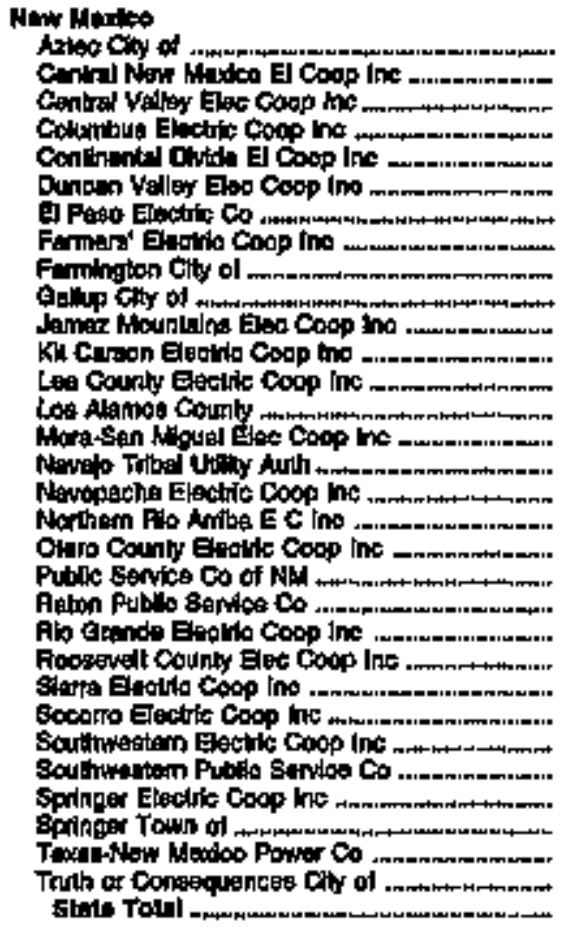 & 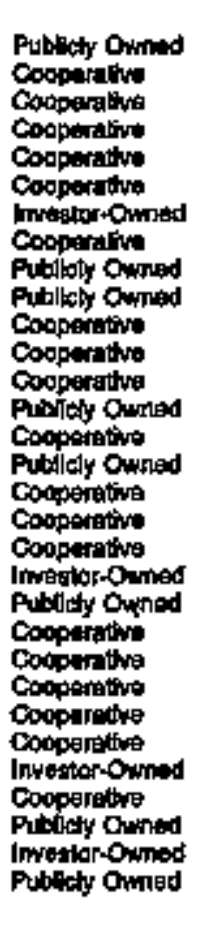 & 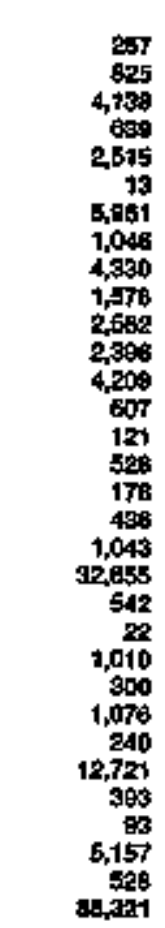 & 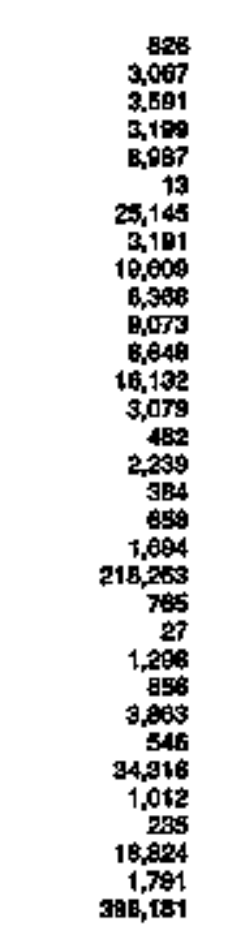 & 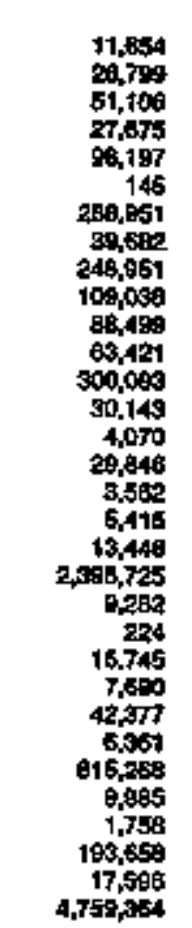 & 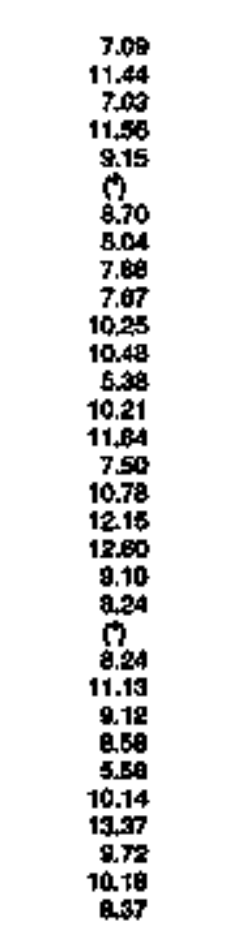 \\
\hline 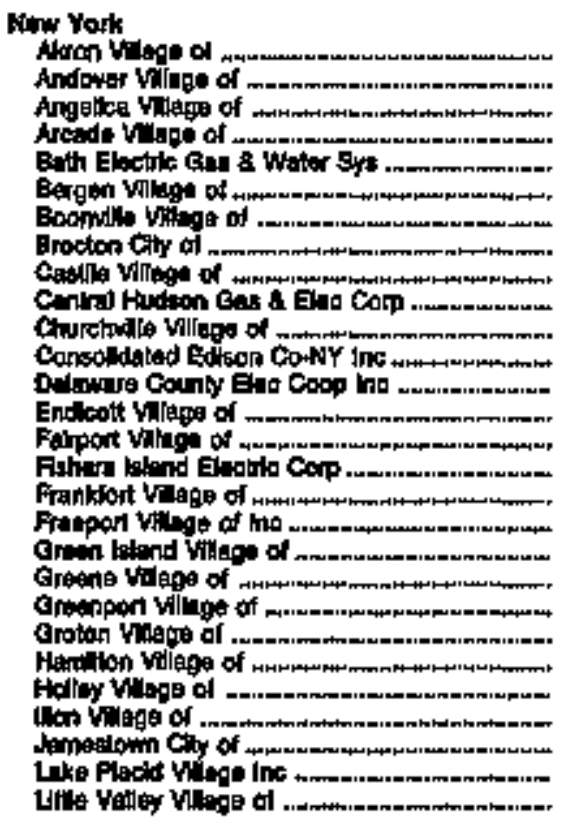 & 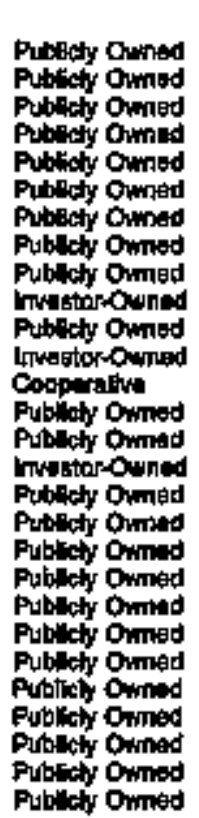 & 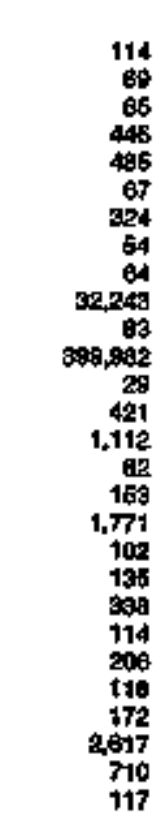 & $\begin{array}{r}55 \\
78 \\
88 \\
689 \\
467 \\
100 \\
187 \\
31 \\
38 \\
101,950 \\
241 \\
3,017,538 \\
62 \\
242 \\
2,168 \\
184 \\
243 \\
6,605 \\
629 \\
467 \\
641 \\
138 \\
213 \\
304 \\
104 \\
5,301 \\
1,694 \\
294\end{array}$ & 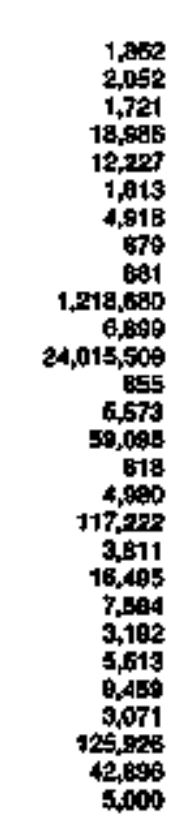 & 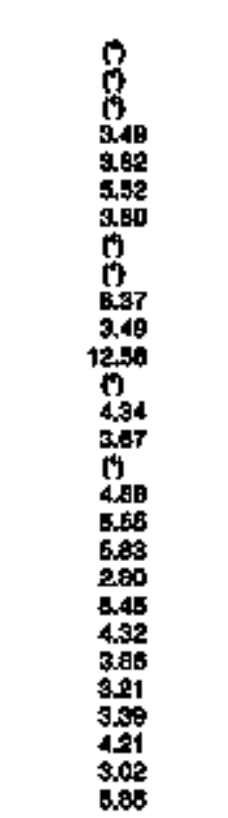 \\
\hline
\end{tabular}

Ses fophotas at and of babl. 
Table 15. Class of Ownershlp, Number of Ultimate Conbumers, Revenus, Sales, and Average Revenue per Kllowatthour for the Commerical Sactor by State and Utility, 1993 (Continued)

\begin{tabular}{|c|c|c|c|c|c|}
\hline $\begin{array}{c}\text { stats } \\
\text { Exhotilo UIny }\end{array}$ & 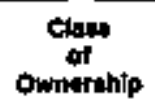 & $\begin{array}{l}\text { Nambar } \\
\text { of } \\
\text { consumers }\end{array}$ & $\begin{array}{c}\text { Revanue } \\
\text { (thougand dollais }\end{array}$ & $\begin{array}{c}\text { tolet } \\
\text { (thouliand } \\
\text { idlomalthourt) }\end{array}$ & 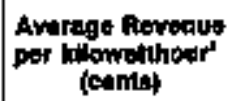 \\
\hline
\end{tabular}

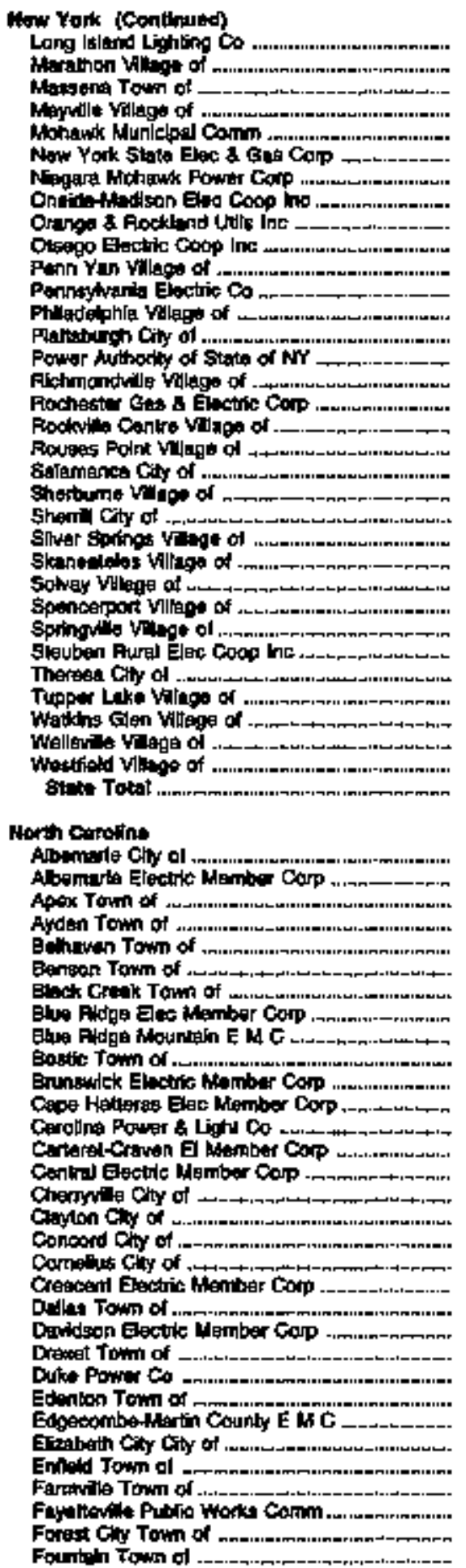

Imeskor-Ommed

Fubllicy Owned

Pudlicky Owmed

Publiely Owned

Pubillady Owrled

movestor-Omied

Invitodor-Oined

Coopertiving

Itwestor Ommed

Cooperative

Publlctr Ounad

Imintslor Onned

Publichy Ownud

Publicts Ownod

Putbly Owned

Publisty Owind

Imveskor-Omned

Publety Ommés

Publity Onted

Publleys Owned

Pudblich Ounned

Pubtaty Omind

Pubitity Ownes

Publert Owned

Pubrich Owried

Rubtecy Omnd

Publety Owned

Goppetativit

Pubioty Owned

Rublicty Ouned

Fublity Ómed

Fubfict owned

Publiats Ounned

Publicty Owned

Cooperafive

Pubtity Omed

fubient Ormed

Publichy Owned

Publuty Oumed

Pubicty Omed

Cocparaltre

Coopertive

Publich Owned

Cosperatur

Cooperatture

Imeghtion-Owrupd

Cosperallue

Cooperzaltive

Pubulty Onned

Pubcety Omed

Publicty Onaned

Pubuldy Omifod

Cooperalive

Publisty Omed

Cooperalive

Publicty Omored

Investorowned

Publuch Owned

Cocoperative

Putlindy Onsted

Pubucty Owned

Pubicty Omined

Publioly Owned

Publely Owned

Putlichy Owned

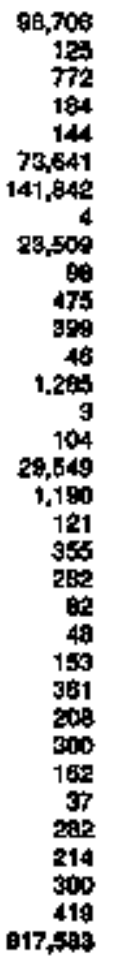

551.939

685

179

170

334,221

1,237,560

86,985

$16 ?$

379

0,191

14

3.258

0

196.100

4,062

309

800

71

21

118

127

989

648

20

164

158

225

$5,563,929$

1.700

354

304

164

325

75

1,2067

11

1,086

125,0098

2,021

1,272

245

1,489

142

1,240

311

770

199, 0997

470

542

1,357

254

305
$\$, 102$

$\$, 102$
6,549

1398

2,128

275

1,560

183

7,000

1,194

160

2,285

495,910

7.448

1,a日7

1,316

610

0,215

770
8,516

1,182

3,843

216

1,263

1.899

0,785

748

34,424
5,127
$3,554,915$

1,420
+2811

5,167

5,187

3.297,714

$12,007,136$

139
08497

2,301

9.523

15,822

1,550

169,145

13:

1,500

1,957,047

0,48

9,260

7,267

16,650

2,511

544

2,000

20,000

3,017

29,746

$6,4 \$ 3$

500

5,463

3,626

4,920

$7,2 \pi$

$47,720,275$

01,901

9,804
11,510

21,675

2,048

16,325

1,350

85,253

13,649

287

84,205

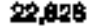

7,262,196

at. 8 be

10,1 $19 \mathrm{~B}$

17,883

5,917

140,560

10,700

112,618

1E,424

$47,0 \mathrm{ds}$

$3,3 \mathrm{BO}$

13,320.006

13,200

32,123

115,666

7,960

6000

521,360

80,077
16.62

4.60

3.3

3.27

10.13

12.47

B.19

3.97

7.5

(')

5.39

th

9.97

3.77

2.84

325

it)

4.

258

4.7

3.36

6.48

(1)

00

4.96

4.57

11.60

7.60

0.47

12.08

9.73

13.38
9.51

12,07

a. 04

a.s1

(t)

10.01

6.89

9.10

9

7,44

10.31

0.6

7,20

756

7.24

8.02

6.43

5.80

9.61

$\$ .72$

a.44

9.40

12.91

seo

500

Ste tooblotitas al tond of tabla. 
Table 15. Clags of Ownership, Number of Uttmate Consumers, Revenue, Sales, and Average Fevente per Kllowatthour for the Commerlcal Sector by State and Utillty, 1998 (Continued)

\begin{tabular}{|c|c|c|c|c|c|}
\hline 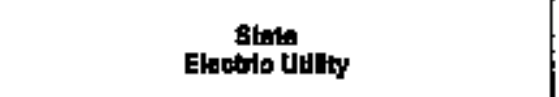 & 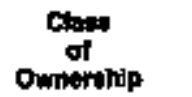 & $\begin{array}{c}\text { Kumbor } \\
\text { of } \\
\text { Conpumars }\end{array}$ & $\begin{array}{c}\text { Rinwinta } \\
\text { thousind dofteret }\end{array}$ & 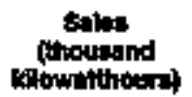 & $\begin{array}{l}\text { Average fiowemun } \\
\text { par kitownithour' } \\
\text { (cents) }\end{array}$ \\
\hline 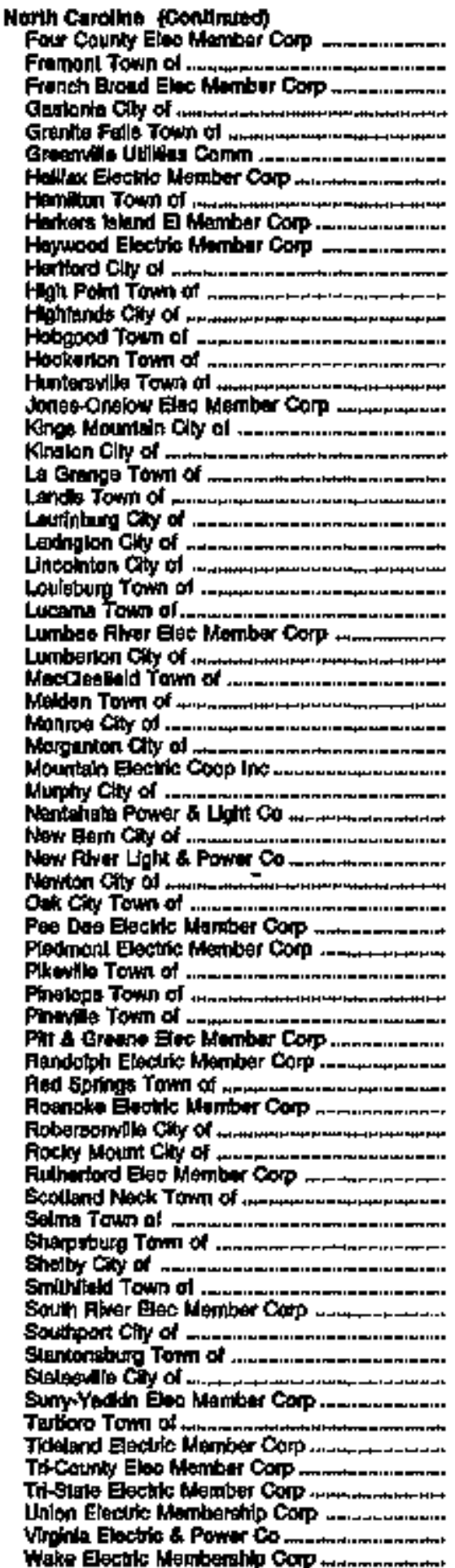 & 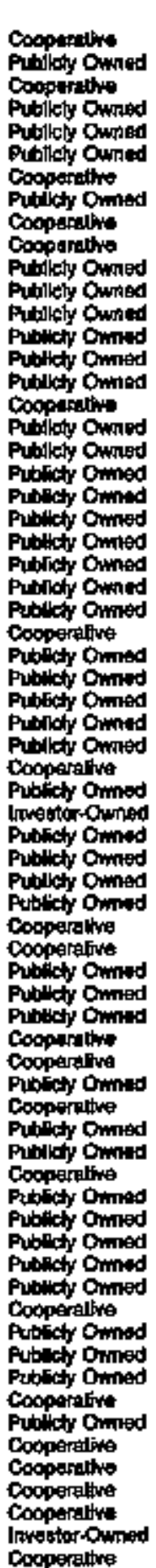 & 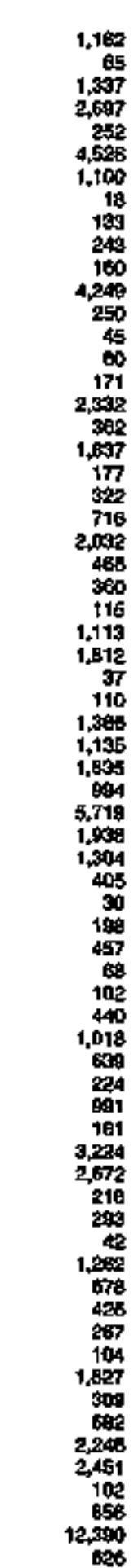 & 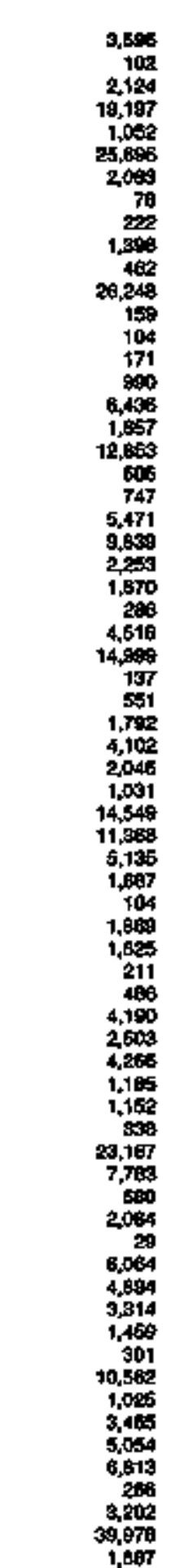 & 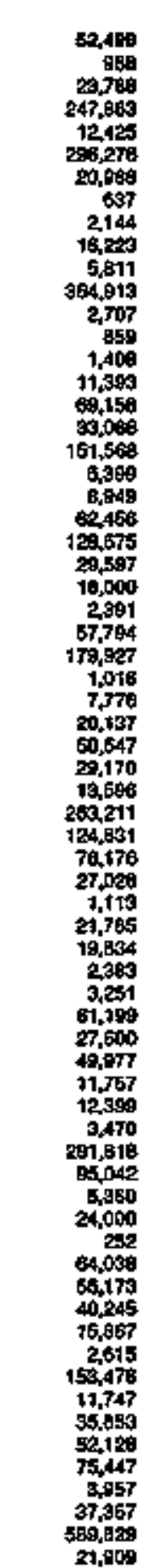 & 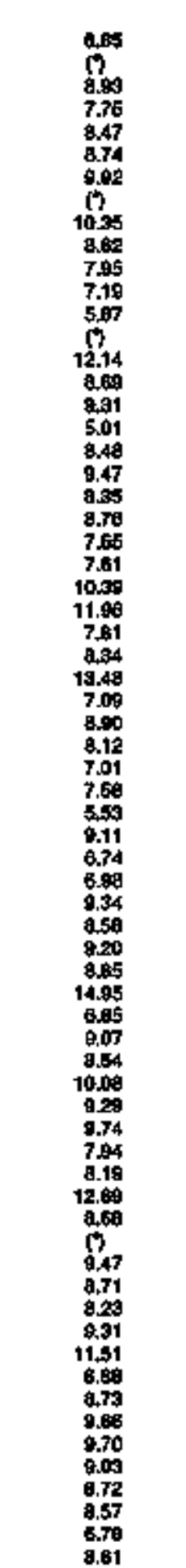 \\
\hline
\end{tabular}

Bes loolvotes at end of tatib. 
Table 16. Class of Ownership, Number of Ulthate Consumers, Revenue, Sales, and Average Fevenue per Kllowathour for the Commericed sector by State and Utility, 1993 (Continued)

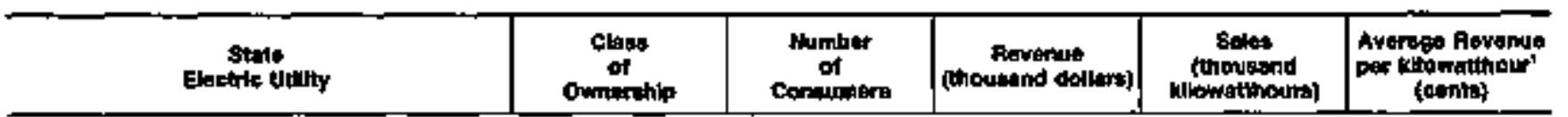

Horth carolnu fContinued)

Walke Foreagt Tom of

Waletonourg Town of

Westingion City of

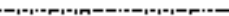

Whangritia Town of

Malson Cly od.

Widsor Tom of .

Narth Dakot.

Baker Eloctrde Coos mo

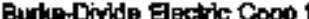

Chphlal Eloctric Coop Inc...

ctuc cocp ins

Cangler Cety of

Cenvellar Runt Elac Coup lac

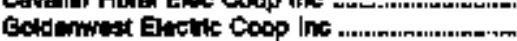

Eration Cay of

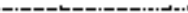

Hope City of

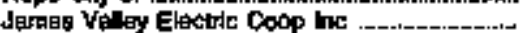

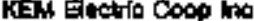

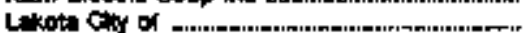

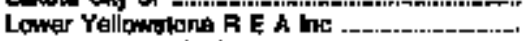

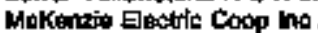

Maleen Eletite Coop ino

Mor-OTan-sow Electric Cocp Ins .................

Mourirail-Wiliants El Coup Int

MOU Reoourcen Crow Ins

Nodalk Electrie Coop line

North Conbal Eloc Cosp ing ......................

Northum Staks Powar Co

Norttmichod Ciny of

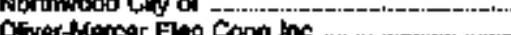

Otive Tall Pown to ......................................

Park Fivis Gity of .

P S A Evertic Codp lo

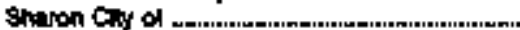

Stheritan Electrit Coop Int ........................

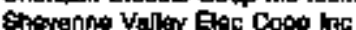

siope Elpertide Coop mo

Stranion City of

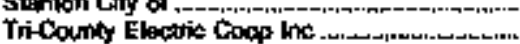

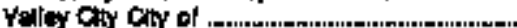

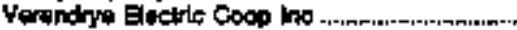

Woat Plaina Electric Coap bic

stab totel.

Ohin

Adums fural Electric Coop ins

Amtrosil City of

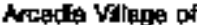

Mosum Chy of

Beach Gity lity of

Eethel Coty of

Glanchester Village of

Glonderia Vileoge of

Bowing Green City al

Grudner Vaage of

Erawster Vinge of

Eryan City ol

Bufkny fural Elec Coop ind

Butier Fural Dectule Coop Ins .

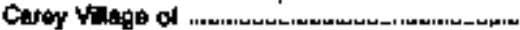

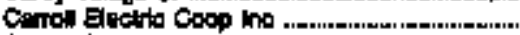

Genne Coly ol .

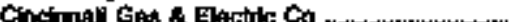

cinvedend City of
Publichy Dwinad

Publick Owmid

Publisty Owmed

Fublitis Owntd

Pubinct Ommad

Publicty Owined

Publitbly Owined

Cooperalve

Cotoperaitive

Coopurativo

Copperatue

Fublichy Dwnec

Coopurative

Coopernthe

Publichy Ownad

eublioly Owned

Punnchy Omined

Cooparalivo

Coopert fwo

Publicisy Ormod

Cosperattre

Cooporative

Cooparative

Cosperiefint

coopertitis

invetor-Owned

Conplérative

Coopertitir:

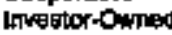

Pubicy Onté

cooperative

Invesior-Owned

Publich Ornied

Cooponattre

Pubichy Gumad

Cocpentivis

Cooperattret

Coopuratye

Pullety Qwisd

Conperativis

Putildy Ournod

Cooperetur

Copperative

Cooperative

Fubllaly owrind

Publicty Owated

Putlloly Owhed

Publiety Dwnad

Fublexis Owind

Putblaly Ownea

Putulutin Domed

Fublely Qwined

Pullaby Ouniad

Publlet ownat

Fublesh Owmax

Coopuratur

Coopurathy

Fublest Ownad

Cooperatur

Publist Cownad

Ime:Aor-Ormed

Publith owned

1,247
37
1,657
614
3,519
339
87
112420

247

657

614

389

12420

104

503

740
1.496

31

10

106

20

184

191

76

1,160

153

390

1,161

8,916

168

1,324

11,188

98

11,001

IB1

Bg

54

128

320

559

670

1,005

658

44 ige

19
355

18

BS

157

1 的

56

1,387

41

73
64

54

283

$1 \mathrm{B3}$

656

$7 \mathrm{BT}$

68.676

6.426

1,905
260
5,002
2,000
21,104
1,500
308
$1,743,906$

18,623
245
50,540
28,167
245,087
10,814
3,000
$26,747,461$

10.28
11
9.00
7.36
8.61
7.67
10.09
6.58

1,252

2,052

2,076

4.911

474

452

149

70

202

45

760

158

158

A, 270

340

2,110

4,422

32,600

2,003

4,100

29:5

301

716

16,841

384

10

149

34

1,427

75

1,029

ro9

$1+6,619$

\section{4,06s}

28,708

26.972

103.020

8,707

5,465

t,737

13,345

5.337

2514

to, 4 t9

5.447

3,909

2000

104.489

4,185

20,012

56,640

500,213

31.221

sd, $7 \mathrm{eB}$

375,515

5,674

12,997

250,004

7,00:

1,146

276

1,770

6,396

15,54

1,052

26,865

41,390

62,65

10,ke4

7, 930,750

8.41

7.6

7.73

4.73

a.29

8.28

5.20

416

7,0

6.79

4.07

8.98

7.92

8.10

7.27

780

653

6.81

7.48

5.90

6.50

B.5

6.71

6.47 
Table 15. Class of Ownerahip, Number of Ulitmate Consumers, Revenue, Sales, and Average Revenue per Khowatthour for the Commerical sector by State and Utilly, 1993 (Continued)

\begin{tabular}{|c|c|c|c|c|c|}
\hline $\begin{array}{c}\text { stale } \\
\text { Elacinis dulty }\end{array}$ & $\begin{array}{c}\text { clate } \\
\text { of } \\
\text { ounethotp }\end{array}$ & 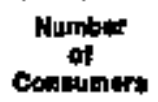 & (taousund doflare) & 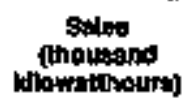 & 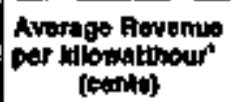 \\
\hline
\end{tabular}

Chlo tcomitions?

Gricland tetectito Itum Co Clyde City of

Colunblane viluga of

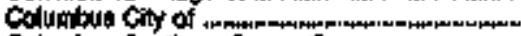

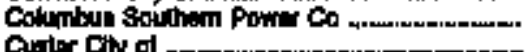

Cyatroga Falis cth of

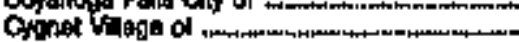

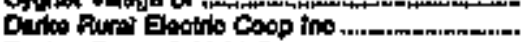

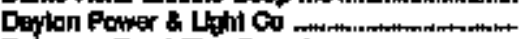

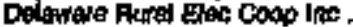

Doalile City of ..-...-.

Dover Cthy of

Edouton Villoget of

Ectorado Vidage of

Einote Vilage of

Firelands Eadito Cocp Inc ........................

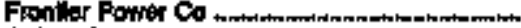

Gallon Chy of

Gamos Vilagh of

Cloufter vilage of

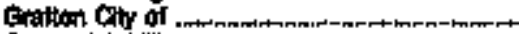

Eroentut sh vilage of .

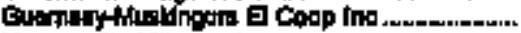

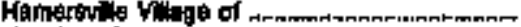

Harallon cly of menow

Hulthe Villige of ..................................

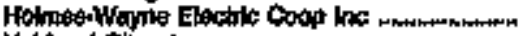

Hultowerd of of

Hudion Cor of

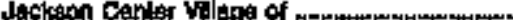

Jakeson Cly ol .

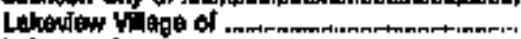

Lebanan toy of

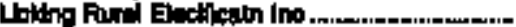

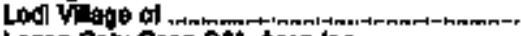

Logan Cnly Coop PAL Noan Ino ..............

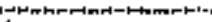

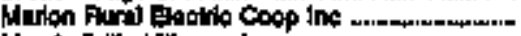

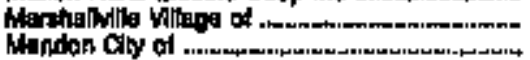

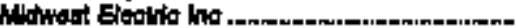

Man valoge of

hipater Vhilage of i

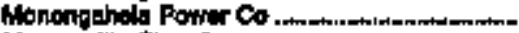

Monroevile $\mathrm{dly}$ of

Honlpelle Vilage of

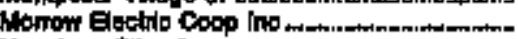

Niposen Clly of

Now Bromm vituog of

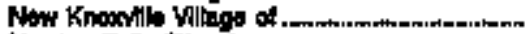

Niving Fala C Cly of

Noa Chy of

Noth contro

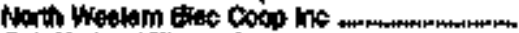

Oak Huther Villoge of

oberint city of

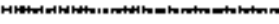

Ofla Editan Ca

Ohis Pawion Co

Ohio Power Co

Orille cin of

年

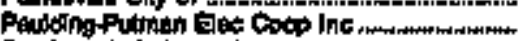

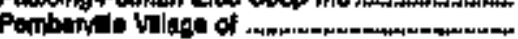

Plonet Rurnl Ene Coon ho
Imvation-Omined

Publety Owned

Putillesy Owrated

Dublikly Owrted

Inwerlorounes

Publloly Owned

Publaty Onwiod

Punilaty Ounsed

Cooperative

Impolor owned

Cooperetive

Publudy Owned

Publldy Owned

Putlicty Onnerd

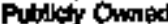

Pubicty Onimed

Cocperable

Cooperative

Pubich Onined

Publicty Oimed

Publedy Omined

Publicky Dimod

Fublicty Onmed

Fubicty Omod

Coopuratye

Fublety Ownod

Fubloty Omad

Cooporalisio

Pubicly Onnud

Cooperaltive

Pulich Ormad

Publety Owned

Fublets Ounned

Pubicty Owned

Fublety Ownod

Fublity owned

Cooperatho

Publety Ownod

Cooperativis

Cooperitive

Publety owned

Coopestativit

Publity Owned

Publict Ommed

Coophtiso

Publtety Owned

Publith Dwited

timealor-Omed

Publity Owned

Pubilicty Ouned

Cooperithe

Pubiloly Owned

Pusticy owned

Pubilaty Owned

Publety Owniad

Pubilcty Owine.

Cooperethve

Cocperative

Putticly Owned

Publitaly Owned

Putilck Ommid

riwestar-Owned

Imoalor-Omned

Prolicly owned

Publlady Owrad

Coopertitive

Publicty Owhes

Cooperetive
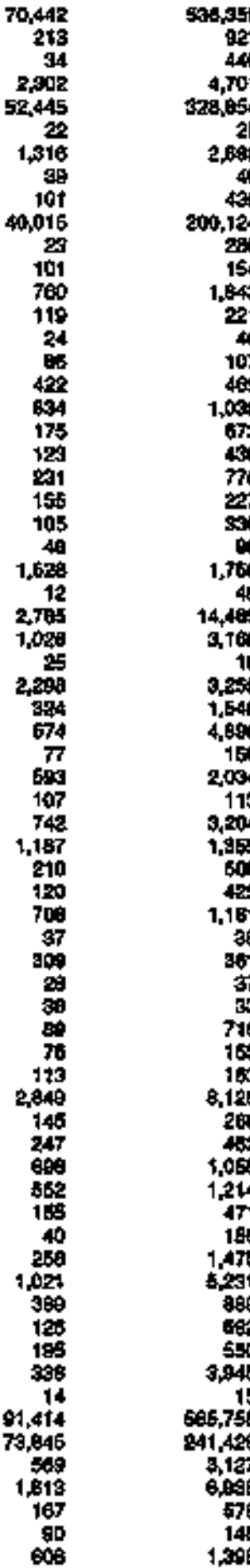

4,55동

46

$4,70 y$

25

2,690

45

200,124

280

154

221

46

107

400

1,009

673

458

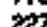

50

in

1,760

14,46

3,76

3,250

1.549

4,690

150

2,004

113

3,204

1,955

5010

1,161

39

301

30

718

165

100
8,128

260

402

5,055

1,214

ari

188

5251

989

60

500

606,758

241,498

3,127

Q AOS

579

148

$1,30 \mathrm{~B}$
5,603,021

0.416

6,502

02974

5,418,065

39,500

705

7,160

3,000,974

$3,4,27$

2,598

3,248

1,451

B,300

12,661

10,102

5,465

15,301

3,238

6,595

1,797
29,746

728

171,250

47,572

170

64,328

19.146

6400

2537

29,B18

1,750

51,204

20,08月

Q.213

S.68:

19, 290

412

4689

574
500

to,7gs

1,612

$2,3 \times 3$

148,400

4,000

4 AfAB

12400

18,042

QS⿰冫欠

2,414

Tops

13,058

10,460

6.444

60,057

5, 058,008

$4,413,200$

$46,7,2$

02,181

0,794

2,164

20,040

9.52

9.7B

7,4

(4)

7.32

(1)

8.1t

7

say

B.g日

Bat

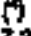

3.3?

7.42

t.on

8.6

7.98
$\mathrm{~s} 0 \mathrm{~T}$

5,00

(1)

709

(1)

8,68

(j)

8.00

7.09

591

7,11

6.46

820

, 
Table 15. Class of Ownership, Number of Uitlmate Consumers, Revenue, Sales, and Average Reventle per Khowatthour for the Commerioat Suctor by State and Utillty, 1993 (Continued)

\begin{tabular}{|c|c|c|c|c|c|}
\hline $\begin{array}{c}\text { stabt } \\
\text { electio tidaty }\end{array}$ & $\begin{array}{c}\text { Anos } \\
\text { of } \\
\text { owmerahip }\end{array}$ & $\begin{array}{c}\text { tumber } \\
\text { of } \\
\text { Consumer: }\end{array}$ & $\begin{array}{c}\text { Arvtmat } \\
\text { (thousand doliara) }\end{array}$ & 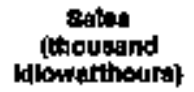 & 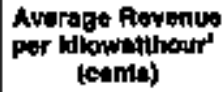 \\
\hline
\end{tabular}

Chlo (Continued)

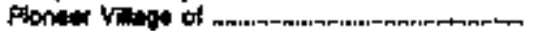

Pious city of.

Flymouth Viliege of .......................

Pfoaptat Corp

Repubric Vildge of

Ripley Gity of

sheloy Chy of

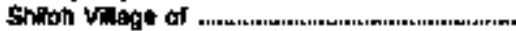

South Centrat Powner to

South Venor Carpotratian

Southeactern Mrition FEC Mn n..................

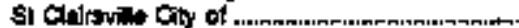

अ Maryo Chy of

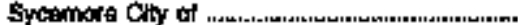

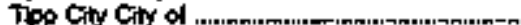

Tolodo Edisan Co

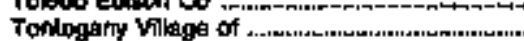

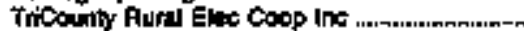

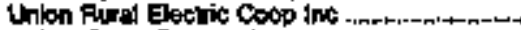

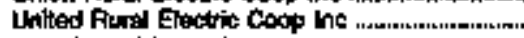

veralles vinlese of

Wactsimath City ol ,

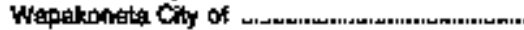

Wastinglon Eivedits Coop ine ........................

Wellinition Gity of

Weateri a city of

Wharton valace of

Woodalied fity of

Woodvlle Vilgge of ....

Yobow spoingt VIlene of

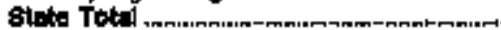

Citanome

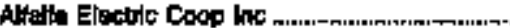

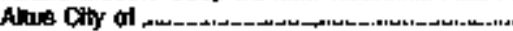

Anadako Puble Wotks Auth .

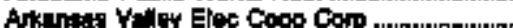

Błgokwid City of ............

Antin

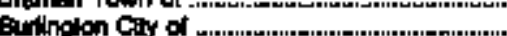

Caddo Elactut Coop las

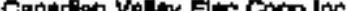

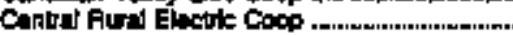

Choctaw Eloctrit Coop ine

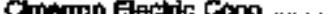

Claremore Coty of

Collinivilia Cly of

Corranchas chy of

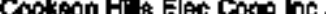

Copen Putble Wotis A

cordin otty of

Conton Bectite toop ins

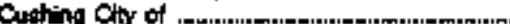

Duncegn Cly of ,

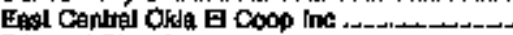

Edmond Gly of

Emphe Dtstutet Eloctulc Co

Falliving $\sigma_{\text {ty }}$ of

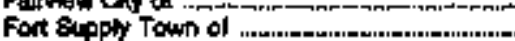

Fiedoden Cly of

(1)

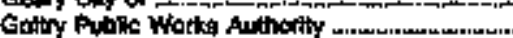

Crend Fiver Dam Authorty.

Grenite Cot of ._-_._-

Hatmon Eiactrio Asan Ino ...........................

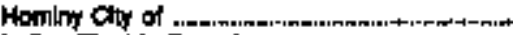

lndin Elatute Coop In:

8ot toolnotes al and of thble
Publecty Owited

Aulion owmind

Ptbilsly owned

Pubitity Omed

Putchlory Owhed

Publicty Dmmod

Publlcly Oumed

Autrot owned

Publlaly owmad

Gooperative

Puthisly Owines

Cooperathe

Publy dy Qming

Putrity omined

Publlaly owned

Publuck Omad

Investord Omined

Publes Dumad

Cooperathe

Copoerative

Cooperathre

Puthlly Owned

Aublity ominos

Aublely Owned

Cocperathe

Publloly Owtad

pubtes owned

Pubtlely Owned

Publity Omined

Publliely Owind

Publicy owmed

Cooperrithe

Ablity Owmed

Publich Owned

Cocperative

Publicy Ombed

Pubritr Omod

Pubich Dumed

Copperative

Copptartive

Coopurative

Cooperathe

Copourntive

Publedry Omed

Pubichy Puned

Publity Onmed

Gooperaltwe

Fubloby Cwned

Pubiely Dimid

Cooperative

Prbines Omed

Puthoty Omed

Coposertive

Fublety Omind

Invaptor-Owned

Futrot Omed

Publicy Omed

Publicy Ounned

Pubrity Onined

Publetr Owhod

Publey Owmad

Pubretr Cumed

Cocorative

Pulaty Omigd

Cocpertivive

74
1,006
20
67
26
136
181
516
26
5,690
29
33
904
457
603
390
28,122
24
174
71
64
207
1,134
616
1,140
212
1,307
29
227
120
228
170,231

166

6.290

14

27

277

1,143

12,579

12,579

B.

B45

1,065

1,049

17, 晒?

62

1,044

1,044
+13

TEd

4.040

2,

85

710

7,200

120

555

228

2, $-27,100$

4,115

1.158

300

242

401

37

1.659

1,836

1,446

511

200

174

704

44

241

3,480

1,062

4,088

2,177

B13

260

18

4.

18
40

420

25

3,293

50

1,494

597

996

1,790

5,072

4,342

2,067

3,64]

1,695

Bs1

347

1,300

90

641
0,345

B, 345

2,445

2,408

16,050

1,262

B?0

ard

857

77
17
1,215

116

467

443

6,605

onyorthedural (came)

2086

86,680

240
1,107

436

4.182

4,144

1 19.6\%

$18+360$

2.000

1,170

15,100

39,645

1200

19,645

$1,671,675$

1,233

17,600

1,580

14,509

67,0007

61,305

7,010

0,069

120,846

1,594

1,200

a,171

6,958

8\%,290000

5.61

50

(b)

870

6.70
7.40

6.17

69

8.25

C)

4.94

(1)

5.34

10.70

7,22

5.96

7.11

6.40

7.08

4.76

0.40

790

5.68

7.63

$\$ .71$

7.18

7.50

84,618

10,354

20,919

9.421

11,592

345

19,700

79,70

65,928 
Table 15. Class of Ownership, Number of Utimate Consumers, Revenue, Sales, and Average Revenue per Kllowathour for the Commorlcal Sector by State and Vtllly, 1993 (Continued)

\begin{tabular}{|c|c|c|c|c|c|}
\hline Eltit & Ownirihip & $\begin{array}{l}\text { Daribor } \\
\text { of } \\
\text { Conumert }\end{array}$ & 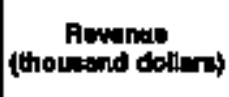 & $\begin{array}{c}\text { Balke } \\
\text { (thoupind } \\
\text { kilowatinoters) }\end{array}$ & 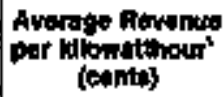 \\
\hline 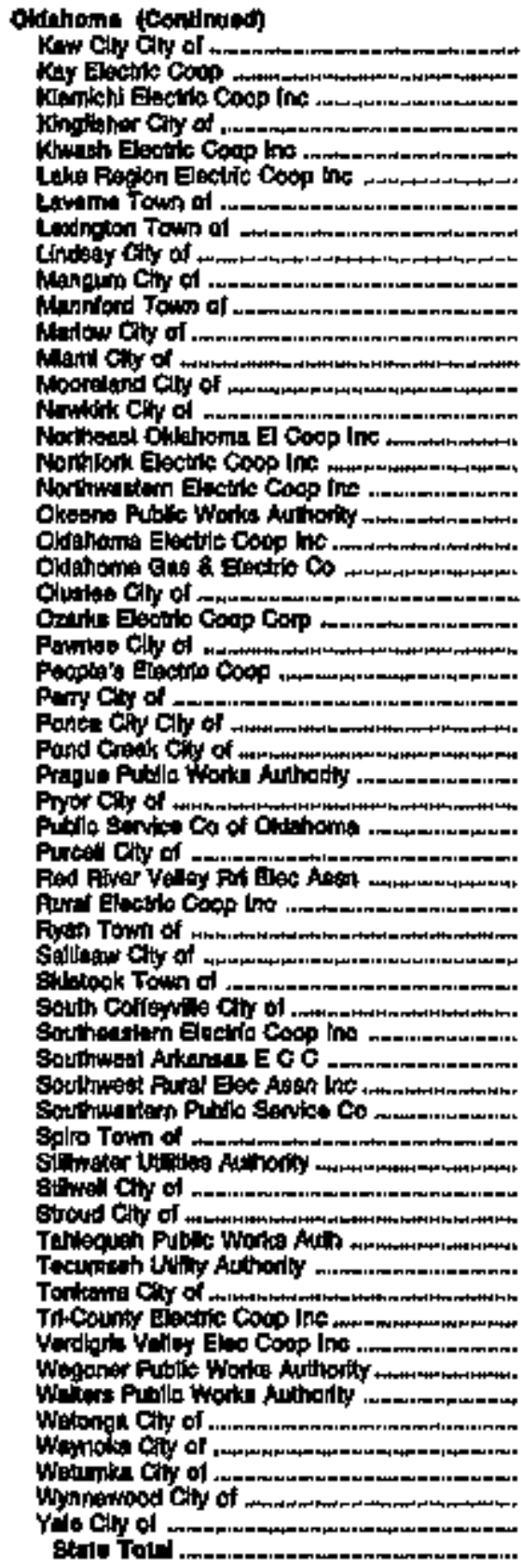 & 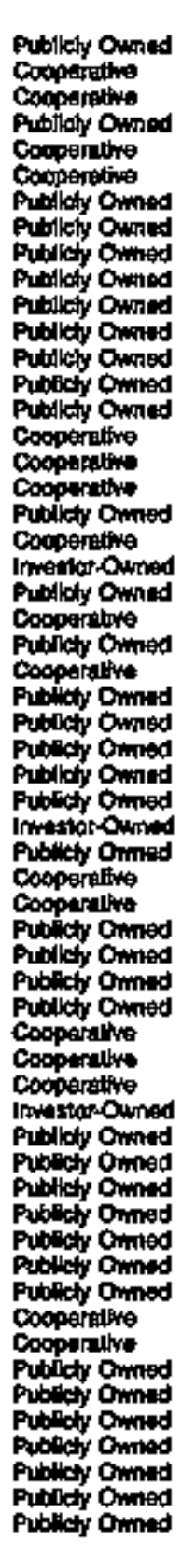 & 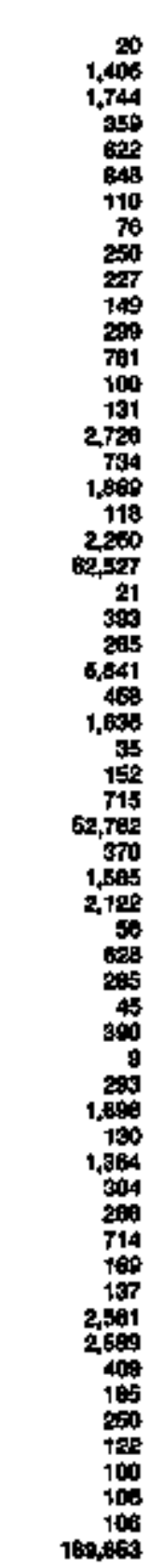 & 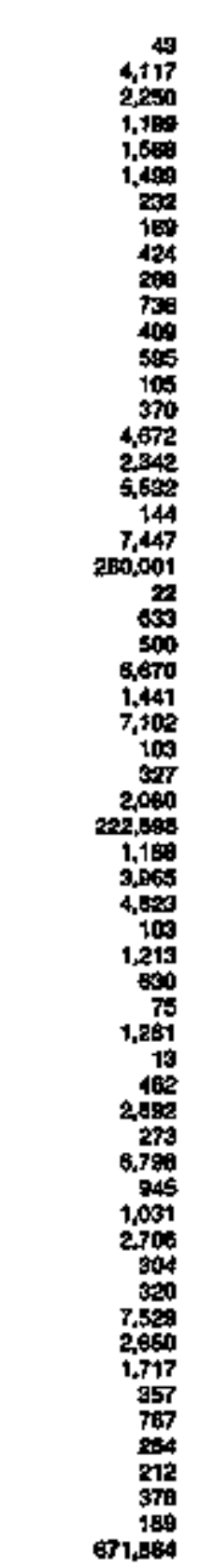 & 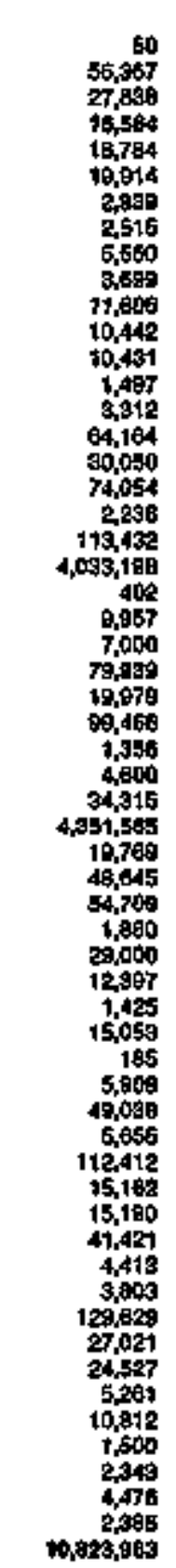 & 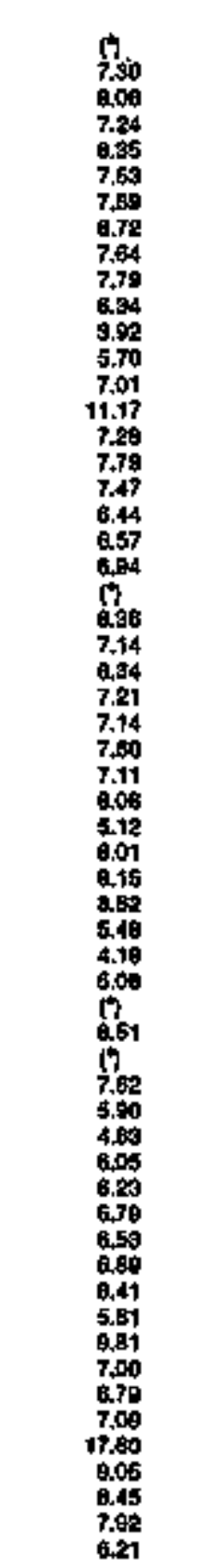 \\
\hline 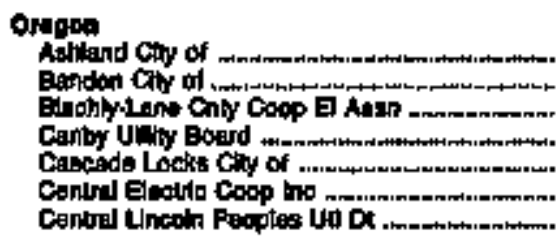 & 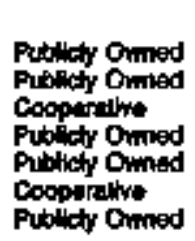 & $\begin{array}{r}1,052 \\
467 \\
106 \\
426 \\
44 \\
1,219 \\
4,149\end{array}$ & $\begin{array}{r}2,635 \\
837 \\
1,319 \\
1,281 \\
300 \\
3,234 \\
8,586\end{array}$ & $\begin{array}{r}62,401 \\
17,409 \\
28,207 \\
30,041 \\
6,918 \\
62,607 \\
195,515\end{array}$ & $\begin{array}{l}4.22 \\
4.40 \\
4.67 \\
4.89 \\
4.37 \\
6.14 \\
4.99\end{array}$ \\
\hline
\end{tabular}

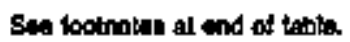


Table 15. Class of Ownership, Number of Uitimate Consumers, Pevenue, Sales, and Average Revenue per Kilowatthour for the Commerioal Sector by State and Utility, 1993 (Contimuod)

\begin{tabular}{|c|c|c|c|c|c|}
\hline $\begin{array}{l}\text { State } \\
\text { Eteotro Uniting }\end{array}$ & $\begin{array}{c}\text { tans } \\
\text { of } \\
\text { cumarthip }\end{array}$ & $\begin{array}{c}\text { Aumbor } \\
\text { of } \\
\text { Con:umars }\end{array}$ & firvenue & 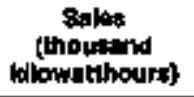 & 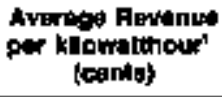 \\
\hline 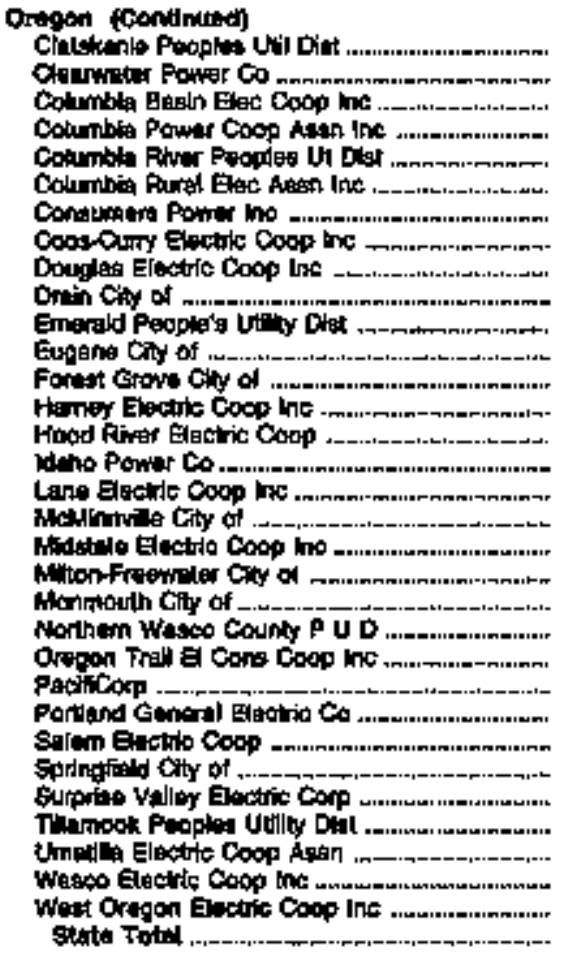 & 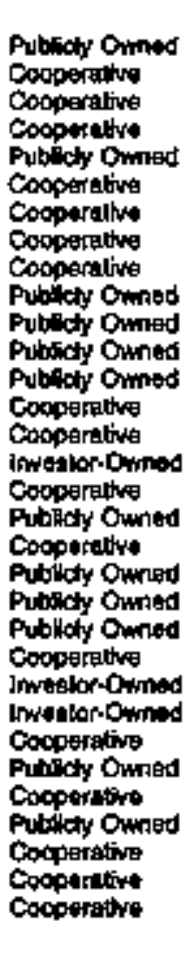 & 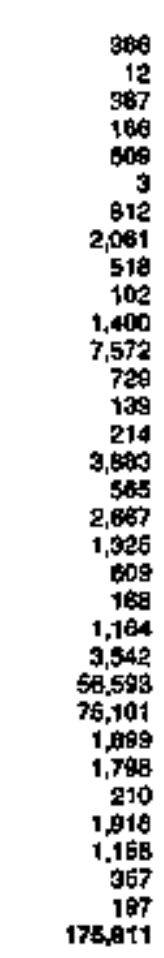 & 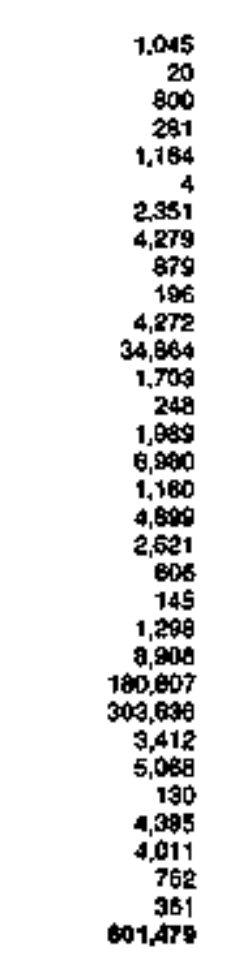 & 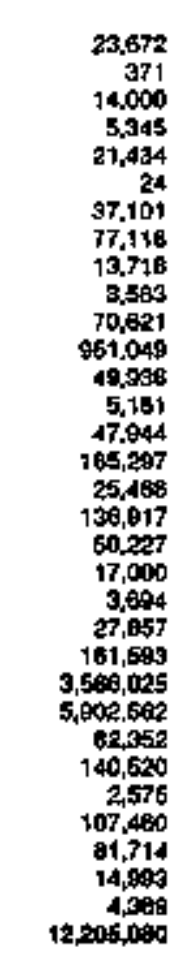 & $\begin{array}{l}4.41 \\
17 \\
5.71 \\
5.26 \\
5.43 \\
77 \\
6.34 \\
5.65 \\
6.41 \\
5.47 \\
6.05 \\
3.67 \\
3.41 \\
4.79 \\
4.15 \\
4.28 \\
4.63 \\
3.58 \\
5.22 \\
3.68 \\
3.29 \\
4.68 \\
5.61 \\
6.07 \\
5.14 \\
4.14 \\
3.61 \\
6.05 \\
4.09 \\
4.94 \\
5.02 \\
8.23 \\
4.93 \\
\end{array}$ \\
\hline 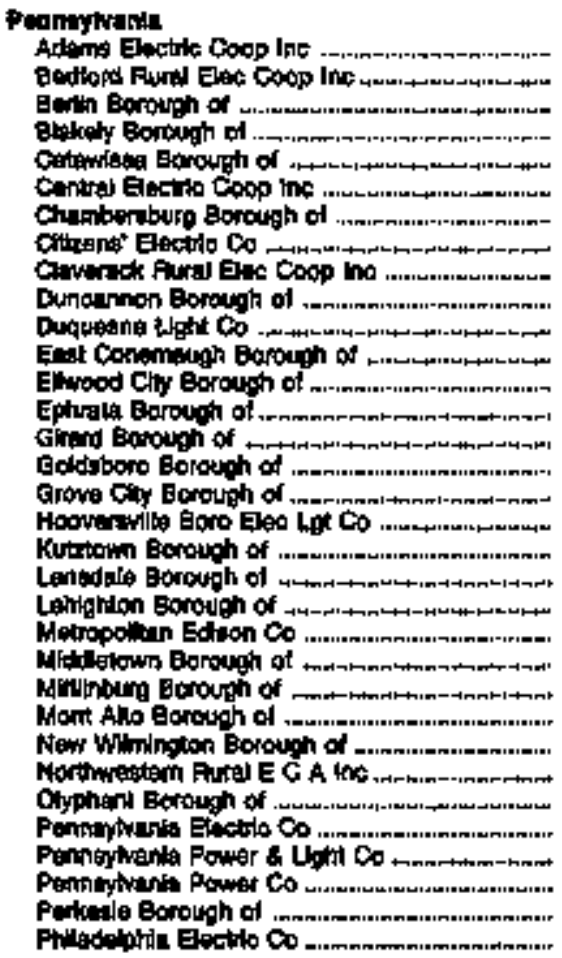 & 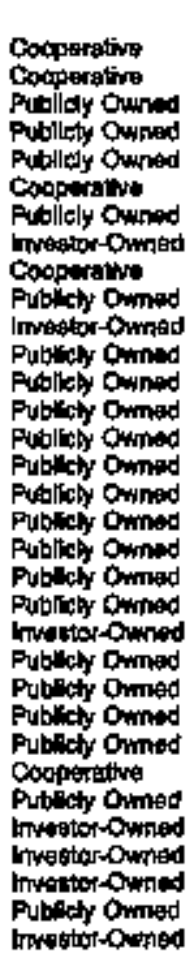 & 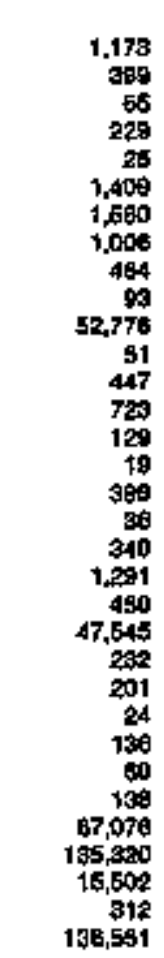 & 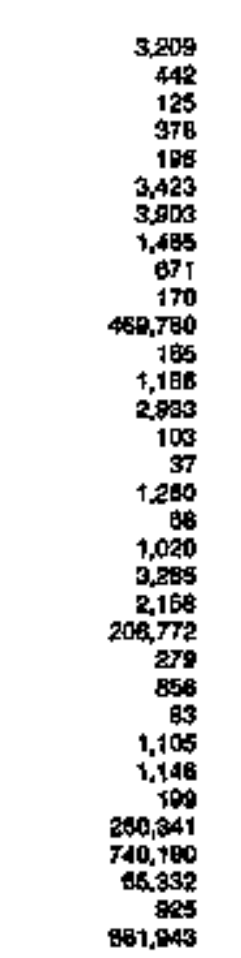 & 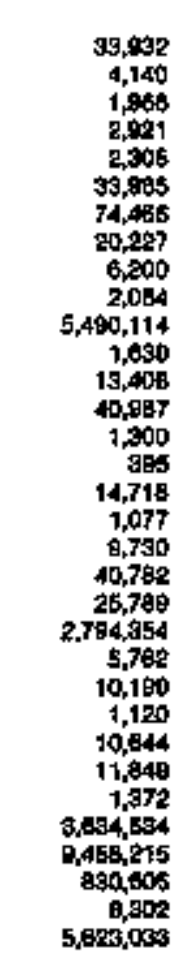 & 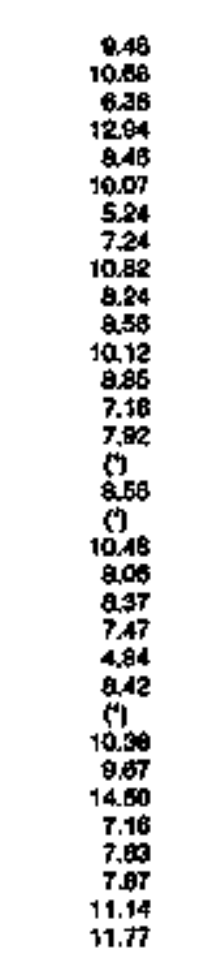 \\
\hline
\end{tabular}

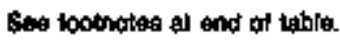


Table 15. Class of Ownershtp, Number of Utimate Consumers, Revente, Sales, and Average Fevenue per Kllowatthour for the Commerleal Sector by State and Utility, 1998 (Continued)

\begin{tabular}{|c|c|c|c|c|c|}
\hline State & of & $\begin{array}{l}\text { Anmisar } \\
\text { of } \\
\text { condumare }\end{array}$ & (tholitad dolturs) & $\begin{array}{c}\text { Enipes } \\
\text { (trousand }\end{array}$ & 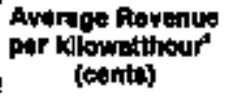 \\
\hline 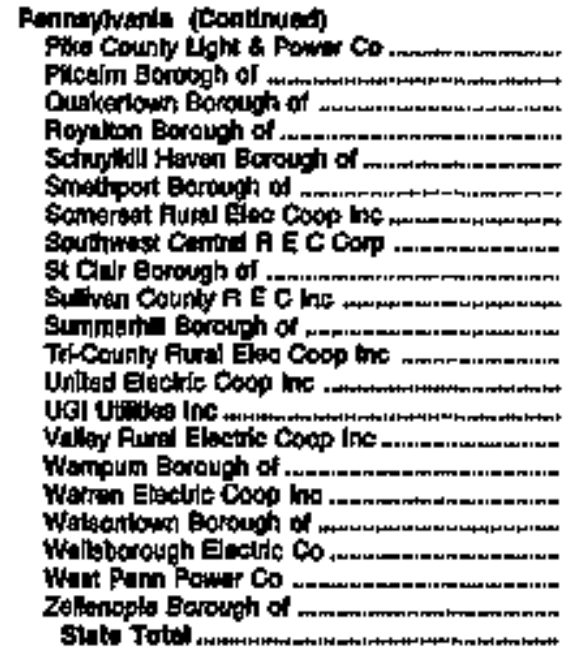 & 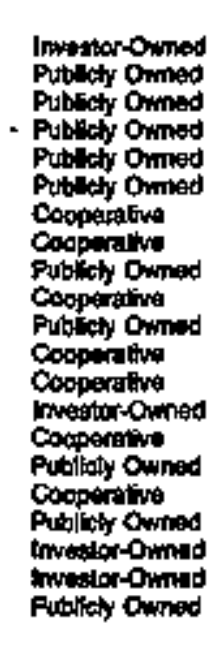 & 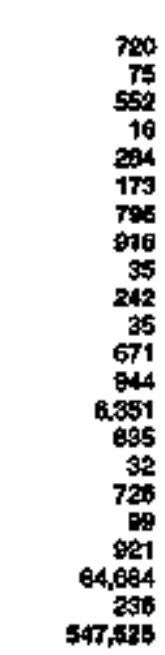 & $\begin{array}{r}1,855 \\
204 \\
1,177 \\
75 \\
577 \\
409 \\
3,042 \\
3,749 \\
243 \\
677 \\
58 \\
850 \\
3,572 \\
21,283 \\
676 \\
42 \\
690 \\
320 \\
1,674 \\
104,773 \\
917 \\
2,473992\end{array}$ & 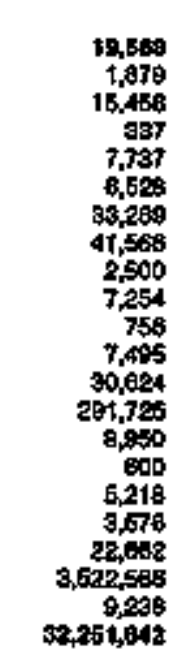 & 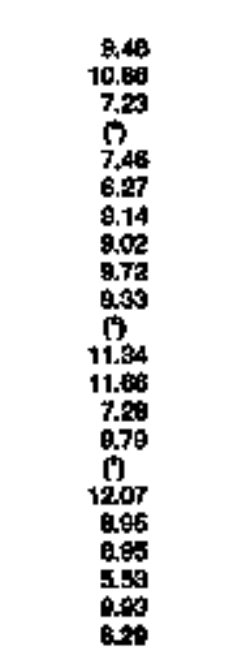 \\
\hline 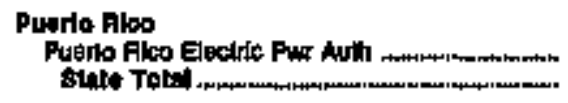 & Publ|cly Oungd & $\begin{array}{l}109,451 \\
199,451\end{array}$ & $\begin{array}{l}57,289 \\
57,245\end{array}$ & $\begin{array}{l}5,452,0028 \\
5,452,001\end{array}$ & $\begin{array}{l}10.69 \\
10.58\end{array}$ \\
\hline 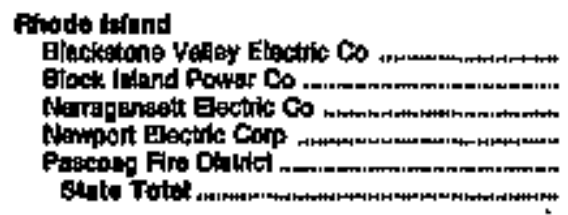 & 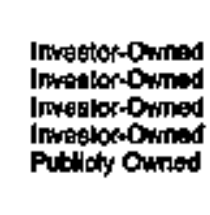 & $\begin{array}{r}6,495 \\
282 \\
31,617 \\
4,141 \\
328 \\
44,977\end{array}$ & $\begin{array}{r}49,218 \\
6 \times 7 \\
190,186 \\
22,655 \\
247,690\end{array}$ & $\begin{array}{r}401,751 \\
3,804 \\
1,081,377 \\
190,915 \\
4,398 \\
2,002,213\end{array}$ & $\begin{array}{r}10.75 \\
24.65 \\
0.85 \\
11.87 \\
13.64 \\
10.17\end{array}$ \\
\hline 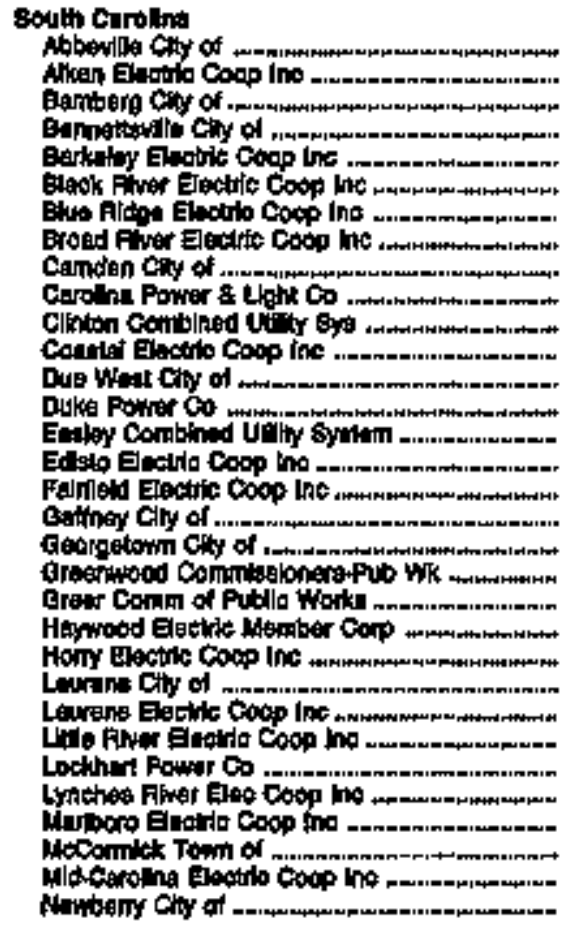 & 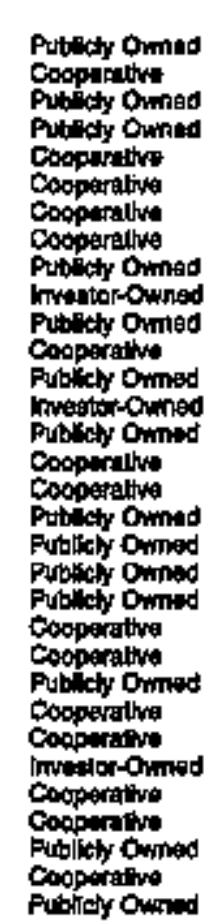 & 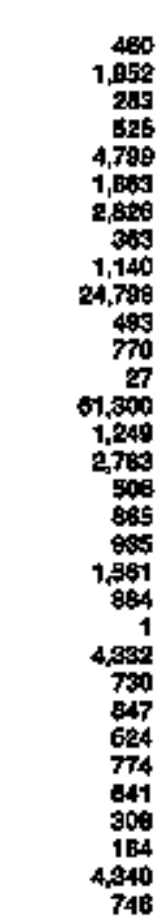 & 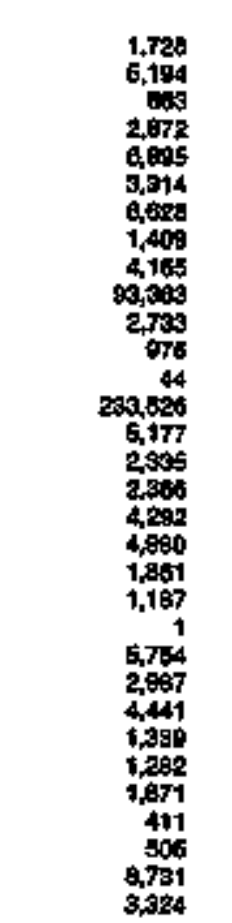 & 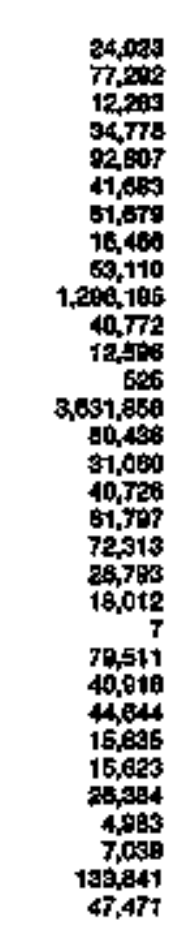 & 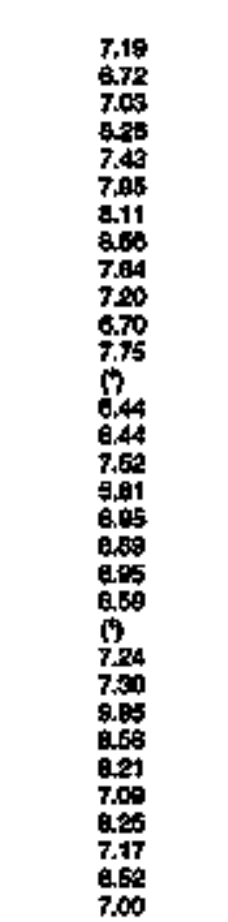 \\
\hline
\end{tabular}

Seo foolnoteo ot end of table. 
Table 15. Class of Ownerahlp, Number of Uttimate Consumers, Revenue, Saleb, and Average Fevenue per Kllowatthour for the Commerical Sector by state and Utifty, 1993 (Continuod)

\begin{tabular}{|c|c|c|c|c|c|}
\hline $\begin{array}{c}\text { 8toto } \\
\text { enectic utury }\end{array}$ & $\begin{array}{l}\text { Clatas } \\
\text { of } \\
\text { Ormitofenip }\end{array}$ & $\begin{array}{l}\text { Number } \\
\text { of } \\
\text { Consulaners }\end{array}$ & $\begin{array}{c}\text { Mowenus } \\
\text { (Thousend dollare) }\end{array}$ & 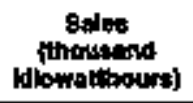 & 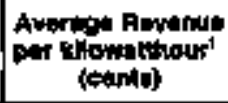 \\
\hline 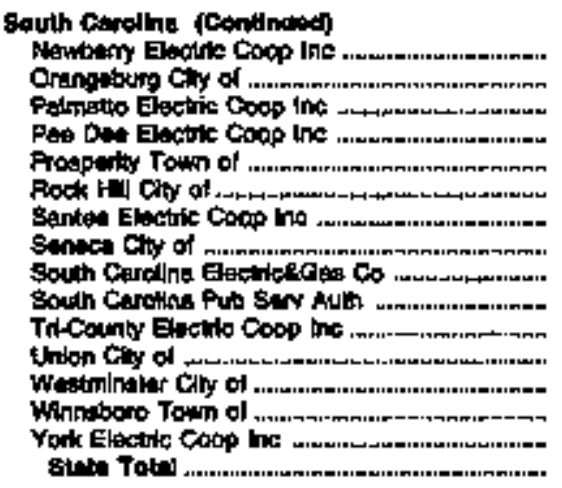 & 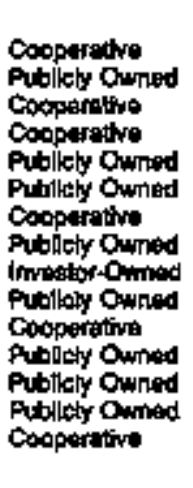 & $\begin{array}{r}460 \\
2,702 \\
4,057 \\
1,070 \\
1,02 \\
2,541 \\
1,876 \\
703 \\
60,728 \\
15,373 \\
552 \\
1,004 \\
212 \\
1,285 \\
1,284 \\
217,906\end{array}$ & $\begin{array}{r}1,396 \\
7,983 \\
18,176 \\
2,962 \\
181 \\
20,111 \\
4,044 \\
3,789 \\
27,171 \\
00,002 \\
2,073 \\
2,511 \\
699 \\
2,587 \\
3,973 \\
90,1144\end{array}$ & 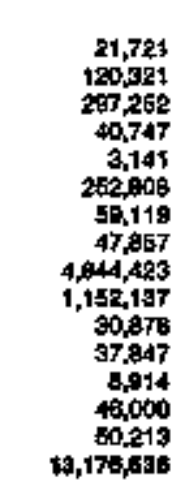 & $\begin{array}{l}6.30 \\
6.63 \\
6.11 \\
7.20 \\
6.76 \\
7.06 \\
6.04 \\
7.80 \\
5.80 \\
6.28 \\
6.71 \\
6.00 \\
7.61 \\
5.80 \\
7.81 \\
6.20\end{array}$ \\
\hline 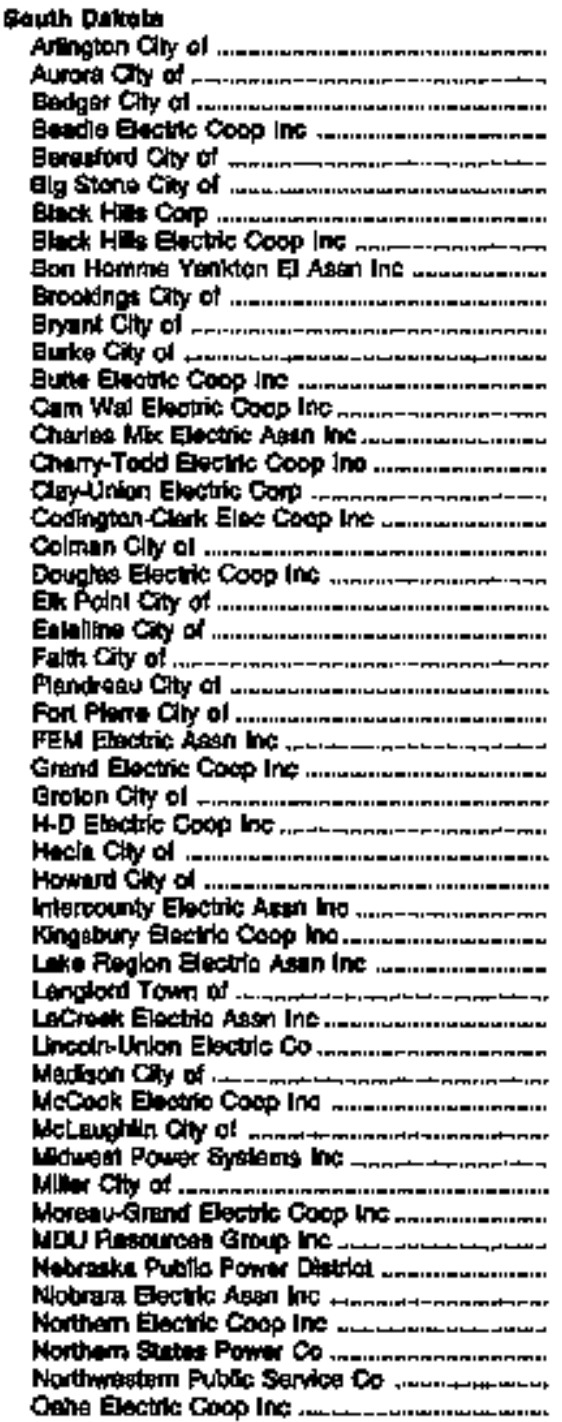 & 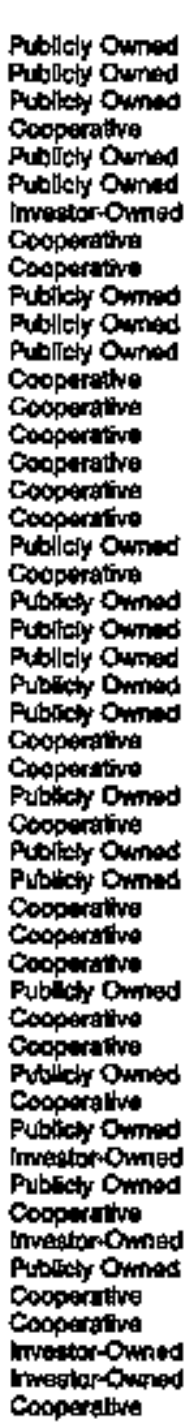 & 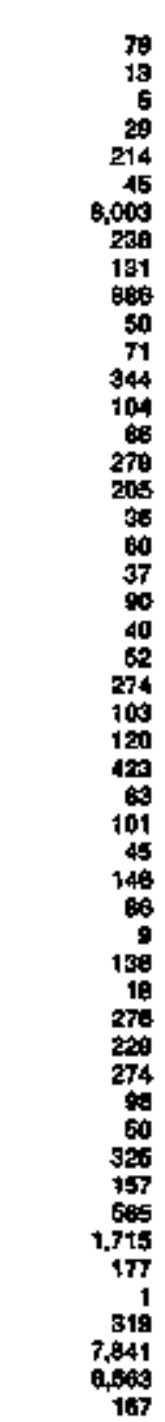 & 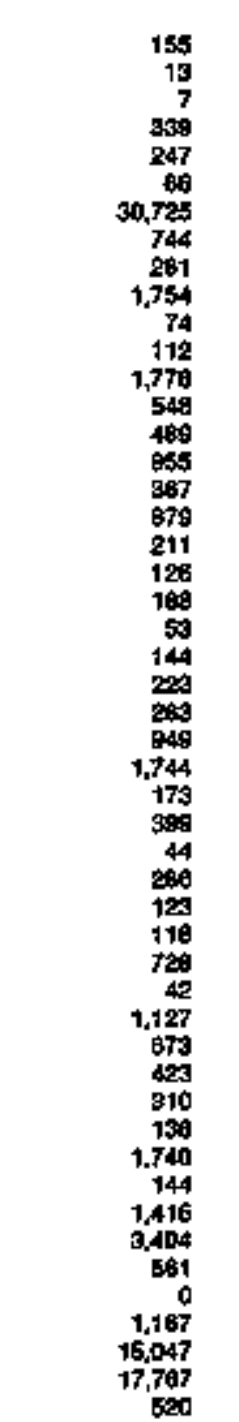 & 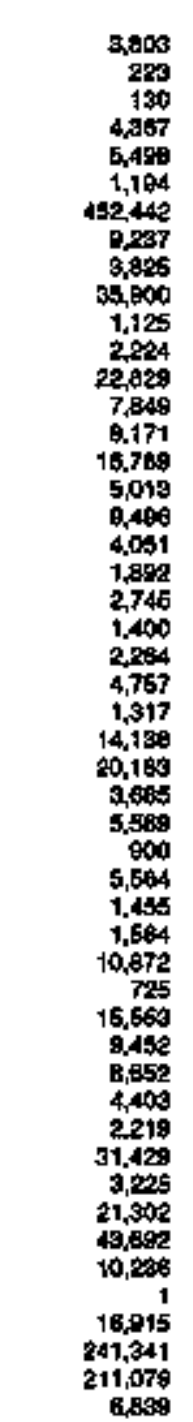 & 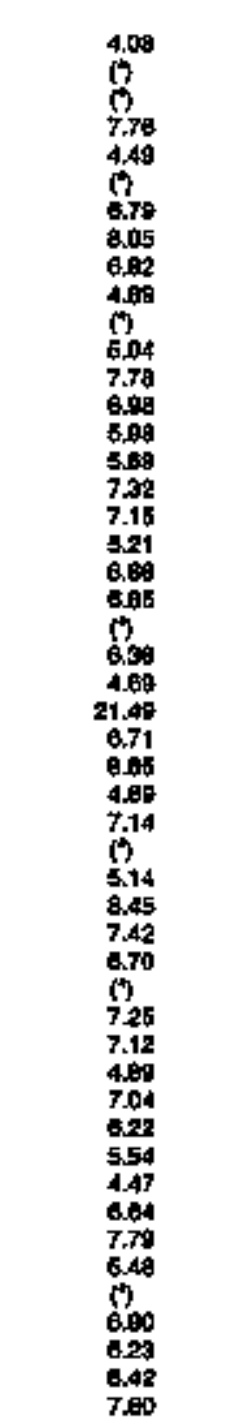 \\
\hline
\end{tabular}

See toobrotes al ind of tuble. 
Table 15. Class of Omnershyp, Number of Ulitinate Consumers, Aevenue, Sales, and Average Revenue per Kilowatthour for the Commerted Soctor by State and Utillty, 1993 (Continuod)

\begin{tabular}{|c|c|c|c|c|c|}
\hline $\begin{array}{l}\text { Sicte } \\
\text { enpitio Uitity }\end{array}$ & Clate & $\begin{array}{l}\text { Momber } \\
\text { of } \\
\text { contubatere }\end{array}$ & 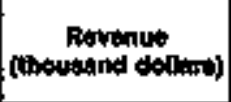 & $\begin{array}{l}\text { Balat } \\
\text { hownithourd }\end{array}$ & 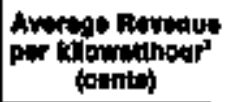 \\
\hline 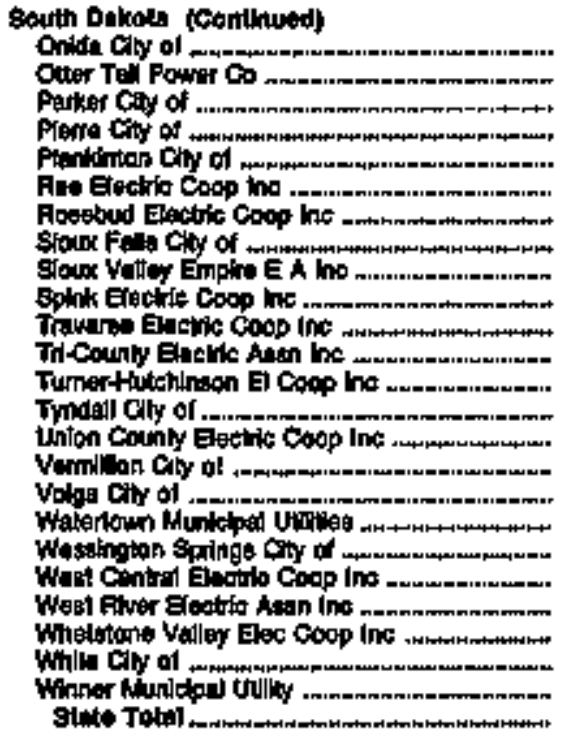 & 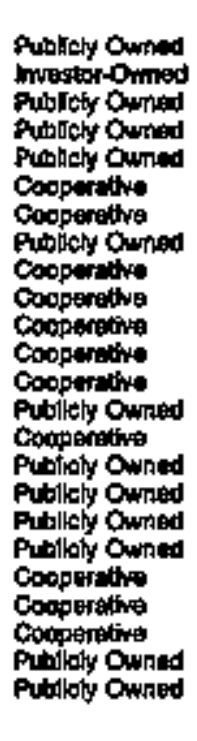 & $\begin{array}{r}50 \\
2,381 \\
108 \\
690 \\
50 \\
61 \\
447 \\
361 \\
974 \\
28 \\
10 \\
165 \\
308 \\
88 \\
49 \\
438 \\
88 \\
1,280 \\
67 \\
752 \\
704 \\
110 \\
42 \\
340 \\
43,111\end{array}$ & 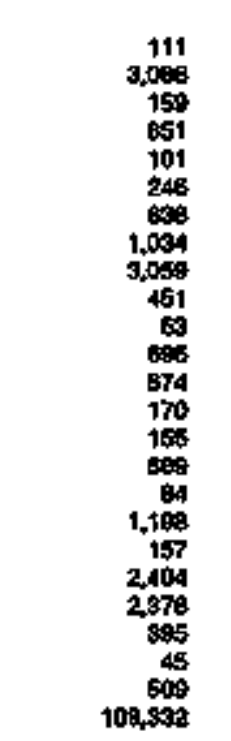 & 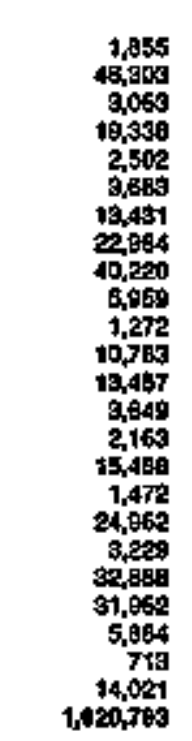 & 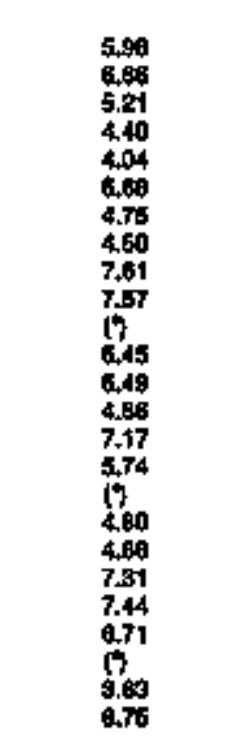 \\
\hline 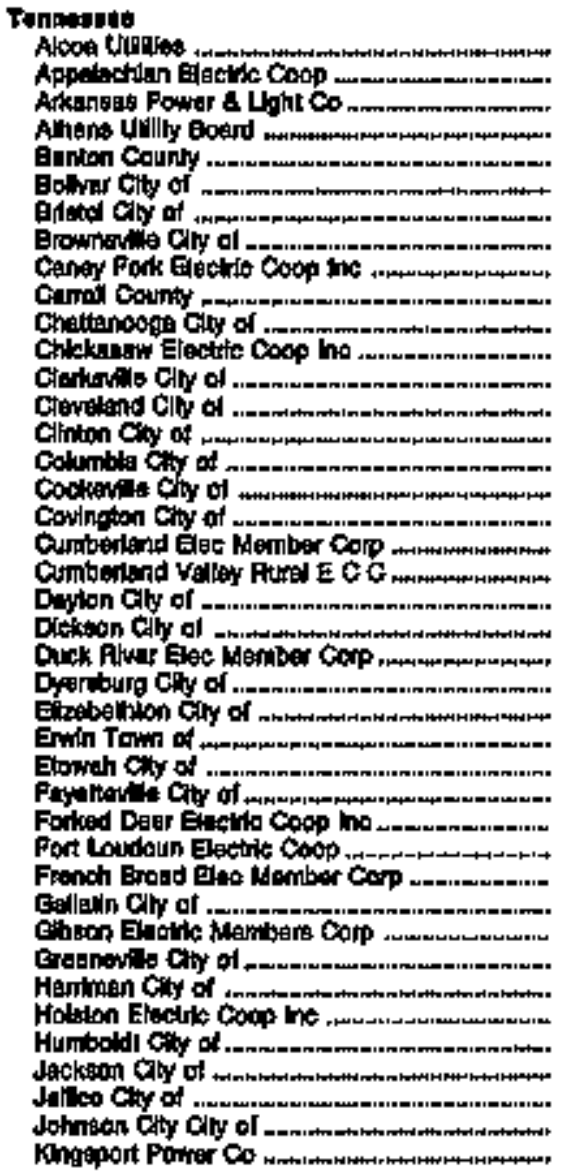 & 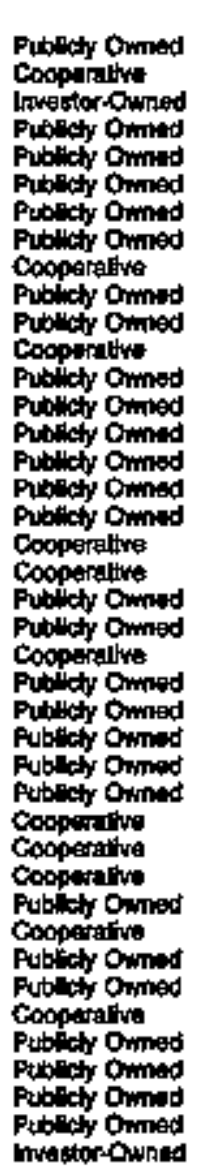 & 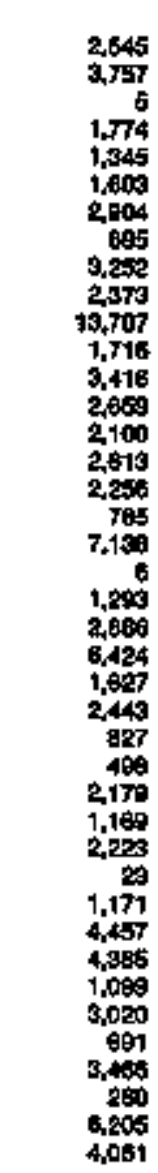 & 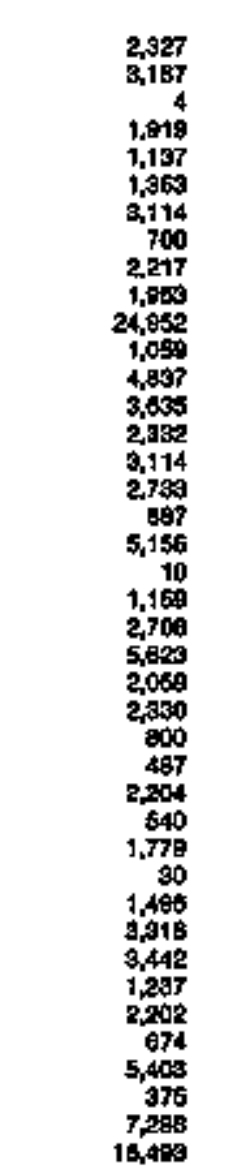 & 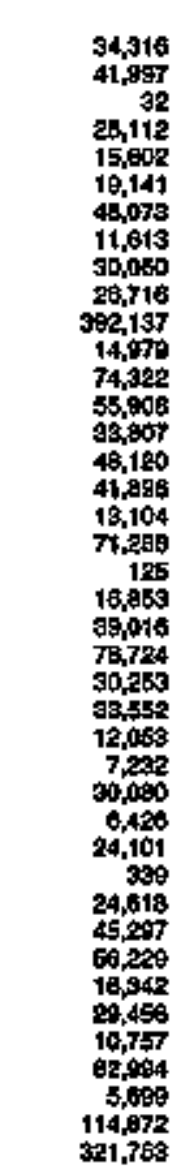 & 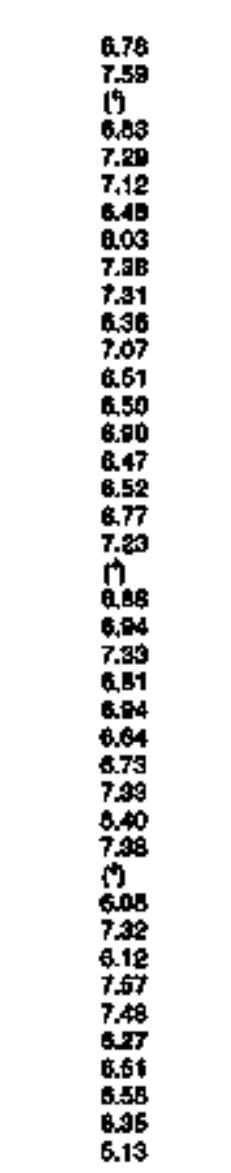 \\
\hline
\end{tabular}

Sed kolnotes at end of tabta. 
Tabje 15. Class of Ownorship, Number of Ultimate Consumers, Revenue, Sales, and Averege Rovenue per Kllowatthour tor the Comumerical Sector by State and Utilty, 1998 (Continued)

\begin{tabular}{|c|c|c|c|c|c|}
\hline Gitk & of & $\begin{array}{c}\text { Aumbar } \\
\text { of } \\
\text { Contaymines }\end{array}$ & (thousand doltere') & 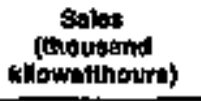 & 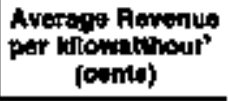 \\
\hline 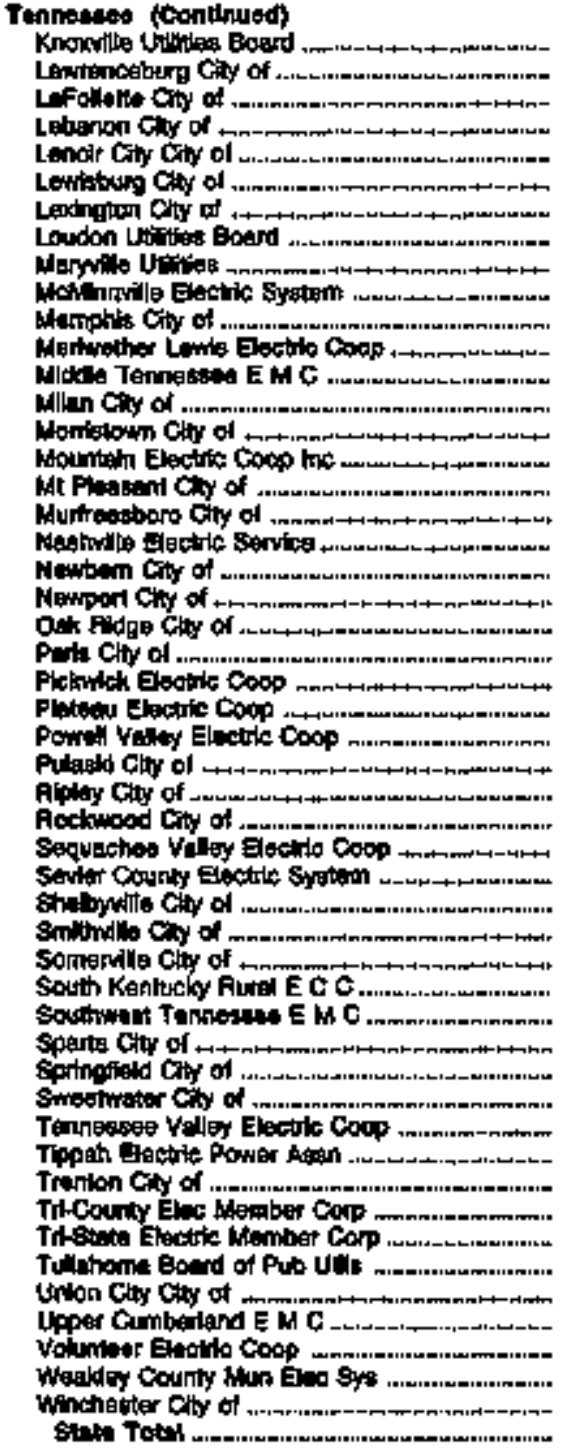 & 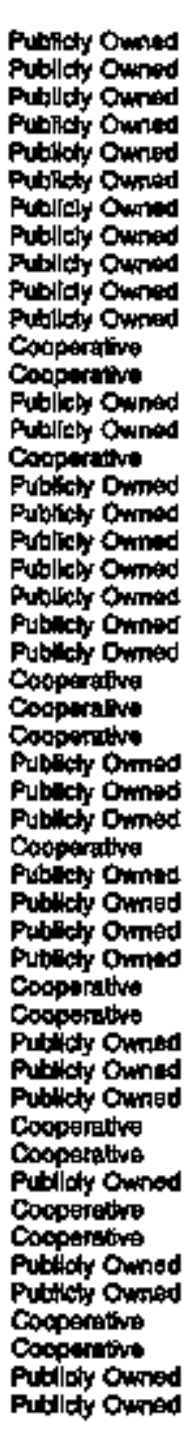 & 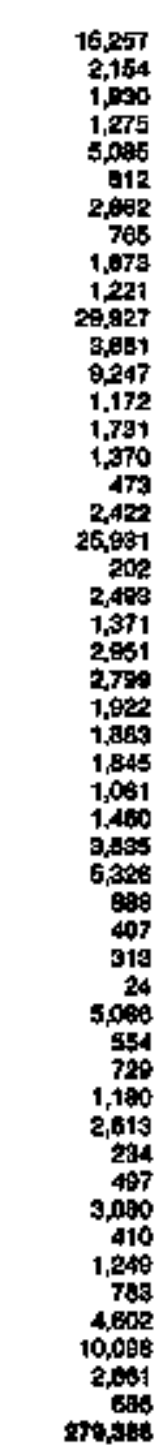 & 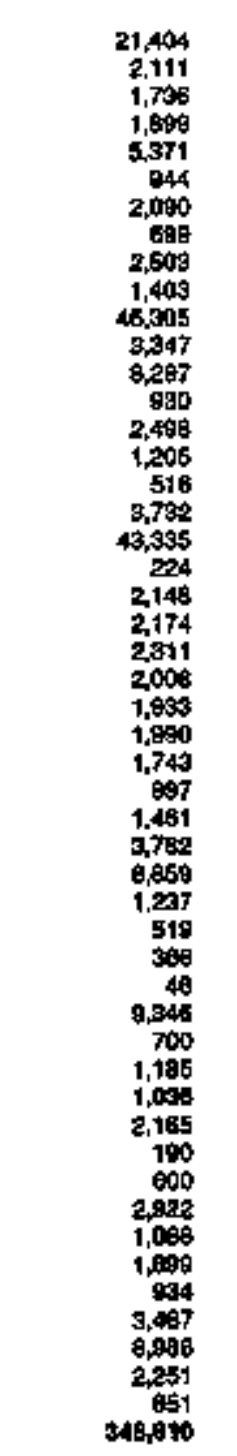 & 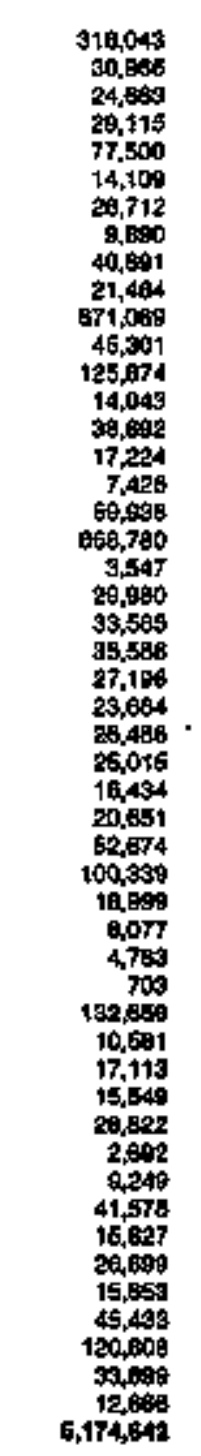 & 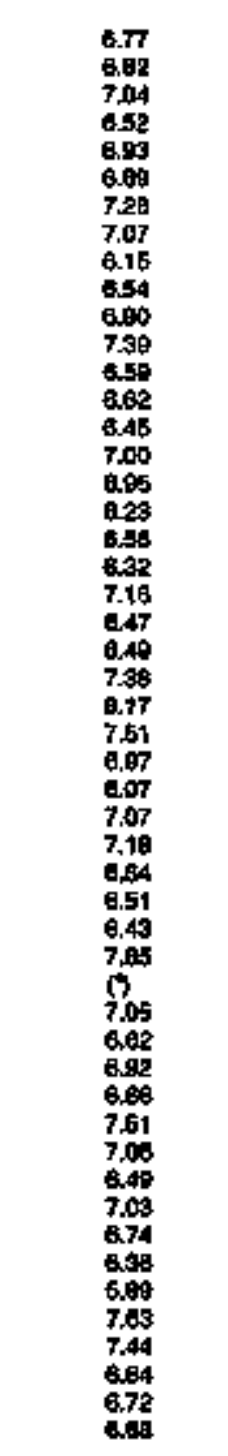 \\
\hline 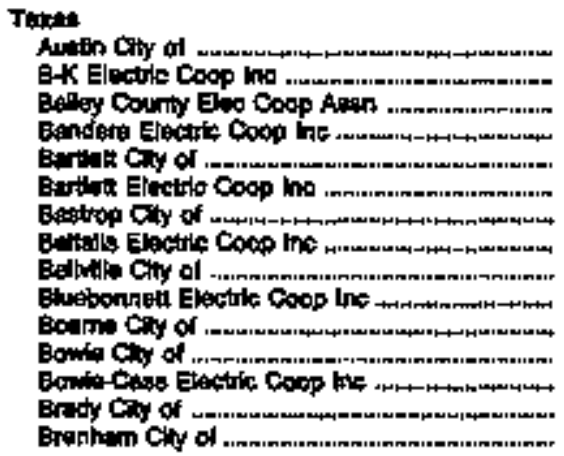 & 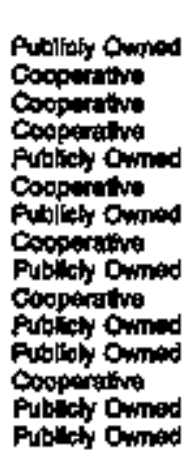 & 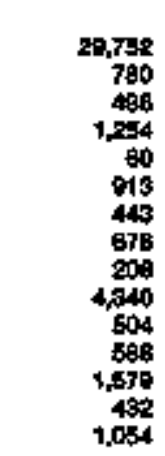 & $\begin{array}{r}207,140 \\
3,792 \\
1,894 \\
3,688 \\
283 \\
474 \\
1,710 \\
1,118 \\
186 \\
4,577 \\
1,887 \\
1,458 \\
4,198 \\
700 \\
3,187\end{array}$ & 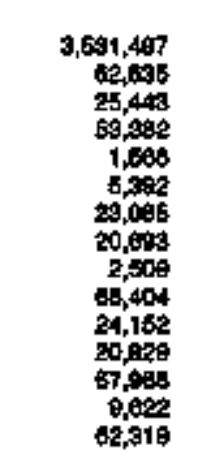 & $\begin{array}{r}6.71 \\
6.04 \\
7.80 \\
6.70 \\
18.18 \\
8.70 \\
7.41 \\
5.40 \\
7.81 \\
6.60 \\
7.90 \\
6.90 \\
6.08 \\
7.38 \\
5.11\end{array}$ \\
\hline
\end{tabular}

Ses tootnotes at end of teble. 
Table 15. Cinses of Ommerehlp, Number of Uitimate Consumers, Revenus, Sales, end Average Fevenue per Klowsthour for the Commerical Sector by state and Utility, 1998 (Continued)

\begin{tabular}{|c|c|c|c|c|c|}
\hline gitato & $\begin{array}{c}\text { ciat: } \\
\text { of } \\
\text { Dimerishlp }\end{array}$ & comber & 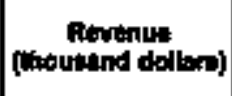 & $\begin{array}{c}\text { Eakt } \\
\text { (thowtind } \\
\text { titowithours) }\end{array}$ & 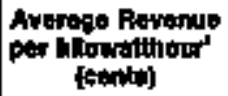 \\
\hline 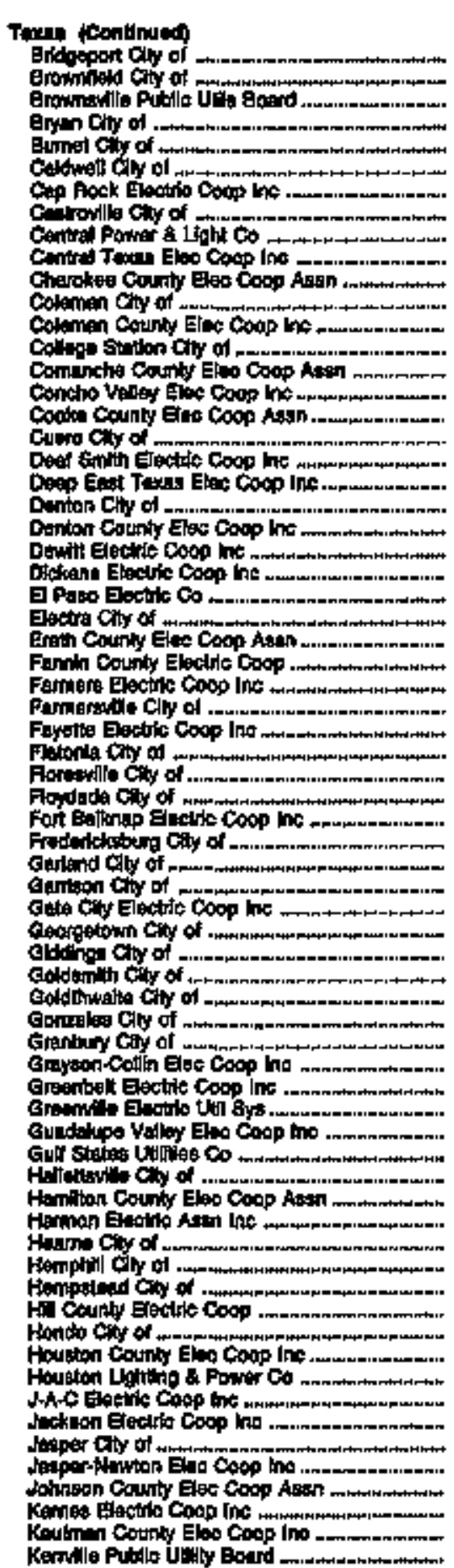 & 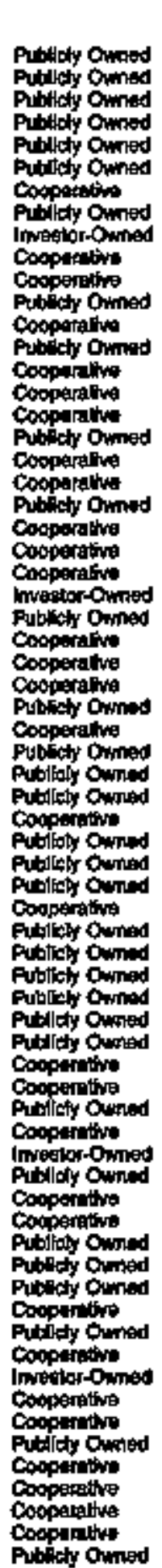 & 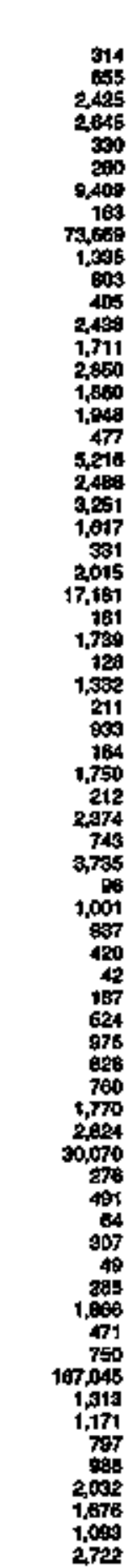 & 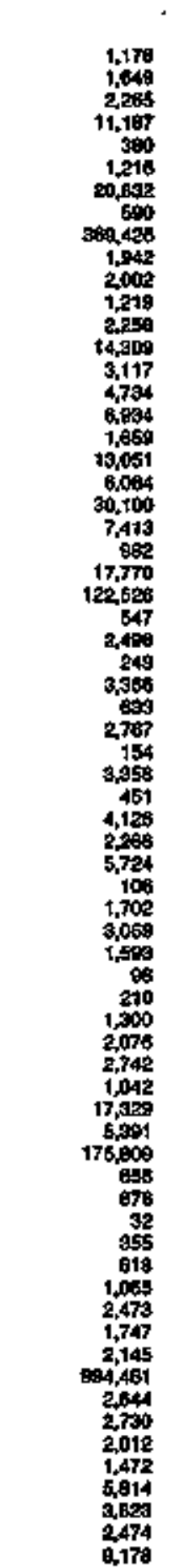 & 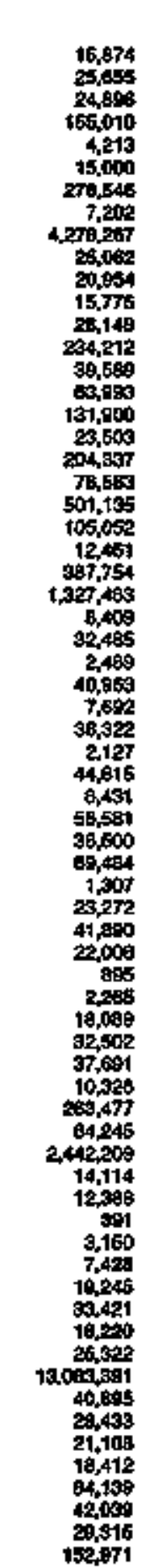 & 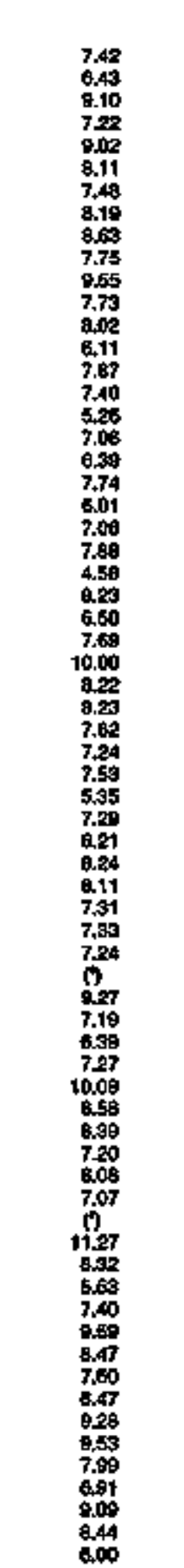 \\
\hline
\end{tabular}

Sea toothotas al and of tabla. 
Table 15. Clase of Ownershlp, Number of Uitmate Consumert, Revenue, Sales, and Average Revenue per Kilowatthour tor the Commerical Sector by State and Utility, 1993 (Continued)

\begin{tabular}{|c|c|c|c|c|c|}
\hline $\begin{array}{c}\text { Slate } \\
\text { Elaptric Utiny }\end{array}$ & $\begin{array}{c}\text { Ctist } \\
\text { of } \\
\text { onthershp }\end{array}$ & $\begin{array}{l}\text { Number } \\
\text { of } \\
\text { Conitimar: }\end{array}$ & 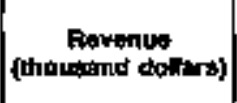 & $\begin{array}{l}\text { stat } \\
\text { (thoumend } \\
\text { tholwthourd) }\end{array}$ & 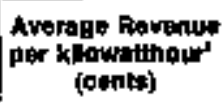 \\
\hline 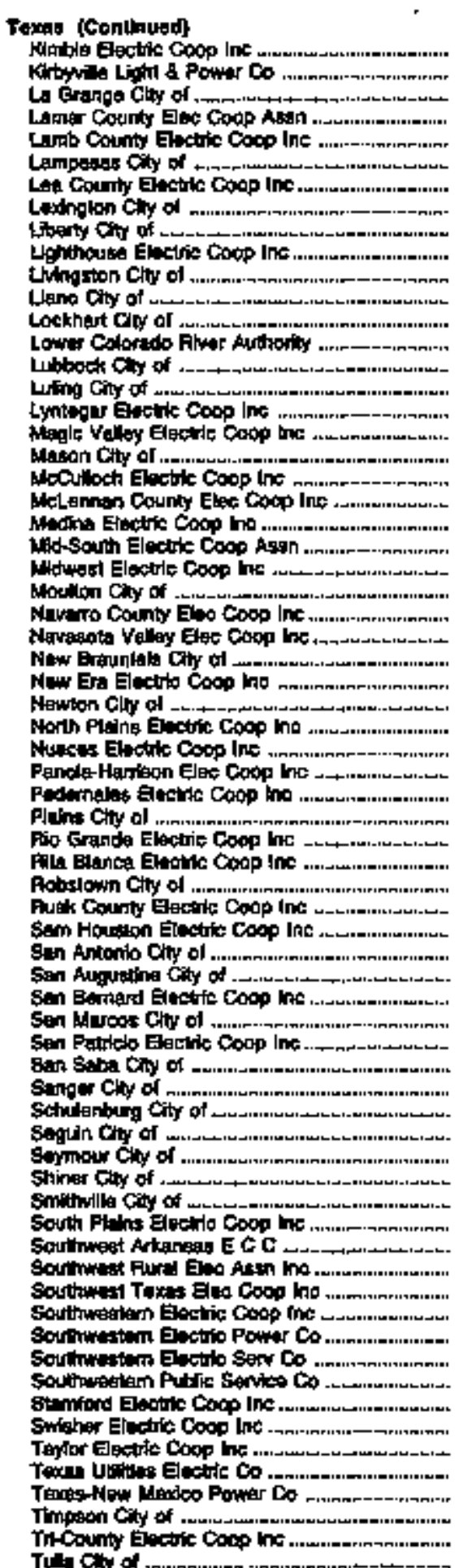 & 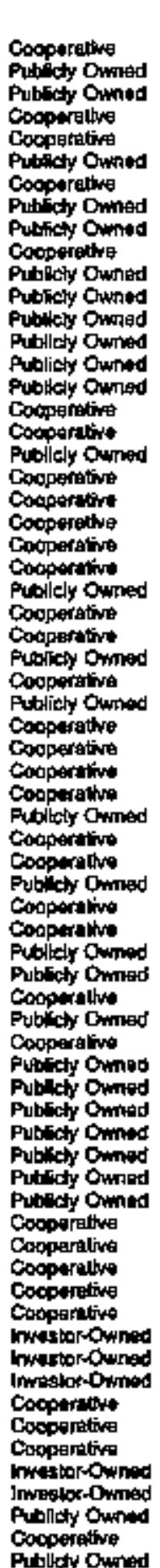 & 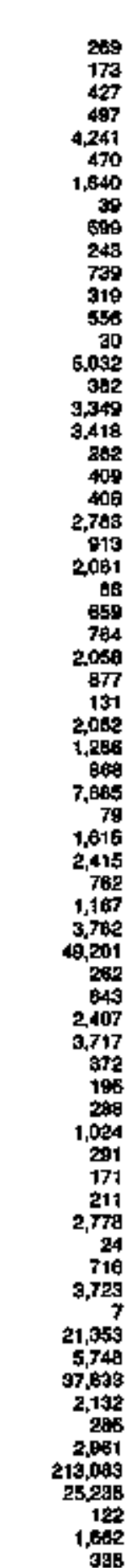 & 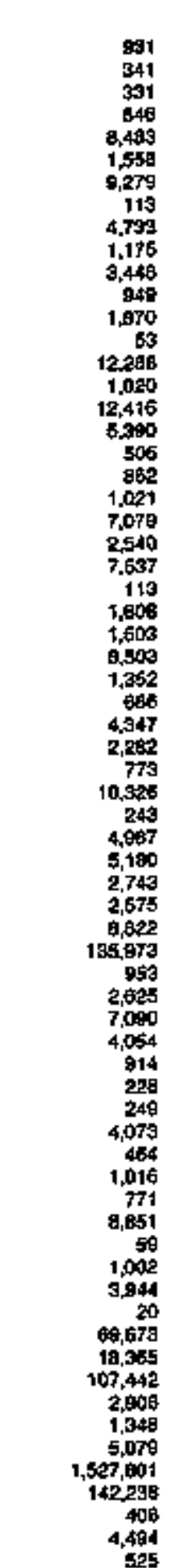 & 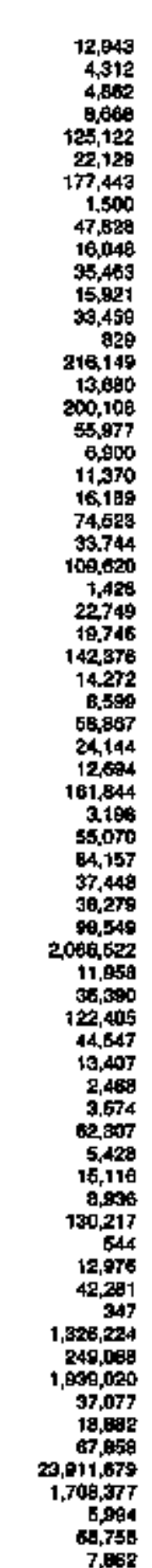 & 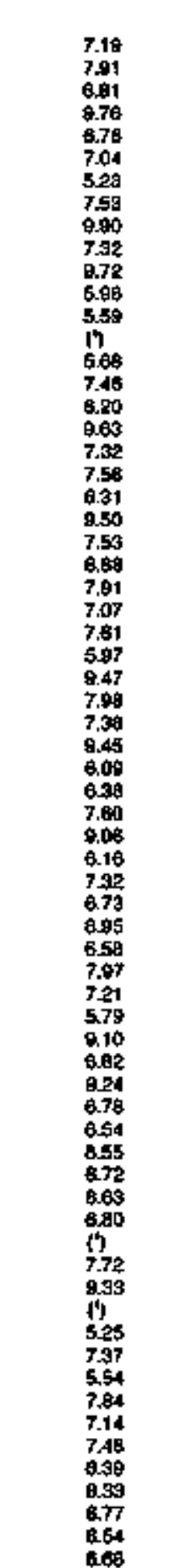 \\
\hline
\end{tabular}

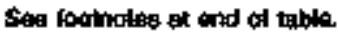


Table 15. Class of Omnership, Number of Utimate Consumers, Revenue, Sales, and Ayarage Revenue per Kilowatthour for the Commerical Sector by State and Utillty, 1993 (Continued)

\begin{tabular}{|c|c|c|c|c|c|}
\hline 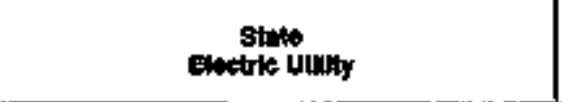 & 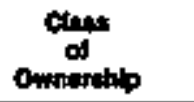 & $\begin{array}{l}\text { Number } \\
\text { of } \\
\text { Connumers }\end{array}$ & Rowoulo & 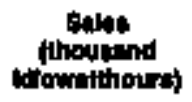 & 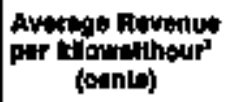 \\
\hline 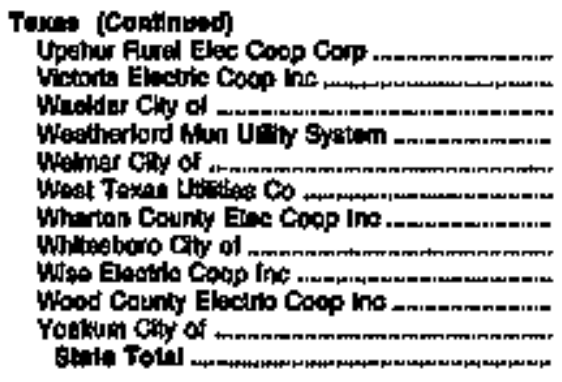 & 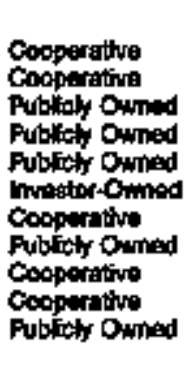 & $\begin{array}{r}2,691 \\
1,624 \\
76 \\
980 \\
185 \\
25,904 \\
642 \\
155 \\
1,492 \\
2,711 \\
321 \\
695,502\end{array}$ & 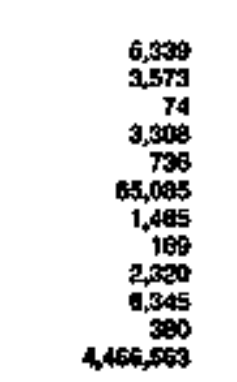 & $\begin{array}{r}98,016 \\
44,487 \\
0112 \\
19,380 \\
11,382 \\
1,087,410 \\
17,701 \\
2,065 \\
34,756 \\
109,702 \\
4,211 \\
4,991,490\end{array}$ & $\begin{array}{l}6.45 \\
6,03 \\
17 \\
7.13 \\
6.47 \\
5.69 \\
0.39 \\
9.22 \\
6.69 \\
5.77 \\
9.02 \\
6.94\end{array}$ \\
\hline 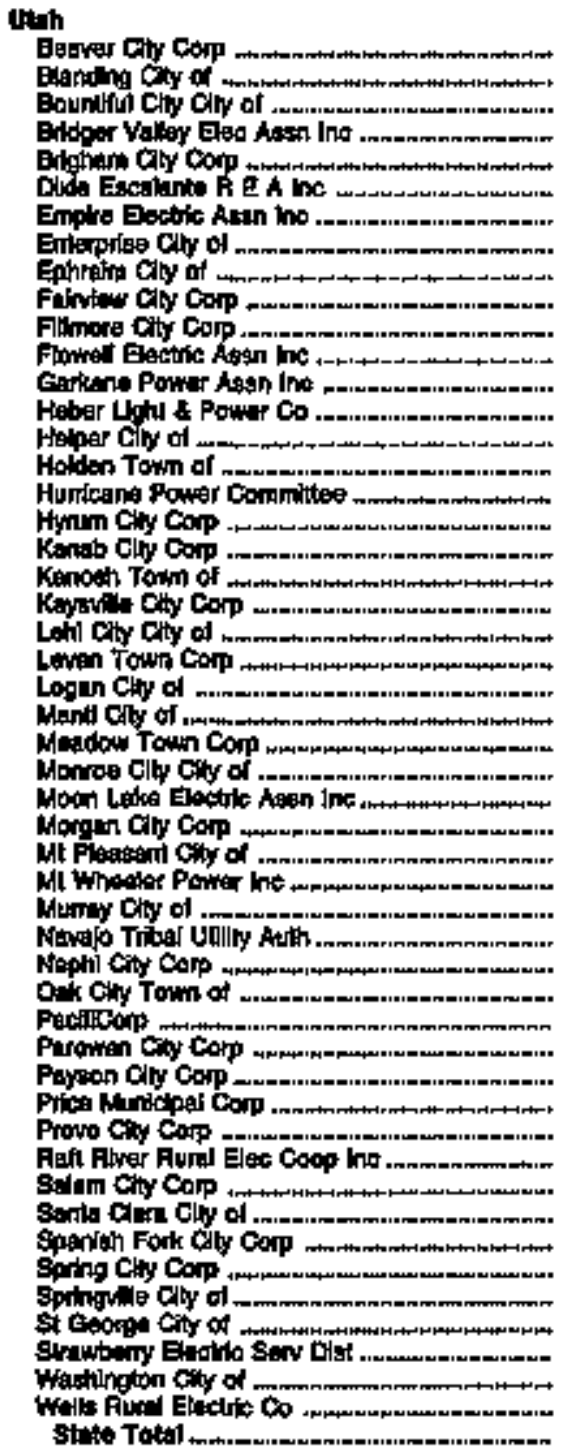 & 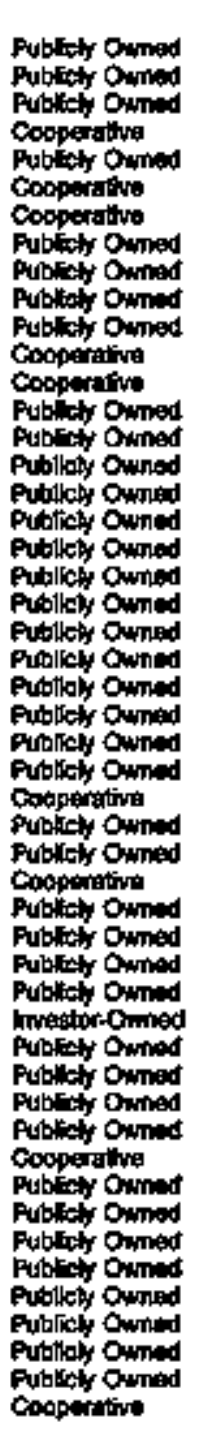 & 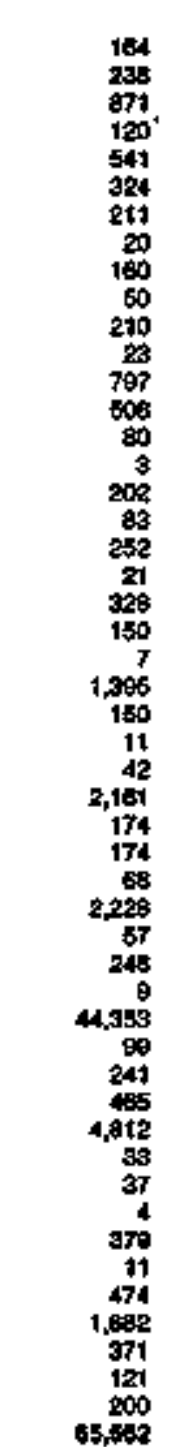 & 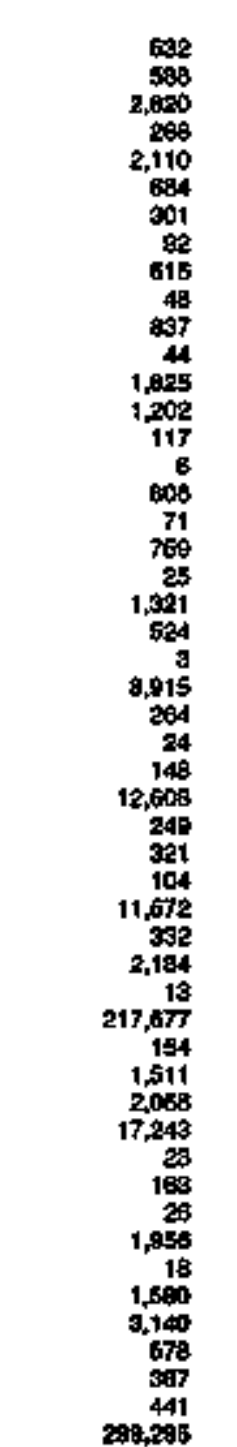 & 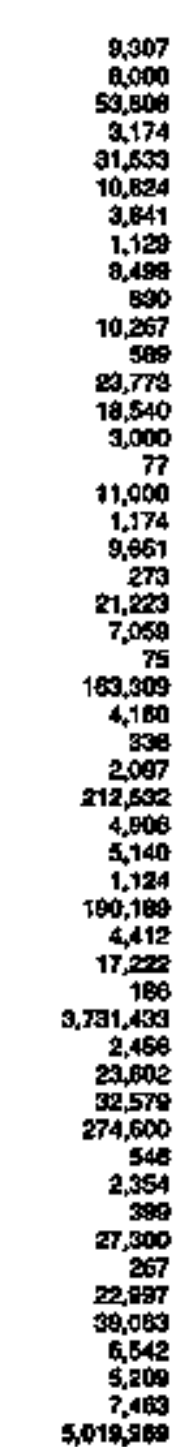 & 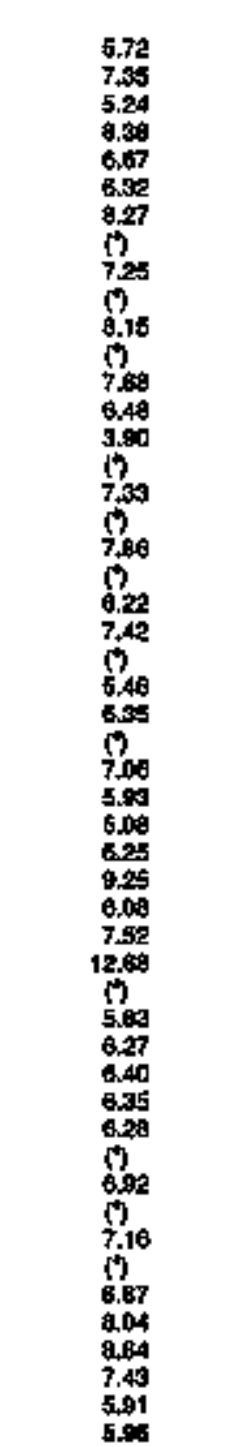 \\
\hline
\end{tabular}

8.0 looknote: at and of thits. 
Table 15. Class of Ownership, Number of Uttimate Consumers, Revenue, Sales, and Average Revenue per Kllowatthour for the Commerical Sector by State and Utility, 1993 (Continued)

\begin{tabular}{|c|c|c|c|c|c|}
\hline Etch & 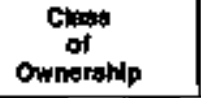 & $\begin{array}{l}\text { Number } \\
\text { of } \\
\text { Gonganamera }\end{array}$ & $\begin{array}{c}\text { Atwinch } \\
\text { \{thousand donery }\end{array}$ & $\begin{array}{l}\text { Salea } \\
\text { (thourand } \\
\text { klowsthoursy }\end{array}$ & $\begin{array}{l}\text { Avarage Agrenun } \\
\text { par Mllowatihour' } \\
\text { (conig) }\end{array}$ \\
\hline 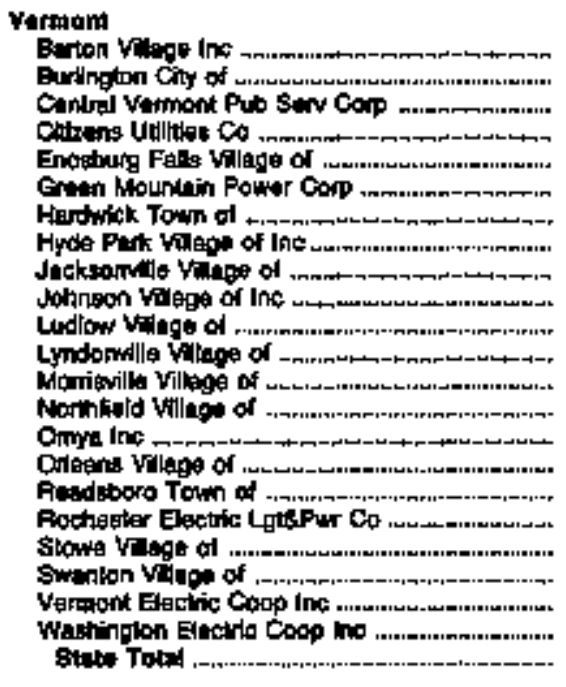 & 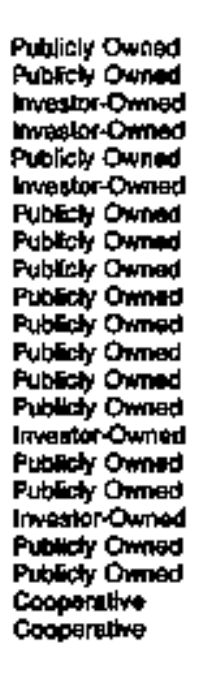 & $\begin{array}{r}137 \\
2,888 \\
16,409 \\
1,968 \\
106 \\
11,447 \\
321 \\
97 \\
50 \\
94 \\
189 \\
588 \\
371 \\
178 \\
54 \\
54 \\
42 \\
112 \\
519 \\
234 \\
440 \\
189 \\
30,776\end{array}$ & $\begin{array}{r}250 \\
2,707 \\
60,098 \\
6,308 \\
300 \\
47,310 \\
413 \\
128 \\
68 \\
375 \\
885 \\
1,030 \\
672 \\
202 \\
311 \\
143 \\
99 \\
161 \\
3,001 \\
374 \\
860 \\
371 \\
146,187\end{array}$ & 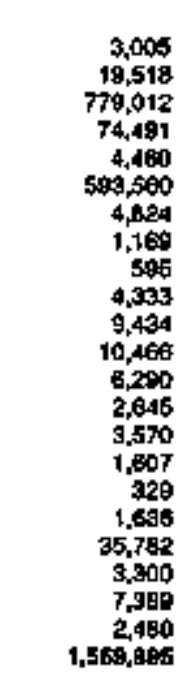 & 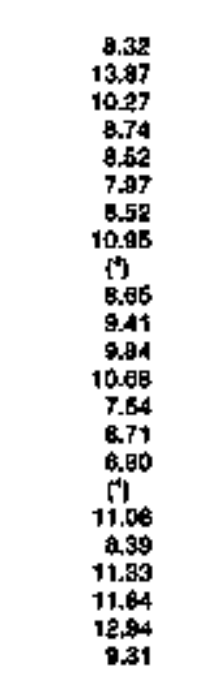 \\
\hline 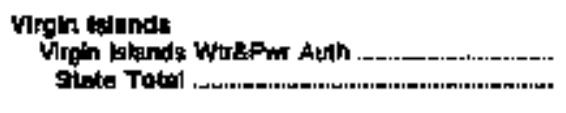 & Publicty Owned & $\begin{array}{l}7,171 \\
7,171\end{array}$ & $\begin{array}{l}10,502 \\
16,502\end{array}$ & $\begin{array}{l}111072 \\
111,072\end{array}$ & $\begin{array}{l}14.94 \\
t 4,94\end{array}$ \\
\hline 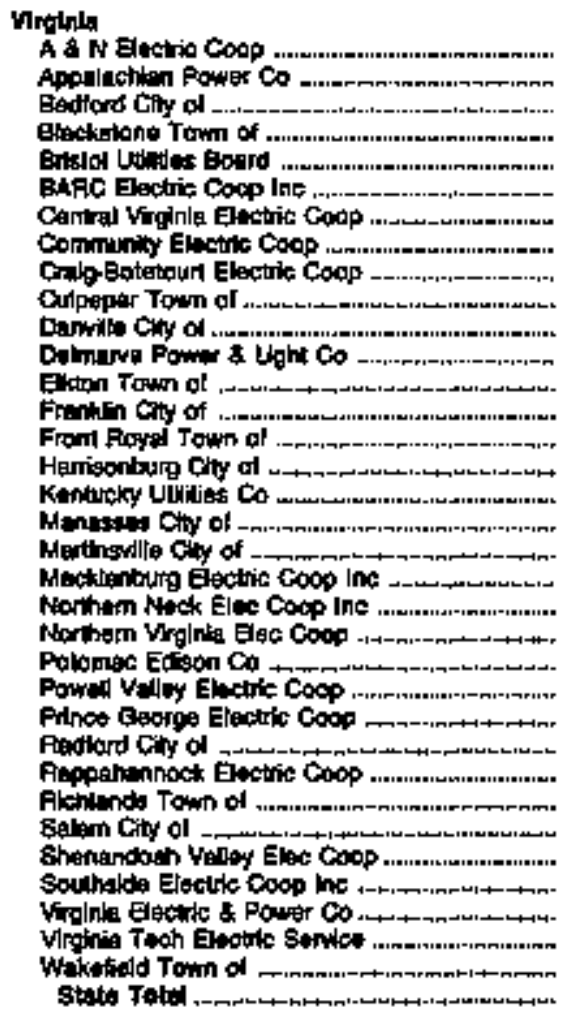 & 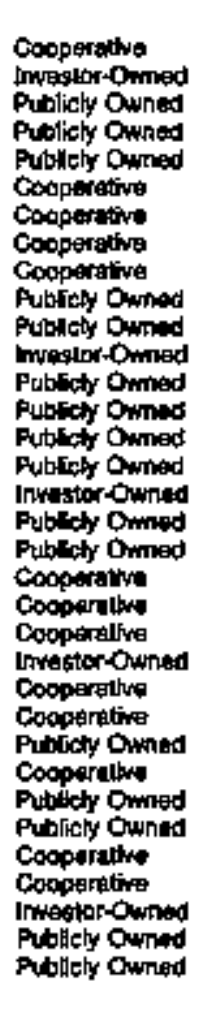 & 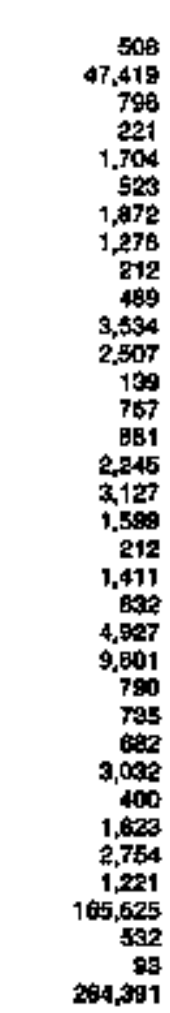 & 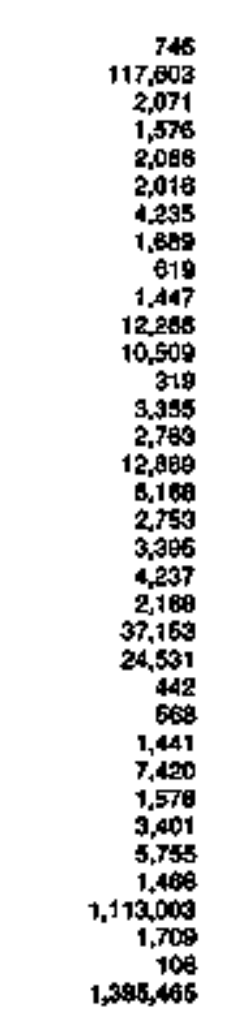 & 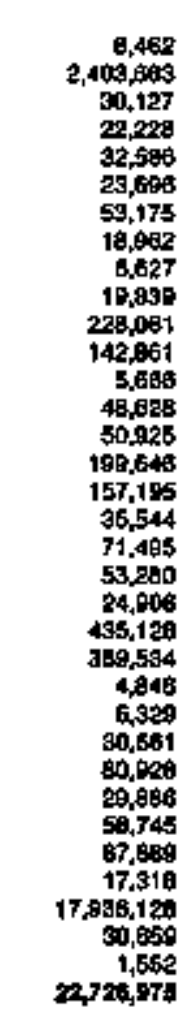 & 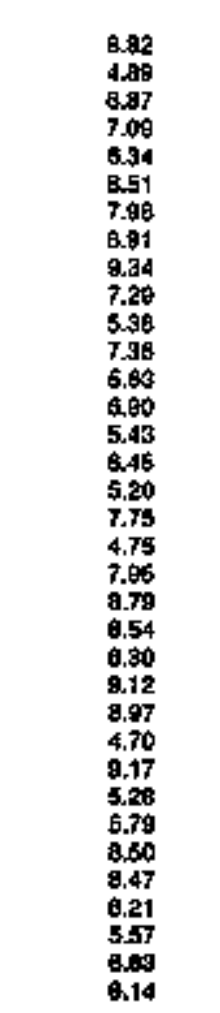 \\
\hline
\end{tabular}

8w tooknoles et and of tuble. 
Table 16. Class of Ownership, Number of Vtimate Consumers, Revenue, Sales, and Average Aevenue per Kllowatthour for the Commerical Sector by State and Utility, 1993 (Contnued)

\begin{tabular}{|c|c|c|c|c|c|}
\hline $\begin{array}{c}\text { Stoto } \\
\text { Elothis Utilty }\end{array}$ & $\begin{array}{l}\text { Chas } \\
\text { of } \\
\text { Owniritip }\end{array}$ & $\begin{array}{l}\text { Numbar } \\
\text { of } \\
\text { Connemers }\end{array}$ & Finemint & 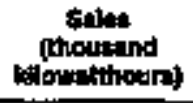 & 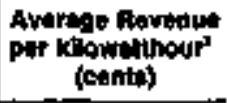 \\
\hline 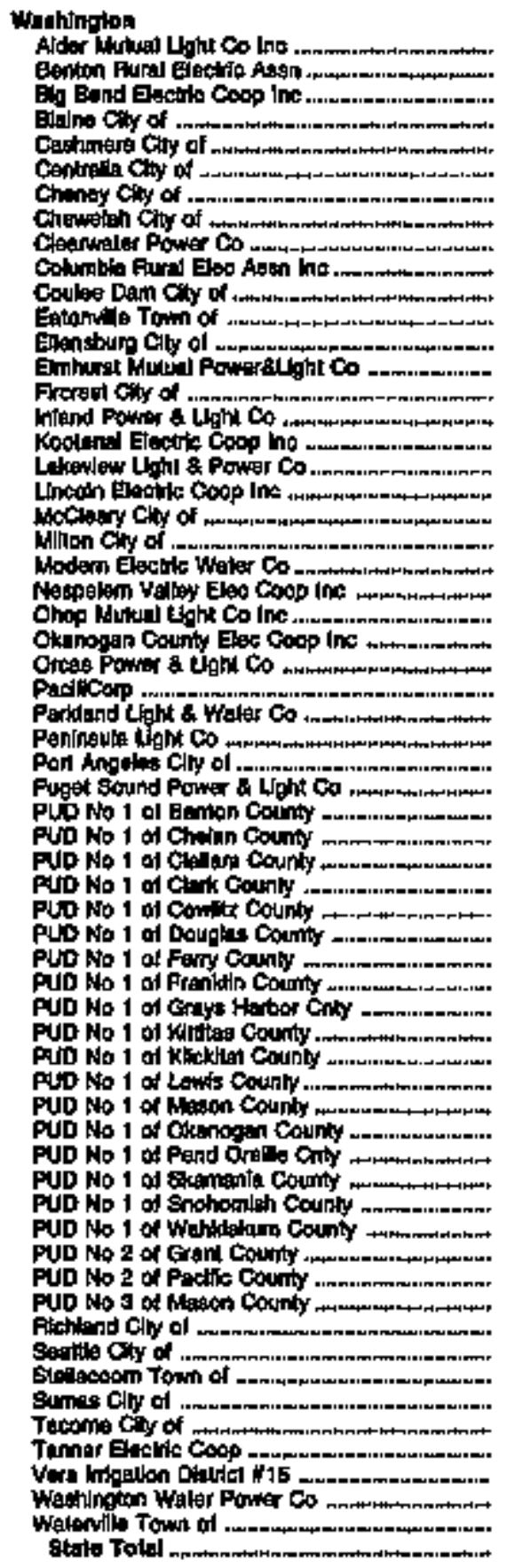 & 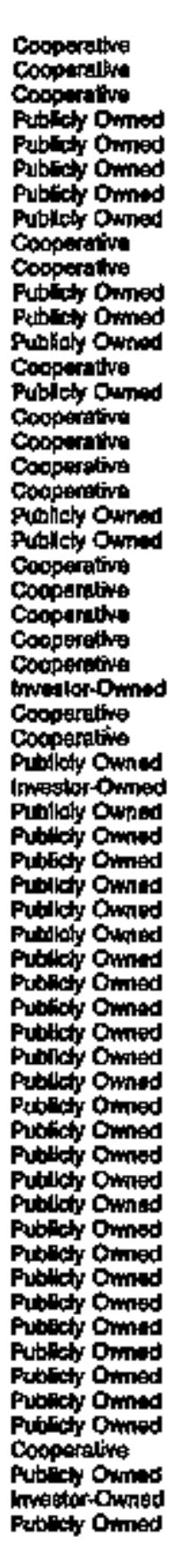 & 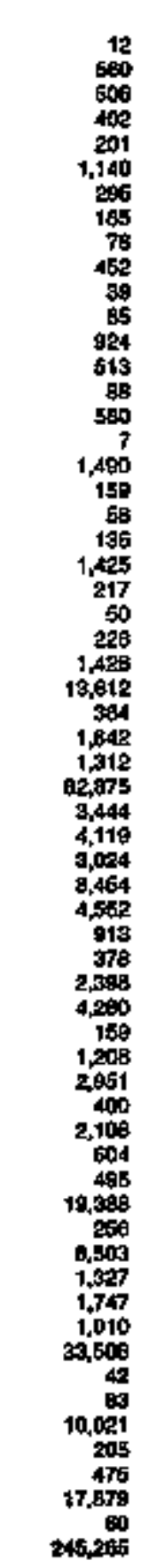 & 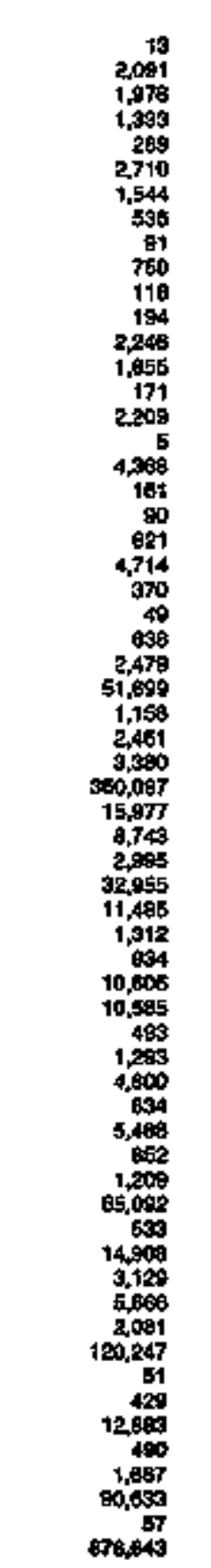 & 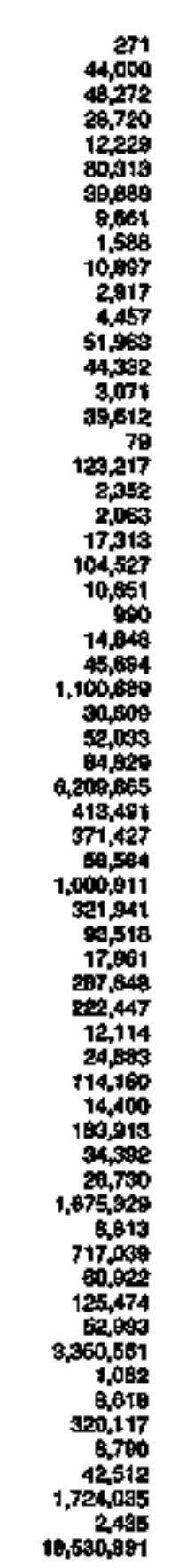 & 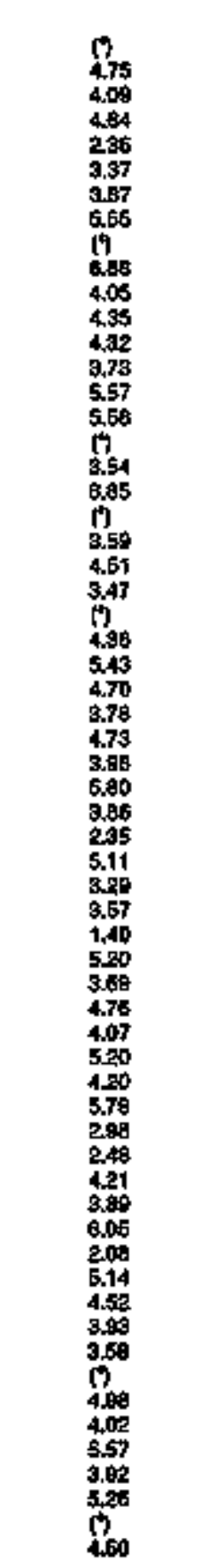 \\
\hline 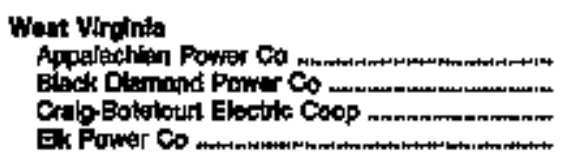 & 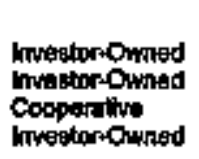 & $\begin{array}{r}49,250 \\
134 \\
16 \\
205\end{array}$ & $\begin{array}{r}155,544 \\
274 \\
509 \\
609\end{array}$ & $\begin{array}{r}2,600,042 \\
4,145 \\
484 \\
6,045\end{array}$ & $\begin{array}{l}5.59 \\
6.81 \\
19 \\
7.29\end{array}$ \\
\hline
\end{tabular}

She foolnotes at end of twbis. 
Table 15. Class of Ownershlp, Number of Ultimate Conuumers, Revenue, Sales, and Average Revenue per Klowatthour for the Commerical Sector by State and Utillty, 1993 (Continued)

\begin{tabular}{|c|c|c|c|c|c|}
\hline Evite & $\begin{array}{l}\text { Clase } \\
\text { of } \\
\text { Onmeinhlp }\end{array}$ & 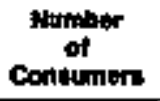 & Retortus & 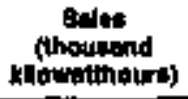 & $\begin{array}{c}\text { Awreos Rovinut } \\
\text { Por xilpwathour' } \\
\text { (otents) }\end{array}$ \\
\hline 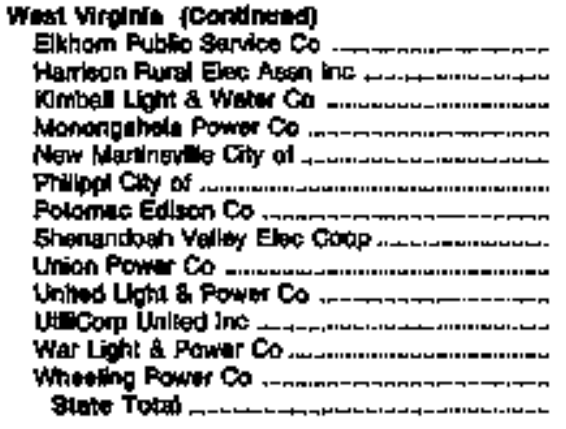 & 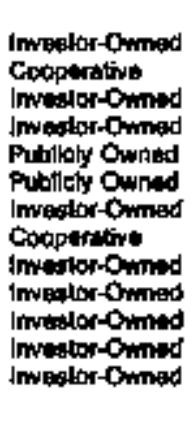 & $\begin{array}{r}20 \\
324 \\
66 \\
31,495 \\
390 \\
295 \\
10,375 \\
298 \\
238 \\
170 \\
2,081 \\
186 \\
4,645 \\
1,060\end{array}$ & $\begin{array}{r}28 \\
693 \\
56 \\
102,698 \\
698 \\
684 \\
27,658 \\
390 \\
302 \\
740 \\
7,776 \\
227 \\
18,455 \\
316,065\end{array}$ & $\begin{array}{r}300 \\
7,379 \\
848 \\
1,879,718 \\
14,704 \\
10,384 \\
474,054 \\
4,074 \\
4,672 \\
11,782 \\
113,414 \\
3,298 \\
345,142 \\
5,40,456\end{array}$ & 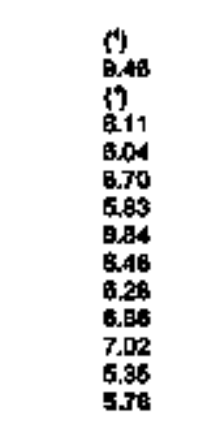 \\
\hline 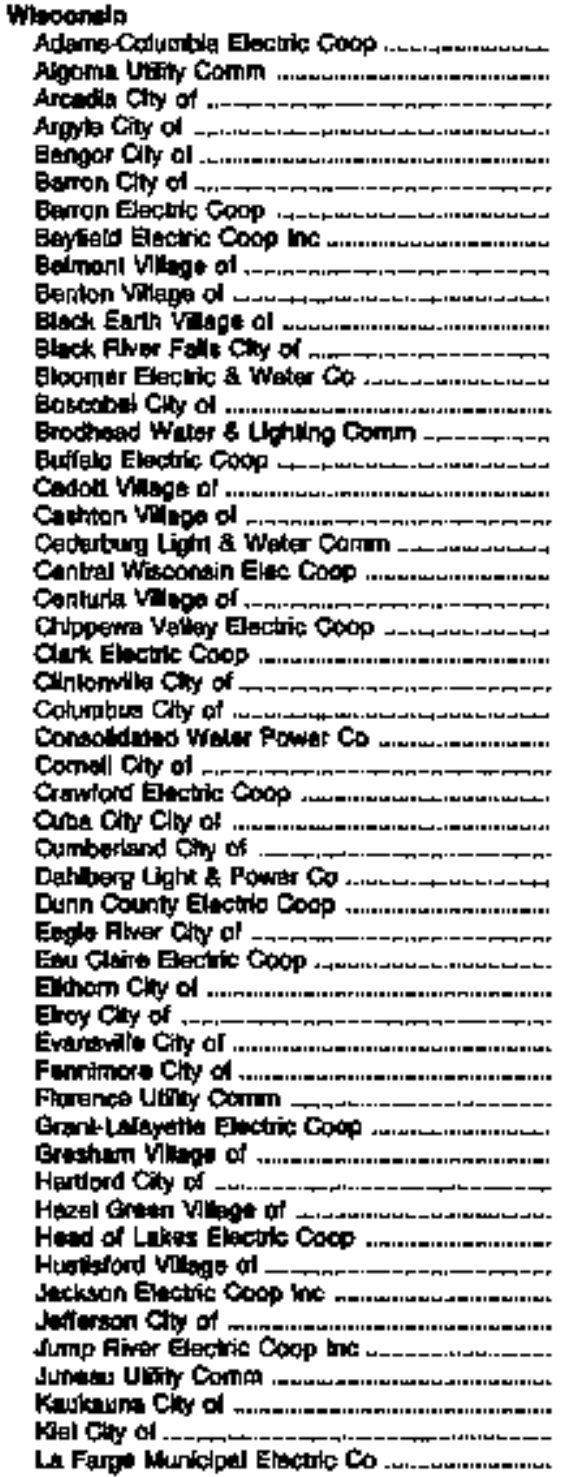 & 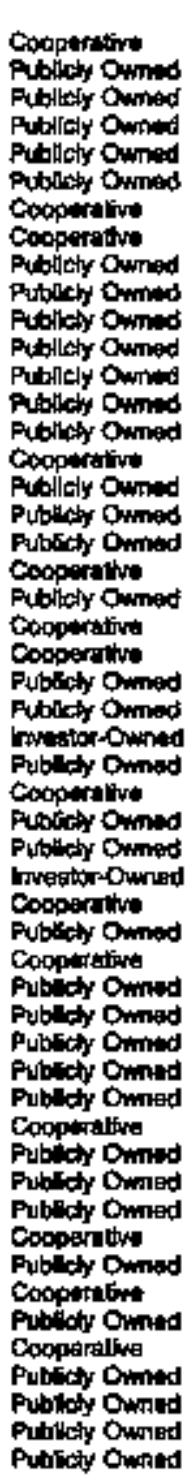 & 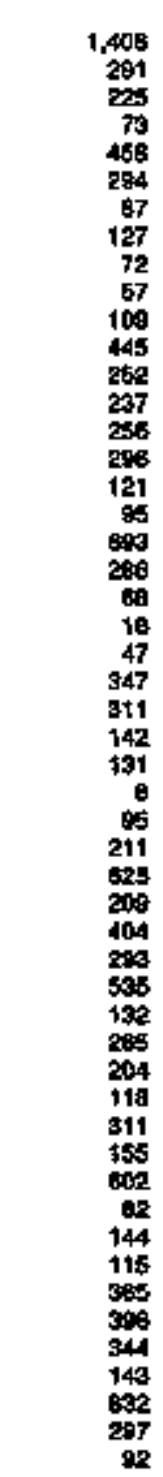 & 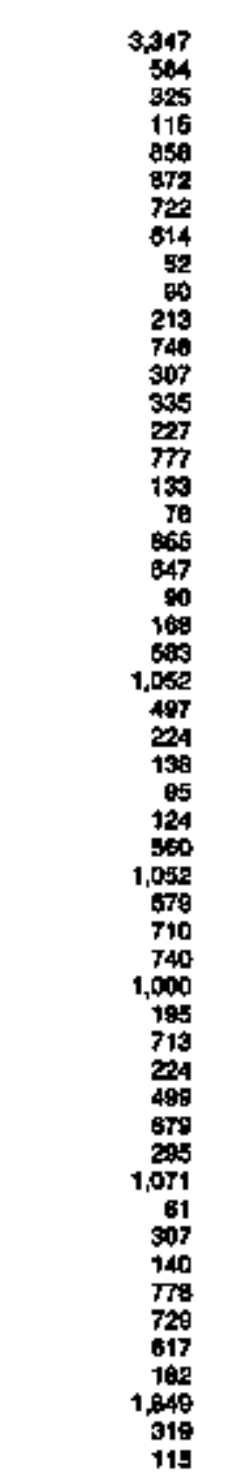 & 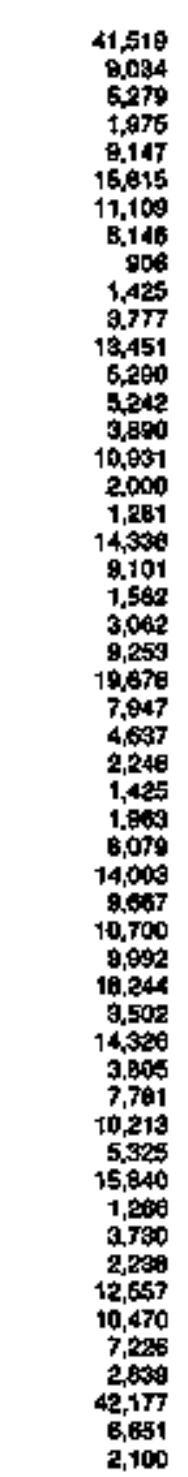 & 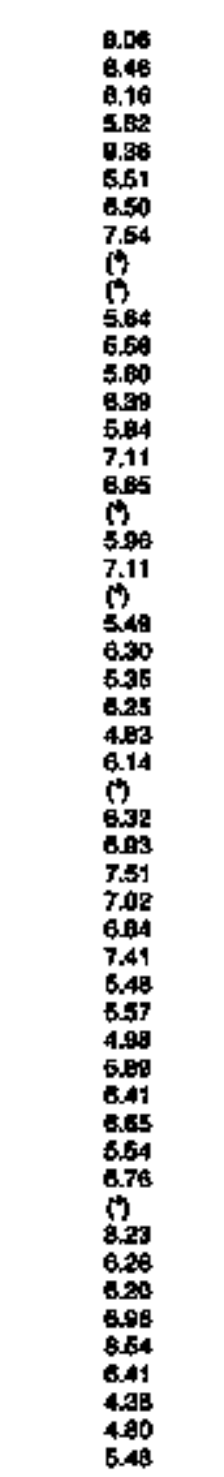 \\
\hline
\end{tabular}

Sev lootnoles at end of ublo. 
Tabte 15. Class of Ownershlp, Number of Ultimate Congumers, Revenue, sales, and Average Revenue per Kilowatthour for the Commerteal 8ector by State and Utillty, 1993 (Continued)

\begin{tabular}{|c|c|c|c|c|c|}
\hline 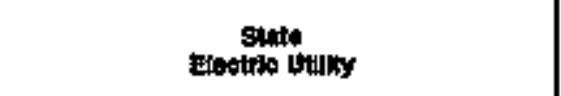 & $\begin{array}{c}\text { Civer } \\
\text { of } \\
\text { onmonitp }\end{array}$ & $\begin{array}{c}\text { Animber } \\
\text { of } \\
\text { conwumers }\end{array}$ & Btrinto & 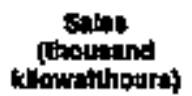 & 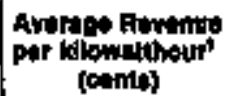 \\
\hline 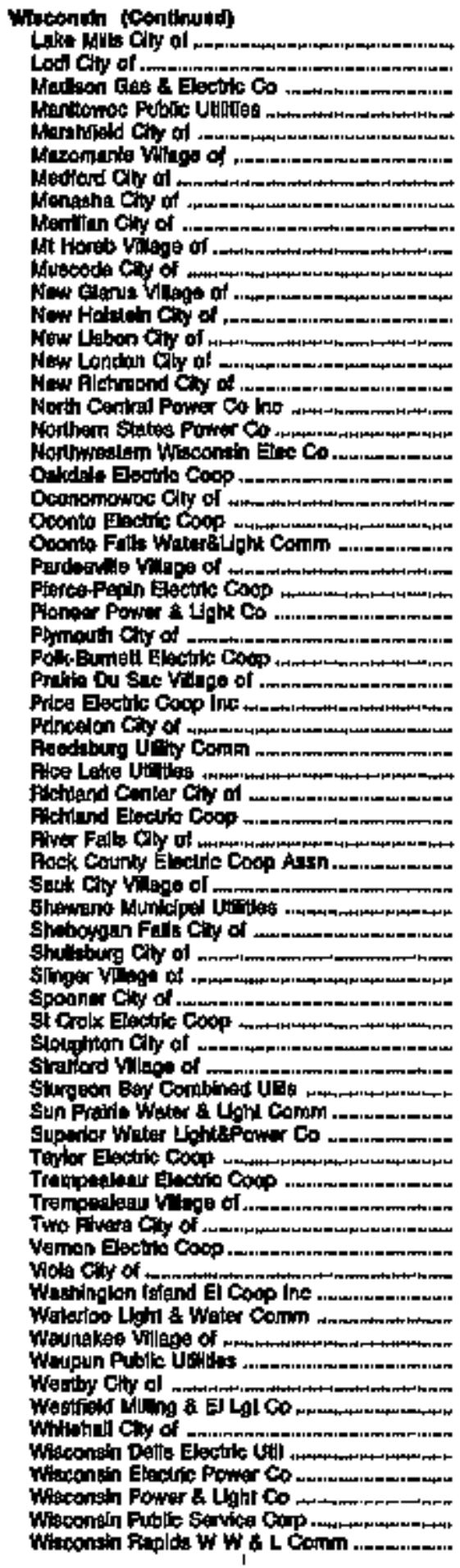 & 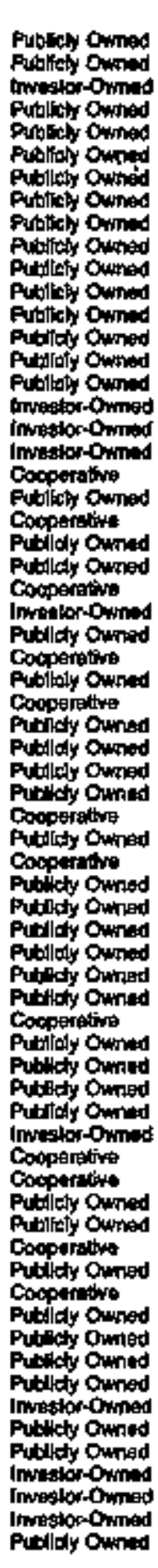 & 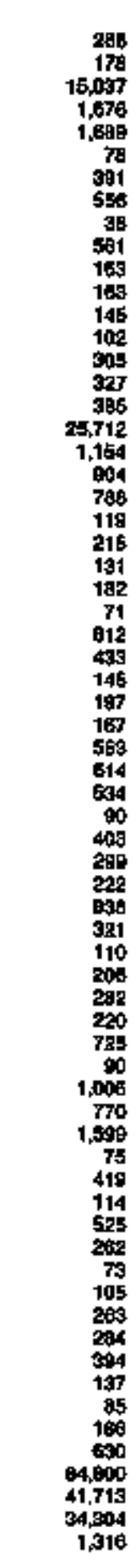 & 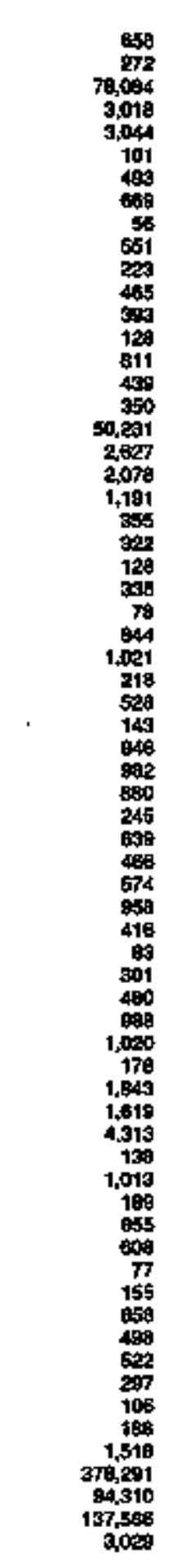 & 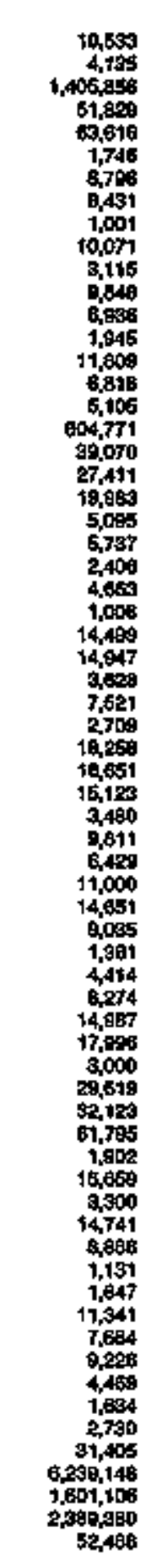 & 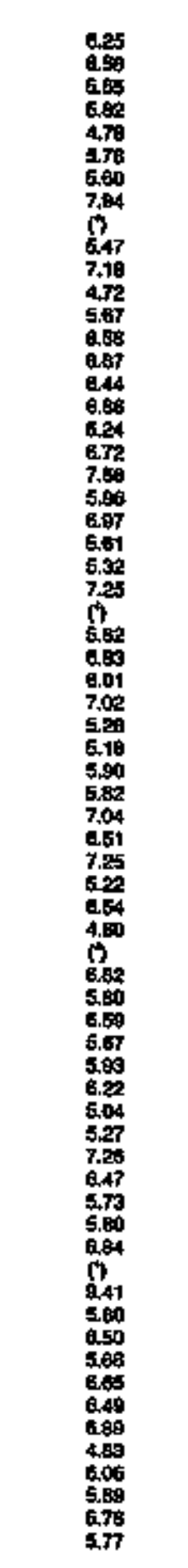 \\
\hline
\end{tabular}

See kooholes at and of tabla 
Table 15. Class of Ownerahip, Number of Ulthate Coneumers, Revenue, Sales, and Average Revenue per Kllowatthour for the Commerical Sector by State and Utillty, 1993 (Continued)

\begin{tabular}{|c|c|c|c|c|c|}
\hline $\begin{array}{c}\text { State } \\
\text { Hoetic dunty }\end{array}$ & $\begin{array}{c}\text { Chopg } \\
\text { of } \\
\text { Ownerahtp }\end{array}$ & $\begin{array}{l}\text { Number } \\
\text { of } \\
\text { congumert }\end{array}$ & 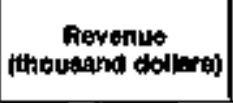 & $\begin{array}{l}\text { seles } \\
\text { (thoutand } \\
\text { kinwothourds) }\end{array}$ & 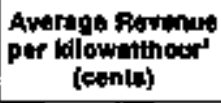 \\
\hline 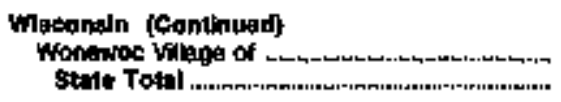 & Puttalchy Owrod & 241,679 & 015,272 & $13, \begin{array}{r}1,409 \\
13,10,449\end{array}$ & 6.95 \\
\hline 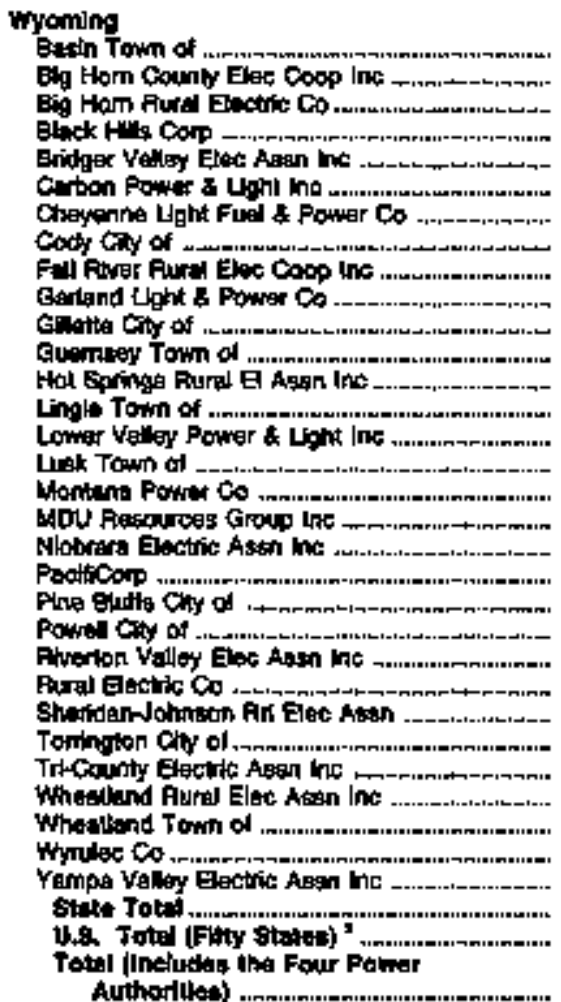 & 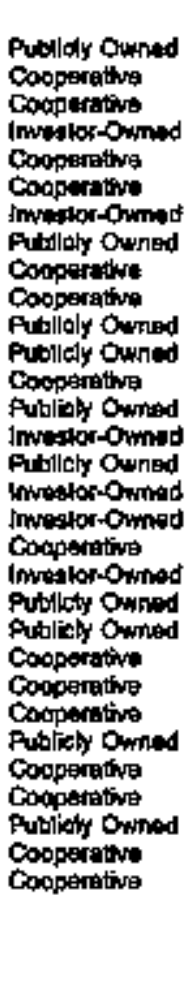 & 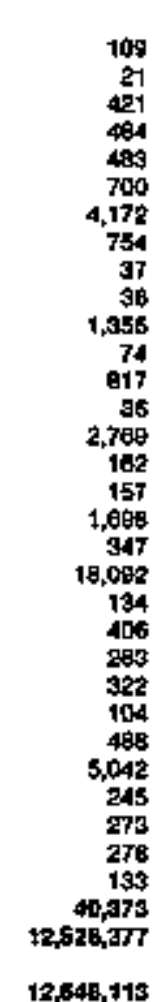 & 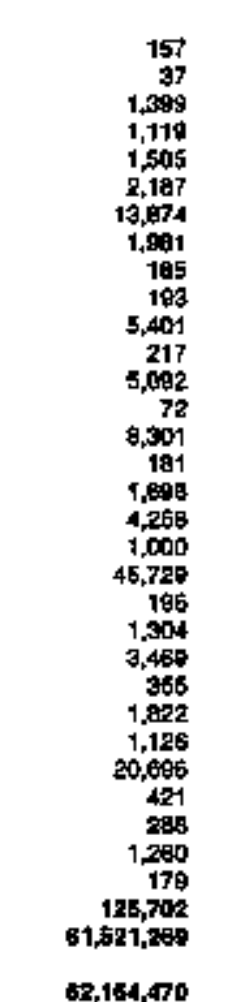 & 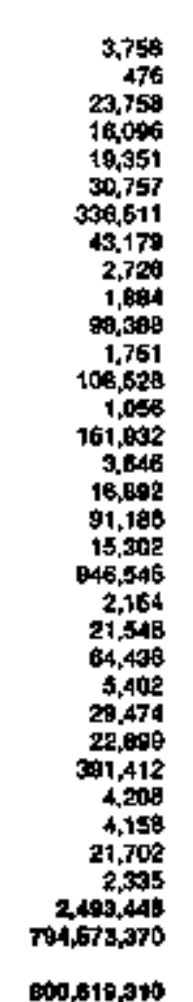 & 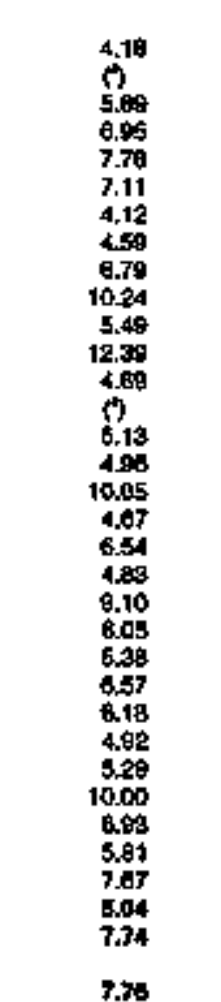 \\
\hline
\end{tabular}

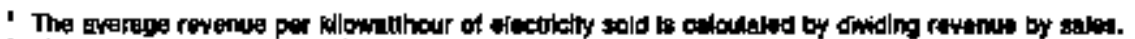

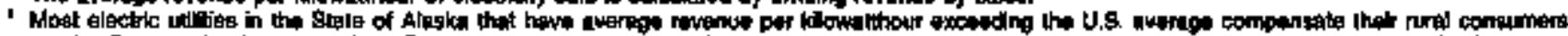

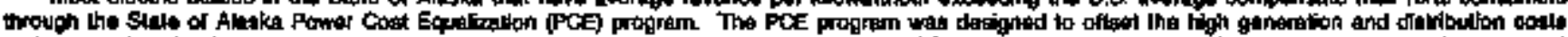

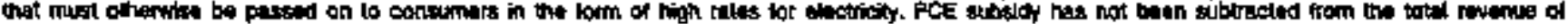

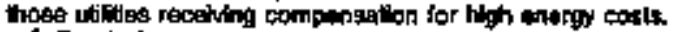

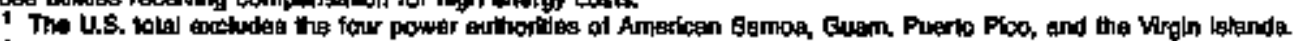

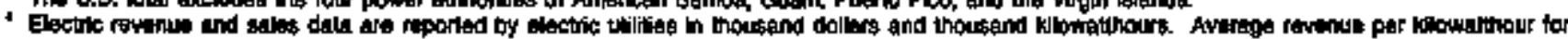

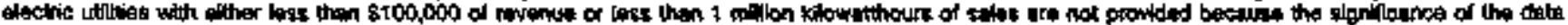

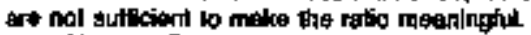

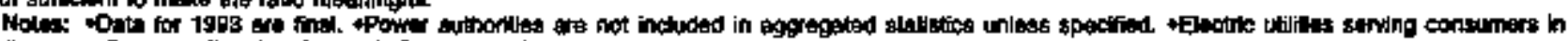

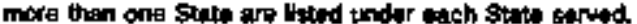

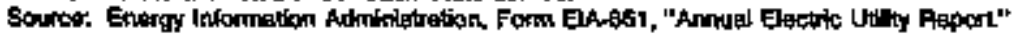


Table 16. Chass of Owmershdp, Number of Untmate Consumers, Revenue, Safes, and Average Revenue per Kilowatthour for the Industrial Sector by State and Utility, 1993

\begin{tabular}{|c|c|c|c|c|c|}
\hline Elste & Olotit & $\begin{array}{c}\text { Numbor } \\
\text { of } \\
\text { Conotmmers }\end{array}$ & Alhopoand dolartis & 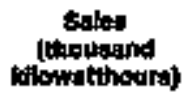 & $\begin{array}{l}\text { Average Aowenue } \\
\text { por kilowsithour" } \\
\text { (centis) }\end{array}$ \\
\hline 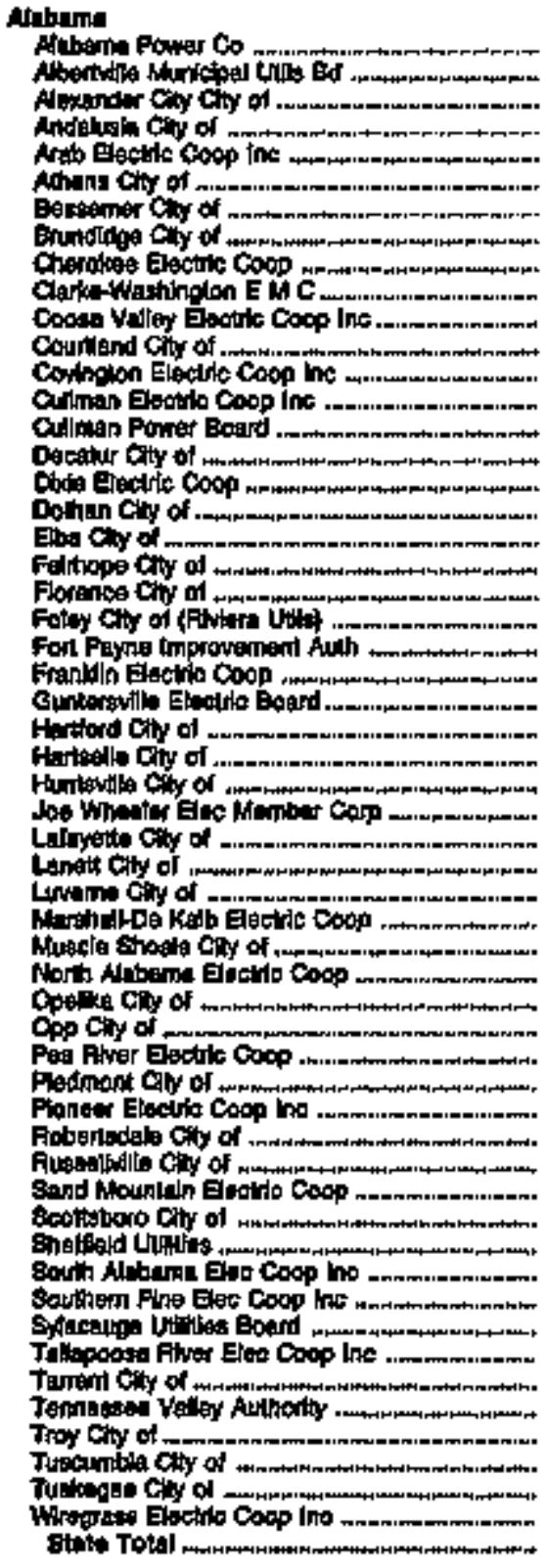 & 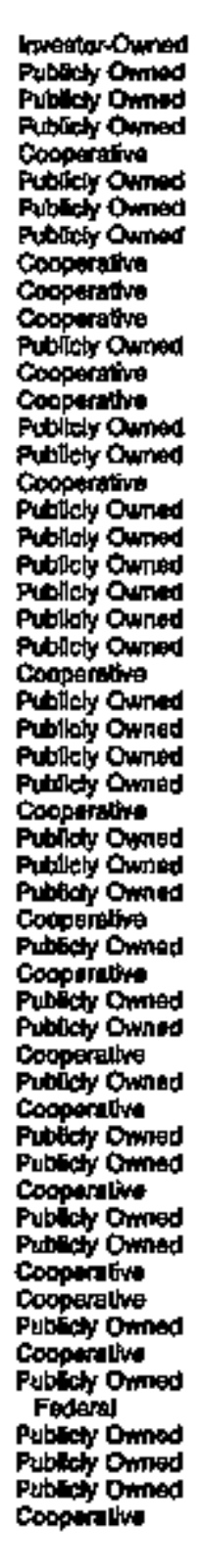 & 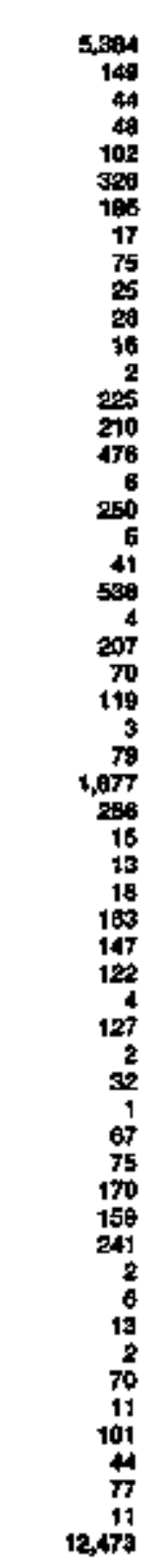 & 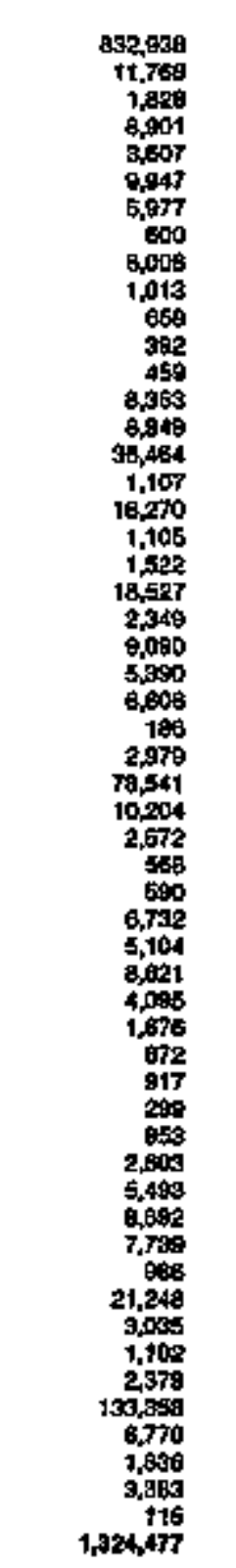 & 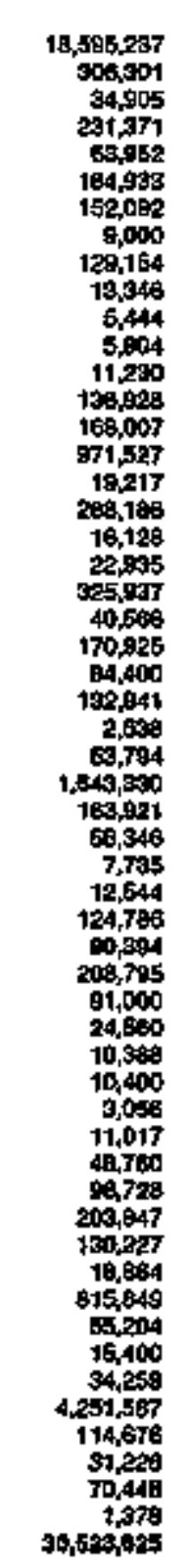 & 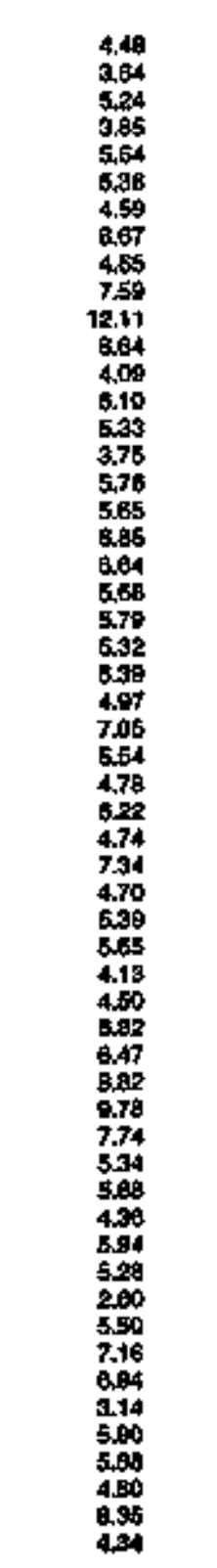 \\
\hline 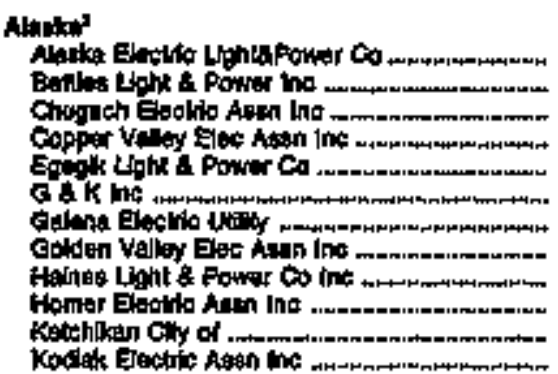 & 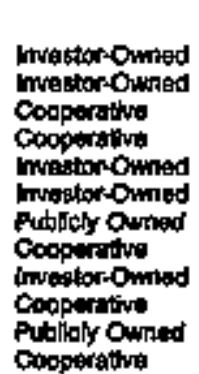 & $\begin{array}{r}70 \\
14 \\
5 \\
2 \\
1 \\
1 \\
1 \\
15 \\
4 \\
20 \\
11 \\
65\end{array}$ & $\begin{array}{r}2,748 \\
815 \\
2,984 \\
1,405 \\
87 \\
142 \\
7,00 \\
0,154 \\
260 \\
8,210 \\
894 \\
0,568\end{array}$ & $\begin{array}{r}47,770 \\
600 \\
40,616 \\
13,490 \\
9,50 \\
302 \\
6,5,47 \\
123,711 \\
1,658 \\
116,400 \\
13,972 \\
71,065\end{array}$ & $\begin{array}{c}0.60 \\
17 \\
6.82 \\
10.91 \\
17 \\
17 \\
11.84 \\
7.40 \\
15.08 \\
6.84 \\
6.80 \\
13.39\end{array}$ \\
\hline
\end{tabular}

Sop rookoles at and of tuble. 
Table 16. Clabs of Ownerahlp, Mumber of Ultimate Consumers, Revenue, Sale8, and Average Revenue por Killowatthour for the Induetrial Sector by State and Utillity, 1993 (Continued)

\begin{tabular}{|c|c|c|c|c|c|}
\hline Etato & Clate & $\begin{array}{l}\text { Munber } \\
\text { of } \\
\text { conaument }\end{array}$ & $\begin{array}{c}\text { Prwenul: } \\
\text { (theouand dollars) }\end{array}$ & 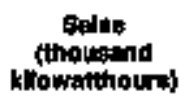 & 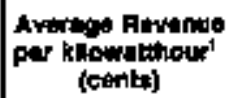 \\
\hline
\end{tabular}

Abatke" (Conthinuter)

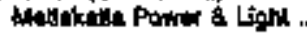

Noleson Lagoon Eloc Coop he

Nightomitio City of

ughaguk Electo Coop Ine

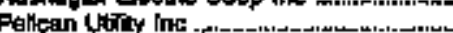

Patomburg Cot of

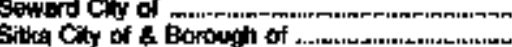

Totolot anctio Ually.

Thuigrika chy of

Wranol City of

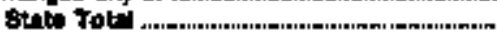

Amtrkean same

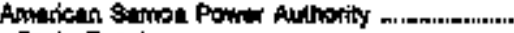

Btate Totol

Aprons:

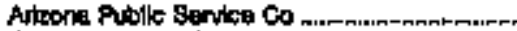

Clizent Untotien Co ...................................

Colorido Alyer Indtien int Prol.

Ouncen Valuer Elec Coco he

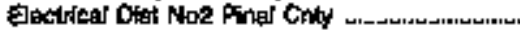

Electron Dlat Not Pinal Gny

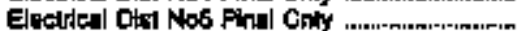

Electitegl Dlist Nos Pirgl Conty ....................

Fredonia $C$ y of

Garkase Rown Asun Inc n...m

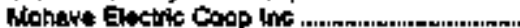

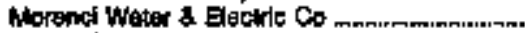

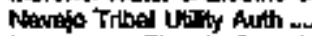

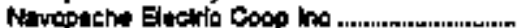

Sationd Cin of

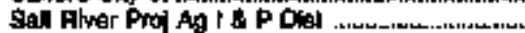

Stlpher Springs viluy $E$ C inc ..........................

Totron OrOdhen Ualíty Auth

Triog Electic Coop the

Tucson Elecisto

USaidasien Cartos Projegt ...........................

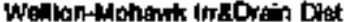

Stato 7oled

Artanat:

Artancas Power Lotu Co.

Alkansas Velloy Eles Coop Corp...........ר.

Eenton City of .

Bentoming City ol.

cromitentio Copo

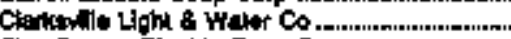

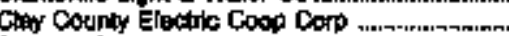

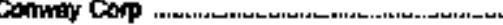

Grelghand Eloctide Coop Cor ...n......................

Empita Digtriet Electric $C_{0}$.

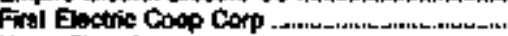

Hope City of

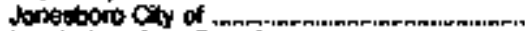

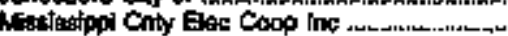

North Aikensas Eloc Coop ho ......................

Porth Litile Rock city of

Odaheme tats 8 Electric $C_{0}$..............

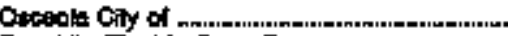

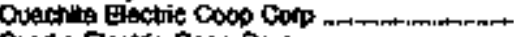

Oxthe Glactic Coos Corp

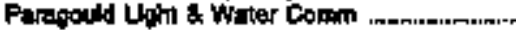

Paris Caly of .r.

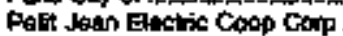

Publety Owned

Cooperativis

Publicly Owned

Cooperstho

Iowselor-Owned

Publicty Owmes

Publichy Owned

Invelor.ownec

Publichy Owriod

Putallyly Owned

Publicty Owned

Itrugator Comed

Inweslor-Owned

Publely Owned

Cooperative

Publicly Owined

Publedy Owned

Publlely Owmed

Putbly Owined

Publily Owined

Coopertatise

Cospentitive

Cocperalive

Inerlor Owned

Pulsicty Omed

Cocpenatis:

Fubilcty Oened

Publley Owniad

Cosporative

Putuldy Owngod

Cooppentition

Intestor-Owined

Federa!

Putaly Owhed

Inkestopowind

Coopriture

Puthicty Owhed

Publialy Owned

Cooperalve

Coxprertitim

Publialy Ownted

Cooporture

Publlchy Owner

coopantule

Intoptortomed

Cosperative

Publloky Owned

Putracty Onried

Cospentitire

Coconralue

Pubilaly Ownod

Itweetoromest

Publialy Owned

Cooperetwo

Cospentition

Publity Owred

Publicly Owined

copperative 1,003e

2

2

18

2

so

1

11

348

125

128

5
8
772
995
272
1,161
1,147
374
3
1.182
174
41,052

7,509

17
4
6,699

4,025

2,104

14,005

14,730

4,998

0,089

4,598

501,059

1389

3025

0,078

32,258

16.84

5,078

$9,002,78 x$

188,441

10,498

305
48,350

2870

102

1,2 ge

63

378

162

2,167

68

13,340

2,406

155,018

155,018
1,000

5,902

407

197,467

2377

38

697,719

115,e0s

Q.00B

1,169972

30518

1,546

10689

17,397

765

5094

1,900

50,036

7,098

232,705

27,097

$3,118,408$

24,001

121,108

60 (998

$2,202,339$

$49.1 \% 9$

5402

6,136

$0,909,057$

336,960

6.671

2,193

$5+06$

378

5,647

6,640

1,510

12514

1,283

1,920

6,190

4,425

13,503

50,348

3,505

16,793

$30,98 B$

6,116

4035

6,959

4,728

69

955

$5,669,710$

168,6]5

32,010

152,473

5,198

13,728

121,015

22,701

264,582

21,214

51,688

$257, n 7$

0601

392007

1,704,685

6,707

25, alas

647,531

104,046

58,742

128040

152,464

16,000

(4)

11.54

17.97

1203

7

7.46

()

11,86

3.76

Q. 19

6.25

0.02

5.06

4.13

7.4

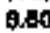

5.27

7.42

17

B.33

6.00

4.53

D44

5.73

B. Bs

1)

4.67

5,24
4,87

5.

7

4.83

Q.72

B.50

See footnoles at and of leble. 
Table 16. Claws of Ownership, Mumber of Utimate Consumers, Revenue, Ssles, and Average Revenue per Kilowatthour for the Industrial Sector by Stote and Utility, 1893 (Continuted)

\begin{tabular}{|c|c|c|c|c|c|}
\hline 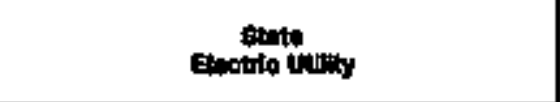 & $\begin{array}{c}\text { Clact } \\
\text { of } \\
\text { Omininthip }\end{array}$ & $\begin{array}{c}\text { Number } \\
\text { of } \\
\text { coterthere }\end{array}$ & Bthrempl & (thoutiond & 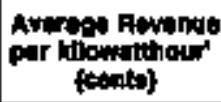 \\
\hline 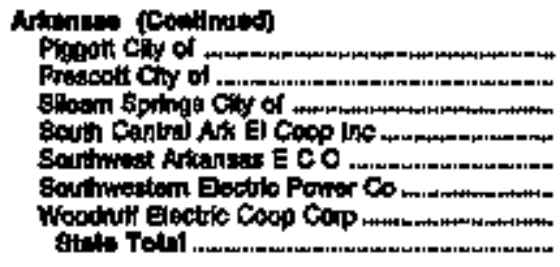 & 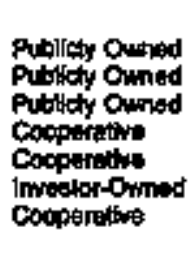 & $\begin{array}{r}68 \\
7 \\
4 \\
4 \\
4 \\
40\end{array}$ & $\begin{array}{r}600 \\
0,581 \\
6,613 \\
3,372 \\
5,735 \\
60,390 \\
2,169 \\
611,160\end{array}$ & $\begin{array}{r}17,747 \\
61,560 \\
124,904 \\
7,029 \\
125,185 \\
1,402,047 \\
43,493 \\
17,609,0198\end{array}$ & $\begin{array}{l}4.56 \\
5.36 \\
4.49 \\
4.96 \\
4.58 \\
4.24 \\
4.98 \\
4.6\end{array}$ \\
\hline 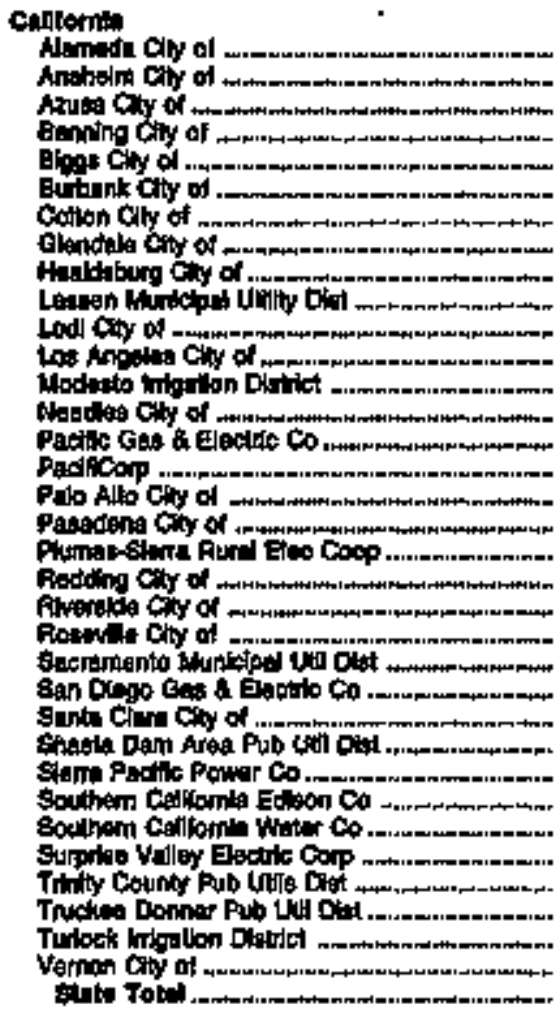 & 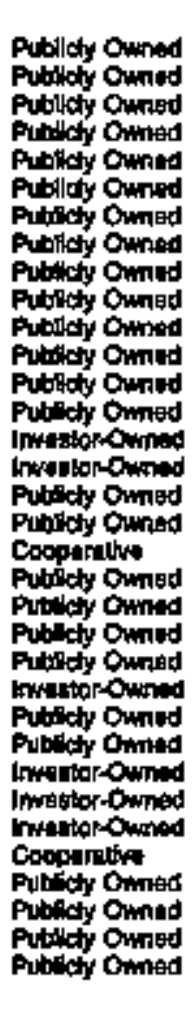 & $\begin{array}{r}3 \\
674 \\
28 \\
7 \\
1 \\
218 \\
18 \\
181 \\
1 \\
5 \\
25 \\
17,185 \\
1,370 \\
35 \\
1,198 \\
949 \\
191 \\
917 \\
16 \\
581 \\
195 \\
30 \\
7310 \\
5098 \\
1,606 \\
8 \\
38 \\
4,000 \\
4 \\
16 \\
3 \\
28 \\
878 \\
852 \\
30,408\end{array}$ & 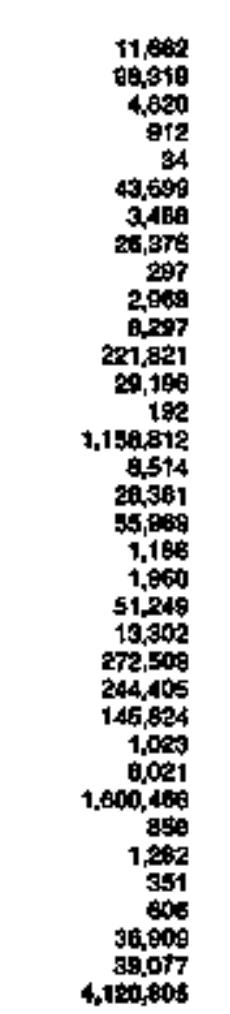 & 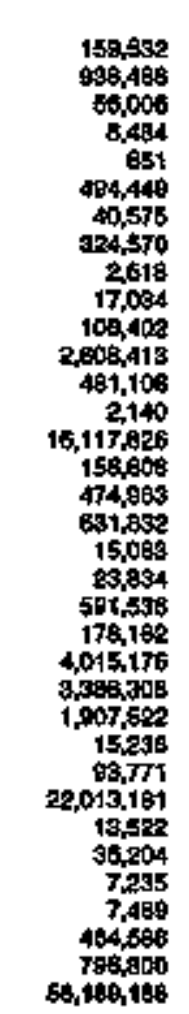 & 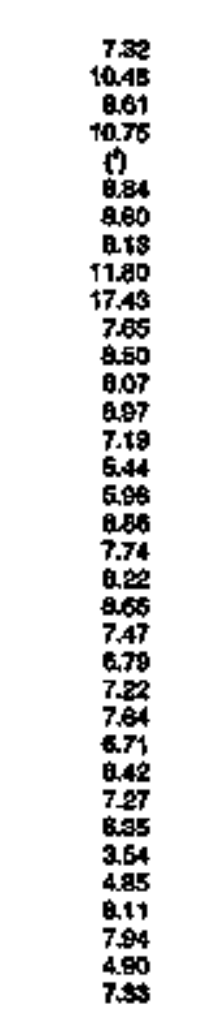 \\
\hline 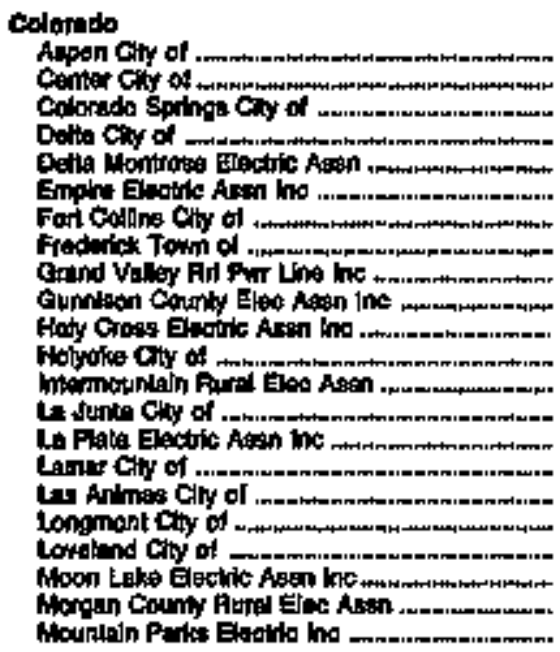 & 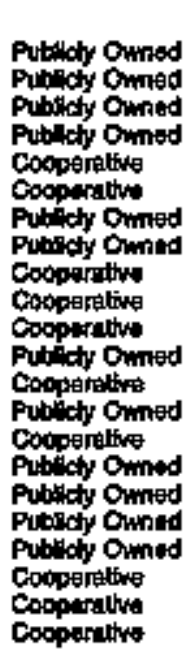 & $\begin{array}{r}1 \\
67 \\
397 \\
39 \\
129 \\
98 \\
11 \\
3 \\
3 \\
4 \\
9 \\
23 \\
10 \\
64 \\
7 \\
9 \\
2 \\
7 \\
184 \\
7 \\
20 \% \\
93\end{array}$ & $\begin{array}{r}41 \\
146 \\
50,447 \\
1,000 \\
1541 \\
0,505 \\
11,324 \\
61 \\
295 \\
445 \\
1,200 \\
186 \\
2,860 \\
1,802 \\
10,184 \\
601 \\
6 \\
3,075 \\
10,544 \\
10,056 \\
2002 \\
2.716\end{array}$ & 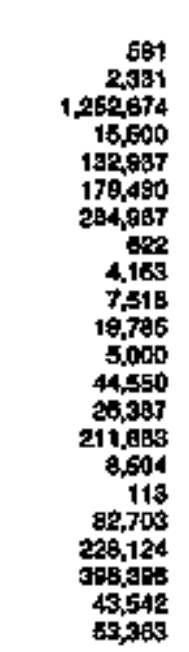 & $\begin{array}{l}10 \\
6.28 \\
4.00 \\
6.46 \\
6.42 \\
6.80 \\
3.97 \\
19 \\
7.09 \\
6.92 \\
6.47 \\
3.72 \\
6.42 \\
7.32 \\
4.81 \\
7.30 \\
19 \\
3.72 \\
4.62 \\
4.83 \\
6.76 \\
5.08\end{array}$ \\
\hline
\end{tabular}

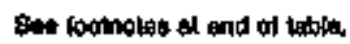


Table 16. Class of Ownorship, Mumber of Ultimate Consumers, Revenue, Sales, and Average Fevenue per Kllowatthour for the Industrial Seclor by State and Utillty, 1993 (Continued)

\begin{tabular}{|c|c|c|c|c|c|}
\hline 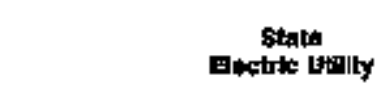 & clath & $\begin{array}{c}\text { Nunber } \\
\text { of } \\
\text { Contumiter }\end{array}$ & $\begin{array}{c}\text { Arwento } \\
\text { (Whouland dollare) }\end{array}$ & $\begin{array}{l}\text { Sales } \\
\text { funbupend } \\
\text { Mlownlthours) }\end{array}$ & $\begin{array}{l}\text { Awarago Ravenua } \\
\text { per lifowatihour' } \\
\text { (cenis) }\end{array}$ \\
\hline
\end{tabular}

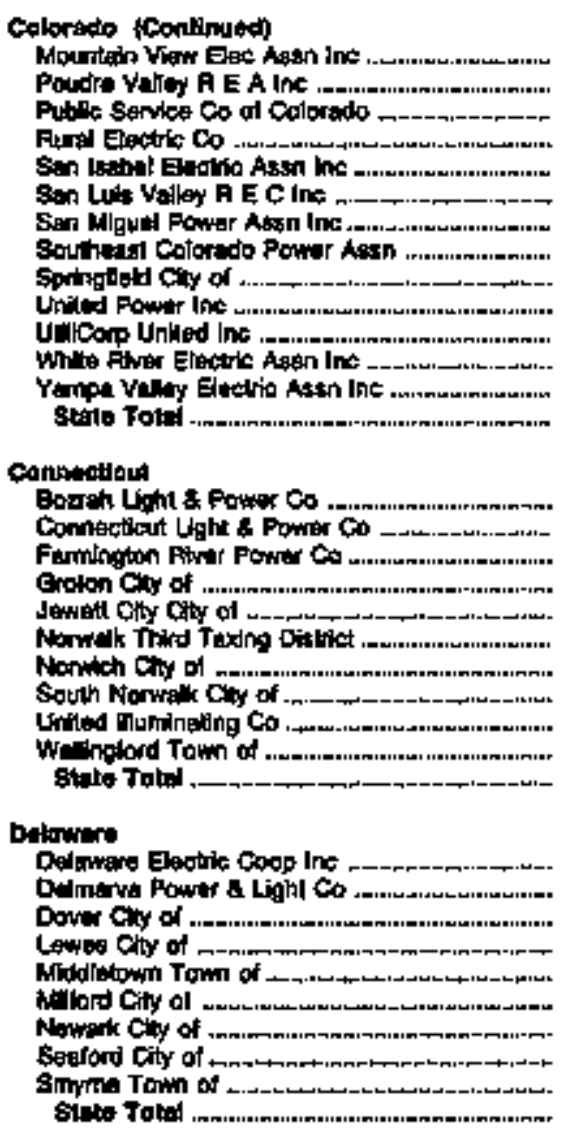

Dintilet of Columbirs

Fororage Esetic Powar Co

Ftortde

Bartow City ol

Contrat Huride Glec Conp inc

Choptrehochet Chy ol ....................................

Choctanhatohs Eloc Doop ine ...........................

Cay thetrice Coop tine

Chintalon ciny of

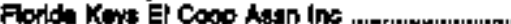

Flordda Power \& Ligit Co

Fondt Power corp.

Fionda Publuc Uedites Co

Fort Miade Chy of

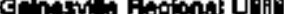

Clades Electic Coop he .................................

Groen Cove Springs Chy of

Gut Pomat Co

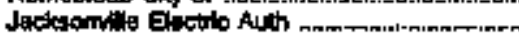

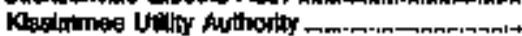

Late Worth Cly of ........................................

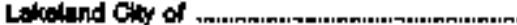

Lee Coumty Electrit Coop hes,

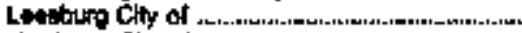

Newbery City ol

Ses toptunteg al and of tabla.
Cocoperative

Cooperative

Imealor-Omined

Copoptative

Cooperitue

Cooperative

Cocpurativis

Cooperathe

Fublict Owmed

cooperalive

innostor-Omingod

Cocoperalive

Cooperative

Wweptor-Owned Investor-Owring howtstor-Ownad

Publichy Onmed

Publity Owned

Publicty Omed

Publichr Ormed

Pubichty Ommed

Intastor-Owined

Publicty Ommed

Cocperaltwe

Itreslorowned

Pubilkty Omirg

Publuty Owned

Puscity Ontred

Putdlaty Omind

Publecty Ommod

Putildy Owied

Pubticty Oined

Imoaker.0uned

Putaley Owind

Cocteseditiva

Pubiloby Ownes

Coopurnthe

Cocperative

Putilety Owined

Cooperative

Impestor-Cmined

imvelorer omined

investor-Onned

Piblity Owred

Publlaby Owited

Cooperethe

Pubilety owned

motestor-Oment

Pubfest Owited

Publyty Dwnod

Puthlof Owined

Publlely Owind

Publcty Owned

Goppertative

Publey Owned

Publyty Dumed

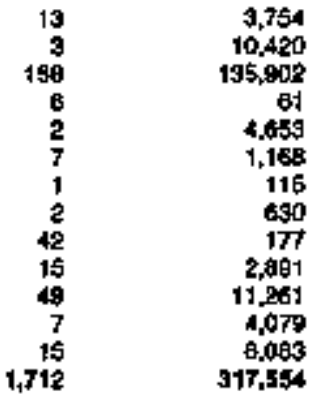

1.5444
320.5300

4,197

1

की

23.987

45
0.43

3.857

1,963

ㅂ, 4 依

13.767

464,001

827

131,206

13,064

1,674

4.97

9,748

3,535

1,812

199,898

170,012

$$
\begin{array}{r}
166 \\
+66
\end{array}
$$

176,012

a,388

1,066

1,050

5,602

493

0,022

210,478

181,928

7,945

9

7,002

1,982

1,526

67,547

5 月13

114,384

4,697

6,121

20,206

5.117

10,018

979
79,551

281,724

082,04

25,120

14,256

1,498

a, 908

2,501

65.952

255,123

00,248

93,65d

$7,024,007$

17.499

$3,055,113$

26,800

375,484

472
7.058

489

12,478

$1,038.547$

218.584

$6,697,349$

10,322

2,891.000

197.922

21.950

9,016

67,551

151,400

45,120

20,620

$3,416,611$

$2,878,390$

2, 176,300

591

105,462

4.78

3.98

4,41

(1)

8.10

7.66

7.01

B.10

$4 . \mathrm{as}$

4.77

6.50

4.52

8.85

6.31

6.35

10.73

7.95

10.

9.40

6.36

a,20

6.07

4.5.4.

e.81

7,69

6.44

3,4

a.70

4.00

14,004

24,255

91,034

7,200

+50,513

$3,800,134$

$3,300,796$

128, 189

34

131,577

17,994

64,540

2029000

70,640

$1,072,359$

73,250

15, 3ap

377,475

01,300

142446

14,044 
Table 16. Class of Ownershlp, Number of Ultimate Consumers, Revenue, sales, and Average Fevenue per Klowatthour for the Industrlal Sector by State and Utily, 1993 (Continued)

\begin{tabular}{|c|c|c|c|c|c|}
\hline $\begin{array}{l}\text { State } \\
\text { Elenteris Uutity }\end{array}$ & $\begin{array}{c}\text { Clan } \\
\text { of } \\
\text { Qumneratp }\end{array}$ & $\begin{array}{c}\text { Munber } \\
\text { od } \\
\text { Contmmart }\end{array}$ & 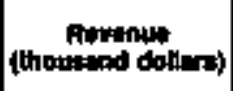 & 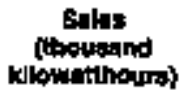 & 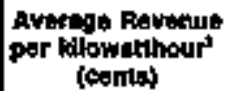 \\
\hline 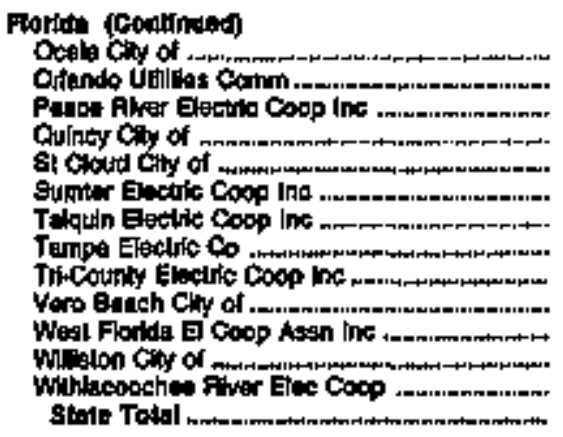 & 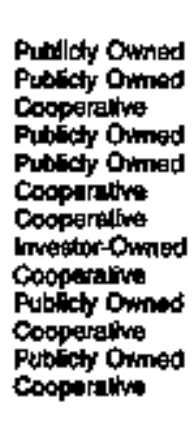 & $\begin{array}{r}632 \\
30 \\
88 \\
26 \\
112 \\
292 \\
3 \\
506 \\
30 \\
1 \\
35 \\
2 \\
15 \\
21,057\end{array}$ & 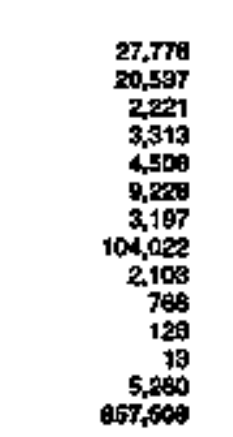 & 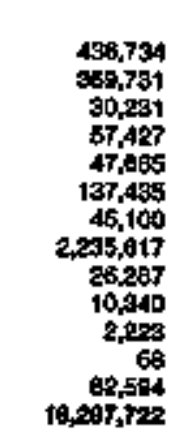 & $\begin{array}{l}6.30 \\
5.65 \\
7.95 \\
5.77 \\
9.46 \\
6.71 \\
7.09 \\
4.66 \\
6.00 \\
7.49 \\
5.67 \\
17 \\
6.37 \\
5.26\end{array}$ \\
\hline 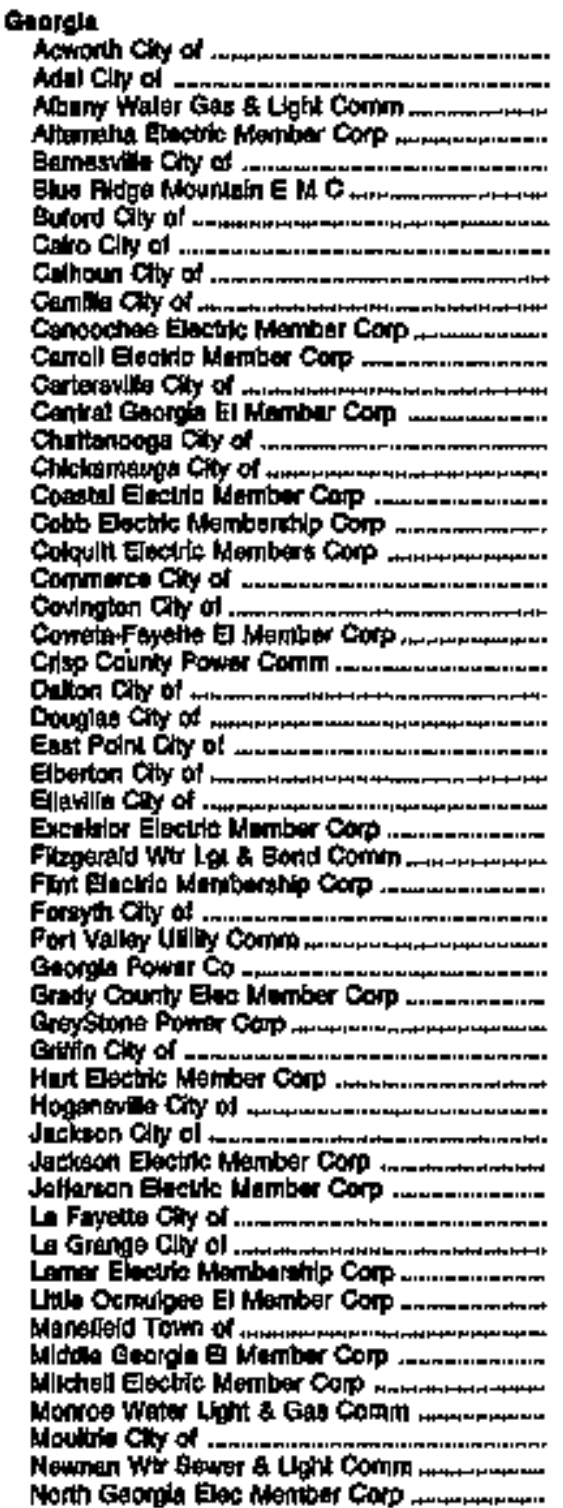 & 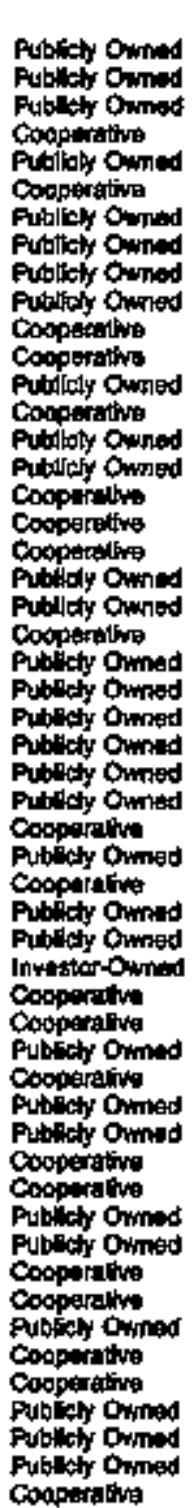 & 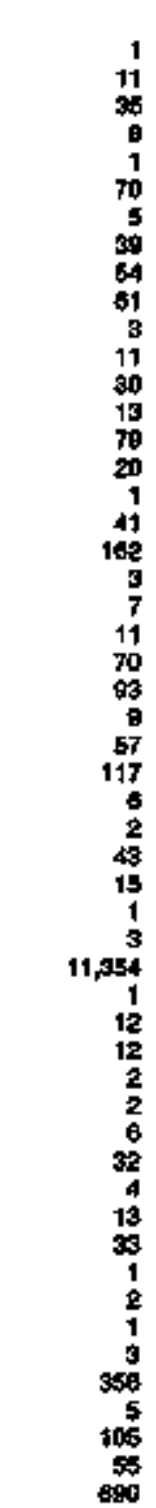 & 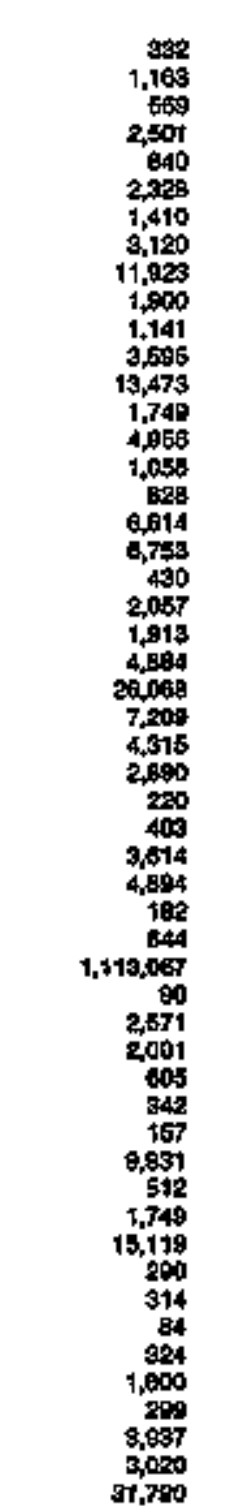 & 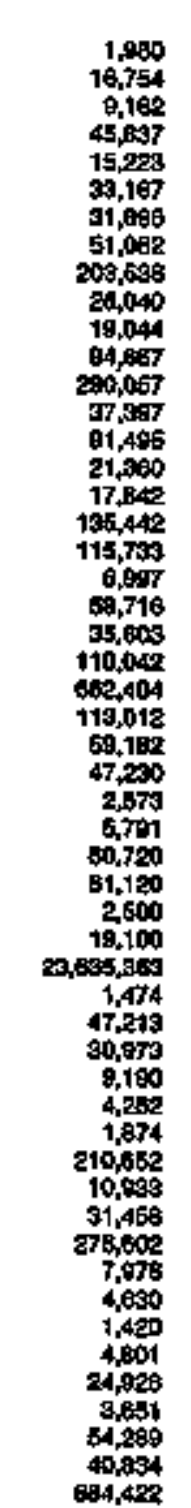 & 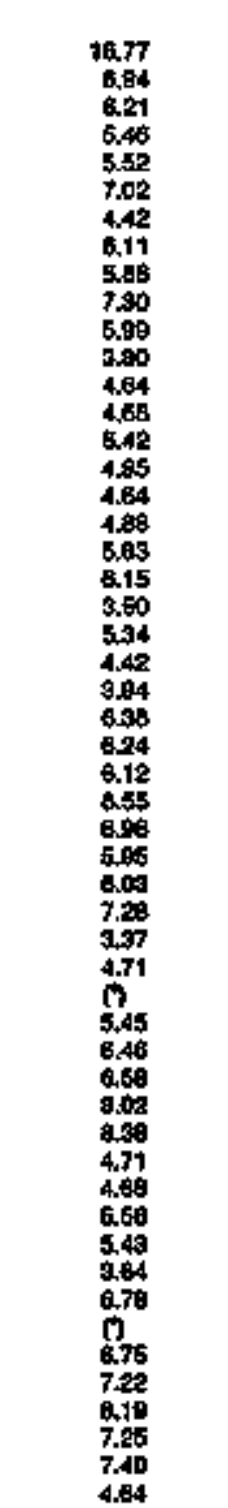 \\
\hline
\end{tabular}

Sor looknotes at and of teble. 
Table 16. Class of Ownorship, Number of Ultimate Consumers, Revenue, Sales, and Average Revenue per Klowatthour for the Industrilat Sector by state and Utillty, 1993 (Continued)

\begin{tabular}{|c|c|c|c|c|c|}
\hline $\begin{array}{c}\text { Btatt } \\
\text { Electild Utifty }\end{array}$ & $\begin{array}{c}\text { ote:p } \\
\text { of } \\
\text { Dembinthtp }\end{array}$ & $\begin{array}{c}\text { Wumber } \\
\text { of } \\
\text { coneungrs }\end{array}$ & Rewonus & 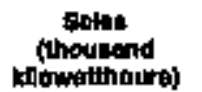 & 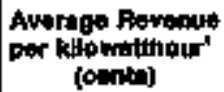 \\
\hline
\end{tabular}

Coorgla ctominuad;

Oxones Electric: Marnibar Conp .........................

Paimelo Caly of

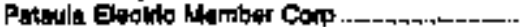

Rayle Eactric Klembarthlp Corp .......................

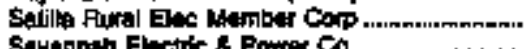

Savannal Elactric 4 Pomer Co

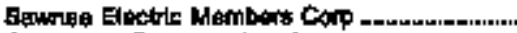

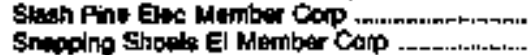

Sufdier Elactric Sdambnt Corp ...........................

Symania tivy of

Thomagkon City of

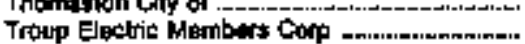

Upson Count Elwo Alarmber Corp

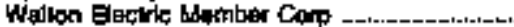

Washington City of .......................................

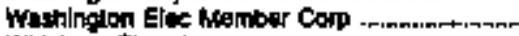

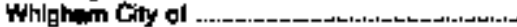

State Totel

Homal

Otizend Utitios Co

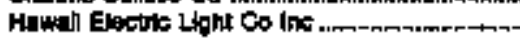

Hawallan Electrle $C o$ Int ..

Mani Electrio Co Lte

stats Todnl

Land

Bonners Fanry Ciny of

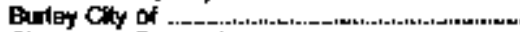

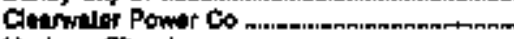

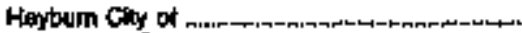

Idaho Fila City af

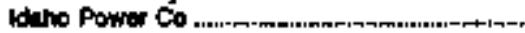

Indend Power \& Lught Co.

Kootenta Electric coop Inc ...............

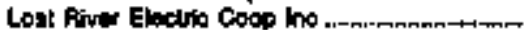

Lower Valley Fowrer of Lidgt ing

Northem Lighis he

Paciricorp

Phummer Gify of

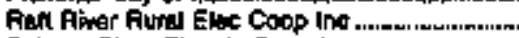

Salmon Fiwer Electric CoOp Int

Slota Sprthos Clty of

Washington Waler Rowar Co ............................

Slato Totod

Illinots

Atiming Eactricen Coop

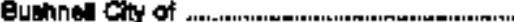

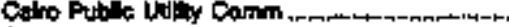

Carbila Coty or

Casey city of

Central llinots Loht Co

Central Ilinois Pub Serv Co .......................

Chathas vilken of ...

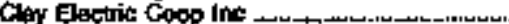

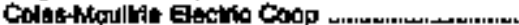

Commonwantls Edison Co.

Eatstem I"ri Electric Coopp

Goyplant lenctic Cosp Asth

Fainilaid Chr ol

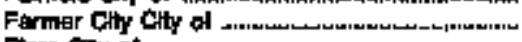

Fore Cry of

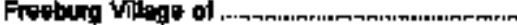

Genow City of

Gerame chy of .

Highlend Cthy of
Cooperative

Putaldy Owined

Coopersters:

Cooperathe

Cooperithe

Inveetion Owned

Coopenettve

Goopentive

Coopertivis

Cooperative

Putpldy Owied

Putblity Owned

Cooperather

Cooperative

Cooperstive

Publicly Ownod

Couperativt

Publety Owned

Imectoromined impestor-Omed investor-Owned

impostor-Gined

Publesty Oumad

Publikty Owneo

Cooperative

Putichty Orined

Pubrect Owned

Irvegtor-Omid

cocpiralive

Cocperathe

Cooperative

Inwastrow-Owned

Cooperathe

Invation-Owned

Puscicty Oimed

Coopurative

Cooperalive

Puttlaty Oumed

Intestor-0wned

Cooperntive

Pubuty Oined

Publlaty Ouned

Putwlely Ownod

Publicty Owned

Publlaty Owned

Imvelar Owned

Imeator-Omed

Publloly Owned

Coxperitus

Cosperalliv:

Imeitor-Onnod

Conperative

Coopentive

Putillty Owrited

Fublity Owned

Putlety owned

Publety Owned

Publicly Owined

Publety ownes

Publity Owned

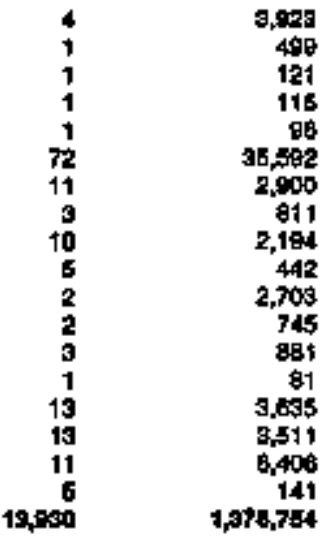

10,421

2) 001

250 sed

33.988

s\$7,239

207

664

1,570

1,349

2.168

1,047

02,048

cos

75

1,694

g2,143

(4)

597

605

35,79

203,002

$3,7+1$

292

3,736

2298

430

306

65,394

112,362

20

140

1,076

$1,4 \times 7,680$

588

964

273

1.a16

351

2 , 455

3,362
B4,720

15,230

4.704

2,119

3,121
B5,325

61,644

15,540

38,580

8,515

sin 700

15,731

10,337

1,003

79,295

61,002

138,065

1.750

20,094;21

193,842

105,700

3.135.214

$3+6250$

$\$, 769,796$

1120

11.20
8.37

10.58

ass

20.229

44,275

30,424

79,604

5,245

$3,791,829$

302

27,769

1,425

44,509

16,441

$2,070,646$

16,721

11,000

13,396

335

1,084,708

$7,228,097$

4.27

3.65

4.43

2.75

(1)

3.63

ti)

300

424

3.77

4.48

0.02

4

201

6.39

5.51

6.81

4.69

6.83

7.64

3.62

4,67

7.17

5.69

6.5.

6.27

7.02

7.57

7.97

4.10
5,79

57

6.00
6.63

Soe foothoters of end at lablo. 
Table 16. Class of Ownershlp, Mumber of Ulimate Consumers, Revenue, Salea, and Average Fevenue per Kllowatthour for the Industrlal Sector by state and Utilly, 1903 (Continued)

\begin{tabular}{|c|c|c|c|c|c|}
\hline Silte & 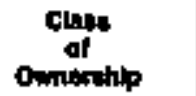 & 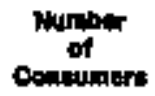 & Ronsinge & 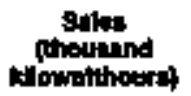 & 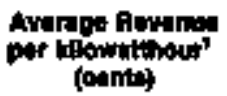 \\
\hline 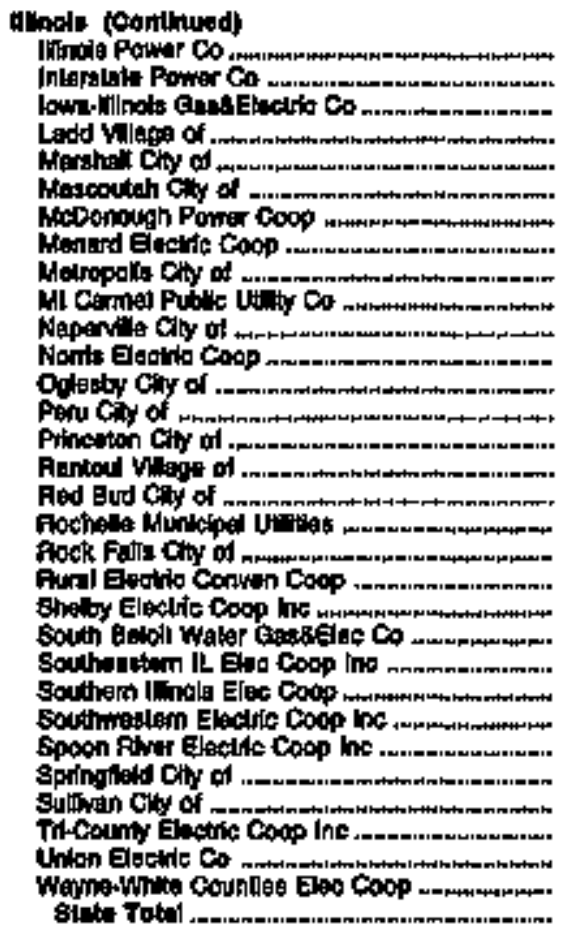 & 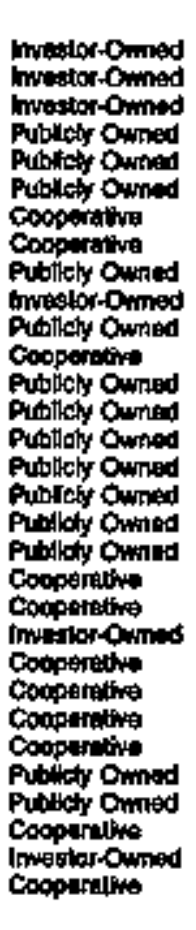 & $\begin{array}{r}409 \\
2 B \\
28 \\
1 \\
6 \\
3 \\
3 \\
1 \\
6 \\
141 \\
25 \\
380 \\
17 \\
37 \\
76 \\
80 \\
2 \\
18 \\
48 \\
2 \\
10 \\
28 \\
130 \\
2 \\
2 \\
14 \\
1 \\
58 \\
18 \\
970 \\
298 \\
4750\end{array}$ & 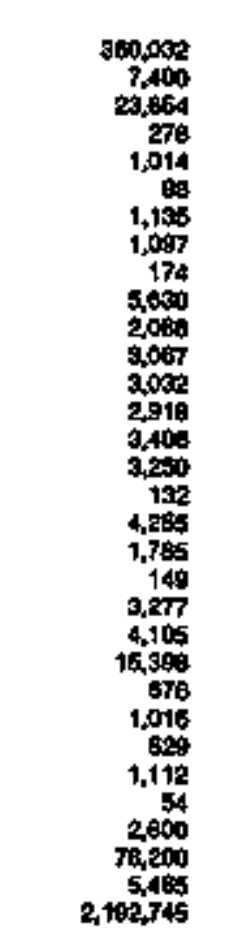 & 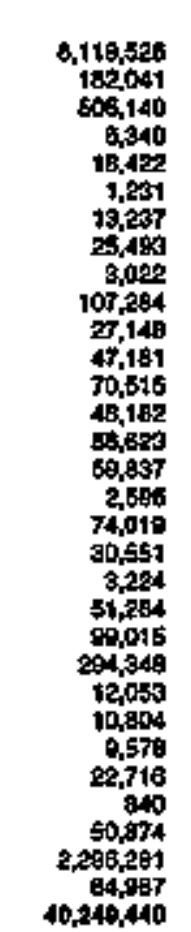 & 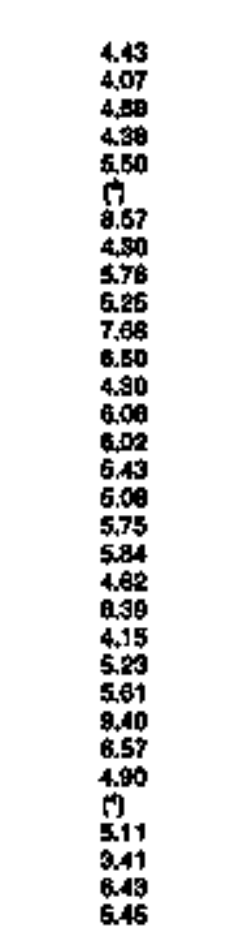 \\
\hline 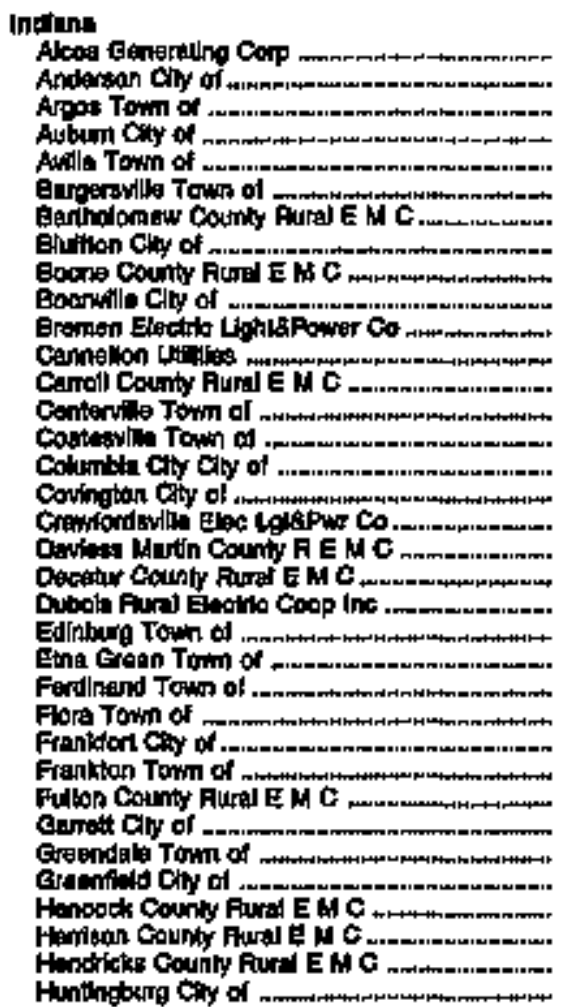 & 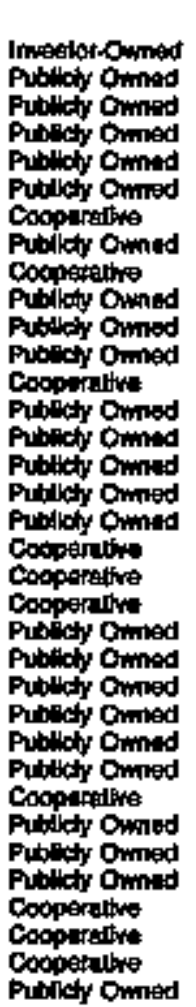 & $\begin{array}{r}1 \\
35 \\
8 \\
74 \\
18 \\
42 \\
6 \\
30 \\
1 \\
18 \\
21 \\
7 \\
2 \\
27 \\
5 \\
30 \\
23 \\
118 \\
42 \\
13 \\
30 \\
78 \\
2 \\
57 \\
12 \\
89 \\
16 \\
1 \\
30 \\
11 \\
28 \\
2 \\
5 \\
46 \\
57\end{array}$ & 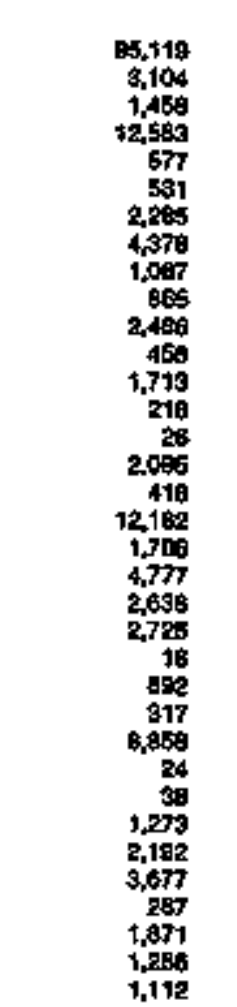 & 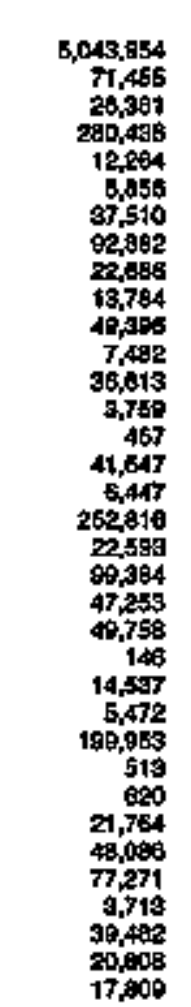 & 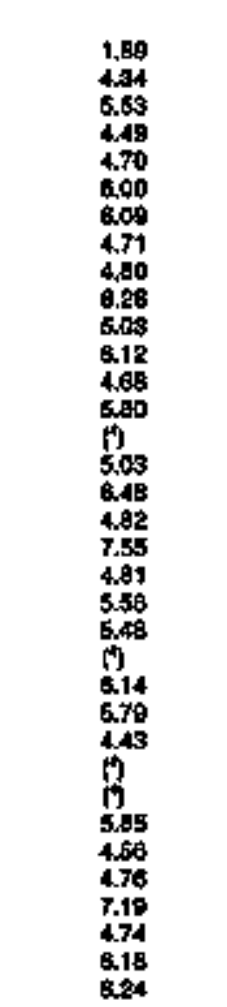 \\
\hline
\end{tabular}

Sen toctnolat at and of table. 
Table 16. Clase of Ownerahip, Number of Ultimate Constumers, Rovenue, Salos, and Average Revenue per Kilowatthour for the Industrial Sector by State and Utility, 1993 (Continued)

\begin{tabular}{|c|c|c|c|c|c|}
\hline $\begin{array}{l}\text { stat: } \\
\text { Enetrilc Utunty }\end{array}$ & $\begin{array}{c}\text { Clas: } \\
\text { of } \\
\text { Ownerthip }\end{array}$ & $\begin{array}{l}\text { Number } \\
\text { of } \\
\text { Connumers }\end{array}$ & 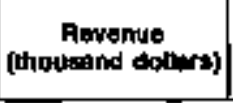 & $\begin{array}{c}\text { Sales } \\
\text { tubousund } \\
\text { kllpwothours }\end{array}$ & 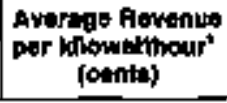 \\
\hline 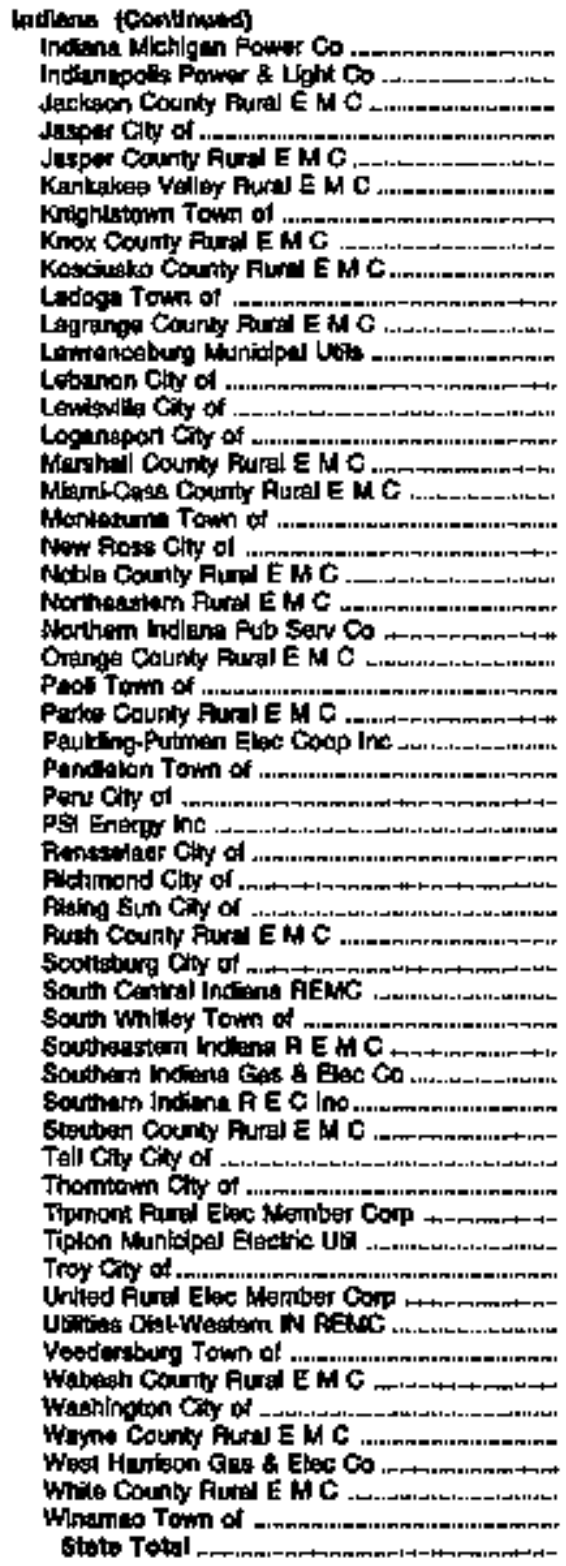 & 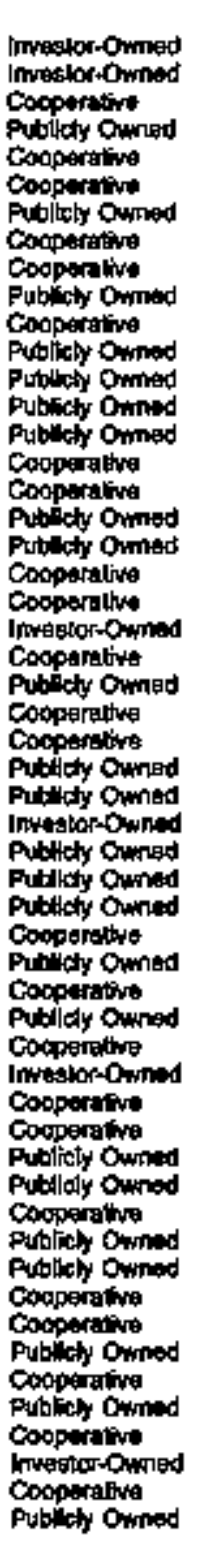 & 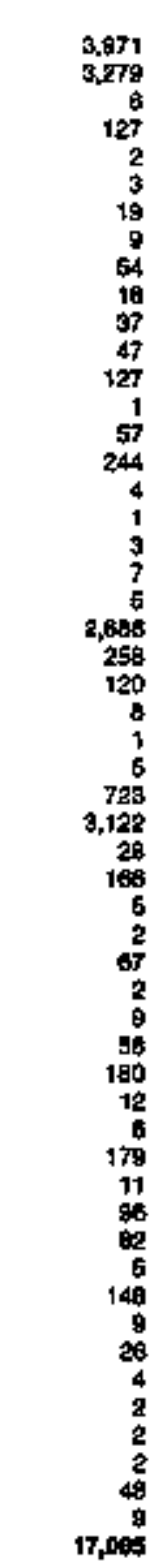 & 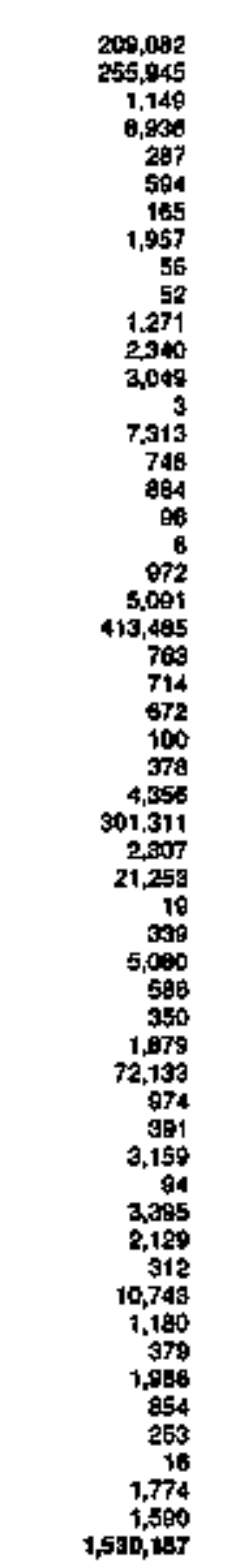 & 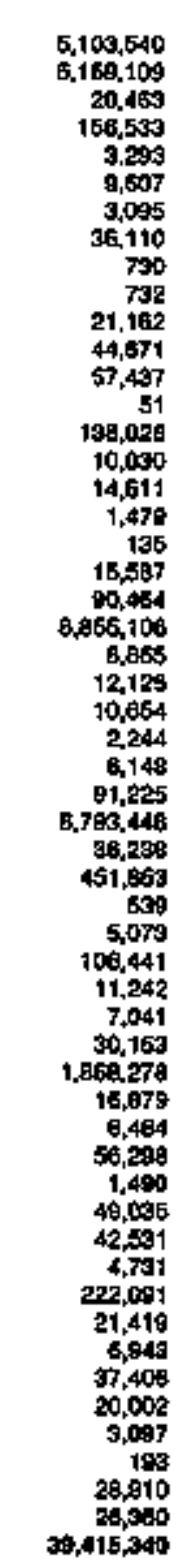 & 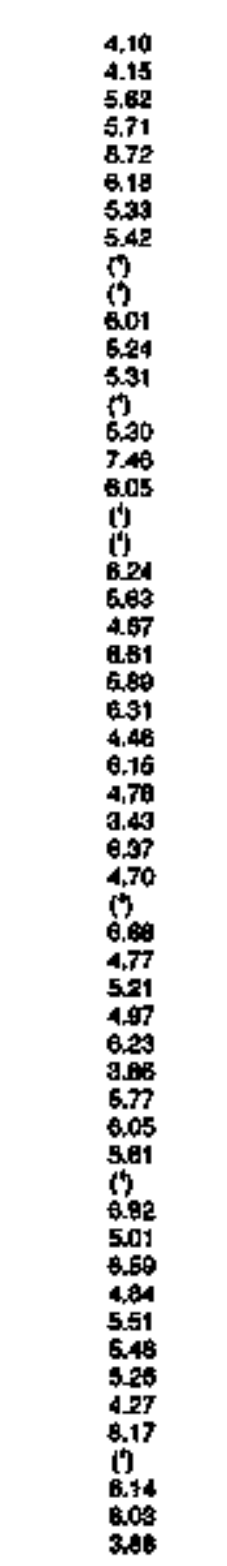 \\
\hline 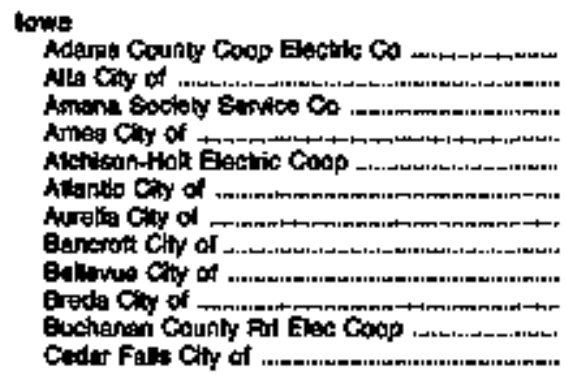 & 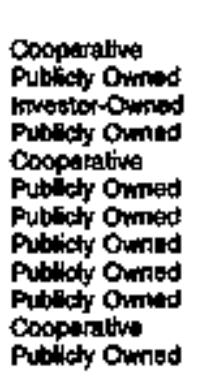 & $\begin{array}{r}1 \\
7 \\
1 \\
251 \\
1 \\
63 \\
16 \\
11 \\
8 \\
38 \\
2 \\
41\end{array}$ & $\begin{array}{r}233 \\
110 \\
3,208 \\
7,094 \\
6 \\
1,205 \\
129 \\
145 \\
160 \\
74 \\
444 \\
1.742\end{array}$ & $\begin{array}{r}4,149 \\
2,170 \\
6,1,591 \\
140,046 \\
7 \\
94,214 \\
2,138 \\
2,527 \\
2,206 \\
1,490 \\
8,273 \\
14,837\end{array}$ & $\begin{array}{l}5.62 \\
5.05 \\
4.98 \\
5.04 \\
07 \\
3.70 \\
6.09 \\
5.74 \\
6.70 \\
0 \\
5.37 \\
3.09\end{array}$ \\
\hline
\end{tabular}

See foctinotea al and of labla. 
Table 16. Class of Ownershlo, Number of Uitimate Conaumers, Revenue, Salos, and Average Fevenue per Kllowathour for the Industrtal Sector by State and Utulity, 1993 (Continued)

\begin{tabular}{|c|c|c|c|c|c|}
\hline Siak of & OAlat & $\begin{array}{l}\text { Mintery } \\
\text { al } \\
\text { Congumitrs }\end{array}$ & 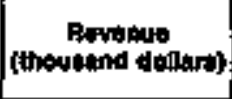 & 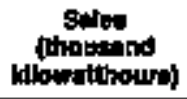 & 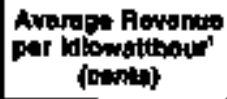 \\
\hline 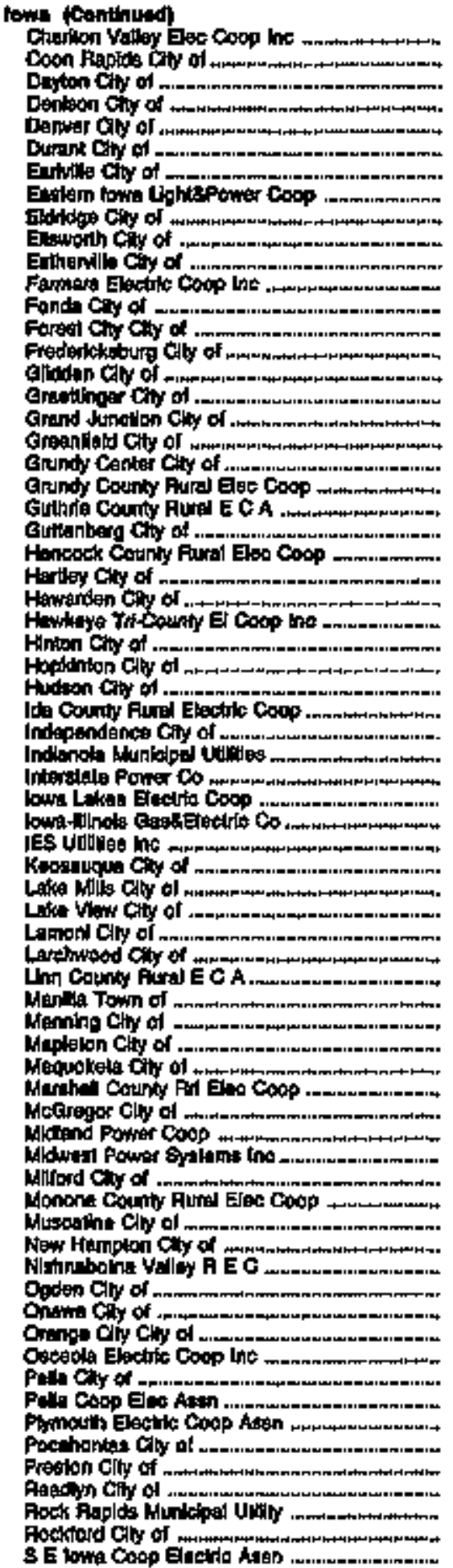 & 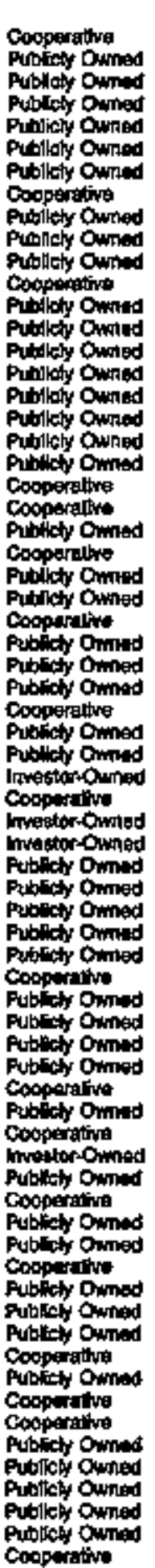 & 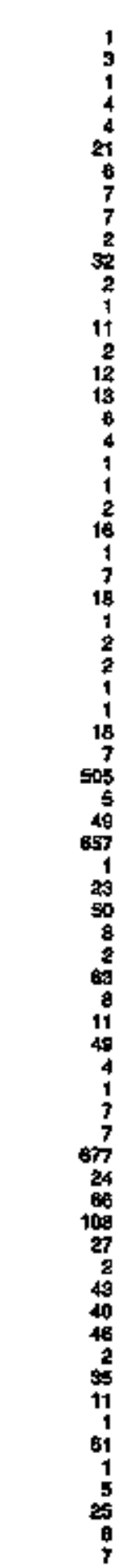 & 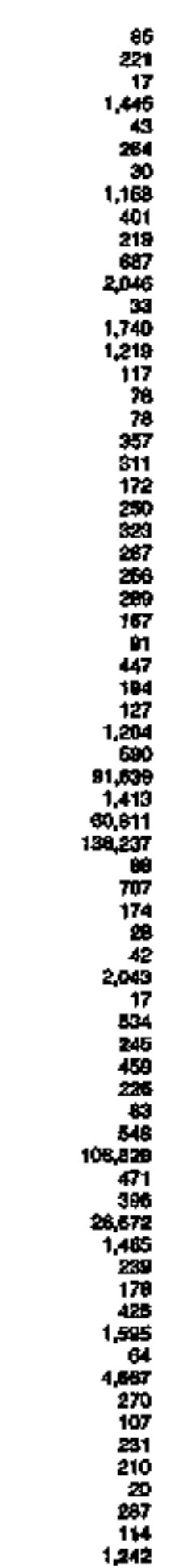 & 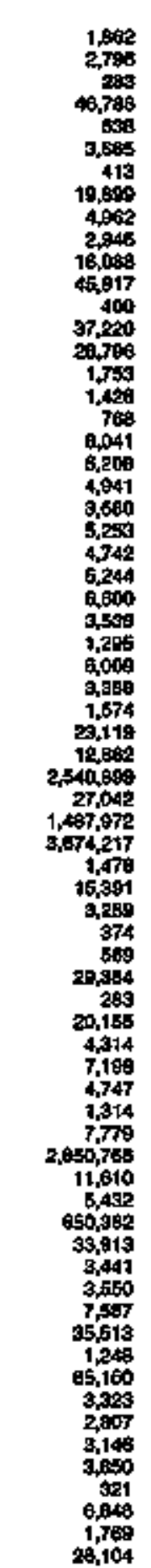 & 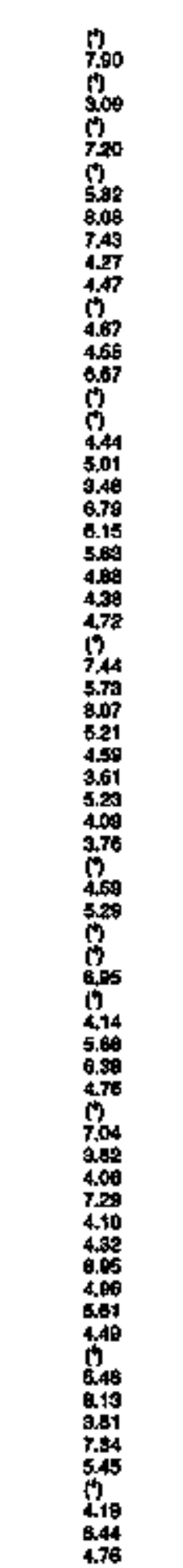 \\
\hline
\end{tabular}

5oo toolnoters al and af teldes. 
Table 16. Cless of Ownershlp, Number of Ulthate Contumera, Revenus, Sales, and Average Revenue per Kilowatthour for the Industrial Sector by State and Utility, 1993 (Continued)

\begin{tabular}{|c|c|c|c|c|c|}
\hline Eecticte todity & $\begin{array}{c}\text { Clate } \\
\text { of } \\
\text { Oumbinhlp }\end{array}$ & $\begin{array}{c}\text { Number } \\
\text { of } \\
\text { Contoutatot }\end{array}$ & $\mid \begin{array}{c}\text { Favanus } \\
\text { (thousund dolimes) }\end{array}$ & 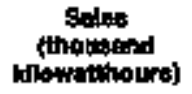 & 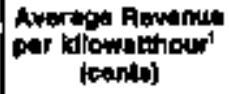 \\
\hline 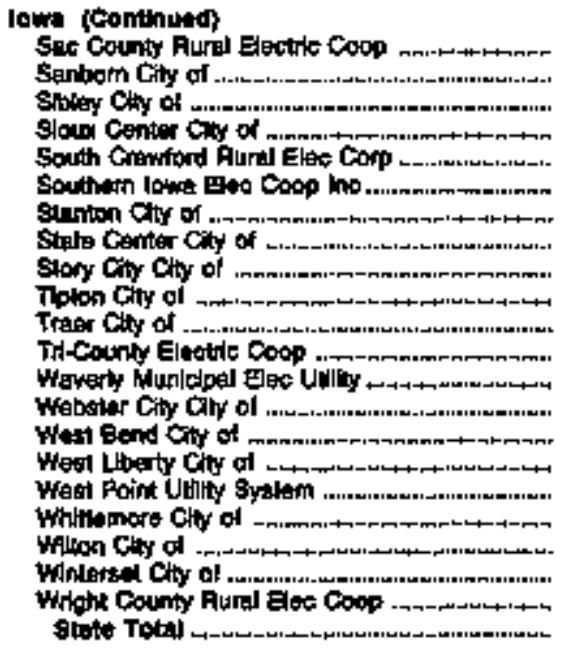 & 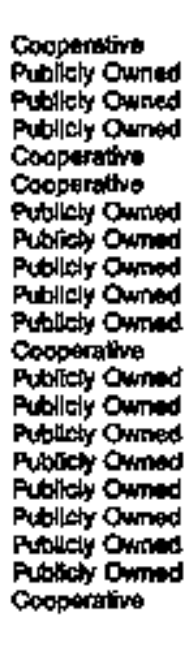 & $\begin{array}{r}14 \\
3 \\
2 \\
119 \\
2 \\
2 \\
1 \\
6 \\
39 \\
4 \\
1 \\
1 \\
41 \\
15 \\
7 \\
1 \\
7 \\
2 \\
7 \\
9 \\
3 \\
3.671\end{array}$ & $\begin{array}{r}67 \\
208 \\
358 \\
1,620 \\
852 \\
243 \\
13 \\
40 \\
7 \% 1 \\
241 \\
248 \\
1 \\
2,677 \\
3,052 \\
154 \\
1,447 \\
26 \\
82 \\
372 \\
466 \\
4,470 \\
406,427\end{array}$ & 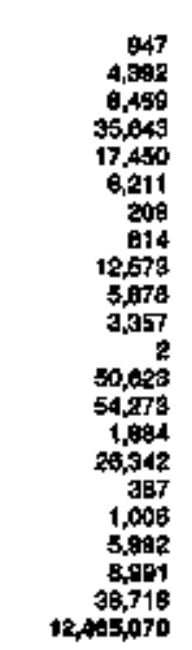 & 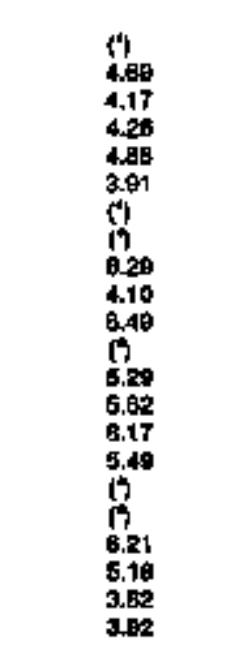 \\
\hline 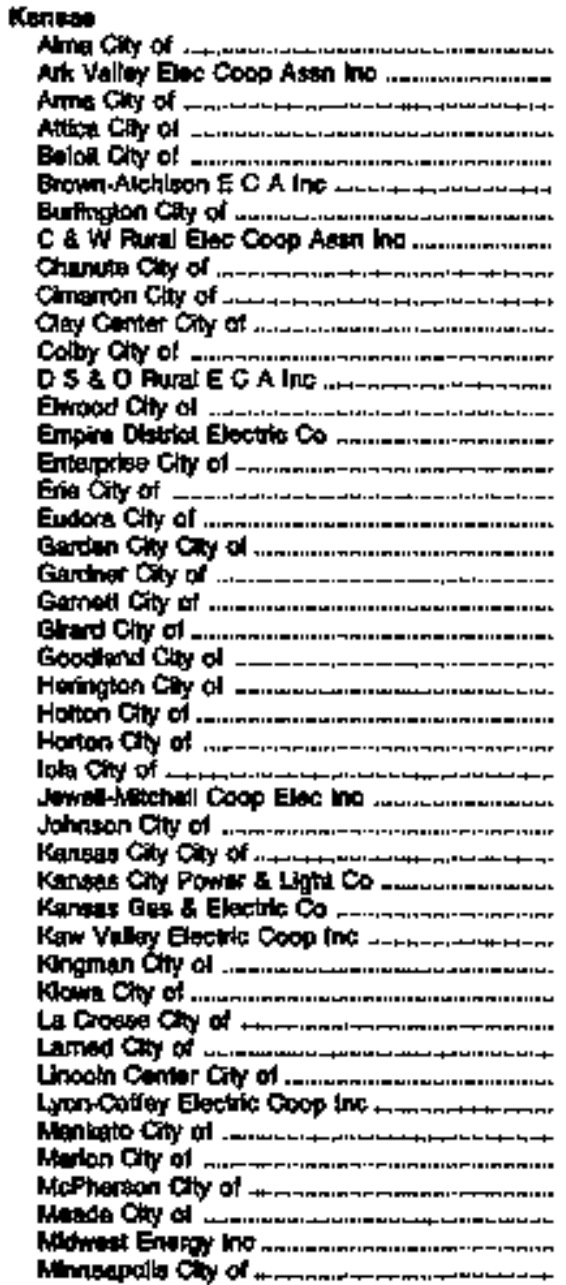 & 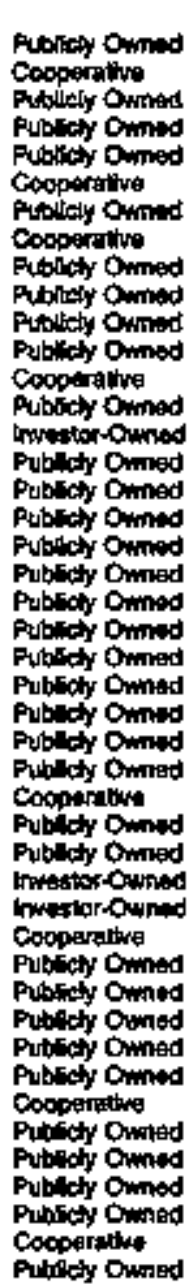 & 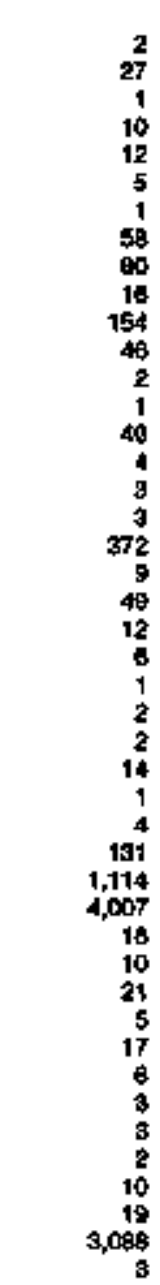 & 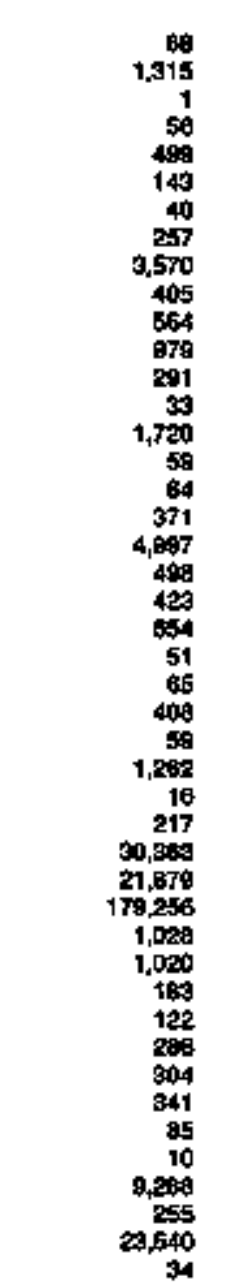 & 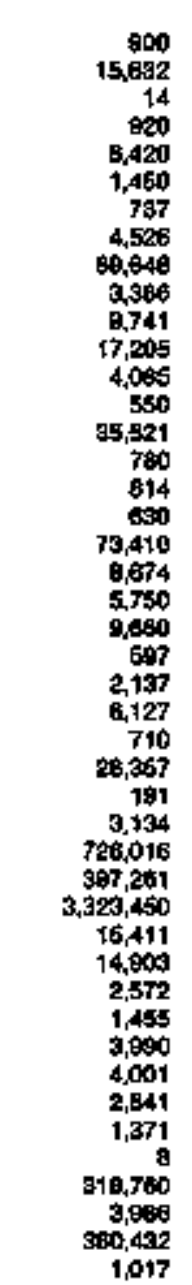 & 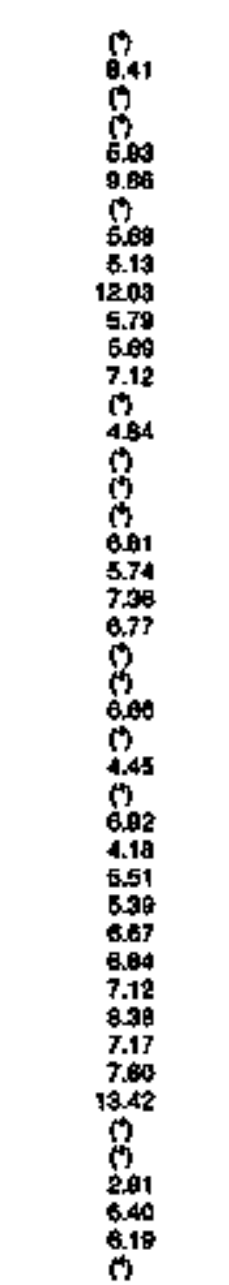 \\
\hline
\end{tabular}

Ses footnoles al end of tabia. 
Table 16. Clase of Ownershdp, Number of Utimate Conaumers, Revenue, Sales, and Average Revenue per Kllowatthour for the kndustrlal Sector by Slate and Utllty, 1998 (Continued)

\begin{tabular}{|c|c|c|c|c|c|}
\hline State & cias: & 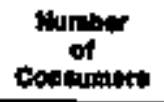 & fhousind dollores & 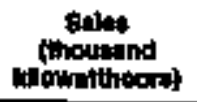 & 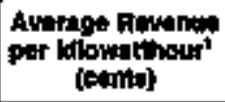 \\
\hline
\end{tabular}

Kanana (Conthovd

No Nondidge City of

Noodenha Cly of

Nhtoocth Furd ec $A$ in

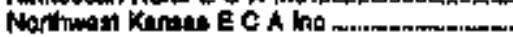

Oniser Chy of

Quage Clity city of .

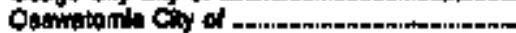

Ottomin Chy of .

Cithere City of

(1)

Plonegr Bedvit Coop he .

Piatt ciny of

Fuanatl Cly of

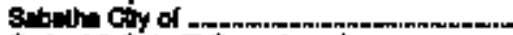

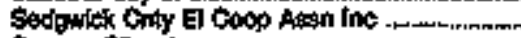

Gentece chy of

Eharon Saings Coty of .

Enoty Hill Elec Coep Aam bne nnm.m........

Southugotern Pubere senvico Co

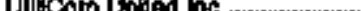

Vietary Ehotilo Coop Asen tho .......................

Wemego Chy of

Wentingion $C^{2}$ of

Westem Coop Elecile Alsi Ine ..............

Weatam Finources inc.

Whatiand Eetive Coop Ins

WhItêld City of

thate Toll

Kanivelsy

Babourville Ctr of

Bactoloun C Thy of

Banlen Cin of .

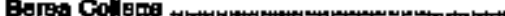

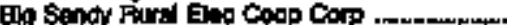

Bus Brars furd E Coop Corp .

Bowiling timen chy of .

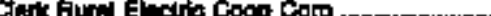

Corblin Coty Lthine Camm

Contherland Vallo futral E O C

Elactro Entroy Int

Falmoulh Cty of

Fimment Rurd Eleo Coop Cop ....

Fleming Atason Rural E G C.

Fox Creak Fumé Bec Cacp CorP

Frenkfort Cly of -

Frevllim Clty of

c겸onow Chy of

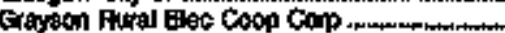

Creen Furer Electro Coxp...

Henderson Cty Uto Conm

Hendertontuninn fural ECC

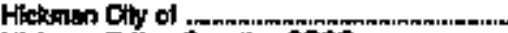

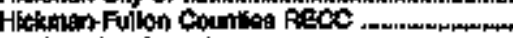

Himitansitia Coty of

knler County Pund E C C

Jaskation County Furgl E C C .

Jackoon Purchise 5 Coop Corp

Je-co Cly of

Kentretion Poner

Kaputuaky Uullitan $\infty$

Lcking VAloy furs E C C.

Loulm $\mathrm{R}$.

Moritelt Cily of

mondieelo cany of

Murray Cly of
Fublut Ounied

Coopertitue

Publichy Omingd

Cooperative

Cooperative

Fublity Ommed

Pubrict Omed

Pubichy Omed

Publict Onned

Pusticty otined

Publity Ounnd

Cooperathe

Putifey Owned

Putbloty Owned

Putblely Owned

Cooperaliva

Pultint Owned

P(t)ing Ownd

Coopuritue

Imettor-Owingd

imvactortowned

Cooparallue

Fubluty Dumed

Puthoy Omined

Coopuratue

Imvestor-Omined

Cocoperefiviva

Publisty Owited

Pubtch Owined

P(itifiefy owned

Putbly Owned

Innepalow-Omitad

Cooperatus

Cooperativa

Futitin Owhod

Cooperthe

Puntury Ommed

Cooperstra

imvestor-Omed

Pubsteh Owined

Cooperathe

Goposerdthe

Cocporative

Publlet Owned

Fubity Owned

Putch Owned

Putbles Dined

Gooperditia

Cooperathe

Publicty Drmed

Cocantity

Publely Ownod

cooperative

Pubicky Omed

Cocoperativa

Copoperative

Cooperathe

Futhos Ourled

me:tor Omined

mrestor-Onined

Fooperatine

Imuetor-Onarod

Pubicts Dmod

Pitsey Ownod

Putich Ommed

Futich Owned

22
206
43
1
10
4
3
4
29
06
21
26
36
287
60
1
49
20
24
15
69
2
13
27
251
1,781
10
104
106

149

10

44

3

40

$\$$

6

$\mathbf{5}$

2

281

51

162

1
154

10

19

205

198

7
1.850

$1,8 \div 0$

2

363

721

131
297

281

1,296

704

120

10s

24

341

204

3,650

323

的1

1,118

17

495

110

由72

343

29,109

1,917

212

1,098

0.,64

11,575

B.77

430,107

1,944

2504

1,171

1,762

207

4,203

20,48:

132

68

6585

200,047

44

000

a,

423

11,608

6,194

1,012

0,903

15,308

15,308
2,748

04,79

390

3,038

0,700

80

1,650

3,086
200

$\mathbf{0 0 5 0 1}$

130,567

650

104,506

3,675

2,015

6,46

$1,4=5$

5.132

8,300

15, 037

Q.641

7,849

2000

1,87

4,608

42,323

2,360

60.67

1.252

15, 360

10,500

1,624

6.50

587
0.240

7,519

509.494

35,800

3.,01

20,008

$1,077,176$

215.479

140.764

0,702:519

5.79

g.13

5.50

a.97

6.47

(1)

7.43

7,13

96

6.42

5,17

6.30

5.73

10.79

7,00

(1)

4.15

4.08

$5 \mathrm{se}$

5.50

6.41

(i)

5.50

4.70

4.94

34.4164

50,582

19,004

40,75e

15,381

07,002

321,497

1,565

16,067

125002

$11,000,004$

T1

13,716 
Table 16. Class of Ownership, Number of Ultimbte Consumers, Revenue, Sales, and Average Revenue per Kllowatthour for the Industrial Sector by State and Utility, 1993 (Continued)

\begin{tabular}{|c|c|c|c|c|c|}
\hline 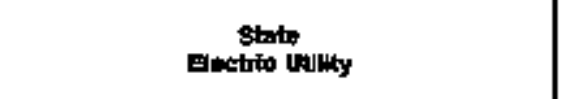 & $\begin{array}{c}\text { Clat: } \\
\text { of } \\
\text { Otherthlp }\end{array}$ & 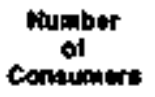 & Prownise & 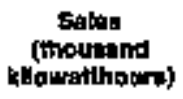 & $\begin{array}{l}\text { Average howenue } \\
\text { per linowathour' } \\
\text { (conts) }\end{array}$ \\
\hline 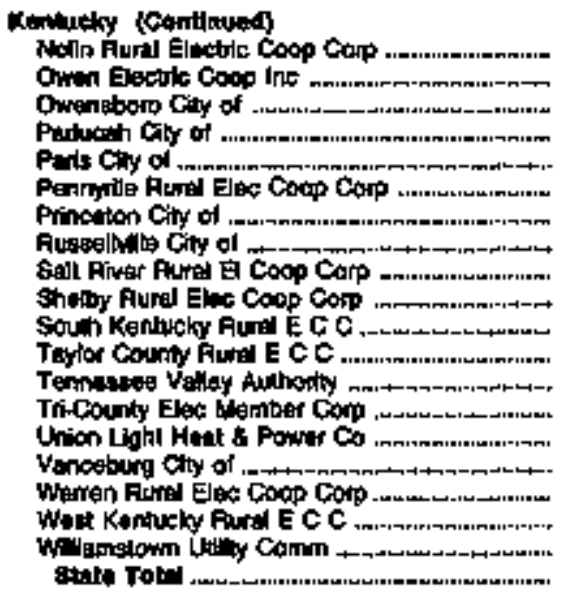 & 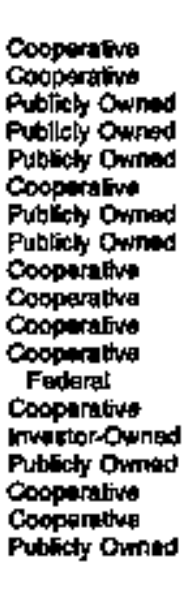 & $\begin{array}{r}3 \\
1,972 \\
475 \\
23 \\
364 \\
51 \\
85 \\
216 \\
5 \\
235 \\
4 \\
16 \\
159 \\
406 \\
5 \\
347 \\
189 \\
10 \\
10,866\end{array}$ & $\begin{array}{r}9,517 \\
909 \\
19,659 \\
19,776 \\
335 \\
15,474 \\
2,007 \\
3,487 \\
6,487 \\
3,988 \\
5,646 \\
537 \\
151,958 \\
10,276 \\
37,439 \\
427 \\
16,306 \\
12,491 \\
1,179 \\
1,197,681\end{array}$ & 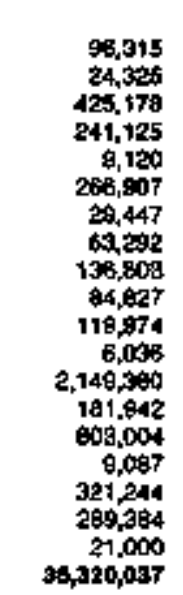 & $\begin{array}{l}3.66 \\
4.09 \\
3.21 \\
6.71 \\
5.67 \\
5.80 \\
6.92 \\
5.51 \\
4.71 \\
1.00 \\
5.54 \\
5.58 \\
7.01 \\
5.85 \\
4.67 \\
4.70 \\
5.70 \\
4.64 \\
5.81 \\
3.50\end{array}$ \\
\hline 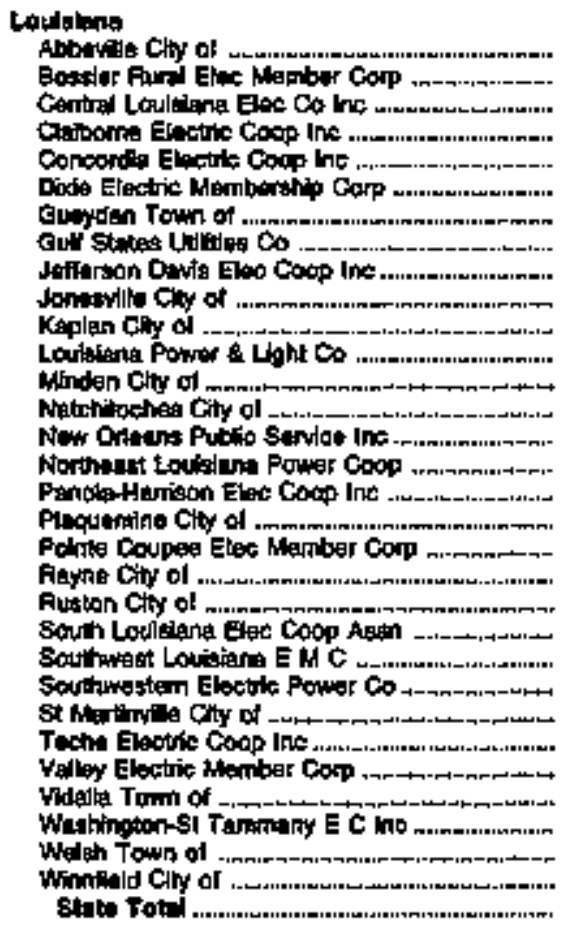 & 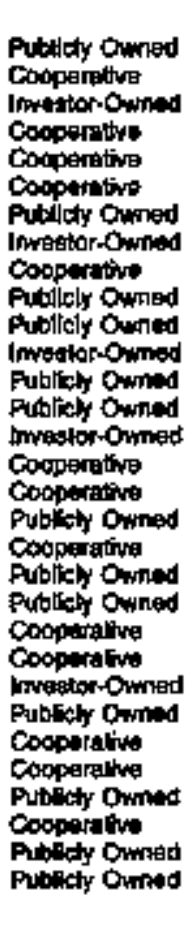 & $\begin{array}{r}44 \\
689 \\
70 \\
23 \\
2 \\
291 \\
31 \\
3215 \\
49 \\
6 \\
25 \\
6,143 \\
234 \\
9 \\
620 \\
90 \\
39 \\
209 \\
7 \\
7 \\
67 \\
20 \\
11 \\
25+3 \\
204 \\
3 \\
1 \\
2 \\
8 \\
12 \\
4 \\
15,429\end{array}$ & 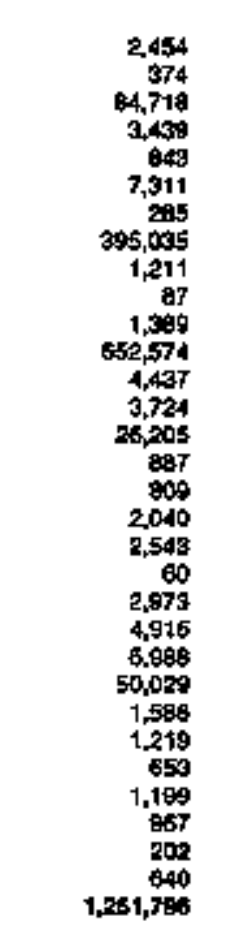 & 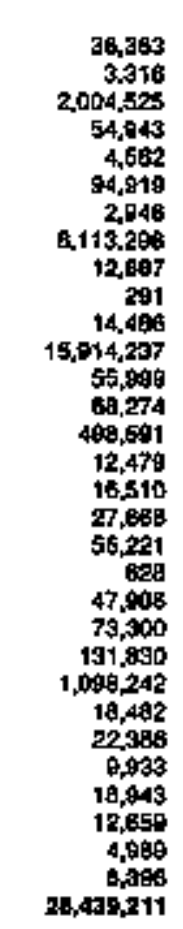 & 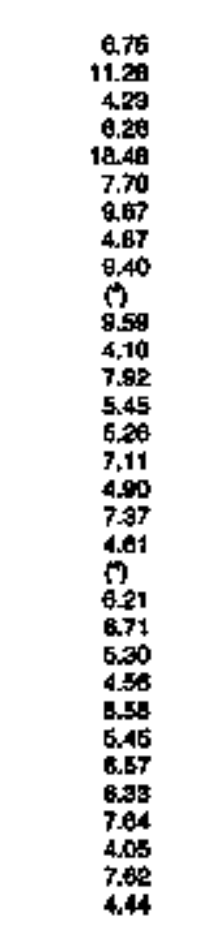 \\
\hline 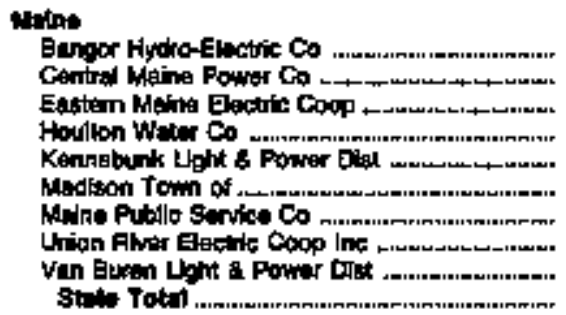 & 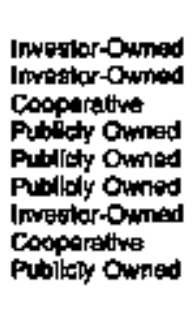 & $\begin{array}{r}1,215 \\
1,494 \\
1 \\
80 \\
9 \\
17 \\
16 \\
7 \\
16 \\
2,794\end{array}$ & $\begin{array}{r}69,527 \\
283,192 \\
1,064 \\
4,083 \\
2,462 \\
674 \\
9,553 \\
24 \\
360,840\end{array}$ & $\begin{array}{r}906,172 \\
3,8,33,342 \\
25,525 \\
48,350 \\
\$ 1,161 \\
6,447 \\
135,029 \\
164 \\
3,431 \\
5,099,830\end{array}$ & $\begin{array}{r}7.67 \\
6.70 \\
4.13 \\
6.34 \\
7.90 \\
10.46 \\
7.09 \\
61 \\
9.30 \\
6.66\end{array}$ \\
\hline
\end{tabular}

sed foontops at and ol tank 
Table t6. Class of Ownershlp, Mumber of Uhtmate Consumerg, Fevenue, Sales, and Average Revenue per Kllowathour for the Industrial Sector by State and Ut|llty, 1993 (Continued)

\begin{tabular}{|c|c|c|c|c|c|}
\hline $\begin{array}{l}\text { Stats } \\
\text { Enotito tudity }\end{array}$ & $\begin{array}{l}\text { Olate } \\
\text { of } \\
\text { Oumerthip }\end{array}$ & $\begin{array}{l}\text { tunber } \\
\text { of } \\
\text { Conaumere }\end{array}$ & 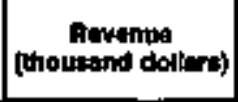 & 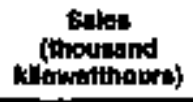 & 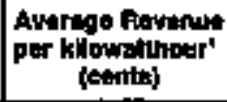 \\
\hline 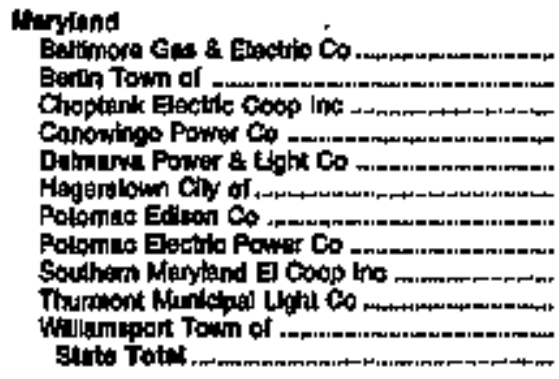 & 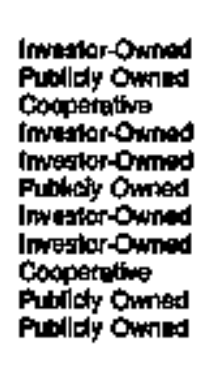 & 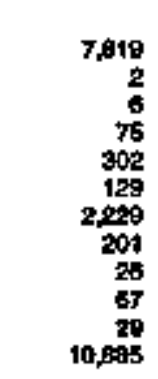 & $\begin{array}{r}802,659 \\
1,000 \\
3,690 \\
16,740 \\
18,649 \\
5,080 \\
100,401 \\
121,216 \\
9,009 \\
1,439 \\
3,100 \\
1,100,112\end{array}$ & 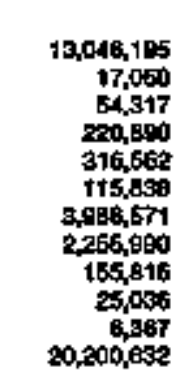 & $\begin{array}{l}6.15 \\
6.35 \\
7.14 \\
7.58 \\
5.88 \\
4.39 \\
3.02 \\
5.37 \\
5.76 \\
4.55 \\
0.50 \\
6.46\end{array}$ \\
\hline 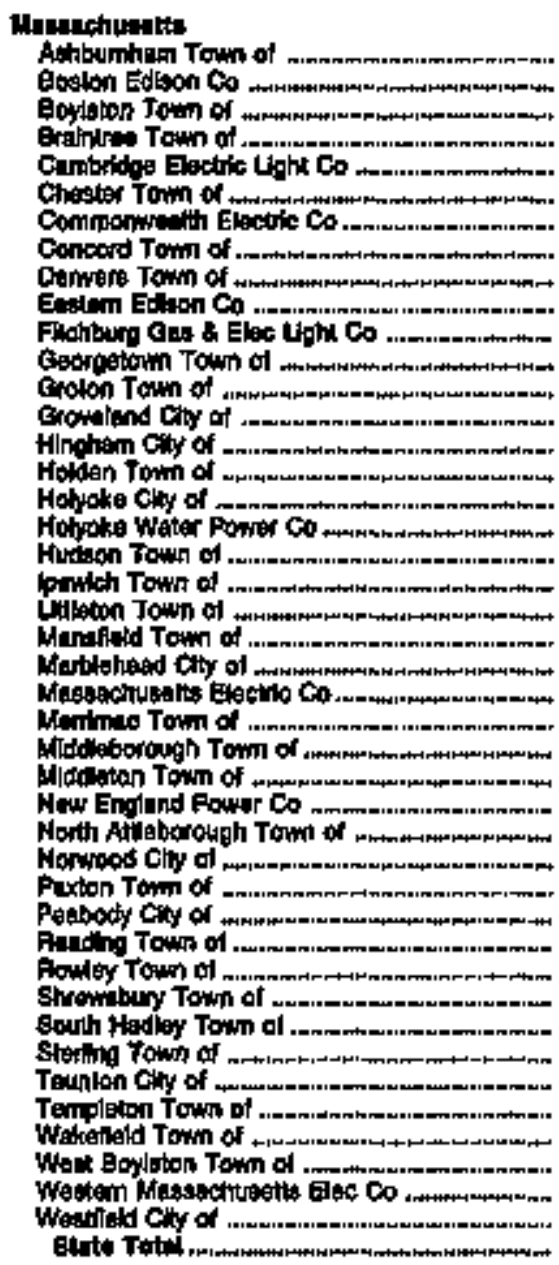 & 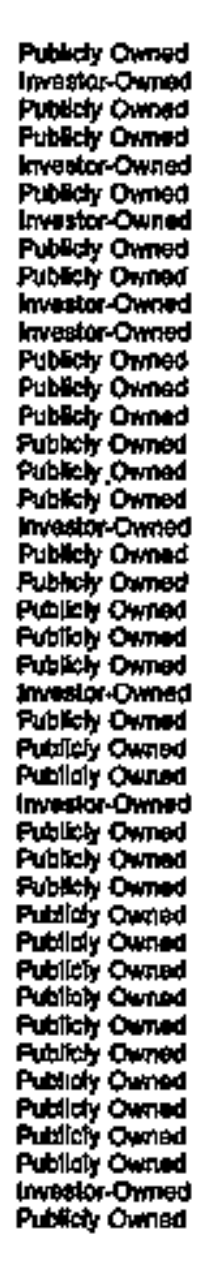 & 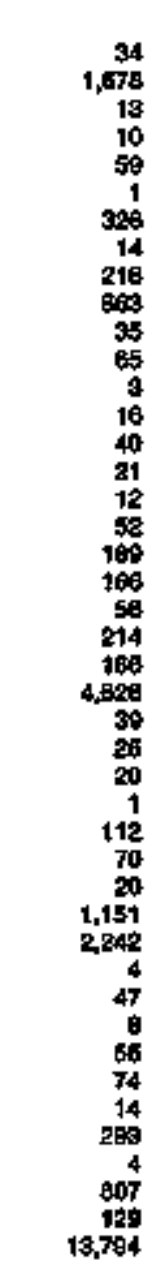 & 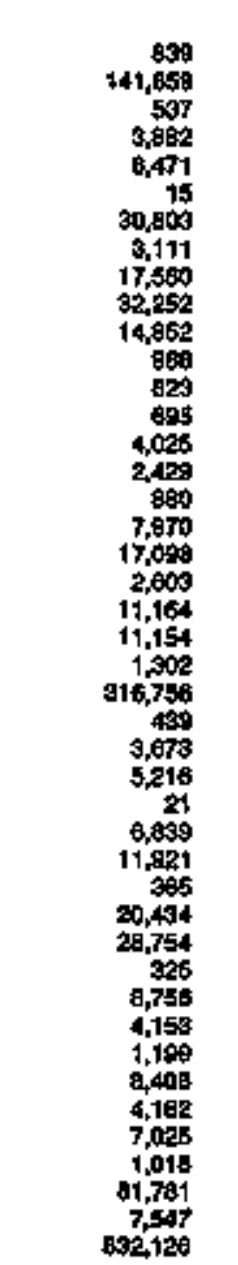 & 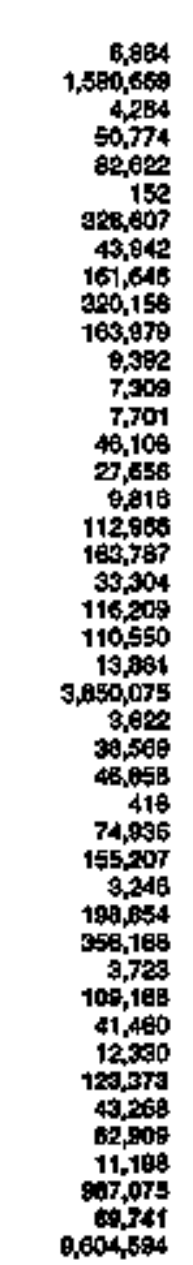 & 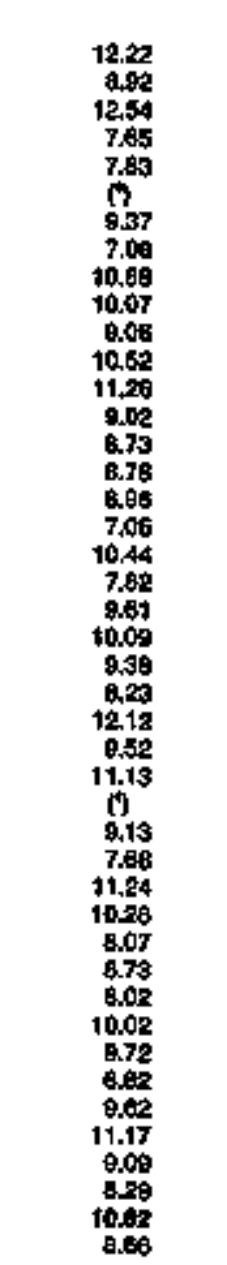 \\
\hline 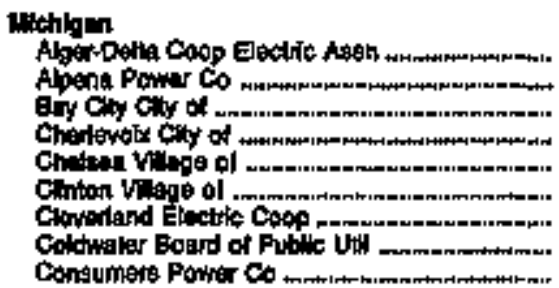 & 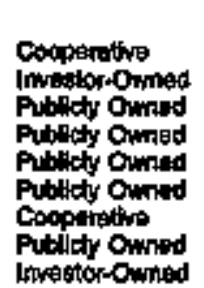 & $\begin{array}{r}1 B \\
5 \\
58 \\
18 \\
5 \\
15 \\
4 \\
160 \\
9,078\end{array}$ & $\begin{array}{r}707 \\
4,560 \\
2,009 \\
609 \\
615 \\
701 \\
1,005 \\
6,168 \\
028,400\end{array}$ & 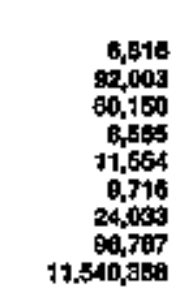 & $\begin{array}{l}10.37 \\
4.93 \\
4.64 \\
6.97 \\
6.32 \\
6.14 \\
4.51 \\
6.37 \\
5.49\end{array}$ \\
\hline
\end{tabular}

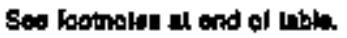


Table 18. Class of Ownership, Number of Uitimate Conuumera, Revenue, Sales, and Average Fevenue per Kilowatthour for the Industrial Sector by State and Utilly, 1993 (Contlnued)

\begin{tabular}{|c|c|c|c|c|c|}
\hline 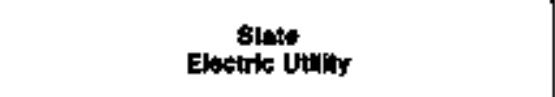 & $\begin{array}{l}\text { Chats } \\
\text { of } \\
\text { Omperstip }\end{array}$ & $\begin{array}{l}\text { Kulpber } \\
\text { of } \\
\text { Conotimary }\end{array}$ & 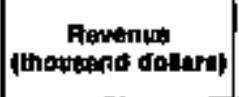 & 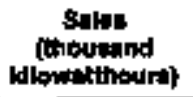 & $\begin{array}{l}\text { Avereas Revarus } \\
\text { per knowitheur' } \\
\text { (onme) }\end{array}$ \\
\hline 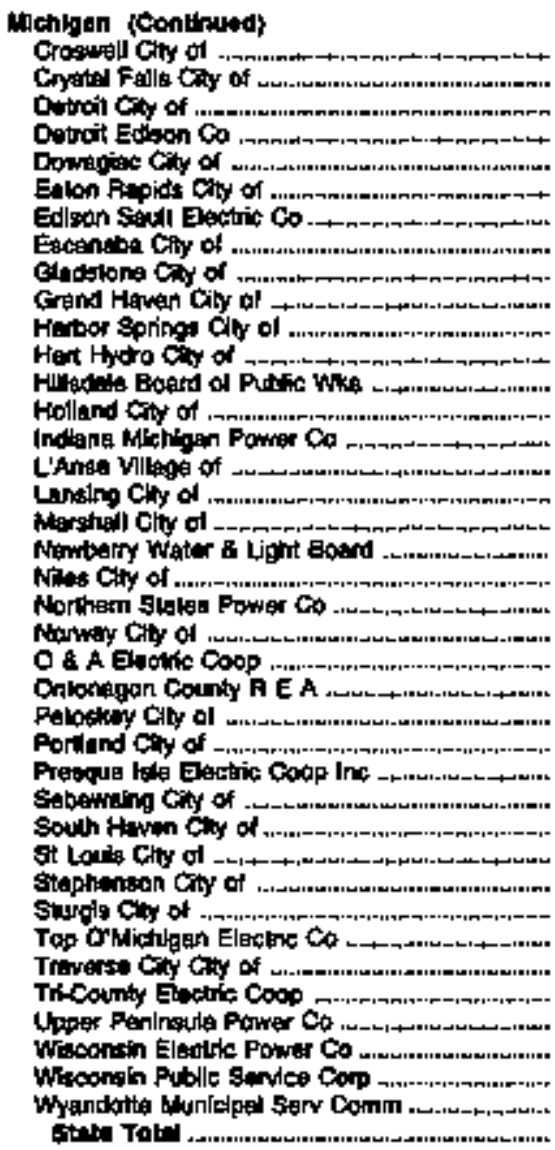 & 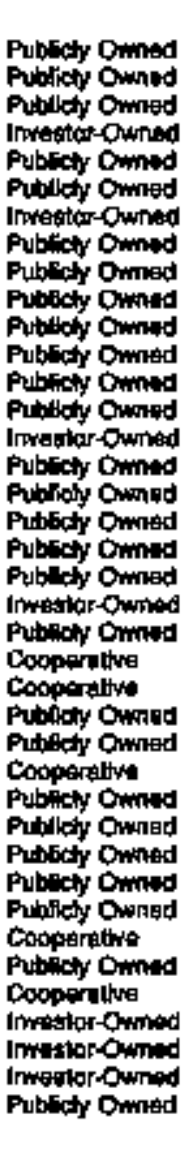 & $\begin{array}{r}19 \\
1 \\
9 \\
027 \\
29 \\
6 \\
4 \\
21 \\
19 \\
128 \\
2 \\
5 \\
20 \\
106 \\
11121 \\
7 \\
162 \\
54 \\
13 \\
18 \\
21 \\
12 \\
45 \\
20 \\
14 \\
17 \\
7 \\
7 \\
50 \\
1 \\
1 \\
75 \\
5 \\
19 \\
50 \\
16 \\
9 \\
5 \\
197 \\
12997\end{array}$ & 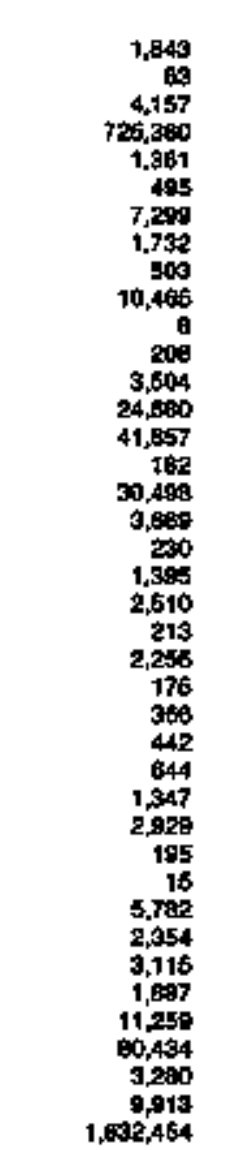 & 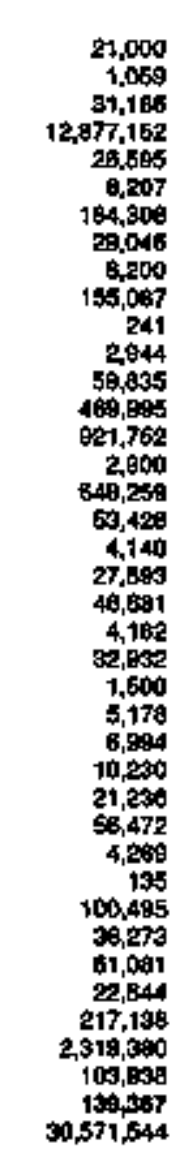 & 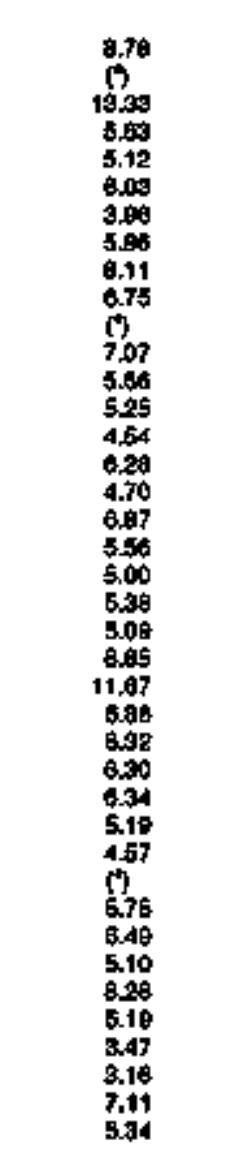 \\
\hline 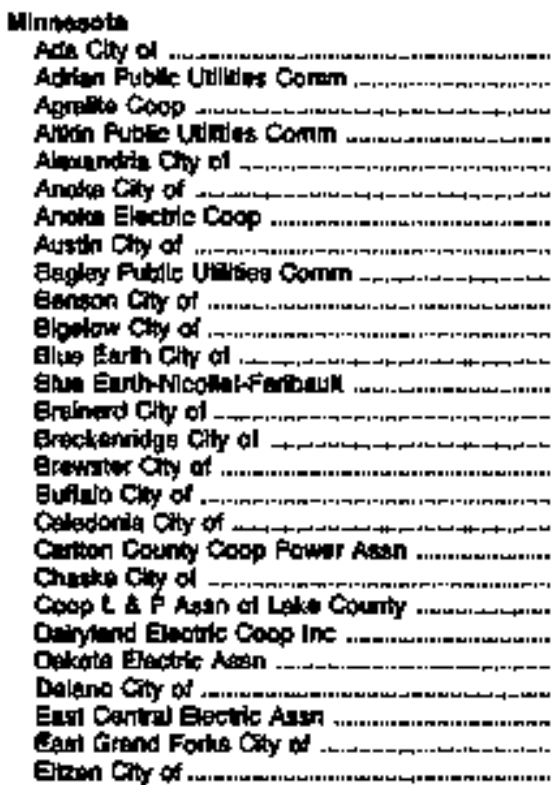 & 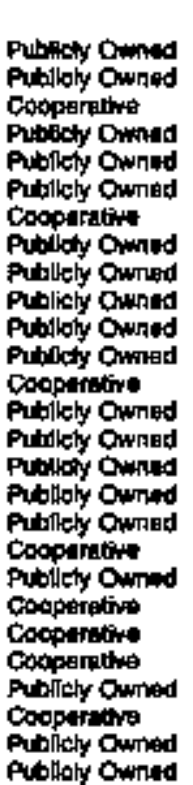 & $\begin{array}{r}8 \\
7 \\
1 \\
40 \\
46 \\
17 \\
11 \\
1 \\
51 \\
42 \\
1 \\
48 \\
1 \\
15 \\
18 \\
1 \\
5 \\
57 \\
1 \\
186 \\
13 \\
1 \\
145 \\
50 \\
5 \\
11 \\
3\end{array}$ & 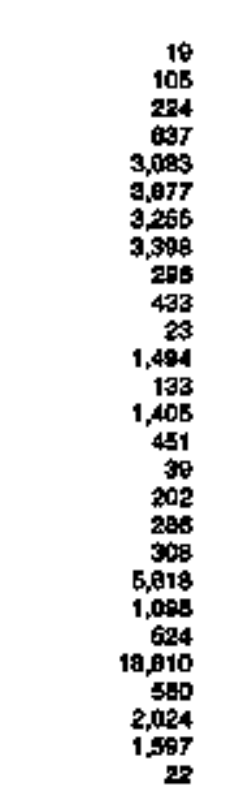 & 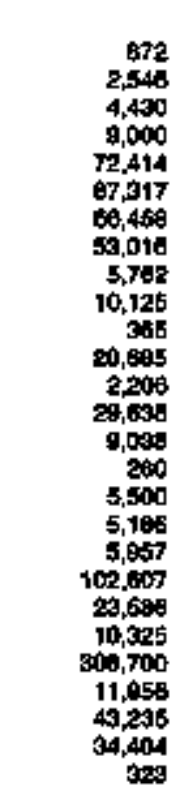 & 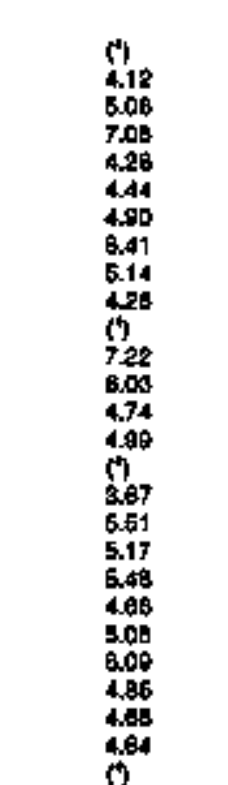 \\
\hline
\end{tabular}

See tootnotes at and of uble. 
Tabje 16. Class of Ownership, Number of Utimate Conatumers, Revenue, Sales, and Average Revenue per Kllowatthour for the Industrial Sector by State and Utillty, 1993 (ContInued)

\begin{tabular}{|c|c|c|c|c|c|}
\hline Atots & $\begin{array}{c}\text { Cats: } \\
\text { of } \\
\text { Ommarthipp }\end{array}$ & $\begin{array}{l}\text { Number } \\
\text { of } \\
\text { Consumars }\end{array}$ & 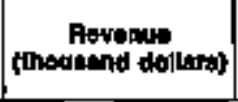 & $\begin{array}{l}\text { Bwles: } \\
\text { (thationd } \\
\text { (diawithours) }\end{array}$ & 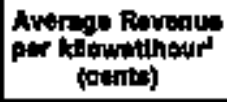 \\
\hline
\end{tabular}

Whnuata condinued]

Elk Alwer Cly of.

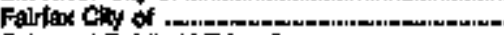

Fatrout Publo Itimites Corar

Fotiston Chy of

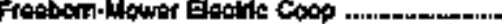

Glencos Lipht \& Power Comrp

Grand Pap ts Putre Uia Catm -

Gipontto Falls Coly of . .

Geove Cly Cly of ....-.

Halled cth of

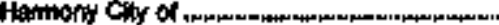

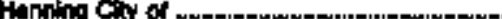

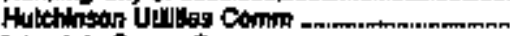

Interatals Power to

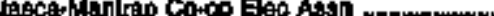

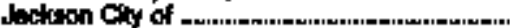

Jensenilit Chy of

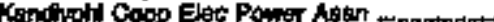

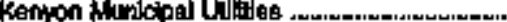

taks Cily Cly ol

Inte Pridk Cily of

teke Pen Con Elog Aasn

Le Sucter Cly of -

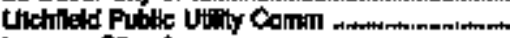

urverne City of

Madison Cly of

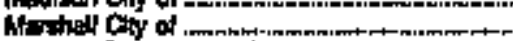

Mateod Copp Pown A A

Aresos Pubte Uthes

Mile Lacs Elethic Coop

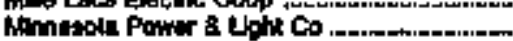

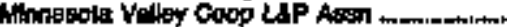

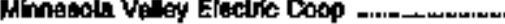

Hoonow Chy of

Mtora Caty of

Mounlain Iran Coy of

Houninin tone Cli

Now Pregis Mun bras com

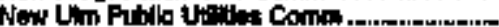

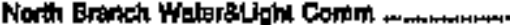

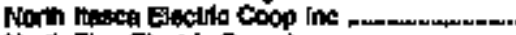

North Fing Elesist Coop ho.

Northem Elecilt Coop As:

Northein States Pown Co o

Olwh Coty of

Ortomile Ciny of

Otat Tat powter Co

Owitonne Clyy ol

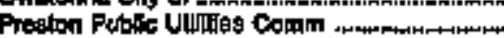

Prncelon Putrie Lit: Comn

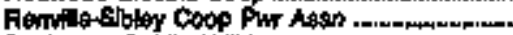

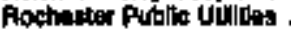

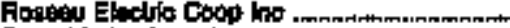

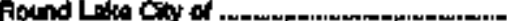

Fumastone Eluotrle ABSn ............................

Fustriond ctay of

Sethl Polet con el ...

Sack Centr Chy of

Sisepy Eye Putib thity Gomm

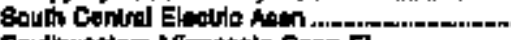

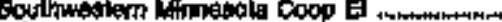

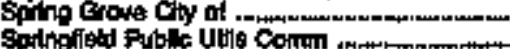

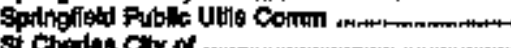

st Chentas coly of

stionos Ciny of

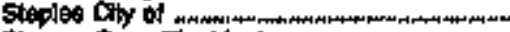

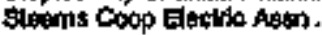

Pubicty Onnod Puticly Omed Pubtcty Owned

Publety Omind

Cosperaltura

Pubichy Omined

Putichy Omied

Pubicty Omiad

Pubicly Ounnd

Publcty Ouned

Publictr Onned

Publety Omind

Pubicty Owned

ineoftor Owited

coopurative

Publes Drned

Publdy Omed

Cooperalive

Putblety Oumad

Publicty Omned

Publuty Ommed

Cospuratue

Publich Cunad

Publity owned

putbicty Omined

Fublect Ounad

Publecy Oumed

Publesty Onnad

Cooptstitivo

Publicts Owned

Coopurative

imestor-Oinud

Cooperalutive

Cooperative

Pusthely Owned

Fublict ommed

Pulbicty Omod

Publichy Dumod

Puthltaly Owrod

Putitely Owned

Fubits owned

Cooperallye

coopersthe

Cooperative

imve:alor-Omint

Puticley Ounned

Fubliby Ommed

muaster-Owist

Plbllely Ouned

Futhinty Owned

Pubstiy Oumed

Coopercithe

Coxporalve

Pestely Ouned

Ccoporrative

Aublict Ownod

Cooperathe

Pulliwy Oaned

Aubltely Ourned

Publedy owned

Pubrisly Oumad

Cooperatrve

Cooperative

Pithly Ouned

Publusty awned

Fubitit Ownod

Publes Downd

Publicty Onened

coopustivis

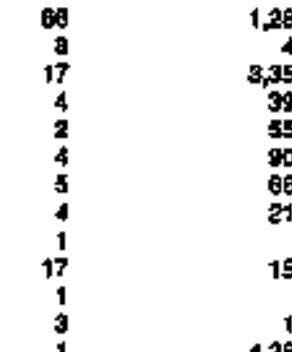

1,290

3,350

394

900

687

1

158

17

4,297
10,247

gap

20

392

2,108

3)

2,650

929

977

72

9,457

$\$ 16$

2, nos5

900

241,582

a42

464

6.286

1,500

104

1204

3205

138

14:

348

1,603

625,353

563

41,203

8 895

243

1,108

178

757
24,496

370

2ss

500

255

648

39

2.1

163

36

515

024

40

205
10,755

092

59,067

0,472

13,517

18,460

18,460

10,212

14
2,350

100

301

120,616

204,038

15,360

20,656

20

4,509

5,546

40,354

49

6979

001500

26,576

13,691

1,315

277 , 991

9.173

54,680

17,739

$6,341,40$

17,248

11,45

171,038

2,17

1,599

3.307

20,636

60,300

1,650

2,500

Q.020

37,0e0

$13,700,820$

0,518

9,610
126
4,779

824,77:

152,309

2,940

16,158

2625

1 1.

S05,356

1234

5005

11,401

4,712

19.70

14,343

13.737

0,000

5,185

3940

6005

0.380

27.20

0,111

7,801

65

(1)

o.

4.11

4.0.

431

s.0:

(b)

(t) 
Table t6. Class of Ownership, Number of Ultimate Consumers, Revenue, Sales, and Average Revenue per Kilowatthour for the Inougtrtal Sector by State and UNilty, 1993 (Continued)

\begin{tabular}{|c|c|c|c|c|c|}
\hline $\begin{array}{l}\text { Siste } \\
\text { atuatirle Utiny }\end{array}$ & Clang & $\begin{array}{c}\text { Nomber } \\
\text { of } \\
\text { Consoumars }\end{array}$ & (thoverind doatars) & $\begin{array}{l}\text { Salos } \\
\text { (thpusand } \\
\text { wlownthoust }\end{array}$ & $\begin{array}{l}\text { Averugo Reveruve } \\
\text { per Winowathour } \\
\text { (oints) }\end{array}$ \\
\hline 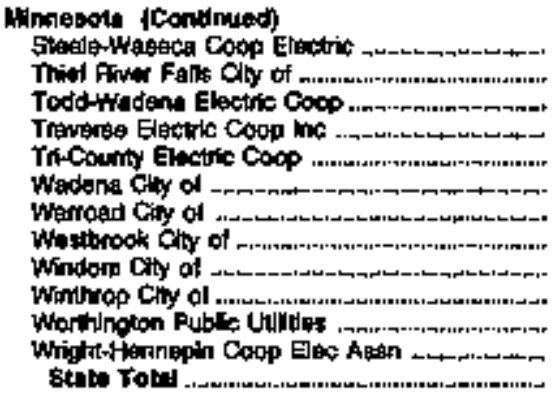 & 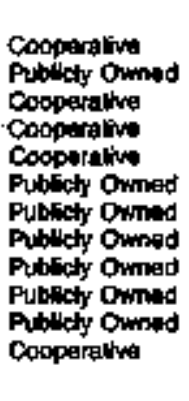 & $\begin{array}{r}2 \\
27 \\
12 \\
1 \\
66 \\
20 \\
1 \\
2 \\
17 \\
1 \\
5 \\
5 \\
10,349\end{array}$ & $\begin{array}{r}498 \\
1.680 \\
699 \\
309 \\
691 \\
719 \\
559 \\
21 \\
754 \\
106 \\
t .640 \\
1,919 \\
1,072,081\end{array}$ & 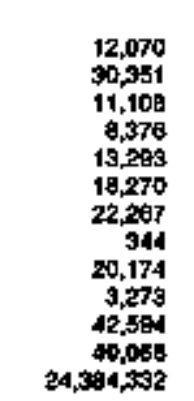 & $\begin{array}{l}4.11 \\
5.21 \\
5.75 \\
3.09 \\
7.23 \\
4.10 \\
2.51 \\
17 \\
3.71 \\
5.07 \\
4.55 \\
4.70 \\
4.40\end{array}$ \\
\hline 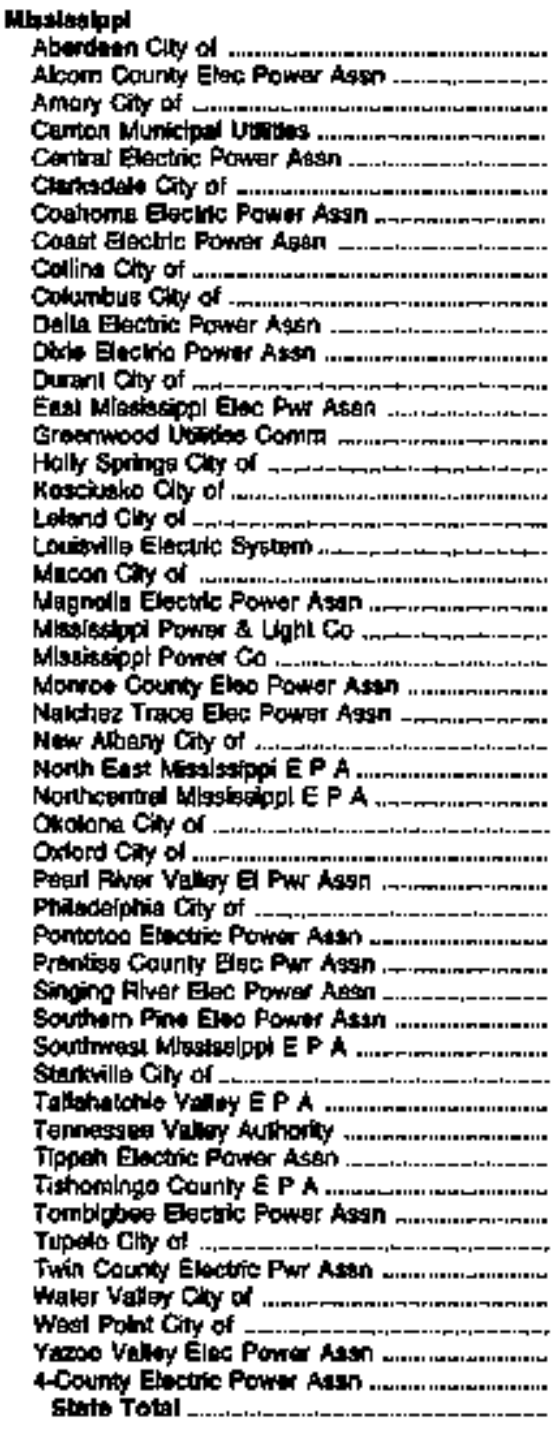 & 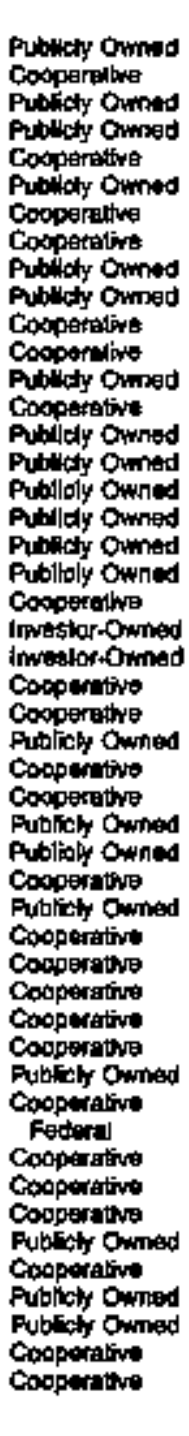 & 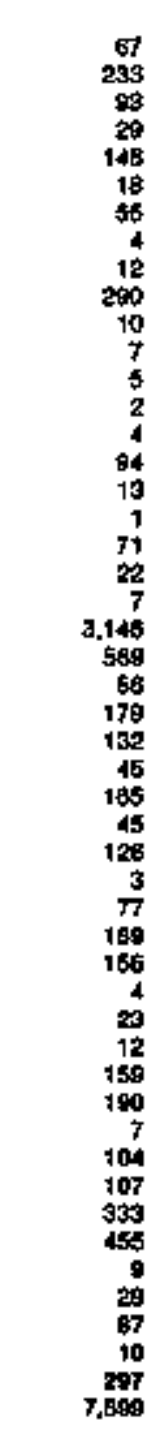 & 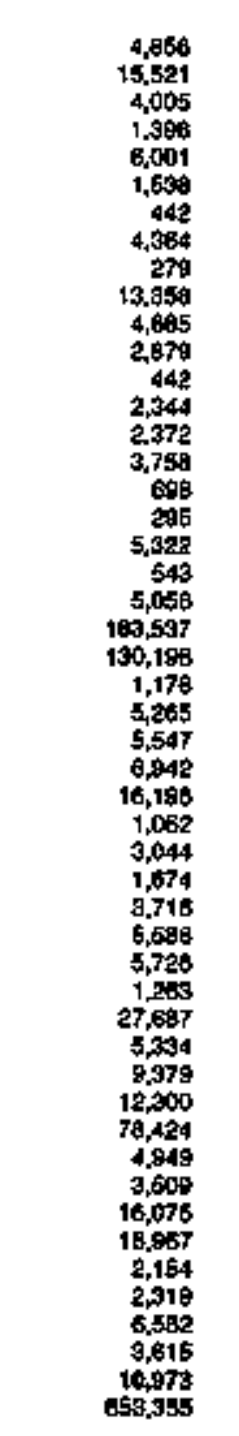 & 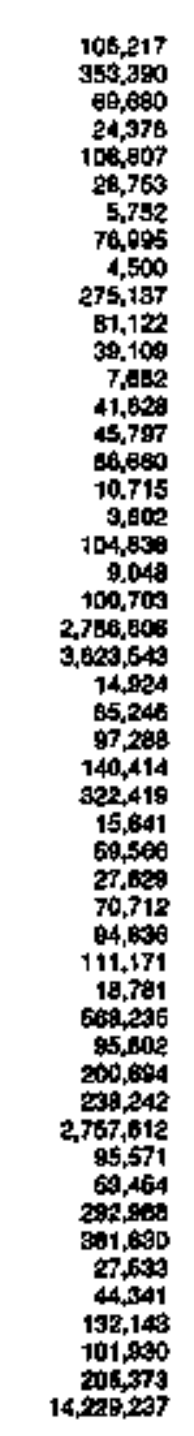 & 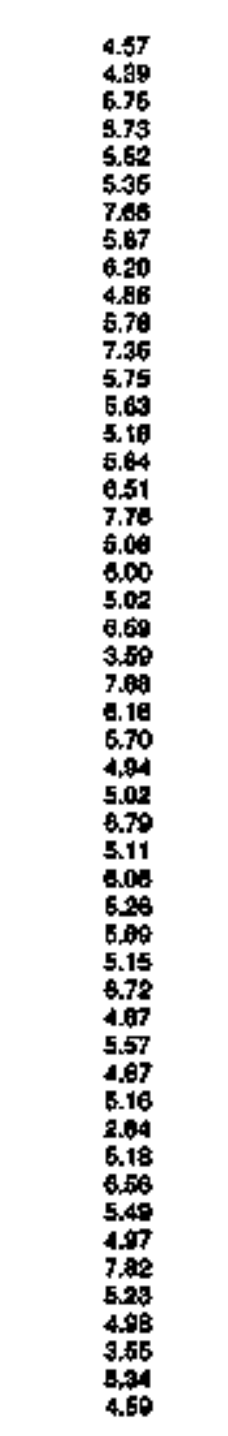 \\
\hline 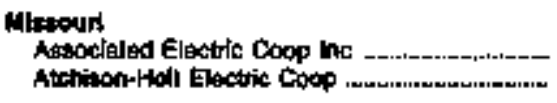 & $\begin{array}{l}\text { Copperalive } \\
\text { Copperative }\end{array}$ & $\begin{array}{l}\mathbf{1} \\
\mathbf{2}\end{array}$ & $\begin{array}{r}42,41 B \\
132\end{array}$ & $\begin{array}{r}1,292,345 \\
2,647\end{array}$ & $\begin{array}{l}3.31 \\
6.18\end{array}$ \\
\hline
\end{tabular}

Ser hootnotes gr and of toble. 
Table 16. Class of Owmership, Number of Uitimate Consumers, Revenue, Sales, and Average Revenue per Kfiowatthour for the industrial Sector by State and Utillty, 1993 (Continued)

\begin{tabular}{|c|c|c|c|c|c|}
\hline sintis & $\begin{array}{c}\text { chases } \\
\text { od } \\
\text { onoratolp }\end{array}$ & $\begin{array}{l}\text { Humbit } \\
\text { of }\end{array}$ & Rithousend diplarsis & 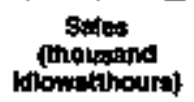 & $\begin{array}{l}\text { Arerape Aowemue } \\
\text { por (dlowatliour" } \\
\text { [camls) }\end{array}$ \\
\hline
\end{tabular}

Whave (Combinad)

Avaciny of

Bun Electric Coop -

Getriny City oid

Bback Fiver Eloctio Coop ...

Boons Elocisk Coop

Colbow chity of

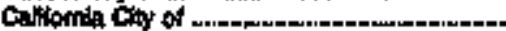

Compition Bourd of Pubtio Whas ..................

Cethugen Ciny of

Cintifethe cty of

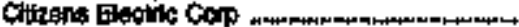

Colvmble Chy of ..

Conooldated Degtrlc Cooph

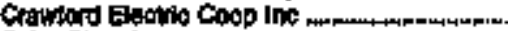

Cubl Chy of

E Dorado spings Chy of .

Emalie oistict Electric co

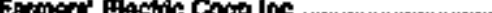

Farminglon Cyy of ....

Frodericklown Cly of ..............................

Pitlon clly of

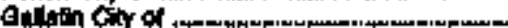

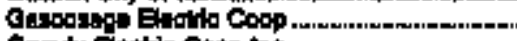

Anndy Elettito coop ho.

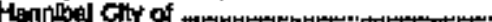

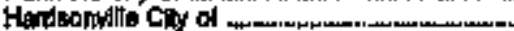

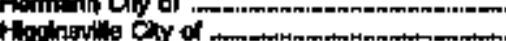

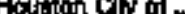

Indopondence $C$

interbotinly Elactic COOp ABgon

Jiakson Ciny of

Kahow Cin of .

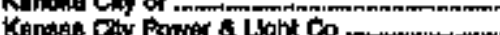

Kannet City of

kalkombod city of

Lebanon Clly of

Macon city of

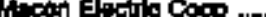

Moldan City of

Marcethe Cly of

Morthell City of

Mren Chy of

Manett City of

Monioe Cly ciy of

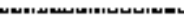

Moumin Vow Cony of .

New Hedid City of

Nixa Cty of .

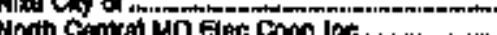

Didota Chy of

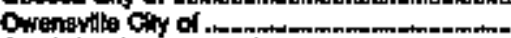

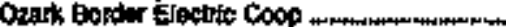

Paimyra Chy of .

Patis Chy of .

Penlocol-Dindin fiec coos inc

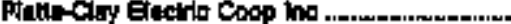

Poplat Bhuti chty of

Rolle Ciny or

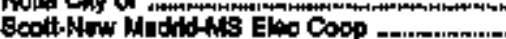

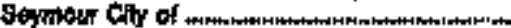

Shetrin City of -

sinerion clity of

tinles oly of

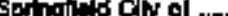

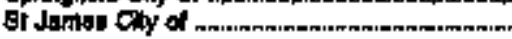

Pubicty Ommed

Cooperatho

Pulbilty Owriod

Cooperarive

Cooperative

Fublikly Owned

Pusticty Owned

fustles Owned

Fublity Owned

Putalest Ounted

Cocopretive

Poblety Owend

Coopperetive

conperative

Putility Owned

Putulely Owned

timestotomes

Cooperstivis

Pubiloty Owned

Putthy Omined

Putlisty Owered

Publisy Owned

Cooperatro

Cooperathe

Pubicty Oumind

Pululy Oined

Pubilety Omned

Pubinty Omined

Pulidy Orined

Pubichy Onmed

Cooperisther

Putiliety Omed

Pubich Oimed

Irretetor-Ouned

Pubicty Ommad

Publicty Onned

Pubichr Otmod

Cooperalwo

Publicty Ommed

Pubistr Otmod

Coopenturs

Pubicty Owned

Pubichy Oimot

Publety Onmed

Fulbicht Owmad

Coopurative

Fublibty Owned

Pulatict Omant

Putbled Omed

Fublety Dwned

Pratich owned

Putricy Owined

Cooperative

Adblety Owined

Putbitily owned

cooperalivo

Pitilicty Owine

Fublicly Owmed

cocperalive

Coopertitu

Publicy Drmed

Putilety O4tied

Cooperatvis

Publich Dwiod

Putillely Owined

putilicy owned

Putplity ounded

Putillely Owind

Publisty Owned

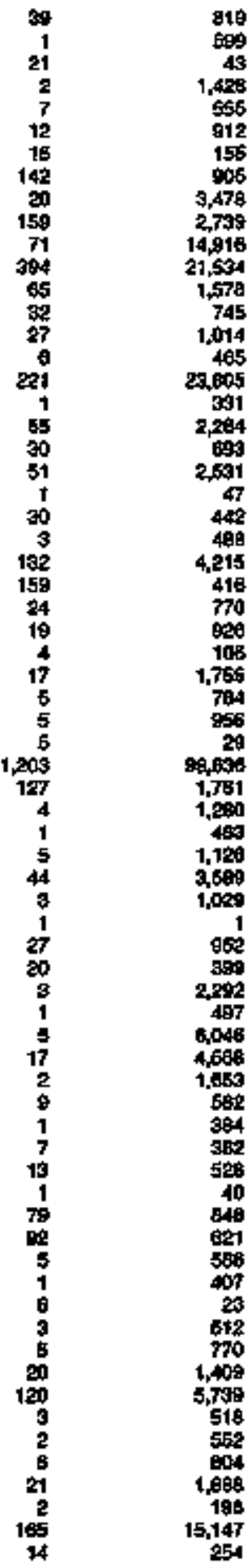

810

43

1,428

912

3,478

14,916

1,534

745

1,014
405

$2 \times, 605$

2,264

60

47

498

4,215

70

Qeed

1,755

794

29

1,781

1,290

1,120

,609

1,009

95

7002

4686

1.053

384

362

648

벅

407

65:

70

1,408

5,795
518

552

1,688

198

254
20053

11,300

1,095

37,724

9,140
15,392

2,010

15,912

97,214
40,596

309,0:5

$3 \mathrm{Bg}, 290$

36,494

8,120

15,141

4.575

$\$ 37,385$

7,849

39,507

10.711

41,168

1,000

8.873

7,692

62,740

5,159

15, 260

73,639

1,207

41,470

14,492

16.949

506

2,100,044

57,500

25,081

$11,5+2$

21,903

67.127

2B, 129

24,028

7,006

41,7

13,880

184,707

84,662

27,052

10,710

7,154

0.50

16,50

534

14,344

9,504

12,67\%

9.5요

$\$ 78$

12,611

$18,31 \%$

40,651

100334

0.002

10398

14,212

42,651

404,472

5,174

4.00

500

3.3

6.07

5.50

5.69

3.90

6.75

4.04

5.85

4.32

6.70

3.75

4.10

5.77

6.47

6.15

(1)

6.35

5.00

a,c6

5.04

0.69

8.57

4.23

6.41

5.84

(1)

4.72

261

5.10

4,20

5. 
Table 16. Class of Ownerahlp, Number of Ultimate Consumers, Revenue, Sales, and Average Aevenue per Klowatthour for the Industrlal Sector by Stale and Utility, 1999 (Continued)

\begin{tabular}{|c|c|c|c|c|c|}
\hline 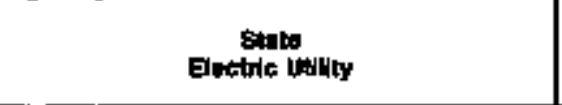 & $\begin{array}{c}\text { Claps: } \\
\text { of } \\
\text { Ohwmernhlp }\end{array}$ & $\begin{array}{c}\text { Nimber } \\
\text { of } \\
\text { Congupters }\end{array}$ & $\begin{array}{c}\text { Reveanut } \\
\text { (thousand dotlary) }\end{array}$ & 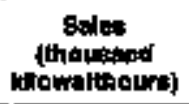 & $\begin{array}{c}\text { Awertipa Revaming } \\
\text { par mllowabibour' } \\
\text { foenke) }\end{array}$ \\
\hline 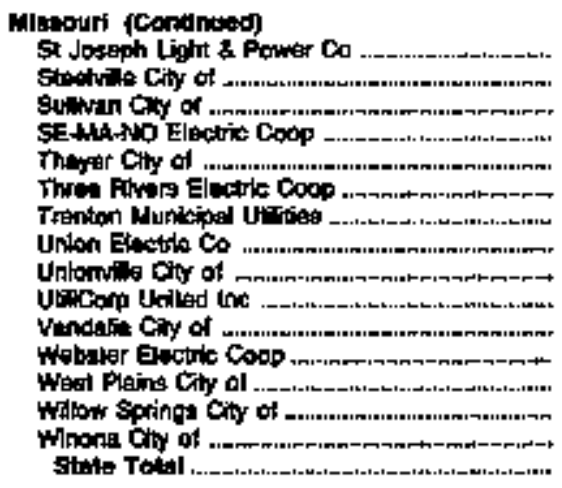 & 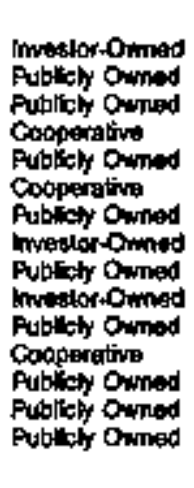 & $\begin{array}{r}104 \\
3 \\
17 \\
1 \\
7 \\
5 \\
1 \\
7,385 \\
9 \\
149 \\
9 \\
2 \\
124 \\
50 \\
10 \\
11,800\end{array}$ & $\begin{array}{r}18,286 \\
353 \\
999 \\
189 \\
200 \\
1,732 \\
738 \\
295,153 \\
97 \\
27,160 \\
160 \\
347 \\
3,961 \\
305 \\
189 \\
641,942\end{array}$ & 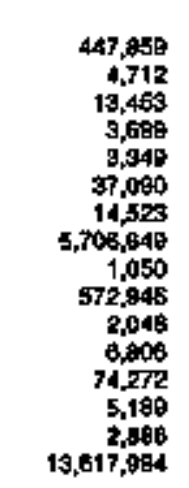 & $\begin{array}{l}4.08 \\
7.49 \\
5.20 \\
5.41 \\
6.67 \\
4.67 \\
5.03 \\
5.47 \\
(1) \\
4.74 \\
7.07 \\
5.01 \\
5.20 \\
5.89 \\
5.62 \\
4.71\end{array}$ \\
\hline 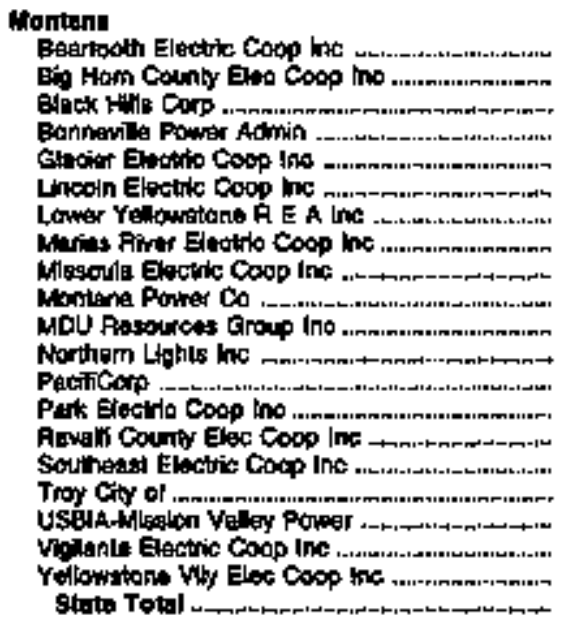 & 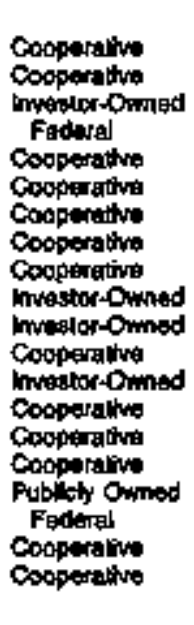 & $\begin{array}{r}50 \\
1 \\
3 \\
1 \\
4 \\
0 \\
260 \\
5 \\
4 \\
3,079 \\
973 \\
1 \\
211 \\
158 \\
3 \\
1 \\
6 \\
1 \\
6 \\
402 \\
4,078\end{array}$ & 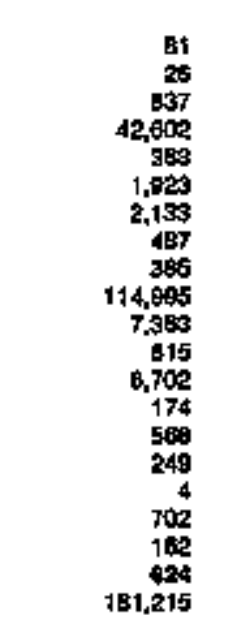 & 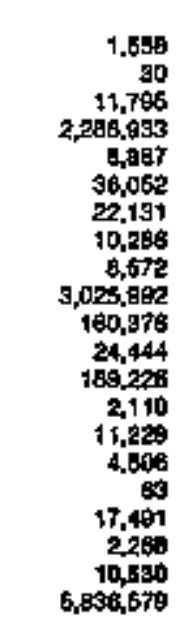 & 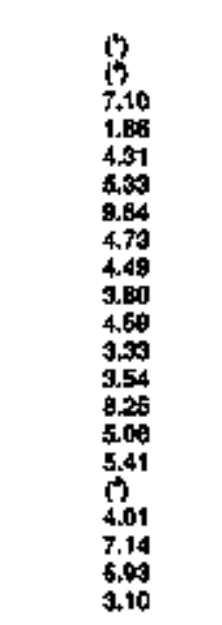 \\
\hline 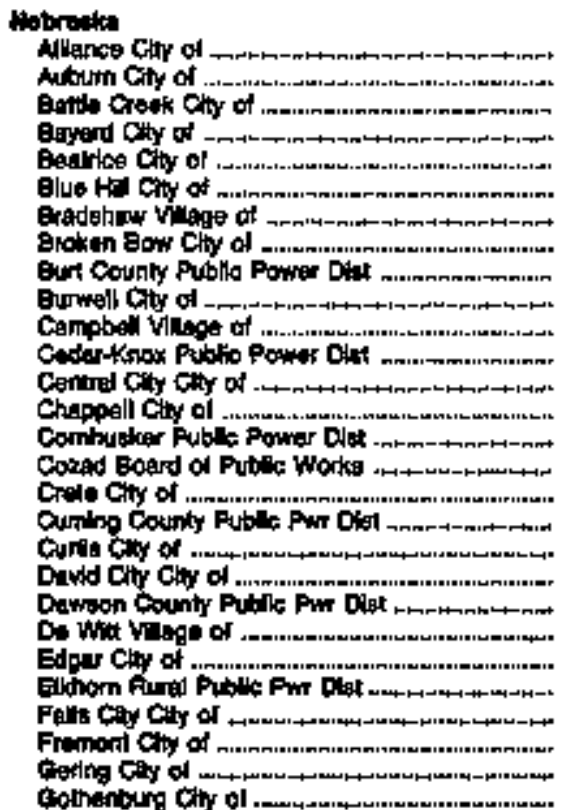 & 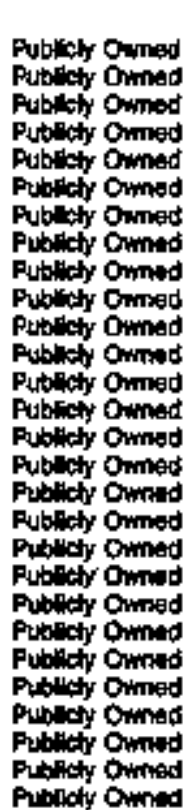 & $\begin{array}{r}2 \\
20 \\
2 \\
5 \\
119 \\
3 \\
9 \\
3 \\
19 \\
1 \\
1 \\
49 \\
7 \\
2 \\
5 \\
7 \\
5 \\
1 \\
8 \\
28 \\
5 \\
1 \\
6 \\
17 \\
29 \\
428 \\
48 \\
11\end{array}$ & 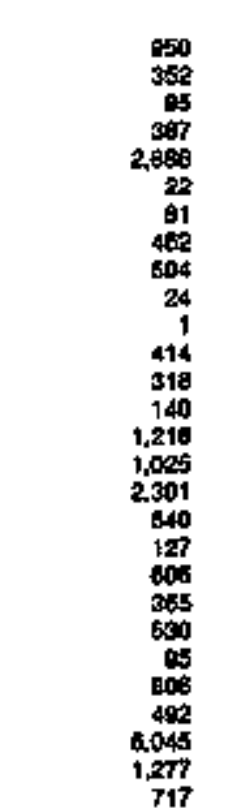 & 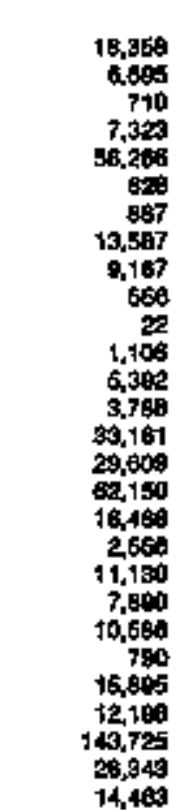 & 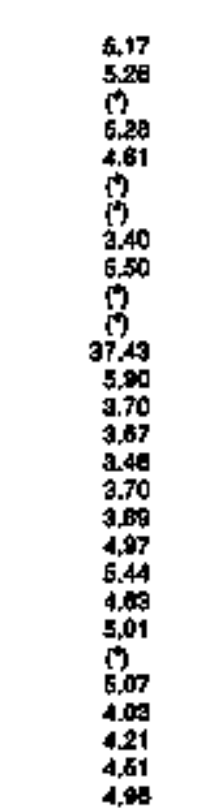 \\
\hline
\end{tabular}

Sea lootnolas al and of bable. 
Table 16. Clase of Ownerahip, Number of Uthinate Contumers, Revenus, Sted, and Average Revenue per Kllowathour for the Indurgtrla Sector by State and Utilty, 1993 (Continued)

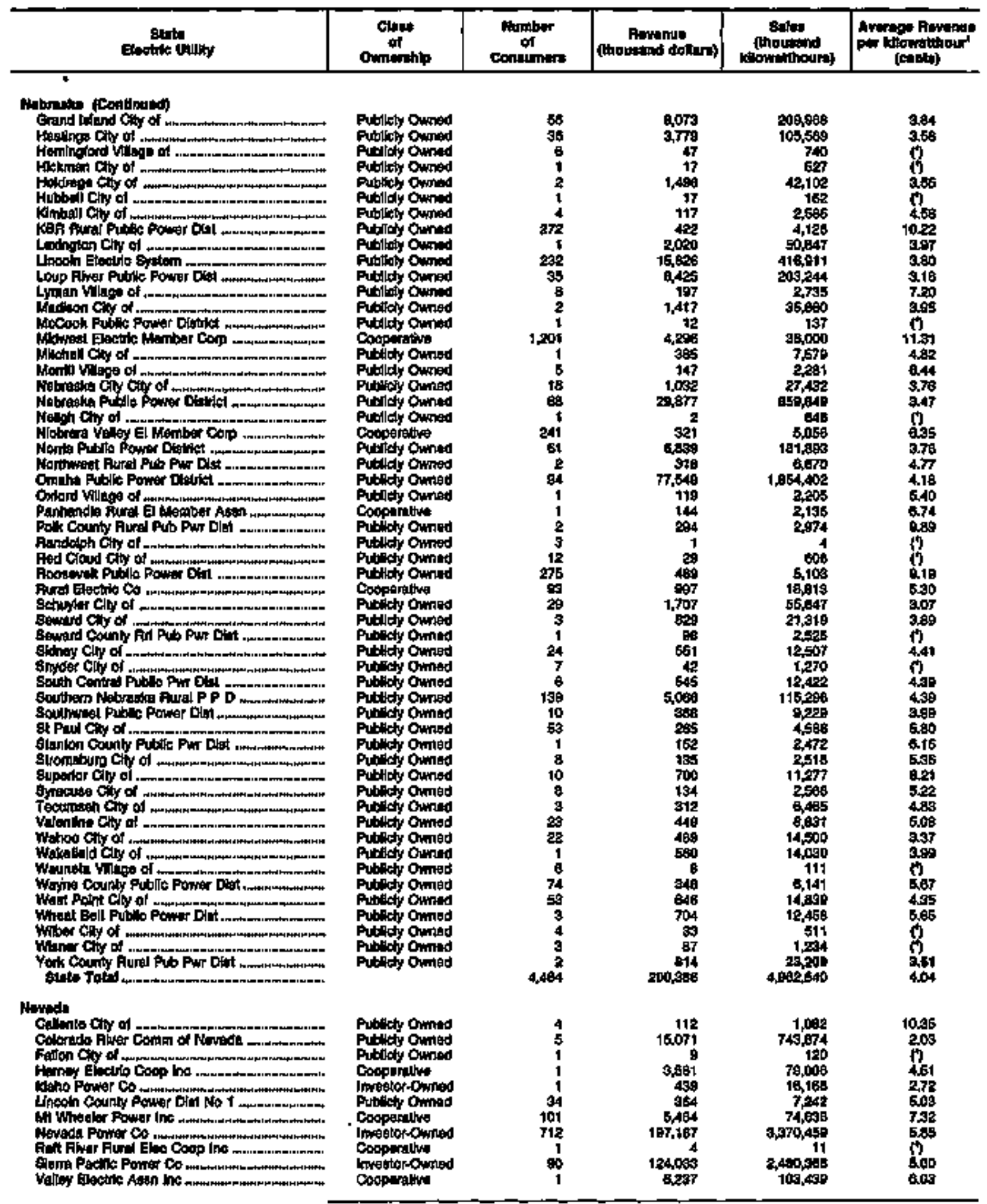

Soo loobroks at and of tabla. 
Table 16. Class of Ownership, Number of Uitimate Consumers, Revenue, Sales, and Average Revenue per Kllowatthour for the Induetrlal Sector by State and Utilty, 1993 (Continued)

\begin{tabular}{|c|c|c|c|c|c|}
\hline $\begin{array}{l}\text { 8tols: } \\
\text { Electrle Uilfty }\end{array}$ & Ofes: & $\begin{array}{l}\text { Mumbor } \\
\text { of } \\
\text { Cansopropra }\end{array}$ & Astremuls & 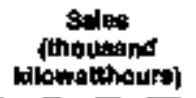 & 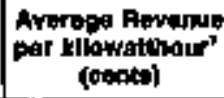 \\
\hline 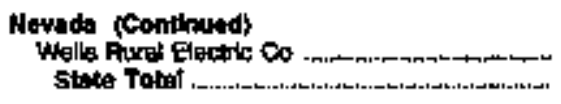 & Cooperothe & st & $\begin{array}{r}9,552 \\
382.0833\end{array}$ & $\begin{array}{r}304,706 \\
7,180,913\end{array}$ & $\begin{array}{l}3,56 \\
5.04\end{array}$ \\
\hline 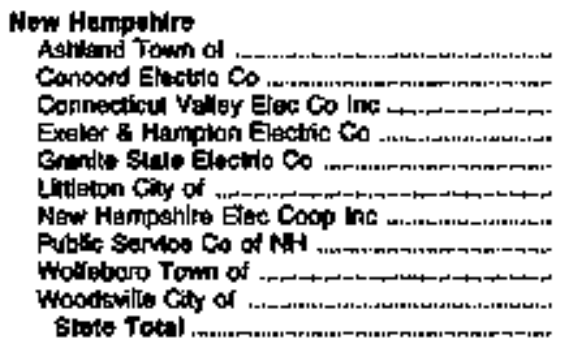 & 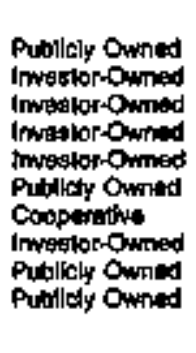 & $\begin{array}{r}3 \\
49 \\
8 \\
40 \\
243 \\
16 \\
9 \\
1,217 \\
17 \\
10 \\
1,642\end{array}$ & $\begin{array}{r}1.249 \\
6,093 \\
3,115 \\
9.169 \\
7,504 \\
1,809 \\
2029 \\
244,159 \\
638 \\
479 \\
200,165\end{array}$ & $\begin{array}{r}15,627 \\
103,990 \\
39,042 \\
111,510 \\
91,140 \\
25,940 \\
\$ 5,707 \\
2,658,192 \\
9,661 \\
9,634 \\
3,090,592\end{array}$ & $\begin{array}{l}7.84 \\
6.80 \\
7.86 \\
8.25 \\
9.23 \\
6.97 \\
0.20 \\
9.10 \\
\text { 9.76 } \\
5.55 \\
9.04\end{array}$ \\
\hline 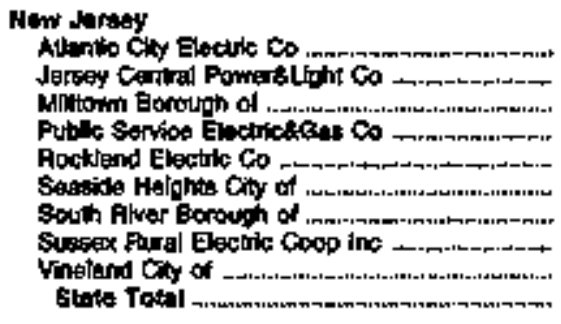 & 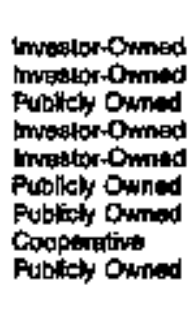 & $\begin{array}{r}971 \\
3,292 \\
12 \\
0,316 \\
200 \\
1 \\
49 \\
3 \\
61 \\
13,868\end{array}$ & $\begin{array}{r}100,748 \\
320,454 \\
100 \\
707,159 \\
39,740 \\
198 \\
498 \\
621 \\
19,850 \\
1,100,451\end{array}$ & $\begin{array}{r}1,258,405 \\
3,689,469 \\
902 \\
9,077,009 \\
355,246 \\
1,009 \\
6,618 \\
5,310 \\
200,845 \\
14,595,879\end{array}$ & $\begin{array}{c}8.01 \\
8.09 \\
19 \\
7.79 \\
8.50 \\
16.94 \\
7.49 \\
11,06 \\
1.40 \\
8.00\end{array}$ \\
\hline 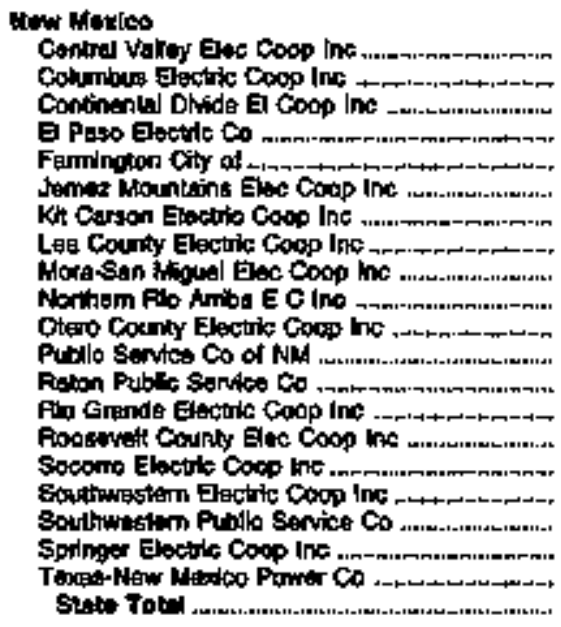 & 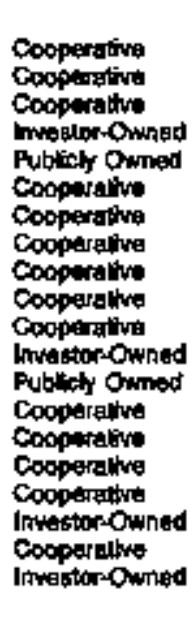 & $\begin{array}{r}419 \\
1 \\
7 \\
4 \\
3 \\
1 \\
9 \\
19 \\
1 \\
50 \\
99 \\
390 \\
35 \\
2 \\
104 \\
6 \\
104 \\
4,251 \\
1 \\
3 \\
5,408\end{array}$ & 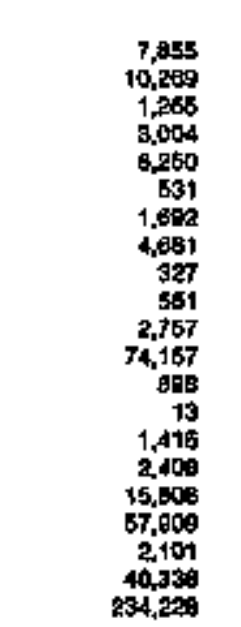 & 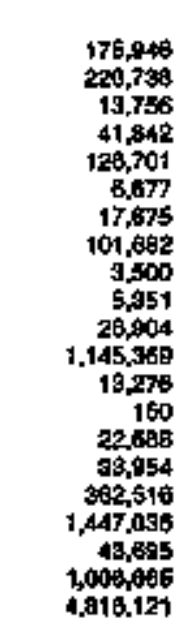 & 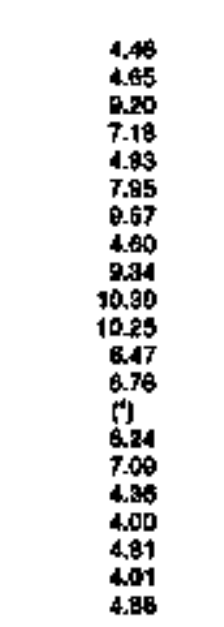 \\
\hline 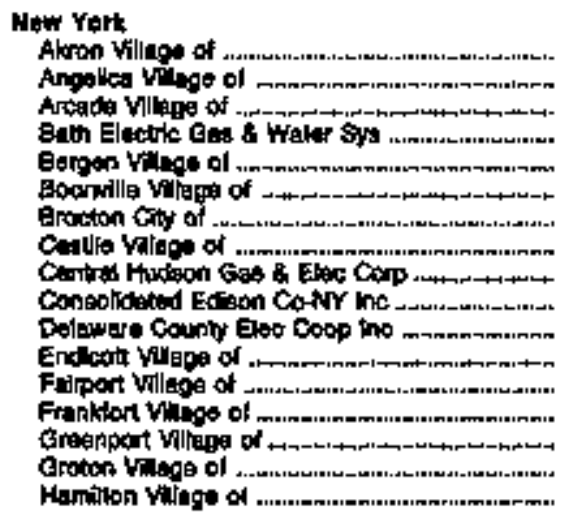 & 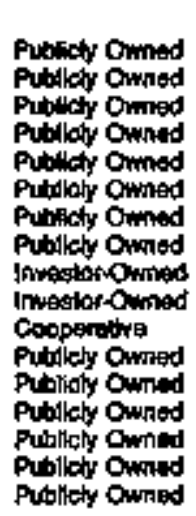 & $\begin{array}{r}40 \\
2 \\
18 \\
24 \\
8 \\
57 \\
10 \\
18 \\
606 \\
632 \\
5 \\
57 \\
37 \\
8 \\
8 \\
6 \\
57\end{array}$ & 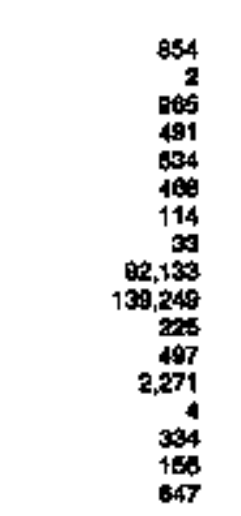 & 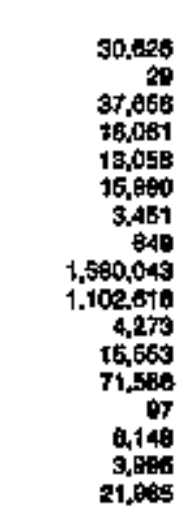 & 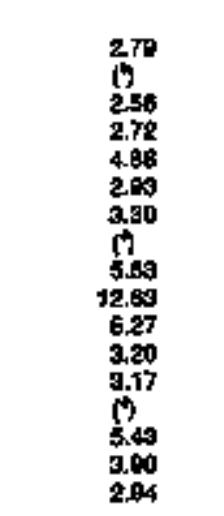 \\
\hline
\end{tabular}

Seg touthoulas at end of telde. 
Table 36. Chase of Ownorahlp, Number of Ultimate Consumers, Revenue, Sales, and Average Aevenue per Kllowathour for the Industria Sector by State and UtIlly, 1993 (Continued)

\begin{tabular}{|c|c|c|c|c|c|}
\hline $\begin{array}{l}\text { State } \\
\text { Elotulc Udity }\end{array}$ & $\begin{array}{l}\text { Chats } \\
\text { of } \\
\text { Ownirahip }\end{array}$ & $\begin{array}{l}\text { Nurnober } \\
\text { of } \\
\text { Connemen }\end{array}$ & 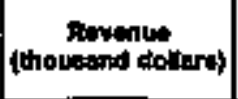 & 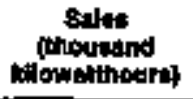 & 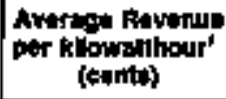 \\
\hline 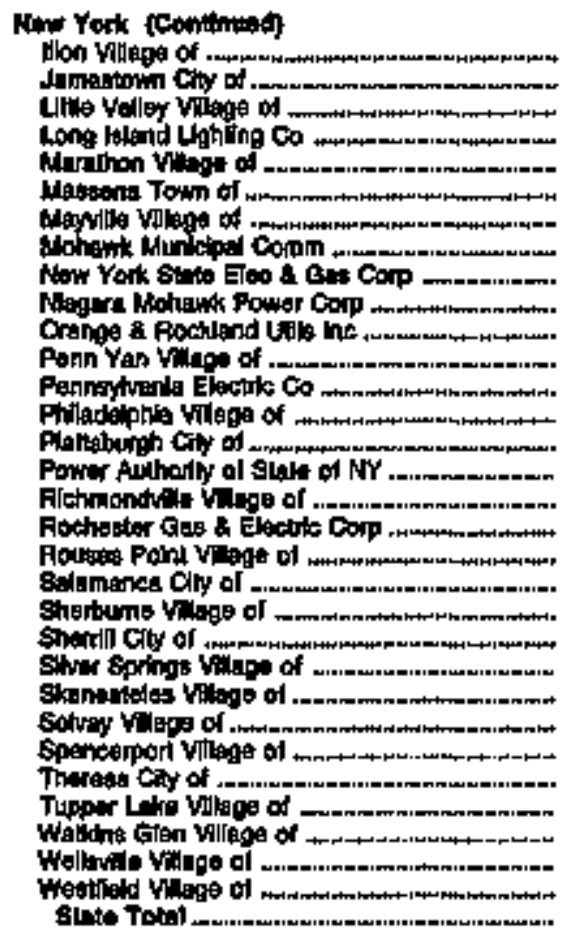 & 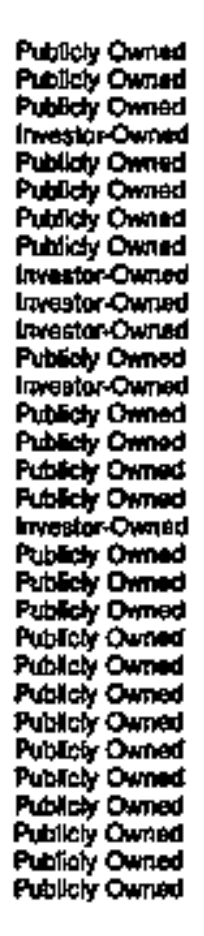 & $\begin{array}{r}114 \\
128 \\
10 \\
3,549 \\
34 \\
160 \\
0 \\
2 \\
1,624 \\
2,194 \\
359 \\
110 \\
12 \\
1 \\
9 \\
19 \\
19 \\
1,360 \\
1 \\
52 \\
1 \\
18 \\
17 \\
19 \\
19 \\
70 \\
6 \\
41 \\
65 \\
61 \\
19 \\
11,991\end{array}$ & 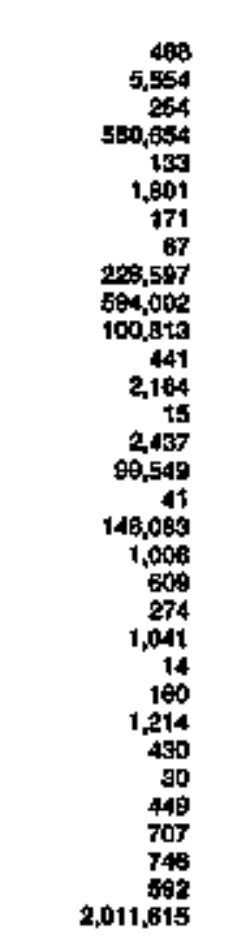 & 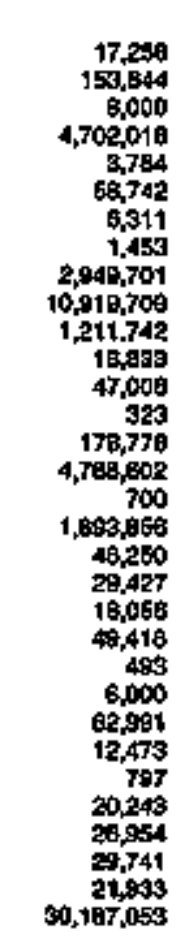 & 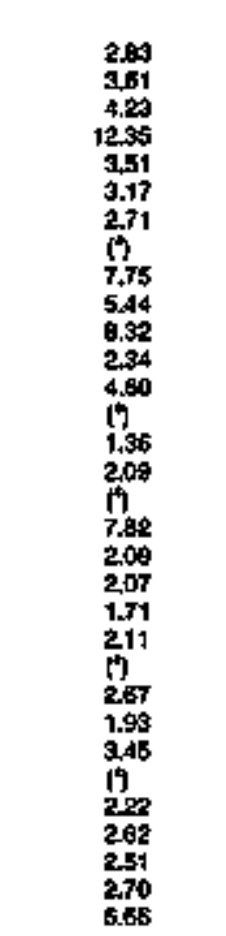 \\
\hline 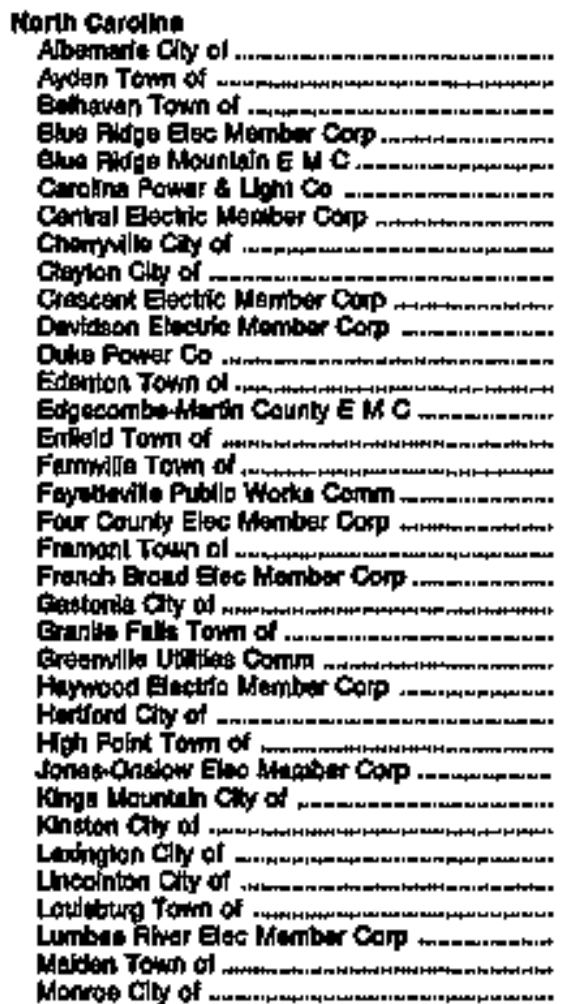 & 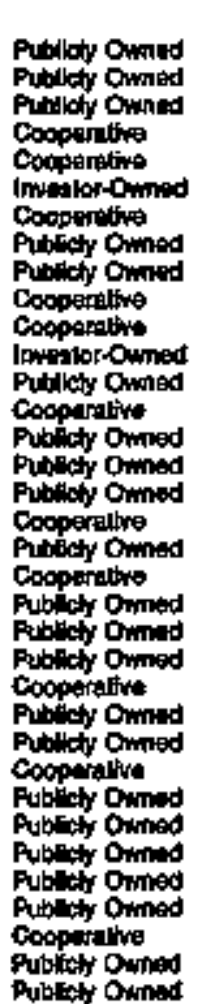 & $\begin{array}{r}24 \\
1 \\
48 \\
155 \\
73 \\
4,002 \\
5 \\
13 \\
19 \\
25 \\
6 \\
6,040 \\
95 \\
4 \\
1 \\
15 \\
10 \\
8 \\
8 \\
80 \\
72 \\
16 \\
10 \\
6 \\
7 \\
325 \\
134 \\
20 \\
8 \\
61 \\
4 \\
5 \\
4 \\
32 \\
460\end{array}$ & 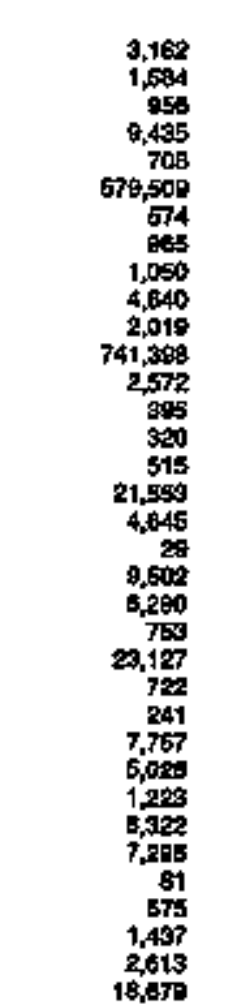 & 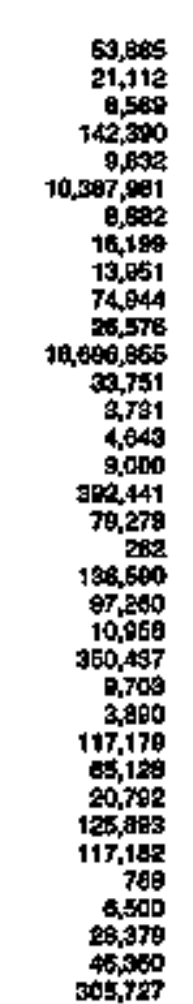 & 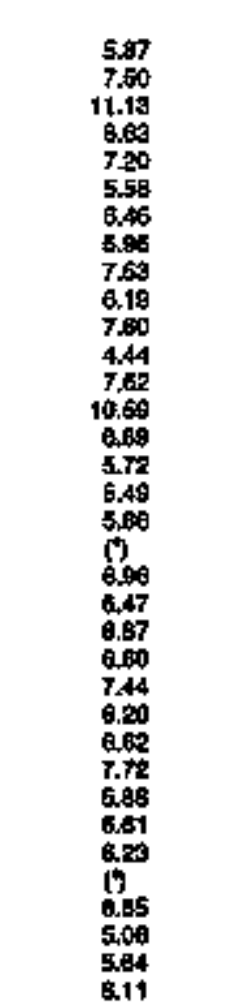 \\
\hline
\end{tabular}

See loolnotes al end of tibte. 
Tabie 16. Class of Ownershlp, Number of Ultimate Consumers, Revenue, Sales, and Average Revenue per Kllowathour for the findustrial Sector by State and Utility, 1993 (Continued)

\begin{tabular}{|c|c|c|c|c|c|}
\hline $\begin{array}{c}\text { Shate } \\
\text { Exetric Undy }\end{array}$ & $\begin{array}{l}\text { Chas: } \\
\text { of } \\
\text { Owiperatop }\end{array}$ & $\begin{array}{l}\text { Momber } \\
\text { of } \\
\text { Copaumerts }\end{array}$ & $\begin{array}{c}\text { firvemule } \\
\text { thounard do|lam) }\end{array}$ & 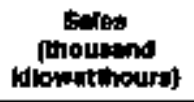 & 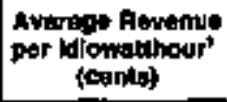 \\
\hline 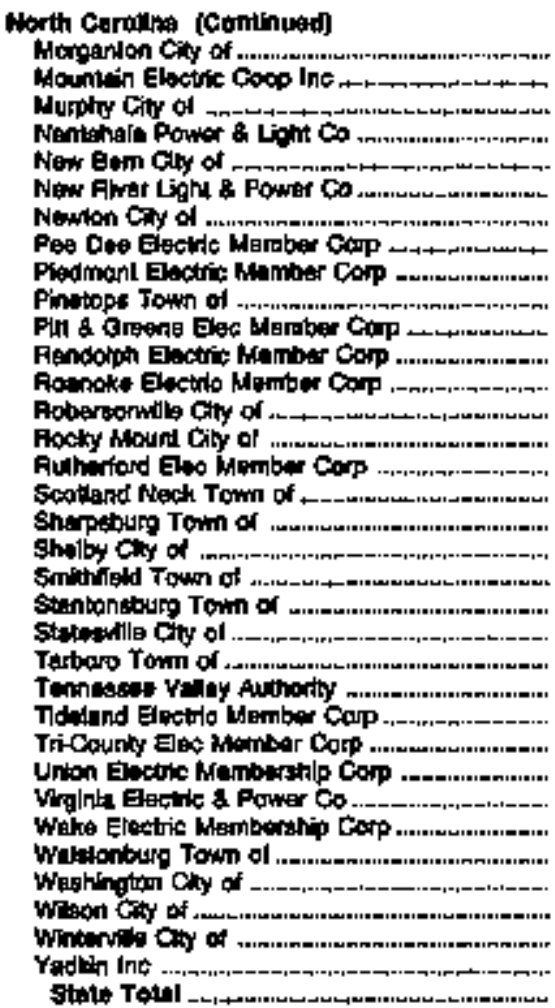 & 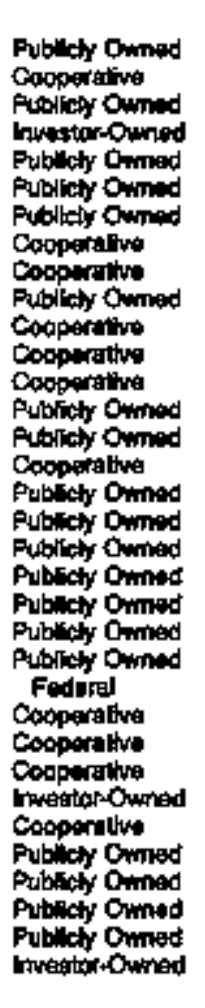 & 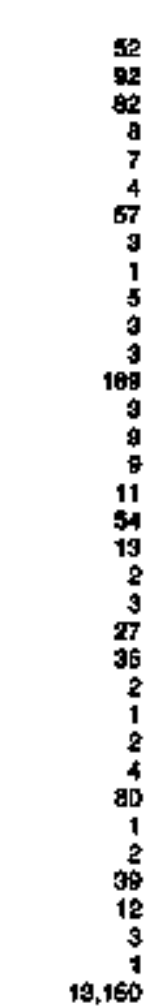 & 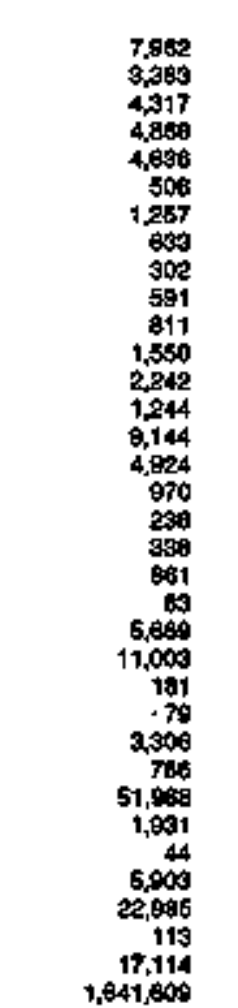 & 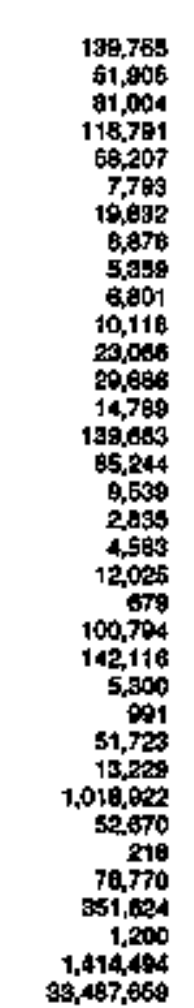 & $\begin{array}{l}6.70 \\
6.48 \\
5.33 \\
4.10 \\
7.98 \\
6.52 \\
6.30 \\
7.13 \\
5.84 \\
6.69 \\
6.02 \\
6.71 \\
7.60 \\
6.41 \\
6.55 \\
5.79 \\
10.17 \\
6.40 \\
7.41 \\
7.16 \\
0 \\
6.62 \\
7.74 \\
3.42 \\
69 \\
6.09 \\
5.04 \\
5.10 \\
3.67 \\
67 \\
7.69 \\
6.54 \\
9.42 \\
1.21 \\
4.90\end{array}$ \\
\hline 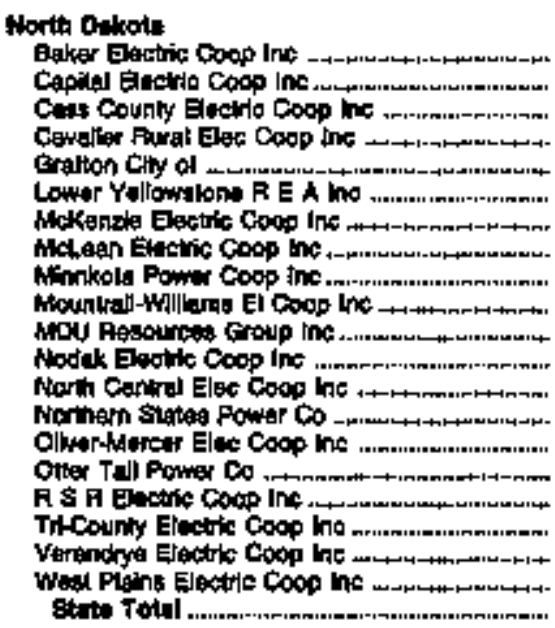 & 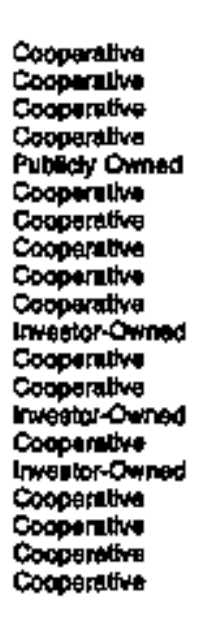 & $\begin{array}{r}3 \\
10 \\
00 \\
42 \\
82 \\
5 \\
1 \\
1 \\
1 \\
197 \\
64 \\
1 \\
580 \\
6 \\
690 \\
30 \\
1 \\
4 \\
707 \\
2,640\end{array}$ & 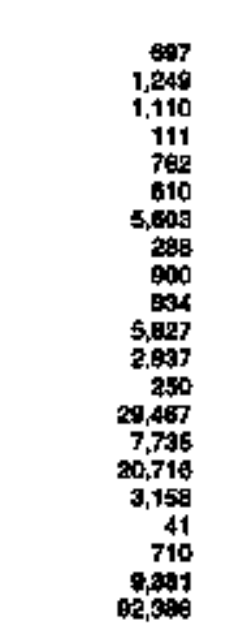 & 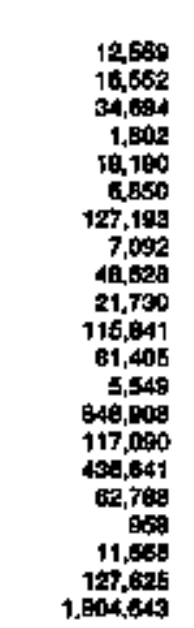 & 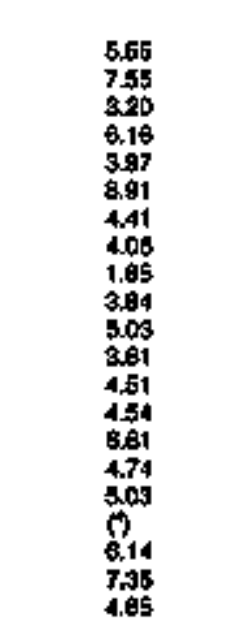 \\
\hline 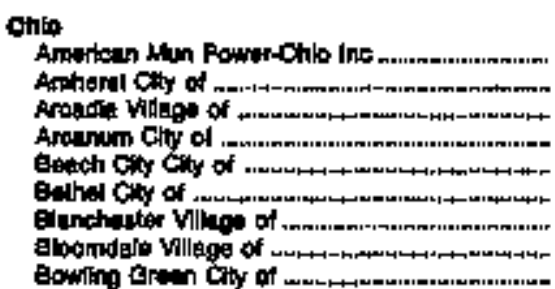 & 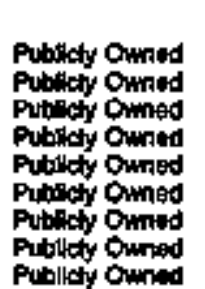 & $\begin{array}{r}1 \\
39 \\
6 \\
13 \\
22 \\
61 \\
42 \\
3 \\
67\end{array}$ & $\begin{array}{r}13,290 \\
1,006 \\
51 \\
25 \\
70 \\
406 \\
404 \\
54 \\
6,601\end{array}$ & 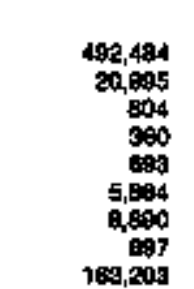 & $\begin{array}{l}270 \\
505 \\
0 \\
60 \\
650 \\
650 \\
450 \\
692\end{array}$ \\
\hline
\end{tabular}

Sine lootnoters at and of tublo. 
Table 16. Clags of Ownership, Number of Ultimate Consumers, Hevenue, Sales, and Average Revenue per Kllowatthour for the industrial Sector by State and UtIIty, 1993 (Continued)

\begin{tabular}{|c|c|c|c|c|c|}
\hline $\begin{array}{c}\text { Suto } \\
\text { Enectile Utility }\end{array}$ & $\begin{array}{l}\text { Cina: } \\
\text { of } \\
\text { Oninering }\end{array}$ & 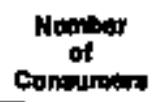 & $\begin{array}{c}\text { Revenuse } \\
\text { (thoritend dollets) }\end{array}$ & $\begin{array}{l}\text { galot: } \\
\text { (theweind } \\
\text { iflowatthound) }\end{array}$ & 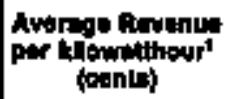 \\
\hline
\end{tabular}

ahlo tConinued

Erediner Ylage of

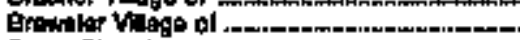

Enan Cly al .

Builes Pural Electic Cocp Inc

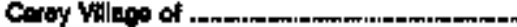

Celling coly of

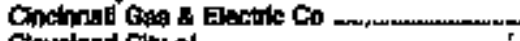

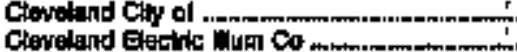

Cleveland Elestits Wuat Co

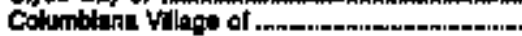

Columbur Ciny of ...-

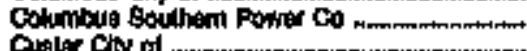

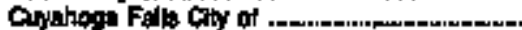

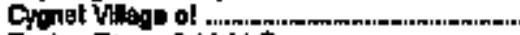

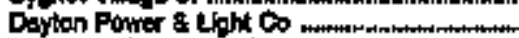

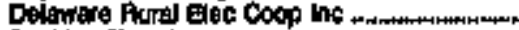

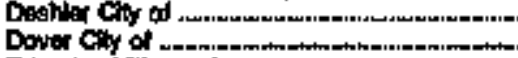

Edperton Vllage of

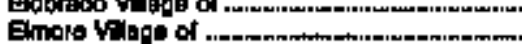

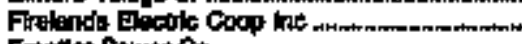

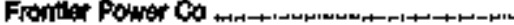

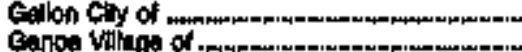

Carpo viluge of

Erenowith Vopos of

Hiandion Cry of - of

Hanpack thood Elactik coap ine . .

piastidns villoos of

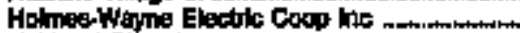

Huthbard Clty of

Jagken Conter villapo of

Jeckson Caly of trisn

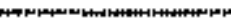

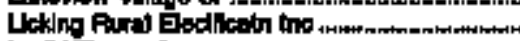

Lod Vllaps of

Logan Gity Coop PBL Asin ho ...............

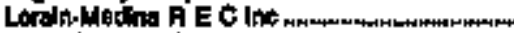

Liles Viage of

Mouhatsile viluge of .

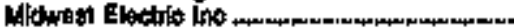

Niliun Villages of .

Winster Vinge of

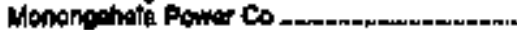

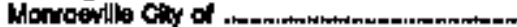

Montpediar viluga of

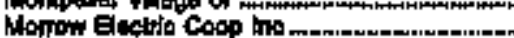

Mapolen Ciny of t.

(1)

New Knowil vilo of

Now coty of

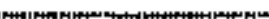

North Westem Eloc Coop Int

Dest Hatbor Villaps of

Onle Chy Vllioge of _._.

Ond Eilison Co

Ollo Power Co

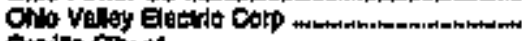

Cinite ciny of

Palnegitle City of -

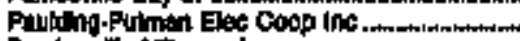

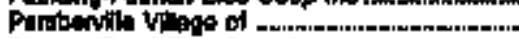

Ploneer Rurd Gis Coop hit

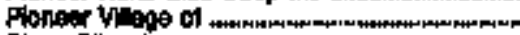

Piatele Oly of -

Pipmoulh village of

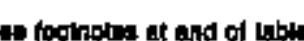

Publicty Omed

Pubicy Omed

Publicty Omined

Cooperaline

Publety Ormed

Putulyty Omined

Imestats Owised

Futrely Ownes

investor-Omered

Publity omed

Pubich Owned

Fublicty Omned

Inveltor-Ondiad

Pulutchy Otrned

Pupley Omat

Fubtey Owned

investor-Omned

Cooperathre

Publint ouned

Fublicty Owned

Publicty Ouned

Pubien Owned

Fubicly Owned

Cooperitiva

Cocpenative

Putritcly Owned

Purttely owned

Publecty owned

Publets Orined

Pubtey Owned

Copoperative

Publtaly Owned

Cooperretsa

Pubilcty Owned

Putilaty Owned

Publich ound

Pubtitsy Owned

cooperethre

Publicy owned

Cocperatus

Cooperatina

Putiloty Owned

Publoby Owned

Coapentitivo

Pictitaly Owned

Publiks Owried

intelor-Omed

Puttley Owned

Puinibly Owned

Cosperative

Pubillely Orried

Pubilcty Owaned

Putliaty owned

Publich Ounted

Caperontive

Cooperathe

Publichy Ononed

Publuty Onted

Itwerlor-Omed

Invetior-owned

Imitor-Owned

Pubilety Owisted

Putillaty Owned

Cooperietiva

Putstely Owned

Cooperentio

Pinildy owned

Publlaly Ourrod

Putalesty Ondited
1 (1)

2730

18
7,854

i

งิ5

2914

130

2,401

2

80

1

30

44

7

3

昰

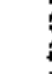

14

13

16

(

3

5

9.0

6

33

5

3
6
6

4

2
2
7

3,305

8,034

15

.

2
8
5
8

5
8
8

2
188

951

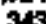

2,207

3,404

2xa,aso

18.549

cototes

4,260

21,044

137,400

45

6,075

205,998

454

658
4,021

$5 \$ 0$

12
318

570

2,409

公

210

200

9,463
4,644

68

625

372

618

105

329

513

ass

tise

19.

610

160

2550

15, 7 tet

1,278

1,5950

1 161

3,609

1,759

172

4,09

3,740

205

204

50

618, 如

222.117

$\mathbf{B} 3$

1,300

463

450

5,023

635

4,474

2400

20,658
142,766

5.111

503

71,200

$5,20 \times 1,59$

ara, 592

7,011, stat

107,704

10,03:9

428,200

2,840,148

BOA

113,259

181

6,094

10,440

100,000

0,706

156

3,809

B,943

15,551

44,224

254

2,084

5.199

150,271

15a,5s

4

16,560

7.900

20

2000

4.967

0,700

20,787

13,281

1.175

t3,160

1,542
13,160

56,950

442. 6 ?

29,000

27,208

2,985

75 , tee

3050

2,047

$77,7 \mathrm{~s}$

126,082

405

4,346

2047 
Table 16. Class of Ownerghlp, Number of Uthithte Consumers, Revenue, Sales, and Average Revenue per Klowatthour for the Industrial Sactor by State and Utility, 1993 (Continued)

\begin{tabular}{|c|c|c|c|c|c|}
\hline ginto & $\begin{array}{l}\text { Chats } \\
\text { of } \\
\text { Omenthlp }\end{array}$ & $\begin{array}{l}\text { Wumber } \\
\text { of } \\
\text { contumert }\end{array}$ & (thousand dollare) & $\begin{array}{l}\text { Oalow } \\
\text { (1houtand } \\
\text { killowathours) }\end{array}$ & 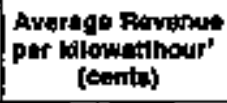 \\
\hline
\end{tabular}

Ohio (Conthowed)

Prospanct Gopp

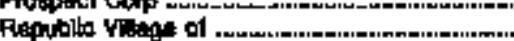

Riploy Ciy of.

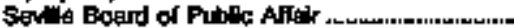

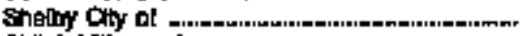

Shitoh vitupe of

Boulh Contrit Fowir Co ............................

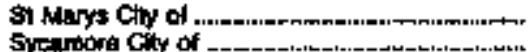

Tip Ciny Cly of

Tdinto Edion $\mathbf{6}$.

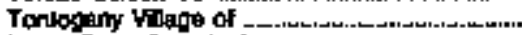

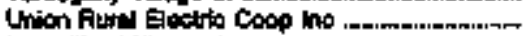

Verullegs Villinges of

Whadgmert $\mathrm{Chy}$ of

Wepakonet $C \mathrm{y}$ of

Wellintor any of

Westentie city of

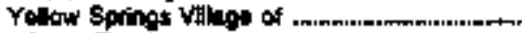

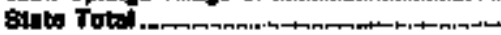

Olonhome

Altus City of

Blaktwal Gity of 1

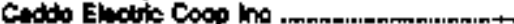

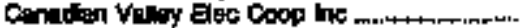

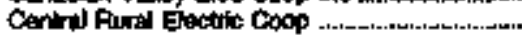

Choolnw Enctrid Coop ino .

Clminion Electide Coop ...

Glaremore Caty of ...............................

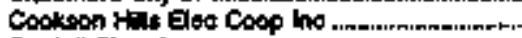

corden Oty of

Collon Elatute Coop tint

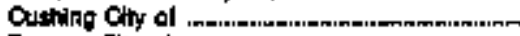

Duncun City of

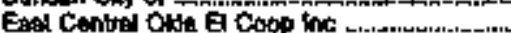

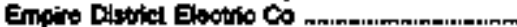

Faindew City of

Finderick Chy of

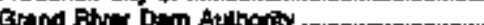

Hermon Electric Asson Inc

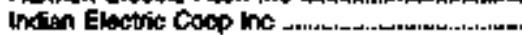

Kur Electio Coop .

Kand Elonty Com los

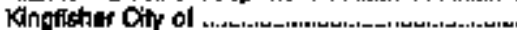

Kintath Electrole Coop mo nrmm.................

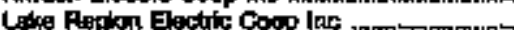

Lindsay Coty of . .

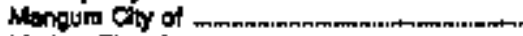

Markow City of .

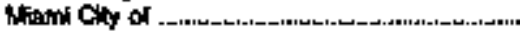

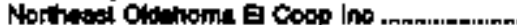

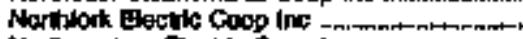

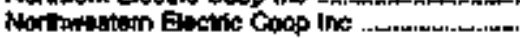

Otroens Publo Wesks Atthorty n...m.n.......m.

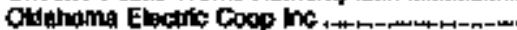

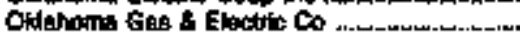

Oratio Electio Coop Con

Poodo's Eloctic Coop

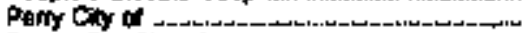

Ponce ciny ofy of

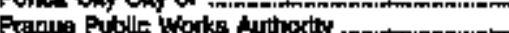

Pulste Barke Co of Oldahoma .....................

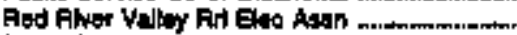

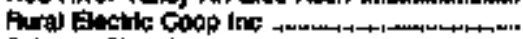

Salisew City of

Stolook Tom of

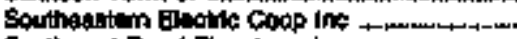

Southwest Rural Elec Asen inc.
Publecty Ormed

Publich Owmad

Pubicty Owmed

Pulbichly Omed

Publicty Demed

Puthictr Ómined

Coopurative

Publictr Omed

Pubsicty Owned

Publicty Omined

Livestror Onited

Pubicty Omined

Cooperaltua

Publicty Omerd

Publucty Omred

Publlety Ommed

Putincty Onmed

Publledy Ouned

Putithy Ormed

Put|cty Owine:

Putllety Ouned

Cooperethe

Comperative

Cooperituve

Cooperethe

Comperentives:

pitilety Owined

Cooperetiva

Putslithy Owined

Cooperasu

P(tolity Owned

Publitit Owined

Cooperative

imvestor-Oined

Puljistr Owinad

Publity Owned

Puthers Onnod

Copparatum

Cooperalve

cooperatse

Cooperature

Puticty Omont

Cooperature

Conperatre

Publicty Orired

Publety Owned

Putility Owned

Anticty Ouned

Cocperature

cosperathe

Cocparative

Publihy Omos

Coxperstra

Intettorowned

Cooperatve

Coopertitive

Pirilichy Owined

Publlaty onned

Publikty Ouned

timalor Omed

Cooperntive

Cosperation

Outtley Owined

Publifiy owned

Copperative

Cooperative

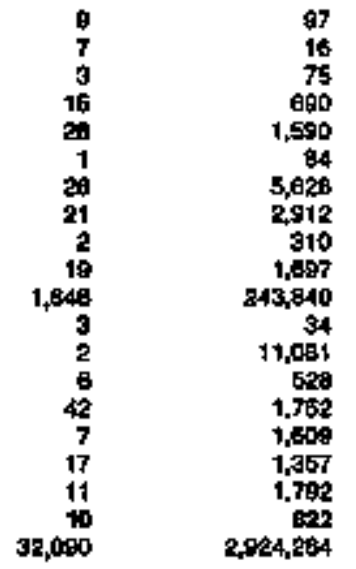

1.169

1,152

10,590

30,206

1,474

107,677

73,918

6,000

41,202

$3,76,978$

454
2760

12,276

61,422

42,801

10,357

41,000

16,037

68, 91,023

(1)

i)

85.

4,78

i)

523

3.94

S.17

4.05

B.48

1)

4.01

4.90

287

3.73

7.04

4.37

4.34

4.25

104

2.677

9,803

64

477

1,500

728

5098

eaz

52

1,328

1,447

1.160

1.455

111

1,260

18.323

1,425

1,105

202

707

115

526

1,012

285

241

145

3,124

100

304

2,120

20

1,473

247,757

1,589

574

1,040

2064

2004
49
402

149,762

1,000

4.171

741

121

290
4,637

46,486

14.760

9.494

37,047

17,478

109,794

18,464

64

107,005

$18,6 \mathrm{az}$

29,465

21,788

36,158

1,600

24,613

BA5:50

26,032

20,997

4,5

13,849

2,074

5,051

14,055

3.760

5,344

2,439

65.707

7.45

7,888

43,487

4,016

33,385

6. 157.184

27,022

11.300

16.363

36,3:

1,300

4,44,7a4

41, as9

70,316

18000

6,7

2439

$3.6 \pi$
397

5.50

6.

4 故

4.93

4,16

4,17

400

6.30

(1)

7.57

$4, \$ 1$

5.94

3.07

4.17

5.13

200

5.69

5.5B

6.64

5.76

5.54

4.77

7.18 
Table 16. Class of Ownershlp, Number of Utimate Consumers, Revenue, Sales, and Average Revenus por Klowatthour for the Induetrial Sector by State and Utilly, 1993 (Continued)

\begin{tabular}{|c|c|c|c|c|c|}
\hline Eectist toulty & ofidip & $\begin{array}{l}\text { muber } \\
\text { of } \\
\text { conoumars }\end{array}$ & (thousand dohers) & (thousind & 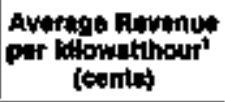 \\
\hline 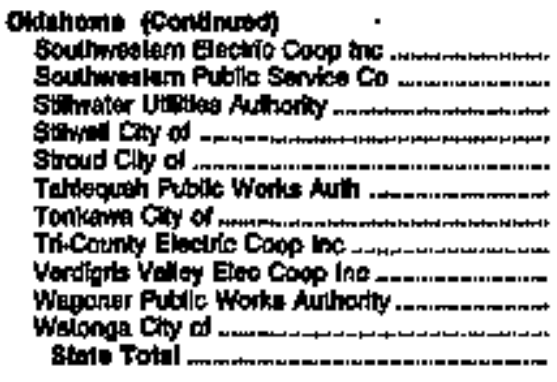 & 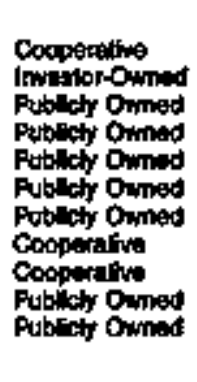 & $\begin{array}{r}138 \\
5 \\
3 \\
6 \\
149 \\
14 \\
5 \\
300 \\
3 \\
10 \\
10.74\end{array}$ & 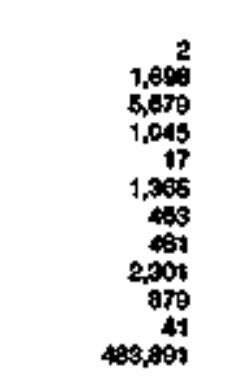 & 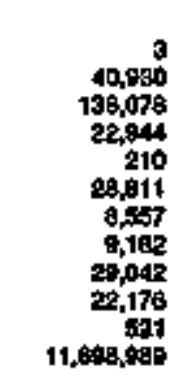 & $\begin{array}{l}17 \\
4.15 \\
4.17 \\
4.55 \\
10 \\
4.74 \\
5.41 \\
5.02 \\
7.02 \\
3.08 \\
40 \\
4.14\end{array}$ \\
\hline 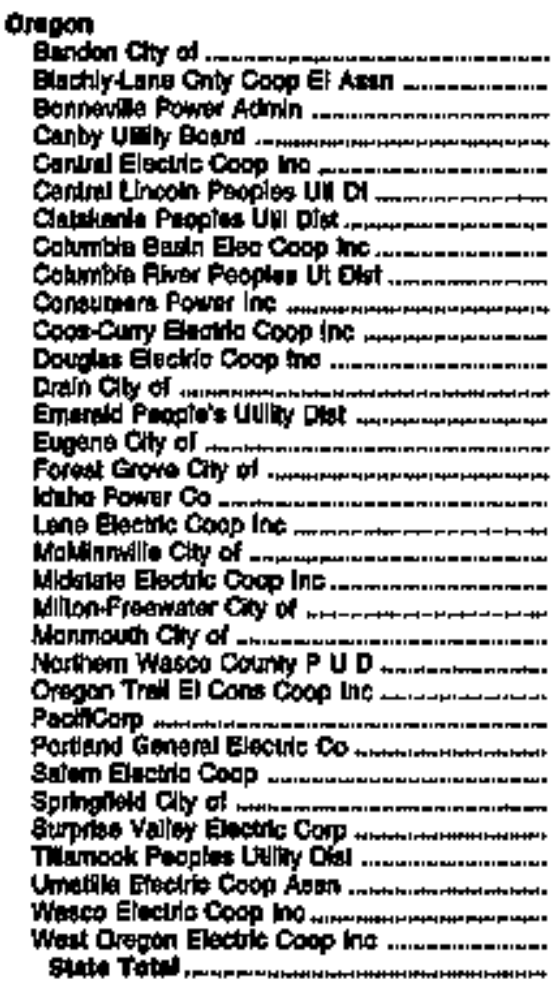 & 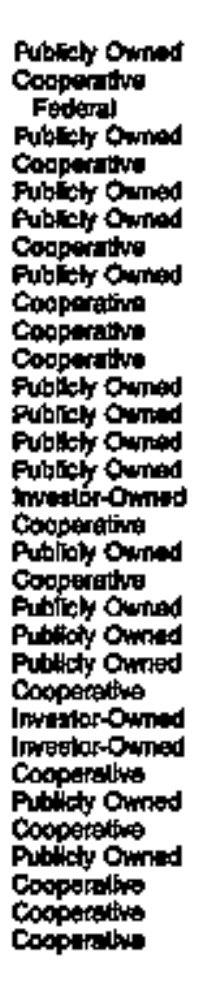 & 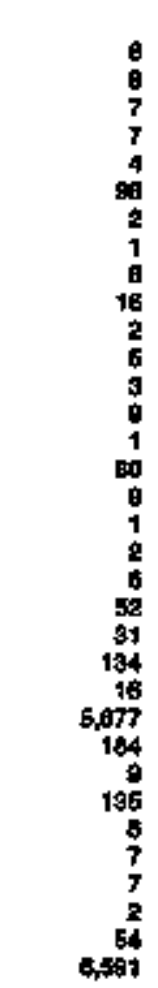 & 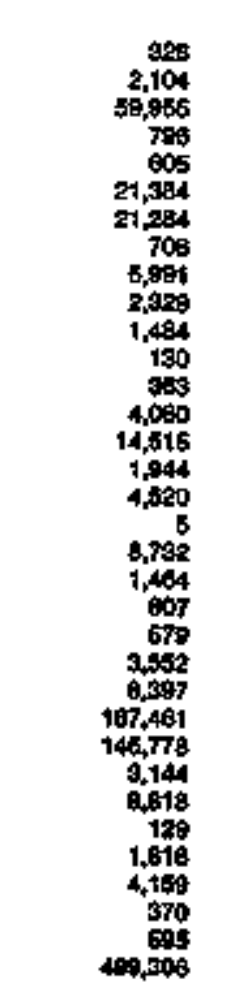 & 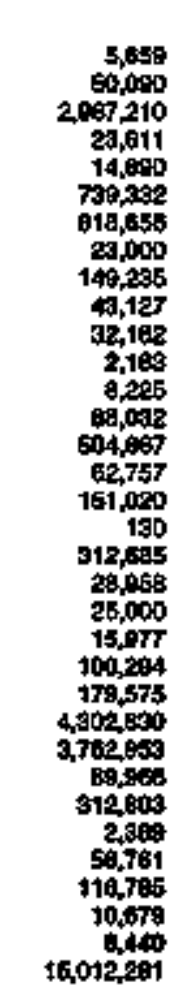 & 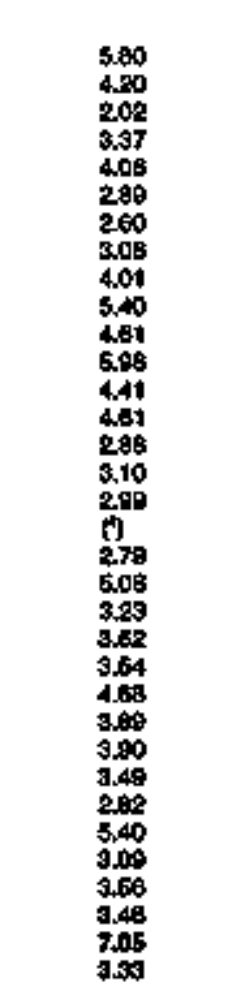 \\
\hline 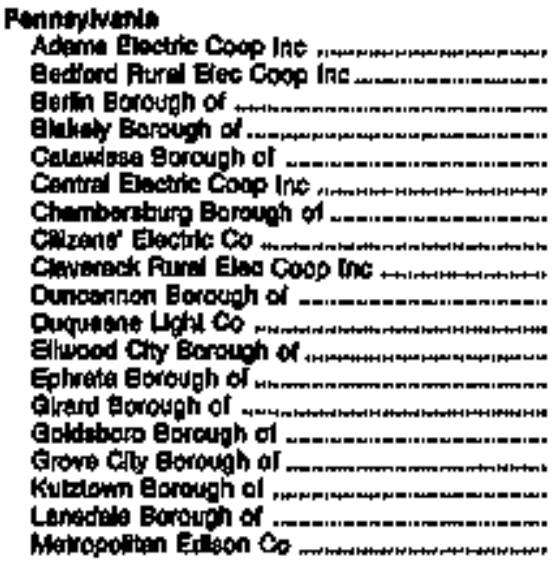 & 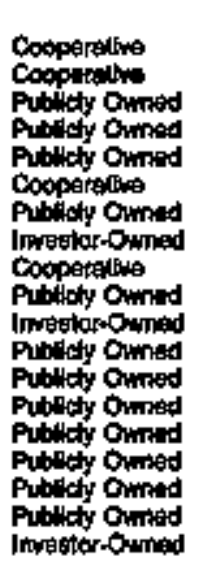 & $\begin{array}{r}2 \\
1 \\
10 \\
7 \\
1 \\
20 \\
34 \\
19 \\
3 \\
1,903 \\
9 \\
23 \\
72 \\
1 \\
7 \\
9 \\
30 \\
2,198\end{array}$ & 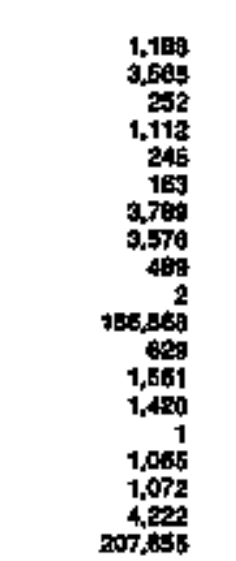 & 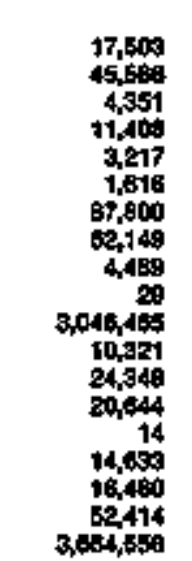 & 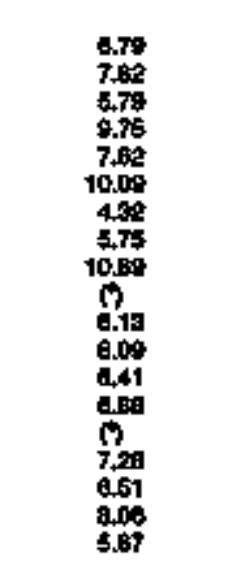 \\
\hline
\end{tabular}

Ses loolnotes at end of tabta. 
Table 16. Class of Ownership, Number of Ultimate Consumers, Revenue, Sales, and Average Rovenue per Kilowatthour for the Industrial Sector by State and Utility, 1993 (Continued)

\begin{tabular}{|c|c|c|c|c|c|}
\hline State & $\begin{array}{l}\text { Clanes } \\
\text { of } \\
\text { Onimerathp }\end{array}$ & $\begin{array}{l}\text { Numbor } \\
\text { of } \\
\text { Conorimers }\end{array}$ & Revontus & 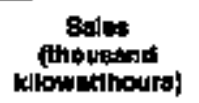 & 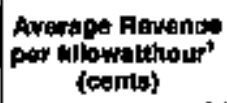 \\
\hline
\end{tabular}

Pansoplwanla contruics

utdoliown Borough of

inilnoung Barough at

Manil Altip borough of

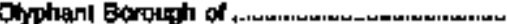

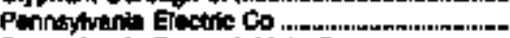

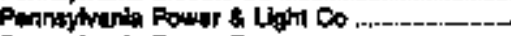

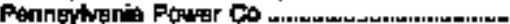

Pothadis Borough of

Phlodiphle Eloctulc Co

Pow County Lighd \& Folwor Co

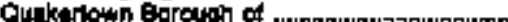

Schuryllal Havan Borouth of

Simethport Bortaugh of .

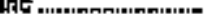

sil Cl: Barouph of .

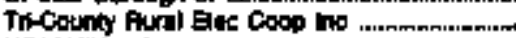

Led Jititles he

Valloy Furd Ebctric Coop ino ........................

Wationterm Barough of

Wegtiderty Bcoungh of

Wedlotoriouh Eloctric Co .............................

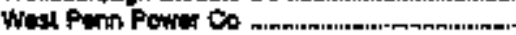

Stubla Tot:

Puorio Rico

Puark Flos Electide Fur Auth

state Tobl.

Panode totind

Blackstone Valoy Electrit $C_{0}$

Naragunate Evotido Co

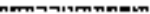

Nemport Electic Carp

Pascoag Fre bialriet w...................................

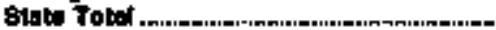

Eeuth tirents

Almen Electde Coop ins .................................

Bamberg City of

Gotkelny 텅trio Codo ho

Elock Rivor Eectic coop ins.......

Bue Ridgr Elextruc Coop lic

Brond Alwar Eleotrito coop Int

Camdon Chry of

Ceralinta Power 8 Light Co

Cinton Combinad uitity syt

Dus wasl City of

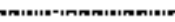

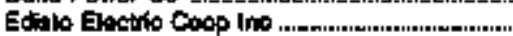

Fairlend Electite coop inc

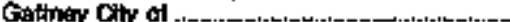

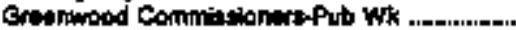

Criger Conan of Putlo Works .

Hotis Eledtric Coxp Ine

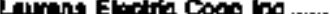

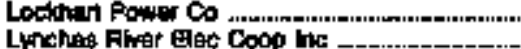

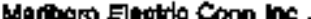

Mromick Toun ol

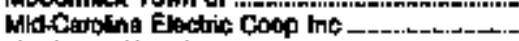

Numberiy Cly of .

Nowberry Eloctile coop Inc n.m.....

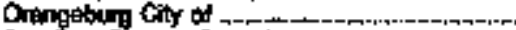

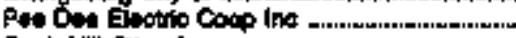

Poek Hill City of .

Santes fivectio Comp inc

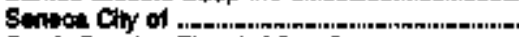

South Gering Electibstes: Co
Pulaticty Oritiad

Putrich Onmed

Puburs Ommod

Cooperative

Publaty Owned

Inrostorowiled

Inwelitar Owned

investor-Owhed

Publictr Owmed

inverter-Ouwed

Inwostor-Dined

Publly Ormad

Putricty Oinned

Pubredy Onmod

Cosparathe

Coxparatute

Pubchly Oined

Cooperalture

Imvestor-Owned

Coeperetho:

Publuty Orned

Putecty Ouned

Investor-owned

howsotorowned

Puticty Ormed

Inwontor-Owned

Itrestion-Ownad

Inveskromed

Publldy Owned

Cooperative

Putelcty Owred

Cospermity

Cooporathe

Comperativis

Cocperative

Putilley Owastad

Invediar-Owned

Putsicly Owned

Publits Owmed

Imvestor.Oined

Cooperative

Cooperative

Pubich Ounted

Publey owned

Putolist Durned

Copperative

Cooperstive

imvestor-Onmod

Copoptialing

Conptotive

Publebs Owned

Cooperativa

Aubliky Owhed

Coopurathe

Fubietr Oumad

Cooperative

Rubich Owmed

Cooperaliva

Pubioly Owned

investor Owited

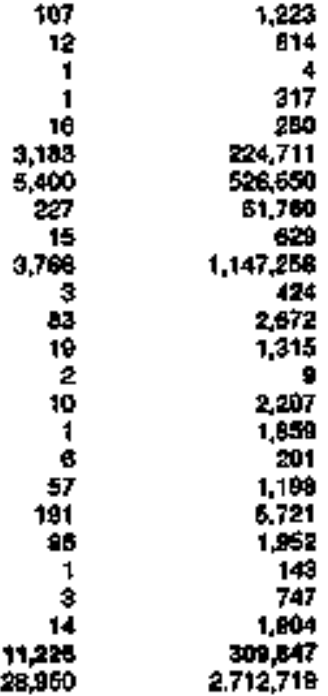

2,3948

2,356

297,496

$297,40=$

561

26

78,08

3,126

0,001

$2,4=13$

1.439

528

3,60

2,689

1,330

750

341

164,507

1,952

47871

698

7,495

050

6, 678

2400

$5,8 \pi 7$

580

11,016

897

870

1,557

14.071

8,933

1,600,

4.700

1,735

191,349

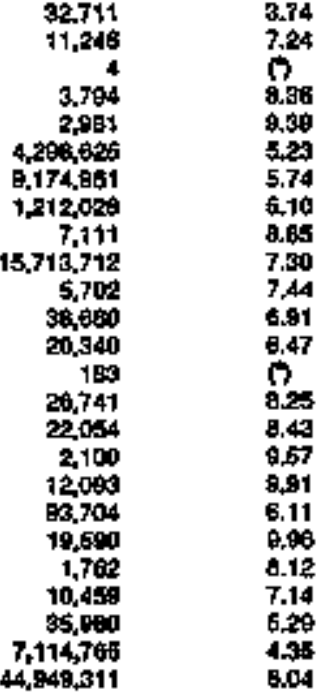

Exic

B.80

9.98

0.51

10.70

12.16

$\begin{array}{rr}7,680 & 12018 \\ 418,673 & 8.05\end{array}$

28,127

6,972 5.55

S.to

$21.361 \quad 6.28$

75157.80

3,760

3169,757

35,75

$11,570.957$

14,3Ea $\quad 4.87$

$198,318 \quad 3.75$

38.228

$10 \mathrm{~d}, 324$

$50.129 \quad 4.97$

$8,203 \quad 6.17$

$47.493 \quad 7.68$

$137,4=0$

13.3475 .00

3as,ass 2.8.

454

15.648

$42,970 \quad 5.65$

30,328 5

308.524

220,a20

22,064

128.517 a.6:

sd.456 5.04

$4,607,290$

Sed loobtotes al end of table. 
Table 16. Class of Ownership, Number of Utimate Consumors, Revenute, 8ales, and Average Revenue per Kllowatthour for the Industrial sector by State and Uthlty, 1993 (Continued)

\begin{tabular}{|c|c|c|c|c|c|}
\hline Etation Utinty & of & $\begin{array}{c}\text { Humbor } \\
\text { ef } \\
\text { eonismere }\end{array}$ & Roverut & 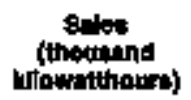 & 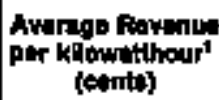 \\
\hline 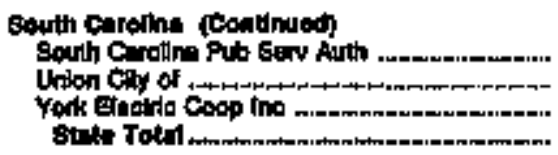 & 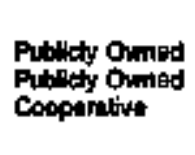 & $\begin{array}{r}32 \\
8 \\
6 \\
4,370\end{array}$ & $\begin{array}{r}168,930 \\
428 \\
1,148 \\
1,090,797\end{array}$ & 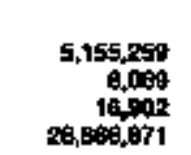 & $\begin{array}{l}5.27 \\
5.29 \\
6.50 \\
4.08\end{array}$ \\
\hline 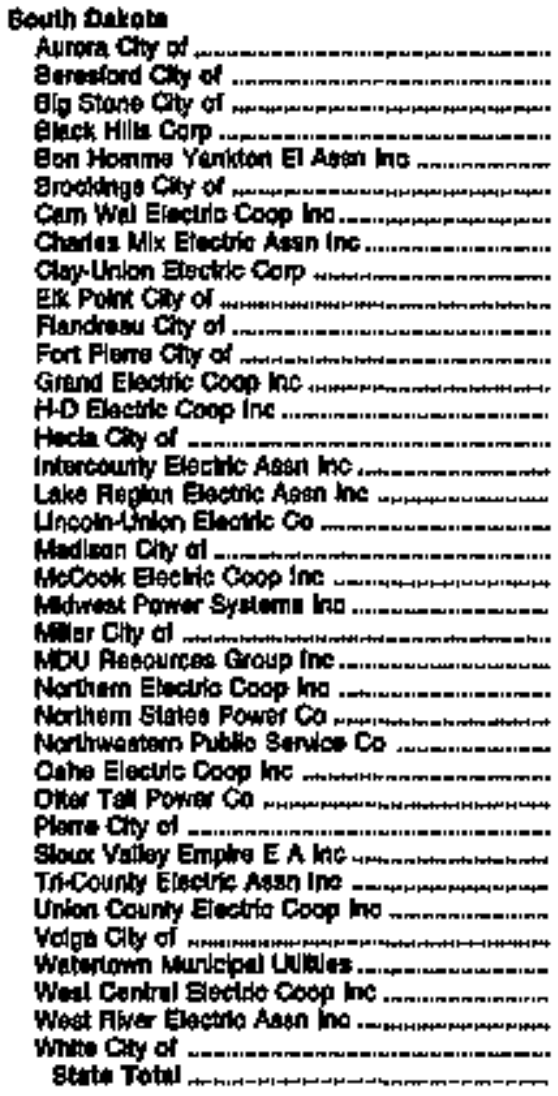 & 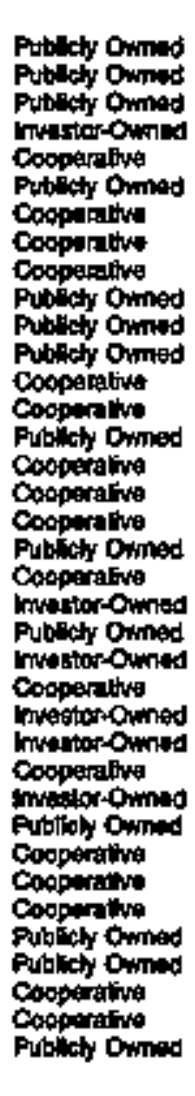 & 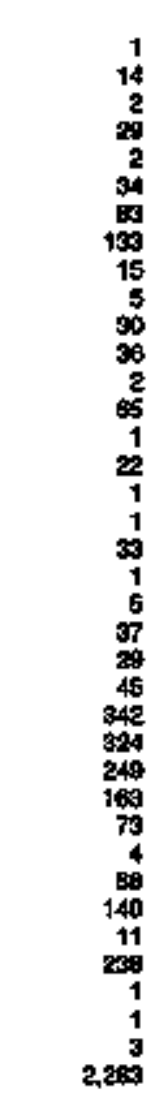 & 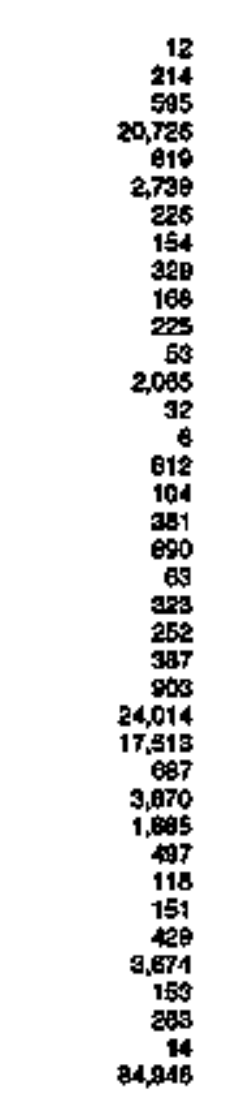 & 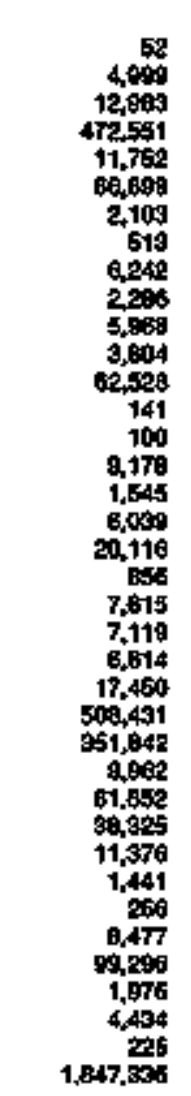 & 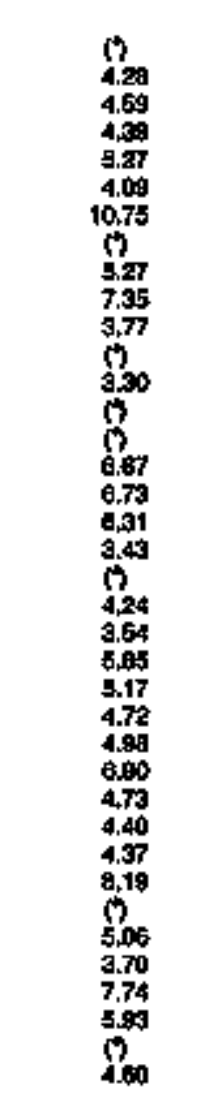 \\
\hline 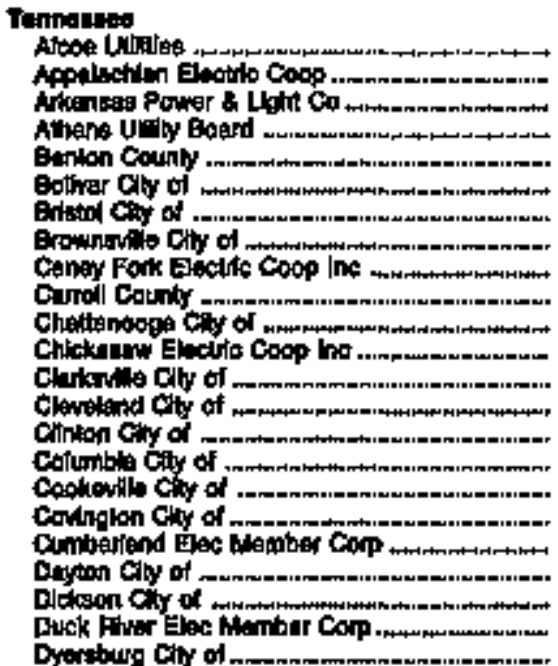 & 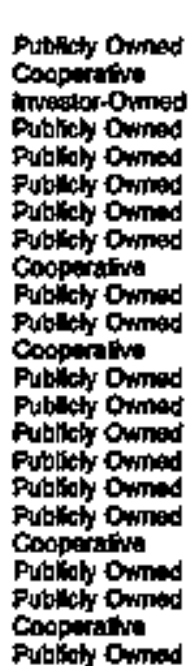 & $\begin{array}{r}204 \\
284 \\
17 \\
221 \\
70 \\
00 \\
470 \\
80 \\
174 \\
170 \\
1715 \\
60 \\
460 \\
174 \\
280 \\
282 \\
937 \\
100 \\
447 \\
04 \\
251 \\
405 \\
100\end{array}$ & 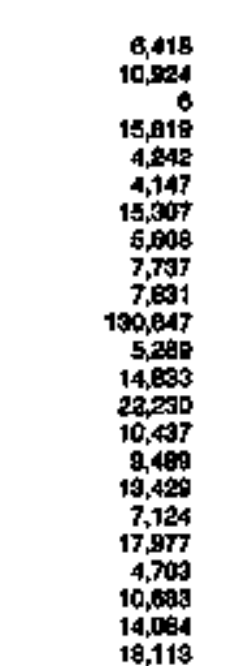 & 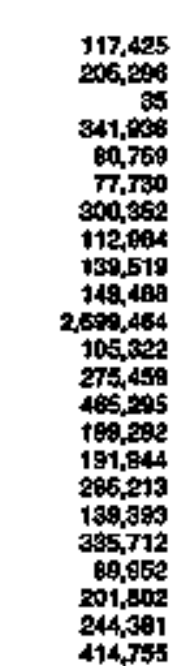 & 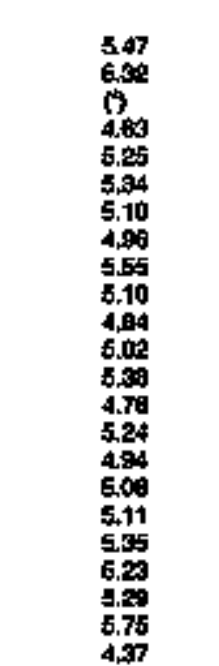 \\
\hline
\end{tabular}

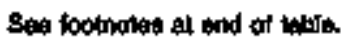


Table 16. Class of Ownershlp, Number of Ulimbte Consumers, Revenue, Sales, and Average Revenue por Kllowatthour for the Inductrlal Sector by State and Utility, J993 (Continued)

\begin{tabular}{|c|c|c|c|c|c|}
\hline 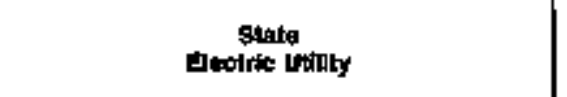 & $\begin{array}{c}\text { clata } \\
\text { of } \\
\text { Orenterthip }\end{array}$ & $\begin{array}{l}\text { Nuniber } \\
\text { of } \\
\text { certitumer }\end{array}$ & $\begin{array}{c}\text { bowenue } \\
\text { (thousand dollarel) }\end{array}$ & 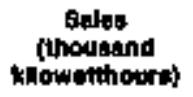 & 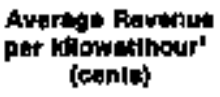 \\
\hline 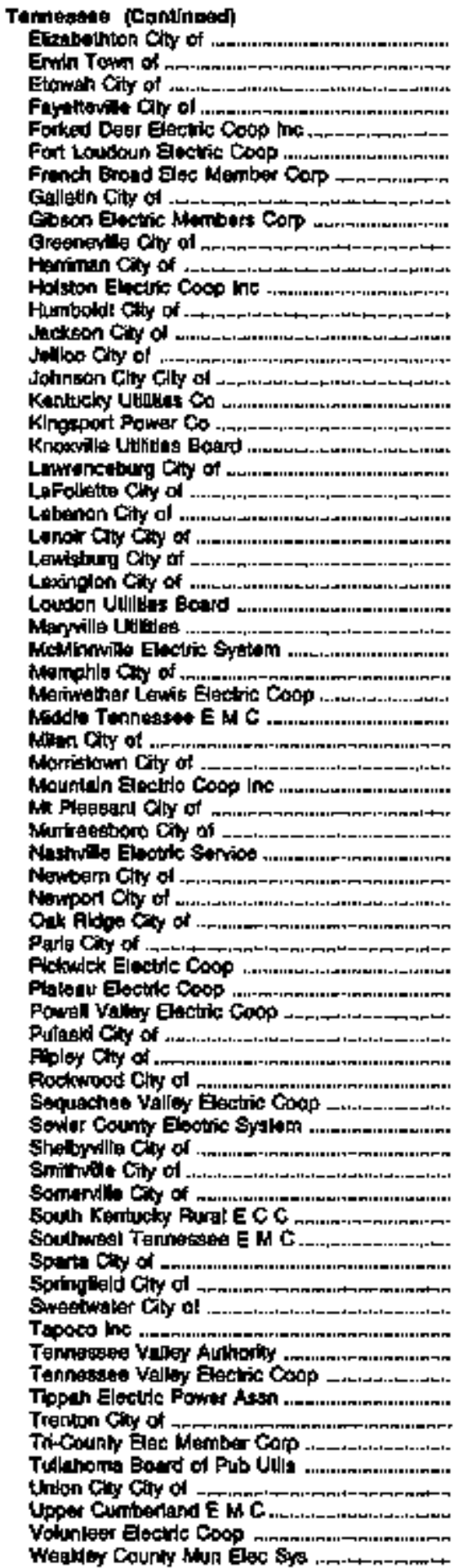 & 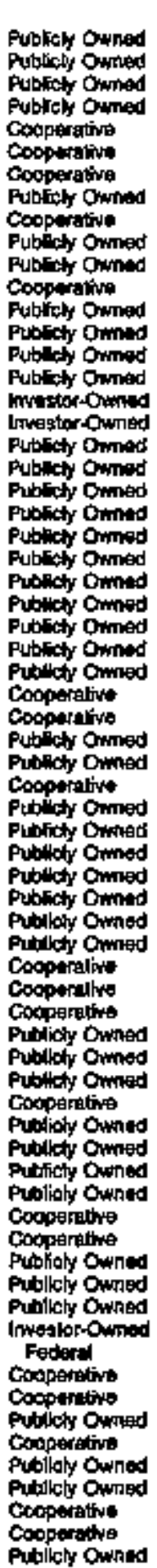 & 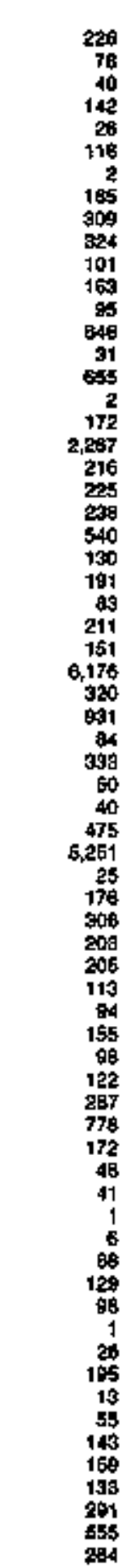 & 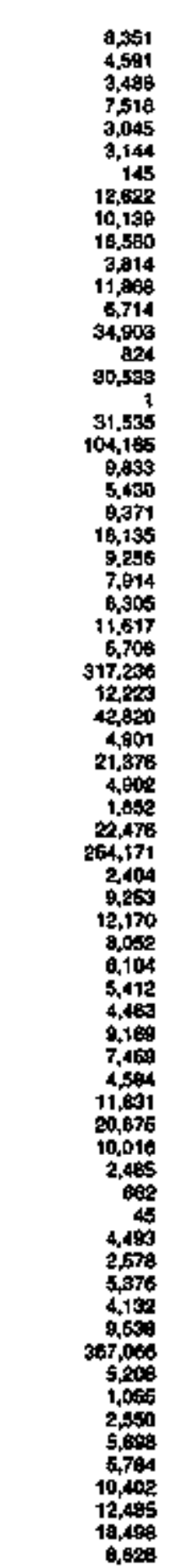 & 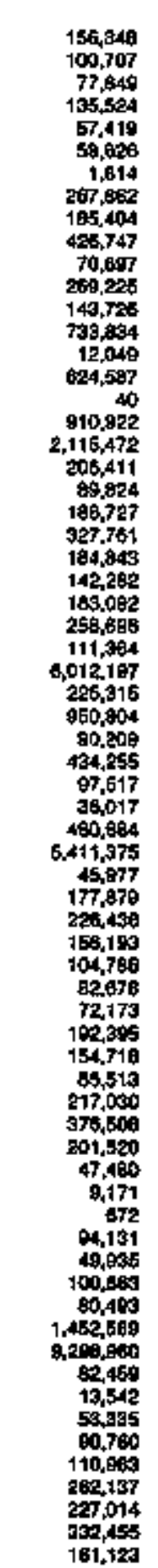 & 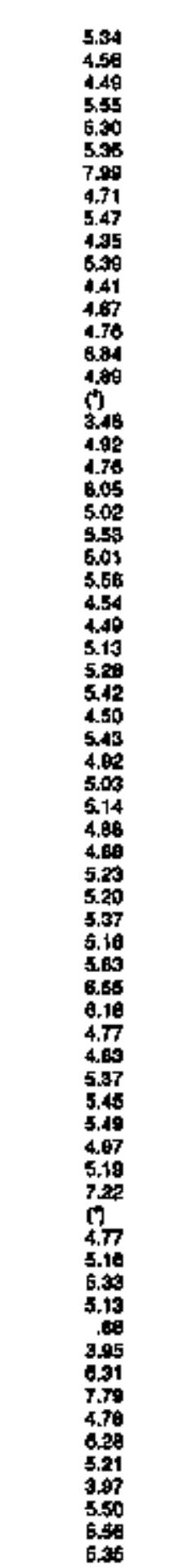 \\
\hline
\end{tabular}

Ses toobnotes at and of thile. 
Table 16. Class of Ownershlp, Mumber of Utitmate Constmers, Revenue, Sales, and Average Fevenue per Kilowalthour for the Industrlat Sector by State and Utilly, 1998 (Continued)

\begin{tabular}{|c|c|c|c|c|c|}
\hline Gectite terty & $\begin{array}{l}\text { OHas: } \\
\text { of } \\
\text { Onnerahip }\end{array}$ & $\begin{array}{l}\text { Number } \\
\text { of } \\
\text { contumers }\end{array}$ & (thousand dollast) & $\begin{array}{l}\text { siats } \\
\text { (qhoupond } \\
\text { kDowithours) }\end{array}$ & 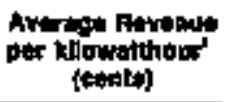 \\
\hline 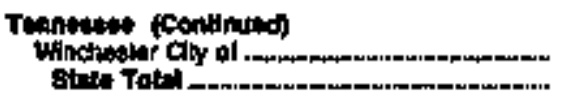 & Putbly Owoted & 38,74 & $2,011,554$ & $\begin{array}{r}50,759 \\
49,500,694\end{array}$ & $\begin{array}{l}6.15 \\
4.62\end{array}$ \\
\hline 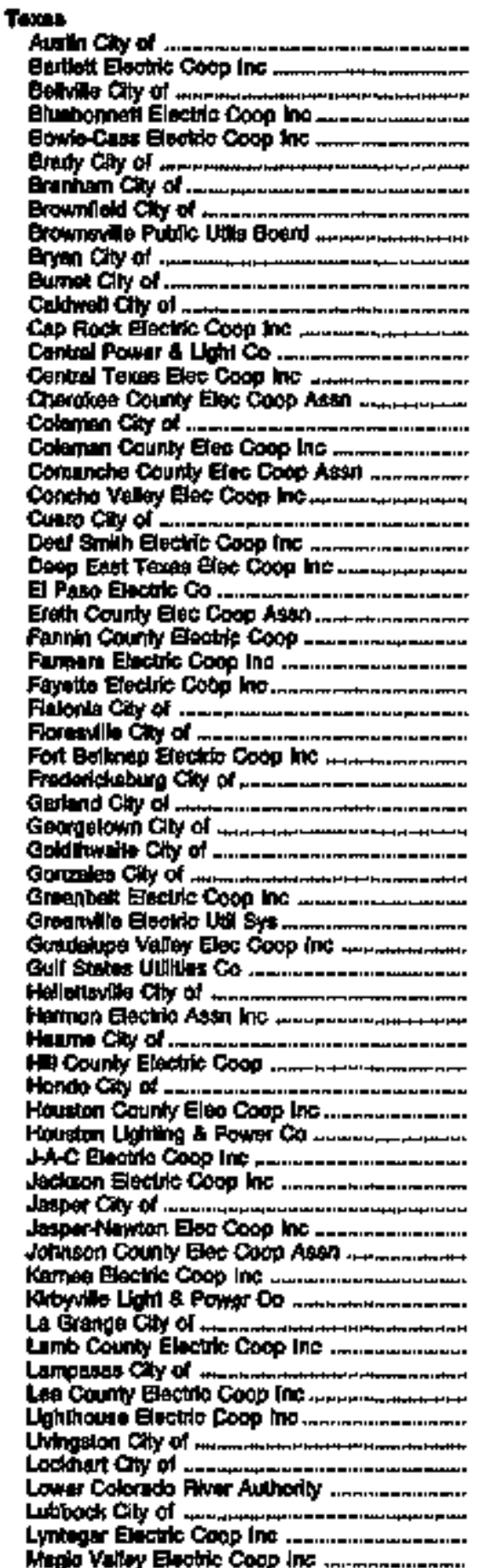 & 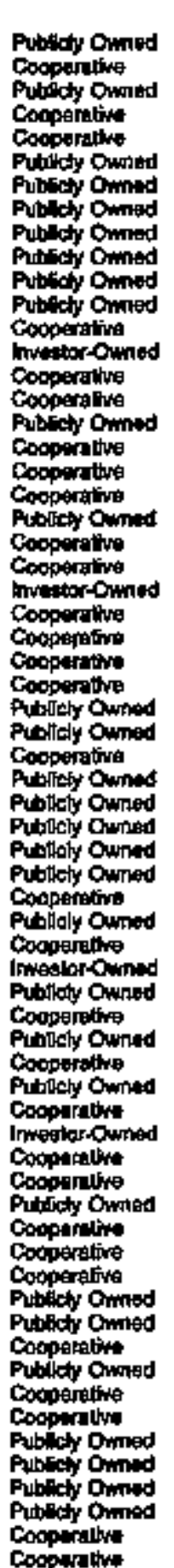 & 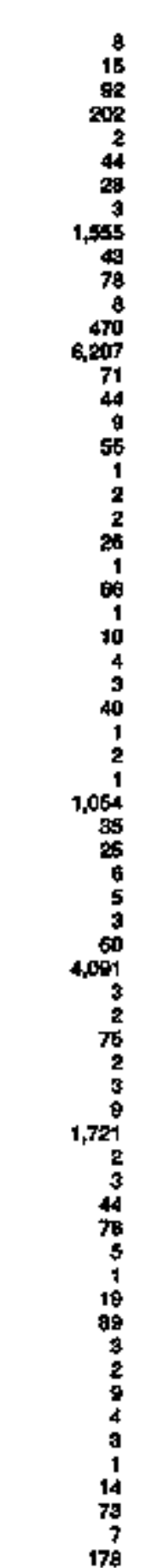 & 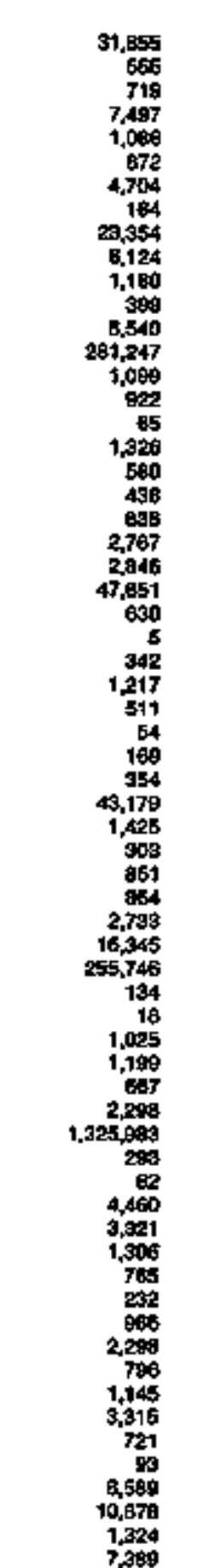 & 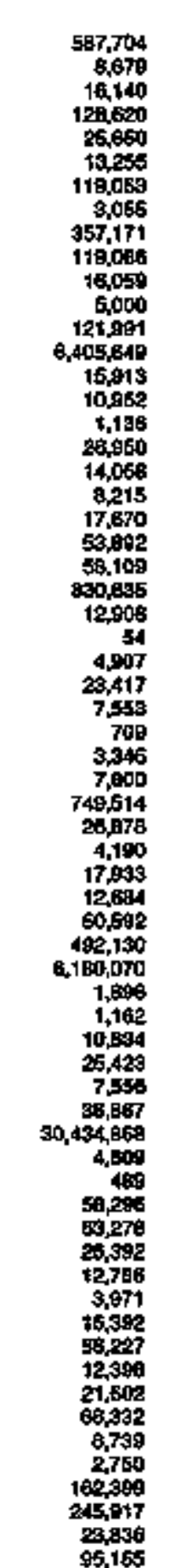 & 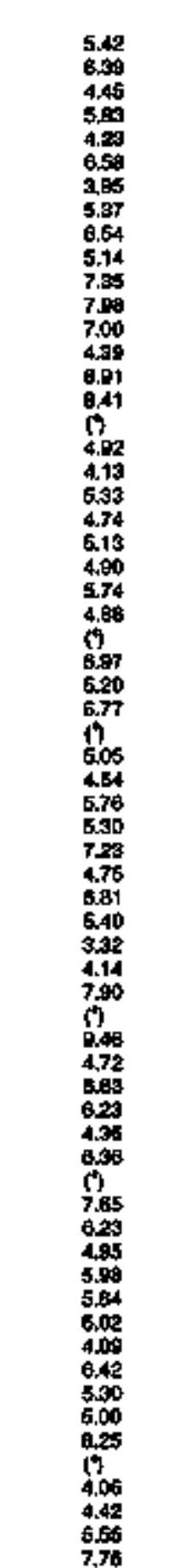 \\
\hline
\end{tabular}

sec tootnotes af end of table. 
Table 16. Class of Ownership, Number of Ultimate Consumers, Revenue, Salea, and Average Revenue per Kllowatthour for the Industrial Sector by State and Utility, 1993 (Continued)

\begin{tabular}{|c|c|c|c|c|c|}
\hline State & $\begin{array}{c}\text { Cros: } \\
\text { 여 } \\
\text { Ownerenp }\end{array}$ & $\begin{array}{l}\text { Munther } \\
\text { of } \\
\text { Consumers }\end{array}$ & $\begin{array}{c}\text { Pevenute } \\
\text { (thouldend dolara) }\end{array}$ & $\begin{array}{c}\text { Salen } \\
\text { (thopond } \\
\text { dolowithours) }\end{array}$ & $\begin{array}{l}\text { Avarage Reyromus } \\
\text { per ldiowethour' } \\
\text { (centsy }\end{array}$ \\
\hline
\end{tabular}

Taxpos (Combinuted]

Meculoch Elocinle Coop inc

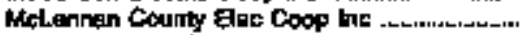

Hbedina Eactric Coap inc .....................................

Mid-South Electric Coop issm

Moultan City of

Navamo Counly Eles Cocp ine

Navapold Valla Eloce Coop ins

Now Branufels Gly ol

New Er Eloctic Cocp Ine ............................

North Flains Elective Goop int

Nubes Elactic torop inc

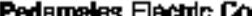

Pis Giande Electic Coos ins

fill Elunce Eloctide coop ins.

Fusk Coundy Etectric Coon lnc

Sem loutston Eloctic con

sers Antonlo Ciby of

San Bemand Electric Coop be

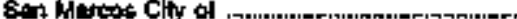

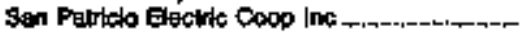

Sencor Cly of

Schudonburd City of ,

Seguln Chy of

Sermour City of

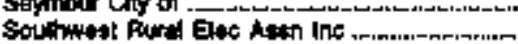

Southwe日t Tecoes Elerc Coop ITG.

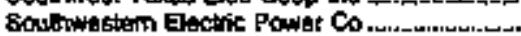

Soutwestom Eoolic SoN Co ,

Fouthwastem Publle service Co

Stamford Elactite Coop Inc

Sulaher Eloctsio Coop

Toykt Elpotric Coop hro

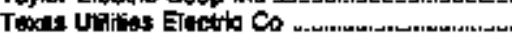

TamoNow Mpodeo Ponver Co .

Tritcoenty Electric Goop lnc

Tuca city of .

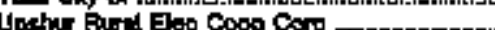

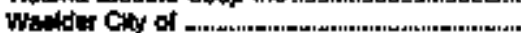

Watherlord Mum Uillity Sprear

Me'star Coty of

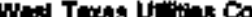

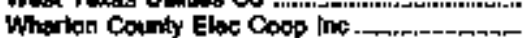

Wintustorn City 어

Wise Elecirto Coop ho ......................................

Wood County Elecike Goop Int _-

Youktun Gity of

Sath Total

Utin

Bewner Cly Com

Aountert Cin City of

Enoham City Cosp.

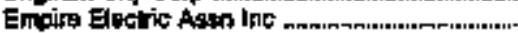

Eohri:n Cily of

Flowell Eocitio Atst ine

Hrnum Cily Corp

Kana'b City Com

Kughilla Gity Cam .

Meradon Tawn Comp

Hoon Leke Electite kadn InG n......................

Mit wheolor Powiti inc.

Pacticorp

Bath Fiver Rurd Eeo toop he m................

Spentah Fank City COR .

Spinomian of

si coores ciny of

Ene Inotnoles fat ind ol table.
Copperathe

Cooperalive

Cooporature

Cocoperativa

Publisty Ouined

cooperithe

Copplálisía

Publishy Owned

Cooperaltwe

cooperafive

Coopurarivo

Cooperallya

Cooperative

coopuratro

Copperaltu

Cooparalive

Cooperalfwa

Publichy Owmed

Copparationa

Publichy Ommod

Copperative

Pebilety Dimat

Publicty Ommed

Publictr Onmed

Pubichy Orined

Coopardute

Cooperzitum

howation Ownes

Investor-Owned

Irvestor Ownted

Cooperative

Cosperzature

Cooperettut

Inwastor-Owned

Inverter. Ownad

Cooparattre

Publichy Orined

Coxpertilue

Cooperedrat:

Publinty Orined

Pubnchy Orwied

Publithy Orwitid

Investor oumed

Cocpardive

Puthichy Ouwitad

Coopensedbrt

Cospenetve

Publicty Orested

Puthlly Dindid

Publicly Ourted

Putichy Ouned

Cocoprature

Puticly Owned

cocpertiont

Coonerithe

Pullichy Owned

Publlely Owined

Pullicty Ourred

Fulich Owned

Conperative

Conperpitre

Imbetor-Omned

Cooperativi

Puticty Owtud

Putloty owred

Publezty Owned

\subsection{3}

860

3.697

161

3.104

4,575
14,874

1.169

1.169

391

15,494

768

587

3.620

3.620
100,270

3,897

3.074

573

500

028

2.604

458

416

141,404

41,404
9,265

217,100

32

69

605

17,124

5,604

24

69

300

255

347

53,700

2,055

178

385

421
1,511

$3,7,6,85$

68.275

9,70,

$\begin{array}{rr}1 & 67 \\ 1 & 1,472 \\ 2 & 1,447 \\ 13 & 257 \\ 29 & 43 \\ 1 & 30 \\ 1 & 1,357 \\ 25 & 1,995 \\ 4 & 20 \\ 1 & 69 \\ 2 & 3 \\ 11 & 4,160 \\ 3 & 45 \\ 10,587 & 214,351 \\ 16 & 59 \\ 10 & 1,445 \\ 25 & 2,492 \\ 192 & 4,966\end{array}$

\section{7,299}

$18.077 \quad 4,46$

$58.930 \quad 6.67$

$40,271 \quad 4,60$

$1.7 \mathrm{BeC} \quad 0.14$

$71,144 \quad 4,33$

$114,170 \quad 4,01$

$374.056 \quad 9.97$

$10,830 \quad 7.12$

22,700

$5,650 \quad 7.79$

$329.731 \quad 470$

$7.028 \quad 9.95$

$9,891 \quad 6.68$

25.690 413

56.9800 .24

3,547, 2स1 5,00

$04.707 \quad 4.60$

$51.202 \quad 0.00$

$12,676 \quad 4,50$

7.0127 .19

$77,818 \quad 5.81$

46.893 6.35

$6,003 \quad 7.60$

$\begin{array}{rr}6,612 & 8.25 \\ 0.620 & 4.55\end{array}$

$3,622.379 \quad 5.91$

163,698

6,066,004 3.00

318

17,007

12,731

21.339 .748

$1,225,995$

122,752

3.898

22,431

3,608

$\mathbf{3 , 9 7 5}$

45,325

7,304

$1,231,181$

32,078

3,406

8,297

10,468

$19,67 \%$

$60,932,759$

(i)

6.87
4.75

4.11

6.04

4.73

6.16

3.06

6.67

6.29

4.75

4 (3)

B.41

5.14

4.64

4.01

7.32

$\begin{array}{rl}1.189 & \text { (') } \\ 51,682 & 2.84\end{array}$

2095? 4.89

3,600

600

437

24,500

43,363

254
697

72

589

$5,011,702$

1,158

24,500

52,200

웅, 056

7.9

1

6.53

4.45

0

it

522

3.60

(")

6.00

4.77

6.20

tape. 
Table 16. Class of Ownership, Number of Ultimbte Consumers, Revenute, Sales, and Average Fevenue per Kilowatthour for the Industrial Sector by State and Utility, 1998 (Continued)

\begin{tabular}{|c|c|c|c|c|c|}
\hline $\begin{array}{c}\text { stata } \\
\text { enctule todity }\end{array}$ & $\begin{array}{l}\text { Co:a } \\
\text { on }\end{array}$ & $\begin{array}{l}\text { Humbiar } \\
\text { ol } \\
\text { Contopitars }\end{array}$ & $\begin{array}{c}\text { Revonug } \\
\text { (thoutand dotarsal }\end{array}$ & 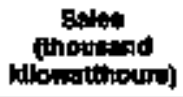 & 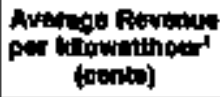 \\
\hline 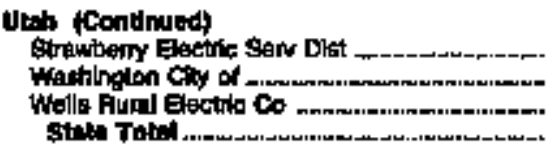 & 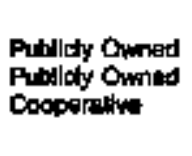 & $\begin{array}{r}6 \\
1 \\
3 \\
10,038\end{array}$ & $\begin{array}{r}295 \\
15 \\
617 \\
254.969\end{array}$ & $\begin{array}{r}3,018 \\
296 \\
12,160 \\
6,221,063\end{array}$ & $\prod_{3.78}^{7.48}$ \\
\hline 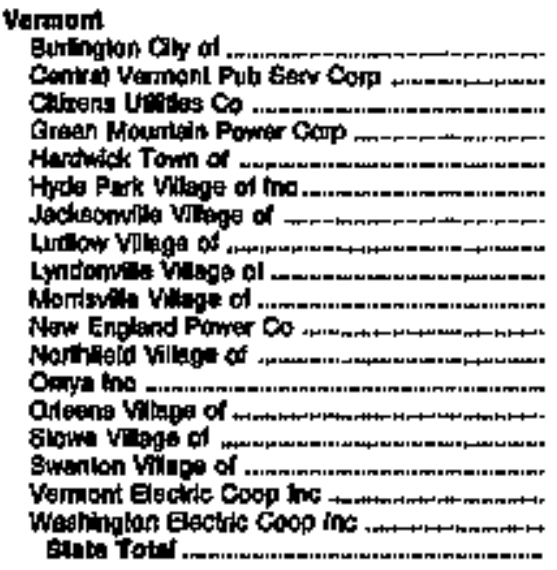 & 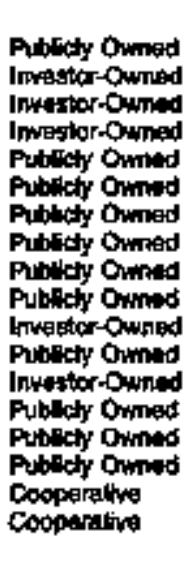 & $\begin{array}{r}697 \\
37 \\
6 \\
25 \\
16 \\
2 \\
4 \\
8 \\
28 \\
40 \\
2 \\
15 \\
3 \\
1 \\
11 \\
71 \\
42 \\
12 \\
1.027\end{array}$ & $\begin{array}{r}24,106 \\
27,687 \\
5,094 \\
31,060 \\
340 \\
108 \\
107 \\
1,277 \\
1,914 \\
1,802 \\
308 \\
601 \\
7,864 \\
979 \\
26 \\
2,624 \\
1,287 \\
308 \\
167,308\end{array}$ & 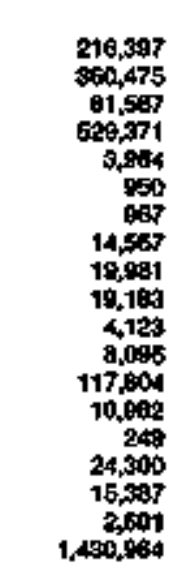 & $\begin{array}{c}11.14 \\
7.06 \\
6.23 \\
6.06 \\
8.56 \\
0 \\
6 \\
6.77 \\
9.58 \\
8.59 \\
9.41 \\
7.48 \\
6.17 \\
8.01 \\
10 \\
10.35 \\
8.36 \\
1.65 \\
7.50\end{array}$ \\
\hline 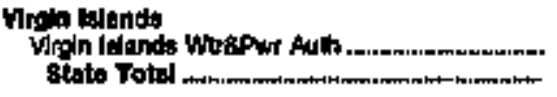 & Pubilety Ormed & $\begin{array}{l}\text { a17 } \\
817\end{array}$ & $\begin{array}{l}26,573 \\
24,973\end{array}$ & $\begin{array}{l}237,456 \\
2 \times 7,438\end{array}$ & $\begin{array}{l}11.35 \\
1138\end{array}$ \\
\hline 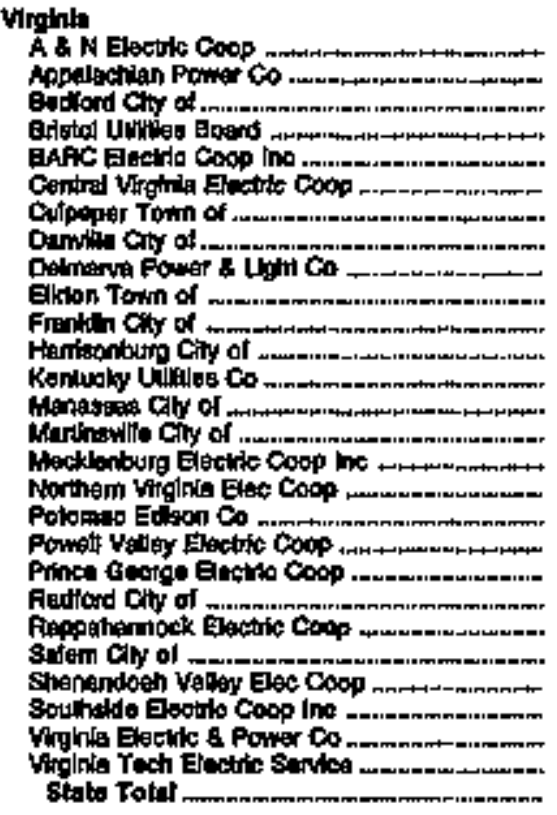 & 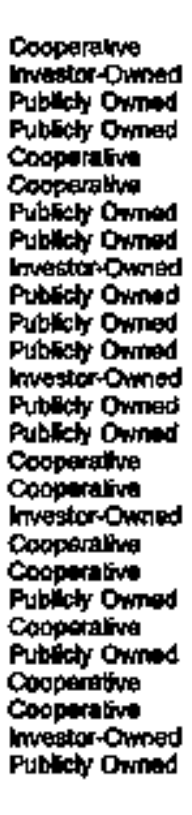 & $\begin{array}{r}30 \\
1,8 \% 2 \\
29 \\
260 \\
2 \\
6 \\
7 \\
74 \\
61 \\
6 \\
5 \\
11 \\
\$ 66 \\
115 \\
5 \\
6 \\
10 \\
1,013 \\
29 \\
39 \\
20 \\
129 \\
97 \\
12 \\
160 \\
769 \\
2 \\
4,900\end{array}$ & 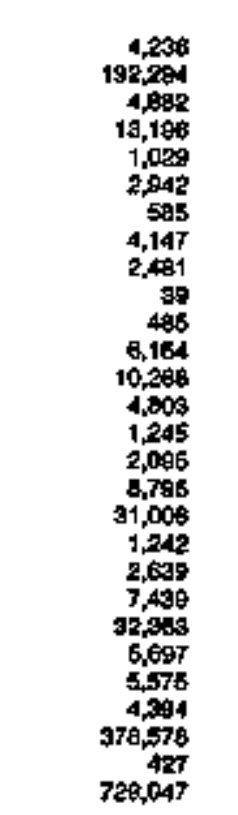 & 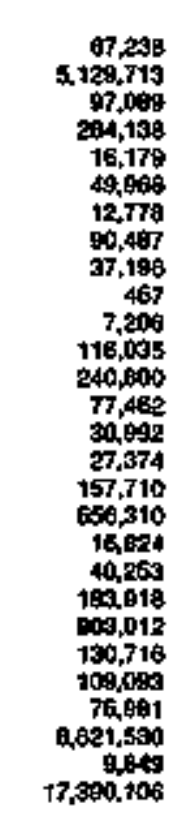 & 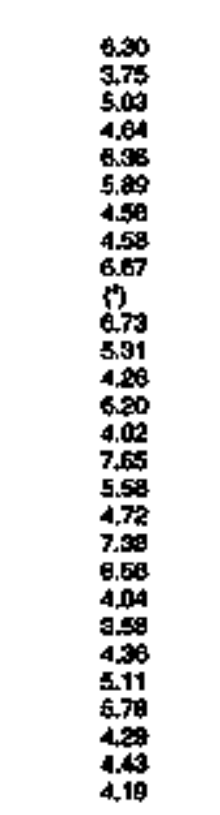 \\
\hline 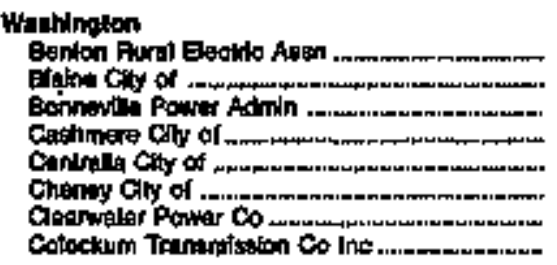 & 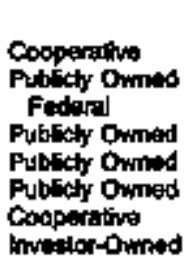 & $\begin{array}{r}4 \\
10 \\
9 \\
10 \\
45 \\
4 \\
9 \\
1\end{array}$ & $\begin{array}{r}1,406 \\
176 \\
294,094 \\
279 \\
607 \\
413 \\
195 \\
9,430\end{array}$ & $\begin{array}{r}41,000 \\
3,332 \\
16,473,239 \\
18,036 \\
209,009 \\
11,695 \\
3,688 \\
1,699,728\end{array}$ & $\begin{array}{l}3.49 \\
5.28 \\
1.80 \\
1.59 \\
3.60 \\
3.79 \\
3.69 \\
.55\end{array}$ \\
\hline
\end{tabular}

Seg roolnoles al ens of thith. 
Table 16. Class of Ownership, Number of Ultimate Consumers, Revenue, Sales, and Average Rovenue per Klowatthour for the Industrial Sector by State and Utility, 1993 (Continued)

\begin{tabular}{|c|c|c|c|c|c|}
\hline State & 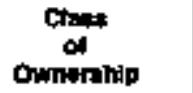 & $\begin{array}{l}\text { Number } \\
\text { of } \\
\text { Contumer. }\end{array}$ & $\begin{array}{c}\text { Arownte } \\
\text { (thotatard dolaral }\end{array}$ & 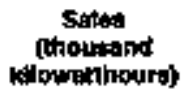 & 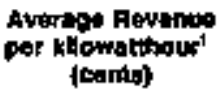 \\
\hline 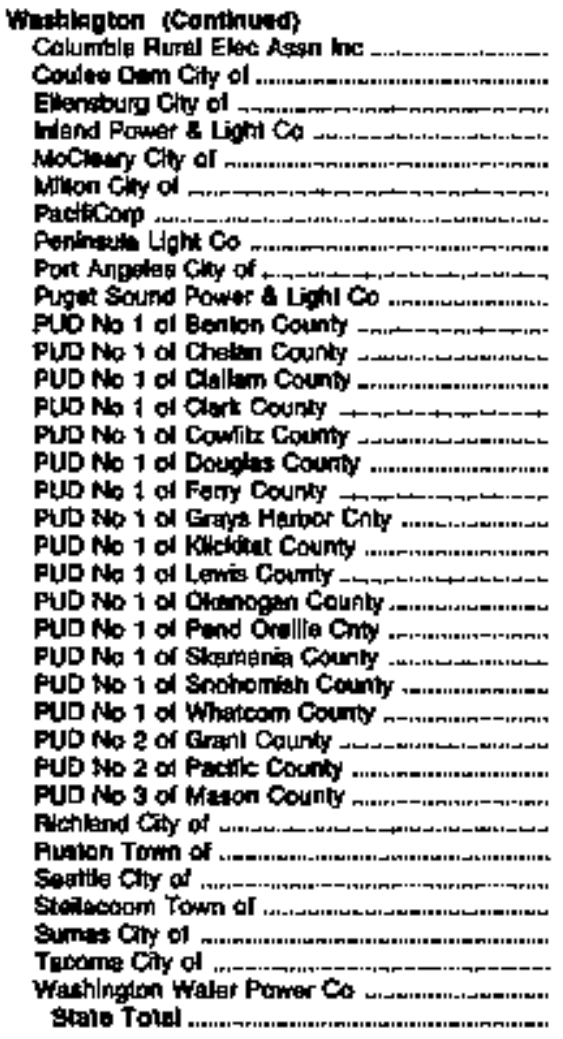 & 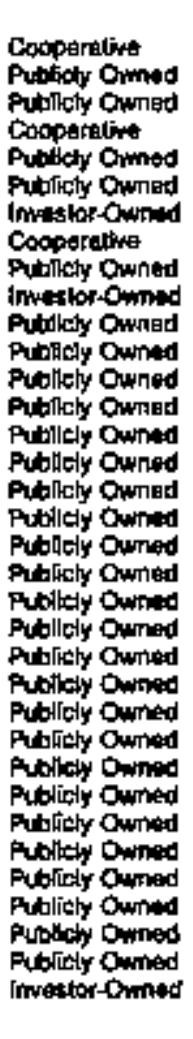 & $\begin{array}{r}2 \\
8 \\
1 \\
19 \\
4 \\
91 \\
3,285 \\
12 \\
8 \\
3,716 \\
763 \\
26 \\
188 \\
15 \\
87 \\
1 \\
6 \\
42 \\
143 \\
93 \\
5 \\
10 \\
3 \\
84 \\
1 \\
58 \\
2 \\
1 \\
211 \\
1 \\
301 \\
20 \\
9 \\
1,554 \\
613 \\
11,404\end{array}$ & 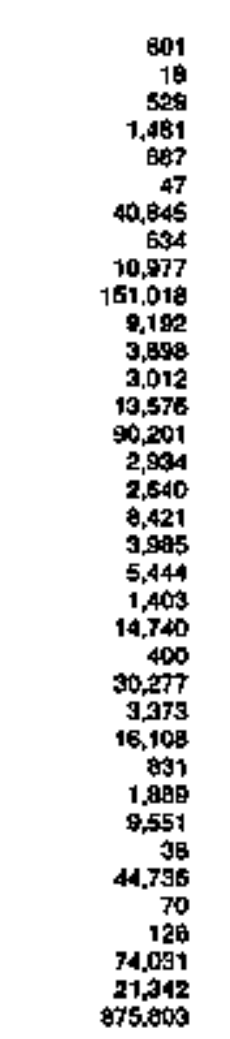 & 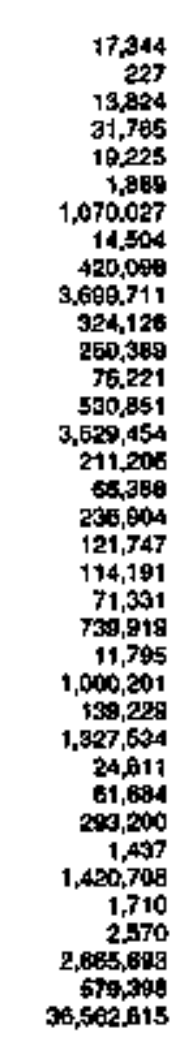 & 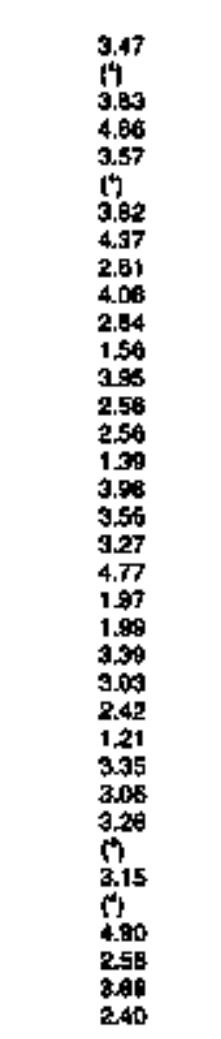 \\
\hline 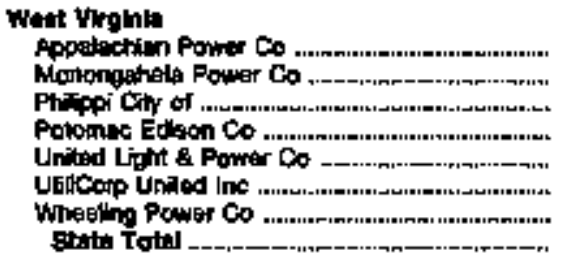 & 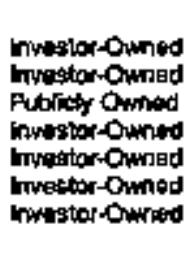 & $\begin{array}{r}2.395 \\
7.139 \\
7 \\
1,239 \\
5 \\
2 \\
313 \\
11,094\end{array}$ & $\begin{array}{r}167,600 \\
17 \times, 807 \\
270 \\
24,494 \\
9 \\
1,690 \\
37,606 \\
403,650\end{array}$ & $\begin{array}{r}1,249,305 \\
4,213,084 \\
5,178 \\
552,026 \\
134 \\
36,028 \\
7,132,915 \\
10,187,147\end{array}$ & 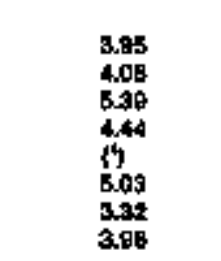 \\
\hline 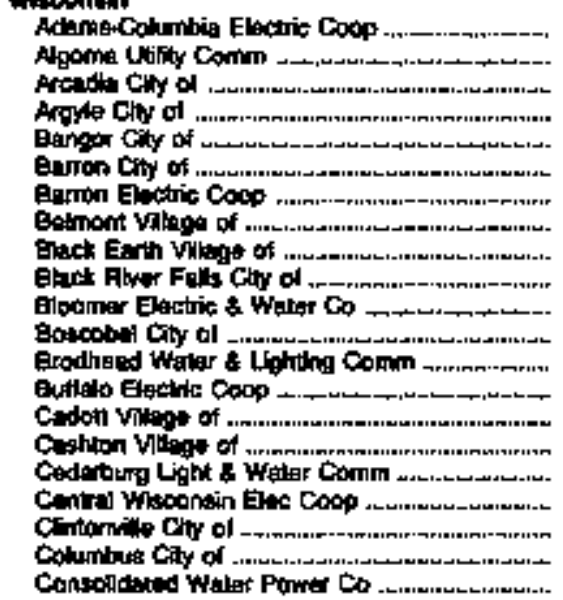 & 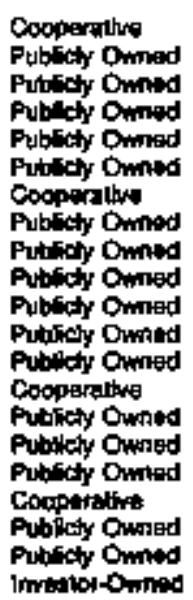 & $\begin{array}{r}6 \\
9 \\
30 \\
5 \\
23 \\
10 \\
6 \\
7 \\
8 \\
26 \\
32 \\
14 \\
14 \\
1 \\
10 \\
8 \\
41 \\
1 \\
6 \\
31 \\
1\end{array}$ & $\begin{array}{r}1.476 \\
1,060 \\
2,030 \\
64 \\
510 \\
609 \\
3,092 \\
82 \\
50 \\
824 \\
1,029 \\
690 \\
576 \\
46 \\
196 \\
84 \\
2,451 \\
44 \\
1,427 \\
5,270 \\
34,394\end{array}$ & 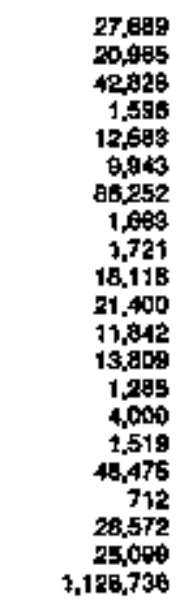 & 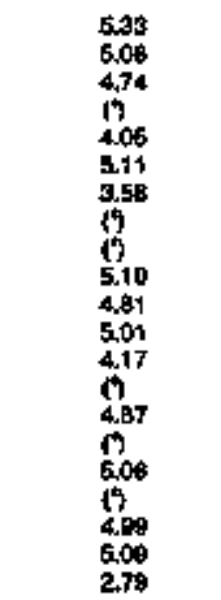 \\
\hline
\end{tabular}

She footnoles at and ol ubiv. 
Table 16. Clags of Ownershlp, Number of Utimate Consumers, Revenue, Sales, and Average Revenue per Khowatthour for the Industrial sector by State and Utility, 1993 (Continued)

\begin{tabular}{|c|c|c|c|c|c|}
\hline Elpotilo Uanty & 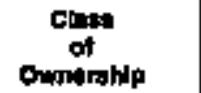 & $\begin{array}{l}\text { Murpbir } \\
\text { of } \\
\text { conloming }\end{array}$ & Funames & $\begin{array}{c}\text { salas } \\
\text { (thousand } \\
\text { kilowithours) }\end{array}$ & 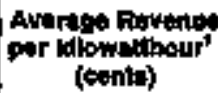 \\
\hline 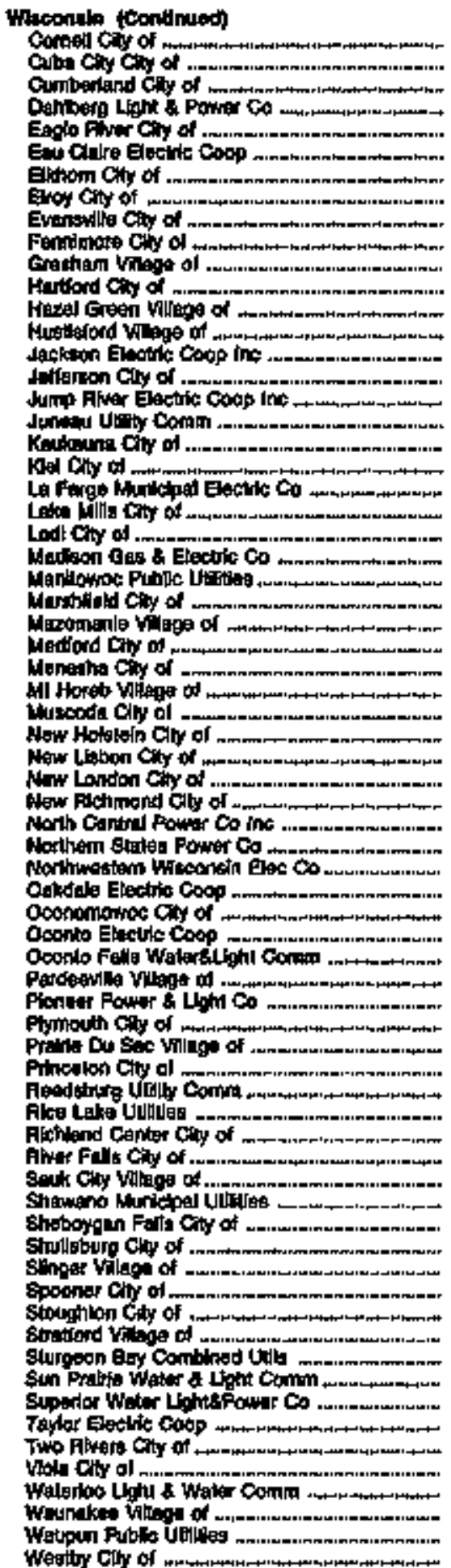 & 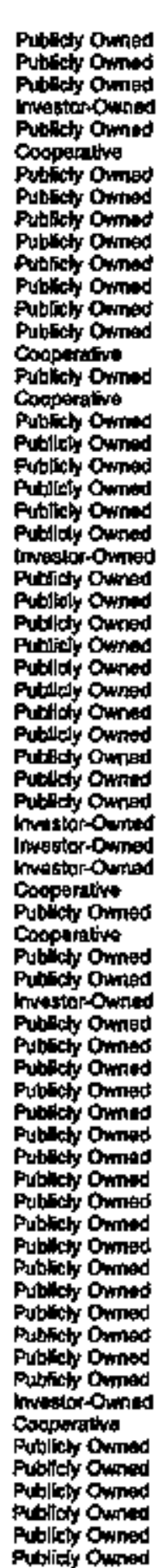 & 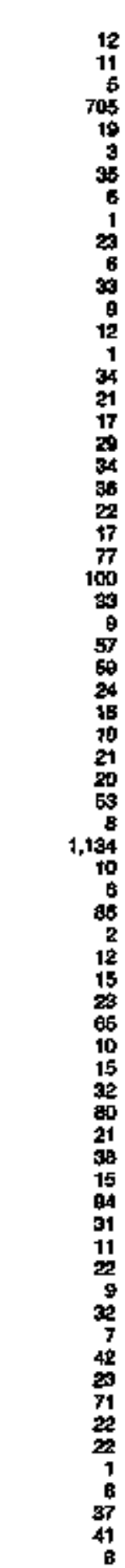 & 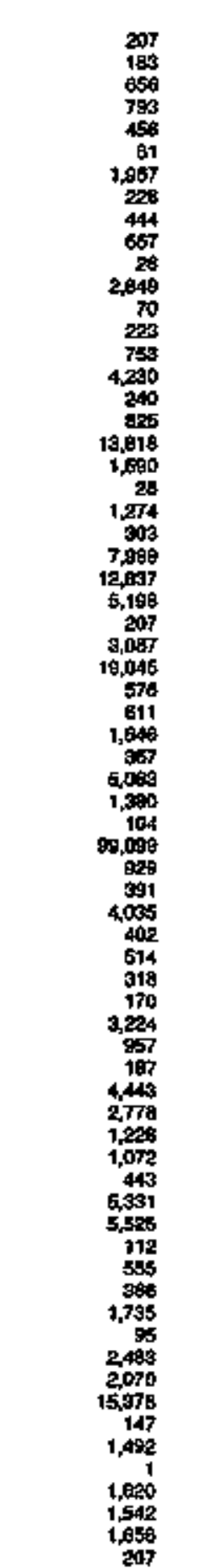 & 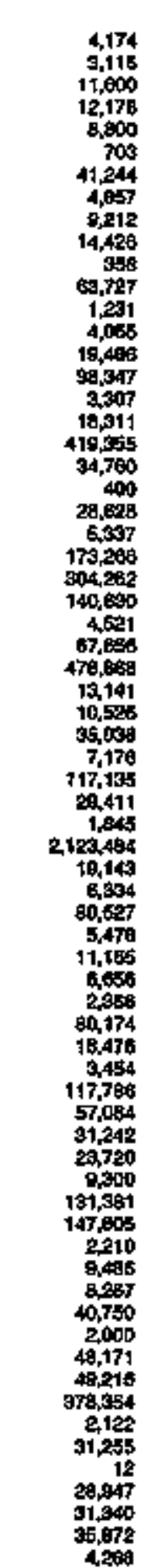 & 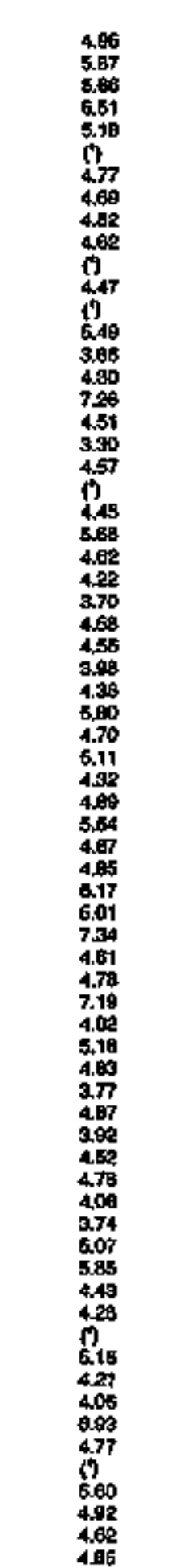 \\
\hline
\end{tabular}

See loolnotes at and of tublt. 
Table 16. Class of Ownership, Number of Ultimate Consuners, Revenus, Sales, and Average Fevenue per Kllowatthour for the Industrial Sector by State and Utility, 1999 (Contimued)

\begin{tabular}{|c|c|c|c|c|c|}
\hline $\begin{array}{l}\text { State } \\
\text { enctric Uisity }\end{array}$ & $\begin{array}{l}\text { Cinge } \\
\text { of } \\
\text { Oninterthlp }\end{array}$ & $\begin{array}{l}\text { Numbar } \\
\text { of } \\
\text { Condeutaters }\end{array}$ & (1houlsund dotiars) & $\begin{array}{c}\text { 8rites } \\
\text { (thosestad } \\
\text { lolowathours) }\end{array}$ & 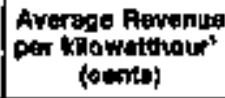 \\
\hline 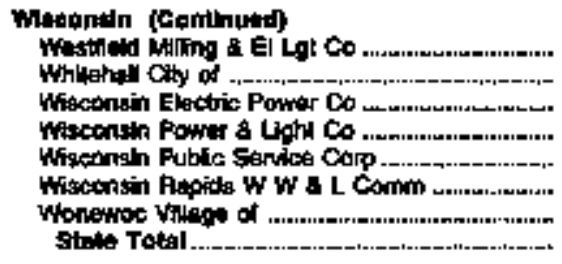 & 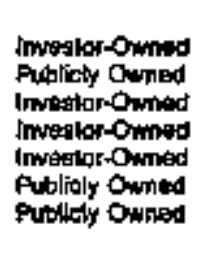 & $\begin{array}{r}20 \\
14 \\
688 \\
681 \\
192 \\
27 \\
5 \\
5,460\end{array}$ & $\begin{array}{r}243 \\
807 \\
300,047 \\
128,796 \\
120,640 \\
2,281 \\
65 \\
052,544\end{array}$ & $\begin{array}{r}4,278 \\
22,011 \\
7,452,0003 \\
3,441,006 \\
3,192,316 \\
61,221 \\
1,004 \\
21,410,441\end{array}$ & $\begin{array}{l}6.48 \\
4.59 \\
4.03 \\
3.74 \\
3.78 \\
4.46 \\
1.7 \\
3.48\end{array}$ \\
\hline 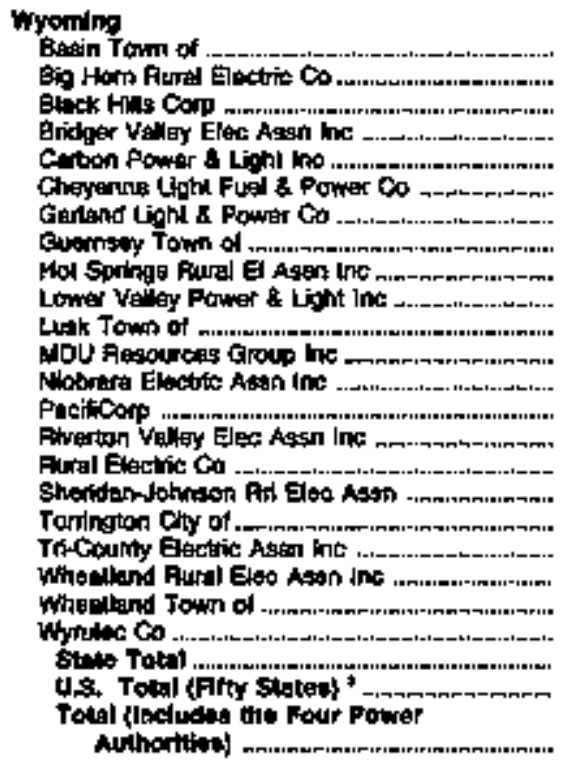 & 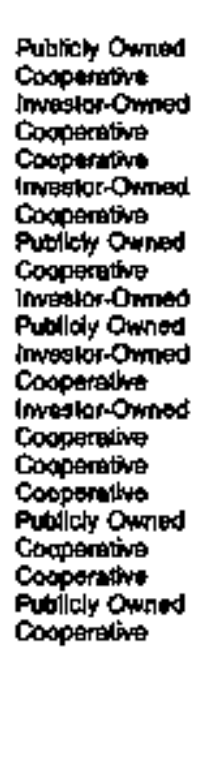 & $\begin{array}{r}1 \\
2 \\
6 \\
5 \\
4 \\
11 \\
35 \\
1 \\
11 \\
3 \\
17 \\
29 \\
3 \\
2,777 \\
7 \\
48 \\
2 \\
1 \\
21 \\
63 \\
56 \\
1 \\
3.097 \\
068,281\end{array}$ & $\begin{array}{r}60 \\
967 \\
4,698 \\
1,151 \\
604 \\
6,244 \\
88 \\
3 \\
6,964 \\
412 \\
126 \\
456 \\
655 \\
198,206 \\
2,287 \\
458 \\
1,217 \\
649 \\
32,055 \\
2,392 \\
467 \\
91 \\
257,607 \\
47,357,146\end{array}$ & 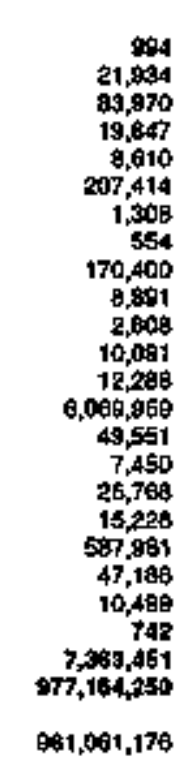 & $\begin{array}{l}0 \\
1.49 \\
4.47 \\
5.80 \\
7.02 \\
3.01 \\
90 \\
40 \\
4.08 \\
4.03 \\
4.45 \\
4.54 \\
5.33 \\
3.23 \\
4.44 \\
8.42 \\
4.70 \\
4.28 \\
5.45 \\
5.05 \\
4.25 \\
51 \\
7.60 \\
4.05\end{array}$ \\
\hline
\end{tabular}

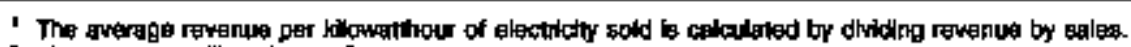

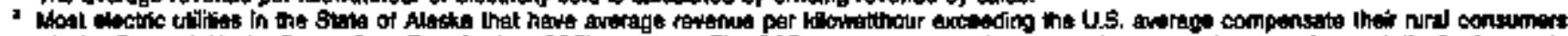

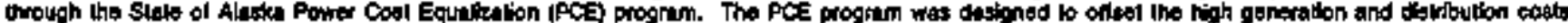

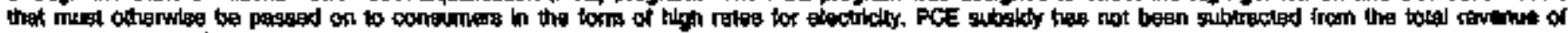

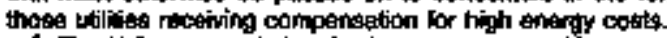

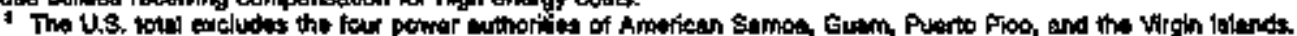

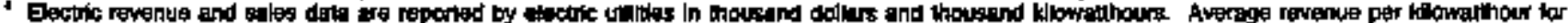

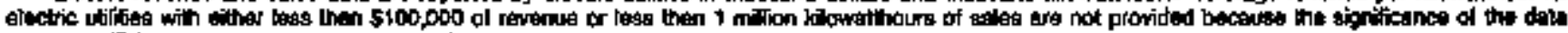
are nol surticint to make the ratio maningtel.

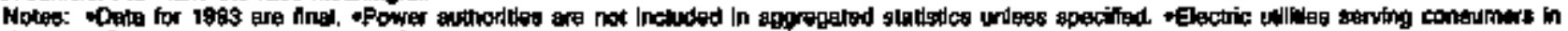

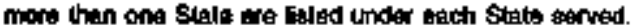

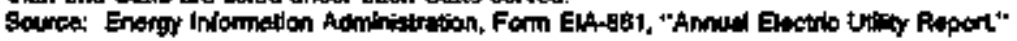


Table 17. Class of Ownershlp, Number of Ultimate Consumers, Rovenue, Salos, and Average Revenue per Kiowatthour for All Sectors by State and Utility, 1993

\begin{tabular}{|c|c|c|c|c|c|}
\hline 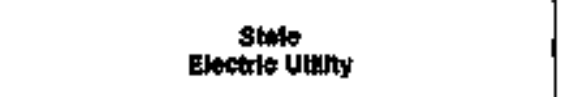 & 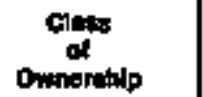 & $\begin{array}{c}\text { Huntper } \\
\text { of } \\
\text { Coneunese }\end{array}$ & Roweaus & 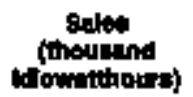 & 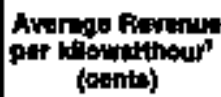 \\
\hline 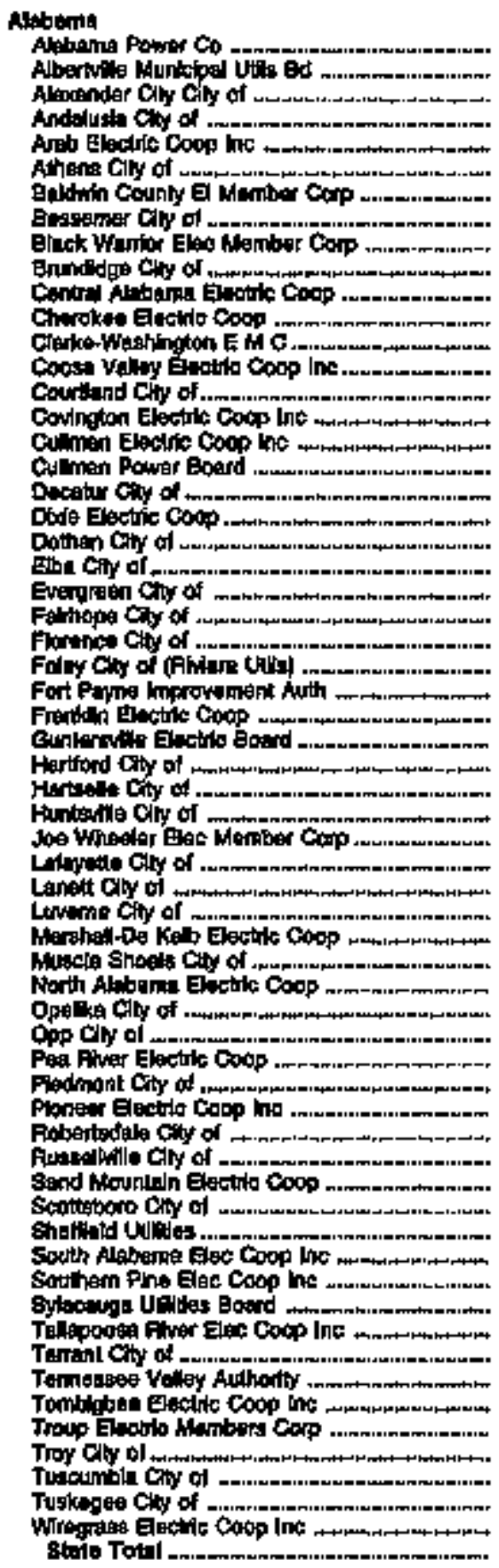 & 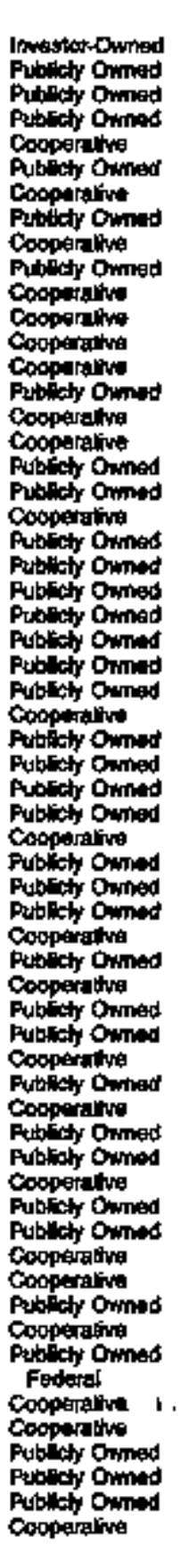 & 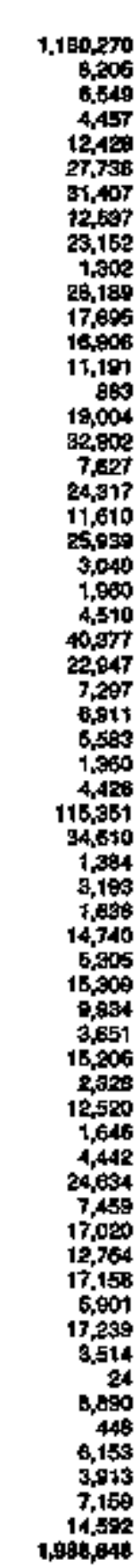 & 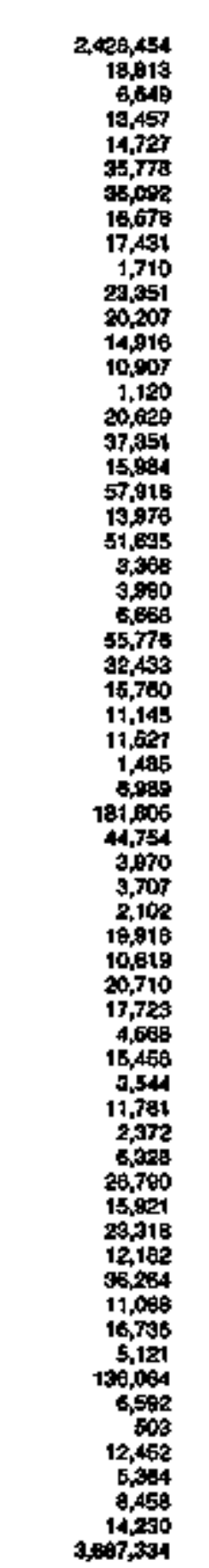 & 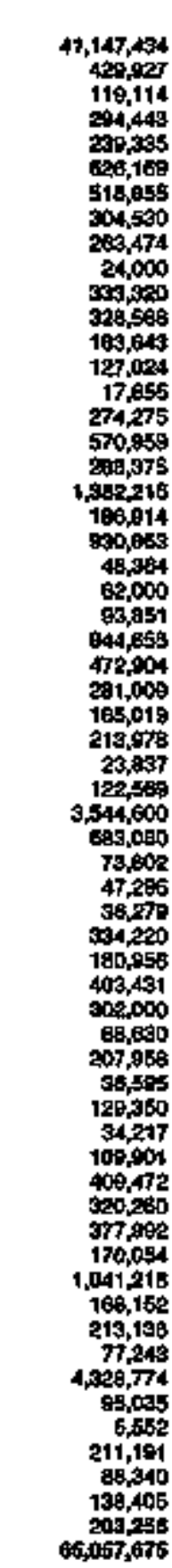 & 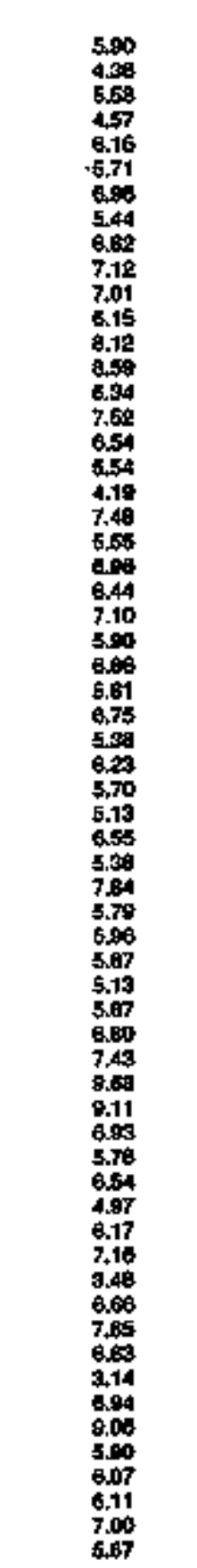 \\
\hline 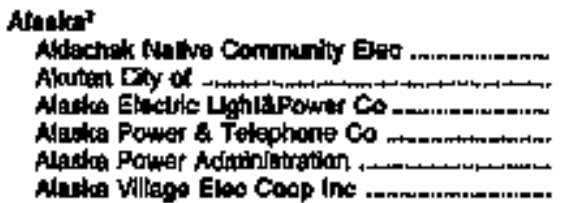 & 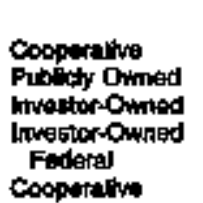 & $\begin{array}{r}173 \\
37 \\
12,851 \\
2,420 \\
2 \\
5,680\end{array}$ & $\begin{array}{r}342 \\
104 \\
20,600 \\
4,700 \\
60 \\
16,727\end{array}$ & $\begin{array}{r}673 \\
282 \\
26,848 \\
25,711 \\
3,294 \\
40,604\end{array}$ & $\begin{array}{c}17 \\
77 \\
17.60 \\
377\end{array}$ \\
\hline
\end{tabular}

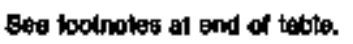


Tabje 17. Class of Ownership, Number of Ultimate Consumers, Revenute, Soles, and Average Revenue per Kilowatthour for AN Sectors by State and Utillty, 1993 (Continuad)

\begin{tabular}{|c|c|c|c|c|c|}
\hline Eteriste Utity & $\begin{array}{l}\text { Cla:s } \\
\text { of } \\
\text { Oinnerahlp }\end{array}$ & $\begin{array}{l}\text { Hunbur } \\
\text { of } \\
\text { connumara }\end{array}$ & (thousand dolyart) & 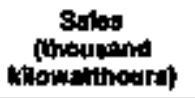 & 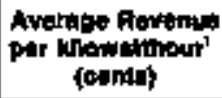 \\
\hline 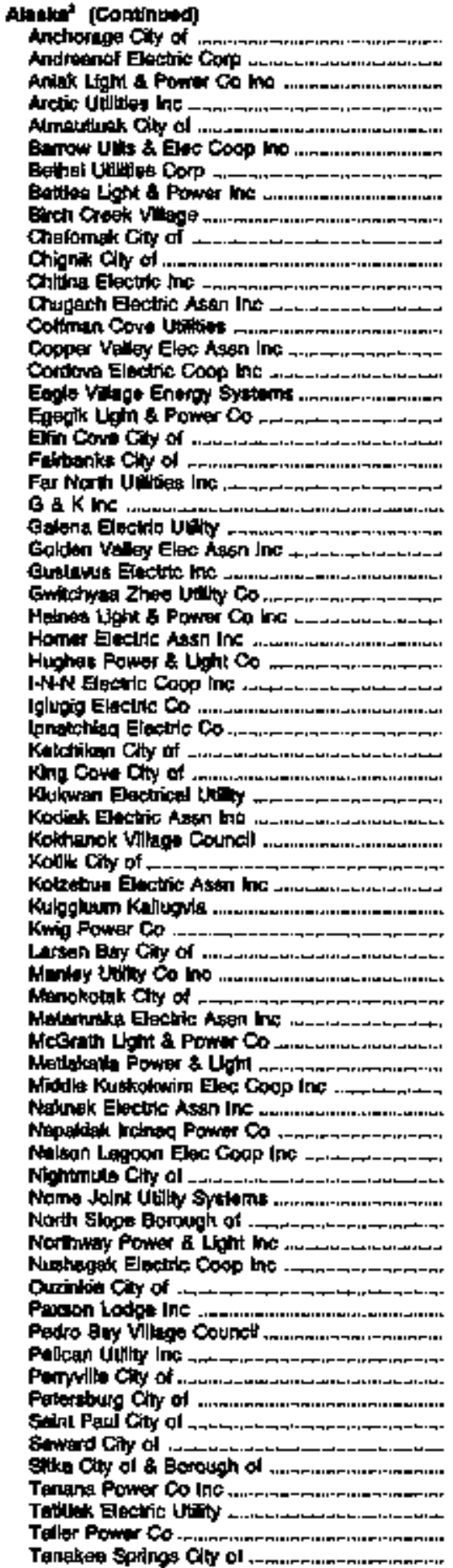 & 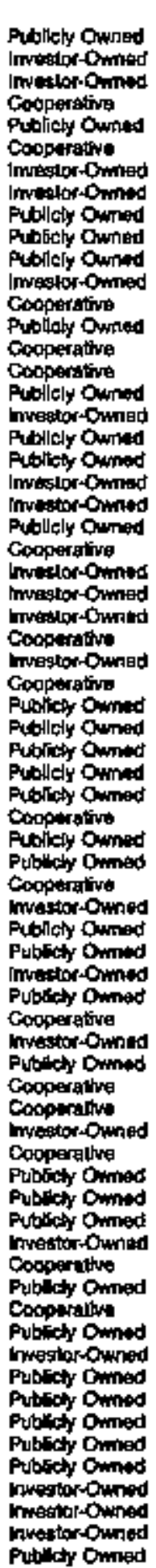 & 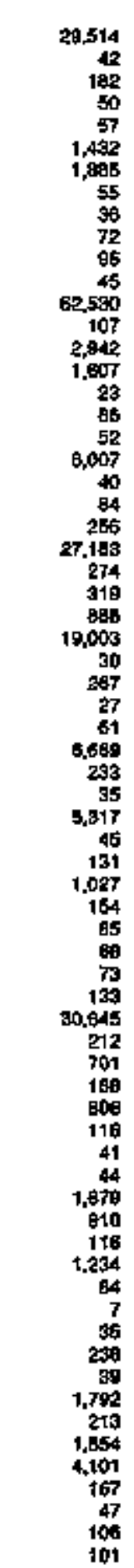 & 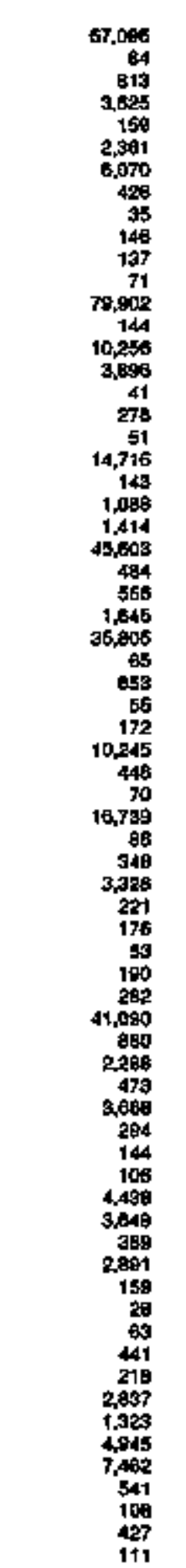 & 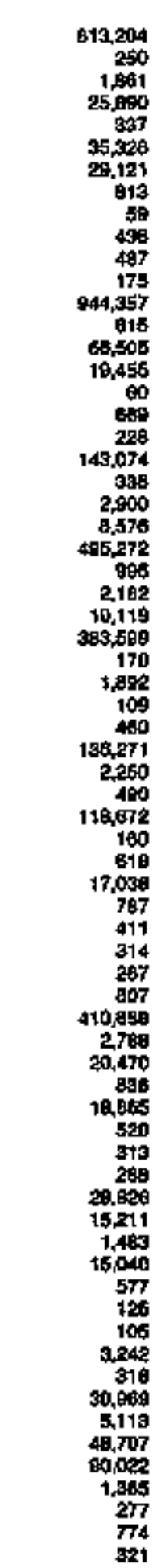 & 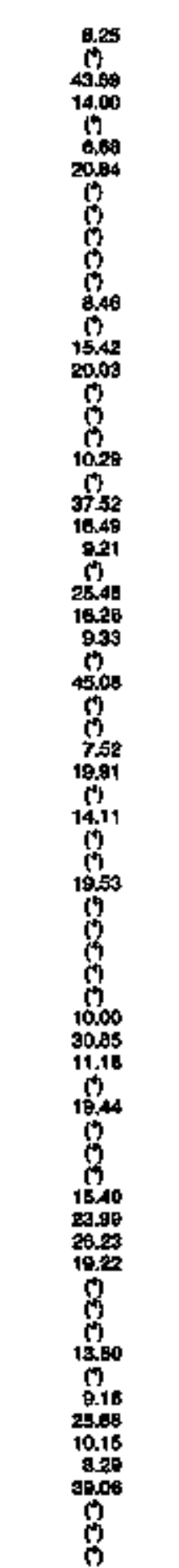 \\
\hline
\end{tabular}

Sea tootnaks al end al teble. 
Tabie 17. Class of Ownership, Number of Ultimate Consumers, Revenue, Sales, and Average Revenue per Kilowatthour for All sectors by state and Utilty, 1993 (Continuted)

\begin{tabular}{|c|c|c|c|c|c|}
\hline Etas & of & $\begin{array}{l}\text { Aumbar } \\
\text { of } \\
\text { contumar: }\end{array}$ & Revemus & 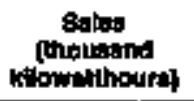 & 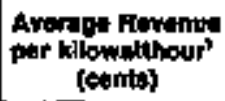 \\
\hline 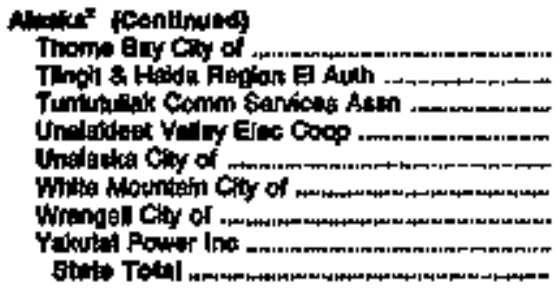 & 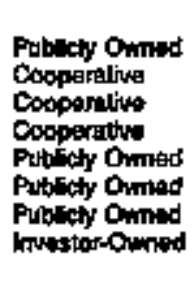 & $\begin{array}{r}235 \\
1.299 \\
77 \\
311 \\
549 \\
81 \\
1,310 \\
307 \\
299,716\end{array}$ & $\begin{array}{r}470 \\
3,306 \\
240 \\
860 \\
3,006 \\
250 \\
1,816 \\
1,044 \\
442,909\end{array}$ & $\begin{array}{r}1,870 \\
12,204 \\
549 \\
3,016 \\
20,912 \\
527 \\
21,495 \\
5,024 \\
4,934,047\end{array}$ & $\begin{array}{l}2500 \\
27,45 \\
6 \\
2984 \\
16,14 \\
19 \\
6,47 \\
20.78 \\
10.12\end{array}$ \\
\hline 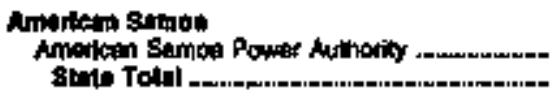 & Puterisy Owmad & $\begin{array}{l}8,418 \\
6,418\end{array}$ & $\begin{array}{l}16,454 \\
16,454\end{array}$ & $\begin{array}{l}91,673 \\
91, \text { st3 }\end{array}$ & $\begin{array}{l}17,05 \\
17.05\end{array}$ \\
\hline 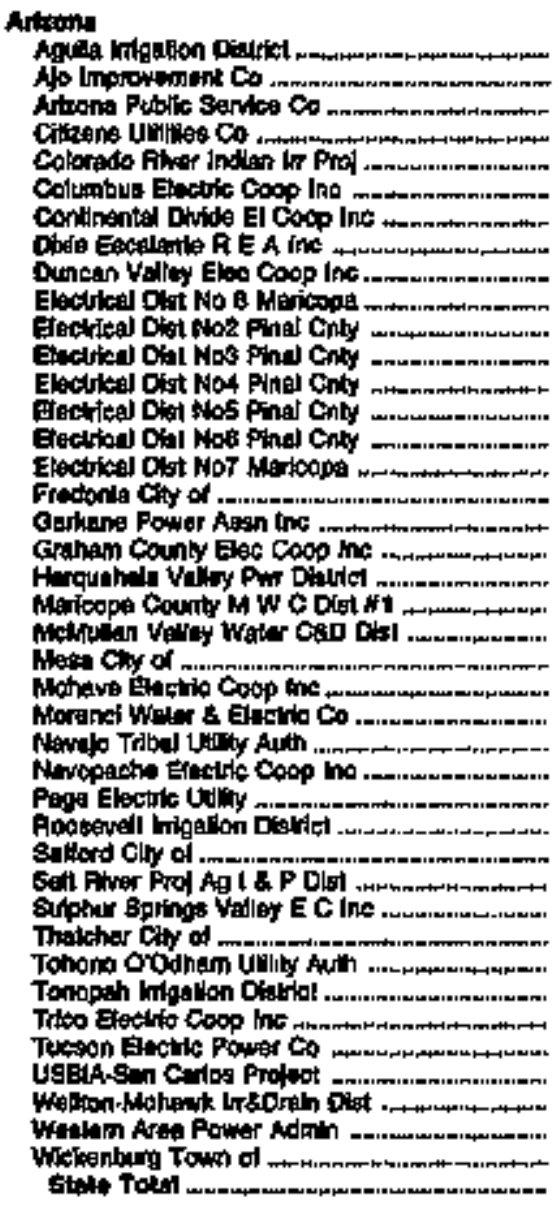 & 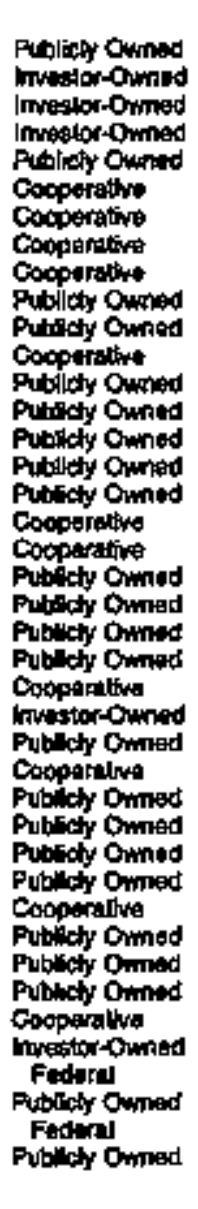 & 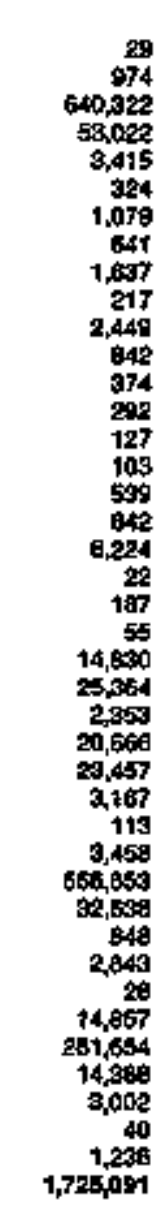 & 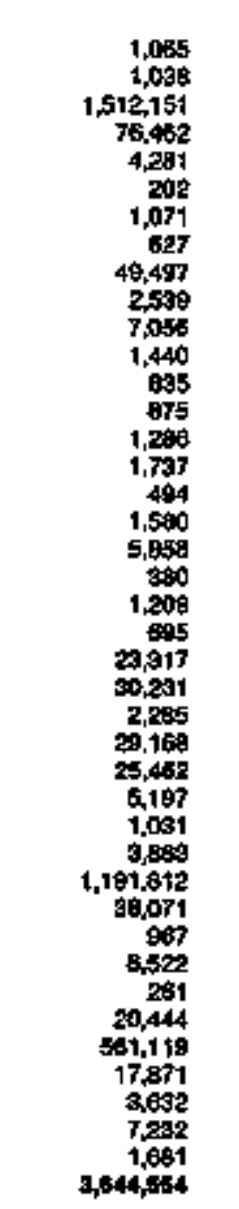 & 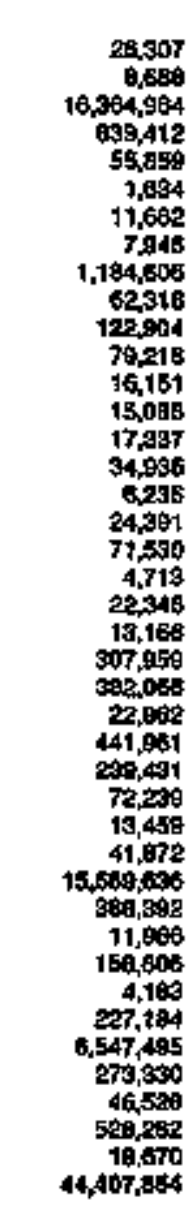 & 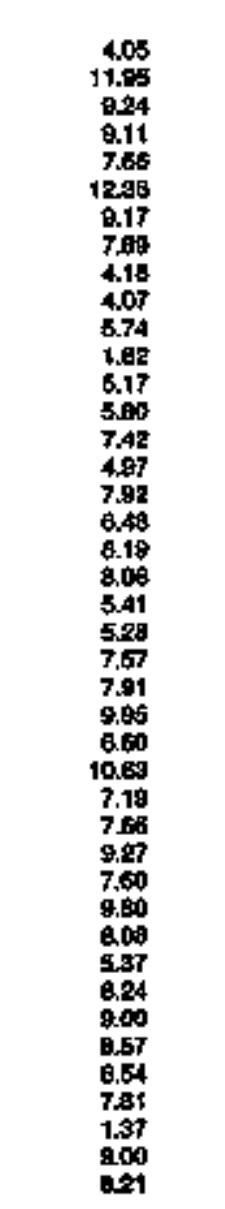 \\
\hline 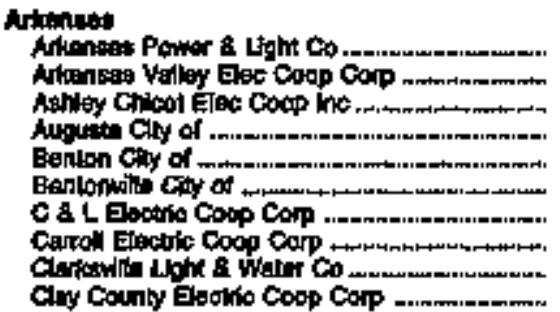 & 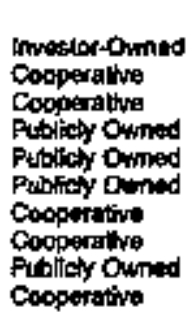 & $\begin{array}{r}590,571 \\
35,036 \\
4,317 \\
1,100 \\
6,572 \\
5,728 \\
17,348 \\
41,827 \\
3,0927 \\
10,701\end{array}$ & $\begin{array}{r}1,188,958 \\
40,995 \\
4,549 \\
1,028 \\
14,322 \\
9,177 \\
17,720 \\
49,997 \\
8,678 \\
11,008\end{array}$ & 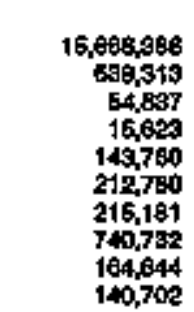 & 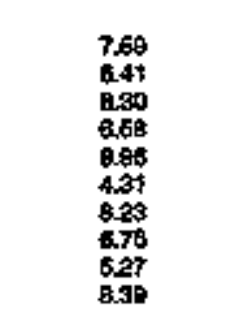 \\
\hline
\end{tabular}

Sere townolis at end of thise. 
Table 17. Class of Ownership, Number of Uttmate Consumers, Povenute, Salea, and Average Revenue per Klowatthour for All Sectors by State and Utllity, 1993 (Continued)

\begin{tabular}{|c|c|c|c|c|c|}
\hline $\begin{array}{c}\text { Stote } \\
\text { Electric Iilitity }\end{array}$ & $\begin{array}{c}\text { Cteog } \\
\text { of } \\
\text { Owperstitop }\end{array}$ & $\begin{array}{c}\text { Numbar } \\
\text { os } \\
\text { Cantumers }\end{array}$ & 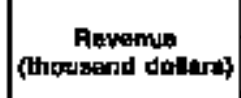 & $\begin{array}{c}\text { Salwa } \\
\text { (thoureand } \\
\text { balowithoura) }\end{array}$ & 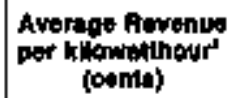 \\
\hline
\end{tabular}

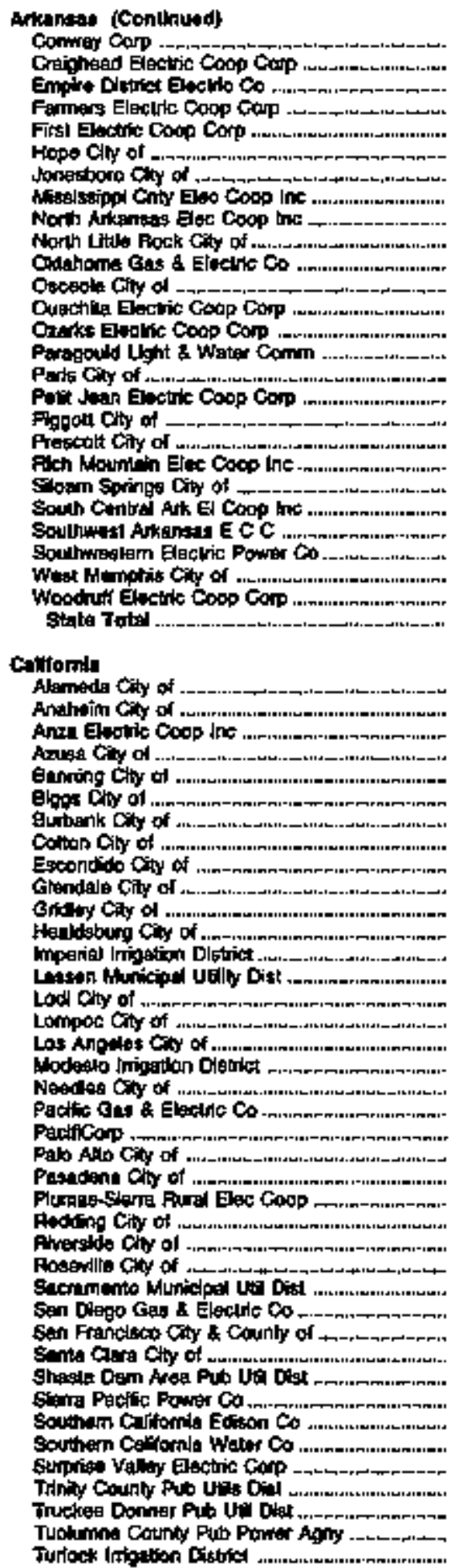

Pubcaty Ommed

Cooperallwe

Inwebtar-Owned

Copparalione

Coopereltyo

Pubscty Omined

Publicty Omned

Coopuratbr

Cooperafive

Putbicy Orwad

Investor Onwhod

Publocky Owned

Cocperallye

Cooperative

Puticty Onwerd

Publialy Onwied

Cooperrtlre

Publichy Owated

Pubricty Ohined

Cocperstime

Putbicty Dhwied

Cocparative

Cooperalive

Implester-Owmed

Puttichy Ohined

Cooperstive

Puticly Owingd

Putbedy Omned

Cooparation

Publicty Owitid

Publely Onwat

Putich Omped

Publichy Owried

Putrlesy Ownerd

Publichy Owmen

Publicky Owatad

Publely Omnded

Publichy Owrod

Putichy Owmed

Publichy Owand

Publlty Ownod

Publichy Owmed

Publlely Owint

Publecly Owmod

Putricly Owined

Imptor-Omed

Impother-Gmino

Publicly Owined

Publlyly Ownid

Cooparathe

Putricty Owned

Publely owned

Publicty Qumed

Publicly Owned

investor-Omed

Publlcty Owned

pubity dunted

Publlcly Oumed

imsator-Omined

Investor-Oment

invistor-Onined

Copperative

Pusich Omond

Publlcly owmin

Pubritit Qmined

Pubres owned
13.794

21,660

3,251

4,900

54,218

B, 5000

20.009

3,512

25,03\%

35,320

60.292

3,715

4.795

29,216

10,401

1,65

14,460

2,212

1,720

5,924

4,398

8. 110

21,0089

6),23:

11,313

18500

$1,193,947$

31,390

$+019,433$

9.497

14,194

8, 748

621

50,292

18,322

02,185

1,900

4.684

80,867

9.46日

21,

14,102

$1,357,679$

87,391

2,002

4,307,165

38, $\$ 15$

27,085

57,300

6.405

94.964

89,5的

24.917

469,181

1,125.부연

6
45,553

3.910

47.579

4,116,429

20,040

3,569

6.497

8,016

30
25,734

20,55

5,049

5,527

97.688

11515

32,16 舟

54,192

30,401

64,790

84,320

9,348

15,006

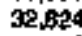

11,722

2,170

14,280

1,800

5,754

6,055

9,128

10,309

29,917

147514

18 ;99

20,918

$2,008,400$

43,550

201,522

4.320

17,16

10,493

635

88,158

$17,7 \mathrm{bB}$

92.715

2,430

6.927

173,290

13,966

$\$ 4,654$

12,111

$1,901,206$

127.204

4,149

$7,542,460$

52,551

83.68

97,681

B.ae日

485

152,771

44,051

512,85:

1,40e.014

24.462

171369

$\$, 706$

36,590

$7,0 \mathrm{~g} 2,014$

10,220

4,541

3,753

$B, 060$

1200
-7.814
174,367

292.009

109,280

60,40

1,0\$1,582

179,358

770.211

$1,357,144$

401,138

612341

$1,981,480$

173.951

189,350

488,374

350,304

43000

181,672

4), 4 \& 4

85,314

T1873

175,770

164,795

490377

2.

$290,75 \%$

258,659

$31,692,033$

475,265

1.409\%,016

29.769

18cie日a

100.639

5.980

062,160

204,527

124

998,212

27,000

61.9g9

2,135,060

127,774

381,890

116.789

$21,135,657$

$1.764,013$

61.518

$71,106,960$

745. ES4

1.045,009

$1,06-1,651$

68.438

636.5영

$1,545,424$

543,460

B.448.219

$45,065,549$

557.523

2. 214,915

59,114

480,634

60.565. 336

106,284

100.608

50,325

295.

21,300

1.170.1 18

54

B.0.

4.6

6.42

$4+14$

10

7.80

4.81

527

8.01

B.7

ज.56

S.05

7.80

4 朝

5.74

B.42

5.19

B.87

B.89

5.10

Bst2

GAS 
Table 17. Class of Ownership, Number of Uttimate Consumere, Revenus, Sales, and Average Revenue per Kllowatthour for All Sectors by State and Utllty, 1988 (Continued)

\begin{tabular}{|c|c|c|c|c|c|}
\hline 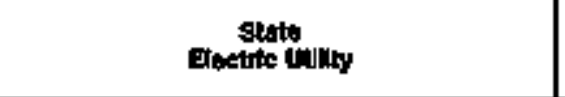 & $\begin{array}{l}\text { Cta: } \\
\text { of } \\
\text { Ommeraldp }\end{array}$ & $\begin{array}{l}\text { Numbar } \\
\text { of } \\
\text { Connumers }\end{array}$ & 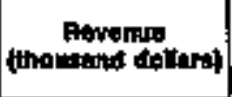 & 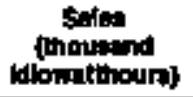 & 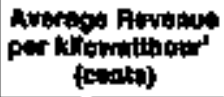 \\
\hline 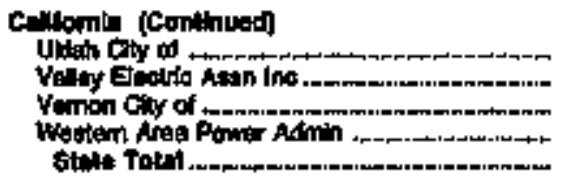 & 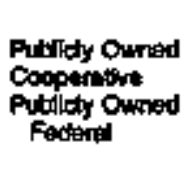 & $\begin{array}{r}7,710 \\
95 \\
2,052 \\
98 \\
12,300,812\end{array}$ & $\begin{array}{r}10,164 \\
293 \\
63,874 \\
72,213 \\
20,401,476\end{array}$ & $\begin{array}{r}89,545 \\
5,5 \% 9 \\
1,029, \mathrm{BO} \\
4,1+0,700 \\
210,498,060\end{array}$ & $\begin{array}{r}11.48 \\
5.29 \\
5.28 \\
1.76 \\
0.09\end{array}$ \\
\hline 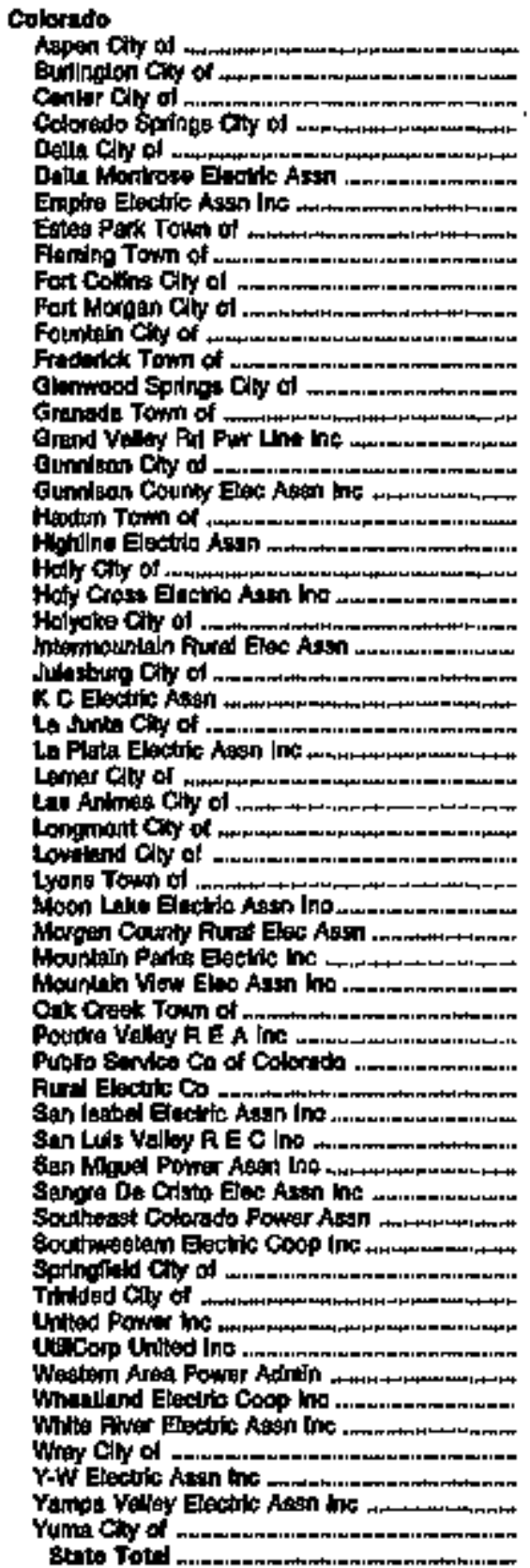 & 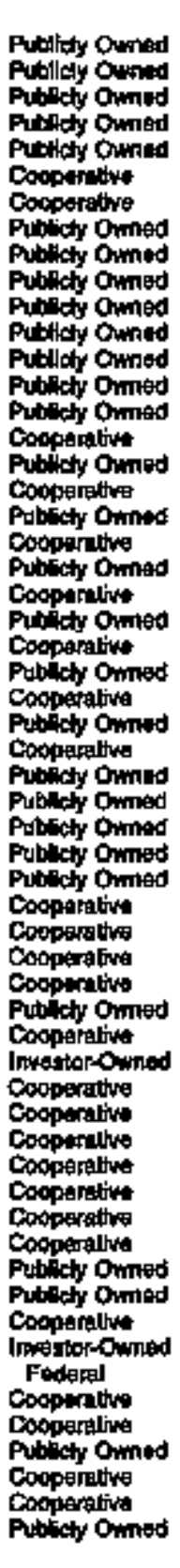 & 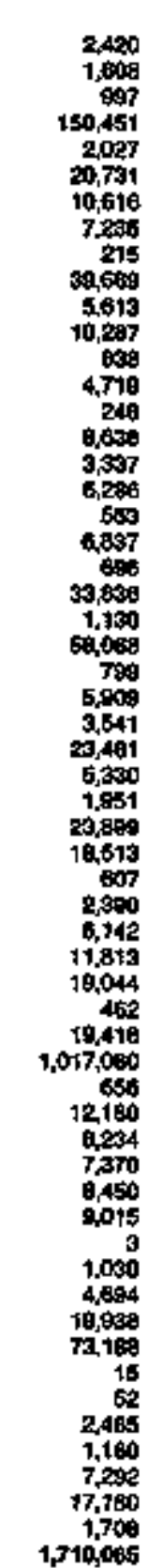 & 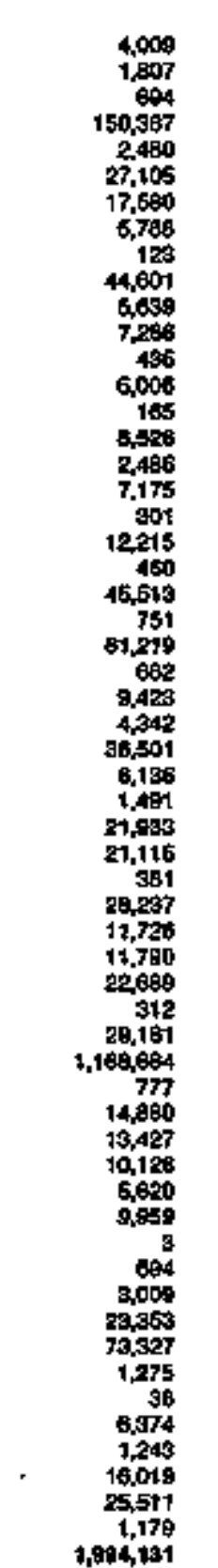 & 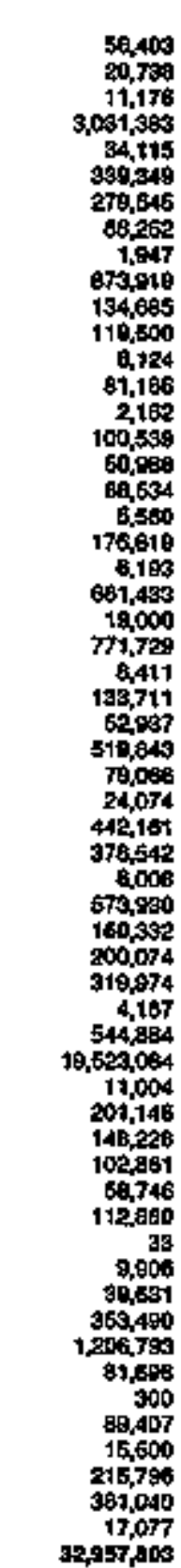 & 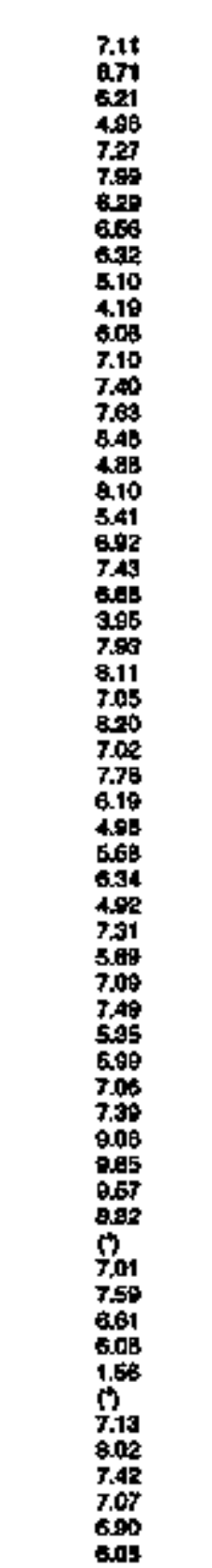 \\
\hline
\end{tabular}

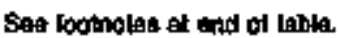


Table 17. Class of Ownershlp, Number of Ulitimate Consumers, Revenue, Sales, and Average Revenue per Kllowatthour for All Sectors by State and Utllity, 1993 (Continued)

\begin{tabular}{|c|c|c|c|c|c|}
\hline $\begin{array}{c}\text { Stake } \\
\text { Eleotrilo U1ality }\end{array}$ & 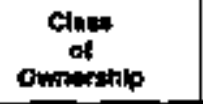 & $\begin{array}{c}\text { Nymber } \\
\text { of } \\
\text { Condumers }\end{array}$ & $\begin{array}{c}\text { Rovenure } \\
\text { (thourand tollara) }\end{array}$ & 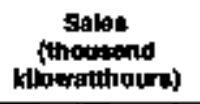 & 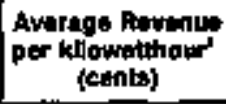 \\
\hline 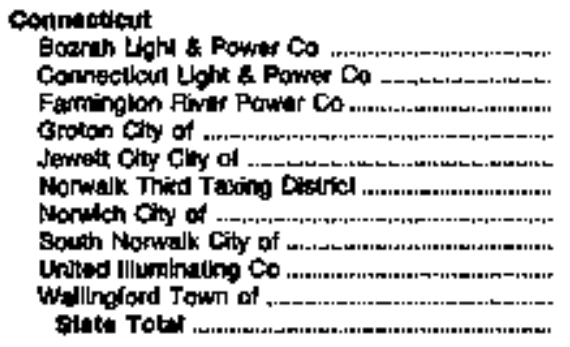 & 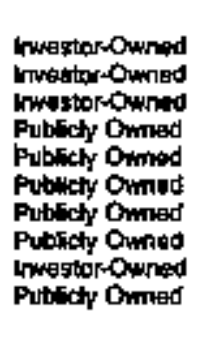 & $\begin{array}{r}2,225 \\
1.078,906 \\
1 \\
11,145 \\
1,904 \\
3,484 \\
17.701 \\
5,700 \\
304,854 \\
21,570 \\
1,447,692\end{array}$ & $\begin{array}{r}3,752 \\
2,062,100 \\
619 \\
44,804 \\
1,906 \\
5,657 \\
24,865 \\
7,372 \\
609,559 \\
40,377 \\
2,706,000\end{array}$ & $\begin{array}{r}38,672 \\
20,307,372 \\
26,060 \\
623,574 \\
19,197 \\
65,139 \\
207,010 \\
72,754 \\
5,290,328 \\
597,630 \\
27,230,497\end{array}$ & $\begin{array}{r}0.70 \\
10.15 \\
2.30 \\
7.10 \\
10.59 \\
10.28 \\
0.31 \\
10.13 \\
11.41 \\
7.51 \\
10.25\end{array}$ \\
\hline 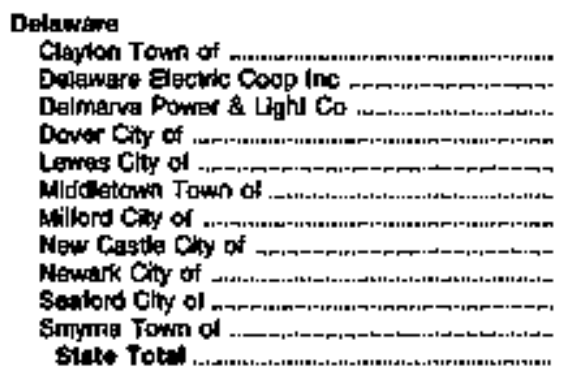 & 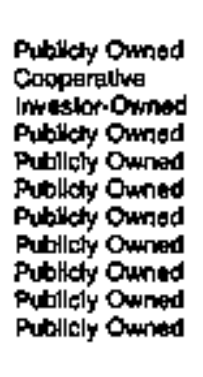 & $\begin{array}{r}\$ 11 \\
40,160 \\
209,137 \\
16,3 \% 6 \\
2,091 \\
1,847 \\
\$, 576 \\
2,066 \\
9,2672 \\
3,020 \\
2,418 \\
392,347\end{array}$ & $\begin{array}{r}639 \\
64,760 \\
467,214 \\
43,413 \\
4,062 \\
2,499 \\
+0,667 \\
2,291 \\
20,273 \\
7,210 \\
4,2013 \\
630,912\end{array}$ & $\begin{array}{r}6,009 \\
587,619 \\
7,360,309 \\
528,739 \\
51,094 \\
25,790 \\
138,729 \\
26,100 \\
274,240 \\
80,989 \\
44,512 \\
9,121,134\end{array}$ & $\begin{array}{r}10.43 \\
0.22 \\
6.62 \\
6.21 \\
7.64 \\
9.63 \\
7.05 \\
9.09 \\
7.42 \\
8.01 \\
9.44 \\
0.00\end{array}$ \\
\hline \multicolumn{6}{|l|}{ Dbatritet of Colpmbila } \\
\hline 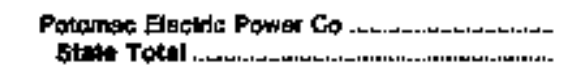 & imestpr-Omied & $\begin{array}{l}200,542 \\
220,642\end{array}$ & $\begin{array}{l}703,077 \\
703,077\end{array}$ & $\begin{array}{l}10,374,6,22 \\
10,374,622\end{array}$ & 6.78 \\
\hline 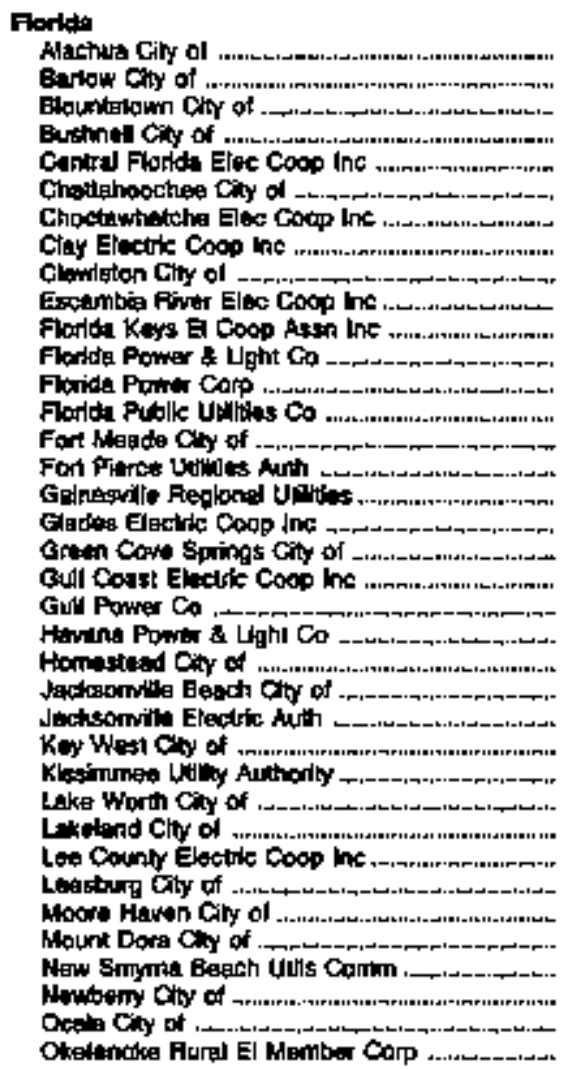 & 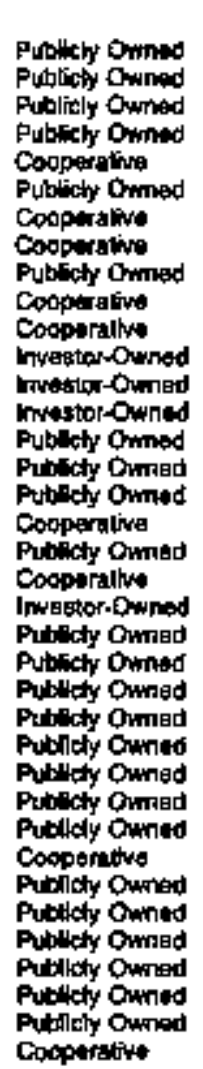 & 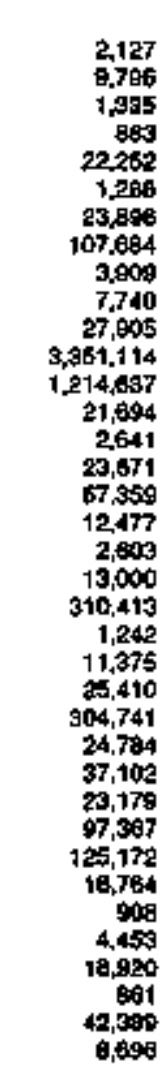 & 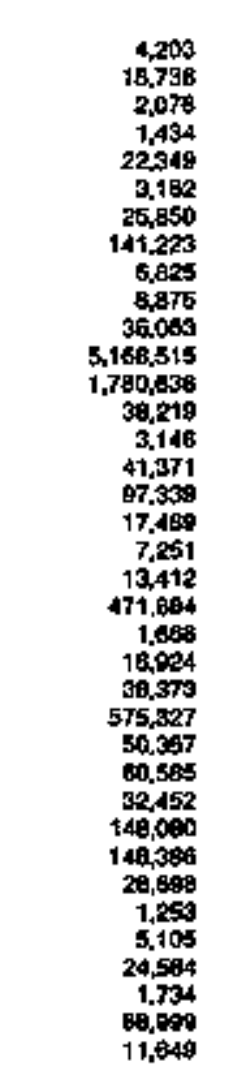 & 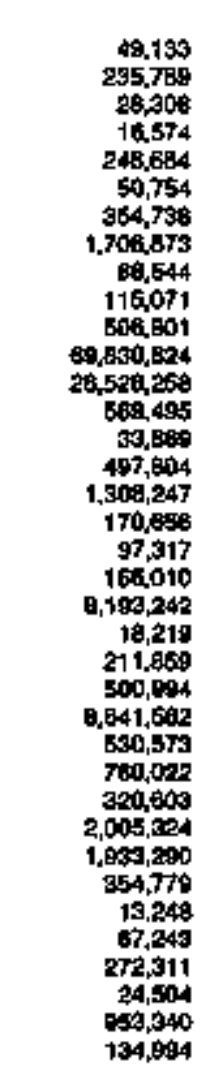 & 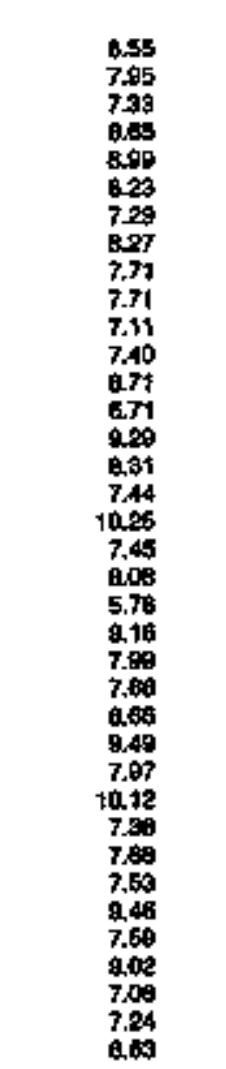 \\
\hline
\end{tabular}

Spe footnotis at and of table. 
Table 17, CAess of Ownershlp, Number of Ultimate Consimers, Revenue, Sales, and Average Revenue per KHowntthour for All Sectors by State and Utility, 1993 (Continued)

\begin{tabular}{|c|c|c|c|c|c|}
\hline Etrith & $\begin{array}{c}\text { Clais: } \\
\text { of } \\
\text { Oinntith }\end{array}$ & $\begin{array}{c}\text { Punits } \\
\text { of } \\
\text { Contoptitrs }\end{array}$ & Fiowenus & 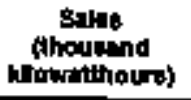 & 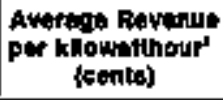 \\
\hline 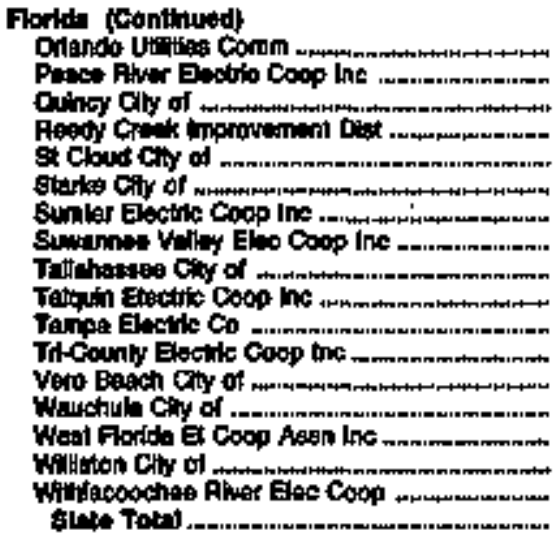 & 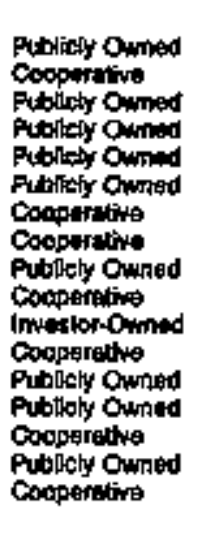 & 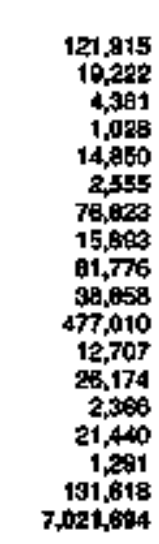 & 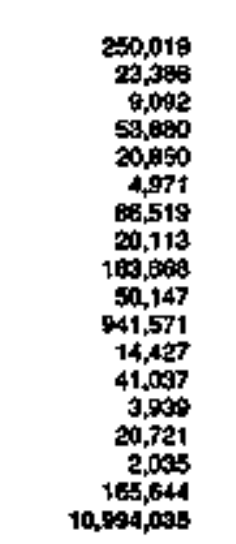 & 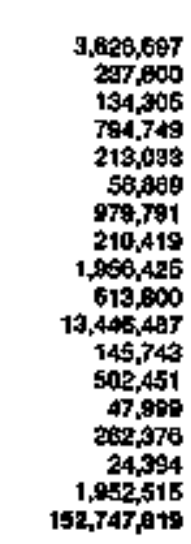 & 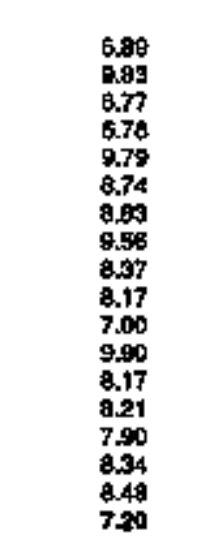 \\
\hline 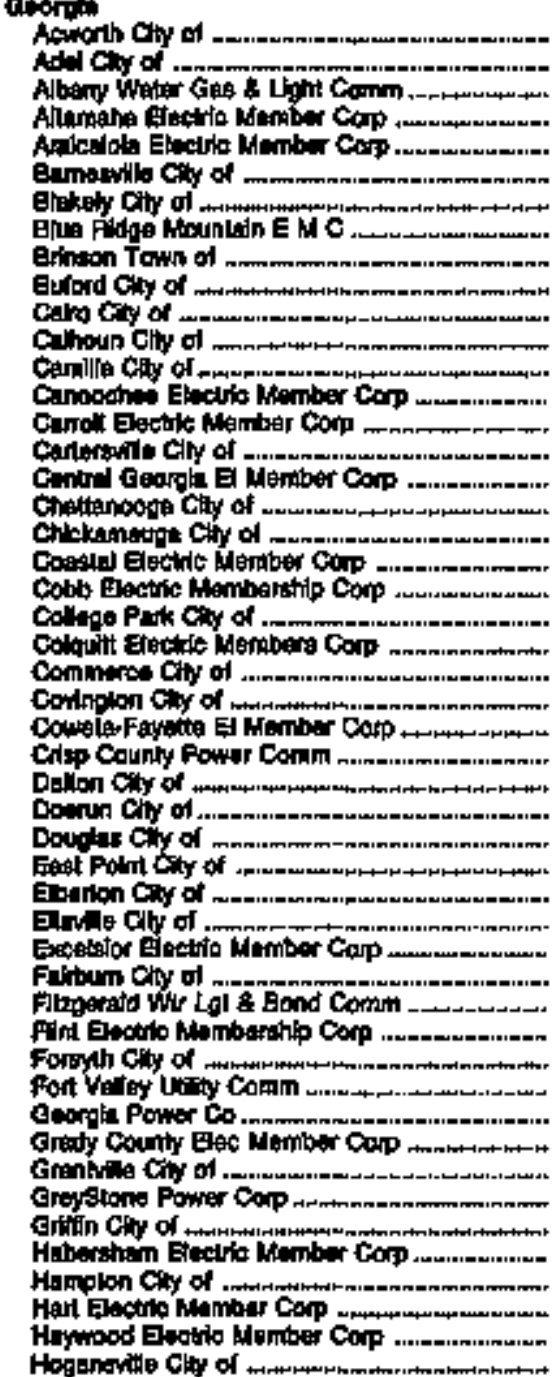 & 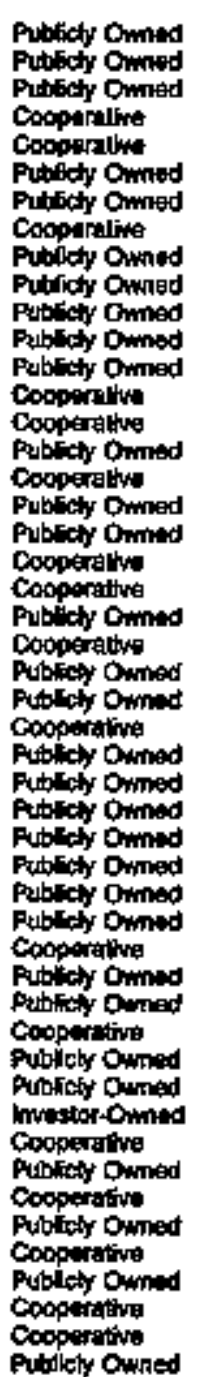 & 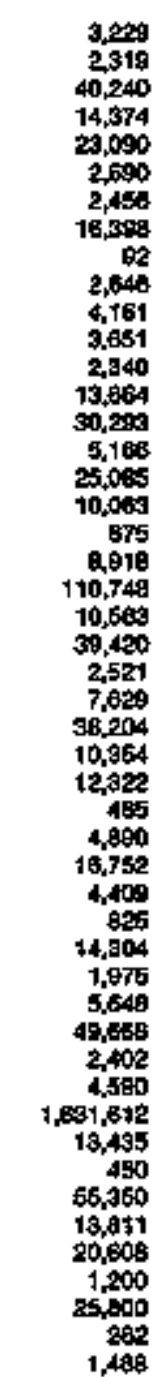 & 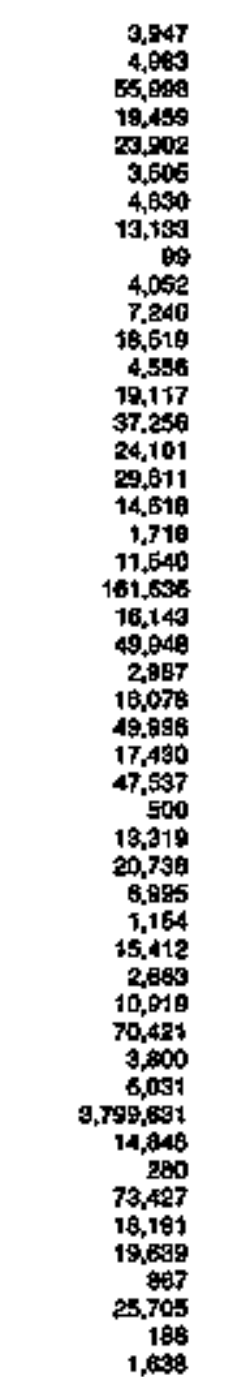 & 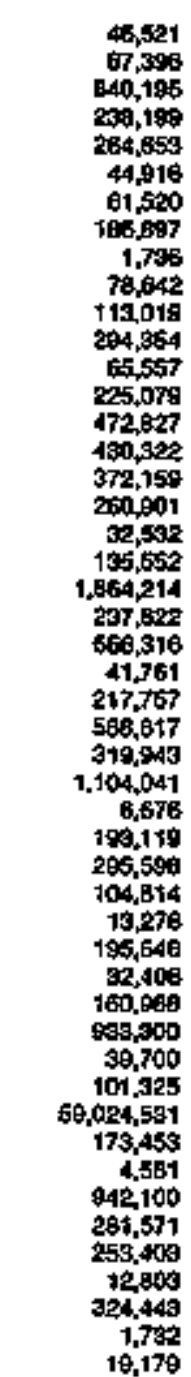 & 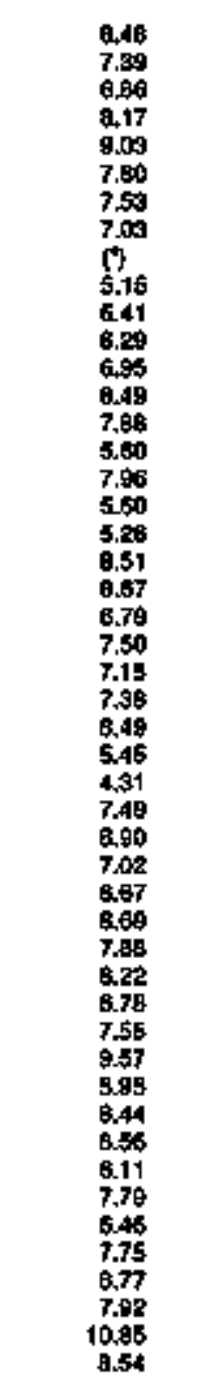 \\
\hline
\end{tabular}

Soet foptnoles at and of labls. 
Table 17. Class of Ownerahlp, Number of Ultimate Consumers, Revenue, Sales, and Average Revenue per Kllowattiour for All Sectors by State and Uthlty, 1998 (Continued)

\begin{tabular}{|c|c|c|c|c|c|}
\hline $\begin{array}{l}\text { stats } \\
\text { Elneile vituity }\end{array}$ & $\begin{array}{l}\text { Clatid } \\
\text { of } \\
\text { Onnerthip }\end{array}$ & $\begin{array}{l}\text { Mumber } \\
\text { of } \\
\text { Conumert }\end{array}$ & $\begin{array}{c}\text { Giavenus } \\
\text { (thoubiand dolters) }\end{array}$ & 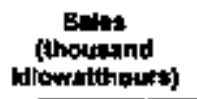 & 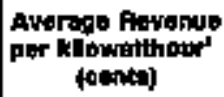 \\
\hline 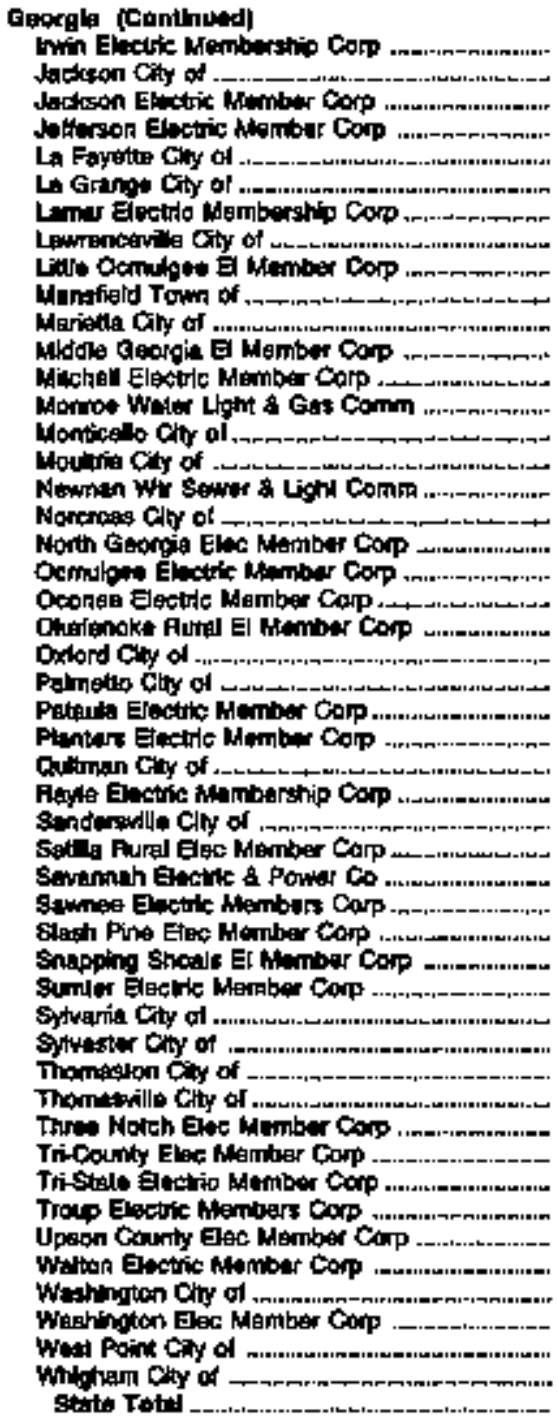 & 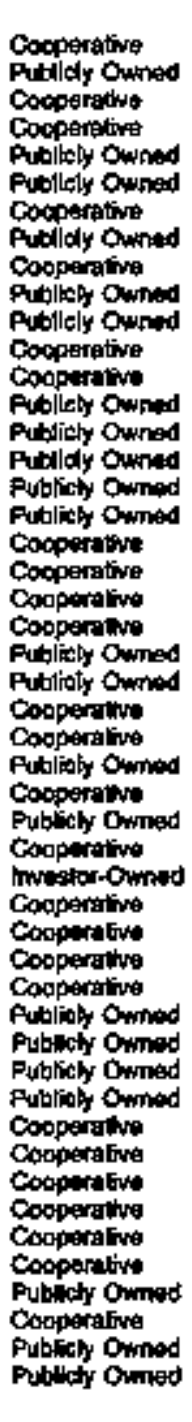 & 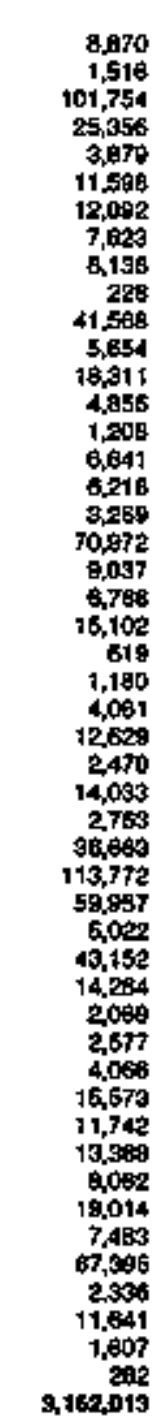 & 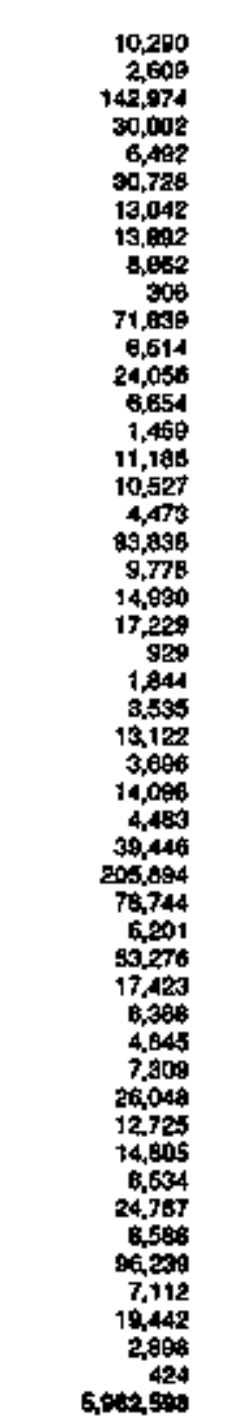 & 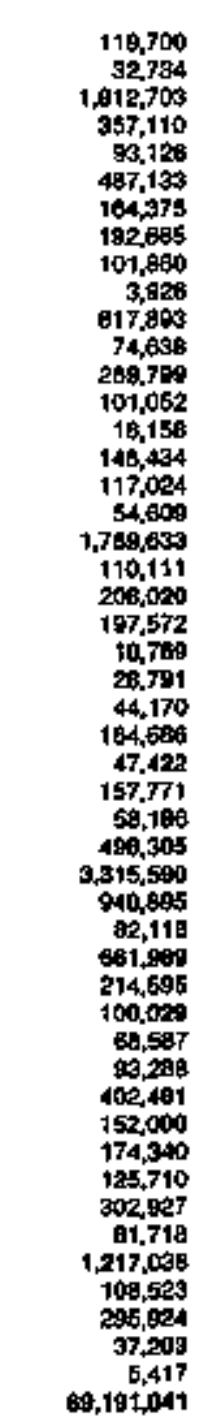 & 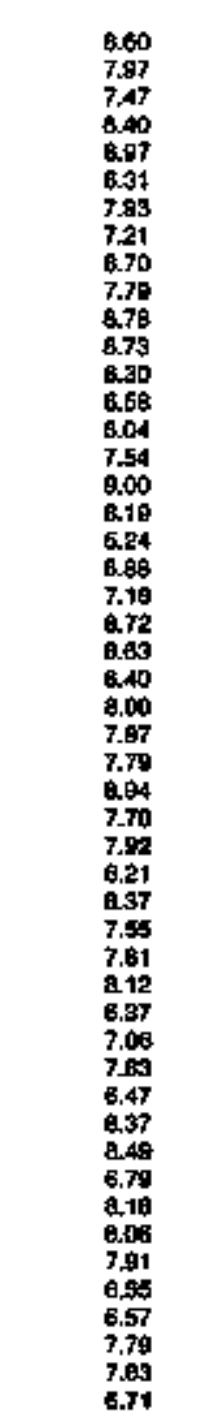 \\
\hline 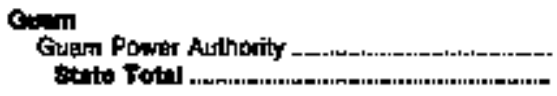 & Publicky Onied & $\begin{array}{l}37,838 \\
37,+8 ; 0\end{array}$ & $\begin{array}{l}135,777 \\
139,777\end{array}$ & $\begin{array}{l}1,492,119 \\
1,492,1 \div 8\end{array}$ & $\begin{array}{l}9.18 \\
0.10\end{array}$ \\
\hline 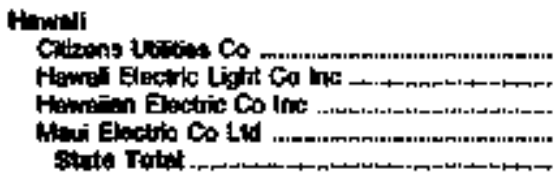 & 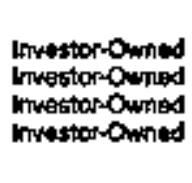 & $\begin{array}{r}20,340 \\
55,978 \\
200,113 \\
60,840 \\
300,2,04\end{array}$ & $\begin{array}{r}52,7190 \\
112,987 \\
644,0,99 \\
113,019 \\
02,797\end{array}$ & $\begin{array}{r}392,678 \\
802,070 \\
6,607,424 \\
915,760 \\
9,057,901\end{array}$ & $\begin{array}{r}16.87 \\
14.08 \\
1.75 \\
12.34 \\
16.90\end{array}$ \\
\hline 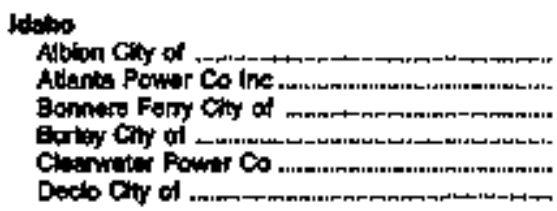 & 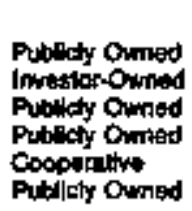 & $\begin{array}{r}175 \\
47 \\
2,011 \\
3,002 \\
7,000 \\
108\end{array}$ & $\begin{array}{r}97 \\
46 \\
2,729 \\
4,247 \\
1,2,29 \\
111\end{array}$ & $\begin{array}{r}2,130 \\
152 \\
34,110 \\
111,327 \\
137,327 \\
3,462\end{array}$ & $\begin{array}{l}\phi \\
0.20 \\
3.01 \\
0.22 \\
3.30\end{array}$ \\
\hline
\end{tabular}

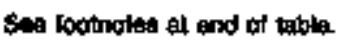


Table 17. Class of Ownershlp, Number of Ultimate Consumers, Revenue, Stles, and Average Revenue per Kllowathour for All Sectors by State and Utillty, 1993 (Continued)

\begin{tabular}{|c|c|c|c|c|c|}
\hline Etata & $\begin{array}{l}\text { Clase } \\
\text { of } \\
\text { Owmernhip }\end{array}$ & $\begin{array}{l}\text { Number } \\
\text { of } \\
\text { Congumbirs }\end{array}$ & 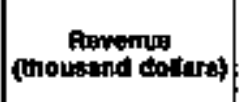 & 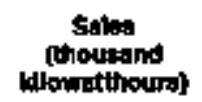 & 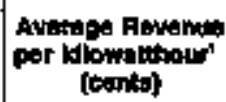 \\
\hline
\end{tabular}

idno tContinued

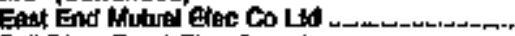
Fall Fiver Rural Elac Coop tho n..................

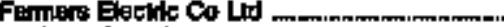

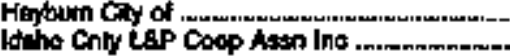

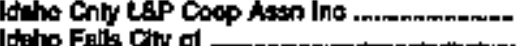
thino Fulis Chy of

Ithino Pown Co Kootent Electur Coop ins.

Low Biver Eloctric coop the

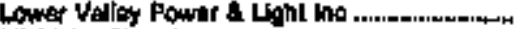
dinteloka chy of

Mresould Electric Coop ho .

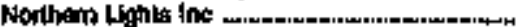

Pacesticon

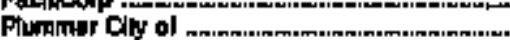

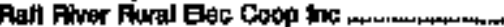

Fupest Chy of

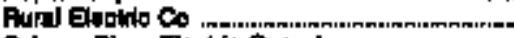

Behmon Phver Elactric Cocp Ine

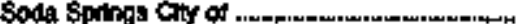

South SIto Electifo Lows ho .........................

Unily Loltx \& Fower Co .

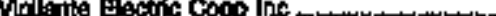

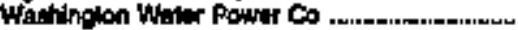

Weisar City of

Stete Toth

Ithole

Adems becincal Coop

Alany Cet of

Allandala City of

Atomani cay of

Betwovit Cly of ..

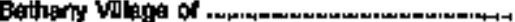

Eresis Cis of

Bushnell Chy of .

Chiro Publo Uth Comm .

Centise Cly of

Carill cily or

Cosoy City ol

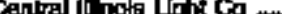

Cenlral Ithola Pub Sarv Co ..................

Chaham Vilogo of

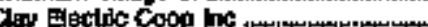

Clinion County enc Coop tho ...

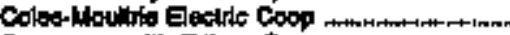

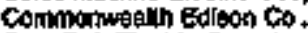

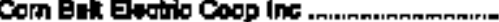

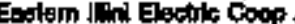

Edyg Elactrtc Cogp Assn.

Egpinn Eleotio Coop Asen

Fairitald Cty of

Faphers Muhul Electide Co

Flom Ciy of .

ing vilioge of

Genesos Cty or

Benture Cly of

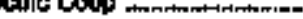

Helakend Ciny of

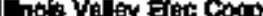

interstats Powat $C_{0}$

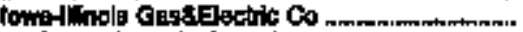

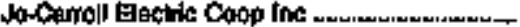

Ladd vilupe of
Copperative

Cooperative

Cooperative

Puttichy Oumed

Cocopefative

Putilery Owned

imvegion-Oimed

Cocoperativis

Cooperative

Cocoprathe

investor-Omine

furltely Owand

Cooporiliws

Coopenation

Inweilor-owned

Publicty Ownad

Cooperalive

Puhicly Owined

Coopuratise

Cooperalive

Putrichy Orwnest

Cosparatis:

Cocperabive

Coopentive

Intosior Onned

Publety Owned

Coopenative

Pubtlety Owned

Putilety Ownsed

Putlkely Owant

Publloly Onwred

Pitilkty Owrod

Publlay Owned

Pubillely Owosęd

Publlaly Owited

Publely Ownod

Pubinty Owned

Pubtholy Owned

Invesior-Omed

Impestor-Owmed

Pubtloly Owned

Cooperative

Cosperetive

Cooperative

Imisstor-Ommed

Cooperative

Cooperajive

Gooperentivis

Cooperative

P(b-lely Ownod

Punticly Owned

Cocperative

Putnthy Owinted

fustoly Owined

Pubiely Ourted

Pubicly Ownted

Cooperative

Pubuchy Owinad

Publloty Ouned

Itweglot:Owried

Cooperalim

Cooperative

Impolprowned

Impaker-Owmed

Cocopenatime

Pubirisly Owitud
540

6,702

124

1,117
3,377

20,809

295

802

11,340

2,093

1,155

54

9,170

55,814

709

1,819

2,70 영

2,8ed

2, 195

1,555

100
2,093

18

78,419

2,200

516,49

B,687

30

1,209

0,005

68

1,688

1,501

2,292

1,665

3,613

$1,8 \times 3$

悯, 300

314,345

2,616

2, 455

4,642

7,105

3, 2096,604

14,169

12,712

4,851

11,746

3,274

1,178

1,293

2,906

1,402

1,209

B,577

12

4,760

557,290

9.575

5,569

10,500

91,772

5,160

627
790

7.400

130

2,969

2,50:

20,169

400, BCF

768
12,014

2,469

2,346

46
65

B.

119,867

1,268

5,681

3.585

3.384

3.2.16

1.209

1,217

2840

115,579

1,044

749,568

$10+332$

200

214

1,445

13,08

$5 \times 3$

2,038

1,611

5,191

2,340

3,576

2,018

297936

518,200

2,364

4,234

10,146

12,277

$6,243,125$

22, ह18

20.557

7,072

14,379

4,14

1,021

1,605

4,007

1,717

3,101

0,097

14

79

7,360

$1,116,481$

12,121

6.2 21

15,400

110,539

5.775

720

17,131

101,173

3,984

100,541

55,608

612,6526

10.6757

13,159

212,020

0,603

S4,671

744
1.171

17730

3,035, 800

31,802

139,135

79,911

83,000

5 s.oen

22,386

5,500

71,514

107

2509,889

43,675

$19,719,640$ 
Table 17. Class of Ownership, Number of Ultimate Consumers, Revenue, Seles, and Average Revenue per KIlowatthour for All Sectors by State and Utility, 1993 (Conlinued)

\begin{tabular}{|c|c|c|c|c|c|}
\hline $\begin{array}{c}\text { 8tate } \\
\text { Finetrit Luthy }\end{array}$ & $\begin{array}{c}\text { clate } \\
\text { of } \\
\text { onmiershp }\end{array}$ & $\begin{array}{c}\text { mober } \\
\text { of } \\
\text { Computhers }\end{array}$ & $\begin{array}{c}\text { Rtwitnua } \\
\text { (thourand dolars) }\end{array}$ & $\begin{array}{l}\text { Salke } \\
\text { (Uhouteind } \\
\text { athowithoure) }\end{array}$ & 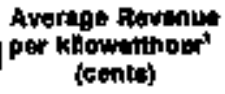 \\
\hline 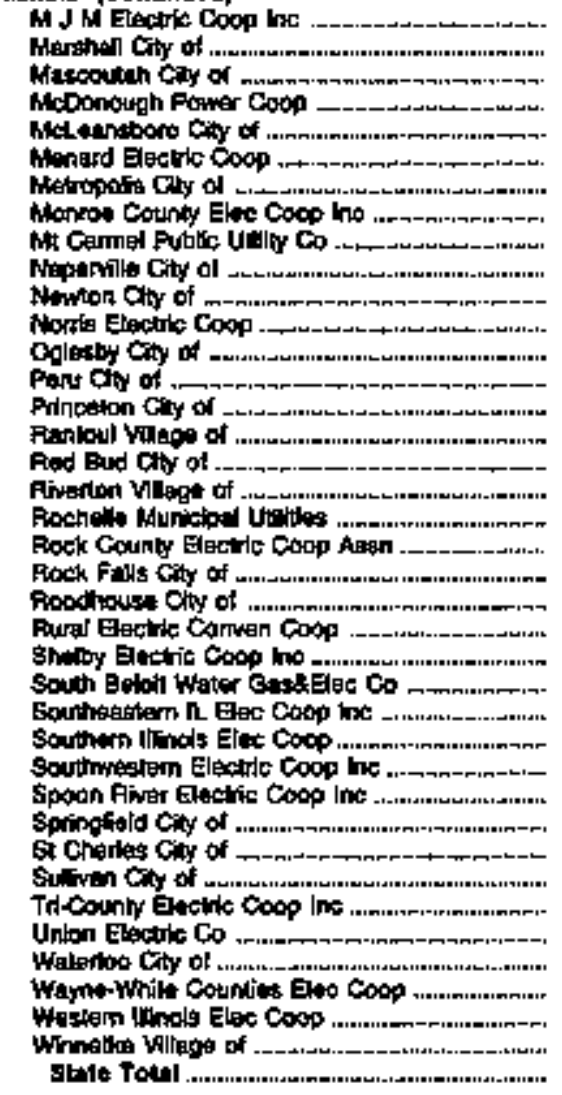 & 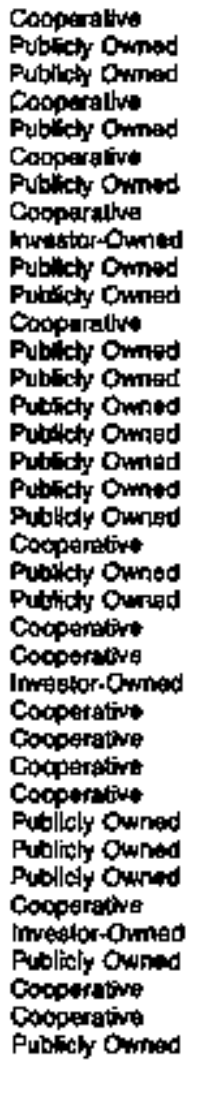 & 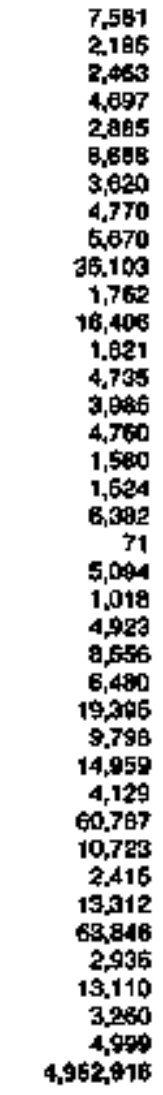 & 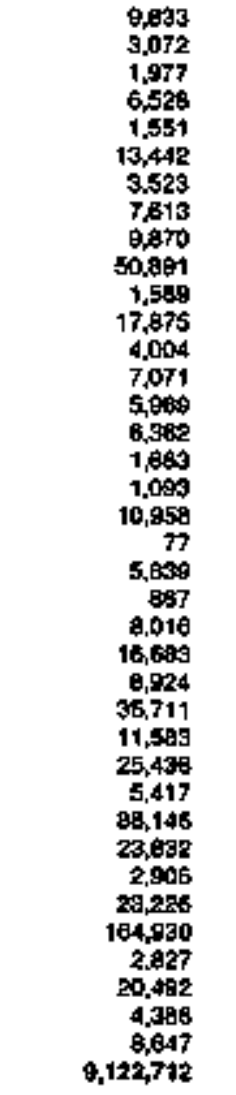 & 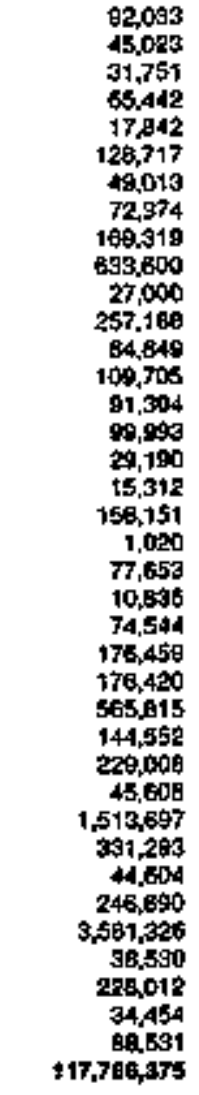 & 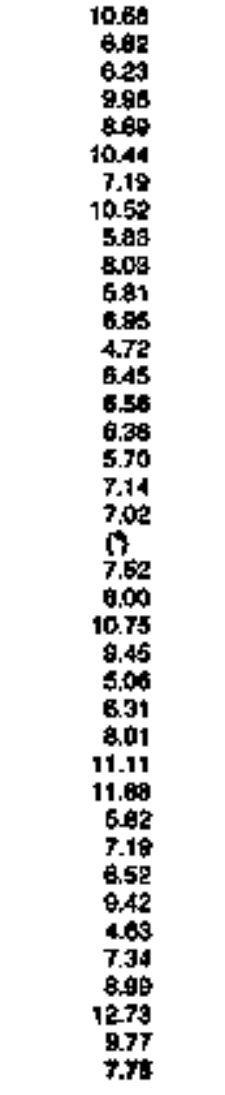 \\
\hline 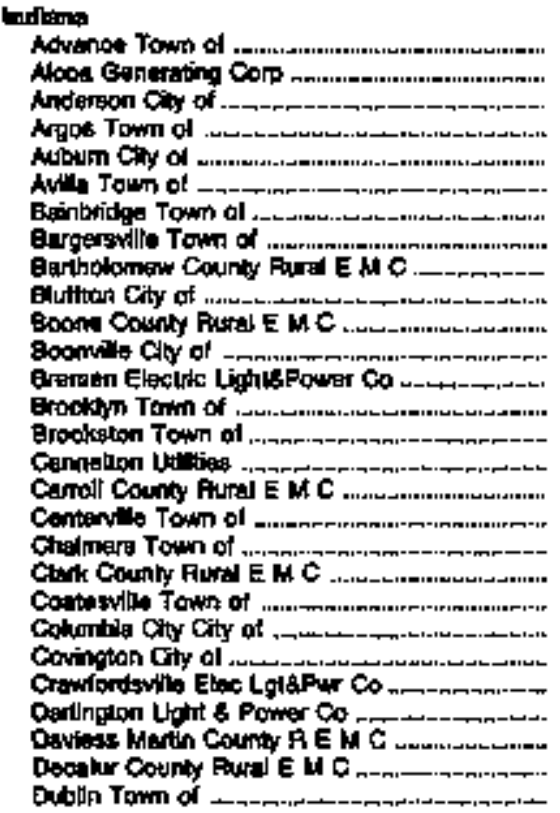 & 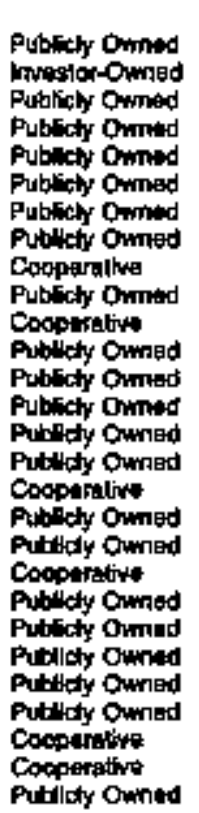 & 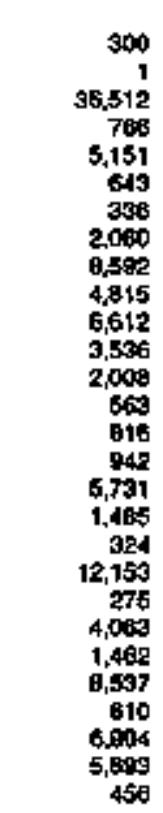 & $\begin{array}{r}300 \\
95,119 \\
38,228 \\
2,031 \\
15,617 \\
964 \\
270 \\
2,160 \\
12,077 \\
8,317 \\
10,155 \\
4,250 \\
4,071 \\
490 \\
589 \\
1,125 \\
7,655 \\
1,146 \\
205 \\
15,250 \\
215 \\
5,185 \\
1,402 \\
16,760 \\
805 \\
9,446 \\
11,641 \\
256\end{array}$ & 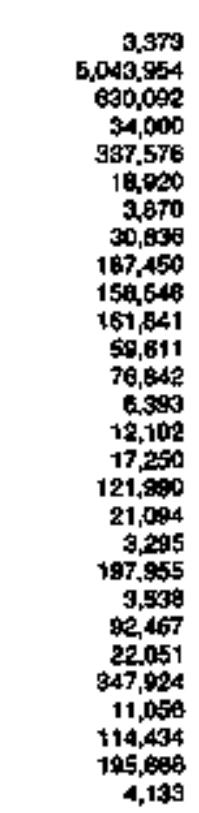 & $\begin{array}{l}0.89 \\
1.89 \\
5.75 \\
5.87 \\
4.63 \\
6.20 \\
6.96 \\
7.02 \\
7.21 \\
5.85 \\
6.28 \\
7.13 \\
5.50 \\
7.57 \\
5.49 \\
6.52 \\
6.42 \\
6.44 \\
6.22 \\
7.71 \\
6.09 \\
5.62 \\
6.49 \\
5.40 \\
7.55 \\
6.25 \\
5.60 \\
6.19\end{array}$ \\
\hline
\end{tabular}

Sae fooknotes at end or table. 
Table 17. Class of Ownershlp, Number of Uitimate Consumers, Revenue, sales, and Average Fevenue per Kllowalthour for All Sectors by State and Utllty, 1998 (Continued)

\begin{tabular}{|c|c|c|c|c|c|}
\hline Entiblo Utinty & $\begin{array}{l}\text { Cota: } \\
\text { of } \\
\text { Onineming }\end{array}$ & $\begin{array}{c}\text { Mumber } \\
\text { ou } \\
\text { Connumara }\end{array}$ & 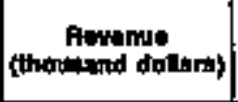 & 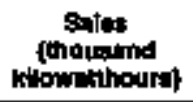 & 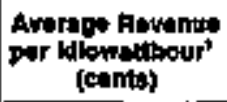 \\
\hline
\end{tabular}

Iaciane tcontinuwds

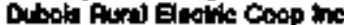

Dursinh Tom of

Enil croun Tor

Feyratio-Union County R E M C .

Ferdintend Jown of

Fians Town of

Frenldort chy of

Fremition Town of

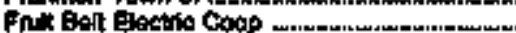

Fution Cotink Rural E M C

Bermath Cly of

Gat Chy cory of .

(1)

Hegertown Towi of

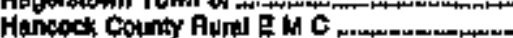

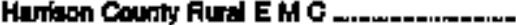

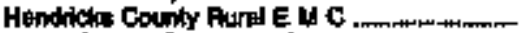

Hency County futral E M C.

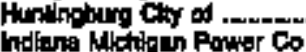

-

Indiantapolis Power \& L L ghit to

Jactoson Cotinly Pural E M C

Juriatomin Chy of

Jasper County Fural E U C

Jony County furad e is C

Johnsan County Rurd E M G.

Kankakipe Valey Furel E H C

Kingulord Holghis Town of

Kninhtown Tom of

Knox County Fur: E H C.

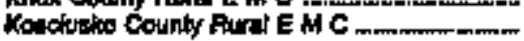

Ladoga, Tomm of

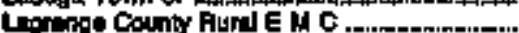

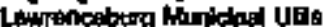

Lebmon $\mathrm{C}$ y of

Lewiswlits ciry of

Unton chl of .

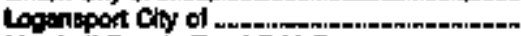

Metshid County Rurt E MC

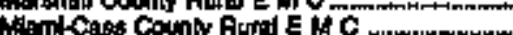

Matdelown Town of

Mishathot Cony of

Hen Gotible Town ol

Now Ross Cony of

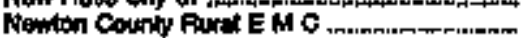

Noblo County Fund E M C

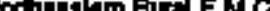

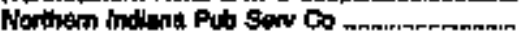

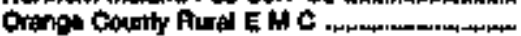

Puall Town al.

Perke Counky Raral E M O

Puldinopulman Goe Coop ho

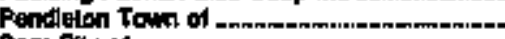

Pend Coty of .

Proboro Toun of

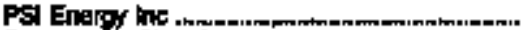

Rensistiter Gity of

Plohmond $\mathrm{Coy}$ of

Alomg oin Cat of

Packetitis Toin of

Ruth County Rurd E M C

scotieburg City of

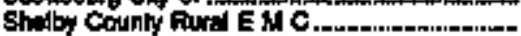

Sowh Central Indiand FEMC

South whitey Town of
Cooparative

Pubtuty Ominod

Putsictr Omined

Putichy Owned

Cooperativa

Pubictromed

Putichy Omed

Pulluch Dwned

Pisticty Owmed

Cosperativo

Cooperative

Putitiets Omned

Publety Owinad

Publecty Dwned

Fusilcter Owned

Publicty Oungod

Cocperative

Cooperatus

Cooperative

Cooperalive

puttldy Owned

Imestor-Owned

Investort Ommed

Comeratue

Publity Owated

Publledy Owred

Cocperation:

Cocoperatire

Cocperathre

Cooperetive

Putuldy Ouned

Putllaty Oured

Cocpentive

Cooperathe

Putildy Ommed

Coconretwo

Pulindy Ourned

Putllaty Onned

Puthly Onmed

Pubicty Omined

Pubscly Oumed

Coopenative

Coxperative

Publichy Domed

Puthicty Onimod

Putscty Ommed

Publicty Owned

Pubsicts dimed

Coopurathe

Cooparaltve

Cocperature

Invertor-Owned

Cooperative

Publicky Ownad

Cooperpifine

Cooperative

Putbety Owned

Publicly Owmed

Publich Omind

havertor-Owned

Putricty Ommed

Aubicly Omed

Pubichr Onmed

Publicty Onted

Cooperative

Publithy Owned

Cocoperatur

Cooperative

Fublisty Omad
10,197

151
2410

342

4,194

750

8,615

1,053

191

5.372

2011

2.856

1,865

5.653

I511

7,280

16,428

15,0.45

8028

2,900

400,145

395.671

18,654

536

5,591

6,220

5,024

11,601

18,848

696

1,604

8787

13,040

615

3,500

B,089

202

3,70

12,144

5,604

3.078

1,397

22,444

61

1,276

(1) 49

14,275

989,059

5,007

2614

10420

2317

1,085

10.353

还

610 t10

2759

20,657

1.434

1,484

4,783

2,962

6.821

24.790

97
12,655

79
4.256

20

4,702

1,412

1,293

14,

174

4.974

3,376

2604

3,773

8,721

1,170

9,040

19,770

18,102

Se17

4,500

642300

615,020

19,9g7

412

15,499

7,533

6,492

14,773

12,7e1

340

12,169

14,007

$44 a$

4 BA

7,249

118

3,400

18, 60

5,701

5,715

$\infty$

24.699

55

137

$1,7 t 7$

0,5as

20.974

019039

5.926

2,108

11,479

259

2,131

0.974

358

940.481

4,228

40.69

$1.20 \mathrm{~B}$

1,574

6,440

7518

ans

26,53

879

192608

1,173

2,480

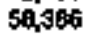

22005

20,812

29.102

16,05

2450

69,874

$\$ 1,221$

47,875

76,0 bio

165,606

20,276

120,434

295, 152 
Table 17. Class of Ownerahlp, Number of Ulitimate Consumers, Revenure, Sales, and Average Revenwe per Kllowatthour for All Sectors by State and Utility, 1993 (Continued)

\begin{tabular}{|c|c|c|c|c|c|}
\hline $\begin{array}{c}\text { State } \\
\text { Ebutetrle Uting }\end{array}$ & $\begin{array}{c}\text { Clot: } \\
\text { of } \\
\text { Ominstip }\end{array}$ & $\begin{array}{l}\text { Number } \\
\text { of } \\
\text { Contemers }\end{array}$ & Athoratend dollars) & $\begin{array}{c}\text { Saléd } \\
\text { (thousand } \\
\text { kilomathoura) }\end{array}$ & $\begin{array}{l}\text { Avorage fievenue } \\
\text { per hilonatihour' } \\
\text { (cama) }\end{array}$ \\
\hline
\end{tabular}

Iadioni (Combued)

Boundablain Indlavie P E M C

Southem indlang Gus 4 Etac Co

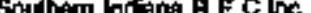

Solcolond Toum ol

Sileuben Counly Rurgi E M G

Siraughn Tom of .

Stivin Count Pue E W C

Tell City City ol

Thornderm City of

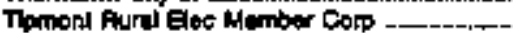

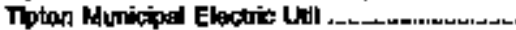

Troy City of ..............................................

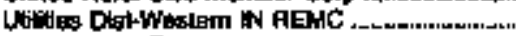

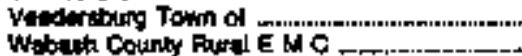

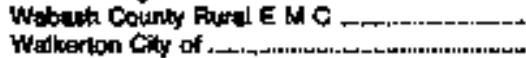

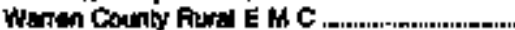

Waren Town of

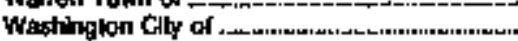

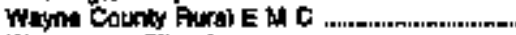

Wangrion City of

Whast Hamipon Go: a Ero Co.......................

Whili Counk Rural E M c

Wrlaminot City at

Winthitc Town of ,

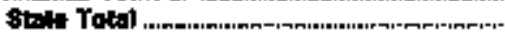

Ithat

Adoms County Coop Elextide Co ...r.

Atten City of

Akrin City of .

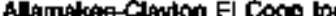

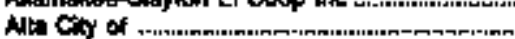

Alta Vota $C^{2}$ ty of

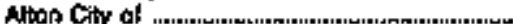

Amana Soclety Senvize Co

Aumos City of

Anila Gity of ...

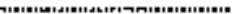

Aptinglion City of

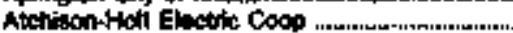

Altanite Cas of

Nytown Gity of _L__-

urratia ciny of

Bencrot Cty of

Ballontm cris

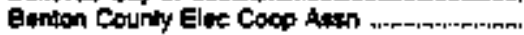

algalow ciny of .

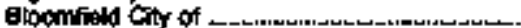

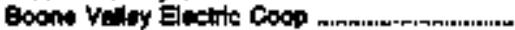

Bagda Givy of .

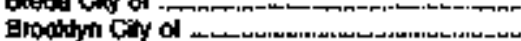

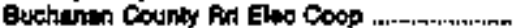

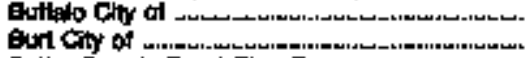

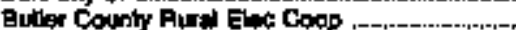

Calhoun Gawity Elac Coop Agan +

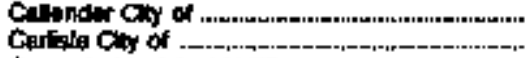

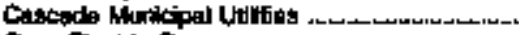

Can: Euction Coop

Cedar Falik Cty al ,...,

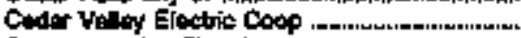

Chitton Yaloy Elec Coco InC .....................

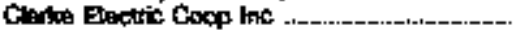

Cospon ciny of ....

Coon Rierids Chy of
Cooparaltwa

Imvegttic Owined

Coopurtity

Publecty Ounged

Copparative

Putrictr Omined

Cocoeratur

Publety Ountid

Pubilety Oinad

Cocparaltur

Pubcacty Onmined

Putildy Oungd

Coopertation

Coconentat

Publely Ormigd

Coopatativa

Publidy Ohwhed

Cooperative

Publkty Ounied

Puticty Oimed

Coeperathe

Putlicly Owned

Invteror-Owmed

Cooperative

Pulicly Ounod

Publitis owned

Cocpostativa

Publaty owned

Publebs owned

Puticisly Owhed

Cocperathin

Pubitis Dwmad

Publect Dimnd

Fublich Owned

invessor-Orwed

Publisy owned

Publest Owmed

Fubllaty Ommad

Publect Ownad

Cooperatur

Publicty Owmad

Pulpicty Onime

Pubrent Owmed

Publecty Owmas

Publaty Cunad

Cooperative

Publety Ommed

Pubictry Oimned

Cooperaliny

Publety Owmat

Pubraty Cirted

Cooperntwa

Pubichly Ommed

Putcict Gimad

Cooporative

Coxpraraline

Pubilery Orined

Pullcty Owrad

Pubricty Omined

Cooperetho

Phildy Owned

Cospotitint

Cocperrethr

Coxpetatis

Publioly Owined

Putulky Ouprad
117,303

7,411

7,307

174

5,692

3,059

841

15,707

4,5000

373

g,600

16,2017?

1,133

A,

1,256

4,0991

725

6,800

5,609

506

7,158

的1

1,098

3,555,796

1,egs

462

819

3,536

8,02:1

tos

180

$8+2$
17.122

617

x6:

513

1.006

4.958

172

BS4

Brs

1,454

$\mathbf{3 . 4 4 1}$

16

1,445

20

77

3.490

496

乡?

4,707

1,655

1.476

B54

82

$14,6 \times 62$

2,

5. ऊ

4,207

글

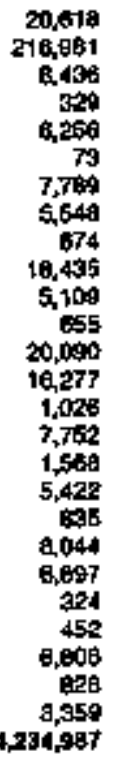

2.,445

369

8,616

590

1 르

SBS

4 ats

20,112

378

339

1.147

4,030

119

171
548

1.10

4.590

18

1, 2 宛

142

280

693

6.137

240

7,179

2, 200

I41

1.0

1.009
819
05

13,081

3,970

\$.139

5,147

345

15.5

280,540

4.254,673

115,391

6,366

a 241

1,210

1) $30 \mathrm{Bg}$

94,264

11, 820

250,580

91,393

8.542

357,312

200,792

17,048

116,905

66,002

64,047

14.208

144,842

01,024

4.006

6,101

60,866

9.245

53.22?

61,831,AB0

25,487

5,035
$+0,265$

B5,481

117,534

11, RE?

1, 1960.

7.479

79,50 ?

\$67,269:

7,627

4,806

4, 920

14,202

Bว,910

1,303

7,208

9,377

14,655

57,500

260

2,250

4,200

a,

79,194

\$,471

3,330

8)

24.

3,805 
Table 17. Class of Ownershlp, Number of Utimate Consumers, Revenus, Sales, and Average Aevenue per Kilowatthour for All sectors by State and Utility, 1993 (Continued)

\begin{tabular}{|c|c|c|c|c|c|}
\hline $\begin{array}{c}\text { Btedo } \\
\text { Elechidt Utipty }\end{array}$ & $\begin{array}{l}\text { Cila: } \\
\text { of } \\
\text { Ommerthip }\end{array}$ & $\begin{array}{c}\text { hurnhor } \\
\text { of } \\
\text { Contument }\end{array}$ & thourand dottand & 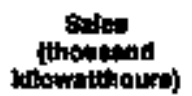 & 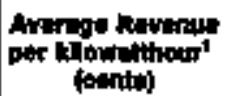 \\
\hline
\end{tabular}

tow tcontinuta

coming City of

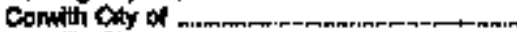

Damitl Ciny of

Daryon City of .

(1)

Demper Cy of .......-.

Dike Chy of .

Durank Coy of

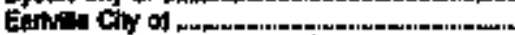

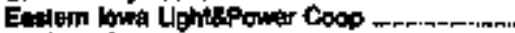

Eldidet Ciny of

Elaworth City of -

Esthenalo Cay or

Fitibank Ciny of ..-.-_.

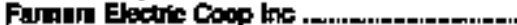

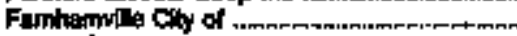

Fonda Cisy of

Fonlanots Cly of

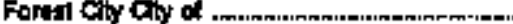

Frarkiln Rurat Eloctric Cocp

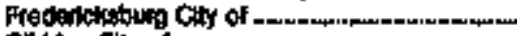

cindutun Ciny of .

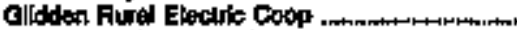

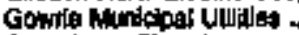

Greatinger Chy of

Ginfion Ciy of

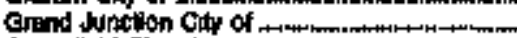

Geentivid Ciny of

Gendy Conter Givy of

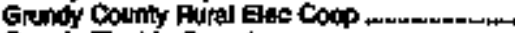

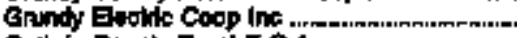

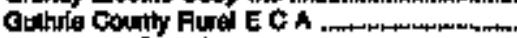

Gitionbatg Chy of

Henoodk Courly Rurel Eloc Coop ....-...nm...m

Herlan City of .

Harisan Count fit Elog coop ................

Hartley chy of

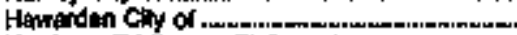

Henkerse TitCounty El Coop inc n...m.

Hintalt Cly al

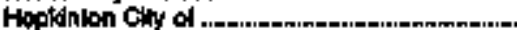

Hutson City of

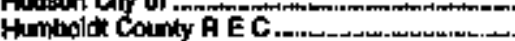

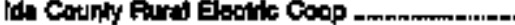

indsperiotons City of

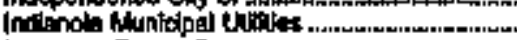

Interedelo Powrer Co

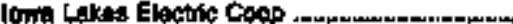

Iowa-llinols GessElwotic Co ...................

IES UITing lite

Keosannis Coty of

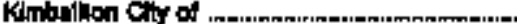

La Parta Chty ofty of

Lele Hits Ciny of .

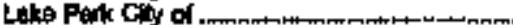

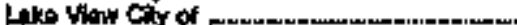

Lamonl Clty of

Latchwod $C_{t y}$ of

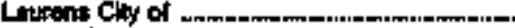

Lauler cay or

Lohigh chy of

Lonex Coty of

Unn Count Plut E c A

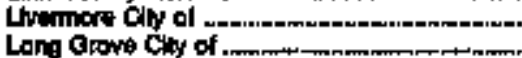

Lyin Auml Eletelto Coop

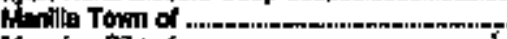

Manning Cily of
Pubticty Owned

Pulithy Oumed

Publlety Onined

Putaldy Owned

Puthlicty Owoned

Pubiloty Owined

Publut onned

Putlyy Ormed

Publaty Oninted

Pubalcty owned

Cooperathe

Publlady Ounted

Pubully Gimat

Putrity Oumed

Publkty Omined

Coopersulve

Putildy Ouned

Pustcty owmed

Pubiluy onmend

Fubloct Onmed

Cooperzitive

Pubtlaty orimed

Publkty Orwed

Cooperathe

Pukichty Ónned

Publichy Ormed

Fubichy Onmed

Pulticty onned

Publlety Onened

Fubledy Ommod

Cooperaliva

Cooperithy

Conperalitue

Pubiluty Oimend

Cooperathe

Pubichly Ownod

Cooparathe

Pudicty Ommed

Putley Oumed

Coopurstero

Pubristy Owned

Pubinty Owned

Putalicty Omed

Cooperalive

Cooparalys

Putily Oumed

Publuty Oined

Imvestor-Omaned

Cooperalina

Imwitor-Owned

Irwestor-Owned

Pubity Owned

Fublichy Ownes

Publicty Owmats

Publicty Onmod

Puscicts Ounned

Pubicty ormed

Fublety Ouned

Pubrifly Owmed

Publictr Owned

Futsichy Owmed

Pubicty Omind

Fukilety Oumat

Cocperativit

Fublucty owned

Futhlaty Ommed

Cooparalive

Pubilcty Onined

Pubulty Owned
1,006

216

432

3.179

3.779

389

B41

72

394

19,654

1,378

tra

3.185

497

4,618

207

487

2,051

1.458

499

595

t.659

616

489

149

597

trats

1.359

2,271

368

4,510

1,276

1.816

2,742

2,902

BSo

1,304

5,639

25

389

654

1,837

1,169

281

5,111

110,813

$17,05 \%$

118,018

\$25, 876

Ext

212

1,005

7.180

1,081

347

888

200

285

786

11,381

252

201
1.708

1.708

842

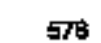

178

50

357

4,002

510
364

856

ses

297

24,289

1,549

484
2,188

ase

7,599

291

250

3,089

3,043

1,680

990

2,607

530

324

s9e

1.480

1,518

9.672

273

5,201

1,037

3,284

2,987

3,774

811

1,200

7,745

285

785

90
8.101

2A71

3,200

4,158

178 . fits

$17, a$ are

190,247

409,551

653

108

742
1,845

4

1,843

1,2010
284

806

194

188

19,757

215

127

1,473

$15,7 / 25$

1,868

4,704

4,612

115,051

9,119

4 ,en

11,309

7,770

3,854
73,108

18,474

5,88 ;

45,190

B,429

78,379

$3,82 \%$

4,570

5,614

60,207

34,450

35,019

7,880 
Teble 17. Class of Ownership, Number of Uitimate Consumers, Revenue, Sales, and Average Revenue per Kllowatthour for All Sectors by State and Utiltity, 1993 (Continued)

\begin{tabular}{|c|c|c|c|c|c|}
\hline $\begin{array}{c}\text { State } \\
\text { Clecte Ut:ity }\end{array}$ & 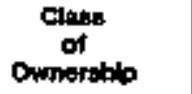 & $\begin{array}{c}\text { Number } \\
\text { of } \\
\text { Conouniters }\end{array}$ & $\begin{array}{c}\text { Ravanut } \\
\text { [thourend dotiars] }\end{array}$ & $\begin{array}{c}\text { 821as } \\
\text { (thpriptind } \\
\text { idlowethours) }\end{array}$ & 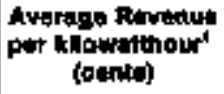 \\
\hline 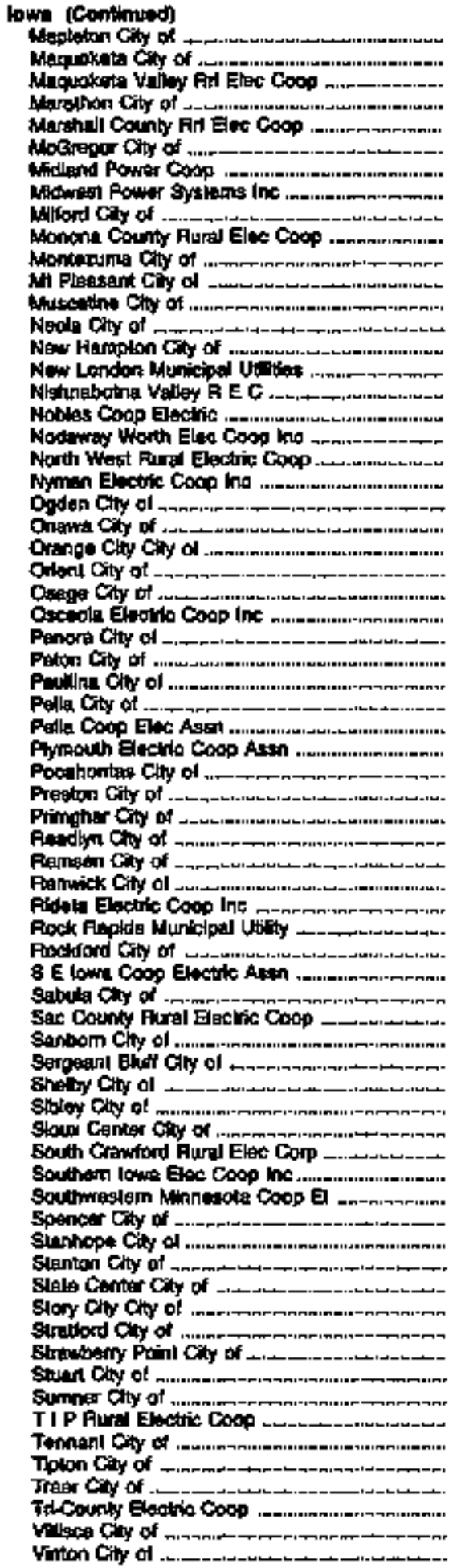 & 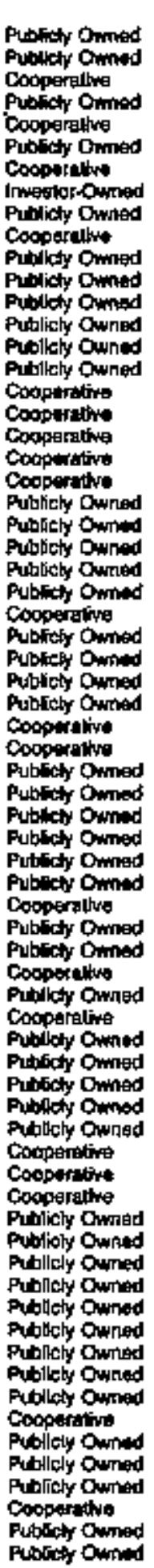 & 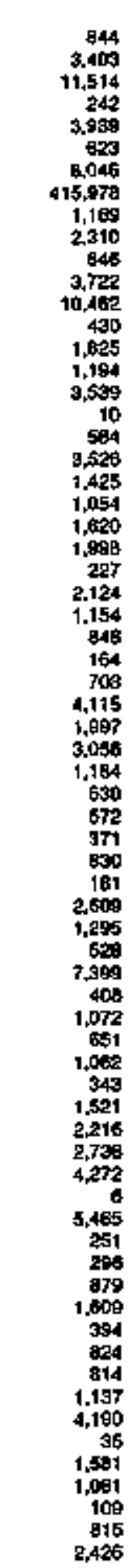 & 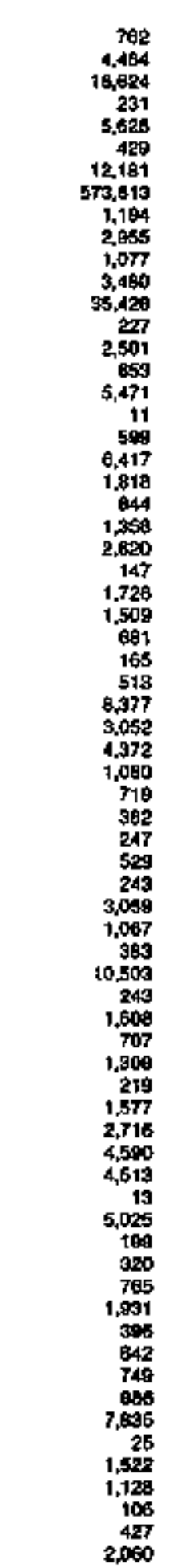 & 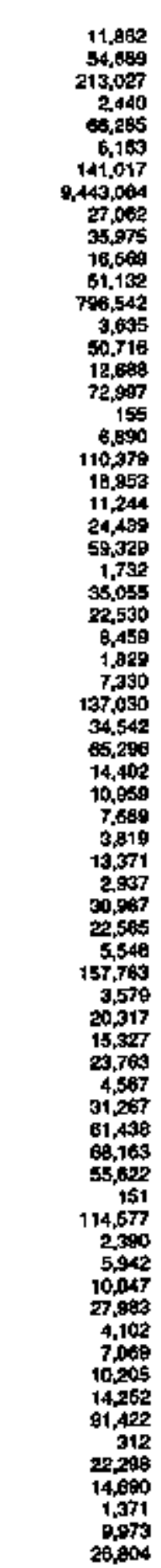 & 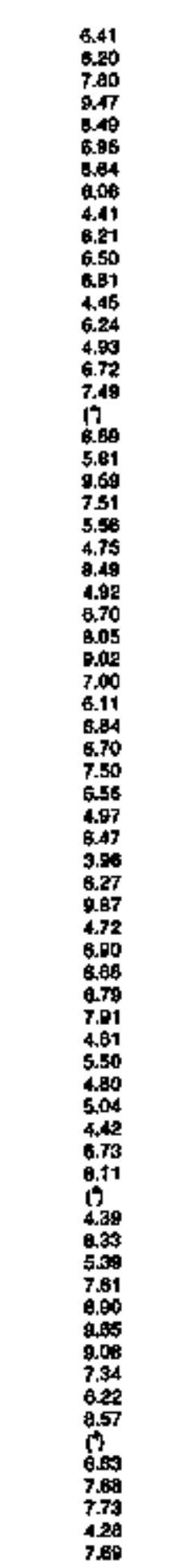 \\
\hline
\end{tabular}

Ses botnoter al end of table. 
Table 17. Clags of Ownerghlp, Number of Ultimate Congumers, Revenue, Sales, and Average Revenue per KHowatthour for All Sectors by State and Utilty, 1993 (Contlnued)

\begin{tabular}{|c|c|c|c|c|c|}
\hline 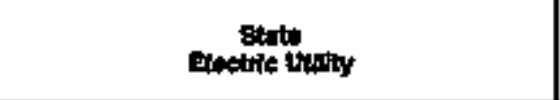 & $\begin{array}{c}\text { glate } \\
\text { of } \\
\text { Orwiternip }\end{array}$ & $\begin{array}{c}\text { lintabet } \\
\text { of } \\
\text { Contiumers }\end{array}$ & Renonve & 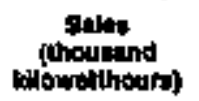 & 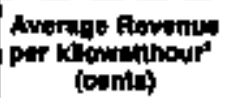 \\
\hline 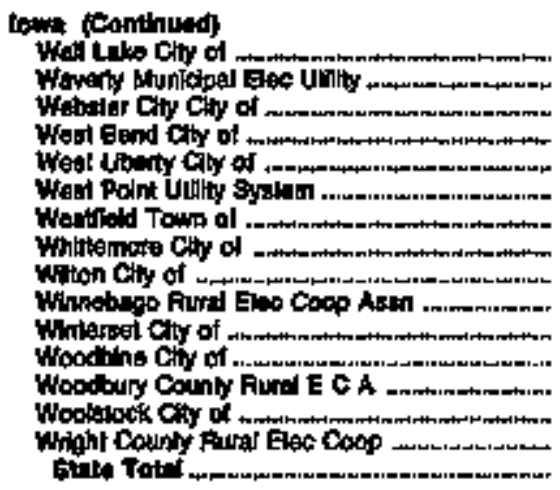 & 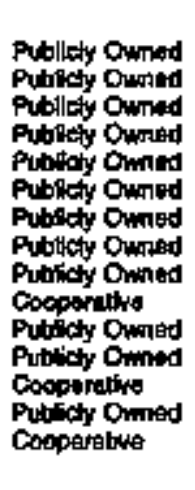 & 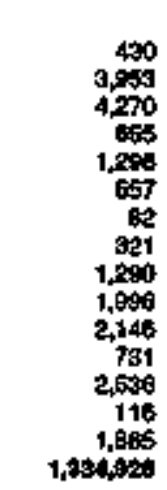 & $\begin{array}{r}390 \\
6,006 \\
7,137 \\
754 \\
2,568 \\
770 \\
70 \\
269 \\
1,960 \\
2,670 \\
2,122 \\
545 \\
4,120 \\
129 \\
4,756 \\
1,915,430\end{array}$ & 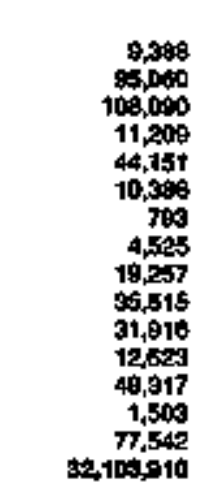 & $\begin{array}{l}4.10 \\
6.40 \\
6.80 \\
6.73 \\
5.51 \\
7.49 \\
19 \\
6.80 \\
7.118 \\
7.96 \\
6.06 \\
4.52 \\
0.37 \\
0.50 \\
6.13 \\
5.97\end{array}$ \\
\hline 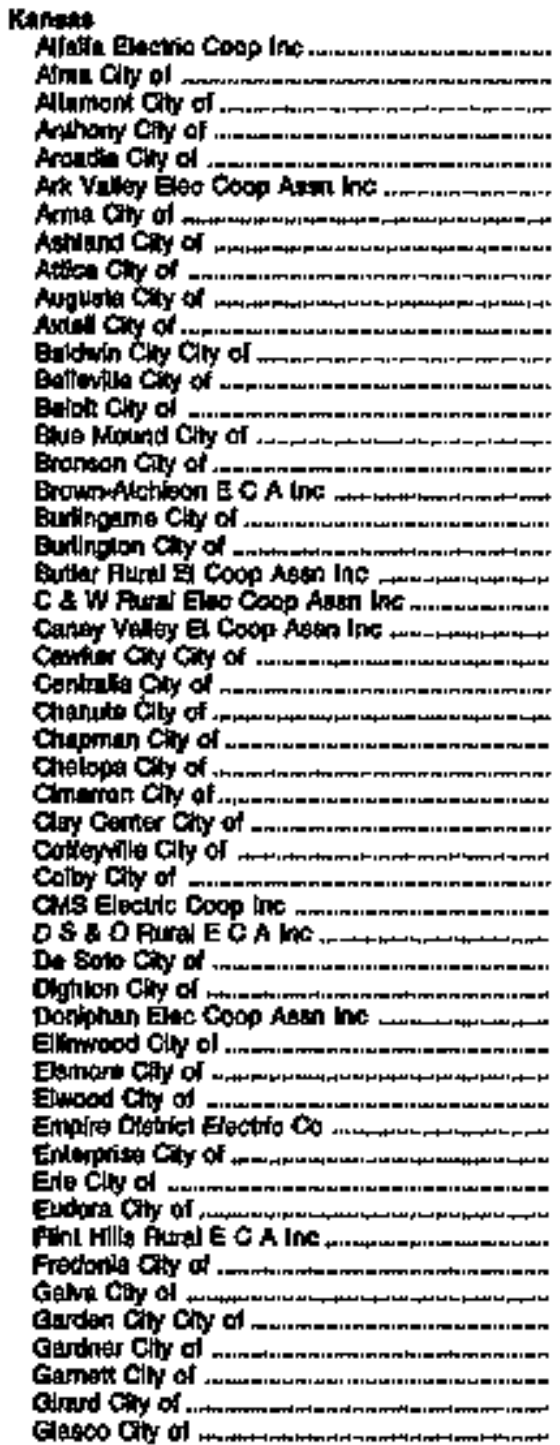 & 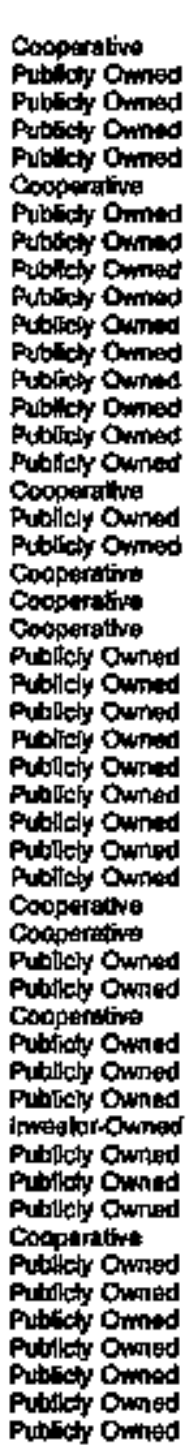 & 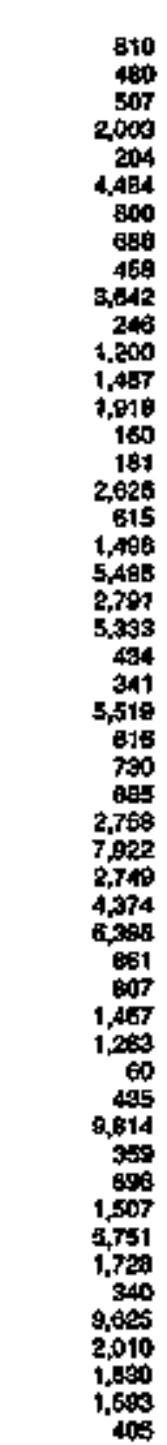 & 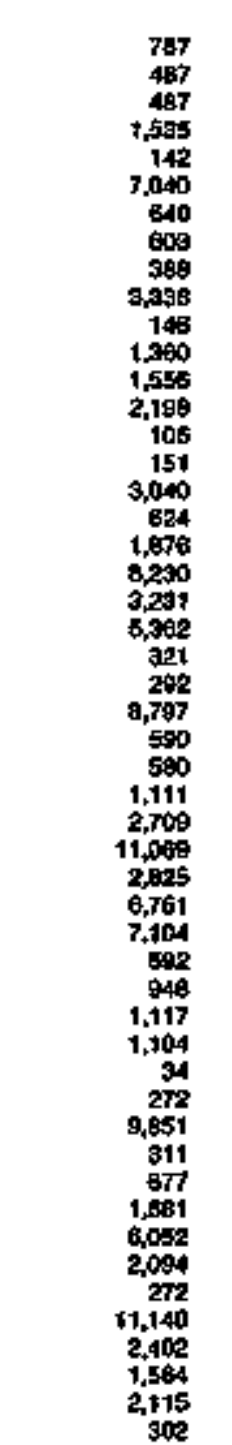 & 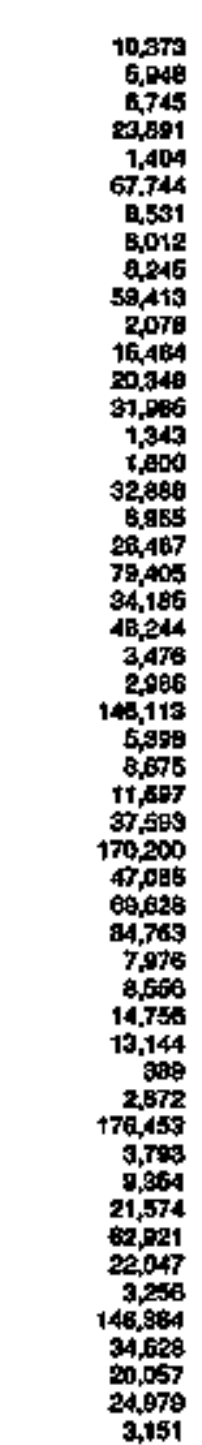 & 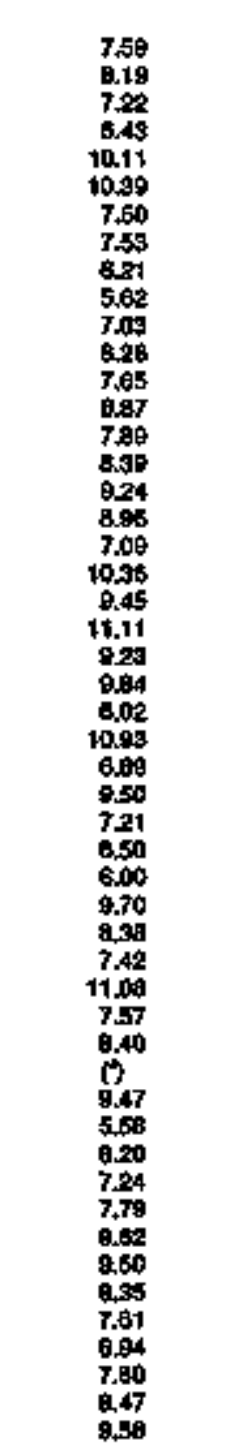 \\
\hline
\end{tabular}

Sos footnotes at and ol tabla 
Table 17. Class of Ownership, Number of Uitlmate Consumens, Revenue, Salte, and Average Revenue per Kllowatthour for All Sectors by State and Utility, 1993 (Conkinued)

\begin{tabular}{|c|c|c|c|c|c|}
\hline 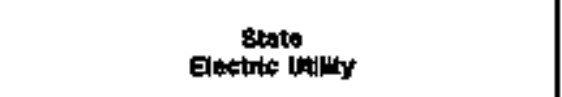 & $\begin{array}{c}\text { Clapa } \\
\text { of } \\
\text { Onwmermhip }\end{array}$ & $\begin{array}{c}\text { hamber } \\
\text { of } \\
\text { Congumaps }\end{array}$ & $\begin{array}{c}\text { Ferferut } \\
\text { (thousind dodins) }\end{array}$ & 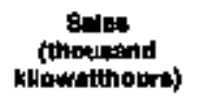 & $\begin{array}{l}\text { Average Rovenue } \\
\text { ptr klkowbithour' } \\
\text { [conte] }\end{array}$ \\
\hline 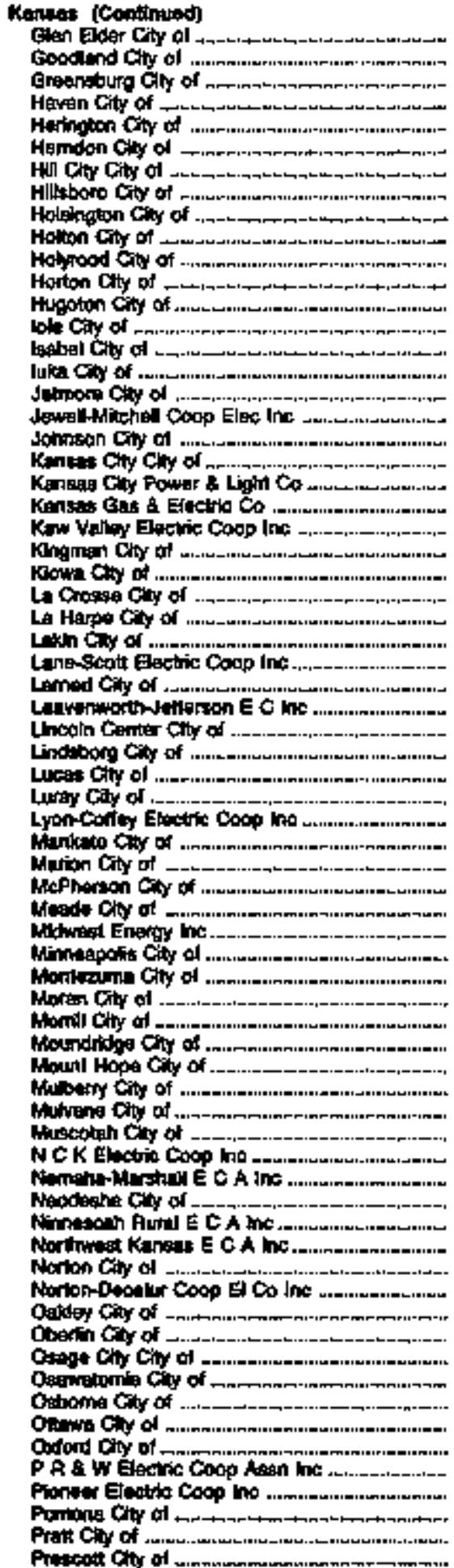 & 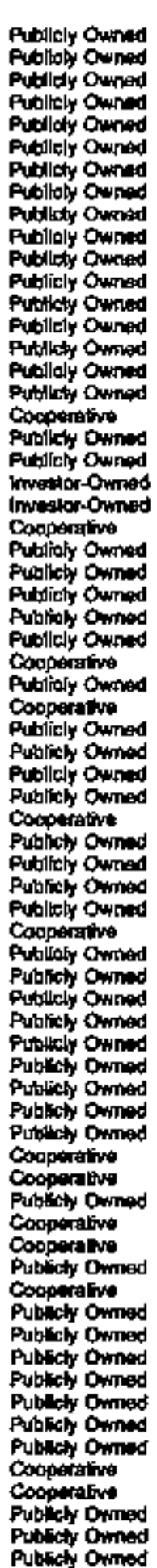 & 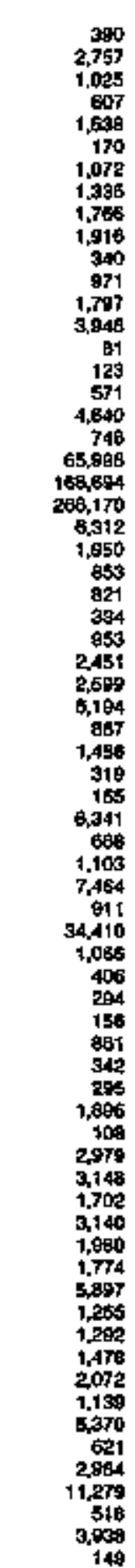 & 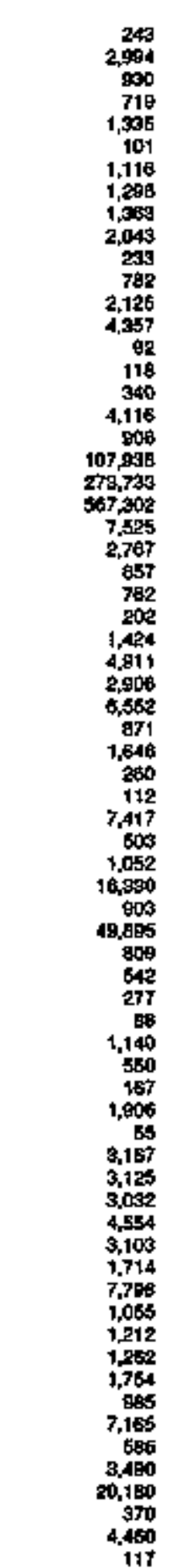 & 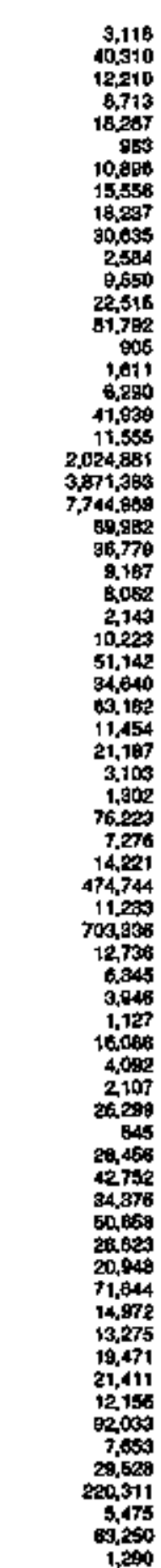 & 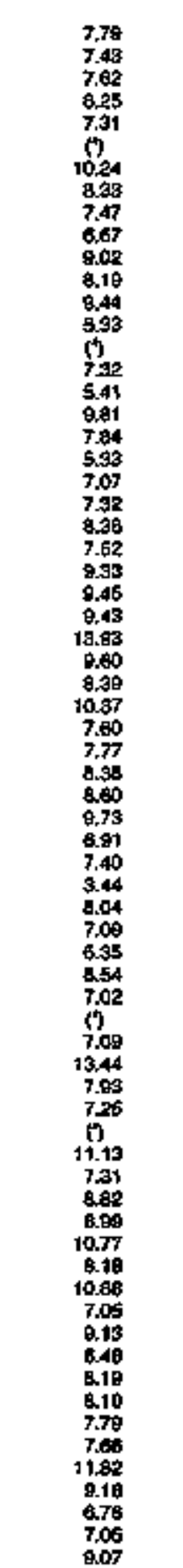 \\
\hline
\end{tabular}

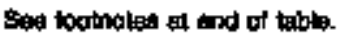


Table 17. Class of Ownership, Mumber of Uttmete Consumors, Reveruie, Sales, and Average Revenue per Kllowatthour for All Sectors by State and Utility, 1993 (Continued)

\begin{tabular}{|c|c|c|c|c|c|}
\hline 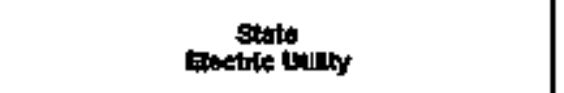 & $\begin{array}{c}\text { Clatim } \\
\text { of } \\
\text { Ommentip }\end{array}$ & $\begin{array}{c}\text { Tumber } \\
\text { od } \\
\text { Conbuntrs }\end{array}$ & 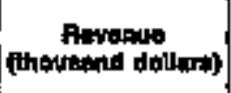 & Ithorated & 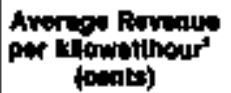 \\
\hline 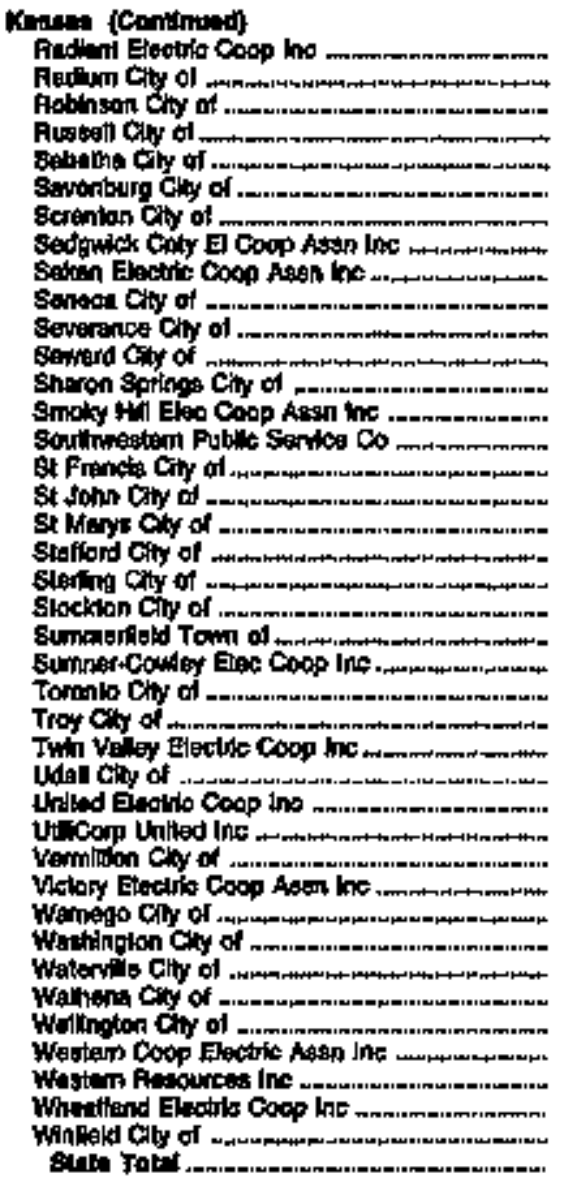 & 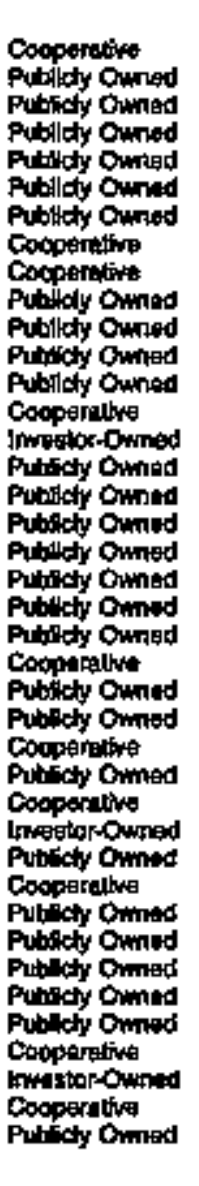 & 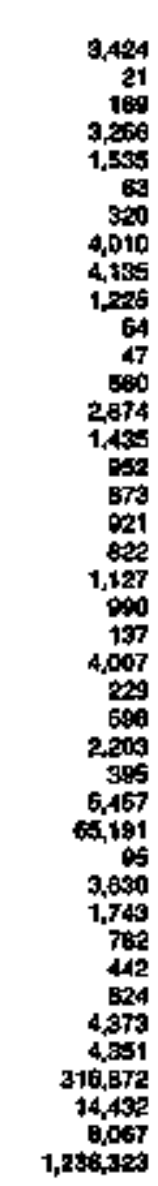 & 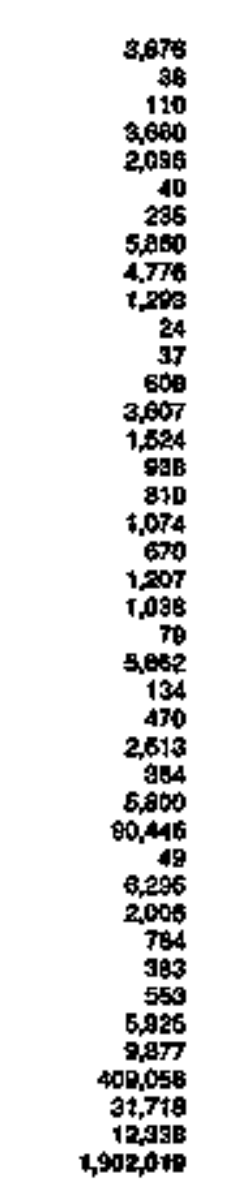 & 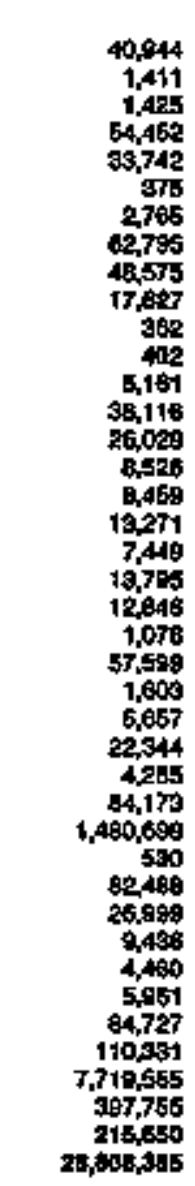 & 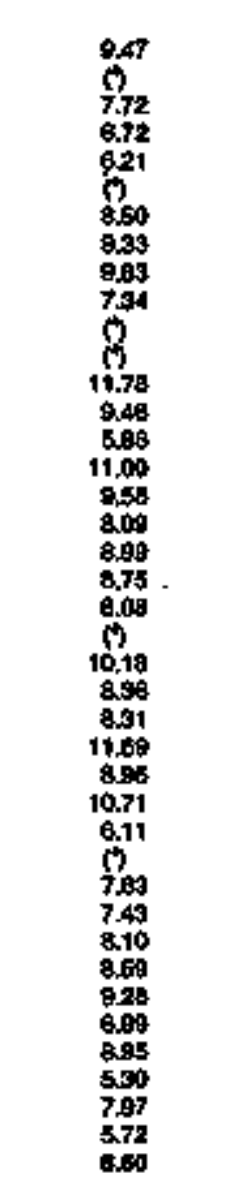 \\
\hline 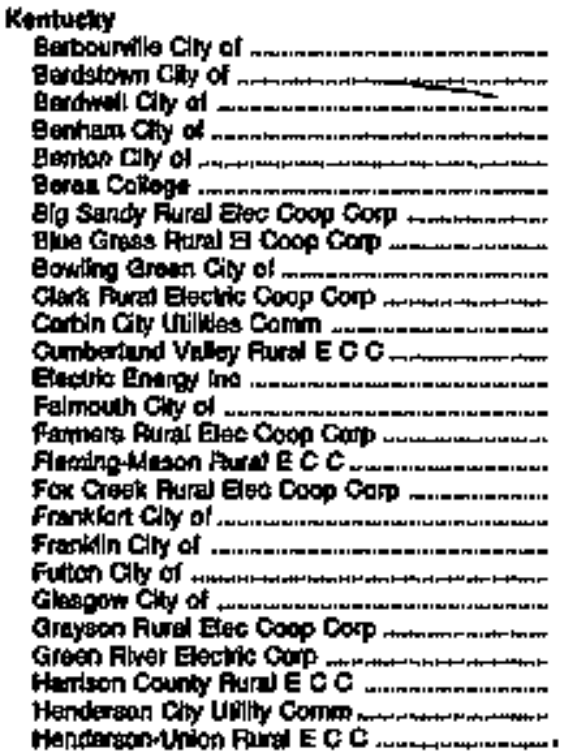 & 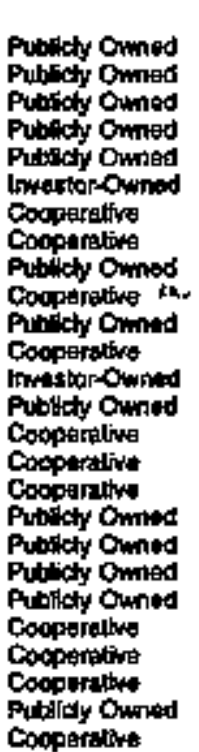 & 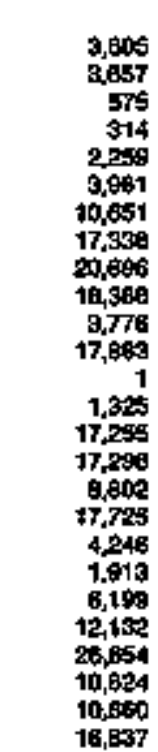 & 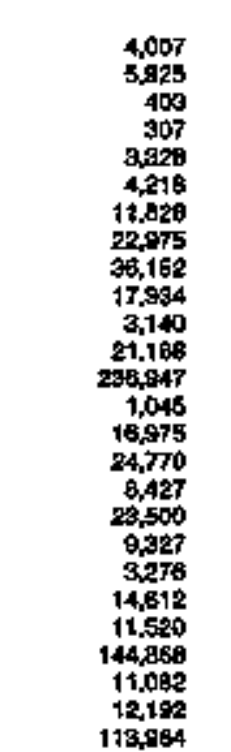 & 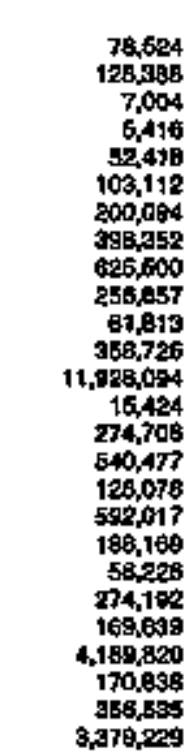 & 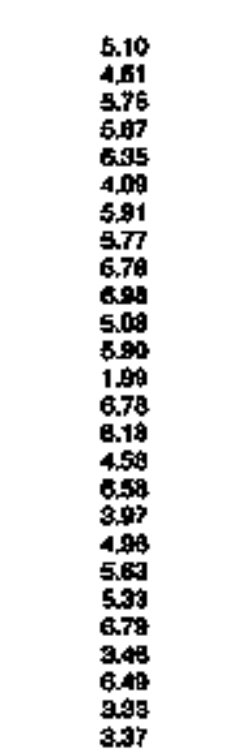 \\
\hline
\end{tabular}

Sap ioolnoles ot and of lable. 
Table 17. Class of Ownershlp, Number of Ultimate Consumers, Revenue, Sales, and Average Revenue per Kilowatthour for All Sectors by State and Utlity, 1993 (Continued)

\begin{tabular}{|c|c|c|c|c|c|}
\hline Btinte & $\begin{array}{c}\text { clate } \\
\text { of } \\
\text { Ownershlp }\end{array}$ & $\begin{array}{c}\text { humber } \\
\text { of } \\
\text { Contumen }\end{array}$ & $\begin{array}{c}\text { Prowinte } \\
\text { (thoupand doters) }\end{array}$ & 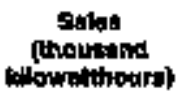 & 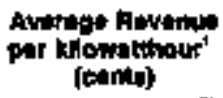 \\
\hline 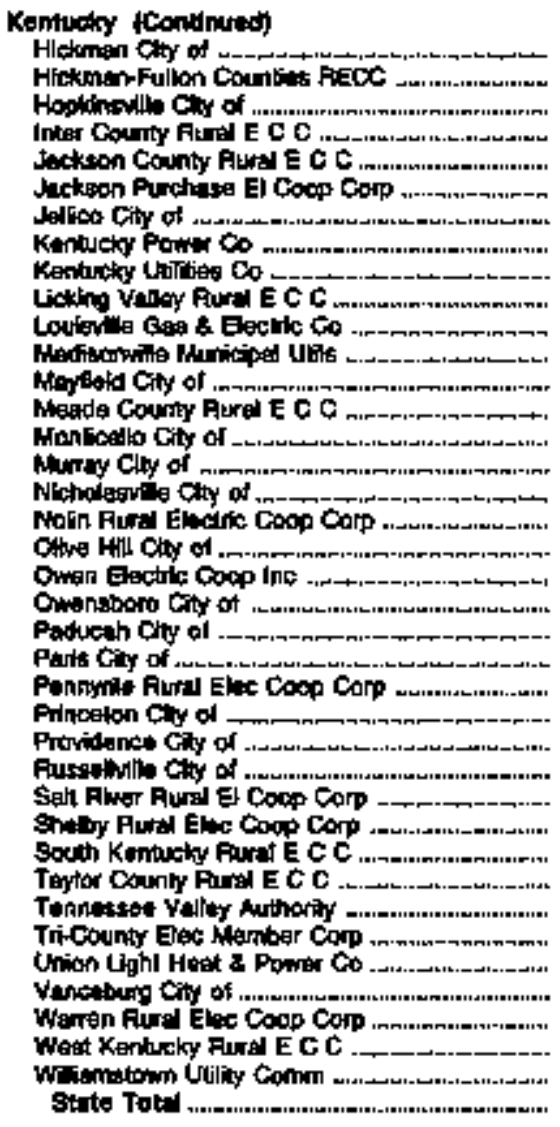 & 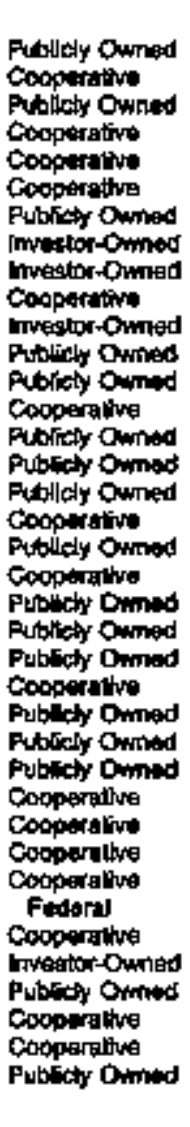 & 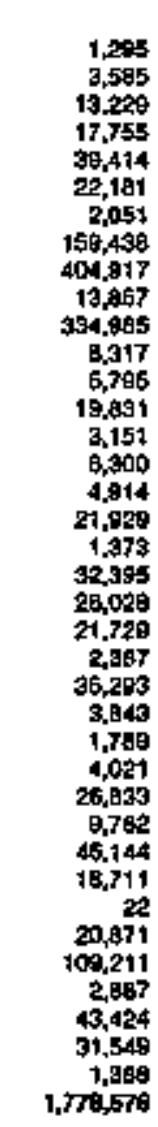 & 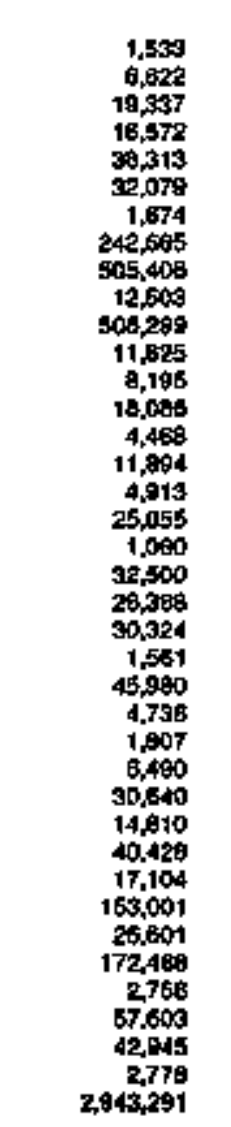 & 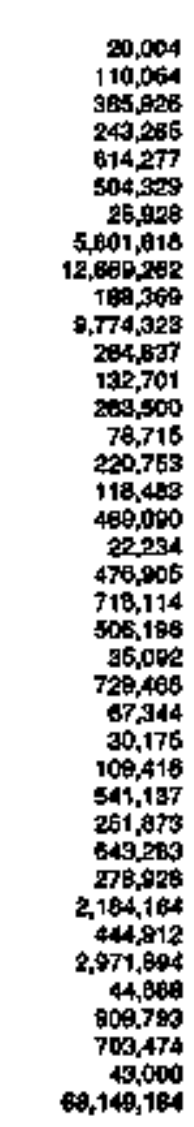 & 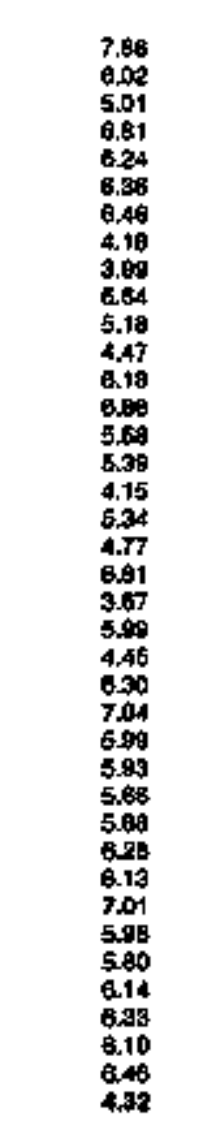 \\
\hline 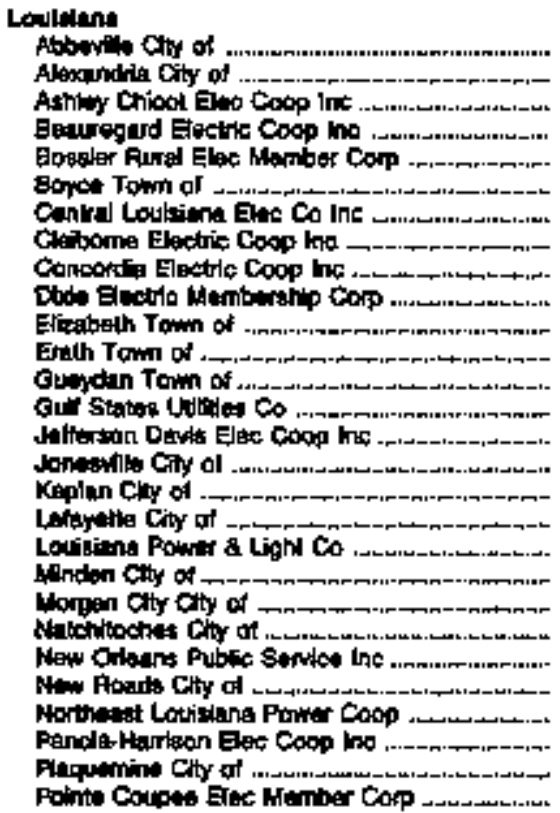 & 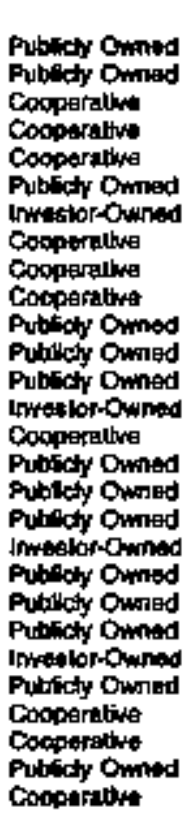 & 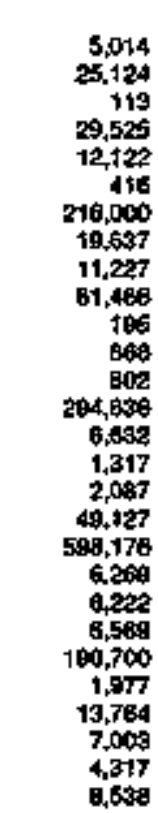 & 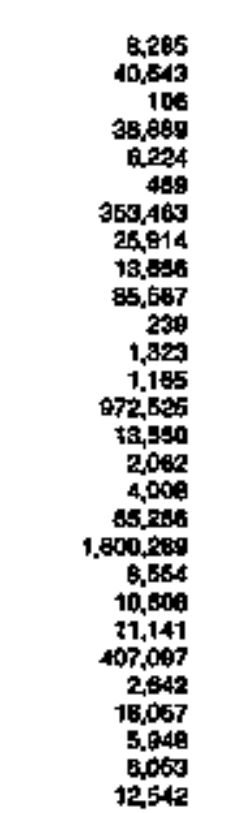 & 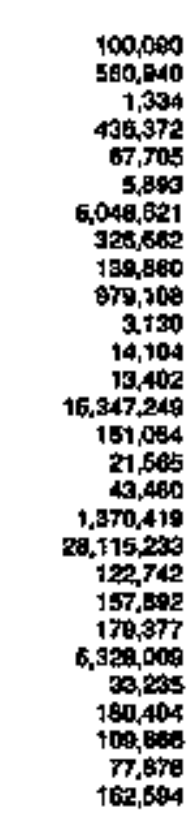 & 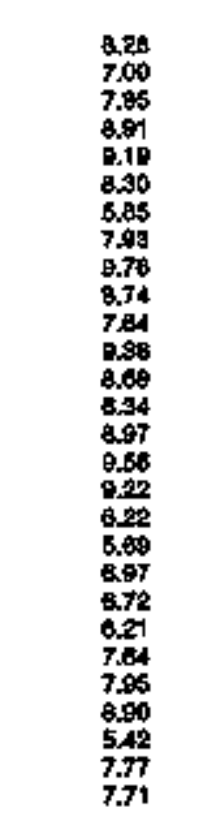 \\
\hline
\end{tabular}

She locinoted at end of lable. 
Table 17. Claes of Ownerahlp, Number of Ultimate Consumers, Rovenus, Sales, and Average Rewante per Kllowatihour for All Sectors by State and Utility, 1993 (Continued)

\begin{tabular}{|c|c|c|c|c|c|}
\hline 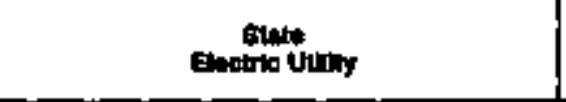 & 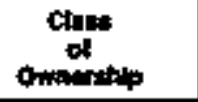 & $\begin{array}{l}\text { Number } \\
\text { opl } \\
\text { conturnatim }\end{array}$ & (thousund doling) & 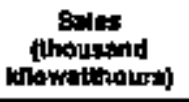 & 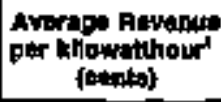 \\
\hline 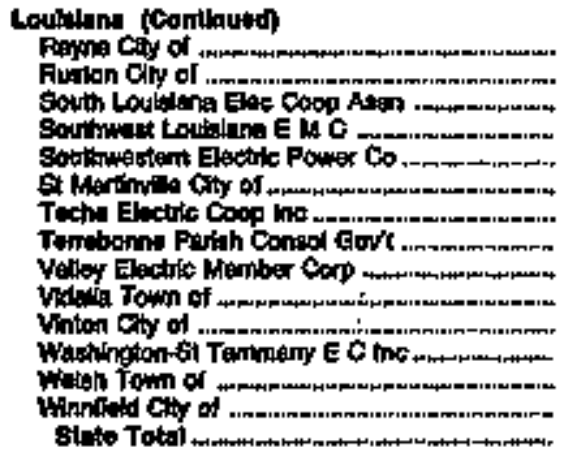 & 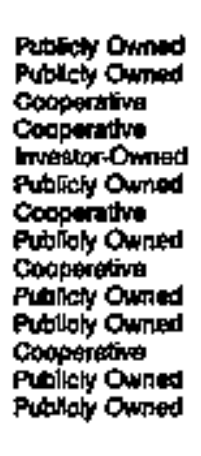 & 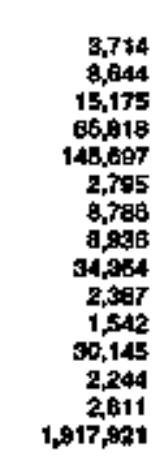 & $\begin{array}{r}4,497 \\
11,195 \\
28,945 \\
91,644 \\
245,983 \\
3,858 \\
12,529 \\
14,544 \\
39,004 \\
4,709 \\
2,360 \\
4,476 \\
1,604 \\
4,500 \\
4,94,004\end{array}$ & 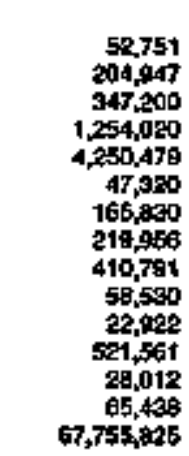 & $\begin{array}{r}6.52 \\
5.46 \\
7.73 \\
7.31 \\
6.70 \\
8.14 \\
8.04 \\
6.61 \\
0.45 \\
7.00 \\
10.30 \\
0.60 \\
5.37 \\
6.60 \\
6.20\end{array}$ \\
\hline 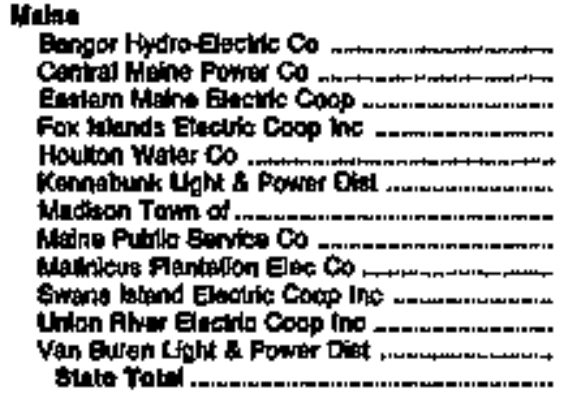 & 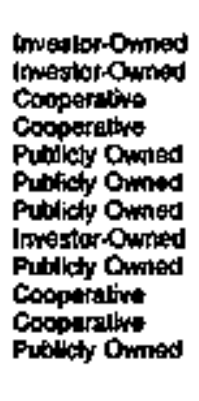 & $\begin{array}{r}116,376 \\
501,6009 \\
11,276 \\
1,587 \\
5,068 \\
4,699 \\
2,201 \\
34,707 \\
100 \\
469 \\
1,740 \\
1,462 \\
61,409\end{array}$ & 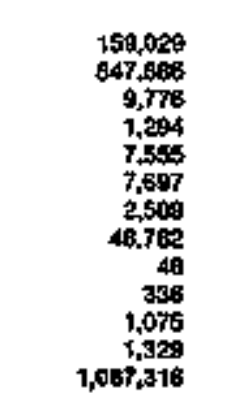 & 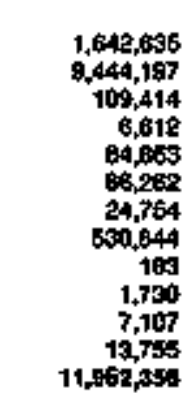 & $\begin{array}{r}0.69 \\
9.80 \\
8.95 \\
19.67 \\
8.90 \\
9.92 \\
10.14 \\
9.19 \\
19 \\
19.42 \\
15.13 \\
0.66 \\
2.10\end{array}$ \\
\hline 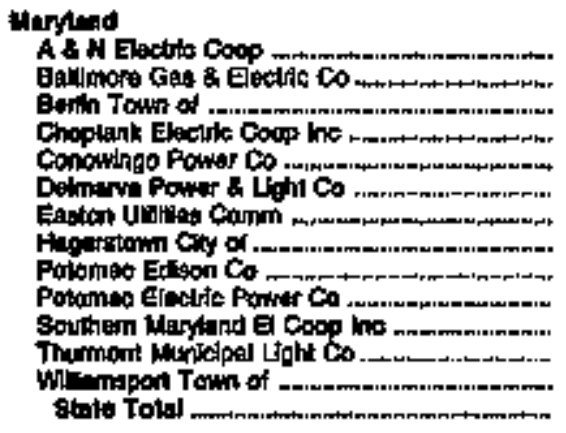 & 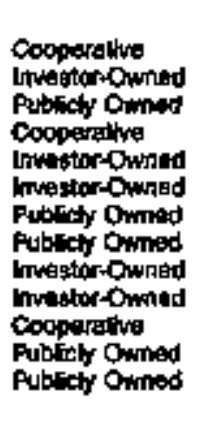 & 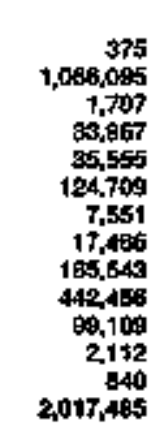 & $\begin{array}{r}909 \\
2,000,519 \\
3,296 \\
51,469 \\
75,559 \\
196,074 \\
12,959 \\
16,675 \\
346,816 \\
895,178 \\
167,760 \\
2,813 \\
667 \\
3,741,336\end{array}$ & 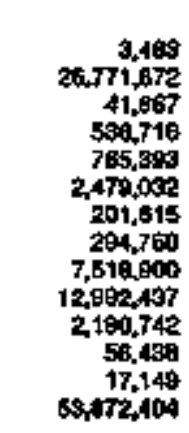 & $\begin{array}{l}0.87 \\
7.47 \\
7.87 \\
9.59 \\
9.87 \\
7.87 \\
6.49 \\
6.92 \\
4.61 \\
6.81 \\
7.20 \\
4.81 \\
6.68 \\
6.98\end{array}$ \\
\hline 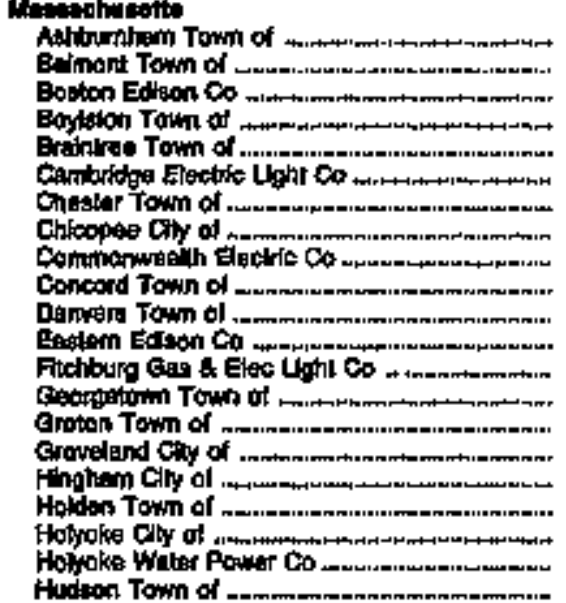 & 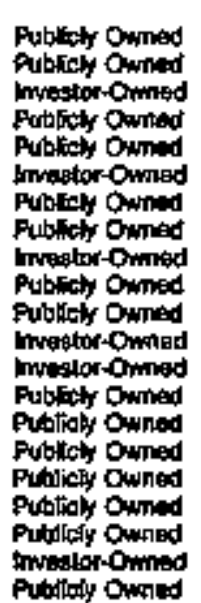 & 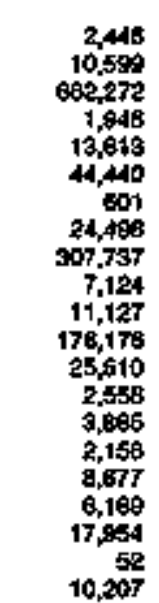 & 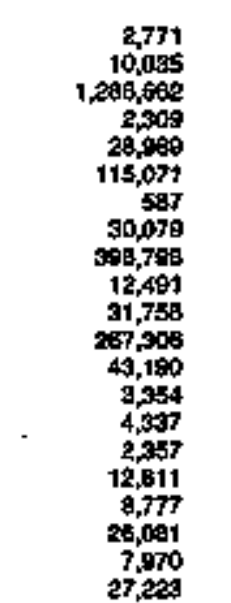 & 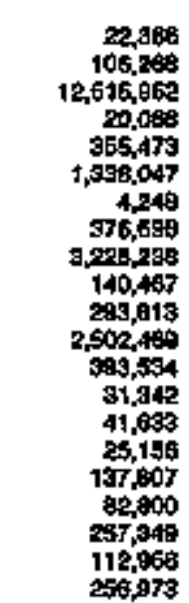 & $\begin{array}{r}12.98 \\
0.44 \\
10.28 \\
1+.51 \\
8.15 \\
6.60 \\
13.92 \\
7.98 \\
12.98 \\
6.98 \\
10.81 \\
10.68 \\
10.97 \\
10.70 \\
10.48 \\
8.97 \\
9.30 \\
10.60 \\
10.13 \\
7.06 \\
10.59\end{array}$ \\
\hline
\end{tabular}

Sae Inotinoles at ard or lables. 
Table 17. Class of Ownershtp, Number of Ultimate Consumers, Revenub, Sales, and Average Revenue per Kllowatthour for All Sectors by State and Utility, 1993 (Continued)

\begin{tabular}{|c|c|c|c|c|c|}
\hline $\begin{array}{c}\text { State } \\
\text { Elactrle Uithy }\end{array}$ & $\begin{array}{c}\text { Cinst } \\
\text { of }\end{array}$ & $\begin{array}{c}\text { Nember } \\
\text { of } \\
\text { Conturntets }\end{array}$ & $\begin{array}{l}\text { Peveout } \\
\text { (thoursid dollary) }\end{array}$ & 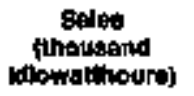 & 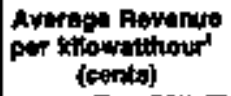 \\
\hline 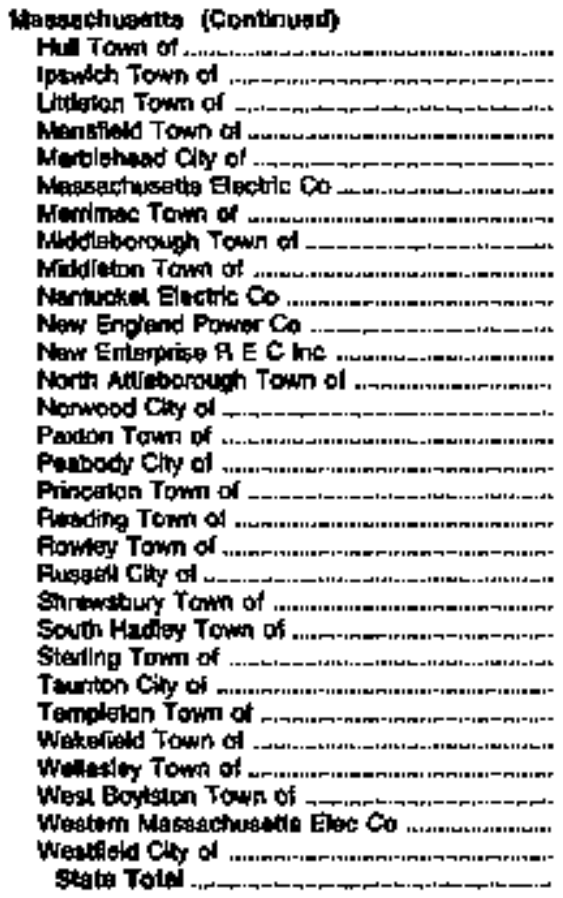 & 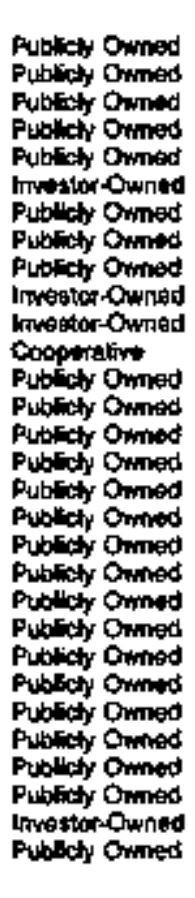 & 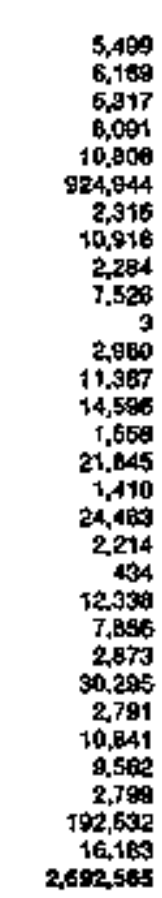 & 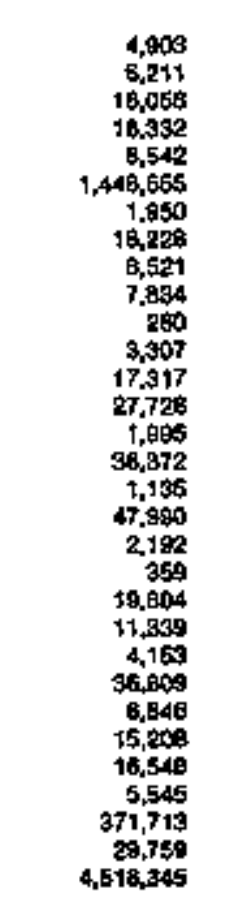 & 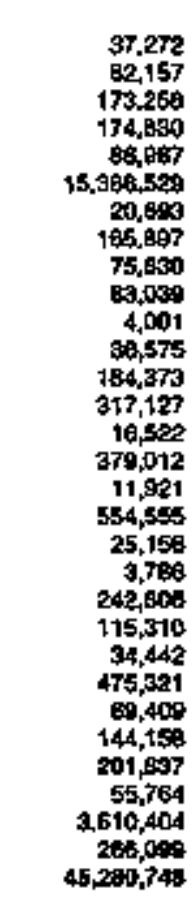 & $\begin{array}{r}13.15 \\
7.68 \\
9.27 \\
10.49 \\
0.92 \\
9.41 \\
9.42 \\
0.78 \\
11.24 \\
0.43 \\
6.60 \\
9.04 \\
9.39 \\
6.74 \\
12.07 \\
10.12 \\
9.59 \\
6.65 \\
0.71 \\
9.48 \\
8.18 \\
9.68 \\
12.06 \\
7.74 \\
9.86 \\
10.55 \\
8.20 \\
9.04 \\
10.30 \\
11.18 \\
9.989\end{array}$ \\
\hline 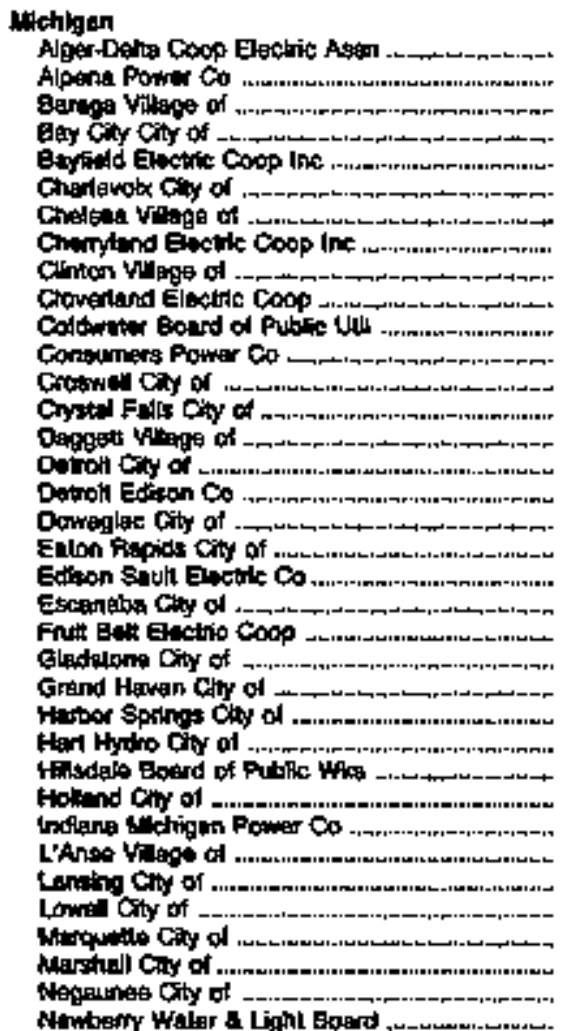 & 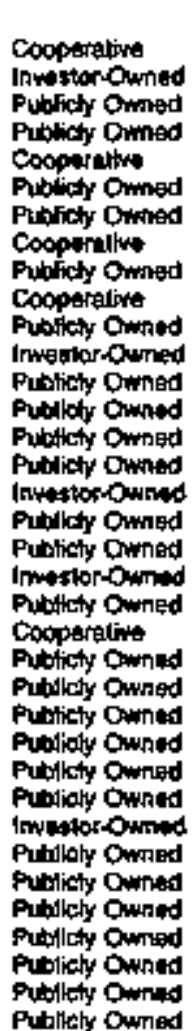 & 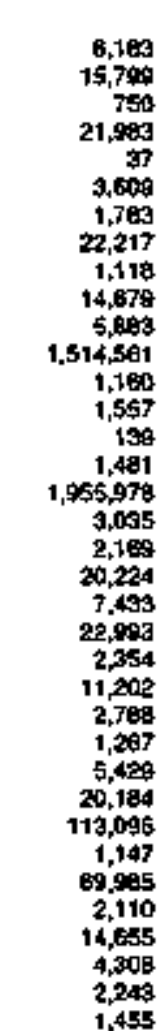 & 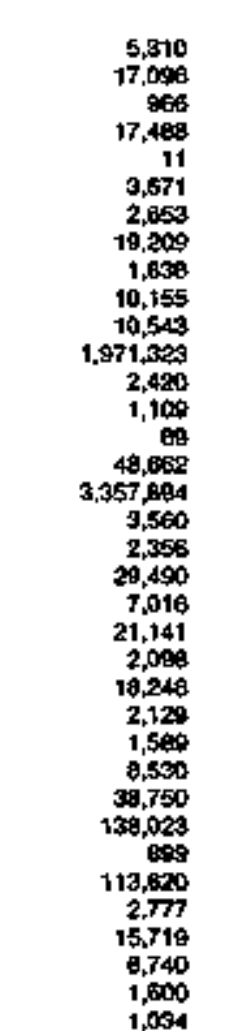 & 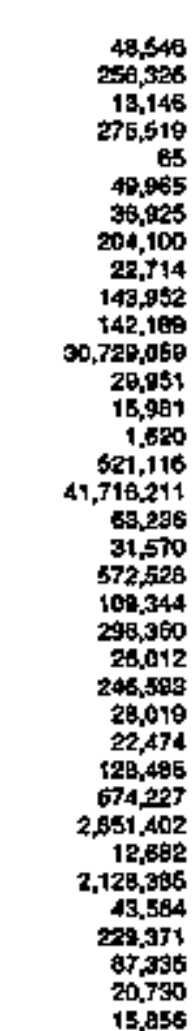 & 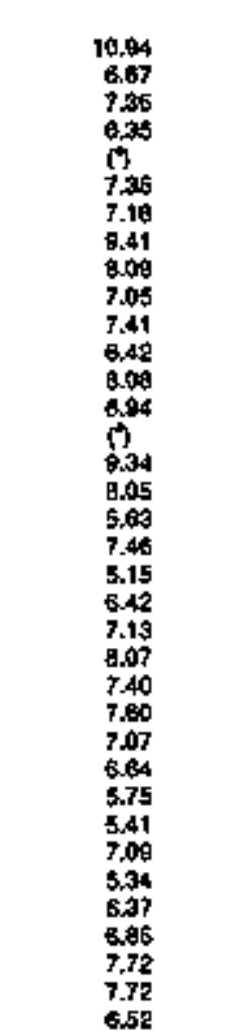 \\
\hline
\end{tabular}

See tootroled at and of lable. 
Table 17. Clags of Ownership, Number of Ulimate Consumers, fevenue, Sales, and Average Revenue per Kilowatthour for All Sectors by State and Utility, 1993 (Continued)

\begin{tabular}{|c|c|c|c|c|c|}
\hline etetels ditnky & chas: & $\begin{array}{c}\text { Nmbar } \\
\text { of } \\
\text { Contoumtort }\end{array}$ & 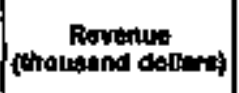 & polpoutend & 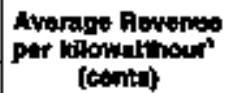 \\
\hline
\end{tabular}

Uichlion (contiound

Nites Coy of

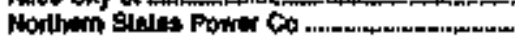

Norway cily of

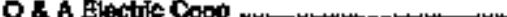

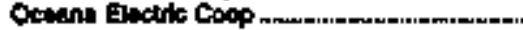

Ontonagon Gounty $P$ E A

Prw Fow Vinge of

Pelooktry cily of

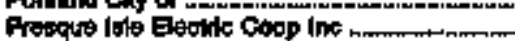

sobenting City od

South Heven Chy of

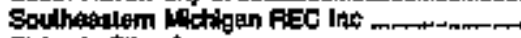

Et Lorits ets of

Slephwisar City of

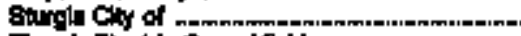

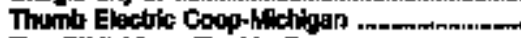

Top OrNichigen Electite Co

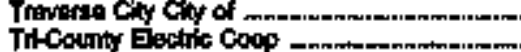

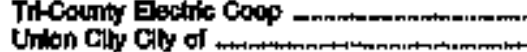

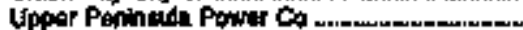

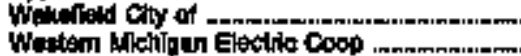

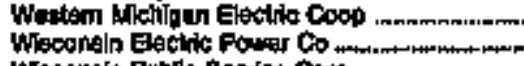

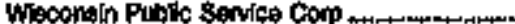

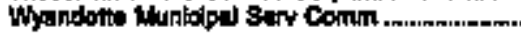

Zeelind Gity of

Ftall Tetr ...

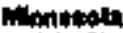

Ade Cly ol

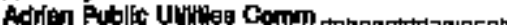

Arafle Cacp ...

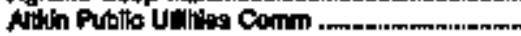

Alextandifia $G$ of

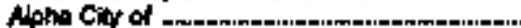

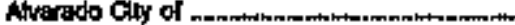

Arowe Giny ol

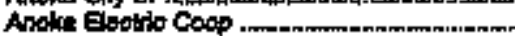

Avilindion $\mathrm{CO}^{2} \mathrm{y}$ of

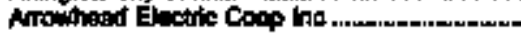

Auth CHy of

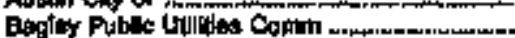

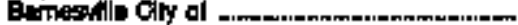

Bapdatla Clly of

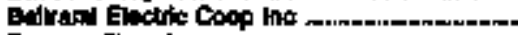

Banson Ciny of

Elably Chy of

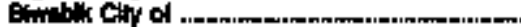

Elooming Fratio Cily of

Guo Eath City of

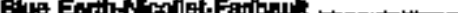

Brainterd C.7y of

Brofownides coty of

Brinmitis City of

(20)

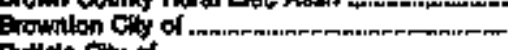

Butfelo of thy of

BTh City of

Caladonis Cory or

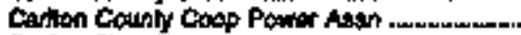

Ceylon Ciny of

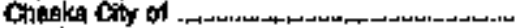

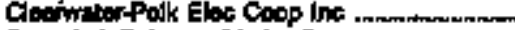

Coop L \& P Aasn of Leice Counly ............m.

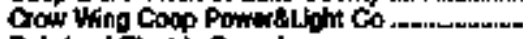

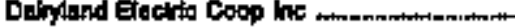

Datrola Electlle Assh
Pubinch Omod

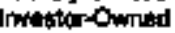

Puthicy Owmot

Cooperplive

Cooperafive

Coopratro

Publetr Ommod

Pubsaty Oined

Pubinty awnod

Cooporative

Pubictr Omined

Pubsisy Owned

Cooperative

Putisy Drmed

Pubsiay Quned

Putbilety Dimed

Cooperailue

Cooperative

Publety Owned

Cooperdilug

Publity Dwined

mrester-Omind

Publich Owned

Cooporativa

Inigstar-Omed

metstar-Cinted

Putlict Owned

Putplicty Owhod

Publety Owned

Publets Duned

Coupenetinro

Putllety Owned

Puthich Owited

Publicty Owat:

Pudlucty Owhed

Putriest Owtid

Cooperative

Pudilely Owitad

Cooperatre

Pulbiley Oumad

Publlkly Owrat

Publety Owred

Pubiets owned

Codpentintin

Publity Owned

Publity Ounid

Putlicly Owned

Publlebs Qwned

Pubfely Owined

Goperative

Puolichy Ournad

Publesy owned

Publikhy Owation

Cooperative

Pubilety Ounad

Pusilicly Owned

Puncly Ownes

Pullicty Ound

Coppetintive

Puthety Qwaded

Mublicly Owined

Cooperatue

Gooperitiva

Cooperatho

Coopergitus

Coppentotivg

\subsection{5}

8.05:

26,850

5,517

3,010

1,802

4,412

2,007

20,420

125

8,169

3,438

1,735

487

6,689

10,400

42,479

8,658

19,411

1,485

61,701

1,0086

11.056

25,023

0,915

13,505

3,205

$1215,75 y$

1,021

4,705

1,691

7270

es

236
10,418

75901

85

$2, \mathrm{BgB}$

11,235

tas?

1,043

61 1

13,964
1.697

64

945

2,002

10,1日?

6,575

1 195

3,381

352

2,954

1,416

10,284

271

5,077

3,391

4,278

28,540

1292.

67,009

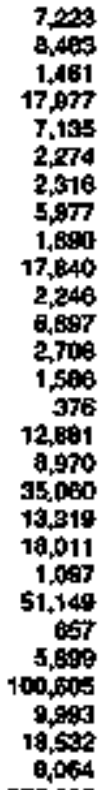

6.p69,300

76

6.276

1,627

7,440

76

280

11.498
74.265

67

2,049

17,764

ac:

685

609

13,049
1,157

85

356

1,334

2,874

13,000

7.220

$1,60 \%$

20

5,8

208
3,846

20

1,030

7,805

140

6.314

3,357

3,931

10,467

11,457

78,505

112,052

191,865

24,091

203058

74,297

18,681

6, 0002

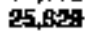

171,

32,007

107,26s

2.76

21,757

5.714

200.478

102,768

379.247

200,500

189,805

13,397

675,137

12,438

50,205

2,565,414

218,907

201,Bes

144.971

67,800,602

19,875 
Table 17. Class of Ownershtp, Number of Ultimate Consumers, Revenut, Sales, and Average Revenue per Kilowatthour for All Sectors by State and Utilly, 1993 (Continued)

\begin{tabular}{|c|c|c|c|c|c|}
\hline $\begin{array}{c}\text { State } \\
\text { Electute lallity }\end{array}$ & $\begin{array}{c}\text { Clats: } \\
\text { of } \\
\text { Gemitrohp }\end{array}$ & $\begin{array}{l}\text { Humbor } \\
\text { of } \\
\text { contumora }\end{array}$ & Finande & 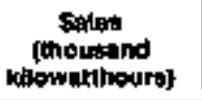 & 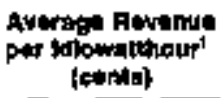 \\
\hline 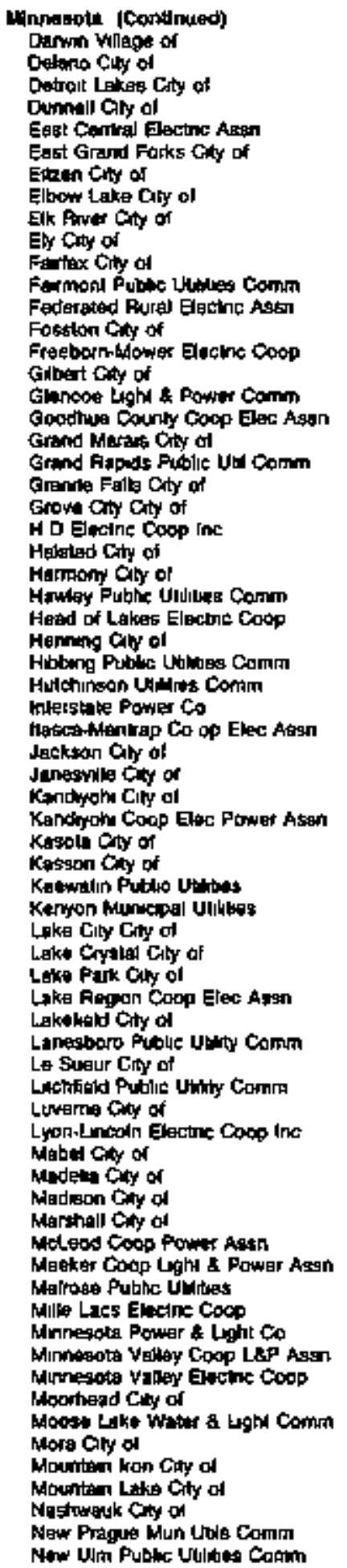 & 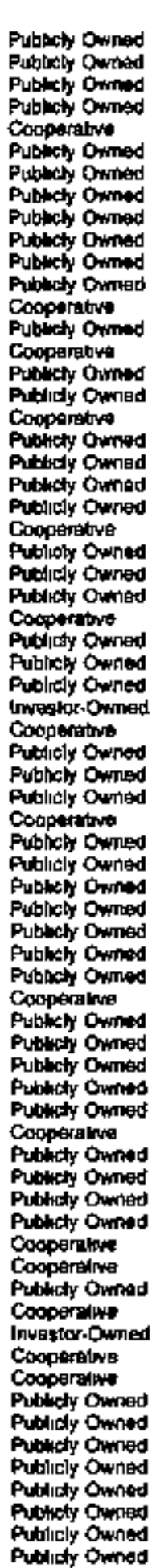 & 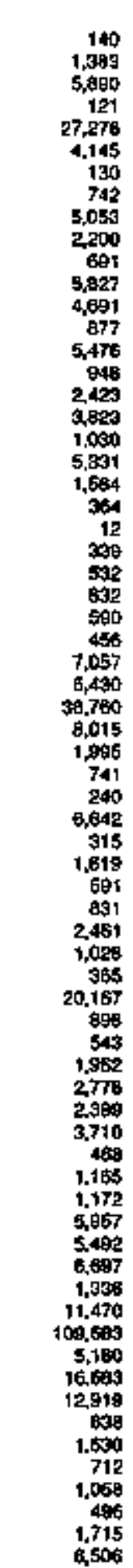 & 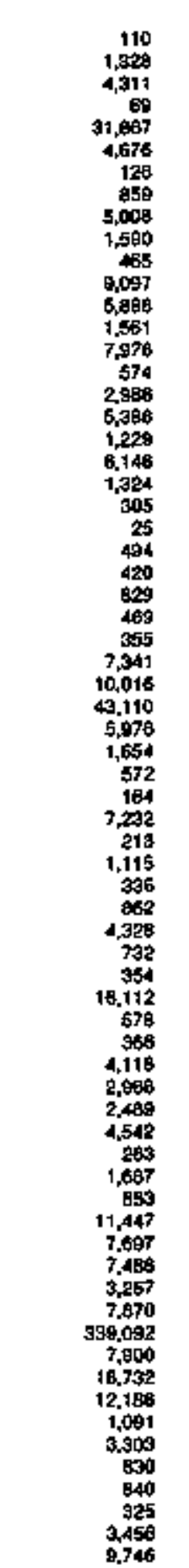 & 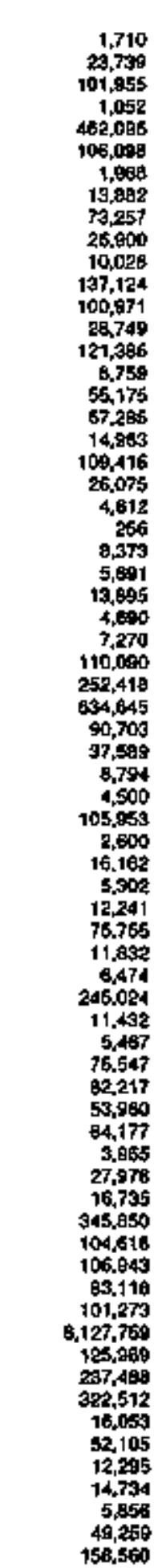 & 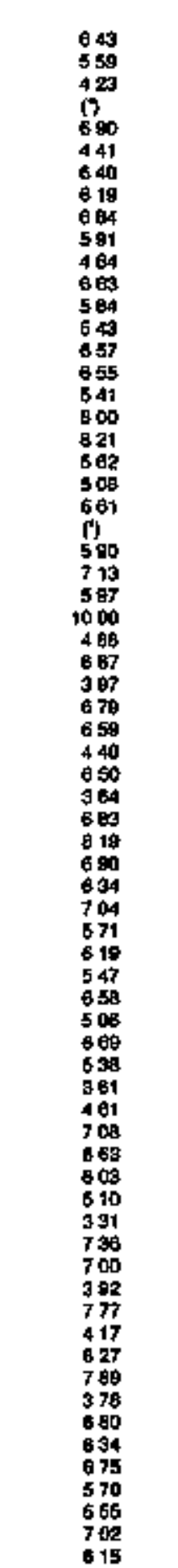 \\
\hline
\end{tabular}

Sae ficolnotes at end of table 
Tabie 17. Class of Ownershlp, Number of Uitimate Consumers, Revenue, Sales, and Average Hevenue per Kilowatthour for Al Sectors by State and Utility, 1993 (Continued)

\begin{tabular}{|c|c|c|c|c|c|}
\hline Eneterto vinty & Chase & Monger & 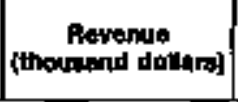 & $\begin{array}{l}\text { States } \\
\text { (thoutand } \\
\text { ldiomalthoure) }\end{array}$ & 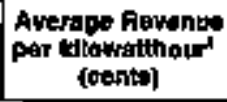 \\
\hline
\end{tabular}

Whineote (Tonthued)

Nawdedin CNy of

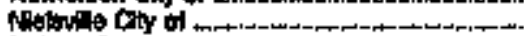

Noblow Cosp Enovito

Korth Branch Weters Loht Comm nnm

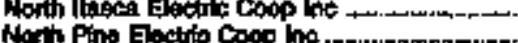

North St Pat Chy of

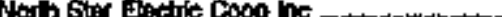

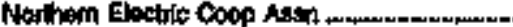

Nortiem SInlä Powier Co .

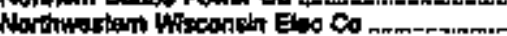

Colvila City of

Giomiti Cxy of .

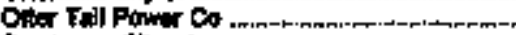

Omatemns chy of

P X M Eleditic Coop tho .

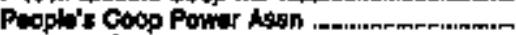

Petersan Caty of

Puer ctin 어

Pitredion Futale U't

Proctor Platic vilities Cornm

Pand cll City of

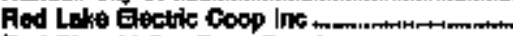

Fed Rivar Valoy cocp Pwt Ason

Fedwood Eectits CoOp -

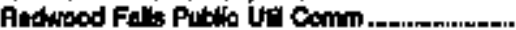

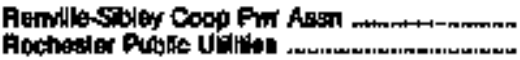

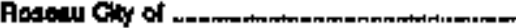

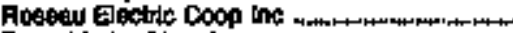

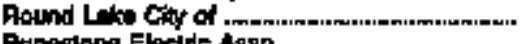

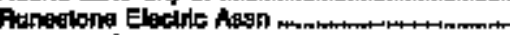

Aythlond Ciny of

Pustinore City of ....

Selni Pelar City of

Sark Centre Cry of

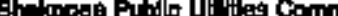

thenty coly of

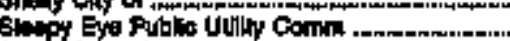

South CAtral Electic Aast.

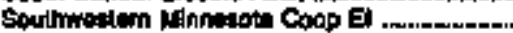

Sping Growe Chy of

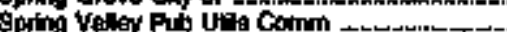

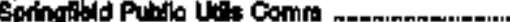

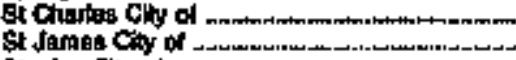

siegles Cin of

Stem coop Elowte Netrin

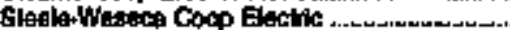

sisphen Cly ol.

Thiof A Twe Palls City of

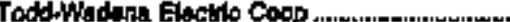

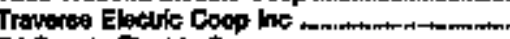

Tritcount Electric coop

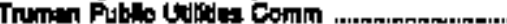

Two Habora Chy ol

Tyter ciny of .

virglnie City ol

themen

Weroind City of

Wastect Cir of -

White ciny of

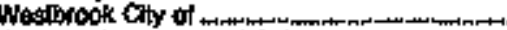

Woolom Arat Powter Adplin .....................

Whalan City of .

Wid Pice Electitis CoOp Int

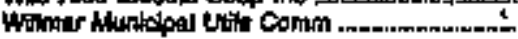

Publety Onnied

Publity Omined

Cosporation

Pubichy Onrod

Cosperative

Cooperathe

Puticty Owneos

Cooperaliva

Cosperariso

Investorowned

invastor-Owned

Pubictr Ownod

Publedy Owmod

impostem- Ourted

Pthplich Onmest

Cocopsative

Coopuratura

Pajbility Owned

Pub:at Owned

Fubled Dwned

Fublicty Owmat

Publicy Owned

Fublic): Ownod

Cooperative

Cooperative

Cooperative

Putblety Owned

Cooperative

Putilikly Owned

Publter, Ownad

Gooptrativa

Publichy Owind

Cocoprativo

Putslesty Ouret

Publloly Ownpod

Putalefly Ouned

Poticy ownet

Pubtdy Owned

Pusthdy Ouneal

Puthdy Ouned

Cooperative

Conoentive

Pubily Onned

Punnty Ontrat

Pusiloty Omes

Puttldy Owerted

Putichy Owined

Publisty Omed

Cooperettrop

Cooperetion

Putllaty Oined

Putaldy Owreal

Cooperative

Cooperative

cooperattus

Pubilety Onwas

Publly Owned

Publesty Ounet

Publich anned

Publaty Omros

Punich Owrom

Publaty Ownet

Pubfery Oimet

Puticty Omoo

Pullichty Ommed

Fodors

Publichr Omed

Cospenative

Putifict Orinod

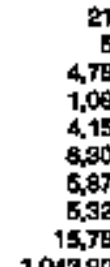

$1,043,907$

70

1,353

1,306

55,254

B, 538

3,707

13,708

120

488

84t

1,885

1,428

4702

4,217

2,995

2,476

1,948

32,698

1,248

5,149

252

10,094

77

3,316

1,78

0,000

140

1,600

3,706

2,006

704

1,307

1,178

1,3 s

2,167

1.417

16,0

6,917

421

3 ,901

6.298

1,0

11.204

630

2,007

5.745

2,391

910

850

4,013

1,353

490

5

10,968

B.549

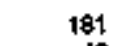

43

5,892

305

6,496

4,216

B.39B

13,658

$1,358,076$

38
1,3928
1,002

1,002

$\mathbf{1 , 0 0 2}$

15,620

5,154

14,190

a7

497

2,759

694

228

5,754

6,545

3,608

2,096

5,670

64,419

1,570

6.151

602

8.391

677

131
4,945

1,780

$7,0,50$

10 ?

1,000

5,750

4,210

65

1.379

1,27

1,194

2,298

1,354

19014

9.772

501

5095

7,659

2,611

13.120

709

1,25

547

6,797

2,270

800

1.569

4,201

1,608

1,008

480

50

9,700
10,848

\section{3,766}

86,179

36,036

26.671

78,742

03,875

70,653

1603,a5s

24,a00,245

48

23.286

21,734

$1,033,029$

227.242

B1.906

200,993

1,226

7.040

11.148

36,464

14,148

3.502

69,186

$10 \mathrm{BS} .462$ 
Table 17. Clans of Ownershlp, Number of Ultimate Consumers, Revenus, Salos, and Average Fevenue per Kilowatthour for All Sectore by State and Utllity, 1999 (Continued)

\begin{tabular}{|c|c|c|c|c|c|}
\hline $\begin{array}{c}\text { stat: } \\
\text { Desiric viluty }\end{array}$ & $\begin{array}{c}\text { Clato } \\
\text { of } \\
\text { ownenthip }\end{array}$ & $\begin{array}{l}\text { Nymaber } \\
\text { ol } \\
\text { conpumare }\end{array}$ & 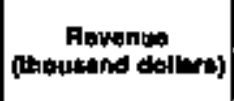 & $\begin{array}{l}\text { salat } \\
\text { (thourand }\end{array}$ & 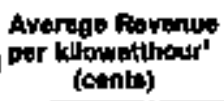 \\
\hline 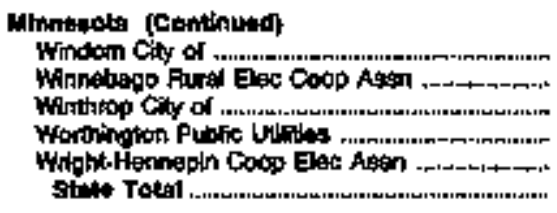 & 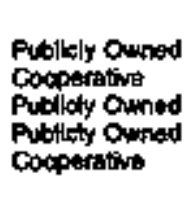 & $\begin{array}{r}2,200 \\
20 \\
759 \\
5,049 \\
25,734 \\
2,071,974\end{array}$ & $\begin{array}{r}1,975 \\
212 \\
727 \\
6,677 \\
27,679 \\
2,767,667\end{array}$ & $\begin{array}{r}40,316 \\
257 \\
15,050 \\
128,540 \\
390,049 \\
4210,670\end{array}$ & $\begin{array}{l}4,00 \\
19 \\
5.57 \\
6.26 \\
6,95 \\
5.60\end{array}$ \\
\hline 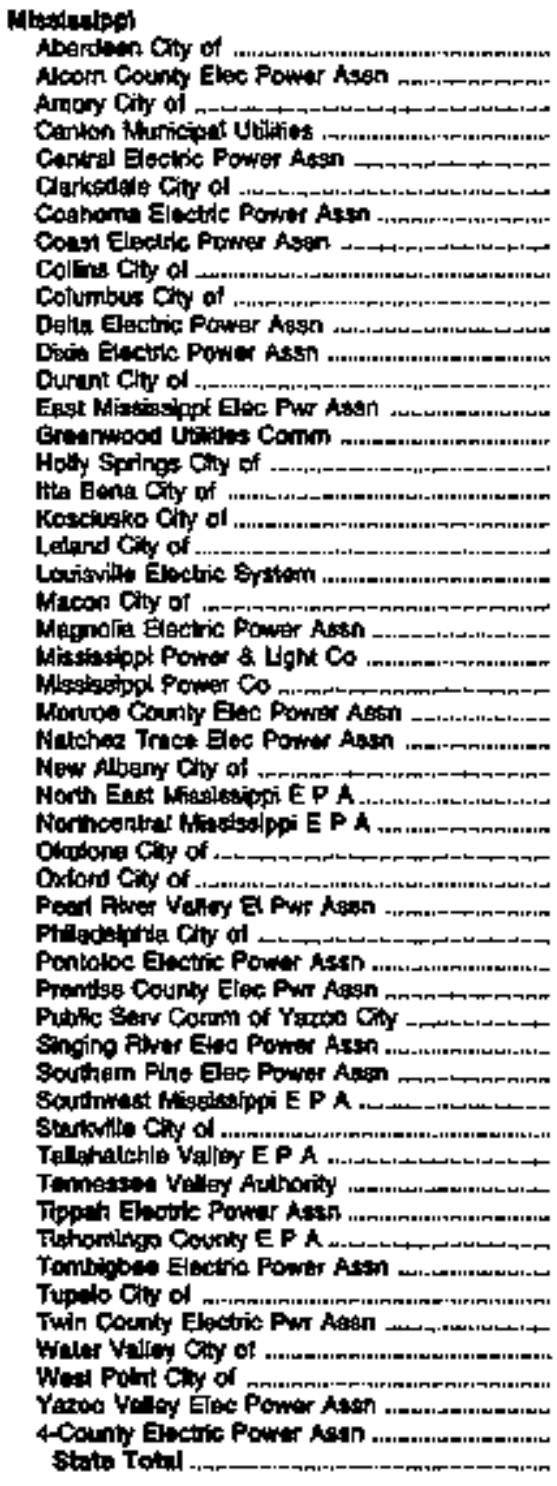 & 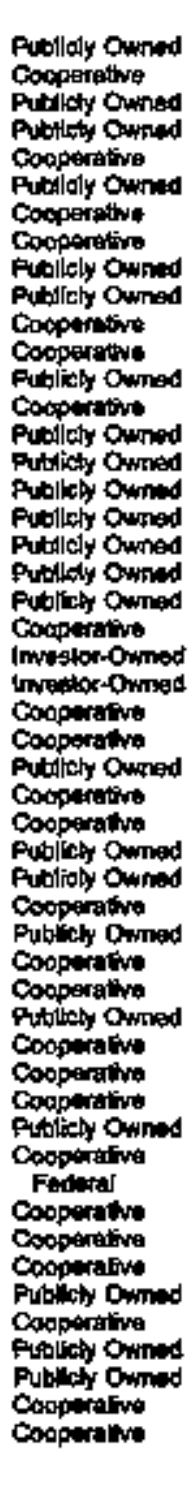 & 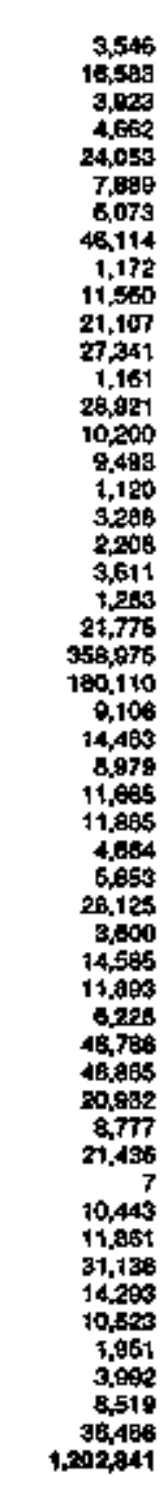 & 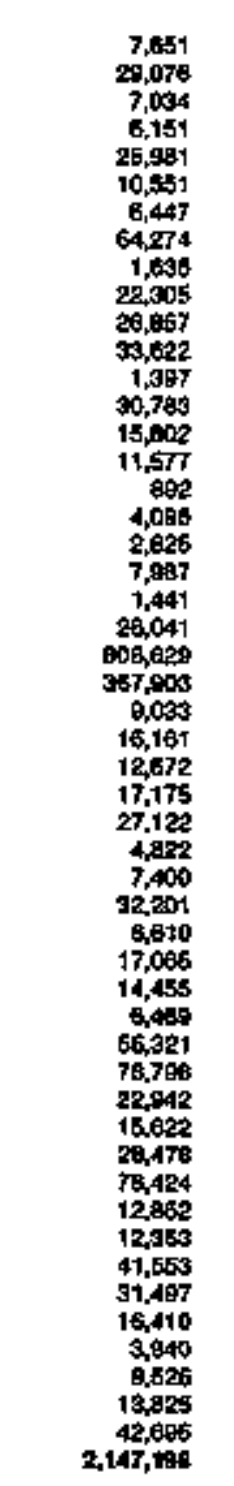 & 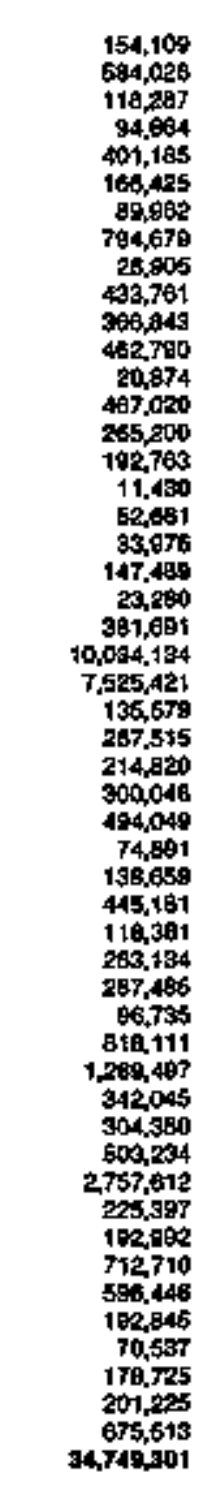 & 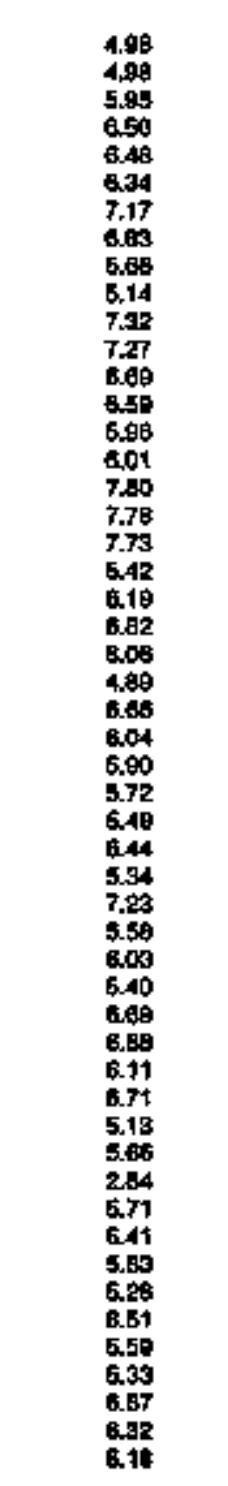 \\
\hline 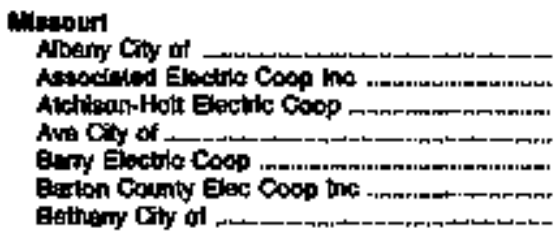 & 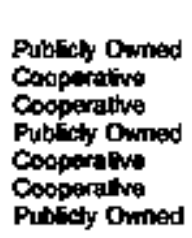 & $\begin{array}{r}1,007 \\
1 \\
2,602 \\
1,427 \\
7,540 \\
5.134 \\
1,510\end{array}$ & $\begin{array}{r}796 \\
42,418 \\
2.676 \\
1,086 \\
7,830 \\
5.404 \\
1,657\end{array}$ & $\begin{array}{r}14,143 \\
1,282,345 \\
36,440 \\
34,199 \\
124,332 \\
71,344 \\
26,749\end{array}$ & $\begin{array}{l}6.63 \\
3.3 t \\
7.35 \\
6.51 \\
6.50 \\
7.51 \\
6.20\end{array}$ \\
\hline
\end{tabular}

Seo looboles al ind of thets. 
Table 17. Class of Ownership, Number of Ulitmate Consumers, Revonus, Sates, and Averags Revenuse per KIlowatthour for Alt Sectors by state and Utilty, 1993 (Continued)

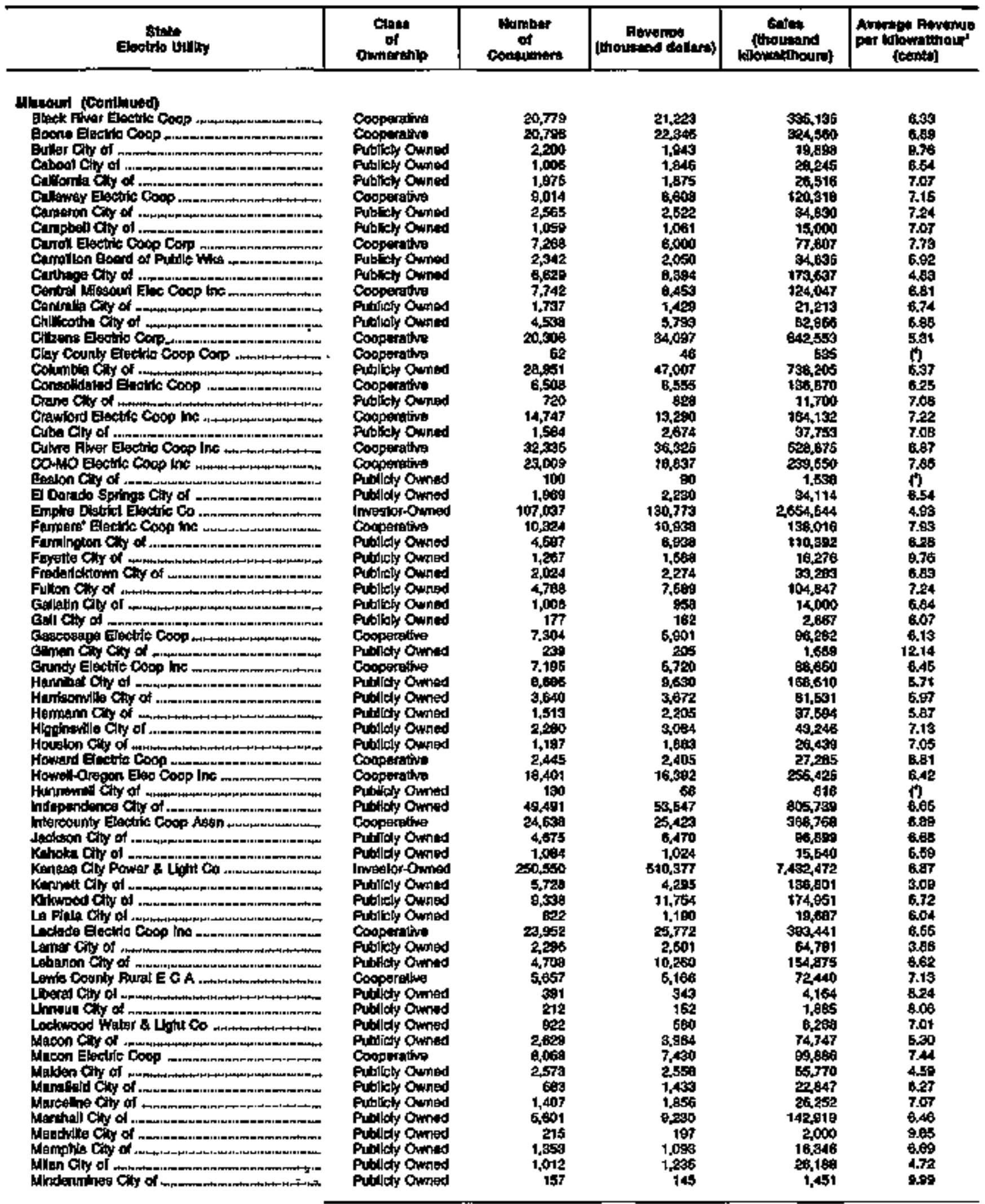

So toonotes at end of lehk. 
Table 17. Class of Ownershlp, Number of Ultimate Consumers, Revenue, Sales, and Average Revenue per Kllowatthour for All Sectors by State and Utility, 1993 (Continued)

\begin{tabular}{|c|c|c|c|c|c|}
\hline 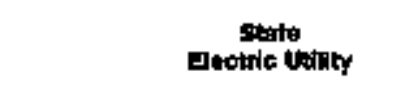 & $\begin{array}{l}\text { Cta:a } \\
\text { off } \\
\text { Oiwniftehlp }\end{array}$ & Contumart & 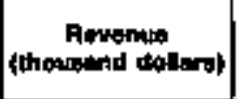 & $\begin{array}{l}\text { stubt } \\
\text { Ekoulind } \\
\text { klowethoutial }\end{array}$ & 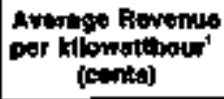 \\
\hline 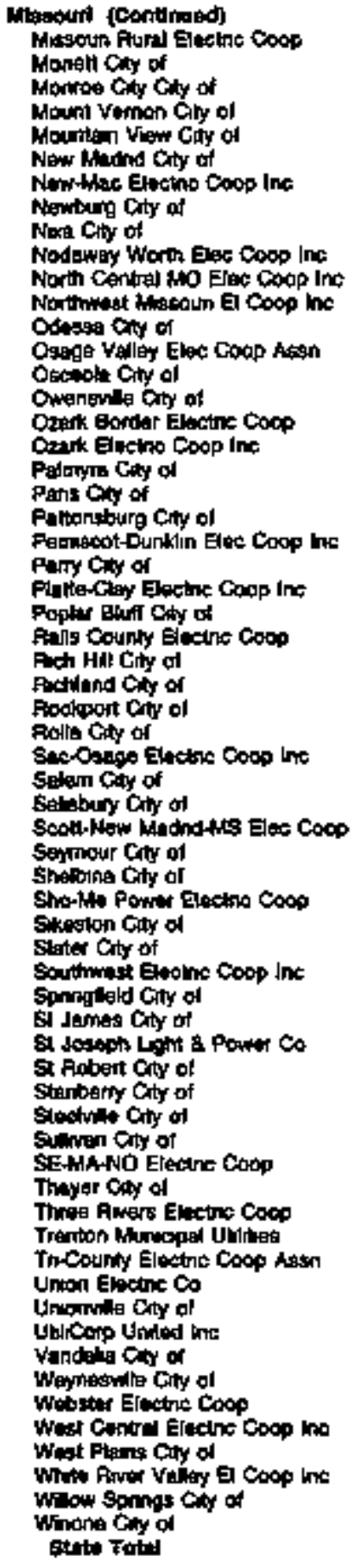 & 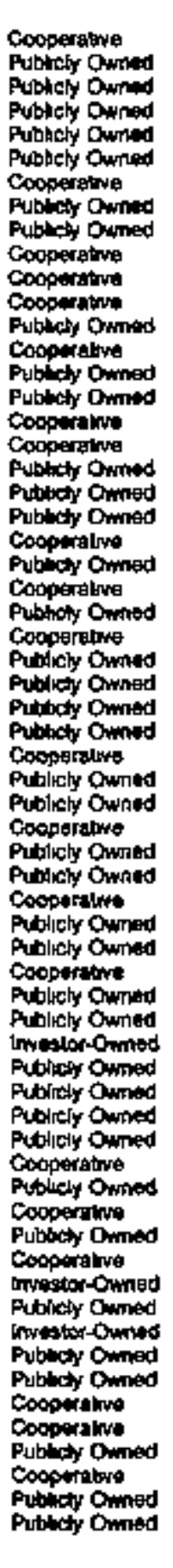 & 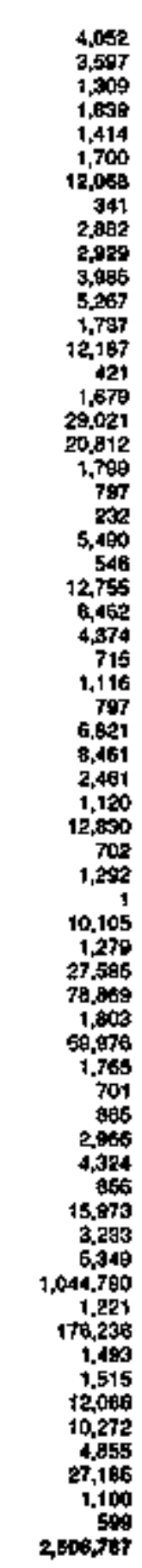 & 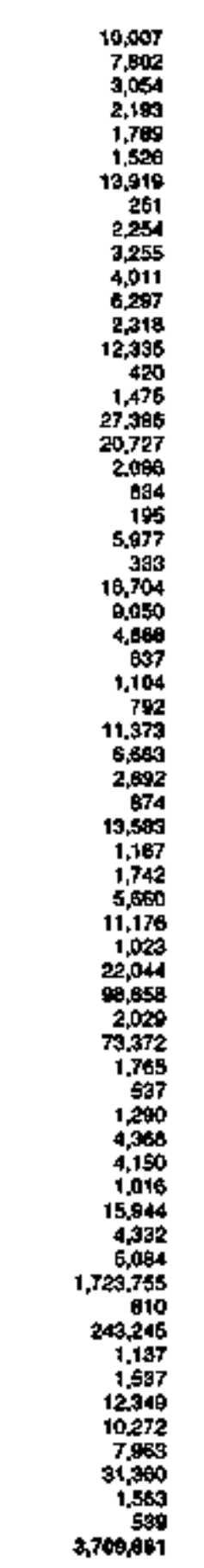 & 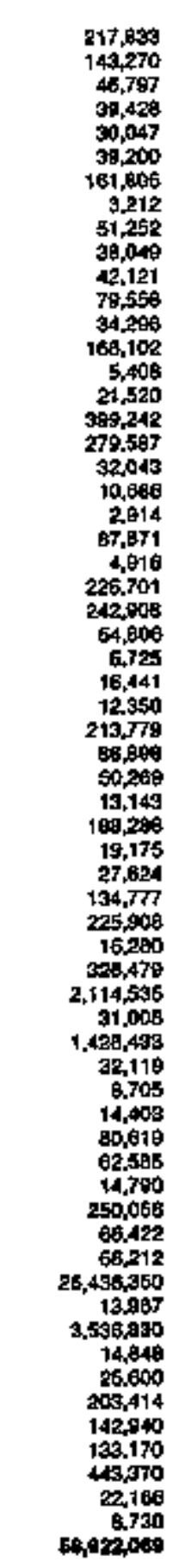 & 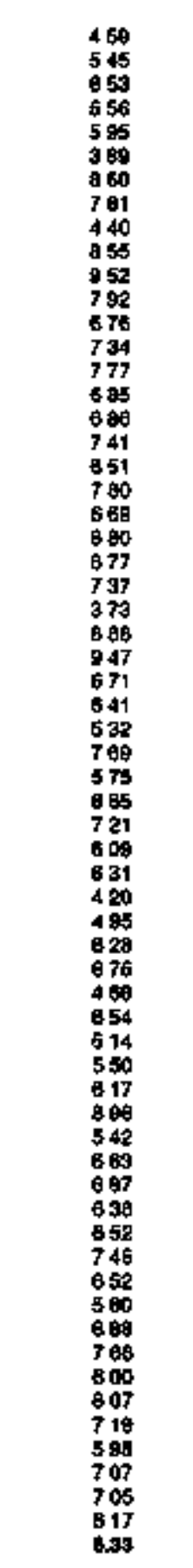 \\
\hline 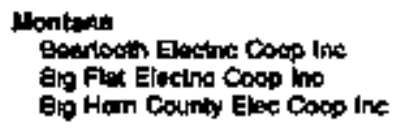 & $\begin{array}{l}\text { Cocperaber } \\
\text { Cooporatiwe } \\
\text { Cooperalowe }\end{array}$ & $\begin{array}{l}3,774 \\
1,569 \\
3,007\end{array}$ & $\begin{array}{l}2,802 \\
0,026 \\
2,871\end{array}$ & $\begin{array}{l}4,102 \\
58,178 \\
42,080\end{array}$ & $\begin{array}{l}857 \\
5 \$ 9 \\
672\end{array}$ \\
\hline
\end{tabular}

Sta forinotes at and at uble 
Table 17. Class of Ownerehlp, Number of Utimate Consumers, Revenue, Sales, and Average Revenue per Kllowatthour for An Sectors by State and Utilly, 1993 (Conthued)

\begin{tabular}{|c|c|c|c|c|c|}
\hline Etath & of & $\begin{array}{l}\text { Number } \\
\text { of } \\
\text { canoumars }\end{array}$ & Rovetinuts & 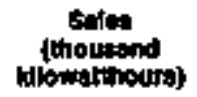 & 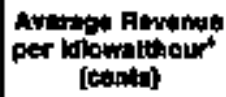 \\
\hline
\end{tabular}

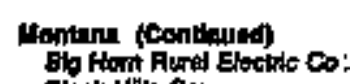

ilats Hit Comp

Gannerile Panier Admin

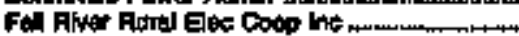

Fingut Elmotrit Coxp ino.

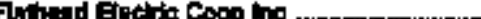

elucier Elatino Coop tho

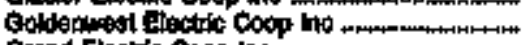

Gind Elactio coop he

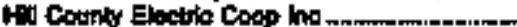

Uacoh Electric Coop linc

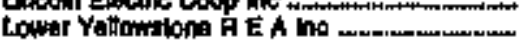

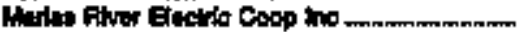

MeCond Electris Coop his

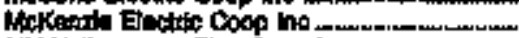

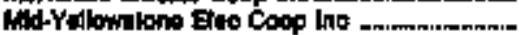

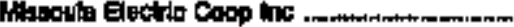

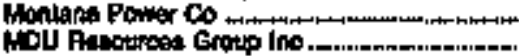

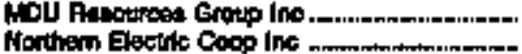

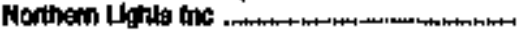

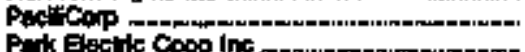

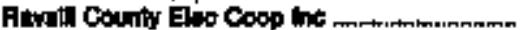

Bhoddan Etoctric Coop itc

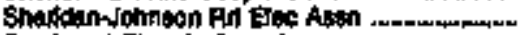

Southunt Electic Coop lo

Bu Ftre Elactric Coop hit

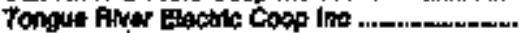

Troy chy od.

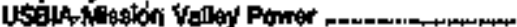

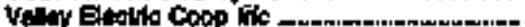

Vontertiv Electro Coos int

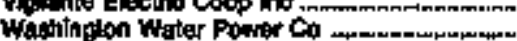

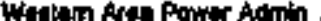

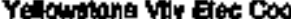

\$tits Totel .

Achatio

Nitoros Coty of

Arelay City of

imold viling of

Aldintin Ciby ol .

Bertion vilngo of .

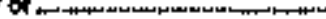

Eetiven $C$ ity of

Gewiver Ciny Cofy of

Aenivimat Coby of .

Evas iflis of of

Endahew Villuge of

Grabiand village of

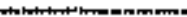

Brolina Bow City of

But County Puble Power Dist

Bunit: cits of

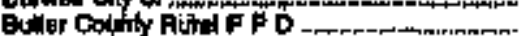

collewer vitiofo of

Cambridut City of .

Cumbbell Vilaria or

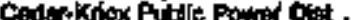

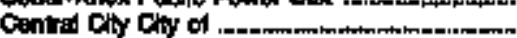

Chaped city of

Chory-Todd Eloctris Casp ins

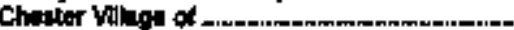

Chimner Roch Publin Power Dist

Ctudion City of

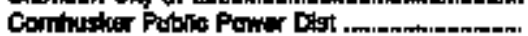

Cooparattive

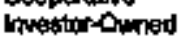

Feding

Cooperafiva

cooperativa

Coopentive

Cooperative

Coppitaliva

Cocperathia

Cooporndive

Gooperitiva

Cocpratua

Cooperative

Copopotitiva

Cooperathe

Cooperative

Cooperative

Imrester-Omed

invertor-Owned

Cocperathe

Cocperative

Imnetor-Omad

Cocporalive

Cocperativa

Cucperative

Cocperatho

Cocoperative

Copoporative

Coopurature

Pubility Ourtod

Fodon:

Cocperable

Cocperetivi

Inveslor-Oming

Foderat

Cucperative

Purngly Ouned

Putricty Owlet

Puthly Ounted

Putildy Ounsed

Publety Oward

Publicty Owned

Publity Ounod

Pubtcty Outhed

Publly Omined

Pubticly Omined

Pubrich Cinnd

Pullach Omiled

Pubtely Ouned

Putisd Omined

Puncely Ounted

Pubrich Ouned

Putrity Omad

Publicty Ouned

Puthcty Ouned

Putich Omied

Pubtedy Cinnd

Puticer Owned

Pubicty Omitad

Putecty Omined

Putedy Owned

Cooperative

Puticty Ormat

Alvinty Omad

Prbiles Dined

Puliles Omed

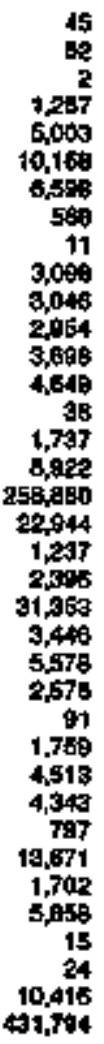

(2)

47005

2,010

4,686

8,721

7,953

581

3,006

4,678.

4,805

$3,49 d$

4,57:

1,370

$7 \mathrm{ARS}$

390955

27,378

2031

9,001

30,650

3,190

5.578

4,877

1.0

1,40

4,519

6:0

13,309

1,472

S,ext

19

6, 640

So4,학

4,847

308

507

2,708

214

67

4, 827

40

84t

464

200

B87

2.207

3,750

758

4.160

305

349

$4.46 \mathrm{X}$

1,750

Bre

240

2,255

413

7,649
5.806

216

\$21

2, 09

148

557

76

6.700

अ

674

202

200

674

1,84

4820

589

405

273

602

168

5000

1,553

stas

76

160

2594

10,05s
1,05

12063

2,487,741

50,670

70.981

$165 ? 00$

142.850

5,702

85

$\$ 5,554$

55602

76,00?

$55,55 t$

T78

2001

132540

7,122032

470,741

28,752

60.170

761,105

43,304

a9,3es

70,845

498

18.158

g5ess

78,657

13,945

200,054

20,348

95215

25

27.535

147.715

topetst

0,2ac

a, ass

6,278

48,175

1,800

8.778

18,70 어

t20,157

5,505

B. 044

6,212

2,674

3,131

12,941

44,154

75,ars

9.00\%

62789

B.971

6.601

2,844

04,060

20,272

11,295

10914

2,754

25,002

9.567

181.557 
Table 17. Class of Ownermhip, Number of Utimate Constumers, Revenue, Sales, and Average Feverue per Kllowatthour for All Sectors by State and Utility, 1893 (Contlnued)

\begin{tabular}{|c|c|c|c|c|c|}
\hline State & $\begin{array}{l}\text { Clops } \\
\text { of } \\
\text { Cwaratip }\end{array}$ & $\begin{array}{l}\text { Number } \\
\text { of } \\
\text { Contumars }\end{array}$ & $\begin{array}{c}\text { Fowenus } \\
\text { (thourtind dollara) }\end{array}$ & $\begin{array}{l}\text { sales } \\
\text { (thougend } \\
\text { idlowatthecura) }\end{array}$ & 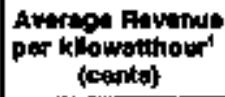 \\
\hline
\end{tabular}

Habrasce toondinued

Cornd Boand of Publle Worticg ..--_...--....-...

Coteb Gity of ....................................................

Cuming County Pubic PM Dal

Curtis City of

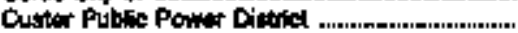

Dewenpor Vilgoge of

Davis Cin City of

Doweon Count Pube Put Dit

Do Wha vila ol

Dexphr Vilage of

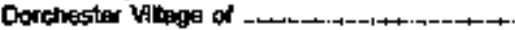

Eilgar Chy of .

Ex Cratk villaga of

Emestion Chy of .

End"colt Vilopge al

Feitury city al

Formanl this of of

Fatis Ciny city of

Filay Clly of $u$.

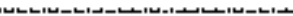

Framoni ciny of

Filand City di .

eviner City of .

Eothenbuig City 하

Crand lsind finy of.

Crand Cöby in

Gretamood City of

Hempton VIliog of

Heqing Coty of

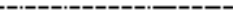

Heninglord viloge ol ........................................

Herkonan (Ct) of .

Hotine Eloctric Aten

Hidroth Villapa of

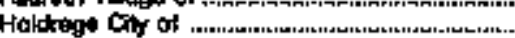

Howed Ciregloy Rural \& P D

Hutbol city of

imperial city of

Indininola Giny of

Nmbill Cor or

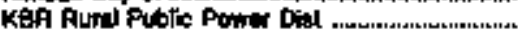

Lured Givy of

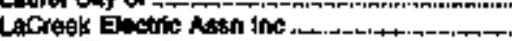

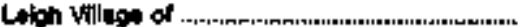

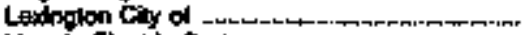

Linooin Electio Sytion

Lodoupols chy of

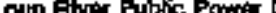

Loup Valors Rurn! P P D.

Lymin Vatipg of

Lyong Gity of .

Ningers Civ

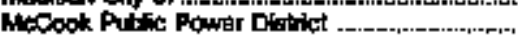

Afinmeat Batrito Momber Corp

Mindien Gity of

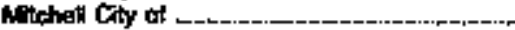

hordl vithos of

Mullen vaty of

Nebraka Cory Cot of

Nobrtak Publo Power Dlotrict ......................

Neilgh City of

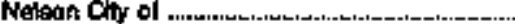

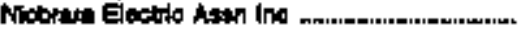

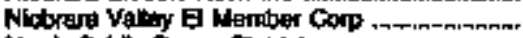

Norita Putsic Pown Districl ..

North Contral Publlo Pur Otst..
Pubricty Cimied

Publict Dimod

Prbicy owmed

Pubticy Owned

Pubitsy owned

Publesy owmed

Publith Ounted

Putbist Ommod

Publlaly Owned

Fublisy awmd

Putblesy Owmen

Puthlaty Owned

Publicy awned

Publely Owmed

Fublenty Owritd

Fubllaly Owned

Publeby Cimed

Publicty Cimed

Pubcer Dmad

Publecy OMmed

Publiptr Omind

Pubatir Dimod

Pub'cy Owmed

Fublecty Oimid

Pribles Dimed

Pulbitisy Omed

Publity Dimed

Pubtery Ommad

Putbinty Oment

Pubicty Owned

Publicy Dwmed

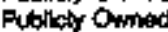

Aubrtit Ownta

Putbliby Owmed

Fubliaby Owited

Cooptrative

Fubicty Dinied

Publicty Oimed

Pubcety Omod

Pusticty Dimat

Pulatey Oimed

Pubsery Omod

Pubricty Dumied

Publity Omed

Pubrery Cwned

Cooperalive

Publity Ouned

Pulbich Omed

Pubcty Cims

Pubras Cimed

Prbicky Omad

Publey Cimad

Pubricy OMmed

Publey Omad

Pubcy armad

Publey Qumed

Cooperative

Pubiely Ownod

Publect Omied

Publecty Dimina

Publicty Omined

Publity Omied

Pubcary Oimed

Publety Owned

Publicty Onhed

Cooperature

Cooperaltwe

Pubity Comed

Pubsich Ouned
25

2425

20199

ro1

8,403

290

1,322

18,010

348

Eดी

292

งตำ

408

7

4,083

320

2 .832

100

702

12.429
844

3,491

197

1,650
20,263

775

asta

247

1,035

Be?

457

1.8B1

200

3,075

3,116

1,061

345

1.546

3.228

562

179

3.751

08.914

267

15.629

3,731

267

938

4.464

4,742

1.492

1.014

son

5.100

107,080

1,006

367

750
4,702

12,820

3,606
2.862

$3, \$ 94$

3.925

6EB

7,144

108

1,442

13,723

eos

3 a2

250

304

68
200

7,200

300

37

270

2,065

2005
89
656

12.

511
3340

211

1,891

23,644

813

270

218

12.

965

460

568

3,472

140

3,869

2,327

$\$ 1$
1.193

1.193
270

1,160

2780

253

230

5.212

104,437

148

22.419

2,980

$34 \mathrm{~B}$

2,375
B.2315

7,464

1.300

979

716

144,749

D87

270

559

6,365

20.160

3,765

58.140

6E.600

7 고오

0.617

101,568

2500

26.296

218,713

14,938

3.200

6,523

4,200

5.112

1006

116,042

5.228

59,606

4.602

44,465

1,090

7,089

286,099

0,040

60,005 
Toble 17. Clags of Omnershlp, Number of Uttlmate Congumers, Revenue, Salea, end Average Revenue per Kllowathour for All Sectors by State and Utility, 1993 (Continued)

\begin{tabular}{|c|c|c|c|c|c|}
\hline Sinto & 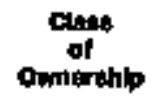 & $\begin{array}{l}\text { Number } \\
\text { of } \\
\text { Conpuners }\end{array}$ & thactend dolares & 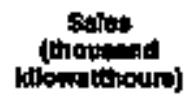 & 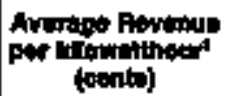 \\
\hline
\end{tabular}

Nabranle (Contimuted)

Nowl Pinte Cory of

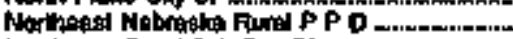

Narthant Furd Pub Pir Olst.

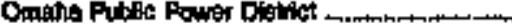

Crd Chy of ...

partinta wiple of

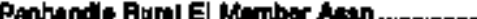

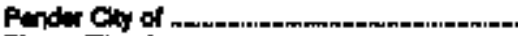

Pinces City of

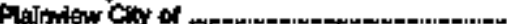

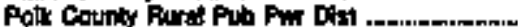

Poitk Vileges of t.

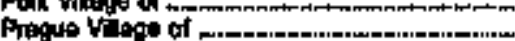

Findoloh City of

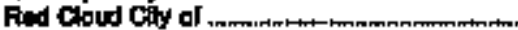

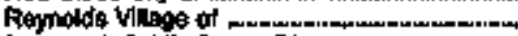

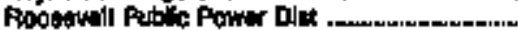

Furtl Elctéc Co

Bagint Cly of

schutin tin of

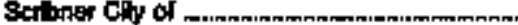

Beward che of

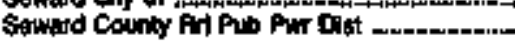

Stchloy tinapa of.

Sidney cint of .

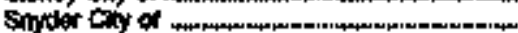

South Condid Puble Pwr Dist

Bouth sibux City Caly of

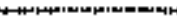

Aural $P$ P D

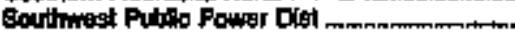

spesting Viliage of

Spanest Clyy or

(l)

Stamion Cosnk Pubso PWr Olat ................

Stretion cthy of

Stromstyvg City of

Shit Ciry ol

Supaitor Cily of

Sition Chy of

Srucuse City id .

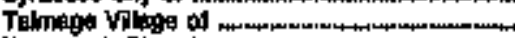

Tecumbin Chy of .

Trention Clty of

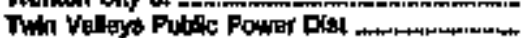

Valen'ine Cily of

Whos Chy of

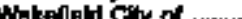

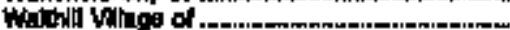

Wunate Viloge of

Hiante Cty of

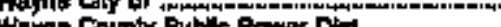

West Point Cly of

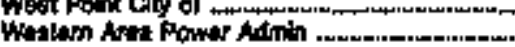

Wheat Batt Publis Powar Dist........m-mint.

wilber city of .

Witeox vilage of

Winste vilang of

Winar Cly ol

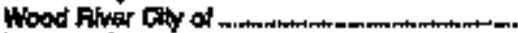

Nimore ons of

Hinuto og

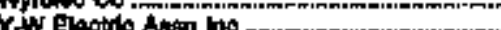

York Counh Furd Pab Pur Dist

Stats Tabil

Fublick Owind

Fublay owned

Fubrity Owned

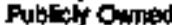

Publed, Owined

Pustaty Owmed

Publicty Ouxiod

Cooperative

Publoty owned

Fublicts ownd

Publety Quntad

Publley ownod

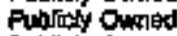

Publioly Owated

Publich owned

Publich Owmed

subtely Ound

Publets Owned

Cocoprefive

Putrely Quried

Pustloty Qwined

Publicky Owned

Futricly owned

Futticit owned

Publlty Owned

Puttey Owined

Putaldy owned

Putlely Ownas

Publitey Owend

Futtioty Owned

Publlty Oined

Publloty Orined

Publldy Owner

Putifiely Gurced

Publoly Owned

Putblecty Ownad

Purlidy Ormed

Putldy Oined

Pulicly Qwred

Puthledy Ownes

Putrely Owand

Putilety Oumbat

Publey Ouned

Pubidy Ounod

Putlity Gured

Putaldy Owed

Putalely owned

Publety Owned

Publly Oinind

Publly Gimed

Pubaldy Orwited

Puthlety Ownod

Putilicty ouried

Fodict:

Publtcty Onred

Pullety Ormed

Publledy Oined

Punicty Owwed

Pubildy Owned

Putdly Owrod

Pubichy Ciwred

Coopordin

Cocparedre

Pubdly Ouned

Publety Owned

Putaldy Oined
13,64

2.744

2,74

250,468

1,394

514
101

act?

由78

1,008

604

2,540

192

6.7

776

78

2,362

2,540

44

2,193

2,504

2,616

255

200

4,317

4.864

18,682

5,190

305

1,085

273

852

359

1,492

바1

159

1,200

430

4,591

1,772

2,201

504

360

462

2.151

2,599

1.12:4

13

4,326

tola

210

285

924

441

64

Bat,275

64

6,20?
10.60

3,010

3, 所

sed, 760

1,060

500

6. nges

646

B82

600

2617

282

643

B.20

50
2803

5,013

s40

3,161

575

2.161

3,189

207

2.291

276
4.510

5.505

20.485

6.596

251

324

2662

205

601

282

1,091

634

111

1,283

3.365

1,027

1.409

50

330

548

2.74

2,007

1.497

1,589

7,842

584

167
152

152

500

647

810

다

1,016,746

484
6,082

110,810

508

5.00

40

5Th

63

1.:

4 (e)

6.74

7.7

B.3.

862

$6 \leq$

634

(b)

5.16

9.73

60

1.90

6.70

608

A.er

5.82

G.17

7.05

9.03

G.65

6.41

B.34

b.23

6.09

6.31

412 
Table 17. Class of Ownershlp, Number of Ultimsto Consumers, Revenue, Sates, and Average Rovenup per KIlowatthour for All Sectors by State and Utility, 1993 (Continued)

\begin{tabular}{|c|c|c|c|c|c|}
\hline $\begin{array}{c}\text { 5talt } \\
\text { Enectele Utrity }\end{array}$ & $\begin{array}{c}\text { clest } \\
\text { of } \\
\text { Omerahlp }\end{array}$ & $\begin{array}{c}\text { Number } \\
\text { of } \\
\text { Contunters }\end{array}$ & Revenut & $\begin{array}{c}\text { salos } \\
\text { (thodgend } \\
\text { ittownthours) }\end{array}$ & 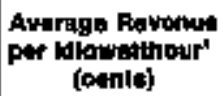 \\
\hline 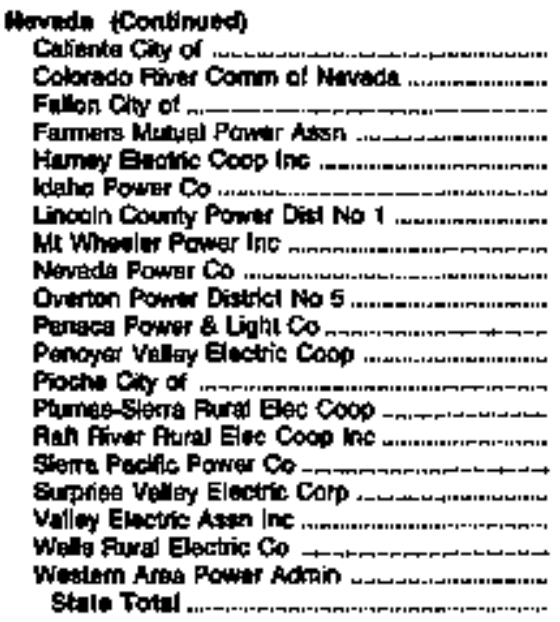 & 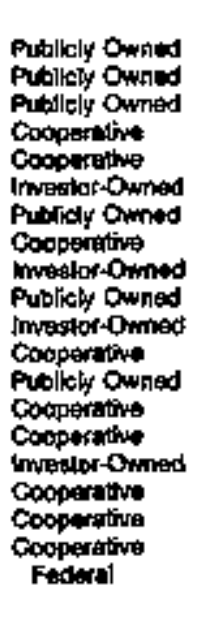 & $\begin{array}{r}519 \\
7 \\
3,701 \\
49 \\
1,266 \\
1,185 \\
169 \\
5,740 \\
392,779 \\
3,645 \\
370 \\
52 \\
340 \\
148 \\
200 \\
211,577 \\
3 \\
0,043 \\
4,195 \\
3 \\
640,922\end{array}$ & 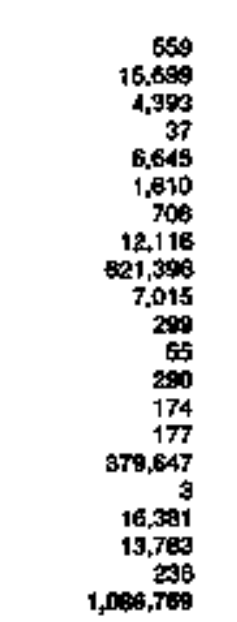 & 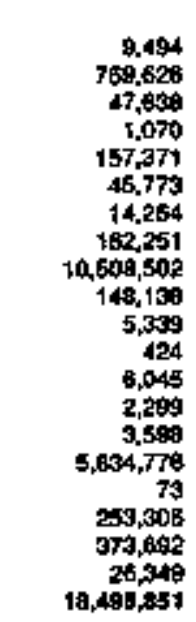 & $\begin{array}{l}5.80 \\
2.01 \\
9.28 \\
4.72 \\
4.52 \\
4.85 \\
7.47 \\
5.81 \\
4.74 \\
5.80 \\
17 \\
4.00 \\
7.67 \\
4.82 \\
6.61 \\
17 \\
6.47 \\
3.60 \\
5.60\end{array}$ \\
\hline 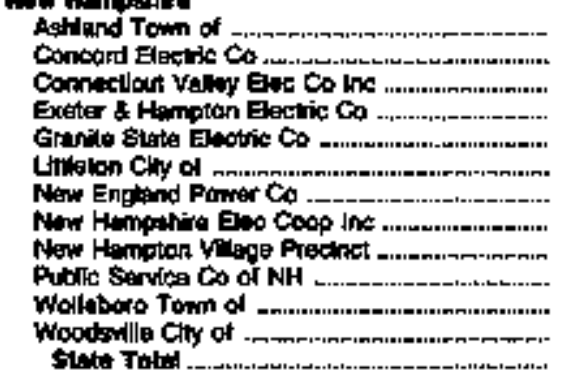 & 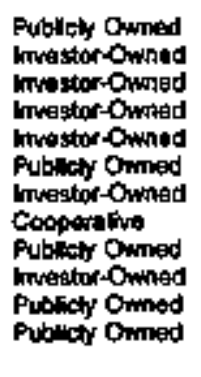 & $\begin{array}{r}1,092 \\
26,802 \\
10,275 \\
35,680 \\
34,806 \\
3,240 \\
1 \\
63,126 \\
141 \\
9 \times 7,252 \\
6,102 \\
5,005 \\
5 r 4,620\end{array}$ & 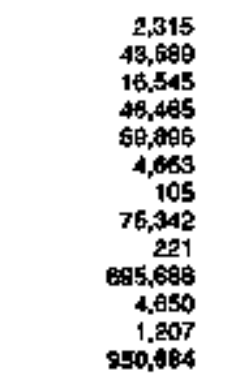 & 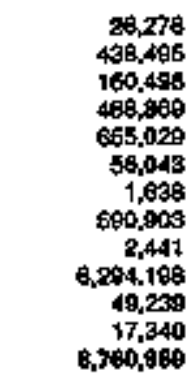 & $\begin{array}{r}8.81 \\
0.94 \\
10.31 \\
0.91 \\
0.14 \\
0.32 \\
6.41 \\
12.75 \\
9.05 \\
11.05 \\
0.44 \\
8.98 \\
10.95\end{array}$ \\
\hline 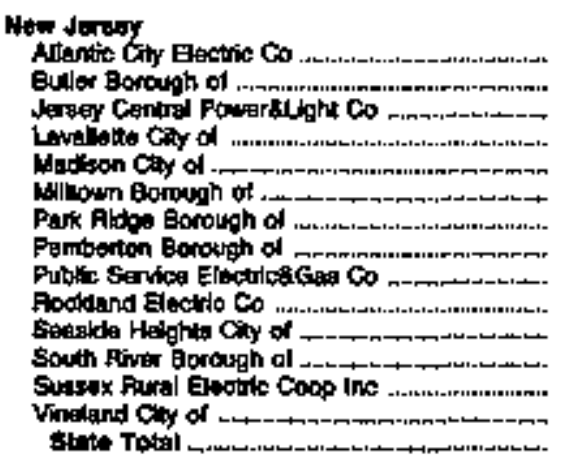 & 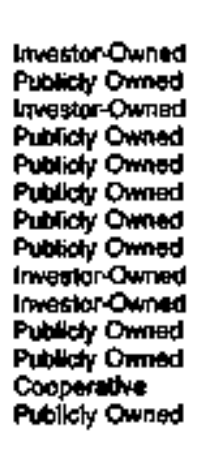 & 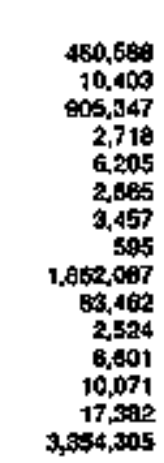 & $\begin{array}{r}820,370 \\
11,743 \\
1,974,755 \\
1,406 \\
13,944 \\
3,064 \\
5.299 \\
651 \\
9,628,471 \\
133,700 \\
3,301 \\
4,585 \\
12,679 \\
40,590 \\
6,554,408\end{array}$ & 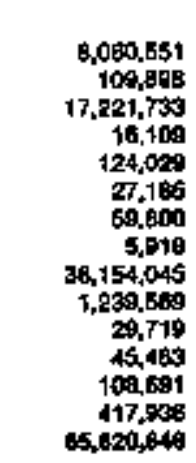 & $\begin{array}{r}10.10 \\
10.85 \\
10.98 \\
6.72 \\
11.24 \\
11.27 \\
6.85 \\
11.00 \\
9.61 \\
10.79 \\
11.11 \\
10.09 \\
11.67 \\
9.70 \\
0.90\end{array}$ \\
\hline 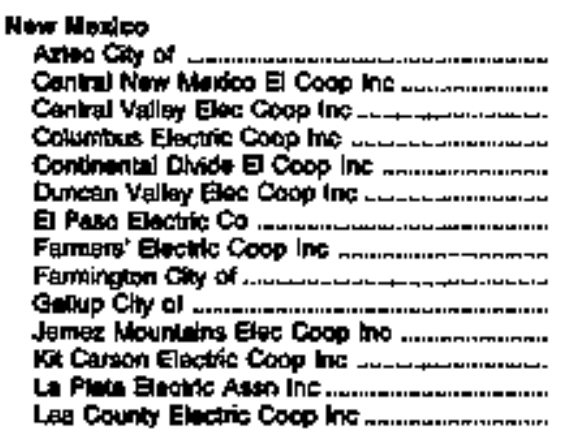 & 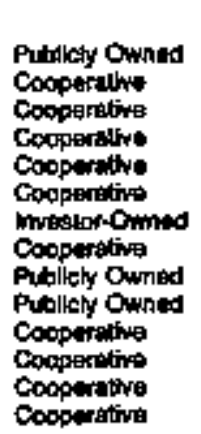 & 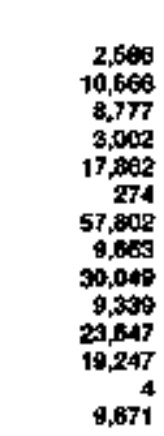 & 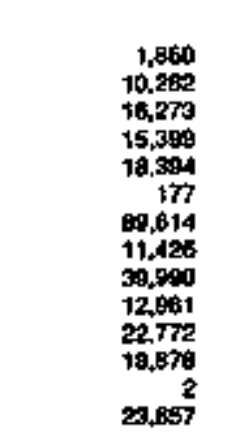 & $\begin{array}{r}24,072 \\
94,735 \\
90 \$, 041 \\
208,113 \\
104,385 \\
2,350 \\
1,005,058 \\
140,570 \\
598,674 \\
199,660 \\
217,080 \\
171,209 \\
27 \\
454,981\end{array}$ & $\begin{array}{r}7.54 \\
11.05 \\
6.27 \\
5.85 \\
8.48 \\
7.69 \\
6.65 \\
6.13 \\
7.45 \\
7.04 \\
10.49 \\
11.02 \\
16 \\
5.24\end{array}$ \\
\hline
\end{tabular}

Sev toobrotos all end of tible. 
Table 17. Class of Omnership, Number of Ultimate Consumers, Reverue, Sales, and Average Revenue per Kifowathour for All Sectors by State and Utility, 1993 (Continued)

\begin{tabular}{|c|c|c|c|c|c|}
\hline sirto & $\begin{array}{c}\text { Fise } \\
\text { of } \\
\text { Onntenhing }\end{array}$ & $\begin{array}{c}\text { Numbor } \\
\text { at } \\
\text { Comiumare }\end{array}$ & 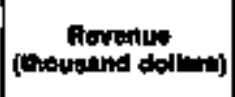 & 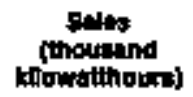 & 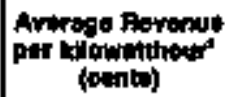 \\
\hline
\end{tabular}

Anw Heriog (Contiaund)

Los Alomas County

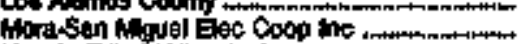

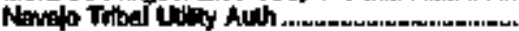

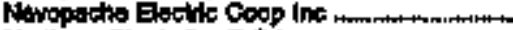

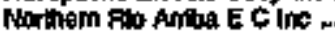

Otan County Bentic Coop inc

Publi Senvos Co of NM.

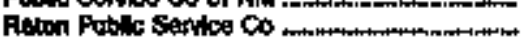

Rib Qrende Eurtic Coop ins ....................

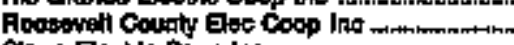

Slinta Eectile coop lint:

Bocorro Eectio coop inc

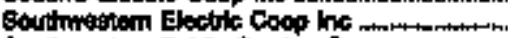

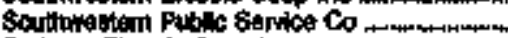

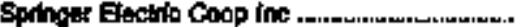

Spinger Tom of

TexBs New Hexico Fow

Thuth or Coneequincas Cin of

Wontern Arat Powter Admin

Steto Tot:l

\section{Now York}

Nom Viliage od

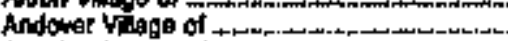

Angaber valage of

Areids Vilone of .

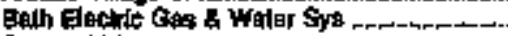

Borgen viluge of .

Boomille Viliega of

Eracton City of

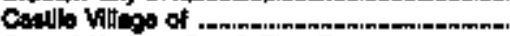

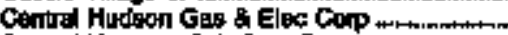

Cenirst Vermonl Pub Sen Corp ...................

Churctuille viloss of

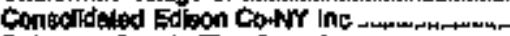

Oelouad County Eeo Coop Ino .................

Endicott Villege of

Fuport Viage of

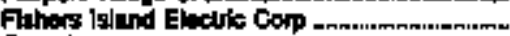

Franktor viliege of.

Frowport Villeog of Ins -

Green tatend Vilage or

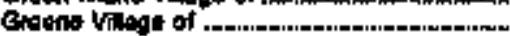

Gaenport Villoge ol

Goten viluge of

Hamliton vilegs of

Holloy Villoge of

linom vinge ol -

Jempelowar City of

Like Ptand viltog the

Uitit Volny valiog of

Long leland Uthiting $C_{0}$

Murtithen vitige of

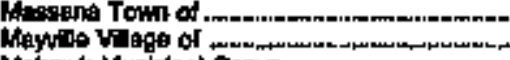

Mohum Hunlolpal Comm ....n...................

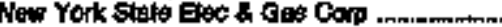

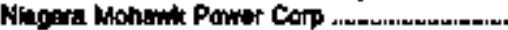

Oneldotiladson Elo Coop th................

Orengs 8 Focklend Utits inc tom

Otatio Enetric coop lne .............................

Penr Yan Villege ol

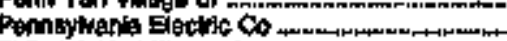

Ftiadolphin wlace ol

Plattofiprot City of

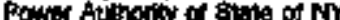

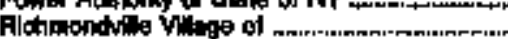

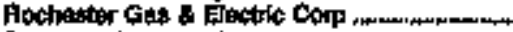

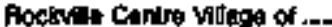

Publity Ommad

Cooptiding

Plibily Owned

Cooperathe

Cooperative

Cooperatse

invedor-Owned

Publisty Owntod

Coopurative

Cooperethe

Gouperative

Cooperabse

Cooperative

Impetortomnad

Coopinelats

Putilesy Owned

Imestor-Omed

Pubticty Oment

Eoder:

Putwly Owared

Publety Omed

Pullaty Ormed

Publly Onined

Publity Ourter

Pubtety Onmed

Pubucty Onmed

Puticty Ormed

Publucty Owriod

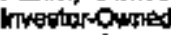

Investortowner

Publuty Oined

Investers Owried

Cocpurative

Pubict Ourrad

Psibictr Ormat

Invortor-Owised

Putichty onned

Publichy Ownod

Pubicis Ommed

Pubichy Orned

Pulbilly Owmed

Putsict Omned

Publict Ownod

Putblaty Ommed

Pubicty Ómed

Publich Dwmod

Pulaticty Ommod

Pubicty Ownes

inveption-Ouned

Fublesty Owitad

Pasblety Dunned

Fublict owned

Publich Owned

investor-Omined

threstor-Ommed

Cooperalrue

imvestor-Ommod

Coopurative

Publecty Domed

Imveator-Omed

Puttody ownes

Fulility owned

Putllohy Owined

Pubillely owned

rmestor-Ommed

Pronloby Ouned

7,096
7,369
5,190
1,195
3,134
1,730
300,325
4,014
280
5,221
2,912
8,629
1,497
99,019
2,449
4096
40,697
3,760
716,391

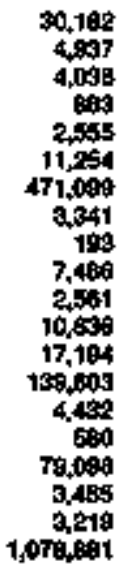

1,442

549

3,446

4.723

65

2034

971

tea

259,600

2

2884714

4.252

3,274

14,386

894

1,671

14,874

1.254

1,253

2884

1,029

$\$, 487$

of

3.948

20,107

4398

1,957

1,012,001

904

8,946

1.004

1,352

789,709

$1,544,159$

$+500$

168,480

3,444

2,790

9,6028

720

8,970

104

09

344,921

10,0092
1,300

250
340

3214

2450

1,127

1,674

414

652

397,674

015

4,900,406

3,106

1,806

092

a72

13,765

667

sigs

784

1,471

609

2,017

17,204

3,922

1,040

$2,028,246$

s12

$5,76 \%$

695

1,347,621

3,046,07T

1,257

345,105

2,792

1,500

5,601

620

10,35:

850,202

15

9.469

457,473

41,270

59.713

8,602

20,756

90,694

6,446,788

48,305

1,511

107,275

1829

118, 352

375.499

2868,195

64,0904

4,302

$1,409,020$

st5ed

151,870

14,026,67? (onts)

(a)

46,476

$6703 \quad 37$

$7,101 \quad 4.79$

113,416 28

71,220

21,7675

69.489208

1253013.50

$4,703,769$

3

16.037

35.240,999

40,614

49.637

340,707

4,565

19,301

295,407

$11, \mathrm{BS}$ 9

31,757

23,771

21,010

48,133 
Table 17. Class of Ownerahlp, Number of Utimate Constumers, Rovenue, Sales, and Average Revenue per KIlowatthour for All Sectors by State and Utility, 1993 (Continued)

\begin{tabular}{|c|c|c|c|c|c|}
\hline stabs & $\begin{array}{c}\text { Clate: } \\
\text { of } \\
\text { Otmermip }\end{array}$ & $\begin{array}{c}\text { Humper } \\
\text { of } \\
\text { condupers }\end{array}$ & 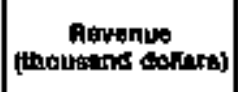 & 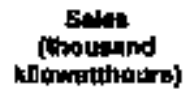 & 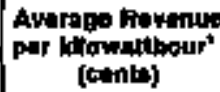 \\
\hline
\end{tabular}

Now York (Cominued

Fouspes Point Vilage of

Salinince tor of

Shatume viteos of

Shemill Ciny or

Sitrof springs Valage of

orano:

Sctury Vilape of.

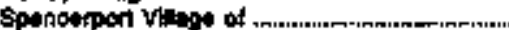

Springrit thage ol

Staphan Fural Elect Coop Int

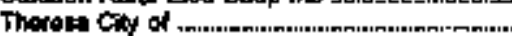

Tupper Lake villege of

Wging Gin Villog of

Wolswili valige of .

Wrestilids Vinge ol .

stite Tolat

North Caroling

Aberiate Gily or

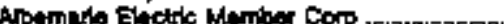

Apex Tawr of

Ayden Town of

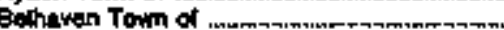

Bengon Trmm of .

Black Craak Town of

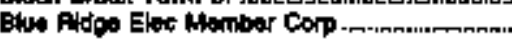

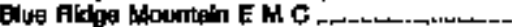

Bostic Tom of

Broud Fiver Elocinto coop inc

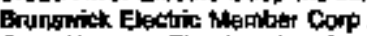

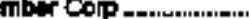

Cegin Power 8 Lolal Co

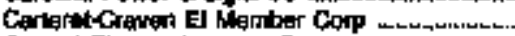

Canind Elactric Member Corp

ciomying chty of

Clayton Chy of

Conitird Chy of

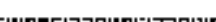

Creseanl Elactric Member Comp - -

Dellas Tomm ol ...

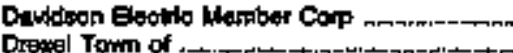

Diraigl Toim of

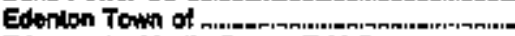

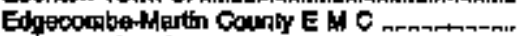

Eratew Gy City of

Enfatd Town of

Fampilis Tomm of .

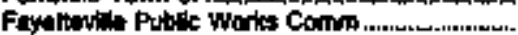

Forat Cly Tom of nom

Fouml:-1 Town of .

Four Counb Elac Mamber Corp

Frerrount Toun of

Franch Bingad Elec Mambe Corp

Cictitenta Cory of

Crantie Fille Town of

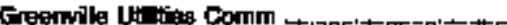

Halex Elactric Nomber Corp

Humbion Toim of

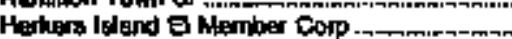

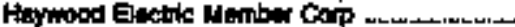

Hertiond chy of

High Folit Towl of

Firlands City of

Hobood Toum of

Hoblutiton Town of

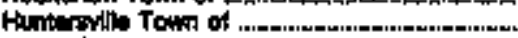

Jonar Onatow Elac Woriber Corp

Iongs Mountaln City of

Sog lootnoles al and of table.

Fublicly Ouroded

Pullohy Owined

Publthty Owriad

Publisty Owited

Publloly Owined

Putllcty Owoned

Publey Owined

Publtebr owned

Pubdidy Owortad

Coaperative

Fublety Ownod

Publidy Owed

Publicty Ouned

Publloly owned

Publldy Quped

Putlaly Owned

Cooperathe

Fublict Oenud

Fubllaly Owned

Putllaty Quinad

Fublith Qumded

Publoly Owned

Cooperative

Goaptrotive

Futlobs owned

Cooperative

Copperative

Cooperative

Imvestor-Oymed

Coposintirte

Coaperative

Puthlely Owned

Publicay Ounted

Pubtiay Owtid

Publieb owned

Cooperative

Pubicky Owned

Cooperathe

Putulely Owinted

Intealos-Ownes

Fubliny Owned

Cooperatha

Putilely Oumed

Publich Ownod

Publitis ownad

Publecty Ouned

Pusilitis Owned

Publety Owmad

Cuaperativi

Puthlaby Outued

Cooperatura

Pubsey Dewned

Putilety ownod

Publuky Ownad

cocprative

Publebs ownod

Cooperathe

caponiario

Eubliels Owned

Putilleb owited

PubFry Owm

Publaty Owmed

Publicty Owmad

Pubichy Oumed

Cooperathe

Pulblich Owmid

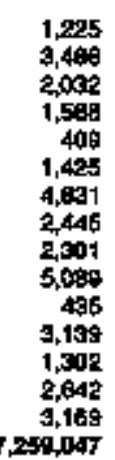

$7,259,047$

10,648

8,3028

3,151

3,230

1,241

1,714

608

42,000

9,978

173

182
47.705

4858

a? 155

26,440

10,651

2,353

2,608

18500

1,212

40,912

2,015

32,080

1,278

1.299,312

3561

0,901

$800 \mathrm{~d}$

1847

2,709

B,251

4.277

385

802

25.16

23.485

1,850

\$9.0S5

9589

273

1.100

16394

1 , 18

ง1,306

2,300

$\$ 43$

345

1,238

30,677

s.t.2
1,839

1,760

1,014

1,676

161

80

$3,5 \times$

1.761

1.008

4,574

267

2169

1,302

1,652

1,694

$13,000,395$

19,658

8,762

4,719

7,247

2,200

2,003

E⿹

5496

7,2:1

142

223

60,18

6,577

$1,850,497$

32,973

14,006

4,310

4,658

25,126

1,065

5, $000 \mathrm{~d}$

3,132

11,110

1,199

2,613,679

7,777

19,134

20,608

2,342

4,018

115,489

7,674

$\$ 40$

1,469

28,845

$45,54 B$

3,178

01,005

10,308

378

1,202

16. 169

2,245

50,800

$1,5 \%$

490

$\$ 10$

2,082

54,430)

6.015e

40,420

$72,22:$

80,032

70,425

4,590

24,400

140,104

5 2,280

51, 27:

54,199

G.48

7,695

40,623

53,410

$130,170,17$.

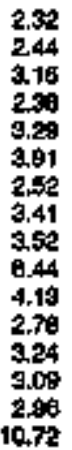

2012,942

109,598

4),420

77,005

19,896

31, 638

7,620

649,958

102,081

2242

2,454
50,406

$6 \times 897$

2S, 440,302

367,356

149,206

56.54 
Table 17. Class of Owmershlp, Numbers of Ultimate Consumers, Revenue, Sales, and Ä̌rerage Rovenue per Kilowatthour for All Sectors by State and Utillty, 1993 (Continued)

\begin{tabular}{|c|c|c|c|c|c|}
\hline $\begin{array}{c}\text { stato } \\
\text { Elactite dinty }\end{array}$ & $\begin{array}{c}\text { Clatal } \\
\text { of } \\
\text { Ormantolp }\end{array}$ & $\begin{array}{c}\text { Humbar } \\
\text { of } \\
\text { consumers }\end{array}$ & Roverits & 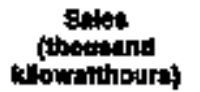 & 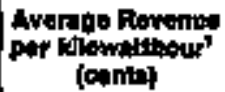 \\
\hline 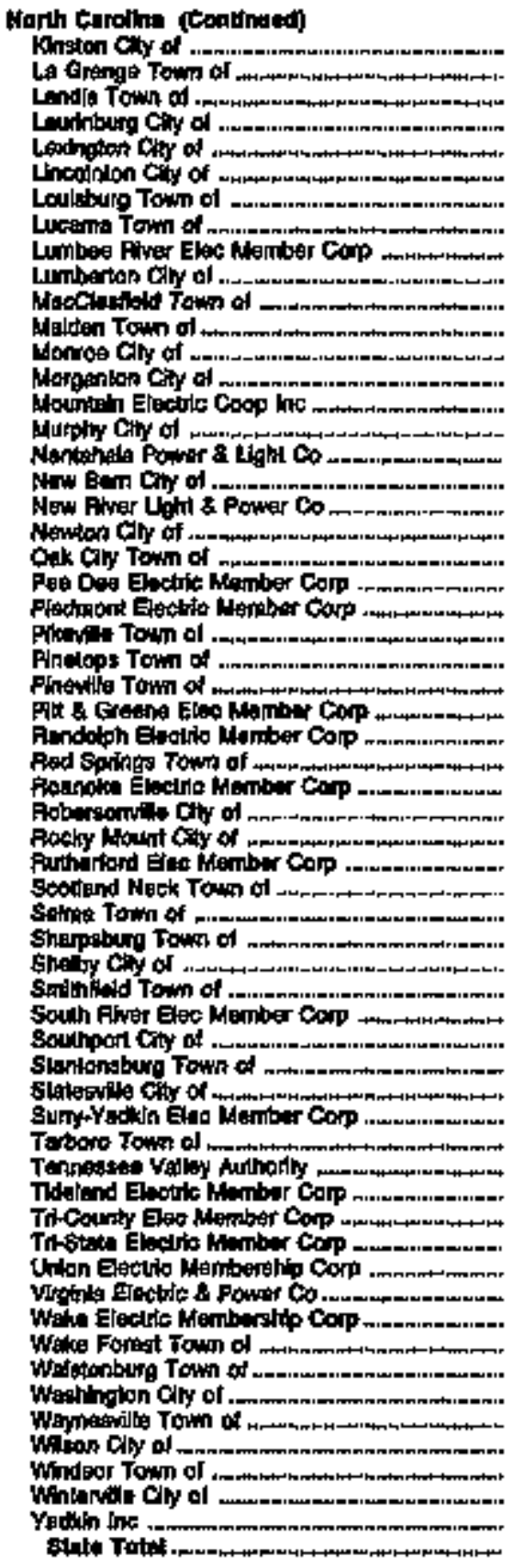 & 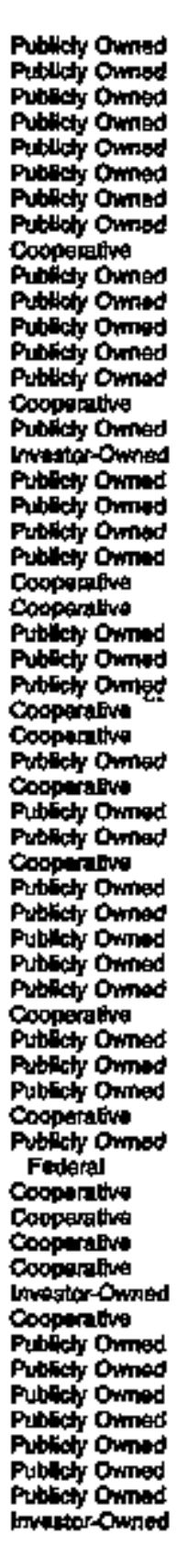 & 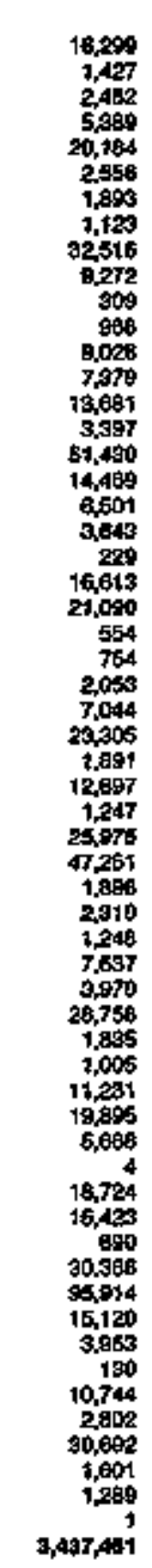 & 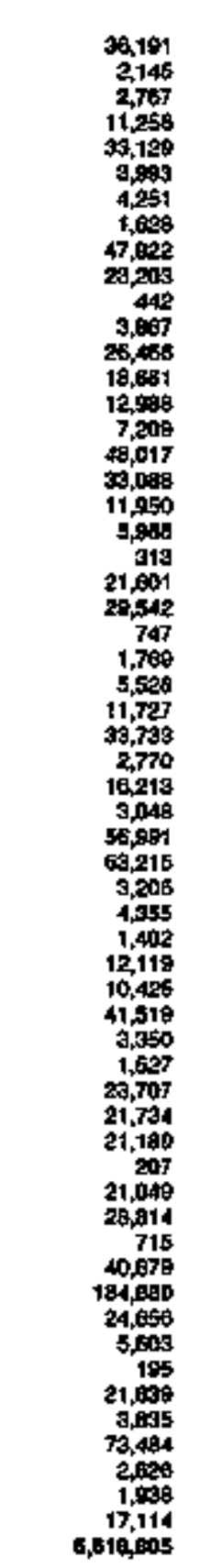 & 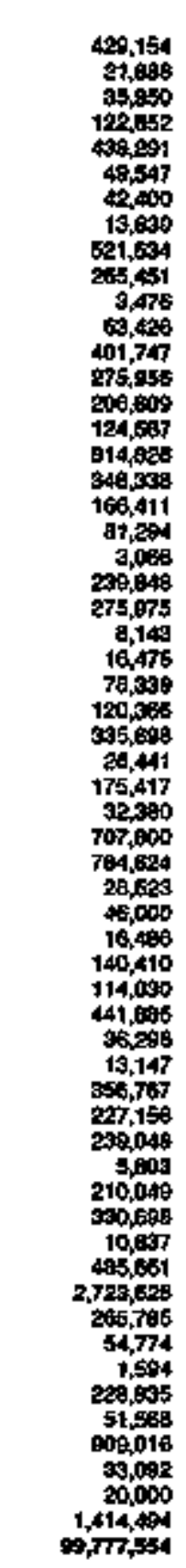 & 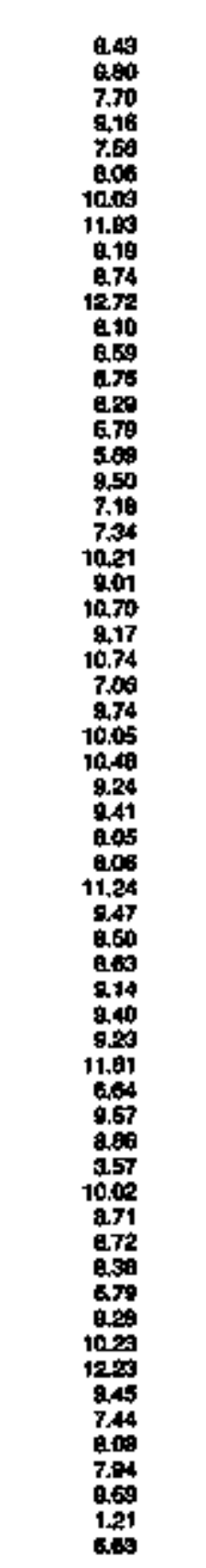 \\
\hline 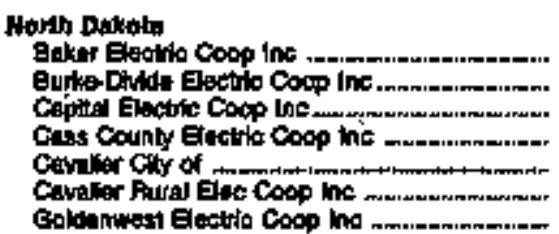 & 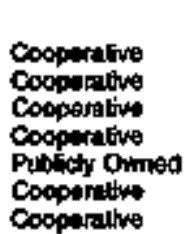 & $\begin{array}{r}5,624 \\
2,331 \\
7,155 \\
14,646 \\
918 \\
1,531 \\
542\end{array}$ & $\begin{array}{r}0,650 \\
3,460 \\
8,294 \\
21,178 \\
0.54 \\
2,505\end{array}$ & $\begin{array}{r}128,200 \\
49,606 \\
108,075 \\
414,848 \\
18,152 \\
38,263 \\
6,718\end{array}$ & $\begin{array}{l}0.75 \\
7.11 \\
7.79 \\
5.11 \\
4.70 \\
6.20 \\
0.42\end{array}$ \\
\hline
\end{tabular}

Seg tootnolas al end of tate. 
Table 17. Class of Ownership, Number of Ultimate Consumers, Revenue, Sales, and Average Revenue per Kilowatthour for All Sectors by State and Utllty, 1993 (Continued)

\begin{tabular}{|c|c|c|c|c|c|}
\hline $\begin{array}{c}\text { Sidte } \\
\text { Elowetric Utility }\end{array}$ & 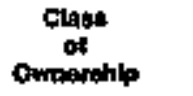 & $\begin{array}{c}\text { Aumbif } \\
\text { of } \\
\text { Coptoplarts }\end{array}$ & 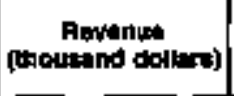 & 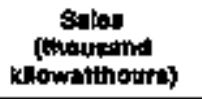 & 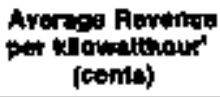 \\
\hline 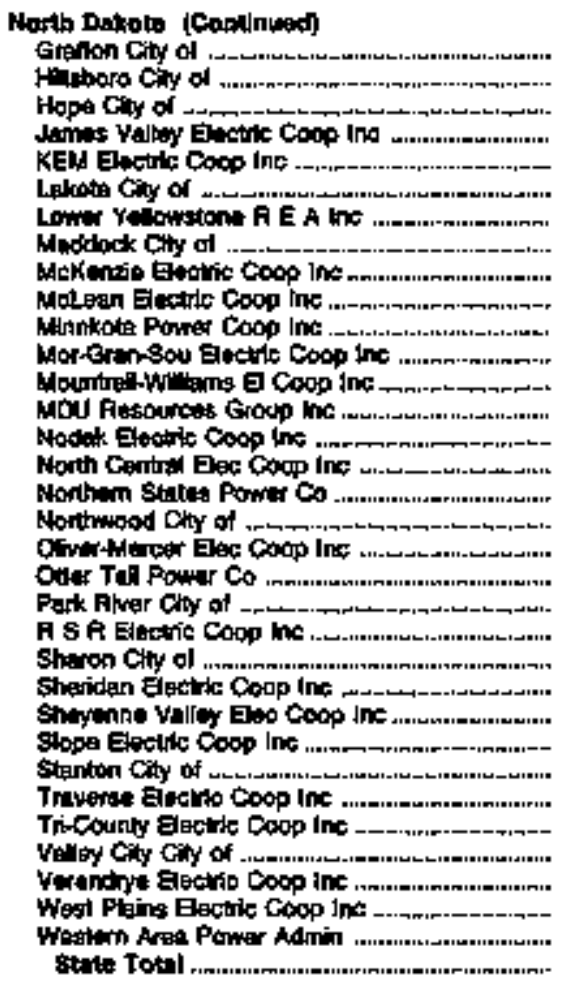 & 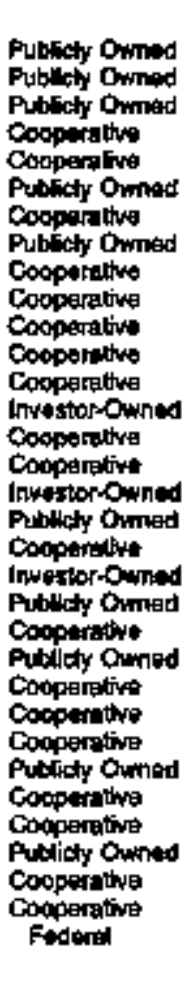 & 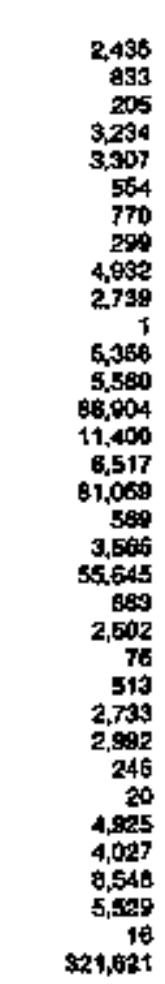 & 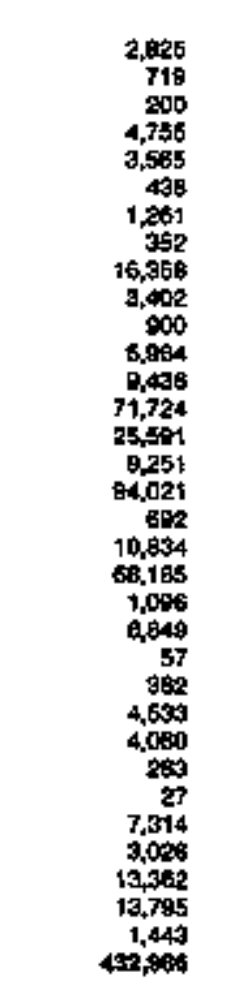 & 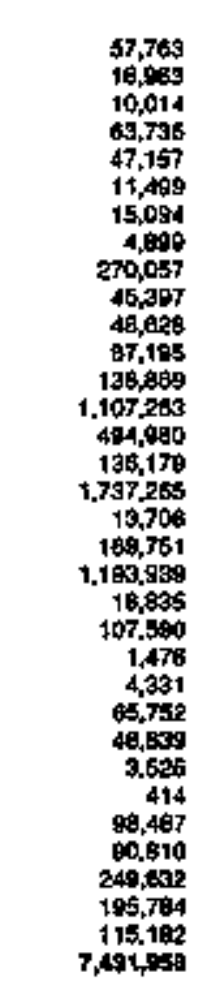 & 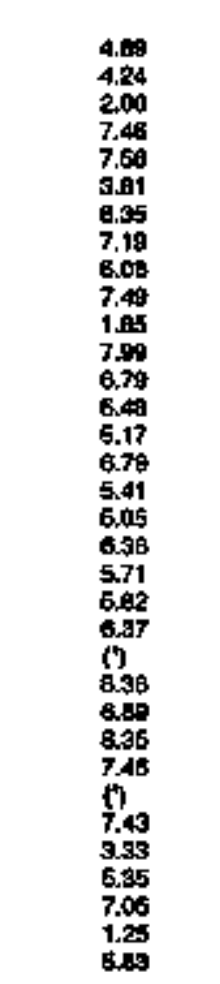 \\
\hline 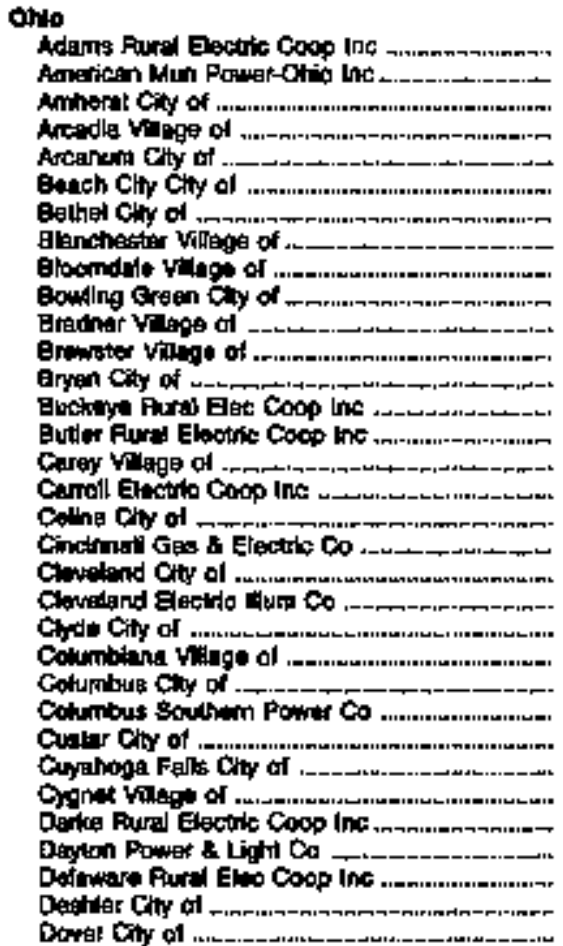 & 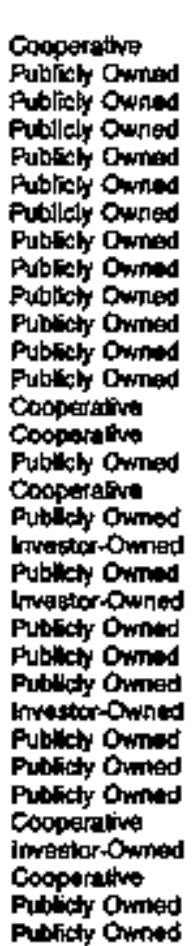 & 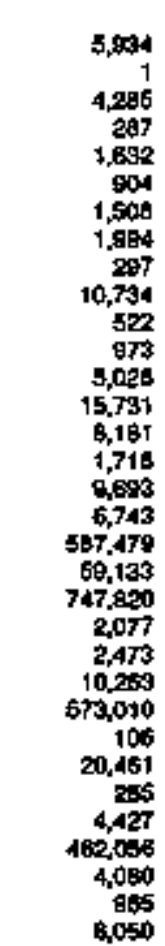 & 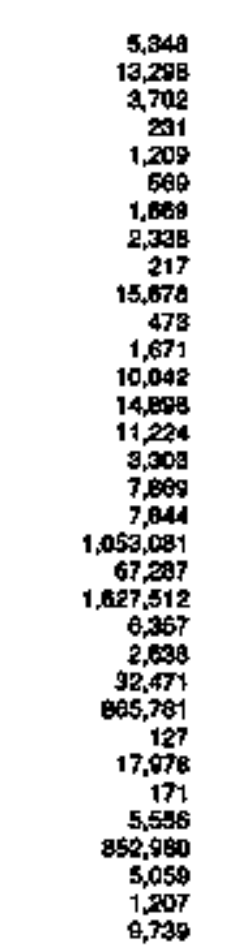 & 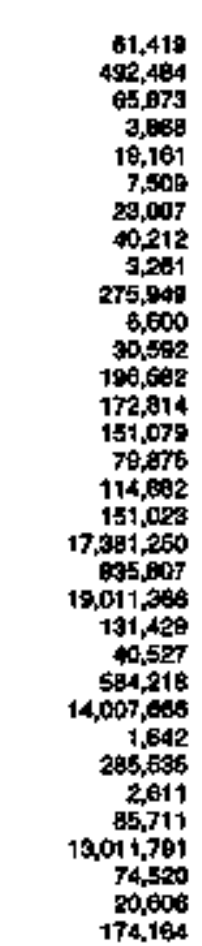 & 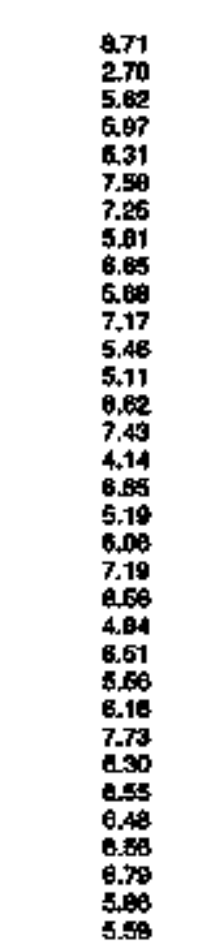 \\
\hline
\end{tabular}

See lookolos at end of labig. 
Tabie 17. Class of Ownership, Number of Ultimate Consumers, Fevenue, Sales, and Average Revenue per Kllowatthou for All Sectors by State and Utility, 1998 (Conlinued)

\begin{tabular}{|c|c|c|c|c|c|}
\hline Etale & $\begin{array}{l}\text { Clact } \\
\text { of } \\
\text { Omiteretils }\end{array}$ & $\begin{array}{c}\text { Humber } \\
\text { of } \\
\text { Conuumer }\end{array}$ & fioremit & $\begin{array}{c}\text { gedet } \\
\text { (thoctent } \\
\text { tllowathourt) }\end{array}$ & 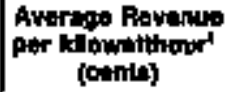 \\
\hline
\end{tabular}

Ohls trotinutiol

Edgenton Villoge of

Entorado vilep of

Clmort village of

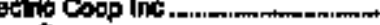

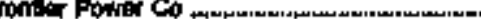

erilan con of

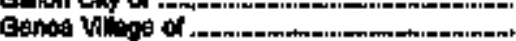

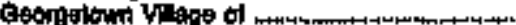

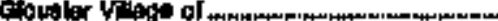

Er tton Cby of

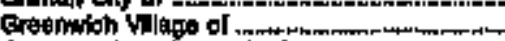

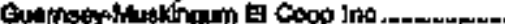

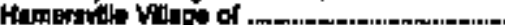

Hainilon ciny of .

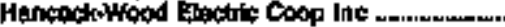

Hotdis Vilugg of._.

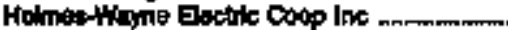

Hibberd city of

Hodion Ciny of

Inotem Corler vetige ol

Jactoun Clty of

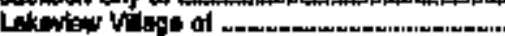

Lobnon Chy of

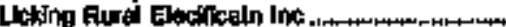

Lodi triage ol ..

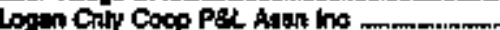

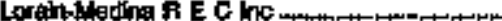

Livetr Viage ol

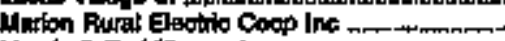

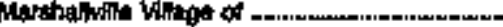

Mendon Cty of -.....

Mhwa

titun vilage of .

Minter Vheres of

Manangatrata Poluter Co ........................

Momosing City of

Montovilar wlape of

anow Elecind coop in

kepoloon Caty of

Niv Gemen viliaga of

New Knowadta Vilinge of.

Nives Ciy of

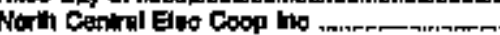

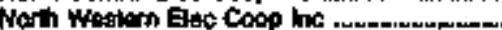

Onk Habor Vllage of ...........................

toberfin ceity of

Ohlo Cly Villgge of ...-...-.

Oho Edlsan Co

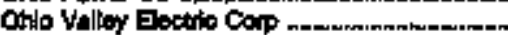

Drvine Cily of

Pe'hefvila Cisy of

PuldinaPulman Eled Coop ho _...mnn..._...m

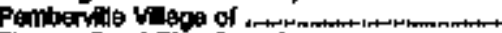

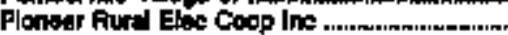

Pionger Villoga of

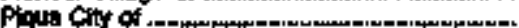

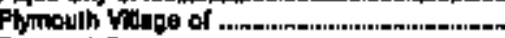

Frospect Cort

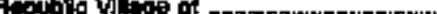

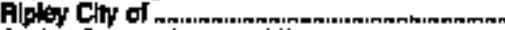

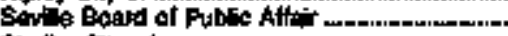

onviby Cay of

shion Valos of

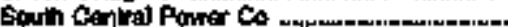

South Vianne Corporalon

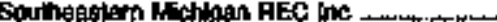

si Clittrite City of ...
Putliely owned

Fublitity Owned

Pubicly Ownid

Copplatisa

cocostative

Putilety Owned

Publictr Owned

Futhict Owined

Fublich Oumted

Putsildy Ouned

Publith Ouned

Cacperative

Putllaty Owned

Publidy Oumbd

Cocperation:

Publldy Ounod

Cocperative

Putidy Omined

Pubikcty Ournat

Pubtty Ouned

Publey Ourtad

Publety Omind

Putply Omad

Cocopenative

Purinty Ouned

Fooparativ.

Cosparalture

Pubidy Oimed

Cosperafture

Putsicty Omnod

Pubichly Cunsed

Cooperzeltue

Pubtely Omined

Puticty Omined

Investo towint

Pulbly Omed

Pulticty Ominad

Cocperalutu

Futbist Owmed

pulbich owned

Fubilety Dunod

Pubicty Oumed

fubles owned

Copperatho

conoperative

Pubilchy Omed

Pubficty Dimad

Publinely Owined

investor-Omied

hrestor-Omined

muestor-Ouned

Pubthly Owhed

Publitely Owtid

Coopertive

Publity Owmad

Cooperative

Publesy owned

Publiclir owned

Putblict owned

Publitty Gived

Publlaly Owned

Publly ly Owned

Putlitaly Owned

Punlisit Owand

Publcty Ousind

cocpention

Publtcty Owmod

Gooperative

Publidy Owined 9g9

798

7,276

B,797

6,160

976

1,760

1,008

980

605

12,398

267

26,077

0,127

268

12,601

3,464
5,587

$6 \mathbf{0 8}$

3,910

Bos

5.414

17,05

1,704

3,650

11,460

321

3,3:27

421

331

0,568

899

7.192

850

2155

B. 000

5446

1284

412

290

Tf, eg:

8,300
4,790

1,321

23as

$4 \mathrm{~B}$

92,386

653,601

5.11

10.001

7,677

046

13,187

60

5,053

exp

6.

294

1,003

1,404

5,023

Eiv

79,039

201

2,842
1,147

$1 \mathrm{a}$

ด19

7,191

6,201

5,040

039
2,002

64

1.247

608
10.474

240

ตล. 916

T店

ㄴ)

11,790

3.746

072

862

4,710

574

8.031

16.431

1,784

4,847

11805

991

0,456

381

291

10.116

278

37,996

1.933

3.000

7.00

7,707

2.489

514

2,684

15,372

11,895

5,357

1290

5010

40末

$1,678,249$

1,257545

202,117

13,473

15,215

8,428

901

20,343

1004

14,350

648

519

ons

1,794

6.105

$2 \pi$

70,851
270

788

2,164

20,041

3,265

9.765

86,613

97,676

209,500

11,576

32,958

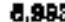

16,659

12,144

130,625

3,730

$5 \%$, 700

321,358

1, g52

206,340

44. 340 
Table 17. Class of Ownership, Number of Litimate Consumers, Revenue, Sales, and Averoge Rovonue por Kilowatthour for All Sectors by State and Utilty, 1993 (Continuod)

\begin{tabular}{|c|c|c|c|c|c|}
\hline Eloctorlo Udity & $\begin{array}{c}\text { Cinas } \\
\text { of } \\
\text { Ownerahlp }\end{array}$ & $\begin{array}{l}\text { Nipipor } \\
\text { of } \\
\text { Consumers }\end{array}$ & 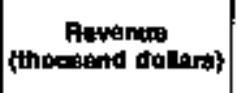 & 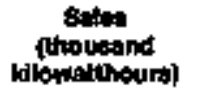 & 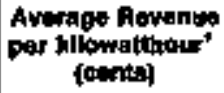 \\
\hline
\end{tabular}

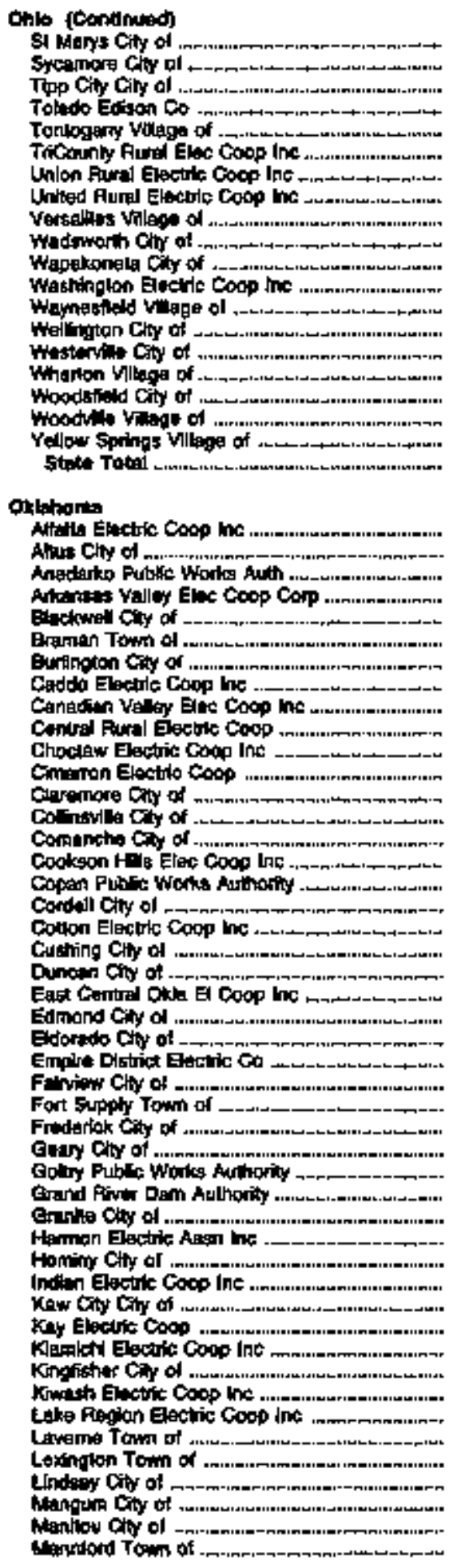

Publudy Oursed Pubilely Ownet Publedy Onwerd Irwaslar-Owined Publicty Oinned Cooperntive

Cooparellive

Coopureter

Pubflty Owried

Putalchy Owried

Pubdlaly Owned

Cooperalve

Putiticly Owried

Pustloly Owned

Putilcty Omined

Putricty Ouned

Puolleby Owned

Pubilcly Owined

putllaty Owthed

Coopertive

Putilthy Ovited

Publicky Owned

Cooperativa

Putwlety Owned

Publicty ouned

Publect Dened

Cocoperitivis

Cooperative

Gopperathre

Cooperitivis

Cooperative

Putplecly Ownex

Pubficy owned

Publety Owned

coxoperethis

Pusticly Owne:

Publetis Owmad

cocoperative

Publery Owned

Ruthicty Ownod

cocoperativt

Publier Owno.

Publets Qwnod

invastor-Owated

Publick owned

Pubfith Owned

Pubfory Owried

Pubtichy Ownet

Publickly Ommes

Publity Owind

Publety Ownor

Cooperalture

Pubfity Oomed

Cosperathe

Pubtity Ontred

Cooperative

Cooparnthe

Pubity Ombad

Cocperaline

Cooperathe

Publictyr Oumed

Pubicty Ommed

Puthlicty Owined

Pubichy Ommed

Publich Omed

Publicty Omined

\begin{tabular}{|c|c|}
\hline $\begin{array}{r}3,695 \\
497\end{array}$ & $\begin{array}{r}6,725 \\
616\end{array}$ \\
\hline $\begin{array}{r}3,571 \\
305,500\end{array}$ & $\begin{array}{r}4,509 \\
608.7\end{array}$ \\
\hline $\begin{array}{r}206 \\
3605\end{array}$ & $\begin{array}{r}18 \mathrm{e} \\
\mathbf{3} 300\end{array}$ \\
\hline 4,708 & $\begin{array}{r}17.549 \\
3.205\end{array}$ \\
\hline 1,567 & 2110 \\
\hline 9,048 & 10,662 \\
\hline $\begin{array}{l}4,711 \\
a, 402\end{array}$ & $\begin{array}{l}6,559 \\
6,037\end{array}$ \\
\hline 490 & 338 \\
\hline 2,027 & 3,171 \\
\hline 12,236 & $17, \mathrm{BSO}$ \\
\hline 1,590 & $\begin{array}{r}109 \\
1,146\end{array}$ \\
\hline 827 & 625 \\
\hline $1,0, \theta^{2}$ & 1,013 \\
\hline & $9,259,617$ \\
\hline
\end{tabular}

146,-108

11,310

92,071

$7,07,260$

2,000

47,578

3as

45,601

41,552

190,796

140,600

72,815

4,835

41,560

318,584

2,798

17,070

5,005

39,935

$144,570,606$

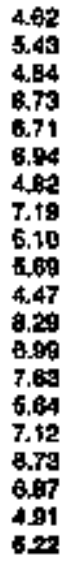

7,000

0,033

3,260

3,456

4,000

173

14,958

17,866

13.918

14,217

10,720

Q.195

1,847

972

16,132

395

1.630

17,735

4,442

B.151

24,340

21,958

415

5,233

1,029

194

2.504

720

81

9.3

2,77

1,606

16,049

280

5,849

15.646

2083

5. 775

17.965

714

784

1.567

1.90

12

88 a.113

8,874

4,268

a,200

$5.6 \%$

148

105

17,27

apes4

17,804

14,965

12,359

12,57.

1,924

a71

13,47

298

1.505

27.771

4770

2056

28,980

26,606

240

5.432

1,060

180

3,367

689

120

20.6日8

307

1,387

21,067

141
7.670

2,821

5,462

17.367

699

610

1.522

1,400

65
1.2052
107,867

140,497

53,178

47.477

85.510

1.660

1,022

18,302

295475

281.054

218,610

162.387

204,357

20050

$\$ 1.072$

171,702

6044

22018

41 하영

64.358

150,870

342009

424,731

2,683

104,479

29.005

(2004

63,826

5,698

1,601

674, 70

6,25?

43,450

15.713

269.015

$1, \mathrm{B12}$

109,369

180194

36,45

19.20

217,400

7,862

6,785

19,65

21,504

118

19,567
7.51

8.32

o.03

s.75

860

540

10.27

7.98

7.07
7.08

6.86

7.61

B.16

7.82

7.26

7.24

6.73

7.4

bon

7.70

0.35

5.20

s.92

6.24
9.99

7.14

3.04

6.35

7.10

Q.s

7.83

6.75

7.52

7.56

7.20

7.69

0.00

B. 38

7.04

7.67
6.94

17

Ses lootnotas at and of lablo. 
Table 17. Class of Ownershlp, Mumber of Uitimate Consumers, Revenue, Sales, and Average Revenue per Kilowathour for Al Sectors by State and Utibity, 1993 (Continued)

\begin{tabular}{|c|c|c|c|c|c|}
\hline 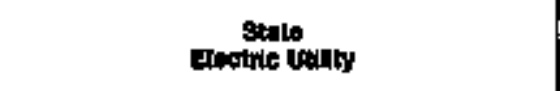 & $\begin{array}{c}\text { cloas } \\
\text { of } \\
\text { onmondplp }\end{array}$ & $\begin{array}{c}\text { Hitapeyt } \\
\text { of } \\
\text { conoumers }\end{array}$ & Raverure & 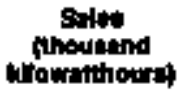 & 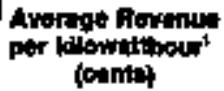 \\
\hline 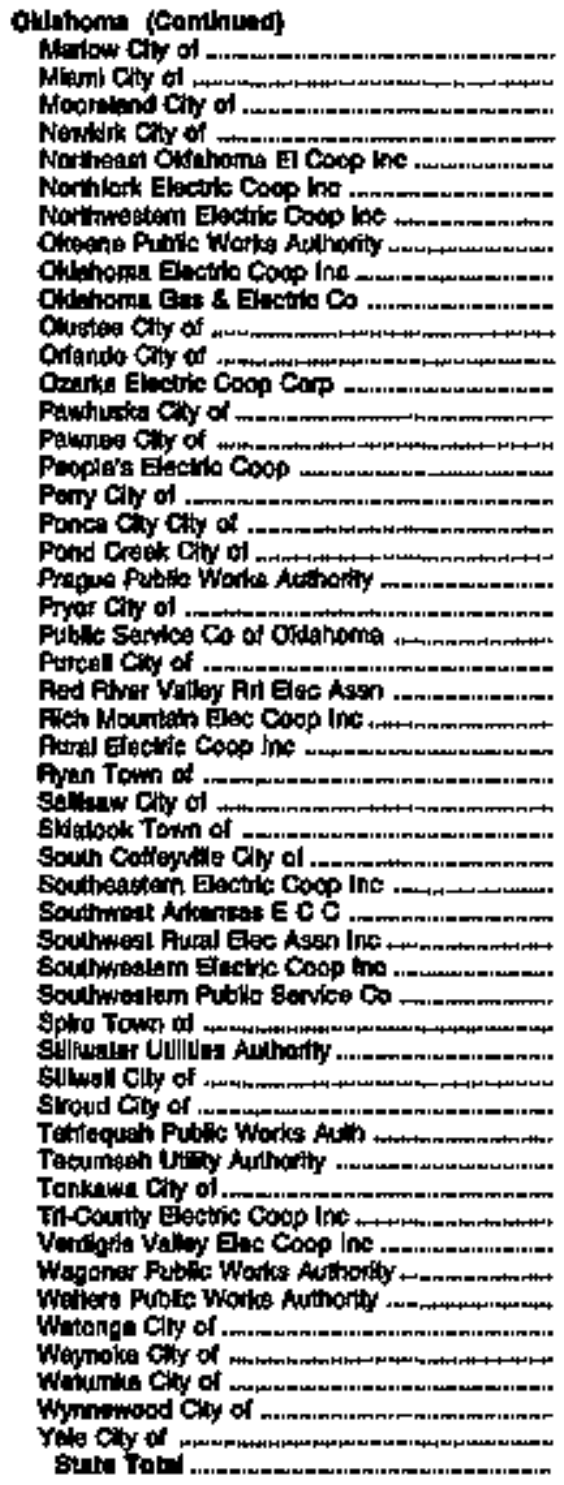 & 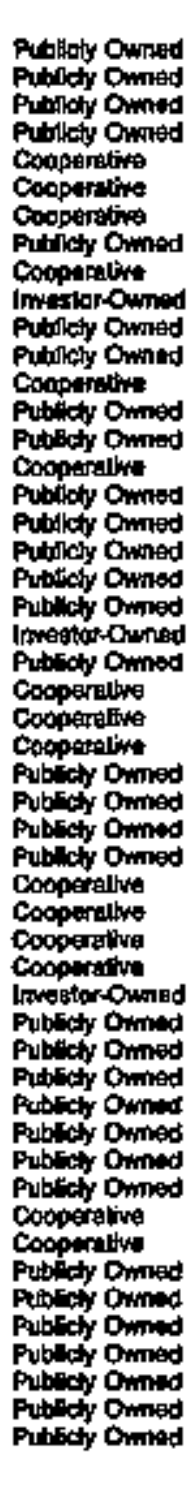 & 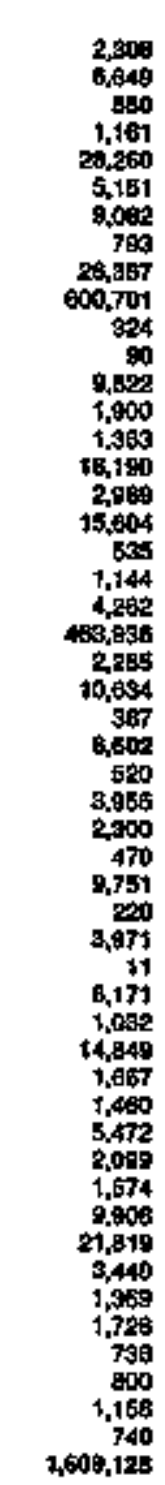 & 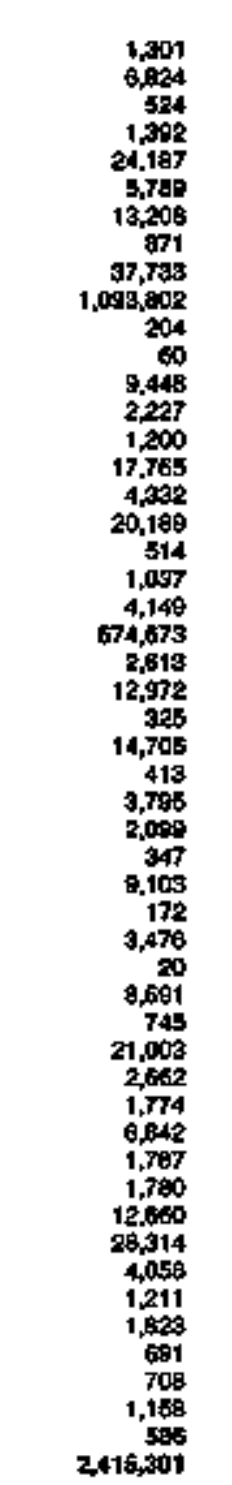 & 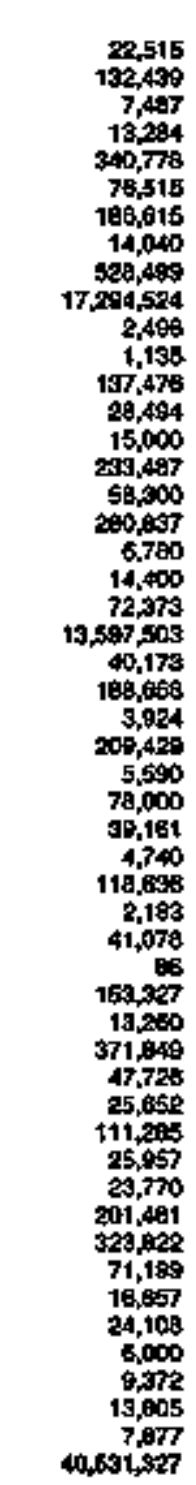 & 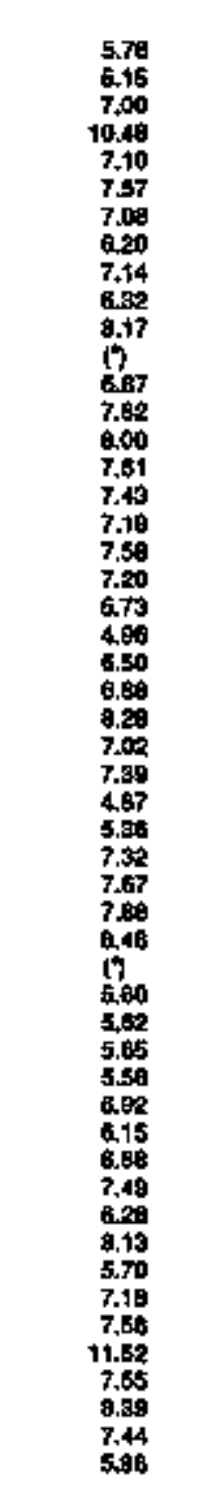 \\
\hline 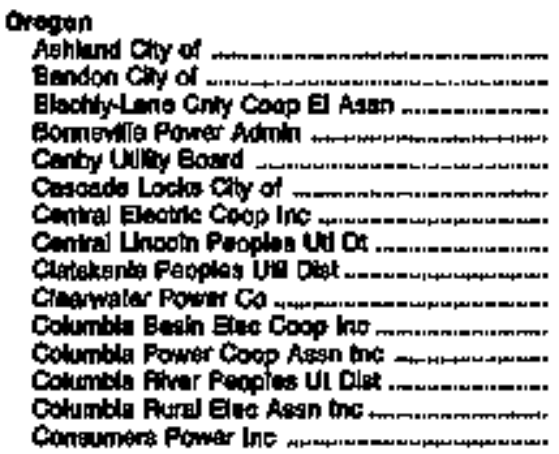 & 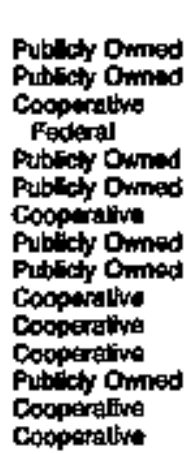 & 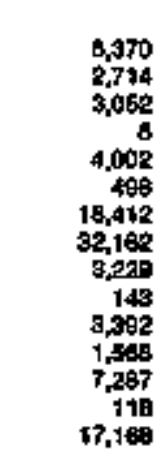 & $\begin{array}{r}6,978 \\
7,017 \\
6,021 \\
60,102 \\
4,735 \\
901 \\
19,790 \\
48,737 \\
24,200 \\
128 \\
5,102 \\
1,302 \\
11,892 \\
175 \\
19,442\end{array}$ & 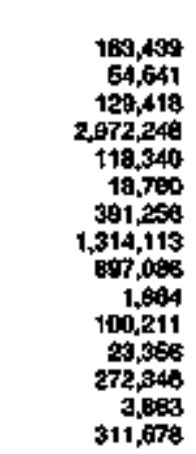 & $\begin{array}{l}4.27 \\
5.16 \\
4.65 \\
200 \\
4.00 \\
4.00 \\
5.06 \\
3.71 \\
2.70 \\
6.64 \\
5.09 \\
6.67 \\
4.46 \\
4.69 \\
0.24\end{array}$ \\
\hline
\end{tabular}

Seg footnolat at end of table. 
Table 17. Class of Ownership, Number of Ultimate Consumerg, Revenut, Sales, and Average Revenue per Kilowatthour for All Sectors by State and Utllity, 1998 (Continuted)

\begin{tabular}{|c|c|c|c|c|c|}
\hline $\begin{array}{c}\text { Etate } \\
\text { Eleqtais Utilty }\end{array}$ & $\begin{array}{c}\text { Clmse } \\
\text { os } \\
\text { Ownargihlp }\end{array}$ & $\begin{array}{c}\text { Nunber } \\
\text { of } \\
\text { Congumer }\end{array}$ & $\begin{array}{c}\text { Fentenus } \\
\text { (Jbousand dotlare) }\end{array}$ & $\begin{array}{l}\text { sales } \\
\text { (thoutpand } \\
\text { Wllowathound) }\end{array}$ & 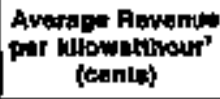 \\
\hline 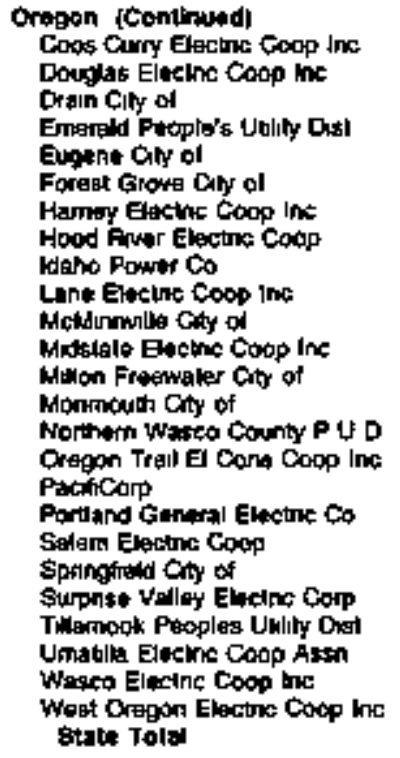 & 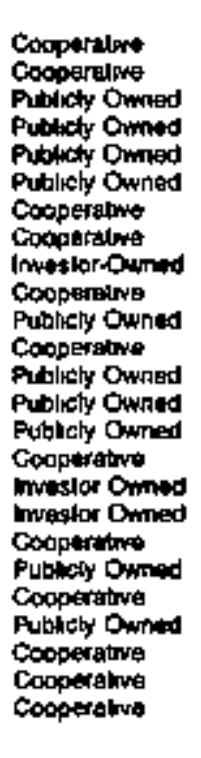 & 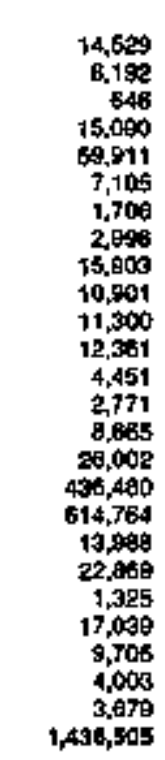 & 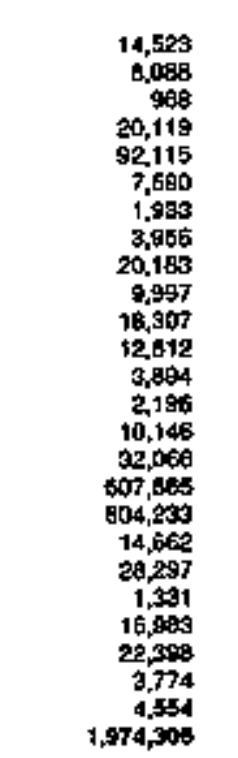 & 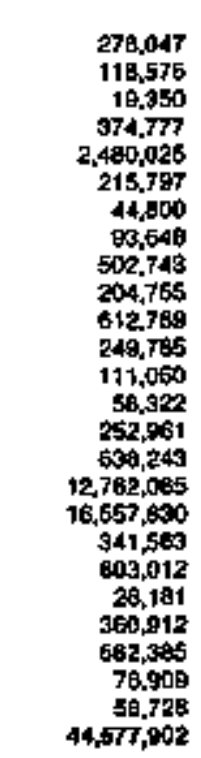 & $\begin{array}{l}522 \\
682 \\
500 \\
637 \\
371 \\
352 \\
439 \\
420 \\
101 \\
498 \\
298 \\
613 \\
354 \\
377 \\
401 \\
502 \\
476 \\
486 \\
420 \\
362 \\
500 \\
443 \\
306 \\
491 \\
775 \\
443\end{array}$ \\
\hline 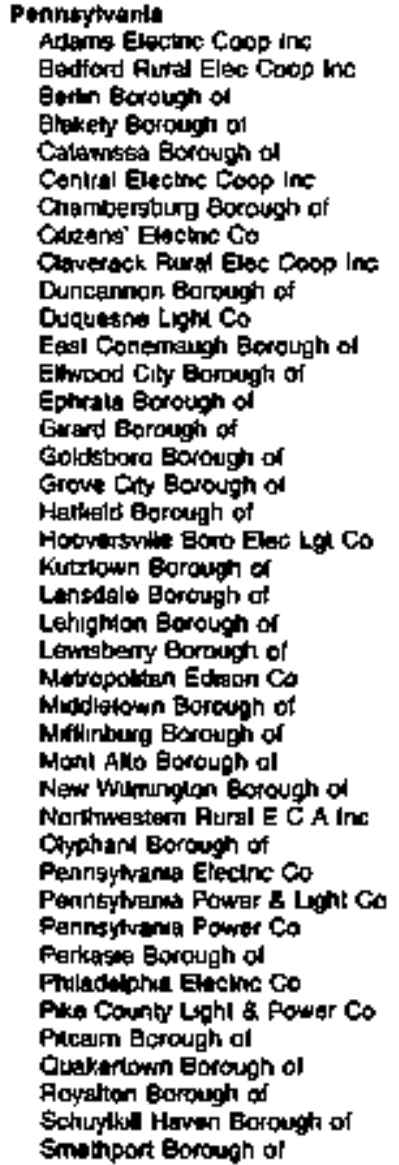 & 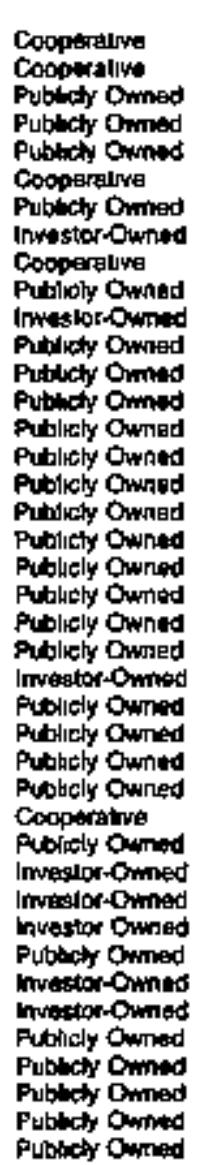 & 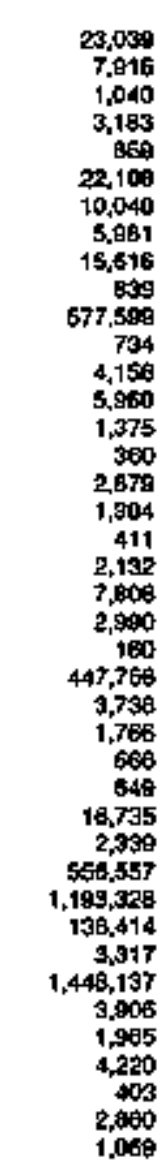 & 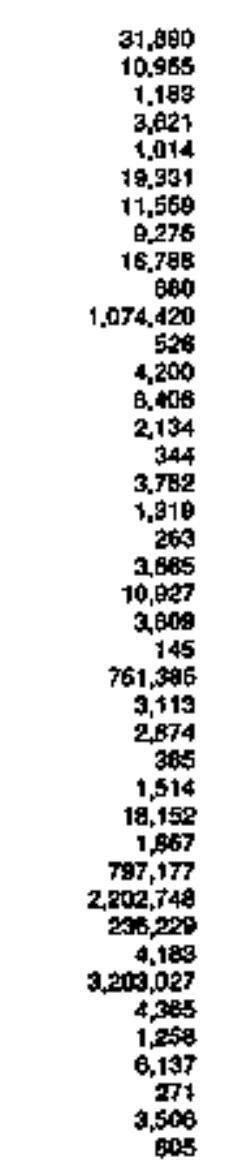 & 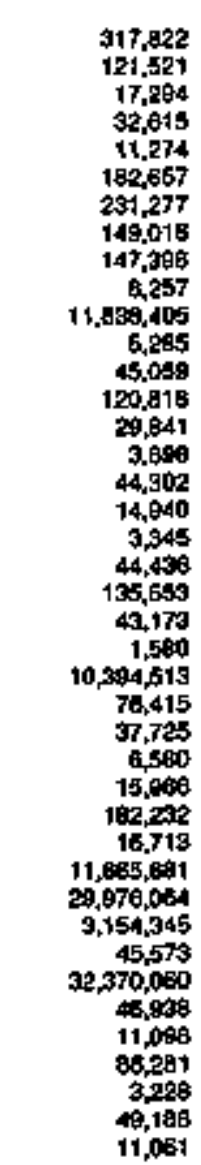 & 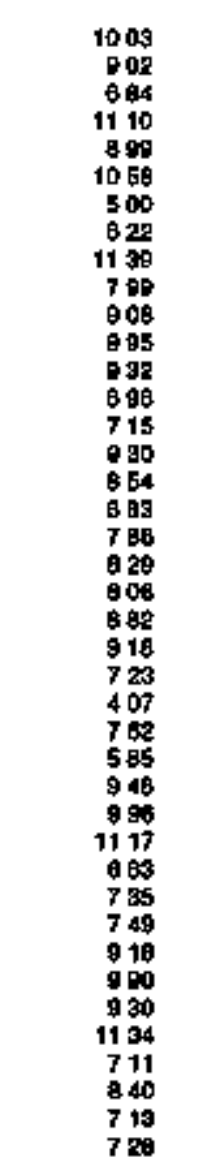 \\
\hline
\end{tabular}

Sad fookndes at end ol lable 
Table 17. Class of Ownorahip, Number of Utimate Consumers, Revenue, Sales, and Average Revenue per Kilowatthour for All Sectors by State and Utility, 1999 (Continued)

\begin{tabular}{|c|c|c|c|c|c|}
\hline guble & of & $\begin{array}{l}\text { Numbor } \\
\text { pant } \\
\text { eantumara }\end{array}$ & 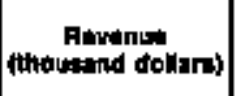 & $\begin{array}{l}\text { salon } \\
\text { (theugnd } \\
\text { Wowathourt) }\end{array}$ & $\begin{array}{l}\text { Average Ravenut } \\
\text { per klinwatlhour' } \\
\text { (conta) }\end{array}$ \\
\hline 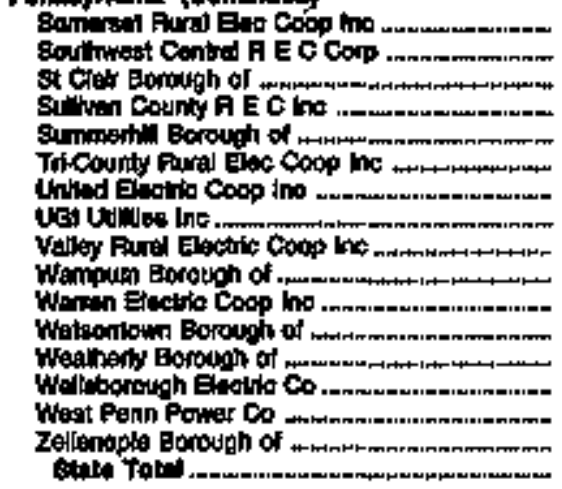 & 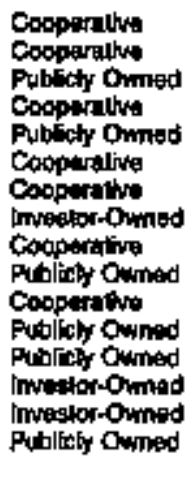 & 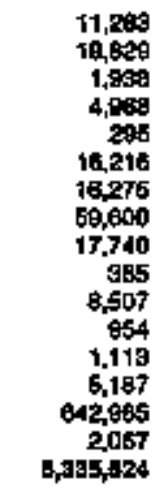 & $\begin{array}{r}14,498 \\
23,047 \\
1,397 \\
4,200 \\
204 \\
14,410 \\
14,840 \\
62,683 \\
18,775 \\
278 \\
4,898 \\
1,072 \\
1,625 \\
5,588 \\
870,081 \\
2,237 \\
0,409,024\end{array}$ & $\begin{array}{r}155,692 \\
246,199 \\
19,700 \\
39,415 \\
2,910 \\
119,699 \\
119,428 \\
860,217 \\
180,609 \\
3,000 \\
43,590 \\
11,300 \\
20,476 \\
90,694 \\
16,360,845 \\
22,469 \\
119,184,494\end{array}$ & $\begin{array}{r}1.31 \\
1.38 \\
10.20 \\
10.68 \\
7.01 \\
12.67 \\
12.43 \\
7.37 \\
10.40 \\
9.27 \\
11.25 \\
9.46 \\
7.04 \\
6.16 \\
5.32 \\
9.95 \\
7.08\end{array}$ \\
\hline 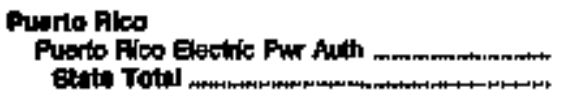 & Pubicty Ouned & $\begin{array}{l}1,213,093 \\
1,213,003\end{array}$ & $\begin{array}{l}1,327,042 \\
1,327,042\end{array}$ & $\begin{array}{l}14,423,617 \\
14,423,617\end{array}$ & $\begin{array}{l}\text { 0.20 } \\
\text { 9.20 }\end{array}$ \\
\hline 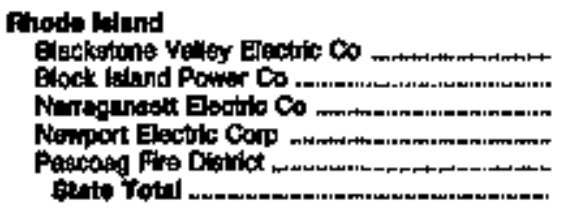 & 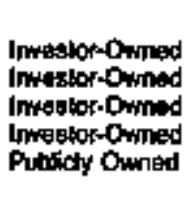 & $\begin{array}{r}84,005 \\
1,293 \\
321,225 \\
31,710 \\
3,995 \\
492,049\end{array}$ & $\begin{array}{r}136,575 \\
1,678 \\
477,678 \\
61,136 \\
3,987 \\
600,958\end{array}$ & $\begin{array}{r}1,252,892 \\
6,836 \\
4,716,170 \\
537,066 \\
33,414 \\
6,540,498\end{array}$ & $\begin{array}{l}10.90 \\
24.55 \\
10.12 \\
11.38 \\
11.06 \\
10.40\end{array}$ \\
\hline 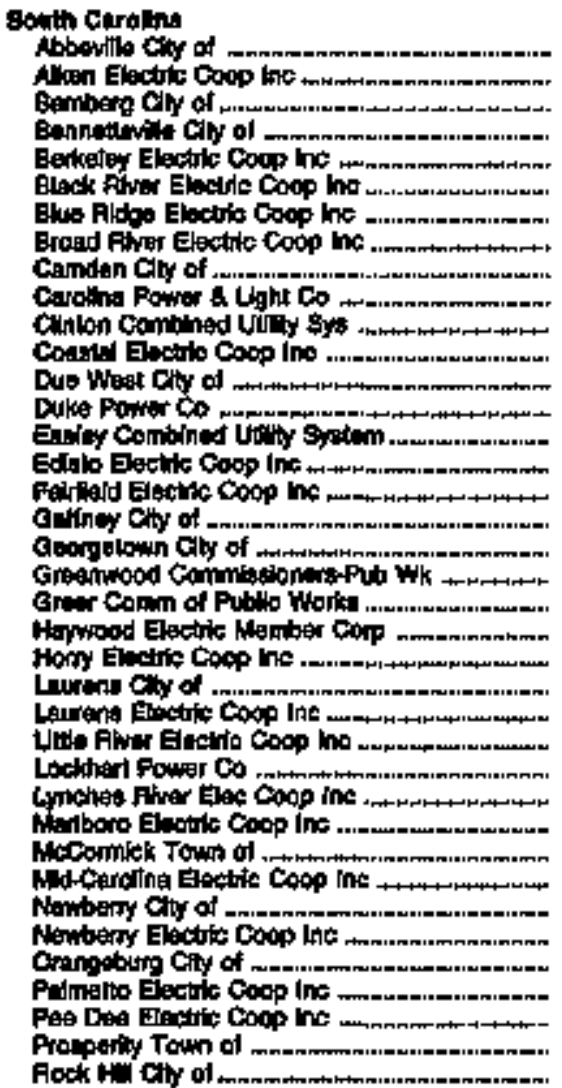 & 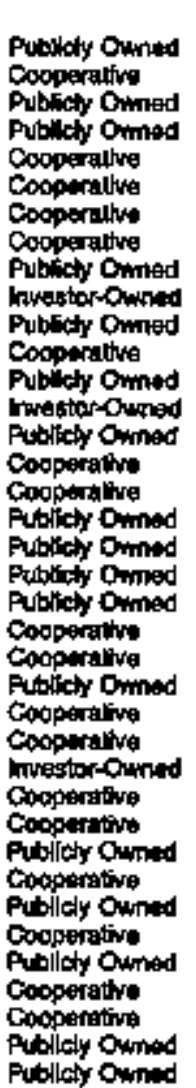 & 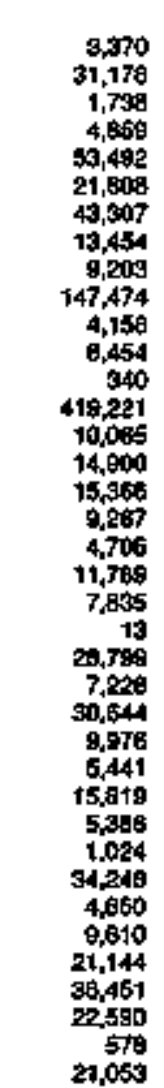 & 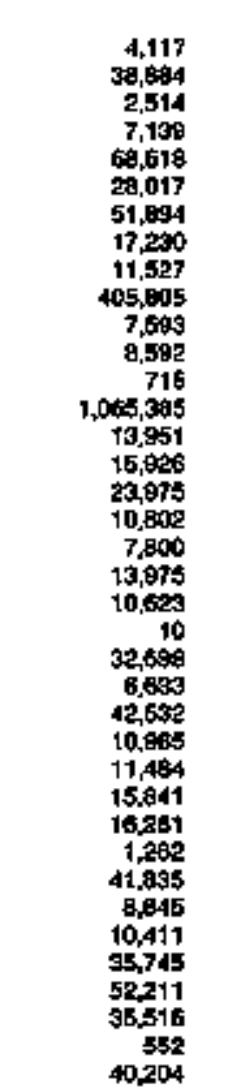 & 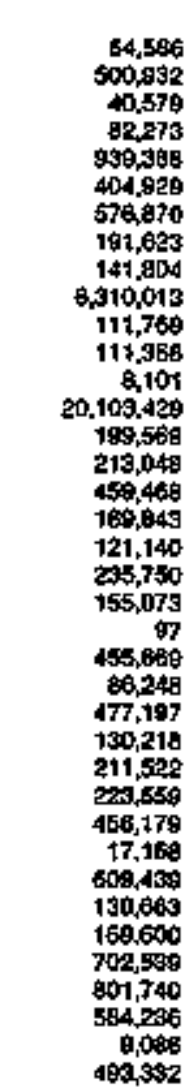 & $\begin{array}{l}7.54 \\
7.78 \\
6.20 \\
6.60 \\
7.30 \\
6.82 \\
9.00 \\
8.99 \\
6.13 \\
8.49 \\
6.79 \\
7.71 \\
8.83 \\
6.30 \\
6.99 \\
7.49 \\
5.22 \\
6.38 \\
6.44 \\
5.99 \\
6.85 \\
17 \\
7.18 \\
7.69 \\
8.91 \\
8.44 \\
5.43 \\
7.09 \\
3.56 \\
7.35 \\
6.66 \\
6.77 \\
6.14 \\
5.09 \\
6.51 \\
6.08 \\
6.08 \\
6.15\end{array}$ \\
\hline
\end{tabular}

Sea toptnoles at end of baht 
Table 17. Class of Ownership, Number of Ultimate Consumers, Rovwnue, Sales, and Average Revenue per Kilowatthour for All Sectors by State and Utility, 1993 (Continued)

\begin{tabular}{|c|c|c|c|c|c|}
\hline $\begin{array}{c}\operatorname{sinto} \\
\text { [alectric titilly }\end{array}$ & $\begin{array}{c}\text { clites } \\
\text { of } \\
\text { omitanhlo }\end{array}$ & $\begin{array}{l}\text { Wumber } \\
\text { os }\end{array}$ & Pavinus & $\begin{array}{c}\text { salo: } \\
\text { (thouteand } \\
\text { witoritihoerst }\end{array}$ & 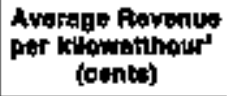 \\
\hline 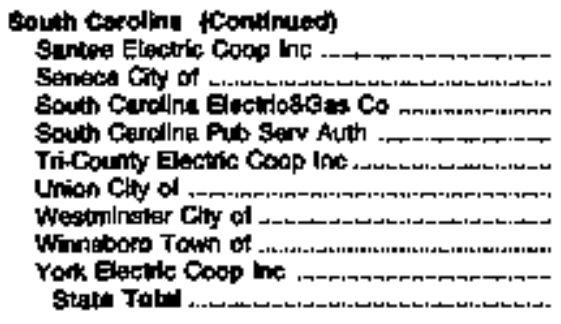 & 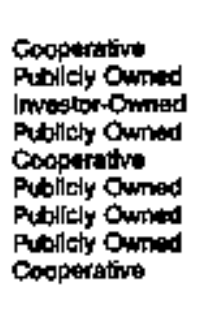 & $\begin{array}{r}34,845 \\
5,161 \\
485,673 \\
98,975 \\
13,760 \\
7,161 \\
1,692 \\
3,146 \\
21,494 \\
1,739,389\end{array}$ & $\begin{array}{r}41,034 \\
9,124 \\
090,064 \\
781,424 \\
14,742 \\
7,549 \\
1,842 \\
4,474 \\
31,761 \\
3,471,052\end{array}$ & $\begin{array}{r}102,564 \\
134,700 \\
15,600,539 \\
7,370,730 \\
201,246 \\
115,750 \\
22,044 \\
79,000 \\
379,498 \\
1,50,725\end{array}$ & $\begin{array}{l}6.39 \\
6.77 \\
5.81 \\
3.95 \\
7.39 \\
6.59 \\
8.11 \\
6.19 \\
8.40 \\
5.64\end{array}$ \\
\hline 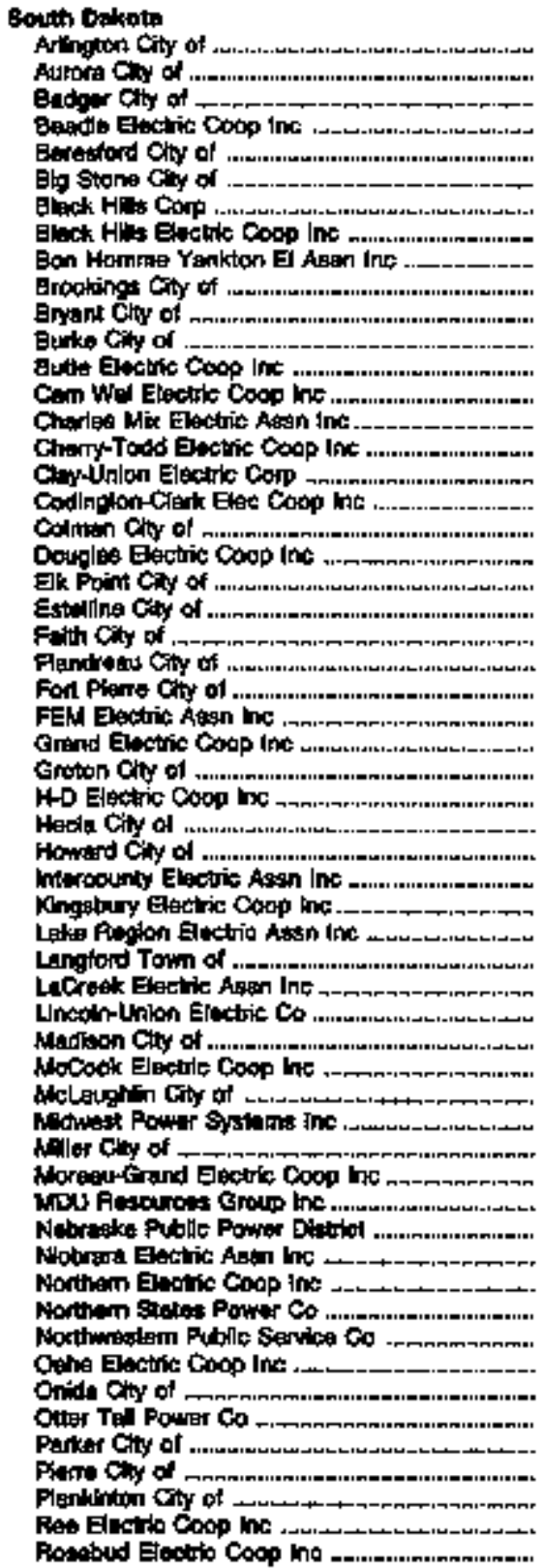 & 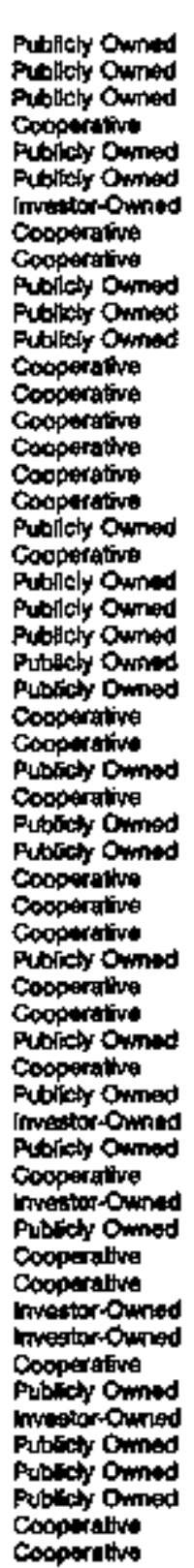 & 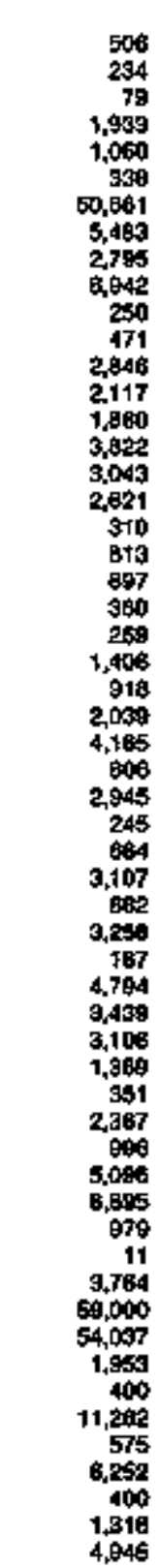 & 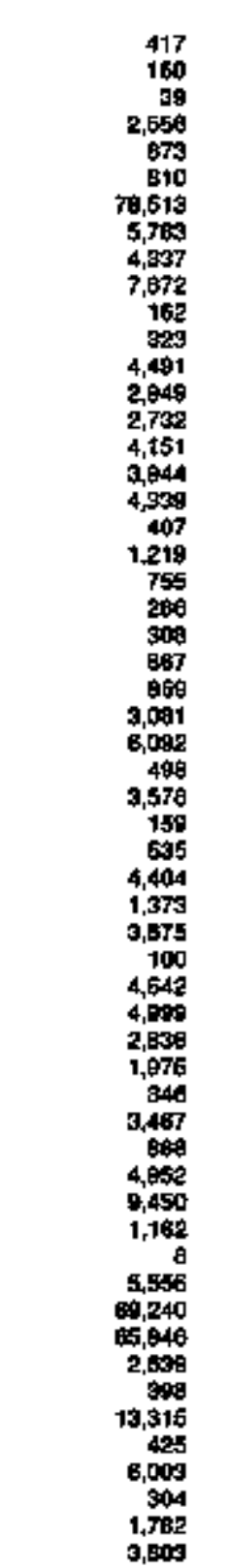 & 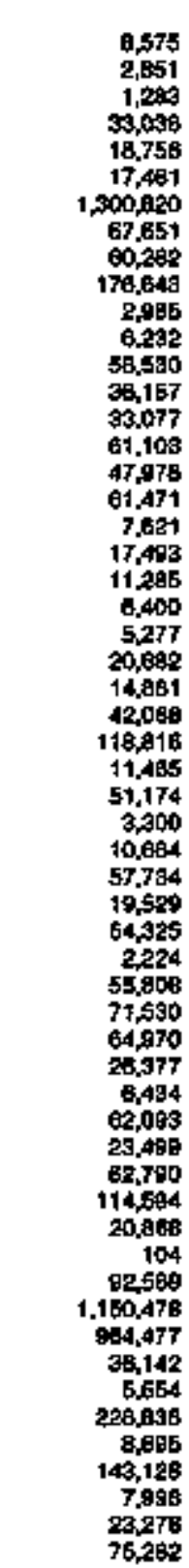 & 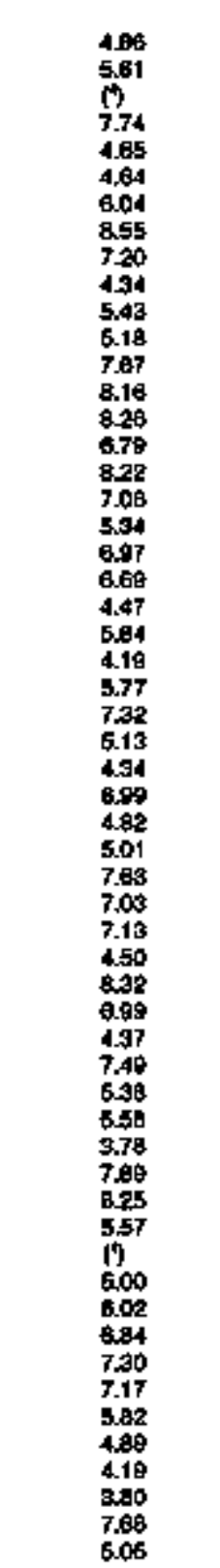 \\
\hline
\end{tabular}

See tootholes ot end of teble. 
Table 17. Class of Ownership, Number of Ultimate Consumers, Revenue, sales, and Averege Revenue per Kilowatthour for All Sectort by State and Utility, 1993 (Continued)

\begin{tabular}{|c|c|c|c|c|c|}
\hline Etalk & $\begin{array}{c}\text { clast } \\
\text { of } \\
\text { owmarindp }\end{array}$ & $\begin{array}{c}\text { Number } \\
\text { of } \\
\text { pentumars }\end{array}$ & Ethotetind dollars? & 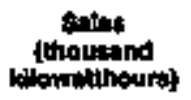 & 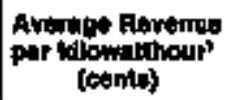 \\
\hline 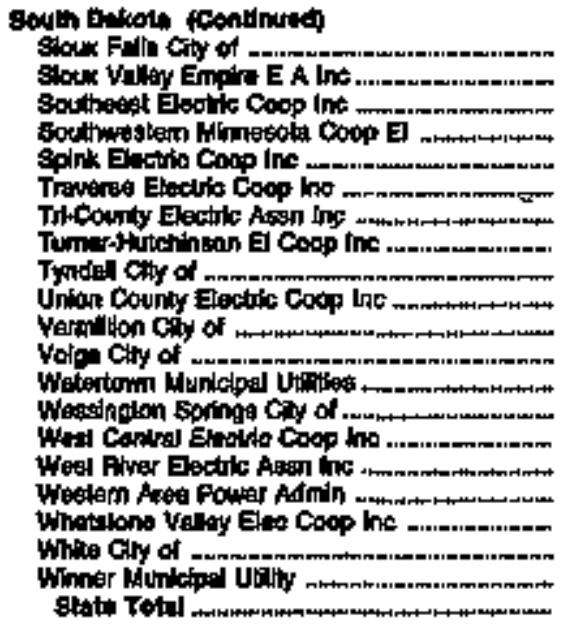 & 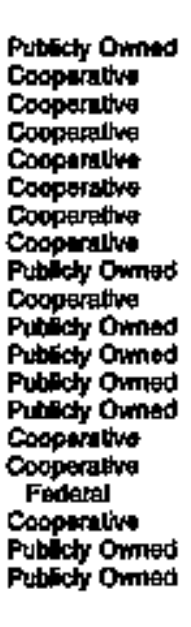 & 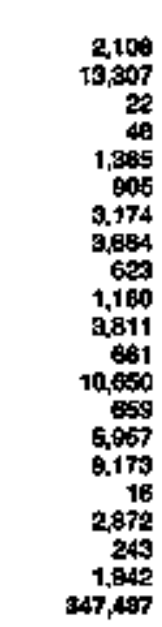 & $\begin{array}{r}3,980 \\
17,200 \\
30 \\
62 \\
2,097 \\
1,121 \\
4,197 \\
5,302 \\
509 \\
1,570 \\
2,442 \\
850 \\
7,867 \\
462 \\
8,869 \\
19,125 \\
1,677 \\
3,776 \\
174 \\
1,443 \\
429,076\end{array}$ & 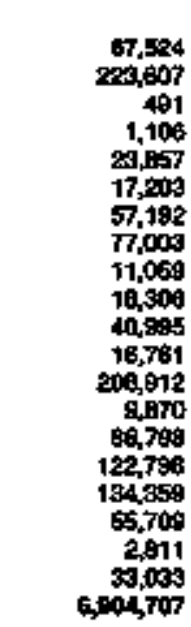 & 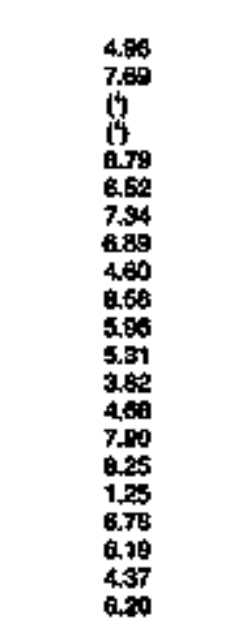 \\
\hline 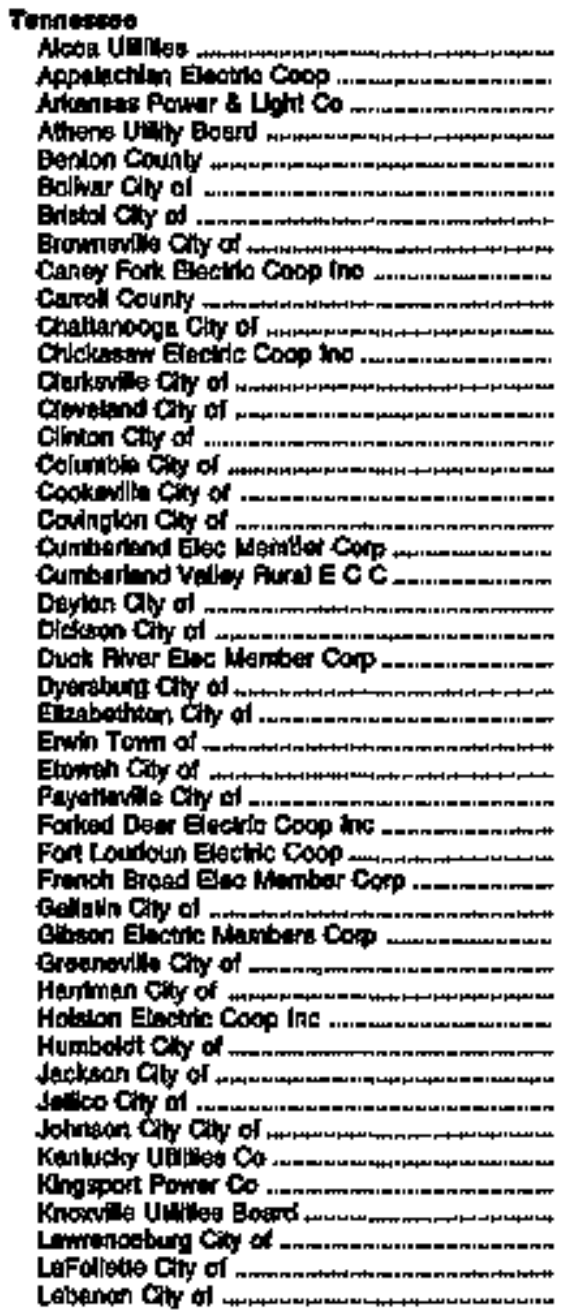 & 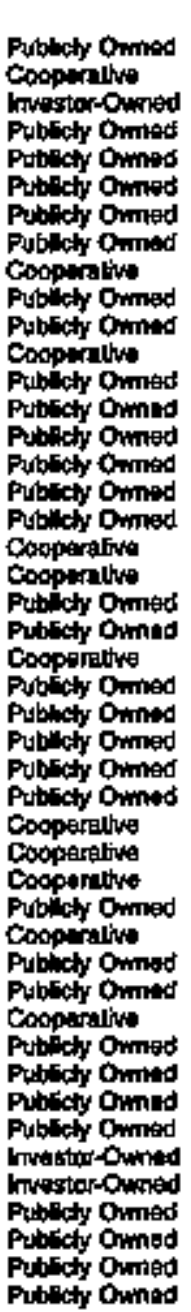 & 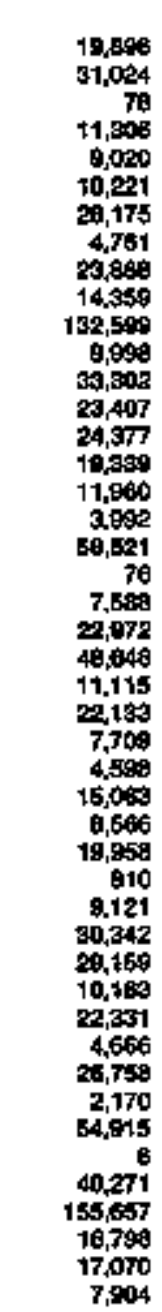 & 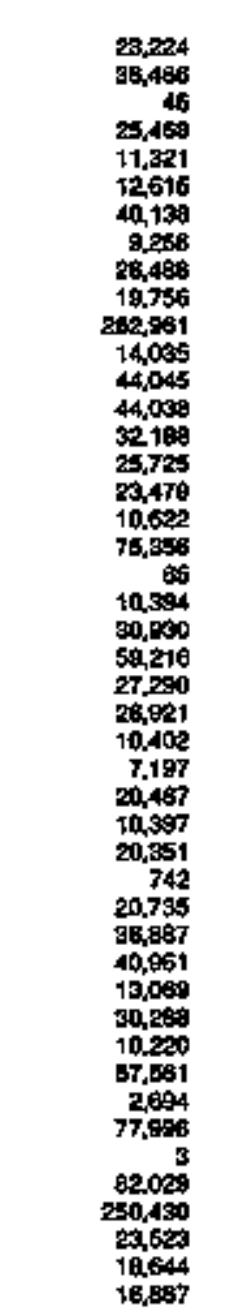 & 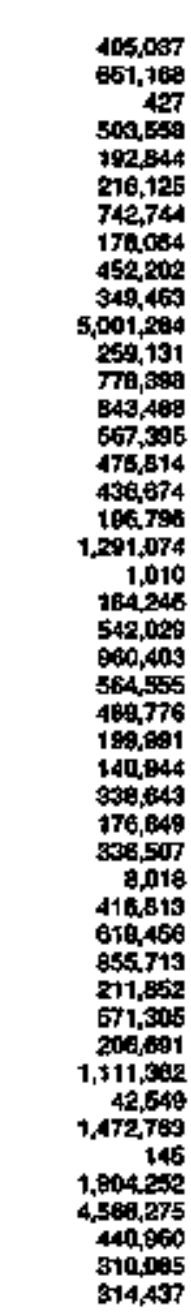 & 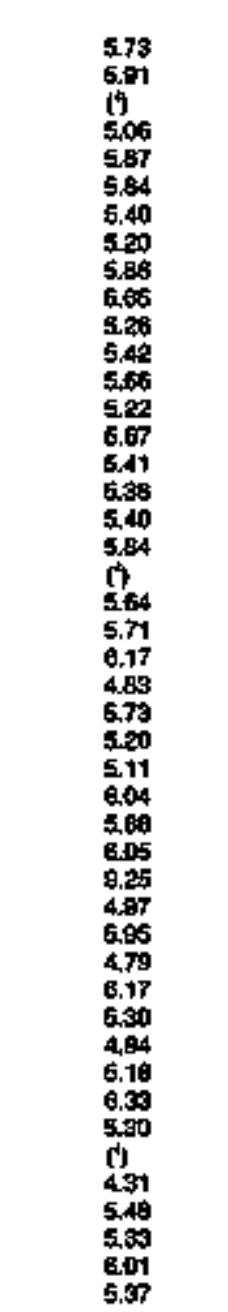 \\
\hline
\end{tabular}

See foolnotee at and of table. 
Table 17. Class of Ownershlp, Number of Ultimate Consumers, Revenue, Sales, and Average Revenue per Kilowatthour for All Sectors by State and Utility, 1993 (Continued)

\begin{tabular}{|c|c|c|c|c|c|}
\hline $\begin{array}{l}\text { State } \\
\text { Eectric ually }\end{array}$ & $\begin{array}{c}\text { Class } \\
\text { of } \\
\text { dumerehlo }\end{array}$ & $\begin{array}{c}\text { Nin'bit } \\
\text { of } \\
\text { Confunduers }\end{array}$ & $\begin{array}{c}\text { Revenut } \\
\text { (thousand dolaral }\end{array}$ & 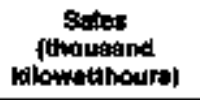 & 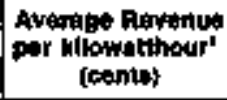 \\
\hline 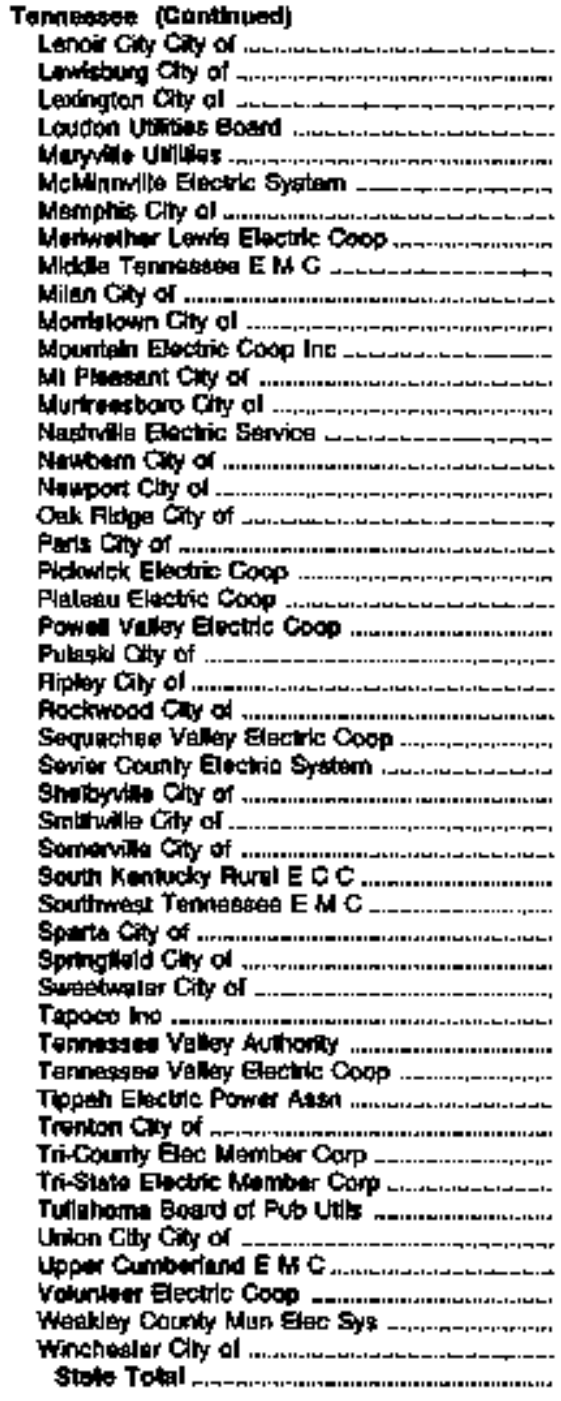 & 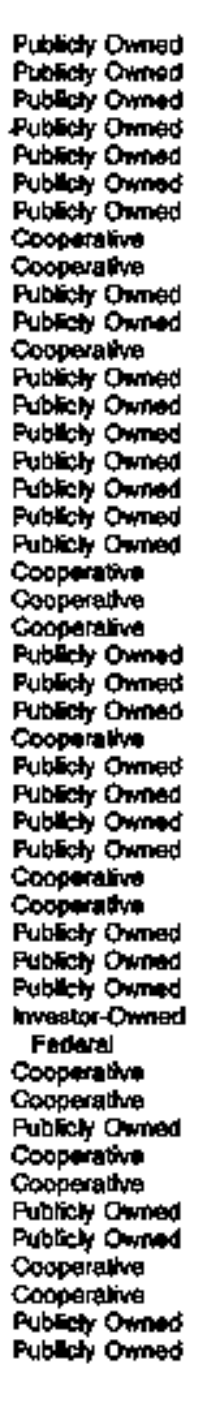 & 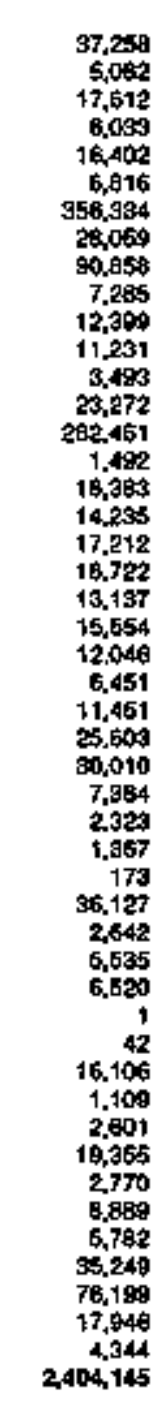 & 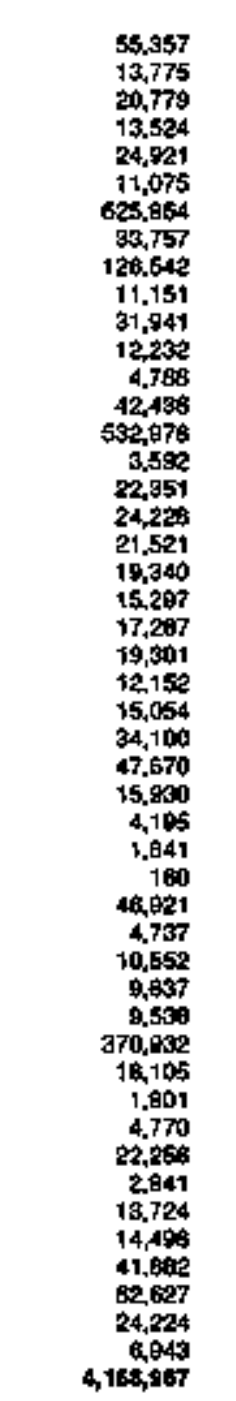 & 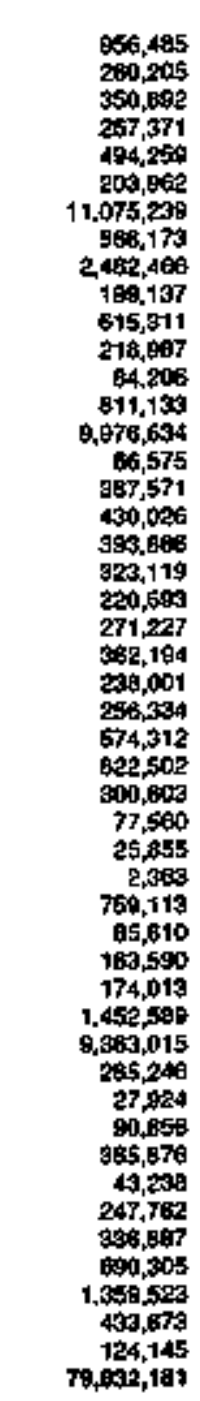 & 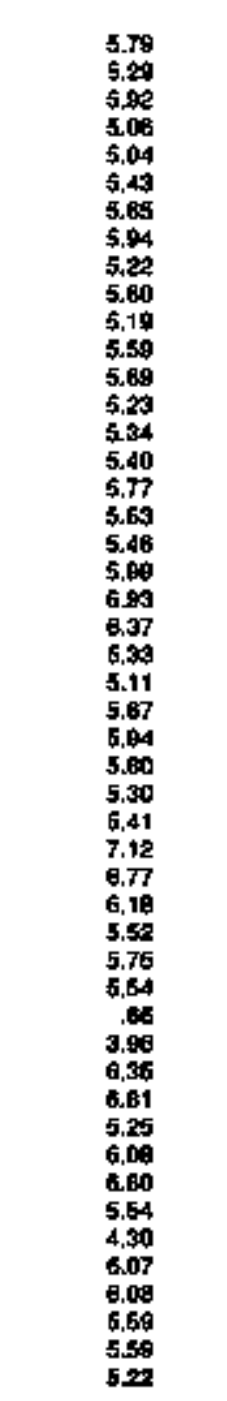 \\
\hline 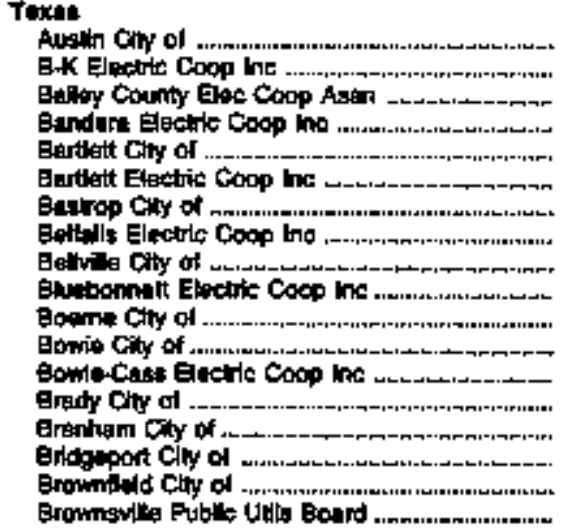 & 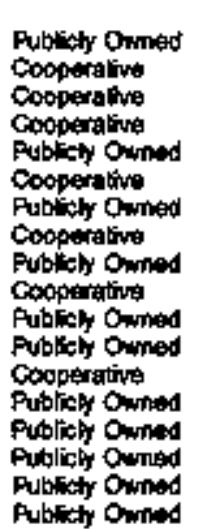 & $\begin{array}{r}284,282 \\
4,873 \\
5,641 \\
17,792 \\
9,60 \\
6,674 \\
2,001 \\
4,447 \\
1,635 \\
48,079 \\
2,674 \\
2,965 \\
28,363 \\
3,019 \\
6,496 \\
1,682 \\
4,138 \\
30,630\end{array}$ & $\begin{array}{r}482,094 \\
5,489 \\
10,045 \\
17,467 \\
779 \\
5,168 \\
2,987 \\
4,299 \\
2,608 \\
53,437 \\
3,659 \\
3,193 \\
29,440 \\
3,650 \\
11,745 \\
2,445 \\
3,691 \\
44,200\end{array}$ & 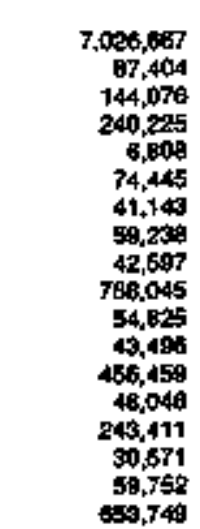 & $\begin{array}{r}6.68 \\
6.29 \\
6.97 \\
7.20 \\
11.49 \\
6.04 \\
7.29 \\
7.19 \\
5.69 \\
6.79 \\
6.67 \\
7.94 \\
6.45 \\
7.60 \\
4.69 \\
7.67 \\
6.16 \\
6.77\end{array}$ \\
\hline
\end{tabular}

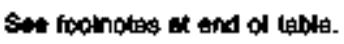


Table 17. Class of Ownershlp, Mumber of Uthimate Consumers, Revenue, Sales, and Average Aevenue per Kilowatthour for All sectors by State and UtIllty, 1993 (Continued)

\begin{tabular}{|c|c|c|c|c|c|}
\hline beite ty & of & $\begin{array}{c}\text { Eumber } \\
\text { of } \\
\text { Contuitert }\end{array}$ & fthoustend doltarly & 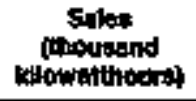 & 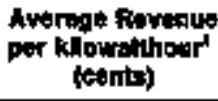 \\
\hline
\end{tabular}

Twat continued

Enyan Cly of .

Bumel chy of

calthall City of

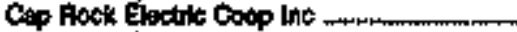

Cesbordlly Chy of

Central Pontor \& Lote Co

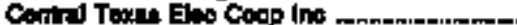

Gheroked County Eed Coop Assm n..........

Coleman Dity of

Colonin Count Eteo Coco Ino

Collepe Station City of

Comenche County Elec Cocp Ason .mnn

Concho Vet tor Eloc Coop Int.

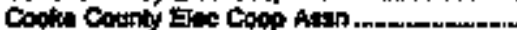

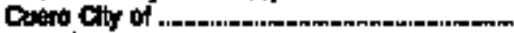

Doed sinth Electic Coop his

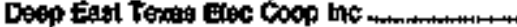

Denton Chy d d.

Dandt Electro Coop he -

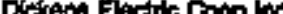

ef Paso Encticic

Exotr Chy of

Erith Couth Gec Coop Avon

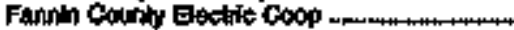

Fatinien Elactirc coop Ine.

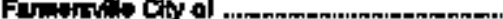

Fryette Eectso Coop he .

Fralonda Cyy of .

Forotwill $C$ H of

Foydada cty of

Fort Belkrap Eectric CoOp bo

Frododcksiburg Chy of nm

Gierlind City of

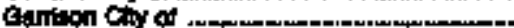

Cate Cily Eectrio Coop lno nnr.r.m.n.m.

coorgolown Cin or .

Cidtinas Chy of

coldignilh Cin of

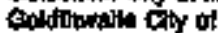

Corrinial Ciy of

Gronbury city of

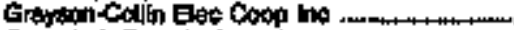

Grembek Eecito coop ino

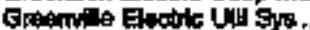

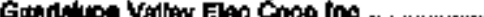

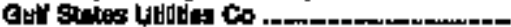

Helietlonite chy of

Hamitan Country Geoc Coop Asen

Hurman Elocifo Assm ins .

Heisme city of

Hemphtil City of

Hompedpad con of

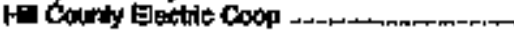

Honds $C S y$ of _..._.

Houston Counky Elec Coop Inc

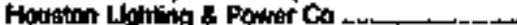

WhG Electlo coop $1 \mathrm{n}$.

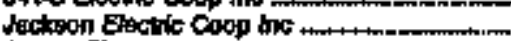

tasper Con of

leoperthenton Eeo Coop In

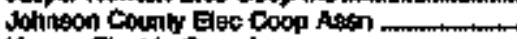

Kanis Eledite coop ho

Kuufman County Eoe Coop Inc ._.

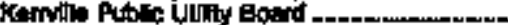

Kandlo Eleorite Coop lng ......................

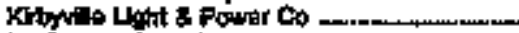

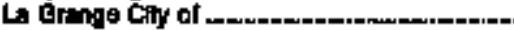

Laner Courhy Elec Coop Assn .................
Pubicty Omed

Pubicty Omined

Publlchy Omined

Cooperaline

Pubilcty Omied

In the thenowined

Cocperaltes

Cooperathe

Putitly Owned

Cooperative

Fublect Owned

Cooperaline

Cooperative

Coopetalive

Futbicty Omned

Cooperative

Cooporituing

oublet Owined

Cooperative

Cooperrithe

Cooperithy

Imitughor-Chined

pubfey Owned

coloproturs

conperition

Comperative

Pubildy Owmed

Coopenothes

Pubilly Owned

Pubrcty Owned

Fubilety Owned

cosperativa

Pubility Omod

Punindy Omed

Purfigy Oimed

Coopuretwe

Purlity Omined

Purticty Oined

Publloty Owtrod

Punledy Omaned

Putwisty Owoned

Pupticty Onned

Coxperative

Coopereetibs

Pulitsty Onnod

Cocperzitiose

Iprestrotowned

Puldkdy Ouned

Cosperative

Cospective

Fatplichy Ouned

Puslaty Ommed

Publect Onntrd

Cooperalve

Putisy Onined

Cooperathe

misotir-Ourted

Cooperrative

Cooperathe

Putitity Omied

Cooperathe

Cocperalve

Cooperathe

Cocparatur

Publets Oimed

Cooperathe

Pustict Ommed

Publety Ounes

Cooperatus
24,154

$1,0 \pi$

1,742

20730

1,042

BE0, 14:

201000

t3,83:2

3,282

7,65

20,180

t4,754

BAs?

10,153

3,473

0.826

31.006

25,604

30,370

6216

4884

200,749

1 䍂

16,516

$5,5 e$

28,538

1,348

9,470

946

10215

1,228

4,781

4449

60.,976

591

1,765

7,974

2,128

167

1,158

2,033

2451

14 3014

3,759

11,684

30.568

297,6028

1.558

10,947

283

2,340

694

1,860

12494

2684

14,69

1,440,15:

4,000

11,68

4

$28+197$

11,762

2006

(2)

16,509
4,042

910

TT6

8.624
3,013

3,342

42,366

1,347
16006

18,952

18,100

2,066

6 s.55

28,494

11,954

10,654

12,342

4,616

18,724

39.77

54,3z:

48,143

5.94

18,952

\$31,600

1,357

16,9t9

6,295.

29,469

1,556

11,098

1,198

10,16:

948

6,014

5,243

129,601

521
2371

2,371
11,208

2,684

211

1,071

4,059

4 Tin

19.290

3,802

29,601

40.035

701,954

1,744

7,748

112

2,919

1,020

2.197

14.819

4,246

17,642

9.922,87

6,47

1120

10.359

2,162

36,156

11,500

e7 20 ?

21,352

3,604

1,570

280

0,854

577896

36,044

41,000

562,197

16,274

16,780,914

255,838

191,573

36,069

Ba,490

436

148,650

136,697 
Table 17. Class of Ownership, Number of Ultimate Consumers, Reventhe, Sales, and Average Rovenue per Kilowatthour for All Sectors by State and Utalty, 1993 (Continued)

\begin{tabular}{|c|c|c|c|c|c|}
\hline 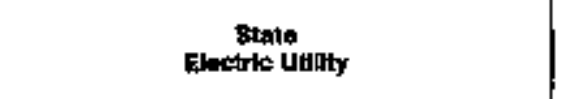 & $\begin{array}{c}\text { Clats } \\
\text { of } \\
\text { Qumfermhtp }\end{array}$ & $\begin{array}{c}\text { Number } \\
\text { af } \\
\text { Contumers }\end{array}$ & Rowernw & 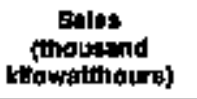 & 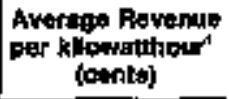 \\
\hline 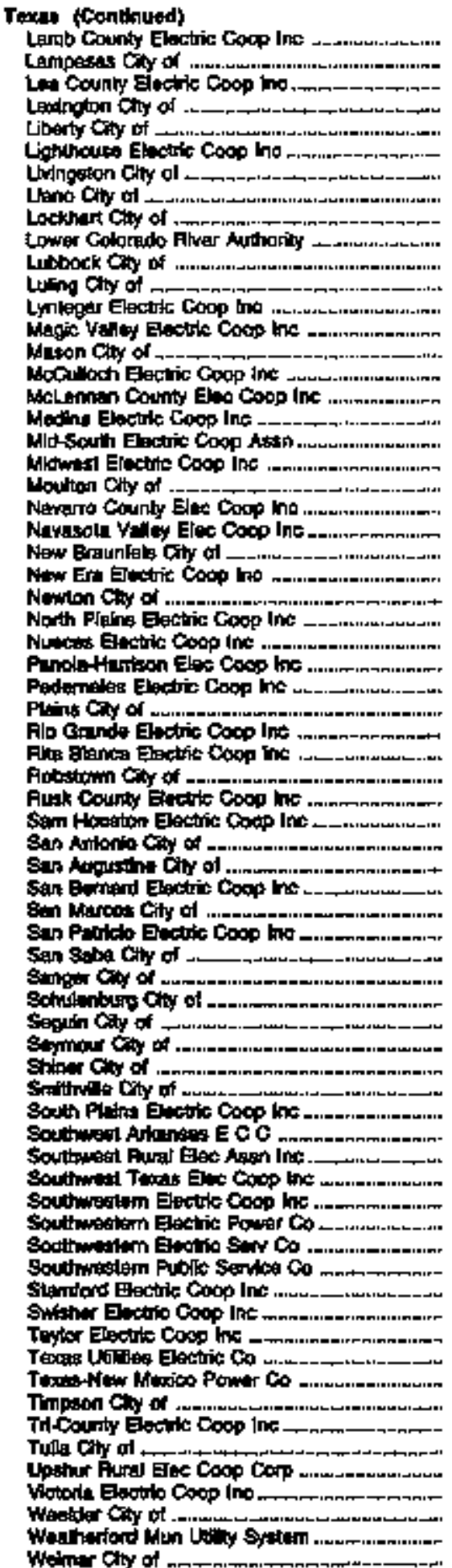 & 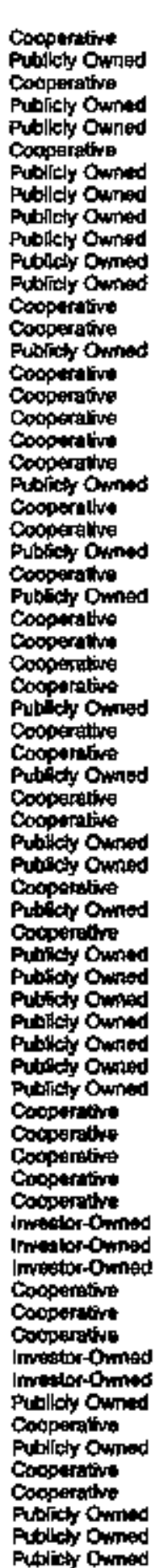 & 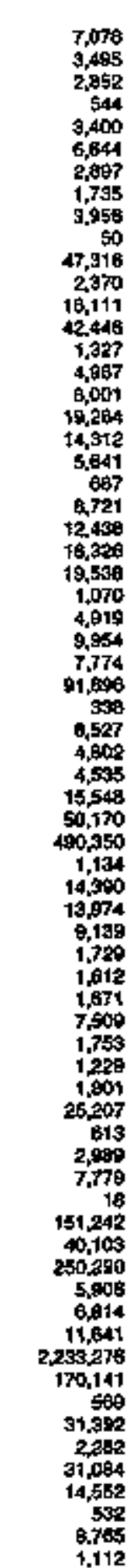 & 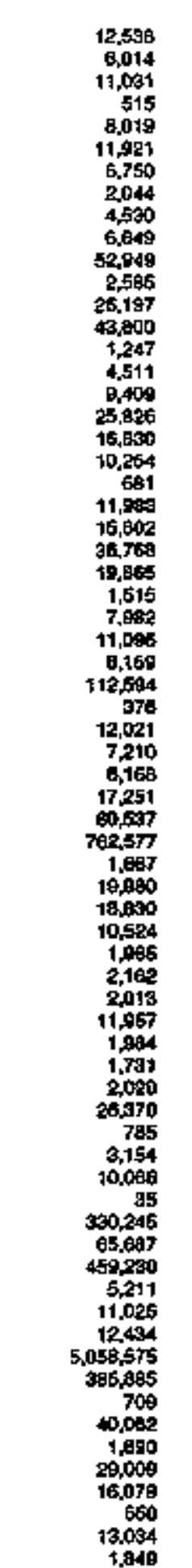 & 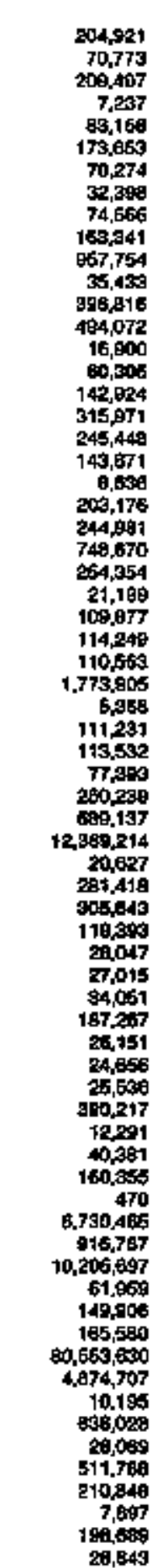 & 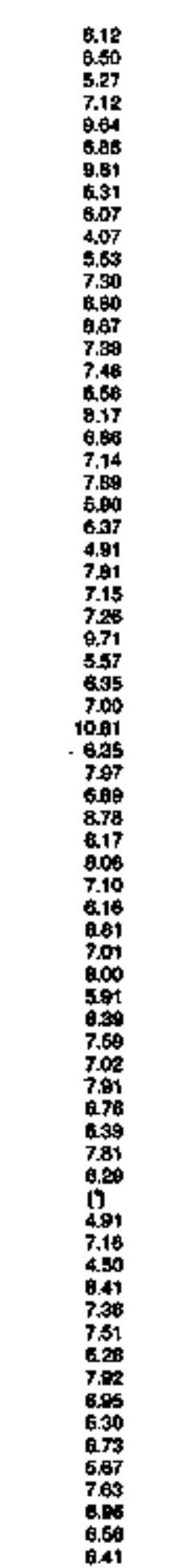 \\
\hline
\end{tabular}

See toptrotis at end of talle. 
Table 17. Class of Ownership, Numbèr of Ultimate Constumers, Pevenue, Sales, and Average Revenue per Kllowatthour for A. Sectors by State and Utility, 1993 (Contimued)

\begin{tabular}{|c|c|c|c|c|c|}
\hline $\begin{array}{l}\text { 8tito } \\
\text { Esocifle (wally }\end{array}$ & of & $\begin{array}{l}\text { Mumbar } \\
\text { of } \\
\text { Consoument }\end{array}$ & $\begin{array}{c}\text { Arwenus } \\
\text { (thousend dotlare) }\end{array}$ & 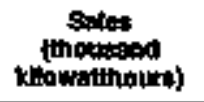 & 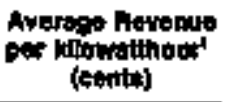 \\
\hline 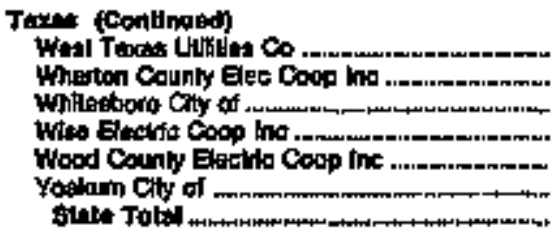 & 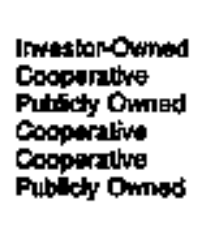 & $\begin{array}{r}181,461 \\
4,870 \\
1,715 \\
12,839 \\
22,582 \\
2,020 \\
7,080,416\end{array}$ & $\begin{array}{r}285,220 \\
8,128 \\
5,746 \\
12,047 \\
24,483 \\
3,069 \\
15,903,948\end{array}$ & $\begin{array}{r}4,304,210 \\
105,995 \\
24,190 \\
176,920 \\
398,789 \\
48,624 \\
250,064,419\end{array}$ & $\begin{array}{l}6.16 \\
7.72 \\
7.24 \\
6.101 \\
6.84 \\
7.04 \\
600\end{array}$ \\
\hline 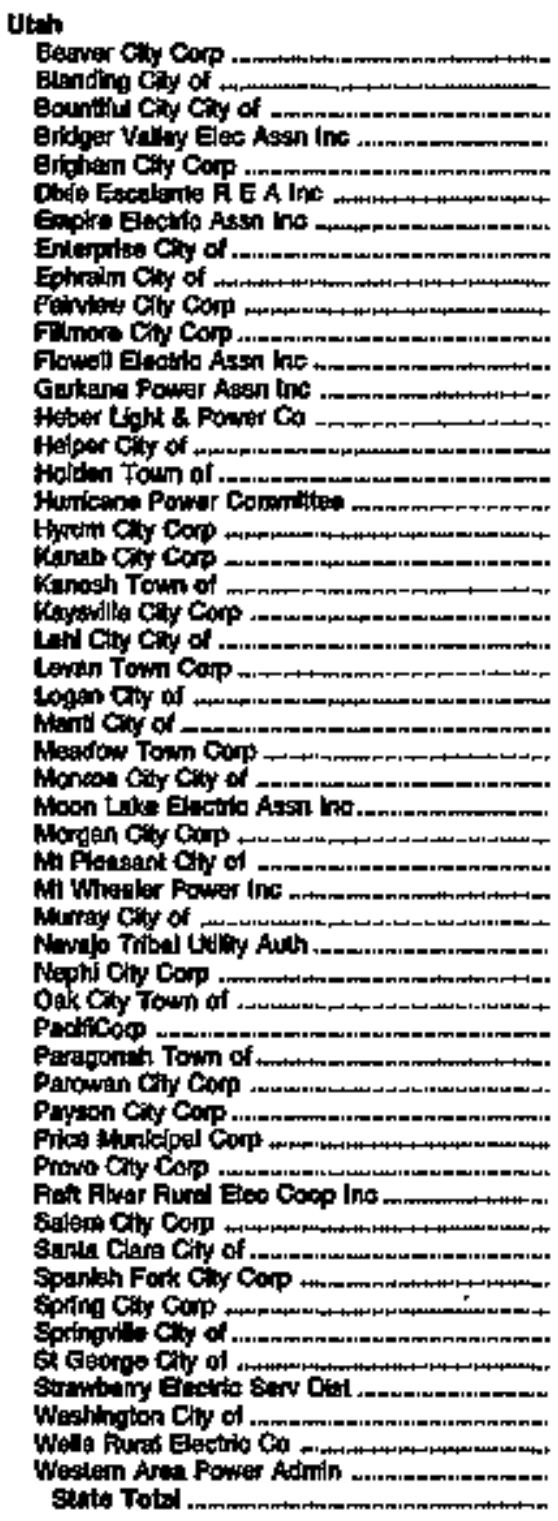 & 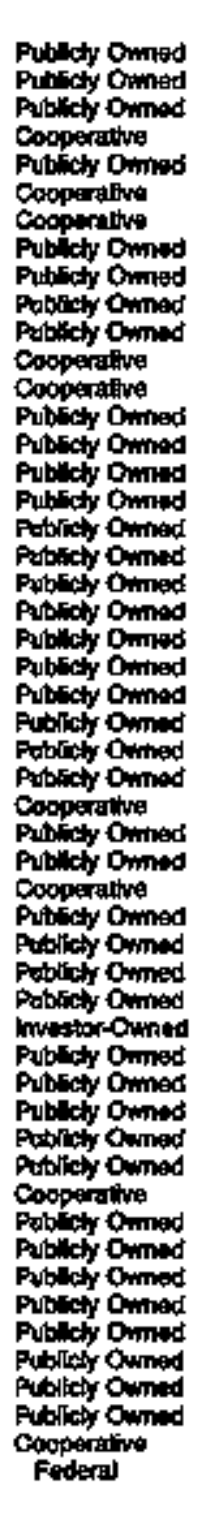 & 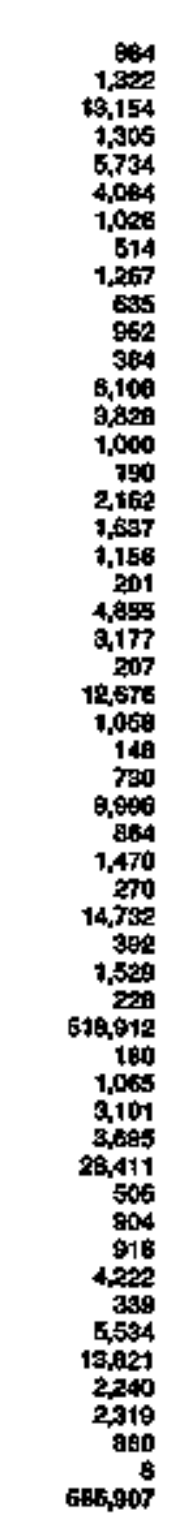 & 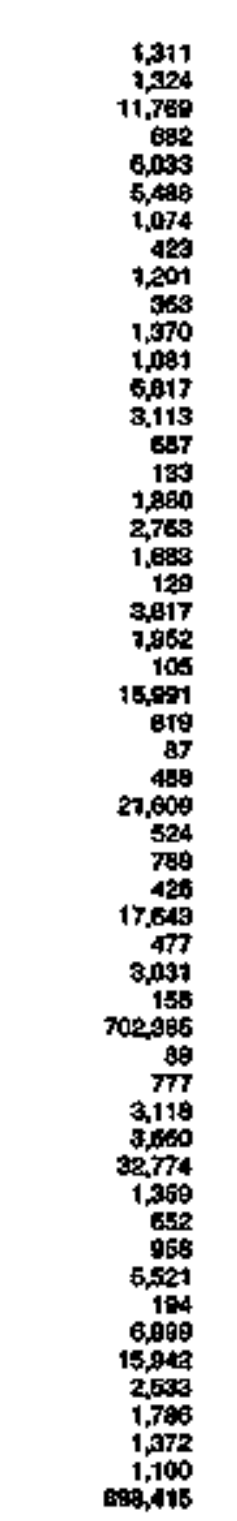 & 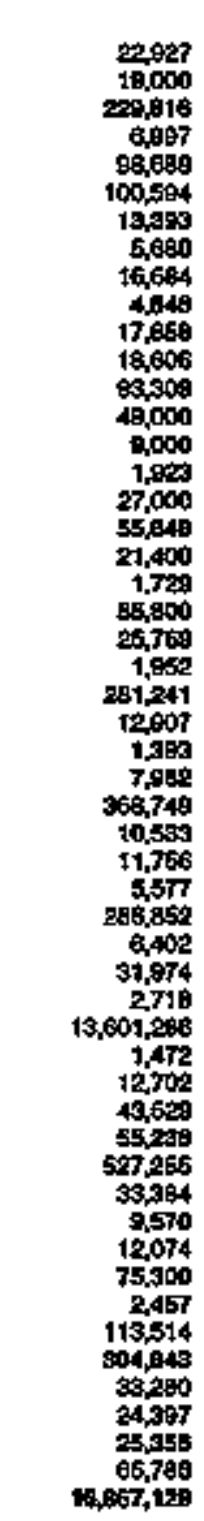 & 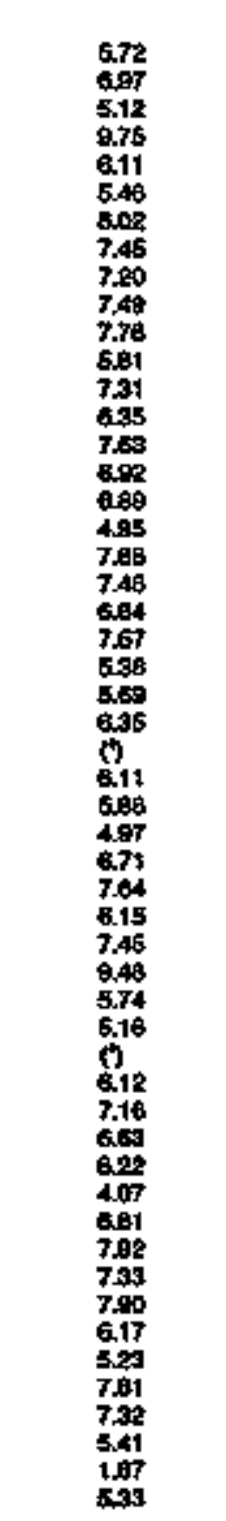 \\
\hline 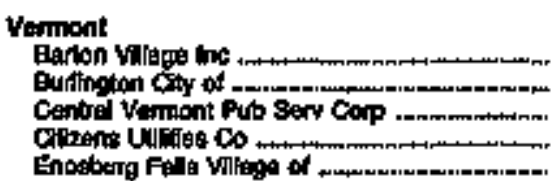 & 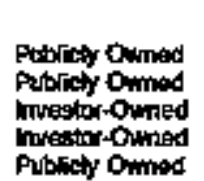 & $\begin{array}{r}1,055 \\
18,578 \\
135,245 \\
10,462 \\
1,325\end{array}$ & $\begin{array}{r}1,128 \\
34,724 \\
199,097 \\
24,053 \\
1,549\end{array}$ & $\begin{array}{r}14,578 \\
323,093 \\
2,022,143 \\
280,297 \\
17,802\end{array}$ & $\begin{array}{r}7.74 \\
10.72 \\
9.99 \\
8.58 \\
8.84\end{array}$ \\
\hline
\end{tabular}

Sea lootnotas at end ol table. 
Table 17. Class of Ownership, Number of Utimate Consumers, Revenue, Sales, and Average Rovenue par Kilowatthour for All Sectors by State and Utilty, 1983 (Continued)

\begin{tabular}{|c|c|c|c|c|c|}
\hline $\begin{array}{c}\text { stata } \\
\text { Eleotile } 2 \text { fetty }\end{array}$ & $\begin{array}{l}\text { Claps } \\
\text { of } \\
\text { ownetship }\end{array}$ & 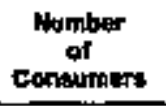 & 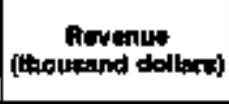 & 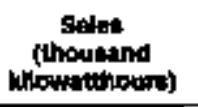 & 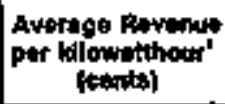 \\
\hline 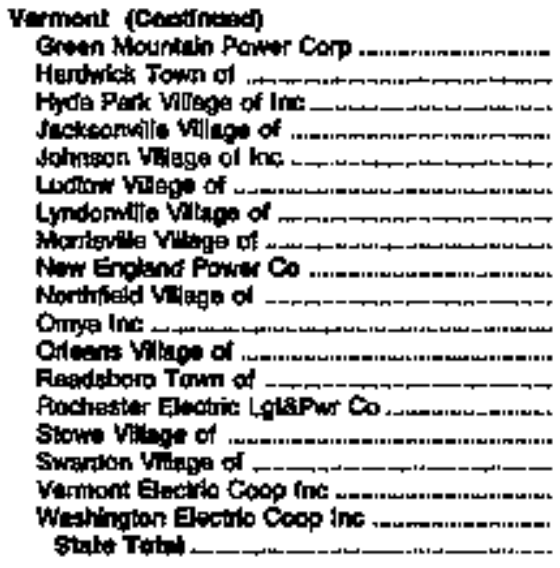 & 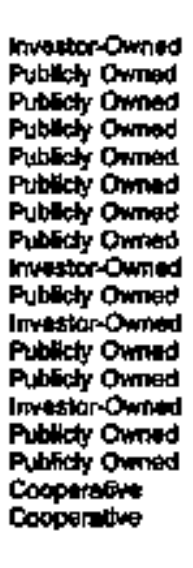 & 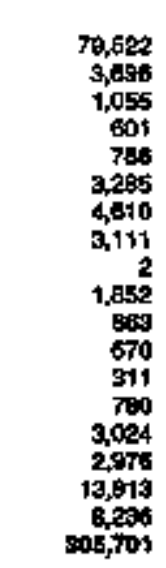 & 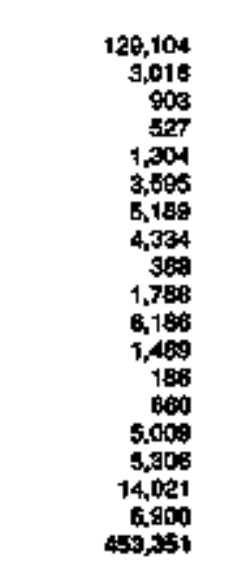 & $\begin{array}{r}1,684,779 \\
30,892 \\
0,213 \\
4,745 \\
1,969 \\
40,454 \\
58,603 \\
43,442 \\
4,120 \\
2,863 \\
129,003 \\
17,090 \\
2,000 \\
6,000 \\
55,175 \\
50,000 \\
127,205 \\
55,254 \\
5045,554\end{array}$ & $\begin{array}{r}7.68 \\
0.70 \\
9.80 \\
11.11 \\
7.60 \\
6.69 \\
6.86 \\
9.96 \\
9.41 \\
7.86 \\
6.39 \\
0.72 \\
0.16 \\
10.69 \\
9.99 \\
10.61 \\
11.02 \\
12.49 \\
0.04\end{array}$ \\
\hline 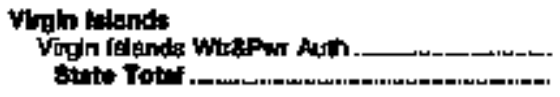 & Pulicty Owhed & $\begin{array}{l}47,200 \\
4,200\end{array}$ & $\begin{array}{l}71,375 \\
71,376\end{array}$ & $\begin{array}{l}577,202 \\
677,202\end{array}$ & $12: 7$ \\
\hline 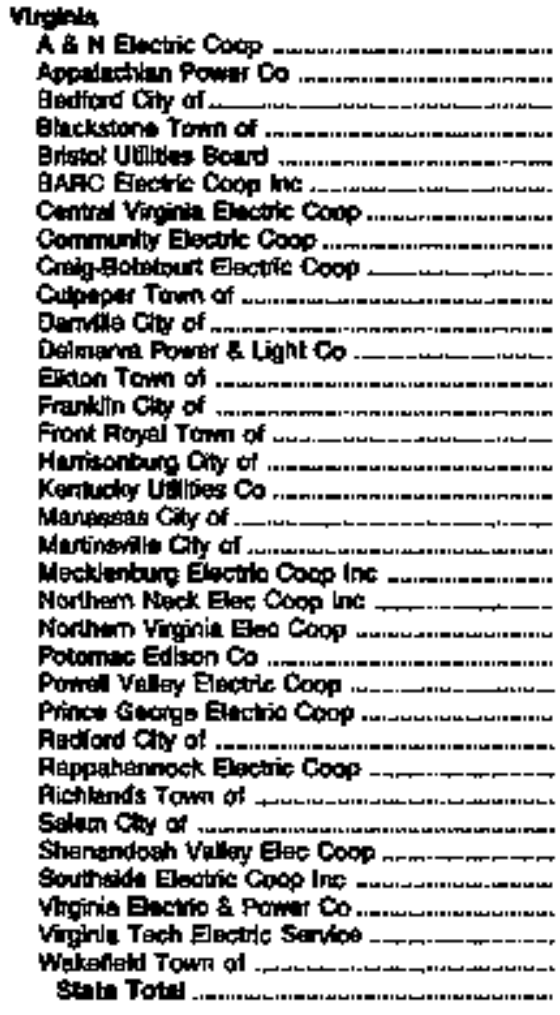 & 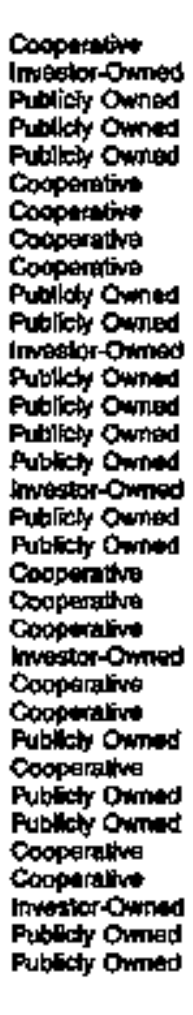 & 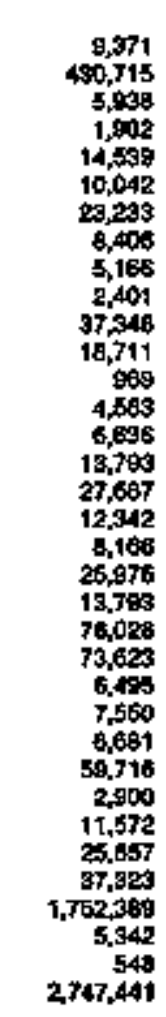 & 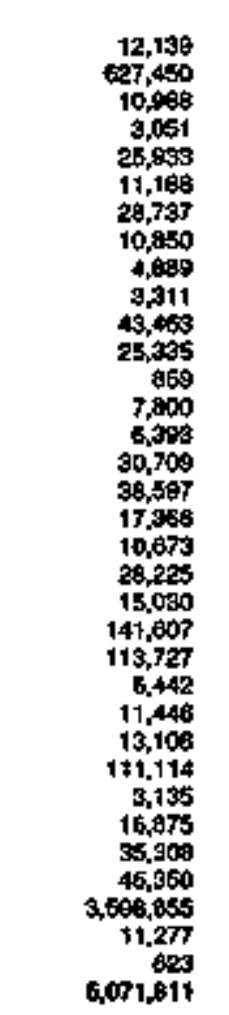 & 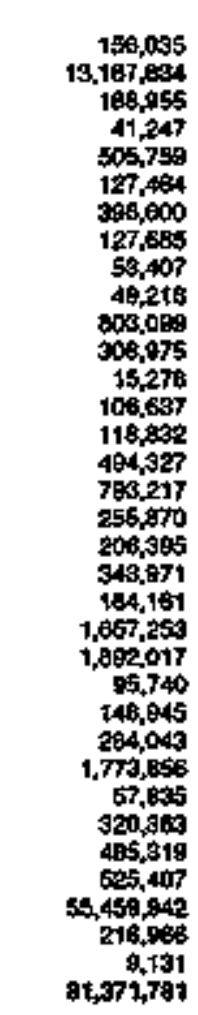 & 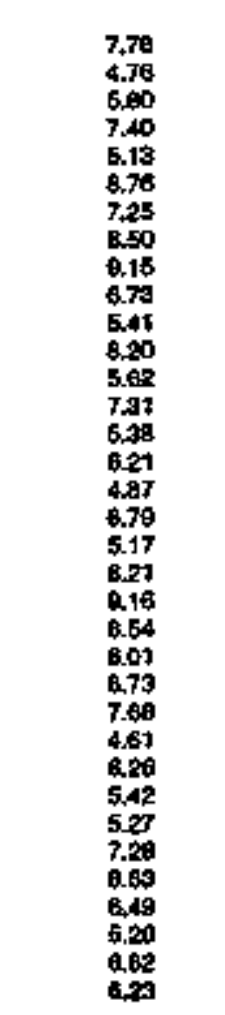 \\
\hline 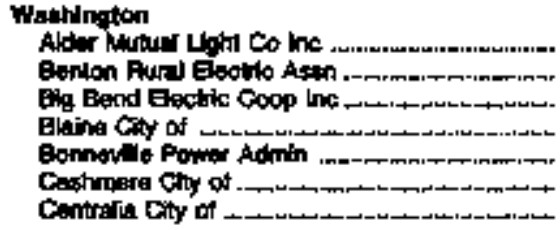 & 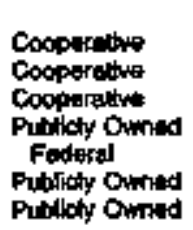 & $\begin{array}{r}190 \\
0,700 \\
6,571 \\
1,902 \\
10 \\
1,104 \\
6,260\end{array}$ & $\begin{array}{r}127 \\
16,120 \\
52,815 \\
2,705 \\
314,140 \\
7,464\end{array}$ & $\begin{array}{r}2,706 \\
32,000 \\
347,562 \\
60,057 \\
18,292,776 \\
51,514 \\
219,168\end{array}$ & $\begin{array}{l}4,80 \\
5.01 \\
3.80 \\
4.00 \\
1.00 \\
1.01 \\
3.42\end{array}$ \\
\hline
\end{tabular}

Sact toptroles at wrod of tablo. 
Table 17. Class of Ownerahlp, Number of Uttimate Consumers, Revenue, Seles, and Average fevenue per Kilowatthour for All Sectors by State and Utility, 1993 (Continued)

\begin{tabular}{|c|c|c|c|c|c|}
\hline Etate & $\begin{array}{l}\text { ciats } \\
\text { of } \\
\text { Onnthenth }\end{array}$ & 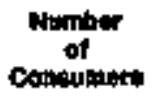 & Ratitur & 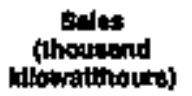 & 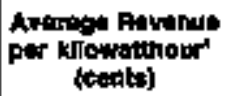 \\
\hline 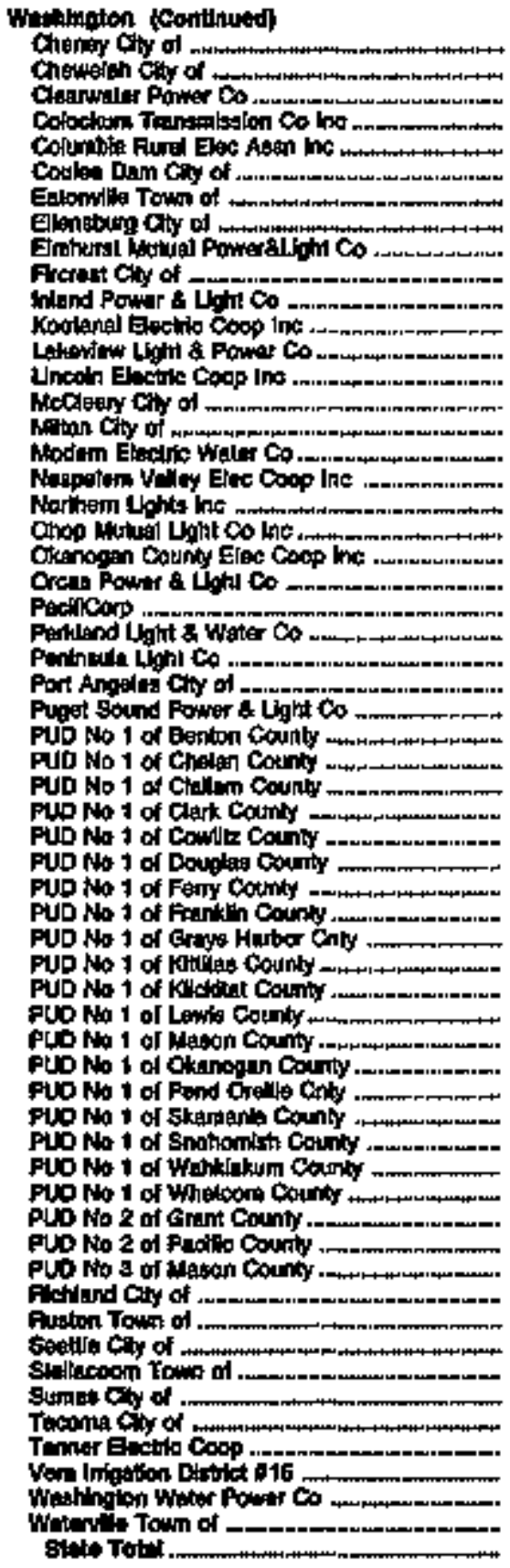 & 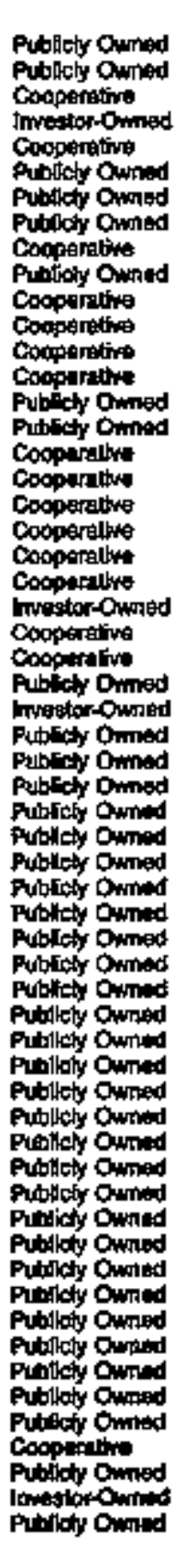 & 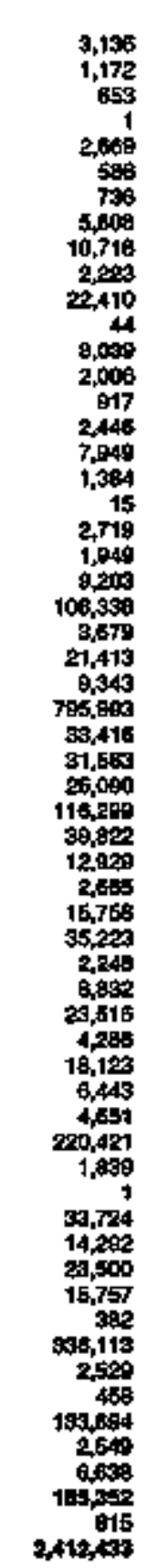 & 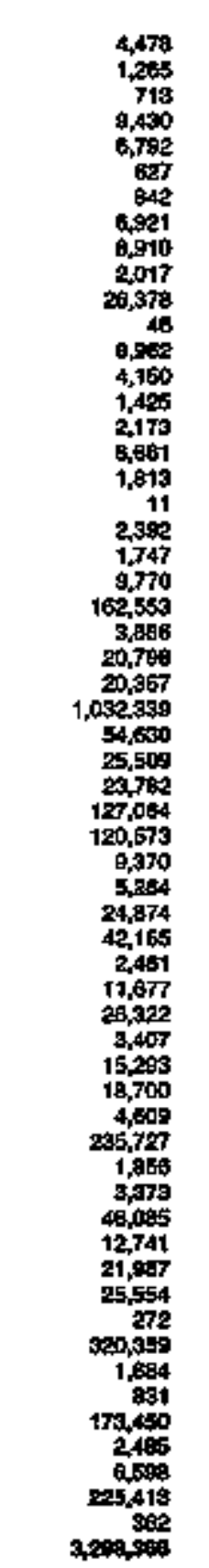 & 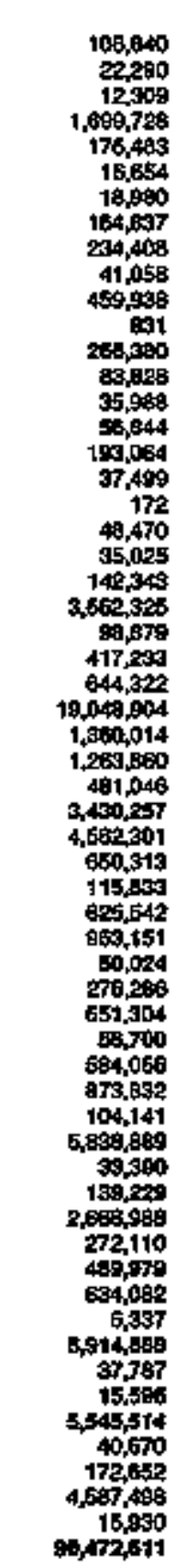 & 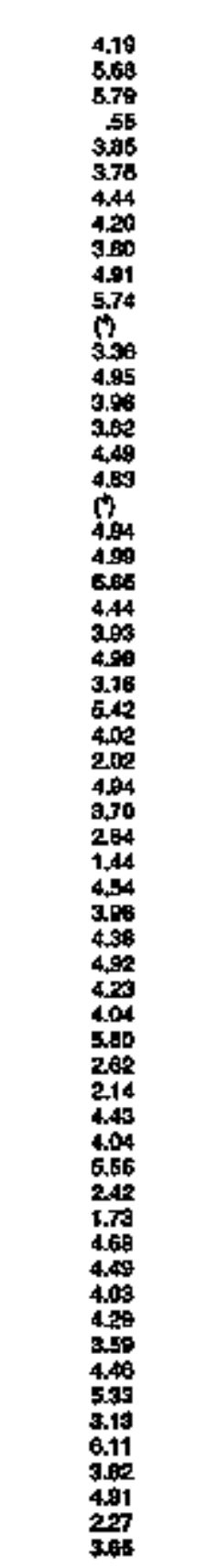 \\
\hline 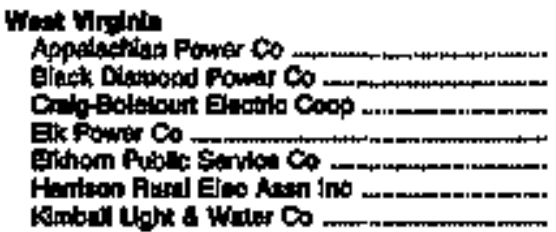 & 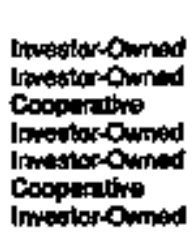 & 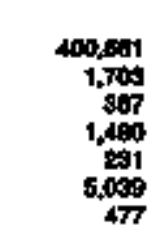 & $\begin{array}{r}56,703 \\
1,072 \\
240 \\
1,094 \\
164 \\
3,803 \\
918\end{array}$ & $\begin{array}{r}11,595,360 \\
17,845 \\
2,508 \\
15,690 \\
2,052 \\
43,984 \\
5,203\end{array}$ & 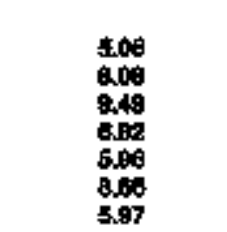 \\
\hline
\end{tabular}

Hea foxingles at and of tobin 
Table 17. Class of Ownerthip, Number of Uitimate Consumers, Rovenup, Sales, and Average Revenue per Klowatthour for All Sectors by State and Utility, 1993 (Contlnued)

\begin{tabular}{|c|c|c|c|c|c|}
\hline Stake & otsen & $\begin{array}{c}\text { Nunibar } \\
\text { of } \\
\text { Consumere }\end{array}$ & $\begin{array}{c}\text { Pavenut } \\
\text { (thoupand ditlara) }\end{array}$ & 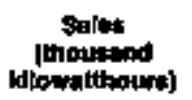 & $\begin{array}{c}\text { Avtrage howenue } \\
\text { per tillowalingur } \\
\text { (covip) }\end{array}$ \\
\hline 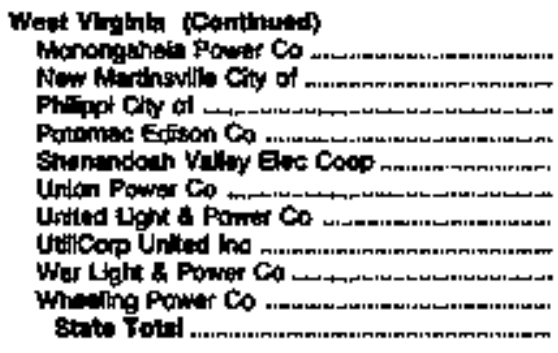 & 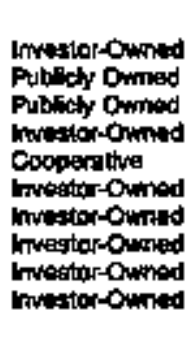 & $\begin{array}{r}311,128 \\
1,856 \\
1,668 \\
91,16 \% \\
2,371 \\
1,500 \\
1,276 \\
24,751 \\
1,091 \\
41,174 \\
087,507\end{array}$ & 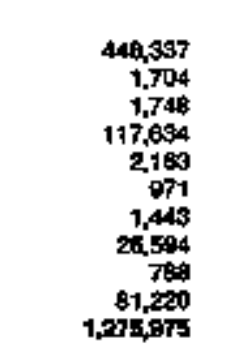 & $\begin{array}{r}6,374,269 \\
27,070 \\
27,246 \\
2,042,199 \\
24,278 \\
16,429 \\
24,456 \\
384,204 \\
12,061 \\
1.054,340 \\
24,44,595\end{array}$ & $\begin{array}{l}5.35 \\
6.09 \\
6.42 \\
5.75 \\
6.81 \\
5.91 \\
5.00 \\
7.30 \\
6.49 \\
4.30 \\
5.20\end{array}$ \\
\hline 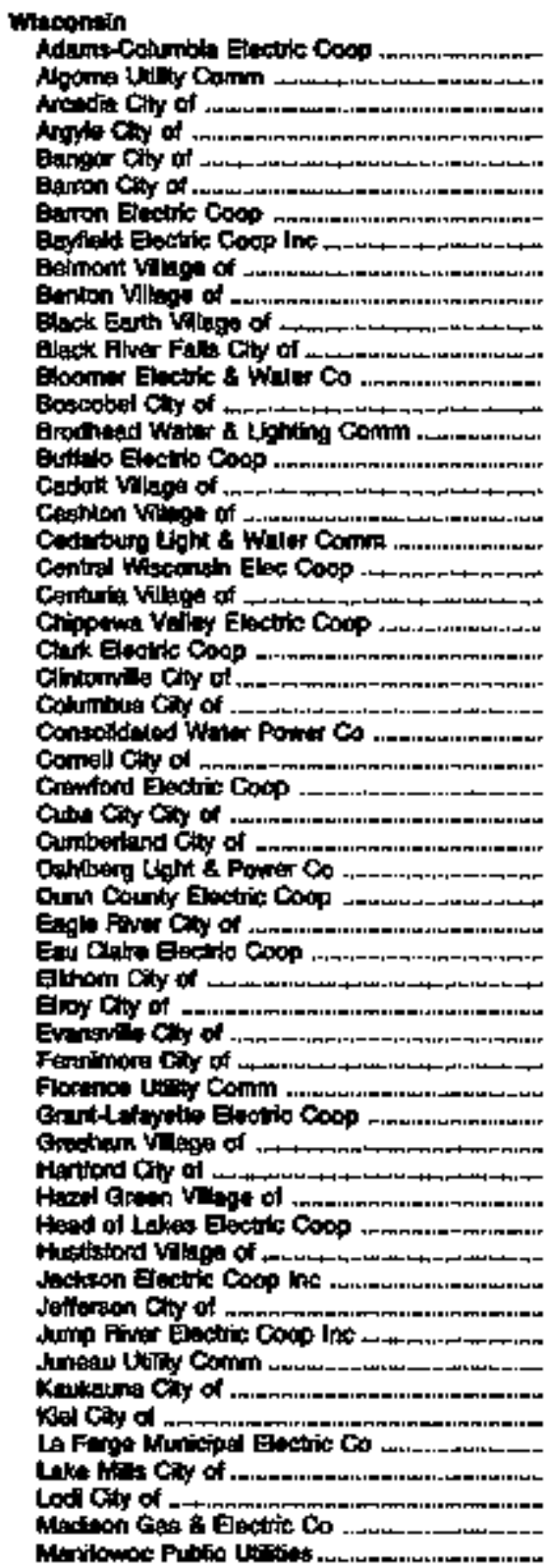 & 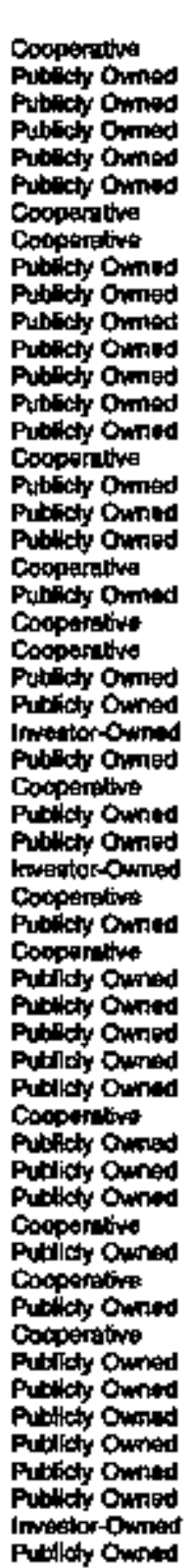 & 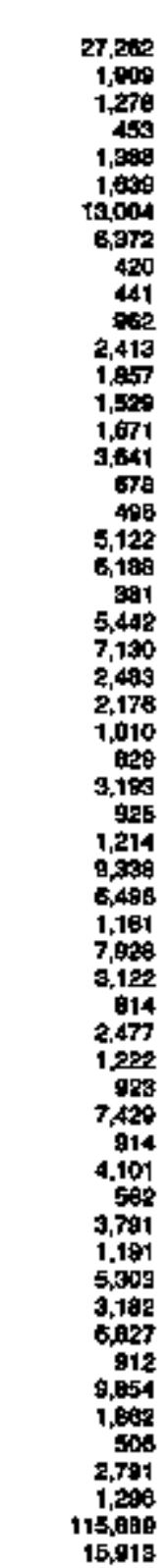 & 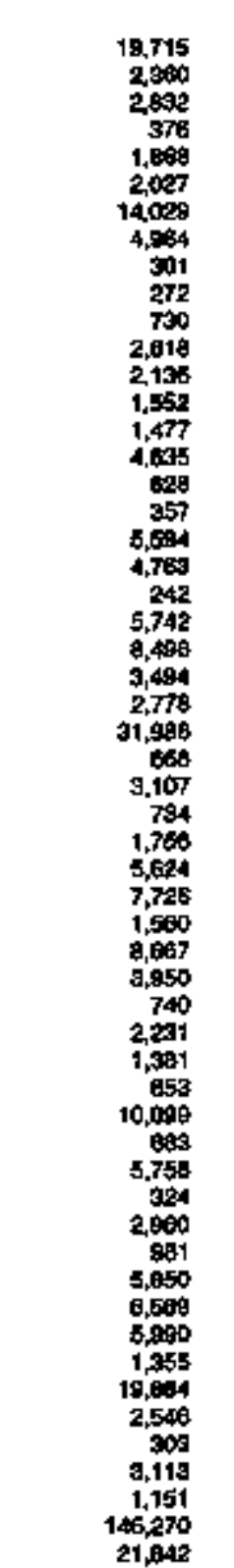 & 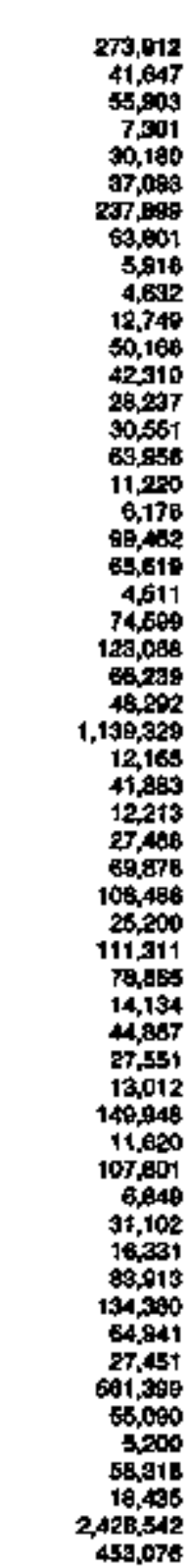 & 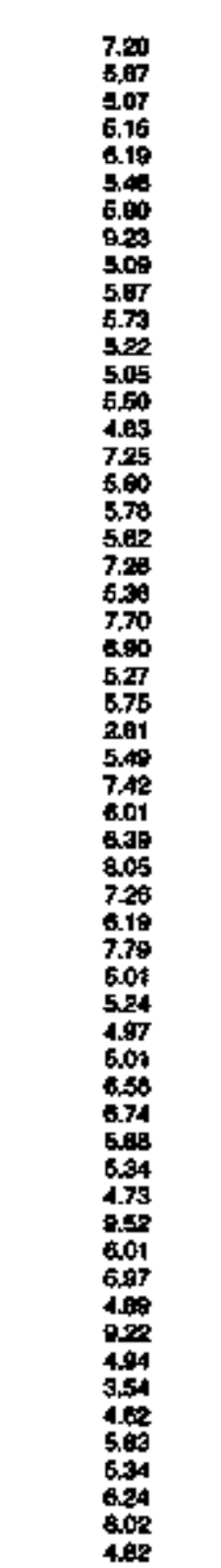 \\
\hline
\end{tabular}

See tootnotes at end of tabls. 
Table 17. Class of Ownership, Numbert of Ultimate Consumers, Fevenue, Sales, and Average Revenue per Kilowatthour for Au Sectors by State and Utility, 1993 (Continuted)

\begin{tabular}{|c|c|c|c|c|c|}
\hline $\begin{array}{l}\text { stinte } \\
\text { Electukt ulitity }\end{array}$ & $\begin{array}{c}\text { Clath } \\
\text { of } \\
\text { ommernthp }\end{array}$ & $\begin{array}{l}\text { Cumber } \\
\text { of } \\
\text { Contamer }\end{array}$ & phourand doilianst & $\begin{array}{l}\text { siles } \\
\text { (thoumand }\end{array}$ & 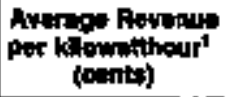 \\
\hline 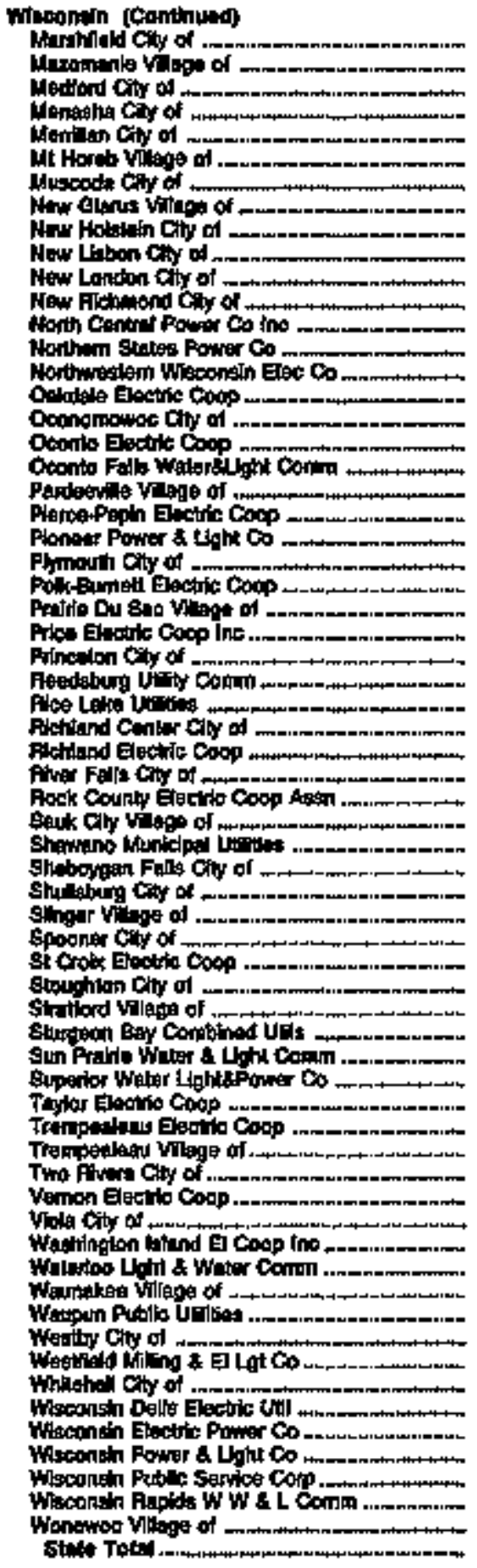 & 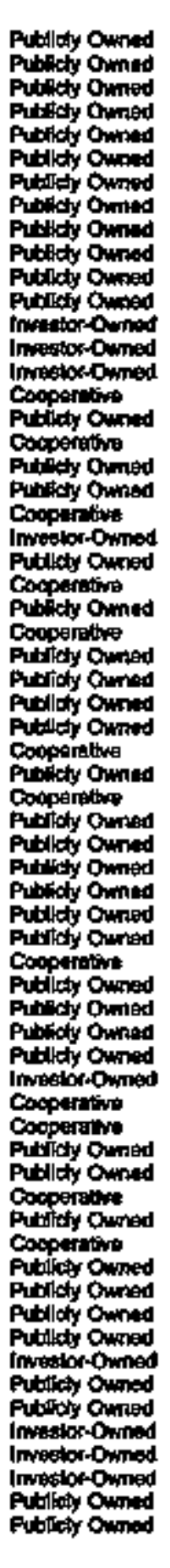 & 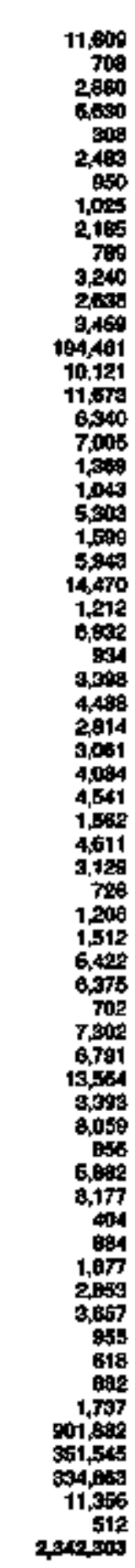 & 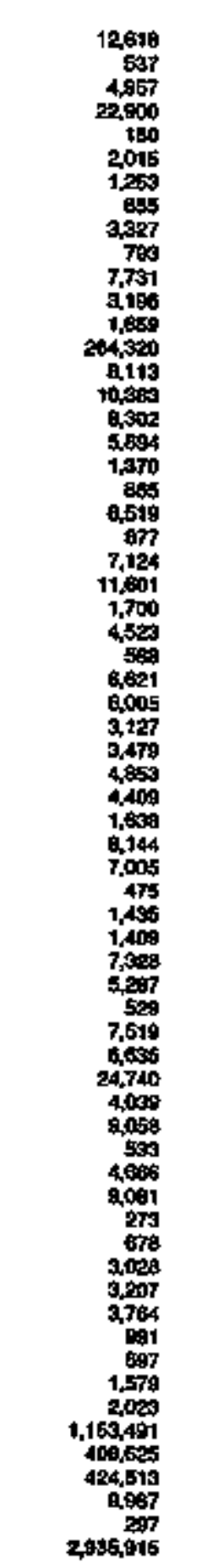 & 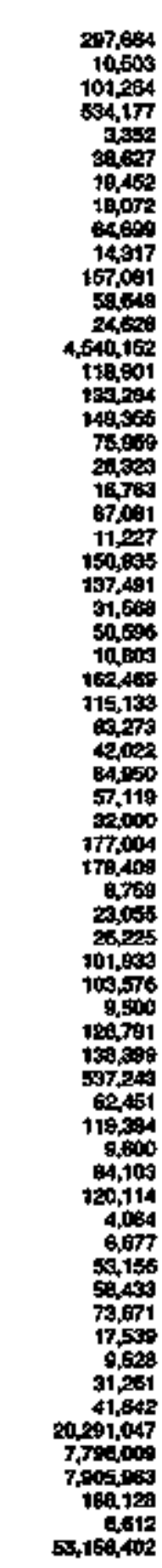 & 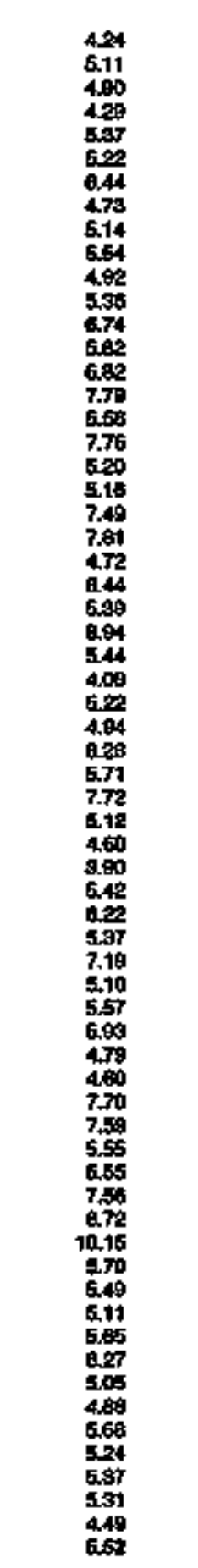 \\
\hline 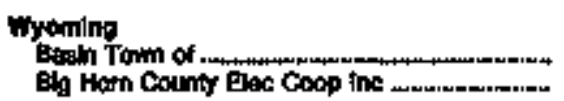 & $\begin{array}{l}\text { Puntohy Owind } \\
\text { Cooperntwe }\end{array}$ & $\begin{array}{l}\text { 的1 } \\
28\end{array}$ & $\begin{array}{l}445 \\
245\end{array}$ & $\begin{array}{l}7,0609 \\
3,513\end{array}$ & $\begin{array}{l}\mathbf{5 6 0} \\
\mathbf{6 . 9 7}\end{array}$ \\
\hline
\end{tabular}

soe foolnotes ot end of lable. 
Table 17. Class of Ownership, Number of Uttmate Consumers, Revenue, Sales, and Average Revenue per KIlowatthour for All Sectors by State and Utility, 1993 (Continued)

\begin{tabular}{|c|c|c|c|c|c|}
\hline 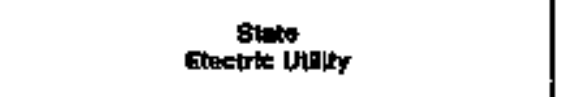 & 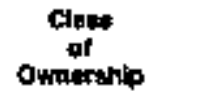 & 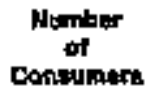 & (thoyseand dolbars) & $\begin{array}{c}\text { Soles } \\
\text { (therditidd } \\
\text { Whowrithourst) }\end{array}$ & 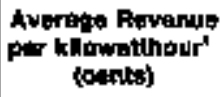 \\
\hline 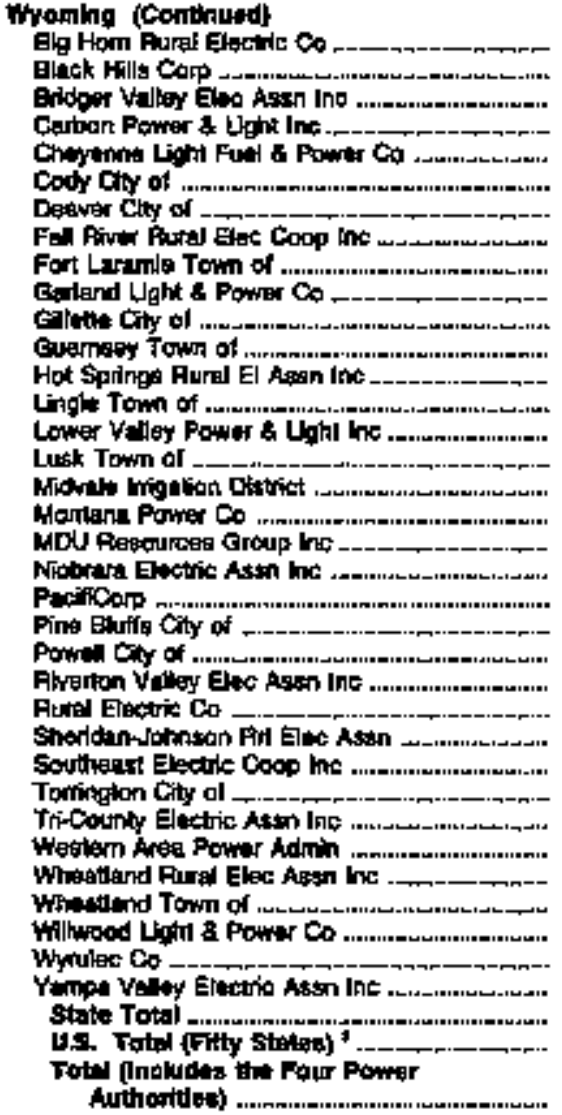 & 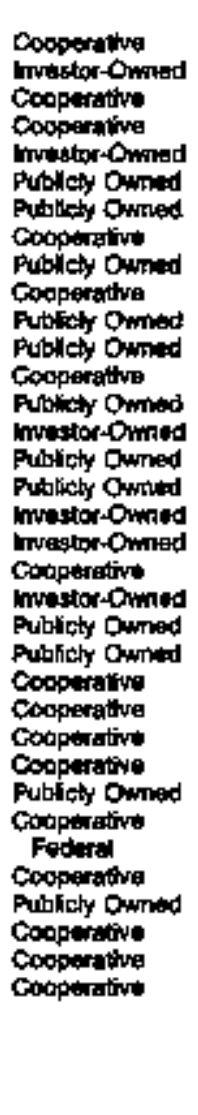 & 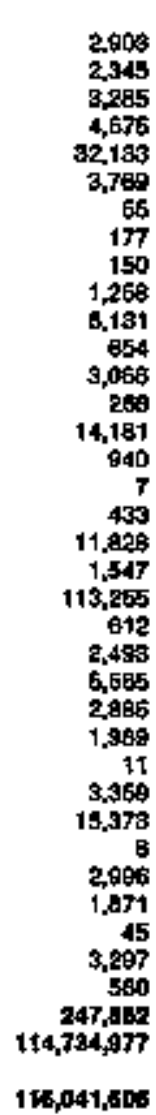 & 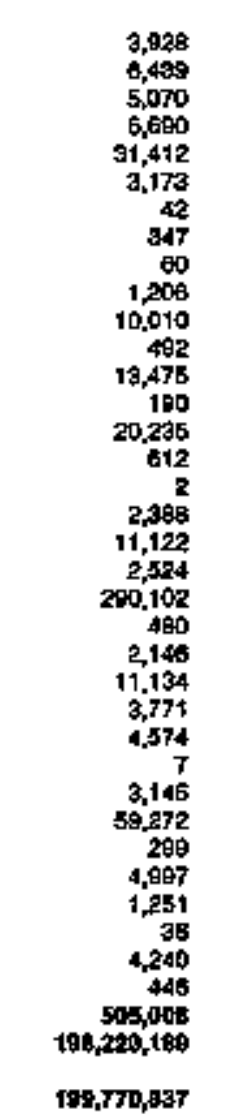 & 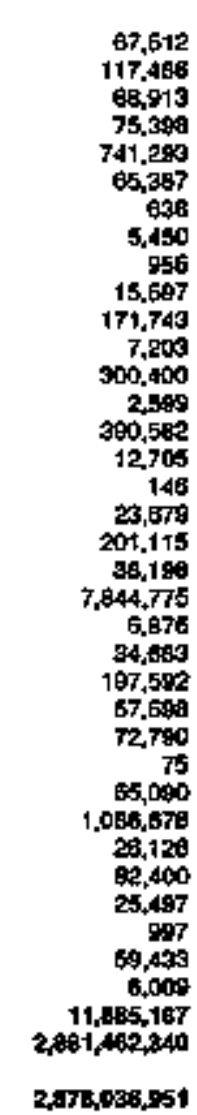 & 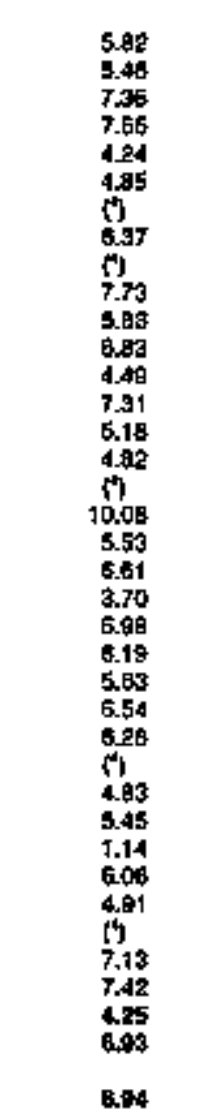 \\
\hline
\end{tabular}

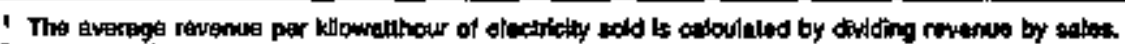

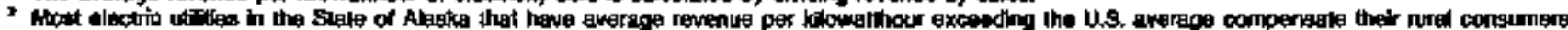

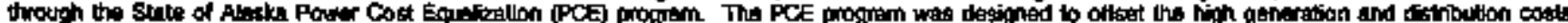

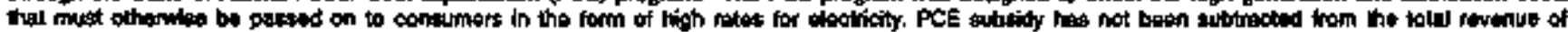

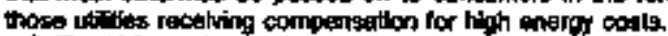

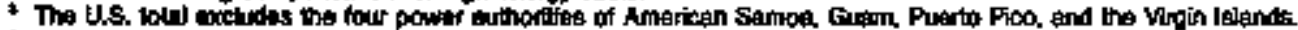

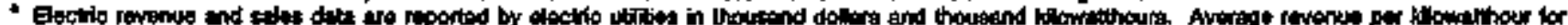

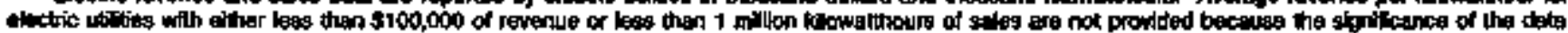

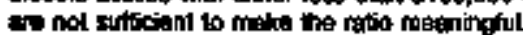

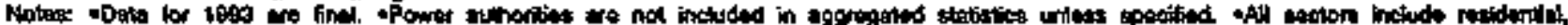

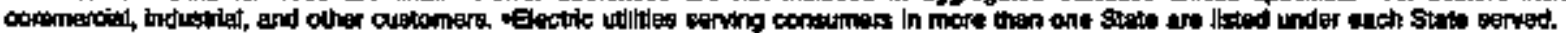

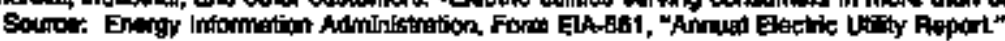


Appendix A

Electric Utllities Serving UItImate Consumers in More Than One State 



\section{Electric Utilities Serving Ultimate Consumers in More Than One State}

This appendix contains information from the Form EIA-861, "Annual Electric Utility Report," regarding electric utilities that serve ultimate consumers in more than one State (Table Al). Additionally, the percent of sales of electricity is each State is included in Table A1. The information can be used as a reference to Tables 14 through 17. For example, information contained in Table Al indicates that the Appalachian Power Company serves consumers in Virginia and west Virginia. Tables 14 through 17 contain the respective sales, revenue, and average revenue by each sector and for all sectors for this electric utility in both States. The majority of utilities that serve ultimate consumers in more than one State are the investor-owned electritc utilities. These electric utilities generally have large franchise service areas and historically have served re. tail consumers in more than one State. Publicly owned electric utilities nsually are chartered by municipal of State governments and usually provide service within the cities or counties where they are located.

Table A1. Electric Utillties Serving Uttmate Consumers In More Than One State, 1993

\begin{tabular}{|c|c|c|}
\hline $\begin{array}{l}\text { Enotrto } \\
\text { Ltity }\end{array}$ & $\begin{array}{l}\text { Stetes } \\
\text { Servitd }\end{array}$ & $\begin{array}{c}\text { Paroent of Totel } \\
\text { Soles }\end{array}$ \\
\hline A 1 N Elactrio Cosp & $\begin{array}{l}\text { Vigirala } \\
\text { Mrytand }\end{array}$ & 触 \\
\hline Nfilfa Enotio Coop ine & $\begin{array}{l}\text { Oktanome } \\
\text { Krisa: }\end{array}$ & 9 \\
\hline Appotachlar Power Co & Whoint Worda & $\frac{53}{47}$ \\
\hline Nkenawe Powar a Loh Co & Alkerists & 100 \\
\hline Nkerted Valloy Eas Coop Corp & $\begin{array}{l}\text { Alkents:s } \\
\text { Ohthoma }\end{array}$ & 98 \\
\hline Antey Criook Ens Coop no & $\begin{array}{l}\text { Allan:as } \\
\text { Loughan }\end{array}$ & 的 \\
\hline 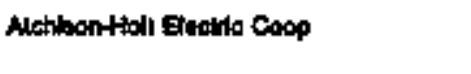 & $\begin{array}{l}\text { Mosour } \\
\text { Moma }\end{array}$ & $\begin{array}{l}72 \\
29\end{array}$ \\
\hline Boyletd Bectio Cocp Ino & $\begin{array}{l}\text { Wacosnain } \\
\text { heliogur }\end{array}$ & 100 \\
\hline E[ Hom County Ees Coop ino & $\begin{array}{l}\text { Mondans } \\
\text { Wronalng }\end{array}$ & 92 \\
\hline Big Hom Furid Eloctse Co & $\begin{array}{l}\text { Wroming } \\
\text { Mtonlang }\end{array}$ & $\underset{\mathbf{2}}{\boldsymbol{\theta}}$ \\
\hline Elgetow Chty of & $\begin{array}{l}\text { Nhmingots } \\
\text { lowt }\end{array}$ & $\begin{array}{l}85 \\
15\end{array}$ \\
\hline Elatk Hin cop & 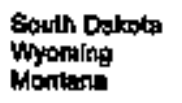 & $\begin{array}{r}01 \\
0 \\
0\end{array}$ \\
\hline
\end{tabular}


Table A1. Electric Utilitie Serving Ultimate Consumers In More Than One State, 1990 (Continued)

\begin{tabular}{|c|c|c|}
\hline $\begin{array}{l}\text { Elentrke } \\
\text { utinty }\end{array}$ & $\begin{array}{l}\text { gtoies } \\
\text { Sarved }\end{array}$ & $\begin{array}{l}\text { Percesm of Toul } \\
\text { Saleiv }\end{array}$ \\
\hline Bhe Ridgs Mountein E M C & $\begin{array}{l}\text { Gewingie } \\
\text { Horth Cerosinn }\end{array}$ & $\begin{array}{l}65 \\
35\end{array}$ \\
\hline Bonnemile Power Admin & $\begin{array}{l}\text { Wastinglon } \\
\text { Oregon } \\
\text { Udonters }\end{array}$ & $\begin{array}{l}75 \\
14 \\
11\end{array}$ \\
\hline Enidger Valley Elec Asan Inc & $\begin{array}{l}\text { thaning } \\
\text { Utalh }\end{array}$ & $\begin{array}{r}\text { B1 } \\
9\end{array}$ \\
\hline Erat Plwer Elactric Coop kre & $\begin{array}{l}\text { South Gargilne } \\
\text { North C.mlinh }\end{array}$ & 1 \\
\hline Cerolina Powiof 8 Light Co & $\begin{array}{l}\text { North Cerollne } \\
\text { south crmorina }\end{array}$ & $\begin{array}{l}62 \\
10\end{array}$ \\
\hline Canoll Electic Coop Cap & $\begin{array}{l}\text { Altunses } \\
\text { Allospouri }\end{array}$ & 91 \\
\hline Cantral Vemanl Pub Serv Cos & $\begin{array}{l}\text { Vommont } \\
\text { New Yort }\end{array}$ & 100 \\
\hline Chittinoogat City of & 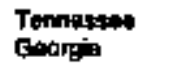 & 5 \\
\hline Chany-Todd Eectric Coopp Inc & $\begin{array}{l}\text { Solth Deplota } \\
\text { Nutrastig }\end{array}$ & 95 \\
\hline Ctibens U'tiats Co & 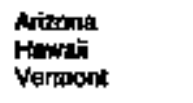 & $\begin{array}{l}59 \\
28 \\
19\end{array}$ \\
\hline Chay County Eloctric Coop Corp & Attansias & 100 \\
\hline Clearualar Panrer Co & $\begin{array}{l}\text { ldeho } \\
\text { Waghington } \\
\text { Oregon }\end{array}$ & $\begin{array}{c}90 \\
0 \\
1\end{array}$ \\
\hline Columbin fiurd Elec Asen me & $\begin{array}{l}\text { Wreshington } \\
\text { Orepon }\end{array}$ & 8 \\
\hline Cobunbus Gectrie coop Ine & $\begin{array}{l}\text { Newn Mearico } \\
\text { Avibont }\end{array}$ & 1 \\
\hline Condinented Dhide G coos unc & $\begin{array}{l}\text { Narr Meexical } \\
\text { Acteons }\end{array}$ & 94 \\
\hline Crals-Botetourt Electutc Coop & $\begin{array}{l}\text { Vhoginia } \\
\text { West Viroginle }\end{array}$ & 6 \\
\hline Ounterland Velley Rurd E G C & $\begin{array}{l}\text { Kontucky } \\
\text { Tonnessyee }\end{array}$ & 100 \\
\hline 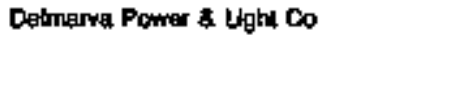 & 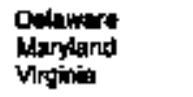 & $\begin{array}{r}73 \\
24 \\
3\end{array}$ \\
\hline Dixal Escalante R E A he & $\begin{array}{l}\text { Whath } \\
\text { Aritonat }\end{array}$ & $\frac{08}{7}$ \\
\hline Duke Power Co & $\begin{array}{l}\text { Morlh Gerr"lne } \\
\text { South Carellnse }\end{array}$ & $\begin{array}{l}69 \\
3 t\end{array}$ \\
\hline Ouncan Valloy Eloc Coop he & Aurzona & 100 \\
\hline El Paso Electric Co & $\begin{array}{l}\text { Texas } \\
\text { Mlow Marteos }\end{array}$ & $\begin{array}{l}79 \\
21\end{array}$ \\
\hline
\end{tabular}


Table A1. Electric Utilitles Serving Ulitmate Consumers In More Than One State, 1993 (Contlnued)

\begin{tabular}{|c|c|c|}
\hline $\begin{array}{l}\text { Eootrk } \\
\text { Utilly }\end{array}$ & stntas & $\begin{array}{l}\text { Pareent of Tolal } \\
\text { 3:10is }\end{array}$ \\
\hline 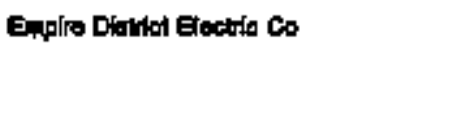 & 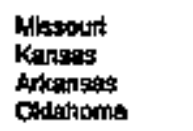 & $\begin{array}{r}67 \\
6 \\
4 \\
3\end{array}$ \\
\hline Empipe Esotho Asor Ino & $\begin{array}{l}\text { Coloratio } \\
\text { Ulah }\end{array}$ & $\$ 6$ \\
\hline Fal Fher fised Eno Coop Inc & $\begin{array}{l}\text { Idaho } \\
\text { Montang } \\
\text { Wyoping }\end{array}$ & $\begin{array}{r}74 \\
22 \\
4\end{array}$ \\
\hline Franoh Broed bion Whober Corp & $\begin{array}{l}\text { North Curotina } \\
\text { Tennosseg }\end{array}$ & se \\
\hline Fns Bat Elachto $\cos$ & $\begin{array}{l}\text { Mlichiogn } \\
\text { Indieng }\end{array}$ & 9 \\
\hline Gakand Power Motn Inc & $\begin{array}{l}\text { Ulath } \\
\text { Afizons }\end{array}$ & $\begin{array}{l}78 \\
21\end{array}$ \\
\hline Coldatwost Elactrio Coop loc & $\begin{array}{l}\text { North Dekola } \\
\text { Wortenta }\end{array}$ & $\begin{array}{l}54 \\
48\end{array}$ \\
\hline Crand Etecule Coop ha & $\begin{array}{l}\text { South Dakola } \\
\text { Momitante }\end{array}$ & 100 \\
\hline Crandeleyen Elactic Cocp & $\begin{array}{l}\text { Wisconsin } \\
\text { Whnols }\end{array}$ & 100 \\
\hline Entanty Electric coop Int & $\begin{array}{l}\text { Massoutro } \\
\text { lowid }\end{array}$ & 98 \\
\hline Eut Stalne Ubrias Co & $\begin{array}{l}\text { Lockistent } \\
\text { Texigs }\end{array}$ & $\begin{array}{l}55 \\
45\end{array}$ \\
\hline H,D Elecilit Coop ins & $\begin{array}{l}\text { South Dakola } \\
\text { IAlnesota }\end{array}$ & 100 \\
\hline Harmon Eactro Aasn tho & $\begin{array}{l}\text { Oklanomn } \\
\text { Toxas }\end{array}$ & 96 \\
\hline Humey Eeato Coop ino & $\begin{array}{l}\text { Nevida } \\
\text { Oregon }\end{array}$ & $\begin{array}{l}78 \\
28\end{array}$ \\
\hline Hnymood Enolita Meraber Coip & $\begin{array}{l}\text { Morth Guriline } \\
\text { Georgla } \\
\text { South Caroling }\end{array}$ & $\begin{array}{r}90 \\
-1\end{array}$ \\
\hline Hend of Lates Glectio Coop & Wheongin & $\begin{array}{l}97 \\
13\end{array}$ \\
\hline Hlophe Extrilc Assor & Colorodo & $\frac{62}{10}$ \\
\hline Whio Fown $C$ & $\begin{array}{l}\text { lolato } \\
\text { Oregan } \\
\text { Nevata }\end{array}$ & 9 \\
\hline indiang turchlogen Fower Co & $\begin{array}{l}\text { tndiagra } \\
\text { Nilchigan }\end{array}$ & 89 \\
\hline intand Powar $4 \omega_{\text {aftu }}$ co & $\begin{array}{l}\text { Whohingion } \\
\text { Idthe }\end{array}$ & 97 \\
\hline 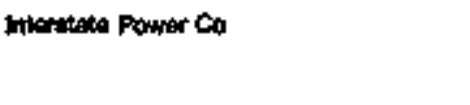 & 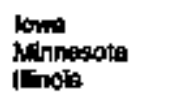 & $\begin{array}{r}80 \\
19 \\
7\end{array}$ \\
\hline lowadlinola Gestelectrid Co & tome & 57 \\
\hline Jellios Chy of & $\begin{array}{l}\text { Tipnipstases } \\
\text { Kentucky }\end{array}$ & $\begin{array}{l}92 \\
30\end{array}$ \\
\hline
\end{tabular}


Teble A1. Electric Utilties Serving Ultimate Consumers in Wore Than One State, 1093 (Continued)

\begin{tabular}{|c|c|c|}
\hline $\begin{array}{l}\text { Elemptr } \\
\text { Ututy }\end{array}$ & $\begin{array}{l}\text { Spples } \\
\text { sonued }\end{array}$ & $\begin{array}{l}\text { Parount of Toted } \\
\text { 8ates }\end{array}$ \\
\hline Kantsina Ciny Pounet \& Ligh Co & $\begin{array}{l}\text { HAlsowound } \\
\text { Kaneses }\end{array}$ & 34 \\
\hline Kentuckly Utities Co & $\begin{array}{l}\text { Kentucky } \\
\text { Virgitia } \\
\text { Teinosides }\end{array}$ & 8 \\
\hline Kootonal Electite Coop he & $\begin{array}{l}\text { Idaho } \\
\text { Wastington }\end{array}$ & 100 \\
\hline La Fiela Elactuc Asgan he & $\begin{array}{l}\text { Colibrado } \\
\text { New Mesalco }\end{array}$ & 100 \\
\hline LaCreak Electre Assan ine & $\begin{array}{l}\text { South Cakole } \\
\text { Mabresshp }\end{array}$ & ed \\
\hline Lea County Goutte Coop he & $\begin{array}{l}\text { Now Moodeo } \\
\text { Texas }\end{array}$ & $\begin{array}{l}\mathbf{6 a} \\
\mathbf{3 a}\end{array}$ \\
\hline Lower Velly Power \& Light tho & $\begin{array}{l}\text { Whorring } \\
\text { htahos }\end{array}$ & $\begin{array}{l}89 \\
12\end{array}$ \\
\hline Lower Yatlowetone RE A Inc & $\begin{array}{l}\text { Monttiong } \\
\text { North Dakole }\end{array}$ & $\begin{array}{l}79 \\
21\end{array}$ \\
\hline Mekengla Electide Coop Inc & $\begin{array}{l}\text { North Dakocta } \\
\text { Wontane }\end{array}$ & 100 \\
\hline Midweol Power Syratemá inc & $\begin{array}{l}\text { lown } \\
\text { South Delkols }\end{array}$ & 99 \\
\hline Mescoult alectrte Coop Inc & 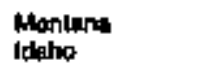 & 89 \\
\hline Wanongahewa Pawar $C_{0}$ & $\begin{array}{l}\text { What Virginle } \\
\text { Ohlo }\end{array}$ & $\theta$ \\
\hline Momtara Putrer Co & $\begin{array}{l}\text { Wontand } \\
\text { Wyoming }\end{array}$ & 100 \\
\hline Mtoon Latce Elontric Aston boc & $\begin{array}{l}\text { Colorido } \\
\text { Utigh }\end{array}$ & 61 \\
\hline Mounlain Enctric Coop Ins & $\begin{array}{l}\text { Twancoswe } \\
\text { Nuprot Caroling }\end{array}$ & $\begin{array}{l}61 \\
40\end{array}$ \\
\hline Whe Whester Power ine & $\begin{array}{l}\text { Noveadn } \\
\text { Utiah }\end{array}$ & 97 \\
\hline HOU fistources Group Ine & $\begin{array}{l}\text { North Dakola } \\
\text { Monkang } \\
\text { Wroming } \\
\text { South Dakote }\end{array}$ & $\begin{array}{c}69 \\
25 \\
11 \\
6\end{array}$ \\
\hline Mavain Jrtbel Whility Atuh & 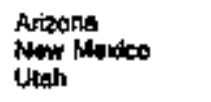 & $\begin{array}{r}80 \\
\$ 1 \\
1\end{array}$ \\
\hline Navopache Eectio Coop inc & $\begin{array}{l}\text { Adrons: } \\
\text { New Mtexice }\end{array}$ & 97 \\
\hline Nabresica Fuble Pownar Clatilet & 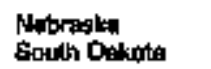 & 90 \\
\hline Naw England Popwer Co & 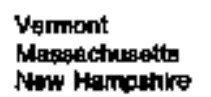 & $\begin{array}{l}42 \\
41 \\
17\end{array}$ \\
\hline
\end{tabular}


Table A1. Electric Utilities Serving Uatimate Consumera In More Than One State, 1993 (Continuted)

\begin{tabular}{|c|c|c|}
\hline $\begin{array}{l}\text { Eroctito } \\
\text { Uillity }\end{array}$ & $\begin{array}{l}\text { Etatiod } \\
\text { Eerwed }\end{array}$ & $\begin{array}{c}\text { Ftritont of Tottis } \\
\text { Sates }\end{array}$ \\
\hline Nobrapa Electic Asen lne & $\begin{array}{l}\text { Whoming } \\
\text { Nobraskip } \\
\text { South Oakota }\end{array}$ & $\begin{array}{l}85 \\
14\end{array}$ \\
\hline Nobled CoOp Esectib & 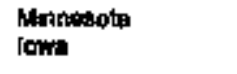 & 100 \\
\hline Nodianay Wonth Elect Coap Las & $\begin{array}{l}\text { Hustotit } \\
\text { lowit }\end{array}$ & $\begin{array}{l}95 \\
15\end{array}$ \\
\hline Alathem Udite Ine & $\begin{array}{l}\text { ldaho } \\
\text { Monhtana } \\
\text { Warkhtigton }\end{array}$ & $\begin{array}{l}71 \\
28\end{array}$ \\
\hline Northam Hatea Power Co & $\begin{array}{l}\text { Woconaln } \\
\text { Mtrigan }\end{array}$ & $\begin{array}{r}97 \\
3\end{array}$ \\
\hline Narthem Stalne Power Co & $\begin{array}{l}\text { Wrinmaots } \\
\text { North Datroln } \\
\text { South Dalsola }\end{array}$ & $\begin{array}{r}6 \\
4\end{array}$ \\
\hline 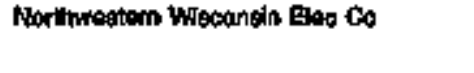 & $\begin{array}{l}\text { Wiscohsin } \\
\text { Minnis:ola }\end{array}$ & 100 \\
\hline Olodenoke furral E Meatiber Corp & Faonta & $\begin{array}{l}59 \\
41\end{array}$ \\
\hline OH:hona Gas 8 Elotila Co & $\begin{array}{l}\text { Oklatioma } \\
\text { Hringons }\end{array}$ & to \\
\hline Dust Tre Power Co & 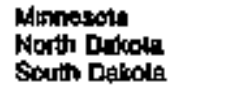 & $\begin{array}{r}59 \\
39 \\
7\end{array}$ \\
\hline Oratks Electrio Coop Cop & $\begin{array}{l}\text { Aukangos } \\
\text { Othahomina }\end{array}$ & $\begin{array}{l}78 \\
22\end{array}$ \\
\hline Pacticonp & 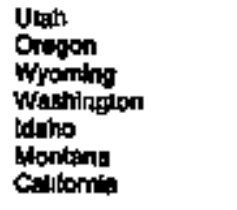 & $\begin{array}{r}32 \\
30 \\
18 \\
9 \\
7 \\
2 \\
2\end{array}$ \\
\hline Panolattanteon telec Cosp lnc & $\begin{array}{l}\text { Tequad } \\
\text { Lotatara }\end{array}$ & $\begin{array}{l}50 \\
50\end{array}$ \\
\hline Pauldigfortmen Elac Coop inc & $\begin{array}{l}\text { Ohio } \\
\text { indianta }\end{array}$ & $\begin{array}{l}78 \\
28\end{array}$ \\
\hline 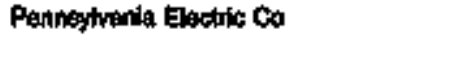 & $\begin{array}{l}\text { Pernofighanla } \\
\text { Non: York }\end{array}$ & 9 \\
\hline Puntagstara Fum Elac Cagp & $\begin{array}{l}\text { Caltotipia } \\
\text { Movado }\end{array}$ & $\begin{array}{r}97 \\
3\end{array}$ \\
\hline Potomad Etion Co & 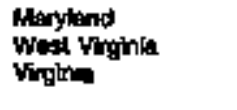 & $\begin{array}{l}88 \\
18 \\
17\end{array}$ \\
\hline Potomac Etectios Power Co & $\begin{array}{l}\text { Moryland } \\
\text { Duptriat of Columbia }\end{array}$ & 69 \\
\hline Powell Ve"ley Electric Coop & $\begin{array}{l}\text { Tennostes } \\
\text { Wiglitic }\end{array}$ & $\begin{array}{l}74 \\
26\end{array}$ \\
\hline
\end{tabular}


Tabte A1. Electric Utistes Serving Ultimate Consumers in More Then One State, 1993 (Continued)

\begin{tabular}{|c|c|c|}
\hline $\begin{array}{l}\text { Jativis } \\
\text { utilly }\end{array}$ & $\begin{array}{l}\text { tinted } \\
\text { Served }\end{array}$ & 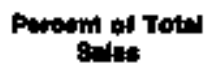 \\
\hline Regt Rbegr Rufal Ekec; Coop Ine & $\begin{array}{l}\text { ldatho } \\
\text { Utan } \\
\text { Nowndin }\end{array}$ & $\begin{array}{l}78 \\
20 \\
2\end{array}$ \\
\hline 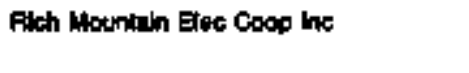 & $\begin{array}{l}\text { Whenses } \\
\text { Oklahomid }\end{array}$ & 6 \\
\hline Fin Grinde Electic Goop Ins & $\begin{array}{l}\text { Tate: } \\
\text { Now Mlodico }\end{array}$ & $\infty$ \\
\hline Rock Counly Electic Coop Assh & $\begin{array}{l}\text { Wloconsin } \\
\text { Dlintise }\end{array}$ & 2 \\
\hline Furfl घhactic Co & $\begin{array}{l}\text { Nebratelp } \\
\text { Wyoming } \\
\text { Codoredo }\end{array}$ & $\begin{array}{r}64 \\
30 \\
7\end{array}$ \\
\hline Shronatogh Vplory Ene Coop & 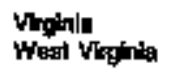 & 5 \\
\hline Sheridan Electric Coop the & $\begin{array}{l}\text { Montonin } \\
\text { North Dalditita }\end{array}$ & 6 \\
\hline Sheridaralatingon Rry Elac Asta & $\begin{array}{l}\text { Wyoming } \\
\text { Mortana }\end{array}$ & 1 \\
\hline 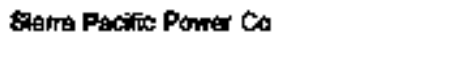 & $\begin{array}{l}\text { Newartid } \\
\text { Callowinit }\end{array}$ & 6 \\
\hline South Kentucisy fiural E C C & Kentuxly & 100 \\
\hline Southast Elootic Coop ins & $\begin{array}{l}\text { Montane } \\
\text { South Oinkole } \\
\text { Wyoudte }\end{array}$ & $\begin{array}{r}97 \\
3\end{array}$ \\
\hline Southwost Artombes E GC & $\begin{array}{l}\text { Arkarsags } \\
\text { Texas } \\
\text { OHdahomal }\end{array}$ & $\begin{array}{r}97 \\
9 \\
1\end{array}$ \\
\hline Southwes Rurd Eles Ason Inc & $\begin{array}{l}\text { Dlankma } \\
\text { Texus }\end{array}$ & 50 \\
\hline Bouthrealem Electik coop lnc: & 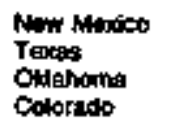 & $\begin{array}{c}100 \\
:\end{array}$ \\
\hline Southwe:pern Electrlo Power Co & $\begin{array}{l}\text { Tocis } \\
\text { Loukglan: } \\
\text { Adkensag }\end{array}$ & $\begin{array}{l}A 0 \\
31 \\
21\end{array}$ \\
\hline 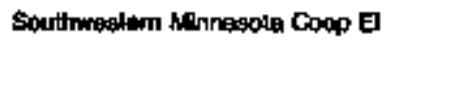 & $\begin{array}{l}\text { Ninnesot: } \\
\text { South Dikital } \\
\text { lown }\end{array}$ & $\begin{array}{r}88 \\
2\end{array}$ \\
\hline Southutedim Futlu Service Co & $\begin{array}{l}\text { Texpes } \\
\text { Now Moxico } \\
\text { Otdohorat } \\
\text { Rentens }\end{array}$ & $\begin{array}{r}77 \\
27 \\
1\end{array}$ \\
\hline Surprist Vallor Eactric Gup & $\begin{array}{l}\text { Gefiemla } \\
\text { Oregen } \\
\text { Nowade }\end{array}$ & $\begin{array}{l}70 \\
21\end{array}$ \\
\hline
\end{tabular}


Table A1. Electric Utufties serving Ulimate Consumers in More Then One State, 1993 (Contunued)

\begin{tabular}{|c|c|c|}
\hline $\begin{array}{l}\text { Eteotito } \\
\text { titidy }\end{array}$ & $\begin{array}{l}\text { Strito: } \\
\text { Sonred }\end{array}$ & $\begin{array}{c}\text { Pureesat of Tocal } \\
\text { Sales }\end{array}$ \\
\hline Tennesees Valloy wuthortly & 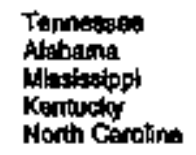 & $\begin{array}{l}50 \\
29 \\
16 \\
12\end{array}$ \\
\hline Teneos New Mexdco Poner Co & $\begin{array}{l}\text { Texag } \\
\text { New Maxico }\end{array}$ & $\begin{array}{l}78 \\
23\end{array}$ \\
\hline Tppat Eectio Power Am & 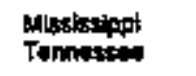 & 89 \\
\hline 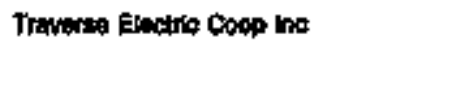 & 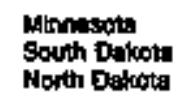 & $\begin{array}{l}70 \\
28 \\
1\end{array}$ \\
\hline THCounhy Elec Hember Corp & $\begin{array}{l}\text { Konively } \\
\text { Tamosteo }\end{array}$ & 56 \\
\hline TitCounhy Electio Coop & $\begin{array}{l}\text { Whnnerota } \\
\text { butat }\end{array}$ & 6 \\
\hline Tis-tate Gectio Mentber Corp & 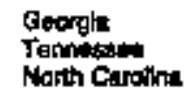 & $\begin{array}{l}70 \\
24 \\
8\end{array}$ \\
\hline Troup Electule Homben Cosp & $\begin{array}{l}\text { Georgle } \\
\text { Alghalina }\end{array}$ & $\frac{69}{2}$ \\
\hline Unton Elodtic Co & $\begin{array}{l}\text { Mllsgourt } \\
\text { linotis }\end{array}$ & $\begin{array}{l}08 \\
12\end{array}$ \\
\hline Utecop Ukated tho & $\begin{array}{l}\text { Nilsogoul } \\
\text { Kant:s } \\
\text { Colorado } \\
\text { West Wirgina }\end{array}$ & $\begin{array}{r}64 \\
22 \\
16 \\
6\end{array}$ \\
\hline Velloy Electio Asan tho & $\begin{array}{l}\text { Hovads } \\
\text { Cofftaris }\end{array}$ & $\frac{6}{2}$ \\
\hline Viplentilo Electuk Coop Ins & $\begin{array}{l}\text { Monitana } \\
\text { blathd }\end{array}$ & 100 \\
\hline Vrghila Electric \& Power Co & $\begin{array}{l}\text { Wrointa } \\
\text { Noith Cartina }\end{array}$ & 6 \\
\hline Wastinglon Water Power co & $\begin{array}{l}\text { Waghingten } \\
\text { milino } \\
\text { Montana }\end{array}$ & 3 \\
\hline Wolle Ford frectif Co & $\begin{array}{l}\text { Novade } \\
\text { What }\end{array}$ & 9 \\
\hline Wasterit Arou Pomer Adrilla & 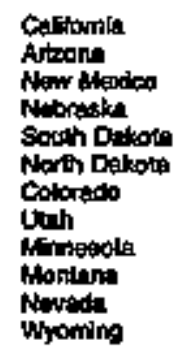 & $\begin{array}{r}7 \\
10 \\
3 \\
3 \\
2 \\
2 \\
1 \\
1 \\
1 \\
1 \\
\vdots\end{array}$ \\
\hline Whactend Elestro Cocos Ino & $\begin{array}{l}\text { Kunses } \\
\text { Colketaly }\end{array}$ & 100 \\
\hline 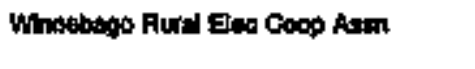 & lowe & $\begin{array}{r}99 \\
1\end{array}$ \\
\hline Wisconain Electio Power co & $\begin{array}{l}\text { Mioconoin } \\
\text { Mishigen }\end{array}$ & $\begin{array}{l}69 \\
11\end{array}$ \\
\hline
\end{tabular}


Table A1. Electrtc Utiltite Serving Utimate Consumers In More Than One State, 1993 (Continued)

\begin{tabular}{|c|c|c|}
\hline $\begin{array}{l}\text { Exctsc } \\
\text { Utilty }\end{array}$ & $\begin{array}{l}\text { Ethet } \\
\text { Sorwed }\end{array}$ & $\begin{array}{l}\text { Purcent of tolv } \\
\text { Salpis }\end{array}$ \\
\hline Whatonsin Puble Servite Carp & $\begin{array}{l}\text { Worconsin } \\
\text { Mechigan }\end{array}$ & 87 \\
\hline Whindac Co & $\begin{array}{l}\text { Wyomitip } \\
\text { Nobraska }\end{array}$ & $\begin{array}{l}84 \\
18\end{array}$ \\
\hline Y.W Elactio Asten the & $\begin{array}{l}\text { Colorado } \\
\text { Anobratk. }\end{array}$ & $\stackrel{56}{2}$ \\
\hline Yempe Valley Eectro Assin ino & $\begin{array}{l}\text { Coloresto } \\
\text { Wyorming }\end{array}$ & $\stackrel{88}{2}$ \\
\hline
\end{tabular}

- Value kast than 0.5 gareant.

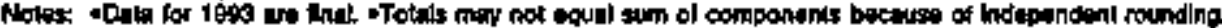

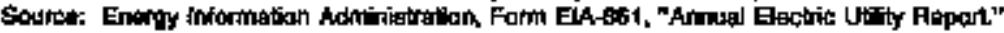


Appendix B

\section{Technical Notes}





\section{Technical Notes}

\section{Souress of Data}

The Electric Sales and Revenue (ESR) is prepared by the Survey Management Division, Office of Coal, Nuclear, Electric and Alternate Fuels (CNEAF), Energy Information Administration (EIA), U.S. Department of Energy. Data published in the ESR are compiled fron the EIA statistical Form EIA-861, "Avinual Electric Utility Report." A brief summary of the form is presented below.

\section{Farm ELA-861}

The Form EIA-861 is a mandatory census of electric utilities in the United States. The survey is used to collect information on power production and sales data from approximately 3,200 electrio utilities. The colllected date are nsed to maintain and update the electric utility frame data base of the EIA. This data base sapports queries from the Executive Branch, Congress, other public agencies, and the general public. Summary data from the Form EIA-861 are also contained in the Electric Power Monthly, the Electric Power Anturi, the Funancial Statistles of Mojor U.S. Invertor-Owned Elec tric Utitities and the Financial Stotistics of Mojor U.S. Publicly Owned Electric Utilitiex These reports present aggregate totala for ekctric utilities at the rational, regional, and State levels by consumer chasses. Data for the four power authorities of the Virgin Islands, American Saroos, Guam, and Puerto Rico are included only in Tables 14 tirongh 17, and not in the summary statistics.

Instrament and Desiga Histary. The Form EIA-861 was implemented in January 1985 to collect data as of year-end 1984. The Pederal Energy Administration Ast of 1974 (Public Law 93-275) deffmes the legislative authority to collect these data.

Data Procesaing. The Form EIA.861 is mailed to the respondents in January to collect data as of the end of the preceding calendar year. The completed forms are to be returned to the EIA by May 1 . These data are manually edited before being entered into the interactive on-line system. Internal edit checks are performed to verify that current data total across and between schedules, and are comparable to data reported the previous year. Edit checks are also performed to compare data reported on the Form EIA-861 with similar data reported on the Forms EIA-826, EIA-412, and Federal Energy Regulatory Commission Form 1. Respondents are contacted by telephone to obtain clarlfication of reported data or to obtain missing data.

Form EIA-861 figures shown in this publication difter somewhat from the surn of the monthly Form EIA-826 figures for sales published in the Electric Power Monthly and in issues of the Electric Power Annval prior to 1988. Both forms use sectorial classifications (residential, commercial, and industrial) based on the usual classification of consumers by the electric utiltites. At the national level, the Form EIA-861 and Form EIA-826 figures corresponded closely (within 3 percent) for all ead-use sectors. Some larger differences were observed at the State level, particulariy for Tennessee, due to inconsistent classification of commercial and industrial data between the Form EIA-826 and the Form EIA-861 surveys.

Differences between the Form ELA-861 and Form EIA-826 itgures can result from the Form EIA-826 sampling procedure and/or from nonsampling ertor in either data collection. One source of nonsantping error in the Form EIA-826 stems from the practice of filing estimates for monthly sales and revenue when the actual figures are not yet available. Although respondents are instructed to file revisions, revisions are not always filed. The respondents are instructed to review their Form EIA-826 figures at the end of the reporting year to ensure consistency with Form EIA.861 tigures. Survey analysts at the EIA compare these data series and attempt to resolve any differences. Nevertheless, some reporting differences remain.

Electric, revenue and sales dath are rounded and roported by electric utilities in thousand dollars and thousand kilowatthours. For this publication the average revenue per unit cost of electricity sold is calculated by dividing total electrits revenute by the total sales of electricity. For Tables 14 through 17 the average revenue per kilowatthour for electric utilities with either Les than $\$ 100,000$ of revenue of less than 1 million kilowathours of seles, is not provided because the significance of the data is not sufficient to make the ratio nteaningfill. 


\section{Qualty of Data}

The CNEAF office is responsibie for routine data improvement and quality assurance activities. All opera. tions in this office are done in accordance with formal standards established by the EIA. These standards are the measuring rod necessary for guality statistics. Data improvement efforts include verification of data-keyed input by automatic computerized methods, editing by subject matter specialists, and followup on nonrespondents. The CNEAF oftice supports the quality assurance efforts of the data collectors by providing advisory reviews of the structure of toformation requirements and of proposed designs for new and revised data collection forms and systems. Once implemented, the actual performance of working data collection systems is also validated. Computerized respondent data files are checked to identify those who fail to respond to the survey. By law, nonrespondents nay be fined or otherwise penalized for not filing a mandatory EIA data form. Before invoking the Jaw, the EIA tries to obtain the regaired information by encouraging cooperation of nonrespondents.

Completed forms received by the CNEAF office are sorted, screened for completeness of reported information, and keyed onto computer tapes for storage and transfer to random access data bases for computer processing. The infomation coded on the computer tapes is manually spot-checked against the forms to certify accuracy of the tapes. To ensure the quality standards established by the EIA, formulas that use the past history of data values in the data base have been designed and implemented to abtomatically check data input for errors. Data values that fall outside the ranges prescribed in the formulas are verified by telephoning respondents to resolve any diserepancies.

\section{Dats Editing System}

Data from the Form EIA-86] are edited on an annual basis using automated systems. The edits include both deterministic checks, in which records are checked for the presence of required fields and their validity, and statistical checks, in which estimation techniques are used to validate data according to their tehavior in the past and in comparison to other current fields.

\section{Conftdentiallity of the Data}

The data collected on the forms used for input to this report are not confideptial.

\section{Formulas}

Average Revenue per KHlowatthour. The average revenue per unit cost of electricity sold is calculated by dividing tota] annual revenue by total arunal sales of electricity.

Calculation Effects of Average Revenue Per Kilowatthour and Sales on Revenwe.

$\Delta r=\underset{\text { Change in average revenue from pear } r_{1} \text { to }}{\text { yor }}$

$\Delta S=$ Change in sales from year, to year $2=S_{2}-S_{1}$

$\Delta R$ a Change in revenue from year ${ }_{1}$ to year $r_{2}=R_{2}-R_{1}$

$\Delta R=\left(S_{2}\right) \Delta r+\left(r_{1}\right) \Delta S$

Effect of change in average reventie on change in

$$
\text { revenue }=\frac{\left(S_{2}\right) \Delta r}{\left(S_{2}\right) \Delta r+\left(r_{1}\right) \Delta S} * 100 \%
$$

Effect of change in sales on change in revenue $=$ $\frac{\left(r_{1}\right) \Delta S}{\left(s_{2}\right) \Delta r+\left(r_{1}\right) \Delta S} * 100 \%$

\section{Rounding Rules for Data}

Given a number with $r$ digits to the left of the decimal and $d+t$ digits in the fraction part, with $d$ being the place to which the number is to be rounded and $t$ being the remaining digits that will be tnuncated, this number is roundeo to $\mathrm{r}+d$ digits by adding 5 to the $(\mathrm{r}+\mathrm{d}+1)$ th digit when the number is positive or by subtracting 5 when the number is negative. The $t$ digits are then trancated at the $(r+d+1)$ th digit. The symbol for a rounded number truncated to zero is (*).

\section{Data Correction Procedure}

The CNEAF office has adopted the following policy with respect to the revision and correction of the Form EIA-861 data published in this report ard made avaitable in a machine format:

- Publication of these data results in their finalization at which point the data base is closed.

- Revisions or corrections are made only in the event a substantial (greater than 1 percent) report. ing change is received or a substantial (greater than 1 percent) error is discovered.

- No revisions will be made without the approval of the Director, Survey Managentent Diviston. 


\begin{tabular}{|c|c|c|c|}
\hline the & & tequfratean & \\
\hline 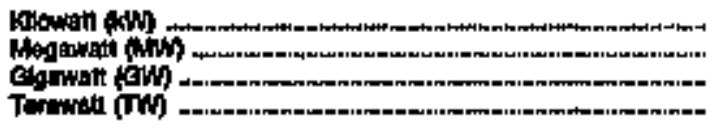 & $\begin{array}{r}1,000 \\
1,000,000 \\
1,000,000,000 \\
1,000,000,000,000\end{array}$ & 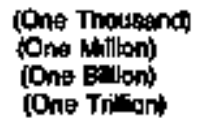 & $\begin{array}{l}\text { Wathos } \\
\text { Watta } \\
\text { Walts } \\
\text { Wetts }\end{array}$ \\
\hline 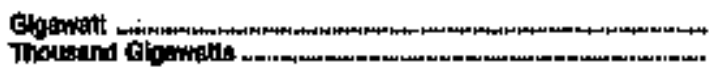 & $\begin{array}{r}1,000,000 \\
1,000,000,000\end{array}$ & $\begin{array}{l}\text { (Cone Mllian) } \\
\text { (Cons ETlon) }\end{array}$ & $\begin{array}{l}\text { Khlowats } \\
\text { Kitowatis }\end{array}$ \\
\hline 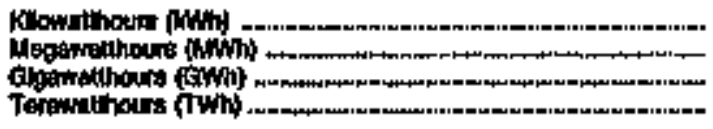 & $\begin{array}{r}1,000 \\
1,000,000 \\
1,000,000,000 \\
1,000,400,000,000\end{array}$ & 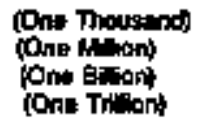 & $\begin{array}{l}\text { Wathours } \\
\text { Wathours } \\
\text { Wathotits } \\
\text { Wabnours }\end{array}$ \\
\hline 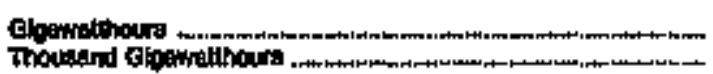 & $\begin{array}{r}1,000,000 \\
1,000,000,000\end{array}$ & 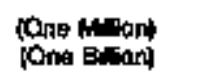 & 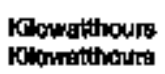 \\
\hline
\end{tabular}

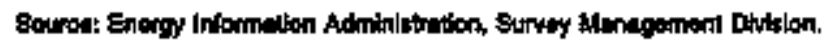

\section{Obtalning Coples of Data or the Form}

Upon EIA approval of the Electric Soles and Revente, the dats become available for public use Computer listings may be obtained by submitting a written request to:

Energy Information Adrninistration, EI-521

Forrestal Building

U.S. Department of Energy

Washington, DC 20585

These data are also available on machine-readabile tapes. Tapes may be purchased by usinte Visa, Master Card, or American Express cards, as well as money orders or checks payahle to the National Technical Information Service (NTIS), Purchasers may also use NTIS and Govefument Printing Office depository ac- counts. To place an order, contact:

National Technical Information Service (NTIS) Ofice of Data Base Services U.S. Department of Commerce 5285 Port Royal Road Springfield, Virginia 22161 (703) $487-4650$

Copies of the form and its instructions may be obtained from the Nattonal Baergy Infortation Center (NETC). The address and telephone number appear below.

National Energy Information Center, EI-23̂1

Energy Information Admimistration

Forrestal Building, Room IF 4048

Washington, DC 20585

(202) $586-8800$ 



\section{Glossary}

Base Bil: A charge calcolated by taking the rate from the appropriate electric rate schedule and applying it to the level of consumption.

Buge Rote: A fixed per kilowatthour charge for electricily consumed that is independent of other charges and/or adjustments.

Block Rate Schedules An electrtc rate schedule with a provision for charging a different unit cost for various increasing blocks of demand or emergy. Usually a redaced price is charged on suoceeding blocks.

Census Difisions: The nine geographic divisions of the United States esteblitshed by the Bureau of the Censua, U.S. Department of Commerce for statistical analysis. The boundaries of Census divisions coincide with State boundaries. The Pacifie Division is subdivided into the Pacific Contiguous and Pacific Noncontignous areas

Clasves of Service: Consumers grouped by similar characteristics in order to be identified for the purpose of setting a common rate for electric service. Usually classified into groups identified as residential, commercial, todustrial and other.

Claga Rate Schedule: An electric rate schedule appilcable to one or more specified classes of service, groups of businesses, or customer uses.

Commercini Sector. Ths commercial sector is generally deftmed as nonmannfacturing business extablishments, inchuding hotels, motels, restaurants, wholesale businesses, retail stores, and health, social, and educa. tional institutions. Electric ntilities may classify com* mercial service as service that includes all consumers whose demand or annual use exceeds some specified limit. The Himit may be set by the utility based on the rate schedule of the utility. Consumers (t.e. farms and irrigation) that the utility has no system for separating into residential, commercial, and industrial classifica- tions, should be classified based on the schedule they most closely resemble. If there is no rate schedule distinction, utilities may define commercial consumers as those having a demand of less than 1000 kilowatis.

Consumer Charge An amount charged pertodically to a consumer for such utility costs as billing and meter reading, without regard to demand or energy consumption.

Cooperative Electric Utility: A group organized under the law jnto a utility company that will generate, transrait, and/or distribute supplies of electric energy to a specified area not being serviced by another utility. Such ventures are generally exempt from the Federal income tax laws. Most electric cooperatives have initially been financed by the Rural Electrification Administration, U.S. Department of Agriculture.

Cost of Service: A ratemaking concept used for the design and development of rate schedules to ensure that the tiled rate schedules recover only the cost of providing the electric service at issue. These costs include operating and raaintenance expenses, depreciation and amortization expenses, and inconse and other taxes found just and reasonable by the regulatory agency for ratemaking purposes plus, in the case of privately owned electric utilities, an allowance for a return on capital (usualiy computed by applying a rate of retum to the rate base). This concept attempts to equate the cost incurred by the utility to the revenue received for the service provided to each of the consumer classes.

Demand (Flectrfe); The rate at which electric energy is delivered to or by a system, part of a system, or piece of expipment, at a given instant or averaged over any designated period of time.

Demand Charget That portion of the consumer's bill for electric service based on the consumer's maximum electroc capacity usage and calculated based on the 
billing demand charges under the applicable rate schedule.

Energy Information Administration (EIA): An independent agency within the U.S. Department of Energy that develops surveys, collects energy data, and does analytical and modeling analysis of energy issues. The Agency must meet the requests of Congress, other elements within the Depariment of Energy, Foderal Energy Regulatory Commission, the Executive Branch, its own independent needs, and assist the general public, or other interest groups, without taking a policy pasition.

Eonnom of Scale: The principle that larger production facilitites have lower unit costs than smaller facilities.

Electric Power Industry: The privately, publicly, federally and cooperatively owned electric utilities of the United States taken as a whole. This includes all electric systems serving the public: regulated investorowned electric utility companies; Federal power projects; State, municjpal, and other governmentowned systems, including electric public vtility districts; electric cooperatives, including generation and transmission entities. Exciuded from this definition are the special purpose electric facilities or systeras that do not offer service to the prblic.

Electric Rate: The price set for a specified anount of electricity in an electric rate schedule or sales contract

Electric Rate Schedule: A statement of the electric rate and the terms and conditions governing its application, including attendant contract terms and condilions that have been accepted by a regulatory body with appropriate oversight authority.

Electric Utility: An enterprise that is engaged in the generation, transmission, or distribution of electric energy primarily for use by the public and that is the unajor power supplier within a designated service area. Electric utilities include privately owned, publicly owned, cooperatively owned, and government-owned (muricipais, Federal ageucies, State projects, and public power districts) systems.

Energy: The capacity for doing work as measured by the capability of doing work (potential entrgy) or the conversion of this capability to motion (kinetic ent- trgy). Energy has several forms, some of which are easily convertible and can be changed to another form useful for work. Most of the world's convertible toergy comes from fossil fuels that are burned to produce hest that is then used as a transfer mediurn to mechapical or other means in order to accompplish tasks. Electrical energy is ustally measured in kilowatthours, while heat energy is usually measured in British thermal units.

Federal Eaergy Regulatory Commission (FERC) A quasi-independent regulatory agency within the $\mathrm{De}$. partment of Energy having jurisdiction over interstate electricity sales, wholesale electric rates, hydroelectric licensing, natural gas pricing, oil pipeline rates, and gas pipeline certification.

Flat and Meter Rate Schedule: An electric rate schedvle consisting of two components, the first of which is a service charge, and the second a price for the exergy consumed.

Flat Denand Rate Sckedule: An electric rate schedule based on billing demand that provides no charge for energy.

Gignotitt (GW): One billion watts.

Gigawatthour (GWh): One billion watthours.

Industrial Sector: The industrial sector is generally defined as incluéing manufacturing, construction, mining, agriculture, fishing, and forestry establishments under Standard Industrial Classification (SIC) codes ol-39. The utility may classify industrial service using the SIC codes, or based on demand or annual usage exceeding some specified limit. The limit may be set by the utility based on its own rate schedule. Sales for consumers (t.e. farms and irrigation) that the utility has no system for separating isto residential, commercial, and industrial classifications, should be classified based on the classification of their rate most closely resembles. If there is no rate schedule distinction, utilities may define industrial consumers as those heving a demand equat to or greater than 1,000 kilowatts.

Interdepartmental Sales: Includtes amounts charged by the electric department at tariff or other specified rates for electricity supplied by it to other utility de. partiments. 
Investor-Onited Blectric Utility: A class of utility that is investor owned and organized as a tax paying business, ustally financed by the sales of securities in the capital market.

Killowatt (kW) One thousand watts.

Kllowatthour (kWh): One thousand watthours.

Megowatt (MW): One million watts.

Megawatthour (MWh): One million watthours.

Other Sector Electricity supplied to public street and highway lighting, other service to public authorities, servics to railroads and and railways, and interdepartmental service.

Power (Clectricoly: An electric measurement unit of power called a voltampere is equal to the product of I volt and 1 ampere. This is equivilent to 1 Watt for a direct eucrent system and a unit of apparent power is separated into real and reactive power. Real power is the work-prodncing part of apparent power that measures the rate of supply of enerpy and is tenoted as kilowatts (KW). Reactive power is the portion of apparent power that does no work and is referred to as kilovars; this type of power must be supplied to most types of magnetic equipment, such as motors, and is supplied by generator or by electrostatic equipment. Voltamperes are usually divided by 1,000 and called kilovoltamperes (kVA). Energy is denoted by the product of real power and the length of time utilized; this product is expressed as kilowatthours.

Public Authorities: Electricity supplied to musicipalities or divisions or agencies of State and Federal governments, usually under specid contracts or agreements that are applicable only to public authorities.

Public Street and Hiphway Lghting Servicen Electricity supplied and services rendered for the purpose of lighting streets, highways, parks, and okher public places; or for trafific of other signal system service, for mupicipalities, or other divisions or agencies of State or Federal governments.

Pohlicly Omed Electric Utility: A class of utility that includes those utilities operated by municipalities, po. litical subdivisions, vtility or power districts, and State and Federal power agencies.

Rallroad and Roilway Electric Service: Electricity supplied to raijrosds and interurban and street railways, for general railsoad use, including the propulsion of cars or locomotives, where such electricity is supplied under separate and distinct rate schedules.

Rate Base: The value of property upon which a utility is permitted to earn a specified rate of return as established by a regulatory authority. The rate base generally represents the value of property used by the utility in providing service and may be calculated by any one or a combination of the following accounting methods: fair value, prodent investment, reproduction cost, or onginal cost. Depending on which method is used, the rate base includes cash, working capital, materials and supplies, and deductions for accumulated provisions for depreciation, contributions in aid of construction, customer advances for construction, accumulated deferred income taxes, and accumalated deferred investuant tax credits.

Residential Sector: The residential sector includes private household establishuents that consume energy prtmarily for space heating, water heating, air conditioning, Itghting, refrigeration, cookding, and clothes drying. The classification of an individual consumer's accoutut, where the use is both residential and connmercia], is based on princlpal use. Apartment houses are included.

Retaits Sales covering electrical energy supplied for resideatial, commercial, and isdustrial end-use purposes. Other small classes, such as agriculture and street lightting, aiso are included in this category.

Rural Blectrificotion Admintstration (REA): A lending agency of the U.S. Department of Agriculture, the REA makes self-liquidation loans to qualified borrowers to finance electrit and telephone service to raral areas. The REA also finances the comstruction and operation of generating plants, electric trensmission and distribution lines, or systems for the furnishing of initial and continued adequate electric fervices to per. sons in rural areas not rectiving central station service.

Sales: The amount of kilowatthours sold in a given period of time; usually grouped by classes of service, such as residential, commercial, industrial, and other. Other sales include public street and highway lighting, 
other sales to public authorities, sales to railroads and railways, and interdepartmental sales.

Schedules A statement of the pricing format of electricity and the terms and conditions governing its applications.

Sersonal Rotas: Different seasons of the year are structured into an electroc rate schedule whereby an electric utility provides service to consumers at different rates. The electric rate schedule usually takes into account denand based on weather and other factors.

Special Contract Rate Schedule: An electric rate schedule for an electric service agreement between a utility and another party in addition to, or independent of, any standard rate schedule.

Special Purpose Rate Schedule; An electric rate schedule limited in jts application to some particulat purpose or process within one, or more than one, type of industry or business.

Tariff: A published volume of rate schedules and general terms and conditions under which a product or service will be supplied.

Time-of-Day Rater The rate charged by an electric utility for service to vartons classes of customers. The rate reflects the different costs of providing the service at different times of the day.

Watt: The electrical unit of power. The rate of eneryy transfer equivalent to 1 ampere lowing under a pressure of 1 volt at unity power factor.

Watthour (Wh): An electrical energy unit of measure equal to 1 watt of power supplied to, or taken from, ant tectric circtuit steadily for 1 hour. 